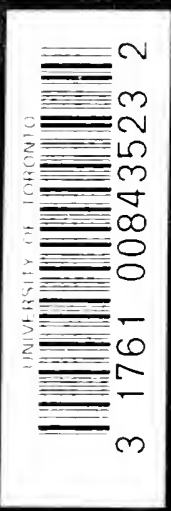



Digitized by the Internet Archive in 2007 with funding from Microsoft Corporation 




\title{
BIOCHEMICAL CATALYSTS IN LIFE AND INDUSTRY
}

\section{PROTEOLYTIC ENZYMES}

\author{
BY \\ JEAN EFFRONT \\ PROFESSOR IN THE NEW UNIVERSITY \\ AND DIRECTOR OF THE INSTITUTE OF FERMENTATIONS \\ OF BRUSSELS
}

TRANSLATED BY

SAMUEL C. PRESCOTT

PROFESSOR OF INDUSTRIAL MICROBIOLOGY

MASSACHUSETTS INSTITUTE OF TECHNOLOGY

ASSISTED BY

CHARLES S. VENABLE

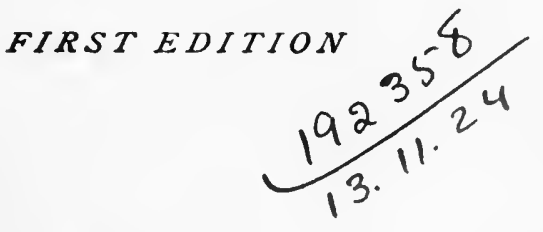

NEW YORK

JOHN WILEY \& SONS, INC.

LONDON: CHAPMAN \& HALL, LIMITED

1917 


\section{Copyright, 1917}

BY

SAMUEL C. PRESCOTT

\section{Stanbope press}

F. H. GILSON COMPANY BOSTON, U.S.A. 


\section{TRANSLATOR'S NOTE}

The present volume occupies in a measure the position of companion volume to the "Enzymes and Their Applications," which was published several years ago, and which I had the pleasure of translating. Dr. Effront has done me the honor to request that $I$ render this volume also into English. In doing this I have attempted to make the translation a fairly literal one and to set forth the meaning of the author as accurately as possible. The only changes made by the translator are in the cutting off of insignificant figures in certain of the tables and in the occasional use of names which have gained wide currency in the literature of the subject in English and seemed to be, therefore, more suitable than the literal translation of the French words.

The translator acknowledges with thanks the great assistance of two of his former students. Dr. Charles S. Venable has rendered invaluable service in the preparation of the material and Mr. Elmer H. Heath, Jr., has ably assisted in the reading of the proofs.

SAMUEL C. PRESCOTT.

Massachusetrs Institute

or Technology,

Seplember, 1917. 



\section{TABLE OF CONTENTS.}

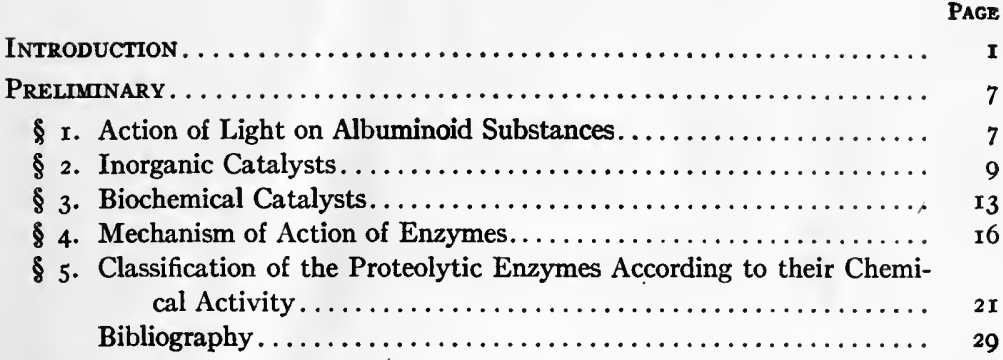

PART I.

\section{COAGULATING ENZYMES}

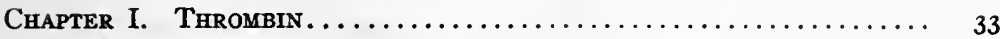

$\S$ I. Preparation of Thrombin and Fibrinogen ................ 33

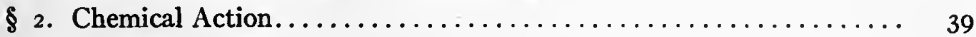

3. Theory of Coagulation of Blood ..................... 42

4. Blood Plasmas not Spontaneously Coagulable............... 46

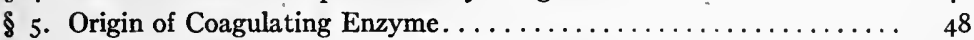

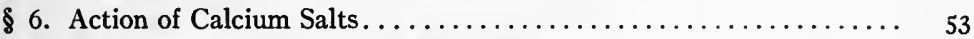

\$. Prothrombin. Conditions for the Transformation of the Pro-enzyme

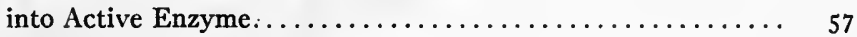

8 8. Phenomena of Anti-coagulation.............................. 65

8. General View of the Phenomena of Coagulation of Blood ........ 79

§ro. Analysis of Blood: Thrombin and Fibrinogen............. $8 \mathrm{r}$

Bibliography on Thrombin........................ $8_{3}$

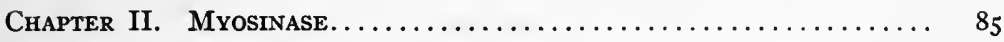

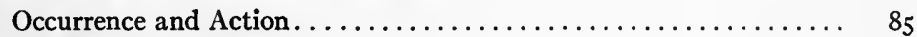

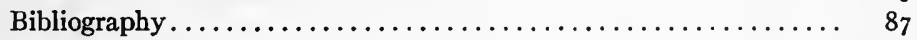

Chapter III. Rennet.............................. 88

$\S$ I. Distribution. Preparation. Properties................ 88

2. Influence of Temperature ......................... 93

3. Influence of the Reaction of the Medium ................ 100

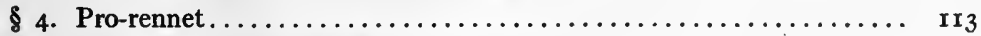

5. Law of Action of Rennet..................... $\mathrm{Ir}_{7}$ 
\$ 6. The Identity of Different Rennets.................. 123

$\S 7$. Chemical Action of Rennet......................... $3_{30}$

8. Anti-rennet.............................. 39

§ 9. Determination of Rennet....................... $\mathbf{1 4 2}^{2}$

Bibliography on Rennet..................... 145

\section{PART II.}

\section{PEPSIN}

Chapter I. . . . . . . . . . . .

\& 1. History. Distribution. Preparation. Chemical Composition.... I5I

$\S$ 2. Dissolving and Peptonizing Powers of Pepsin ............ I58

$\S$ 3. Effect of Temperature......................... I6 $\mathbf{I}$

$\S$ 4. Effect of Light and Shaking. Adsorption of Pepsin.......... 167

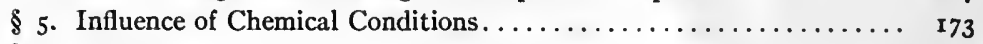

$\S$ 6. Pepsinogen ................................... 88

$\S$ 7. Law of Action of Pepsin ....................... r 89

8. The Possible Identity of Pepsin and Rennet............... I99

§ 9. Chemical Activity of Pepsin..................... 206

§ro. Study of Different Albumoses by the Method of Fractional Precipitation........................... 2 ro

§Ir. Peptones and Complex Non-biuretic Compounds Resulting from the Action of Pepsin: "Formaldehyde Nitrogen" and Amido Nitrogen........................... 237

§1 2. Anti-pepsin ................................... 244

\$13. Determination of Pepsin........................ 249

\$1 4. Gastric Juice.................................. 256

Bibliography on Pepsin...................... ${ }_{26} 6 \ldots$

Chapter II. Reversible Action of Enzymes. Synthetic Action of

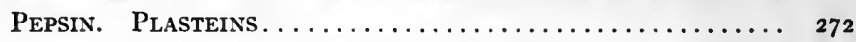

Bibliography on Reversible Action of Enzymes........... 289

PART III.

\section{TRYPSIN}

Chapter I. Pancreatic Trypsin....................... 293

$\S$ r. Distribution and Preparation....................... 293

$\S$ 2. Characteristic Products of Tryptic Digestion................ 298

3. Effect of Temperature and Other Physical Agents............ 300

$\S$ 4. Influence of the Reaction of the Medium............... 304

5. Influence of Antiseptics........................ 309

$\S$ 6. Law of Action of Trypsin............................. $3^{13}$

8. Chemical Activity of Trypsin...................... 316

8. Methods of Analysis for Tryptic Products............... 32 I 
9. Progress of Tryptic Peptonization.................... 328

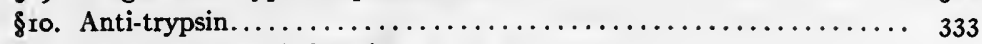

\$1r. Determination of Trypsin......................... 339

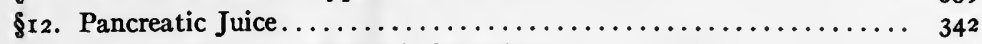

\$r3. Excitation of the Pancreatic Secretion................. 347

\$r4. Zymogen and Trypsinogen. Enterokinase............... $35 \mathrm{r}$

815. Activation of Pancreatic Juice by Calcium................ 355

8r6. Other Enzymic Properties of the Pancreatic Juice............ 359

Bibliography on Pancreatic Trypsin.................. 364

Chapter I. (Continued) Functioning of the Digestive Glands..... 371

\&. Variations in the Secretion of the Gastric and Pancreatic Glands... 37 1

2. Mechanism of Secretion....................... 377

3. Chemical Influences on the Gastric Secretion.............. 386

4. Chemical Influences on the Pancreatic Secretion............. 396

5. Nutrition from the Secretorial Point of View............. 401

Bibliography on Functioning of the Digestive Glands......... 404

Chapter II. Trypsins of Various Origns.................. 406

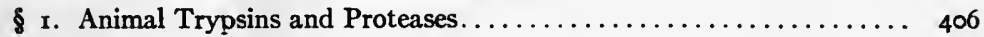

8. Vegetable Proteases............................ 408

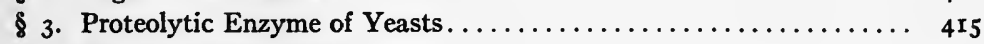

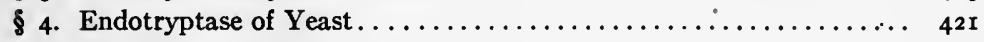

5. Bacterial Proteases.............................. 429

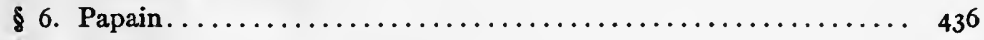

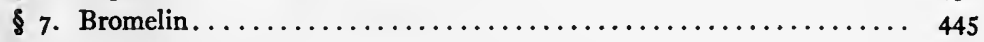

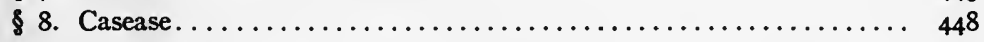

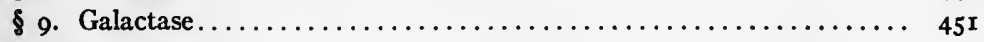

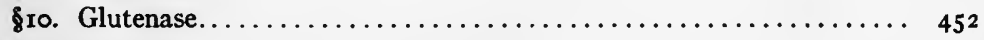

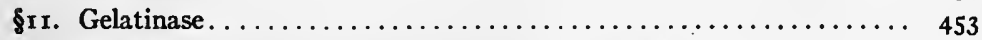

Bibliography on Different Trypsins and Proteases.......... 457

Chapter III. Antigens. Antibodies. Complement............ $46 \mathrm{r}$

Products of Vital Defense and of Immunization. . . . . . . . .

Bibliography on Antibodies...................... 484

\section{PART IV.}

\section{EREPSINS}

Chapter I. Intestinal Erepsin $\ldots \ldots \ldots \ldots \ldots \ldots \ldots \ldots \ldots \ldots \ldots \ldots, 487$

I. Preparation and Properties of Erepsin. .............. 487

§ 2. Actions Exerted by Proteolytic Enzymes upon Each Other....... 498

Bibliography on Intestinal Erepsin................. 500

Chapter II. Peptolytic Enzymes...................... 502

Preparation and Properties..................... 502

Bibliography on Peptolytic Enzymes................ 512 
Chapter_III. Enzymes of the Nucleo-protens.............. 513

Preparation and Properties...................... 513

Bibliography on Enzymes of the Nucleo-proteins........... 52I

Chapter IV. Arginase.............................. 522

Properties............................ 522

Bibliography on Arginase $\ldots \ldots \ldots \ldots \ldots \ldots \ldots \ldots \ldots \ldots \ldots \ldots, 524$

Chapter V. Creatino-creatase, Creatase, Creatinase......... 525

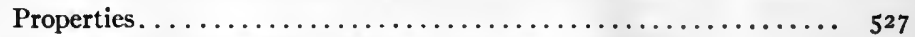

Bibliography on Creatino-creatase, Creatase, Creatinase. ...... 527

PART V.

\section{AMIDASES}

\&. Historical. Distribution. Properties................ 53 I

8. Chemical Activity of Amidases..................... 539

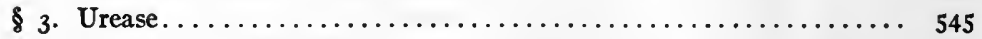

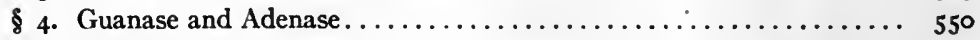

5. Butyro-amidase.............................. 554

Bibliography on Amidases. . . . . . . . . . . . . . . . . $55^{8}$

PAR'T VI.

\section{APPLICATIONS}

I. Officinal Pepsin: Its Therapeutic Use. Commercial Peptones..... 563 Bibliography................................ 573

§ 2. Chemical and Enzymic Exploration of the Stomach.......... 574 Bibliography............................ $5_{582}$

8. Role of Proteolytic Enzymes in the Conservation of Grains and Flour,

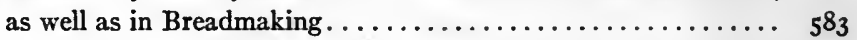
Bibliography............................. $55^{87}$

\& 4. Role of Proteolytic Enzymes in Brewing............... $5 \ldots \ldots$ Malting. . . . . . . . . . . .

Treatment in the Kiln....................... 591

Brewing ....................... 59

Fermentation. ......................... 593

Impregnation with Hops......................... $6_{02}$

Preservation. ............................... $6_{02}$

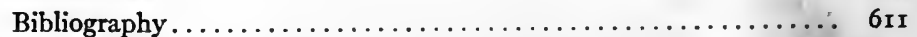

§ 5. Role of Proteolytic Enzymes in Grain Distillation........... 6r 2

Manufacture of Compressed Yeast.................... 616

8 6. Enzymes in the Cheese Industry..................... 620

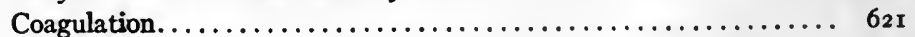

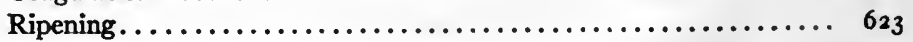


Page
Page

Examples............................... 628

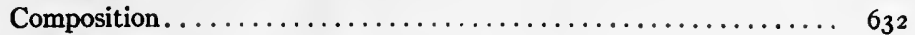

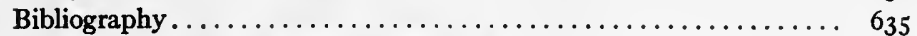

8 7. Role of Proteolytic Enzymes in Tanning ................ $66_{36}$

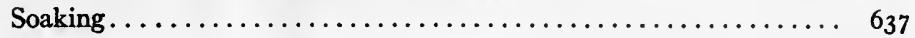

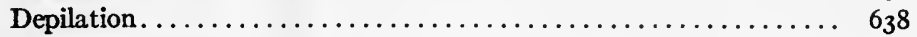

Excrement Tubs. . . . . . . . . . . . . . .

Bibliography ............................ 647

8. Rôle of Amidases in the Formation of Petroleum . . . . . . . . . 648

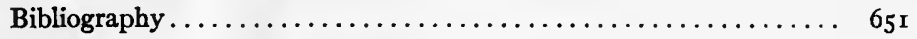

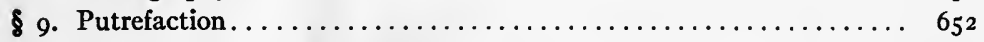

Chemical Activity in the Course of Putrefaction . . . . . . . . . 654

Variation in the Products Which Appear. . . . . . . . . . . 662

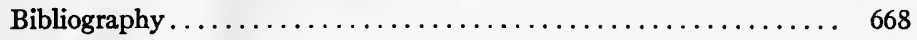

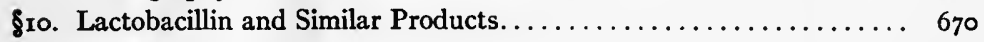

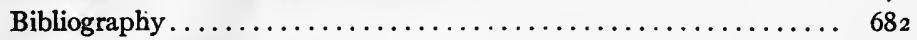

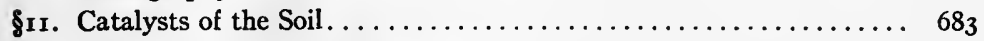

Bacterial Flora of the Soil. . . . . . . . . . . . . . . . . . 683

Chemical Reactions in the Soil. . . . . . . . . . . . . . 684

Ammoniacal Fermentation of the Soil. ............... 688

Nitrification. ........................... 690

Assimilation of Atmospheric Nitrogen. . . . . . . . . . . . $66_{92}$

Denitrification in the Soil. . . . . . . . . . . . . . . . . . 697

Chemical Nitrogenous Fertilizers and Green Fertilizers . . . . . . . . 700

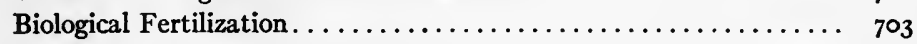

Future of Biological Fertilization. . . . . . . . . . . . . . 707

Bibliography ........................... 709

8r2. Recovery of Nitrogenous Wastes................... 7 ro

Bibliography ......................... 7 r8

8r3. Artificial Nitrogenous Foods. Nutritive Value of Products of Advanced Hydrolysis of Protein Substances............ 7 I8

Synthesis of Albuminoid Substances in the Organism......... 718

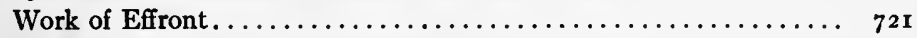

Work of Abderhalden on the Assimilation of Nitrogenous Substances. $\quad 729$

Bibliography ............................. ${ }_{73}^{8}$

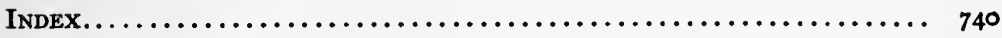





\section{BIBLIOGRAPHICAL ABBREVIATIONS.}

Amer. Journ. Physiol. The American Journal of Physiology.

Ann. inst. Past . . . Annales de l'Institut Pasteur.

Apothek. Zeit. . . . Apotheker-Zeitung.

Arch.f. Anat. u. Phys. Archiv für Anatomie und Physiologie (Waldeyer u. RUBNER). Physiologische Abteilung.

Alti. della Accad. . . Atti della Reale Accademia dei Lincei. Rendiconti (Roma).

Ber. d. Deuts. Ch. Ges. Berichte der Deutschen Chemischen Gesellschaft.

Bioch. Centb. . . . Biochemisches Zentralblatt.

Bioch. Zeits. . . . . Biochemische Zeitschrift.

Bull. Acad. Belg. . - Bulletin de l'Académie royale de Belgique.

Bull. Soc. Chim. . . Bulletin de la Société Chimique de France.

Chem. Zeit. . . . . Chemiker-Zeitung.

C. $R$. . . . . . . Comptes Rendus de l'Académie des Sciences (Paris).

Deuts. med. Woch . . Deutsche medizinische Wochenschrift.

Dingler's . . . . . Dingler's Polytechnisches Journal.

Hofm. Beitr. . . . . Hofmeister's Beiträge zur chemischen Physiologie und Pathologie.

Landw. Vers. Stat . . Die landwirtschaftlichen Versuchs-Stationen.

Maly's Berichte . . . Maly's Jahresberichte für Tierchemie.

Mon. Scient . . . Moniteur Scientifique Quesneville.

Pflïg. Arch. . . . . Archiv für die Gesamte Physiologie des Menschen und die Tiere (PFLüger).

Proc. Roy. Soc. . . . Proceedings of the Royal Society (London).

Soc. Biol. . . . . Comptes Rendus de la Société de Biologie.

Virch. Arch. . . . . Virchow's Archiv für Pathologie.

Zeits.f. Hyg. u. Infekt. Zeitschrift für Hygiene und Infektionskrankheiten.

Zeits. f. physiol. Chem. Hoppe Seyler's Zeitschrift für physiologische Chemie.

The other signs are sufficiently clear. 



\section{BIOCHEMICAL CATALYSTS IN LIFE AND INDUSTRY}

\section{INTRODUCTION.}

The successive transformations of living matter which constantly occur during the greater part of the cycle accomplished in nature by the elements which compose it offer a limitless field to the researches of investigators. Unfortunately, the reactions which take place in the organism present a considerable complexity, resulting in great part from the very great diversity of chemical bodies which occur in the living cell. The analysis of organic tissues reveals to us, in fact, the presence, not only of four constant elements, carbon, hydrogen, oxygen, and nitrogen, united in manifold combinations, but also of a certain number of other simple bodies which, although often existing only as traces, play nevertheless an important rôle in the functioning of the cell.

Formerly, before the difficulty of explaining biological phenomena, it was considered that living beings possess a special vital energy, and to this alone was attributed the initial cause of all the transformations observed in the organism. The effort of the modern biologist is to free science so far as possible from this metaphysical conception and to bring it to a more positive theory by showing that most vital manifestations are regulated by purely physical and chemical laws. The task is vast and difficult, and the moment has certainly not arrived for writing a book of physiology based solely on physico-chemical reactions. But science progresses, and the mystery which still envelops vital manifestations is being gradually made clear. From day 
to day is disclosed the mechanism of transformations which previously had appeared to obey only unknown forces, in short, the number of phenomena which can be explained only by vital activity constantly diminishes.

Particularly, if we consider only the transformations relative to the three principal elements of the organism, such as the assimilation by the plant of carbon, hydrogen, and oxygen, and their condensation into carbohydrates (glucose, starch, cellulose, and others), the origin of fats, etc., the decomposition of these products of vegetable synthesis into simpler materials, and, finally, their return to the inorganic states, water and carbonic acid, it is found that our knowledge represents a certain number of definitely acquired results, in spite of certain points still obscure. If, however, we pass to quaternary substances, we are obliged to admit that the definite ideas which we possess in regard to them are much less extended. Recently, without doubt, important work has been done, and we know, if not exactly, at least in a very general way, the constitution of albuminoid matter, the seat and support of life. It is known that, by its decomposition under the influence of the agents of digestion, it gives rise to a whole series of bodies, the nature of which it has been possible to establish, and even to determine approximately. Finally, it has been shown that the numerous derivatives of protein degradation, substances which can moreover be obtained by a purely chemical method, are susceptible, when united, of being absorbed in their turn by the organism and are consequently capable of providing the nitrogenous food for animals.

These are important and very encouraging results, but there still remains much to be accomplished. In answer to a certain number of questions one is always reduced to giving out hypotheses. In what does living albuminoid matter differ from dead albuminoid matter? How is the nitrogenous substance secreted in the plant or in the animal organism? In particular, how is the nitrogen, drawn from the great atmospheric reservoir by the symbiotic bacteria of Hellriegel and Willfarth, or assimilated in the form of nitrates, ammonia, or of vegetable mould, 
transformed within the plant into protein substance, and how is the albumin, absorbed by the animal, changed into muscular tissue? Finally, by what mechanism is the albuminoid adapted to the cell which shelters it? We still seek the key to the enigma, or, rather, see ourselves obliged to abandon the solution of these puzzling problems to the occult influences of vital activity.

Thus, according to the logic of things, it is especially in the analytical direction that progress has been noticeable. The chemistry involved in the digestion of the different food materials, as well as in the dissolving and utilizing of the reserves contained in the germinating seed; the mechanism according to which the coagulation of various fluids of animal or vegetable origin take place; the way in which the transformation of a certain number of substances of the organism is effected, are now known to us in their general outlines. We know that all these reactions are brought about under the influence of marvelous agents, which, by a remarkable relation of cause and effect, are accurately secreted by the living cells concerned in these transformations. These biochemical reagents have been isolated and studied apart. It has been found that all, in spite of their individuality, have a certain number of common characteristics, resulting from their sensitiveness to heat, and to the reaction of the medium. Their fundamental property, however, is the ability to produce, even in very small amounts, very large effects, without having their own matter disappear, for example, in themselves combining in a definite manner with one of the products of the transformation.

This disproportion between cause and effect, as well as this immutability, which is found with all these active substances, justifies their being gathered into a well-characterized group. To this class of bodies has been given various names, such as soluble ferments, diastases, or better still, enzymes. A detailed study shows that all these substances offer a close analogy to the so-called catalysts of inorganic chemistry. It therefore appears to us that the term biochemical catalysts would be a most suitable 
one for designating these special substances, which play so dominating a rôle in the cell life.

Their diversity is very great. Some are the normal products of the organism. Proceeding by means of cleavage or oxidation, they regulate the essential manifestations of life, i.e., the functions of nutrition and of respiration. But, in connection with these catalysts of decomposition there have been observed in some cases reversed reactions - that is to say synthetic reactions - sufficiently obvious to permit the generalisation of the reversibility of these catalytic effects and to regard the formation of original material and the reconstruction of living tissues as being likewise the result of this phenomenon of catalysis. In addition to these catalysts, there are others which represent rather the products of organic defense, and of which the proportion present increases under the influence of various causes: maladies, introduction of foreign substances, etc. These are the antibodies and the immunizing substances.

All, so far as chemistry yet permits us to say, have compositions similar to those of the albuminoids. However, they are accompanied by mineral matter whose presence has been recognized, in certain cases, as being useful, or even necessary - as, for example, manganese in the process of oxidation, or calcium in the coagulation of blood - so that one ends by asking if the rôle of these substances, in vital manifestations in general, is not even a greater one than has hitherto been supposed. The constant presence of iron in the hemoglobin, of magnesium in the chlorophyll, or of iodine in the thyroids, is perhaps an indication of unknown catalytic action, which would thus explain the physiological importance of these substances, from the points of view of respiration, the assimilation of carbon dioxide and the normal functioning of the organism, respectively.

It is seen that a complete account of the biochemical catalysts would have to be a veritable treatise on biological chemistry and physiology. The author has limited his task to a less comprehensive plan, and has occupied himself exclusively with the catalysts for nitrogenous substances. The chemists will 
find in this book data on all the proteolytic enzymes. The action of rennet on milk, the action of pepsin and the various trypsins, erepsins and amidases, of vegetable or animal origin, on albuminoid matter, and the study of the products which result have all been carefully described. Most of the facts cited have been verified again by us, and many of the numerical data are taken from experiments especially conducted at our laboratory. We have likewise controlled the methods of analysis which serve as a measure of the activity of these various enzymes. The study of the coagulation of blood has been made the subject of a particularly important chapter, benefiting from recent acquisitions, very many publications having appeared recently on the subject. Finally, we have described the different antienzymes known as antirennet, antipepsin, etc., and have shown how the formation of all these substances results from the same principle, that of vital defense, a principle upon which rest all the phenomena of immunization.

The chemist will find, in addition to these references of a more general nature, an exposition of certain applications of enzymic reactions to industry. The important place which the biochemical catalysts will take in the near future is just beginning to be realized. We have therefore passed rapidly over in review the various branches of industry in which proteolytic enzymes take part. Bread making and the conservation of flours, the manufacture of compressed yeast and that of the nutritive substances for yeast and other micro-organisms, brewing, cheese making, tanning, the recovery of by-products from distilleries, etc., has given us an opportunity to show the more or less important rôle played by the enzymes for nitrogenous substances.

From another point of view, we think that this volume may be consulted with interest by physicians who wish to study the phenomena of digestion, as well as the mechanism of gastric and pancreatic secretions. They will see, in particular, how one can apply present analytical methods to the products of proteolysis in order to follow the course of digestion and in an 
efficient manner to explore the stomach. They will find, furthermore, information on the pharmaceutical applications of pepsin and trypsin, as well as the commercial products derived from them. Finally, the chapter on the nutritive value of the derivatives of the advanced hydrolysis of nitrogenous substances contains numerical data worthy of being considered. 


\section{PRELIMINARY.}

\section{$\S \mathrm{I}$.}

\section{Action of Light on Albuminoid Substances.}

A solution of sterilized peptone undergoes fundamental transformation when it is exposed, at ordinary temperature, to the action of light. The biuretic products disappear gradually, amino-acids being first formed, which then decompose into ammonia and volatile acids. These bodies, in their turn, slowly oxidize, to form nitrates and carbon dioxide as final products. This phenomenon amounts to a decomposition of the most complex nitrogenous substance, that of albuminoid matter. It is a disintegration analogous to that which takes place in nature when any protein is left to itself: under the action of the numerous micro-organisms characteristic of putrefaction, the substance becomes soluble, decomposes, and is transformed into successively simpler bodies, of which the ultimate forms are $\mathrm{CO}_{2}, \mathrm{H}_{2} \mathrm{O}, \mathrm{NH}_{3}$, and $\mathrm{N}_{2}$.

What is the initial cause of these phenomena? Observing that the peptone solution remains intact when protected from the action of light, we are at once led to relate the transformations noticed to the photochemical influence of the solar rays and to classify these reactions in the same group as those studied by D. Berthelot, which are directly caused by the action of the ultra-violet rays.

However, in studying more closely the mechanism of the degradation of peptone, it is observed that the effect produced, while being due to the action of the light, is not an immediate result of the same, but that still another very important factor is concerned. In fact, the albuminoid solution exposed to the sun gives a positive reaction with iodo-starch in iron sulphate 
solution, thus revealing the presence of hydrogen peroxide. The intensity of the reaction is moreover subject to great variation. At certain times it is very strong; at others, it disappears, to reappear later. There is, then, in the liquid a successive formation and destruction of hydrogen peroxide: the presence of this unstable body, capable of acting both as an oxidizing and as a reducing agent, allows us to suppose that it is this which intervenes directly in the hydrolysis of the albuminoids, the light serving only to assure its formation.

In fact, experiment fully confirms this opinion, for solutions of peptone with the addition of $\mathrm{H}_{2} \mathrm{O}_{2}$ act in the dark in the same manner as the solutions without $\mathrm{H}_{2} \mathrm{O}_{2}$ exposed to the light. The hydrolysis occurs, in the first case, even more easily than in the second, and the resulting products are identical in the two experiments. These facts lead to the conclusion, that the autolysis of the peptone in the presence of light is caused by the hydrogen peroxide formed under photochemical influence, a synthesis known to be possible under these conditions.

But let us go further and ask in what manner the hydrogen peroxide reacts with the albuminoid substance. We shall see that all the chemical change produced in the peptone solution in the course of its disintegration consists in a hydrolysis. The albuminoid molecule, by addition of water, divides into less complex products, which themselves, by hydrolysis, are farther split, yielding amino-acids, and finally volatile acids and ammonia.

The first idea which occurs, then, is that $\mathrm{H}_{2} \mathrm{O}_{2}$, in decomposing, furnishes a molecule of water in the nascent state, a state consequently endowed with a special activity, and that it is this water that produces the hydrolysis. This hypothesis demands, as we shall see in a later chapter, the interaction of 38 grams of water, that is to say of $7 \mathrm{I}$ grams of $\mathrm{H}_{2} \mathrm{O}_{2}$, for the complete conversion of 100 grams of albumin to the ammonia form. To be sure, it is granted that in the liquid submitted to autolysis there is a liberation of oxygen, indicating a decomposition of $\mathrm{H}_{2} \mathrm{O}_{2}$. It is indeed for this reason that in the course of the process of disintegration carried on in the dark, it is necessary to add $\mathrm{H}_{2} \mathrm{O}_{2}$ 
little by little, and not all at once. However, the quantity of water fixed during hydrolysis is always considerably more than the quantity of $\mathrm{H}_{2} \mathrm{O}_{2}$ used in the reaction. In careful experiments, one often observes a relation of 8 to I, that is to say that eight times as much water enters into the combination as that which $\mathrm{H}_{2} \mathrm{O}_{2}$ can furnish.

These facts in no way justify the preceding hypothesis. This is all the less probable, since with a given quantity of $\mathrm{H}_{2} \mathrm{O}_{2}$ the minimum quantity that is undecomposed in the liquid during a certain time, one can cause a very marked hydrolysis, and since there is no direct relation between this quantity of $\mathrm{H}_{2} \mathrm{O}_{2}$ employed and the effect produced. Thus, there is a disproportion between the cause and the effect, an effect which is moreover not instantaneous, but increases with the duration of the action. Evidently, the hydrogen peroxide does not react through the molecule of water which it may bring, but through its mere presence.

\section{$\S$ II. \\ INORganic Catalysts.}

The reactions which are produced by hydrogen peroxide in an albuminoid solution come under the category of "actions of presence," to which Berzelius first drew attention. At the present time we know of a large number of transformations that are due to catalytic power. They are produced by the action of various substances, which, nevertheless, do not belong to a definite chemical class. Among these are, according to circumstances, water, acids, carbon compounds, metals, oxides, and salts. The following are some of the chemical actions which catalysts may bring about.

I. Hydrolysis. Ex.: the transformation of saccharose into glucose and levulose, or that of starch into glucose, both under the influence of dilute acids.

2. Oxidation. Ex.: the combination of $\mathrm{SO}_{2}+\frac{1}{2} \mathrm{O}_{2}$ in pres- 
ence of platinum-sponge, or again the transformation of $\mathrm{NH}_{3}$ into $\mathrm{HNO}_{3}$, by the aid of the same agent.

3. Direct combination. Ex.: $\mathrm{N}_{2}+3 \mathrm{H}_{2}$ in the presence of a metallic catalyst.

4. Condensation. Ex.: $3 \mathrm{C}_{2} \mathrm{H}_{2}=\mathrm{C}_{6} \mathrm{H}_{6}$, in the presence of nickel above $180^{\circ}$.

5. Decompositions. Ex.: $\mathrm{C}_{2} \mathrm{H}_{2}=2 \mathrm{C}+\mathrm{H}_{2}$, in the presence of nickel at high temperature.

6. Reversible reactions. Ex.: $2 \mathrm{CO} \rightleftarrows \mathrm{CO}_{2}+\mathrm{C}$ (with a metallic oxide), or again $\mathrm{CH}_{3} \mathrm{CHO}+\mathrm{H}_{2} \rightleftarrows \mathrm{CH}_{3} \mathrm{CH}_{2} \mathrm{OH}$ (with nickel).

In many reactions, it is the water which plays the rôle of the catalyst. It is thus that, $\mathrm{H}_{2}+\mathrm{Cl}_{2}, \mathrm{H}_{2}+\frac{1}{2} \mathrm{O}_{2}, \mathrm{NO}+\frac{1}{2} \mathrm{O}_{2}, \mathrm{NH}_{3}$ $+\mathrm{HCl}$, etc., do not combine under the conditions under which they are ordinarily made, if these substances are kept entirely free from moisture.

According to Moissan, dry carbon dioxide and pure potassium hydride combine at about $54^{\circ}$ to give $\mathrm{HCO}_{2} \mathrm{~K}$. But a trace of moisture considerably lowers the temperature necessary for the reaction, since then this reaction can be accomplished at $-85^{\circ}$. For this purpose an exceedingly small quantity of water suffices, that which corresponds to the vapor pressure of ice at this low temperature.

In addition to water, we have seen that there are many other catalysts. Their rôle consists either in accelerating the velocity of certain reactions, or even in determining a combination between substances which would not have taken place [to a perceptible degree] under ordinary conditions. Following the expression of Simon, in the last case the catalysts would act as a mechanism which sets off a stiff spring. By their action, one passes from a metastable to a stable state.

Ordinarily, a catalyst is not limited to a single reaction. Thus finely divided platinum catalyzes the hydrogenating reactions just as well as the oxidizing reactions; $\mathrm{HCl}$ catalyzes hydrolytic reactions just as well as polymerizations; as to nickel, the experiments of Sabatier and Senderens have shown that it 
is capable of causing a large number of reactions. Inversely, a definite reaction can be catalyzed by several different agents: the mineral acids may be substituted for each other in the hydrolysis of saccharose; likewise, nickel can be replaced by other metals in some organic syntheses. Although the catalyst may not be specific, it has nevertheless a directing influence on the course of the reaction. Thus acetylene, $\mathrm{C}_{2} \mathrm{H}_{2}$, heated with nickel above $180^{\circ}$, gives benzene, $\mathrm{C}_{6} \mathrm{H}_{6}$, while it is converted into cuprene, $\mathrm{C}_{7} \mathrm{H}_{6}$, if kept in presence of copper at $180^{\circ}-250^{\circ}$. Likewise, it is found that alcohol can be transformed into aldehyde, $\mathrm{CH}_{3} \mathrm{CHO}$, or into ethylene, $\mathrm{C}_{2} \mathrm{H}_{4}$, according to whether a hydrogenating or hydrating catalyst is used.

Now by what path does the catalyst intervene? First of all, it is not a source of energy, since it is found at the end of the reaction in the same condition as it was at the beginning. It does not modify the energy called into play in a transformation, since all the reactions made with catalysts can equally well be made without them under other experimental conditions, it is true, but without any difference in the quantity of heat liberated. If the reaction is limited, the catalyst modifies the velocity, but does not change the direction of the reaction, nor the condition for equilibrium. In this respect, it is seen that in the transformation the catalyst does not play the part that an elevation of temperature would play.

At present, to explain catalysis, there are two kinds of theories. The first calls in the physical properties of the catalyst. The catalyst acts, not by its mass, but by its surface, which, in contact with the body to be transformed, is the seat of the various phenomena, either by solution, by occlusion, or by an electrical phenomenon.

The other theories claim the formation of intermediate compounds between the catalyst and the substances upon which the catalyst exercises an effect. This view is based moreover on experimentation and in a large number of cases it has been possible to isolate the intermediate substance, which is formed and which is decomposed, in turn, to cause the reaction under 
consideration. It is by such actions, among others, that the decomposition of $\mathrm{H}_{2} \mathrm{O}_{2}$ by metallic oxides is explained, or the liberation of oxygen by calcium hypochlorite in presence of a trace of a cobalt salt. In organic chemistry, the use of aluminium chloride in the Friedel and Crafts method for preparing aromatic hydrocarbons, or again the action of $\mathrm{H}_{2} \mathrm{SO}_{4}$ when added to ordinary alcohol in the preparation of ether, are also characteristic examples of this kind of reaction.

Matignon even assumes from these results that every time a body brings about a reaction by its presence, we may conclude that there must exist an intermediate compound. For him, the action of catalysts is explained by an analogy drawn from mechanics. Assuming first of all that many reactions are not produced under ordinary conditions, contrary to thermodynamic considerations, he asserts that this discordance results from internal frictions. The catalysts would have the effect of substituting for a given reaction, which does not occur alone because of a too great friction, two other reactions, which are no longer in the zone of friction, although in the vicinity of ordinary temperature, and which have for result the action in question.

Let us take the combination $\mathrm{H}_{2}+\frac{1}{2} \mathrm{O}_{2}$. This reaction, while theoretically possible, does not occur at ordinary temperatures. Let us, on the contrary, bring the hydrogen in contact with a platinum-sponge at ordinary temperature. There is formed a hydride which, when placed in oxygen, is transformed into $\mathrm{H}_{2} \mathrm{O}$ with liberation of platinum. This latter has now returned to the same condition as at the beginning, and it is possible with the same quantity of material to continue the combination indefinitely. We have thus replaced a reaction by friction by two others, which do not undergo friction under the same conditions.

This second explanation of catalysis, based on the existence of intermediate reactions, gives rise to some objections. One of these comes from the fact that this theory postulates that the catalyst at the beginning and at the end of the reaction is identical. Now, this is not usually the case. We find almost 
always that the catalyst is changed: when fresh, it is not immediately apt to effect the reaction; then, after a certain length of service it becomes exhausted, and finally ceases to act. This slackening and arrest of the activity of the catalyst, due to a change in its physical condition, is evidently more comprehensible in the first hypothesis than in the second.

Another objection is drawn from the mechanism of the action itself. As Ostwald notes, it is not sufficient to show that there are intermediate reactions, but it must also be established that these have a greater velocity than that of the direct action. This excess must moreover be sufficient to make up for the disproportion which exists between the mass of the catalyst and that of the products which it helps to form. So long as it has not been proved that this is the case, one should speak of accessory reactions, and not employ the expression intermediate reactions. Finally, at present it is difficult to generalize either one of these explanations. It is evident that, in certain cases, there are undoubtedly intermediate reactions, but in many others, we find ourselves in the domain of pure hypothesis. It is probable that, for these last effects, physical actions are those which predominate.

\section{$\S$ III.}

\section{Biochemical Catalysts.}

The transformations which light produces in albuminoid material, transformations whose mechanism we have previously studied, are of special interest from the viewpoint of the chemistry of the living cell. In fact, reserve nitrogenous substances, as well as protoplasmic substance itself, are consumed in the organism in undergoing a series of transformations, due to successive hydrolyses, which bring them to the same final products as those which result from the action of light on the albumin itself. The analogy between the two phenomena is all the more striking in that it has to do not only with the identity of the effect produced, but also with the forces put into play to 
accomplish the change. In the case of the disintegration of albuminoid substances in the presence of sunlight, we see appear, under this photochemical action, a catalyst which brings about the reaction, and directs it in a definite direction. In the living cells factors of the same nature intervene. Under the influence of the physical and chemical conditions of the medium, there are produced, during life, biochemical catalysts which regulate most of the cellular reactions. These active bodies are known under the name of enzymes. They may be regarded as products intermediate between living matter and mineral matter. They are organic substances in the colloidal state. Neither their composition nor their chemical structure is known, but the mechanism of their action, their general characteristics, and their individual properties are sufficiently established to leave no doubt as to their existence.

At the present time we possess certain data on a considerable number of biochemical catalysts accelerating well-characterized reactions. These act, some as oxidizing agents, others as reducing agents, but the majority as hydrolyzing agents. The biochemical catalysts, being essentially specific, affect bodies of a well-determined chemical structure and configuration.

The essential and characteristic properties of the enzymes are as follows:

I. The enzymes, while causing a reaction, are not found in the products resulting from this reaction: as a consequence, a minimum quantity of an enzyme can effect an unlimited transformation.

2. Enzymes are soluble in water and glycerin, and are rendered inactive when their solutions are brought to a temperature near $100^{\circ}$.

3. These bodies may be precipitated from their solutions, either by alcohol, or by salts, such as ammonium or zinc sulphates.

4. They are easily fixed on fibrin, silk, elastin, etc., and are carried down by different precipitates.

5. They are very sensitive to the physical and chemical conditions of the medium. 
Many of these properties recall those of "organized ferments." Enzymes are however distinguished, first in that they can be filtered, although incompletely, through a porous filter, and next because they offer a certain resistance to antiseptics.

6. It has been clearly demonstrated that some enzymes are capable of exerting a reversible activity, and there is good reason for thinking that all are capable of acting thus when the conditions permit.

These very curious properties have been discovered in the inorganic catalysts, especially with the metallic colloids studied by Bredig. This experimenter prepares colloidal platinum in the following manner: the electric spark produced by a current of 8 to 12 amperes at 30 to 40 volts is passed through pure water between two platinum threads. Under this action the metal disintegrates and the liquid takes on first a yellow and then a brown color. It filters easily and under the microscope insoluble particles are not distinguishable. The platinum appears, therefore, to have been dissolved. However this is not the case, for the liquid presents none of the physical properties of a true solution (lowering of the freezing point, existence of an osmotic pressure, etc.). The material is simply in a state of extreme division, the particles formed having a size estimated at only some hundred thousandths of a millimeter (0.0000 $\mathrm{mm}$.). Likewise, pseudo-solutions of gold, silver, cadmium, iridium, etc., have been prepared.

It is found that platinum, thus brought to a colloidal state, using I g. to 300 liters of water, possesses the properties of a true enzyme, those of an oxidase. It decomposes $\mathrm{H}_{2} \mathrm{O}_{2}$, reddens aloin, turns guaiac blue, and is characterized by an optimum temperature. It is activated, then inhibited by a trace of alkali. Certain poisons, as prussic acid, retard it. These properties are identical with those of enzymes, both behaving as catalysts.

In the course of cellular life the different biochemical catalysts present are influenced by each other, either directly,- or by reason of the products formed. The chemical work produced 
in the cell is the result of a very complex action of the catalysts, which regulate their activity in a manner favorable to life. After death the catalysts, remaining intact, continue to act and are capable of producing the same result as in the living cell; but the total result is not exactly the same, either from the quantitative or qualitative point of view. The equilibrium between the catalysts is disturbed, new reactions come into play, and we observe an effect in one direction only. Thus it is that a dead organ, left to itself in the presence of an antiseptic, undergoes an autolysis leading to dissolution and a complete disintegration. On the contrary, in a living organism, the synthetic catalysts, as well as the antibodies, counterbalance the destructive action of the catalysts of decomposition, and we observe a dynamic equilibrium resulting from the complex action of all the enzymes.

\section{$\S \mathrm{IV}$.}

\section{Mechanism of Action of Enzymes.}

We may now inquire how enzymes effect these results. It is not easy to answer this question, since the nature of these bodies is unknown. Even in the case of the inorganic catalysts, whose composition is known, we have not been able to offer a general theory as to their manner of action. Here, the answer is still more difficult to give.

Opinions are divided as to the real nature of the biochemical catalysts. Some regard the enzymes, not as material bodies, but as special forms of energy, which are associated with certain substances, notably the albuminoids. This conception of enzymic force is rather favored by certain physiologists, and so far appears to be more a theory than an actual expression of observed facts. Others, on the contrary, especially chemists, give allegiance to a more positive conception. For them the enzymes are substances that, if not identical with, are at least related to the albuminoids. 
Whatever is the exact nature of these bodies, it is certain that there exists a direct relation between enzyme and albuminoid, a relation which causes the first to partake in a large measure of the properties of the second. In particular, their sensitiveness to heat, which destroys them by coagulation, their change in physical condition under the influence of acids, bases, or salts, which precipitate or redissolve them, and other properties are the result of a condition common to enzymes and to proteins, namely, their colloidal condition.

It has been known for years that the study of the colloids has held an important place in physical chemistry. One entire school has attacked the question of enzymes from this special point of view. Although many results have already been published, the total, however interesting it may be, has not yet led to very important conclusions. We will, however, review some of the facts thus acquired.

In a general way, colloidal particles have special properties differing only in degree from the chemical molecules studied under other conditions. These properties result from two characteristics inherent in colloids.

In the first place it has been established that colloidal particles, suspended in a liquid, possess an electric charge. This fact can be shown by the orientation which these particles undergo when a current is passed through their pseudosolution. According to whether the colloidal substance is charged positively or negatively, it will be directed towards the cathode (cataphoresis) or towards the anode (anaphoresis). Moreover the sign of the charge is indicated in another set of phenomena. An electropositive colloid is precipitated by an unstable electronegative colloid, for example a $0.3 \%$ solution of colloidal arsenious sulphide, an electronegative colloid, is precipitated by an electropositive colloid, such as a $0.2 \%$ solution of colloidal ferric hydroxide. These charges explain the sensitiveness of colloids towards electrolytes, which are known to dissociate into ions when in solution. Acids react especially on electropositive colloids, bases on electronegative colloids. Salts 
react either through their anion or their cation. Further, the precipitating effect will vary with the valence of the reacting ions.

The second characteristic relates to the extreme minuteness of the colloidal particles. It is known that at the surface of all bodies is found a layer of molecules which, by reason of their asymmetric position, are submitted to different forces from those which act on the molecules situated in the interior. Here the superficial layer assumes a rôle of great importance to the whole molecular structure. It has been established that the thickness of the first is of the same order of size as the diameter of the second, from 20 to 50 millionths of a millimeter. In addition, around the colloidal particle is found an adsorption layer, formed by adherent liquid, which forms a sort of envelope isolating the colloidal substance from the rest of the surrounding medium. The composition of the adsorption layer is not always the same as that of the mass of the liquid. There may be in this region a concentration of the dissolved parts, or, on the other hand, a dilution, so that the material considered, protected by this double envelope - superficial layer and adsorption layer - no longer reacts as it would have done had it preserved its normal condition.

To summarize, the special properties characteristic of the colloidal condition are the result of:

I. The electrification of the particles, which, to a certain degree, may be contrary to the laws of chemical combination.

2. The extreme division of these particles, which lends a great importance to the phenomena of contact.

Some consequences of the preceding are as follows:

Pepsin, according to Iscovesco, as dialyzed gastric juice, containing only electropositive colloids, will act in the following manner: In the bottom of a $\mathrm{U}$ shaped tube pour egg-albumin, which is coagulated on the spot. Then, above, in each branch, place a solution of pepsin hydrochloride. Pass through an electric current of 0.3 to 0.6 centivolts and $0.000 \mathrm{I}$ to 0.0005 amperes, and it will be found that, at the end of an hour, egg- 
albumin has been attacked on the positive side. This attack increases, and reaches a maximum at the end of from eight to nine hours, while no reaction occurs on the negative side. The current then pushes the pepsin, on the positive side into the albumin, which is in part dissolved and peptonized, while on the other side the pepsin leaves the albumin and moves towards the negative electrode.

On the other hand, the pancreatic juice of the dog previously dialyzed, contains only electronegative colloids. If the pure gastric juice of a dog is mixed with pure or dialyzed pancreatic juice, a precipitate is formed, which, however, would not be the case if the pure gastric juice had been replaced by dialyzed juice. The positive colloid of the gastric juice has formed with the negative colloid of the pancreatic juice a compound, which is soluble in a neutral medium, but which is insoluble in a medium very slightly acidified with $\mathrm{HCl}$. Hence one is forced to admit that the paralyzing action of the gastric juice on the pancreatic juice is not due, as is generally stated, to the acidity of the gastric juice, but, according to the preceding, to the fixation of the negative pancreatic enzymes by the positive enzymes of the gastric juice.

The supporters of the physical theory have sought to explain a certain number of the biological phenomena which we shall study in this volume. The following is their manner of regarding the anti-ferments. According to them, the neutralizing action which serum of dog's blood exerts on the gastric juice would not be due to an anti-pepsin contained in this serum, but more simply to a precipitating effect on the positive colloid of the pepsin by a negative colloid brought by the blood, a precipitation which would render the gastric enzyme inactive by fixing it in an insoluble compound.

As yet there has not been found an interpretation from the point of view of colloidal chemistry for the phenomena of the coagulation of blood or of milk. According to Iscovesco, the blood contains two serum albumins, one positive, the other negative, and two globulins, one positive, the other negative. 
Now, although the plasma contains both positive and negative globulins, the serum contains only positive globulins. Coagulation would therefore result from the precipitation of a colloidal compound, formed by the union of positive and negative globulins, a compound which is none other than fibrin. This coagulation would be due simply to physical or chemical agents, which would thus modify the electrical conditions of the medium.

From these examples it is seen that these considerations are very suggestive. Without doubt, the data on which they depend are as yet too incomplete for the explanations which they furnish to be entirely satisfactory. But they nevertheless represent a new viewpoint for the investigations in biochemistry, and, on this account, deserve to be noted here.

Guided by these general considerations, let us try, finally, to get an idea of the nature and of the mode of action of these special substances, the enzymes.

An enzyme is always formed by a protein substance containing a considerable quantity of inorganic matter. In water solution it acts like an albumin colloid associated with an inorganic colloid. This latter is in some way the active part of the enzyme, that is, according to the expression of Bertrand, the co-enzyme. It has the albuminoid colloid for support. The specificity of the action, which evidently assumes an appropriate configuration for the corresponding catalyst, would result from the very nature of the co-enzyme. This will act according to the manner of intermediary reactions. As to the albuminoid colloid which serves to protect the active co-enzyme, it influences the properties of the whole in either one direction or another, by virtue of its special colloidal condition, but it does not participate in the true chemical action. It is, in fact, difficult to understand how a molecule as large as that of albumin, and thereby endowed with a certain inertia, could play a similar rôle in effecting a transformation. The biochemical catalysts then differ from the purely inorganic catalysts by a greater complexity. We see in this fact the reason for a more perfect 
adaptation to the work which they perform, and consequently we find here the explanation of their more pronounced specificity. In fact, while by the aid of hydrogen peroxide we may get all the nitrogenous substances derived from albuminoids in the state of ammonia and of volatile acids, the catalysts of living matter cannot accomplish all this work by the aid of a single agent. - To produce this whole transformation there is needed an assembling of a series of catalysts, each of which accomplishes a strictly defined part.

In this volume, we shall study in detail this progressive hydrolysis of albuminoid matter caused by different enzymes and leading to a complete disintegration of the molecule. But, first, it is necessary to classify the different enzymes which furnish the successive stages of this process.

\section{$\S \mathrm{V}$.}

\section{Classification of the Proteolytic Enzymes According} to their Chemical Activity.

The important work of Schutzenberger on albuminoid substances, as well as some recent investigations which have been made on the same subject, which have in great measure confirmed the hypotheses of the French savant as to the constitution of these substances, indicates that albumin, which has a molecular weight of about 5700 , has a composition which corresponds approximately to the formula: $\mathrm{C}_{250} \mathrm{H}_{409} \mathrm{~N}_{67} \mathrm{O}_{84}={ }_{5}^{6} \mathrm{I}$. This very large molecule, with 250 carbon atoms $\left(\mathrm{C}_{250}\right)$, would result from the condensation, with a molecule of tyrosin, $\mathrm{C}_{9} \mathrm{H}_{11} \mathrm{NO}_{3}$, of three identical compounds $\mathrm{C}_{60} \mathrm{H}_{100} \mathrm{~N}_{16} \mathrm{O}_{20}$ and of a base $\mathrm{C}_{61} \mathrm{H}_{98} \mathrm{~N}_{18} \mathrm{O}_{21}$.

Different considerations, based on the manner of progressive cleavage of albuminoid material, as well as on the final products of the hydrolysis, led Schutzenberger to give to the grouping $\mathrm{C}_{60}$ the following structure: 


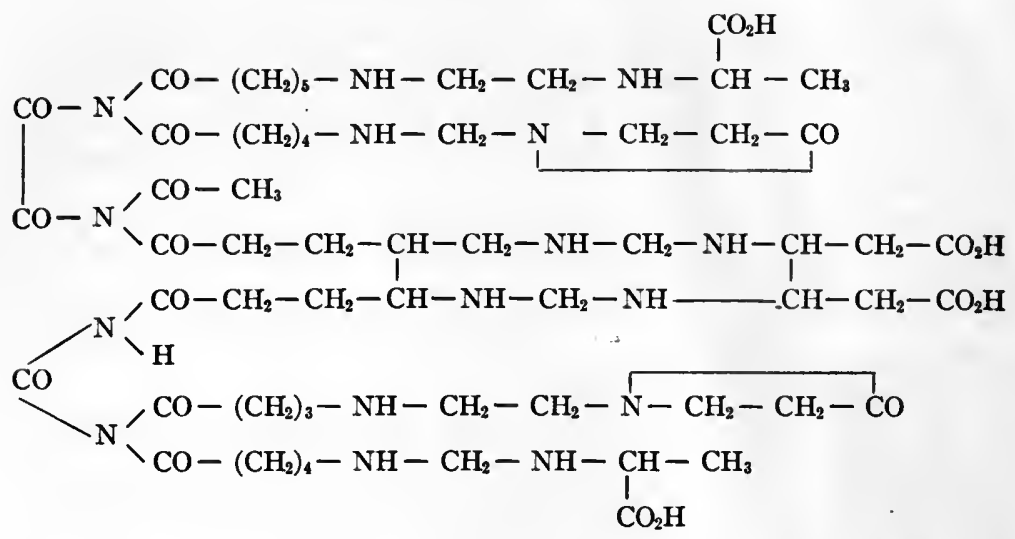

The grouping in $\mathrm{C}_{61}$ is indicated as a derivative of $\mathrm{C}_{60}$. It differs only by the addition of $\mathrm{C}+\mathrm{N}_{2}+\mathrm{O}$ and the loss of $\mathrm{H}_{2}$, but its general structure is the same. The four compounds, three in $\mathrm{C}_{60}$ and one in $\mathrm{C}_{61}$, as well as tyrosin, which form the albuminoid, are found related to each other through many dehydrations. The radicals themselves, as is shown in the preceding scheme, result from the union, by loss of water, of a certain number of amino-acids, each containing only a few atoms of carbon.

Thus, according to Schutzenberger, the bodies which possess the fundamental properties of albuminoid matter would result from the union of several compounds, which in turn would arise from the union of simple groupings all having analogous structures, those of the amino-acids. Various criticisms of detail may be directed at this conception. First of all, the regarding of albumin as a ureid and an oxamid of complex radicals, united in the same molecule, is not entirely consistent with experimental data. It is true that the presence of arginin, in an almost constant proportion, in the products of proteolysis, indicates that the urea grouping is present in the albuminoid molecule, but it possibly does not play as important a rôle as that which Schutzenberger has attributed to it. Further, tyrosin, which in some manner represents the keystone of the whole edifice, is not the only benzol derivative which is obtained 
in the course of the decomposition. There are likewise other aromatic nuclei, like tryptophane, which appear in the course of hydrolysis and which however do not figure in the preceding scheme.

From the work of Kossel, and especially from that of $\mathrm{E}$. Fischer on the polypeptides, we conclude that it is more exact to regard the albuminoid as a very long chain of a large number of different links, amino-acids joined to each other with loss of water. While according to Schutzenberger the union is made by the groups $-\mathrm{CH}_{2}-\mathrm{NH}-$, which, after rupture, become $-\mathrm{CH}_{2} \mathrm{OH}$ and $-\mathrm{NH}_{2}$, according to Fischer, it would be made by $-\mathrm{CO}-\mathrm{NH}-$, which, by hydrolysis, would give $-\mathrm{CO}_{2} \mathrm{H}$ and $-\mathrm{NH}_{2}$.

All these criticisms are especially in the domain of pure chemistry. They in no way change the conception which we must make of the albuminoid molecule, as being a very voluminous aggregation of fragments of unequal resistance, which are formed by a greater or lesser number of different amino-acids. This internal structure, which has been revealed to us by Schutzenberger, without admitting accuracy in all its development, nevertheless corresponds fairly well to our present knowledge of this subject. Guided by it, we may regard the mechanism of hydrolysis as follows:

Under the influence of hydrolytic agents, inorganic or biological, the albuminoid molecule is broken up: the chain, as a result of the attaching of the elements of water is broken in one or several places, but the fragments which are formed are still very large. If desired, by adhering to the formula of Schutzenberger, we can account for the liberation of one or of several of the four principal compounds in $\mathrm{C}_{60}$ or $\mathrm{C}_{61}$, without, however, the tyrosin ceasing to remain associated with one of them.

Then hydrolysis takes place; these large fragments in their turn undergo cleavage, the elements of water are fixed at the points of least resistance, liberating those amino-acids which are detected as soon as the attack becomes rather intense, namely tyrosin and leucin. At the same time, the products subdivide 
more and more, according to the successive hydrolyses taking place. When the process is sufficiently advanced, there will remain in the liquid only very simple molecular groupings, corresponding almost entirely to the constitution of the aminoacids: glycin in $\mathrm{C}_{2}$, others in $\mathrm{C}_{3}, \mathrm{C}_{4}, \mathrm{C}_{5}$, leucin in $\mathrm{C}_{6}$, tyrosin in $\mathrm{C}_{9}$, tryptophane in $\mathrm{C}_{11}$, hexonic bases, etc. Finally, by a still more intense action, the groupings $\mathrm{NH}_{2}$ will in their turn be split off from the molecules which have appeared, and ammonia will be formed on the one hand, and fatty acids on the other.

Now we shall consider the direct consequences of this theory.

I. First of all there is observed a progressive diminution in the average molecular weight of the substance undergoing hydrolysis. We have stated that albuminoid substances appear to be compounds of very high molecular weight; Schutzenberger has attributed to albumin the value of $569 \mathrm{I}$; Sabonijeff, by applying the cryoscopic method to various solutions of albuminoids, found 15,000 . However that may be, successive hydrolyses of this molecule must furnish derivatives of decreasing molecular weight. The first derivatives, still very complex, will have properties similar to those of the original material, while their structure will remain practically the same. These are the albumoses. Then we have the peptones, or the natural polypeptides. Their molecular weight will be about 2400 for the first products and about 400 for the second. The average molecular weight will continue to decrease progressively, to finally reach a value between 75 , corresponding to glycin, and 240, which represents cystin, amino-acids which appear in the extreme decomposition of albuminoids.

2. In the albumin molecule, there is none of the grouping $\mathrm{NH}_{2}$ as such. Every time that water enters into the combination $-\mathrm{NH}-\mathrm{CO}-$ a $\mathrm{NH}_{2}$ group appears, as well as a $\mathrm{CO}_{2} \mathrm{H}$ group. Now, the method of Sörensen, with formaldehyde, permits us to determine the number of $\mathrm{CO}_{2} \mathrm{H}$ groups, and consequently of $\mathrm{NH}_{2}$ groups which are formed. We shall therefore find that as the hydrolysis proceeds, the "formaldehyde" nitrogen increases. This formaldehyde nitrogen corresponds thus to the 
amino form. It serves as a measure of the quantity of aminoacids present in a hydrolyzed liquid. But it is necessary, from now on, to determine this definitely. From the beginning of the action, formaldehyde nitrogen has a value which is not zero. This does not signify that there are real amino-acids already formed of a simplicity greater or at least equal to that of leucin. These bodies do not appear in the first phase of the disintegration, any more than tyrosin or other benzene or indol nuclei. However, the first compounds which are liberated at this time, albumoses for example, already contain, from the rupture of the linkage $-\mathrm{NH}-\mathrm{CO}-$, a $\mathrm{NH}_{2}$ function which will titrate with formaldehyde. From this there might be a possible error of interpretation. The remaining natural polypeptides, albumoses, and peptones, are nothing else than compound amino-acids.

3. The albuminoid molecule already contains amide groupings, of the form $-\mathrm{CO}-\mathrm{NH}_{2}$. These, by boiling with $\mathrm{MgO}$, are decomposed and liberate $\mathrm{NH}_{3}$. Now, as the proteolytic disintegration proceeds, whether the number of groups $-\mathrm{CO}-$ $\mathrm{NH}_{2}$ increases, or whether the peptides produced afford a greater sensitiveness to the action of the alkali, it is found that the proportion of $\mathrm{NH}_{3}$ which the boiling with $\mathrm{MgO}$ liberates, increases. This quantity, to be sure, is not very great in the major part of the enzymic action. On the contrary, in the last phase of the hydrolysis of the amino-acids, all the $\mathrm{NH}_{3}$ may be liberated by $\mathrm{MgO}$, provided that at this point there are produced ammoniacal salts easily decomposed by this base.

To summarize, all these variations in the properties of the products formed result from a single cause, the progressive hydrolysis of the protein molecule. Now, this is susceptible of a valuation, which if not exact, is at least sufficiently definite to form a basis of classification. In fact, from what we have just stated with regard to the formaldehyde method, that which is measured is not amino-nitrogen, but the $\mathrm{CO}_{2} \mathrm{H}$ groups which have appeared. Now, their number is just equal to the number of water molecules introduced, each $\mathrm{CO}_{2} \mathrm{H}$ measured corresponding exactly to the introduction of $\mathrm{I}$ molecule of $\mathrm{H}_{2} \mathrm{O}$. The 
experimental results furnished by the method of Sörensen give us a very clear idea as to the extent of the hydrolysis of albuminoid matter.

If we take the molecule of albumin, whose weight is, according tc Schutzenberger, 5691, and which contains 67 nitrogen atoms, we see that for a total hydrolysis, which ought to bring all the nitrogen to the state of $-\mathrm{NH}_{2}$ (admitting that this would be possible theoretically and that there would be no di-amino-acids), would require 67 molecules of water, or a weight equal to $2 \mathrm{I} . \mathrm{I} \mathrm{g}$. for $100 \mathrm{~g}$. of albumin. Moreover, if one must hydrate all the mono-amino-acids to decompose them into $\mathrm{NH}_{3}$ and into fatty acids, it would take in addition $2 \mathrm{I} \mathrm{g}$. of water, or in all $42 \mathrm{~g}$. for $100 \mathrm{~g}$. of albumin.

Now, experiment shows that, by reason of the formation of di-amino-acids and other nitrogenous products not correctly measurable by the formaldehyde method, one cannot in practice measure in this way more than $90 \%$ of the total nitrogen. Thus in reality only $90 \%$ of the water which has been estimated for the reaction, or $21 . \mathrm{I} \times .90=18.99$, in round numbers, I9 g. of water, has been introduced per I00 g. of albumin, a quantity which represents $67 \times .90=60$ molecules of water. A complete disintegration would require a determined value of $38 \mathrm{~g}$. of water, or I 20 molecules.

Therefore, if in the products of hydrolysis, the formaldehyde method gives $32 \%$ of the total nitrogen, there has been introduced, for I molecule of albumin: $60 \times \frac{32}{9} \frac{2}{0}=2 \mathrm{I} \cdot 3$ molecules of water, or I9 $\times \frac{32}{90}=6.7 \mathrm{~g}$. per $100 \mathrm{~g}$. albumin.

So far as the biochemical catalysts are concerned, it is found that these can be differentiated according to the extent of the proteolysis produced, that is to say according to the quantity of water introduced. Each of these is capable of producing a maximum result, where the degree of degradation is characteristic of the hydrolytic agent employed, or rather of the family to which it belongs. Thus, the different known enzymes for nitrogenous substances can be grouped into five classes, corresponding to the following maximum quantities of water introduced. 
Classification of Proteolytic Enzymes According to the Quantities of Water which they are Capable of Introducing into Albuminoid Material.

\begin{tabular}{|c|c|c|}
\hline Family of enzymes. & $\begin{array}{l}\text { Number of } \\
\text { molecules of } \\
\text { water fixed by } \\
\text { a mol. of } \\
\text { albumin. }\end{array}$ & $\begin{array}{l}\text { Grams of water } \\
\text { fixed by 100 } \\
\text { grams of } \\
\text { albumin. }\end{array}$ \\
\hline 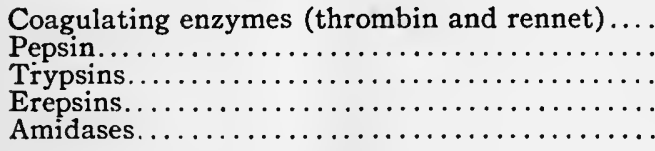 & $\begin{array}{r}0 \\
21 \\
40 \\
60 \\
120\end{array}$ & $\begin{array}{l}0 \\
6.7 \\
12.6 \\
19 \\
38\end{array}$ \\
\hline
\end{tabular}

These figures are evidently not absolute; they are, however, sufficiently characteristic to establish a very clear difference between the various classes of proteolytic enzymes. However, it must be noted that the enzymes which produce a very great hydrolysis do not act on albuminoid matter, but on the products of its decomposition, a decomposition which can even, as in the case of the amidases, be very advanced. Nevertheless, for these enzymes, we have related not the quantities of water introduced into compounds on which the enzymes in reality act, but that quantity introduced into the initial albumin.

If we now examine the variations of each of the three properties of which we have previously spoken, properties which are in direct correlation with the quantity of water introduced, we find that the order according to which the enzymes arrange themselves is almost the same as that which is realized by adopting the basis of classification which we propose. Thus the diminution of molecular weight and the increase of amino-acid nitrogen occurs in the same direction as the hydrolysis. The variation of nitrogen that may be liberated by magnesia is not as regular. Here is what is actually observed: 
Variations of Properties. Products of Proteolysis of Albumin.

\begin{tabular}{|c|c|c|c|}
\hline Family of enzymes. & $\begin{array}{l}\text { Molecular weight } \\
\text { of last nitrogen } \\
\text { products formed. }\end{array}$ & $\begin{array}{l}\text { "Formaldehyde" } \\
\text { nitrogen (amino- } \\
\text { acids formed), \% } \\
\text { of total N." }\end{array}$ & $\begin{array}{l}\text { Nitrogen } \\
\text { which can } \\
\text { be freed by } \\
\text { MgO.*\% of } \\
\text { total N. }\end{array}$ \\
\hline $\begin{array}{l}\text { Coagulating enzymes (throm- } \\
\text { bin and rennet) } . . . \cdots \cdots \cdots\end{array}$ & $\begin{array}{l}\text { No change appre- } \\
\text { ciable in the } \\
\text { weight mol.t }\end{array}$ & $\circ$ & $\circ$ \\
\hline 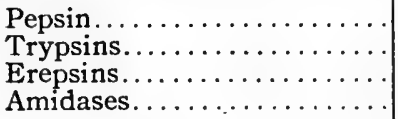 & $\begin{array}{l}2400-400 \\
2400-75 \\
240-75 \\
17\end{array}$ & $\begin{array}{r}32 \\
60 \\
90 \\
0\end{array}$ & $\begin{array}{l}5.4 \\
4.6 \\
10 \\
80-100\end{array}$ \\
\hline
\end{tabular}

- The quantities of nitrogen which can be liberated by $\mathrm{MgO}$ vary with the albuminoid material used. The figures given here relate to white of egg. With casein, they would be higher, but the relative proportions would remain the same.

t In all cases, if, among the products formed, there is any whose molecular weight is less than that given, these are not found in appreciable quantities.

This basis of classification gives entire satisfaction so far as the three first families of enzymes are concerned. As to the two last families, the figures cited are more difficult to interpret. We have profited by this fluctuation to put into these classes a certain number of enzymes which belong to them only in an indirect way, for instance the nucleo-proteases, arginase, creatinase, urease, etc.

The following order has been adopted for this description: In the first part, we shall study the coagulating enzymes, thrombin, myosinase, and rennet; in the second part, pepsin; in the third, the different trypsins, of animal or vegetable origin, papain, bromelin, casease, etc.; in the fourth, the erepsins, intestinal erepsin, the nucleo-proteases, arginase, etc.; in the fifth, the amidases and urease; finally in a sixth and last part we shall describe the principal applications of these different catalysts to industry. 


\section{BIBLIOGRAPHY}

G. BREDIG. Ueber anorganische Fermente, Leipzig, I899.

NeUBerG. Bioch. Zeit. $(36)$, 1911, p. 39.

Effront. Compt. Rend., IgI2 (I), p. 1111 et I296; Bull. Soc. Chim. France, 1912, p. 744. Les Enzymes, Paris, 1899.

Duclaux. Microbiologie, Paris, 1900.

Reynolds Green. Die Enzyme, Berlin, Igor.

C. Oppenheimer. Die Fermente, Leipzig, rgoo.

Iscovesco. Soc. Biolog., 1906 (1), p. 783.

Matignon. Leçons sur la catalyse faites au Collège de France.

Simon. Conférence sur la catalyse, Bull. Soc. Chim. (29-1), I903.

V. Henri. Leçons de chimie physique, Paris, Igog.

E. Zunz. Fermente, pp. 538-668 in E. Abderhalden's Biochemical Hand Lexicon, Vol. V, Berlin, rgr r. 



\section{PART I.}

\section{COAGULATING CATALYSTS.}





\section{CHAPTER I.}

\section{THROMBIN.}

\section{$\S I$.}

\section{Preparation of Thrombin and Fibrinogen.}

The blood of mammals, upon issuing from the veins, remains liquid from 5 to ro minutes, according to the conditions, and then, quite suddenly, is transformed into a jelly which, after a certain time, contracts, giving a red clot floating in a clear transparent liquid called serum. The clot is formed by fibrin, which encircles in its filaceous net the blood globules, hemocytes and leucocytes.

The velocity of coagulation varies largely with the animal species. The blood of the horse, in particular, which coagulates rather slowly, lends itself, for this reason, better than any other to the observation of the phenomenon. When this blood is left at a temperature of $5^{\circ}$ or $10^{\circ}$, red globules are soon seen to be deposited at the bottom of the vessel, which are clearly more dense than the surrounding liquid. On this layer a second is superimposed, formed by the yellowish gray cells, the leucocytes. The blood fluid, separated from the elements described, is called the plasma. The plasma, possessing a distinctly yellow color, remains liquid for some time, then thickens, curdles, and finally contracts, forming a clot from which exudes the serum.

The coagulation of blood, which we have just described, due to the appearance of precipitated fibrin, results from the action of a special enzyme on certain albuminoids of the blood. The enzyme which takes part in this action has been described by different authors under various names, especially in German literature, where it is called fibrin-ferment. Duclaux has adopted the term of plasmase, but it is also designated by the names of 
fibrinase, thrombin, or even of thrombase. We shall adopt the term thrombin, since, by reason of the different theories of coagulation given out, it leads to less confusion than any other.

Thrombin was discovered by Buchanan in 1835. This enzyme, or rather one of the constituent parts of this enzyme, is found in abundance in the white corpuscles of the blood. It is also found in the red corpuscles, though in much smaller proportion, and in many tissues. It exists in the lymph, which has approximately the same composition as blood, and in pus, which is a sort of serum in which float the white corpuscles that have undergone fatty degeneration. Much discovered the presence of thrombin in staphylococcus aureus, and Higuchi has, by the aid of physiological salt solution, isolated this enzyme from the soft mass of the placenta.

To obtain thrombin, it is possible to use either a maceration of leucocytes, or extracts of muscles or of lymphatic ganglia. Schmidt first isolated the enzyme from defibrinated blood by precipitation with alcohol. To one part of serum he adds twenty parts of alcohol. A precipitate of albuminoid substances forms, which carries with it the thrombin. The contact with alcohol is prolonged for two or three months, which renders the coagulum almost entirely insoluble in water. Then the precipitate is filtered and is dried in vacuo over $\mathrm{H}_{2} \mathrm{SO}_{4}$. On treating with warm water, the enzyme dissolves and not the albuminoids. The solution so obtained shows great activity, and if a little is added to plasma, there results a coagulation infinitely more rapid than that which results under ordinary conditions.

Hammarsten prepares thrombin by another method. Some fresh horse blood is first centrifuged in order to separate the blood corpuscles. Then $0.3 \%$ potassium oxalate is added and the precipitate of calcium oxalate is removed by centrifuging and filtering. The filtered liquid is then diluted with two or three volumes of water, filtered again to eliminate the last traces of insoluble matter, and then acidified with acetic acid. A precipitate is formed, which contains thrombin. This is col- 
lected and purified by dissolving in water made slightly alkaline, and reprecipitating with acetic acid. To obtain a sufficiently pure product, it is necessary to repeat this treatment a number of times. Finally the product is redissolved in a dilute solution of sodium chloride. The enzyme so obtained is free from calcium.

The thrombin may also be extracted from fibrin which is precipitated during the coagulation. The blood, freshly collected, is first of all diluted to prevent a sudden coagulation. It is left undisturbed for some time, and then is whipped with a small brush to produce defibrination. The fresh fibrin is then treated with a $0.5 \%$ solution of $\mathrm{NaCl}$, a concentration sufficient to dissolve the enzyme, but too dilute to attack the fibrin. Thus is obtained a very active solution of thrombin.

Finally, Pekelharing proposes the following method for preparing an active solution of thrombin. He takes $300 \mathrm{~g}$. of the soft mass of calf's spleen, which he treats with $600 \mathrm{~g}$. of chloroform water and the next day centrifuges the whole. The liquid, after filtration, is slightly acidified with acetic acid. The precipitate formed is separated in the centrifuge, dissolved in water, made slightly alkaline, and is again precipitated by acetic acid. This treatment is repeated several times until finally there results an alkaline solution which, when neutralized, upon the addition of $\mathrm{CaCl}_{2}$ and added to the fluid from a hydrocele which is known not to be spontaneously coagulable, very rapidly causes the formation of solid fibrin. This active solution also causes the coagulation of blood which has been kept liquid by artificial means.

The processes of preparation of thrombin which we have just enumerated are, however, far from being perfect. The product obtained is active only under certain conditions of experimentation. The coagulation of blood, as will be seen later, is a very complex phenomenon in which many different factors take part. The active substance, obtained by one or the other of the preceding processes cannot be considered as a complete thrombin, but rather as one of its constitutents. True thrombin is obtained only after the beating of blood extracted 
from blood-vessels, and it is the serum so obtained which should really serve for studying the phenomenon of coagulation, although the isolation of thrombin from such a serum presents many difficulties, due to the nature of this enzyme.

To obtain an active serum, one may proceed in the following manner. Rabbit's blood drawn. from the veins is diluted $\mathrm{I}$ to 3 with a $20 \%$ solution of $\mathrm{NaCl}$. The mixture, containing $5 \%$ $\mathrm{NaCl}$, is centrifuged and thus a non-coagulable plasma is collected, which is non-coagulable because of its high concentration of salt. However, if the solution is diluted with 4 volumes of water, it rapidly curdles. It is necessary only to eliminate the fibrin to have a clear serum having all the properties of a solution of thrombin. To show the activity of the serum thus prepared, use is made of a plasma diluted and made $0.2 \%$ in sodium oxalate; this plasma; because of the presence of the oxalate, does not coagulate spontaneously, even after dilution. It acts up to a certain point like a solution of fibrinogen, and curdiles to a mass when active thrombin or serum is added. Thus, by a rather clever device, it is possible to separate from blood the two factors which take part in its coagulation: on the one hand, the serum containing the enzyme, on the other the plasma deprived of active thrombin but nevertheless containing fibrinogen.

The action of thrombin can, however, be studied outside of blood, by using the albuminoid principle contained in the plasma, the fibrinogen, the material on which the enzyme acts and which is considered to be the mother substance of fibrin.

Fibrinogen occurs not only in blood and lymph, or white blood, but also in watery exudations, normal or pathological, such as peritoneal and pericardial exudations, hydrocele fluid, the liquid of œdema, etc. These exudations are not spontaneously coagulable, but they contain fibrinogen and coagulate if thrombin is added to them.

There are different methods of isolating fibrinogen. The first on record is due to Denis (I857), who, by adding to saline plasma a great quantity of $\mathrm{NaCl}$, precipitated albuminoid substances. The coagulum, next treated with a dilute salt solution, 
gave up to the liquid a part of its fibrinogen. However, Schmidt first succeeded in isolating this body by starting out with the hydrocele fluid. To prepare fibrinogen, four volumes of horse blood, taken directly from a vein, are added to one volume of a saturated solution of $\mathrm{MgSO}_{4}$. The blood, in the presence of this salt, remains uncoagulated. The corpuscles are removed by centrifuging and filtering, and the plasma so obtained is mixed with one volume of a saturated $\mathrm{NaCl}$ solution. Under these conditions the fibrinogen precipitates, is collected on a filter, dried between double leaves of paper, and is purified by dissolving in an $0.8 \%$ solution of $\mathrm{NaCl}$ and precipitating by saturating with $\mathrm{NaCl}$. After several successive treatments, a product is obtained which, when dried on paper, is easily soluble in distilled water, as a result of the $0.2 \% . \mathrm{NaCl}$ which it still contains. The fibrinogen can be separated from this salt by dialysis in water made slightly alkaline.

Fibrinogen can also be prepared by the aid of oxalated plasma. Blood from the blood-vessels is mixed with an equal volume of a $0.6 \%$ solution of sodium oxalate, is centrifuged and left standing for twenty-four hours at $0^{\circ} \mathrm{C}$. The clear liquid is decanted and a saturated solution of $\mathrm{NaCl}$, containing $0 . \mathrm{I} \%$ of oxalate, is added, until the salt content is $15 \%$. The fibrinogen then separates into a coagulum, which is collected and purified as previously, by successive solution and reprecipitation with $\mathrm{NaCl}$. Reye, to separate fibrinogen, mixes twelve parts of fluoride plasma with thirty parts of water and sixteen parts of a saturated solution of ammonium sulphate. Under these conditions, the fibrinogen is precipitated, and is collected and purified according to the usual method.

Precipitated fibrinogen appears in the form of a sticky mass, adhering to glass. Insoluble in boiled distilled water, it readily dissolves in water which has not been boiled, and its solubility increases with the supply of oxygen in the latter. It also dissolves in a weak solution of $\mathrm{NaCl}$, but is precipitated upon dilution with water, in consequence of the small concentration of the chloride ion. 
A solution of fibrinogen in a dilute solution of $\mathrm{NaCl}$ becomes turbid at a temperature near $53^{\circ}$. Fibrinogen coagulates at $5^{\circ}$. According to Bordet and Gengou, salt plasma, freshly diluted to $\mathrm{I} \%$ and heated for ten minutes at about $53^{\circ}$, still coagulates under the influence of thrombin. It will not coagulate if the temperature is raised for the same time interval to about $54^{\circ}$. The liquid becomes opalescent as a consequence of the deterioration of the fibrinogen. A solution of fibrinogen, upon the addition of thrombin, acts in the same way as blood issuing from the blood-vessels. The liquid soon changes its consistency and there is produced a coagulum more or less resistant according to the concentration of fibrinogen.

As to thrombin, it possesses the characteristics of an enzyme. It is precipitated from its solutions by alcohol, and is dragged down by certain precipitates formed in the liquid. It is very sensitive to temperature and to the condition of the medium. Moreover, in its action on fibrinogen there is observed, under favorable conditions, a constant relation between the velocity of the action at the beginning and the quantity of enzyme used. It is found furthermore that there is a disproportion between the cause and the effect, a very slight amount of enzyme being able to coagulate a very large quantity of blood. However, this action is not unlimited, for thrombin, though not exhausted by the reaction, is nevertheless absorbed by the fibrin, which retains it and prevents it from following the general laws of the action of enzymes.

Thrombin is destroyed, according to Hayem and Schmidt, by heating for three-quarters of an hour at $58.5^{\circ}$; according to Bordet, the temperature at $56^{\circ}$ is sufficient to cause a serum to lose its coagulating power. Cold considerably retards the activity of this enzyme, for whereas salt plasma freshly diluted and made $\mathrm{x} \%$ in oxalate, upon the addition of an equal volume of serum $\mathrm{I} \%$ in oxalate, coagulates at $30^{\circ}$ in five minutes, it will not coagulate in less than twenty minutes if the mixture is kept at $0^{\circ}$. The optimum temperature is between $38^{\circ}$ and $40^{\circ}$. By diluting a plasma, the coagulation is retarded. Certain salts, 
$\mathrm{NaCl}$ among others, also counteract the coagulating action of fibrinogen, the effect being directly proportional to the concentration of salt used.

Influence of Salt Concentration on the Transformation of Fibrinogen. 2 c.c. plasma $2 \% \mathrm{NaCl}+2$ c.c. serum $2 \% \mathrm{NaCl}$ : Coag. takes place in $\mathrm{I}$ h.

2 c.c. plasma $3 \% \mathrm{NaCl}+2$ c.c. serum $3 \% \mathrm{NaCl}$ : Coag. takes place next day.

2 c.c. plasma $4 \% \mathrm{NaCl}+2$ c.c. serum $4 \% \mathrm{NaCl}$ : Coag. takes place 2 nd day after.

On the contrary, a I\% solution of sodium oxalate does not paralyze the action of thrombin.

Thrombin, like many other enzymes, possesses the property of being adsorbed by certain precipitates. One of them especially, calcium fluoride, is endowed to a high degree with the power of adsorbing simultaneously both the active substance and the fibrinogen of the plasma. We shall have occasion to return to its action in connection with fluoride plasma. Other precipitates, such as barium sulphate and carbonate and calcium oxalate, likewise have an anti-coagulating power. They are, however, less active than calcium fluoride, especially from the point of view of adsorption of fibrinogen, though they can retain all the thrombin. Thus it is that 4 c.c. of serum obtained from a normal coagulation of dilute plasma, upon the addition of 2 c.c. of a milky emulsion of $\mathrm{BaSO}_{4}$, loses, after agitation, centrifuging, and decantation of the clear liquid, all coagulating power towards an oxalated plasma.

\section{$\S$ II.}

\section{Chemical Action.}

We now come to the chemical action of coagulation. In what way does thrombin act on fibrinogen? At the present time two explanations are proposed, and although the second has the greater number of adherents, the tendency is to revert to the first, when given in a slightly modified form.

Some have considered coagulation to be due solely to a change in the physical condition of fibrinogen. According to 
Duclaux, who has supported this opinion, fibrin is merely the passing of that albuminoid constituent of blood which is nearer to a state of suspension than a state of solution into a visible form. Others, on the contrary, consider that coagulation results from an actual decomposition of fibrinogen. Some former experiments of Hammarsten and the more recent ones of Arthus, show, in fact, that fibrinogen splits under the influence of plasmase into two substances, one a globulin, which remains dissolved in the serum, the other, fibrin, which is precipitated. According to Hammarsten the globulin does not previously exist in the plasma and differs from fibrinogen in that the latter is coagulated at $56^{\circ}$ while globulin only coagulates at $64^{\circ}$. This fact is moreover confirmed by Arthus, who has shown that the weight of fibrin formed is always less than that of fibrinogen contained in this plasma.

Indeed, the question is far from being solved. As Duclaux observes, the quantities of nitrogenous matter involved are very slight. The weight of fibrin collected from I liter of plasma, dried, and weighed, is from 2 to 4 grams. Now the weight of fibrinogen obtained by coagulation at $56^{\circ}$ is no larger. It is true that heating does not release all the fibrinogen contained in the plasma. The fact, then, that the weight of fibrin collected is slightly less than that of fibrinogen obtained by coagulation leads only to the conclusion that this weight of fibrin is clearly smaller than the actual weight of fibrinogen contained in the plasma. But this reasoning is not an actual proof. On the other hand, Bordet and Gengou have shown, while studying the comparative action of an emulsion of $\mathrm{BaSO}_{4}$ on serum exuded from a clot and on serum obtained by defibrination of blood in process of coagulation, that, in the first case the mixture remains turbid indefinitely, while in the second the precipitate is rapidly deposited, due to an actual clarifying effected by the fibrin remaining in solution in the blood. These authors thus maintain that by defibrinating with a rod a plasma which is coagulating, one does not necessarily release the total quantity of fibrin actually present, 
this being entirely deposited only at the end of several days. Finally, the fact that the temperature of coagulation of serum by heat is slightly higher than that of the plasma, is not an irrefutable argument in favor of the formation of a new globulin, the phenomena of coagulation being too easily influenced by the conditions of the medium.

To summarize, there is nothing to prove that there is really a breaking up of albuminoid matter into two components. We shall find an analogous case in the coagulation of milk, and the arguments which we shall then introduce apply equally well here. There is every reason to believe that we have to do with a purely physical modification, or at least with a chemical transformation affecting the molecule only to a very slight extent, a transformation not followed by division.

In addition to these two theories with regard to coagulation, we must also speak of another possible origin for fibrin. It is known that there exists in the blood, besides the red and white corpuscles, a third organized component, designated under the various names: free granulations, plates of Hayem, or globulin. These elements are much more unstable outside the bloodvessels than the other cells of the blood, and the idea has occurred that perhaps fibrin would result from their decomposition. Duclaux has refuted this assertion by establishing the fact that there is no relation between the destruction of the plates and the formation of fibrin.

However, Bürker has returned to this conception. According to him, coagulation of the blood is intimately related to the decomposition of the plates. All the conditions which influence thrombin, and consequently coagulation, such as temperature, contact, chemical products, act equally on the transformation of the plates. Sodium metaphosphate, magnesium sulphate, ammonium oxalate, all of which hinder coagulation, also preserve the blood components. This decomposition of the plates would take place under the action of a trypsin contained in the blood. Once these particles are destroyed, coagulation would appear. The quantity of fibrin formed is then in direct proportion to the 
quantity of plates transformed. The rôle of these plates does not then consist in secreting a special coagulating enzyme, but probably in the forming of fibrin even at their own expense. It is known that the number of plates in the blood is very great, about 400,000 per cubic millimeter. Bürker, by an approximate calculation, has determined that the weight of fibrin formed is of the same order as the weight of decomposed plates.

It is perhaps inadvisable to pretend that fibrin comes only from the transformation of the plates. It appears, however, from a certain number of observations that we shall present later that these organized components of the blood actually play a part in coagulation, but only in the elaboration of the active substance, and not of the fibrin which is precipitated. Therefore they will not be considered at this point.

\section{§ III.}

\section{Theory of Coagulation of Blood.}

Coagulation of blood has been the subject of very many investigations, and yet the data which we possess at the present time is far from being complete. One can even say that of all the proteolytic enzymes, thrombin is the least understood. For a long time this enzyme was regarded as a single, well-defined substance. Then, as the phenomenon of coagulation of blood was studied more closely, it was perceived that the problem was becoming more and more complicated, and the simplicity of the constitution of thrombin was becoming less and less certain.

The work of Hammarsten and Arthus has established the fact that calcium salts are indispensable in the transformation of fibrinogen into fibrin. This discovery changed completely the direction of investigations and led to different interpretations. According to A. Schmidt, coagulation of blood is caused by secretions arising from the leucocytes and the plates. The substance secreted is not in reality an enzyme, but is pro-throm- 
bin, which, under the influence of certain zymoplastic substances also produced in the cells, is transformed into thrombin or active enzyme.

On the contrary, according to Pekelharing, the leucocytes already contain the completed plasma, which, in presence of calcium salts, acts on fibrinogen. According to this investigator, the nucleo-proteins, which are obtained from the tissue extracts, are endowed with the same properties as the plasmase which comes from the leucocytes. Both, in the presence of calcium salts, coagulate fibrinogen. Clotting is then the combined result of the action of plasmase and of that of calcium salts. This phenomenon would moreover be complicated by the fact that the leucocytes, as well as the extracts of an organ, contain in addition to coagulating enzymes, anti-coagulating substances, that is to say anti-bodies. It is to be noted, in passing, that these conclusions are based on experiments made with solutions of pure fibrinogen and solutions of enzyme isolated according to the previously described method.

According to Moravitz, thrombin as such is not found in blood. The plasma contains a pro-enzyme or zymogen which Moravitz calls thrombogen or serum-agent, whose transformation into thrombin requires the coöperation of lime salts and of a substance which exists in all cells and tissue extracts, and which normally is brought by the leucocyte secretion, a substance to which Moravitz has given the name of thrombokinase. Thus, according to this last conception, three factors intervene in the formation of thrombin: the zymogen, calcium salt, and thrombokinase. Of the three constituents which enter into the composition of thrombin, two have various names according to the authors who have studied them. Let us enumerate them for-record: thrombogen [or thrombinogen: Editor] is thus called pro-thrombin, plasmazyme, serozyme; thrombokinase is likewise designated as zymoplastic or cytozyme substance.

According to the accepted theory, the phenomena observed are interpreted in different ways. Here, for example, is a blood which does not coagulate spontaneously, but which clots when 
a little organic extract is added. This is the case of blood of a bird, as we shall see later. The adherents of the existence of thrombokinase explain this fact by saying that the blood examined contains much serozyme and calcium salts but that it is lacking in thrombokinase. Coagulation occurs immediately on the addition of organic extract, for this introduces the third indispensable constituent. The adversaries of this opinion answer that it is not at all necessary to admit the intervention of a third factor. The blood is not caught (caught in the sense of a single substance) in mass because it does not contain enough thrombin. By adding a tissue extract the enzyme needed is supplied, and coagulation takes place immediately.

The partisans of the unitary theory of plasmase find a support for their conception in the fact that a solution of pure fibrinogen, which does not coagulate spontaneously, does clot, on the contrary, when there is added to it an extract of tissue containing sufficient calcium salts. To this experiment, apparently conclusive, the objection is raised that the fibrinogen which has been used cannot be regarded as a pure substance. Prepared by precipitation, it contains one of the constituents for producing thrombin, namely serozyme. Its solution does not coagulate because it contains but one of the two constituents for thrombin. If now tissue extract is added the absent constituent is supplied, and coagulation is at once produced.

The experiments of $\mathrm{E}$. Zak furnish a serious argument for the theory of the duality of the constituents producing the coagulating enzyme. It is known that oxalated plasma remains non-coagulable in consequence of insufficient calcium salts, whereas it curdles in mass as soon as this indispensable constituent is added. E. Zak treats oxalated plasma with petroleum ether: He thus dissolves out a lipoid body, and finds that the plasma, after addition of lime, now coagulates more slowly, or else not at all, according to the degree of extraction of the liquid. On the contrary, this same plasma regains all its original properties when the substance removed is replaced or is substituted by ethereal organic extracts of the lecithins of 
the brain. The extract with petroleum ether is in reality the thrombokinase of Moravitz.

Nolf ascribes the process of the coagulation to a rupture of the equilibrium between the colloidal substances contained in blood. According to this author, there are found in blood plasma three principal colloids which react for the formation of fibrin, namely: fibrinogen, thrombogen, and thrombozyme. These three constituents are maintained in solution in an unstable equilibrium. Mechanical excitation, contact with foreign bodies, as well as a chemical action such as introducing substances extracted from the tissues, may destroy this equilibrium. At this time, the three constituents mentioned above combine to give two different compounds. One is solid, and is fibrin, the other remains in the liquid, and is thrombin. The originality of this theory consists in the fact that the usual enzyme of coagulation is a product of the reaction, and not the determining cause of the transformation. Moreover, according to this conception, thrombin which is formed as a result of coagulation and which remains in the liquid after the precipitation of the fibrin, possesses the properties of a mixture of thrombogen and thrombozyme. It is, therefore, a thromboplastic substance, which, with the fibrinogen of another solution, will again be able to react to form solid fibrin and dissolve enzyme. But here the term enzyme is not used, since the thrombin does not act as a catalyst. According to Nolf, thrombogen, which he also calls hepatothrombin, has its origin in the liver. As to thrombozyme or leucothrombin, it comes from the leucocytes, as well as numerous tissues.

This theory, however interesting it may be, does not explain to us the phenomenon of coagulation in all its details. It has, however, the merit of introducing new ideas founded on the colloidal condition of the substances in question, a condition which undoubtedly plays a very large part in the whole transformation, and which has been neglected in the other theories. In the chapters which follow we shall have occasion to return to the origin of thrombin and to consider the various conditions which favor or prevent coagulation. 


\section{$\S I V$.}

\section{Blood Plasmas not Spontaneously Coagulable.}

In the preceding chapter we became familiar with the principal constituents which come into play in the phenomenon of the coagulation of blood. We have studied thrombin and fibrinogen, and established the relation which exists between the two. We now return to the question and, excluding the theories which we have just described, will try to penetrate the actual mechanism of coagulation by analytical methods. Let us ask first of all why the blood, liquid in the blood-vessels, coagulates on leaving them. What is the ultimate cause of this curdling? To find the answer more easily, we will describe the processes used to preserve the blood in the liquid state. The special precautions to be taken to obtain this result will require a study of the influence of each factor on the transformation, and will thus lead, by contrast, to a knowledge of the indispensable conditions for the manifestation of the phenomenon.

Different means exist for obtaining a plasma not spontaneously coagulable, or at least capable of remaining liquid for different intervals of time.

I. One may cut a section of the blood-vessel of an animal, after having ligatured the two extremities. If this operation is practiced on the jugular vein of a horse, whose blood corpuscles are appreciably more dense than the plasma in which they float, these corpuscles, if the vein is kept vertical, will dispose themselves at the lower extremity, and it is possible, after contraction of the blood-vessel above the deep layers, to preserve a certain quantity of limpid and non-coagulable plasma.

2. Again the plasma of a horse may be obtained by maintaining at low temperature, about $0^{\circ}$, blood freshly collected. The deposition of corpuscles takes place and at the end of a certain time a clear liquid is obtained, which can be decanted and preserved. Its stability is much further increased by working quickly, and by filtering the plasma obtained at $0^{\circ}$.

3. Delezenne has shown that the blood of all the verte- 
brates containing nucleated red corpuscles (birds, reptiles, batrachians, fish) coagulates very slowly when pure, that is to say, not mixed with the juice of broken tissues. This condition is found to be fulfilled, for example, in introducing into a goose's artery a tube which is held in such a way as to avoid scraping the walls of the blood-vessel. With certain precautions, and by centrifuging the blood thus obtained, a plasma is obtained which remains liquid for a month or more: However, this goose plasma, with addition of even a very small quantity of the juice of bruised tissues, at once coagulates.

4. To prepare a rabbit plasma capable of being preserved several hours without coagulating, advantage is taken of the retarding influence which paraffin exerts on the appearance of thrombin. Withdrawing the blood by means of paraffined tubes and centrifuging in receptacles likewise paraffined, there is obtained a plasma which, preserved in vessels coated with this substance, keeps liquid for four or five hours, sometimes even for twenty-four or thirty hours. It ends, however, by coagulating, which proves that it already contains a certain quantity of thrombin, which has appeared in the course of manipulation.

5. Blood with addition of a certain quantity of neutral salts, $\mathrm{NaCl}, \mathrm{Na}_{2} \mathrm{SO}_{4}, \mathrm{MgSO}_{4}$, etc., ceases to be spontaneously coagulable. If, for example, blood is received in an equal volume of a $10 \% \mathrm{NaCl}$ solution, the mixture can be centrifuged, and the plasma so obtained, containing $5 \%$ salt, can thus be kept indefinitely without coagulating.

6. Arthus and Pagès, in I888, showed that coagulation of blood can likewise be prevented by adding to it a $0.1 \%$ solution of a neutral alkali oxalate. We shall see that this salt acts by precipitating the calcium of the plasma, the presence of which is indispensable for the elaboration of thrombin.

For the same purpose, one can also use sodium fluoride (0.15\%-0.3\%), alkali soaps, sodium metaphosphate (0.2\%), and finally sodium or potassium citrate $(0.2 \%-0.3 \%)$.

Blood, thus treated, can be centrifuged and furnishes plasmas 
not spontaneously coagulable. They have the advantage over those obtained by the aid of neutral salts of containing only very small quantities of foreign substances.

7. If Witte peptone dissolved in ten times its weight of salt water $(0.7 \%)$ is injected into the jugular vein of a dog in the proportion of $0.3 \mathrm{~g}$. per $\mathrm{kg}$. of animal, it is found that five to ten minutes after the introduction of the solution, the blood which is collected is no longer spontaneously coagulable. This can then be centrifuged and a peptonized plasma obtained.

It should be noted, in passing, that these different plasmas, not spontaneously coagulable, are, however, rich in fibrinogen, and that they can serve, especially the goose plasma and the oxalated plasma, as reagents to show the presence of thrombin in a liquid. Arthus has even shown that fluoride plasma can be utilized as a quantitative reagent for this enzyme.

\section{$\S \mathrm{V}$.}

\section{Origin of Coagulating Enzyme.}

As we have just seen in the preceding chapter, there exist numerous methods for rendering blood not coagulable spontaneously. These are of very diverse nature. Some of them are purely physical, others chemical, and, finally, some, like that in which peptone is used, are of a physiological nature. All act to prevent the enzyme from forming, either by paralyzing or by eliminating one or the other of its constituents.

Among the purely physical processes are: No. 2, which has recourse to cold; No. I, based on the isolation of the jugular, and No. 4, where paraffin is used. The efficacy of cold is easily explained, biological manifestations always requiring a certain temperature in order to become evident. As to the other two processes, if we examine them carefully, we see that both arise from the same principle, namely, that of contact which plays a very important part in the phenomenon of coagulation.

Blood remains liquid in the organism because it does not 
contain any coagulating enzyme. If, in fact, the latter existed in blood, it would pass, like blood-amylase, into the transudates, and would be recovered there, which is not the case. It appears, however, if the blood is received in a vessel, and especially if it is beaten with rods, since these very quickly cause a curdling in the mass. The impression is thus given that thrombin is not equally distributed in blood, but that it is enclosed in fragile little vessels, which, at the least injury, are broken and pour out their contents. This idea corresponds to a certain degree to the facts. One of the constituent products of thrombin, thrombokinase, is found deeply imprisoned in the leucocytes. In the normal state, in the circulating stream, the white corpuscles, by a kind of adaptation to the nature of the walls of the bloodvessels in which they circulate, do not give up their enzyme. Its appearance outside corresponds to a certain excitation,- to a weakening, or even to the death of the cells. Under the influence of various causes, and especially under the influence of contact other than that of the wall of the blood-vessels, contact which modifies the surface tension of the blood corpuscles, there is an abundant excretion of the active substance, not because the cells are dislocated or broken, but simply because a certain mechanical excitation results from such contact.

It can thus be shown that thrombin has its origin in the blood corpuscles. If the blood of a horse is collected in a long tube and is left at a temperature of from $5^{\circ}$ to $10^{\circ}$, as already stated, the cells will be deposited and at the end of a certain time, coagulation begins. It is observed that this coagulation begins in the deep layers in contact with the corpuscles. It is even possible to demonstrate that it is especially the leucocytes which are the producers of thrombin. From the deposit formed at the bottom of the tube described, two samples may be taken, one consisting of red corpuscles, the other, of white corpuscles. These samples, mixed in water, are put in contact with a liquid rich in fibrinogen, hydrocele fluid for example, which is known to be not spontaneously coagulable. While the mixture containing the white corpuscles, or even simply a filtered macera- 
tion of these, coagulates very rapidly, the other curdles only with extreme slowness.

The secretion of the active substance by the leucocytes is not instantaneous; it takes place slowly, and is hindered by cold. It appears very sensitive, as we have seen, to the influence of the wall of the vessel in which the blood is preserved. Blood, in contact with paraffin or vaseline, curdles more slowly than if it touches glass or porcelain. It is very probable that the phenomenon of molecular adhesion enters to accelerate the enzymic secretion, but it is necessary to observe that this explanation of the part played by the wall in the coagulation of blood is not exclusive. Bordet and Gengou, in experiments which we shall describe later, have, in fact, shown that plasma, deprived of every cell, is likewise much slower to coagulate in a paraffined vessel than in a glass vessel. It appears that other factors, which we shall study later, enter into this phenomenon.

From the data just given it is concluded that the active coagulating substance comes from the leucocytes. It must, however, be remarked that this constituent of thrombin is also found in the plates. According to Lesourd and Pagnier, the plates are much richer in thrombokinase than the leucocytes. Their part in the coagulation of blood is entirely predominant. A maceration of plates in water coagulates the liquid of hydrocele and furnishes a retractile clot. The blood, separated from the plates by fractional centrifugation, coagulates much more slowly than normal blood. It has also been shown that the content of the serum in thrombin depends on the richness of the plasma in plates. By coagulation of a plasma rich in plates, a serum rich in thrombin is obtained, and the absence of plates in a plasma leads, on the other hand, to a serum poor in active substance. Thus it is that non-coagulability, or rather the very slow coagulation of a bird or fish plasma, which is usually explained on the hypothesis that their leucocytes permit with difficulty the diffusion of their active substance, must actually be referred to the lack of plates in this liquid. According to Bordet, who has studied more closely the nature 
of the active substances of the plates, thrombokinase from the latter does not differ at all from that taken from the leucocytes, except as to the quantity secreted. Furthermore, the tissue extracts, such as the peptone solutions coming from flesh previously neutralized, likewise contain the same substance.

It is due to this latter circumstance that bird-blood collected without precaution coagulates very rapidly. In fact, in this case there occur two superimposed reactions, the one, very slight, due to the thrombokinase of the leucocytes; the other, very strong, which results from the thrombokinase secreted by the broken tissues. Wooldridge has noted, in fact, that the addition of various extracts of an organ to blood hastens coagulation. Delezenne, using the blood of birds, and Spangaro, the blood of mammals, have shown that contact with the wound, or, better, mixture with the juice of tissues, for example crushed muscular tissue, favors in a very pronounced manner the curdling of blood, due to the contribution of thrombokinase.

We now possess rather precise data on the accelerating components of these tissue extracts, as well as on that of the plates. This material, which is thrombokinase, is diffused from the plates into the plasma rather slowly. The plates, diluted in water, give up its active substance only with difficulty. Diffusion, however, increases with the salt concentration, and a salt plasma furnishes, after being centrifuged, a serum very rich in thrombokinase. The thrombokinase of the plates can be heated to $100^{\circ}$ without losing its activity. However, it is considerably weakened by heating to a temperature of $120^{\circ}$. Muscle extract acts almost in the same way. The thrombokinase of muscle or of the plates dissolves in alcohol, petroleum, and chloroform, as well as in acetone solution. Toluene dissolves this substance when it has been previously treated with absolute alcohol.

To isolate thrombokinase from the muscle, Bordet and Delarge propose the following method: The muscles are dried in vacuo over metallic potassium. They are then extracted with toluene to remove the fatty material. The product thus 
freed from fat is treated with absolute alcohol and the filtrate is evaporated. The residue so obtained rapidly coagulates bird plasma and also considerably hastens the coagulation of freshly extracted blood. Thrombokinase contains lecithin and there is every reason to believe that its activity is due to this substance. In reality, every reagent which precipitates or decomposes the lecithin when added to plasma prevents coagulation. Quinine and cocaine, which precipitate lecithin, show themselves to be anti-coagulants, whereas morphine and atropine, which do not act on lecithin, do not influence coagulation. Tests made with commercial lecithins have also shown that they actually possess to a high degree the power of accelerating coagulation.

These facts concerning the nature of thrombokinase just mentioned indicate the presence of a lipoid containing phosphorus or phosphatide, which is largely distributed in all organic extracts, as well as in the plates, and is also present in the leucocytes. This substance lacks all properties of enzymes. It is, however, indispensable in the process of the coagulation of blood.

What rôle then does this thrombokinase play in the coagulation? The plasma contains an active substance which is destroyed at $56^{\circ}$. This substance, serozyme, possesses the properties of an enzyme, but it is deprived of the ability to produce coagulation. It is the union of this principle with thrombokinase which produces active thrombin. Serozyme, contained in the plasma, is carried down with a barium sulphate precipitate as well as by other precipitates. A plasma deprived of serozyme is incapable of coagulating, even after the addition of thrombokinase. The combination, serozyme and thrombokinase, requires the presence of calcium salts to react. Under favorable conditions, and in presence of these three constituents, thrombin is formed very rapidly, but this reaction is not instantaneous. Serozyme is always abundant in the plasma. Coagulation, more or less rapid, will depend on the presence of calcium ions, as well as thrombokinase. In the serum, one finds, besides thrombin as such, either an excess of serozyme, or an excess of thrombokinase. The serum obtained by the aid of perfect blood contains none or 
very little serozyme but prolonged contact with the plates has brought an excess of thrombokinase, which removes all the serozyme of the blood. On the contrary, the serum obtained from a plasma deprived of plates will always be found rich in serozyme, provided the latter does not possess sufficient thrombokinase. It has been, in fact, experimentally shown by Bordet and Delange that serozyme, once combined with thrombokinase, no longer reacts with the latter.

We have mentioned previously that one can also prevent coagulation by various chemical means. The addition of alkali oxalates in small quantities, or that of neutral salts $(\mathrm{NaCl}$, $\mathrm{MgSO}_{4}$, etc.) in large quantities, leads to this result. By these means the blood becomes non-coagulable because the constituents producing thrombin cannot combine. In the case of salts, it is the strong ion concentration in the liquid which is of influence, the salt plasma coagulating upon dilution. The action of the oxalates results from the fact that oxalates remove the calciumions, which is also an indispensable element for the curdling of blood. It is the mechanism of this action that we shall study more closely in the following chapter.

\section{$\S \mathrm{VI}$.}

\section{Action of Calcium Salts.}

Hammarsten, as well as Green, has determined that in coagulation of blood, lime salts play a very important part. Green has shown that the plasma of horse blood, dialyzed with physiological salt solution so that it loses the whole of its calcium salt while keeping $0.6 \%$ of $\mathrm{NaCl}$, may be kept for a long time without clotting, while coagulation is produced rapidly if there is added to the liquid a very slight quantity of calcium sulphate. Arthus and Pagès later prepared a plasma equally non-coagulable by adding to blood freshly prepared potassium oxalate. This salt precipitates the calcium, with which blood is always sufficiently provided, and the liquid obtained by centrifuging does 
not coagulate. However, it still contains the essential constituents for thrombin, for it is only necessary to add a little $\mathrm{CaCl}_{2}$, in an amount slightly higher than that necessary to precipitate the remaining oxalate, to cause curdling. Pekelharing and Hammarsten have observed the same phenomenon with macerations of certain organs. These extracts are inactive towards fibrinogen, but they act on it as soon as $\mathrm{CaCl}_{2}$ is added. These data on the function of calcium and on the absolute necessity of its presence have been thoroughly discussed. It is, in fact, often very difficult to remove entirely this element, and the coagulating enzyme shows in this respect a much greater sensitiveness than our chemical reagents. To prevent the formation of thrombin it is necessary to remove the calcium ions very rapidly from solution. Under all the experimental conditions where the calcium oxalate does not appear at once in the oxalated plasma, or where the precipitate is only partially formed, coagulation takes place, though the quantity of oxalate added would necessarily lead to the conclusion that the solution has been deprived of its calcium ions.

Thus, in presence of $\mathrm{MgCl}_{2}$, oxalated blood is coagulated by the addition of a trace of calcium salts, insufficient to precipitate the excess of oxalate. It is known, in fact, that in presence of magnesium chloride, traces of calcium salts do not precipitate as oxalate. Likewise, the addition of a certain volume of a solution of sodium oxalate to a salt plasma, which is then diluted, prevents coagulation, while the addition of a similar amount of oxalate to the same plasma, previously diluted, does not prevent it, because, in the first case the calcium oxalate is formed, although in the second this salt, in a more dilute solution, has not precipitated.

After having determined that the anti-coagulating power of oxalates is due solely to the fact that these salts make calcium insoluble, we were much surprised to find that sodium fluoride, from this point of view, does not behave - or at least does not appear to behave - in the same manner as the oxalates.

Experiments by Arthus and Pagès have indicated that the 
addition of $3 \% \mathrm{NaF}$ solution to blood direct from blood-vessels prevents coagulation, but that the final addition of $\mathrm{CaCl}_{2}$, even in large proportion, does not cause coagulation. However the fibrinogen has not been changed, for if one adds to the fluoride plasma fibrinogen coming from a normal serum, curdling is produced. To explain this difference between the oxalated plasma and the fluoride plasma, these experimenters believe that, while the first contained thrombin, the second was deprived of it and that its absence was due to the addition to the blood of fluoride, which is a cell poison, killing the leucocytes and thereby preventing the secretion of enzyme.

Bordet and Gengou have also taken up this study and have shown that this hypothesis as to the absence of enzyme in the fluoride plasma was not necessary to explain its non-coagulability. First of all, they determined that salt plasma of a rabbit, deprived of cells but still containing all the constituents of thrombin, behaves, in the presence of $\mathrm{NaF}$, exactly as normal blood, and that, as a consequence, the true cause of the anticoagulating power of the fluoride does not consist in the toxicity which it shows toward the cellular component. Fluoride, like oxalate, is a decalcifying agent and it is this precipitation of the calcium ions that makes plasma non-coagulable. But why is it that on the final addition of $\mathrm{CaCl}_{2}$, coagulation no longer takes place, as is the case with oxalated plasma? This difference comes from the fact that calcium fluoride, in being precipitated, carries with it, to a more or less extent, not only fibrinogen but also serozyme, the latter even more completely than fibrinogen.

A dilute oxalate plasma, and therefore non-coagulable, is prepared by mixing 7 c.c. $5 \%$ salt plasma, 28 c.c. distilled water, and 5 c.c..$\%$ sodium oxalate solution. In tube $(A)$ put ro c.c. of this dilute plasma +2 c.c. of a turbid emulsion of $\mathrm{CaF}_{2}$ in water. In tube $(B)$ place ro c.c. of this same plasma +2 c.c. of the supernatant liquid obtained by centrifuging and decanting an emulsion of $\mathrm{CaF}_{2}$. The tubes are allowed to stand for several hours, then the two mixtures are centrifuged and the clear 
liquids decanted. It is then found that the first liquid $(A)$, which has been in contact with calcium fluoride, with addition of $\mathrm{CaCl}_{2}$ or thrombin, does not coagulate, and when heated to $60^{\circ}-65^{\circ}$, it is no longer turbid. The fibrinogen as well as the active substance has been carried down by the precipitate. On the other hand, the second liquid $(B)$, which has not been in contact with the calcium fluoride, is coagulated by addition of $\mathrm{CaCl}_{2}$, or of serum, and when heated to $60^{\circ}-65^{\circ}$ becomes very turbid.

This experiment then explains the two following facts: A plasma, that contains just sufficient fluoride to render it noncoagulable, curdles upon dilution, in fact the precipitate of $\mathrm{CaF}_{2}$, which was first formed, and which is strictly insoluble only in a weak solution of $\mathrm{NaF}$, is in part redissolved and has thus permitted the enzyme to act. On the other hand, if $\mathrm{CaCl}_{2}$ is added to a fluoride plasma, no coagulation results, for there is produced a great quantity of calcium fluoride, which eliminates all the serozyme which remained after the first precipitation. In place of favoring coagulation the addition of calcium chloride tends to check it.

Thus the anti-coagulating power of fluoride is of the same nature as that of oxalate, both acting by precipitating calcium ions. However it should be noticed that not only is it necessary that this compound be found in the plasma in a soluble state, but also, as Sabbatani has shown, it is necessary that soluble calcium salts should be sufficiently dissociated into ions to play their proper rôle in coagulation. It is because they diminish the degree of ionization of the calcium salts normally contained in the blood, that strong salt concentrations $\left(\mathrm{NaCl}, \mathrm{Na}_{2} \mathrm{SO}_{4}\right.$, $\mathrm{MgSO}_{4}$ ) prevent coagulation. It is probably for an analogous reason that alkali citrates $(0.2 \%-0.3 \%)$ are equally effective. These salts, in fact, do not precipitate calcium salts and cannot be properly considered as decalcifiers. They must act by forming with the calcium ions a soluble complex not undergoing dissociation. 


\section{$\S$ VII.}

\section{PRO-THROMBIN.}

Conditions for the Transformation of the Pro-enzyme into Active Enzyme.

We have just seen that in the coagulation of blood, calcium ions play an indispensable part. Let us now seek to discover in what way these ions react. Three hypotheses have been successively presented. The first asserted a superimposed action of the two coagulating agents, the enzyme and the calcium ion. The transformation of fibrinogen into fibrin would be produced by each of the two factors, and coagulation would appear as the result of their combined action. The second hypothesis attributed to the calcium ion a double rôle. First, it permitted the action of thrombin on fibrinogen, and then it entered into the constitution of the fibrin formed. These first two explanations of the functioning of the calcium ion have been abandoned, for they do not agree with experimental data, which, on the contrary, are very favorable to the third conception, which regards calcium ions as the generators of thrombin.

First of all it is an established fact that the action of calcium in coagulation is not instantaneous. The contact must be prolonged for a certain time, and if this element is eliminated too soon, no result will be obtained.

It even appears from the following experiment that this action takes place sharply, though after a certain lapse of time. To show this, Bordet and Gengou make use of a $5 \%$ salt plasma, which they bring to a $\mathrm{NaCl}$ content of $\mathrm{I} \%$ at the moment of using by the addition of 4 volumes of distilled water. In such a plasma, diluted and salty, coagulation is very slow and the phenomenon can easily be followed. Let $\frac{9}{10}$ c.c. of this plasma, freshly prepared, be placed in several tubes. One of the tubes is kept without any further addition; the others receive, at the end of various times $\left(2,10,20,30,35 \mathrm{~min}\right.$.) $\frac{1}{10}$ c.c. of a $1 \%$ solution of sodium oxalate. Thus the calcium is eliminated after having prolonged its action for a greater or less time. The 
tubes where coagulation takes place are then examined with the following results:

Action of Calcium ions in Coagulation.

Liquids

Appearance of coagulation

Ist. Salt dilute plasma (control) $\ldots \ldots \ldots \ldots \ldots \ldots$ At the end of $40 \mathrm{~m}$.

2nd. " " " add. oxal. after $2 \mathrm{~m} . . . . . .$. . No coagulation.

3rd. " "

4th. " "

5 th. " "

6 th.

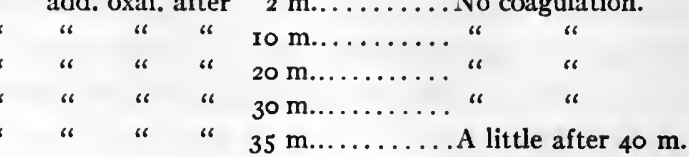

In the control experiment (No. r), where the calcium has not been eliminated, coagulation occurs at the end of 40 minutes. In test No. 5 the calcium is eliminated after $30 \mathrm{~m}$., and no curdling is observed. Then, even after $30 \mathrm{~m}$., the action of calcium ions has been zero or insufficient, thrombin is not formed, and up to this point the precipitation of this element still renders the plasma non-coagulable. On the other hand, after $35 \mathrm{~m}$., the calcium has produced its effect, and $5 \mathrm{~m}$. is enough then to bring about the clotting of the liquid. As, in this last test; coagulation has not been appreciably longer than in the control test where the calcium has not been eliminated, it must be admitted that this element has exercised its action between the thirtieth and the thirty-fifth minute and that, during this short time, it has produced all its effect.

Furthermore, as Pekelharing has shown, oxalate plasma, with addition of serum coming from a normal coagulation, which is consequently rich in thrombin, coagulates, even though oxalate was added to the serum before the latter was added to the plasma. Therefore the calcium ions, which are necessary at a given moment, are not necessary at others. It has been thought possible to explain this anomaly by supposing that the enzyme concerned is not, properly speaking, a coagulating enzyme but a pro-enzyme, a pro-thrombin [or Thrombinogen: Editor]. This, to become active and to be transformed into thrombin, would require the aid of calcium ions. Once the transformation is produced, the ions would be of no further use. It can be imag- 
ined, therefore, that a fibrinogen, free from calcium, can nevertheless coagulate under the influence of a thrombin likewise deprived of this element, if, unknown to the experimenter, the enzyme has been able previously to form with the assistance of traces of calcium salts. We shall see later that the rôle of calcium ions in the activation of the pancreatic juice presents a certain analogy with what is observed here, both from the standpoint of the opportuneness of this substance as well as the course of the phenomenon.

To summarize, calcium ions necessary for the appearance of thrombin do not take part at once in the actual coagulation. Such, however, is not the opinion of Stassano and Daumas, who have shown that in the coagulation of blood and of lymph, calcium ions play a double rôle and act successively at two different periods in the phenomenon.

In the first place, calcium is indispensable in the formation of thrombin, and it acts on the two albuminoid generators of the latter, of which we have already spoken, thrombogen (or prothrombin) and thrombokinase, The following are some details which define more clearly this interaction. The authors prepared a $5 \%$ salt plasma which they deprived of all its calcium salts by a dialysis of 20 days or thereabouts on a $5 \%$ solution of $\mathrm{NaCl}$, the latter being frequently renewed. The plasma thus obtained, diluted with water, is no longer spontaneously coagulable. It requires the assistance of a calcium salt in order to clot, but - and this is the characteristic point - this assistance must be given under definite conditions and in definite proportions.

The addition of very slight amounts of $\mathrm{CaCl}_{2}$ to salt plasma, completely dialyzed as above, and then diluted to 3 vol. in such a way as to bring its concentration to $1.25 \%$ (a concentration insufficient to prevent the coagulation of an ordinary plasma), is followed by no external manifestation, the plasma preserving its limpidity and all its fluidity. But it is only necessary to add to it, some hours later, a considerably larger quantity of $\mathrm{CaCl}_{2}$, $\mathrm{SrCl}_{2}$, or $\mathrm{BaCl}_{2}$, to make it coagulate considerably in advance 
of the control samples of this same plasma, - controls which were prepared by adding to the plasma the same total amount of calcium salt as soon as the salt plasma was diluted.

The most favorable proportion of $\mathrm{CaCl}_{2}$ for this internal action corresponds to about $0.000,02 \mathrm{I}$ g. per c.c. of plasma. The addition of less than $0.000,013 \mathrm{~g}$. of $\mathrm{CaCl}_{2}$ is without effect. Above this optimum quantity, coagulation is produced only with a considerable retardation. It is due to this internal action, caused by a very small amount of calcium, that the thrombin appears, but this action is not in evidence unless a much greater amount of calcium salt is added to the plasma, coagulation then taking place very rapidly. This same action can be revealed in still another way by comparing the effect upon the peritoneal fluid of a horse (spontaneously non-coagulable) of two samples of the same salt plasma, completely dialyzed and diluted, one of which has received the optimum trace of $\mathrm{CaCl}_{2}$ several hours previously and the other of which receives it only at the moment of the control test. Under these conditions it happens that the first sample coagulates the fluid in much less time than the second, sometimes by more than 2 or 3 hours. At this same optimum molal concentration $\mathrm{SrCl}_{2}$ and $\mathrm{BaCl}_{2}$ also act, but much more slowly, especially the latter. The rôle of calcium ions is not absolutely exclusive therefore, but it shows itself much more energetically.

Calcium, according to Stassano and Daumas, aids also in the action of thrombin on fibrinogen, but this time, instead of acting in infinitesimal amourts, it assists only when present in rather large proportions. These authors show this by the following experiment. Serum, freshly extracted from a clot of blood, contains thrombin already formed. On the other hand, the peritoneal fluid of the horse, perfectly non-coagulable, can be considered as a solution of fibrinogen. If several drops of the first liquid are added to the second, the mixture coagulates at the end of a certain time, varying from several minutes to several hours according to the proportions and the quality of the serum added. Now this moment arrives 
much sooner if $\mathrm{CaCl}_{2}$ or some other chloride is added to the mixture. The optimum concentration for each of these corresponds to 0.133 c.c. of a $\frac{1}{10} N$ solution per I c.c. of diluted salt plasma, or $0.00 \mathrm{I}, 48 \mathrm{~g}$. $\mathrm{CaCl}_{2}$ per I c.c. of plasma. The other chlorides likewise act at this molal concentration and are, in order of activity, $\mathrm{SrCl}_{2}, \mathrm{BaCl}_{2}, \mathrm{KCl}$, and finally $\mathrm{MgCl}_{2}$. It is also possible to show the accelerating action of calcium ions by use of undialyzed salt plasma - this would coagulate spontaneously in two hours after dilution. On the contrary, it clots in only a few minutes, following the addition of $\mathrm{CaCl}_{2}$ in the proper concentration.

However, it must be noted that, if it is true that calcium takes part in the two phases of coagulation of blood, it does not appear to be really indispensable save in the first part, or that which corresponds to the transformation of the pro-enzyme into active thrombin. Its accelerating action in the transformation of fibrinogen, in presence of the enzyme already formed, could perhaps arise quite simply from the fact, as we shall see later, that $\mathrm{CaCl}_{2}$ is capable of destroying the antagonistic substances which appear in a serum even after only an hour of keeping, thus weakening very rapidly the coagulating power. Calcium ions, in decomposing these, reactivate the serum used, but their effect would be only indirect. However this may be, it is evident from this exposition that the action of calcium ions, known in its broad outlines, still presents many obscure points, and that this important question still calls for new investigations.

Let us now see some of the other conditions under which the combination of serozyme with thrombokinase takes place. First of all, it appears from the experiments of Bordet and Gengou that in oxalate plasma the serozyme does not exist in the functional state, but that it acquires the property of reacting on the cytozyme or thrombokinase only after a kind of activation. Further, it is known that that which delays clotting is the formation of the thrombin, the transformation of fibrinogen into fibrin being very rapid. If to salt plasma just diluted, normally requiring about 40 minutes to coagulate spontaneously, a little 
serum rich in thrombin is added, it is found that coagulation takes place in a few minutes.

This formation of thrombin is moreover greatly influenced by the other physical and chemical conditions. Thus, due to the action of cold, at $0^{\circ}$ plasmase would appear much less quickly than at $30^{\circ}$. To show this, it is but necessary to prepare with $3 \%$ salt a plasma, which is diluted so as to bring its $\mathrm{NaCl}$ content to $1 \%$, one sample of which is then kept at $30^{\circ}$, the other at $0^{\circ}$. At the end of 17 minutes, the first curdles. The second, after 37 minutes of stay in the ice, is still liquid. Upon adding $0.1 \%$ of oxalate it is found that even when replaced in the incubator at $30^{\circ}$, it remained non-coagulable. Thrombin was not yet formed. In fact a third sample, similar to the other two, kept entirely at $0^{\circ}$, would coagulate in $\mathrm{I}$ hour and $\mathrm{I} 5$ minutes.

Concerning the influence of the conditions of the medium on the production of thrombin, very little is really known. We have already seen that the presence of sufficient quantities of neutral salts hindered the coagulation of blood. In particular, $5 \% \mathrm{NaCl}$ (Hewson), and even $2 \%$ (Bordet), proves efficient, not because it is opposed to the secretion of a pro-enzyme, as was believed, but because it checks the transformation of this pro-enzyme into the active enzyme. In fact dilution of the salt plasma, separated from the cells, causes it to curdle. A concentration of $0.8 \%$ to $1.25 \% \mathrm{CaCl}_{2}$ only retards the reaction. That strong salt concentrations ast by repressing the dissociation of the soluble calcium salts naturally contained in the blood, and thus retard the appearance of the plasmase, has already been noted.

\section{Effect of Contact.}

The blood presents a very curious characteristic as regards the nature of the walls of the receptacle in which it is kept. The fact has already been noted that blood collected in a vessel whose walls have been previously oiled, vaselined, or coated with paraffin coagulates much more slowly than if it is received in a clean glass vessel. As regards the origin of the coagulating 
enzyme, it has been stated that it was probable that this difference of result came from the fact that the glass, by its contact, produced on the blood cells a special mechanical excitation, which was not produced by fatty or paraffined surfaces, and that from this different mechanical action resulted a secretion of thrombokinase much greater in the one case than in the other.

In addition, it has been found that plasma, like blood, is sensitive to the influence of the walls. While rabbit plasma, completely deprived of corpuscles, coagulates in a few minutes in glass, it can be kept for 24 to 30 hours before curdling if it has not been subjected to contact other than that of paraffin. Still liquid after 20 hours, for example, it coagulates instantaneously if it is touched with glass. Contact with a body capable of being wet, such as glass, acts, not only on the cells, but also on the substances which control coagulation, that is to say on fibrinogen, thrombin, or its constituents. We have to deal, here, with a non-biological phenomenon, this time a physico-chemical one, apparently of the nature of molecular adhesion.

In studying more closely the rôle of glass in coagulation, one finds that this body acts by accelerating the production of thrombin, in other words the absence of coagulation in paraffined tubes is due to the fact that the enzyme is not formed, and not that this principle, after having appeared, has been paralyzed through some agency. If the coagulation of a diluted salt plasma (which is known to coagulate more slowly than pure plasma) is studied, the plasma being placed in a glass vessel which is not agitated, it is found that coagulation appears at the end of about a half-hour, starting at the wall, and in such a way that a real sheath of solid fibrin is formed about the plasma which still remains liquid in the center of the vessel. It is observed that coagulation begins at the precise spot where the immediate contact of the glass would most actively influence the liquid, that is to say at the edge of the meniscus.

If, in a liquid plasma kept, for example, in a paraffined watch crystal, a modification of the surface tension in certain places is produced, either by touching the liquid with glass 
rods or by throwing on it some powder or fine drops of water, it is found that coagulation rapidly occurs about these points of contact. However, the surface tension, when the active surface is increased, can act in the manner of a contact. Thus it is that by agitating dilute salt plasma for several seconds in such a way as to cause the formation of bubbles, giving to the surface of the liquid a persistent froth, coagulation is very plainly accelerated. This begins with the bubbles, the froth becoming solid, and soon a curdling is communicated to the underlying liquid.

If dilute salt plasma is preserved in a glass vessel, and, at the moment when the liquid begins to coagulate, or after about 30 minutes, is stirred with a glass rod, almost instantaneous curdling results. The production of thrombin, under these conditions, takes place in an explosive manner. The phenomenon is rather complex, the velocity of the reaction being the result of several coincident causes: I. It scatters through the mass the small quantity of plasma already formed along the walls, and the enzyme which remained is removed by the fibrin that has appeared; 2. The contact with glass is extended and thus the appearance of a fresh quantity of thrombin is hastened; 3. Finally, in the case where the plates still remain in the plasma, their reappearance in the mass of liquid favors clotting by simple contact.

It is easy to show that this influence due to the nature of the walls does not affect fibrinogen, but only the combination of the constituents of the active thrombin. It is known, in fact, that fibrinogen is slightly more sensitive to heat than serozyme. If salt plasma, freshly diluted with $\mathrm{r} \% \mathrm{NaCl}$, is heated for Io minutes at $54^{\circ}$, the liquid becomes slightly opalescent and no longer coagulates, due to the change in the fibrinogen. Thus results a non-coagulable plasma, but one in which, since the serozyme is not destroyed, the production of thrombin follows gradually its usual course.

When this liquid is kept in a glass vessel, at the end of about 45 minutes it acquires the property of coagulating oxalate 
plasma. On the other hand, if it is kept in a paraffined receptacle, the spontaneous production of thrombin is prevented.

To summarize, the rôle of paraffin in coagulation of plasma is purely passive. This substance, not being wet by the blood liquid, does not influence the formation of thrombin, but it does not prevent this taking place if brought about through another agency. In other words, it does not hinder the decomposition of fibrinogen if the thrombin is already formed. Thus it is that coagulation of a dilute salt plasma by the addition of fresh serum, rich in thrombin, takes place as quickly in a paraffined vessel as in glass.

In all these experiments, it is necessary that the paraffin be rigorously incapable of being wet by the plasma, and that this rolls on the wall coated with this substance in the manner of mercury on glass. There are good and bad samples of paraffin and Bordet recommends the use of a mixture of I part paraffin and of 2 or 3 parts liquid vaseline.

It is almost superfluous to add that glass plays no specific rôle and that any other substance capable of being wet, such as porcelain or platinum, may replace it.

\section{$\S$ VIII}

\section{Phenomena of Anti-coagulation.}

I. Antagonistic Serums of Bordet. - The phenomena of anti-coagulation may be observed in many instances. One of them depends on the existence of antagonistic serums. Bordet and Gengou, in fact, have shown that an animal $a$ of a species $A$, a guinea-pig, for example, injected several times with plasma or serum of an animal $b$ of a different species $B$, such as a rabbit, furnishes a serum which neutralizes in vitro the thrombin of the serum of the animal $b$, and therefore renders the latter incapable of causing the clotting of a plasma not spontaneously coagulable, that of the goose, for example, prepared as previously described.

In order to show the antagonistic substance contained in 
the serum of a guinea-pig so treated, first of all this serum is heated to $58.5^{\circ}$, a temperature at which the thrombin which it normally contains will be destroyed. Then, upon adding fresh rabbit serum which has not been so treated, it is found that the mixture is without action on the goose plasma. In comparison, the following control experiment is made. To fresh guinea-pig serum, heated to $58.5^{\circ}$, is added fresh rabbit serum which has not been heated. This time the mixture quickly coagulates the goose plasma. The fact is that in the first case the thrombin of the rabbit had been rendered inactive by the prepared guinea-pig serum, while in the control experiment the thrombin of the rabbit, not neutralized, had been able to act freely. The guinea-pig serum so treated contains, then, an antibody for the thrombin of the rabbit, an antibody which resists heating to a temperature of $58.5^{\circ}$. To entirely remove the coagulating power from I part of fresh rabbit serum requires, however, a fairly strong quantity, about 6 parts, of treated guinea-pig serum.

Bordet and Gengou have recognized that this neutralizing action is clearly specific, though not to an absolute degree. This fact is all the more remarkable since the thrombin is not itself specific, rabbit serum being able to coagulate equally well the plasma of a guinea-pig, goose, sheep, or dog. This apparent contradiction, however, is explained by assuming that the fibrinogen of these various plasmas, rabbit, goose, etc., is not capable of showing the difference which really exists between the thrombin of various origins, while the organism, by means of a more sensitive analysis, can, by means of a vital action, show the specificity of thrombin by elaborating antagonistic substances capable of adapting themselves singly to each enzyme.

In conjunction with these clearly anti-enzymic actions, there are similar phenomena coming from properties of precipitins. Thus it is that the serums of animals $A$, specially prepared with plasma of species $B$ (and not with serum), precipitate the plasma of species $B$. According to Camus, the anti-coagulating action of these serums on plasmas results from 
the fact that, by precipitating fibrinogen, they have removed its capacity for coagulation. Although this interpretation rests on an exact observation, it is probable that the serums hinder coagulation more in that they eliminate the thrombin than in that they transform fibrinogen.

2. Anti-coagulating Action of Extract of a Leech's Head, of Peptone: Thrombase. - Duclaux has given the name of thrombase to a de-coagulating enzyme, i.e., capable of redissolving the coagulum, or thrombus, formed in the vessels. In reality, this enzyme has not been studied from this direction, but simply by reason of the fact that its action opposes that of thrombin. It is rather then an antibody, an anti-thrombin. However, this thrombase of Duclaux, which comprises a certain number of substances capable of preventing coagulation, does not correspond to a well-defined enzyme. Moreover, in view of the fact that various writers have wrongly used this name of thrombase to designate thrombin, a coagulating enzyme, it seems best definitely to abandon this term, which, as we have just said, does not even answer to the conception of Duclaux, on which rests all his nomenclature of enzymes. We shall, therefore, adopt, for all of these antibodies, the name of anti-thrombin or anti-coagulating substances.

A certain number of substances of organic origin are known, capable of opposing in vitro the coagulation of blood. The best known is extract of leech's head. Haycraft first found that the buccal secretion of leeches contains an anti-coagulating substance, which he isolated. Leeches' heads are macerated in alcohol in order to dehydrate them. Then they are reduced to powder, and the product is macerated in water and filtered. The liquor obtained possesses the property of preventing plasma from coagulating. This anti-thrombin can withstand prolonged boiling without being destroyed. However, heated to $140^{\circ}$, it loses its activity. The active substance drawn from leeches is not specific and is effective with every kind of plasma; it acts in vitro, but, injected into the circulation, it likewise renders the blood non-coagulable. 
A similar property has been detected in the juice of crab's liver, in the product of the papainic digestion of the liver, and in the extracts of certain organs. Lilienfeld has detected the presence of anti-coagulating substances in bird's blood, in the red corpuscles, in the leucocytes, and in the lymphatic ganglia. Finally, the histone of Kossel likewise possesses this power of arresting in vitro the clotting of blood.

In addition to these substances, there are others which, after injection into an organism, render the blood of the latter non-coagulable. This type of body is the product commercially designated under the name of peptone, which is known to be a mixture of different products of peptic hydrolysis. If an intravenal injection of Witte's peptone is made into a dog, 0.2 to $0.3 \mathrm{~g}$. of peptone per $\mathrm{kg}$. weight of animal, it is found that the blood which is collected can stand for a day without coagulating. This fact, discovered by Albertoni in 1878 , has been studied more in detail by Fano, Conte Jean, and others. It should be noted that the same amount of peptone in vitro gives no result. One can, however, obtain with this product a retarding action, but very large quantities have to be used. The anti-diphtheria serum of Roux, certain extracts of the organs like the juice of fresh muscle, and the alkaloid atropine, act, as Doyon has shown, in a manner similar to peptone. However, atropine added to the blood in vitro is without effect.

This result is due to the production in the organism of an anti-coagulating substance, for the addition of this blood or of its plasma to normal blood hinders coagulation of the latter: Delezenne has recognized that the anti-coagulating substance, which is thus formed under the influence of peptone, endures a temperature of $100^{\circ}$. Gley and Pachon have shown that the seat of the formation of these antibodies is in the liver. The injection of peptone after a destruction or extirpation of the liver is without effect, and, furthermore, as Delezenne has found, artificial circulation through the liver of a serum, with addition of peptone or organic extract, furnishes, on leaving the liver, a liquid possessing energetic anti-coagulating power. 
Anti-coagulating Power of a Serum with Addition of Organic Extract, after Passage Through the Liver.

\begin{tabular}{|c|c|c|}
\hline \multirow[b]{2}{*}{ Circulating mixture. } & \multicolumn{2}{|c|}{$\begin{array}{l}\text { Time of coagulation of a solution } \\
\text { of fibrinogen. }\end{array}$} \\
\hline & $\begin{array}{c}\text { Mixture which has } \\
\text { not passed through } \\
\text { liver. }\end{array}$ & $\begin{array}{l}\text { Mixture passed } \\
\text { through liver. }\end{array}$ \\
\hline $\begin{array}{l}\text { Io serum + I extract.. } \\
\text { I3 serum + } 1 \text { extract. } \\
20 \text { serum }+ \text { i extract.. } \\
40 \text { serum }+ \text { i extract.. }\end{array}$ & $\begin{array}{ll}3 & \text { minutes } \\
3 \frac{1}{2} & \text { minutes } \\
4 & \text { minutes } \\
4 & \text { minutes }\end{array}$ & $\begin{array}{l}2 \text { days } \\
40 \text { hours } \\
\text { I } 2 \text { hours } \\
3^{\frac{1}{2}} \text { hours }\end{array}$ \\
\hline
\end{tabular}

Thus, whereas a mixture of Io serum $+\mathrm{I}$ extract causes coagulation of a hydrocele liquid in 3 minutes, on the other hand, if made to circulate through the liver, it no longer produces the curdling of this same solution of fibrinogen in less than 2 days' time. The production of an anti-coagulating substance is manifest, and it is all the greater, the stronger the proportion of extract with respect to the serum.

3. Anti-coagulating Substances in the Serum. - When serum is kept for some time, it is found that its coagulating power weakens very rapidly. Pekelharing has followed the diminution of the quantity of active thrombin in a serum by measuring the time necessary for the coagulation of a certain volume of a solution of fibrinogen, to which has been added the same quantity of serum, taken after different intervals of standing.

Loss of Coagulating Power of a Serum with Time.

\begin{tabular}{|c|c|}
\hline . & $\begin{array}{l}\text { Duration of coagula- } \\
\text { tion of the solution } \\
\text { of fibrinogen. }\end{array}$ \\
\hline $\begin{array}{l}\text { Fresh serum..... } \\
\text { Serum I day old. }\end{array}$ & $\begin{array}{l}\ldots \quad 3 \mathrm{~min} . \\
\ldots . \\
\text { ro } \min .\end{array}$ \\
\hline
\end{tabular}

Thus serum, 6 days old, has a coagulating activity only one-fifth as strong as when it is fresh.

Bordet and Gengou have confirmed this fact and have even found a much greater change. These investigators make use 
of a serum obtained by the dilution and spontaneous coagulation of salt plasma, and take oxalate as reagent for the coagulating power. To different tubes containing plasma diluted with $1 \%$ $\mathrm{NaCl}$ and made one-tenth per cent in oxalate, there is added, after differing intervals of time, the same quantity of serum coming from a coagulation made at the beginning of the experiment. Further, 7 minutes before mixing, $0.1 \%$ of oxalate is added successively to the different previous preparations of serum. It is then found that the coagulations are slower, the older the samples of serum used.

Weakening of Coagulating Power of a Serum with Time.

Plasma + serum coming from a coag. dating from $\frac{1}{4} \mathrm{~h}$. Coag. less than $3 \mathrm{~m}$.

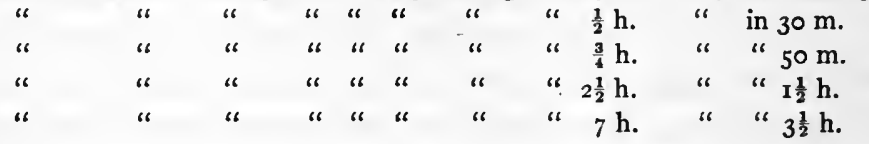

The coagulating power of the serum then weakens very rapidly. This weakening is sometimes so sudden, that keeping for to minutes a freshly prepared serum may suffice to increase tenfold the lapse of time which it takes to coagulate an equal volume of oxalate plasma.

- The disappearance of the thrombin is much more rapid at $37^{\circ}$ than at ordinary temperature.

Influence of Temperature on the Alteration of Serum.

Length of coagulation.

Serum of 5 days kept at ordinary temp............ 30 minutes.

Serum of 5 days kept for 6 hours at $38^{\circ} \ldots \ldots \ldots \ldots \ldots \ldots, 15$ hours.

While serum kept 5 days at ordinary temperature brings about the coagulation of a solution of fibrinogen in 30 minutes, if this same serum has been maintained 6 hours at $38^{\circ}$, clotting takes place only in 15 hours. We see, therefore, how a slight elevation of temperature may enormously injure the preservation of a serum.

We have just seen that the alteration of the serum found by Bordet is much greater and more rapid than that observed by Pekelharing. But these two experimenters worked under differ- 
ent conditions. The first used oxalate plasma as source of fibrinogen, while the second used pure fibrinogen. The diminution of activity of the old serum, indicated by Bordet, may be explained by the great sensitiveness with regard to the precipitates produced therein which the serum acquires in ageing. The new serum resists relatively well the action of calcium oxalate, whereas in an old serum, the precipitate which is formed there carries off and removes a more or less large part of the active substance. The results obtained are, therefore, the consequence of two causes which are superimposed: I. A real alteration; 2. A disappearance of the enzyme by being made insoluble.

However, the alteration due to ageing cannot be attributed to a using up or a destruction of the active substance. In reality, in a serum weakened by time, the enzyme is in some way masked by an antagonistic body. It can be regenerated, either by the addition of calcium salt to the serum, or by treating the serum successively by alkali and acid. The addition of $\mathrm{CaCl}_{2}$ is not very effective. To be sure, it raises again the coagulating power, but it does not bring it back to its original value. On the contrary, very good results are obtained by the second process.

In the following manner it is possible to reactivate an old serum: To roo cc. of serum are added $40 \mathrm{cc}$. of sodium hydroxide $(N / 4)$. This is allowed to act for some time, then $40 \mathrm{cc}$. $\mathrm{HCl}(\mathrm{N} / 4)$ is carefully added to the mixture. In this treatment, it must be remembered that the fibrinogen, in alkaline medium, is destroyed slowly, and that, on the other hand, the serum cannot remain more than 30 to 40 minutes in presence of an excess of alkali without undergoing a change.

Regeneration of an Old Serum by Treatment with NaOH and $\mathrm{HCl}$.

Length of coagulation.

Fibrinogen +6 hour serum..................... 3 r m.

Fibrinogen + the same serum treated................ $4 \mathrm{~m}$.

The regenerated serum again loses with time the power of coagulating fibrinogen and this alteration takes place, according 
to Pekelharing, even more strongly in this case than with an untreated serum.

Comparative Alteration and Regeneration of a Normal Serum and a Treated Serum.

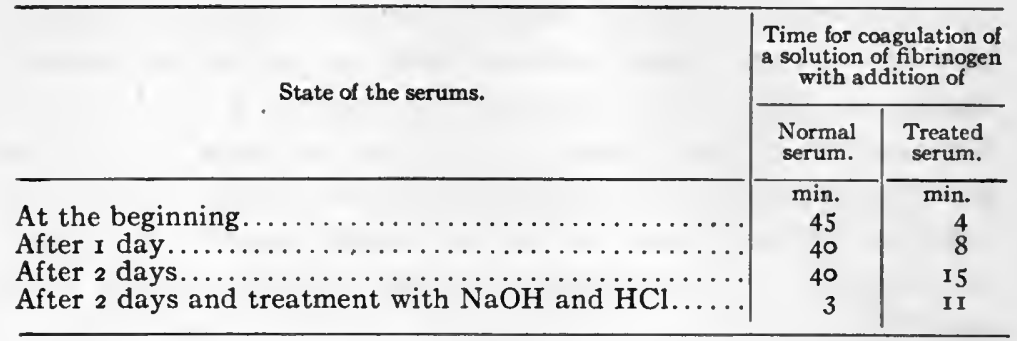

In this experiment, we take on the one hand an old serum, which we call normal serum, whose coagulating power is stationary, since, after I or 2 days additional preservation, the coagulation of a solution of fibrinogen to which it is added still takes place in 40 or 45 minutes. On the other hand, the treated serum is formed by regenerating the normal serum. The duration of coagulation, which was 45 minutes before the addition of the sodium hydroxide and of the acid, has now been reduced to 4 minutes. Then slowly the coagulating power lessens and clottings are accomplished in 4,8 , and ${ }_{5}$ minutes respectively.

At the end of two days, these two serums are reactivated and it is found that the first, which received the treatment for the first time, shows itself very sensitive and returns from 45 to 3 minutes while the second falls to in minutes. The active substance in the second case appears, therefore, to be actually changed.

It should be noted, also, that serum, which has been weakened by the action of prolonged heating at $38^{\circ}$, no longer allows itself to be activated by the treatment with alkali and acid.

action of the Treatment on a Serum Kept at $38^{\circ}$.

Time for coagulation of fibrinogen.

A. Serum of 6 days kept at ordinary temp......... Io minutes.

B. Serum of 6 days and then kept 4 h. at $38^{\circ} \ldots \ldots \ldots$ I 2 hours.

C. Serum of $\mathbf{B}$, which has undergone further treatment. No coagulation after I2 hours. 
How shall we explain these facts? Schmidt, Moravitz and Pekelharing have studied very closely the conditions for revivification of thrombin. For the first two investigators, the results mentioned above are easily understood if it is assumed that the serum contains a certain quantity of pro-enzyme, in addition to thrombin already formed. It is this which would be quickly destroyed. In a serum several days old there is only very little thrombin remaining, but the pro-enzyme is still found in good condition, capable of being activated either by calcium ions, or by treatment with sodium hydroxide and hydrochloric acid. After several treatments there is no more proenzyme and the regeneration of the serum is no longer possible.

Moravitz, from the fact that the addition of calcium ions produces only a rather weak result, while with treatment by alkali and acid the reactivation is much more complete, concludes that there are in the serum two pro-enzymes. He calls the one transformed by the $\mathrm{CaCl}_{2}$ pro-enzyme $(\alpha)$, and designates by pro-enzyme $(\beta)$, or metathrombin, the one activated by alkali.

Pekelharing is of an opposite opinion. To explain the revivification, he claims the presence of antagonistic substances in the serum at the beginning, and their subsequent formation during the course of preservation. The treatment with sodium hydroxide would result merely in the destruction of these injurious bodies which hide the presence of thrombin. This opinion agrees well enough with the fact that old serum, consequently changed, always acts as paralyzer of the active thrombin.

Action of Serum Brought to $38^{\circ}$ on Thrombin.

\begin{tabular}{|c|c|c|}
\hline Quantity of thrombin. & $\begin{array}{l}\text { Quantity } \\
\text { of } \\
\text { fibrinogen. }\end{array}$ & $\begin{array}{l}\text { Time for } \\
\text { coagulation. }\end{array}$ \\
\hline $\begin{array}{r}\text { I. } 3 \text { c.c. }+2 \text { c.c. } \mathrm{NaCl}, \mathrm{N} / 8 \ldots \ldots \\
\text { II. } 3 \text { c.c. }+2 \text { c.c. serum heated to } 38^{\circ} \ldots \ldots \\
\text { III. } 3 \text { c.c. }+2 \text { c.c. serum heated to } 38^{\circ}, \text { then } \\
\text { treated with } \mathrm{NaOH} \text { and } \mathrm{HCl} \ldots \ldots \ldots\end{array}$ & $\begin{array}{r}\text { c.c. } \\
5 \\
5 \\
5\end{array}$ & $\begin{array}{l}30 \mathrm{~m} \text {. } \\
\text { No coagulation. } \\
30 \mathrm{~m} \text {. }\end{array}$ \\
\hline
\end{tabular}


Thus, the addition of 2 c.c. of a serum, heated to $38^{\circ}$, to 3 c.c. of a solution of active thrombin prevents all coagulation. But if the same serum, heated to $38^{\circ}$, has been treated by sodium hydroxide and hydrochloric acid, it no longer exerts any injurious action. This fact is all the more interesting since the serum rendered inactive by heating to $38^{\circ}$ no longer is activated by the alkali treatment, as seen above. The efficacy of the treatment resides in this case exclusively in the destructive action which it exerts with regard to the antibodies contained in the serum, and not at all in the transformation of the proenzyme into enzyme; this is why we find again in test III the same time required for coagulation as in $\mathrm{I}$.

The anti-coagulating action of the heated serum can likewise be observed if, in place of causing it to act on a solution of thrombin, as we have just seen, we use fresh serum. The activity of this latter will be found to be considerably weakened by the antibodies contained in the old serum kept for several hours at $38^{\circ}$.

Action of Serum Heated to $38^{\circ}$ on a Fresh Serum.

Time for coagulation of fibrinogen.

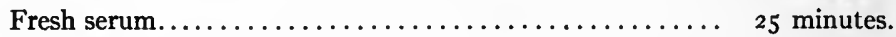

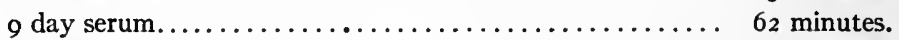

9 day serum heated to $38^{\circ} \ldots \ldots \ldots \ldots \ldots \ldots \ldots \ldots \ldots$ II hours.

Fresh serum +9 day serum heated to $38^{\circ} \ldots \ldots \ldots \ldots \ldots, 45$ minutes.

The addition of old changed serum to fresh serum has therefore retarded the coagulation from 25 to 45 minutes.

The presence of antagonistic substances in the plasma itself and their sufficiently quick formation are considered by certain physiologists as important in the phenomenon of coagulation. According to them, in the circulating blood there is a little thrombin already formed. Of the numerous leucocytes, some are changed, others dead, and these let their enzyme exude, which the calcium ions of the plasma soon make active. How is it, then, that under these conditions the blood does not clot in the blood-vessels? It is because these vessels normally contain anti-coagulating substances. These, while in the 
organism, exert a predominating action. The leucocytes, and in a general way all the cells, contain at the same time these two kinds of substances, some of which favor, some of which hinder the curdling of blood. Those favoring curdling appear to be more specially localized in certain organs, the abdominal viscera for example, and those hindering, in others, such as the lungs. Thus Pawlow has shown that the blood of a dog loses at the end of a short time its coagulability if the circulation is limited to the heart, the large blood-vessels, and the lungs, or again, if only the abdominal viscera are excluded from the circulation.

In man, coagulation of blood normally takes place in I minute 44 seconds. It may, however, in certain pathological cases, be accelerated or much retarded. The diet, certain microbic infections, or inflammation of the wall of the vein from which the blood is extracted, render the blood much more rapidly coagulable. On the other hand, infantilism with insufficient secretion of the thyroid body, diabetes, and tuberculosis of the third degree, give to the blood a relative non-coagulability. These opposite characteristics of the blood would result, according to these same physiologists, from the presence in the plasma of very different proportions of thrombin and of anti-coagulating substances.

The fact just established, namely that there exists in the old serum substances antagonistic to thrombin, appears to be in absolute contradiction to the experiments of Bordet and Gengou, who recognized, on the contrary, in old serum the power of considerably accelerating, in a plasma not deprived of calcium salts, the transformation into active enzyme of the pro-enzyme which this plasma contains, a property which they call excitoproduction.

The following is one of their experiments to show this activating action of a serum, 5 hours old, towards a salt plasma diluted to $\mathrm{I} \%$. In tests (I) and (2) dilute salt plasma is used; in test (3) the same plasma is used, but oxalated, in order to remove the constituents of the thrombin present in this plasma. 


\section{Excito-productive Action of an Old Serum.}

(Bordet and Gengou.)

I. Dilute salt plasma $(\mathrm{r} \%)$ coagulates in............. $40 \mathrm{~m}$.

2. ro c.c. dilute salt plasma $(\mathrm{r} \%)+$ r c.c. 5 h. old serum coagulates in ........................... 3 or $4 \mathrm{~m}$.

3. Io c.c. dilute salt plasma oxalated $(0.1 \%)+$ ro c.c. 5 h. old serum oxalated coagulates in ................ 2 or $3 \mathrm{~h}$.

The first two tests seem to show clearly that the addition to a plasma of $\frac{1}{10}$ of its volume of old serum enormously accelerated curdling, and yet this rapid coagulation does not appear to be due to the thrombin remaining in the old serum, since test (3) coagulates very slowly. This last test indicates, furthermore, that calcium ions are necessary for the manifestation of this excito-productive property.

Bordet and Gengou explain this action of old serum by the presence of free serozyme, which, with the thrombokinase contained in the plasma, gives active thrombin. The contradiction between these results and those of Pekelharing is in reality more apparent than real.

In the first place, it must be noted that the preceding experiment leads to a criticism identical with that which we have made before concerning these same experimenters. Old serum, in (2), has undoubtedly caused the coagulation of the plasma, but nothing proves that this serum does not still contain a certain quantity of active enzyme, considering that test (3), which is destined to establish this fact, is a very bad witness. The experimental conditions under which it was made are different, notably in that a precipitate of calcium oxalate has been produced in this serum and it is known that this precipitate mechanically removes a fraction of the coagulating enzyme, the greater the fraction the older the serum. It is, therefore, erroneous to draw the conclusion that old serum no longer contains thrombin, but it is, on the contrary, very probable that it still contains some, and that it is by reason of this thrombin that the coagulation of (2) was rendered more rapid.

Yet the contradiction in the results found by the two experi- 
menters in question remains intact. How may this be explained? Without doubt, it is well known that the conditions under which they have worked were not the same. Pekelharing worked with a solution of fibrinogen and Bordet studied the phenomenon with a dilute plasma. Both used an old serum, but one waited I to 5 days to utilize it, while the other made use of it after a lapse of hours only.

Hence, to explain this contradiction, would lead to the consideration that old serum possesses indeed the two antagonistic substances observed, and that, according to the conditions of the experiment, one or the other predominates. However, an objection arises. It has already been noted that a solution of fibrinogen coagulates upon the simple addition of tissue extract, and it has been said above that this coagulation could not be explained according to the dualistic theory of the formation of thrombin, except by considering that the fibrinogen employed is not pure, but contains serozyme, carried away at the moment of the preparation.

If then, as Bordet believes, it is really the serozyme which is excito-producer of old serum, and if it is to serozyme that we owe an acceleration of coagulation, we must expect to see this old serum remain inactive when it is made to act on a solution of fibrinogen, since it introduces only one of the constituents of thrombin, a constituent which already exists in the fibrinogen, and does not furnish the second, thrombokinase, which would be necessary here. In fact, it is the opposite that is observed, there being clearly a positive action.

In addition, it must be agreed that the study of the phenomenon of coagulation presents a very great complexity and that it is often very difficult to draw a conclusion from tests made even under good conditions. Serum is not a substance of constant composition. According to its origin, it may contain, besides thrombin, either serozyme or thrombokinase. Further, a weakened serum, like a fresh serum, may contain antibodies in varying quantities. Serums may also differ in their calcium ion content. If, therefore, there is added to a fresh serum, 
containing an excess of calcium ions, a weak serum deficient in these ions, the coagulating power of the mixture will be increased because the ions of fresh serum will activate the weakened serum, and the result produced will be the resultant of the action of fresh serum with reactivated old serum.

It is apparently to an effect of this kind that must be attributed the contradiction which exists between the results of Bordet and those of Pekelharing. In the experiments of the former it is not the old serum which would accelerate the transformation of the thrombin contained in the fresh plasma, but rather the calcium ions of this plasma, which revivify the old serum, restoring its activity. Evidently the result produced would be exactly the same.

It is possible to arrive at the same result by adding to fresh serum, containing very little calcium ions, heated serum, which has had its thrombin destroyed, but which is rich in calcium ions. When introducing the antibodies, the activity of the thrombin of the fresh serum is intensified by the bringing in of an excitant, here calcium ions, and if the overproduction of coagulating enzyme which results exceeds the losses due to the antagonistic substances, the coagulating power of the mixture will be increased. It would be quite otherwise, however, if to a fresh serum containing a very slight excess of calcium ions or none at all, an old serum, also poor in these ions, is added. The antibody of this second serum would come into play, there would no longer be a counterbalancing of actions, and there would be observed a weakening in the coagulating power of the mixture.

However, it is evident that the presence of calcium ions is not the only factor to be considered in this sort of phenomenon. Thrombin, like all the other enzymes, is very sensitive to the physical and chemical condition of the medium, and if we expect to obtain check results by mixing serum with distilled water, it is much to be feared that we shall obtain irregular and even contradictory results if instead of distilled water we take another serum, especially an old serum, since the chemical composition 
of the liquids will not fail to modify, in one direction or the other, the properties of the mixture.

\section{$\S \mathrm{IX}$.}

\section{General View of the Phenomena of Coagulation OF BLOOD.}

From the numerous data, often contradictory, considered in the preceding discussion, there results a series of positive conclusions. In the coagulation of blood the following factors are involved:

I. An albuminoid substance, fibrinogen, at the expense of which the insoluble fibrin is formed.

2. Serozyme, a substance contained in the plasma, possessing the properties of an enzyme, destructible at $5^{\circ}$, soluble in water, insoluble in alcohol, and subject to mechanical removal from its solutions by barium sulphate or other precipitates.

3. Thrombokinase, a substance lipoidal in nature, rich in phosphorus, soluble in alcohol, petroleum ether and chloroform, stable toward heat, contained in abundance in the plates, the leucocytes and the tissue extracts.

4. Calcium ions, always present in blood.

5. A mechanical excitation, due to contact. Under the combined influence of the last four factors the coagulating enzyme or thrombin is formed and the fibrinogen of the plasma coagulates and is transformed into fibrin.

In circulating blood, the conditions indispensable for the production of this thrombin are not fulfilled. The thrombokinase, retained inside the plates and the blood corpuscles, is not directly in contact with the other elements, for the contact effect is lacking as a consequence of the viscous and slimy nature of the walls of the blood-vessels, and finally, the existence in the blood of anti-coagulating bodies opposes the action of the traces of thrombin which may nevertheless be formed.

Outside the organism, the blood is under conditions more 
favorable to its coagulation. The thrombokinase diffuses in the plasma, and the contact with the wall of the vessel where the blood is received starts the reaction, which then takes place very rapidly.

The specific coagulating substance is the serozyme. The thrombokinase exerts only a secondary influence and can be replaced by substances of similar nature, such as lecithins and peptones, peptides and amino-acids.

Calcium ions play a predominant rôle, but do not take part at the decisive moment, that of coagulation. They merely prepare, by the aid of materials brought by the blood, the active substance, the thrombin. Once this preliminary work is accomplished, the calcium can be eliminated without affecting the coagulation.

The rôle of contact is also indispensable. It influences not only the diffusion of the active substance contained in the plates and leucocytes, but also aids in the coagulation of a plasma deprived of these same organized constituents. It, therefore, assists, on the same par with the calcium ions, and concurrently with them, in the formation of thrombin from its constituents.

These facts are well established. The importance of each of these conditions can be experimentally shown and their exactness leaves no room for doubt. These are very positive results. But if now we ask in what order these different factors intervene in coagulation, by what means the action of calcium ions and that of contact is promoted, and in what state a preformed enzyme, or pro-enzyme, and the serozyme are found in the plasma, we are reduced to giving hypotheses in answer to these various questions. Without doubt, the theory as to how the serozyme and the thrombokinase combine, with the intervention of calcium ions and contact, to form active thrombin, explains in a satisfactory manner a whole series of experimental facts, but it must not be forgotten that here we are in the domain of pure speculation and that the agreement of all these facts could perhaps be explained by a different mechanism. However, thanks to the labors of Moravitz, Pekelharing, Bordet, and others, 
great progress has been made in this part of the study of biological phenomena, and if it is true that there still remains a certain number of obscure or unknown points, nevertheless we have come to have a very satisfactory notion of the phenomenon as a whole.

\section{$\S \mathrm{X}$.}

Analysis of Blood.

\section{Quantitative Determination of Thrombin and Fibrinogen.}

I. Determination of Thrombin. - The principle of the method of Wohlgemuth consists in determining the minimum quantity of serum necessary to produce the coagulation of a given volume of plasma. The plasma to be analyzed is prepared from rabbit blood or dog blood by the method of $\mathrm{A}$. Schmidt. Three volumes of blood are introduced in I volume of a solution of $\mathrm{MgSO}_{4}$, obtained by dissolving I part of salt in 3 parts of water. The mixture, after cooling, is shaken and the blood corpuscles are separated from the plasma by centrifuging. Under these conditions, the solution can be kept in a refrigerator for some time without any change in fibrinogen content. In the analysis of the blood-serum, the latter is defibrinated by beating with a glass rod. The clear decanted liquid will serve as a measure of the thrombin.

Determination of Thrombin.

\begin{tabular}{|c|c|c|}
\hline Amount of serum. & $\begin{array}{l}\text { Quantity of } \\
\text { thrombin. }\end{array}$ & $\begin{array}{l}\text { State of coagulation after } \\
24 \mathrm{~h} \text {. at } 0^{\circ} \text {. }\end{array}$ \\
\hline 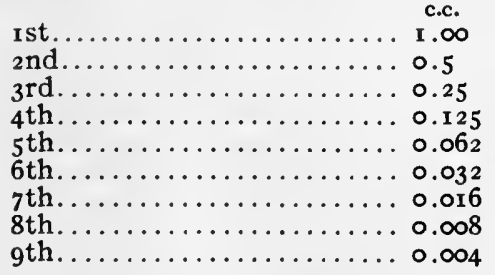 & $\begin{array}{l}\text { c.c. } \\
2 \\
\cdots \cdots \\
\cdots \cdots \\
\cdots \cdots \\
\cdots \cdots \\
\cdots \cdots \\
\cdots \cdots \\
\cdots \cdots\end{array}$ & $\begin{array}{l}\text { Complete coagulation. } \\
\text { Complete coagulation. } \\
\text { Complete coagulation. } \\
\text { Complete coagulation. } \\
\text { Not complete. } \\
\text { Partial. } \\
\text { Commencement. } \\
\text { Commencement. } \\
\text { Nothing. }\end{array}$ \\
\hline
\end{tabular}


The determination is made in the following manner: In a series of test tubes are poured different quantities of serum, and all the tubes are brought to the same volume by the suitable addition of a I $\%$ solution of $\mathrm{NaCl}$, free from calcium; 2 c.c. of plasma is then added in each of them. The tubes thus prepared are placed on ice and are examined at the end of 24 hours. To judge the state of coagulation, the tubes are placed, without shaking, in a horizontal position. Those solutions which have received the maximum of serum are completely coagulated; in others, coagulation is advanced, without being complete; finally, some show only a trace of coagulation.

No. 8, containing 0.008 c.c. of serum, is apparently the limit of action. This extreme case is taken as the enzyme unit and the coagulating force of serum, or its content in thrombin, is rated by the number of units corresponding to I c.c. of serum. In the example cited, we see that 0.008 c.c. serum represents I unit. I c.c. serum will contain I 25 enzyme units and therefore the value $(t)$ in thrombin of the serum is 125 .

2. Determination of Fibrinogen. - The blood to be analyzed is treated, as in the preceding, with one-third its volume of a solution of $\mathrm{MgSO}_{4}$, containing I part of salt to 3 parts of water. After centrifuging, the plasma obtained is distributed in increasing quantities in a series of test tubes and all of the volumes equalized by the suitable addition of a I per cent solution of $\mathrm{NaCl}$. To each is added I c.c. of serum diluted I to ro, they are shaken, and then left 24 hours on ice.

\begin{tabular}{|c|c|c|c|}
\hline Volume of plasma. & & $\begin{array}{l}\text { Serum diluted to. } \\
\mathbf{I} / \mathbf{I 0}\end{array}$ & $\begin{array}{l}\text { State of coagulation } \\
\text { after } 24 \text { h. at } 0^{\circ} \text {. }\end{array}$ \\
\hline 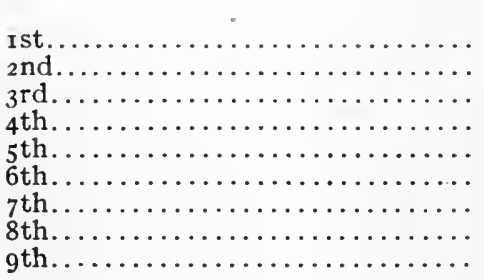 & $\begin{array}{l}\text { c.c. } \\
\text { I } \\
0.5 \\
0.25 \\
0.125 \\
0.062 \\
0.032 \\
0.016 \\
0.008 \\
0.004\end{array}$ & $\begin{array}{l}\text { c.c. } \\
\text { I } \\
\cdots \cdots \\
\cdots \cdots \\
\cdots \cdots \\
\cdots \cdots \\
\cdots \cdots \\
\cdots \cdots \\
\cdots \cdots\end{array}$ & $\begin{array}{l}\text { Nothing } \\
\text { Nothing } \\
\text { Partial } \\
\text { Coagulat. } \\
\text { Coagulat. } \\
\text { Partial } \\
\text { Traces } \\
\ldots \ldots \\
\ldots \ldots\end{array}$ \\
\hline
\end{tabular}


In the first three tests, the formation of fibrin did not take place or was retarded. In the two following tubes coagulation took place. It was complete in (4) and (5), and then was only partial with the diminishing content of fibrinogen.

If we compare these results with those of the preceding table, we see that they are entirely different; the experimental conditions are no longer the same, due, probably, more to the respective content in fibrinogen and serum than to the proportion of salts. In the example cited, No. 7 represents the minimum quantity of plasma capable of coagulating in presence of an excess of thrombin. The unit of fibrinogen contained in the blood analyzed will therefore be o.or6. Thus, I c.c. of plasma will contain 62.5 units. The value $f g$ in fibrinogen of the plasma will then be 62.5 .

\section{BIBLIOGRAPHY ON THROMBIN.}

Buchanan. On the coagulation of the blood and other fibrinoferous liquids, London Medical Gazette, 1845 .

Denis. Mémoire sur le sang, 1859 .

Alberton. Med. Centralbl., 1878, p. 641; 1879.

Rauchenbach. Ueber die Wechselwirkung der Protoplasma u. Blutplasma, Diss., Dorpat, 1883.

G. Fano. Arch. Italiennes de Biolog. (2), 1882, p. 146.

J. Green. Journ. of Physiol., 1887, p. 354.

LowIr. Ueber Blutgerinnung, Prag. Med. Wochen, 1889.

Morawitz u. Rehn. Zur. Kenntniss der Entstehung des Fibrinogens, Arch. f. exper. Pathol. u. Pharmak., (58), p. I4I, 1907.

Arthus. Recherches sur la coagulation du sang, Thèse, Paris, I89o.

Arthus et Pages. Coagulation du sang, Arch. de Physiol. et Path., (5), 739, r8go. Wooldridge. Die Gerinnung des Blutes, Leipzig, I89r.

Pekelharing. Untersuchung über Fibrinferment, Verhand. d. K. Akad.d. Wiss., Amsterdam, 1892.

ARTHUS. Fibrinogene et Fibrine, Soc. Biol., 1894, p. 306.

LILIENFEld. Ueber Blutgerinnung. Zeits. f. physiol. Chem., I895, (26), p. 89.

O. Hammarsten. Ueber die Bedeutung des lösl. Kalksalzes für Gerinnung,

Zeits. f. physiol. Chem., 1896, (22), p. 335.

Retrger. Die Coagul. d. Blutes, Amer. Journ. Physiol., (24), p. 406, 1909.

A. SCHMidT. Pflüg. Arch., (6), I872, p. 445.

Dastre et Floresco. Contr. à l'étude du ferment coagul. du sang, C. R., I897, 94.

Delezenne. Archiv. de Physiol., 1896, p. 666; 1897, pp. 338, 568; 1898, pp. 508, 568.

Roge. Diss., Strassburg, r898. 
Bordet et Gengou. Sur la coagul. du sang, Ann. Inst. Pasteur, 190r, p. 129; 1903, p. 822; 1904, pp. 26, 98; 1913, p. 345.

Bürker. Blutplättchen u. Blutgerinnung, Pftüg. Arch., 1904, (102), p. $3^{6 .}$

Fuld et Spiro. Beit. z. Physiol. u. Pathol., I904, (5), p. I80.

Camus. Recherches s. fibrinolyse, $C . R$., 1901, (1), p. 215 .

P. Nolf. B. Acad. Roy. de Belg., I906, p. 7 I.

H. Much. Vorstufe des Fibrinferments im Staphylococcus aureus, Bioch. Zeils, 1908, (14), p. 143.

Doyon. Sur l'origine du fibrinogène, C. R., I9ro, (r), p. 348.

Pekelharing. Bem. über Fibrinferment, Bioch. Zeits, rgo8, (I I), p. 7.

Hrguch. Fibrinenzym d. Placenta, Bioch. Zeits, I909, (22), p. 339.

Stassano et Daumas. Actions des sels de chaux, C. R., rgio, (I), p. 937.

Wohlgemuth. Best d. Fibrinferments im Fibrinogen. Bioch. Zeils, 1910, (25), p. 81.

EMIL ZaK. Studien über Blutgerinnungslehre, Arch. f. Experiment. Patholog. u. Pharmak., (70), p. 27, I9I 2.

Le Sourd et Pagnier. Recherches sur le rôle des plaquettes, Jour. de Physiol. et de Pathol. Génér., 1909, (XI), I.

Mellamby. Jour. of Physiol., vol. 38, Nos. I and 6. 1908 and I909. 


\section{CHAPTER II.}

\section{MYOSINASE.*}

Muscle, soft and elastic during life, changes in consistency after death and becomes rigid and hard. This transformation is due to the coagulation of certain albuminoid substances, coagulation caused by a special enzyme, myosinase. Kühne was one of the first to study this enzyme. He found that living muscle, thoroughly chilled and submitted to strong pressure, produces a thick yellowish liquid, muscular plasma, which, if the temperature rises, becomes jelly-like and contracts, forming a clot from whence exudes a serum, thus acting like blood plasma. However, this is only so in appearance, for the coagulation observed with muscular plasma differs radically from that of blood plasma. In this case, there is no formation of fibrin. The substance which coagulates is soluble in salt water, and is much less resistant than fibrin.

To obtain muscular plasma, Kühne proceeds in the following manner. He injects into the aorta of a frog a solution of $\mathrm{NaCl}$ $(0.7 \%)$, in order to take all the blood from the blood-vessels. The animal is then cooled to $-10^{\circ}$, the muscles are cut with a cold instrument, the product is ground in a mortar, and the ground mass, still at $\circ^{\circ}$, is submitted to a very strong pressure. The liquid which runs off, of syrupy consistency, has a slightly alkaline reaction, but rapidly becomes acid. It jellies as soon as the temperature rises. Halliburton used the process of Kühne to study the muscular plasma of a rabbit, as well as that of other animals. According to this investigator, it is possible to separate from muscular plasma the active agent causing coagulation and the albuminoid substance which under-

* The term Myosinase is substituted for the term Myosin-ferment used by Effront: Edilor. 
goes the action. The enzyme is therefore called myosinase, and the substance on which the enzyme acts myosinogen.

To obtain the enzyme, the mass of coagulated ground muscle is first treated with absolute alcohol, and the pulverized product is treated with water. The liquid so obtained coagulates muscular plasma in a much shorter time than if the alcohol is not added. The myosinase; obtained by precipitation of this water extract, presents the characteristics of a deuteroproteose. This product does not act on blood plasma, but acts on muscle plasma, as well as on myosinogen. Myosinase is much more resistant to heat than thrombin, and the solution of the former can be maintained at $90^{\circ}$ for fifteen minutes without destruction. However, this solution, heated to $100^{\circ}$, becomes inactive.

The muscular plasma contains, according to Halliburton, four albuminoid materials:

I. Myosinogen, a substance coagulable at $56^{\circ}$. It is precipitated from its solutions by $36 \% \mathrm{NaCl}$, as well as by $\left(\mathrm{NH}_{4}\right)_{2} \mathrm{SO}_{4}$.

2. Paramyosinogen, which is precipitated from its solutions by the addition of $50 \%\left(\mathrm{NH}_{4}\right)_{2} \mathrm{SO}_{4}$ or $28 \% \mathrm{NaCl}$. The temperature of coagulation of this body differs somewhat according to its origin, the substance taken from the frog coagulating at $47^{\circ}$, while the paramyosinogen of birds coagulates at $5 \mathrm{I}^{\circ}$.

3. A myoglobulin, coagulable at $63^{\circ}$ and capable of precipitation by $\mathrm{NaCl}$ or saturated $\left(\mathrm{NH}_{4}\right)_{2} \mathrm{SO}_{4}$.

4. A myoalbumin, coagulable at $73^{\circ}$ and not capable of being precipitated by saturated $\mathrm{NaCl}$.

In the coagulum which is produced as a result of the transformation of muscle plasma, we find again the first two albuminoids. This coagulum, dissolved in $5 \%$ magnesium sulphate coagulates with heat at from $47^{\circ}$ to $56^{\circ}$. Furthermore, it is possible to separate the myosinogen from the paramyosinogen by the method of fractional precipitation. Only the former is coagulable by myosinase. Paramyosinogen is, on the contrary, mechanically carried down in this precipitation. We can thus conclude that in the action of myosinase on myosinogen, there 
is no division or formation of new material, but simply a passage from one state to another. Indeed, myosinogen, coagulated by the enzyme, can be redissolved in salt water and precipitated again by the enzyme.

However, according to Furth, the first two albuminoids: myogen (or the myosinogen of Halliburton) and myosin (or the paramyosinogen of Halliburton) both undergo the enzyme action. Myosin produces, at the moment of coagulation, a clot of myosin-fibrin, and myogen, a clot of myogen-fibrin; but in the second case there is also a division, since in addition to this precipitate a globulin appears in the solution, soluble myogenfibrin, whose coagulation temperature is $30^{\circ}$ to $40^{\circ}$.

Furth suggests the following scheme for the coagulation of plasma:

Myogen (myosinogen)

Myogen-fibrin Soluble myogen-fibrin
Myosin (paramyosinogen)

Myosin-fibrin

Finally, according to Cavazzani, potassium oxalate would retard the action of myosinase.

\section{BIBLIOGRAPHY.}

DaNILEWSKy. Abhängigkeit der Contractionsart der Muskeln von den Mengen. verhältn. einig. ihr. Bestandteile, Zeits. f. physiol. Chem., I88I (5), p. 158.

W. D. Halliburton. Journ. of Physiol., I887, p. I32

Grubert. Kugler, Thèse, Dorpat, I888.

Cavazzani. Maly's Jahresbuch, 1892 (22), p. 333.

V. Furth. Ueber die Eiweisskörper d. Muskelplasma, Arch. f. experim. Patholu. Pharmak., 1895 (36), p. 321 .

Christine TeBb. Journ. of Physiol., I904 (30), p. 25.

K. BÜ̈RER. Blutplättchenzarfall, Blutgerinnung u. Muskelgerinnung, Centralbl.f. Physiologie, 1907 (2I), p. 65I. 


\section{CHAPTER III.}

\section{$\S$ I. RENNET.}

\section{Distribution. Preparation. Properties.}

Distribution. - Rennet is the active principle which determines the transformation of casein. Introduced into milk, this enzyme causes coagulation, the milk becoming gelatinous and compact. A clot of caseum is formed which carries with it the fatty particles of the milk and the phosphates. Left to itself, this clot contracts, expelling a transparent liquid which is the serum.

Rennet is secreted by the gastric glands of mammals and is very abundant in their stomachs. It is found in large quantities especially during the period of lactation, the rennet content gradually diminishing as the animal grows. Rennet is also normally found in the gastric juice, as well as in the pancreatic juice, of animals of every age. It is constantly present in the small intestine, and in the autolytic liquids of a certain number of organs, such as the liver, the lungs, the kidneys, etc. It has also been found in the digestive secretions of birds, reptiles, fish, mollusks, actinia, and other animals.

This property of coagulating milk belongs equally well to many vegetable juices. The agent producing this action is an enzyme similar to, if not identical with, animal rennet. We find this enzyme present in the leaves, fruits, and seeds of various plants. The coagulating properties of Galium verum and of Pinguicula vulgaris are utilized by dairymen of certain countries for making cheese. Fig juice, artichoke flowers, and darnel are also very rich in rennet. The seeds of Withania coagulans are extremely rich in rennet and can even serve as raw material for the preparation of this enzyme. Rennet has also been discovered in many micro-organisms, such as molds and bacteria. In fact this enzyme is one of the most widely distributed enzymes in nature. 
Preparation. - To prepare rennet, the inner lining of the true stomach of a calf is generally used. Maceration in water at $30^{\circ}-35^{\circ} \mathrm{C}$. brings the active substance into solution. The procedure is as follows: The lining is freed from the lumps which they enclose, washed thoroughly in water, allowed to swell for some time, and then is dried for ten days. The dried linings are cut in small pieces and macerated in water with addition of 4 per cent boric acid. The maceration is made at $30^{\circ} \mathrm{C}$. and lasts from 4 to 5 days. About roo grams of dried linings are taken per liter of water. In place of boric acid, sodium chloride, 3 to 6 grams per liter, can also be used. The salt aids the extraction, and the maceration under these conditions can be made at $15^{\circ}-20^{\circ} \mathrm{C}$. The preliminary drying of the linings is to coagulate certain substances and avoid a high viscosity of the liquid. For the same purpose, the linings are separated from the neighboring parts of the pylorus. The product obtained by this method is very active, but it contains a great deal of foreign matter. In addition to the rennet, there are also present other proteolytic enzymes.

To purify the commercial solution, the method of fractional precipitation with alcohol is utilized. To the solution of rennet a quantity of alcohol sufficient to produce a first light precipitate is added. This first deposit is separated, and an additional quantity of alcohol is added, causing a more voluminous precipitate. This precipitate is rapidly collected and dried in a vacuum over sulphuric acid. The product obtained is a white very active powder, but is not free from other enzymes.

In order to obtain a purer product, free from foreign enzymes, Hammarsten adopts the following method: The source of rennet is preferably the mucous membrane of the stomach dried at a temperature of $35^{\circ}-40^{\circ} \mathrm{C}$. As in the process used by Ebstein and Grutzner, the stomach is divided following the line of small curvature, and stretched, the mucous membrane being underneath. The muscular layer is then cut away with a razor, the pouch spread out on filter paper, the mucous membrane outside, and is dried at $35^{\circ}-40^{\circ}$. After drying, the paper is taken away. The horny substance thus obtained is cut in small 
pieces, which keep in a closed flask for a very long time without losing their activity. To obtain a solution of rennet, some fragments of this material are macerated with water or glycerin, usually taking a gram of dried mucous membrane for 50 to roo cubic centimeters of water. The product obtained is very active; an extract coming from one gram of mucous membrane thus preserved can, in 30 minutes, under favorable temperature conditions, coagulate from 5 to ro liters of milk.

Van Hasselt advises a preliminary drying of the calf stomach lining in the air, this drying, according to him, increasing the yield by 50 per cent. The dried stomach lining is then macerated in water containing o.I per cent $\mathrm{HCl}$ for 4 days, and is then filtered. The clear liquid is precipitated by magnesium sulphate, the coagulum is dissolved in water, and the whole is dialyzed against water acidulated with $\mathrm{HCl}$. Thus a very active product is obtained, but one very sensitive to alkali, so that, in order to have it react, it is necessary to very slightly acidify the milk.

To prepare vegetable rennets, Gerber suggests the following general method: The plants are partially dried at $40^{\circ}$, are crushed, and then are more thoroughly dried. In this way they can be kept a long time without diminishing their coagulating power. To obtain the enzyme, the powder, previously macerated for 24 hours, is submitted to a lixiviation with a 5 per cent solution of $\mathrm{NaCl}$, with the addition of several drops of essence of mustard, the operation taking place at a temperature of $0^{\circ}-5^{\circ} \mathrm{C}$. The clear liquid obtained is saturated with ammonium sulphate. The coagulum formed is collected, redissolved in $\mathrm{NaCl}$, and then is reprecipitated by ammonium sulphate. This is again filtered, and the product, suspended in water in sacs of collodion, is dialyzed thoroughly, in running water. The precipitate is first redissolved, then formed again. Finally it is collected, washed in distilled water and dried at $40^{\circ}$, or better, in a vacuum. Thus is obtained a gray or greenish gray product.

General Properties. - Researches of Javillier, and especially those of Gerber, show that rennet is widely distributed in the vegetable kingdom. At the present time, this enzyme has 
been found in pawpaw, woad, white Chinese mulberry (Broussonetia papyrifera), fig, prickwood, amadou fungus, vasconella, chardonella, spurge, the Compositæ, the Solanaceæ (tomato, belladonna), Cruciferæ, Rubiaceæ, Renunculaceæ, Basidiomycetes, brown algæ. All these rennets differ among themselves, nor are they identical with those obtained from ruminants. Each possesses a distinctive behavior towards exterior agents. Thus it is that vegetable rennets better resist the action of heat, and consequently act at a higher temperature than those of animals. Some coagulate unheated milk better than boiled milk; others do the reverse. Some are calciphile or oxyphile; others, are neither, etc. At any rate, it appears that these distinctive characteristics, albeit quite delicate, come rather from secondary influences than from a real specificity, and they do not appear to be of such a nature as to contradict the generally admitted idea of the unity of rennet. We shall later return to these peculiarities.

It has been stated above that rennet, in the stomach of ruminants, is present in especially large amounts at the time of lactation, diminishing afterwards. Gerber has likewise found a variation in the rennet content of a plant at different phases of its evolution. The quantity of coagulating enzyme increases in summer, decreasing in winter, and then increasing again the following summer, etc.; but the different maxima and minima are less and less pronounced and tend towards a mean value which then remains constant during the whole year. Diana Brusch has found similar variations in the resistance of the enzyme to heat. According to this observer, the optimum temperature decreases with age in the same plants, the variation amounting to a score of degrees. While the rennet of a young plant has $55^{\circ}$ for the most favorable temperature, that of a mature plant will act particularly well at $35^{\circ}$.

The variable resistance of different rennets to heat is indicative of the adaptation of the organisms secreting them to climatic influences. Gerber and Daumézon, having studied rennets of ascidians, a family of the group of the Tunicata, intermediary between the vertebrates and the invertebrates, found that 
the ascidians which are submitted to variations of temperature have a rennet much more resistant that those which live at a constant temperature. This fact corresponds to the differences of the same kind, noted by Gerber, between rennet of vegetables and of decapod crustaceans inhabitating the region uncovered at low tide, on the one hand, and the rennets of mammals whose temperature is constant, on the other hand. The study of rennets taken from Basidiomycetes has likewise given analogous results to the same investigator. Certain rennets are destroyed at about $50^{\circ}$, and act like those of animal origin. These come from mushrooms having their mycelium parasitic inside roots, trunks of trees, etc., and develop in the course of the autumn, only between rather narrow limits of temperature. Others resist a temperature of $85^{\circ}$ for Io minutes and resemble vegetable rennets. They come from mushrooms having a much less sheltered existence.

The characteristics of a rennet, however, depend on other conditions than those which could result from its habitat. Thus it is that Gerber, in a comparative study of rennet enzymes of a parasitic mushroom, Plerotus ostreatus, and of the plant on which it grows, Broussonetia papyrifera, found that they are very different. The rennet of the mushroom is very calciphile, very oxyphile, exceedingly sensitive to alkalis, and little resistant to heat; that of the white mulberry, on the contrary, is moderately calciphile, moderately oxyphile, little sensitive to alkalis, and very resistant to heat.

On the other hand, with the Amanitas, it is found that a certain relation exists between the rennet activity of these mushrooms and their degree of toxicity. A. phalloides, extremely poisonous, coagulates acidulated boiled milk ten to eleven times as quickly as $A . m a p p a$ or citrina, and fifty times as quickly as $A$. muscaria. As to edible Amanitas $(A$. rubescens, $A$. vaginata), they are still less coagulating than $A$. muscaria. According to Gerber the excessively rapid coagulation by $A$. phalloides can even be utilized in doubtful cases to recognize this mushroom among the other Amanitas; in fact, I c.c. of this juice 
coagulates in a few minutes 5 c.c. of unboiled or boiled milk, not sensitized by $\mathrm{HCl}$, at $55^{\circ}$ as well as at $40^{\circ}$, a behavior characteristic of no other Amanita.

\section{$\S$ 2. INFLUENCE OF TeMPerature.}

The action of rennet on milk is characterized by very striking external manifestations. With I g. of active product, 50 liters and more of milk can be coagulated in 30 to 40 minutes under favorable conditions. The velocity with which the coagulation takes place in a determined volume furnishes a relative measure of the power of the active substance, and most of the studies made on rennet from the point of view of its mode of action are based upon the coagulation produced in milk, although the phenomenon, as we shall see later, may be much less simple than one might at first believe.

Among the various physical and chemical agents which can influence milk-coagulation, temperature comes first. Temperature may not only modify the rapidity of the reaction, but may also act separately on the enzyme or on the milk. Let us first consider its sphere of action, when coagulated milk is exposed to different temperatures, it is found that the time which elapses up to the moment of coagulation differs notably according to the temperature to which the milk is brought during the experiment. If one works with calf rennet, the temperature of $40^{\circ}-$ $4 \mathrm{I}^{\circ}$ may be considered as optimum. It is at this point that the action of the rennet is the most pronounced, and with the same quantities of rennet and of milk we get coagulation in the least time. The influence of temperature on coagulation can also be verified by determining at different temperatures the maximum amount of milk which a unit of rennet is capable of coagulating in the same interval of time. The same result is obtained by determining, at different temperatures, the minimum quantity of rennet which must be used to cause the coagulation of the same volume of milk. In all cases, the optimum temperature will be the same. 
Time Required for Coagulation at Different Temperatures.

\begin{tabular}{|c|c||c|c|}
\hline Temperature. & $\begin{array}{c}\text { Time } \\
\text { required. }\end{array}$ & Temperature. & $\begin{array}{c}\text { Time } \\
\text { required. }\end{array}$ \\
\cline { 2 - 3 } & Min. & & Min. \\
\cline { 2 - 3 } I5 & $\ldots \ldots$ & 39 & 6.26 \\
20 & 32.17 & 40 & 6.15 \\
25 & 14.00 & 41 & 6.06 \\
30 & 8.47 & 42 & 6.12 \\
31 & 8.15 & 43 & 6.24 \\
32 & 7.79 & 44 & 6.44 \\
33 & 7.47 & 45 & 6.74 \\
34 & 7.19 & 46 & 7.16 \\
35 & 6.95 & 47 & 7.72 \\
36 & 6.74 & 48 & 8.44 \\
37 & 6.55 & 49 & 10.00 \\
38 & 6.39 & 50 & 12.00 \\
\hline
\end{tabular}

The course of coagulation according to the temperature is given in the preceding table by Fleischmann. This experiment is made with a milk, with addition of $\frac{1}{1000}$ parts of neutral calf rennet, exposing to temperatures varied by means of suitably regulated water-baths.

Thus the influence of temperature is indicated by the time necessary to obtain coagulation. At the temperature of $15^{\circ} \mathrm{C}$., the rennet used has no action. Between $20^{\circ}$ and $40^{\circ}$, the activity gradually increases; beyond $42^{\circ}$, the rapidity of the reaction diminishes; at $50^{\circ}$, the activity is not higher than that which rennet shows at $25^{\circ}$. At $66^{\circ}$, we find the same negative result as at $15^{\circ}$. However a notable difference exists between these two temperatures; the rennet, maintained in milk for to minutes at $66^{\circ}$, has definitely lost the property of coagulating the caseinogen, while in milk at $15^{\circ}$, the rennet undergoes no deterioration, and, when reheated afterward to $40^{\circ}$, can cause caseification. Furthermore, it can be experimentally shown that during contact at $15^{\circ}$ a preliminary enzyme action takes place, not ending in coagulation, but facilitating the later accomplishment of this. Milk treated at $15^{\circ}$ with rennet coagulates in presence of amounts of acid which would not produce the coagulation of normal milk. Further, this milk, then brought to $40^{\circ}$, coagulates at this last temperature more rapidly 
than the milk not exposed previously to the action of rennet at $15^{\circ}$.

The data in the table of Fleischmann furnish a clear picture of the comparative effect of the different temperatures. It is to be noted that these tests have been made with large amounts of neutral rennet, and in such a way that the time elapsing before reaching coagulation, at temperatures of $40^{\circ}-50^{\circ}$, does not exceed ${ }_{5}$ minutes. These precautions are absolutely indispensable in this kind of experimentation. With a rennet of acid reaction, or with small quantities of enzyme, different results would be attained. The reasons for these differences lie in the fact that the effect of temperature is considerably influenced by the reaction of the medium, and also that the temperature, while favoring the action of the rennet, in the long run exerts a very bad influence from the point of view of the preservation of the active substance. Between $35^{\circ}$ and $45^{\circ}$, we see a stronger destructive action on the enzyme, and to such an extent that when coagulation is already produced at a temperature of $45^{\circ}$, there is a considerable fraction of the active substance which is destroyed. At this moment, that portion really in action is less than in the experiment conducted at $40^{\circ}$. The optimum temperature observed has then a great relative significance, since we measure not constant quantities of substance in activity but very different quantities.

To show the complex effect of temperature, its influence on the rennet itself will first be considered and then its influence on non-coagulated milk.

Influence of Temperature on Rennet. - Rennet in solution keeps at $0^{\circ} \mathrm{C}$. for a very long time without losing its activity. At a temperature of $20^{\circ}-30^{\circ}$, a perceptible diminution of enzymic power is perceived, a diminution still more noticeable between $35^{\circ}$ and $40^{\circ}$. The following is an experiment which relates to this observation: In a series of test tubes there is introduced Io cubic centimeters of solution of a rennet freshly extracted from calf stomach linings. The tubes are placed in waterbaths regulated to different temperatures, where they are kept 
for a certain time, and then all are cooled to $15^{\circ}$. Then, from each tube, the same quantity of liquid is taken, which is put in Io c.c. of milk kept at $38^{\circ}$. The action of the temperature on the rennet is estimated by the time elapsing before coagulation. The following figures have been obtained by Effront by exposing rennet for 4 hours to different temperatures. It should be observed that the keeping of rennet solution at $40^{\circ}$ causes it to lose two-thirds of its enzymic activity.

Influence of Previous Heating on Rennet.

\begin{tabular}{|c|c|}
\hline $\begin{array}{c}\text { Rennet kept } 4 \\
\text { hours at a tem- } \\
\text { perature of }\end{array}$ & Time of coagulation. \\
\hline & Min. Sec. \\
$15^{\circ}$ & 20 \\
$20^{\circ}$ & 21 \\
$30^{\circ}$ & 32 \\
$35^{\circ}$ & 415 \\
$40^{\circ}$ & 62 \\
$50^{\circ}$ & 90 \\
$55^{\circ}$ & No coagulation after 6 hours. \\
\hline
\end{tabular}

In another test, the duration of the experiment was prolonged for 12 hours. Now the examination of samples taken at different moments showed that at temperatures running from $35^{\circ}$ to $50^{\circ}$ the destruction of coagulating power takes place progressively with the exposure to the heat. Between $15^{\circ}$ and $30^{\circ}$, the loss in rennet is only perceptible in the first 6 hours, and in the second period of 6 hours no variation was found.

Influence of Dilution. - Camus and Cléry recognized the fact that the effect of heat on rennet is considerably accentuated with its degree of dilution in water. A concentrated rennet can be maintained at $40^{\circ}$ at least Io minutes without perceptibly losing its activity. The same rennet, previously diluted with water, immediately becomes much more sensitive to the effect of the temperature. The influence of dilution is felt when very small quantities of water are added, and gradually increases with the quantity added. Camus and Cléry heat a known 
volume of rennet at $40^{\circ} \mathrm{C}$. for 2 minutes. They then add this rennet to milk maintained at $40^{\circ}$ and determine the velocity of coagulation. In a similar manner they add to the same quantity of rennet variable quantities of water, expose it likewise to $40^{\circ}$ for 2 minutes, and then determine the time of coagulation:

Influence of a Previous Heating on Solutions of RENNET Diluted with Water.

\begin{tabular}{|c|c|}
\hline $\begin{array}{l}\text { Degree of dilution } \\
\text { of the rennet. }\end{array}$ & $\begin{array}{l}\text { Time for } \\
\text { coagulation. }\end{array}$ \\
\hline 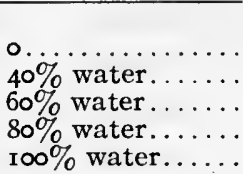 & $\begin{array}{rl}\text { Min. Sec. } \\
3 & 30 \\
4 & \text { I5 } \\
\text { I0 } & \\
\text { I } 2 & \\
\text { I9 } & \end{array}$ \\
\hline
\end{tabular}

According to this table a dilution of the rennet solution with an equal volume of water makes it much more sensitive to the effect of the temperature. After 2 minutes at $40^{\circ}$, this diluted rennet has already become six times less active. The influence of dilution is also observed at the temperature of $30^{\circ}$, but in a much less appreciable extent than at $40^{\circ}$.

The data, borrowed from Camus and Cléry, were probably obtained with a rennet of alkaline reaction, or else a rennet contaminated by foreign proteolytic enzymes, which can, in certain cases, influence its action perceptibly. With commercial rennet, as with rennet obtained from dried calf stomach linings, such a pronounced degree of sensitiveness toward dilution was not observed.

The sensitiveness of rennet to the temperature depends largely on the reaction of the medium, and it differs also according to whether the rennet is diluted with water or with glycerin. Lorcher dilutes I volume of rennet to 4 volumes with water, with $0 . x \% \mathrm{HCl}$, and with glycerin. $\mathrm{He}$ exposes the three solutions for to minutes at different temperatures, and then determines, using the same volumes of each diluted rennet, the time of coagulation for a given volume of milk maintained at $40^{\circ}$. 
Influence of a Previous Heating on Solutions of Rennet Diluted WITH VARIOUS LIQUids.

\begin{tabular}{|c|c|c|c|c|}
\hline Diluent. & Control. & $\begin{array}{l}\text { Test } \\
\text { at } 45^{\circ} \text {. }\end{array}$ & $\begin{array}{l}\text { Test } \\
\text { at } 50^{\circ} \text {. }\end{array}$ & $\begin{array}{c}\text { Test } \\
\text { at } 55^{\circ} \text {. }\end{array}$ \\
\hline 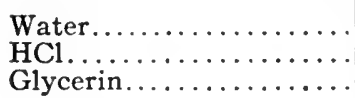 & $\begin{array}{l}\text { Coag. in } 13^{\prime} \\
\text { Coag. in } 11^{\prime} 15^{\prime \prime} \\
\text { Coag. in } 14^{\prime}\end{array}$ & $\begin{array}{l}15^{\prime}, 5^{\prime \prime} \\
14^{\prime} \\
18^{\prime}\end{array}$ & $\begin{array}{l}16^{\prime} \\
18^{\prime} \\
18^{\prime}\end{array}$ & $\begin{array}{l}2 \text { h. } 30^{\prime} \\
54^{\prime} \\
\text { I } 8^{\prime}\end{array}$ \\
\hline
\end{tabular}

It is obvious that dilution of rennet with water is the reason why this enzyme does not withstand a temperature higher than $40^{\circ}$. After ro minutes at $45^{\circ}$, there is a perceptible weakening. The preservation of the enzyme is accomplished much better in an acid medium and if, on the other hand, glycerin is used in place of water, there is no longer a deterioration of the active substance, even at $55^{\circ}$. The conclusion is that water exercises an injurious action on rennet, a conclusion all the more justified in that a water solution of rennet, maintained for ${ }_{5}$ minutes at $66^{\circ}$, becomes inactive, while rennet previously dehydrated can withstand a temperature of $130^{\circ}$.

The results obtained with acidulated water cannot be explained except by experimental conditions, especially by the origin of the rennet used. Usually there is observed a contrary result. In fact, the gastric juice, which is always acid, when kept at $40^{\circ}$ very rapidly loses it coagulating power, while its proteolytic power is preserved. Previously neutralized, it withstands much better the effect of this temperature. The difference arises from the fact that, in this last case, it is pepsin which destroys the rennet, while in the experiment of Lorcher pepsin does not interfere.

All the data just mentioned lead to the conclusion that the phenomena observed in the study of the influence of temperature on rennet are of an exceedingly delicate nature. The contradictory results obtained are explained by the influence of different factors, which are often very difficult to realize. Rennet always contains proteolytic enzymes and foreign substances which influence the effect of temperature. Finally, the 
reaction of the medium and the dilution play a rôle which is not negligible in the question of resistance toward temperature.

Influence of Temperature on Milk Intended for Caseification. - In studying the progress of coagulation of milk treated with rennet, it has been shown that in passing the temperature of $4 \mathrm{I}^{\circ}$, the time necessary for coagulation increases with each degree, and that at $50^{\circ}$ it requires a time double that required at the optimum temperature. As previously stated, the slowing up in the progress of coagulation at temperatures running from $40^{\circ}$ to $50^{\circ}$ must be attributed to the partial destruction of the active substance. But may not temperature also act directly on the casein of the milk by making it harder to coagulate? Samples of the same milk were taken at different temperatures, their temperatures brought to $40^{\circ}$, and the same quantity of rennet was added. Results obtained by Effront with milk kept an hour at different temperatures are as follows:

Influence of a Previous Heating of Milk on its Coagulation.

\begin{tabular}{|c|c||c|c|}
\hline $\begin{array}{c}\text { Temperature of } \\
\text { milk. }\end{array}$ & $\begin{array}{c}\text { Time for } \\
\text { coagulation. }\end{array}$ & $\begin{array}{c}\text { Temperature } \\
\text { of milk. }\end{array}$ & $\begin{array}{c}\text { Time for } \\
\text { coagulation. }\end{array}$ \\
\hline $30^{\circ}$ & $21^{\prime}$ & $70^{\circ}$ & $28^{\prime}$ \\
$40^{\circ}$ & $21^{\prime}$ & $80^{\circ}$ & $31^{\prime}$ \\
$50^{\circ}$ & $24^{\prime}$ & $90^{\circ}$ & $41^{\prime}$ \\
$60^{\circ}$ & $26^{\prime}$ & $100^{\circ}$ & $48^{\prime}$ \\
\hline
\end{tabular}

We see that milk heated between $80^{\circ}$ and $100^{\circ}$ coagulates with much more difficulty than normal milk. Boiled milk takes a time double that of fresh milk. Yet, at temperatures intermediate between $30^{\circ}$ and $50^{\circ}$ the influence is very slight. The retardation of the coagulation of milk, produced by boiling, apparently results from the precipitation of calcium ions, whose strongly accelerating power is known. However, boiling also coagulates the albumins of the milk, which themselves are retarders. There are here two contrary effects, and, according as the variety of rennet under consideration is more sensitive to the one or the other influence, we shall find that it coagulates unboiled milk more easily than boiled milk, as is the case of calf 
rennet, or, inversely, boiled milk more easily than unboiled milk, as is the case of fig rennet.

\section{§ 3. Influence of the Reaction of the Medium.}

Effect of Acids. - The coagulation of milk by rennet goes on more rapidly in an acid milk than in a normal milk. The sensitiveness of rennet to acid is such that infinitesimal amounts, not detected in direct analysis, produce very manifest results. In the effect of acids on rennet we find again the same complications as in the effect of temperature. The acid affects the coagulation by different routes. It especially favors the action of the rennet itself, by acting as an excitant, but, in addition, the coagulation is also activated as a result of the action of the acid on the milk. Caseinogen, in the presence of increasing quantities of acid, becomes more and more apt to coagulate. The velocity of its coagulation by rennet in the presence of acid results from effects which influence each other in a reciprocal manner.* The effect of the acidity on the caseification is shown in the following table, borrowed from the experiments of Effront:

Influence of Acidity on Caseification.

\begin{tabular}{|c|c|c|c|}
\hline Nos. & $\begin{array}{l}\text { Milligrams of } \mathrm{HCl} \\
\text { per liter of milk. }\end{array}$ & $\begin{array}{c}\text { Time for } \\
\text { coagulation. }\end{array}$ & $\begin{array}{l}\text { Value of } \\
\text { the rennet. }\end{array}$ \\
\hline I & 0 & $45^{\prime}$ & $R=100$ \\
\hline 2 & I & $44^{\prime} 06^{\prime \prime}$ & 102 \\
\hline 3 & 5 & $40^{\prime} 06^{\prime \prime}$ & I I I \\
\hline 4 & Io & $37^{\prime} 30^{\prime \prime}$ & 120 \\
\hline 5 & 20 & $3 \mathbf{I}^{\prime}$ & 245 \\
\hline 6 & 40 & $24^{\prime} 05^{\prime \prime}$ & $18 \mathrm{I}$ \\
\hline 7 & 100 & 10, $30^{\prime \prime \prime}$ & 402 \\
\hline 8 & 200 & $4^{\prime} 36^{\prime \prime}$ & 960 \\
\hline 9 & 225 & $3^{\prime} 24^{\prime \prime}$ & 1300 \\
\hline
\end{tabular}

The value $R$ expresses the comparative course of coagulation. It is represented by the figure 100 in normal milk (test

* Moreover it must be taken into account that the action of the acid shows itself in the dissolving of the calcium salts, which, we have seen, also influence coagulation. 
No. I). In this milk, with addition of a milligram of acid (test No. 2), coagulation takes place more rapidly, the value of $R$ is I02, and this value expresses the relation between the coagulation of normal milk and that of acidulated milk. In tests $2,4,5$ and 6 , we find a direct relation between the quantity of acid added and the velocity of coagulation. For each milligram of acid added to the milk, the value of $R$ increases about 2 per cent. When the quantity of acid passes 50 milligrams, the increase of $R$ becomes larger and ceases to be proportional to the quantity of acid. This is probably the result of two actions which at first are superimposed and then become separated, namely: casein, under the influence of the acid, becomes more apt to be coagulated, and rennet increases in activity. But when we pass certain limits, rennet is found to be unfavorably influenced.

The quantity of acid which rennet can endure at the ordinary temperature without deterioration is relatively very large. Liquid rennet endures an acidity of 4 grams of $\mathrm{HCl}$ per liter without showing diminution in enzymic power. Rennet may be left in the presence of these quantities of acid for Io or I 5 days, and after neutralization there is found the same activity as before acidification.

Effect of Alkalis. - Rennet is, on the contrary, much more sensitive to the action of alkalis than to that of acids. A solution of rennet, diluted and made $\frac{1}{500} N$ with sodium hydroxide, loses about 50 per cent of its activity after 20 minutes. A contact of 48 hours in the presence of this same quantity of alkali causes all activity to disappear (naturally a test for the enzyme has been made after neutralization). The sensitiveness towards alkalis depends up to a certain point on the concentration of the solution of rennet, concentrated solutions being a little less sensitive than dilute solutions.

To study the influence of alkalis on the coagulation of milk, Lorcher proceeds as follows: He brings milk to different degrees of alkalinity and determines coagulation with the same rennet in normal milk and in the alkaline milk. The term "per cent alkalinity" means the quantity of alkali used per ıoo c.c. of 
milk. The influence upon coagulation exerted by each concentration of alkali is given by the diminution of the figure of the coagulating power, indicated in an inverted fashion by the increase in the time required for coagulation. Thus, we see that 4-milligram per cent of alkali causes the coagulating power, represented by the figure 100 in normal milk to fall to the figure of 70.8 ; a quantity of 6 -milligram per cent of potassium lowers this figure to about $50 \%$ indicating by this that it has taken for coagulation under these conditions a time about double that which was necessary to coagulate normal milk with the same rennet:

Influence of Sodium and Potassium Hydroxides.

$\mathrm{NaOH}$ (mol. wt. $=40$ ). $\quad \mathrm{KOH}$ (mol. wt. $\left.=5^{6}\right)$.

Control milk, without alkali: $R=$ roo.

\begin{tabular}{|c|c|c|c|c|}
\hline \multirow{2}{*}{$\begin{array}{l}\text { Alkalinity } \\
\text { in mol. } \\
\text { mg. per } \\
\text { liter of milk. }\end{array}$} & \multicolumn{2}{|r|}{$\mathrm{NaOH}$} & \multicolumn{2}{|r|}{$\mathbf{K O H}$} \\
\hline & $\begin{array}{l}\text { Per cent } \\
\text { alkali. }\end{array}$ & Coagulating power. & $\begin{array}{l}\text { Per cent } \\
\text { alkali. }\end{array}$ & Coagulating power. \\
\hline $\begin{array}{r}1 \\
2 \\
10\end{array}$ & $\begin{array}{l}0.004 \mathrm{~g} . \\
0.008 \\
0.04\end{array}$ & $\begin{array}{r}R=70.8 \\
=60.7\end{array}$ & $\begin{array}{l}0.006 \mathrm{~g} . \\
0.012 \\
0.056\end{array}$ & $\begin{array}{r}\qquad \begin{array}{r}R=54.8 \\
=44.7\end{array} \\
\text { Coag. flaky, incompl. } \\
\text { after several hours }\end{array}$ \\
\hline
\end{tabular}

The alkali carbonates act similarly on rennet, though in a less energetic fashion:

Influence of Carbonates.

$$
\left(\mathrm{Na}_{2} \mathrm{CO}_{3}=\text { ro6. }\right) \quad\left(\mathrm{K}_{2} \mathrm{CO}_{3}={ }_{13} 8 \text {. }\right)
$$

Control milk, without addition: $R=$ roo.

\begin{tabular}{|c|c|c|c|c|}
\hline \multirow{2}{*}{$\begin{array}{l}\text { Alkali in } \\
\text { mol. mg. } \\
\text { per liter } \\
\text { of milk. }\end{array}$} & \multicolumn{2}{|r|}{$\mathrm{Na}_{2} \mathrm{CO}_{3}$} & \multicolumn{2}{|r|}{$\mathrm{K}_{2} \mathrm{CO}_{3}$} \\
\hline & $\begin{array}{l}\text { Per cent } \\
\text { alkali. }\end{array}$ & Coagulating power. & $\begin{array}{l}\text { Per cent } \\
\text { alkali. }\end{array}$ & Coagulating power. \\
\hline$I$ & $0.011 \mathrm{~g}$. & $R=82.3$ & $0.014 \mathrm{~g}$. & $R=100$ \\
\hline 2 & 0.020 & $=70$ & 0.028 & $=73.1$ \\
\hline 4 & 0.042 & $=53.8$ & 0.045 & $=43.7$ \\
\hline 10 & 0.106 & $\begin{array}{l}\text { No coag. perceptible } \\
\text { after several hours }\end{array}$ & 0.138 & $\begin{array}{r}\text { No coag. perceptible } \\
\text { after several hours }\end{array}$ \\
\hline
\end{tabular}


We see in test (I) that with II-milligram per cent of $\mathrm{Na}_{2} \mathrm{CO}_{3}$, the coagulating power has decreased 18 per cent, while with I4-milligram per cent of $\mathrm{K}_{2} \mathrm{CO}_{3}$, coagulation took place in the same length of time as in normal milk; the coagulating power is then not lowered, and is that expressed by the figure 100. On the contrary, a quantity ten times larger almost completely prevents all coagulation.

Bicarbonate of soda likewise retards the action of rennet.

INFLUENCE OF BiCARBonate OF SODA.

$\mathrm{NaHCO}_{3}(84)$.

\begin{tabular}{|c|c|c|}
\hline $\begin{array}{l}\text { Alkalin. in mol. } \\
\text { mg. per liter of } \\
\text { milk. }\end{array}$ & $\begin{array}{l}\text { Per cent } \\
\text { salt. }\end{array}$ & Influence on coagulation. \\
\hline I & $\begin{array}{l}\text { grams. } \\
0.008\end{array}$ & $R=94.7$ \\
\hline 2 & 0.017 & $=90$ \\
\hline 10 & 0.08 & $=47 \cdot 3$ \\
\hline 20 & 0.17 & $=22.27$ \\
\hline 100 & 0.84 & $\begin{array}{l}\text { No coagulation after ro } \\
\text { hours. }\end{array}$ \\
\hline 500 & 4.2 & $\begin{array}{l}\text { No coagulation; de- } \\
\text { composed. }\end{array}$ \\
\hline
\end{tabular}

Comparing the different results obtained with the alkalis, we find:

I. That caustic alkalis are more energetic paralyzers than the alkali carbonates; sodium hydroxide influences coagulation more unfavorably than potassium hydroxide.

2. Bicarbonate acts less strongly than neutral carbonates.

There results from these tests a practical indication of great importance, namely, that when it is a question of neutralizing rennet, the preference should be given to bicarbonate which has less action on the enzyme than the other alkalis.

It should be noted that all these results, for acid as well as for alkali, refer to ordinary rennet, extracted from lining of calf stomach. With rennet of other origins, quite different results will be obtained. There are rennets much more oxyphile than others; some have a much greater resistance to alkali than that just found. Gerber, in particular, cites rennet of prickwoods (Euonymus europaeus and E.japonicus), which is exceed- 
ingly basiphile, the optimum of alkalinity at every temperature being $40 \mathrm{~mol} . \mathrm{mg}$. per liter of milk, which represents a quantity of soda equivalent to $\mathrm{r} .6 \mathrm{~g}$. per liter of milk.

Influence of Salts. - Lorcher has made a systematic study of the influence of salts on the course of coagulation by rennet. $\mathrm{He}$ adds to the milk different quantities of salts which have been dehydrated, representing fractions of their molecular weight, and compares the results with those of a normal coagulation without the salt addition. In the table which follows is found a summation of these tests. Each concentration is expressed in milligram moles per liter (a concentration of $\mathrm{I}$ then being equal to a solution one thousandth normal) as well as in the quantity of salt added Io c.c. of milk. The coagulating power, calculated as previously, shows the retarding or accelerating action of salts in different concentrations. The time which milk takes to coagulate, without addition of salts, is expressed in each test by the figure 100 . The figure 50 , for example, indicates that the coagulation in the presence of salt requires a time double that of milk without salt. In all the tests, the salt is first added to the milk, then the rennet, and the whole is exposed to a temperature of $38^{\circ}$. The time required for curdling is noted.

The data obtained by Lorcher agree in general with those obtained by Duclaux. The influence of salts on the coagulation of milk treated with rennet will differ according to the nature of the latter. Certain salts accelerate the coagulation; others retard it considerably. Among the accelerating salts are barium nitrate, chlorides of barium, calcium, strontium, cadmium, and aluminium. In the group of retarding salts belong especially the sulphates, iodides, bromides, chlorides, and fluorides of potassium and sodium, as well as a whole series of other salts. There is also a marked difference of effect according to the concentration of the salt solutions. With most of the retarding salts the effect is increased with the increase of the salt content. There are isolated cases, on the contrary, where an accelerating effect is produced in weak concentrations, and a retarding with stronger, notably in the case of $\mathrm{CaCl}_{2}$. 
Influence of Different Salts on Coagulation.

\begin{tabular}{|c|c|c|c|c|}
\hline \multicolumn{3}{|c|}{ Concentration of milk in salts. } & \multicolumn{2}{|c|}{ Coagulating power. } \\
\hline \multirow{2}{*}{$\begin{array}{l}\text { Mol. mg. } \\
\text { per liter. }\end{array}$} & \multicolumn{2}{|c|}{ Per cent content. } & \multirow{2}{*}{ Sodium salts. } & \multirow{2}{*}{$\begin{array}{l}\text { Potassium } \\
\text { salts. }\end{array}$} \\
\hline & Sodium salt. & Potassium salt. & & \\
\hline
\end{tabular}

I. - SUlphates: $\mathrm{Na}_{2} \mathrm{SO}_{4}=\mathrm{I}_{42} ; \mathrm{K}_{2} \mathrm{SO}_{4}=\mathrm{I}_{74}$.

\begin{tabular}{r|l|l|r|r}
$\mathrm{I}$ & $0.014 \mathrm{~g}$. & $0.017 \mathrm{~g}$. & $\mathrm{R}=78 . \mathrm{I}$ & $\mathrm{R}=82.6$ \\
10 & 0.142 & 0.174 & 70.0 & 70.0 \\
20 & 0.284 & 0.348 & 66.6 & 66.6 \\
100 & 1.420 & 1.740 & 43.8 & 38.8 \\
500 & 7.100 & 8.700 & 15.2 & $<10$
\end{tabular}

II. - Nitrates: $\mathrm{NaNO}_{3}=85 ; \mathrm{KNO}_{3}=$ IOI.

\begin{tabular}{r|l|l|l|r}
10 & $0.085 \mathrm{~g}$. & $0.101 \mathrm{~g}$. & $\mathrm{R}=90.0$ & $\mathrm{R}=94.3$ \\
20 & 0.170 & 0.202 & 85.5 & 85.5 \\
100 & 0.850 & 1.01 & 52.6 & 54.7 \\
500 & 4.250 & 5.05 & 7.7 approx. & 8.3
\end{tabular}

III. - Phosphates: $\mathrm{Na}_{2} \mathrm{HPO}_{4}={ }_{142} ; \mathrm{K}_{2} \mathrm{HPO}_{4}={ }_{174}$.

\begin{tabular}{r|l|l|l|r}
$\mathrm{I}$ & $0.014 \mathrm{~g}$. & $0.017 \mathrm{~g}$. & $\mathrm{R}=85.4$ & $\mathrm{R}=\mathrm{II4}$ \\
5 & 0.070 & 0.085 & $75 \cdot 3$ & 164 \\
10 & 0.142 & 0.174 & $33 \cdot 3$ & 200 \\
100 & $\mathbf{1 . 4 2}$ & 1.74 & No coag. & 256
\end{tabular}

IV. - IODATES: $\mathrm{NaI}={ }_{150} ; \mathrm{KI}=\mathrm{I} 66$.

\begin{tabular}{r|l|l|l|r}
10 & $0.150 \mathrm{~g}$. & $0.166 \mathrm{~g}$. & $\mathrm{R}=78$ & $\mathrm{R}=82.6$ \\
20 & 0.300 & 0.332 & 70.0 & 70.0 \\
40 & 0.600 & 0.664 & 63.7 & 61.0 \\
100 & $\mathrm{I} .5$ & 1.66 & $4 \mathrm{I} .2$ & $45 . \mathrm{I}$ \\
500 & 7.5 & 8.30 & No coag. & $3 . \mathrm{I}$
\end{tabular}

V. - BRomides: $\mathrm{NaBr}=$ I03; $\mathrm{KBr}=\mathrm{II} 9$.

\begin{tabular}{r|l|l|r|r}
10 & $0.103 \mathrm{~g}$. & $0.119 \mathrm{~g}$. & $\mathrm{R}=97.0$ & $\mathrm{R}=85.6$ \\
20 & 0.206 & 0.238 & 89.4 & 66.6 \\
40 & 0.412 & 0.476 & 80.5 & 58.9 \\
100 & 1.03 & 1.19 & 58.9 & 18.3 \\
500 & 5.15 & 5.95 & 16.7 & 8.3
\end{tabular}

VI. - Chlorides: $\mathrm{NaCl}=58.5 ; \mathrm{KCl}=74.5$.

\begin{tabular}{r|l|l|r|r}
10 & $0.058 \mathrm{~g}$. & $0.074 \mathrm{~g}$. & $\mathrm{R}=80.6$ & $\mathrm{R}=94.4$ \\
20 & 0.116 & 0.149 & 80.6 & 94.4 \\
100 & 0.58 & 0.745 & 70.0 & 71.0 \\
500 & 2.92 & 3.72 & 37.9 & 29.9 \\
1000 & 5.8 & 7.4 & 23.9 & 20.9
\end{tabular}

VII. - Fluoride and Oxalate: $\mathrm{NaF}=42 ; \mathrm{K}_{2} \mathrm{C}_{2} \mathrm{O}_{4}=166$.

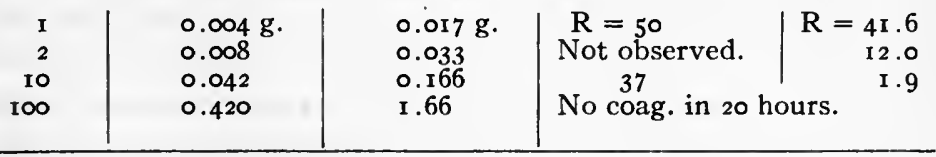


Salts Other than Sodium and Potassium.

Conc. in mol. mg. per liter.

Coagulating power.

I. Chloride of Lithium: $\mathrm{LiCl}=42.5$
$\mathrm{R}=106.5$
5
10

II. - Alkaline-earth Hydroxides: $\mathrm{Ca}(\mathrm{OH})_{2}=74 ; \mathrm{Ba}(\mathrm{OH})_{2}=171$.

\begin{tabular}{r|r|r} 
I & $\mathrm{Ca}: 84.6$ & $\mathrm{Ba}: 80$ \\
2 & 66.6 & 66.6 \\
$\mathrm{IO}$ & 20.9 & 33.3
\end{tabular}

III. - BARIUM Nitrate: $\mathrm{Ba}\left(\mathrm{NO}_{3}\right)_{2}={ }_{2} 61$.

\begin{tabular}{r|l}
2 & 100 \\
10 & 133 \\
20 & 163 \\
100 & 178 \\
300 & 106.6
\end{tabular}

IV. - Chlorides: $\mathrm{CaCl}_{2}=\mathrm{III} ; \mathrm{SrCl}_{2}=159 ; \mathrm{BaCl}_{2}=.208$.

\begin{tabular}{r|r|r|r}
1 & $\mathrm{Ca}:$ I33 & Sr: I14 & Ba: I22 \\
2 & 145 & 133 & 133 \\
10 & 295 & 227 & 250 \\
20 & 526 & 266 & 400 \\
100 & 345 & 179 & 526 \\
500 & 28.5 & 66 & 69.5
\end{tabular}

V. - Magnesium Chloride: $\mathrm{MgCl}_{2}=95 ; \mathrm{ZINC}_{\mathrm{In}}$ Chloride: $\mathrm{ZnCl}_{2}=\mathbf{1}_{3} 6$.

\begin{tabular}{r|c|c}
1 & $\mathrm{Mg}: 65 \cdot 9$ & $\mathrm{Zn}: 128$ \\
2 & 70.5 & 137 \\
10 & 100 & 149 \\
20 & 270 & 175 \\
100 & 476 & 37.4
\end{tabular}

VI. - Cadmium Chloride: $\mathrm{CdCl}_{2}=183$; Aluminium Chloride:

$\mathrm{AlCl}_{3}=$ I39.9.

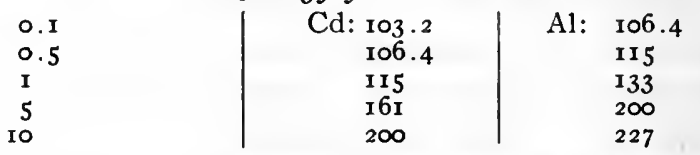

VII. - Magnesium Sulphate: $\mathrm{MgSO}_{4}=120$; Barium Nitrate:

$\mathrm{Ba}\left(\mathrm{NO}_{3}\right)_{2}=26 \mathrm{I}$.

\begin{tabular}{r|c|c}
$\mathrm{I}$ & $\mathrm{Mg}: 106.6$ & $\mathrm{Ba}: 100$ \\
2 & 115 & 106.6 \\
10 & 163 & 133 \\
100 & 115 & 175 \\
500 & 13.3 & Not observed. \\
\hline
\end{tabular}


In addition to the salts mentioned, borax, in the concentration of 0.1 g. per liter, considerably retards coagulation. At a borax content of I gram per liter, milk coagulates four times less quickly than normal milk; at 2 grams per liter, coagulation takes place six times less quickly than without the addition of this salt.

To explain the effects produced by salts the following should be taken into consideration:

I. The injurious influence of alkali and the favorable action of acid. Salts such as $\mathrm{AlCl}_{3}, \mathrm{CdCl}_{2}$, hydrolyze and cause acceleration of coagulation by the acid which they liberate.

2. The influence which salts exert directly on rennet.

3. The action which salts may have on the phosphates and the casein of the milk.

However, in general, data on the mode of action of salts is lacking. With regard to the chemical activity of rennet, we shall have occasion to return to the effect exercised in the course of caseification by potassium oxalate and sodium citrate, as well as by $\mathrm{NaCl}$, alone or in presence of either or both of these salts. Apparently, in this particular case the mechanism according to which these substances act is satisfactorily explained.

Influence of Various Substances. - Albumoses and peptones added to milk considerably retard the action of rennet. Thus it is that 0.5 per cent of peptone retards coagulation, and that in the presence of 5 per cent, rennet has almost no action on the milk. This paralyzing effect is partially explained by the possibility that peptones may enter into combination with acid phosphates of the milk and render the action of the rennet more difficult by changing the reaction of the medium.

Rennet is very sensitive to the action of antiseptics. Solutions of rennet, saturated with chloroform or thymol, are very rapidly rendered inactive. Yet milk, containing chloroform, affords a normal action with rennet not treated with chloroform, the coagulation being not at all retarded. With formaldehyde this difference is not observed. This last may be considered as 
the best paralyzer of rennet. It acts weakly if put in the solution of rennet or if it is first added to the milk. Milk containing formaldehyde at a concentration of a gram per liter has its rennet coagulation considerably retarded. With three grams per liter, the rennet has hardly any effect. Boric acid, one gram per liter of milk, renders the coagulation 20 times more difficult than in normal milk. It should be observed that, as a paralyzer, this body acts much more energetically than borax. Saccharin, in very dilute amounts, accelerates the action of rennet, but paralyzes it in larger quantities. Iodine in dilute concentrations is without effect on rennet. Morgenroth utilizes this property of iodine to sterilize solutions of rennet. He obtains appreciable results, from the viewpoint of the preservation of the enzyme, by adding to ro c.c. of a solution of rennet I c.c. of $N /$ ro iodine at the ordinary temperature. At the end of an hour he removes the free iodine with sodium thiosulphate. The rennet is hardly weakened at all and the liquid thus obtained is practically sterile.

Work of Gerber. - In the course of a long study on the different vegetable rennets, Gerber has made a certain number of interesting observations, some of which have already been mentioned. Others, relative to the reaction of the medium on enzyme action are as follows: First of all it should be noted that milk being a very complex mixture of variable composition, especially in salty constituents whose accelerating or retarding action is known, the rapidity of its coagulation may be modified by its own quality. This has been previously noted by Duclaux and is also shown by an observation of Van Dame. The latter having noticed that certain milks offer an anomaly from the point of view of coagulation, shows that this results from an insufficient content of collodial calcium in the milk. In fact, if cows, giving a non-coagulable milk, are made to take $50 \mathrm{~g}$. of calcium diphosphate a day, their milk soon becomes normal and perfectly coagulable. Moreover, the manner of preservation of the same milk may also influence the conditions of the curdling. If the same quantity of solution of rennet acts at $28^{\circ}$ for different 
lengths of time on unboiled milk, collected from the udder of the cow in an aseptic manner, and kept at $7^{\circ}$ up to the moment of treating with rennet, large differences in the time necessary for caseification are observed.

Influence of Age of Milk on Its CoAgulability.

\begin{tabular}{|c|c|c|c|c|c|c|}
\hline \multirow{2}{*}{$\begin{array}{l}\text { Thousandths of c.c. } \\
\text { of rennet solution. }\end{array}$} & \multicolumn{6}{|c|}{ Hours elapsed after treatment. } \\
\hline & I & 8 & 24 & 72 & 98 & 120 \\
\hline $\begin{array}{l}80 \\
20\end{array}$ & $\begin{array}{l}2^{\prime} \\
4^{\prime} \times 5^{\prime \prime}\end{array}$ & $\begin{array}{l}26^{\prime} \\
17^{\prime} 30^{\prime \prime}\end{array}$ & $\begin{array}{l}28^{\prime}, 55^{\prime \prime} \\
22^{\prime} \text { ' } 55^{\prime \prime}\end{array}$ & $\begin{array}{l}23^{\prime}, 15^{\prime \prime} \\
16^{\prime} 40^{\prime \prime}\end{array}$ & $\begin{array}{l}4^{\prime} \\
4^{\prime} \text { I } 5^{\prime \prime}\end{array}$ & $\mathrm{O}^{\prime}, 20^{\prime \prime}$ \\
\hline
\end{tabular}

Thus, at the end of 24 hours, the milk coagulates less easily than an hour after the treatment. On the contrary, if the same milk is kept at ordinary temperature, it is found that at the end of ten hours it shows no notable modifications in its resistance to rennet-action. The difference in these results comes from the fact that, in the preservation at ordinary temperature, the lactic microbes have been able to develop and the activating action of the acid formed counterbalances the increasing resistance of the pure milk with age. The proof of this hypothesis is that if the lactic bacteria are prevented from developing, either by the use of formaldehyde, of mercury bichromate, or chloride, added to the milk at the temperature of the surrounding air, or quite simply by maintaining the milk at a temperature of $58^{\circ}$, we see that this milk acts like that kept in the refrigerator. This observation shows the precautions necessary to carry on experiments results of which shall be comparative.

To come now to the influence of salts on caseification, with respect to the effect of alkali phosphates, the two writers who have studied the question do not agree. Duclaux found that $\mathrm{Na}_{2} \mathrm{HPO}_{4}$ is an accelerator, then a retarder, while Lorcher describes it as a retarder. As to $\mathrm{K}_{2} \mathrm{HPO}_{4}$, Duclaux found it to be a retarder, then accelerator, while Lorcher considers it to be an accelerator. These contradictory results were 
obtained with rennet of calf stomach lining. Gerber, in taking up this study with lab-ferment, found that the two phosphates act in the same way and are retarders, while with vegetable rennets they are both accelerators, then retarders. This difference comes from the fact that, the lab-ferment being more strictly calciphile than the others, the neutral salts of potassium or of sodium change the medium and render it less favorable to the action of animal rennets than to that of vegetable rennets. The importance of calcium in caseification by animal rennets is known. If pains be taken that the calcium, instead of being precipitated, remains in solution, we should find an identity of conduct between the two kinds of enzyme. This is fully in accord with the experiments. If, in place of neutral salts acid phosphate of potassium or of sodium is used, and it is observed that small quantities of these salts act as accelerators for animal rennet (as neutral phosphate would have done on vegetable rennet), and that stronger doses act as retarders.

The same phenomenon is observed with sulphates of sodium or of potassium. Having compared, with respect to fresh milk, the effect of these two neutral salts on rennets of calf and of hog, on the one hand, on that of white mulberry, on the other, Gerber finds that they act as retarders in the first case, and as accelerators and then retarders in the second. Gerber thinks that these two different effects, as with neutral alkali phosphates, come from the precipitation of calcium by the salts, this base being much more necessary to coagulation of milk in the case of animal rennets than in the case of vegetable rennets. To prove it, he takes milk poor in calcium, for example boiled milk, so that the addition of $\mathrm{Na}_{2} \mathrm{SO}_{4}$ or of $\mathrm{K}_{2} \mathrm{SO}_{4}$ does not precipitate the calcium and finds that, under these conditions, the two rennets act in the same way, that they are at first accelerated, then retarded by the sulphates. The same result is obtained if these two kinds of rennets act in presence of $\mathrm{NaHSO}_{4}$ and of $\mathrm{KHSO}_{4}$ on fresh milk. First activation is noticed and then inhibition.

If acid sulphates act on boiled milk, in every case, just as 
the acid phosphates, up to quite large concentrations, an accelerating effect is produced. The lactalbumin and the lactoglobulin being the only substances which differentiate fresh and boiled milks, one is led to attribute to them the retarding effect observed in the coagulation of fresh milk in the presence of large quantities of phosphate or of acid sulphate. In reality, when various rennets act on different milks, coagulation takes place in variable times, but the duration is greater the more albumin and globulin the milk contains. The content in casein has no effect, or at least only a very slightly retarding effect. The following are some of the results:

Retarding action of Albuminoids of Mrck on the Caseification of this Liquid BY RENNETS.

5 c.c. of milk: Milks A

Enzyme: 2 drops

B

20 drops - of parachymosin.
C

25 drops $\{$ To coagulate in $14 \mathrm{~m}$. at $26^{\circ}$.

Analysis of these Milks.

\begin{tabular}{|c|c|c|c|}
\hline & A & B & C \\
\hline Ash (per liter). & 7.05 & 6.80 & 7.85 \\
\hline $\mathrm{CaO} \ldots \ldots \ldots$ & 1. 56 & I. 44 & I. 65 \\
\hline 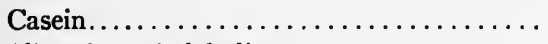 & 26.40 & 22. 10 & $36 . \infty$ \\
\hline Albumin and globulin................ & 4.90 & 6.30 & 7.80 \\
\hline
\end{tabular}

If these milks are boiled, only the casein remains in solution. Now the time for coagulation becomes the same, although the content in casein may be very different.

Coagulation of these Borled Milks by the Same Quantity of Fig Rennet AT $\mathrm{T}=55^{\circ}$.

A B C

Time I4 m. $5 \mathrm{~s}$ I3 m. $50 \mathrm{~s}$. I6 m. $50 \mathrm{~s}$.

Thus, the albumin and the globulin of the milk are substances very plainly antagonistic to rennet. Since, on the other hand, their inhibitory power disappears on boiling, it might well be that the anti-rennets encountered by certain investigators in fresh milk are simply the coagulable albuminoids of the milk. This hypothesis is all the more admissible since one of these antibodies, that which corresponds to fig rennet, 
is progressively destroyed at from $65^{\circ}$ to $80^{\circ}$, temperatures at which lactoglobulin and lactalbumin coagulate. We shall find later similar facts with rennet of pawpaw. Furthermore the same writer has found that serum-albumin, egg-albumin, serum-globulin, egg-globulin, and myosin are almost all retarders both of animal and vegetable rennets, acting on fresh-boiled milk. These results put us on guard against certain antirennets which have been noted perhaps a trifle hastily in serums, white of egg, muscle extracts, etc.

Gerber found that borax, which Duclaux mentions as a paralyzing substance, is, on the contrary, an accelerator towards rennets which coagulate boiled milk more easily than raw milk (broussonetia, fig, etc.). As to boric acid, which Duclaux considers a more active paralyzer than borax, Gerber finds that it is, on the contrary, an accelerator for all the animal rennets. With respect to rennets which coagulate boiled milk more easily than fresh milk, the acceleration takes place only for boiled milk, and that very slightly; fresh milks are, on the contrary, retarded. Gerber explains his disagreement with Duclaux by the supposition that the boric acid used by the latter was probably not pure. It is only necessary, in fact, to add to pure boric acid a trace of white of egg, a substance which is often added to the acid to obtain good plates, to have no longer an acceleration but a retardation.

Lorcher, like Duclaux, found that $\mathrm{NaCl}$ was a retarder; Gerber found that $\mathrm{NaCl}$ is an accelerator in dilute concentrations, a retarder in medium quantities, and finally an accelerator in large quantities. Gerber further studied the effect of alkali salts of some organic acids, as well as the effect of a large number of metallic salts, those of $\mathrm{Zn}, \mathrm{Cu}, \mathrm{Hg}, \mathrm{Ag}, \mathrm{Au}, \mathrm{Pt}$, etc., on the coagulation of milk in presence of rennets of various origins, and published a number of tables, from which it appears that the phenomenon is very irregular and often very complex.

With respect to the action of hydrogen peroxide on different rennets, we observe also results that vary with the type of enzyme considered. Some rennets, like that of fig or of pawpaw, 
are very sensitive to this antiseptic; others, like the rennet of calf or parachymosin, are not influenced except by relatively large quantities; finally, the rennets of Broussonetia or of pancreatic juice show themselves very resistant, even to large quantities of $\mathrm{H}_{2} \mathrm{O}_{2}$ (500 c.c. of $\mathrm{H}_{2} \mathrm{O}_{2}$ per liter of coagulating juice).

In general, aside from certain reagents which are plainly accelerating, like the acids, or plainly retarding, like alkalis or certain salts, we may say that the effects produced are variable, if not in their direction, at least in their intensity, and that this effect depends much on the origin of the rennet under consideration.

In addition to the effect of temperature and of chemical reagents, rennet, as is shown by the works of Aguhlon, is also considerably influenced in an unfavorable manner by ultra-violet rays in presence of oxygen or in a vacuum. On the contrary, it is not sensitive to the luminous part of the spectrum.

\section{§ 4. Pro-Rennet.}

In the mucous membranes of the stomach, rennet is found in two different states, that of active enzyme or rennet properly so-called, and that of pro-enzyme or pro-rennet. Under the influence of very small quantities of acid at the optimum temperature, the pro-rennet is rapidly transformed into active rennet. The presence of pro-rennet in the glycerin extract of dried mucous membranes is easy to show. A similar glycerin extract is added to a volume of o.I per cent $\mathrm{HCl}$ and is left at the optimum temperature for I hour. For control, I volume of the glycerin extract is mixed with I volume of water, which is also left for I hour at the optimum temperature. Then in the two solutions the coagulating power with regard to milk is determined. The glycerin extract, when acidulated, shows considerably more activity than the extract diluted with water. The activity gained under the influence of the acid is explained by the transformation of pro-rennet into rennet. 
The following is an experiment of Lorcher relating to this transformation: In a series of tubes is poured ro c.c. of milk and different amounts of o.I per cent $\mathrm{HCl}$. To each sample of milk, thus acidified, is added $\frac{1}{2}$ c.c. of the glycerin extract of mucous membranes and the time required for coagulation at $40^{\circ}$ is determined. This series of tests (series A) gives us data on the influence of quantities of acid used on the time required for coagulation. In a second series (series B), the experimental conditions are changed, while using the same proportions of rennet, milk, and acid. In place of adding the acid directly to the milk, it is added to the glycerin solution of mucous membranes and the whole left two hours at the optimum temperature. Then the glycerin extract is added to the milk as in series A. In the two series, the rennet acts in presence of the same quantities of acid.

Transformation of Pro-Rennet into Rennet Under the INFLUENCE OF $\mathrm{HCl}$.

\begin{tabular}{|c|c|c|c|c|}
\hline \multirow{2}{*}{ Extract of rennet. } & \multirow{2}{*}{$\begin{array}{l}\text { 0.1 per cent } \mathrm{HCl} \\
\text { (cubic cent.) }\end{array}$} & \multirow{2}{*}{$\begin{array}{l}\text { HCl per I liter } \\
\text { of milk (milli- } \\
\text { gram.) }\end{array}$} & \multicolumn{2}{|c|}{ Time for coag. } \\
\hline & & & Series A. & Series B. \\
\hline I. 0.5 cu. cent. . & 0.00 & 0 & $23^{\prime}$ & $23^{\prime}$ \\
\hline 2. 0.5 cu. cent.. & 0.05 & 5 & $19 \frac{1}{2}$ & 2 \\
\hline 3. 0.5 cu. cent.. & 0.08 & 8 & $18 \frac{1}{2}$ & 2 \\
\hline 4. $0.5 \mathrm{cu}$. cent... & 0.1 & Io & 17 & 2 \\
\hline 5. $0.5 \mathrm{cu}$. cent.. & 0.5 & 50 & I $5 \frac{1}{2}$ & I \\
\hline
\end{tabular}

In series $B$, in the presence of 0.05 c.c. of acid, milk was coagulated in 2 minutes, while without addition of acid it took 23 minutes. In series $\mathrm{A}$, the same quantity of acid produced a relatively slight action, one falling only from 23 minutes to $18 \frac{1}{2}$ minutes. The radical difference in the results obtained, according to whether the acid is added directly to the milk or first to the rennet, shows that the rapidity of coagulation found in series $B$ cannot be explained by the change of reaction of the medium. We ought then to assume that there is formed under these circumstances a greater quantity of active substance in the glycerin extract.

The existence of pro-rennet is strongly contested by Duclaux. 
This investigator does not admit that there exists in the mucous membranes, and still less in the extract of mucous membranes, a particular inactive substance, capable of becoming active simply by the chemical change of the medium, for example, by addition of acid. The experiments of Lorcher are explained, according to Duclaux, by the presence, in the glycerin extract, of granulations or cellular débris which retain the rennet and prevent its solution in the milk. The appearance of rennet, following the contact with acid, would be due to the fact that the rennet under the influence of the acid detaches itself from the granulations, where it had remained in an insoluble state, and thus passes to the soluble state. This opinion, however, is in contradiction to the fact that filtered extract of mucous membranes, perfectly clear, acts in the same way as extract which is not filtered. In the two cases dilute acid considerably increases the content in active substance:

According to Hédin, the preceding facts may be interpreted in another way. The pro-rennet would be a combination of rennet with a substance antagonistic to the first, this combination nevertheless being able to retain a very small proportion of active lab-rennet. If the pro-rennet be treated with dilute $\mathrm{HCl}$, the rennet is set free and the antagonistic substance destroyed, and hence its activity is lost. On the contrary, the solution of prorennet, treated with very dilute ammonia at $37^{\circ}$, loses all its rennet already free, while the antibody remains unchanged, so that by adding active rennet to this treated liquor, the latter is rendered inactive. Then if this antagonistic body is treated with very dilute $\mathrm{HCl}$, it is destroyed, and the solution, even after neutralization, can no longer act on the rennet. Whatever may be the originality of such an explanation, it appears, nevertheless, that the existence of a pro-rennet, the mother substance of rennet, is very probable, assuming that we find similar facts with other enzymes.

Therefore, with this conception, it is possible to explain the passage from one state to the other as the consequence of a hydration which is facilitated by the presence of acid. The 
experiments of Lorcher have been made on extract of dried mucous membranes. The pro-rennet is also found in fresh mucous membrane, although in much less proportion than in dried mucous membrane. The extract of fresh mucous membrane shows itself twice as active as the extract of the same quantity of mucous membrane previously dried. It contains, therefore, twice as much rennet. But the treatment of this extract with dilute acid proves that it contains little pro-rennet. The extract of dried mucous membrane, on the contrary, is rich in pro-rennet and relatively poor in rennet.

From the increase in the pro-rennet content of mucous membranes by drying, it may be concluded that a dehydration takes place which transforms the rennet into pro-enzyme. Experiments of Effront have confirmed the retrogression of rennet into prorennet. It is to a phenomenon of the same order that must be attributed the diminution of activity which is observed in fresh rennet during the first two months of its preservation. [NotE. - According to some authors the reaction zymogen $\rightarrow$ enzyme is considered non-reversible. - ED.]

The transformation of pro-rennet into rennet by contact with acid takes place sharply. By using a unit volume of rennet per unit volume of o.I per cent acid, transformation is complete after 30 minutes. The following is a test showing the time required for the transformation of pro-rennet into rennet. One part of the rennet solution is mixed with I part of o.I per cent $\mathrm{HCl}$. From this mixture 0.1 c.c. is removed at different intervals and is introduced into Io c.c. of milk maintained at $40^{\circ}$.

Rapidity of the Transformation of Pro-RenNet Under THE INFLUENCE OF $\mathrm{HCl}$.

\begin{tabular}{|c|c|c|c|}
\hline $\begin{array}{c}\text { Duration of } \\
\text { contact with } \\
\text { o.I per cent } \\
\text { HCl. }\end{array}$ & $\begin{array}{c}\text { Time for } \\
\text { coagulation. }\end{array}$ & $\begin{array}{c}\text { Duration of } \\
\text { contact with } \\
\text { o.I per cent } \\
\text { HCl. }\end{array}$ & $\begin{array}{c}\text { Time for } \\
\text { coagulation. }\end{array}$ \\
\hline Minutes. & Minutes. & Minutes. & Minutes. \\
\cline { 1 - 2 } & 43 & Io & 6.5 \\
2 & 24 & 20 & 5.5 \\
3 & I9 & 60 & 5.5 \\
5 & II & $\cdots$ & $\cdots$ \\
\hline
\end{tabular}


The pro-rennet resists better the action of alkalis than rennet; it also withstands higher temperatures. In a mixture of prorennet and rennet kept at $70^{\circ} \mathrm{C}$., the rennet is eliminated almost completely, while the pro-rennet is much less affected.

\section{§ 5. LaW of Action of RenNet.}

In the chapter relating to pepsin, the general law of action of enzymes will be formulated. It will then be shown that enzymes obey a logarithmic law expressed by the relation:

$$
t=\frac{S}{m} \log \frac{S}{S-s}
$$

a relation in which $t$ represents the time required for the action, $S$ the total quantity of material to be transformed, $s$ the quantity already transformed at the time $t$, and $m$ a factor proportional to the quantity of enzyme used.

It results from this equation that if we compare two enzyme actions, applied on the same quantity of material $S$, but caused by different quantities of enzymes, $m$ and $m^{\prime}$, at the moments $t$ and $t^{\prime}$ so that the fraction of substance not transformed is equal in the two cases $\left(s=s^{\prime}\right)$, we shall have:

$$
t=\frac{S}{m} \log \frac{S}{S-s} \text { and } t^{\prime}=\frac{S}{m^{\prime}} \log \frac{S}{S-s},
$$

whence

$$
\frac{t}{t^{\prime}}=\frac{m^{\prime}}{m}
$$

In other words, the time necessary to transform the same quantity of substance, in solutions of equal concentration, is inversely proportional to the quantities of enzymes present. Of course, according to the equation, the complete transformation of the substance would require infinite time, but, in fact, we know that the reaction always has an end. In reality it approaches the limiting value rapidly enough to warrant neglecting variations between actual and final values. Therefore, we can consider those reactions as completed in which there remains only immeasurable quantities of substances untransformed. This is 
done in studying the coagulation of milk with rennet. But it is important then to define exactly what is meant by end of the reaction. Practically, caseification is considered as finished when, the experiment having been made in test tubes or graduated vessels, the coagulum formed is sufficiently curdled for one to be able to turn the receptacle over without the contents escaping. Other criteria are the aspect of the gash which a knife blade makes or of a finger print made in the jelly of the coagulated milk. The edges must be sharply cut and the liquid which collects (quickly) in the wound must be transparent.

Under these conditions, working at constant temperature, the time required for coagulation is inversely proportional to the quantities of rennet used.

We shall have:

$$
m t=m^{\prime} t^{\prime}=m^{\prime \prime} t^{\prime \prime}=\ldots
$$

This result constitutes what is called the law of Segelcke and Storch. The following is a verification of it borrowed from the work of Lorcher. In a series of test tubes containing Io c.c. of milk kept at $37^{\circ}$, increasing quantities of calf rennet are added and observations are made on the time required for complete caseifications.

Verification of the Law of Segelcke and Storch.

\begin{tabular}{|c|c|c|}
\hline $\begin{array}{c}\text { Quantity of } \\
\text { rennet, } m .\end{array}$ & $\begin{array}{c}\text { Time of } \\
\text { coagulation, } t .\end{array}$ & $\begin{array}{c}\text { Product, } \\
m t .\end{array}$ \\
\hline c.c. & Minutes. & \\
0.02 & 245 & 490 \\
0.04 & 126.5 & 485 \\
0.06 & 78 & 468 \\
0.08 & 63 & 504 \\
0.10 & 43 & 430 \\
0.2 & 24.5 & 490 \\
0.3 & 16 & 480 \\
0.4 & 12.5 & 500 \\
0.5 & 10 & 500 \\
0.8 & 7.5 & 600 \\
1.0 & 6 & 600 \\
\hline
\end{tabular}

To summarize, for moderate quantities of rennet, the verification is satisfactory; for small quantities of enzyme, the results are in general too small; for large quantities, they are too 
large. These irregularities can be explained in part by the fact that in the case of large dilutions the enzyme deteriorates more easily, and that it is slower in producing the reaction, without doubt in consequence of the internal friction. On the other hand, when large quantities of rennet are used, not only is the velocity of the transformation made almost instantaneous, but, in addition, foreign substances are introduced which disturb the results.

If we seek to verify this law, under the preceding conditions, with rennet or commercial pepsins, we find that the inverse proportionality is greater. This is, moreover, one of the reasons for considering rennet taken from the gastric juices of a hog or of man as being different from rennet taken from calf stomach lining, and hence the name of parachymosin for the former. Thus it has been stated that parachymosin can produce only very rapid coagulations. If a quantity $A$ causes a coagulation in 7 minutes, the quantity $\frac{A}{2}$ will not cause coagulation at all. Gerber showed that this anomaly disappears if the experiment is carried out under different conditions of temperature and reaction of the medium. With increasing quantities of a solution of pepsin in plates acting on 5 c.c. of fresh cow's milk at a temperature of $25^{\circ}$, he finds:

Action of Parachymosin on Milk at $25^{\circ}$.

\begin{tabular}{|c|c|c|}
\hline $\begin{array}{c}\text { Drops of } \\
\text { parachymosin. }\end{array}$ & $\begin{array}{c}\text { Velocity of } \\
\text { coagulation. }\end{array}$ & Products. \\
\cline { 2 - 3 } I & Minutes & 29.40 \\
2 & 14.45 & 29.6 \\
3 & 10.20 & 29.5 \\
4 & 7.35 & 31 \\
5 & 7.35 & 30.3 \\
6 & 5.30 & 30.3 \\
7 & 4.30 & 33 \\
8 & 3.40 & 31.5 \\
9 & 3.10 & 29.3 \\
10 & 2.55 & 28.5 \\
& & 29.2 \\
\hline
\end{tabular}

The law of Segelcke and Storch is then verified, although all the coagulations are far from being rapid, since one drop of enzyme coagulates milk in $29.40 \mathrm{~min}$. 
In reality, the difference between these results and those formerly found by the author is due only to the influence of the temperature, this having a large influence on the curve of the activity of rennets. In fact, if increasing quantities of a I per cent solution of pepsin in plates act on 5 c.c. of fresh cow's milk at temperatures between $25^{\circ}$ and $45^{\circ}$, it is observed that the law of inverse proportionality does not hold beyond $30^{\circ}$.

action on Parachymosin on Milk at Different Temperatures.

\begin{tabular}{|c|c|c|c|c|c|c|c|}
\hline \multirow{2}{*}{$\begin{array}{l}\text { Quantity } \\
\text { of rennet } \\
\text { used. }\end{array}$} & \multicolumn{7}{|c|}{ Time necessary for coagulation of milk. } \\
\hline & $25^{\circ}$ & $30^{\circ}$ & $33^{\circ}$ & $36^{\circ}$ & $39^{\circ}$ & $42^{\circ}$ & $45^{\circ}$ \\
\hline $\begin{array}{l}\text { c.c. } \\
0.005 \\
0.010 \\
0.020 \\
0.030 \\
0.050 \\
0.100\end{array}$ & $\begin{array}{l}30^{\prime} 20^{\prime \prime} \\
14^{\prime} 45^{\prime \prime} \\
7^{\prime} 30^{\prime \prime} \\
4^{\prime}, 40^{\prime \prime} \\
3^{\prime} \\
x^{\prime} 40^{\prime \prime}\end{array}$ & $\begin{array}{l}29^{\prime} \\
1 I^{\prime} 30^{\prime \prime} \\
5^{\prime} \\
2,50^{\prime \prime} \\
I^{\prime} 50^{\prime \prime} \\
x^{\prime}\end{array}$ & $\begin{array}{l}\text { Nothing } \\
7^{\prime} \\
2^{\prime} 30^{\prime \prime} \\
1^{\prime} 40^{\prime \prime} \\
I^{\prime} 10^{\prime \prime} \\
0^{\prime} 40^{\prime \prime}\end{array}$ & \begin{tabular}{|} 
*Nothing \\
Nothing \\
3 '1 $5^{\prime \prime}$ \\
$1^{\prime} 30^{\prime \prime}$ \\
$0^{\prime} 55^{\prime \prime}$ \\
$0^{\prime} 30^{\prime \prime}$
\end{tabular} & $\begin{array}{c}\text { Nothing } \\
\text { Nothing } \\
5^{\prime} 30^{\prime \prime} \\
1_{40}^{\prime \prime} \\
0^{\prime} 40^{\prime \prime} \\
0^{\prime} 25^{\prime \prime}\end{array}$ & $\begin{array}{l}\text { Nothing } \\
\text { Nothing } \\
\text { Nothing } \\
\text { Nothing } \\
2^{\prime} 05^{\prime \prime} \\
0^{\prime}=5^{\prime \prime}\end{array}$ & $\begin{array}{l}\text { Nothing } \\
\text { Nothing } \\
\text { Nothing } \\
\text { Nothing } \\
\text { Nothing } \\
0^{\prime} 45^{\prime \prime}\end{array}$ \\
\hline
\end{tabular}

- No coagulation at the end of 360 minutes.

Thus, starting with $39^{\circ}$, we find only rapid coagulations for relatively high quantities of rennet. It is necessary then to work with parachymosin at temperatures between $25^{\circ}$ and $30^{\circ}$ in order to observe the law of Segelcke and Storch. Under these conditions, it is found that parachymosin acts like other animal rennets. This is a general phenomenon, which is found even in the case of vegetable rennets. Gerber has shown that one of the most evident characteristics of rennets from mammals consists in not obeying the law of Segelcke and Storch except at relatively low temperatures, below $30^{\circ}$ for hog rennet, below $40^{\circ}$ for that of calf. Above these temperatures, the time required for coagulation varies, being much longer for small amounts of the enzyme than the law requires. Hence the impossibility of observing coagulations which do not take place in a very short time, and the necessity of using much enzyme. Briot thought that this anomaly came from the existence of an antibody in the milk. Gerber has refuted this opinion by drawing attention to the fact 
that boiled milk acts in the same way as fresh milk with regard to all the rennets, animal or vegetable.

The cause of this deviation must then be the same for all the coagulating juices and is found in the two kinds of milk; in fact, it resides in the small proportion of mineral matter in the milk itself. Rennets of mammals require, as we have already seen, in order to produce curdling of solutions of casein, that they shall be strongly mineralized. It is necessary, for example, to add to the milk a certain quantity of $\mathrm{NaCl}$, I $_{5}$ to $\mathrm{r} 8$ per cent, in order that coagulation follows the law, even at high temperatures. Similar results are obtained with alkaline-earth salts and with acids. But the degree of mineralization of the milk necessary is less with alkaline-earth metals than with alkali metals. Acids, capable of dissolving calcium salts which occur in suspension in boiled milk under the form of the neutral phosphate, may likewise assist in rendering the coagulation of boiled milks regular under the influence of animal rennets.

With regard to the fact that vegetable enzymes act at high temperatures, Gerber shows that three cases are possible:

(I) The enzymes (Ex. Broussonetia) act like animal rennets in presence of fresh milk, while being much more resistant to high temperatures. They follow the law. In the presence of boiled milk, they follow it likewise, but only with the addition of $\mathrm{CaCl}_{2}$.

(2) The enzymes (Ex. fig) do not, so to speak, obey the law in the case of fresh milk, and only partially in the case of boiled milk. The effect of $\mathrm{NaCl}$ or of $\mathrm{CaCl}_{2}$ is almost null.

(3) The enzymes (Ex. pawpaw) are as resistant as the first to high temperatures, and as little obedient to the law as the second.

It results from these facts that at present there still exist rennets for which we have not succeeded in regulating their functioning at high temperatures. This impotence is not surprising. Just as it was sufficient to lower the temperature a little or to add a trace of acid or of calcium salts to make regular the action of rennets which deviate from the common law, in the same way we can foresee that in certain cases a very small 
quantity of the impurities, which disastrously accompany the enzyme examined, will suffice to disturb its mode of action and to give an entirely different turn to its course.

This opinion finds support in the experiments of Pawlow, who observed with the same rennet a very different course of coagulation, according to the dilution or the reaction of the medium in which it is placed. With gastric juice neutralized by bicarbonate, the progress of the coagulation follows the law of inverse proportionality.

Gastric Juices Neutralized by $\mathrm{NaHCO}_{3}$.

\begin{tabular}{|c|c|c|}
\hline $\begin{array}{c}\text { Quantities of } \\
\text { gastric juice. }\end{array}$ & $\begin{array}{c}\text { Time for } \\
\text { coagulation. }\end{array}$ & $\mathrm{mt}$. \\
\hline c.c. & min. & 2.00 \\
0.8 & 2.50 & 2.00 \\
0.4 & 5.00 & 2.06 \\
0.2 & 10.33 & \\
\hline
\end{tabular}

The results are entirely different when the same gastric juice is strongly diluted and neutralized by barium carbonate.

Gastric Juice Neutralized by $\mathrm{BaCO}_{3}$, Diluted to Times.

\begin{tabular}{|c|c|c|c|}
\hline $\begin{array}{c}\text { Quantity of } \\
\text { gastric juice. }\end{array}$ & $\begin{array}{c}\text { Time for } \\
\text { coagulation. }\end{array}$ & $\begin{array}{c}\text { Averages of } \\
\text { time. }\end{array}$ & $m f^{2 .}$ \\
\cline { 1 - 2 } c.c. & min. & I IO & II \\
I & 6.50 & 36 & 108 \\
3 & 4.25 & 18 & 108 \\
6 & & & \\
\hline
\end{tabular}

We see that here the quantities of enzyme are inversely proportional to the square of the time required for coagulation. Now, the same juice, without neutralization or dilution gives the following results:

ACID JUice.

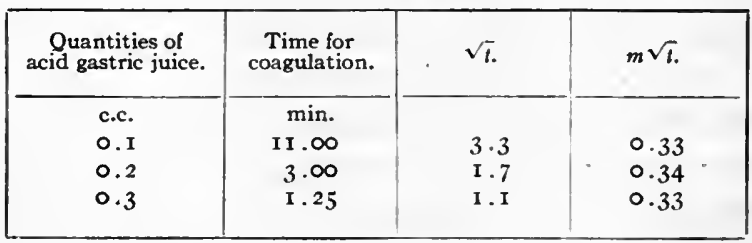


We see this time that the quantities of enzyme are inversely proportional to the square root of the time required for coagulation. This law is also maintained for gastric juice neutralized previously with $\mathrm{BaCO}_{3}$, without dilution:

Gastric Juice Neutralized by $\mathrm{BaCO}_{3}$, but not Diluted.

\begin{tabular}{|c|c|c|c|}
\hline $\begin{array}{c}\text { Quantities of } \\
\text { gastric juice. }\end{array}$ & $\begin{array}{c}\text { Time for } \\
\text { coagulation. }\end{array}$ & $\sqrt{t .}$ & $m \sqrt{t .}$ \\
\cline { 1 - 2 } & min. & & \\
\hline c.c. & 95.0 & 9.75 & 0.97 \\
0.1 & 23.0 & 4.80 & 0.96 \\
0.2 & 10.0 & 3.20 & 0.96 \\
0.5 & 3.5 & 1.90 & 0.95 \\
\hline
\end{tabular}

Thus, with the same gastric juice we can obtain three different processes of coagulation, according to the physical and chemical conditions of the medium. These facts show very well the complexity of the phenomenon, and indicate that the influence of temperature and that of the reaction of the medium affect simultaneously both the rennet and the coagulation.

\section{§ 6. The Identity of Different Rennets.}

Rennets of different origin are not equally influenced by the physical and chemical conditions of the medium. According to their origin, their optimum temperature varies, they are more or less resistant to heat, are more or less sensitive to alkalis, are more or less activated by acids or by calcium salts, and finally, they follow more or less exactly the law of Segelcke and Storch. In a general way, we can say that vegetable rennets act on a scale of temperature more extended than animal rennets, and that their optimum temperature is higher. We have previously seen that ordinary rennet, taken from calf stomach lining, acts especially well at about $40^{\circ}$, that at $15^{\circ}$ it no longer acts, and that at $70^{\circ}$ it is destroyed.

The following is the behavior of an enzyme taken by Mesnil from the mesentery filaments of actinia, and given by him the name actino-rennet. Its optimum temperature differs little from 
that of ordinary rennet, but, though the latter does not act at all at $15^{\circ}$, with actino-rennet a perceptible action is observed even at $6^{\circ}$.

Coagulation Causepd by Actino-Rennet at

Different Temperatures.

\begin{tabular}{|c|c|}
\hline Temperature, ${ }^{\circ} \mathrm{C}$. & $\begin{array}{c}\text { Time for } \\
\text { coagulation. }\end{array}$ \\
\hline 38 & Io $\mathrm{min}$. \\
35 & I2 $\mathrm{min}$. \\
20 & $45 \mathrm{~min}$. \\
15 & 105 $\mathrm{min}$. \\
8 & $3 \mathrm{~h} .30 \mathrm{~min}$. \\
6 & $4 \mathrm{hours}$ \\
\hline
\end{tabular}

Gerber, in studying rennet from brown algæ, found that this coagulating juice can still act on milk at a temperature of $0^{\circ}$; but more curious still is the special way in which it acts. The active substance of brown algæ, which can be extracted, instantaneously coagulates milk (more easily boiled than fresh milk), and the quantity of casein precipitated is proportional to the quantity of active substance used. This action takes place just as well at low temperatures $\left(0^{\circ}\right)$ as at medium temperatures (up to $60^{\circ}$ ). The activity gradually diminishes at high temperatures, but it does not entirely disappear even at $100^{\circ}$. The behavior of this enzyme towards milk is very different from that of ordinary rennets. It would be difficult to find here the catalytic character of enzymes, and we should be tempted to put it in the class of precipitins, if its resistance at $100^{\circ}$ and the absence of an optimum temperature were not very evident. It appears more likely that these two typical characteristics, namely, the phenomenon of instantaneous coagulation and resistance at $100^{\circ}$, are the result of the mucilaginous nature of the. material which accompanies it or composes it, and from now on we draw this conclusion, which will be developed later, that the foreign substances which always contaminate enzymes may sometimes be the causes of very great disturbances in the manifestation of enzymic action. 
We have just seen that the rennet of brown algæ acts even at $0^{\circ}$. Gerber has shown that this manifestation of activity at a temperature so low does not constitute a very important distinctive characteristic, since all rennets are capable of coagulating milk at $0^{\circ}$ if the precaution is taken of sensitizing the system by increasing the quantity of alkaline-earth salts which are already present. But while the salts of calcium are extremely active, the acids and the other alkali salts have only very slight activating actions, or even none at all.

It must be noted, however, that pawpaw rennet is also capable of producing the curdling of a non-calcified milk at $0^{\circ}$. This rennet is further characterized by a resistance to high temperatures; it possesses an optimum temperature of about $80^{\circ}$, and is not completely destroyed by an exposure of several minutes to a temperature of $100^{\circ}$. Gerber has found, in fact, that a solution of papayotin, heated only ro minutes in a boiling water-bath, is still capable, when placed in milk at $40^{\circ}$, of not coagulating it but of transforming it in such a way that the latter, afterwards boiled, coagulates. Rennet of papayotin, acting on boiled milk, obeys very well the law of Segelcke and Storch. But with fresh milk, we find that the long coagulations which can be still obtained at $40^{\circ}$, compared with the short coagulations obtained at the same temperature with larger quantities of enzyme, are longer than the law of inverse proportionality warrants. They deviate the more the further the temperature deviates from $40^{\circ}$. At $50^{\circ}$, there are only short coagulations, and they follow the law very poorly. With boiled milk, nothing of the sort takes place. The long coagulations are observed just as well above $40^{\circ}$ as below it. There is then in fresh milk a destruction of the coagulating agent, the more rapidly the higher the temperature.

Gerber thinks that it is the albuminoids of the milk (lactalbumin and lactoglobulin) that are the cause of the absence of long coagulations, and, consequently, of the destruction of the coagulating enzyme. The proof of this is given directly by the fact that we can heat fresh milk to $67^{\circ}$ without changing its special manner of caseifying, while after heating a half 
hour at $72^{\circ}$ (partial coagulation of the lactoglobulin), it now behaves differently, and that after $\frac{1}{2}$ hour at $78^{\circ}$ (complete coagulation of the two albumins), it becomes capable of giving coagulations almost as long as boiled milk, coagulations presenting the same characteristics. Later we shall find similar facts with regard to the digestion of natural albumins by papayotin. This close analogy between the coagulating properties and the proteolytic properties of pawpaw juice leads Gerber to the belief that perhaps we have here one and the same enzyme, an opinion conforming to a certain theory which will be developed in more detail in another chapter.

To return to the effect of heat on rennets, this resistance just described, however exceptional it may seem, is not characteristic solely of the rennet of the pawpaw, since it is found to a no less degree with the coagulating juice of belladonna (Atropa belladonna). This last rennet, also vegetable, is, in fact, the most resistant of all those known. Its optimum temperature is near $90^{\circ}$, and it is possible to obtain quite a good coagulation, even with boiled milk, if enough rennet is taken for the coagulation to be rapid. However, if the rennet is heated, not with the milk but alone, it is less resistant, 30 minutes at $100^{\circ}$ causing it to lose all activity and 30 minutes at $78^{\circ}$ reducing by a half its coagulating power.

Time Necessary for the COAgulation of 5 c.c. OF Fresh MilK OR BoILEd MiLK.

\begin{tabular}{c|r|c|c|c|c}
\hline & \multicolumn{4}{|c}{ Quantities of belladonna juice. } \\
\cline { 2 - 5 } $\begin{array}{c}\text { Temperature of } \\
\text { coagulation. }\end{array}$ & \multicolumn{2}{|c|}{0.48 c.c. } & \multicolumn{2}{|c}{0.16 c.c. } & 0.053 c.c. \\
\cline { 2 - 5 } & Fresh milk. & Boiled milk. & Fresh milk. & Boiled milk. & Boiled milk. \\
\hline & min. & min. & min. & min. & min. \\
$20^{\circ}$ & 280 & 240.00 & $\ldots$. & $\ldots$ & $\ldots$ \\
$45^{\circ}$ & 37 & 24.00 & 108 & 45.00 & $\ldots$ \\
$55^{\circ}$ & 25 & 11.00 & 70 & 22.00 & $\ldots$ \\
$65^{\circ}$ & 18 & 6.00 & 40 & 13.00 & $\ldots$ \\
$75^{\circ}$ & $\ldots$ & 4.30 & $\ldots$ & 10.30 & 27 \\
$85^{\circ}$ & $\ldots$ & 3.15 & $\ldots$ & 8.00 & 21 \\
$90^{\circ}$ & $\ldots$ & 2.30 & $\ldots$ & 7.00 & $\ldots$ \\
$95^{\circ}$ & $\ldots$ & 2.00 & $\ldots$ & $10.3 \circ$ & $\ldots$ \\
$100^{\circ}$ & $\ldots$ & 1.30 & $\ldots$ & 25.00 & $\ldots$ \\
\hline
\end{tabular}


This table indicates that belladonna-rennet coagulates boiled milk more rapidly than fresh milk.' In this it acts like rennets of the Crucifera and of the fig. The albuminoids of milk, coagulable by heat, have also a very evident retarding effect on its coagulation by the juice of belladonna, but the albuminoids of the white of egg or of horse serum have none at all. To conclude the peculiarities of this rennet, it is strongly basiphile in presence of neutral salts of alkali metals, slightly oxyphile and calciphile, and finally almost indifferent to neutral salts of the alkali metals.

Diana Brusch has confirmed some of the results found by Gerber concerning vegetable rennets. This investigator has found, among other things, that the coagulating enzyme of Ricinus communis resembles closely the lab-ferment. Its optimum temperature is at about $47^{\circ}$, the limit being at $67^{\circ}$. This enzyme acts exclusively in acid media. On the other hand, the rennet of Ficus carica acts in neutral or slightly acid media. Its maximum action takes place at $90^{\circ}$; and its destructive temperature is situated between $95^{\circ}$ and $100^{\circ}$.

It is seen that the characteristic properties of rennets vary to a rather large degree with their origin. Oxyphile rennets, in general, are likewise strongly calciphile, but there are also some, like pawpaw rennet, which are oxyphile and only slightly calciphile. Ordinarily, rennets are very sensitive to alkali. However, we have just noted that the rennet of belladonna is basiphile, and previously we have described prickwood as being capable of resisting a dose of 1.6 gram of soda per liter of media.

The irregular course of coagulation has also been an argument invoked in favor of the existence of several rennets. We have just given the modern conception of this. But before these various results were published, the fact of the non-observance of the law of Segelcke and Storch could have been taken into consideration. Bank, having encountered in the gastric juice of man and of the hog a rennet differing from ordinary rennet by a certain number of characteristics, among others the one cited 
above, gives it the name of parachymosin, reserving chymosin for the rennet taken from calf stomach linings.

I. Calf rennet is destroyed by the action of pepsin, while parachymosin resists pepsin in a slightly acid medium. After neutralization by sodium carbonate, the solution acts on normal milk.

2. Calf rennet is destroyed at $70^{\circ} \mathrm{C}$, the parachymosin at $75^{\circ}$ only.

3. Parachymosin is more sensitive to the action of alkalis. It is destroyed after 24 hours in the presence of one centigram per cent of alkali.

4. Parachymosin and rennet show perceptible differences to the action of $\mathrm{KCl}$ and of $\mathrm{CaCl}_{2}$. $\mathrm{KCl}$ paralyzes coagulation and $\mathrm{CaCl}_{2}$ accelerates it to a greater extent with parachymosin than with ordinary rennet, very small quantities of $\mathrm{CaCl}_{2}$ rendering parachymosin much more active.

- 5. Bubbling through $\mathrm{CO}_{2}$, which activates coagulation of milk with ordinary rennet, sensitizes milk with respect to parachymosin to a much larger degree. Example:

Fresh milk not treated: 5 c.c.

Pepsin solution ( $1-5): 2$ drops.

\} In complete coag. in $22 \mathrm{~min}$.

Fresh milk treated: 5 c.c.

Pepsin solution (I-100): 2 drops.

Coagulation in $5^{\frac{1}{2}} \mathrm{~min}$.

This sensitizing effect of $\mathrm{CO}_{2}$ takes place just as well with boiled milk as with fresh milk.

6. Finally, parachymosin does not obey the law of Segelcke and Storch. It cannot produce any but very rapid coagulations. Furthermore, from this point of view, rennet of gastric juice of a dog acts a little like parachymosin.

To these differences already existing between parachymosin and ordinary chymosin, Briot adds another, drawn from the existence of an anti-rennet of parachymosin, different from that of calf rennet. While the coagulating action of anti-parachymosin is retarded by mixing with $\mathrm{CO}_{2}$, that of anti-rennet is not influenced. Further, the action of heat is different. While anti-rennet is sensitive to a temperature of $60^{\circ}$, anti-parachy- 
mosin is much more thermostable, and can be left 20 minutes in a boiling water-bath without losing perceptibly its retarding power.

Irregular Action Due to Parachymosin and to Dog Rennet.

\begin{tabular}{|c|c|c|c|c|c|}
\hline \multicolumn{3}{|c|}{ Parachymosin. } & \multicolumn{3}{|c|}{ Gastric juice of a dog. } \\
\hline $\begin{array}{l}\text { Milk } \\
\text { (c.c.) }\end{array}$ & $\begin{array}{c}\text { Enzyme } \\
\text { (c.c.) }\end{array}$ & $\begin{array}{l}\text { Time for } \\
\text { coagulation. }\end{array}$ & $\begin{array}{l}\text { Milk } \\
\text { (c.c.) }\end{array}$ & $\begin{array}{l}\text { Gastric juice } \\
\text { (c.c.) }\end{array}$ & $\begin{array}{l}\text { Time for } \\
\text { coagulation. }\end{array}$ \\
\hline $\begin{array}{l}\text { 10 } \\
10 \\
10 \\
10\end{array}$ & $\begin{array}{l}\text { I } \\
\frac{1}{2} \\
-\frac{1}{4} \\
\frac{1}{8}\end{array}$ & $\begin{array}{r}2^{\prime}, 30^{\prime \prime} \\
7^{\prime}, 30^{\prime \prime} \\
40^{\prime} 0^{\prime \prime} \\
\text { No coag. }\end{array}$ & $\begin{array}{l}10 \\
\text { 10 } \\
\text { 10 } \\
\cdots\end{array}$ & $\begin{array}{l}0.20 \\
0.10 \\
0.05 \\
\ldots .\end{array}$ & $\begin{array}{l}\mathbf{r o}^{\prime} 5^{\prime \prime} \\
34^{\prime}, \mathbf{r o}^{\prime \prime} \\
\text { r } 59^{\prime} \infty^{\prime \prime} \\
\ldots \ldots\end{array}$ \\
\hline
\end{tabular}

Finally, the consideration of antibodies has allowed Hedin to distinguish four classes among the animal rennets: (I) calf rennet; (2) man rennet; (3) guinea-pig rennet; (4) pike rennet. This investigator gives as reason for this classification the fact that the infusions of these four rennets, treated by weak ammonia, and then neutralized, furnish four liquids containing the four antibodies which are specific only for enzymes of the same origin.

In the presence of all these findings, namely, a different sensitiveness toward chemical and physical conditions of the medium according to the origin of the active substance or of its antibody, anomalies observed in the course of the action according to the quantity of enzyme used, etc., it is generally concluded that the enzymes from various sources are not identical. Without denying the data brought in favor of this opinion, these still need to be confirmed, and, until proof to the contrary is offered, we hold this conception to be improper. In fact, in the study of the action of enzymes one cannot separate the action of the active substances from that of the medium in which they are found. We do not know any method permitting the isolation of enzymes in a pure state since they are always accompanied by other substances which influence their activity, either favorably or unfavorably. We have given the proof of this in many instances. (See also chapter on Pepsin.) 


\section{§ 7. Chemical Activity of Rennet.}

Up to now we have studied the action of rennet on milk, under various conditions of temperature and of reaction of the media, without considering the actual mechanism of the action. Let us now ask by what process caseinogen, which was originally in solution in the milk, is transformed into an insoluble clot. When the phenomenon is studied a little more closely it. is found that coagulation has not been directly caused by rennet. What we observe is only a reflection of the direct action, the curdling of milk being a result of enzymic action, but not representing the first stage of this action.

In the first place, the nitrogenous matter which coagulates under the influence of rennet differs, in its composition and its properties, from the caseinogen that was dissolved in the milk and from the casein that is precipitated when the latter is acidified. The first always leaves by calcination an ash residue, while the second and the third are free from mineral matter. Furthermore, the rotary power and the solubility in alkalis are not the same for both substances. It is, moreover, for this reason that we often give to the phenomenon of enzymic coagulation the special name caseification.

Further, as the result of the work of Hammarsten and that of Arthus and Pagès, we know that calcium ions play an important part in the phenomenon of caseification. This is clearly shown by the following classic experiment: To two samples of the same milk, each 100 c.c., is added a sufficient quantity of oxalate or of fluoride of sodium to precipitate all the calcium ions contained in the milk. Then to each of the two liquids is added the same quantity of rennet, a quantity just sufficient to cause in 20 minutes the coagulation of the milk not thus decalcified. It is found that the decalcified milk does not coagulate at all at the end of this time, nor even after a much longer action. Furthermore, coagulation does not take place if the quantity of rennet is increased. However, the rennet added to the decalcified milk has not remained inactive, the caseinogen having undergone a transformation. 
As evidence of this, if a small quantity of $\mathrm{CaCl}_{2}$ is added to the milk, the milk instantly curdles to an elastic and trembling clot, entirely identical with that produced by rennet and which is characteristic of caseification. From this, a double conclusion is reached. Rennet, in absence of calcium salts, does not coagulate caseinogen, but the latter is prepared for coagulation by the action of these salts.

To explain these purely experimental facts, two theories are at hand, one based on a chemical activity of the rennet; the other, on a physical activity.

Theory of Division. - This theory, due to Arthus, may be summed up as follows. Rennet divides the caseinogen into two albuminoid substances, one called caseogen substance or paracasein; the other, lactoserum-proteose. Only the first enters into the coagulum, the second remaining in solution. Calcium ions take part by combining with the paracasein formed to give the insoluble caseum which is precipitated. This theory of division of caseinogen by rennet depends on two observations by Arthus: (I) The weight of the coagulum is always less than that of the caseinogen of the milk. (2) In the serum is found an albuminoid substance, which is absent from the milk that has not undergone the action of rennet. This new material, which was not coagulated by boiling and which is not precipitated by acids, is a proteose.

The arguments produced in favor of this opinion are, however, far from being conclusive. In fact, if in this reaction there is really a production of a soluble albuminoid material at the expense of the caseinogen, we should have to find in the serum, after the action of the rennet, a very perceptible constant increase of albuminoid matter, an increase that is not verified by analysis. We know that natural milk will filter through porcelain filters, giving a filtrate free from casein. Consequently, by filtering the same milk, before and after caseification, we ought to find an increase of albuminoid matter in the liquid filtered. Now, the difference in composition of the two filtrates is not constant, and in every case, the figures obtained do not permit 
the conclusion that there is an increase. It has then been thought that this lactoproteose does not form in the course of caseification. If afterwards found present, it is because it was there originally, the existence of this product in normal milk being capable of being hidden by the large amount of caseinogen present.

But, even while admitting the formation of this substance in the course of coagulation, one can interpret this fact in an entirely different manner. The lactoproteose found in the caseified milk may come from the constant presence of pepsin contained in the rennet. Many investigations have been undertaken with the view of separating in an indisputable manner the action of these two enzymes that are always found associated. So far, the conclusions which have been reached have never been above criticism, and the question as to the origin of the proteose still remains pending. How could it be otherwise, since the supposition that rennet has a distinct individuality apart from pepsin or trypsin is disputed by many of even the better known physiologists?

Moreover, lactoproteose could also result from the action of other proteolytic enzymes, and especially from the enzymes of bacterial secretions. According to Couvreur, proteose, which is likewise formed in coagulation of milk by auto-acidification, a phenomenon known to be different from caseification, results from hydrolysis of albuminoids other than caseinogen. It is found present in a milk that is not fresh but still is not yet coagulated, while fresh milk, rapidly coagulated with rennet in the presence of an antiseptic like bichloride of mercury or in aseptic media, does not give lactoproteose. The appearance of this substance would then be a secondary effect due to the presence of bacteria. It is true that Arthus doubts this hypothesis, because, as he says, the solutions of purified caseum submitted to the action of the calcium-rennet give, as does milk, a precipitated caseum and a serum containing a newly formed proteose. It will be remarked that this answer is not itself above reproach in that the rennet used perhaps contained a trace of pepsin. 
We see that the discussion, lacking decisive argument, is not yet closed, and yet the importance of the evidence submitted is not negligible. Without doubt, the two fractions of the molecule of caseinogen which are separated by the rennet are of unequal importance. It is claimed that the insoluble part corresponds to about 95 per cent of the original albuminoid matter, while only 5 per cent passes into the form of lactoproteose. But, as cow's milk contains about $40 \mathrm{~g}$. of nitrogenous material per liter, the serum, after caseification, would contain just 2 g. per liter, to which should be added the $5 \mathrm{~g}$. which already existed there in the form of lactalbumin and lactoglobulin. A parallel increase of concentration could not pass unperceived. A still graver objection to this theory of division comes from the very size of the molecules formed. The lactoproteose which appears, and which is an albumose and not a peptone, has apparently a molecular weight equal at least to 2500 . The most favorable hypothesis at present being that the molecule of caseinogen divides into a molecule of paracasein and a molecule of proteose, we see that the molecule of caseinogen, which is 20 times larger than the molecule of proteose, would possess a weight which would be at least 50,000 , a figure much above the value generally given for albuminoid matter. If, on the contrary, we accept for caseinogen a molecular weight of 10,000, we conclude from it that the liberation of a single molecule of proteose, with a weight of 2500 , would bring about the conversion of 25 per cent of nitrogen into a soluble form, a result not at all conformable to experience. Thus, simply from the theoretical point of view, it appears hardly probable that the action of rennet on caseinogen is primarily in the nature of a division.

Moreover, the precipitated caseum always contains calcium. Hammarsten thought that this calcium resulted from the simple precipitation of the insoluble calcium phosphate of the milk with the paracasein. Arthus believes, on the contrary, that the caseinogen combines with the calcium salts of the milk to give an insoluble calcium complex. This hypothesis of the formation of a calcium complex in the coagulum, although 
very widely held, is not based on experiments whose conclusions are above reproach. First of all, the argument that calcium is indispensable to coagulation is very doubtful. In fact, what has been established is that oxalate or fluoride of sodium hinders the formation of the coagulum, but as, on the other hand, it is known that these salts are antagonistic to rennet, it is possible that the absence of caseification is due to an inhibition by an excess of these bodies. Furthermore, if, in reality, the paracasein combined with the calcium, the content of calcium in the coagulum should be constant. However, the contrary is observed, the analyses of the caseum showing variable quantities of calcium. These results indicate a mixture rather than of a definite chemical combination. Finally, we have said that decalcified milk, which has been then treated with rennet, coagulates instantly upon the addition of $\mathrm{CaCl}_{2}$. If we suppose that at this moment a calcium complex of casein is formed, it would be necessary to assume that the caseogen, or rather its alkali salt, decomposes the $\mathrm{CaCl}_{2}$ to take possession of the calcium. This is not impossible, but it appears more probable that the reaction takes place between the calcium chloride and the alkali phosphates which keep the casein in solution. Thus there would be produced calcium phosphate which would also carry down the caseogen. Furthermore, the hypothesis as to the formation of a calcium complex of casein is weakened by the fact that the presence of a calcium salt is not absolutely indispensable to cause the coagulation of milk oxalated and treated with rennet. Sodium chloride can produce the same result, although to a slightly less pronounced degree.

To summarize, from all the data just noted, there is a single fact which deserves to be remembered, namely, that the caseinogen has undergone a transformation under the influence of the rennet. The nature of this transformation still remains to be explained.

Physical Action of Rennet: Hypothesis of Duclaux. - In opposition to the hypothesis of the chemical activity of rennetdivision of caseinogen and subsequent precipitation of caseogen 
in the form of insoluble complex, Duclaux offers another conception. First of all, he finds that neutral alkaline-earth salts coagulate milk in a way similar to that of rennet. In the action of salts, as in that of rennet, a definite time is required to cause coagulation. The duration of contact necessary for coagulation diminishes with the proportion of salt. The reaction becomes more and more active as the temperature rises. In the case of salts, the optimum temperature is $100^{\circ}$, in that of rennet it is $40^{\circ}$ since heat in this case would destroy the active substance. The following figures are instructive:

Coagulating Quantities of Some Salts and Corresponding Temperatures.

\begin{tabular}{|c|c|c|}
\hline Substances added. & Quantity. & $\begin{array}{l}\text { Temperature of } \\
\text { coagulation. }\end{array}$ \\
\hline $\begin{array}{cc}\text { Crystallized } \mathrm{CaCl}_{2} . \\
“ " \\
\text { “" } \\
\end{array}$ & $\begin{array}{l}12.0 \% \\
4.0 \\
0.9\end{array}$ & $\begin{array}{r}15^{\circ} \\
40^{\circ} \\
100^{\circ}\end{array}$ \\
\hline $\begin{array}{c}\mathrm{SrCl}_{2} \ldots \ldots \ldots \ldots \ldots \ldots \\
\quad “ \quad \ldots \ldots \ldots \ldots \ldots \ldots \\
\quad \cdots\end{array}$ & $\begin{array}{l}8.0 \% \\
4.0 \\
0.5\end{array}$ & $\begin{array}{l}15^{\circ} \\
5^{\circ} \\
100^{\circ}\end{array}$ \\
\hline 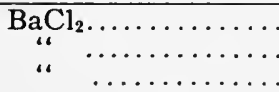 & $\begin{array}{l}8.0 \% \\
4.0 \\
0.5\end{array}$ & $\begin{array}{l}15^{\circ} \\
50^{\circ} \\
10^{\circ}\end{array}$ \\
\hline $\begin{array}{r}\mathrm{Ba}\left(\mathrm{NO}_{3}\right)_{2} \ldots \ldots \ldots \\
\ldots \ldots\end{array}$ & $\begin{array}{l}20.0 \% \\
0.5\end{array}$ & $\begin{array}{r}80^{\circ} \\
100^{\circ}\end{array}$ \\
\hline
\end{tabular}

To cause coagulation of milk in a few minutes, 0.5 per cent crystallized calcium chloride is added at $100^{\circ}$. To obtain the same result at $40^{\circ}, 4$ per cent is necessary; and at $15^{\circ}, \mathrm{I} 2$ per cent must be used.

Coagulation obtained with salt is caused exclusively by the change in salt concentration of the medium. It cannot be said that there is a true chemical reaction. A similar phenomenon is moreover observed in many other coagulations. The substance originally in solution changes its state under the influence of the salts added. Its physical properties are changed, among others its solubility. It is then precipitated, but coagulation has produced no change in the chemical composition. The coagula- 
tion of milk, whether with salts or with rennet, is explained by its very nature, that is to say by the physical state in which the caseinogen and the phosphates are found. The caseinogen is for the most part dissolved, but a fraction is also maintained in the milk in a state of suspension. The calcium phosphate is likewise only partially dissolved, a certain quantity being found in colloidal form.

The stability of these two substances is found to be interdependent, each exerting a reciprocal influence on the other. By changing the stability of the phosphates, one changes that of the casein, and the precipitation of the casein carries down the phosphates also. It can be shown experimentally that the stability of the casein is influenced by a whole series of conditions. Each time that we effect a physical change, whether of casein or of phosphates, we arrive at new conditions of coagulation. With boiled milk another process is observed than with natural milk. The difference becomes still more striking when we approach the conditions in which colloidal phosphate passes to the soluble form or to the crystalline form. The change of stability of substances in suspension in milk can also be caused by changes of density or of chemical composition of the milk. By the addition to the milk of alkaline-earth salts, coagulation is brought about, because the equilibrium which existed between the casein and the various inorganic substances contained in the milk has been disturbed.

Thus the action of rennet can be explained by the modification which it causes in the structure of the caseinogen. This change, entirely physical in nature, considerably weakens its stability in the solution. The casein, modified by the rennet, still remains in the milk, but its sensitiveness towards chemical and physical agents is greatly increased. In normal milk, casein sensitized by rennet is precipitated, carrying with it the calcium phosphate. In oxalated milk, the modified casein remains in suspension, but coagulation is produced by the addition of quantities of $\mathrm{CaCl}_{2}$ or of $\mathrm{NaCl}$, that were without action on the normal milk or on oxalated milk not treated with rennet. 
To summarize, the coagulation of milk by rennet is caused by a physical modification of the casein, and the coagulum is composed of a modified casein mixed with calcium phosphate. This opinion of Duclaux finds a quite pertinent argument in the work of Briot. This investigator has studied the reciprocal influence of phosphates and casein in the phenomenon of coagulation. The experiments which he cites on sodium chloride and citrate throw a special light on the different methods by which the course of coagulation can be influenced. The effect exerted by sodium chloride and citrate as well as by potassium oxalate on the velocity of coagulation have been tabulated as follows:

Influence of the Oxalate, Citrate, and Chloride of Sodium on CaseiFICATION.

\begin{tabular}{|c|c|c|}
\hline Nature of mixture. & $\begin{array}{l}\text { Phenomenon } \\
\text { observed at } 40^{\circ} \text {. }\end{array}$ & Observations. \\
\hline I. Natural milk... & Coagulates in $8^{\prime}$ & \\
\hline $\begin{array}{l}\text { 2. A. Milk }+ \text { r. } 25 \% \mathrm{NaCl} \ldots \ldots \\
\text { B. Milk }+2.5 \% \mathrm{NaCl} \ldots \ldots\end{array}$ & $\begin{array}{l}\text { Coagulates in } 10^{\prime} \\
\text { Coagulates in } 15^{\prime}\end{array}$ & \\
\hline $\begin{array}{l}\text { 3. A. Milk }+0.13 \% \mathrm{Na} \text { citrate } \\
\text { B. Milk }+0.4 \% \mathrm{Na} \text { citrate }\end{array}$ & $\begin{array}{l}\text { Coagulates in } 29^{\prime} \\
\text { Does not coagulate }\end{array}$ & $\begin{array}{l}\text { Coagulation incompl. } \\
\text { Coagulates at } 100^{\circ}\end{array}$ \\
\hline 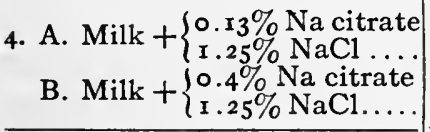 & $\left\{\begin{array}{l}\text { Coagulates in } \mathbf{r}^{\prime} \\
\text { Coagulates in } 32^{\prime}\end{array}\right.$ & \\
\hline 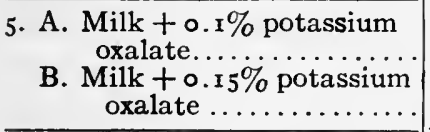 & $\begin{array}{l}\text { Coagulates in } 23^{\prime} \\
\text { Does not coagulate }\end{array}$ & $\begin{array}{l}\text { Coagulate at boiling } \\
\text { or by adding of very } \\
\text { little } \mathrm{CaCl}_{2}\end{array}$ \\
\hline 6. Milk $+\left\{\begin{array}{l}0.15 \% \text { oxalate } \ldots \\
1.25 \% \mathrm{NaCl} \ldots .\end{array}\right.$ & Coagulates in $40^{\prime}$ & \\
\hline
\end{tabular}

These figures show the retarding influence exercised by sodium chloride on coagulation. Normal milk coagulates in 8 minutes with rennet (experiment $\mathrm{I}$ ); the same milk treated with the same quantity of rennet coagulates in 15 minutes if 2.5 per cent of $\mathrm{NaCl}$ is added to it. Sodium citrate and potassium oxalate (experiments 3 and 5) act in a similar manner. Thus it is that 0.15 per cent of oxalate or 0.4 per cent of citrate are enough to prevent 
coagulation, even after a prolonged interval, and even though the quantity of rennet used is increased.

The three salts mentioned cause a similar effect in that they are all three retarders. However, the data of the table show that there is an essential difference in the internal mechanism of the action of these salts. The oxalate forms calcium oxalate, deriving its calcium from the colloidal phosphate. In consequence of the absence of this last body in the oxalated milk, coagulation of casein already transformed is hindered. The action of the sodium citrate is explained by its power to dissolve calcium phosphate. Vaudin has shown that normal milk contains $\mathbf{I . 2}$ to I.5 grams of citric acid per liter. The phosphate is kept in partial solution in the milk, due to the combined action of the alkali citrate and the lactose. By increasing the content of the citrate in the milk, we increase the stability of the solution of phosphate, and in this way prevent coagulation from taking place. Therefore, the oxalate, as well as the citrate, influences coagulation by modifying the stability of the phosphates of the milk, but the rennet is not at all influenced. The oxalated milk or citrated milk ( $3 \mathrm{~B}, 5 \mathrm{~B})$ coagulates at $100^{\circ}$, proving that the rennet has acted on the casein in spite of the addition of salts.

On the other hand, the milk treated with rennet, by addition of oxalate or of citrate in sufficient quantities to prevent coagulation (experiments 4 and 6), coagulates with the supplementary addition of weak quantities of sodium chloride. However, we have seen the retarding influence of sodium chloride (experiment 2). The fact is that the retarding action of the salt $(\mathrm{NaCl})$ has a quite different mechanism from those of citrate and oxalate. Here we have a direct reaction of sodium chloride on rennet. The salt $(\mathrm{NaCl})$.weakens the rennet, thus retarding the coagulation. But in the case of experiments 4 and 6, the action is more complex. This time there comes into play the property possessed by sodium chloride of aiding the coagulation of the modified casein. The salt then acts in two different directions. It retards the coagulation of the casein by weakening the rennet, but at the same time it aids coagulation by acting on the modified casein. 
In conclusion, rennet acts on the caseinogen of milk by modifying the chemical structure. This modification, whose exact nature is unknown, does not appear greatly to affect the molecule, but even less does it appear to divide it into two albuminoid fragments. The caseinogen, which this modification has rendered more sensitive to the action of substances which normally accompany it in the milk, coagulates spontaneously. Becoming insoluble, it carries with it the calcium phosphate, but the caseum formed seems rather to be a mixture of organic matter and of calcium salt than a true compound.

\section{§ 8. ANTI-RENNET.}

We have known for some time that normal horse serum inhibits the action of rennet. This action has been attributed, first of all, to the alkaline reaction of the serum, and it is only of late that the mechanism of this action has been studied at all closely. Briot and Morgenroth, simultaneously and independently of each other, have established the existence in the serum of an antibody which can inhibit the action of rennet. This enzyme has received the name of anti-rennet. To isolate it, normal horse serum is saturated with powdered ammonium. sulphate. A precipitate is obtained which is washed with a saturated solution of $\left(\mathrm{NH}_{4}\right)_{2} \mathrm{SO}_{4}$, and is then dissolved in an equal volume of distilled water. The $\left(\mathrm{NH}_{4}\right)_{2} \mathrm{SO}_{4}$ is eliminated by dialysis, and thus there is obtained a solution of anti-rennet of the same activity as the serum used, the $\left(\mathrm{NH}_{4}\right)_{2} \mathrm{SO}_{4}$ being without effect on this substance.

Anti-rennet withstands a temperature of $58^{\circ} \mathrm{C}$. At $60^{\circ}$ there is an alteration, which increases with the duration of the heating. At $62^{\circ}$, the destruction takes place more rapidly than at $60^{\circ}$, and after three hours at this temperature, anti-rennet becomes inactive. Alcohol precipitates anti-rennet from its solutions, but the precipitate obtained deteriorates very quickly and this mode of preparation of the anti-rennet does not give good results. 
The non-coagulation of milk treated with rennet, in presence of anti-rennet, must be attributed to the formation of a combination between rennet and anti-rennet, a combination having no action on the casein. This opinion is confirmed by the fact that there exists a constant proportion between the quantity of rennet and that of serum necessary to neutralize it. This proportion is shown by the following test: Put into a series of test tubes the same quantity of rennet of known value, and different quantities of serum. Let them stay for some time at ordinary temperature, then add to each tube so c.c. of milk and determine the point of coagulation according to the method of Morgenroth. Repeat the same test with different quantities of rennet:

Neutralization of Rennet by Anti-Rennet.

\begin{tabular}{c|c|c|c||c|c|c|c}
\hline Nos. & $\begin{array}{c}\text { I\% solution of } \\
\text { rennet (c.c.) }\end{array}$ & $\begin{array}{c}\text { Serum of a } \\
\text { horse (c.c.) }\end{array}$ & Results. & Nos. & $\begin{array}{c}\text { I\% solution of } \\
\text { rennet (c.c.) }\end{array}$ & $\begin{array}{c}\text { Horse } \\
\text { serum (c.c.) }\end{array}$ & Result.* \\
\hline & 0.5 & 0.80 & 0 & 6 & 0.05 & 0.070 & 0 \\
1 & 0.5 & 0.75 & 0 & 7 & 0.05 & 0.065 & 0 \\
2 & 0.5 & 0.70 & 0 & 8 & 0.05 & 0.060 & 0 \\
3 & 0.5 & 0.65 & 0 & 9 & 0.05 & 0.055 & + \\
4 & 0.5 & 0.60 & + & $\cdots$ & $\cdots$ & $\cdots$ & $\ldots$ \\
5 & 0.5 & 0.6 & \\
\hline
\end{tabular}

- The sign o means that there is no coagulation; the sign + that there is coagulation.

To neutralize 0.5 c.c. of I per cent rennet solution 0.6 c.c. of serum has been used; to neutralize 0.05 c.c. of rennet, practically a tenth as much is required. The existence of a constant proportion between the unit of rennet and the quantity of serum is likewise found when we mix different quantities of rennet with a definite quantity of serum. By adding to rennet the quantity of anti-rennet sufficient to render it inactive, we obtain a neutral mixture without action on the rennet or the milk. The addition of fresh rennet causes, in the milk thus treated, the same phenomenon as in normal milk.

The effect produced by anti-rennet on rennet is not instantaneous. As in all the actions of enzymes, the time factor is very important. The milk, containing serum, coagulates as soon as rennet is added, because the action of rennet on casein is more rapid than that of anti-rennet on rennet. 
To measure the anti-rennet value of serum, it is necessary to place the rennet in contact with the serum for some time. This reaction takes place at ordinary temperature and is completed after an hour. To estimate the anti-coagulating power of the serum, Briot takes as a standard the quantity of rennet at I : ro,, 00 , whose effect can be neutralized by I c.c. of serum. The content of the serum in anti-rennet differs according to the kind of animal. Furthermore, perceptible differences are observed in the serum of the same animal according to age:

Content in Anti-Rennet of Different Serums.

\begin{tabular}{|c|c|c|c|}
\hline Animal species. & $\begin{array}{l}\text { Quantity of rennet } \\
\text { neutralized by I } \\
\text { c.c. of serum. }\end{array}$ & Animal species. & $\begin{array}{l}\text { Quantity of rennet } \\
\text { neutralized by I } \\
\text { c.c. of serum. }\end{array}$ \\
\hline 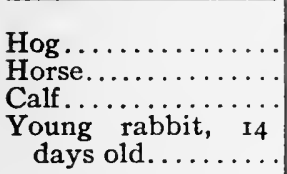 & $\begin{array}{l}\text { c.c. } \\
\text { From } \frac{1}{18} \text { to } \frac{1}{20} \\
\text { From } \frac{1}{19} \text { to } \frac{1}{25} \\
\text { From } \frac{1}{300} \text { to } \frac{1}{500} \\
\frac{1}{800}\end{array}$ & 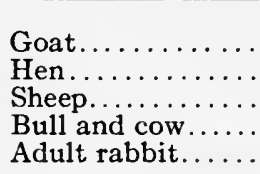 & $\begin{array}{l}\text { c.c. } \\
\frac{1}{2000} \\
\frac{1}{500} \\
\frac{1}{700} \\
\frac{1}{700} \text { to } \frac{1}{500} \\
1 \frac{1}{200}\end{array}$ \\
\hline
\end{tabular}

The quantity of anti-rennet contained normally in the blood can be increased considerably by injections of rennet taken from the animal under consideration. These injections can be made under the skin, in the veins, or even in the peritoneal cavity. They are repeated at intervals of ro days approximately. After Io injections, we get the maximum formation of anti-enzyme. Briot has treated rabbits with subcutaneous injections of Hansen's liquid rennet, which is previously freed of its salts by dialysis and then filtered through a Chamberland filter. $\mathrm{He}$ has found that the proportion of anti-rennet in serum increases with each injection. While at the beginning, I c.c. of serum was capable of neutralizing I c.c. of rennet diluted I to I 200 , after 8 injections, I c.c. of serum could neutralize I c.c. of rennet diluted only I to 50 .

If, in its turn, we inject anti-rennet several times into an animal, we cause in the blood of the latter the appearance of a new antibody which will neutralize the anti-rennet inoculated. Therefore, an anti-anti-rennet has thus been formed. In a 
general way, the organism always reacts against every foreign introduction, by the secretion of a body capable of neutralizing those which one seeks to impose upon it.

\section{§ 9. Determination of RenNet.}

Rennet is found in commerce in the form of a solution or of a powder. To determine the value of liquid rennet, a dilution is made I to I00. The solid product must be carefully dissolved in water to a I per cent concentration and the solution should be clear. In test tubes are introduced Io c.c. of milk, which are heated to $38^{\circ}$ in a water-bath kept at this temperature. As soon as the content of the tubes is at $38^{\circ}, \frac{1}{2}$ c.c. of a I per cent rennet solution is added, and the number of minutes which elapse before coagulation is determined.

\begin{tabular}{|c|c||c|c|}
\hline $\begin{array}{c}\text { Time for } \\
\text { coagulation. }\end{array}$ & $\begin{array}{c}\text { Value of } \\
\text { the rennet. }\end{array}$ & $\begin{array}{c}\text { Time for } \\
\text { coagulation. }\end{array}$ & $\begin{array}{c}\text { Value of } \\
\text { the rennet. }\end{array}$ \\
\hline Minutes. & 4000 & $\begin{array}{c}\text { Minutes. } \\
7\end{array}$ & 11000 \\
20 & 6000 & 6 & 13000 \\
15 & 8000 & 5 & 16000 \\
I0 & 10000 & $\ldots$ & $\ldots \ldots$ \\
8 & & \\
\hline
\end{tabular}

The value of the rennet is expressed by the number of c.c. of milk which a gram of rennet can coagulate in 40 minutes at a temperature of $38^{\circ} \mathrm{C}$. A $\frac{1}{2}$ c.c. of solution of rennet used corresponds to 5 milligrams of product analyzed. Introduced into so c.c. of milk, we get a proportion between the rennet and the milk of $I$ to 2000 . If we obtain, under these conditions, the coagulation in 20 minutes, we can calculate the quantity of milk which a gram of rennet can coagulate in 40 minutes in the following manner:

$\mathrm{X}: 2000=40: 20$, whence the value of the rennet, $X=4000$.

The Unit Method. - The preceding method is especially applicable to the analysis of commercial products of rennet. More accurate results can be obtained by using the method called 
" rennet unit." Effront uses as a rennet unit the quantity of enzyme which causes coagulation of ro c.c. of milk at $38^{\circ}$ in 40 minutes.

To obtain the value of a rennet, expressed in units above, proceed as follows: Fill a series of tubes with ro c.c. of milk, then bring them to $38^{\circ}$. Add in each tube I c.c. of active liquid previously brought to different dilutions with water. Observe in which tube the coagulation takes place in exactly 40 minutes.

Comparative Value of Several Products Containing Rennet.

\begin{tabular}{|c|c|c|c|c|c|}
\hline Origin. & $\begin{array}{l}\text { Nature of } \\
\text { dilution. }\end{array}$ & Units. & Origin. & $\begin{array}{l}\text { Nature of } \\
\text { dilution. }\end{array}$ & Units. \\
\hline $\begin{array}{l}\text { Dry rennet of calf } \\
\text { stomach lining.. }\end{array}$ & $I-I 500$ & I 500 & $\begin{array}{l}\text { Gastric juice neu- } \\
\text { tralized by bicar- } \\
\text { bonate }\end{array}$ & & O \\
\hline $\begin{array}{l}\text { Liquid commer- } \\
\text { cial rennet...... }\end{array}$ & $I-900$ & 900 & $\begin{array}{c}\text { Gastric juice not } \\
\text { neutralized....... }\end{array}$ & I-38 & 38 \\
\hline
\end{tabular}

Method of Morgenroth. - The " unit method" gives comparative values of different rennets, but it cannot be used to estimate very small quantities of active substance. In this case, even in working with reduced volumes of milk, the time required for coagulation can be prolonged for days, and a positive result can be then attributed to an infection, as a negative result can be imputed to the destruction of the enzyme at the temperature of $38^{\circ}-40^{\circ}$.

Morgenroth, to avoid these difficulties, proceeds in the following way: He shakes the milk energetically with I per cent of chloroform, then he cools to a temperature near $0^{\circ} \mathrm{C}$. He then adds for each ro c.c. of milk o.I c.c. to I c.c. of liquid to be analyzed and allows the whole to stand for 12 hours at about $0^{\circ}$. He then puts the samples in a water-bath at $38^{\circ}$. The action of the rennet when cold considerably diminishes the time of the action at $38^{\circ}$. Thus he avoids the injurious influence of the prolonged optimum temperature. Even in the presence of traces of rennet, coagulation is produced in the first three hours. The verification of the Morgenroth method has shown that for the measure of 
very small quantities of rennet it is four times more sensitive than ordinary methods.

W. Sawlajow suggests for the analysis of rennet a special apparatus which gives an accurate determination of the moment of coagulation of milk. Milk, treated with rennet, flows through capillary tubes at constant temperature. At the moment of coagulation, the outflow of droplets is checked.

Method of Blum and Fuld. - The exact determination of the coagulating power often presents real difficulties, for the origin of the milk plays an important rôle in these analyses. We have seen, among other things, that cooked milk is more difficult to coagulate with ordinary rennet than fresh milk. The degree of coagulability depends not only on the freshness of the milk but also on its composition. Thus with two samples of normal milk, not adulterated but of different origin, we may obtain quite different results.

Blum and Fuld, to avoid causes of error and to establish a scale for reference, use milk powder of Ekenberger as a standard. They weigh a definite quantity of this powder, dilute in 9 times its weight of distilled water and, while shaking it, heat the mixture for one minute at $80^{\circ}$. Then the liquid is cooled to $15^{\circ}$ and left to settle. The decanted solution can be kept in ice for I or 2 days, but it is better to use it at once for analysis. When ready to use, this artificial milk is made 0.4 per cent in $\mathrm{CaCl}_{2}$ by adding to 98 c.c. of the solution 2 c.c. of a 20 per cent solution of $\mathrm{CaCl}_{2}$. This done, it is well to let the liquid settle again for I to 2 hours at $15^{\circ}$ and then to decant. The product thus obtained behaves towards rennet like fresh milk. For the analysis, place 4.5 c.c. of artificial milk in a series of reaction tubes, and add 0.5 c.c. of the enzyme liquid to be analyzed, which has been previously diluted to different degrees. The whole is left 2 hours at $15^{\circ}$, then 5 minutes at $37^{\circ}$, and then the tubes are examined. The rennet value of the juice is estimated according to the extreme dilution still capable of producing coagulation under the conditions indicated.

Before definitely making the determination, it is well to make 
a preliminary test in the following way: Into 3 tubes place 4.5 c.c. of artificial milk, and into each 0.5 c.c. of the rennet to be analyzed, previously diluted from I to ro, from I to roo, and from I to I000. The test is made without previously leaving the tubes at $15^{\circ}$, but by keeping them directly for 5 minutes at $37^{\circ}$. The resulting coagulation gives an approximate idea of the value of the rennet examined. In the final test, which requires a preliminary heating for 2 hours at $15^{\circ}$, use a dilution 4 times as great as that found necessary in the approximate determination. If, for example, the coagulation at the time of the rapid test is produced even in the tube corresponding to a dilution of $\mathrm{r}$ to 1000 , one should use for coagulating liquids in the final test dilutions between the values I to roo0 and I to 4000 .

If we find that the extreme dilution capable of causing curdling of the milk is I to 3000 , the rennet value of the juice examined is 3000 units. In fact, considering the unit of rennet as that quantity which coagulates 4.5 c.c. of artificial milk used under the preceding conditions, we deduce from it that $\frac{1}{3000}$ of the rennet considered contains precisely $\mathrm{I}$ unit of rennet and that consequently I c.c. of the rennet to be analyzed is equivalent to 3000 units.

The method of Blum and Fuld is especially recommended for the determination of the coagulating power of gastric juice. A normal gastric juice requires, for analysis, a dilution of between 3000 and 7000 . There are very rich juices which require dilutions higher than 7000 , and juices are also met which are so poor in rennet that their content is below roo.

\section{BIBLIOGRAPHY.}

Arthus et Pagés. Arch. de Physiol., I890, (2), pp. 331, 540.

ARtHus. Soc. Biol., 1903, p. 795.

Abderhalden et Friedel. Absorption du lab. Zeits. f. physiol. Chem., (7r), p. 449 , I9I I.

Allemann et Muller. Chemismus der Labwirkung in d. Käsefabrication, Milchw. Centralbl., (7), p. 385 , 19 I .

O. Allemann. Die Bedeutung der Wasserstoffionen für die Milchgerinnung, Bioch.Zeits., (45), p. 346, I9I 2. 
Agulhon. Action de la lumière sur les diastases, Ann. Inst. Pasteur, IgI 2, p. 47.

BOKORNY. Chem. Zeitung, (25), p. 3, I901.

Brauler, R. Einfluss von Labmengen u. Temperatur auf die mikroskopische Structur des Caseïngerinsel, Pflüg. Arch., (133), p. 519, 1910.

Bang. Ueber Parachymosin, ein neues Labferment, Pfliug. Arch., (79), 1900, p. 425 .

Bang. Chemischer Vorgang. Skandinav. Arch.f. Physiol., (25), p. 105, 1911.

BRгот. (I) Études sur la présure et l'antiprésure, Thèse, Paris, 190. (2) C. R., (144), p. 1164, 1907.

BAGINSKy. Zeits.f. physiol. Chem., (7), pp. 209 et 22 I, 1882.

Blum et Boehme. Ueber das Verhalten des Lab, Beil. z. chem. Physiol. u. Path., pp. 9 et 74 , I906.

Blum Ex Fuld. Ueber eine Methode der Labbestimmung und über das Verhalten des Magenlabs unter normalen und pathologischen Zustanden. Berl. klin. Woch., r905, no. 44 .

Belgowski, I. Experimentelle Untersuchungen an Kälbern, Pflüg, Arch., (148), p. 320 , I91 2.

B. Bienenfeld. Frauenmilch, Bioch. Zeits., (7), p. 262, 1907.

A. Burr U. Berberich. Käufliche Labpräparate, Chem. Zeits., 1908, (32), p. 313 .

Camus et Gley. Arch. de Physiol., 1897, p. 764.

Conn. Cenl.f. Bacleriol., 1892, (1 2), p. 223.

Courant. Kuh u. Frauenmilch, Thèse, Breslau, r891.

Couvreur. Soc. Biol., 1906, (2), p. 512; I910, (2), p. 579; I9II, (I), p. 23.

Delezenne. Soc. Biol., 1907, (2), p. 187 .

Diana Bruschi. Atti. Accad. Roma, igo7, (i6), II, p. 360.

Deschamps. Dingl. Polyt. Journ., 1840, n ${ }^{\circ} 78$, p. 445.

Duclaux. (I) Traité de Microbiologie, II. (2) Sur le phosphate du lait, Ann. Insl. Pasleur, $1893, \mathrm{I} 6$.

Engel. Vergleichende Untersuchungen über das Verhalten von Frauenmilch zu Säure und Lab, Bioch. Zeils., (13), p. 89, 1908.

EDmunds. Journ. of Physiol., p. 466, (r9).

EFrront. Sur la présure, Monil. Scient., 1909, p. 305.

FrIEDBURG. Pharmaceut. Journal, 1889, p. 527.

Fuld et Wohlgemuth. Natur der Labfermentwirkung, Bioch. Zeits., (5), p. Ir8, I907.

C. Funck U. Niemann. Ueber die Filtration von Lab und Pepsin, Zeits. f. physiol. Chem., (68), p. 263, r9ro.

Fuld U. Hirayama. Ausscheidung von Lab u. Pepsin durch den Urin, Zeits. $f$. experiment. Pathologie u. Therapie, I91 2, (10), p. 248.

Lours Gaucher. Sur la digestion de la caséine. C.R., Igri, (2), p. 89 I.

Grognot. Industrie du lab, Revue génér. de Chimie pure el appl., mai 1907, p. 177 .

Gerber, C. Voir C. R. Acad. Sc. et Soc. Biol., de rgo7 á I9iz.

N. Georgiades. J. pharm. Chem., (9), p. 519, 1899.

GewIN. Zeils. f. physiol. Chem., 1908, (54), p. 32. 
S. Hedin. (1) Ueber Hemmung d. Labwirkung, Zeits. f. physiol. Chem., 1909, (60), p. 365. (2) Ueber das Labzymogen des Kalbsmagens, Zeits. f. physiol. Chem., I9I I, (72), p. 187. (3) Ueber spezifische Hemmung der Labwirkung, in verschiedenen Labenzymen, Zeils. f. physiol. Chem., I9I I, (74), p. 242; I91 2, (77), p. 229.

Halliburton AND Brodie. Journ. of Physiol., i896-97.

Hammarsten. (1) Malys Berichte, 1872, 1874. (2) Zur Kenntniss des Kaseins und der Wirkung des Labs, Dissert., Upsala, 1877 . (3) Zeits. f. physiol. Chem., (22), p. 103, 1897.

Herzog. Beziehung zwischen Pepsin u. Lab, Zeits. f. physiol. Chem., (60), p. 306, 1909.

H. HoFt. Labprüfung, Milchw. Centralbl., I908, (4), p. 489; I9ıо, (6), p. 49.

Hawk. Amer. Journ. of Physiol., (ro), p. 37, r9o3.

Van Herwerden. Magenverdauung d. Fische, Zeits. f. physiol. Chem., (56), p. 453,1908 .

Hofte. Labgerinnsel u. Säuremilchgerinnsel, Milchw. Centralbl., 1908, p. 293; I910, p. 533 .

Van Herwerden. Zeits. f. physiol. Chem., (52), p. 184, 1907.

Javillier. (I) Contribution à l'étude de la présure chez les végétaux, Thèse pharmacie, Paris, 1903. (2) Sur la recherche et la présence de la présure chez les végétaux, $C . R$., 1902, (r), p. 1373 .

M. JACOBY. Fermente und Antifermente, Bioch. Zeits., 1907, (4), pp. 21, 47I, 48 I.

Kreidl u. Neumann. Labgerinnung im Säuglingsmagen, Centralbl. f. Physiol., 1908, p. 133 .

Korschun. Lab. u. Antilab, Zeits. f. physiol. Chem., (36), p. I4I, 1902.

R. Kobert. Enzyme wirbelloser Tiere, Pfliig. Arch., (99), p. I I6, 1903.

A. KREId U. E. LeNK. Verhalten steriler u. gekochter Milch zu Lajo, Bioch. Zeits., (36), p. 357, I9r I.

Korschun. Lambolekül. Zeits.f. physiol. Chem., (37), p. 366, I903.

Kuraeff. Beit.z. chem. Physiol. u. Path., (2), p. 41 I, 1902.

Loeв. Bakterielles Lab. Centralbl. f. Bact. u. Parasitenk., (32), p. 471, 1908.

Lawrow. Zeits.f. physiol. Chem., (51), p. 1, 1907.

E. Laqueur. Theorie d. Labwirkung, Beil. z. chem. Physiol. u. Path., (7), p. 273, 1905; Biochem. Cent., (4), 333, 1905.

Langley. Journ. of Physiol., I881, p. 246.

LEA. Journ. of Physiol., 1885, p. 136.

Michaelis Et Rona. Beziehung zw. Lecithin u. Mastix, Osterreich. Pharm. Gesellsch., Wien, 1907.

Morgenroth. Antikörper des Labenzyms, Centralbl. f. Bact. u. Parasitenk., (26), p. 349, 1899; (27), p. 721, 1900.

Meunier. J. Pharm. Chem., I900, p. 460.

Pekelharing. Ueber Pepsin, Zeits. f. physiol. Chem., (35), p. 8, 1902.

F. Prylewski. Milchw. Centralbl., pp. 3 et $8 \mathrm{r}$, r907.

Popper. Ueber den Einfluss der Labgerinnung auf die Verdaulichkeit der Milch, Pfliig. Arch., (92), p. 605 .

Petry. Hofm. Beitr., 1906, p. 339, (8).

Peters. Ueber Labferment, Thèse, Rostock, 1894 . 
Pawlow et Paratschuk. Zeits. $f$. physiol. Chem., igo4, (42), p. 4 I5.

Pfleiderer. Pepsin u. Labwirkung, Pflitg. Arch., 1897, (66), p. 605.

R. RAPP. Hefelab, Contralbl. f. Bact. u. Parasitenk., (9), p. 625, 1903.

Reichel et Spiro. Beit. z. chem. Physiol. u. Pathol., 1906, (7), p. 485; 1906, (8), p. 15 .

Roberts. On Pancreatic Extracts, Proc. Roy. Soc., 1879, p. 160; 1881, p. 145.

Rosertr. Cynarase, das Koagul. Enzym d. Cynara Cardunculus, Chem. Cent., I899, (I), p. I3I.

SCHERN K. Hemmung d. Labwirkung, Bioch. Zeits., Igo9, (20), p. 23 I.

Signe und Sigval Schamid-Nielsen. Zeits.f. physiol. Chem., (60), p. 426, I909; (68), p. 317, I910.

Sigval Schmidt-Nielsen. (I) Radiumstrahlen, Beit. z. chem. Physiol. u. Path., 1904, (5) p. 398. (2) Paracasein, Beit. z. chem. Physiol. u. Path., 1907, (9) p. 322. (3) Zerstörung durch Licht, Beit. z. chem. Physiol. u. Path., 1909, (58), p. 233.

A. Scala. Constitution chimique du lab, Stas. Sperim. agrar. Roma, I904, (36), p. $94 \mathrm{I} ; 1907,(40)$, p. 129.

Stowzow. Labgerinnung d. Milch. Beit. z. chem. Physiol. u. Path., 1906, (9), p. 149.

P. Sommerfeld. Formalinmilch, Zeits. f. Hyg., I905, (50), p. 153.

Chana Smeliansky. Arch.f. Hyg., (59), p. $187,1906$.

SPIRO. Hofm. Beitr., I906, (8), p. 365.

Sawjalow. Zeits. f. physiol. Chem., 1905, p. 307, (46).

SIEGFeld. Die Einwirkung mässiger Wärme auf Labferment, Milchw. Centralbl., 1907, (3), p. 426.

Sellier. (I) Sur le pouvoir antiprésurant du sérum sanguin des animaux inférieurs, C. R., I906 (I), p. 409. (2) Soc. Biol., Ig06, (2), p. 449.

Teichert-Kurt. Ueber Bereitung von Labkugeln, Milchw. Centralbl., I9II, (7), p. 74 .

Van DaM. Handelslab, Landw. Vers.-Station, Hoorn, Holland, 19г2. Labgerinnung, Zeits. f. physiol. Chem., 1908, (58), p. 330; 1909, (58), p. 295; 1909, (6r), p. I47.

Van Hasselt. Zeils. $f$. physiol. Chem., I910, (70), p. 182.

Vaudin. Sur l'acide et le phosph. de chaux en dissolution dans le lait, Ann. Inst. Pasteur, 1894, p. 502, (8).

G. Werncken. Theorie der Milchgerinnung, Zeits. f. Biol., (52), p. 47, 1908.

F. WeIss. Zeits. f. physiol. Chem., I900, (31), p. 78.

A. Winogradow. Pflug. Arch., 1901, (87), p. 170.

Wohlgemuth U. Roeder. Lab und Pepsin, Bioch. Zeils., I906, (2), p. 42 I.

Wohlgemuth. Ueber das Labferment, Bioch. Zeits., I906, p. 350. 


\section{PART II.}

\section{PEPSIN.}





\section{CHAPTER I.}

§ I. History. Distributron. Preparation. Chemical Composition.

Pepsin is the active substance of the gastric juice. It possesses the property of dissolving and of peptonizing albuminoid matter. The discovery of this enzyme is intimately connected with researches made on digestion, the mechanism of which has for a long time occupied the world of scientists. At the very beginning, digestion was considered the result of a purely physical action, and was explained by the friction of food particles under the influence of pressure exerted by the walls of the stomach. Réamur (I683-I 757) first showed the influence of the stomachal juice in the phenomenon of digestion. His experiments consisted in causing falcons to swallow metallic tubes pierced with holes and filled with foods. He found that at the end of a certain time, these substances, although protected from all friction, were perfectly digested. About the same period, Spallanzani established the fact that the gastric juice outside of the stomach still preserved its power for dissolving flesh. To procure gastric juice, he caused a tame eagle to swallow a sponge fastened to the end of a cord. By drawing the sponge out and squeezing it, he obtained the juice sought.

However, the very clear data of Réamur and of Spallanzani were not accepted by the scientific world of the period. Experiments, which contradicted each other, considerably beclouded the question, so that physiologists for a long time disagreed on the subject of the mechanism of digestion. Some attributed it to the effect of vital and nervous force; others indeed assumed the intervention of the gastric juice, though this was then considered only a salivary liquid having merely undergone an acidification. The study of digestion, abandoned for some time, was actively 
taken up again between 1820 and 1840 . Tiedmann, Gmelen, Leuret, and Lassaigne, repeating the experiments of Réamur and of Spallanzani, showed anew that digestion is due to the gastric juice. They further established the fact that this liquid is not to be confounded with the salivary liquid, but is, on the contrary, secreted by the peptogen glands which furnish an acid juice possessing the property of digesting albuminoid material. In 1834, Beaumont took gastric juice from the stomach of a sick person having a fistula. About the same time, Eberlé prepared an artificial gastric juice by macerating pieces of stomach membrane in water very slightly acidified with hydrochloric acid. In 1839 , Wasmann isolated the active substance from a similar maceration. This, moreover, had already been foreseen by Schwann, who had given the name of pepsin to the active principle of the gastric juice.

Distribution. - Pepsin is an enzyme of animal origin exclusively. The analogous products which are found in vegetables differ from it perceptibly in their properties. This enzyme is found associated with other proteolytic enzymes in the digestive glands of invertebrates and, especially, in the vertebrates. Its principal source is the stomach; but it is also met in the blood, the muscles, the urine, etc. In the stomach, pepsin is secreted by glands with branching tubes. These are distributed in the stomachal mucous membrane and can easily be drained by maceration. For the preparation of pepsin, it is better to use the mucous membrane of a hog's stomach. One can also utilize the stomach lining of a calf or sheep.

Preparation. Method of Van Hasselt. - To obtain a solution of artificial pepsin free from other enzymes, the procedure is as follows: The stomachal mucous membranes of a hog, well minced, are macerated at ordinary temperature for Io to 15 days in a 5 per cent solution of $\mathrm{NaCl}$. The filtrate is saturated with $\mathrm{NaCl}$, and the precipitate, rich in coagulating enzyme, is removed. The filtrate, dialyzed to free it of the greater part of the $\mathrm{NaCl}$ which it contains, is then saturated with ammonium sulphate. The precipitate obtained, redissolved 
in water, is dialyzed with a 0.2 per cent solution of $\mathrm{HCl}$ to eliminate the ammonia salts. The solution thus obtained no longer coagulates milk and is rich in pepsin. Van Hasselt recommends the use of mucous membranes previously dried. Thus much less foreign material is dissolved, and a liquid of greater activity obtained.

Method of Petit. - To prepare a product possessing a large digestive power, proceed in the following manner: Hog stomachs are carefully washed and the mucous membranes separated by scraping. The pieces, reduced to a paste, are macerated in a 5 per cent alcohol solution, one part of mucous membrane for four parts of liquid. It is frequently shaken during maceration, which lasts 4 hours. Then it is filtered and evaporated at low temperature.

Method of Kühne. - Hog mucous membranes, previously minced, are put to macerate with water containing 2 to 3 grams of $\mathrm{HCl}$ per liter. A real digestion is produced and when the greater part of the albumoses has disappeared ammonium sulphate is added to the filtrate until no further effect is produced. The precipitate collected, containing pepsin and albumoses, is pressed, then dissolved in water slightly acidulated. It is left to digest for some time in such a way that the small quantity of albumose contained in the liquid is transformed into peptones not precipitable by ammonium sulphate. Again saturating with ammonium sulphate, the precipitate collected is submitted to the same treatment as before until it no longer contains a trace of albumose. At this point the precipitate, composed solely of pepsin, is dissolved in water. The product is purified by dialysis, and is then precipitated by alcohol.

To extract the pepsin from stomachal mucous membranes, the use of glycerin is also recommended, or rather of glycerin with addition of $\mathrm{HCl}$. Glycerin has the power of dissolving pepsin without dissolving great quantities of albuminoid matter. To precipitate pepsin from the macerations of mucous membrane, we can also utilize the property which enzymes possess of being 
precipitated by inert bodies or of being carried down by precipitates formed evenly throughout their liquid solutions. Thus, by adding to a maceration of a gastric mucous membrane sodium phosphate and then calcium chloride, a precipitate of calcium phosphate is obtained, which, dissolved in dilute $\mathrm{HCl}$ and then dialyzed, gives a solution containing the greater part of the pepsin contained in the initial liquid. In the same way we can extract pepsin from the gastric juice by mechanical precipitation or by means of cholesterin.

Industrial pepsin is prepared in the following manner: To roo kg. of hog mucous membrane, well minced and cleaned, are added about 125 liters of water, I liter of concentrated $\mathrm{HCl}$, and $150 \mathrm{~g}$. of chloroform. The whole is kept cool, and is often stirred. After 24 hours the mixture is filtered and the liquid is reduced by evaporation at $40^{\circ}$ to $\frac{1}{4}$ of its volume. It is again filtered, and is concentrated in a vacuum on large surfaces at a temperature as low as possible. $100 \mathrm{~kg}$. of mucous membrane gives 8 to ro $\mathrm{kg}$. of commercial pepsin.

The method of Van Hasselt, as a result of using dried mucous membrane, affords the great advantage of furnishing an enzyme much less contaminated by foreign substances and also almost free from rennet. This method, however, does not lend itself well to the preparation of a powdered pepsin; in fact, the enzyme thus obtained withstands evaporation very badly, so much so that products showing little activity are obtained when the solutions are concentrated. The method of Petit gives a very active product. Yet, in spite of the slight duration of the maceration and the presence of alcohol, the pepsin obtained still contains much ash as well as foreign organic substances. The method of Kühne does not yield a very active pepsin.

Chemical Composition. - Pepsin appears in the form of a white powder, soluble in acidulated water, as well as in 5 per cent alcohol. Pepsin is not dialyzable: when we seek to dialyze a solution of pepsin in 0.2 per cent of $\mathrm{HCl}$ in the presence of ordinary water, we find that the enzyme is precipitated in the form 
of little sticky transparent granules when the acidity falls to 0.02 per cent. The preparations of well-purified pepsin do not give the color reactions of the albuminoids. Their solutions are precipitated by an addition of ammonium sulphate and zinc sulphate, and this precipitation is already far advanced after the addition of a quantity of salt equal to half of that necessary for saturation. Absolute alcohol likewise precipitates pepsin. This enzyme, submitted to acid hydrolysis, yields tyrosin, leucin, alanin, as well as many other amino-acids, and consequently acts like an albuminoid.

The product which is taken from the gastric juice, as well as that obtained by the maceration of stomach mucous membrane, contains phosphorus and ash up to I.5 per cent. Its content in nitrogen is from 14 to 15 per cent. It also contains sodium and chlorine. By purification, we suceed in considerably reducing the ash content, in eliminating all the phosphorus, and in obtaining a more constant nitrogen per cent. But a criterion of purity is lacking for a given pepsin, as is the case for any enzyme. In fact, pepsin, properly speaking, may be considered as a mixture of a really active substance and inert material. A well-conducted purification ought gradually to eliminate the foreign substances without altering the active body. The final product, which with the minimum weight would produce the maximum effect, could be considered as the true pepsin. This, submitted to analysis, would give definite data on the constitution of this substance.

Unfortunately, this method, which recalls that followed by the scientists who isolated radium, cannot be rigorously applied here. The precipitations obtained by means of salts or of alcohol free the enzyme from a part of the inert substances, but at the same time they considerably weaken the active substance. In the final product we do not find an increase in percentage activity corresponding, to the quantity of impurities taken away. In other words, the purest pepsins are not always those which are the most active. Thus a crude pepsin, obtained by the method of Petit, can dissolve $100,000 \mathrm{~g}$. 
of fibrin for I $\mathrm{g}$. of enzyme By purifying this product according to the best existing methods, 80 per cent of substance is removed, and the product obtained possesses an activity of only Io per cent to 20 per cent greater than that of the initial product. While I $g$. of this purified pepsin should be able to dissolve $500,000 \mathrm{~g}$. of fibrin, it dissolves barely II0,000 g. to I $20,000 \mathrm{~g}$.

If we compare the percentage compositions of a pepsin before and after purification, we do not find perceptible differences, with the exceptions of the salts. This result can be interpreted in two ways. Either the impurities and the active substances are of analogous composition, and then the enzyme belongs to the albuminoid group, or else the actual quantity of active substance is so small that it does not influence the composition of the inert matter. This last hypothesis is not at all improbable. It is known that enzymes have the power of adhering to a number of substances. In the mucous membranes and in the gastric juice there are albuminoids to which the pepsin would be attached, and all the separations attempted would not be able to part them. Salts would precipitate them in different proportions, but always together:

We owe to Nencki and Siber, to Samanoski and to Pekelharing a series of careful analyses of pepsin. These have always been made on purified products possessing a fairly high enzymic power. The methods of purification used were very different and the pepsins obtained were standardized according to their activity. Nevertheless, it is very curious

Analyses of Pepsin.

\begin{tabular}{|c|c|c|c|c|}
\hline & Pekelharing. & $\begin{array}{l}\text { Nencki and } \\
\text { Siber. }\end{array}$ & Samanoski. & White of egg. \\
\hline 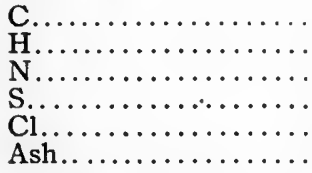 & $\begin{array}{r}51.99 \\
7.07 \\
14.44 \\
1.63 \\
0.49 \\
0.10\end{array}$ & $\begin{array}{r}51.26 \\
6.74 \\
14.33 \\
1.50 \\
0.48 \\
\ldots . .\end{array}$ & $\begin{array}{r}50.37 \\
6.88 \\
\mathrm{r} 4.55 \\
\mathrm{r} .35 \\
\circ .89 \\
\ldots\end{array}$ & $\begin{array}{r}54.30 \\
7.10 \\
15.80 \\
1.80 \\
\cdots \\
\cdots\end{array}$ \\
\hline
\end{tabular}


to find that the figures given by these investigators agree very well and are entirely comparable to those furnished by the analysis of white of egg.

Pekelharing considers his pepsin as a pure enzyme deprived of inert substance. He believes that this enzyme is an albuminoid, or rather that it is found intimately fixed on albuminoid matter; he justifies his opinion by the following considerations:

I. Pepsin becomes inactive if heated to $100^{\circ}$; now, at the same temperature the albuminoid substance coagulates, and this transformation involves the destruction of the enzyme.

2. When a solution of pepsin is half saturated with ammonium sulphate the albuminoid material is precipitated together with the active substance adsorbed by it.

3. The solutions of acidified pepsin are coagulated by heat, and the quantity of coagulum which is obtained is proportional to the activity of the liquid; the more active the solution the larger the coagulum.

The demonstration of Pekelharing does not completely solve the problem; it is, in fact, difficult to believe for the reasons given above that purified pepsin is a pure substance.

It should be remembered, however, that phosphorus does not enter into the composition of pepsin; that on the contrary, all the samples examined contain chlorine; finally, that the ash can be reduced to $0 . \mathrm{I}$ per cent and that iron is always detected in this ash. So far, it has not been possible to obtain pepsin free from inorganic matter. It is true that the presence of o.I per cent ash represents a very slight content, but if we take into consideration the fact that the preparation analyzed by Pekelharing was far from being a pure pepsin, we are led to believe that true enzyme, free from foreign matter, has a much greater proportion of ash.

Moreover, it has not been proved that we should consider these inorganic substances as impurities. The data furnished by Bertrand and Lisbonne justifies the hypothesis regarding mineral matter as an essential part of the active substance. Bertrand has shown the constant presence of manganese in 
laccase. This element is a constituent of this enzyme; in any case, it functions as activator and as conveyor of oxygen. According to this investigator we must regard the oxidases as true combinations of manganese with proteolytic substances of an acid function. The study of amylase leads to similar results. Lisbonne has found that the salivary, pancreatic and vegetable amylases when completely free from inorganic substances do not act on starch free from salts. These enzymes can again be rendered active if sodium or calcium chloride is added. These experiments, which relate to two different classes of enzymes, indicate that the inorganic substance must be regarded as a co-enzyme. It is very probable that when we study more closely the constitution of pepsin, we shall discover the importance of inorganic substances and especially of iron, whose presence is always detected in preparations of this enzyme.

\section{$\S$ 2. Dissolving and Peptonizing Powers of Pepsin.}

Demonstration of Dissolving Power: Effront Method. - To show the dissolving power of pepsin, we may use an albumin milk coagulated and containing 4 per cent of albumin.* Into each of 6 flasks put 20 c.c. of this liquid, which has previously been passed through a very fine sieve, and add to each $\mathrm{I} .5$ c.c. of $\mathrm{HCl}(\mathrm{N})$. In the first 5 flasks place $\mathrm{I}$ to 5 c.c. of a I per cent solution of pepsin; the sixth receives nothing and will serve as control. Then bring all the liquids to a volume of 40 c.c. with distilled water and place the whole in a water-bath at $50^{\circ}$. The control flask will stay white during the entire experiment. The flask which has received 5 c.c. of pepsin solution will very rapidly become clear as the result of the solution of the albumin. As to the other liquids, they clarify progressively, the complete solution of albumin in the first 5 flasks taking place in the space of 20 to 30 minutes.

Method of Gützner. - This method is based on the property

* For the manner of preparation of this milk see the chapter relating to the analysis of pepsin (method of Effront). 
which fibrin possesses of adsorbing coloring matter. Colored fibrin in dissolving colors the liquid, and according to the intensity of its coloration we judge of the extent of the solution of the fibrin. To perform this experiment, the fibrin is made to swell in an ammoniacal solution of carmine; then the product is washed in water and is macerated in 0.2 per cent $\mathrm{HCl}$. Thus is obtained a gelatinous mass, translucent and colored, which is dried on paper. For the tests, 0.25 to $0.50 \mathrm{~g}$. of colored fibrin is suspended in 50 c.c. of 0.2 per cent $\mathrm{HCl}$ and the pepsin is added. The liquid, which is colorless at the beginning, takes on a more and more pronounced tint.

For a demonstration before an audience, one may proceed thus: Fill a funnel with colored fibrin and then scatter over the gelatinous mass a solution of pepsin. Liquefaction begins at once and the liquid which flows out of the funnel is colored. In place of fibrin meat may also be used; choose lean minced meat, and work with to parts 0.2 per cent $\mathrm{HCl}$. The solution is then quite rapid, but it is not complete and the liquid remains turbid.

Demonstration of Peptonizing Power: Method with Edestin. - The peptonizing power of pepsin is exerted much less quickly than the dissolving power. Under the action of pepsin, a progressive hydrolysis is effected which results in the formation of acid albumins and albumoses, bodies which are precipitated by saturation of the liquor with zinc sulphate or ammonium sulphate, and complex polypeptides. The chemical transformations which albuminoid matter undergoes under the influence of pepsin will constitute the subject of another chapter.

For the demonstration of peptonizing power the method of Fuld with edestin can be utilized. A 0.5 per cent solution of edestin in $\mathrm{N} / 30 \mathrm{HCl}$ is prepared, filtered, and ro c.c. are placed in each of a series of test tubes. Add to the control tube ro c.c. $N / 30 \mathrm{HCl}$, while in the other tubes place 0.5 c.c., I c.c., 2.5 c.c., and 5 c.c. of a 0.5 per cent solution of pepsin in $N / 30 \mathrm{HCl}$ and bring the volumes to 20 c.c. with $N / 30 \mathrm{HCl}$. After Io or 15 
minutes at the ordinary temperature, add in all the tubes 5 c.c. of ro per cent $\mathrm{NaCl}$. In the control tube, the edestin is precipitated and the liquid becomes completely opaque; on the contrary, in the tube which has received the maximum of pepsin, no turbidity is observed, and the intermediate tubes give turbidities inversely proportional to the amount of pepsin they have received.

Remarks. - I. The dissolving and peptonizing powers of pepsin are always found in all the active preparations; these two properties are ordinarily shown with an equal intensity, a product which liquefies rapidly having also the faculty of peptonizing in a short time. However, this rule is not general. We shall see, in fact, that the conditions which favor the manifestation of one of the properties are not always those which favor the manifestation of the other, and that, furthermore, in the pepsins secreted by bacteria or molds, we find cases where the two properties do not occur on an equal footing.

2. Tests made with edestin show that the peptonizing action is rapid even at the ordinary temperature. However, what is observed here corresponds only to the very first phase of the transformation. To reach the end of peptonization, there remains still a very long road to travel, representing a considerable chemical change. Unfortunately we do not possess reagents capable of indicating the end of the peptonizing reaction. At the point where the digestive action is completely arrested, we still find in the liquid some of the molecular products formed at the beginning of digestion.

Use of the Tannin Reagent. - These fundamental transformations which the albuminoids undergo under the influence of pepsin can be followed by the aid of a solution of tannin with addition of tartaric acid, a solution which precipitates the natural albuminoids as well as the albumoses.* At the

* This solution is prepared by dissolving $100 \mathrm{~g}$. of tannin in I liter of water, adding 100 c.c. of normal $\mathrm{NaOH}$ and 30 c.c. of to per cent tartaric acid and then bringing the whole to a volume of 2 liters. I 50 c.c. of this solution is used for each mixture. The precipitate is washed on a filter with fresh solution of tannin and then the nitrogen is determined by the Kjeldahl method. 
beginning of peptonization almost all the nitrogenous material is precipitated by this reagent. Then as digestion advances the precipitate becomes less and less abundant. The following are figures on a peptonization of acidified albumin in the presence of a small amount of pepsin at a temperature of $40^{\circ}$.

Variation of Tannin Nitrogen in the Course of Digestion.

Length of digestion.

Per cent of total nitrogen precipitated.

At the beginning. $\ldots \ldots \ldots \ldots \ldots \ldots \ldots \ldots \ldots \ldots \ldots \ldots \ldots \ldots,{ }_{91} \%$

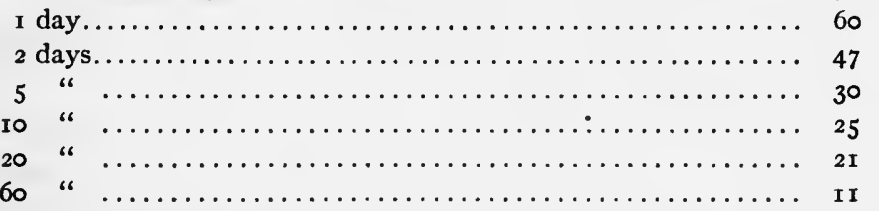

It is observed that after 24 hours 60 per cent of the total nitrogen is still precipitable by tannin, while at the end of 60 days there is not over II per cent precipitable. However, this remaining precipitable nitrogen is still in large part susceptible to digestion, and to transformation into non-precipitable nitrogen without ever arriving at the freeing of the liquid from all the substances precipitable by tannin or zinc sulphate.

\section{§ 3. EFfect of Temperature.}

Influence of Temperature on the Dissolving and Peptonizing Powers. - The two properties of pepsin, that of dissolving and digesting, are considerably influenced by temperature, but to a very different extent. When a solution of pepsin remains for some time at a temperature of $68^{\circ}$, the liquid loses almost wholly its peptonizing power, while its action on coagulated albumin or on fibrin is still very pronounced. Effront, in determining the time required for the solution of albumin at different temperatures, has arrived at the following results: 
Influence of Temperature on the Time of Solution of Albumin. Temperature

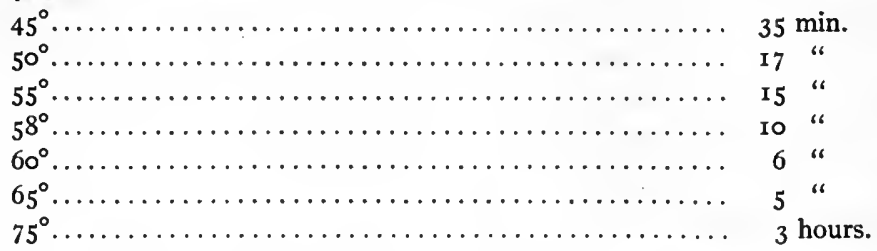

The tests were made with coagulated albumin finely divided and sifted. With this product sufficiently homogeneous, the optimum temperature was found to be $65^{\circ}$; at $50^{\circ}$ the pepsin acted three times so slowly.

The optimum temperature is very near the destructive temperature. At a temperature of $65^{\circ}$ pepsin acts very rapidly at first, but soon the action is retarded, due to the destruction of the active substance. The influence of a prolonged heating is summarized in the following table:

Influence of a Previous Heating on the Dissolving Power of Pepsin.

\begin{tabular}{|c|c|c|c|}
\hline \multirow{2}{*}{ Numbers. } & \multirow{2}{*}{ Temperature. } & \multicolumn{2}{|c|}{ Dissolving power. } \\
\cline { 4 - 4 } & & After I hour. & After 6 hours. \\
\cline { 3 - 4 } & & & \\
I & $40^{\circ}$ & 100 & 100 \\
2 & 50 & 100 & 100 \\
3 & 60 & 100 & 80 \\
4 & 65 & 91 & 75 \\
5 & 70 & 27 & 0 \\
\hline
\end{tabular}

The solution of pepsin is kept at temperatures varying from $40^{\circ}$ to $70^{\circ}$ for I and 6 hours. After these exposures I c.c. of enzyme is removed, a certain quantity of albumin milk is added, and the mixture is left at $50^{\circ}$. Thus in (I) and (2), where the pepsin has been previously heated to $40^{\circ}$ or $50^{\circ}$, the liquefying power survives even after 6 hours of heating. On the other hand, a stay of 6 hours in a bath at $60^{\circ}$ lowers the liquefying power to 80 .

In these tests the pepsin has shown a fair resistance to the action of temperature, due to the fact that the solutions have 
previously been exactly neutralized. On the other hand, in the case of a slight acidity, the enzyme keeps much less satisfactorily even at perceptibly lower temperatures.

\section{Effect of Preliminary Heating on a Pepsin}

Very Slightly Acidified.

\begin{tabular}{|c|c|c|}
\hline Temperatures. & $\begin{array}{c}\text { Time in } \\
\text { minutes. }\end{array}$ & $\begin{array}{c}\text { Liquefying } \\
\text { power. }\end{array}$ \\
\hline & & 100 \\
$30^{\circ}$ & 15 & 83 \\
50 & 15 & 78 \\
50 & 30 & 71 \\
50 & 60 & \\
\hline
\end{tabular}

With rather large quantities of acid, pepsin shows a greater sensitiveness to the effect of temperature, this sensitiveness increasing considerably with the acidity. On the other hand, the way in which the optimum temperature varies with varying quantities of $\mathrm{HCl}$ is shown below:

With $\mathrm{r} .5 \mathrm{~g}$. $\mathrm{HCl}$ per liter, the optimum temperature is.......... $65^{\circ}$

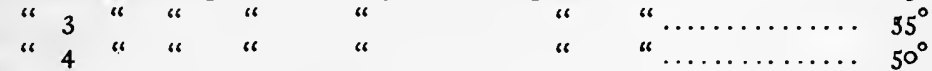

The optimum temperature is also influenced, up to a certain point, by the concentration of the liquids and their content in salts. For albumin, this depends again on its state of coagulation. Thus with an albumin coagulated at $85^{\circ}$ we observe an optimum higher by $6^{\circ}$ than that shown by an albumin coagulated at $70^{\circ}$.

All these facts, which show the great sensitiveness of pepsin toward physical or chemical agents, explain why the data furnished by the writers on the optimum temperature vary. The reason for such variations is that each time the conditions are different. According to Pawlow and Jacobi, pepsin would be very sensitive to the effect of a temperature of even $45^{\circ}$. These investigators found almost a complete destruction of pepsin after a heating of 10 minutes at $5 \mathrm{r}^{\circ}$. The optimum for the peptonizing power of pepsin lies between $40^{\circ}$ and $45^{\circ}$. In' a digestion of short duration it is the temperature of $45^{\circ}$ which is found to be most appropriate; on the contrary, a thorough pep- 
tonization, requiring a prolonged action, takes place preferably at $40^{\circ}$.

According to Agro and Sago, pepsin shows activity towards ricin at a temperature near $0^{\circ}$. At the temperature of $8^{\circ}$, the action is very pronounced. On the other hand, we know that edestin is transformed at ordinary temperature. As to the albumin and the fibrin, they are hardly at all attacked by pepsin at low temperatures. These facts show then that the action of the proteolytic enzyme depends largely on the nature of the bodies on which it acts.

The great sensitiveness which pepsin offers towards heat is due particularly to the water which accompanies it. Pepsin in powdered form can withstand for 20 minutes a temperature of $108^{\circ}$ without losing its digestive power. The solutions of pepsin with addition of peptone, of sugar, or of other materials, withstand heat better than pure solutions of pepsin. The ability which pepsin in powdered form possesses to withstand high temperatures is utilized to conduct experiments in sterile conditions. The liquid to be peptonized is sterilized separately, as well as the pepsin placed in a little tube. Then, with the desired precautions, the two products can be mixed.

Effect of Temperature on the Physical State of Pepsin. The influence which heat can exercise on the solutions of pepsin cannot always be controlled by direct analysis, and yet it is real. Thus a solution of pepsin, slightly weakened by rapid heating, offers a much lower resistance to physical and chemical conditions of the medium. The optimum temperature, the most favorable quantity of acid, etc., are changed. We also notice a greater sensitiveness to chloroform, to fluorides, and to the other antiseptics. In a general way, we may say that heat considerably changes the relations existing between the pepsin and the antiseptics. The modifications undergone by pepsin are also influenced by the fact that the solutions withstand shaking to a less extent after heating than before. A pepsin heated to $45^{\circ}$ and then shaken very vigorously loses almost all its enzymic power. 
Finally, there are facts of another sort which are also very conclusive. When horse serum is added to a solution of pepsin, the latter is rendered inactive by a sort of neutralization. This action is regular; if, for example, one part of serum neutralizes a certain quantity of pepsin, or rather a certain number of pepsin units, we find that a double quantity of serum neutralizes a double number of pepsin units. Now this constancy between the active substance of the pepsin and the anti-peptic power of the serum is no longer found when a pepsin, previously kept at $50^{\circ}$, is used. One part of serum is able to neutralize more heated pepsin than non-heated pepsin. The quantity of pepsin which neutralizes the same amount of serum increases with the exposure of the enzyme at $50^{\circ}$, and in a proportion considerably larger than that warranted by the destruction which analysis indicates. The antiseptic is, from this point of view, a much more sensitive reagent than the albumin for indicating a diminution of activity due to a moderate heating. We shall find absolutely analogous facts in the study of anti-trypsin.

Regeneration of Enzymes. - It is generally admitted that in the neighborhood of $100^{\circ}$ enzymes are definitely destroyed. The destructive temperature of enzymes of vegetable origin is ordinarily found at about $80^{\circ}$, while for enzymes of animal origin it is situated at about $70^{\circ}$. These data, which have been considered definite, do not however apply to certain oxidases which withstand a temperature of $100^{\circ}$. In 1907, G. Bertrand and W. Mutermilch observed that the solution of tyrosinase, heated to $95^{\circ}$, becomes inactive, and that this inactivity is only temporary. In the solution, after treatment at $95^{\circ}$, is observed a sort of revivification of the enzyme, and soon the solution regains its ability to oxidize tyrosin. Also, in 1908, Kulsohns showed that the oxidase of horse-radish is destroyed at $100^{\circ}$, but that it forms again when the liquid is left at ordinary temperature. This regeneration is also observed with solutions of the enzyme heated to II $5^{\circ}$. In I9I0, Gramentzki observed a similar phenomenon with the takadiastase, the maltin of Merck, pancreatin, and invertin. 
The takadiastase contains an enzyme converting starch into sugar and which is destroyed at $80^{\circ}$. The solution, when heated to $95^{\circ}$ and then kept at ordinary temperature, again becomes active.

\section{REgENERATION OF TAKADIASTASE}

Enzyme power.

Liquid not heated............................. 12.

Liquid heated to $95^{\circ}$ and analyzed after a few $\min \ldots \ldots \ldots \ldots \ldots \ldots$

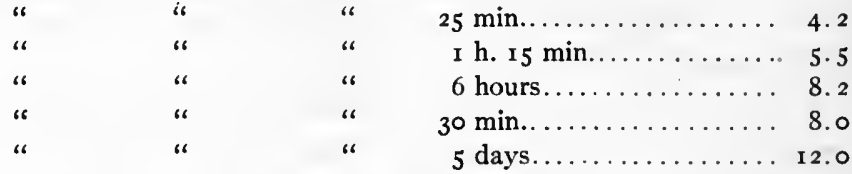

Regeneration takes place also in a liquid heated to $\operatorname{II} 5^{\circ}$ for I5 minutes:

Enzyme power

Liquid heated to $115^{\circ}$ and immediately analyzed.............. 0.0

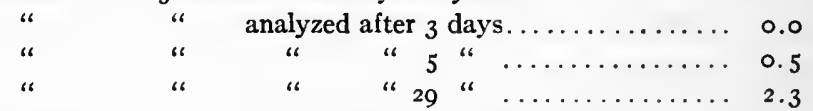

From data furnished by Gramentzki it appears that the regeneration depends on the temperature to which the liquid has been brought at the beginning; the liquid heated to $95^{\circ}$ is easily regenerated, but, on the contrary, the one heated to I $5^{\circ}$ demands more time for regaining a certain activity, and the final product obtained corresponds to a weaker enzymic power. The regeneration also depends on the temperature at which the liquid is left after heating. At the temperature of $50^{\circ}$ there is no regeneration; at $45^{\circ}$ it is very imperfect; at $40^{\circ}$ it is very rapid but never complete. On the other hand, at ordinary temperatures, it is slow but complete. The regeneration takes place in strongly diluted liquids more easily than in liquids containing much enzyme. Invertin, amylase, and pancreatin are regenerated with more difficulty than the takadiastase, but the results obtained with these products are just as convincing.

The new datum introduced by the Russian scientist opens an entirely unforeseen field in the study of enzymes. Without 
being able to confirm the results of Gramentzki, Effront cites an observation which relates to this question. Effront has observed that by exposing to the action of light a sterile solution of albuminoid derivatives, there is obtained in the end a pronounced peptonization, as well as the formation in the liquid of small quantities of hydrogen peroxide. These tests have dealt with the products of a moderate hydrolysis, obtained on one hand with acids, on the other with pepsin. Now, with these last products, there is always found a more rapid action than with the products coming from a digestion with acid. It remains to be seen whether these results are not due to the combined effect of light and a regenerated pepsin.

\section{§ 4. Effect of Light and of Shaking. Adsorption of PEPSIN.}

Effect of Light. - Pepsin in solution, exposed to light, undergoes a marked deterioration. The active substance is destroyed and we even find a change in the character of the albuminoid substance. Before being exposed to the sun's rays the pepsin solutions are precipitable by zinc sulphate. A prolonged exposure prevents the formation of this precipitate. The work done is not an auto-digestion, properly speaking. The action is more fundamental, for in the products formed we find ammonia and fatty acids, substances whose formation exceeds the limit of action of even the pepsin.

The following is the method followed by Effront and the results found in his experiments on the effect of light: $5 \mathrm{~g}$. of powdered pepsin, contained in a tube, are sterilized at $110^{\circ}$ for 20 minutes. The tube is poured into 500 c.c. of sterilized water. The mixture, plugged with a stopper of wadding, is left to the action of the light. On the other hand, as control test, $5 \mathrm{~g}$. of pepsin are dissolved in 500 c.c. of water, and the solution sterilized for 20 minutes at $110^{\circ}$. After three months exposure the liquids are analyzed. 
Effect of Light on Sterilized Solutions of Pepsin.

\begin{tabular}{|c|c|c|}
\hline & $\begin{array}{l}\text { Liquid with pepsin } \\
\text { sterilized dry. }\end{array}$ & $\begin{array}{l}\text { Liquid with pepsin } \\
\text { sterilized in solution. }\end{array}$ \\
\hline $\begin{array}{l}\text { Ammonia nitrogen } \ldots \ldots \ldots \ldots \ldots \ldots \\
\text { Volatile acids... }{ }^{2} \ddot{S O}_{4} \ldots \ldots \ldots \ldots \ldots \ldots \\
\text { Precipitated by } \\
\text { Active substance } \ldots \ldots \ldots \ldots \ldots \ldots\end{array}$ & $\begin{array}{l}\text { mg. } \\
110 \\
430 \\
\circ \\
0\end{array}$ & $\begin{array}{r}\text { mg. } \\
92 \\
370 \\
\circ \\
\circ\end{array}$ \\
\hline
\end{tabular}

In the two liquids is found a very pronounced molecular degradation of albuminoid material. However, in the test with dry sterilized pepsin the action seems to be a little more advanced.

The inhibition of pepsin by light has been studied by Tappeiner, Iodelbauer, Schmidt-Nielsen, and others. Dreyer and Hannsen have sought to establish the law of the effect of light, and they have found that the weakening progresses regularly under the influence of a continuous exposure to light. If $a$ expresses the quantity of substrate at the beginning, $x$ the quantity which has changed during a time $t$, and $k$ a constant, the destruction of the enzyme is represented in a very satisfactory manner by the formula for a monomolecular reaction: $\frac{d x}{d t}=k(a-x)$. Furthermore, this weakening is due primarily to the ultra-violet rays retained by the glass.

Effect of Shaking. - Proteolytic enzymes undergo important modifications under the influence of shaking.

This phenomenon has been observed by Abderhalden and Guggenheim with trypsin, pancreatic juice, and the extract of yeast, by Schmidt-Nielsen with rennet, by Horlow with ptyalin, by Shaklle and Meltzer with pepsin. The results, however, depend on experimental conditions under which this shaking takes place. Duration and rapidity, the content of the liquid in enzyme, and the temperature play an important rôle in this phenomenon. An active solution, introduced into a reaction tube and agitated for 2 minutes, can lose as much as 75 per cent of its activity. After 5 minutes the disappearance is almost 
total and there remains only 3 to 4 per cent of active substance. The effect of the speed of the movement is manifest; a liquid shaken at the rate of 300 revolutions a minute becomes inactive four times as quickly as when the shaking was done at the rate of roo revolutions per minute. There is also here the influence of temperature. Inhibition by shaking takes place in a perceptible way at $0^{\circ}$, it increases with the temperature, and at $30^{\circ}$ it is four times as strong as at $0^{\circ}$.

Inhibition by shaking is especially rapid in a neutral or alkaline medium. The addition of 0.5 c.c. $\mathrm{N} / \mathrm{I} \circ \mathrm{HCl}$ immediately retards the phenomenon. Moreover, all acids do not act to the same extent. The maximum effect is obtained with $\mathrm{HCl}$. Lactic, oxalic, tartaric acids, in equivalent quantities, act much less strongly. Acetic acid is without action; phosphoric and citric acids produce a less effect than lactic acid. Finally, enzyme solutions strongly diluted are destroyed more rapidly than more concentrated solutions.

An endeavor has been made to explain this disappearance of the activity of enzymes upon shaking by changes in their molecular structure produced by the mechanical action. There was seen in these facts an analogy to those observed with living cells. In reality, this phenomenon is due to surface tension. Under the influence of shaking the substance in solution is differently distributed in the liquid, and in addition sticks to the walls of the vessel. When a sample of a pepsin solution submitted to shaking is examined, it is found that at a given moment the liquid is deprived of activity. However, this disappearance is not permanent. Allowed to stand, the liquid again little by little becomes active, and at the end of an hour it possesses anew a very strong digestive power, although one lower than that at the beginning.

The regaining of activity corresponds to the complete disappearance of the froth; from this moment the liquid no longer becomes active upon standing. It is then in the froth that pepsin is concentrated. If this part of the liquid is collected and left for some time until it is resolved into water, there is found in 
the liquid formed a content in active substance much higher than that fo!nd in the liquid from which it comes. The regeneration of the active substance, because of the disappearance of the froth, corresponds only to 50 per cent or 70 per cent of the initial quantity of enzyme. A quite considerable part does not become regenerated. A certain quantity, it is true, is found again in the form of precipitate, produced by the shaking in the very heart of the liquid, but this never constitutes the whole of the deficit. Moreover, the majority of enzyme solutions remain completely transparent in spite of shaking.

It is possible to show that a part of the active substance becomes adherent to the walls of the vessel. A tube containing a pepsin solution, whose digestive power has been determined, is shaken until all enzymic activity has disappeared. Then the liquid is thrown away, the tube is washed with water several times, and then finally filled with distilled water, which is analyzed from time to time. The sample taken in the first quarter of an hour does not contain enzyme. But after 2 or 3 hours, we find again in the liquid a quantity of enzyme corresponding to almost 20 per cent of the entire quantity of active substance first found in the tube.

We may then conclude that the phenomena observed after shaking are of the same nature as those noted with regard to adsorption in that they both result from the play of molecular forces. The dissolved substance is separated from the liquid; a part penetrates into the froth, another adheres to the walls. The first is rapidly regenerated, and reënters into the liquid after a certain period of rest. The other, invisible to the naked eye, is very slowly redissolved and more quickly so in ordinary water than in the solution from which it came. Thus we have to deal here with a phenomenon of temporary precipitation of enzymes which, in other circumstances, as in the blood circulation, may have important consequences.

In conclusion, it has been thought that this very curious phenomenon could be utilized for the preparation of pure en- 
zymes. Unfortunately experiments of this kind are not very easy; the agents which cause the formation of the froth, as, for example, saponin, in place of favoring the phenomenon, retard it by removing the albumin and the enzymes from the surface of the liquids.

Adsorption of Pepsin. - When moist fibrin is introduced into a solution of pepsin, we find that a considerable part of the active substance becomes associated with the albuminoid matter, and that by submitting the latter to repeated washings we only with difficulty remove the adhering pepsin. In this particular the different albuminoid substances do not all act alike. As excellent adsorbing material Abderhalden mentions elastin, which with great ease adsorbs proteolytic enzymes; it is moreover by virtue of this property that this substance is utilized for the exploration of organs in order to determine how the enzymes are distributed there.

This adsorbing power towards enzymes possessed by albuminoid substances, and especially by the connective tissue, plays a very important part in the phenomenon of digestion. The nitrogenous substances not yet digested adsorb the pepsin which is thus found, in a certain measure, exposed to the injurious action of the medium if the latter becomes alkaline. The enzyme can exercise here its action longer than in the stomach, and reach the intestine, where there is already a definite excess alkalinity. Here proteolysis goes on more completely, for it is then the result of the combined actions of pepsin, trypsin, and erepsin. Moreover, this chemical decomposition is also more complete in that the products which in vitro arrest the action are here eliminated by a rapid adsorption.

This fixation of active substance on protein matter has for a long time been regarded as a proof of the existence of a transitory combination of the enzyme with the body on which it acts. Recent investigations have overthrown this opinion. In reality, pepsin is fixed on a great number of substances which are in no way changed by it. Michaelis and Ehrenreiche have studied more closely this phenomenon of adsorption. They 
introduced into a 5 per cent solution of pepsin different inert bodies and analyzed the filtered liquids. In these tests, they used 8 to ro g. of adsorbing substance to 20 c.c. of pepsin solution. They found that the solution became inactive or perceptibly lost its activity, in presence of charcoal, kaolin, aluminium hydroxide, or of talcum. Blood charcoal showed twice the adsorbing power shown by bone charcoal. The reaction of the medium also very plainly influenced adsorption; thus aluminium hydroxide retained pepsin much better in acid than in neutral media. Moreover, according to $\mathrm{Zunz}, \mathrm{BaSO}_{4}$ adsorbs pepsin incompletely.

The phenomenon of adsorption depends, according to Freudlich, on the surface tension of the liquid. Michaelis, on the contrary, sees in adsorption a result of the chemical and electrochemical nature of enzymes. This last investigator finds that trypsin is adsorbed by kaolin while pepsin is not. These two enzymes are, on the other hand, both retained by aluminium hydroxide. Pepsin, according to Michaelis, would have an acid character while trypsin would be amphoteric, that is to say, endowed with both acid and basic functions. $\mathrm{He}$ moreover supports this opinion by a whole series of tests made with different enzymes. The difference observed in adsorption according to the nature of the medium and the fact that the various enzymes act differently towards adsorbents explain to us why the method indicated by Brücke, which consists in precipitating an enzyme from its solution by the formation of a precipitate of calcium phosphate, cannot be applied to the preparation of all the enzymes. As to pepsin, its removal by a precipitate of calcium phosphate, produced in the same medium with a slightly acid reaction, is realized well enough, but for each enzyme it is necessary to seek suitable conditions and sometimes even to change the nature of the precipitant.

In this connection, a similar behavior is observed when we attempt to filter a solution of pepsin through a Chamberland filter. Holderer, in fact, recognized that the reaction of the medium plays a very large part in the filtration of this enzyme. 


\section{§ 5. Influence of Chemical Conditions.}

Effect of Acids. - Pepsin does not act in an alkaline medium. The optimum enzymic effect is obtained in a distinctly acid medium. The gastric juice of a dog has an acidity in $\mathrm{HCl}$ of 0.38 per cent to $0.5^{6}$ per cent, or an average of 0.54 per cent. The gastric juice of man varies between 0.1 per cent and 0.3 per cent. In the course of digestion in the stomach the acidity coming from the gastric juice is considerably diluted, and the solution and the peptonization of albuminoid substances are carried on in presence of a relatively small quantity of acid. In vitro gastric juice is most active when it contains 0.2 per cent $\mathrm{HCl}$. In the tests made with pepsin solutions we find, on the contrary, different values for optimum acidity according to the albuminoid material used. From the experiments of Petit it appears that albumin is best digested in media containing o.I 5 per cent $\mathrm{HCl}$, while fibrin requires a double quantity of acid. The quantities of acid required also depended, according to Kanovaloff, on the purity of the pepsin used, pepsins with little activity requiring generally a larger quantity of acid than pepsins of good quality. According to Zunz, pepsin will attack the sero-albumin slowly in solution $\mathrm{Na}_{2} \mathrm{HPO}_{4}$ weakly acid to phenolphthalein and alkaline to litmus.

Hydrochloric acid can be replaced, up to a certain point, by other mineral or organic acids:

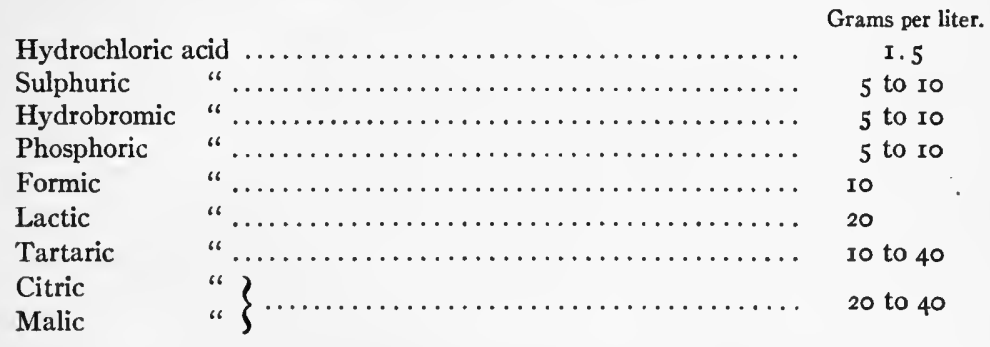

Anhydrous phosphoric acid, acetic, butyric, valeric, and succinic acids have no effect in 4.0 per cent solutions.

The parallel effect of hydrochloric and sulphuric acids has 
also been determined by Effront for fibrin and for albumin, with the following results:

Peptonization of Fibrin in Presencf of Hydrochloric Acid and OF SUlPHURIC ACID.

\begin{tabular}{|c|c|c|}
\hline & Quantities. & Fibrin dissolved. \\
\hline 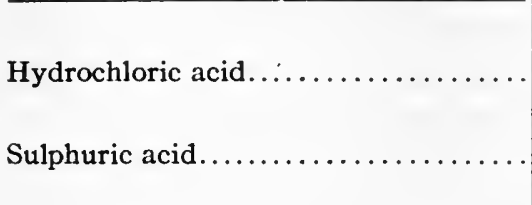 & $\begin{array}{l}\text { Per cent. } \\
\left\{\begin{array}{l}0.1 \\
0.15 \\
0.2\end{array}\right. \\
\left\{\begin{array}{l}0.3 \\
0.5 \\
0.7\end{array}\right.\end{array}$ & $\begin{array}{l}\text { g. } \\
2.00 \\
2.65 \\
2.57 \\
1.25 \\
1.30 \\
1.20\end{array}$ \\
\hline
\end{tabular}

The optimum acidity for $\mathrm{HCl}$ would then be 0.15 per cent, while for $\mathrm{H}_{2} \mathrm{SO}_{4}$ it is 0.5 per cent. For this determination, $20 \mathrm{~g}$. of moist fibrin were used, containing $3 \mathrm{~g}$. of dry substance. The digestion was made in presence of 60 c.c. of dilute acid and $2 \mathrm{mg}$. of pepsin; it lasted 3 hours at a temperature of $50^{\circ}$.

For the experiment with albumin were used $5 \mathrm{~g}$. of white of egg, coagulated and reduced to very small fragments, 30 c.c. of acid, and $5 \mathrm{mg}$. of pepsin; the operation lasted 2 hours at $50^{\circ}$.

Peptonization of Albumin in Presence of Hydrochloric Acid and of SulPHURIC ACID.

\begin{tabular}{|c|c|c|}
\hline & Quantities. & Albumin dissolved. \\
\hline 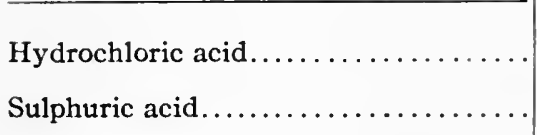 & $\begin{array}{l}\text { Per cent. } \\
\left\{\begin{array}{l}0.15 \\
0.3 \\
0.5 \\
1.0\end{array}\right.\end{array}$ & \begin{tabular}{l}
\multicolumn{1}{c}{ g. } \\
2.7 \\
4.75 \\
3.0 \\
2.3
\end{tabular} \\
\hline
\end{tabular}

Sulphuric acid is less effective with fibrin and albumin than hydrochloric acid, which is the agent that everywhere accompanies pepsin and which, consequently, can be replaced only with difficulty. The optimum obtained for coagulated albumin corresponds to a solution 0.3 per cent in $\mathrm{HCl}$.

These figures are not at all absolute. They may vary according to the degree of coagulation of the albumin and 
also according to the origin of the pepsin. However, it should be observed that quite different figures are obtained if, while using the same coagulated albumin and the same pepsin, the state of division of the albumin is different. Coagulated albumin, suspended in water and passed through a very fine filter, gave the following figures:

$\mathrm{HCl}$

Albumin dissolved.

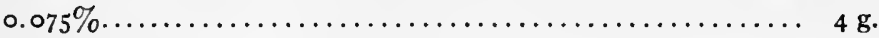

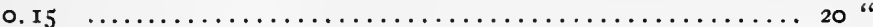

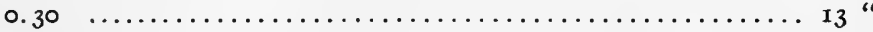

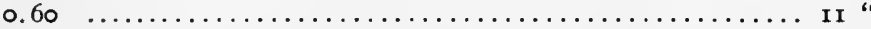

On the other hand, the experiments of Roger and Garnier show that the optimum quantity of acid depends on the quantity of pepsin used:

Variation of the Optimum of Acidity with the Quantity of Pepsin Used.

\begin{tabular}{|c|c|}
\hline $\begin{array}{c}\text { Quantity of pepsin, } \\
\text { per rooo. }\end{array}$ & $\begin{array}{c}\text { Optimum quantity of } \\
\text { HCl, per roo. }\end{array}$ \\
\hline & \\
0.25 to 8 & 2.5 \\
I6 & 2.5 to 5 \\
32 to 64 & 5 \\
I 28 & I0 \\
\hline
\end{tabular}

It is to be noticed that an excess of pepsin, as well as an excess of acid, may embarrass digestion. However, in presence of very small or very large quantities of acid, strong doses of pepsin always give the best results. Thus a concentration of 6 per cent of pepsin is just as active for 0.16 per cent $\mathrm{HCl}$ as for 0.5 per cent $\mathrm{HCl}$.

Effect of Alkalis. - When a solution of gastric juice is made alkaline with potassium carbonate $(0.45 \mathrm{~g}$. of carbonate to Io c.c. of liquid), the active substance is perceptibly changed. If, at this point, $\mathrm{HCl}$ is added in such a way as to bring this juice to a normal acidity, it is observed that the latter is almost entirely deprived of its digestive power. However this loss is not final. To cause the proteolytic power to reappear, in place of rapidly acidifying the originally alkaline juice, it can 
first be brought to a neutral state, allowed to stand for some time, and then acidified.

Tichomirof, who has studied this phenomenon, believes that by neutralization the free acid contained in the gastric juice is removed, while by making it alkaline we destroy the combination formed by the acid and the enzyme, the combination which represented the active principle. To bring the enzyme from its latent state to its active state, the liquid must be kept in a neutral state for a certain time, so that the enzyme combination can reform. The following is an experiment on this point: Gastric juice, previously neutralized, is made alkaline by adding $0.45 \mathrm{~g} . \mathrm{K}_{2} \mathrm{CO}_{3}$ per 100 c.c. solution. Then it is carefully neutralized and the liquid left for some time. Then it is acidified and the digestive power determined.

Influence of a Temporary Alkalinity on the Digestive POWer of PePsin.

\begin{tabular}{|c|c|c|c|}
\hline $\begin{array}{l}\text { Time in a neutral state } \\
\text { before acidification. }\end{array}$ & $\begin{array}{l}\text { Digestive } \\
\text { power. }\end{array}$ & $\begin{array}{l}\text { Time in a neutral state } \\
\text { before acidification. }\end{array}$ & $\begin{array}{c}\text { Digestive } \\
\text { power. }\end{array}$ \\
\hline $\begin{array}{l}30 \text { seconds } . . . \ldots \\
\text { 30 minutes } . . \ldots \\
\text { I hour.......... }\end{array}$ & $\begin{array}{l}\circ \\
\frac{1}{1 \frac{1}{3} g} \\
\frac{1}{40}\end{array}$ & $\begin{array}{l}3 \text { hours..... } \\
4 \text { hours.... } \\
\text { Control.... }\end{array}$ & $\begin{array}{l}\frac{1}{25} \\
\frac{1}{12} \\
1\end{array}$ \\
\hline
\end{tabular}

The control, which has not been made alkaline, has a digestive power taken as unity. The test; which has been made alkaline and then acidified after 30 seconds, does not react. On the contrary, if the liquid is left 4 hours in a neutral state before the addition of acid, it is found that the digestive power is already perceptibly regained, since it represents $\frac{1}{12}$ of that of the control. The loss of activity of a pepsin so treated can be reduced to a very small proportion. This is what happens when a gastric juice, previously alkaline, is treated with a quantity of acid corresponding to $\frac{4}{5}$ of its alkalinity, which is thus left 4 to 6 hours, and then acidified.

Mechanism of the Action of $\mathbf{H C l}$. - The investigations of W. Gurber, Erb, and of Arrhénius have shown that the 
albuminoid substances can enter into reaction with hydrochloric acid and form combinations analogous to ammonium chloride. The existence of these can be verified by the constant lowering of the freezing point of the solutions of the products of hydrolysis of albuminoid substances by $\mathrm{HCl}$. By this method it has been possible to establish the fact that we have here a question of true molecular combinations:

\begin{tabular}{lllll} 
I albuminoid molecule absorbs & \multicolumn{1}{c}{ mol. $\mathrm{HCl}$} \\
I albumose " & " & 3 & " \\
I peptone & " & " & 2 & "
\end{tabular}

Now, it has been recognized that nitrogenous matter, combined with hydrochloric acid, is much less stable than when in its usual form, and it is precisely in this instability produced by the acid molecule that pepsin finds a greater ease of attack. Furthermore, most investigators believe that the action of pepsin is favored by free $\stackrel{+}{\mathrm{H}}$ ions.

Schütz has, however, demonstrated that free $\stackrel{+}{\mathrm{H}}$ ions are not at all indispensable for the action of pepsin, and that peptonization actually takes place in neutral media. He bases this opinion on the following experiment. He first determines for a solution of albumin the acid required for neutralization by adding to ro c.c. of the solution deci-normal acid up to slight acid reaction with Congo paper. Then he puts different fractions of this same liquid to digest, adding increasing quantities of acid, the first quantities being insufficient to neutralize the liquid.

Digestion of Albumin in Different $\mathrm{HCl}$ Concentrations.

Acid Required for Neutralization of the Solution of Albumin: Io c.c. $=$ ז 3 c.c. $N /$ ro.

\begin{tabular}{|c|c|c|c|c|c|}
\hline $\begin{array}{l}\text { Solution } \\
\text { of albumin. }\end{array}$ & $\begin{array}{l}\text { Hydrochloric } \\
\text { pepsin. }\end{array}$ & $N /$ ro $\mathrm{HCl}$ & Total $\mathrm{HCl}$. & Volume. & $\begin{array}{l}\text { Albumin } \\
\text { digested. }\end{array}$ \\
\hline $\begin{array}{l}\text { Io c.c. } \\
\text { Io c.c. } \\
\text { Io c.c. }\end{array}$ & $\begin{array}{l}\text { I c.c. } \\
\text { I c.c. } \\
\text { I c.c. }\end{array}$ & $\begin{array}{r}4 \text { c.c. } \\
9 \text { c.c. } \\
\text { I9 c.c. }\end{array}$ & $\begin{array}{l}5 \text { c.c. } N / \text { Io } \\
\text { 10 c.c. } N / \text { ro } \\
20 \text { c.c. } N / \text { Io }\end{array}$ & $\begin{array}{l}\text { I00 c.c. } \\
\text { 100 c.c. } \\
\text { I00 c.c. }\end{array}$ & $\begin{array}{l}2.8 \mathrm{mg} \text {. } \\
21.0 \mathrm{mg} \text {. } \\
91.0 \mathrm{mg} .\end{array}$ \\
\hline
\end{tabular}


We see that the optimum of digestive action is obtained when the quantity of acid exceeds that required for neutralization, or 20 c.c. in place of 13 c.c. But peptonization is already very pronounced for quantities lower than that required for neutralization. The course of peptonization in presence of small quantities of acid is shown still more clearly by the following figures:

Digestion of Albumin in Different Concentrations of $\mathrm{HCl}$.

Required for Neutralization: 10 c.c. $=14$ c.c. $N /$ io.

\begin{tabular}{|c|c|c|c|c|c|}
\hline $\begin{array}{c}\text { Solution } \\
\text { of albumin. }\end{array}$ & $\begin{array}{l}\text { Pepsin } \\
\text { HCl. }\end{array}$ & $\mathrm{N} /$ io $\mathrm{HCl}$. & Total HCl. & Volume. & $\begin{array}{l}\text { Albumin } \\
\text { digested. }\end{array}$ \\
\hline $\begin{array}{l}\text { c.c. } \\
\text { Io }\end{array}$ & $\begin{array}{l}\text { c.c. } \\
\text { I }\end{array}$ & $\begin{array}{l}\text { c.c. } \\
9\end{array}$ & $\begin{array}{l}\text { c.c. } \\
\text { Io }\end{array}$ & $\begin{array}{l}\text { c.c. } \\
50\end{array}$ & $\begin{array}{l}\mathrm{mg} . \\
\mathrm{I} 8\end{array}$ \\
\hline 10 & I & 9 & 10 & 25 & 25 \\
\hline 10 & I & 9 & I0 & 100 & $2 \mathrm{I}$ \\
\hline 10 & 4 & 6 & 10 & 50 & 25 \\
\hline I0 & 4 & 6 & 10 & 100 & 24 \\
\hline
\end{tabular}

We see that the effect of the acid is proportional to the albumin present and that dilution is without effect. It is also to be noted that in a medium poor in acid, we do not noticeably accelerate the reaction by increasing the proportion of pepsin. The results of Schütz explain why the optimum quantity of acid varies with the different albuminoids. The acid required for neutralization is not the same for all these substances.

It is evident that the maximum activity coincides with a quantity of acid exceeding that required for neutralization. In fact, pepsin acts exclusively on a hydrochloric combination; it is necessary then that a liquid to be peptonized should receive an excess of acid, the excess being destined to prevent the products forming in the course of digestion, substances of basic nature, from decomposing the combination of albuminoid with hydrochloric acid. The free acid would thus play a protecting part without directly intervening in the mechanism of digestion.

The formation in the course of peptonization of basic products slackens the progress of the transformation. The medium, 
which at the beginning had a small quantity of acid sufficient for its normal progress, loses this progressively, and, on the other hand, the digestion cannot begin with a large excess of acid, for pepsin could not act under these conditions. Relying on these observations, it has been proposed to conduct peptonization in such a way that the acid should be progressively added in the course of the transformation. This method is especially rational for thorough digestions and those of long duration.

Action of Salts, Antiseptics, Alkaloids and of Other Organic Substances. - In the action of inorganic salts on peptonization, different factors must be considered. Certain salts precipitate albumin in great quantities; but even before the quantity used is sufficient to produce precipitation, these already exert an effect on the albuminoid by changing its sensitiveness towards the proteolytic enzyme. The influence of the salts on the digestion is exerted in this case by an indirect method. It is not that the pepsin has been retarded, but rather that the protein substance has become less susceptible. Thus it is that in the presence of certain quantities of $\mathrm{NaCl}$, pepsin with difficulty digests albumin, but a solution of pepsin with addition of sea salt appears very active after dialysis.

Inorganic salts can also exert a direct action on pepsin, either by changing the medium, by displacing the hydrochloric acid, or by changing the active substance. We possess quite a mass of data on the action of inorganic salts, but these are far from being complete and the mechanism of the action is made clear in very few cases. A. Petit was one of the first who has studied this question. In his tests he used fibrin in presence of .03 per cent $\mathrm{HCl}$, and a quantity of pepsin in large excess over that required to dissolve the fibrin used. The effect of the different salts on such a peptic digestion is given in the following table: 
Effect of Salts on the Digestive Power of Pepsin.

\begin{tabular}{|c|c|c|c|}
\hline Salts used. & $\begin{array}{l}\text { Inactive quantities, } \\
\text { thousandths. }\end{array}$ & $\begin{array}{l}\text { Quantities which re- } \\
\text { tard, thousandths. }\end{array}$ & $\begin{array}{l}\text { Quantities which } \\
\text { arrest, thousandths. }\end{array}$ \\
\hline 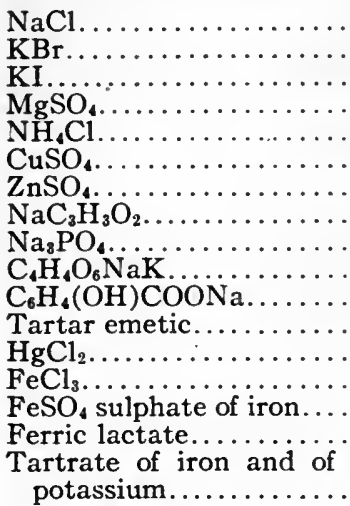 & $\begin{array}{l}\text { Io to } 80 \\
\text { 10 to } 80 \\
\text { 10 to } 80 \\
\text { 10 to } 160 \\
\text { 10 to } 40 \\
\text { 10 to } 40 \\
\text { 10 to } 40 \\
\ldots \ldots \\
4 \\
\text { 10 } \\
\ldots \ldots \\
2 \\
2 \text { to } 4 \\
2 \text { to } 40 \\
2 \text { to } 20 \\
2 \\
2\end{array}$ & 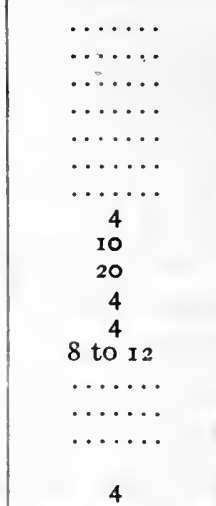 & 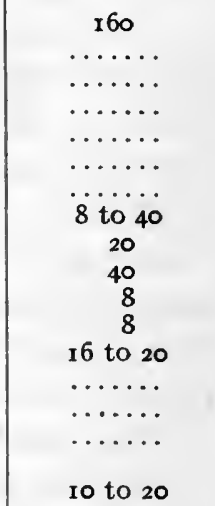 \\
\hline
\end{tabular}

Langley and Edkins, Schütz, Pawlow, as well as many other investigators, have taken up this study. It appears from their results that the neutral salts inhibit pepsin, while alkaline salts produce a much more pronounced effect, being able even, in certain quantities, to destroy it.

The effect of salts and their influence with respect to the displacement of hydrochloric acid of the medium has also been studied by Effront. He finds that peptic digestion is considerably influenced by the salt content of ordinary water, and that, in particular, small quantities of calcium sulphate cause a very perceptible retardation in the dissolving of coagulated albumin.

Influence of Caso, on the Peptic Solution of Albumin.

\begin{tabular}{|c|c||c|c|}
\hline $\begin{array}{c}\text { Quantity calcium } \\
\text { sulphate per I00.c. } \\
\text { (mg.) }\end{array}$ & . Minutes. & $\begin{array}{c}\text { Quantity calcium } \\
\text { sulphate per ID0c.c. } \\
\text { (mg.) }\end{array}$ & Minutes. \\
\hline 0 & 31 & 50 & 133 \\
10 & 50 & 60 & 175 \\
20 & 59 & 80 & 210 \\
40 & 102 & $\ldots$ & $\ldots \ldots$ \\
\hline
\end{tabular}


This retarding effect is likewise found with other sulphates:

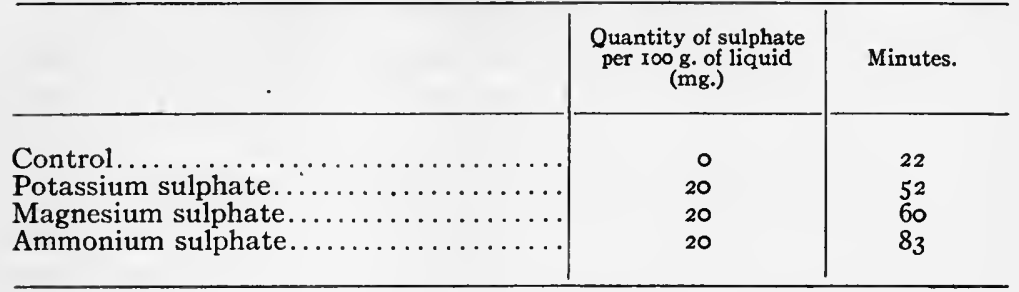

In the presence of the $20 \mathrm{mg}$. of ammonium sulphate, the solution of albumin takes place almost four times as slowly as in the experiment carried on without sulphate.

The retarding effect of sulphates has been likewise found in tests with fibrin. $15 \mathrm{~g}$. of moist fibrin, containing $5 \mathrm{~g}$. of dry substance, are put in contact with pepsin dissolved in 60 c.c. of $\mathrm{I} .5$ per cent $\mathrm{HCl}$, to which has been added increasing quantities of sodium sulphate. After a digestion of two hours at $50^{\circ}$, it is diluted with water, the undissolved fibrin is collected and weighed, after drying at $100^{\circ}$.

Quantity of sulphate, milligrams.

Fibrin not dissolved, grams.

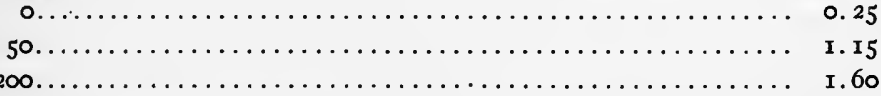

In all these tests we find a constant relation between the quantity of sulphate used and the effect produced. This action is explained by the reaction which takes place between the hydrochloric acid preëxisting in the liquid and the sulphate added. It is for this reason that the action of the sulphate is the more pronounced the less rich in acid the medium, the whole amount of hydrochloric acid then being transformed.

The following table gives the course of solution of three

\begin{tabular}{|c|c|c|}
\hline HCl per liter. & $\begin{array}{c}\text { Without } \\
\text { sulphate. }\end{array}$ & $\begin{array}{c}\text { With } 40 \mathrm{mg} . \\
\text { sulphate. }\end{array}$ \\
\cline { 2 - 3 } g. & min. & min. \\
I.5 & 29 & I20 \\
2.5 & 36 & 92 \\
3.0 & 60 & 90 \\
\hline
\end{tabular}


emulsions containing identical proportions of albumin, pepsin, and sulphate, but acidulated to different degrees.

While for an acidity of 0.3 per cent the retardation is in the ratio of $I$ to $I .5$, for an acidity of 0.15 per cent the retardation appears in the ratio of $I$ to 4 .

The effect of antiseptics has been studied by A. Petit.

\begin{tabular}{|c|c|c|}
\hline & $\begin{array}{l}\text { Inactive quantity } \\
\text { (thousandths). }\end{array}$ & $\begin{array}{l}\text { Quantity which re- } \\
\text { tards (thousandths). }\end{array}$ \\
\hline 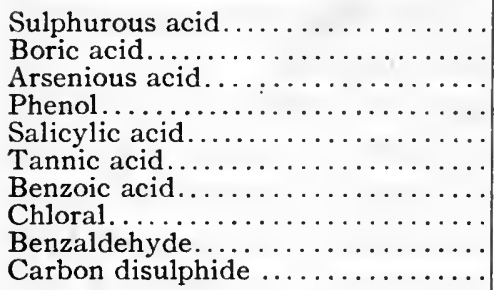 & $\begin{array}{l}2 \text { to } 5 \\
10 \text { to } 20 \\
5 \text { to } 20 \\
\ldots \ldots \ldots \\
0.5 \\
\ldots \ldots \\
2 \\
\text { I } \\
\ldots \ldots\end{array}$ & $\begin{array}{l}8 \text { to } 10 \\
\ldots \ldots \ldots \\
\ldots \ldots \ldots \\
20 \text { to } 100 \\
1 \text { to } 2 \\
0.5 \text { to } 4 \\
4 \text { to } 20 \\
\text { 10 to } 100 \\
2 \\
\text { below } 2\end{array}$ \\
\hline
\end{tabular}

We see in particular that boric acid in antiseptic quantities does not prevent the action of pepsin.

Pekelharing, Sawomara, Bliss and Novy have experimented with the effect of formaldehyde. It is known that formaldehyde enters into combination with albuminoid matter and changes its nature completely; this antiseptic cannot be used as means of preservation of liquids submitted to the action of pepsin. But formaldehyde, in quantities still very active as an antiseptic, can at least serve to preserve pepsin from all infection, as pepsin with addition of formaldehyde does not deteriorate. It has been desired to draw from these facts an argument in favor of the idea that pepsin is not an albuminoid material. But Sawomara has shown that in large quantities (Io per cent for example) formaldehyde acts and destroys pepsin.

As current antiseptic, to protect liquids during the course of digestion against all microbic interference, thymol is used with success, also xylol, toluol, and chloroform. Van de Velde proposes a solution of Io per cent chloroform in acetone for the same purpose, using roo g. per liter of liquid to be digested. 
According to Effront, ammonium fluoride does not retard the action of pepsin when used at concentrations of 30 milligrams per liter. But in presence of 150 milligrams, it requires four times as much pepsin to produce the same effect. The use of fluoride is of real interest, for, even in the minimum quantity of 30 milligrams, this salt acts in acid media as a powerful antiseptic.* The same investigator has found that salicylic acid produces a retarding effect, even at the concentration of 25 milligrams per liter. In the presence of 150 milligrams, it is necessary to use double the pepsin to attain the same result.

Tests on digestion of short duration can be conducted in presence of chloroform in small quantities, 5 to 6 drops per roo c.c. of liquid. When it is a question of experiments of longer duration, the use of antiseptics does not always give the desired results; either the quantity is insufficient to prevent deterioration, or else it is too large, and the pepsin is found to be deteriorated. Thymol always affords an insufficient protection against bacteria, but it does not influence pepsin. Toluol, on long exposures, destroys pepsin. Chloroform acts as antiseptic for the first few days, but, in the end, the liquid almost always becomes infected. The reagent of Van de Velde, at the concentration indicated by the investigator, is more convenient to use, but it weakens the enzyme after a certain interval of contact.

The danger of infection is always very great when digestion takes place in presence of little acid, (o.I per cent -.r 5 per cent). It is to be recommended, for tests of long duration, that a greater acidity be used and that chloroform be added from time to

* Arthus and Huber issued in 1892 a Note in Comptes Rendus (t. II5, 0.839), on the action of fluorides on the micro-organisms and the enzymes. These investigators claim that, in the presence of certain quantities of an alkali fluoride, cellular life is arrested while enzymes can still exert their action, and they decide on the use of fluorides to determine the vital effect of chemical action induced by enzymes. The writer calls attention to the fact that he published in 1890 , in the Moniteur Scientifique (pp. 790 and ror3), observations on the action of fluorides on yeasts, putrefactive bacteria, butyric and lactic acid ferments, as well as on various enzymes, and that this memoir comes to the same conclusion as that mentioned above. 
time in small quantities. Infection can also be avoided, while preserving the proteolytic power of the enzyme, by using iodoform.

The alkaloids, in the quantities customary in therapeutics, likewise affect pepsin. Caffeine and theobromine are favorable to pepsin, and yet tea and coffee may be considered as inhibitors. On the other hand, sulphates of quinine and strychnine, chloride of morphine, etc., in doses of several centigrams per liter, are without effect on peptic digestion. Alcohol, up to a concentration of 20 per cent, does not influence digestion; in the long run it exerts a destructive action. According to Papasotiriou, putrefactive bacteria destroy pepsin very rapidly, experiments having been made with $B$. fluorescens and $B$. putridum. Houghton has also studied the influence of dyes on digestion. He finds that these substances at very great dilutions act as inhibitors. Rocoa and turmeric show an effect in a dose of $I$ to 1600 , and this effect is pronounced even in a dose of $I$ to 800 . Saffron begins to act in a dose of $\mathrm{I}$ to $\mathrm{I} 600$. Cochineal and Bismarck brown very greatly retard the proteolysis of albumin, even in very great dilutions; casein appears less sensitive to their action.

The action of infinitesimal quantities of chemical substances on peptonization has been very little studied. However, it is to be foreseen that it is on this side that we must turn if we wish to explain the influence upon stomachal and intestinal digestion of a whole series of foods which, at first sight, appear inoffensive. The influence of very small quantities of different products has already been established for amylase. It has been demonstrated that this enzyme is sensitive to the action of copper sulphate at a dilution equal to $0.000,000,5$. Lactic acid fermentation is influenced by chloride of platinum at a dilution of $0.000,000,00 \mathrm{I}$ and oxychloride of vanadium acts at a dilution of $0.000, \infty 00, \infty 00$, I. Finally, Trillat, by studying the action of gases produced by putrefaction, arrives at extremely small quantities.

Richet explains this action of homeopathic doses by the 
destruction of the material under the influence of a very great dilution and its transformation into electrons. According to him, for example, silver nitrate in infinite dilution cannot be distributed in all the liquid; the molecules are separated from each other, the attraction is diminished, and the material is disintegrated by being transformed into electric force or electrons. The analytical data brought forward in this thesis are not of a nature to justify this theory. However, it remains an established fact that in the action of inorganic catalysts, as well as in the action of enzymes, there are substances which may intervene in quantities which entirely escape chemical analysis, and it is very probable that in normal digestion phenomena of the same nature often take place.

This influence of infinitely small quantities of substances on the mechanism of certain actions is also found in other domains. Apparently it is to this that the therapeutic action of certain mineral waters must be attributed, and also the value of those fattening foods called catalytic by Bertrand. It is known that, with the addition of very small quantities of these products, there is a considerable increase in the effect of phosphates and nitrates introduced, and consequently the entire yield.

Influence of Conditions of Medium on Functioning of Cells. - Assimilation, in general, always takes place through the aid of infinitely small quantities of enzymes, and these very small quantities are influenced by substances in proportions still smaller. Now the study of the physical and chemical conditions of the medium has shown numerous cases where an enzyme may be checked, arrested, or completely destroyed. Most of the functions studied are found in the living cell, where assimilation takes place in the presence, not of an enzyme, but of a whole series of enzymes whose actions are superimposed. The products of decomposition of an enzyme serve as the primary material for the action of another enzyme. We have here a sort of symbiosis which is very characteristic. The regular course of the whole requires a certain constant relation between 
the acting enzymes. The excess or the lack of an active substance may influence the whole phenomenon. Now the formation and the secretion of these enzymes is up to a certain point determined by the actual needs of the cell. To explain the quantitative regulation of the relative proportions of the different enzymes we must assume that there are incessant variations of the physical and chemical conditions of the medium which act as the real regulators of cellular enzymic actions.

The action of an enzyme is checked or arrested completely when the products of the reaction accumulate in the medium. To this arrest corresponds a change in the reaction of the medium. These new conditions favor the action of another enzyme, which, transforming the products produced by the preceding enzyme, permit the latter to act again. The action of the latter will be taken up again until an equilibrium is established, resulting from the functioning of the several enzymes. Moreover, in the establishment of this equilibrium, the phenomenon of osmosis also comes into play, which represents ancther way of considerably changing the reaction of the medium.

In the case of the activity of a single enzyme, as is the case with ptyalin or gastric juice, the rational utilization of these secretions is due in great part to their extreme sensitiveness to the conditions of the medium. During a meal, 200 to $25^{\circ}$ grams of saliva are secreted; this acts in the mouth on starchy substances, but the action is far from being complete on account of its short duration. During mastication, the active substance has penetrated into the food, which has partially neutralized the alkalinity of the saliva, thus rendering the enzyme more stable, and less sensitive to the action of the medium. Neutralization is completed in the stomach. Here enzyme activity passes through a maximum, due to the presence of small quantities of sodium chloride, and the disintegration of the starch is finished in this way before the medium becomes completely acid and inhibitory for the action of ptyalin.

The same phenomenon is observed with gastric juice. Pepsin, sensitive to alkalinity, shows itself much more resistant when it 
has penetrated into the food. Abderhalden has been able to demonstrate the presence of active pepsin throughout the whole length of the intestine, especially in the duodenum, the jejunum, and the ileum. This enzyme being thus protected, its activity, which for a certain time was inhibited in the intestine, in its turn begins to produce a last effort before disappearing.

Finally, there exists another peculiarity on which we shall dwell later, namely, that enzymes and, especially, rennet and pepsin, treated under certain conditions, can acquire the property of anti-enzymes. This fact is very important, for the resistance of living matter to the action of enzymes, which, however, destroy them in the dead state, appears to be due to a reaction of this nature.

To explain the paradoxical phenomenon that gastric and pancreatic juices, so active on all dead animal substances remain, on the contrary, without action on the glands and mucous membranes which are penetrated by them, we sometimes invoke an imperviousness of the protecting plasmic membranes due to the presence of viscous materials on their surface. On the other hand, Claudio Fermi attributes this protection to the special biochemical resistance of living cells. According to him, the enzymes have a much greater "affinity" for dead albumins than for albumins contained in the living tissues. It is evident, in fact, that albuminoid matter is of an extreme mobility. Its state changes with the greatest facility with changes in the reaction and in the physical or chemical conditions of the medium. There would, then, be nothing astonishing in the fact that a reagent of a sensitiveness so perfect as that of the enzyme could make a distinction between living albumin and dead albumin. In addition, the reaction of the living cell being indispensable to the reaction of the surrounding medium, it is conceivable that the cell opposes, by a sort of natural defense, the action of external enzymes. It becomes itself unfit for this attack, as, for instance, under the influence of certain chemical reagents. In the chapters which treat of anti-pepsin and anti-trypsin 
we shall have occasion to return to these questions, which are, from the different points of view, of the greatest interest.

\section{§ 6. Pepsinogen.}

It is quite generally admitted that pepsin is not secreted in active form by the mucous membrane of the stomach, but rather in the state of a pro-enzyme or zymogen, pepsinogen, which is rapidly transformed into pepsin by dilute acids. Podwyssotsky prepares with the same mucous membrane two glycerin extracts; one is acidulated only at the moment of using, the other receives the same quantity of acid a half-hour before its use. It is found that the latter has a digestive power much greater than the former. A similar difference is observed if the extract is made directly with acidulated glycerin, in place of neutral glycerin acidified at the moment of the test.

Another argument in favor of the existence of a pepsinogen arises from an experiment of Langley. This author compares the resistance to alkali of two artificial gastric juices, one coming from a maceration of the gastric mucous membrane of an animal killed while digesting, the other coming from the treatment of a starved animal. Whereas the first very rapidly loses its digestive power under the influence, at $37^{\circ}$, of a very small quantity of sodium hydroxide, the second can be kept much longer in the same conditions of temperature and of alkalinity, without undergoing a perceptible change in its activity. Thus, therefore, the stomach of an animal which has fasted for some time contains a different substance from that which is in the period of activity. Thus we are led to believe that the material produced in the gastric mucous membrane is a pepsinogen, which, after its secretion, becomes active in consequence of a change of medium, being transformed into pepsin.

From the preceding, not only is pepsinogen more resistant to the action of alkalis than active pepsin, but it also resists better the action of acids. Its activation is brought about by a slight 
acidity; it is believed that it is likewise caused by contact with air or by other agents.

As a matter of fact, there is no decisive proof establishing the existence of this zymogen. All the facts which we have just given can be very well explained, as Duclaux has noted, from a consideration of the phenomenon of molecular adhesion. In fact, it might be that pepsin in the stomach is simply fixed on the mucous membrane, as a dye is fixed on the textile fibre. Then we should understand why in this state it is inactive and much more resistant to the external agents than when in solution. Under the influence of dilute acids or other agents, it would leave its support to be diffused in the liquid and would then become active. According to Duclaux, there would be no need at all of assuming the existence of a new substance to explain an activation which would result from a simple change of condition.

\section{§ 7. Law of Action of Pepsin.}

Before entering upon the special study of the velocity of action of pepsin on an albuminoid material, it is useful to recall the different investigations which have been carried out with the idea of establishing a general law of the action of enzymes. The first investigators who have occupied themselves with this question appear to have been O'Sullivan and Tompson. These, in 1890 , by studying the action of sucrase on a solution of saccharose, found that, within certain narrow limits of concentration, the law which expresses the quantity of sugar transformed as a function of the time is the same as that which holds in case of the acids. Now the law of inversion of sugar by acids had been established by Wilhelmy, in 1860, and can be expressed in the following manner:

If we designate by $S$ the quantity of sugar used, by $s$ the quantity of sugar hydrolyzed after a time $t$, (the reaction being incomplete), by $P$ the weight of acid, we get the expression:

$$
d s=K P(S-s) d t
$$


where $K$ is a constant, depending on temperature and the nature of the acid. This relation expresses the fact that the quantity $d s$ of sugar transformed is proportional to the weight of the acid, to the quantity of sugar remaining, and to the time. By integration, it leads to a logarithmic law relating $s$ and $t$, namely:

$$
K=\frac{\mathrm{I}}{P t} \log \left(\frac{S}{S-s}\right) \text {. }
$$

From the differential equation the velocity of hydrolysis in time $t$ is given by the expression:

$$
V_{t}=\frac{d s}{d t}=K P(S-s)
$$

We see, in particular, that at the beginning of inversion we have $V_{0}=K P S$, which shows that the initial rate is proportional to the total quantity of sugar and to the weight of acid. This law, which is well verified by the action of acids on saccharose, does not rigorously apply to the action of sucrase, as O'Sullivan and Tompson claimed.

About the same time, Tammann recognized that enzymes do not follow the laws of action of acids and that the equation which expresses the law of enzymic action is less simple. But it was Duclaux, in I898, who, taking the different facts then known, grouped them to get a general theory of action of enzymes. Noting, first of all, that the hydrolyzing activity of the enzyme is, to a certain degree, diminished by the quantity of material to be transformed, in other words that the products of the reaction exert on sucrase a retarding action, the greater the more sugar in the liquid, Duclaux believes that the reaction takes place as if the enzymic energy was inversely proportional to the quantity of sugar $S$. Then, by expressing that the quantity transformed, $d s$, is proportional to the weight $P$ of enzyme, to the quantity $(S-s)$ of sugar remaining, and to the time $d t$, and inversely proportional to the initial quantity of sugar $S$, the following relation is obtained:

$$
d s=K \frac{P}{S}(S-s) d t
$$


in which the term $\frac{P}{S}$ represents the quantity of enzyme actually active. We then derive:

$$
V_{t}=\frac{d s}{d t}=K \frac{P}{S}(S-s),
$$

an equation which shows that the reaction velocity for the enzyme at the beginning is constant $-V_{0}=K P$, 一 that it depends on the quantity of enzyme used, and not at all on the weight of sugar or of the material to be transformed. In other words, the weight of substance decomposed is, in the beginning of the reaction, proportional to the quantity of enzyme present and to the time.

The differential equation above by integration gives:

$$
K=\frac{S}{P t} \log \left(\frac{S}{S-s}\right)
$$

a logarithmic expression, which O'Sullivan and Tompson maintain to be characteristic for the action of enzymes.

In reality, this law, proposed by Duclaux, is only approximate. V. Henri has changed it slightly by the introduction of coefficients, which, while putting the law in accord with that of mass action, takes into account the irregularities noted by the investigators. He finds:

$$
V_{t}=\frac{K P(S-s)}{\mathrm{I}+m(S-s)+n s},
$$

a relation containing the two coefficients $m$ and $n$, depending on experimental conditions, especially that of temperature.

This formula is interesting, for it harmonizes the two opinions. Enzymes act, according to conditions, either similar to the acids or as Duclaux indicates. It is only necessary to change the coefficients. For example, if we consider the initial velocity

$$
V_{0}=\frac{K P S}{\mathrm{I}+m S},
$$

we see that, for strong concentrations, we have practically $V_{0}=\frac{K P}{m}$, the formula of Duclaux. On the other hand, for 
dilute concentrations we have approximately $V_{0}=K P S$, this being the formula of Wilhelmy.

This law, which is indeed not perfect, however accounts fairly well for the facts observed. It has been verified by V. Henri as regards the inversion of saccharose, the hydrolysis of salicin by emulsin, that of starch by amylase, and also the action of maltase on maltose. Nicloux has verified it using lipaseidin which he obtains from the seed of Ricinus. Finally, V. Henri and Larguier des Bancels have found that the action of trypsin on gelatin likewise follows the law indicated above.

However, the expression of such a law and its verification require certain experimental conditions which the carbohydrates and fats closely fulfil. It is necessary, first of all, that the substance to be transformed shall be in solution, and shall be uniformly distributed throughout the entire liquid. It is also necessary that these substances on which the enzymes act, or the products of the transformation, shall be easily measurable so that one can follow the progress of the reaction. It is by reason of these difficulties, present in the case of nitrogenous substances, that the study of the law of action of proteolytic enzymes has been so long neglected. Without doubt, as regards the influence of the quantity of enzyme on the velocity of the transformation, Brücke made a good attempt to establish such a law by causing different quantities of gastric juice to act on fibrin. However, it is difficult to find in this case an accurate expression for the action produced.

There are several methods used to determine the activity of a pepsin or a trypsin. We shall first indicate one which permits quite well our following the dissolving action of pepsin, and our seeing how this varies with the time and the quantity of enzyme used. When pepsin is made to act in increasing quantities on the same amount of coagulated albumin, and the reaction is arrested at the moment when, for the maximum quantity, the solution is almost complete, without, however, being entirely finished, we find that digestion, while depending on the quantity of active substance present, is not proportional 
to this quantity. The weight of albumin dissolved increases less rapidly than the quantity of enzyme used. This irregular progress of the solution of albumin is expressed in the following table:

Relation Between the Quantity of Pepsin Used and the Weight of Albumin Dissolved.

\begin{tabular}{|c|c|c|}
\hline Quantity of pepsin. & Albumin dissolved. & $\begin{array}{c}\text { Albumin dissolved per } \\
\text { I c.c. of pepsin. }\end{array}$ \\
\cline { 2 - 3 } c.c. & g. & cg. \\
I & 2.2 & 220 \\
2 & 4.3 & 215 \\
3 & 6.0 & 200 \\
4 & 6.4 & I60 \\
8 & 7.2 & 90 \\
I6 & 8.2 & 50 \\
\hline
\end{tabular}

For this experiment, in each test were used ro g. of egg albumin which had been cooked, grated, and diluted in roo c.c. of 0.3 per cent $\mathrm{HCl}$. The temperature was $40^{\circ}$, and the transformation was arrested at the end of 4 hours. The weight of the dissolved albumin was determined by analyzing for nitrogen in the filtered liquid. With small quantities of pepsin, from $\mathbf{I}$ to 3 c.c., the digestion progressed with a certain regularity, from 220 to 200 centigrams of albumin being dissolved per c.c. of enzyme. If, however, this quantity was increased, this weight was no longer constant, until with 16 c.c. only 50 centigrams of the original 220 centigrams were dissolved.

This irregularity is due to different causes. In the first place the physical condition of the albumin should be considered. Cooked albumin is far from being a homogeneous substance, so that some pieces are attacked more easily than others. Moreover, it is necessary to consider the influence of the dissolved products. At the start, the dissolving action takes place in pure water, but soon the liquid becomes concentrated in products of hydrolysis, and the digestive action proceeds the more slowly the more concentrated the liquor becomes in these substances. When very finely divided albumin is used and at a great dilution, 
we avoid to a certain degree this retarding effect and approach a more regular action.

SOlution IN VERY Dilute Liquor.

\begin{tabular}{|c|c|c|}
\hline $\begin{array}{c}4 \% \text { albumin emulsion } \\
\text { coagulated. }\end{array}$ & Pepsin. & $\begin{array}{c}\text { Passage of the liquid to } \\
\text { the transparent state. }\end{array}$ \\
\hline c.c. & mg. & min. \\
20 & I & I23 \\
20 & I .5 & 89 \\
20 & 2 & 61 \\
20 & 3 & 40 \\
20 & 4 & 30 \\
\hline
\end{tabular}

In these tests, use was made of a very finely divided milk of albumin at a dilution of 4 per cent, and the activity of the transformation was measured by determining the time necessary for complete solution. It is seen that for a double quantity of pepsin the rate is twice that for a single quantity, and that to dissolve the same quantity of albumin in one-fourth the time requires four times the quantity of enzyme. Thus, when we take experimental conditions so that the effect produced is relatively small, either by making determinations at the start of the action or by working with small quantities of enzyme, we find that this double proportionality - the quantity of albumin dissolved being in direct ratio to the quantity of pepsin present and to the time - is verified.

The peptonizing power, in evidence during the first phases of digestion, obeys the same law as the liquefying power but the demonstration here becomes still more difficult. In fact, not only do we meet the same difficulties which previously were found in the observation upon the liquefying power, but still others arise. In consequence of the hydrolysis which is manifest from the beginning, there is produced in the liquid a whole series of intermediate substances, different albumino-acids, various albumoses, and other similar products, which, while being progressively transformed, offer to the action of the pepsin a very unequal resistance. Hence a complete absence of homogeneity is observed. Furthermore, it is also true that the reaction of the medium changes constantly in the course of peptonization and 
that we find, consequently, varying degrees of acidity, a factor which also prevents a simple manifestation of enzyme action.

All these difficulties cause us only to recognize a law of proportionality, but only in the beginning of the transformation when the disturbing causes are not as yet influential. Tannin, dissolved in tartaric acid, a reagent which has already been used to follow the degradation of albuminoid matter, can again serve here for studying this law.

Peptonization of Fibrin.

\begin{tabular}{|c|c|c|}
\hline Time. & $\begin{array}{c}\text { Albumoses precipitated } \\
\text { by tannin. }\end{array}$ & $\begin{array}{c}\text { Products, not precipi- } \\
\text { table, formed per hour. }\end{array}$ \\
\hline $\begin{array}{c}\text { hours. } \\
\text { After 1 }\end{array}$ & $\%$ & $\%$ \\
2 & 91.0 & 9.0 \\
3 & 82.3 & 8.7 \\
5 & 73.4 & 8.9 \\
\hline 52.0 & 9.6 \\
\hline
\end{tabular}

This table shows that after 3 hours, for example, the quantity of products hydrolyzed not precipitable by tannin is 26.6 $[100-73.4]$ or per hour $8.9,\left[\frac{26.6}{3}\right]$ a number which is practically the same for the other determinations, which proves that the velocity of peptonization during the 5 hours of the experiment has remained almost constant.

Law of Schuitz-Borissow. - In addition to this very simple method, determination of the weight of the albumin simply dissolved or digested in the form of products not precipitable by tannin, there are others which can also serve for measuring the activity of a proteolytic enzyme. Neglecting the physicochemical methods, which consist in following the variation either of the rotatory power, of the viscosity, or, finally, of the electrical conductivity of the digestion liquid, we find that the one most in use is that called "Mett Tubes." In brief, a series of glass tubes, from I to $2 \mathrm{~mm}$. in diameter, are filled with fresh white of egg, which is coagulated and the tubes cut in fragments of 10 to $15 \mathrm{~mm}$. in length. For the determination place two of these fragments in the enzyme liquid, leaving the 
whole at $37^{\circ}-40^{\circ}$ for ten hours and, at the end of this time, the length of the cylinder gelatinized or dissolved at the two ends of the tube is measured.

What is the value of this method? Experiment shows that the diffusion of the enzyme in the tube is quite regular, at least up to a depth of 4 or $5 \mathrm{~mm}$., or in other words, that the attack, under these conditions, is practically proportional to the time of action. The following are, according to Vassilief, the lengths of albumin dissolved by a pepsin at $27^{\circ}$ during successive intervals of two hours each.

\begin{tabular}{|c|c|c|c|}
\hline Time. & $\begin{array}{l}\text { Lengths } \\
\text { dissolved. }\end{array}$ & Time. & $\begin{array}{l}\text { Lengths } \\
\text { dissolved. }\end{array}$ \\
\hline $\begin{array}{ll}\text { hr. } & \\
\text { From o to } 2 \\
\text { " } & 2 \text { to } 4 \\
، & 4 \text { to } 6\end{array}$ & $\begin{array}{l}\text { mm. } \\
\text { I. IO } \\
\text { I.14 } \\
\text { I.I } 2\end{array}$ & $\begin{aligned} & \text { hr. } \\
& \text { From } 6 \text { to } 8 \\
& \text { " } 8 \text { to } 10 \\
& \text { 10 to } 12\end{aligned}$ & $\begin{array}{l}\text { mm. } \\
\text { I.15 } \\
1.09 \\
1.10\end{array}$ \\
\hline
\end{tabular}

The constancy of the velocity, at the beginning at least, is then established. But how do the dissolved lengths vary when the quantity of pepsin varies? As Duclaux, from whom we borrowed the following argument, remarks, we cannot here expect obedience to the law of proportionality as in the case of the other procedures where the enzyme and the substance which undergoes the action are uniformly distributed in the same volume of liquid. In the experiment in question, the entire cylinder of albumin is not in contact with the active liquor, and is attacked only at its base. It is then the quantity of enzyme, not by unit of volume but by unit of surface, which must be considered.

If then the quantity of enzyme becomes $n$ times greater by unit of volume, it will increase only by unit of surface by the quantity $\sqrt[3]{n^{2}}$, and by calling $l$ and $l^{\prime}$ the lengths dissolved in the first and second case respectively, we shall have, in all probability, everything now being comparable:

$$
\frac{l^{\prime}}{l}=\frac{\sqrt[3]{n^{2}}}{\mathrm{I}}, \text { whence } \frac{l^{\prime 3}}{l^{3}}=\frac{n^{2}}{\mathrm{I}}
$$


For equal intervals of time the cubes of the dissolved lengths should increase as the squares of the quantities of enzyme. If the quantities of enzyme are as I and 8, the lengths of albumin dissolved will be as I and $\sqrt[3]{(8)^{2}}$, or I and 4 .

In reality, experimentation has not absolutely confirmed these theoretical views. By different methods, E. Schütz, who used the polarimetric method, and Borissow, who made use of Mett tubes, found that the rate of digestion, or the lengths dissolved in the same time-interval, are proportional to the square roots of the quantity of enzyme. In other words, we do not have $\frac{l^{\prime}}{l}=\frac{\sqrt[3]{n^{2}}}{\mathrm{I}}$, as previously, but $\frac{l^{\prime}}{l}=\frac{\sqrt{n}}{\mathrm{I}}$; which means, in the example chosen, that the lengths of dissolved albumin will be, not as I and 4 , but as I and $\sqrt{8}$, or I and 2.8 .

According to Duclaux, the difference can be attributed to the fact that the diffusion does not renew fast enough the surfaces inside the tube when the power of the enzyme increases, which results in restricting the length of albumin dissolved.

On the other hand, Julien Schütz has verified the law of Schütz-Borissow and has found that it is exact for solutions of enzyme of medium concentration and in the presence of an excess of the protein substance. It is amusing to find that this last recommendation, made with the idea of allowing digestion to follow the law of E. Schütz, is precisely the reason that the theoretical law of Duclaux is not verified. In fact, according to the experiment of Hedin, which we shall meet again in the discussion of the law of the action of trypsin, an excess of albuminoid substance, by absorbing a part of the enzyme, naturally results in retarding the attack on the albumin. However this may be, if we take into account the irregularities due to the imperfection of the methods used, it appears that the theoretical law of Duclaux is valid, that is to say, that the premises of which he speaks are also applicable here, namely, that the quantity of substance hydrolyzed by the proteolytic enzyme is, at the beginning, proportional to the quantity of enzyme used and to the time. 
If, in place of Mett tubes, emulsions more or less well made are used and the course of digestion followed for a certain time, complications arise. It is evident that with such heterogeneous mixtures, the results obtained must vary with the experimental conditions. Thus it is that Huppert and E. Schütz have found that the quantity of albumin produced in the peptic digestion is directly proportional to the quantity of albumin and to the square root of the product of the weight of the pepsin by the time and by the concentration of acid. This law is not universally accepted and it is more probable that if we worked with pepsin in a homogeneous medium, as has been done with trypsin acting on a solution of casein, we should find a verification of the logarithmic law that governs all enzyme actions.

The Non-exhaustion of Pepsin During the Act of Digestion. Pepsin, as all the enzymes, acts as a catalyst. The work which it performs does not diminish its activity, and, theoretically, with a given quantity of pepsin, one could transform an infinite quantity of albuminoid substance. The non-exhaustion of pepsin can be shown by the following experiment: $5 \mathrm{~g}$. of white of egg, cooked and reduced to small fragments, are introduced into roo c.c. of 0.25 per cent $\mathrm{HCl} ; 0.05$ c.c. of gastric juice are added and the whole left at $40^{\circ}$ until all the albumin is completely dissolved. The solution, in this experiment, required $2 \mathrm{I}$ hours. Then the liquid is boiled to destroy the pepsin, $5 \mathrm{~g}$. of albumin are again added with 0.05 c.c. of gastric juice, and the mixture is kept at $40^{\circ}$. The presence of products of hydrolysis retard the solution. This time the liquid becomes clear only after $3 \mathbf{I}$ hours. Then a second test is made under the same conditions: $5 \mathrm{~g}$. of albumin are dissolved in $2 \mathrm{I}$ hours. At this point $5 \mathrm{~g}$. more of albumin are added, and the reaction allowed to proceed. The time required for digestion is now 32 hours. It is evident that in this double experiment there is no exhaustion of the pepsin. In fact, the solution of the second addition of albumin by the pepsin already used, has not required any more time than when it was brought about by the action of a fresh pepsin.

This experiment also furnishes very interesting data on the 
weight of active substance entering into the phenomenon of digestion. The gastric juice experimented with was taken from a dog. It contained 0.45 per cent of solid substances and 0.15 per cent of active organic substances. Now, 0.05 c.c. of juice dissolved ro g. of albumin. I c.c. of the enzyme solution can then dissolve $200 \mathrm{~g}$. of albumin, and as I c.c. of the solution contains only $0.0015 \mathrm{~g}$. of active substance, it is therefore concluded that I $\mathrm{g}$. of active substance is capable of dissolving in $2 \mathrm{I}+32=53$ hours, $133,000 \mathrm{~g}$. of albumin.

\section{§ 8. The Possible Identity of Pepsin and Rennet.}

In a previous chapter we have shown the difficulties which are met when we wish to pronounce on the identity of rennets of various origins. Now that we are more familiar with pepsin, it is proper to broaden the discussion and ask if rennet and pepsin are really different enzymes. The question as to whether rennet constitutes a definite type of enzyme is quite a complex one. At the beginning, the coagulating properties of rennet appearing to be characteristic, and, in every case, very different from the dissolving and peptonizing properties of pepsin, a separate individuality was willingly granted to this enzyme; and even later, after it had been recognized that a large number of animal or vegetable juices also possessed the power of coagulating milk, though with some differences in the manner of action, the idea of the plurality of rennets, varying according to their origin, was accepted. However, the recent publications which have appeared on the different coagulating or peptonizing juices strongly reflect on this last idea and even place doubt on the individuality of the rennet enzyme.

Unitary Theory. - A school, in fact, has been formed which denies the existence of rennet, and sees in the properties of this enzyme only one of the functions of proteolytic enzymes. The researches made with a view of clearing up the process of coagulation of milk were not at first foreign to this change in opinion. We know that rennet divides the caseinogen of milk into two 
substances: one, casein, which is precipitated, with or without the aid of calcium salts; the other, a sort of albumose, remains in solution. The first action of rennet on milk would then be a protein hydrolysis, and coagulation would be only a consequence.

The partisans of the unitary theory pride themselves further on the fact that rennet is not only found in the digestive juice of animals during lactation, thus answering a real need, but also in vegetable or animal organs where its presence appears not at all necessary. Why, they ask, is rennet encountered, sometimes in rather large quantities, where there is no trace of casein, since generally the secretion of enzymes is always in direct relation with the quantity of substances which they have to transform? On the contrary, they draw attention to the fact that pepsin is always found associated with rennet. They then conclude that rennet and pepsin make one and the same enzyme. This enzyme, possessing both coagulating and peptonizing powers, would act in one direction or the other, according to the conditions of the medium. We know, in fact, that the coagulating action appears in a neutral medium, while the proteolytic action is produced only in acid solutions.

This argument of the simultaneous presence of the two enzymes in nature appeared to be reinforced by the discovery that these two enzymes are furthermore always associated in the same proportion. Blum and Fuld, in fact, believe that they have demonstrated that the secretions of pepsin and of rennet are always found parallel not only in normal gastric juice, but also in the juice obtained in pathological cases. The relation between the two properties of gastric juice would be, according to these investigators, so constant that one could determine the proteolytic power by analyzing for the coagulating power. We shall see in a moment what is to be thought of this statement.

Discussion. - But first, let us review, one by one, the various arguments in favor of the unitary theory. The first is based on the fact that in certain media we encounter rennet, when its presence appears entirely useless. Why therefore 
admit the existence of an enzyme which answers no need? The reply to this objection is based on the investigation of Danilewsky. This investigator has found that peptones obtained by peptic digestion can be transformed, under the influence of rennet, into more complex albuminoid substances, and that this condensation takes place in exactly the conditions which are unfavorable to the action of pepsin. Hence, rennet would appear as a sort of reversed pepsin, whose function is to place in reserve a part of the products formed by the pepsin. (See chapter on Plastein.)

Let us pass on to the second argument. The fact that rennet has never been found in any animal or vegetable organ without finding associated pepsin, is not convincing. It is purely fortuitous. Moreover, it is the usual thing for enzymes always to be found in nature in an associated state. Furthermore, if these two enzymes are identical, this single enzyme would have to have coagulating and proteolytic properties always in the same relation. Now, such is not the case. On the contrary, very considerable variations exist, according to the origin of the products and the conditions in which they have been obtained. Thus it is that if we compare enzymes taken, on the one hand from a gastric mucous membrane of a hog, on the other hand from calf stomach lining, we find:

\begin{tabular}{|c|c|c|}
\hline & $\begin{array}{l}\text { Digestive } \\
\text { power. }\end{array}$ & $\begin{array}{l}\text { Coagulating } \\
\text { power. }\end{array}$ \\
\hline 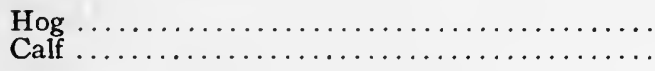 & $\begin{array}{l}\text { I } \\
\text { I }\end{array}$ & $\begin{array}{l}\mathbf{I} \\
9\end{array}$ \\
\hline
\end{tabular}

These results were obtained in the following manner. After having determined the digestive and coagulating powers of the enzyme extracted from the hog, powers which can be taken for units, the enzyme taken from a calf is diluted in such a way that its digestive power is the same as that taken as unity. We then find that the coagulating power of the enzyme from calf stomach lining is 9 times as great as that of the hog extract. 
These data moreover coincide with the experiments of Bang and Hammarsten, who found that there is a difference between the rennets taken from the gastric mucous membranes of man and of the hog and between rennets coming from pike and from calf. Furthermore, the active substances taken from the calf stomach lining also vary according to the age and the manner of nutrition of the animal.

\begin{tabular}{|c|c|c|}
\hline & $\begin{array}{l}\text { Coagulating } \\
\text { power. }\end{array}$ & $\begin{array}{l}\text { Digestive } \\
\text { power. }\end{array}$ \\
\hline 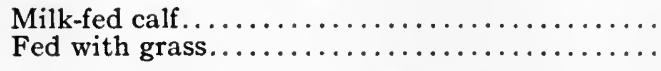 & $\cdot \begin{array}{l}4 \\
1\end{array}$ & $\begin{array}{l}\mathbf{1} \\
\mathbf{2}\end{array}$ \\
\hline
\end{tabular}

We see that the milk-fed calf has a gastric juice rich in rennet and poor in pepsin, while the animal fed with grass gives an enzyme relatively richer in pepsin and poorer in rennet. We again find greater variations if we compare commercial grades of coagulating enzymes.

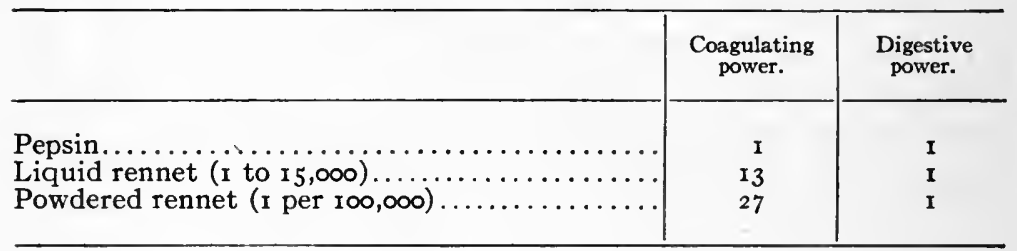

For a unit of digestive rennet in powdered form, we find 27 times more coagulating enzyme than in the same unit of pepsin. In addition, we can, by suitable precipitations, increase one of the two powers to the detriment of the other, in other words, we can roughly obtain a separation of these two enzymes. Thus ammonium sulphate, added to the saturation point to a solution containing the two enzymes, precipitates both, while sodium chloride similarly precipitates almost exclusively the rennet and leaves the pepsin in the solution. By repeating several times this precipitation with sodium chloride we can progressively enrich the precipitate in rennet. 
Van Hasselt precipitates with sodium chloride a maceration of calf stomach lining in water. He collects the precipitate, dissolves it in water, and precipitates again. The progress of the purification is expressed in the following table:

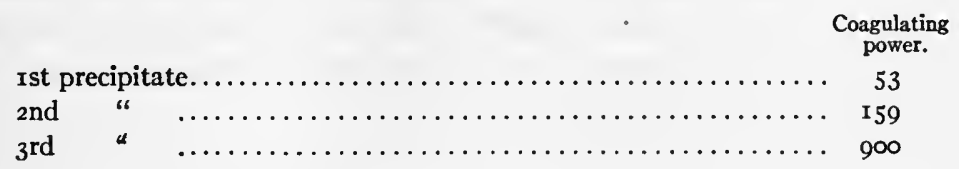

For one digestive unit there are in the first precipitate 53 coagulating units: in the 3 rd, the coagulating power has risen to 900 . Starting with a maceration of the gastric mucous membrane of a hog, made in 5 per cent $\mathrm{NaCl}$, a relatively pure solution of pepsin may be obtained. Saturating the liquid with $\mathrm{NaCl}$ causes a precipitate composed chiefly of rennet, which is eliminated in this manner. Then, dialyzing the filtered liquid to remove the greater part of the $\mathrm{NaCl}$, the pepsin in solution is precipitated by ammonium sulphate. The precipitate obtained is redissolved in water, and the liquor is dialyzed against a 0.2 per cent solution of $\mathrm{HCl}$. The solution thus prepared is exceedingly rich in pepsin and contains only very little rennet.

In order to reach an analogous separation, Hammarsten proposes the use of casein to purify the lab extract of calf stomach lining. Casein precipitates pepsin, while rennet remains in solution. The maceration of stomach lining is made in 0.24 per cent $\mathrm{HCl}$. The precipitation is caused by the addition of a 4 per cent solution of sodium caseate. To r30 c.c. of acid maceration are added, slowly and with shaking, 200 c.c. of a neutral solution of sodium caseate. Then $N /$ ro $\mathrm{NaOH}$ is added until no further precipitation occurs. This precipitate contains the pepsin, while the rennet remains in solution.

These facts are certainly very favorable to the idea of the actual existence of rennet as distinct from that of pepsin, but they do not constitute a proof. A doubt remains, in that there is always found in liquids, in addition to pepsin and rennet, both anti-pepsin and anti-rennet, as well as foreign 
bodies whose unfavorable influence prevents one or the other of the two enzyme properties from being manifested with all the desired intensity. If one produces, for example, an increase of peptonizing power with relation to coagulating power, it can be interpreted equally well by increase of anti-rennet power or by the diminution of anti-peptonizing power, as by the influences of the medium.

In short, the question of the identity or the non-identity of rennet and pepsin remains undecided. From the standpoint of the partisans of the unitary theory, the molecule of pepsin contains two different groupings corresponding, one to the proteolytic action, the other to the coagulating action. Without completely destroying the product, it is possible to weaken one or the other of these groups by the saturation of certain lateral chains, and thus to bring out the other property which has not been changed.

It is certain that this question of the duality of rennet and pepsin, very complex in itself, is rendered still more obscure by the existence of anti-bodies which are produced by immunization, and which in certain cases act at the same time on the two properties. However, hitherto anti-rennet has always been obtained by immunization, with the aid of a rennet also possessing the properties of pepsin. It follows that the antibody thus prepared reacts immediately on the two enzymes. It is therefore necessary to start with purified products in which the two functions have been clearly separated. Then the antibodies formed should be examined. If they are really specific. acting solely on one enzyme to the exclusion of the other, the conclusion from the existence of these two anti-bodies would be that anti-rennet and anti-pepsin exist as two corresponding enzymes. If not, the unitary theory would gain the victory. since it would be improbable that substances which conceal one of the groupings in the inoculated enzyme would be likewise found, fixed in the same place, in the anti-body formed.

One step has already been taken in this direction in the experiments of Hedin. This investigator prepares different 
zymogens by the aid of stomach mucous membranes which he macerates, cooling in water, in the presence of calcium carbonate. The liquid obtained is filtered; one part has added to it 20 c.c. I per cent $\mathrm{HCl}$ for each 100 c.c. of solution, and is left at ordinary temperature. Thus is obtained an active solution, capable of coagulating milk. On the other hand, the same volume of this extract is taken, but is not activated by HCl. 20 c.c. of $\mathrm{N} /$ ro $\mathrm{NH}_{3}$ are added per roo c.c. of solution, and it is left some hours at $37^{\circ}$ and then neutralized. This liquid does not coagulate milk, but it has acquired entirely new properties; it has become an inhibitor in that it diminishes or arrests completely the coagulation of milk, according to the quantity used.

These anti-enzymes have been obtained with zymogens of hog, guinea-pig, calf, and pike. These anti-bodies are specific, that is to say the anti-body, coming from an animal, very actively inhibits the rennet prepared from this same animal, but does not act at all on rennet coming from another animal. The inhibitors which have been obtained with the guinea-pig and the pike do not resist a temperature of $100^{\circ}$, but completely lose their activity. The two others lose it partially; but, curiously enough, they lose their specificity and become inhibitors for the four species. Zymogen, treated with $\mathrm{HCl}$, does not furnish any anti-body when it is afterwards treated with $\mathrm{NH}_{3}$ and then neutralized. The anti-body, treated with $\mathrm{HCl}$ and then neutralized, loses its coagulating property. It becomes itself coagulative up to a certain degree. From these experiments it appears that rennet is always accompanied by an antibody. When zymogen is treated with $\mathrm{HCl}$, the anti-body is destroyed and a coagulating action appears. When treated with $\mathrm{NH}_{3}$, the opposite result is obtained. The rennet is in great part destroyed, and there results an anti-coagulating action.

These data are evidently of a nature to justify the idea of the existence of several rennets, but they do not settle the question of the non-identity of rennet and pepsin. This question cannot be solved until the day when we shall find in a 
plant a single one of these two enzymes, or again, when we shall have prepared by immunization an anti-body applying to a single enzyme, rennet or pepsin, without affecting the other.

\section{§ 9. Chemical Activity of Pepsin.}

Under the action of pepsin, in slightly acid media, albuminoid substances, occurring either in the natural state or as the result of a coagulation, which are insoluble in water, become soluble more or less rapidly. If, at this moment, the liquor is neutralized, we get a precipitate. The filtrate, brought to the boiling point, gives a coagulum. The precipitate obtained by neutralization bears the name of acid-albumin or syntonin, and results from the action of the acid and not at all from that of the pepsin. The coagulum is formed by the albuminoid substance not transformed.

If the digestion is a little more advanced, the liquid is no longer rendered turbid either by neutralization or by heating to $10^{\circ}$. From this moment, the progress of the hydrolysis can be followed by the more or less abundant precipitations which are caused in the liquid by the addition of neutral salts, such as $\mathrm{NaCl},\left(\mathrm{NH}_{4}\right)_{2} \mathrm{SO}_{4}$, or $\mathrm{ZnSO}_{4}$. As peptonization advances, the precipitates obtained diminish. The substances precipitated by the salts bear the name of albumoses or of proteoses; the compounds not precipitated are designated by different writers under the term of peptones.

Kühne and Chittenden have distinguished two classes of albumoses: primary albumoses, which precipitate by $\mathrm{NaCl}$ saturation in neutral media, and the secondary albumoses or deutero-albumoses, which do not precipitate by $\mathrm{NaCl}$ saturation except in acid solution, and then incompletely. Moreover, primary albumoses are divided into hetero-albumoses, insoluble in pure cold water, but soluble in dilute salt solutions, and, consequently, precipitable by dialysis, and proto-albumoses, soluble in pure water and dilute salt solutions.

Primary albumoses, which correspond closely to those 
substances also designated under the name of propeptones, have, in addition, the following three properties:

I. They are precipitated in dilute salt solutions by dilute nitric acid, and this precipitate disappears with heat, to reform upon cooling.

2. Potassium ferrocyanide and acetic acid precipitate their solutions; and this precipitate, soluble upon heating, reappears with cooling.

3. By acidulating cold with acetic acid a mixture of equal parts of a solution of propeptone and a saturated solution of $\mathrm{NaCl}$, a precipitate forms that is soluble upon heating, reappearing with cooling.

The transformations which we have just described can be summarized in the following scheme:
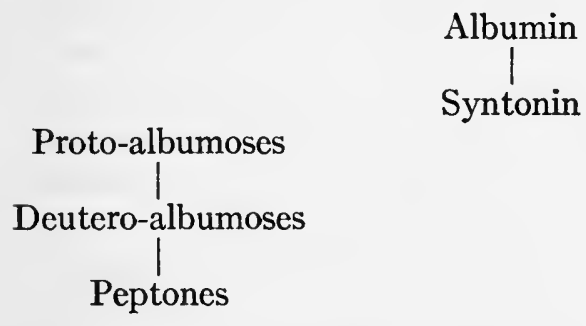

According to Kühne and Neumeister, the peptone formed under the action of pepsin is composed of two fractions, one very resistant to the action of trypsin, which he calls antipeptone; the other, easily hydrolyzed by this second enzyme, with formation of amino-acids, and which he calls hemi-peptone. These authors conclude that anti-peptone comes from the hetero-albumose, which itself corresponds to the part most difficult of attack by the digestive enzymes, while the hemipeptone comes from proto-albumose. The albuminoid molecule is then found divided from the beginning of hydrolysis into two groups: one, hemi; the other, anti. The same mixture of the two peptones, anti and hemi, is noted by Kühne under the name of amphopeptone. This opinion can be represented by the following scheme: 


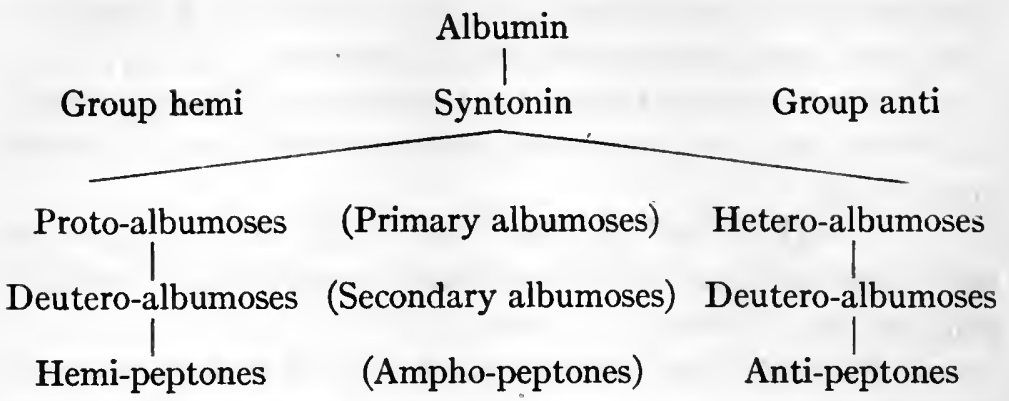

These ideas brought forward by Kühne and Neumeister have contributed much to the development of the chemistry of albuminoids. These writers have based their theory on a series of new facts of a real value, but which have not been capable of saving the erroneous conception of the hemi and the anti group; this, in fact, after having been adopted in an almost general way, cannot, at the present time, be any longer seriously supported.

The knowledge which we now possess as to the advanced hydrolysis of albuminoid matter, under the influence of acids, of alkalis, or of enzymes, indicates an aggregate formed by a very large number of mono- and di-amino-acids, and that, under the action of energetic catalysts, like trypsin and erepsin, this complex molecule, taking up water, successively and integrally divides, and there then remains no anti-group more resistant to trypsin than the others, as Kühne thought.

Pepsin induces the first stage in the hydrolytic degradation of albuminoids. These still retain many of the properties of substances from which they are derived; these are polypeptides of varying complexities: those whose molecular weights are the highest and approach nearest that of the original substance, form the albumoses; the others, more degraded still, represent the peptones.

It is possible to separate the peptones from the albumoses by saturating their solutions, either with zinc sulphate or with ammonium sulphate. Albumose is precipitated while peptone 
remains in solution. Peptone, free from albumose, can be divided into two parts, the one, soluble in phosphotungstic acid, the other precipitated, this latter part corresponding to the complex di-amino-acids. Among the albumoses, we can distinguish different kinds of products, either by the aid of fractional precipitation, by solution in suitable solvents, or again by the use of any other individual property, as we shall see further on.

To take account of the chemical reactions produced in the course of peptic digestion it is necessary to follow the appearance, then the disappearance of the different portions of albumoses, and record the accumulation of the final product of the reaction, which is peptone. But this method, in practice, still leaves much to be desired. The albumoses are not perfectly characterized bodies; nor is peptone a stable and definite term. In peptic digestion, there is never obtained a hydrolysis corresponding to the complete disappearance of the albumoses, and even the peptone which is obtained, as said above, can be divided into two more or less clearly defined portions, comprising compounds incompletely known from the chemical point of view.

However this may be, the working method adopted is capable of giving an approximate idea as to the chemistry involved in the reaction. In outline, we have a phenomenon similar to that of the saccharification of starch. In this latter process to reach the final stage of sugar, maltose or glucose, we pass through a whole series of dextrins which still preserve some of the properties of the initial starch, at the same time being different from starch, and also from each other. The formation of different albumoses which appear in the course of hydrolysis can be compared to the formation of these dextrins. In saccharification of starch, the whole chemical process consists of a series of hydrolyses reducing the original complex compound to a simple substance, sugar. In peptonization, we have a similar transformation; the very complex albuminoid molecule, with a molecular weight of 6000 , or perhaps 15,000 , is degraded until it gives albumoses of molecular weight equal to 2400 and peptones of a value of 400 . These intermediate compounds 
are in a way nitrogenous dextrins which later will be attacked in their turn to produce a large number of relatively simple derivatives, of definite and stable composition.

The various procedures used to differentiate the albumoses and the peptones are all based on the method of fractional precipitation. Before considering how the course of hydrolysis by pepsin is effected, and in order to be able to follow all the changes that occur, it is necessary first to consider various reagents utilized for this study. We will first examine the effect of salts.

\section{§ io. Study of Different Albumoses by the Method of Fractional Precipitation.}

The power which certain salts possess of precipitating albuminoid substances from solution has furnished Hofmeister with the basis for a method of fractional precipitation, with a view of separating the various compounds which are formed in the course of proteolysis. This method is being more and more used, although the results are not equally appreciated by all investigators; it even counts among its adversaries scientific men of recognized ability. However, it can, in certain instances, render great service, and it would be unreasonable to renounce it, all the more since at present we should not know with what to replace it.

General Facts as to the Precipitating Power of Salts. Many salts have the power of precipitating albuminoid substances from solution. The nature of the precipitate differs radically according to the salt used. In certain cases, the precipitate obtained is a metallo-organic compound, produced by a combination of salts with albuminoid substance. This is the case with ferric acetate, neutral or basic lead acetate, copper sulphate, and mercuric chloride. With other salts, such, for example, as with ammonium sulphate, zinc sulphate, potassium acetate, sodium sulphate and chloride, the precipitate is of another nature. The albuminoids precipitated have under- 
gone no change; the precipitate can be easily freed from the salts adhering to it, and is dissolved in water to form a solution identical with the original solution. Thus, according to the salt, two different actions are obtained: one chemical, the other physical in nature.

The physical action of salts especially has attracted the attention of chemists. The power of precipitation, exerted by salts on albuminoids, differs essentially according to the respective nature of these bodies, and they can be divided thus into three groups, according to their power of action.

a. Zinc sulphate, ammonium sulphate and potassium acetate are the most active agents; they precipitate all the natural albuminoids and albumoses. In the same group fall the nitrate and chloride of calcium, as well as a mixture of magnesium and sodium sulphates, except that the precipitate obtained with these last salts changes its nature after a prolonged contact with these reagents. All these salts act very efficiently in relatively dilute concentration; their precipitating powers increase with the density of the liquid, that is to say, the more the solution approaches saturation, the more effective it becomes.

b. Magnesium sulphate also possesses quite a considerable precipitating power, but yet a much less effective one than the preceding.

c. Finally, the chloride, nitrate, and sulphate of sodium. These salts do not precipitate all albuminoids, and it requires a very concentrated and often a saturated solution to obtain complete precipitation.

The chemical and physical conditions of the medium considerably influence the power which salts possess of precipitating albuminoid substances from solution. The presence in the albuminoid solution of foreign substances, the temperature, and the reaction of the medium are factors which have an important bearing on the precipitating power of the salts. In a slightly acid solution, precipitation is more rapid and more complete than in a neutral solution. It must be noted also 
that a part of the acid contained in the liquid is always retained by the precipitate, which leads to the opinion that the acid and the precipitated materials are probably in combination.

It has been sought to establish a relation between the molecular weight of salts and their precipitating powers, but this relation apparently does not exist. Their solubility or their content in water of crystallization also has no marked influence. It is likewise difficult to establish a relation between the other physical properties of salts and their precipitating powers. The mechanism of precipitation still remains to be explained. Salts, which are effective in albuminoid solution, have the same power in solutions of carbohydrate and in that of soap. To certain writers, the separation of substances from solution through the agency of salts, is due to the degree of "affinity" of these substances for water. Put in, contact with substances having little "affinity" for water, the salt withdraws a part of the water indispensable for their remaining in solution; hence, the precipitation of these materials. Hofmeister, who is not content with this explanation, inclines to the belief that the process of dissociation which takes place in the center 'of the liquid plays a part in the precipitation of albuminoids by salts. Posternak develops the idea originated by Hofmeister. According to him, "albuminoid substances cannot be precipitated from solution except with the condition that its nuclei are surrounded by a layer of undissociated salt molecules which protect it against the dissolving action of the ions. But in presence of nuclei dissolved in the liquid, the distribution of the undissociated molecules is not uniform as in the case of a medium free from albuminoids. The nuclei, exerting a more or less great attraction on the undissociated molecules, according to the chemical constitution of the latter, form centers where the concentration of the molecules is greater than in the space between the nuclei. The greater the affinity of the nuclei for any salt, the more easily will the concentration of molecules necessary to precipitate the albuminoid be attained."

The precipitating power of salts towards albuminoids has 
served as the basis for a large number of scientific investigations. Different experimenters use salts to purify, separate, and characterize the different protein substances, as well as the numerous products of their decomposition. For example, a classification of protein substances has been made according to the facility with which salts precipitate them from solution.

Casein and fibrinogen belong to a group of proteins easily precipitable. Casein can be precipitated completely by magnesium sulphate, as well as by a saturated solution of sodium chloride. Fibrinogen is precipitated from solution by the addition of an equal volume of a saturated solution of sodium chloride. The group of globulins forms the lower limit of compounds easily precipitable. They are completely precipitated by a saturated solution of magnesium sulphate, or again, from their neutral solution, by sodium chloride. Albumin is not completely precipitated by sodium chloride, nor by magnesium sulphate, but is completely precipitated by zinc and ammonium sulphates. The products of protein decomposition act very differently towards salts: hetero-albumose, for example, is completely precipitated from its neutral solution by sodium chloride; others, as the deutero-albumose $\mathrm{C}$, do not precipitate except upon saturation with ammonium sulphate in an acid medium.

By changing the reaction of the medium, different results are obtained, which often furnish useful information as to the nature of proteins under consideration. Albumin is not precipitated from its solution by acidifying with acetic acid, or by saturating its neutral solution with sodium chloride. But if, after having saturated the solution of albumin with $\mathrm{NaCl}$, it is then acidified with acetic acid, a precipitate is obtained. The same phenomenon occurs in precipitating albumose with sodium chloride. When a solution of primary albumose is half saturated with sodium chloride, there is no precipitation, but the precipitate appears as soon as the reaction of the liquid is made acid. The precipitate thus obtained is hetero-albumose.

In conclusion, the enzymes, which, from different points 
of view, approach albuminoids, are precipitated by ammonium sulphate.

Differentiation of Natural Albuminoids by the Method of Fractional Precipitation. - As we have just seen, salts act in a very different manner with various albuminoids, and their precipitating power can furnish, in certain cases, very useful information. An advance in this direction has been made by Hofmeister and his pupils. This investigator has demonstrated that we can attain the differentiation of various albuminoids by making use of a single salt, used in varying quantities. The method of Hofmeister is based on the following observations. When to a solution of albumin a solution of ammonium sulphate is carefully added, in the presence of a certain quantity of this salt, the liquid, at first transparent, becomes turbid. If, after a rest, it is filtered, and a fresh addition of salt made, the turbidity reappears in the transparent liquid, and so on, until the albumin is entirely precipitated. The precipitation is then continuous and gradual up to the complete disappearance of albumin from solution. The two limits, at the beginning and at the end of the precipitation, correspond to definite concentrations of salt. The lower limit corresponds to a concentration of salt causing a persistent turbidity; the higher limit, to a concentration of salt giving a complete precipitation.

The two characteristic limits of precipitation are observed not only with solutions of albumin, but with all albuminoids. However, the concentrations of salt, corresponding to the two limits of precipitations differ considerably according to the albuminoids used. Therefore, it is possible to make a differentiation of the different substances belonging to the albuminoid class, by the determination of the two limits of precipitation. To obtain constant results by the method of fractional precipitation, it is necessary to work under definitely determined conditions. A sample experiment is as follows:

The albuminoid solution is brought to a dilution of about 2 per cent. The solution of ammonium sulphate is prepared by saturating in the cold, and has a specific gravity of 1.253- 
1.255. Take a series of numbered reaction tubes and into each pour 2 c.c. of protein solution. To tube No. $\mathrm{I}$ add 6 c.c. of water, mixing the contents. Then add 2 c.c. of the saturated solution of ammonium sulphate, shaking the tube carefully to avoid the formation of foam, and allow to stand. In tube No. 2 and the following add salt solution, increasing the amount each time by $\frac{1}{10}$ c.c., while diminishing the water added, so as to have in all the tubes a volume of Io c.c. The first tube in which appears a lasting opacity will indicate the lower limit of precipitation. This limit will be expressed by the number of cubic centimeters of the sulphate solution used.

To determine the higher limit, let all the tubes showing turbidity stand for 24 hours, after which filter. To the liquid, filtered and completely clear, add 0.2 c.c. of the salt solution. The tube in which no further turbidity appears on contact with the salt solution will indicate complete precipitation and consequently mark the higher limit. The following are results obtained with this method, working on solutions of natural albuminoids:

\begin{tabular}{|c|c|c|}
\hline & Lower limit. & Higher limit. \\
\hline Fibrinogen. & I.9 & 2.8 \\
\hline Casein...... & 2.2 & 3.6 \\
\hline Globulin.. & 2.9 & 4.6 \\
\hline Albumin.. & 6.4 & 9.0 \\
\hline
\end{tabular}

Fibrinogen and casein have the same lower limit of precipitation. The first begins to precipitate when r.9 c.c. of saturated ammonium sulphate has been added to the solution; the second begins to precipitate with 2.2 c.c. of this salt. Globulin does not begin to precipitate except in the presence of a higher concentration of salt. Between globulin and albumin, the difference between the lower limits is still greater, from 2.9 to 6.4. The same gradation is found again in the higher limit; fibrinogen precipitates completely with 2.8 c.c., while albumin precipitates completely in the presence of 9.0 c.c. Still, the 
concentration which causes complete precipitation is always more characteristic than the concentration at which precipitation begins.

We have seen above that, by a judicious choice of salts as precipitating agents, we can make a definite classification of the albuminoids. Fibrinogen and casein are precipitated by sodium chloride, while precipitation of globulin is obtained only with magnesium sulphate. Finally, albumin is precipitated neither by sodium chloride nor by magnesium sulphate. By the method of fractional precipitation a similar classification is obtained. Fibrinogen possesses the lowest lower limit, and albumin the highest.

Differentiation of the Products of Transformation of Albuminoids. - The method of fractional precipitation permits, in many cases, the separating of the various natural albuminoids from their mixed solution. Thus, we can get separation from the same solution of fibrinogen, globulin and albumin. We can also profit by our knowledge of the higher limits for the purification of any albuminoid substance. Finally, Hofmeister and his pupils have applied this method to the separation of products of hydrolysis of protein substances. They have succeeded in isolating and in characterizing different derivatives, and, at present, the method is closely related not only to the ideas which we possess on the progress of peptonization, but also to the prevalent theory as to the molecular grouping of the different albuminoids. If, after having submitted a natural albuminoid to the action of pepsin, the liquor is treated with a solution of ammonium sulphate, it is found that the digestion liquor acts in the same manner as a solution containing a mixture of different substances. The higher limit is no longer a stable limit as was the case for a solution containing only a single substance, but the liquor has several higher limits very easily recognizable.

By applying the method of fractional precipitation to natural albuminoids that have undergone the action of pepsin, we arrive first by the aid of relatively small quantities of sulphate, 
at a higher limit. The liquid, freed of its precipitate, no longer precipitates by a fresh addition of 0.2 c.c. of sulphate; but when we perceptibly increase the quantity of salt, a new precipitate appears, and, by a gradual addition of the salt solution, we arrive at a new higher limit. This is followed by a neutral point where there is no more precipitation, and then comes a new lower limit, where precipitation begins, followed by a third higher limit. The following, according to Pick, is the progress of the fractional precipitation of a 5 per cent solution of Witte peptone.

Existence of Lower and Higher Limits of Precipitation.

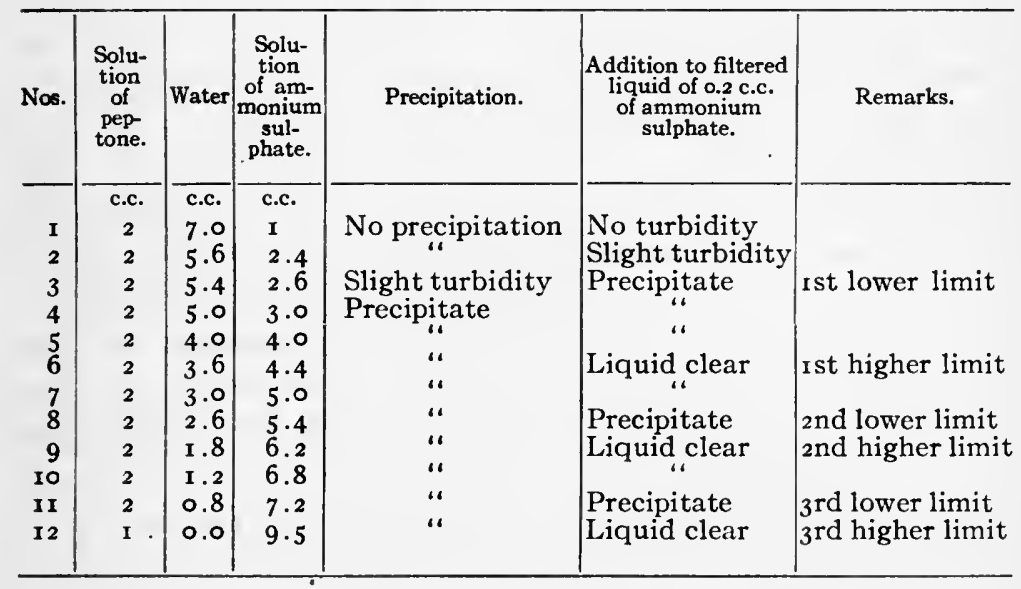

In test No. I, the liquid is composed of 2 c.c. of peptone, 7 c.c. of water and I c.c. of saturated ammonium sulphate. The column "precipitation" indicates whether the mixture remains clear. Precipitation begins only in No. 3, and, consequently, the first lower limit corresponds to 2.6 c.c. of sulphate. Precipitation caused in No. 3 by the sulphate is far from being complete. The column "Addition to filtered liquid" indicates whether or not the liquid to which had been added 2.6 c.c. of sulphate, and then had been freed of precipitate by filtration, gives a new precipitate with 0.2 c.c. of sulphate. In No. 6, 
then, the filtered solution is not precipitated at this point; then 4.4 c.c. is the higher limit: The liquid, freed from the first fraction of precipitate by filtration, begins to be turbid again in the presence of 5.4 c.c. of sulphate. Between the higher limit of the first precipitation and the lower limit of the following is an interval, a neutral point which is also found between 9 and II.

The method of fractional precipitation furnishes, consequently, with peptone three different fractions each presenting. distinct limits.

\begin{tabular}{|c|c|c|}
\hline & Lower limit. & Higher limit. \\
\hline & c.c. & c.c. \\
\hline Ist fraction. & 2.6 & 4.4 \\
\hline 3 rd fraction. & $\begin{array}{l}5.4 \\
7.2\end{array}$ & $\begin{array}{l}6.2 \\
9.5\end{array}$ \\
\hline
\end{tabular}

After having been deprived of these three fractions, the liquid still contains albuminoid substances in solution, which can be divided into two new fractions. The first is precipitated when the liquid, previously saturated with ammonium sulphate, is acidified; the second remains in solution. We have, thus, definitely separated all the products of peptic hydrolysis into five portions:

To make this separation on a larger scale, proceed in the following manner: First, filter the liquor to separate from it the undissolved substances. Then, neutralize to precipitate the syntonin, boil to coagulate the coagulable substances remaining, and bring the filtrate to a dilution of about 5 per cent with respect to albuminoids. In this liquid, make a fractional precipitation by adding increasing quantities of sulphate. To separate the first fraction, add to the liquid of digestion I volume of saturated solution of ammonium sulphate. Let it settle 24 hours, after which the precipitate can be collected on a filter. This precipitate is then washed with a half saturated solution of sulphate, is redissolved in water, and is precipitated again by the same quantities of salt. To get purer products, it is 
necessary to precipitate the substance three or four times, using the same quantity of salt.

The liquid, free from the first fraction, is filtered. To obtain the second fraction, add $\frac{1}{2}$ volume of the salt solution; the precipitate is washed with a salt solution, $\frac{2}{3}$ saturated, is redissolved several times in water, and each time is reprecipitated with the $\frac{2}{3}$ saturated solution. The third fraction is obtained when the filtrate from the second fraction is saturated with powdered ammonium sulphate. The fourth fraction precipitates when to the filtrate from the third fraction is added $\frac{1}{10}$ of a volume of dilute sulphuric acid, saturated with ammonium sulphate. To obtain a complete precipitate, allow this acidulated liquid to settle for two days; wash the precipitate with a saturated and acidified solution of sulphate, and, finally, purify under conditions similar to those used for the purification of the preceding fractions. The fifth fraction remains in solution in the liquid resulting from the four preceding fractions. The first fraction is given the name primary albumose; fractions $2,3,4$, the names deutero-albumoses $A, B, C$; the fifth is peptone.

The five fractions separated in Witte peptone are likewise found in the products of hydrolysis, either with acids, or with enzymes of all protein substances. The limits of precipitation of the four albumoses, according to their different origins, are given in the following table:

Precipitation Limits with $\left(\mathrm{NH}_{4}\right)_{2} \mathrm{SO}_{4}$.

Of the Four Albumoses According to their Origins.

\begin{tabular}{|c|c|c|c|c|c|c|}
\hline Various fractions. & Casein. & Fibrin. & $\begin{array}{l}\text { Egg-al- } \\
\text { bumin. }\end{array}$ & $\begin{array}{l}\text { Album. } \\
\text { serum. }\end{array}$ & $\begin{array}{l}\text { Globul. } \\
\text { serum. }\end{array}$ & $\begin{array}{l}\text { Witte } \\
\text { pep- } \\
\text { tone. }\end{array}$ \\
\hline I. Albumose Slimit lower. & 2.6 & 2.6 & 3.6 & 4.2 & 3.8 & 2.6 \\
\hline primary $\quad\{$ limit higher... & 4.4 & 4.4 & 4.6 & 4.6 & 4.6 & $4 \cdot 4$ \\
\hline II. Deutero $\{$ limit lower.... & 5.2 & 5.4 & 5.6 & 5.4 & 5.6 & $5 \cdot 4$ \\
\hline albumose $A$ \{limit higher... & 7.2 & 6.2 & 6.0 & 6.2 & 7.2 & 6.2 \\
\hline III. Deutreo limit lower.... & 8.2 & 7.2 & 7.0 & 7.2 & 7.8 & 7.2 \\
\hline albumose $B$ \{ limit higher... & $9 \cdot 5$ & $9 \cdot 5$ & 7.8 | & $8.0 \mid$ & 8.6 & $9 \cdot 5$ \\
\hline $\left.\begin{array}{l}\text { IV. Deutreo } \\
\text { albumose } C\{\text { limit lower } \\
\text { limit higher }\end{array}\right\}$. & \multicolumn{6}{|c|}{ Saturated in acid reaction. } \\
\hline
\end{tabular}


The interval between the higher limit of a fraction and the lower limit of the following fraction is everywhere very sharp, and these limits cannot be confused. On the other hand, the corresponding limits differ little from one substance to another. It is only for the lower limit of primary albumose that we observe spaces as great as 2.6 to 4.2. This result can be explained, either by a difference in the nature of the same fraction coming from different substances, or more simply by the degree of purity of the albuminoid substances used.

In reality, the limits of the fractions are subject to certain fluctuations arising from the conditions of the medium. The concentration and the acidity are very important factors in this variation. The part played by concentration of protein substances on the limits of the fractions is very considerable. Thus it is that:

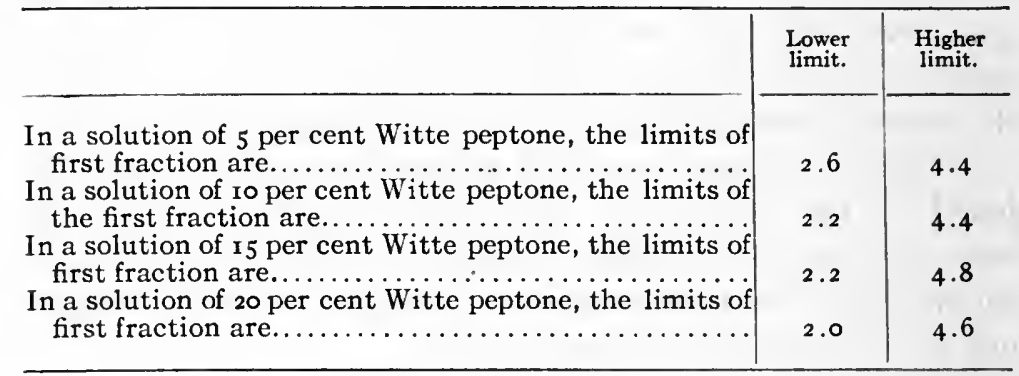

The lower limit is lowered by the concentration. In a 5 per cent solution the lower limit is 2.6 , while in a ro per cent solution it descends to 2.2 .

All albuminoids do not behave in the same manner. Thus, Kauder has found that, in blood serum, the limits of precipitation of globulin vary slightly with the quantity of albuminoid contained in the serum, while the limits of precipitation of albumin of the serum are constant. The chemical reaction of the medium exerts a still more perceptible effect. The following are the limits of three fractions of peptonized fibrin, obtained using a neutral medium, an alkaline medium and an acid medium. 


\begin{tabular}{l|r|r|r|r|r|r|r|r|r|r}
\hline & \multicolumn{2}{|c|}{ First fraction. } & \multicolumn{2}{c|}{ Second fraction. } & \multicolumn{2}{c}{ Third fraction. } \\
\cline { 2 - 7 } & Neut. & Acid. & Alkal. & Neut. & Acid. & Alkal. & Neut. & Acid. & Alkal. \\
\hline Lower limit.. & 2.6 & 1.2 & 2.4 & 5.4 & 4.7 & 5.8 & 7.2 & 6.3 & 7.4 \\
Higher limit. & 4.4 & 4.3 & 4.4 & 6.2 & 5.9 & 6.2 & 9.5 & 7.7 & 9.5 \\
\hline
\end{tabular}

The acid reaction considerably lowers the lower limit of the three fractions. It also exerts an influence on the higher limit, but to a less degree. The alkaline reaction shows a less pronounced effect on these limits of precipitation than the acid reaction.

In addition to the effect produced by the reaction of the medium and by the concentration, the limits can still be influenced by the presence of foreign substances in the solution. Nevertheless, the fractionating limits always appear very clearly defined when working with relatively pure substances in neutral solutions and at a concentration between 2 and 5 per cent.

The results obtained by the method of fractional precipitation using ammonium sulphate have received a new confirmation at the hands of Zunz and Pinkus. These investigators have demonstrated that the five fractions obtained with ammonium sulphate are likewise obtained with sodium sulphate. Zunz, who has studied with much care the action of zinc sulphate, finds an exact analogy between the results obtained with zinc sulphate and with ammonium sulphate. In working with zinc sulphate, the first three fractions are obtained in the neutral solution; the fourth, in an acid solution and after.saturation. The influence exerted on the fractions by the conditions of the medium and the degree of concentration has been previously determined for the two salts. As in certain cases the formation of insoluble compounds of zinc takes place, it is always preferable to choose acid reactions. The acidity most favorable is given by 0.75 per cent sulphuric acid. To maintain the same condition of the medium, it is necessary to bring to the same degree of acidity the solution of albuminoids, 
that of the zinc salts and the water which is used to bring the liquid to a constant volume. The results are then very satisfactory.

The experiments of Zunz relate to the products of transformation of egg-albumin, serum-albumin, serum-globulin, as well as casein; the first three of these products were in a state of almost absolute purity. The following is the procedure: dissolve $2 \mathrm{~g}$. of albuminoid substances in 100 c.c. of liquid containing $30 \mathrm{cg}$. of hydrochloric acid and $4 \mathrm{cg}$. of pepsin. Let it digest at $50^{\circ}$, until a peptone reaction is found. At this point, the liquid is filtered, exactly neutralized, again filtered, and is then acidified with sulphuric acid to a dilution of 0.75 per cent of the liquid. The salt solution is prepared by saturating in the cold; it has a density of 1.450 .

Limits of Precipitation with $\mathrm{ZnSO}_{4}$ of the Four Albumoses According TO THEIR ORIGIN.

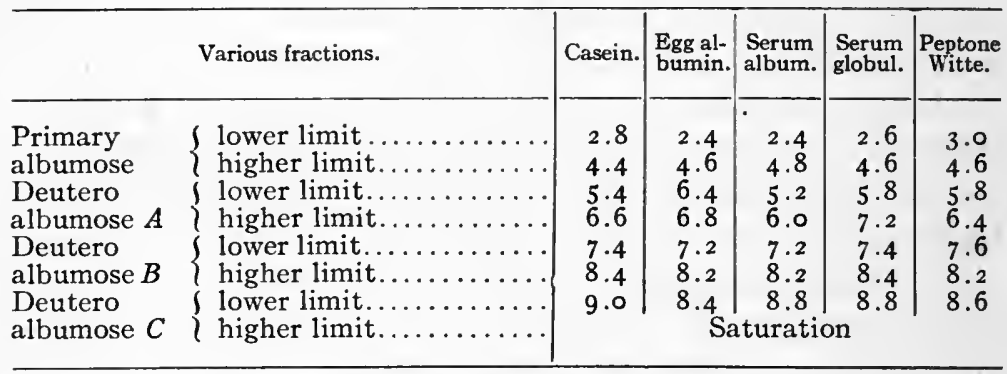

If we compare the limits of the fractions, obtained on one hand with ammonium salt and on the other with zinc sulphate, we find quite noticeable differences; but in the two cases we get the same number of fractions, with a well defined interval between the limits.

Chemical Reactions of the Four Fractions of Albumose. The four fractions obtained by fractional precipitation possess, as we have just seen, constant limits under the same conditions of the medium and with the same concentration. Hofmeister and his pupils consider these fractions as being composed of different substances, and they have applied themselves to the 
task of characterizing them according to their chemical reactions. The first fraction, primary albumose, is composed of the protoalbumose and the hetero-albumose of Kühne. These two substances can be separated by dialysis or by the aid of alcohol. For this, a volume of 95 per cent alcohol is added to a 5 per cent solution of primary albumose and the mixture is maintained at the freezing point for 5 to ro hours. The portion precipitated is hetero-albumose; the albuminoid substance remaining in solution is proto-albumose. The two albumoses of the first fraction differ from each other not only by their solubility in alcohol, but also by other properties characteristic of the heteroalbumose; its solution is rendered turbid by a few drops of dilute nitric acid; it gives an abundant precipitate in presence of a small quantity of copper acetate, as well as with Almen's Reagent (alcoholic solution of tannin with an addition of acetic acid); the precipitate obtained with this latter reagent does not dissolve even in an excess of the reagent.

The fractions 2, 3, 4 are composed of deutero-albumoses $A, B, C$. The two last are believed to be a single substance. The fraction $A$, on the other hand, is composed of two substances $a$ and $b$, which are distinguished by a different sulphur content. The fraction $A a$ gives, on boiling with alkali and lead acetate, a slight browning, while $A b$, treated in the same manner, gives a blackish brown coloration. There is also a difference when a small quantity of copper acetate is added to solutions of the two substances. $A a$ gives an abundant precipitate, $A b$ does not precipitate.

The principal reactions of the different fractions are summarized in the following table: 


\begin{tabular}{|c|c|c|c|c|c|c|}
\hline \multirow{2}{*}{$\begin{array}{c}\mathrm{Re}- \\
\text { agents. }\end{array}$} & \multicolumn{2}{|c|}{ Ist fraction. } & \multicolumn{2}{|c|}{ 2nd fraction. } & \multirow{2}{*}{$\frac{\text { 3rd fraction. }}{\begin{array}{c}\text { Deutero } \\
\text { albumose } B .\end{array}}$} & \multirow{2}{*}{ 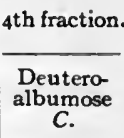 } \\
\hline & $\begin{array}{c}\text { Hetero- } \\
\text { albumoses. }\end{array}$ & $\begin{array}{l}\text { Proto- } \\
\text { album. }\end{array}$ & $\begin{array}{c}\text { Deutero- } \\
\text { albumose } A \text { a. }\end{array}$ & $\begin{array}{c}\text { Deutero- } \\
\text { albumose } A b .\end{array}$ & & \\
\hline I & $++t$ & - & - & - & - & - \\
\hline 2 & ++ & - & + & - & - & - \\
\hline 3 & $+t$ & + & + & - & - & - \\
\hline 4 & + & + & + & & - & - \\
\hline $\begin{array}{l}5 \\
6\end{array}$ & $+t+$ & + & 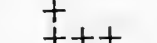 & $+t+$ & $\begin{array}{c}+ \\
+\end{array}$ & - \\
\hline 7 & & & + & $++t$ & ++ & - \\
\hline 8 & + & & ++ & & & \\
\hline
\end{tabular}

Reagents: I, Dilute solution of copper sulphate. 2, Cold nitric acid. $3, \frac{1}{2}$ saturated solution of sodium chloride + nitric acid. 4, One volume of saturated solution of sodium chloride acidified with acetic acid. 5, Saturation with sodium chloride and acidulation with nitric acid. 6, Neutral solution, saturated sodium chloride. 7 , Boiling of the liquid with lead acetate in an alkaline medium. 8, Molische reaction.

The sign + indicates an appearance of turbidity or of a precipitate. The number of these signs gives an idea of the intensity of the effect. The sign - indicates the absence of turbidity or of a precipitate.

The boiling of the liquid with lead acetate, in an alkaline medium (reagent No. 7), is used to indicate the presence of oxidizable sulphur. $(t)$ corresponds to a slight browning of the liquid; $(+++)$ indicates a blackening and a precipitation. The deutero-albumose $A b$ will contain, according to these reactions, the maximum of oxidizable sulphur while the deuteroalbumose $C$ is completely free from sulphur. The reagent of Molische (coloration obtained by $\alpha$-naphthol in presence of sulphuric acid) indicates the presence of a carbohydrate grouping. The first fraction contains but few substances rich in carbohydrates; the following are much richer. The reagent, cold nitric acid, means the addition of one or two drops of dilute nitric acid. The hetero-albumose gives, under these conditions, a precipitate; the deutero-albumose $A a$, a slight turbidity. 
Hofmeister and his pupils attribute a great value to these distinctive reagents. According to them, the fractions obtained are well characterized by a combination of their physical, chemical, and physiological properties. We must, however, insist that the proofs which they bring to the support of their opinion are far from being decisive. The reactions recorded in the table for each of the groups are not very definite. With the possible exception of hetero-albumose, all the fractions are mixtures, and, furthermore, their reactions are not always constant. The properties differ perceptibly, according to the albuminoid material used. Thus, the deutero-albumose $C$ gives no sulphur reaction at all when derived from fibrin, and on the other hand is rich in this element when derived from serum-albumin. The same fractions will give a weak or strong furfurol reaction, according to whether they are produced in the hydrolysis of fibrin or of egg-albumin.

Although the supporters of the method of fractional precipitation recognize this irregularity, they ingeniously explain it by the diverse natures of the primary materials, that is, that the fraction takes special characteristics from substances from which it was derived. However, the chemical differentiation of fractions is based on the colorations or the precipitations. These reactions can, in certain cases, give valuable information. It would, however, be unwise to depend exclusively on these indications to draw a conclusion as to the identity of two substances. This reservation is particularly important in the present instance. We have not as yet obtained albuminoids in a state of absolute purity. By precipitating their solutions, we also carry down the various substances which are mingled there. The precipitation of these substances can also be affected at intervals, according to the concentration of the liquid. We cannot then, even though they evince a certain constancy, make use of certain properties of fractions as indices of a differentiation. Also, the phenomena of precipitations and of distinct colorations can be attributed just as well to impurities likewise precipitated by the salts, as to the albuminoid substance itself 
Objections to the Method of Fractional Precipitation. Our knowledge of the chemical properties of the four albumoses, which are obtained by the method of fractional precipitation, is not extensive at the present time. The reactions of the fractions which we have just studied bring little light to bear upon their chemical nature and, especially, upon their differentiation. Must we conclude that the four fractions are composed of the same substance, that there is no chemical difference between the different albumoses, and that, consequently, the separation obtained is more artificial than real? Some observers are of this opinion and pronounce collectively. against all the results obtained by this method. According to them, the method is absolutely valueless, and the fractions obtained are of a chemical nature created by the manipulation. To confirm this opinion, they quote the very interesting experiments of Duclaux on the crystallization of quinine sulphate, pointing out the analogy existing between the fractional precipitation of this compound and that of an albuminoid.

A saturated solution of basic sulphate of quinine precipitates in the presence of alkali or alkaline-earth sulphate. The precipitation, or rather the crystallization, progresses in a very irregular fashion. By choosing a suitable quantity of ammonium sulphate, one can precipitate a part of the quinine. The filtrate from the crystalline precipitate will again give a precipitation with an additional quantity of ammonium sulphate. The following are some details of these experiments: In a flask containing 100 c.c. of a solution of quinine sulphate, add different quantities of the powdered ammonium salt; allow it to stand for 24 hours and then weigh the precipitate obtained.

\begin{tabular}{|c|c|c|c|c|c|c|c|}
\hline \multicolumn{7}{|c|}{$0.10 \%$ of ammon. sulphate gives a precip. of 0.59 sulph. quin. } & \multirow{2}{*}{$\begin{array}{c}\text { Solution } \\
\text { of salt }\end{array}$} \\
\hline $0.25 \%$ & “ & " & “ & 0.97 & “ & “ & \\
\hline $0.5 \%$ & “ & “ & “ & I.03 & “ & “ & of quin. \\
\hline $1.0 \%$ & “ & “ & “ & x. 17 & “ & “ & 2 grams \\
\hline $2.0 \%$ & “ & “ & “ & I. 18 & “ & " & per liter. \\
\hline $2.0 \%$ & “ & “ & “ & 1.06 & “ & “ & Solution of \\
\hline $4.0 \%$ & " & " & “ & 1. 16 & “ & “ & quin. salt \\
\hline $8.0 \%$ & “ & “ & "، & I. 37 & “ & “ & $1.96 \mathrm{~g}$ \\
\hline $16.0 \%$ & “ & “ & “ & 1. $5^{6}$ & “ & “ & per liter. \\
\hline
\end{tabular}


Between the quantities 0.I per cent and 0.25 per cent ammonium sulphate, there is a very noticeable difference in the crystallization of quinine sulphate. There is no longer the same result upon increasing the amount of ammonium sulphate. With I to $2 \mathrm{~g}$. of sulphate, the weight of precipitate is almost the same, although a large part of the quinine still remains in solution, which will precipitate only with a much stronger dose of ammonium sulphate. This test shows the possibility of obtaining in a solution of quinine a great number of fractions corresponding to different quantities of ammonium sulphate.

It is also seen that the precipitation of quinine can be made in successive stages, with intervals in which the addition of a small quantity of sulphate does not produce any precipitate at all. In reality, if I per cent of the ammonium salt is added to a saturated solution of quinine there is obtained in time a liquid which, filtered from the precipitate, still contains $0.8 \mathrm{~g}$. of quinine salt. To obtain again in this liquid an appreciable precipitate, that is to say a second fraction, requires the use of a quantity of ammonium sulphate two or three times as large as the original. Between the first and second fractions there is then a margin at least of $I$ to 3 . By adopting this technique, it is possible to obtain, at will, a large number of fractions well characterized from the point of view of limits, but all composed of an identical substance. In view of these facts, the adversaries of the method advise a moderation, and suggest, in the future, a greater care in identifying products precipitated by salts.

According to this view, since the action brought into play in this precipitation is of a physical rather than a chemical nature, it is illogical to demand of it a differentiation of species. All these effects are conditional, irregular, and apparently capricious. We must not distinguish materials precipitated in the same liquid by unequal quantities of salts, nor give the same name to materials precipitated in two different liquids by equal quantities of salts.

The experiments with quinine sulphate ${ }^{-}$are not at all of a 
nature to solve the problem, and, moreover, we cannot allow all the conclusions which are drawn from these results. There evidently exists a close analogy between the two phenomena, but this analogy is not absolute. A closer study reveals marked differences of an essential nature. Consider the fractions, obtained by the addition of I per cent ammonium sulphate to solutions of quinine. By dissolving these fractions in water, thus forming a saturated solution, and then by adding to this saturated solution I per cent of the ammonium salt, there is obtained a new precipitate, which will not contain all the quinine in solution. To get a more complete precipitate, the addition of a much greater quantity of salt is necessary. By controlling the quantity of ammonium sulphate, it is possible by successive stages, to obtain a whole serjes of fractions like those obtained with the quinine sulphate of the original solution. The fractions of quinine, then, act as the quinine itself before the fractionation.

We find the case quite different with albuminoid substances. Consider, for example, a solution containing I per cent of globulin and I per cent of albumin. The method of fractional precipitation will give two fractions: the liquid, half saturated with sulphate, will give the precipitate of globulin; in the filtrate from this precipitate the addition of sufficient salt to complete saturation will cause the precipitation of the albumin. The two fractions, when purified, will give solutions acting quite differently from the original mixture. In the globulin solution, a precipitate will appear before the liquid is half saturated; the albumin solution will not precipitate under these conditions. We can obtain the same results with peptonized albuminoids.

By the method of fractional precipitation, the albuminoids can be divided into four fractions, and each of these fractions taken separately and placed in identical conditions, will precipitate in the presence of a definite quantity of salt, which will be different for each fraction. Each of the isolated fractions possesses its two limits of precipitation, constant and stable for the same concentration and the same reaction of the medium. 
These are the peculiarities which characterize the fractions of albuminoid substances, and are peculiarities which are not found in fractions obtained from a solution of quinine sulphate.

Respective Formation of Different Albumoses. - From what we have just seen, we may conclude that the principal argument brought forward to combat the method of fractional precipitation speaks in favor of, rather than against this method. Albuminoid substances, whatever else is said, can, under certain conditions, be characterized and separated by different quantities of the same salt.

The question of the individuality of the four albumoses appears less clear. The distinctive reactions of these fractions are far from being clear and conclusive, and the presence of constant limits for each fraction, while being a favorable index, cannot serve as the basis for a chemical identification of the different fractions. The problem to solve is very delicate since there is very little data on the molecular constitution of albuminoids, and the differences which may exisit among the albumoses are, consequently, very difficult to establish. These compounds, very similar in constitution, perhaps differ in molecular weight alone, and it would not be surprising under these circumstances, that we should not arrive at a differentiation by chemical reagents.

In the study of the products of saccharification of starch, similar difficulties arise. The dextrins obtained do not differ in their rotatory power, nor in their reducing power. They act in an identical manner with the greater number of chemical reagents. The differentiation of the dextrins has been successfully established'by a study of the gradual progress of saccharification, as well as the action of amylase on the different fractions of isolated dextrin.

In the experiments of Zunz on the use of zinc sulphate for the separation of albuminoids, we find interesting indications as to the division of albumose in the different phases of the peptonization of albuminoid substances. Zunz, in his experiments, uses a solution of $3 \mathrm{~g}$. of pepsin per liter in hydrochloric 
acid. In the active solution, he dissolves a certain quantity of albuminoid substance, from I per cent to $\mathrm{I} \frac{1}{2}$ per cent. $\mathrm{He}$ allows it to digest at $40^{\circ}$ and takes samples at different intervals. From these samples, he precipitates four fractions by the aid of zinc sulphate, and determines the total nitrogen contained in each fraction precipitated. This figure for nitrogen serves as basis for the division of the different albumoses and peptones. The following are the results obtained with crystallized serumalbumin.

Division of the Different Albumoses in the Course of Peptonization.

\begin{tabular}{|c|c|c|c|c|c|c|}
\hline \multirow{2}{*}{$\begin{array}{l}\text { Length } \\
\text { of } \\
\text { action, } \\
\text { hours. }\end{array}$} & \multicolumn{6}{|c|}{ Division of nitrogen in the fractions, nitrogen per cent. } \\
\hline & $\begin{array}{l}\text { Prim. } \\
\text { album. }\end{array}$ & $\begin{array}{l}\text { Deutero- } \\
\text { alb. } A \text {. }\end{array}$ & $\begin{array}{l}\text { Deutero- } \\
\text { alb. } B\end{array}$ & $\begin{array}{l}\text { Deutero- } \\
\text { alb. } C .\end{array}$ & $\begin{array}{c}\text { Album. } \\
\text { total. }\end{array}$ & $\begin{array}{l}\text { Deutero-alb. } \\
\text { total. }\end{array}$ \\
\hline 2 & I 7.94 & 4.00 & I 2.92 & I .64 & 36.50 & I $8.5^{6}$ \\
\hline 4 & 7.02 & 8.97 & 8.58 & I. 38 & 25.95 & I 8.93 \\
\hline 8 & I.I 3 & 6.05 & I $5 \cdot 53$ & I. 20 & $23.9 \mathrm{I}$ & 22.78 \\
\hline 24 & I . I 2 & $3 \cdot 71$ & $4 \cdot 47$ & $3 \cdot 52$ & $12.8 \mathrm{I}$ & 11.70 \\
\hline 48 & 0.70 & 0.00 & I. I9 & 7.00 & 8.89 & 8.19 \\
\hline 72 & 0.54 & 0.00 & 0.40 & 5.87 & $6.8 \mathrm{I}$ & 6.27 \\
\hline
\end{tabular}

The first sample is taken after 2 hours. At this moment, the liquid no longer gives an acid albumin precipitate by neutralization. The figure under "total albumose" (6th column) indicates that for $100 \mathrm{~g}$. of nitrogen contained in the liquid, $36.5 \mathrm{~g}$. are found in the albumose. The total albumose diminishes as peptonization advances; after 8 hours $23.9 \mathrm{I}$ remain; after 72 hours only $6.8 \mathrm{r}$. A similar fall is observed in the "primary albumose" column. After 2 hours 17.94 remain; after 4 hours 7.02 remain; and after 48 hours, 0.7 remain. This decrease of the primary albumose is very rapid, and the quantity which has disappeared is higher, at a certain period, than the total quantity of albumose transformed. Thus, in the interval from 4 to 8 hours we find a diminution of 2.04 for total albumose, while the primary albumose transformed in the same interval of time is 5.89. This course in the decrease of primary albumose shows that the deutero- 
albumose forms at the expense of the primary albumose, consequently, in the different phases of the peptonization the liquid must contain varying quantities of deutero-albumose, quantities which will not be in relation to the lapse of time of the digestion. In the column "total deutero-albumose" we find, in fact, this irregular progress. Whereas, after 2 hours we find 18.56 , after 4 hours when peptonization is already much more advanced, the quantity of deutero-albumose has not diminished. After 8 hours, we even get a noticeable increase, 22.78, but thereafter begins the period of decrease and after 72 hours we find only 6.27 .

This ascending and descending progress is found in each of the three deutero-albumoses. Furthermore, we can assert that the different deutero-albumoses are not produced exclusively at the expense of the primary albumose; in fact, between the $4^{\text {th }}$ and 8th hour the deutero-albumose $B$ has increased from 8.58 to $15.53 ; 6.95$ being produced. In this same time, the primary albumose has lost only 5.39 , hence, the deutero-albumose $B$ is then formed in part from the deutero-albumose $A$. The same observation can be repeated with regard to the formation of the deutero-albumose $C$. Between the $24^{\text {th }}$ and 48 th hours, this fraction has increased from $3.5^{2}$ to 7.0 , while the primary albumose has diminished only from I.I 2 to 0.70 .

To have a clearer idea of the division of albumoses during the course of peptonization, we must determine the proportion of each fraction in the total quantity of albumose present at each sampling. The analysis of the samples taken shows that the four albumoses are found at each phase of the peptonization in different proportions. The primary albumose is the product which is formed most abundantly from the beginning of the action of the pepsin, but it disappears rather quickly, leaving a little residue, composed principally of hetero-albumose. The yield increases at the end, due to the transformation of the total albumose into peptone. The deutero-albumose $A$, while arriving at its maximum later than the primary albumose, disappears more quickly than the latter: The deutero-albumose 
$C$ arrives at its maximum when peptonization is more advanced; it having the characteristics of a more degraded product.

Divisions of Albumose in the Different Phases of Peptonization, PER IOO Albumose.

\begin{tabular}{|c|r|r|r|r|}
\hline $\begin{array}{c}\text { Length of } \\
\text { experiment, } \\
\text { hours. }\end{array}$ & $\begin{array}{c}\text { Primary } \\
\text { albumose. }\end{array}$ & $\begin{array}{c}\text { Deutero } \\
\text { albumose } \\
\boldsymbol{A} .\end{array}$ & $\begin{array}{c}\text { Deutero } \\
\text { albumose } \\
B .\end{array}$ & $\begin{array}{c}\text { Deutera- } \\
\text { albumose } \\
\boldsymbol{C} .\end{array}$ \\
\hline & & & & \\
\hline 2 & 49.15 & 10.95 & 35.39 & 4.49 \\
4 & 27.05 & 34.56 & 33.06 & 5.31 \\
8 & 4.72 & 25.30 & 64.95 & 5.01 \\
24 & 8.73 & 28.93 & 34.39 & 27.40 \\
48 & 7.87 & 0.00 & 13.38 & 78.74 \\
72 & 7.92 & 0.00 & 5.87 & 86.19 \\
\hline
\end{tabular}

The differences which are found in the proportions of the various albumoses, the very perceptible increase of albumose $C$, the diminution of primary albumose, the disappearance of deutero-albumose $A$, are additional data in favor of the existence of several varieties of albumoses for the same substance arbitrarily divided could not show such special properties.

From the point of view of the division of albumoses, one can obtain more exact results by studying peptonization under the action of weak acids. By the action of acid on albuminoid matter, we get, according to Goldschmidt, the same albumoses as with enzymes; but the transformation with acids being much slower, it is easier to follow the progress of the hydrolysis and to distinguish the first appearance of each of the albumoses.

Appearance of Different Albumoses in the Course of Hydrolysis WITH $\mathrm{HCl}$.

\begin{tabular}{|c|c|c|c|c|}
\hline 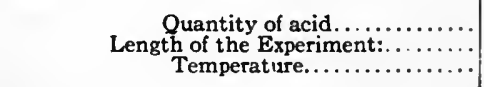 & $\begin{array}{l}+N \mathrm{HCl} \\
\text { after } \mathrm{I} \mathrm{h} . \\
40^{\circ} \mathrm{C} .\end{array}$ & $\begin{array}{l}\frac{1 N \mathrm{HCl}}{\mathrm{N}} \\
\text { after I8 h. } \\
40^{\circ} \mathrm{C} .\end{array}$ & $\begin{array}{l}1 \mathrm{~N} \mathrm{HCl} \\
\text { after } 48 \mathrm{~h} \text {. } \\
40^{\circ} \mathrm{C} .\end{array}$ & $\begin{array}{l}4 \mathrm{~N} \mathrm{HCl} \text {. } \\
\text { after } 16 \mathrm{~h} \text {. } \\
40^{\circ} \mathrm{C} .\end{array}$ \\
\hline 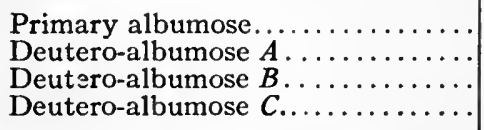 & $\underline{-}$ & $\frac{t}{ \pm}$ & $\begin{array}{l}+ \\
+ \\
+\end{array}$ & $\begin{array}{l}\bar{t} \\
\dot{t} \\
\dot{t}\end{array}$ \\
\hline
\end{tabular}


The sign + marks the presence of albumoses, the sign - the absence. These tests with egg-albumin indicate that, according to the concentration of acid and the duration of the experiment, we can get exclusively deutero-albumose $B$ or primary albumose and deutero-albumose $B$, or again primary albumose and the deutero-albumoses $A$ and $B$.

These results are very favorable to the method of fractional precipitation. Primary albumose and deutero-albumose $B$ are the products which are first formed; deutero-albumose $A$ appears as a secondary product and deutero-albumose $C$ only as a final product of the reaction. This fraction is formed with difficulty in the presence of acids and is not met in appreciable quantity except in the last phases of peptonization.

Comparative Progress of the Transformation of Primary Albumose and the Deutero-albumoses. - As far as may be judged from the experiments described in the preceding paragraphs, primary albumose disappears gradually during the course of peptonization. It was, therefore, interesting to study this point, being given the different progresses of the three other fractions of albumose. Effront made some tests in this respect with white of egg. The following are the conditions under which he undertook these experiments. The whites of egg were. passed through a silk filter. The liquid, diluted with distilled water, was filtered through a filter paper, and then was adjusted to a content of 3 per cent albumin. For each liter of liquid was added 500 c.c. of dilute acid containing $3 \mathrm{~g}$. of hydrochloric acid and $0.1 \mathrm{~g}$. of pepsin. The mixture was allowed to react for a half-hour at $50^{\circ}$, was then cooled, neutralized with soda, and the liquid, free of syntonin, was boiled to coagulate the albumin not transformed. To the filtered liquid was again added hydrochloric acid up to a concentration of $3 \mathrm{~g}$. per liter, then 0.I g. of pepsin, and it was left to digest at $40^{\circ}$ in a closed flask. At different times, samples were taken, in which primary albumose was precipitated. According to the nitrogen content of the precipitate obtained, the quantity of primary albumose present in the liquid at different phases of the peptonization was calculated. (See comparative table, next page.) 
To determine the primary albumose in all the samples, 20 c.c. of liquid are taken, 0.4 c.c. of dilute sulphuric acid added and 17 c.c. of a saturated solution of acidified zinc sulphate. This is left standing 24 hours, then the precipitate is filtered and washed five times with a solution half saturated with zinc sulphate.

Gradual Disappearance of Albumose.

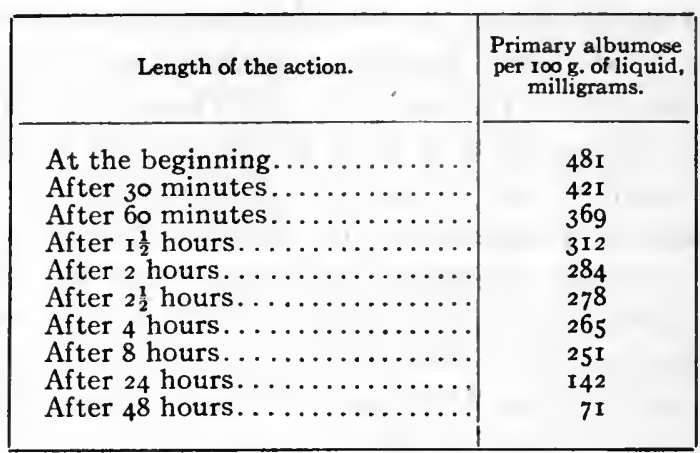

At the beginning of the second peptonization, the liquid, free from untransformed syntonin and albumin contained 690 $\mathrm{mg}$. of total albumose. $48 \mathrm{I} \mathrm{mg}$. were present as primary albumose. In a liquid so rich in primary albumose, its progressive disappearance during peptonization can be easily followed. In the first four samples taken from the beginning to I hour and a half, up to a certain point, a proportionality is observed between the time and the product transformed. In the following sample we already see a very pronounced retardation, and then the quantity of albumose continually decreases. This gradual disappearance is very characteristic of primary albumose. This peculiarity is not found in the three deutero-albumoses; fractional precipitation then gives us a real separation between the products transformed, since, of the four fractions obtained, at least one is absolutely distinct from the three others.

The different natures of the albumoses can also be recognized by the various actions of pepsin on each fraction. The tests 
which Effront has pursued in this direction have given quite satisfactory results. A 5 per cent solution of white of egg is submitted to the action of pepsin. Peptonization is stopped after I 2 hours, and the liquid, after neutralization, boiling, and filtering, is divided into two portions. In the first portion, primary albumose is precipitated separately, then the three deutero-albumoses together. In the second portion, deuteroalbumose $C$ is precipitated, after having separated the liquid from every other albumose. The three precipitates are washed, purified, and each of them is dissolved in the following manner: One and a half grams of substance are dissolved in roo c.c. of 0.3 per cent hydrochloric acid. $0.5 \mathrm{~g}$. of pepsin is added and the mixture allowed to stand in a closed flask at $40^{\circ}$. At different times samples are taken, and the quantity of peptone formed (substance not precipitable by salts) is determined.

Formation of Peptone from the Different Albumoses.

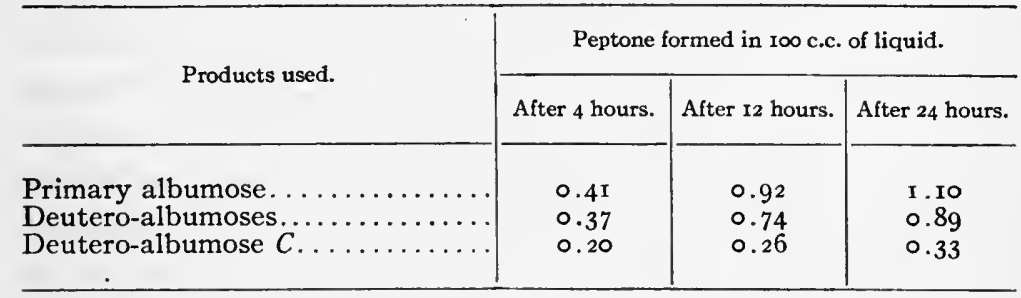

If we compare the quantity of peptone formed from primary albumose with that formed from the deutero-albumoses, we see that peptonization of the two products does not progress equally. Primary albumose peptonizes easily, while the transformation of the deutero-albumoses is much slower. After 12 hours of action, we get $0.92 \mathrm{~g}$. with primary albumose and $0.74 \mathrm{~g}$. with deutero-albumoses. After 24 hours, we have I.I g. with primary albumose and $0.89 \mathrm{~g}$. with deutero-albumoses. We may then conclude that these two fractions are not identical. Furthermore, we may also conclude that the deutero-albumoses are not homogeneous, since the progress of peptonization of deutero- 
albumose $C$ is quite different from that of the mixture of the deutero-albumoses. The deutero-albumose $C$ gives, after 24 hours, $0.33 \mathrm{~g}$. of peptone as compared with $0.89 \mathrm{~g}$. formed by the mixture of the deutero-albumoses.

To summarize, the method of fractional precipitation gives certain results in solutions not containing more than a single species of albuminoid. In certain cases only, can it be applied to the analysis of a mixture of protein substances. In the study of the products of transformation of albuminoid substances by acids or enzymes, it indicates the existence of a certain number of derivatives. The four albumoses which are obtained by the method of fractional precipitation must be regarded as a grouping of distinct compounds, and not as the same substance artificially divided. The individuality of each fraction is attested by the fact that, according to the conditions taken, one may get one or the other albumose, and that the appearance or disappearance of each of these groupings corresponds to different periods, depending on the extent of the hydrolysis. Primary albumose differs from deutero-albumoses in that under the action of pepsin it is gradually transformed, that is to say, the quantity measured in the samples during the experiments diminishes or remains stationary, but never increases. The deutero-albumoses $A$ and $B$, which form at the expense of each other, and also primary albumose, have, on the other hand, during peptonization, two or more maxima. As to deuteroalbumose $C$, it behaves toward the active substance, or toward dilute acid, in a quite different way from primary albumose or the deutero-albumoses $A$ and $B$.

Yet, it is quite true that the four albumoses thus obtained, though they manifest distinctive properties, should not be regarded as strict chemical individuals, but rather as different groupings of compounds. Thus, primary albumose is composed of proto-albumose and hetero-albumose, this last being characterized by the precipitate which it gives with cold nitric acid, or with copper acetate and sulphate. In deutero-albumose $A$, Zunz distinguishes two fractions. Finally, deutero-albumose 
$C$ may be further considered as a single substance, since, under the action of pepsin, it behaves as a mixture of two components, one sensitive and the other insensitive to the action of the enzyme. However, in spite of its imperfections, the method of fractional precipitation, by establishing a distinction, even though a rough one, between the different products of proteolysis, permits study of the formation of these during the course of the action of the digestive enzymes. It is, then, capable of rendering valuable service in the study of the chemical changes involved.

$\S$ ir. Peptones and Complex Non-biuretic Compounds Resulfing from the Action of Pepsin: "Formaldehyde" Nitrogen and Amido Nitrogen.

From the preceding data it appears that in the course of hydrolysis four albumoses are formed which can be regarded as groupings of several distinct compounds, differing in a certain number of properties. The fraction which is not precipitated by zinc sulphate in acid media bears the name of peptone. This important fraction gives the biuret reaction and is composed of complex derivatives of the albuminoids. Further, it precipitates with phosphotungstic acid, although precipitation is not complete. In the precipitated portions are found fractions giving the biuret reaction. They are considered to be composed of the diamino-acids and compound monoamino-acids. In the portion remaining in solution are found still higher polypeptides not giving the biuret reaction. We know that phosphotungstic acid is used readily to separate monoamino-acids of simple structure from compound derivatives. As Wetzel and Kutscher as well as Schmied have already shown, this separation is not complete. The experiments, to have much interest, should be carried out under strictly determined conditions and the results thus obtained have, after all, only a comparative value.

The part of the peptone precipitable by phosphotungstic acid is sometimes designated by the name of true peptone, an 
appellation which must not be confounded with that of commercial peptone, under which is sold the compound product derived from peptic hydrolysis of fibrin or others, and which is largely composed of albumoses. To measure true peptone, add to the digestion liquor, previously separated from albumoses* which it contained, $\frac{1}{2}$ vol. of $\mathrm{H}_{2} \mathrm{SO}_{4}[\mathrm{I}: 3]$. Then a solution of Io per cent phosphotungstic acid (Merck) is introduced little by little with stirring until there is no further precipitation. It is allowed to stand 24 hours at $35^{\circ}-40^{\circ}$ and then is filtered. The difference between the nitrogen of the liquid before the addition of phosphotungstic acid and after filtration gives the nitrogen precipitated. By using successively, as means of fractional precipitation, $\mathrm{ZnSO}_{4}$, then phosphotungstic acid,

Progressive Disappearance of Different Albumoses in the Course of Peptic Digestion of Serum-albumin.

\begin{tabular}{|c|c|c|c|c|c|c|c|c|}
\hline \multirow{3}{*}{ Time of digestion. } & \multicolumn{8}{|c|}{ Nitrogen per roo contained in the Albumoses. } \\
\hline & \multirow{2}{*}{ I. } & \multirow{2}{*}{ II. } & \multirow{2}{*}{ III. } & \multirow{2}{*}{ IV. } & \multirow{2}{*}{ Total. } & \multicolumn{3}{|c|}{$\begin{array}{l}\text { Other products of } \\
\text { digestion. }\end{array}$} \\
\hline & & & & & & v. & VI. & Total. \\
\hline 4 hours & 37.10 & 4.93 & 29.46 & 3.28 & 74.77 & I. 83 & 23.40 & 25.23 \\
\hline 8 hours. & 30.12 & 8.64 & 25.82 & 4.86 & 69.44 & 2.65 & $27.9 \mathrm{I}$ & $30.5^{6}$ \\
\hline 22 hours. & I4.94 & 7.12 & $16.5 \mathrm{I}$ & 5.80 & $44 \cdot 37$ & 5.12 & $50.5 \mathrm{I}$ & 55,63 \\
\hline 2 days. & 7.86 & 5.26 & 23.49 & 6.98 & 43.59 & 7.89 & $48 \cdot 5^{2}$ & $56.4 \mathrm{I}$ \\
\hline 3 days. & $3.7 \mathrm{I}$ & 3.05 & 18.95 & 8.28 & 33.99 & 19.06 & 46.95 & 66.01 \\
\hline 6 days. & I.58 & I.17 & 6.84 & $7 \cdot 95$ & 17.54 & 39.08 & 43.38 & 82.46 \\
\hline Io days. & 0.64 & 0.00 & 2.96 & 6.64 & 10.24 & & & 89.76 \\
\hline I5 days. . & 0.00 & $0 . \infty 0$ & 0.89 & 6.28 & 7.17 & 57.67 & 35.16 & 92.83 \\
\hline 21 days.. & $0 . \infty$ & 0.00 & $0 . \infty$ & 6.17 & 6.17 & $55 \cdot 54$ & 38.29 & 93.83 \\
\hline 30 days. . & $0 . \infty$ & $0 . \infty 0$ & 0.00 & 5.89 & 5.89 & 63.09 & 31.02 & $94 . \mathrm{II}$ \\
\hline
\end{tabular}

\footnotetext{
I. Fraction I: proto-albumose and hetero-albumose.

II. Fraction II: deutero-albumose $A$.

III. Fraction III: deutero-albumose $B$.

IV. Fraction IV: deutero-albumose $C$.

r V. Precipitable by phosphotungstic acid (peptones, etc.).

VI. Not precipitable by phosphotungstic acid.
}

* The determination of albumose nitrogen is made by acidifying the liquor to be analyzed with $\mathrm{H}_{2} \mathrm{SO}_{4}[\mathrm{r}: 4]$ using 2 c.c. per 100 c.c. of liquid, and then saturating the whole with $\mathrm{ZnSO}_{4}$, which is added to excess in the form of crystals. After 24 hours, the precipitate is collected, washed with a saturated solution of acidified $\mathrm{ZnSO}_{4}$, and the nitrogen determined by the Kjeldahl method. 
Zunz succeeded in following sufficiently well the digestive work produced by pepsin acting on crystallized serum-albumin.

This table indicates that true peptone (column V), very slight at the beginning, and corresponding to nitrogen precipitable by phosphotungstic acid, gradually increases in the course of the hydrolysis; the nitrogen, in this form, finally reaching at the end of digestion, after 30 days, 63.09 per cent of the total nitrogen. The nitrogen not precipitable by phosphotungstic acid is already considerable, it being 23.4 per cent, after 4 hours of action. It rapidly increases to attain a maximum, or $50.5 \mathrm{I}$ per cent, then it descends again, to assume at the end a value of 31.02 per cent. If we now examine the first four columns relative to the albumoses, we see that their disappearance occurs in an entirely different way. While primary albumose diminishes almost regularly, the deutero-albumoses show in their descent one or two maxima. Furthermore, fractions I and II disappear quite rapidly and entirely, and, on the contrary, fraction III resists longer, although it ends by being entirely transformed. As to fraction IV, it remains constant after 30 days at 5.89 per cent $\mathrm{N}$. The content of the liquid in these four albumoses is from the beginning, also, very variable; $I$ and III appear in very great quantities after several hours of digestion, while II, and especially IV, form only much later without, moreover, ever reaching very large proportions.

Let us now attempt to follow the progress of hydrolysis. As soon as the action begins, primary albumoses (I), deuteroalbumoses $B$ (III), and bodies poorly defined (VI), not precipitable by phosphotungstic acid, appear. Then these two kinds of albumoses, (I) and (III), are attacked with formation of deutero-albumoses $A$ and $C$, (II) and (IV), of compounds (VI), and of a small amount of peptones (V). This progressive degradation of the albumoses explains the maxima observed with (II), (III) and (IV), and also the high maximum of (VI). After about 24 hours, while the albumoses continue to be attacked, the fraction (VI) in its turn undergoes transformation. While hydrolyzing, it passes from the non-precipitable form to the 
form precipitable by phosphotungstic acid. This is necessarily true, since, during the period of time elapsing between 22 hours and 30 days, the peptone (V) has increased (in nitrogen) from 5.I 2 to 63.09 , or by 57.97 units, while the total albumose has decreased only $44.37-5.89=38.48$ units. The complement comes from the digestion of 'fraction (VI), which has lost just $5^{0.51}-3^{1} .02=19.49$ units. A very interesting fact is thus observed, namely, the immediate appearance, then the passing in the course of hydrolysis, of a fraction in the group of peptones not precipitable by salts nor by phosphotungstic acid, and not giving the biuret reaction; a fraction which would have been considered as a much degraded form and which is, in reality, only a compound, otherwise unknown, of still very large molecular weight. This point deserves to be noted.

To summarize, the final products of peptic digestion are composed of deutero-albumose $C$, products not precipitable by phosphotungstic acid, and peptones. The different results which have just been set forth relate to serum-albumin. Zunz has found that egg-albumin, casein or globulin, treated in a similar way, act in a similar manner. Yet the progress of digestion of these various materials does not show entirely the same course. In general, extensive hydrolysis is reached with these bodies with more difficulty than with serum-albumin.

Peptonization of Different Albuminoids.

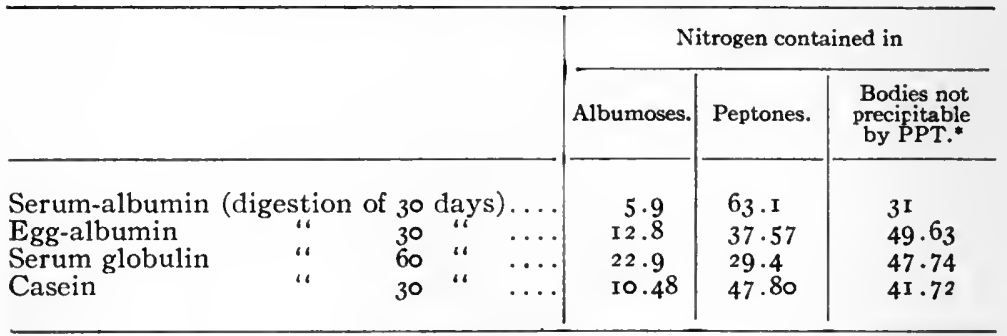

* Phosphotungstic acid.

"Formaldehyde Nitrogen" in Peptic Digestion. - When peptic digestion is allowed to continue for very long periods, 
taking care to add new doses of pepsin from time to time, it is found that the solution finally becomes very rich in nitrogen capable of being titrated with formaldehyde. (See chapter on the analysis of tryptic products.)

Formaldehyde Nitrogen Produced in Peptic Digestions of Long Duration.

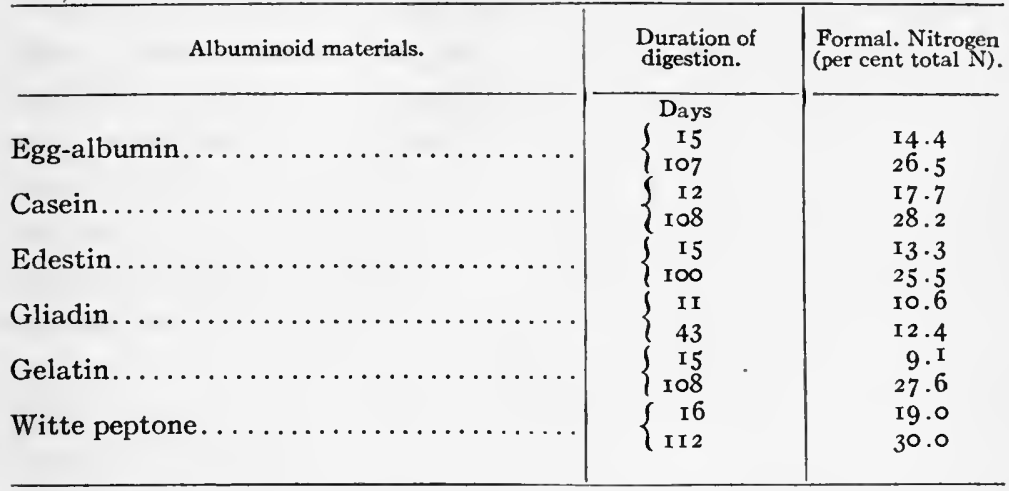

These figures would indicate that pepsin is capable of pushing quite far the dissociation of the albuminoid molecule. Without doubt, the products of advanced digestion furnish the tryptophane reaction; but it would be necessary to show other amino-acids in these products, and also to isolate them, before concluding that they were present. Nevertheless, of 100 units of formaldehyde $\mathrm{N}$ titrated in advanced peptic digestions, about half is still precipitable by phosphotungstic acid, the other half remains in solution. Now, these tungstic filtrates, according to Henriques and Gjaldbaek, treated by $\mathrm{HCl}$, give a much more considerable test of formaldehyde nitrogen than before. They are not then composed of large quantities of simple amino-acids, but of peptides still very complex.

Furthermore, it must be remarked that very prolonged peptic digestions never give very concordant results. Now, we have seen that sterile solutions of peptone, in the long run, hydrolyze under various influences, as that of light for example, without enzymes intervening. Under these conditions a very 
advanced hydrolysis may take place. It is probably to some such cause that must be attributed the presence of crystalline amino-acids which some writers note in the products resulting from a very prolonged peptic action, and which they even profess to have isolated.

Formation of Amid Nitrogen in the Course of Peptic Digestion. - In the course of peptic digestion of albuminoid materials nitrogenous compounds are formed which easily give off ammonia. At the beginning of hydrolysis, in distilling the liquor with $\mathrm{MgO}$, we get only very small quantities of $\mathrm{NH}_{3}$. Then this quantity increases very perceptibly to reach a more or less constant maximum, even before peptonization is completely achieved. The quantity of ammoniacal nitrogen formed depends on the nature of the albuminoid. Gliadin and edestin give the maximum, gelatin, the minimum.

To determine ammoniacal nitrogen, the method of Nencki and Zaleski is used, which consists in distilling the solution to be tested with $\mathrm{MgO}$ in a vacuum. But generally the ammoniacal nitrogen is determined by a simple distillation at ordinary pressure. It is understood that under these conditions the figures obtained are much higher. It is well, on the other hand, to observe that $\mathrm{HCl}, 2$ to $3 \mathrm{~g}$. per liter, eventually causes in

Increase of Amide Nitrogen in the Course of Peptonization.

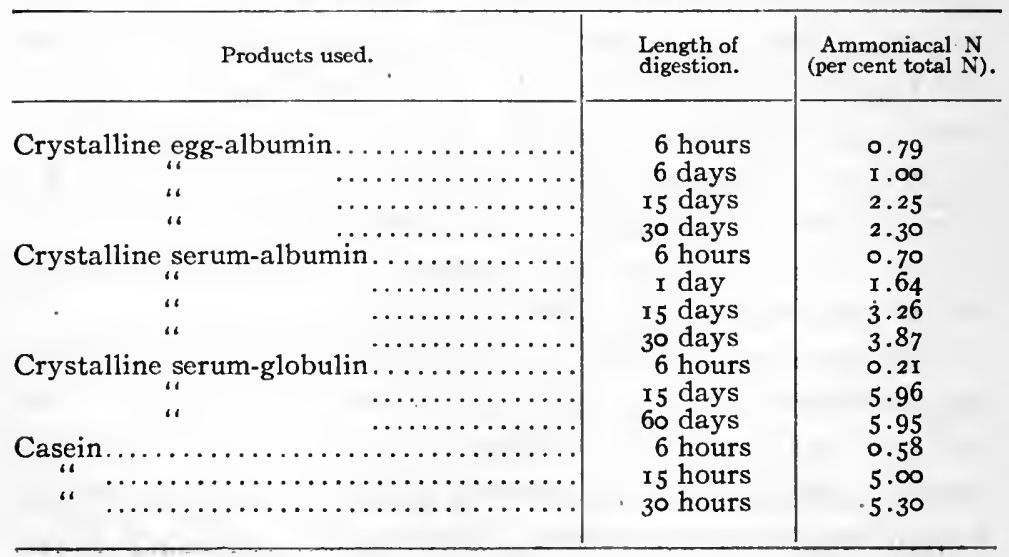


albuminoid substances the formation of amid nitrogen. The quantity of nitrogen thus formed is much smaller than is formed in the presence of pepsin hydrochloride, and very often this fact is not taken into account.

Zunz determined by $\mathrm{MgO}$ the formation of amid nitrogen in the course of different digestions. He worked at ordinary pressure and therefore the data obtained have only a comparative value. They are none the less interesting.

Henriques and Gjaldbaek have made a series of determinations of ammoniacal nitrogen in the products of peptic digestion by distilling the ammonia in a vacuum in the presence of alkalis. Their results show that in very prolonged peptonizations the quantity of $\mathrm{NH}_{3}$ increases considerably, and that in certain cases it even approaches the quantity which is obtained by complete hydrolysis by heating the albuminoid substances 30 minutes at $150^{\circ}$ in the presence of $\mathrm{HCl}(3 \mathrm{~N})$. Moreover, the quantities of $\mathrm{NH}_{3}$ obtained depend on the nature of the albuminoid material used.

Formation of Ammoniacal Nitrogen (Per Cent of Total Nitrogen) in Peptic Digestion.

\begin{tabular}{|c|c|c|c|c|}
\hline $\begin{array}{l}\text { Nature of albuminoid } \\
\text { material. }\end{array}$ & $\begin{array}{l}\text { Length of } \\
\text { peptic } \\
\text { digestion. }\end{array}$ & $\begin{array}{l}\text { Ammon. nitrogen } \\
\text { and formaldehyde } \\
\text { nitrogen. }\end{array}$ & Amm. N. & $\begin{array}{l}\text { Amm. N. } \\
\text { obtained by } \\
\text { complete hydrol- } \\
\text { ysis with } \mathrm{HCl} \text {. }\end{array}$ \\
\hline & days & 20.0 & 3.67 & \\
\hline Egg-albumin...... & $\left\{\begin{array}{r}105 \\
30\end{array}\right.$ & $\begin{array}{l}31.9 \\
24.7\end{array}$ & $\left.\begin{array}{l}5.4 \\
6.1\end{array}\right\}$ & $9 \cdot 5$ \\
\hline Casein.... & $\left\{\begin{array}{r}108 \\
30\end{array}\right.$ & $\begin{array}{l}37.2 \\
23.8\end{array}$ & $\left.\begin{array}{l}9.0 \\
7.2\end{array}\right\}$ & II .9 \\
\hline Edestin. & $\left\{\begin{array}{r}108 \\
6\end{array}\right.$ & $\begin{array}{l}34 \cdot 2 \\
20.4\end{array}$ & $\left.\begin{array}{r}8.7 \\
10.9\end{array}\right\}$ & 14.0 \\
\hline Gliadin. . & $\left\{\begin{array}{l}43 \\
30\end{array}\right.$ & $\begin{array}{l}33 \cdot 3 \\
15.4\end{array}$ & $\left.\begin{array}{r}20.9 \\
1.3\end{array}\right\}$ & 24.8 \\
\hline Gelatin........ & $\{108$ & 29.4 & $1.8\}$ & 3.7 \\
\hline Witte peptone...... & $\begin{array}{rl}1 & 13 \\
112\end{array}$ & $\begin{array}{l}2 \mathrm{I} .0 \\
36.7\end{array}$ & $\begin{array}{l}3.11 \\
6.0\end{array}$ & 8.9 \\
\hline
\end{tabular}

Thus, gliadin gives, by complete hydrolysis with $\mathrm{HCl}, 24.8$ of ammoniacal nitrogen per 100 of total nitrogen, and by complete hydrolysis with pepsin, 20.9. In other words, more than 
80 per cent of nitrogen is already set free by the enzyme. In the case of white of egg, about $5^{6}$ per cent is set free.

To summarize, taking into consideration the various results obtained by the use of precipitating salts, $\left(\mathrm{NH}_{4}\right)_{2} \mathrm{SO}_{4}$ or $\mathrm{ZnSO}_{4}$, of phosphotungstic acid, and by boiling with $\mathrm{MgO}$, it is seen that peptic proteolysis is summed up in the formation of four simplified albuminoid groupings, albumoses, as well as in the appearance of two other series of compounds, some still very complex, which are not precipitable by phosphotungstic acid, and others, the peptones, which correspond to the most advanced stage, and which are precipitated by this latter reagent. As the chemical action goes on, the albumoses, with the exception of a more resistant variety, deutero-albumose $C$, are eliminated and the peptones increase in consequence. An increase of amide nitrogen corresponds to this progressive degradation.

\section{§ I2. ANTI-PEPSIN.}

When blood serum of a horse or dog is added to a solution of pepsin a diminution in the activity of the enzyme is found, a diminution which is in direct proportion to the quantity of serum added. The mechanism of this action, discovered by Schnappauf in 1888 , long escaped exact analysis. If this slackening is attributed to an anti-enzyme, an analogy could be drawn with the similar effect of serum on trypsin where the presence of an anti-enzyme had been established with certainty. To support the idea of the existence of an anti-pepsin, it was, moreover, noted that the anti-peptic power of the serum of an animal increases considerably with the injection of a certain quantity of pepsin. The product synthesized under these conditions by the organism is an anti-pepsin, and it is assumed that this already exists, although in a much smaller proportion, in the serum of the normal animal. This observation, therefore, tended to show that the substance antagonistic to the pepsin is a reaction product of vital defense. The more recent experiments of Cantacuzène appear to justify definitely this opinion. 'This 
experimenter has found in fact, that the serum of a rabbit which has received pepsin by the intravenal path, contains specific anti-bodies capable of fixing the complement; proof that anti-pepsin is indeed the result of a sort of immunization. A later chapter will deal with this phenomenon of deviation of the complement. It is sufficient merely to state here the data of the experiment: He used as antigen, 2 per cent pepsin; as an antibody, prepared rabbit serum; as a complement, fresh serum of a goat; as a hemolytic system, dog corpuscles and goat serum sensitized to dog corpuscles.

To examine the anti-peptic power of the normal dog serum, E. Zunz used the following method: he poured into a reaction tube 2 c.c. of a o.I per cent solution of pepsin (Grübler) acidified with 0.3 per cent $\mathrm{HCl}$; he added 0.5 c.c. of a 0.8 per cent solution of $\mathrm{NaCl}$ and 0.5 c.c. of serum. After mixing, Mett tubes containing coagulated blood serum of a horse or coagulated white of egg were introduced, and the lengths of albumin digested after 24 hours at $38^{\circ}$ were measured.

Presence of Anti-Pepsin in Dog Serum.

\begin{tabular}{|c|c|c|c|c|}
\hline \multirow[t]{2}{*}{. } & & & \multicolumn{2}{|c|}{$\begin{array}{l}\text { Lengths of albumin digested } \\
\text { in } \mathrm{mm} \text {. }\end{array}$} \\
\hline & & & Serum. & White of egg. \\
\hline \multirow{2}{*}{\multicolumn{3}{|c|}{2 c.c. solution $\ldots \ldots \ldots \ldots \ldots \ldots \ldots \ldots \ldots \ldots \ldots$}} & \multirow{2}{*}{$\ddot{20}$} & \multirow{3}{*}{$\ddot{8.0}$} \\
\hline \multirow{2}{*}{\multicolumn{2}{|c|}{$\begin{array}{l}\text { Pepsin hydrochloride +i c.c. } \mathrm{NaCl} \\
\text { to } \\
\text { to.c. } \mathrm{NaCl}+0.5 \text { se- }\end{array}$}} & & & \\
\hline & & $\begin{array}{l}\text { +o.5 c.c. } \mathrm{NaCl}+0.5 \text { se- } \\
\text { rum, fasting }\end{array}$ & \multirow{2}{*}{ II .5} & \\
\hline \multirow[t]{2}{*}{ “ } & \multirow{2}{*}{ “ } & +o. 5 c.c. $\mathrm{NaCl}+0.5$ fast- & & 6.0 \\
\hline & & $\begin{array}{l}\text { ing, serum heated..... } \\
\text { +o. } 5 \text { c.c. } \mathrm{NaCl}+0.5 \mathrm{se}-\end{array}$ & 16.0 & 7.0 \\
\hline " & & $\begin{array}{r}\text { rum, fed } 3 \mathrm{~h} \ldots \ldots \ldots \\
+0.5 \text { c.c. } \mathrm{NaCl}+0.5 \text { se- }\end{array}$ & $9 \cdot 5$ & $5 \cdot 5$ \\
\hline " & “ & $\begin{array}{l}\text { rum, fed } 3 \mathrm{~h} \text {, heated.. } \\
+0.5 \text { c.c. } \mathrm{NaCl}+0.5 \text { se- }\end{array}$ & 15.0 & 7.0 \\
\hline \multirow[t]{2}{*}{ “" } & \multirow[t]{2}{*}{ “" } & $\begin{array}{l}\text { rum, fed } 5 \text { h. } \ldots \ldots \ldots \\
+0.5 \text { c.c. } \mathrm{NaCl}+0.5 \text { se- }\end{array}$ & $10 . \infty 0$ & \\
\hline & & rum fed $5 \mathrm{~h}$ heated & 15.5 & 7.0 \\
\hline
\end{tabular}


The effect of the serum is obvious, but the action is always very slow and very weak. Thus, I g. of Grübler pepsin requires 250 c.c. of serum, and again, with this considerable quantity of serum, the dissolving power of the liquid towards white of egg is reduced only from 8 to 5.5 . In the preceding table we find, furthermore, data on the variation of anti-peptic power of dog serum, according to whether the animal was fasting or had taken raw horse meat. Finally, we see here the effect of temperature on the serum, the term "heated" signifying that the serum, before being added to the pepsin, had been maintained from $\frac{1}{2}$ to I hour at $60^{\circ}-65^{\circ}$. At this temperature, the antipepsin weakens, but does not cause a noticeable coagulation of the albuminoid of the serum. Thus, it is found that heated serum is less effective than natural serum. Here, again, it is found that the effect is far from being very evident. The data lend themselves the less to any conclusion, since under the conditions of the experiment by the addition to a solution of pepsin of 0.5 c.c. of a solution of white of egg or of blood albumin in place of serum, phenomena are observed of just the same nature as are observed with the serum. The digestion of the Mett tubes is unfavorably influenced, and this retarding action diminishes when the solution of albumin is previously heated to $60^{\circ}-65^{\circ}$ for a half an hour. This result is related to that noted earlier (page III), and is, according to Gerber, relative to the anti-coagulating action of various natural albuminoids. In studying papain, it will be seen that the albumin, according as to whether it is fresh or coagulated, is attacked in a very different degree by this enzyme; natural albumin exerting on papain a plainly inhibitory action which does not cease until after a sufficient heating. All these phenomena arise probably from the same cause, and they suggest the reason why living protein substances offer toward enzymes a resistance which dead substances do not possess, and, especially, those which have been destroyed by a definite heating.

V. Oguro, to measure the anti-pepsin in serum, of horse, of rabbit, or of man, uses another method. He depends on data 
furnished by Jacoby and Morgenroth. Instead of mixing the serum directly with the pepsin in an acid medium in the presence of fibrin, he allows the mixture of serum and pepsin to act for a half an hour at the temperature of $37^{\circ}$ without addition of acid. A sort of combination takes place like that which arises between toxins and their antitoxins. 0.4 c.c. of a 0.1 per cent solution of Grübler pepsin are added to o.I to I c.c. of serum. The serum had been previously diluted from $\mathrm{I}$ to $\mathrm{Io}$ with physiological salt solution. The whole is brought to the volume of 2.4 c.c. by the addition of a 0.85 per cent solution of $\mathrm{NaCl}$ and the tubes are left for half an hour at $37^{\circ}$. Then into each of them is introduced some fragments of fibrin colored with carmine, 0.5 c.c. of $\mathrm{N} /$ io $\mathrm{HCl}$ are added, and it is replaced in the incubator.

Anti-Peptic Power of Horse Serum.

\begin{tabular}{|c|c|c|c|c|c|c|}
\hline $\begin{array}{l}\text { Horse } \\
\text { serum } \\
\text { at } \text { 16. }^{2} \text {. }\end{array}$ & $\begin{array}{c}\text { Physiologi- } \\
\text { cal salt } \\
\text { solution. }\end{array}$ & $\begin{array}{c}\mathrm{HCl} \\
N / 10 .\end{array}$ & After $30 \mathrm{~min}$. & After I hour. & After 2 hours. & After 4 hours. \\
\hline c.c. & c.c. & c.c. & & & & \\
\hline$\circ$ & 2 & 0.5 & $\begin{array}{l}\text { Plainly } \\
\text { red }\end{array}$ & $\left.\begin{array}{c}\text { Fibrin } \\
\text { entirely } \\
\text { digested }\end{array}\right\}$ & $\begin{array}{c}\text { Fibrin } \\
\text { digested }\end{array}$ & $\begin{array}{c}\text { Fibrin } \\
\text { digested }\end{array}$ \\
\hline 0.1 & 1.9 & 0.5 & No color & $\begin{array}{l}\text { Slightly } \\
\text { red }\end{array}$ & $\begin{array}{l}\text { Deeper } \\
\text { red }\end{array}$ & $\begin{array}{c}\text { Plainly } \\
\text { red }\end{array}$ \\
\hline 0.2 & I. 8 & 0.5 & “" & No color & " & \\
\hline 0.3 & I. 7 & 0.5 & $"$ " & " & No cólor & " \\
\hline 0.4 & I. 6 & 0.5 & “" & “" & " & A little red \\
\hline 0.5 & 1.5 & 0.5 & “" & “" & ““ & No color \\
\hline 0.6 & I. 4 & 0.5 & “" & “" & “ & “. \\
\hline $\begin{array}{l}0.7 \\
0.8\end{array}$ & $\begin{array}{l}1.3 \\
1.2\end{array}$ & $\begin{array}{l}0.5 \\
0.5\end{array}$ & “" & “" & “ & “، \\
\hline 0.9 & I.I & 0.5 & “ & “. & “" & “، \\
\hline I & I & 0.5 & “ & “" & “" & “ \\
\hline
\end{tabular}

From these data, the serum of the horse possesses an antipeptic power much greater than that found by Zunz for dog serum.

Oguro has experimented in the same way on serums of rabbit and of man, and he has found that to weaken or arrest the action of $\mathrm{I} \mathrm{g}$. of Grübler pepsin, the following quantities of serum are necessary: 


\begin{tabular}{|c|c|c|}
\hline & $\begin{array}{l}\text { Weakening } \\
\text { quantities. }\end{array}$ & $\begin{array}{l}\text { Arresting } \\
\text { quantities. }\end{array}$ \\
\hline & c.c. & c.c. \\
\hline Horse serum. . & 25 & 125 \\
\hline Rabbit serum. & 25 & 200 \\
\hline Man serum $\ldots \ldots \ldots \ldots$ & 50 & 150 \\
\hline
\end{tabular}

In place of fibrin, in the method of Oguro, it is also possible to use, as material to be digested, ricin, which gives even more exact results. Then, in different comparative tests are used 0.4 c.c. of 0.1 per cent pepsin, $O$ to I c.c. of serum at $1 \frac{1}{10}$, r.o to $\circ$ c.c. of physiological salt solution, 0.5 c.c. $N /$ ro $\mathrm{HCl}$, and 2 c.c. of solution of ricin at $5 \frac{1}{\delta} \sigma$. The following are the results found with horse serum:

\begin{tabular}{|c|c|c|c|c|}
\hline Serum. & After 30 minutes. & After I hour. & After 2 hours. & After 4 hours. \\
\hline $\begin{array}{l}\text { c.c. } \\
.0\end{array}$ & & Clear & Clear & Clear \\
\hline 0.1 & $"$ & & & "1 \\
\hline 0.2 & Almost clear & “ & " & “ \\
\hline 0.3 & Trace of turbidity & Almost clear & " & “ \\
\hline 0.4 & Pronounced turbidity & Turbidity & Slight turbidity & “ \\
\hline 0.5 & " & & Turbidity & “ \\
\hline 0.6 & “ & “" & “ & Turbidity \\
\hline 0.7 & “ & “" & “" & " \\
\hline 0.8 & " & " & “” & “ \\
\hline 0.9 & " & “ & “" & “” \\
\hline 1.0 & “ & “" & “" & “ \\
\hline
\end{tabular}

The method used by Oguro is very rapid and, if there should be occasion, is capable of rendering service in the diagnosis of certain maladies. However, it appears that in many pathological cases, the quantities of anti-pepsin found in the serum are almost the same as the normal quantity, at least according to the researches of Rubinstein. This investigator determined the anti-peptic power of healthy man and found figures between 6 and 7 ; that is, 0.6 to 0.7 c.c. of healthy serum diluted $\frac{1}{10}$ neutralizes the action of 0.4 c.c. of Grübler pepsin at roto acting at $37^{\circ}$ in carmine-fibrin with the addition of a little $N /$ Io 
HCl. Now, this anti-peptic index, with a great number of sick persons, of very different kinds, varies very little; much less, as shall be seen later, than the anti-tryptic power.

\section{§ 13. The Determination of Pepsin.}

In a general way, the activity of an enzyme can be measured by the quantity of substance which it is capable of transforming in a given time. This determination is particularly simple when the substrate is simple and the products formed are well characterized and easily measured, as is the case for sucrase; or, again, when the transformation is observed by an external change, as that produced with rennet acting on milk under the usual conditions.

But here the problem is infinitely more complex. First of all, pepsin is capable of transforming not one or two, but a great number of substances whose properties are far from being identical; some are soluble, others insoluble in water; certain ones can be used, either in the natural state, or after coagulation, etc. Furthermore, the chemical action of pepsin is much less obvious than that of the other enzymes. The products which arise are numerous and but little known. If the substrate is insoluble in water, the pepsin will first make it soluble, then digest it. But these two kinds of phenomena are not easy to estimate in an accurate manner. Digestion represents the passage through a whole series of compounds more or less well defined. At what point is the final stage reached, and when, unless arbitrarily, can we consider digestion ended? Solution is doubtless susceptible of being better estimated, but it is not always very easy, and at times it is quite difficult to determine whether complete solution is really reached or is not indeed passed.

The chemical transformations due to pepsin are accompanied by changes in the physical properties of the substrate. We have just spoken of the dissolving of nitrogenous substances. There is also a variation in the rotatory power of the liquid, in its viscosity, in its greater or less aptitude to furnish a persistent 
foam with shaking, and in its electrical conductivity. All these modifications of properties are the result of the same effect, the peptonizing action of the enzyme. However, these effects are not necessarily equivalent. If, by one method, it has been found that the enzyme value of a sample is ten times as great as that of a second sample, it is not at all certain that the same relation would be found using a different method of analysis, the fermenting activity determined according to the dissolving power being often very different from that estimated from the peptonizing power.

From the preceding it appears that the number of methods available for the determination of the enzymic power of a pepsin must be very great. We may, in fact, choose as substrates, fresh or coagulated egg-albumin, moist or dry fibrin, serumalbumin, casein, edestin, ricin, gelatin, and as means of estimating the enzymic value, the degree of solution, or liquefaction; peptonization (disappearance of albumino-acids or albumoses); or variation in rotatory power. It can be stated that no single method has so far been universally accepted. It would, however, be desirable to have experimenters adopt a standardization which would do away with misunderstandings and permit an easy comparison of various results found.

Method of Grützner. - Fibrin, reduced to threads as fine as possible, is dipped for several seconds in an ammoniacal solution of carmine, is then dried and is rinsed in pure water. The product, colored red, is dissolved in a solution of pepsin hydrochloride, the more rapidly the richer the liquid is in enzyme. Therefore, a comparative colorimetric determination is made of the quantity of coloring matter dissolved in a test liquid and in a titrated solution of pepsin, at the end of a given time. The ratio of the shades gives the ratio of the enzymic activities.

Method of Effront. - This method permits the estimation in a quick and accurate manner of the dissolving power of a pepsin. First of all, an albumin emulsion is prepared by dissolving $80 \mathrm{~g}$. of dried commercial white of egg in two liters of water. This solution is exactly neutralized, 20 c.c. of $\mathrm{HCl}(N)$ 
are added, and the liquid is kept at $80^{\circ}$ for to minutes while shaking vigorously. The spongy precipitate of albumin which is formed is collected on a filter, washed several times with distilled water, and is then strongly pressed. The product issuing from the press still retains 80 per cent of water; $50 \mathrm{~g}$. of it are diluted in 250 c.c. of 0.2 per cent $\mathrm{HCl}$, in such a way as to give $4 \mathrm{~g}$. of dry albumin for $100 \mathrm{~g}$. of liquid. To complete the separation of the albumin, the liquid is made to pass a dozen times through a silk sieve.

The estimation of the dissolving power is made with this emulsion in the following manner: In a test tube of $18 \mathrm{~mm}$. diameter, place ro c.c. of the emulsion liquid. Add ro c.c. of water and place it in a vapor-bath at $50^{\circ}$ until it assumes the temperature of the bath. After several minutes, pour into the tube $\frac{1}{2}$ c.c. of a 0.5 per cent solution of pepsin. From time to time vigorously shake the liquid and wait until it becomes completely transparent. The end-point is best determined by attempting to decipher through the tube a small letter written in ink on white paper.

The passage of the liquid from the opaque state to that of transparency is very evident, and it is possible to measure with accuracy the number of minutes which elapse from the moment when pepsin is added to the tube, to the clarification of the liquid. With a pepsin of good quality the white liquid becomes transparent in 20 minutes; the dissolving value is, in this case, expressed by 100 and this datum can be used as a basis for comparison. The value of a pepsin which, under the conditions described above, renders the liquid transparent in 40 or 60 minutes, for example, could be expressed by the figures 50 or 33 respectively.

This method is subject to modification. First of all, dry commercial albumin can be replaced by white of egg. Eggs are kept ro minutes in boiling water after which the white, separated from the yolk, is passed through sieve No. I (Io meshes per $\mathrm{cm}$.). Then an emulsion is made, using ro $\mathrm{g}$. of this very finely divided substance per roo c.c. of ( 0.25 per cent) $\mathrm{HCl}$. The 
mixture is heated to $50^{\circ}$, the pepsin to be measured is added, and the whole is kept at this temperature. The time required for complete solution is determined as before. It is also possible, after a certain duration of action and long after the last portions of coagulated albumin have disappeared, to test the liquid by neutralizing it carefully and seeing how much time is consumed before no further precipitation of albumino-acid is obtained. In this case, the comparison of the enzymic activities is made according to the peptonizing power. Another means of conveniently estimating the end of digestion consists in treating the sample with a little nitric acid. For example, to ro c.c. of the digestion liquor are added $\mathrm{I}_{5}$ drops of pure $\mathrm{HNO}_{3}$. The proteolysis is considered ended when this addition no longer produces a precipitation of hetero-albumose.

A rather convenient modification in the case of a series of determinations is the following. Prepare a certain quantity of a 2 per cent solution of dry commercial albumin in 0.25 per cent HCl. Pass it through a fine cloth to eliminate the undissolved particles and separate it, in Ioo c.c. portions, into different flasks. Bring to $35^{\circ}-40^{\circ}$ and add the peptic liquids to be studied. For each run, a corresponding control is obtained by the addition of the same volume of enzyme; the mixture being boiled. To each of the flasks add I c.c. of chloroform, seal hermetically, and leave in the thermostat for 24 hours. Then, neutralize the liquids, add a little acetic acid, and coagulate by leaving the flasks $\frac{3}{4}$ of an hour in an autoclave at $100^{\circ}$. After cooling, dilute to a volume of 125 c.c., filter, and determine the nitrogen in the clear liquid.

Method of Mett Tubes. - This is the one most commonly used and is particularly suitable for very dilute enzyme solutions. Draw into glass tubes of $\mathrm{I}$ to $2 \mathrm{~mm}$. diameter, albumin taken from fresh eggs and corresponding to the less vesicular part of the liquid, or else blood serum; and coagulate the whole at a temperature of $95^{\circ}$. Then cut these tubes in small fragments of ro to I $5 \mathrm{~mm}$. in length and insert two of them, in order to take an average, into $\mathrm{I}$ or 2 c.c. of various enzyme liquids to be examined. 
The whole is placed in the thermostat at $37^{\circ}-38^{\circ}$ for ro hours. Then, measure the lengths of albumin dissolved at the two extremities of each of the tubes. The sum, expressed in millimeters and fractions of a millimeter, represents the length of the cylinder of albumin dissolved in each test. Experience has shown that, all conditions being equal, the quantities of pepsin in various juices are relatively as the squares of millimeters of albumin which have been digested. For the determination to be valid, it is important that the length of albumin dissolved at the two extremities of the tube shall not be too large. In practice the conditions are favorable when, in $15 \mathrm{~mm}$. of tube, only $5 \mathrm{~mm}$., as a maximum, are dissolved on each side.

Method of Fuld with Edestin. - Edestin, taken from hemp, can serve as a substrate for the rapid determination of the peptonizing power of pepsin. The following procedure is recommended. Preparation of solutions:

$A$. Take 90 c.c. $N /$ Io $\mathrm{HCl}$ and make up to 300 c.c. by adding physiological salt solution or simply water.

$B$. Dissolve $0.2 \mathrm{~g}$. of edestin in 200 c.c. solution $(A)$; dilute well in a little mortar, and when solution is complete, filter to get a perfectly clear liquid.

C. Dissolve $0.5 \mathrm{~g}$. of typical pepsin in 50 c.c. of physiological salt solution. When all is dissolved, filter. The typesolution of I per cent is successively diluted first, to 0.5 per cent by adding an equal volume of a solution containing 60 c.c. $N /$ ro $\mathrm{HCl}$ per IOO c.c., and, then, to 0.05 per cent with a solution $\mathrm{HCl}(A)$.

$D$. Dissolve Io g. of common salt in distilled water and dilute to roo c.c. Filter.

Procedure. - Prepare to tubes of $\mathrm{I} \mathrm{cm}$. diameter, each containing 2 c.c. solution $(B), 0.9,0.8,0.7, \ldots \circ$ c.c. solution $(A)$, and $0.1,0.2,0.3, \ldots$ I c.c. 0.5 per cent or 0.05 per cent enzyme solution. After 30 minutes at ordinary temperature, add to each tube I c.c. solution $(D)$, and note the tube where precipitation no longer takes place.

Example. - Taking a 0.5 per cent solution of typical pepsin, 
there is no precipitation in any of the tubes. By taking a 0.05 per cent solution of pepsin the following results are obtained:

$\begin{array}{lllllllllllll}\text { Pepsin solution, c.c........ } & 0.1 & 0.2 & 0.3 & 0.4 & 0.5 & 0.6 & 0.7 & 0.8 & 0.9 & \text { I }\end{array}$

Precipitation.......... ++++++--

Precipitation ceases to appear with 0.7 c.c. of the 0.05 per cent solution of pepsin.

Calculation. - roo c.c. of 0.05 per cent peptic solution $=0.05$ g. type-pepsin; consequently, 0.7 c.c. of 0.05 per cent peptic solution contains $0.00035 \mathrm{~g}$. type-pepsin. Then, $0.00035 \mathrm{~g}$. pepsin $=2$ c.c. solution $(B)=2 \mathrm{mg}$. edestin, whence $\mathrm{I}$ g. pepsin $=$ $\frac{2}{0.00035}=57 \mathrm{I} 4$ c.c. $=5.7 \mathrm{I} 4 \mathrm{~g}$. edestin. The value of this pepsin, which is taken as a reference, is, therefore, $57^{14}$ units.

Standardization of any solution. - If, for example, it takes 0.8 c.c. of a microbic culture containing pepsin, to prevent precipitation under conditions as described, then 0.8 c.c. culture contains 0.00035 g. type-pepsin, of which I g. of pepsin is contained in 2285 c.c. of culture examined.

Remarks. - I. Dilutions of type-pepsin can be made without practical error in pure water, in place of $\mathrm{HCl}$. Likewise, water can be used to bring the volume of enzyme solution to I c.c.

2. It is necessary to take as tube limit the point where there is no longer any turbidity upon the addition of $\mathrm{NaCl}$. A strong illumination, that from an incandescent lamp, for example, is better than natural daylight for determining the disappearance of turbidity.

3. A fresh solution of edestin is essential. Further, the liquors used must be perfectly clear.

4. It is essential always to precipitate the undigested edestin by constant quantities of $\mathrm{NaCl}$, for example, for experimental conditions indicated, I c.c. of Io per cent $\mathrm{NaCl}$. In fact, greater or less quantities of $\mathrm{NaCl}$ would cause a variation in the limit of non-turbidity.

5. It sometimes happens that the liquid containing the 
pepsin to be measured precipitates of itself the solution of edestin. This is, for example, the case with microbic cultures made on milk and containing little pepsin. The precipitation is due, then, to the inorganic substances contained in the milk, and their coagulating action is felt all the more, the more of the culture is used. In this phenomenon, no enzymic influence intervenes for the precipitation is produced as well with boiled cultures as with cultures not boiled. Naturally, the procedure just described for measuring pepsin does not succeed with such liquids. In fact, the mixture of edestin and of culture to be examined being already turbid, it becomes impossible to see if an addition of $\mathrm{NaCl}$ produces a new precipitate.

According to a verbal communication of Dr. Wallerstein (of New York) this difficulty is often avoided by adding to the solution of edestin a small quantity of sodium citrate. On the other hand, both the sensitiveness and the accuracy of the determination can be increased by the following modification of the method of Fuld. Dissolve 0.I g. of edestin in roo c.c. of $\mathrm{N} /$ ro $\mathrm{HCl}$. Take 2 c.c. of this solution, add 5 c.c. of liquid to be analyzed, and leave in the steam bath at $50^{\circ}$ for 3 hours if determining pepsin, for 24 hours if determining papain. Under these conditions the presence of a quantity of enzyme equal to I or 2 hundred thousandths, or I $g$. of pepsin in roo liters of water can be detected with certainty, the reaction having become about 50 times more sensitive.

Method of Jacoby. - This method is based on the fact that ricin, in dilute $\mathrm{NaCl}$ solution, gives a turbid liquid which becomes transparent upon the addition of a little pepsin, this enzyme exerting its digestive action on the slightly soluble albuminoid compounds (ricino-globulin), which accompany the toxin, without any direct action on the latter.

For the analysis, dissolve I g. of ricin (Merck) in 100 c.c. of water with addition of $1.5 \mathrm{~g}$. of $\mathrm{NaCl}$. Then, add to the liquid a definite quantity of $\mathrm{HCl}$, and, then, the pepsin. The solution becomes very rapidly transparent when there is sufficient pepsin present. In the following tests to 3 c.c. of emulsion 
of ricin $+_{\mathrm{I}}$ c.c. 0.56 per cent $\mathrm{HCl}+$ I c.c. $\mathrm{H}_{2} \mathrm{O}$ were added the quantities of pepsin indicated below:

Effect of Different Quantities of Pepsin on an Emulsion of Ricin.

\begin{tabular}{|c|c|}
\hline Pepsin used. & Clearing up aiter. \\
\hline $\begin{array}{l}\text { mg. } \\
\text { I.0 }\end{array}$ & At once \\
\hline 0.10 & 15 minutes \\
\hline 0.05 & 30 minutes \\
\hline $0.0 \mathrm{r}$ & 4 to 6 hours in the thermostat \\
\hline $0 . \infty 1$ & 36 to 48 hours $\}^{\text {in the thermostat }}$ \\
\hline 0.000 & $\begin{array}{l}\text { Still turbid after long stay in } \\
\text { the thermostat }\end{array}$ \\
\hline
\end{tabular}

This.method is extremely delicate and affords the great advantage of being very quick. It is especially recommended in the case of comparative tests.

We shall have occasion to return to these questions of analysis, in the chapter on Commercial Pepsin, with reference to the determination of the official standard of a pepsin.

\section{$\S$ I4. Gastric Juice.}

Secretion of Gastric Juice. - Gastric juice has lately become a pharmaceutical product. It is easily extracted from dogs which have undergone the operation of œsophagotomy by a technique invented by Pawlow and Mme. SchoumoffSimanowski. The dogs, operated in this manner, are provided with a stomach fistula and are nourished by direct introduction of food into the stomach. They are generally in good health; they increase in weight, have an excellent appetite, and digest properly.

The juice is obtained by fictitious feeding. The animal, kept upright by aid of a special apparatus, receives meat, which it chews with appetite. This nourishment, because of the opening of the œsophagus, does not go to the stomach, but is cast out. Nevertheless, from the beginning of the meal, and even before the dog has touched the meat, the gastric juice begins to flow through the fistula. Naturally, the secretion becomes 
more abundant after some period of mastication. These fictitious meals last from $I$ to $I \frac{1}{2}$ hours. The liquid is collected in a vessel and is then filtered. Konovaloff has the following data on the progress of the secretion in the course of a fictitious meal:

Progress of the Gastric Secretion in the Course of a Fictitious Meal.

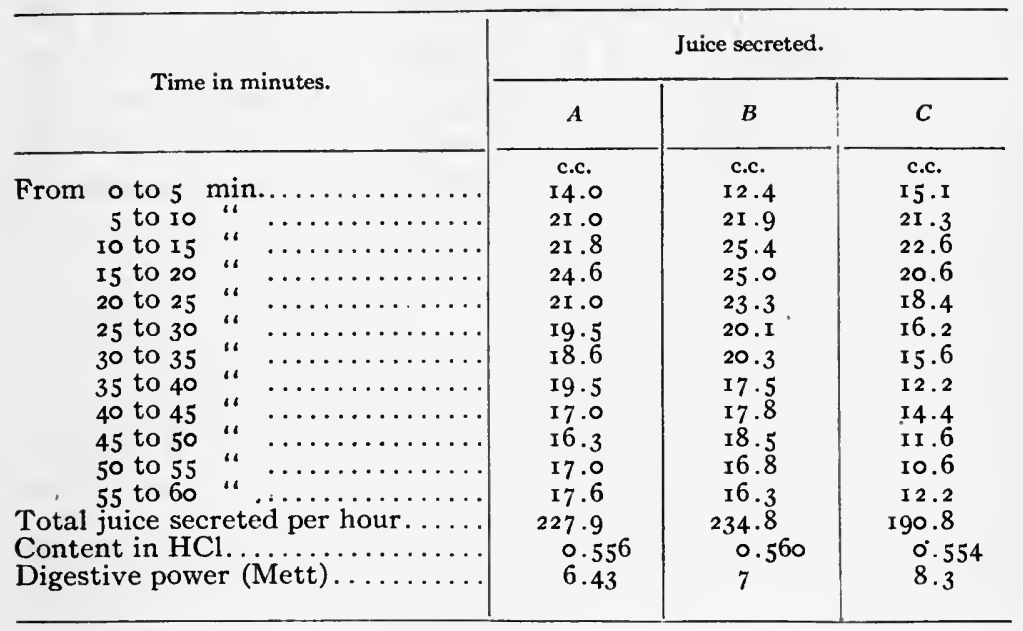

This table gives the volumes of juice collected every 5 minutes. Column A represents the average for a whole series of experiments made on a dog whose juice was taken every three or four days. Column B corresponds to the average of yields from. a dog every 2 days for $\mathrm{I} 6$ days; finally, column $\mathrm{C}$ refers to a dog having a daily fictitious meal for 9 days.

In all these tests, the density of the juice changes little, from 1.00430 to 1.00524 . The acidity varies very little; for a series of experiments lasting 45 days, the average acidity was 0.563 , with a minimum of 0.380 and a maximum of 0.576 . The digestive power varied more, with a minimum of 6 and a maximum of 9. A dog weighing $45 \mathrm{~kg}$. can furnish daily 200 to 300 c.c. of juice. This removal, during the fictitious meal, is not at all injurious to the animal. On the contrary the juice itself loses none of its quality. We have here a sort of excitation which causes an ever stronger secretion, since the diges- 
tive power, which, at the beginning in the series $\mathrm{A}$, was 6.43 , ascended at the end in the series $\mathrm{C}$, to 8.3.

Properties of Gastric Juice. - Gastric juice, as a consequence of its $\mathrm{HCl}$ content, does not putrefy. It keeps for quite a long time, and even the pepsin which it contains deteriorates only very slowly. After being kept for months juices are still very active. Gastric juice of a dog is a colorless liquid, slightly opalescent, and possessing an agreeable taste. It possesses a slight rotatory power to the left; its freezing point is $\Delta=-0.490^{\circ}$ to $-0.638^{\circ}$. The analysis of gastric juice has, according to Rosemann, given the following results:

\begin{tabular}{|c|c|}
\hline & 0.4277 per 100 c.c. \\
\hline Ash........... & 0.1325 \\
\hline Organic matter. & O. 2944 \\
\hline Nitrogen.......... & 0.035 to 0.054 \\
\hline Hydrochloric acid & $0.55^{60}$ to 0.5657 \\
\hline Ammonia......... & 0.0077 \\
\hline Proteins......... & 0.247 to 0.375 \\
\hline
\end{tabular}

The liquid does not contain lactic acid. In the ash are found both $\mathrm{NaCl}$ and $\mathrm{KCl}$, with a predominance of $\mathrm{KCl}$. The relation of the potash to the soda is, in fact, according to Rosemann, I to $0.8 \mathrm{r}$. Small amounts of $\mathrm{Ca}, \mathrm{Mg}$, and $\mathrm{Fe}$ are also present.

With reference to the hydrochloric acid variation of gastric juice under the influence of diet, Dastre and Frouin have shown that the addition of salt to foods renders the subject hyperhydrochloric. On the other hand, Frouin and P. Girard have found that pure gastric juice always contains practically the same quantity of total chloride; if, then, the quantity of free acid begins to increase, that of the fixed chlorides diminishes proportionately. The following are results obtained with a dog having the small stomach isolated (technique of Pawlow, to be described later). This animal normally received every 24 hours $200 \mathrm{~g}$. of rice $+700 \mathrm{~g}$. of horse meat cooked in water without salt + Io $\mathrm{g}$. $\mathrm{NaCl}$. The first day of the experiment the salt was suppressed, the 6 th and 7 th, $5 \mathrm{~g}$. $\mathrm{NaCl}$ were put in his mess. 
Influence of $\mathrm{NaCl}$ on Gastric Secretion.

\begin{tabular}{|c|c|c|c|c|c|}
\hline \multicolumn{3}{|c|}{ Nourishment. } & $\begin{array}{l}\text { Quantity of } \\
\text { juice } \\
\text { secreted in } \\
24 \text { hours }\end{array}$ & $\begin{array}{l}\text { Acidity, } \\
\text { total HCl } \\
\text { per liter }\end{array}$ & $\begin{array}{c}\text { Chloride } \\
\text { expressed } \\
\text { in HCl per } \\
\text { liter }\end{array}$ \\
\hline $\begin{array}{l}\text { Ist day: rice + meat, wi } \\
\text { 2nd } \\
\text { 3rd } \\
4 \text { th } \\
5 \text { th } \\
6 \text { th: addition of } 5 \mathrm{~g} . \mathrm{Na} \\
\text { 7th } \\
\text { I Ith suppression of } \mathrm{NaCl}\end{array}$ & $\begin{array}{l}\text { hout sal } \\
1 \ldots \ldots \\
\ldots \ldots \\
\ldots \ldots\end{array}$ & 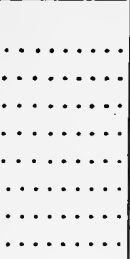 & $\begin{array}{r}\text { c.c. } \\
350 \\
275 \\
115 \\
\text { I } 13 \\
96 \\
185 \\
190 \\
90\end{array}$ & $\begin{array}{l}\text { g. } \\
2.8 \mathrm{r} \\
3.32 \\
3.28 \\
1.97 \\
1.38 \\
3.39 \\
3.06 \\
1.20\end{array}$ & $\begin{array}{l}\text { g. } \\
5 \cdot 55 \\
5 \cdot 57 \\
5.67 \\
5 \cdot 57 \\
5.84 \\
5.98 \\
5.39 \\
5.90\end{array}$ \\
\hline Nature of juice. & $\begin{array}{l}\text { Quantity } \\
\text { of juice } \\
\text { secreted }\end{array}$ & $\mathrm{K}$ per liter & $\begin{array}{l}\text { K secreted } \\
\text { in } \mathbf{2 4} \mathbf{~ h .}\end{array}$ & Na per liter & $\begin{array}{l}\text { Na secreted } \\
\text { in } 24 \mathrm{~h} \text {. }\end{array}$ \\
\hline 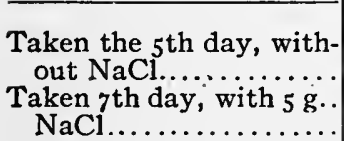 & $\begin{array}{l}\text { c.c. } \\
95 \\
\text { I9o }\end{array}$ & $\begin{array}{c}g . \\
0.150 \\
0.220\end{array}$ & 0.014 & $\begin{array}{l}2.2 \mathrm{I} \\
0.960\end{array}$ & $\begin{array}{c}g . \\
0.212 \\
0.082\end{array}$ \\
\hline
\end{tabular}

We see that, while the acidity varies from 1.20 to 3.39 per thousand, the total chloride hardly varies at all. The writers previously quoted have likewise determined the potassium and the sodium in the juices collected the $5^{\text {th }}$ and 7 th days. They found that the ingestion of $\mathrm{NaCl}$ does not result in increasing the content in sodium. On the contrary, in such a case, as will be seen later, the hydrochloric acid of the salt remains in the gastric juice, while the sodium hydroxide goes to increase the alkalinity of the pancreatic juice. Moreover these results are practically the same when the animal receives $\mathrm{NaCl}, \mathrm{KCl}$, or $\mathrm{BaCl}_{2}$. According to these same investigators, however, the sodium is always the most abundant of the metallic constituents of gastric juice.

Sommerfeld was able to repeat the tests of Konovaloff on a young girl. She, operated upon and furnished with a fistula, reacted each time that she was shown food, secreting gastric juice, and this secretion was the more abundant the more 
palatable the dish to the patient. The effect of chewing food, without its penetrating into the stomach, activated the secretion during the whole fictitious repast which lasted 30 to 45 minutes. The quantity of juice secreted during the fictitious meal was from 20 c.c. to I ro c.c. as a maximum. The acidity of the gastric juice of this young girl was from 0.29 to 0.499 per cent (phenolphthalein) and from 0.29 to 0.5 (Congo paper), or an average of 0.4026 . In one case, Sommerfeld found that in the absence of real appetite, the secretion was much less; then he obtained only 20 c.c., instead of I IO c.c., and the juice with Congo paper showed an acidity of only 0.173 per cent.

Effect of Temperature. - The optimum temperature for the action of gastric juice is not found in the limits of the normal temperature of the body. The most rapid action shows itself at between 50 and $55^{\circ}$; but the optimum temperature is found, as already seen in the study of pepsin alone, in the neighborhood of the destructive temperature. Roeder uses the method of Fuld with edestin to study the effect of temperature on the variation of the digestive power of the gastric juice. In the second column are given the number of c.c. of gastric juice diluted from I to Io which transform in 30 minutes. 2 c.c. of a I per cent solution of edestin renders it non-precipitable by a solution of $\mathrm{NaCl}$. In the third column is recorded the calculated digestive power, that is to say, the number of c.c. of edestin which can transform I c.c. of pure gastric juice.

Variation of Digestive Power of Gastric Jutce With TEMPERATURE.

\begin{tabular}{|c|c|c|}
\hline Temperature. & $\begin{array}{c}\text { Gastric juice di- } \\
\text { luted to if. }\end{array}$ & $\begin{array}{c}\text { Calculated diges- } \\
\text { tive power. }\end{array}$ \\
\hline $30^{\circ}$ & c.c. & 35.7 \\
34 & 0.56 & 47.6 \\
37 & 0.42 & 83.5 \\
40 & 0.24 & 111.3 \\
45 & 0.18 & 154.0 \\
50 & 0.13 & 200.0 \\
55 & 0.10 & 200.0 \\
\hline
\end{tabular}


Thus, the dissolving power of gastric juice increases with the temperature, and at $55^{\circ}$ there is produced almost six times the effect produced at $30^{\circ}$. However, the results found are due to the superposition of two opposed influences. Under the influence of temperature, the enzyme also weakens tremendously; a considerable part of the active substance is destroyed, and it is only that which remains which gives the supplementary effect. It is possible to show these two factors by keeping gastric juice alone at different temperatures for 30 minutes and then putting it in contact with edestin, on which it is made to act at a uniform temperature of $35^{\circ}$ :

\begin{tabular}{|l|c|c|}
\hline $\begin{array}{c}\text { Temperature of } \\
\text { the previous } \\
\text { heating. }\end{array}$ & Gastric juice at $\begin{array}{c}\text { s. } \\
\text { x. }\end{array}$ & Digestive power. \\
\hline $37^{\circ}$ (control) & c.c. & III.0 \\
40 & 0.18 & 62.5 \\
42 & 0.32 & 47.6 \\
43 & 0.42 & 29.9 \\
45 & 0.67 & 18.2 \\
50 & 1.10 & 14.9 \\
55 & 1.34 & 10.0 \\
\hline
\end{tabular}

In the control test, where the juice has been kept only 30 minutes at $37^{\circ}$, it takes o.I8 c.c. of juice diluted to $\frac{1}{10}$ to digest 2 c.c. of edestin solution. The mere tact of having brought the juice to $40^{\circ}$ for 30 minutes lowers its digestive power from III to 62.5 , or almost by a half. If, finally, it is kept 30 minutes at $55^{\circ}$, it is rendered eleven times less active. By comparing these figures with those of the preceding table, it is seen that the optimum and restructive temperatures are almost identical. In the chapter on the secretorial glands we shall have occasion to consider more closely the properties of gastric juice. 


\section{BIBLIOGRAPHY ON PEPSIN.}

\section{HISTORICAL - PREPARATION - CHEMICAL COMPOSITION}

Spallanzani. Expériences sur la digestion, 1784.

RÉanur. Voir Dictionn. de Physiologie, Richet, t. IV.

Tiediann et GMelen. Recherches experim. phys. et chim. sur la digestion, 1827.

Eberle. Physiol. der Verdauung, Würzburg, 1834.

Blondolt. Traitê anatom. de la digestion, p. 371 .

Сн. Sснмidt. Verdaunngsäfte, Leipzig, 1852.

Ebstein et Grützner. Ueber Pepsinbildung im Magen, Pfiig. Arch., (8), p. 127 .

Th. Schwans. Ueber das Wessen der Verdauungsprocessus, Mïller's Arch. $f$. An. Phys. u. Wissens. Medic., 1836, p. 90.

Wasmann. Die Digestion, Berlin, 1879.

Brucke. Beitrage zur Lehre von der Verdauung, Akad. d. Wiss. Wien, r862; Vorlesungen üb. Physiol., r874, (r), p. 294.

voN WrTtich. Ueber eine neue Methode zur Darstellung künstlicher Verdauungsflüssigkeiten, Pflüg. Archiv., (2), p. I93.

C. A. Pekelharing. Mitteilungen über Pepsin, Zeits. f. phys. Chem., (35), p. 8.

Nencki U. Sieber. Beitrage zur Kenntnis des Magnesäftes u. der chem. Zusammensetzung der Enzyme, Zeits. f. physiol. Chem., (32), p. 29 I, 1901.

Petrt. Recherches sur la Pepsine, Paris, I88r.

Friedenthal v. Miyamota. Natur des Pepsins und anderer Verdauungsenzyme, Centralbl.f. Physiol., (16), p. I, I902.

Hugouneng et Morel. Constitution de la Pepsine, $C$. $R$., I908, (2), p. 2 r 2.

Trampedach. Milz und Magenverdauung $u$. der angebliche Pepsingehalt der Milz. Pflïg. Arch.d. Physiol., (14I), p. 59r, I9II.

Wilenko. Zur Kenntnis der Pepsinausscheidung im Harn, Berl. Klin. Woch., (22), p. 1060, r908.

BENFEy. Ueber eiweissspaltende Enzyme im Säuglingsharn, Bioch. Zeits., (10), p. $45^{8}, \mathrm{r} 908$.

\section{EFFECT OF TEMPERATURE.}

Efrront. Sur le dosage de l'albumose, Bull. Soc. chim. France, r899, (2r), p. 680.

J. Oguro. Bioch. Zeits., (22), p. 278, 1909.

H. RøDER. Wirkung thermischer Einflüsse, Bioch. Zeits., (24), p. 499, r9ro.

A. Mayer. Einige Bedingungen der Pepsinwirkung, Zeits.f. Biol., (17), p. 351, I88r.

KLug. Untersuchungen über Pepsinverdauung, Pfiug. Arch., (60), p. 43, r895.

BIERNACKI. Das Verhalten der Verdauungsenzyme bei Temperaturerhöhungen, Zeits. f. Biol., r89r, (28), p. 49.

Kulpsohn. Diss., St-Pétersbourg, 1908.

Finkier. Pflïg. Arch., (I4), p. I 28.

GramenitzKi. Der Einfluss verschiedener Temperaturen auf die Fermenten u. die Regeneration fermentativer Eigenschaften, Zeits. f. physiol. Chem., (69), p. 286, 1910. 
Oнта. Zur Trage der Hitzebeständigkeit von Pepsin u. Trypsin, Bioch. Zeits., (44), p. 472, 1912.

\section{EFFECT OF OTHER PHYSICAL CONDITIONS.}

Signe u. S. SchMidt-Nieisen. Zur Kentniss der Schütteln ü. Aktivität, Zeits. f. physiol. Chem., 1909, p. 427; 1910, p. 360.

Schaklee et Meltzer. Die mecanische Beeinfluss von Pepsin, Centralbl. $f$. Physiol., 1909, (23), p. 3; Americ. Journ. physiol., (25), p. 81.

MichaËLis U. Ehrenreich. Die Absorptionanalyse der Fermenten. Bioch.Zeits., (10), p. 283 , 1908.

Abderhalden u. Otto Meyer. Ueber Nachweiss von activen Pepsin, Zeits. $f$. physiol. Chem., (74), p. 99, I9I1.

GraBER. Journ. of Ind. and Engin. chem., (3), p. 919, I9II.

Georges Dreyer et O. Hanssen. Recherches sur les lois de l'action de la lumière sur les glucosides, les enzymes, les toxines, les anticorps, $C$. $R$., I907, (2), p. 564 .

JodlbaUER ET TAPpeiner. Ueber die Wirkung des ultra violetten Lichtes auf Enzyme, Deutsch. Arch. f. klin. Med., (87), p. 373, 1906.

Jodlbauer et Tappeiner. Ueber die Wirkung von Licht auf Enzyme, Deutsch. . Arch. f. klin. Med., (85), p. $386,1906$.

O. Emmerings. Einwirkung des Sonnenlichtes auf die Enzyme. Ber. Deutsch.

- Chem. Ges., 34, (3), p. 3811 , 1901.

Pekelharing o. Ringer. Zur electrischen Ueberführung des Pepsins, Zeits. $f$. physiol. Chem., (75), p. 282, 1911.

Michä̈lis u. Davidsonn. Die isolectrische Konstante des Pepsins, Bioch. Zeits., (28), p. I, I9ro.

Michä̈iIS. Ueberführungsversuche mit Fermenten, Bioch. Zeits., (I6), p. 486; (17), p. 231, 1909.

Kudo. Ueber den Einfluss der Elektrizität auf die Fermente, Bioch. Zeits., (I6), p. 233,1909 .

Prncussorn. Beeinflussung von Fermenten durch Kolloỉde: Wirkung von anorgan. Kolloïden auf Pepsin, Bioch. Zeits., (8), p. 387, 1908.

AleXANDRE. Journ. Americ. Soc., (32), p. 680.

JACQUeS LeEb. Electrolytische Dissoz. und physiologische Wirksamkeit von Pepsin und Trypsin, Biol. Zeits., (19), p. 535, 1909.

Herzog U. Kasarnowski. Ueber die Diffusion von Kolloïden, Bioch. Zeits., (I I), p. $172,1908$.

Houghton. Journ. Americ. chem. Soc., (29), pp. I351-1 357 .

Möllhausen. Ueber das Eindringen von Albumin in Gelatinegallerten, Zeits., f. Chem. u. Indust. der Kolloüden, (2), p. 325.

Iscovesco. Studien über Kataphorese von Fermenten u. Kolloỉden, Bioch. Zeits. (24), p. 53, 1910.

Dezani. Accad. dell. S. di Torino, (45), p. 14, (47), p. I4, I9I 2.

F. KR ÜGER. Ueber Kenntnis der quantitätiven Pepsinwirkung, Zeit.f. Biol., (4I), p. 378,1901 .

Hancock. Americ. Journ. Pharm., (83), p. 373, 1911.

Holderer. Sur la filtration des enzymes, $C . R$., (150), 1910, p. 790.

E. Zunz. Archiv. d. Physiol., (7), p. 137, 1909. 


\section{INFLUENCE OF THE REACTION OF THE MEDIUM.}

S. Schmidt. Ueber das Wesen des Verdauungsprocesses, Liebigs Ann., (61), p. 3 II, 1847 .

Davibson ex Dieterich. Zur Theorie der Magenverdauung, Arch. f. Anat. $u$. Physiol., 1860, p. 690.

L. LiebermanN U. Bugarsky. Ueber das Bindungsvermögen eiweissartiger Körper für Salzsäure, $\mathrm{NaOH}, \mathrm{NaCl}$, Pfiüg. Arch., (72), p. 5 r.

Krüger. Weitere Beobachtungen über quantitätive Pepsinverdauung, Zeits. $f$. Biol., (4I), p. 467, r9or.

Roger et Garnier. Influence des variations simultanées de la Pepsine et de l'Acide chlorhyd. sur la digestion pepsique, Soc. Biol., I906, (2), p. 314.

Abderhalden U. Wachsmuth. Weiterer Beitrag zur Kenntniss der Wirkung des Pepsins und der Salzsäure auf Elastin und einige andere Proteine, Zeits. f. physiol. Chem., (7I), p. 339, I9I I.

E. Zunz. Hofmeister's Beitr., (3), p. 435, 1902.

\section{PEPSIN.}

Scrütz. Ueber den Einfluss der Pepsin- und Salzsäuremengen auf die Intensität der Verdauung, speziell bei Abwesenheit "freier" Salzsäure, Bioch. Zeits., (22), p. 33, 1908.

Trchoмrrow. Zur Frage nach der Wirkung der Alkalien auf das Eiweissferment des Magensaftes, Zeits. f. physiol. Chem., (55), p. 107, 1908.

Sörensen. Ueber die Messung und Bedeutung der Wasserstoffionen Konzentration bei enzymatichen Processen, Bioch. Zeits., (21), p. 131, 1909; (22), p. $35^{2}, 1909$.

Podwyssotsky. Sur la propepsine, Pfiüg. Arch., (39), p. 62, 1886.

LANGley. Journ. of Physiol., 1881, pp. 246 et 269.

Langley A. Edkins. Journ. of Physiol., 1886, p. 37 .

Abderhalden u. Steinbeck. Wirkung des Pepsins und $\mathrm{HCl}$, Zeits. f. physiol. Chem., (68), p. 293, 1910.

Kıug. Ueber Pepsin, Pflïg. Arch., (60), p. 43, 1895.

F. Klug, Jun. Beiträge zur Pepsinverdauung, Pflüg. Arch., (65), p. 330, r896.

Wróblewski. Zur Kenntniss des Pepsins, Zeits. f. physiol. Chem., (2I), p. I.

Stutzer. Abhängigkeit des Optimums der Verdauung von der Pepsin u. Trypsinmenge, Landwirts. Versuchenst., I891, (38), p. 257; Zeits. f. phys. Chem., (II), p. 530, I887.

\section{EFFECT OF OTHER CHEMICAL CONDITIONS.}

GURBER. Verhand der Wurzburger phys. med. Soc., 1895, p. 67.

Houghton. Journ. Americ. Chem. Soc., (29), p. I35, 1907.

Papasotiriou. Arch. f. Hygien, (57), p. 269, 1906.

Sawomara. Bullet of the College Tokio, (5), p. 265.

Pekelharing. Ueber Pepsin, Zeits. $f$. physiol. Chem., (35), p. 29, 1902.

VAN DE VELDE. Antisept. bei Enzymuntersuch., Bioch. Zeits., (3), p. 3 I6, I907. VAN de Velde U. Poppe. Wirkung von $\mathrm{NaCl}$, Bioch. Zeits., (28), p. 135, 1910.

Grober. Ueber die Wirkung von Antiseptika, Pflüg. Arch., (I04), p. Iog. 
CH. Richet. Ueber die Wirkung schwacher Dosen auf physiolog. Vorgänge, Bioch. Zeits., (I I), p. 273, 1908.

Efrront. Act. des fluorures sur les diastases, Bul. Soc. Chim., (4), p. 627, I8go; Moniteur Scientif., 1890.

Wolberg. Ueber den Einfluss einiger Salze und Alkaloide auf die Verdauung, Pflüg. Arch., (22), 1880, p. 291.

P. Grützner. Pflïg. Arch., (12), p. 285.

A. Sснмшт, Pfiug. Arch., (13), p. 93.

ManN. Diss., Erlangen, 1897 .

Bertels. Einfluss des Chloroforms, Virch. Arch., (130), p. 497.1892.

Dubs. Einfluss des Chloroforms, Virck. Arch., (134), p. 5 I9.

Starkenstein. Ueber Fermentwirkung u. deren Beeinflussung durch Neutralsalze, Bioch. Zeits., (24), p. 2 10, 1910.

Schierber. Arch.f. Phys., (III), p. 357.

Wroblewski. Zeils. f. physiol. Chem., (21), p. I, 1895. Ber. der Deuts. Chem. Ges., p. 1719, 1895.

Gley et Camus. Arch. de Physiol., p. 764, r897.

SALKowski. Bindung von $\mathrm{HCl}$ durch Amidosäuren, Virch Arch., (127), p. 501 .

Burss et Novy. Die Wirkung von Formaldehyd auf Enzyme, J. exper. Med., (4), p. 47,1899 .

\section{GENERAL ACTIONS.}

Van DaM. Die Verdauung des Caseins durch Pepsin von Kalb, Schwein, und Rind, Zeits. f. physiol. Chem., (79), p. 247, 1912.

Thibault. Journ. Pharm. el Chim., (I), p. 480, Igro.

voN GrǘtzNer. Ein einfaches Colorimeter nebst Bemerkungen über die Verdauungskraft von "reinen Pepsin" Pflïg. Arch., (r44), p. 545, I9r2.

Hirayama. Einige Bemerkungen über proteolytische Fermente, Zeits. f. physiol. Chem., (65), p. 290, 1910.

Herzog u. Margolis. Ueber die Einwirkung von Pepsin auf Ovalbumin, Zeits. f. physiol. Chem., (60), p. 298, 1909.

TAKEDA. Ueber das Harnpepsin als differentialdiagnostisches Kriterium zwischen Carcinoma ventriculi u. Apepsia gastrica. Deuts. med. Wochens., (36), p. I807, Igro.

Berg. Amer. Journ. Physiol., (23), p. 420.

Abderhalden u. Frieder. Weitere Beiträge zur Kenntniss der Wirkung des Pepsins. Zeits. f. physiol. Chem., (7 г), p. 449, г9г г.

\section{LAW OF ENZYMIC ACTION.}

O'Sullivan et Tompson, Journ. of the Chem. Soc., I89o, (57), p. 834 .

Wilhelmy. Pogg. Ann., (8I), p. 4I3.

Duclaux. Traile de Microbiologie, (2), p. 136.

V. Henri. Loi d'action des diastases, These, Paris, 1903.

Nicloux. Contribution à l'étude de la Saponification, These, Paris, I906.

V. Henri et Larguier des Bancels. Loi d'action de la trypsine sur la gélatine, C. $R ., 1903,(\mathrm{I}), \mathrm{pp} .1088$ et $158 \mathrm{I}$.

E. ScHüтz. Zeits. f. physiol. Chem., (9), p. 577, r 885 . 
Borissow. Thèse, St-Pétersbourg, $189 \mathrm{r}$.

Huppert et Schútz. Pfiug. Arch., (80), p. 470.

von Grützner. Ueber Fermentgesetz, Pfiig. Arch., (141), p. 63, 1911.

Vassilief. Arch. de Médecine expér. St-Pélersbourg, (2), p. 219, 1893.

J. Schutz. Zeits. f. physiol. Chem., (30), p. I, 1900.

\section{ANTI-PEPSIN.}

OGuro. Ueber eine Methode zum quantitätiven Nachweis des Antipepsins im Serum, Bioch. Zeits., (22), p. 266, I909.

Schwarz. Zur Kenntniss der Antipepsine, Beit. z. chem. Physiol. u. Path., (6), p. 530,1905 .

Schnappaur. Beitrag zur Physiologie des Pepsins, Diss., Rostock, I 888.

BRIot. Sur le mode d'action du sérum sur la pepsine, Soc. Biol., (54), 1902, p. 140.

Perin. Sur le pouvoir antipepsique du sérum sanguin, Soc. Biol., p. 938, 1902.

SAchs. Ueber Antipepsin, Fortschritte d. Medizin, (20), p. 425, 1902.

M. JACoBy. Zur. Kenntniss der Fermente und Antifermente, Bioch. Zeils., 1906, pp. 144, 247; 1907, pp. 21, 471; 1908, pp. 8, 40, 229, 232.

Morgenroth. Berl. klin. Wochens., 1909. (I6), p. $75^{8}$.

J. Cantacuzène et Jonescu-Mihaiesti. Soc. Biol., 1908, (2), p. 273; 1909, (1), p. 53 .

Rubinstein. Soc. Biol., IgII, (I), p. ir6.

DezanI. Estr. Reale Accad. delle Scienze di Torino, r910-11.

\section{PEPSIN.}

MrNaMr. Ueber die Reaction zwischen Fermenten u. Antifermenten, Bioch. Zeits., (39), p. 75, I912.

Zunz. Pouvoir antipepsique du sérum sanguin, Ann. Acad. Belgique, rgo5.

\section{IDENTITY OF PEPSIN AND RENNET.}

Pawlow U. Parastschuk. Ueber proteolytische und milchkoagulierende Wir. kung, Zeits. f. physiol. Chem., (42), p. 423.

van Hasselt. Notiz zur Pepsin-Chymosin-Frage, Zeits. f. physiol. Chem., (70), p. I7I, I9II.

TAyLor. Zur Frage der Identität von Pepsin u. Chymosin, Journ. of Biol. Chem., (5), p. 399, 1909.

SAwisch. Zur Frage über die Identität des Pepsins u. Chymosins, Zeits. f. physiol. Chem., (68) p. 12, 1910.

Hedin. Zeits. f. physiol. Chem., ror I, (74), p. 242.

Blum et Fuld. Bioch. Zeits., (6), p. 473.

Gewin. Pepsin u. Chymosin, Zeits. f. physiol. Chem., (54), p. 32, 1908.

Bang. Pepsin u. Chymosin, Zeits. f. physiol. Chem., (54), p. 359, 1908.

VAN DAM. Zur Frage nach der Identität von Pepsin u. Chymosin, Zeits. f. physiol. Chem., (64), p. 316, rgro.

O. Hammarsten. Zur Frage nach der Identität der Pepsin und Chymosin wirkung, Zeits. $f$. physiol. Chem., (56), p. 18, 1908. 
Mrgay U. Sawitsch. Die Proportionalität der eiweisslösenden und der milchkoagulirenden Wirkung des Magensaftes des Menschen und des Hundes in normalen u. pathologischen Fällen, Zeits. f. physiol. Chem., (63), p. 405, 1909 .

Hammarsten. Ueber die Darsuellung von pepsinarmen oder pepsinfreien Chymosinlösungen, Zeits. $f$. physiol. Chem., (74), p. 142, I91 I.

Rakoczy. Weitere Beobachtungen über Chymosin und Pepsin des Kalbsmagensaftes, Zeits. f. physiol. Chem., (73), p. 453, I9I r.

Porter. Ueber die Frage der Identität von Pepsin u. Lab, Journ. of Physiol., (42), p. 389, I9I I.

Grimmer. Ueber den derzeitigen Stand der Identitätsfrage von Pepsin und Chymosin, Milchw. Centralbl., (7), p. 481, 19ı I.

Hammarsten. Vergleichende Untersuchungen über die Pepsin und Chymosinwirkung bei Hund u. Kalb, Zeits. f. physiol. Chem., (68), p. I 19, I9ro.

Herzoc. Zur Frage zwischen Pepsin u. Labwirkung, Zeits. f. physiol. Chem., (60) p. 306, I909.

Raxoczy. Ueber die milchkoagulirende und proteolytische Wirkung der Rinderund Kalbsmageninfusion und der natürlichen Kalbsmagensaftes, Zeits. $f$. physiol. Chem., (68), p. 421, igro.

J. Sellier. Soc. Biol., I908, (2), p. 754 .

\section{CHEMICAL ACTIVITY.}

F. Alexander. Zur Kenntniss des Caseins und seiner peptischen Spaltungsprodukte, Zeils. f. physiol. Chem., I898, (35), p. $4 \mathrm{II}$.

AdLer. Albumose, Disertation, Leipzig, I907.

Elophe Benech. La question des peptones, Rev. gén. des Sc. pures et appliq., 1899, (то), p. $86_{3}$.

Birchard. Ein Beitrag zur Kenntniss der Protoalbumose, Diss., Leipzig, I 909.

E. Brücke. Beiträge zur Lehre von der Verdauung, Sitzungsber. d. $k$. Akad. d. Wiss. in Wien. Math.-naturw. Cl., 14-7-1859; (43), p. 601, 1861.

A. Bömer. Zinksulfat ein Fällungsmittel für Albumosen, Zeits. f. analyt. Chem., I895, (34), p. 562 .

K. BaUManN U. BöMer. Ueber die Fällung der Albumosen durch Zinksulfat, Zeits. f. Untersuchung des Nahrungs- und Genussmittel, 1898, (1), p. 106.

L. BLUM. Ueber den Nährwerth der Heteroalbumose des Fibrins und der Protoalbumosen des Caseins, Zeits.f. physiol. Chem., I900, (30), p. 2 I.

Bеснноцd. Kolloĩdstudien mit der filtration Methode, Zeits. f. physiol. Chem., (60), p. $257,1907$.

O. Cohnheim. Chemie der Eiweisskörper, Brunswick, I900, p. I72.

J. Efrront. Zur quantitätiven Bestimmung von Ammoniak und Amide, Ber. der Deuts. Chem. Ges., (16), p. 4290.

J. Efrront. Ueber die Bestimmung der Verdauungsproducte des Pepsins, Chemiker Zeitung, $1899,(23), \mathrm{n}^{\circ} 75$.

J. Effront. Sur la solubilité des protéoses et des peptones dans l'alcool, Bull. Soc. Chim. Paris, (21), p. 676.

J. Efrront. Sur le dosage de l'albumose et de la peptone, Bull. Soc. Chim. Paris, (21), p. $68_{3}$, 1899. 
J. Efrront. Sur la méthode de la précipitat. fractionn., Monit, Sc., 1902, p. $24 \mathrm{r}$.

E. Zunz. De la separation des albuminoids par l'emploi des sels. Am. Soc. Sciences Belgique, 1900, t. 9, fasc. 2-3.

Chittenden A. J. A. Hartwell. Crystalline globulin and globuloses or vitelloses, Journ. of Physiol., r89o, p. 435. - The relative formation of proteoses

- and peptones in gastric digestion, Journ. of Physiol., 189r, p. 12.

Chittenden A. G. L. Amerman. A comparison of artificial and natural gastric digestion, together with a study of the diffusibility of proteoses and peptones, Journ. of Physiol., 1893, (14), p. 483.

Chrtrenden A. L. B. Mendel. On the proteolysis of crystallized Globulin, Journ. of Physiol., r894, (17), p. 48.

Chittenden a. Meara. Journ. of Physiol., r894, p. 50r.

S. Dzierzgowski u. S. Salaskin. Ueber die Ammoniakalspaltung bei der Einwirkung von Trypsin und Pepsin auf Eiweisskörper, Centralbl. f. Physiol., 3-8-190r, (25), p. 249.

A. Danilewski. Etude sur la constitution chimique des substances albuminoỉdes, Arch. des Sc. phys. et nat., 1883, (7), pp. 150 et 420.

F. Goldschmidr. Ueber die Einwirkung von Säuren auf Eiweissstoffe, Diss., Strassburg, 1898 .

S. FräNKeI U. L. LanGstein. Ueber die Spaltungsproducte des Eiweisses bei der Verdauung, Akad. der Wissensch. in Wien, Mathem.-Naturw., 1901, (1 10), p. 238; Monats. f. Chem., r898, (rg), p. 8rg.

FriedmanN. Ueber die Bindungsweisse des Stickstoffs in primairen Albumosen, Zeits. f. physiol. Chem., 1900, (29), p. 5 r.

O. Folin. Ueber die Spaltungsproducte der Eiweisskörper, Zeits. f. physiol. Chem., $1899,(25)$, p. 152.

Hasebroek. Ueber erste Producte der Magenverdauung, Zeits. f. physiol. Chem., r887, (r r), p. 348 .

Henriquez u. Gjaldbaek. Ueber hydrolytische Spaltung von Proteine, Zeits. $f$. biol. Chem., (75), p. 377, 1911.

W. Hausmann. Ueber die Vertheilung des Stickstoffs im Eiweissmolekül, Zeits. $f$. physiol. Chem., r900, (29), p. 139 .

PEPSIN.

G. Hopkins. Jour. of Physiol., r900, p. 306.

Haslam. Quantitätive Bestimmung der Hexonbasen in Hetero-Albumosen und Peptone, Zeits. f. physiol. Chem., I90r, (32), p. 54.

Hart. Ueber quantitätive Bestimmung des Spaltungsproducte von Eiweisskörper, Zeits. f. physiol. Chem., rgor, (33), p. 347.

LaNGSTEIN. Weitere Beiträge zur Kenntniss der aus Eiweisskörpern abspaltbaren Kohlenhydraten, Hofm. Beit., r905, (6), p. 349.

W. Kühne U. R. H. ChitTenden. Ueber die nächsten Spaltungsproducte der Eiweisskörper, Zeits. f. Biol., 1883, (19), p. I59.

Kühne u. Chittenden. Ueber Albumosen, Zeits. f. Biol., r884, (20), p. 15. - Globulin und Globulosen, Zeits. f. Biol., 1886, (22), p. 409. 
KüHNE. Erfahrungen über Albumosen und Pepton, Verhand. d. Naturhistor.Med. Vereins, Heidelberg, 1876, p. 236; 1885, p. 286; 1892, p. 308.

Kossel u. Kutscher. Zeits. f. physiol. Chem., (3I), p. 165; (38), p. 39.

F. Kutscher. Ueber die Verwendung der Phosphorwolframsäure, Zeits. f. physiol. Chem., I900, (3r), p, 215.

LeVene. Journ. of biol. Chem., 1905, p. 45; 1910, p. 269.

D. Lawrow. Zur Kenntniss des Chemismus der peptischen und tryptischen Verdauung der Eiweisstoffe, Zeits. f. physiol. Chem., I899, (26), p. 5 r3.

Mralke. Mémoire sur la digestion, C. R., 1846, (23), p. 260.

C.-G. Lehmann. Lehrbuch der physiologischen Chemie, $2^{\mathrm{e}}$ édit., Leipzig, I853, (I), p. 3 I8; (2), p. 45 .

G. MeIssner. Untersuchungen über die Verdauung der Eiweisskörper, Zeits. $f$. rat. Medicin., 3 R., (7), p. I; (8), p. 280; (10) p. 1; 1859-1862.

Osborne A. Harris. Jour. of Amer. Chem. Soc., I905, p. 837.

RAPER. Hofm. Beitr., (9), p. I68, 1907.

Paul Müller. Zur Trennung der Albumosen von den Peptonen, Zeits.f. physiol. Chem., 1898, p. 48.

AL. Rolletr. Ueber die als Acidalbumine und Alkalialbuminate bezeichneten Eiweissderivate, Sitzungsber. der 'k. Akad. d. Wiss. in Wien, juillet 1881, p. 332 .

M. Nencki U. J. Zaleski. Ueber die Bestimmung des Ammoniaks in thierischen Flüssigkeiten und Geweben, Zeits. f. physiol. Chem., I901, p. I93.

W. Sawjalow. Diss. inaug., Dorpat, 1899.

STOOKER. Hofm. Beitr., (7), p. 590, 1906.

A: Schmidt-Mülheim. Beiträge zur Kenntniss des Peptons und seiner physio- logischen Bedeutung, Arch.f. Anat. u. physiol., Physiol. Abth., 1880, p. 33.

K. Spiro U. W. Pemsel. Ueber Basen- und Säurecapacität des Blutes und der Eiweisskörper, Zeits. f. physiol. Chem., 1898, p. 233.

Robertson a. Bidde. Ueber die Zusammensetzung der Substanzen welche entstehen bei Einwirkung von Pepsin auf die Produkte der vollständigen peptischen Hydrolyse des Caseïns. (d'après Chem. Centralb., I9II, (2), p. 218.) Journ. of Biol. Chem., (9), p. 295.

R. Neumeister. Zur Kenntniss der Albumosen, Zeits. f. Biol., (24), 1887, p. $38 \mathrm{I}$.

- Ueber Vitellosen, Zeits. f. Biol., (24), 1887, p. 402. - Bemerkungen zur Chemie der Albumosen und Peptone, Ibid., 1888, p. 267; 1890, p. 324.

Hugounenq et Morel. C. $R$. Acad., Ig08, (I), p. I 29 I.

E. P. Pick. Ein neues Verfahren zur Trennung von Albumosen und Pepton, Zeits. f. physiol. Chem., (28), p. 219, 1899.

M. Praundler. Zur Kenntniss der Endprodukte der Pepsinverdauung, Zeits. $f$. physiol. Chem., I900, (30), p. 93.

Neumann. Ueber Pepton, Zeits. f. physiol. Chem., 1905, (45), p. 2 I6.

Fr. Hofmeister. Zur Lehre vom Pepton, IV. Die Verbreitung des Peptons in Thierkörper, Zeits. f. physiol. Chem., I882, (6), p. 5 I.

Siegr Ried. Antipepton, Ber. Deutsch. Chem. Ges., r 900, p. 285 I.

Rogozinsky. Hofm. Beitr., (II), p. 229, r908.

SwIRlowskı. Einwirkung von Säuren, Zeits. f. physiol. Chem., r906, (48), p. 252. 
H. Schrötter. Ueber die Albumosen des Pepton Witte, Zeits. f. physiol. Chem., I $898,(26)$, p. 338 .

E. Salkowskr. Ueber die Wirksamkeit erhitzter Fermente, den Begriff des Peptons und die Hemialbumose Kühne's, Arch. f. pathol. Anat. u. Physiol., I880; Zeits. f. physiol. Chem., (32), p. 592.

P. Schützenberger. Recherches sur la constitution chimique des peptones, C. R., (II5), 1892, pp. 208 et 764 (V. aussi Dictionn. Wurtz: albuminoides.)

F. UMBer. Die Spaltung des krystallinischen Eier- und Serumalbumins sowie des Serumglobulins durch Pepsinverdauung, Zeits. f. physiol. Chem., I898, p. 258.

D. Van Slyke. Journ. of biol. Chem., IgI I, p. 187.

J. Wenz. Ueber das Verhalten der Eiweissstoffe bei der Darmverdauung, Zeils. $f$. Biol., I886, (4), p. I r.

E. Zunz. Contribution a l'étude de la digestion pepsique et gastrique des substances albuminoides, Ann. Soc. Sc. méd. et nat. de Bruxelles, (I I), 1902.

E. Zunz. Nouvelles recherches sur les protéoses, Bull. Acad. roy. de Belgique, $n^{\circ} 8$, IgII.

E. Zunz. Or colloldal pour caractériser les albumoses primaires, Arch. inter. de Physiol., 1904, p. 427.

E. Zunz. Recherches stalagmométriques sur les albumines et les peptones, Bull. Soc. roy. Sc. med., juin 1906, p. 25 I.

\section{ANALYSIS.}

Fuld U. Levison. Die Pepsinbestimmung mittels der Edestinprobe, Bioch. Zeits., (6), p. 473, 1907 .

M. JACoBy. Zur Kenntniss des Pepsinsnachweiss, Bioch. Zeits., (r), p. 58, $1906^{\circ}$

S. Hata. Uber die Bestimmung des Pepsins durch Aufhebung von Trüben Eiereweisse, Bioch. Zeils., (23), p. 180, 1909.

Waldschmidt. Ueber die verschiedenen Methoden, Pepsin u. Trypsin quantitätiv. zu bestimmen, nebst Beschreibung einer einfachen derartigen Methode, Pfliig. Arch., (143), p. 189, 1912.

O. Gross. Die Wirksamkeit des Pepsins u. eine einfache Methode zu ihrer Bestimmung, Berl. klin. Woch., I908, (13), p. 643.

Cowie u. Dickson. Der Nachweiss von Pepsin durch die Biuretreaction, Pharmac. Journ., [4], (24), pp. I98-r99.

S. KütTnER. Ueber die Volhard'sche Pepsinbestimmung, Zeits.f. physiol. Chem., (52), p. 63, 1907.

Wolf u. Tomaszewsky. Ueber Pepsin und Pepsinbestimmung mittels der Edestinprobe, Berl. klin. Woch., (22), p. 1051, 1908.

M. EinhorN. Ueber eine Vereinfachung der Jacoby-Solmsschen Ricinmethode der Pepsinbestimmung, Berl. klin. Woch., (34), p. 1567, 1908.

Hercod U. Maben. Bestimmung des Pepsins. Vergleichende Studien zur Wertbestimmung des Pepsins nach Vorschrift der verschiedenen Arzneibücher. Schweiz. Wochens. f. Chem. u. Pharm., (49), p. I 7 , Igr I.

Grǘtzner. Ueber eine Methode Pepsinmengen colorimetrisch zu bestimmen. Pflig. Arch., (8), p. 452. 
Erfront. Sur le pouvoir dissolvant de la pepsine, Bull. Soc. Chim., (21), p. 676 .

Efrront. Ueber die Bestimmung der Verdauungsproducte des Pepsins, Chem. Zeitung, (23), p. 75, I899.

Metr. Contribution à la physiologie des sécrétions. Diss. Saint-Pétersbourg, 1889.

Heusrin. Objections à la methode de Jacoby, Am. Soc. Sciences Belgiques, t. 8, fasc. 2,1909 .

\section{GASTRIC JUICE.}

Otro Cohnherm u. G. Dreyrus. Zur Physiologie u. Pathologie der Magenverdauung, Zeits. f. physiol. Chem., (58), 82, 1908-1909.

L. Tobler. Zeits.f. physiol. Chem., 1905, (45), p. 185 .

Nencki et Sieber. Beitrag z. Kenntniss d. Magensaftes u. chem. Zusammensetzung d. Enzyme, Zeits.f. physiol. Chem., I90I, (32), p. 29 I.

Schoumow-Simanowski. Arch.f. experim. Pathol. u. Pharm., (33), p. 336.

Frouin et Girard. Soc. Biol., I9I 2, (1), p. 340.

Rosemann. Pfliig. Arch., (118), p. $467,1907$.

Werchowsky. Dissert. Saint-Pétersbourg, I89o.

Konowaloff. Dissert. Saint-Pétersbourg, 1893.

P. Sommerfeld. Beitrag zur Chem. Zusammensetzung des mensch. Magensaftes. Bioch. Zeits., 1908, (9), p. 352.

(See also literature on the functioning of digestive glands.) 


\section{CHAPTER II.}

\section{REVERSIBLE ACTIONS OF ENZYMES.}

Synthetic Action of Pepsin, Plasteins.

Synthetic Actions in General. - From a study of the mode of action of inorganic catalysts it is found that often they give rise to reversible reactions; the decomposed products can be reconstituted anew, so that between these two contrary forces a certain state of equilibrium is reached, which prevents the reaction from becoming complete. The action of enzymes has been considered for a long time as being quite different from that of the inorganic catalysts. For enzymes the possibility of regenerating substances previously decomposed was not admitted. It was considered a definitely settled fact that these agents are merely capable of effecting an analytical or destructive action.

Recent investigations have brought about a complete change in this opinion. It has been demonstrated that enzymes can, according to the conditions of the medium, produce two distinct actions in opposite directions, and that they are capable, consequently, of intervening in the synthesis of organic substances. The first observation made in this direction we owe to Croft Hill (I898). By allowing maltase, extracted from beer yeast, to act on a concentrated solution of glucose, this investigator found a perceptible change in the rotatory and reducing powers of the liquid, a modification corresponding to the formation of a disaccharide. The discovery of Hill, very much discussed from the point of view of the nature of the new products formed, has been confirmed by the work of O. Emmerling (I9OI). The latter, in the course of his researches, recognized that maltase from yeast is also capable of reconstructing amygdalin starting with a mixture of glucose and amygdo-nitrile-glucoside of Fischer. We have: 


$$
\mathrm{C}_{6} \mathrm{H}_{5}-\underset{!}{\mathrm{CH}-\mathrm{CN}}+\mathrm{C}_{6} \mathrm{H}_{12} \mathrm{O}_{6}=\mathrm{C}_{6} \mathrm{H}_{5}-\underset{\mathrm{O}-\mathrm{C}_{6} \mathrm{H}_{11} \mathrm{O}_{5}}{\mathrm{CH}-\mathrm{CN}}+\mathrm{C}_{12} \mathrm{H}_{21} \mathrm{O}_{10} \mathrm{O}
$$

Amygdo-nitrile-glucoside Glucose Amygdalin Water

At the same time, and before the work of Emmerling appeared, Hanriot brought forward experimental data on the reversibility of lipolytic action, the results of which definitely placed the enzymes among the agents capable of exerting a synthetic action as the result of a molecular condensation accompanied by a loss of water. Hanriot carried out his experiments in the following manner, using serum as a source of lipase. One c.c. of serum, previously neutralized, was introduced into a mixture of ro c.c. of water and ro drops of a solution containing:

Glycerin, 5 g.; isobutyric acid, 2 g.; water, I 25 g.

After a certain time-interval at $37^{\circ}$, he measured the acidity (expressed here in number of drops of a 0.5 per cent solution of sodium carbonate): $1 s t$, in the serum $S ; 2 n d$, in the acid mixture $A$ without serum; $3 r d$, in the mixture of acid and serum $(A+$ $S)$. The loss of acidity of the latter could only come from the combination of glycerin and acid.

Synthetic Action of Sero-lipase.

\begin{tabular}{|c|c|c|c|}
\hline & $\mathrm{h}$. & I h. & It h. \\
\hline $\begin{array}{l}S \ldots \ldots \ldots \\
A \\
A+S \\
\text { Acidity disappeared. } \\
\text { Acidity disappeared (per cent original) }\end{array}$ & $\begin{array}{r}2 \\
47 \\
34 \\
15 \\
30\end{array}$ & $\begin{array}{r}5 \\
46 \\
30 \\
21 \\
44\end{array}$ & $\begin{array}{r}5 \\
48 \\
24 \\
29 \\
54\end{array}$ \\
\hline
\end{tabular}

Therefore, 54 per cent of the butyric acid introduced was combined with the glycerin under the influence of lipase, under conditions of time and of temperature at which direct combination would be almost zero in the absence of this enzyme. Hanriot succeeded in isolating the product formed in the reaction. He found that this compound, which arises from the action of lipase in acid solution, is destroyed by it in neutral solution. In addition, he recognized that this reversal of the action of lipase is general and 
even extends to inorganic acids. About the same time, Kastle and Loevenhart studied the action of the pancreatic enzyme on a mixture of alcohol and butyric acid, and detected likewise an esterification, that is to say, the formation of ethyl butyrate.

Pottevin (I903) succeeded in synthesizing complex fatty substances by the combination of glycerin with the higher fatty acids, using as an enzyme the lipase contained in a glycerin extract of hog pancreas. After having made a mixture of this glycerin extract and pure oleic acid, the whole was allowed to stand at $35^{\circ}$. He found a progressive diminution of the acidity, and at the end of 8 days he succeeded in isolating from the mixture a neutral oil which he analyzed. This oil was found to be identical with the mono-olein of the glycerin. Moreover, this synthetic fat, again placed in contact with glycerin extract of pancreas and an excess of water, could be split into oleic acid and glycerin. Dunlope and Gilbert arrived at similar results using the enzyme from linseed meal, or castor-oil seed free of oil. Fischer and Armstrong, in 1902, by allowing the enzyme of Kephyr granules to act on a mixture of glucose and galactose obtained isolactose. Finally, quite recently, Bourquelot and Bridel have utilized the reversible power of emulsin to reconstruct artificial glucosides, such as methyl-, ethyl-, propyl-, isobutyland allylglucoside.

As to precipitation of starch, which, as Fernbach and Wolff have discovered, can take place under the action of a special enzyme, amylo-coagulase, this does not appear, according to the investigation of Maquenne on the retrogradation of starch, to be really a result of condensation. What is observed is rather a simple change of physical condition caused by a special enzyme.

However, it is seen from the preceding, that in the family of the enzymes acting on complex substances there have already been found several examples of evident synthesis. It is interesting to inquire whether with proteolytic enzymes a similar phenomenon can be found.

Danilewsky Reaction. - In I894, Danilewsky found that rennet produces in a solution of peptone a coagulum whose 
properties are different from those of the substrate. This observation was made the subject of a careful thesis in 1895 at the University of St. Petersburg by Okuneff. Danilewsky and his pupils from the beginning attached great importance to these experiments, for the precipitate produced by rennet was considered by them to be a globulin, and the enzyme action to be of a synthetic nature. The Danilewsky reaction was confirmed later by Lawrow, Sawjaloff and Kurajeff, etc. and served as the basis for numerous researches. It was soon perceived that the power of coagulating a solution of peptone is not possessed by rennet alone. Pepsin, trypsin, papayotin, the tryptase of yeast, and the proteolytic enzymes of various microbes cause the same precipitation. Nürenberg found, further, that extracts of organs, such as the liver, the kidney, as well as the extract of muscle, give a reaction similar to that of pepsin. The precipitate formed by papayotin has been designated under the name of coagulose, while the product of reaction of the other proteolytic enzymes on peptone is called plastein.

The Danilewsky reaction is studied in the following manner. In reaction tubes pour Io c.c. of a 40 per cent peptone solution, acidified with 0.7 per cent $\mathrm{HCl}$. Add a quantity of enzyme, pepsin or rennet, corresponding to 3 per cent of the quantity of peptone used, and keep the tubes in a water-bath at $65^{\circ}$. According to the quality of the peptone used, the liquid becomes gelatinous, curdles, or becomes turbid, in consequence of the formation of an abundant precipitate in a viscous mass. The liquid, diluted with water, leaves on the filter a precipitate, soluble in acid or alkali. The velocity of the reaction depends primarily on the activity of the enzyme used, on the reaction, and the other conditions of medium.

Different hypotheses have been set forth as to the nature of the agents which cause the appearance of plasteins. It has been thought legitimate to conclude that the coagulating or hydrolyzing enzymes, such as rennet, pepsin, or trypsin, are always accompanied by a second enzyme producing the reversed reaction. Now from an observation published by Jacoby, it appears that 
anti-trypsin prevents the formation of plasteins. The serum, then, would act on the two functions of trypsin - on its hydrolyzing power, as well as on its power of condensation - whence the uselessness of assuming a second enzyme, a sort of retro-trypsin.

Influence of Temperature. - We find that the formation of plasteins takes place between $\circ$ and $30^{\circ}$.

\begin{tabular}{|c|c|}
\hline Temperature. & Formation of plastein. \\
\hline $5^{\circ}$ & 5 days \\
$40^{\circ}$ & 26 hours \\
$60^{\circ}$ & 35 minutes \\
$70^{\circ}$ & 20 minutes \\
\hline
\end{tabular}

At low temperature the reaction is very slow and incomplete. At a temperature of $60^{\circ}$, and especially at $70^{\circ}$, the reaction takes place very rapidly, but there is already a destruction of the enzyme, for the quantity of plastein formed does not exceed to per cent of that of peptone used. The optimum temperature is apparently between $37^{\circ}$ and $40^{\circ}$, corresponding to the maximum of plastein which has appeared in the solution.

Influence of the Reaction of the Medium on the Formation of Plasteins.

\begin{tabular}{|c|c|c|c|c|}
\hline \multirow{2}{*}{ Nos. } & \multicolumn{2}{|c|}{ Reaction of peptone. } & \multirow{2}{*}{ Conditions of medium. } & \multirow{2}{*}{$\begin{array}{c}\text { Volume of } \\
\text { plastein } \\
\text { precipitated }\end{array}$} \\
\hline & Litmus paper. & Congo paper. & & \\
\hline $\begin{array}{l}I \\
2 \\
3 \\
4 \\
5 \\
6 \\
7\end{array}$ & $\begin{array}{l}\text { red } \\
", \\
", \\
، \\
\text { neutral } \\
\text { blue }\end{array}$ & $\begin{array}{l}\text { o } \\
\text { brown } \\
\text { bluish-brown } \\
\text { blue } \\
\text { ". } \\
\text { ". }\end{array}$ & $\begin{array}{c}\circ \\
\text { trace } \mathrm{HCl} \\
0.1 \text { per cent } \mathrm{HCl} \\
0.2 \text { per cent } \mathrm{HCl} \\
0.5 \text { per cent } \mathrm{HCl} \\
\text { neutral } \mathrm{Na}_{2} \mathrm{CO}_{3} \\
\text { slight excess } \mathrm{Na}_{2} \mathrm{CO}_{3}\end{array}$ & $\begin{array}{l}2.4 \\
5.6 \\
4.6 \\
0.0 \\
0.0 \\
1.1 \\
0.0\end{array}$ \\
\hline
\end{tabular}

Influence of the Reaction of the Medium. - Rennet and pepsin bring about this formation in a slightly acid medium. In solutions of 20 to 40 per cent peptone, the best results are obtained with an acidity of 0.5 to 0.7 per cent $\mathrm{HCl}$. In solutions of peptone which are weaker the quantity of acid should be 
reduced as it is necessary to avoid the presence of free acid which is very injurious.

The preceding table is the result of experiments made by Lawrow; the solution of peptone which he used was I 5 per cent, the temperature of the experiment was $40^{\circ}$, and its duration 70 hours. At this concentration, we see that the maximum of plastein obtained corresponds to a trace of $\mathrm{HCl}$, or to a brownish tint of Congo paper. Trypsin and pancreatin furnish plastein even in a neutral medium. The maximum yield is obtained with a very slight alkalinity.

Influence of the Concentration and Nature of the Substance to be Coagulated. - While the action of proteolytic enzymes is exerted preferably in dilute solutions of albuminoids, the concentration of the solution plays an essential rôle for the preparation of plasteins. The formation of plastein is easily manifest in a 2.5 per cent solution of peptone. It increases considerably with the concentration to reach a maximum at a concentration of about 40 per cent. The influence of concentration is summarized in the following table:

Influence of Concentration on the Formation of Plasteins.

\begin{tabular}{|c|c|c|}
\hline \multicolumn{1}{|c|}{$\begin{array}{c}\text { Content in nitrogen, mg. per roo c.c. liquid. } \\
\text { solution. }\end{array}$} & $\begin{array}{c}\text { In the filtered } \\
\text { liquid separated } \\
\text { from the plastein. }\end{array}$ & $\begin{array}{c}\text { Nitrogen precipi- } \\
\text { tated (per cent total } \\
\text { nitrogen). }\end{array}$ \\
\hline & 355 & 2.4 \\
364 & 685 & 6.0 \\
729 & I 153 & 20.0 \\
I457 & I 535 & 29.8 \\
2186 & I935 & 33.6 \\
2914 & & \\
\hline
\end{tabular}

The duration of the tests was 96 hours. Thymol had been added to prevent deterioration and the temperature was from $38^{\circ}-40^{\circ}$. In the last test more than 33 per cent of the nitrogen of the peptone was transformed into plastein. This quantity can, moreover, be greatly exceeded by freeing the liquid of the precipitate formed, and following this operation with concentration. Thus, after addition of rennet, a fresh precipitation 
occurs. By repeating this manipulation three times, more than 50 per cent of the nitrogen is transformed into plastein.

Plastein was first obtained with the products of hydrolysis of nitrogenous matter under the influence of pepsin or trypsin, but the products formed by the moderate action of acids or alkalis can serve just as well. The products of advanced hydrolysis by trypsin or acids yield no plastein, or only a very little. According to Kurajeff, the different proteolytic enzymes do not act equally well in the same media. Papayotin gives an abundant deposit of plastein with secondary albumose and very little with primary albumose, while rennet, on the contrary, gives much plastein with the primary and little with the secondary. Below, according to Henriques, is the comparative action of enzymes on the different products of albuminoid hydrolysis:

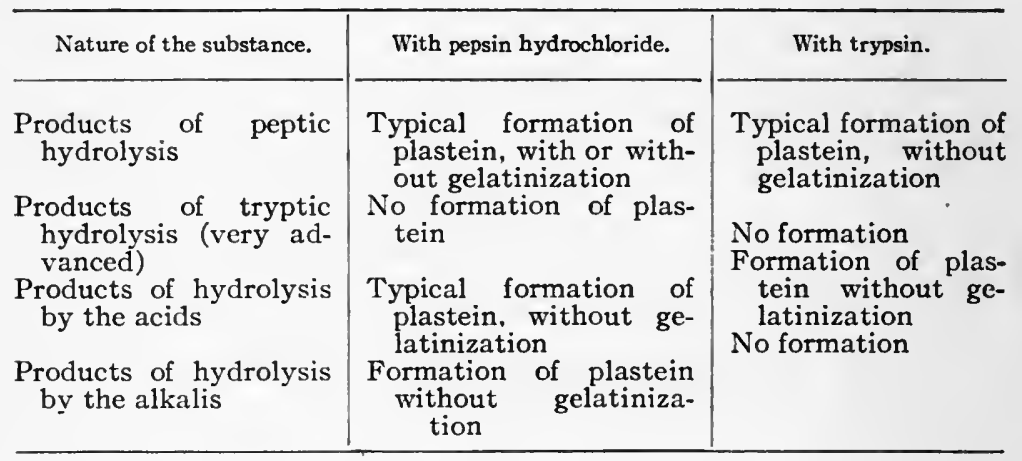

Combined Action of Pepsin and Rennet in the Formation of Plasteins. - We have just seen that we can obtain plasteins with pepsin as well as with rennet. But since the coagulating power is likewise found in all pepsin and since rennet possesses equally the peptonizing power, it was interesting to find out which of the two functions plays the preponderating rôle in the Danilewsky reaction. To this end, Rakoczy studied comparatively the macerations of calf and beef stomach linings, from the point of view of the formation of plasteins. He brought the two active extracts successively to the same coagulating powers, then the 
same digestive powers, and determined in each case the amount of plasteins formed.

Combined Action of Rennet and Pepsin.

\begin{tabular}{l|c|c|c}
\hline & $\begin{array}{c}\text { Coagulating } \\
\text { power. }\end{array}$ & $\begin{array}{c}\text { Digestive } \\
\text { power (Met.). }\end{array}$ & Plastein. \\
\hline r. Extract of calf stomach lining..... & $\begin{array}{c}\text { min. } \\
\text { 2. Extract of beef stomach lining..... }\end{array}$ & 55 & 5.0 \\
3. Extract of calf stomach lining..... & 20 & 0.1 & + \\
4. Extract of beef stomach lining..... & I97 & 4.5 & \pm \\
\end{tabular}

In (I) and (2) the extracts have the same coagulating powers; however, it is only in (I) that plastein appears. In (3) and (4) the conditions are reversed. The two liquids have equal digestive powers, but they differ enormously as to their coagulating powers. This time it is still in (3) that coagulation takes place, the quantity of pepsin alone in (4) being too small to act, as previously in (2) the quantity of rennet alone was insufficient. The Danilewsky reaction is therefore determined, either by pepsin alone, or by rennet alone. In the presence of the two enzymes, the actions are superimposed. When one enzyme is removed from the mixture it is necessary, in order to obtain a result, to replace the quantity eliminated by an equivalent quantity of the other.

Composition and Chemical Nature of Plasteins. - Plasteins give the characteristic reactions of albuminoids. According to Sawjaloff they have a constant composition independent of the original albuminoid material from which they were derived:

Composition of Plasteins.

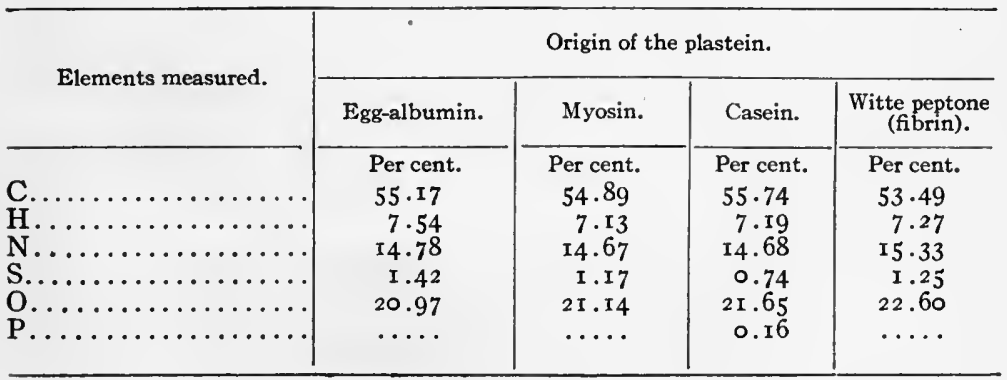


The plasteins, coming from different albuminoid materials, have compositions very similar, but the little difference found is due especially to the fact that the products have all previously undergone the same degree of hydrolysis. Lawrow has shown that in the Danilewsky reaction different products may be obtained, and that the composition of plasteins produced depends on the composition of the medium in which they are formed. From a solution of albumin peptonized by the aid of pepsin, the Russian scientist, by means of fractional precipitations with alcohol, separated different portions which represent in a way the successive phases of the hydrolysis of albumin. With these different fractions, he obtained plasteins, free from mineral matter, whose composition is indicated in the following table:

\begin{tabular}{|c|c|c|c|}
\hline Composition of plasteins obtained with. & c. & н. & N. \\
\hline 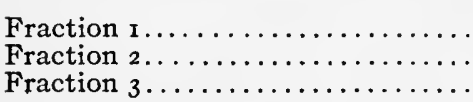 & $\begin{array}{l}54.90 \\
56.46 \\
44.58\end{array}$ & $\begin{array}{l}7.00 \\
7.57 \\
8.07\end{array}$ & $\begin{array}{l}14.54 \\
12.41 \\
12.31\end{array}$ \\
\hline
\end{tabular}

While fractions (I) and (2) precipitate with phosphotungstic acid and are made up of albumoses precipitable by ammonium sulphate, fraction (3) does not precipitate with phosphotungstic acid and is composed of complex amino-acids. The plasteins obtained with these three kinds of products have very different compositions. Plasteins reflect a part of the properties of the substance from which they are derived. Thus it is, that by submitting plasteins from albumoses to a partial hydrolysis under the influence of acids, a product is obtained which partially precipitates with phosphotungstic acid, indicating that albumoses and peptides enter into these plasteins, while the hydrolysis of plasteins (3) gives no substance precipitable by the phosphotungstic reagent.

Chemistry of the Formation of Plasteins. - The data found in literature on the chemical change which accompanies the Danilewsky reaction are quite contradictory. Some regard the 
formation of plasteins as a simple coagulation resulting from physical or chemical changes in the medium. According to this hypothesis, this precipitation has nothing in common with a true synthetic action. It is simply a question of the throwing out of solution of a compound which was in an unstable physical state, a phenomenon analogous to the coagulation of colloidal solutions. The supporters of a synthetic action are divided on the extent of this same synthesis. Some hold there is an advanced retrogression, yielding a globulin. Others maintain, on the contrary, that the final product obtained is not an albuminoid proper, but a derivative of this, assuming that all of the products synthesized in the course of the hydrolysis do not enter into the plastein formed.

The small extent of our knowledge as to the nature of albumoses, and even as to the differentiation of the various natural albuminoids, renders it difficult to quote a single experiment solving the question. The problem is almost solved, however, with data assembled from different sources. It was Levene and van Slyke who first determined quantitatively, according to the method established by E. Fischer, the amino-acids given by hydrolysis with 20 per cent $\mathrm{HCl}$ of a plastein and of Witte peptone from which it was derived.

Comparative HCl Hydrolysis of Plastein and Fibrin.

\begin{tabular}{|c|c|c|}
\hline & $\begin{array}{l}\text { Per } 100 \mathrm{~g} . \\
\text { of plastein. }\end{array}$ & Per $100 \mathrm{~g}$. of fibrin. \\
\hline 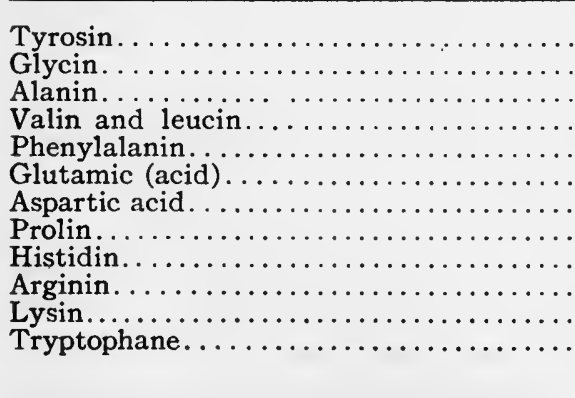 & $\begin{array}{c}\text { g. } \\
3.03 \\
0.50 \\
? \\
15.69 \\
1.00 \\
10.02 \\
2.15 \\
2.55 \\
0.43 \\
2.16 \\
1.42 \\
\text { Present } \\
38.75\end{array}$ & $\begin{array}{r}\text { g. } \\
3 . \mathrm{I} \\
2.2 \\
3 . \mathrm{I} \\
\mathrm{I} 3.0 \\
\mathrm{r} .2 \\
6.8 \\
\mathrm{I} .7 \\
2.4 \\
\ldots \ldots \\
\ldots \ldots \\
\ldots \ldots \\
\text { Present } \\
33.5\end{array}$ \\
\hline
\end{tabular}


In the table above, as Witte peptone comes from a transformation of fibrin, we have given the composition of the products obtained after hydrolysis of the latter with acid. These analytical data show beyond doubt that plastein is a very complex substance, approaching closely the albuminoids in composition. Moreover, of all the products which are found in peptone, only the fraction precipitable by phosphotungstic acid can give rise to a plastein of composition analogous to that given above:

These first results, while indicating the albuminoid nature of plasteins, still do not prove that these compounds are actually synthesized, for they could very well be nothing else than albumose precipitated from its solution. To study more closely the constitution of these plasteins, Levene and van Slyke conceived the plan of measuring the viscosity of their solutions and of comparing it with that of various albuminoids. The substances were dissolved in a normal solution of $\mathrm{NaOH}$, and determinations made at the end of a variable time $S$ of the duration $T$ of the escape of a given volume of solution through the Ostwald viscosimeter. Likewise they determined the duration $T^{\prime}$ of the escape of an equal volume of water $\left(T^{\prime}=126.0 \mathrm{sec}\right.$.). The numbers given in the following table are calculated according to the formula $\frac{100 T}{T^{\prime}}$ and represent the viscosity of these various solutions:

Molecular Condensation of Plastein According to its Viscosity.

\begin{tabular}{|c|c|c|c|c|c|c|c|}
\hline s. & Plastein. & S. & $\begin{array}{l}\text { Hetero- } \\
\text { albumose. }\end{array}$ & S. & $\begin{array}{c}\text { Witte } \\
\text { peptone. }\end{array}$ & s. & Fibrin. \\
\hline $\begin{array}{l}\text { h. } \\
\frac{1}{2} \\
2 \frac{1}{2} \\
8\end{array}$ & $\begin{array}{l}\text { I } 60.7 \\
\text { I } 56.5 \\
\text { I } 56.0\end{array}$ & 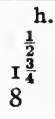 & $\begin{array}{l}\text { I } 66.7 \\
\text { I } 61.6 \\
\text { I } 54.6\end{array}$ & $\begin{array}{l}\text { h. } \\
\frac{1}{2} \\
2 \frac{1}{2} \\
\ldots\end{array}$ & $\begin{array}{l}\mathrm{I} 42.6 \\
\mathrm{I} 4 \mathrm{I} .0 \\
\ldots \ldots\end{array}$ & $\begin{array}{c}\text { h. } \\
\ldots \\
4 \\
8 \frac{1}{2}\end{array}$ & $\begin{array}{r}\ldots \ldots \\
223.1 \\
179.2\end{array}$ \\
\hline
\end{tabular}

These results confirm the fact that plastein possesses a greater molecular complexity than Witte peptone but that it does not exceed that of hetero-albumose, much less that of fibrin. Sawjaloff, to show the synthetic nature of plasteins, calculates by. 
means of acid capacities the respective molecular weights of plasteins and of peptones. He finds for the first a number equal to about 6000 , and for the second, 3000 only.

Okuneff; who is likewise of the opinion that this is a question of a condensation, performs an experiment of quite a different character. After having determined the weight of dry substance contained, on the one hand in a peptone solution, and on the other hand in a pepsin solution, he mixes the two solutions and, after plastein is formed, he evaporates the whole. He finds under these conditions a perceptible diminution in the dry residue. He therefore concludes that the action is the opposite of peptonization, that is to say, that it is indeed a molecular condensation accompanied by a dehydration.

The chemical process followed in the formation of plasteins can again be shown by the aid of tannin. We know that this reagent, added to a solution of peptone, completely precipitates the albumoses, while the products of advanced hydrolysis are not affected. Assuming that there is carried on in the center of the liquid in the course of the Danilewsky reaction a molecular condensation, we should in this case find an increase of the weight of the precipitate obtained with tannin. This is, in fact, verified by experiment. $\mathrm{I} 20 \mathrm{~g}$. of peptone are dissolved in 300 c.c. of 0.7 per cent $\mathrm{HCl}, 3 \mathrm{~g}$. of pepsin are added and the whole allowed to stand at $37^{\circ}$.

Formation of Plasteins as Determined by Tannin Nitrogen.

\begin{tabular}{|c|c|c|}
\hline Duration. & $\begin{array}{l}\text { Content in } N \\
\text { of the tannin } \\
\text { precipitate. }\end{array}$ & State of the mixture. \\
\hline 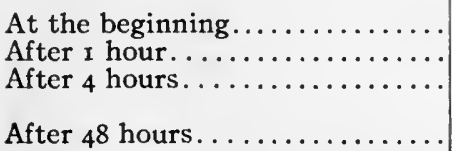 & $\begin{array}{l}79 \cdot 9 \\
80 \cdot 5 \\
81 \cdot 3 \\
82.0\end{array}$ & $\begin{array}{l}\text { Transparent liquid. } \\
\text { Transparent liquid. } \\
\text { Thickening of liquid and } \\
\text { formation of coagulation. } \\
\text { Coagulation. }\end{array}$ \\
\hline
\end{tabular}

It is evident that the quantities of nitrogen precipitated increase as the plastein is formed. The maximum nitrogen 
precipitated by the tannin coincides with the complete coagulation of the liquid. It is also observed that there is already produced quite a perceptible condensation before even the plastein appears. The molecular condensation and the coagulation are, therefore, two different phenomena, one being a direct consequence of the other.

A decisive step in the examination of the nature of plasteins was made by Henriques and Gjaldbaek. These investigators applied to the reaction of plasteins the formaldehyde method of Sörensen for measuring the amino-acids formed in the course of hydrolysis. (See Methods of Analysis of Tryptic Products.) The content in formaldehyde nitrogen of an albuminoid derivative gives us an exact idea of the degree of hydrolysis to which it has been brought. In the course of the hydrolysis, in consequence of the liberation of amino groups, the formaldehyde nitrogen increases considerably; it is therefore to be predicted that synthetic action, resulting from the progressive saturation of these same groupings, will on the contrary coincide with a noticeable diminution of formaldehyde nitrogen. The following example gives us interesting data on the change which takes place in the nature of nitrogen during the formation of plasteins, the nitrogen passing from the amino form to a more complex state. To a 30 per cent solution of white of egg, strongly peptonized, add pepsin and leave the whole at $35^{\circ}$. Take samples from time to time, in which are determined, on the one hand in the turbid liquid still containing the plastein formed, the total nitrogen, the ammoniacal nitrogen, and the formaldehyde nitrogen, and on the other hand in the coagulum collected on a filter, the total nitrogen by the Kjeldahl method.

The following results were obtained: 
Distribution of Nitrogen in the Course of the Formation of Plasteins.

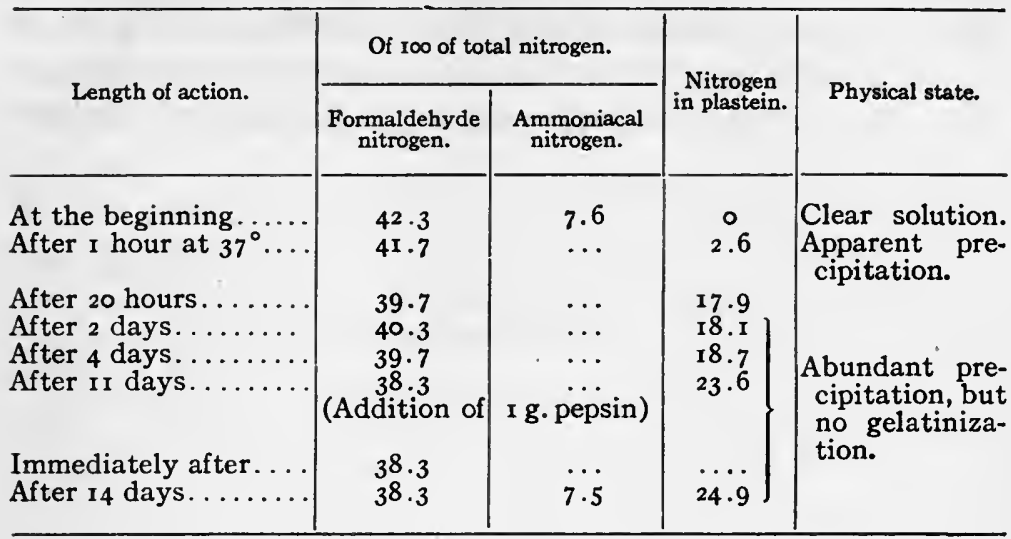

It is seen that plastein appears in the liquid after one hour. After twenty hours, of 100 nitrogen units contained in the original liquid, 17.9 have passed into the precipitate. Of 100 nitrogen units contained in the liquid, 42.3 can be titrated by formaldehyde at the beginning. As the plastein forms, the formaldehyde figure diminishes, to reach 38.3 units after eleven days of action. The progressive diminution of the aminonitrogen indicates very well the nature of the work which has gone on; work opposing that of hydrolysis. But the most convincing proof comes from the fact that it is possible to bring back the amount of formaldehyde nitrogen in the medium where the plastein is produced to the original amount by diluting the liquid and keeping it for some time at $40^{\circ}$. The pepsin, regaining under these conditions its normal activity, hydrolyzes anew the coagulum formed and consequently increases the quantity of formaldehyde nitrogen.

The formation of plasteins is then undoubtedly accompanied by a synthetic action, but it still remains to be seen whether the plastein formed is really a more complex product. It might, in fact, very well be that the molecular concentration relates solely to the soluble parts, and that the precipitate did not participate in this condensation. The method of Sörensen also allows us to 
answer this question. First the formaldehyde nitrogen in the original product resulting from hydrolysis is determined, and then the Danilewsky reaction is started and the formaldehyde nitrogen is determined separately in the liquid and in the precipitate.

Synthetic Nature of Plastein.

\begin{tabular}{|c|c|c|c|c|}
\hline \multirow{2}{*}{ Substances experimented with. } & \multirow{2}{*}{$\begin{array}{l}\text { Degrees of hy- } \\
\text { drolysis accord- } \\
\text { ing to nitrogen. }\end{array}$} & \multirow{2}{*}{$\begin{array}{l}\mathrm{N} \text { of plastein, } \\
\text { per cent of } \\
\text { total nitrogen. }\end{array}$} & \multicolumn{2}{|c|}{$\begin{array}{l}\text { Formaldehyde } \mathrm{N} \text {, per } \\
\text { cent of total } \mathrm{N} \text {. }\end{array}$} \\
\hline & & & $\begin{array}{l}\text { In the } \\
\text { filtrate. }\end{array}$ & $\begin{array}{l}\text { In the } \\
\text { plastein. }\end{array}$ \\
\hline $\begin{array}{l}\text { White of egg hydrolyzed } \\
\text { with pepsin }+\mathrm{H}_{2} \mathrm{SO}_{4} \ldots \ldots\end{array}$ & 38.0 & 24.9 & 39.5 & 134 \\
\hline $\begin{array}{l}\text { Casein hydrolyzed with pep- } \\
\text { sin }+\mathrm{H}_{2} \mathrm{SO}_{4} \ldots \ldots \ldots \ldots \ldots \\
\text { Beef meat hydrolyzed with }\end{array}$ & 38.5 & I 5.4 & 38.4 & 13.2 \\
\hline $\begin{array}{l}\text { pepsin }+\mathrm{H}_{2} \mathrm{SO}_{4} \ldots . . . . \\
\text { Witte peptone hydrolyzed }\end{array}$ & 34.4 & 15.3 & 34.2 & 14.8 \\
\hline $\begin{array}{l}\text { with pepsin }+\mathrm{H}_{2} \mathrm{SO}_{4} \ldots \\
\text { Peptone hydrolyzed with }\end{array}$ & 37.0 & 13.6 & 37.9 & $\mathbf{I 3 . 2}$ \\
\hline $\begin{array}{c}\text { pepsin } \\
\text { Edestin hydrolyzed with }\end{array}$ & 34.6 & 18.7 & 24.5 & 14.0 \\
\hline $\begin{array}{l}\text { pepsin }+\mathrm{HCl} . . . . . . . . \\
\text { Beef meat hydrolyzed with }\end{array}$ & 32.2 & 18.3 & $33 \cdot I$ & 10.3 \\
\hline pepsin $+\mathrm{HCl} . . . \ldots \ldots \ldots$ & 34.6 & 19.7 & $35 \cdot 3$ & I3.4 \\
\hline
\end{tabular}

In the hydrolyzed egg-albumin, the proportion of formaldehyde nitrogen before the formation of plastein was 38.0, which means that 38.0 per cent of the total nitrogen entered into reaction with the aldehyde. In the liquid separated from the plastein, a formaldehyde nitrogen content equal to 39.5 is found, a content perceptibly higher than that at the beginning. This fact indicates very well, therefore, that the molecular condensation has not acted on the soluble parts, but that on the contrary the plastein has been formed in the original liquid, at the expense of substances which were already of a degree of formaldehyde nitrogen lower than the average, in other words, at the expense of the most condensed substances. In the plastein formed, of the total nitrogen we find again 13.4 per cent formaldehyde nitrogen. The synthetic action has then borne exclusively on the product precipitated, and the extent of this work 
is considerable, since the quantity of plastein formed is about 25 per cent, and in this important fraction the formaldehyde standard has been brought from 38.0 per cent to 13.4 per cent.

The preceding table indicates again that the content in formaldehyde nitrogen of plasteins obtained with different hydrolyzed albuminoid substances is variable. This result banishes the idea that it is the case of a well-determined product of constant composition. It also establishes the fact that by starting with the same albuminoid matter, it is possible to arrive at plasteins of different compositions, according to whether the liquid producing the plastein is in a more or less advanced stage of hydrolysis.

Value in Formaldehyde Nitrogen of Different Plasteins.

\begin{tabular}{|c|c|c|}
\hline . & $\begin{array}{l}\text { Formald. value of } \\
\text { experimental } \\
\text { liquid. }\end{array}$ & $\begin{array}{l}\text { Formald. value of } \\
\text { corresponding } \\
\text { plastein. }\end{array}$ \\
\hline 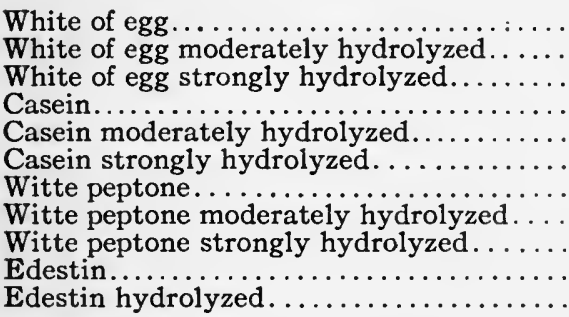 & $\begin{array}{r}2.9 \\
21.0 \\
38.5 \\
9.5 \\
20.8 \\
38.5 \\
13.9 \\
19.0 \\
37.0 \\
2.9 \\
32.0\end{array}$ & 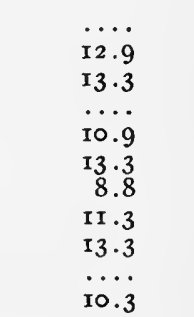 \\
\hline
\end{tabular}

The values of formaldehyde nitrogen of the plasteins obtained vary from 8.8 per cent to $I 3.3$ per cent, the products strongly hydrolyzed giving plasteins having the highest values.

It therefore results from all this data that plasteins are of a very complex protein nature, which approaches in certain points that of natural albuminoids, but which differs from it, however, from the point of view of formaldehyde nitrogen. The special nature of plasteins and their differentiation from the natural albumins appears also from the work of Herrmann and Chain. By inoculation of rabbits with plastein coming from Witte peptone, consequently from fibrin, they obtained an anti-serum which 
shows itself active, not only toward the plastein which served for inoculation, but also toward plasteins coming from egg albumin, edestin or globulin, while it remains inactive toward Witte peptone, as well as toward other natural albuminoid substances. There is, then, a similarity among all plasteins, and a difference between them and the natural albumins.

The Physiological Rôle of Plasteins. - When Danilewsky recognized that rennet was capable of producing in solutions of peptone a precipitate possessing numerous analogies with the albuminoids, it was thought possible to explain by this reaction the rôle of rennet in the organism. In fact, in the study of the conditions for enzyme secretion, it was generally found that the secreted enzymes answer a nutritive need. Now if rennet plays an important rôle in the digestion of casein, and if, consequently, its presence in the digestive juice of animals during lactation corresponds to a real need, we should not know, on the other hand, how to explain the presence of rennet in vegetables, in fishes, in blood, and in the different organs. The researches of Danilewsky appear to be of a nature to give an answer to this question, hitherto unexplained. According to this writer, rennet is a reversible enzyme capable of a synthetic activity, and its rôle, from the physiological point of view, must consist in the formation of albuminoid reserves, not diffusible and not soluble, by means of the products of the hydrolysis of albuminoid material, which products are soluble and diffusible. Moreover, these nutritive reserves could re-enter into the circulation without the new intervention of enzyme solely by a slight change in the reaction of the medium. Plasteins would thus be reserves of nitrogenous substances. They do not represent synthetic albuminoid substances, but rather substances which are easily assimilated and very suitable for this reconstruction.

This concept of Danilewsky as to the rôle of rennet in the organism is not exaggerated. We can even enlarge it. In fact, we have previously seen that pepsin, trypsin, the extracts of organs, etc., possess the same property as rennet. On the whole, all the proteolytic enzymes contribute or can contribute to the 
precipitation and the condensation of the products of hydrolysis. We can then say that in the transformation of nutritive albumin into living albumin, the formation of plasteins plays a predominant rôle. However - and this is the weak point in the whole of the preceding argument-this hypothesis assumes that the reconstruction of living albumin, or that appropriated by the organism, is made with the products of a slightly advanced hydrolysis, that is to say, with albumoses and peptones; substances at the expense of which plasteins are formed. Now these data do not at all accord with our present knowledge on digestion. From the work of Abderhalden and others, it appears that the final products of hydrolysis, products which are no longer capable of giving plasteins, are still very suitable to the reconstruction of living albumin. Furthermore, hydrolysis in the organism is much more advanced than that produced in vitro, because of the presence in the organism of erepsin, and even of amidases, which completely destroy the albuminoid molecule and lead to products very simple in composition.

If then, in the reconstruction of albuminoids enzymes really play a part, it cannot be the digestive enzymes such as pepsin and trypsin, but rather erepsin and the amidases, which apparently must have condensing properties like the preceding, and be, in addition, capable of reacting on the final products of hydrolysis. However, we do not possess at the present time data on the synthetic rôle of erepsin and the amidases.

\section{BIBLIOGRAPHY.}

\section{ON THE REVERSIBLE REACTIONS OF ENZYMES.}

OKunEw. Dissert., St-Petersbourg, 1897 .

Lawrow. Dissert., St-Petersbourg, 1897.

Sawjalow. Eiweissverdauung, Pfiig. Arch., rgor, (85), p. I7I.

LaWrow et SalaSkin. Zeits. f. physiol. Chem., I902, (36), p. 277; 1907, (51), p. I. KuRAJEW. Hofm. Beitr., IgOI, (1), p. I2I; ' I902, (2), p. 4II; I903, (4), p. 476.

Herzog. Zeits. f. physiol. Chem., I903, (39), p. 305.

Nürenberg. Beitr. z. Chem. Physiol. u. Path., 1904, (4), p. 543.

TAYLOR. Journ. of Biol. Chem., 1907, (3), p. 95.

Lawrow. Zeits. f. physiol. Chem., 1907, (53), p. I. 
Delezenne et Mouton. Coagul. de sol. conc. peptone par suc pancréatique activé par Ca, Soc. Biol., 1907, (2), p. 277.

Gerber. Actions comparées des présures végétales sur la peptone et la caséine, Soc. Biol., 1909, (I), p. II 22.

Sacharow. Bioch. Central., I903, (I), p. 233.

Bayer. Hofm. Beitr., I903, (4), p. 555 .

Herrmann u. ChanN. Zeits.f. physiol. Chem., rgr 2, (77), p. 289.

Robertson. Journ. of Biol. Chem., (3), p. 95; (5), p. 493.

Rakoczy. Zeits.f. physiol. Chem., I9I I, (75), p. 273.

JАСОВY. Bioch. Zeits., Ig08, (10), p. 23 X.

Levene et Van Slyke. Bioch. Zeits., 1908, (13), p. $46 \mathrm{r}$.

Robertson and Bidde. Journ. of Biol. Chem., I9I I, (9), p. 295.

Henriques et Gjaldbaex. Zeits. f. physiol. Chem., I9II, (71), p. 485; I9I2, (8I), p. 456.

Hugo Kömmerer. Immunitäls Forschung. u. experim. Therap., 19i1, (II), p. 235.

Croft Hill. Journ. Chem. Soc. London, 1898, (73), p. 634; I903, (83), p. 578.

Emmerding. Synthetische Wirkung der Hefemaltase, Ber. d. Deuts. Chem. Ges. I90I, (34), pp. 600,2206 et 3810.

Potrevin. Sur la réversibilité de l'action lipolytique, $C . R$., I903, (I36), pp. 767 et II 52. - Actions diastasiques réversibles, Ann. Inst. Past., 1906, (20), p. 901 .

HaNRIOT. C. $R$., I9OI, (I) p. 146.

Bourquelot. Synthèse des glucosides avec l'émulsine, Rev. Scient., I9r3, p. I; Confér., Soc. Chim., I9r3.

Messerli. Bioch. Zeits. (54.2) p. 446, I913. 
PART III.

TRYPSIN. 



\section{CHAPTER I.}

\section{PANCREATIC TRYPSIN.}

\section{$\S$ I. Distribution and Preparation.}

Distribution. - Trypsin is the proteolytic enzyme of the pancreatic juice. It acts on the albuminoid material in the same way as pepsin, but its action is distinguished by the two following fundamental characteristics: (I) Tryptic hydrolysis is more advanced, since it results in the production of amino-acids. (2) The maximum result is obtained in a neutral or slightly alkaline medium.

The first data on the enzymes of the pancreatic juice were furnished by $\mathrm{Cl}$. Bernard in 1855 and Corvisart in $185^{8}$. It is to the latter that we owe the name of pancreatin, given to the active constituents of the pancreatic juice. Danilewsky, in 1862, isolated from a maceration of pancreas three enzymes, one amylolytic, a second proteolytic, and finally a third, capable of emulsifying and of saponifying fatty substances. Kühne, in I867, first drew attention to the difference which exists between the chemical action of pepsin and that of the pancreatic enzyme. He showed the production, in this second action, of tyrosin and leucin, and also of those ill-defined residues from the hydrolysis of albuminoids which he called anti-peptones. He then characterized this proteolytic enzyme as a new enzyme, distinct from pepsin, and gave to it the name of trypsin.

Trypsin is very widely distributed in the animal as well as the vegetable kingdom. Trypsins, or very similar active substances, are found not only in the pancreas but also in the spleen, the leucocytès, and the urine. Its presence has been detected in invertebrates, crustacea, and insects. Finally, similar enzymes are found in the beer yeast, in molds, and in bacteria. All these enzymes are not completely identical with trypsin, but they, 
however, approach each other through a certain number of common characteristics which place them in the same family.

Preparation: Method of Danilewsky. - Fresh pancreas, preferably that of hog, is crushed in a mortar with gravel and water. It is allowed to settle and the decanted liquid is filtered through a sieve. To the filtrate magnesia is added, forming a precipitate which carries down the amylase and other impurities. After filtration the liquid, which contains trypsin free from saccharifying enzyme, is acidified with phosphoric acid and then neutralized with lime. The calcium phosphate formed, which partially carries down with it the lipase, is filtered off and the filtrate, rich in trypsin, is evaporated in a vacuum. The separation of trypsin and amylase can also be accomplished with the aid of collodion diluted with an equal volume of a mixture of alcohol and ether. After the addition of collodion, the pancreatic liquid is stirred, the precipitate formed carrying with it the trypsin, while the amylase remains in solution.

Method of Kïhne. - The pancreas, cut into small pieces, is first macerated in alcohol, and is then treated with ether. . The dried product is then kept four hours in water at $40^{\circ}$, with addition of o.I per cent salicylic acid. It is thrown in a sieve and the residue, separated as far as possible from the impregnating liquid, is diluted with a solution of 0.25 per cent $\mathrm{Na}_{2} \mathrm{CO}_{3}$. The residue is left for twelve hours at $40^{\circ}$ and then is again drained. To the liquid obtained mixed with that coming from the preceding filtration, is added sufficient $\mathrm{Na}_{2} \mathrm{CO}_{3}$ to bring the content in this salt up to 0.5 per cent. An antiseptic having been added, the liquid is then left to auto-digest, until a complete or nearly complete disappearance of the albumoses has resulted. The digestion ended, the liquid is put on ice for twenty-four hours and then is filtered. The solution obtained is saturated with ammonium sulphate which precipitates the trypsin. The preliminary digestion was for the purpose of peptonizing the protein substances as completely as possible, in order that they might not also precipitate with ammonium sulphate.

Kühne has also developed a quicker method. To remove the 
albuminoids as far as possible, he seeks to render them insoluble. It is necessary first of all to macerate the pancreas with water at a temperature near $0^{\circ}$. In the filtrate, the trypsin is precipitated with absolute alcohol. The precipitate obtained is washed for some time with absolute alcohol, and then again with water. The mass is acidified with acetic acid until a concentration of I per cent is reached, and is filtered. The clear solution is brought to $40^{\circ}$, filtered, made slightly alkaline with $\mathrm{Na}_{2} \mathrm{CO}_{3}$, again filtered, and then is evaporated at $40^{\circ}$. During evaporation the tyrosin separates out, and is eliminated. The liquid is dialyzed, and then concentrated.

Method of Wittich. - The pancreas, previously washed in water, is crushed in absolute alcohol. After repeated washings in alcohol, the dry product is treated with water and glycerin. The digestion lasts two or three days. It is filtered and alcohol is added to the liquid. The precipitate obtained is again dehydrated with alcohol, put to digest in glycerin, and finally the solution is precipitated with alcohol.

Method of Stutzer. - The following is the method of Stutzer by which a solution of trypsin can be prepared for laboratory uses: Calf pancreas is used that is free from fats and has been passed through a meat grinder. The mass, with addition of sand, is crushed in a mortar, where it is left for twenty-four hours in order to permit the transformation of zymogen into trypsin. Then there is added, for one part of ground mass (without sand), three parts of water made alkaline with lime water and one part of glycerin. After three or four days, the mixture is filtered through flannel and pressed; the juice escaping is filtered and chloroform is added. This liquid, which is of neutral reaction, keeps very well. The addition of lime is to saponify the remaining fats, and also to destroy the lipase which causes the turbidity of the liquid. This addition must be very moderate for an excess may considerably weaken the trypsin.

Pancreatin. - The products obtained by the preceding methods are more or less well separated from the other enzymes that accompany the proteolytic enzyme in the pancreas. In 
addition to trypsin, we also use in therapeutics pancreatin, which contains all the enzymes of the gland. To prepare this pancreatin, the following procedure is adopted. Calf pancreas, isolated twenty-four hours after the death of the animal, is separated from fats and surrounding tissues, minced in small pieces, and then is introduced into 90 per cent alcohol. After twenty-four hours it is decanted, the liquid is replaced by absolute alcohol, and is allowed to stand twenty-four hours. The liquid is then removed and ether added. After some time, the well dried product is reduced to powder and sifted. I gram of this powder can digest in 24 hours, under favorable conditions, Io to 5 grams of Hammarsten casein.

On the other hand, the following is the method by which, according to the French pharmacopœia, officinal pancreatin is prepared (Codex 1895): "Take one part of pancreas and two parts of water. The pancreas, free from the foreign tissues which accompany it, is cut up and suspended in water, a little chloroform being added in order to prevent deterioration. After a short contact, the insoluble residue is separated, pressed, and the liquid obtained is filtered. This is received in vessels offering a large surface and is rapidly evaporated in a current of air at a temperature which does not exceed $45^{\circ}$." The indefinite character of these directions is to be regretted. However, the pancreas used is generally that of the hog. The organs must be taken from animals in the act of digesting, for it is at this moment that the pancreas is the most active, and the pancreas so obtained must be utilized as soon as possible. The duration of the maceration can be fixed at six hours and the evaporation, according to Javillier, is best made in a vacuum at $0^{\circ}$.

Test for Pancreatin. - The pharmaceutical pancreatin is in the form of a powder or of yellowish scales; it is incompletely soluble in water. It possesses the triple power of hydrolyzing the starchy compounds, the fatty compounds and the albuminoids. The test for pancreatin given in the French Codex of 1908 is as follows:

(x) To insure its power of action on protein materials, take: 
Pancreatin: $0.20 \mathrm{~g}$.

$\left.\begin{array}{l}\text { Distilled water: } 60 \mathrm{~g} . \\ \text { Dried fibrin: } 2.50 \mathrm{~g} .\end{array}\right\} \quad$ or else $\quad\left\{\begin{array}{l}\text { Distilled water: } 5^{2.5} \mathrm{~g} . \\ \text { Dried fibrin: ro } \mathrm{g} .\end{array}\right.$

In a water-bath or an oven heated to $50^{\circ}$, place a flask containing the dried fibrin and the water and allow it to digest for half an hour. Add the pancreatin and continue the digestion for 6 hours, taking care at the beginning to shake the mixture quite frequently until a complete solution of the fibrin has been effected, and then about every hour. Filter. Io c.c. of the liquor thus obtained should not become turbid at ordinary temperature upon the addition of 20 drops of laboratory nitric acid (mixture of about I mol. $\mathrm{HNO}_{3}$ with 2 mol. $\mathrm{H}_{2} \mathrm{O}$ ).

(2) To insure the power of action of pancreatin on starchy substances, prepare roo g. of starch paste containing $5 \mathrm{~g}$. of potato flour. Add $0.05 \mathrm{~g}$. of pure pancreatin, heat to $55^{\circ}$ on a water-bath or in the oven, and maintain this temperature for an hour, shaking from time to time. One should thus obtain a liquid, easily filtered, which reduces four times its volume of titrated ammonical copper solution at boiling temperature.

These directions of the Codex necessitate a few remarks. First of all, tryptic digestion takes place in neutral medium, and it leads to compounds strongly biuretic. To make the biuret test, take about 2 c.c. of liquid and add about 0.5 c.c. of concentrated $\mathrm{NaOH}$ and a few drops of a 2 per cent solution of copper sulphate. A beautiful purple violet coloration is observed.

The amylolytic power required by the Codex is quite high. That required in the following test is much weaker. To roo c.c. of a 6 per cent starch jelly add o.r g. of pancreatin, and allow it to stand for 6 hours at $50^{\circ}$. 2.5 c.c. of the filtered liquid, which does not color with iodine, should be able to reduce ro c.c. of standard Fehling solution.

Finally, in the Codex test there is no allusion to the saponifying power of pancreatin. To inform ourselves in this respect, we could allow the powder examined to act on monobutyrin and determine the acidity formed at the end of a certain time, according to the suggestions of Hanriot. An emulsion of fat could also be used. 


\section{$\S$ 2. Characteristic Products of Tryptic Digestion.}

Trypsin very quickly attacks fibrin and digests serum-albumin quite well, though it acts much less quickly on egg-albumin. Peptic and tryptic digestions are distinguished by the reaction of the medium and the extent of the hydrolysis produced. While pepsin reacts only in acid media, trypsin reacts in all the media, but especially in one made slightly alkaline, corresponding to a content of 0.5 per cent of $\mathrm{Na}_{2} \mathrm{CO}_{3}$. In the effect produced by trypsin, tyrosin is detached almost from the beginning from the albuminoid molecule, while in peptic digestion, the tyrosin nucleus is not separated but remains combined in the form of polypeptides. Furthermore, among the early products of tryptic digestion are tryptophane, leucin, and other crystalline amino-acids. Trypsin, like pepsin, dissolves coagulated albumin and fibrin. The first compounds formed, the primary and secondary albumoses, are identical in the two digestions. Also it is very difficult to distinguish a tryptic action from a peptic action by the products of a partial hydrolysis, but the diagnosis, as we shall see, becomes much easier in the case of a more advanced hydrolysis.

Tryptic digestion can easily be followed by the aid of nitric acid. For the tests are required: $20 \mathrm{~g}$. of dried fibrin, $0.5 \mathrm{~g}$. of trypsin and $100 \mathrm{~g}$. of water. Add 5 drops of chloroform and allow the whole to digest at $50^{\circ}$. The solution of fibrin takes place gradually, and without previous swelling. From time to time a sample is taken and filtered. In ro c.c. of clear liquor add a few drops of nitric acid. After five to eight hours of action, a point is generally reached where the acid no longer produces a precipitate or turbidity in the liquid tested.

Crystallization of Tyrosin and Characterization by Means of Tyrosinase. - In such a liquid, not precipitating with nitric acid, tryptic action can be identified by the tyrosin formed. The tyrosin can be detected in two ways: (I) By crystallization; (2) by identification by means of tyrosinase.

In the products of advanced digestion, crystals of tyrosin are 
found at the bottom of the receivers in the form of fine needles grouped in silky little tufts. In the less degraded products, this body can be detected by the addition to the filtered liquor of 5 to to volumes of alcohol. Upon filtering, washing in alcohol, and evaporating the clear liquid, crystals may be obtained. For this experiment, very little liquid must be used and the entire crystallization can be easily followed under a microscope.

The identification of tyrosin can be still more easily accomplished by means of tyrosinase. Bertrand, in studying the chromogen of Russula negricans, found that the red coloration turning to black which appears on broken mushrooms, comes from the action of an oxidizing enzyme, tyrosinase, on tyrosin. The oxidation and the coloration of juice of beet, of potato, and of dahlia, have also been referred to the same cause. Tyrosinase is, then, very sensitive and its application in the identification of a tryptic digestion has been attended with success by Bourquelot and Harlay.

To prepare the solution of tyrosinase Russula delica is used. These mushrooms are crushed with gravel and glycerin. They are left to macerate an hour, and then filtered. Thus a glycerin solution is obtained which contains tyrosinase in a good state of preservation. For the tyrosin test, pour into a reaction tube the digestion liquid which no longer precipitates with nitric acid, then add 5 to 6 drops of the glycerin solution of tyrosinase. The liquid assumes, in a few minutes, a red coloration which turns black, becoming opaque after two or three hours. For the test, it is better previously to neutralize the liquid. Coloration begins in the upper portions, in contact with the air, and progressively descends in the tube. In the products of a peptic digestion we do not get a similar coloration; the reagent gives a red tint, which then passes to a green shade.

Reaction of Tryptophane. - Harlay also recommends the use of tryptophane to identify a tryptic digestion. Several drops of saturated bromine water, added to the liquids resulting from a pancreatic hydrolysis, cause the formation of a reddish yellow 
precipitate which is redissolved by shaking with the production of a violet rose tint. Five c.c. of the liquid obtained in the laboratory test of pancreatin with fibrin are colored a distinct pink by 5 drops of bromine water; 20 to 30 drops give a deep violet red color; beyond 35 drops, the color becomes more dull and the liquid becomes turbid; beyond 60 drops, a violet brown precipitate forms which floats in a yellow liquid. This reaction can be identified only by adding the bromine water little by little. If this addition is made suddenly, the violet tint passes unperceived. This reaction is very characteristic, for with peptic digestions no red coloration is obtained whether the liquid be acid or neutral; at most, there results only a yellow coloration or precipitate. This reaction had been already noted, in $183 \mathrm{I}$, by Tiedeman and Gmelin in the action of chlorine water on the pancreatic juice of a dog. Cl. Bernard, Kühne, and others vainly sought the compound responsible for the red coloration observed. It was Neumeister who showed, in 1889 , that the chromogen was a new compound, which he called tryptophane, and which is produced in all the processes involving the disintegration of albuminoids, such as tryptic digestion, putrefaction, or the action of hot acids or of alkalis. It always accompanies tyrosin and from its presence one can assume that of tyrosin.

\section{§ 3. Effect of Temperature and Other Physical Agents.}

Trypsin, in a dry state, endures very well the effect of a moderate heating. Harlay, by the aid of tyrosinase proved that trypsin, which has been brought to $100^{\circ}$, still digests fibrin very easily with the rapid production of tyrosin. Hufner has found the same result for pancreatin. According to Schmidt, trypsin can be heated for an hour and a half at $112^{\circ}$ without destroying the active substance. Salkowski even finds that dry trypsin resists the temperature of $160^{\circ}$ for an hour and a half, but that it is destroyed at $170^{\circ}$.

On the contrary, in solution trypsin shows itself very sensitive 
to the action of heat. This sensitiveness, according to Schmidt, is in direct relation with the purity of the products. The more foreign matter the trypsin contains, the less it is weakened by heating. Colloidal substances, such as albumoses and peptones, increase its resistance. A 5 per cent peptone solution, with addition of trypsin, can be brought to boiling point without losing entirely the power of digesting fibrin. A similar resistance is conferred by agar-agar and gelatin. The resistance to heat, under these circumstances, is, however, of little duration and a boiling of five minutes effects the complete destruction of the active substance.

Dilute solutions of trypsin or of pancreatic juice cannot be kept at temperatures above $38^{\circ}$ without losing a part of their activity. Roeder found that solutions kept at $40^{\circ}$ weaken very perceptibly.

Effect of Heat on Very Dilute Solutions.

\begin{tabular}{|c|c|}
\hline $\begin{array}{l}\text { Duration of previous } \\
\text { heating at } 40^{\circ} \text {. }\end{array}$ & $\begin{array}{l}\text { Quantity of enzyme necessary } \\
\text { to digest } 2 \text { c.c. of a solution } \\
\text { of casein at } 38^{\circ} \text {. }\end{array}$ \\
\hline $\begin{array}{l}\text { At the beginning... } \\
\text { After } 5 \text { minutes.... } \\
\text { After ro minutes... } \\
\text { After } 30 \text { minutes... }\end{array}$ & $\begin{array}{c}\text { c.c. } \\
0.65 \\
0.65 \\
\text { I. } \\
\text { With I c.c., no digestion }\end{array}$ \\
\hline
\end{tabular}

We see that the solutions of trypsin, maintained 5 minutes at $40^{\circ}$, react like those kept at the ordinary temperature. But the effect of a heating at $40^{\circ}$ for Io minutes is a very perceptible diminution in activity, since to digest 2 c.c. of the solution of casein used requires I c.c. of enzyme, as compared with the $0.6_{5}$ necessary at the beginning.

These results are obtained with very dilute solutions of enzyme. I per cent or 2 per cent solutions of trypsin, however, withstand a temperature of $40^{\circ}$ for two hours without deterioration, although they are weakened by heating to $50^{\circ}$. The following are some data relative to this prolonged action of a temperature of $50^{\circ}$ on a 2 per cent solution: 
Action of Heat on $2 \%$ Solutions.

\begin{tabular}{|c|c|c|}
\hline $\begin{array}{c}\text { Temperature of } \\
\text { previous heating. }\end{array}$ & $\begin{array}{c}\text { Duration of the } \\
\text { previous heating. }\end{array}$ & Enzyme activity. \\
\cline { 2 - 3 } Degrees. & Minutes. & Units. \\
40 & 60 & 100 \\
50 & 15 & 100 \\
50 & 30 & 70 \\
50 & 60 & 60 \\
50 & 90 & 50 \\
\hline
\end{tabular}

Consequently, a similar solution of trypsin kept for $\mathrm{I} \frac{1}{2}$ hours at $50^{\circ}$ weakens considerably, since it loses half of its activity. According to Biernacki, solutions of trypsin that are 0.25 per cent in $\mathrm{NaOH}$ show themselves still more sensitive to the thermal action than neutral solutions. In the presence of this alkalinity at the temperature of $50^{\circ}$, enzyme solutions very rapidly lose all proteolytic power.

The optimum temperature for the action of trypsin, in the tests of a duration of $\frac{1}{2}$ hour to I hour, is found between $50^{\circ}$ and $55^{\circ}$. In tests of longer duration it is found in the neighborhood of $40^{\circ}$. The influence of temperature on the course of the digestion in a relatively short time is summarized in the following table, giving the results of a test on casein, analyses being made by the Fuld method:

Influence OF Temperature ON THE SOlution OF CASEIN.

\begin{tabular}{|c|c|}
\hline Temperature. & $\begin{array}{c}\text { Quantity of } \\
\text { enzyme in c.c. }\end{array}$ \\
\hline Degrees. & 0.65 \\
38 & 0.40 \\
40 & 0.25 \\
48 & 0.15 \\
52 & \\
\hline
\end{tabular}

At a temperature of $52^{\circ}, 2$ c.c. of casein are digested with 0.15 c.c. of a tryptic solution, while at $38^{\circ}$ it takes 0.65 c.c. of the same solution, or four times as much of active liquid, to produce the same effect. 
The influence of temperature appears quite different when it is a case of peptonization of long duration. To a series of bulbs containing roo c.c. of a 2 per cent solution of casein are added equal quantities of trypsin, and the mixtures are left to digest for ten hours at different temperatures. At the end of this time, the casein remaining is precipitated by acetic acid, is filtered, and the nitrogen in solution is determined.

INFLUENCE OF TEMPERATURE ON THE

Peptonization of Casein.

\begin{tabular}{|c|c|}
\hline Temperature. & Nitrogen digested. \\
\cline { 2 - 2 } Degrees. & mg. \\
38 & 255 \\
40 & 240 \\
52 & 160 \\
\hline
\end{tabular}

At the temperature of $52^{\circ}$, only $160 \mathrm{mg}$. of nitrogen have been dissolved, as compared with the $225 \mathrm{mg}$. obtained at $38^{\circ}$. The optimum temperature, under these conditions, is therefore between $38^{\circ}$ and $40^{\circ}$.

Other Physical Agents.- As regards the other physical agents, their effect is found to be practically the same on trypsin as on pepsin. According to Loewenthal and Edelstein, radium emanation favors the autolysis of organs. This influence on trypsin had already been noted by Bergelli and Bickel who, in 1906, recognized that emanation perceptibly activated peptonization. Both trypsin and pepsin are absorbed by different substances, notably by edestin. According to $\mathrm{H}$. L. Holzberg, safranin combines with trypsin to give an insoluble red precipitate. This property may even be utilized to free a liquid from the trypsin it contains. For this purpose add about an equal volume of a 0.8 per cent solution of Grübler safranin; the precipitate which is formed shows great activity while the filtered liquid has lost all its digestive power. 


\section{§ 4. Influence of the Reaction of the Medium.}

Influence of Acids. - Loeb explains the influence of the reaction of the medium by the chemical nature of the enzyme. According to this writer, the conditions favorable to the action of the enzyme are those which facilitate its ionization. Now, the salts formed by the union of a strong acid and a strong base are just those which most strongly undergo electrolytic dissociation. Trypsin, having according to this writer an acid character, combines with strong bases to form salts easily dissociated and consequently much more active.

Trypsin, in general, shows itself much more independent of the reaction of the medium than pepsin. A tryptic digestion can be obtained as well in a neutral or alkaline solution as in a slightly acid solution, containing 0.02 per cent $\mathrm{HCl}$, for example. However, it is generally considered that the maximum activity is realized in the presence of 3 to 4 grams of $\mathrm{Na}_{2} \mathrm{CO}_{3}$ per liter. Yet, according to Kudo, the digestion of casein by trypsin would occur much better in a neutral medium. He finds that a neutral solution of casein, with addition of neutral trypsin, furnishes, after 60 minutes of digestion, the maximum of dissolved nitrogen. The presence of acid or alkali is apparently injurious. The effect of acids, as far as the digestion of casein is concerned, is as follows:

Effect of Acids on the Tryptic Digestion of Casein.

\begin{tabular}{|c|c|c|}
\hline Acids. & $\begin{array}{l}\text { Quantities which } \\
\text { begin to retard. }\end{array}$ & $\begin{array}{l}\text { Quantities which } \\
\text { very plainly retard. }\end{array}$ \\
\hline 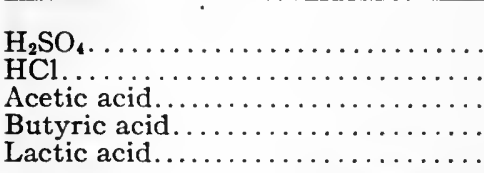 & $\begin{array}{l}\text { g. per liter. } \\
0.016 \\
0.023 \\
0.052 \\
0.05 \\
0.05\end{array}$ & $\begin{array}{c}\text { g. per liter.] } \\
0.045 \\
0.033 \\
0.07 \\
0.08 \\
0.06\end{array}$ \\
\hline
\end{tabular}

The acid may exert its destructive action very rapidly. Thus, a solution of trypsin to which $0.5 \mathrm{~g}$. per liter of sulphuric acid has been added, when immediately neutralized, has already lost a considerable portion of its digestive power. If the acid is 
allowed to act for an hour and then is neutralized, an almost complete destruction of the enzyme is the result. Hydrochloric acid acts in the same manner, but requires greater concentrations. The organic acids, such as butyric acid, at a concentration of $3 \mathrm{~g}$. or $4 \mathrm{~g}$. per liter, exert no action even after three hours. These solutions, after neutralization, again show great activity. Lactic acid acts even less strongly than butyric acid.

Influence of Alkali. - Trypsin is, however, much less sensitive to the action of alkali. A solution of trypsin withstands very well for an hour a concentration of $\mathrm{Na}_{2} \mathrm{CO}_{3}$ as high as $7 \mathrm{~g}$. per liter. When neutralized, it still possesses the same degree of activity. It is only after a long time - after 5 or 6 hours - that a weakening is observed; this is, moreover, also observed with a much weaker dose of alkali, I.4 g. per liter. Peptonization in the presence of an alkali gives the following results:

Effect of Algkali on Tryptic Digestion of Casein.

\begin{tabular}{|c|c|c|}
\hline Alkali. & $\begin{array}{l}\text { Quantities of alkali } \\
\text { which begin to retard. }\end{array}$ & $\begin{array}{l}\text { Quantities which } \\
\text { plainly prevent. }\end{array}$ \\
\hline 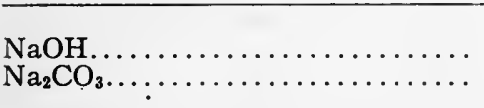 & $\begin{array}{l}\text { g. per liter. } \\
0.143 \\
0.9\end{array}$ & $\begin{array}{l}\text { g. per liter. } \\
\text { O. } 677 \\
\mathrm{I} .3\end{array}$ \\
\hline
\end{tabular}

It, moreover, must be noted that the reaction of the medium influences differently the dissolving power and the peptonizing power of trypsin. Fernbach and Schoen have studied the progress of the solution and the hydrolysis of albumin and fibrin with relation to the reaction of the medium, the latter being varied by the use either of monopotassium phosphate, which is known to be neutral to methyl orange, or dipotassium phosphate, which is alkaline toward this indicator.

This table, relative to the hydrolysis of fibrin; shows that dipotassium phosphate, that is to say, an alkaline reaction to methyl orange, while favoring a greater dissolving action than monopotassium phosphate, is much less favorable to an extensive degradation; in other words, by working in a medium neutral to 
Influence of the Reaction on the Tryptic Digestion of Fibrin.

\begin{tabular}{|c|c|c|c|}
\hline Temperature. & $\begin{array}{l}\text { Length of } \\
\text { digestion. }\end{array}$ & $\begin{array}{l}\text { Nitrogen in } 100 \text { c.c. of } \\
\text { filtered liquid } \\
\text { (in milligrams). }\end{array}$ & $\begin{array}{l}\text { Nitrogen not precipitable } \\
\text { by tannin (per cent of } \\
\text { dissolved nitrogen). }\end{array}$ \\
\hline Degrees. & Hours. & & \\
\hline 40 & 8 & $\left\{\begin{array}{lr}\text { Mono } & 42.7 \\
\text { Di } & 102.2\end{array}\right.$ & $\begin{array}{l}31.1 \\
27.4\end{array}$ \\
\hline 45 & 8 & $\left\{\begin{array}{lr}\text { Mono } & 42.0 \\
\text { Di } & 108.5\end{array}\right.$ & $\begin{array}{l}36.66 \\
29.67\end{array}$ \\
\hline 50 & 8 & $\left\{\begin{array}{lr}\text { Mono } & 47.0 \\
\mathrm{Di}^{2} & 121.7\end{array}\right.$ & $\begin{array}{l}43 \cdot 5 \\
20.7\end{array}$ \\
\hline
\end{tabular}

the indicator used, the percentage of substance which passes to the state of amino-amide nitrogen, not precipitable by tannin, is much higher.

Abderhalden and Koelker have studied more closely the influence of the reaction of the medium on the pancreatic juice activated by the intestinal juices. First of all, they found that Io c.c. of active juice, when neutralized with 8 c.c. $N /$ ro NaOH after 5 minutes contact with 8 c.c. $N /$ ro $\mathrm{HCl}$, loses none of its proteolytic power. The influence of sodium hydroxide was studied in the case of the decomposition of the polypeptides, which are known to be hydrolyzed by trypsin. These substances, in solution, rotate the plane of polarized light and, as the result of their decomposition, this rotatory power varies, thus permitting the progress of the reaction to be followed. The following are the results of two experiments made with solutions of glycyl-1-tyrosin, one at the concentration $\frac{3}{34} \mathrm{~mol}$, , the other at $\frac{3}{32} \mathrm{~mol}$. 
Influence of NaOH on Tryptic Hydrolysis of Glycyl-L-tyrosin. First solution of glycyl-1-tyrosin [r.64\%].

\begin{tabular}{|c|c|c|c|c|}
\hline Time. & \multicolumn{4}{|c|}{ 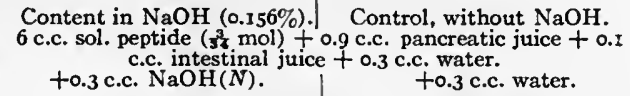 } \\
\hline $\begin{array}{c}\text { Minutes. } \\
7 \\
85 \\
163 \\
205 \\
275\end{array}$ & $\begin{array}{r}\text { Degrees. } \\
+0.73 \\
+0.57 \\
+0.43 \\
+0.36 \\
+0.20\end{array}$ & & & $\begin{array}{l}\text { Degrees. } \\
+0.64 \\
+0.54 \\
+0.40 \\
+0.37 \\
+0.33\end{array}$ \\
\hline \multicolumn{5}{|c|}{ Second solution of glycyl-1-tyrosin [1.44\%]. } \\
\hline Time. & $\begin{array}{l}\text { Content in } \mathrm{NaOH} \\
(0.236 \%) \text {. } \\
4 \text { c.c. sol } \\
+0.37 \text { c.c. } \mathrm{NaOH}(N) \\
+1.23 \mathrm{H} \text {. }\end{array}$ & $\begin{array}{r}\text { Content i } \\
(0.35 \\
\text { peptide }( \\
\text { creatic act } \\
+0.55 \mathrm{~N} \\
+1.05 \mathrm{H}\end{array}$ & $\begin{array}{l}\mathrm{NaOH} \\
\% \text { mol.) }+ \\
\text { mated jui } \\
\mathrm{OH}(N)\end{array}$ & $\begin{array}{l}\text { Control, without } \\
\mathrm{NaOH} \text {. } \\
.67 \text { c.c. } \\
\text { e. } \\
+ \text { r } .6 \mathrm{H}_{2} \mathrm{O} \text {. }\end{array}$ \\
\hline $\begin{array}{r}\text { Minutes. } \\
6 \\
15 \\
41 \\
174 \\
260 \\
378 \\
1428\end{array}$ & $\begin{array}{l}\text { Degrees. } \\
+0.73 \\
+0.75 \\
+0.64 \\
+0.60 \\
+0.54 \\
+0.43 \\
+0.31\end{array}$ & $\begin{array}{l}\text { Deg } \\
+c \\
+c \\
+c \\
+c \\
+c \\
+c \\
+c\end{array}$ & $\begin{array}{l}\text { ees. } \\
.80 \\
.81 \\
.80 \\
.76 \\
.73 \\
.53 \\
.49\end{array}$ & $\begin{array}{r}\text { Degrees. } \\
+0.59 \\
+0.57 \\
+0.48 \\
+0.40 \\
+0.38 \\
+0.23 \\
+0.09\end{array}$ \\
\hline
\end{tabular}

We see that the action of the $\mathrm{NaOH}$ is favorable in experiment (I) where the content in $\mathrm{NaOH}$ is $0.15^{6}$ per cent. But when this content reaches 0.236 per cent or $0.35^{\mathrm{I}}$ per cent, the decomposition of the polypeptide is manifestly less rapid than in the control test without $\mathrm{NaOH}$. It is to be noted that this proportion of $0.15^{6}$ per cent of $\mathrm{NaOH}$ corresponds, relative to the quantity of glycyl-l-tyrosin used, to 0.53 molecule of $\mathrm{NaOH}$, when, according to the chemical equation, to decompose the polypeptide and neutralize the acids liberated, would require two molecules. This last concentration, or 0.472 per cent of $\mathrm{NaOH}$, would be entirely disastrous to the action of trypsin acting on glycyl-1tyrosin at $\mathrm{I} .44$ per cent.

Influence of Salts. - Relative to the action of chemical agents on trypsin and pancreatin we possess very contradictory data, which result from the very different conditions under which 
the experiments have been made. Some take pancreatic juice free from enterokinase, consequently susceptible of being activated by traces of calcium; others work on a crude trypsin, having a greater or less content in impurities. Finally the tests refer to variable albuminoid substrates; this leads to results that are not at all comparable. H. R. Weiss makes his experiments with a pancreatin in powdered form prepared by the method of Kühne. He uses as substrate Hammarsten's casein, which must be digested in the presence of different quantities of salts, the results being referred to the number Ioo, corresponding to the control test without salt.

Effect of Salts on Pancreatic Digestion of Casein.

\begin{tabular}{|c|c|c|c|c|c|}
\hline Salts used. & $0.05 \%$ & $0.1 \%$ & $0.5 \%$ & $\mathbf{I} \%$ & $10 \%$ \\
\hline 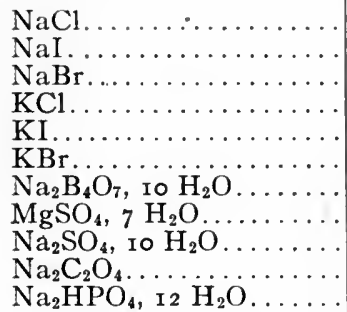 & 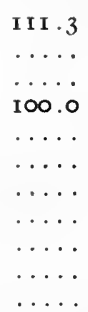 & $\begin{array}{r}\ldots \ldots .6 \\
99.6 \\
100.0 \\
\ldots \ldots .6 \\
99.6 \\
\ldots \ldots .7 \\
\ldots \ldots \\
98.5 \\
99.3 \\
108.2\end{array}$ & $\begin{array}{l}99 \cdot 5 \\
\ldots \cdots \\
\ldots \cdots \\
\cdots \cdots \\
\cdots \cdots \\
\cdots \cdots \\
\cdots \cdots \\
\cdots \cdots \\
\ldots \cdots \\
\ldots \cdots\end{array}$ & $\begin{array}{l}\ldots \cdots \\
\cdots \cdots \\
\cdots \\
\cdots \\
\cdots \cdots \\
\cdots \cdots \\
\ldots \ldots \\
97.0 \\
97.5 \\
97.15 \\
111.7\end{array}$ & $\begin{array}{r}87 \cdot 3 \\
96.0 \\
97.8 \\
98.5 \\
97.2 \\
94.4 \\
100.7 \\
89.9 \\
81.7 \\
\ldots \ldots .2 \\
110.2\end{array}$ \\
\hline
\end{tabular}

Sodium chloride, at a very small concentration, accelerates the activity of the trypsin, but at a higher concentration, it retards it. Disodium phosphate acts, as seen above, by its alkalinity; but its favorable action is limited to solution, without effecting peptonization. According to Kudo, who has experimented with solutions of trypsin, the alkaline phosphates have no action beyond change of the reaction of the medium which they determine. The nitrates and the nitrites have a slight retarding action. The chlorides, in concentrations higher than the preceding salts, inhibit digestion; and the sulphates act as inhibitors only at a still higher concentration. Furthermore, potassium salts generally retard hydrolysis less than those of sodium. Finally, cane sugar, milk sugar, and glucose are without effect. 
On the contrary, starch acts unfavorably, even in very small doses. Moreover, according to Zunz, amino-acids and peptides which do not activate pancreatic juice prevent the action of trypsin.

As previously stated, the varying results just mentioned indicate that the enzyme solutions on which they depend have been incompletely separated from the impurities which they contained. With regard to the effect of a sodium salt, for example, on the action of trypsin, it is not, properly speaking, the quantity which is put with the enzyme which intervenes, but rather the sum of that added with that already present. Now, the impurities of the various inactive substances which accompany the active substances of the pancreatic juice exert a marked activating action on the three enzymes of this juice. Thus it is that pancreatic juice, dialyzed with water, completely loses all amylolytic power, and, on the contrary, regains it when one adds salts, like $\mathrm{NaCl}$ or $\mathrm{Na}_{3} \mathrm{PO}_{4}$. Pancreatic juice would also lose by dialysis its property of becoming activated by addition of kinase. If such a dialyzed juice is examined, it is found that it still contains an enzyme capable of digesting casein or albumoses, as well as other protein substances having already undergone a preliminary attack, but that it is inactive toward albumin. This last enzyme is not trypsin but erepsin, that is to say, an impurity of pancreatic juice.

We see that the study of the influence of foreign substances on pancreatic juices is very complex. It is still more so by reason of the more or less easy activation by enterokinase, which the pancreatic juice possesses in the course of different diets, and also from the very fact that the products of digestion modify the activity of enzymes, as Terroine has shown in the case of the action of amino-acids on pancreatic amylase. We shall return later to this important question.

\section{§ 5. INFLUENCE OF ANTISEPtics.}

The examination of trypsin in the different organs, as well as in vegetables, often offers great difficulties by reason of the 
changes which liquids undergo during the experiments. The neutral or alkaline solutions become infected very quickly, and the results which are obtained in these circumstances are very doubtful. A dissolving of albumin or fibrin is not always proof of the presence of proteolytic enzyme, since it may also be attributed with reason to the action of bacteria. Even the negative results, a non-dissolving of the albuminoid, may also in certain cases be referred to bacteria which are known to be capable of destroying enzymes.

To avoid this great inconvenience, we often have recourse to antiseptics. However, this means is far from being certain. Contrary to current opinion, there is no antiseptic which acts on bacteria and is completely indifferent toward soluble enzymes. In reality, the action of antiseptics is exerted simultaneously on bacteria and enzymes and on the albuminoids contained in solution, so that the result obtained for a given quantity of antiseptic will depend on the relation existing between these three components. Furthermore, the quantity necessary to inhibit bacteria depends primarily on their species, their resistance from this point of view being variable. Finally, for the same species, the lethal dose may vary from I to ro according to the number of organisms contained in the liquid. In the determination of bactericidal doses of different substances, these very important points have seldom been taken into consideration. In addition, in the action of antiseptics on enzymes, account must also be taken of the fact that the sensitiveness of enzymes increases with their dilution. Whereas a concentrated solution may satisfactorily resist a given antiseptic, it is necessary, upon dilution, to diminish the concentration of this antiseptic, or else risk destroying the active substance. The demonstration can be made in the following manner: Solutions of trypsin of different concentrations, after being made slightly alkaline, are saturated with toluol, fibrin is immediately added to them, and the course of digestion is compared with that of controls without toluol. The following are the results for tests with toluol: 
Influence of Toluol on the Progress of a

Tryptic Digestion.

\begin{tabular}{|c|l|}
\hline $\begin{array}{c}\text { Concentration } \\
\text { in trypsin. }\end{array}$ & \multicolumn{1}{|c|}{ Effect of toluol. } \\
\hline mg. per cent. & No effect \\
$\mathbf{I} 2$ & Perceptible retardation \\
8 & Complete destruction \\
\hline 4 & \\
\hline
\end{tabular}

We see, then, that the solution containing $12 \mathrm{mg}$. per cent of trypsin withstands very well for 24 hours toluol to saturation. In this test, the same quantity of dissolved fibrin was found as in the control without toluol. On the contrary, the solution of $4 \mathrm{mg}$. per cent of trypsin does not resist at all the action of the toluol. It no longer digests fibrin, while the control, containing the same quantity of trypsin; still produces a vigorous digestion. In the preceding experiment the fibrin, added at the same time as the toluol, protects up to a certain point the enzyme from the destructive action of the antiseptic. A solution of trypsin saturated with toluol changes much more quickly when there is no albuminoid matter present, even for quite strong concentrations of enzyme.

Effect of Toluol on the Solutions of Trypsin OF VARIOUS CONCENTRATIONS.

\begin{tabular}{|c|c|}
$\begin{array}{c}\text { Concentration } \\
\text { in trypsin. }\end{array}$ & \multicolumn{1}{|c|}{ Action of toluol. } \\
\cline { 1 - 2 } $\begin{array}{c}\text { mg. per cent. } \\
80\end{array}$ & $\begin{array}{l}\text { Slight retardation } \\
\text { Complete destruction }\end{array}$ \\
\hline
\end{tabular}

In this second experiment solutions of trypsin, at concentrations of 80 and $20 \mathrm{mg}$. per cent, after saturation with toluol are kept for 24 hours at ordinary temperature. Then their enzyme power on fibrin is examined simultaneously with the control tests without toluol. As seen, solutions containing $20 \mathrm{~g}$. of trypsin are completely destroyed, while in the experiment given above, with a concentration of $12 \mathrm{mg}$., no injurious action was observed. It is curious to find that enzymes, from this point of 
view, act like bacteria. We know, in fact, that, if the quantity of substrate is increased in a culture, the bacteria are rendered much more resistant to the action of antiseptics.

This relation which exists between the destructive dose of antiseptics and the concentration of enzyme is also observed with substances other than toluol. The following are the concentrations of active trypsin which resist, are weakened, or are destroyed by three common antiseptics:

EfFect of Various Antiseptics on Solutions of Trypsin.

\begin{tabular}{|c|c|c|c|}
\hline . & $\begin{array}{l}\text { No antiseptic } \\
\text { action. }\end{array}$ & Weakening. & Destruction. \\
\hline $\begin{array}{l}\text { Saturated with chloroform........ } \\
\text { Saturated with thymol........... } \\
\text { Saturated with } 2 \% \text { NaF......... }\end{array}$ & $\begin{array}{l}\text { mg. per cent. } \\
200 \\
200 \\
80\end{array}$ & $\begin{array}{c}\text { mg. per cent. } \\
80 \\
\text { 100 } \\
60\end{array}$ & $\begin{array}{l}\text { mg. per cent. } \\
20 \\
20 \\
\ldots\end{array}$ \\
\hline
\end{tabular}

Thus solutions of trypsin, at a concentration of $200 \mathrm{mg}$. per cent, withstand saturating with chloroform or thymol. But at a concentration of 80 or of $100 \mathrm{mg}$. per cent, the chloroform or the thymol begins to weaken the trypsin. With an enzyme concentration of only $20 \mathrm{mg}$. per cent, saturating with chloroform or the thymol completely inhibits the enzyme. Of all the antiseptics, sodium fluoride appears to be the least injurious, only it must be noted that, according to Effront, this salt does not react strongly except in acid media, its action being less in a neutral, and exceedingly weak in alkaline media.

To summarize, we do not possess a sure and practical means of keeping a solution of trypsin protected from all infection for a length of time surpassing 6 to ro hours. If we use large quantities of antiseptic, we run a great risk of weakening or destroying the enzyme; if we are content with small doses, we get no preservative action. In this connection, we must also take into consideration the observation of Effront, according to which the bacterial spores, attenuated either by an antiseptic or by heat, show themselves the more productive of enzyme the more difficult their germination. Under certain conditions and in the presence 
of antiseptics, the spores may produce an intense secretion of enzyme in the liquid without, however, arriving at germination. It appears from all these facts that in the examination of trypsin, those methods must be renounced which require a prolonged exposure in the incubator. Only those which are expeditious, involving a duration of 3 to 6 hours at the most, will be capable of giving data that is worthy of confidence.

\section{§ 6. LAW of Action of Trypsin.}

We have recounted, with regard to the law of action of pepsin, the general laws of the action of enzymes on carbohydrates and have noted why, by reason of the non-homogeneity of the substrates, the law could not apply to the case of proteolytic enzymes. We have indicated, nevertheless, a law - that of Schütz and Borissow - relative to the action of pepsin on the albumin contained in Mett tubes. This law applies equally well to trypsin. The velocity of digestion, or the lengths of albumin dissolved, are proportional to the square root of the quantities of enzyme. Or again: The quantities of enzyme are proportional to the squares of the lengths of albumin dissolved. In other words, if the quantities of enzyme are as $1,4,9,16$, the lengths of albumin dissolved will be as I, 2, 3, 4, respectively. However, all writers are not agreed on the exactitude of this law. Bayliss, and then Hedin, have found that at the beginning of digestion and for small quantities of trypsin, the effect produced is proportional to the quantity of enzyme and to the time. It is therefore the general law which is verified under these special conditions.

If we examine now the influence of the quantity of substrate on the rapidity of digestion, we see that the velocity of digestion relative to the unit of substrate increases when the total quantity of the latter diminishes, and that finally it becomes constant. Hedin, by causing trypsin to act on a solution of casein, found that the proportionality between the time and the quantity of 
enzyme is no longer manifest if the digestion takes place in the presence of fresh egg-albumin.

INfluence of Fresh EgG-Albumin on the Action of TRYPSIN ON Casein.

\begin{tabular}{|c|c|c|c|}
\hline \multirow{2}{*}{$\begin{array}{l}\text { Quantity of } \\
\text { trypsin in c.c. }\end{array}$} & \multirow{2}{*}{$\begin{array}{l}\text { Duration of } \\
\text { action. }\end{array}$} & \multicolumn{2}{|c|}{ Casein transformed. } \\
\hline & & $\begin{array}{c}A, \text { Sol. of } \\
\text { casein alone. }\end{array}$ & $\begin{array}{c}\text { B, Sol. of } \\
\text { casein with } \\
\text { add. albumin. }\end{array}$ \\
\hline c.c. & hrs. & mg. & mg. \\
\hline 30 & 3 & $27 \cdot 5$ & $21 \cdot 3$ \\
\hline I 5 & 6 & 27.6 & 17.25 \\
\hline Io & 9 & $27 \cdot 5$ & 13.18 \\
\hline
\end{tabular}

In the series A, with 30 c.c. of trypsin in 3 hours the same quantity of casein was dissolved as with ro c.c. in 9 hours. There is, then, a proportionality between the effect produced on the one hand, and the quantity of enzyme and the time on the other. In series B, the albumin added to the casein has not undergone transformation, but it has brought about a retardation of the digestion of casein, this retardation being all the more sensitive the poorer liquid being digested is in trypsin. Hedin explains this phenomenon as being due to the adsorption of trypsin by albumin, an adsorption which is, up to a certain point, proportional to the concentration of active substance in the liquid.

In the study of the law of enzyme actions, consideration must be taken of the fact that trypsin is adsorbed by a whole series of substances, and that the enzyme adsorbed may again become active in consequence of the phenomenon of autolysis. The quantity of active enzyme present in a digestive mixture not always being constant in all the phases of the reaction, it is evident that under such conditions we can no longer observe the proportionality of time and of the quantity of enzyme. Thus it is that the addition of charcoal powder to a solution of trypsin causes a very perceptible diminution of digestive power. The charcoal, previously kept in a colloidal liquid, such as a solution 
of saponin, adsorbs much less trypsin than fresh charcoal, and that which has adsorbed the enzyme gives it up again when placed in a solution of saponin. These results prove that the progress of the adsorption of trypsin depends on the colloidal condition of the liquid, a condition which may change, it is known, during the course of digestion. These are the kinds of phenomena which especially hinder the verification of the laws of enzyme action.

By taking different conditions, V. Henri and Larguier des Bancels have nevertheless succeeded in establishing the law of the action of trypsin on gelatin. To follow quantitatively the progress of the hydrolysis, they determined the change in the electrical conductivity of the substrate. By causing pancreatic juice, with an addition of kinase, to act on 2.5 to 5 per cent solutions of gelatin, they found first of all that the conductivities increase quite regularly, more rapidly in the case of the 5 per cent concentration than the 2.5 per cent. But they also found that in the first minutes the variation is the same for the two different gelatin concentrations.

Progress of Tryptic Digestion of Gelatin.

\begin{tabular}{|c|c|c|c|}
\hline \multirow{2}{*}{\multicolumn{2}{|c|}{ Temperature. }} & \multicolumn{2}{|c|}{$\begin{array}{c}\text { Variations in the electrical conductivity } \\
\text { multiplied by } 10^{5} \text {. }\end{array}$} \\
\hline & & Gelatin at $2.5 \%$. & Gelatin at $5 \%$. \\
\hline \multicolumn{4}{|c|}{ Minutes. } \\
\hline & IO & I 5 & 17 \\
\hline & 24 & 25 & 34 \\
\hline & 39 & 30 & 44 \\
\hline & 600 & 65 & 122 \\
\hline \multirow{3}{*}{ I I } & I I & I 2 & I I \\
\hline & 23 & 22 & 24 \\
\hline & 37 & 29 & 34 \\
\hline
\end{tabular}

This first result, indicating that the rate of digestion at the beginning is independent of the concentration of substance to be transformed, relates the action of trypsin on gelatin to the action of enzymes on carbohydrates. However, having recognized that the activity of the reagent (pancreatic juice + kinase) 
does not change after an hour of digestion, but that on the other hand the products of a prolonged digestion of gelatin retard its action, the investigators found that the variation of electrical conductivity can be expressed by the general logarithmic equation:

$$
K=\frac{\mathrm{I}}{t} \log \frac{a}{a-x} .
$$

In this expression, $K$ is a constant, $a$ a number which corresponds to the quantity of gelatin, $x$ the variation of electrical conductivity with time $t$. This formula expresses fairly closely. the progress of tryptic digestion during the first hour. The values of the constant $K$ are as follows:

\begin{tabular}{|c|c|c|c|c|}
\hline \multirow{2}{*}{ Duration. } & \multicolumn{2}{|c|}{$\begin{array}{c}\text { ro c.c. gelatin +o. } 5 \text { c.c. } \\
\text { juice + } 0.5 \text { c.c.kin. }+ \\
\text { r c.c. water. }\end{array}$} & \multicolumn{2}{|c|}{$\begin{array}{l}\text { ro c.c. gelatin + I c.c. } \\
\text { juice }+\mathrm{r} \text { c.c.kin. }\end{array}$} \\
\hline & $x$ & $K \times 10^{4}$ & $x$ & $K \times 10^{4}$ \\
\hline $\begin{array}{c}\text { Minutes. } \\
\text { 10 } \\
20 \\
30 \\
40\end{array}$ & $\begin{array}{l}22 \\
37 \\
45 \\
53\end{array}$ & $\begin{array}{l}\text { I61 } \\
\text { I64 } \\
\text { I } 48 \\
\text { I55 }\end{array}$ & $\begin{array}{l}28 \\
44 \\
55 \\
60\end{array}$ & $\begin{array}{l}222 \\
216 \\
227 \\
213\end{array}$ \\
\hline
\end{tabular}

The law of the action of trypsin on gelatin is then the same as that of sucrase, of amylase, of emulsin, and other enzymes.

\section{§ 7. Chemical Activity of Trypsin.}

Products Formed. - In the reactions involving pepsin it was seen that albumoses and peptones were produced as well as complex non-biuretic bodies not precipitated by phosphotungstic acid but susceptible of crystallization. All these products of high molecular weight come from the partial hydrolysis of the albuminoid material. They also show the characteristics, more or less pronounced, of the substance from which they are derived. With trypsin, a much deeper effect is observed. Without doubt primary albumoses are still formed 
at the beginning of the action, but these rapidly disappear giving deutero-albumoses and peptones. Very rapidly the compounds characteristic of tryptic action, tyrosin, tryptophane and leucin, appear. If the digestion is sufficiently prolonged there is found in the liquid a large number of mono- and diamino-acids. The final products of the reaction are deuteroalbumoses and peptones, both biuretic and precipitable by phosphotungstic acid, and different amino-acids not precipitable by this reagent.

We shall see in a subsequent chapter what is to be thought of the presence here of the albumoses and the peptones. Let us pause for the moment in consideration of the end products, which form the greater part of tryptic hydrolysis. And first of all, it should be noted that these crystalline compounds, non-biuretic and not precipitable by phosphotungstic acid, have absolutely nothing in common with those of the corresponding fraction obtained in peptic action; whereas the former are very simple amino-acids, the latter are compounds, capable even of being transformed by degradation into peptones.

The numerous amino-acids which are met in the products of advanced tryptic hydrolysis are of quite different constitution. Some possess only an acid function and an amine function; others are di-acids and mono-amines, or mono-acids and di-amines. Some have an alcohol function; others are sulphur products. Finally, these compounds are sometimes of straight chain, sometimes of closed chain constitution. Yet, we may say that all answer to the general formula, $R-\mathrm{CH}\left(\mathrm{NH}_{2}\right)-\mathrm{CO}_{2} \mathrm{H}$, which shows that there is at least a grouping $\mathrm{NH}_{2}$ fixed on the $C$ adjacent to carboxyl, $R$ being an acyl, an aromatic or heterocyclic radical. The following is a list of these compounds:

\section{A. Amino-acids Possessing an Acid Function and an Amine Function.}

(I) Glycocoll or glycin (or amino-acetic acid),

$$
\mathrm{CH}_{2}\left(\mathrm{NH}_{2}\right)-\mathrm{CO}_{2} \mathrm{H} \text {, }
$$


a crystalline body first obtained from the products of the hydrolysis of gelatin, where it is found in large amounts.

(2) d-alanin (d- $\alpha$-amino-propionic acid),

$$
\mathrm{CH}_{3}-\mathrm{CH}\left(\mathrm{NH}_{2}\right)-\mathrm{CO}_{2} \mathrm{H} \text {. }
$$

(3) d-valin (d- $\alpha$-amino-isovaleric acid),

$$
\left(\mathrm{CH}_{3}\right)_{2}=(\mathrm{CH})-\mathrm{CH}\left(\mathrm{NH}_{2}\right)-\mathrm{CO}_{2} \mathrm{H} \text {. }
$$

(4) l-leucin (l- $\alpha$-amino-isocaproic acid),

$$
\left(\mathrm{CH}_{3}\right)_{2}=(\mathrm{CH})-\mathrm{CH}_{2}-\mathrm{CH}\left(\mathrm{NH}_{2}\right)-\mathrm{CO}_{2} \mathrm{H} \text {, }
$$

a well-crystallized substance which is found in the products of hydrolysis of a majority of the albuminoid substances. This substance is often mixed with an isomer from which it is difficult to separate it, and which is:

(5) iso-leucin (methyl ethyl $\alpha$-amino-propionic acid),

$$
\begin{aligned}
& \mathrm{CH}_{3} \\
& \mathrm{C}_{2} \mathrm{H}_{5}
\end{aligned} \mathrm{CH}-\mathrm{CH}\left(\mathrm{NH}_{2}\right)-\mathrm{CO}_{2} \mathrm{H} \text {. }
$$

(6) 1-phenylalanin (1- $\beta$-phenyl $\alpha$-amino-propionic acid),

$$
\mathrm{C}_{6} \mathrm{H}_{5}-\mathrm{CH}_{2}-\mathrm{CH}\left(\mathrm{NH}_{2}\right)-\mathrm{CO}_{2} \mathrm{H} \text {. }
$$

(7) 1-tyrosin (l-para-oxyphenylalanin),

$$
\begin{aligned}
& \text { (I) } \mathrm{CH}_{2}-\mathrm{CH}\left(\mathrm{NH}_{2}\right)-\mathrm{CO}_{2} \mathrm{H} \\
& { }_{(4)}{ }^{\mathrm{C}_{6} \mathrm{H}_{4}} . \mathrm{OH}_{\mathrm{OH}}
\end{aligned}
$$

small silky needles, slightly soluble in water, easily recognizable and found in most proteolyses.

(8) 1-prolin (l-pyrrolidin $\alpha$-carbonic acid),

$$
\left.\right|_{\mathrm{CH}_{2}-\mathrm{CH}_{2}} ^{\mathrm{CH}_{2}-\mathrm{CH}-\mathrm{CO}_{2} \mathrm{H}}
$$

(9) 1-Oxyprolin (l-oxypyrrolidin $\alpha$-carbonic acid),

$$
\begin{aligned}
& \mathrm{CH}_{2}-\mathrm{CH}-\mathrm{CO}_{2} \mathrm{H} \\
\mathrm{OH} & -\mathrm{CH}-\underset{\mathrm{CH}}{\mathrm{CH}_{2}}
\end{aligned}
$$


found in the products of hydrolysis of gelatin and of the oxyhemoglobin of the horse.

(I0) Tryptophane (indol $\beta$-alanin),<smiles>NC(Cc1c[nH]c2ccccc12)C(=O)O</smiles>

a compound appearing at the beginning of tryptic digestions, and easily recognizable from the property which it possesses of giving with bromine water a violet red coloration. Trytophane is interesting because of its relationship to indol and scatol, which are met in the products of putrefaction of albuminoids.

(I I) Histidin (or imidazolalanin),

$$
\begin{aligned}
\mathrm{NH}^{\prime} & \mathrm{CH}=\mathrm{C}-\mathrm{CH}_{2}-\mathrm{CH}\left(\mathrm{NH}_{2}\right)-\mathrm{CO}_{2} \mathrm{H} \\
\mathrm{CH} & =\mathrm{N}
\end{aligned}
$$

levorotatory in the free state, but its salts are dextrorotatory.

B. Amino-acids Possessing Two Acid Functions and One Amine Function.

(12) 1-Aspartic acid (1- $\alpha$-aminosuccinic acid),

$$
\mathrm{CO}_{2} \mathrm{H}-\mathrm{CH}_{2}-\mathrm{CH}\left(\mathrm{NH}_{2}\right)-\mathrm{CO}_{2} \mathrm{H} \text {. }
$$

(r3) d-Glutamic acid (d- $\alpha$-aminoglutaric acid), $\mathrm{CO}_{2} \mathrm{H}-\mathrm{CH}_{2}-\mathrm{CH}_{2}-\mathrm{CH}\left(\mathrm{NH}_{2}\right)-\mathrm{CO}_{2} \mathrm{H}$.

C. Amino-acids Possessing One Acid Function and Two Amine Functions.

The simplest of these, di-amino-acetic acid, $\mathrm{CH}\left(\mathrm{NH}_{2}\right)_{2}-\mathrm{CO}_{2} \mathrm{H}$, which has been detected in the products of the action of $\mathrm{HCl}$ on casein, gelatin and conglutin, does not appear to form under the influence of trypsin. 
(14) d-Lysin (d- $\alpha$-diamino acid caproic acid),

$\mathrm{NH}_{2}-\mathrm{CH}_{2}-\mathrm{CH}_{2}-\mathrm{CH}_{2}-\mathrm{CH}_{2}-\mathrm{CH}\left(\mathrm{NH}_{2}\right)-\mathrm{CO}_{2} \mathrm{H}$.

This substance, by putrefaction, gives a known ptomaine, cadaverin, $\mathrm{NH}_{2}-\left(\mathrm{CH}_{2}\right)_{5}-\mathrm{NH}_{2}$, or pentamethylene-diamine, which is met in the putrefaction of various albuminoid materials.

( 5 ) d-Arginin (d- $\alpha$-amino $\delta$-guanido-normal valeric acid), $\mathrm{NH}=\mathrm{C}\left(\mathrm{NH}_{2}\right)-\mathrm{NH}-\mathrm{CH}_{2}-\mathrm{CH}_{2}-\mathrm{CH}_{2}-\mathrm{CH}\left(\mathrm{NH}_{2}\right)-\mathrm{CO}_{2} \mathrm{H}$.

This substance, derived from guanidin, $\mathrm{NH}=\mathrm{C}\left(\mathrm{NH}_{2}\right)_{2}$, is transformed by hydrolysis into urea, $\mathrm{CO}\left(\mathrm{NH}_{2}\right)_{2}$, and into ornithin, $\mathrm{NH}_{2}-\mathrm{CH}_{2}-\mathrm{CH}_{2}-\mathrm{CH}_{2}-\mathrm{CH}\left(\mathrm{NH}_{2}\right)-\mathrm{CO}_{2} \mathrm{H}$, this last giving, by putrefaction, putrescin, $\mathrm{NH}_{2}-\left(\mathrm{CH}_{2}\right)_{4}-\mathrm{NH}_{2}$. But ornithin has not been obtained in vitro by the decomposition of albuminoid materials.

These last substances, lysin and arginin, together with histidin previously cited, form the three hexonic bases, thus named because of their six atoms of $\mathrm{C}$. They are met in the decomposition of most albuminoid materials, and play an important rôle in their constitution. They are all three precipitable by phosphotungstic acid. Various salts, by giving with them metallic derivatives more or less soluble, offer a means of separation.

\section{Other Compounds.}

(I6) r-Serin ( $\beta$-oxyalanin),

$$
\mathrm{CH}_{2} \mathrm{OH}-\mathrm{CH}\left(\mathrm{NH}_{2}\right)-\mathrm{CO}_{2} \mathrm{H}
$$

obtained as a product of the decomposition of silk-protein.

(I7) 1-Cystin-

$$
\begin{aligned}
& \mathrm{S}-\mathrm{CH}_{2}-\mathrm{CH}\left(\mathrm{NH}_{2}\right)-\mathrm{CO}_{2} \mathrm{H} \\
& \mathrm{S}-\mathrm{CH}_{2}-\mathrm{CH}\left(\mathrm{NH}_{2}\right)-\mathrm{CO}_{2} \mathrm{H} .
\end{aligned}
$$

This sulphur compound can be regarded as resulting from the union of 2 molecules of cystein or thio-alanin,

$$
\mathrm{CH}_{2}(\mathrm{SH})=\mathrm{CH}\left(\mathrm{NH}_{2}\right)-\mathrm{CO}_{2} \mathrm{H} \text {. }
$$

Yet cystein is not met among the derivatives of the proteins.

(18) Leucinimide ( $\beta$ - $\gamma$-di-isobutyl $\alpha$ - $\gamma$-diacipiperazin), 
$\begin{aligned} & \mathrm{CH}_{3} \\ & \mathrm{CH}_{3}\end{aligned}>\mathrm{CH}-\mathrm{CH}_{2}-\mathrm{CH}_{\backslash}>\mathrm{CO}-\mathrm{NH}-\mathrm{NO} \backslash \mathrm{CH}-\mathrm{CH}_{2}-\mathrm{CH}^{\prime}>\mathrm{CH}_{3}$

which is a double anhydrid of leucin.

To these various amino-acids, it is necessary to add two other products which are obtained in the tryptic decomposition.

(Ig) Glucosamine,

$\mathrm{CH}_{2} \cdot \mathrm{OH}-\mathrm{CH} \cdot \mathrm{OH}-\mathrm{CH} \cdot \mathrm{OH}-\mathrm{CH} \cdot \mathrm{OH}-\mathrm{CH}\left(\mathrm{NH}_{2}\right)-\mathrm{CHO}$,

(20) and ammonia $\mathrm{NH}_{3}$.

We shall now see how we may, without separating and measuring each of these products, at least reunite them in a certain number of groupings whose relative importance can be approximately determined.

\section{§ 8. Methods of Analysis for Tryptic Products.}

General Method. - To analyze tryptic products the same method must be applied which has served for the study of peptic digestion. The liquid is first of all treated with acidulated zinc sulphate, and the precipitate so obtained contains all the albumoses. In the liquor free from albumoses, the peptones are precipitated by phosphotungstic acid; at the same time the higher peptides of the mono-amino-acids, as well as the hexonic bases, are rendered insoluble. Finally the filtrate, not precipitable by the tungstic reagent, corresponds to the simple monoamino-acids. Moreover, in another part of the liquid, by distillation with magnesia, the amide nitrogen may be determined.

The complete analysis of the products of tryptic digestion would involve, in addition to the separation and the determination of albumoses and remaining peptones, the characterization of the hexonic bases formed, as well as the qualitative and quantitative examination of the various amino-acids appearing. Unfortunately, in the present state of the science, no general method exists which permits, by a systematic course, the attainment of this end. At most, we can merely note the methods of Drechsel, Kutscher, and Kossel for analyzing hexonic bases, and 
the esterification* method of Fischer for the separation and the identification of the various amino-acids. Still, these procedures are so complex that they cannot be regarded as simple analyses; they require, in fact, very numerous and delicate operations requiring much time and material. Further, the results which they furnish, however interesting they may be, cannot be considered as quantitative. We shall therefore refrain from descriptions, but shall indicate in detail a very simple and expeditious method which permits the estimation of the total amino-acids present in the liquor with a very sufficient degree of approximation, a method originated by Sörensen, now commonly spoken of as the formaldehyde method.

Formaldehyde Method of Sörensen. - This method, which can serve just as well for determining amino-acids as for determining the degree of hydrolysis of protein material, is based on the following principle: If, to a solution of an amino-acid, neutral from the fact of internal neutralization of the acid $\mathrm{CO}_{2} \mathrm{H}$ by the basic $\mathrm{NH}_{2}$, formaldehyde, likewise neutral, is added, the latter, reacting on the amino group, removes this basic function and the solution immediately becomes acid. For example, in the case of glycin,

$$
\mathrm{CH}_{2} \backslash \underset{\mathrm{CO}_{2} \mathrm{H}}{>\mathrm{NH}_{2}}+\mathrm{CH}_{2} \mathrm{O}=\mathrm{H}_{2} \mathrm{O}+\mathrm{CH}_{2} \backslash \stackrel{\mathrm{N}=\mathrm{CH}_{2}}{\mathrm{CO}_{2} \mathrm{H}} .
$$

The measure of acidity consequently indicates the quantity of amine in solution. A like phenomenon takes place with the products of proteolysis. The albuminoid molecule being formed by various radicals united by the grouping $-\mathrm{CO}-\mathrm{NH}-$, each time that a molecule of water is introduced at one of these points, the chain opens and an acid carboxyl and a basic amine grouping appear. These two functions mutually neutralizing each other, the reaction of the medium is not perceptibly changed.

* This method consists in transforming all the amino-acids, in alcoholic solution, by means of gaseous $\mathrm{HCl}$ into the ethyl esters, separating these by fractional distillation under reduced pressure and, finally, in saponifying each of them by concentrated $\mathrm{NaOH}$ to set free the acids. 
But if to the perfectly neutral liquid a solution of $\mathrm{HCHO}$ is added the liquid at once becomes acid, due to the fact that the group $-\mathrm{NH}_{2}$, reacting with formaldehyde is transformed into $-\mathrm{N}=\mathrm{CH}_{2}$ which is a neutral form, and the unattached group $\mathrm{CO}_{2} \mathrm{H}$ renders the solution acid. The acidity can be titrated against standard $\mathrm{Ba}(\mathrm{OH})_{2}$ solution. The more advanced the hydrolysis, the more chains will be broken and the greater will be the number of $\mathrm{CO}_{2} \mathrm{H}$ groups to neutralize. Naturally, to each $\mathrm{CO}_{2} \mathrm{H}$ measured there corresponds at least one $\mathrm{NH}_{2}$ group. But as there are also, among the albuminoid residues, constituents containing two or three nitrogens, and since both the quantity and the moment at which they are set free is unknown, we cannot calculate except for mono-nitrogenous derivatives. Thus an error results if, after having converted the number of cubic centimeters of alkali used for neutralization into milligrams of nitrogen titratable with $\mathrm{HCHO}$, we should consider this result as representing the quantity of nitrogen really separated from the albuminoid molecule.

The analytical procedure is as follows:

Reagents. - (I) A solution of phenolphthalein corresponding to 0.5 per cent of phenolphthalein in 50 per cent alcohol.

(2) Very sensitive litmus paper, prepared as follows: Pulverize finely $0.5 \mathrm{~g}$. of azolitmin, which is the coloring matter of litmus, and dissolve it in 22.5 c.c. of $N$ / ro soda, diluting the whole with 200 c.c. of water. After filtration, add 50 c.c. of alcohol and dip rapidly in this tincture strips of paper containing very little ash. After drying the strips are kept in dry brown glass flasks. This paper should have a neutral tone and be slightly tinted, for its sensitiveness is thus greater. It should react in the following manner with these mixtures of the two sodium acid phosphates:

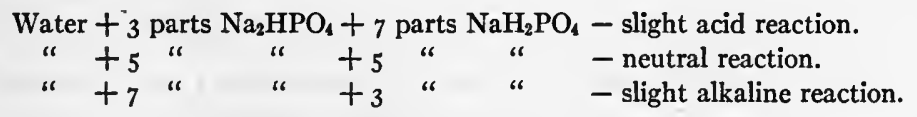

If, by reason of the quality of paper used, the strips which have been prepared do not correspond to the preceding tests, repeat 
the tests after having corrected the tincture of litmus by the addition of an estimated amount of $\mathrm{N} / \mathrm{I} \circ \mathrm{NaOH}$.

(3) A solution of formaldehyde, which should be prepared at the moment of using. For that purpose, to 50 c.c. of commercial formaldehyde add I c.c. of the above solution of phenolphthalein and, drop by drop, that amount of $\mathrm{N} / \mathrm{I} \circ \mathrm{NaOH}$ necessary to produce a pale pink tint. This solution should undergo a correction for the conditions under which it is used. To determine this correction, take 20 c.c. of boiled distilled water. If the liquid of digestion in which the determination is performed is colored, add to this distilled water several drops of a very dilute solution of tropeolin or of Bismarck Brown, in order to bring it to the same tint and thus to make the changes comparable. Then add to this water Io c.c. of the formaldehyde solution, and test this control solution. For that purpose, add about 5 c.c. of $N / \mathrm{r} \circ \mathrm{NaOH}$, and with $N /$ io $\mathrm{HCl}$ bring the coloration produced to a pale red tint. Then add again two drops of $N /$ Io $\mathrm{NaOH}$, which gives to the solution the bright red tint that should be the same for all the analyses.

If, for example, 5.15 c.c. of $N /$ Io $\mathrm{NaOH}$ and 5 c.c. of $N /$ Io $\mathrm{HCl}$ are required, the difference, or 0.15 c.c. of $\mathrm{NaOH}$, will represent the correction for ro c.c. of formaldehyde, which should be subtracted from the result given by the liquid studied. On the contrary, if 0.15 c.c. excess $\mathrm{HCl}$ are used, the correction should be added.

(4) A saturated solution of $\mathrm{Ba}(\mathrm{OH})_{2}$ in methyl alcohol.

To obtain satisfactory results, it is important first of all to use a quantity of substance such as shall require about 5 c.c. of $N /$ Io $\mathrm{NaOH}$, and further it is well to repeat each determination two or three times. If the solution contains ammonia, it is better first to remove it, this reacting only in an incomplete manner in the formaldehyde method. Place the liquid to be analyzed in a tall, narrow vessel, add to it I c.c. of solution of phenolphthalein, $2 \mathrm{~g}$. of $\mathrm{BaCl}_{2}$, and a half-volume of the saturated methyl alcohol solution of $\mathrm{Ba}(\mathrm{OH})_{2}$. Then for several hours cause a rapid current of air to pass through the liquid, and receive the ammonia 
carried away in a flask containing standard $\mathrm{H}_{2} \mathrm{SO}_{4}$, the subsequent titration of which will give the content in ammonia $\mathrm{N}$. The solution, free from ammonia, is then brought to a given volume and filtered; an aliquot part is taken for the determination of the amino-acids. To do this, neutralize the solution carefully with $\mathrm{N} /$ ro $\mathrm{HCl}$, using very sensitive litmus paper as the indicator, and then add ro c.c. of HCHO solution. At this point bring the solution up to the red coloration change with $N / \mathrm{Io} \mathrm{NaOH}$ and add 5 c.c. in excess. Then in turn test with $N /$ го $\mathrm{HCl}$ until a pale red coloration is obtained, and by means of a few drops of $\mathrm{N} /$ ro $\mathrm{NaOH}$ bring the tint equal to that of the control.

The exact neutralization of litmus paper is always quite tedious. If a sufficient quantity of the liquid is prepared and if this is little colored, we may make one half the solution neutral in the presence of several drops of tincture of litmus, and then pour into the other half the same quantity of $N /$ ro $\mathrm{HCl}$ which was used for the first neutralization.

If the solution examined does not contain ammonia, to a known volume add directly I c.c. of phenolphthalein, 2 g. of $\mathrm{BaCl}_{2}$, and $\mathrm{Ba}(\mathrm{OH})_{2}$, until the appearance of the red coloration, and then an excess of 5 c.c. Bring the whole to a volume of 100 c.c., shake vigorously, leave standing $\frac{1}{4}$ hour, filter if necessary, and with an aliquot part proceed with the determination as above.

In the calculation of formaldehyde nitrogen only that quantity of $\mathrm{NaOH}$ is taken which was required to exactly neutralize the solution to litmus. The following are figures which have been obtained by applying this method to a 2 per cent solution of glycin: To to c.c. of this solution, representing 0.2 g. of substance or $0.0344 \mathrm{~g}$. total $\mathrm{N}$, was added ro c.c. of phenolphthalein, $2 \mathrm{~g}$. $\mathrm{BaCl}_{2}$ and 8 c.c. of $\mathrm{Ba}(\mathrm{OH})_{2}$. The solution was then brought to a volume of roo c.c., shaken, left $\frac{1}{4}$ hour, and filtered. 25 c.c. of the filtrate, or 2.5 c.c. of the original solution, required for neutralization in the presence of litmus paper 7.5 c.c. $\mathrm{N} /$ 10 $\mathrm{HCl}$. At this point, there has been added successively, 6.I c.c. of $N /$ Io $\mathrm{NaOH}$ (pale red coloration) +5.5 c.c. of $N /$ Io $\mathrm{NaOH}$ (excess), or in all, Ir.6 c.c. $\mathrm{NaOH}$. Then, 5.75 c.c. $N /$ ı $\mathrm{HCl}$ 
was necessary to produce again the pale red tint. Finally, o.I c.c. of $N /$ I $~ \mathrm{NaOH}$ gave the red tint of the control. In all there was used II.7 c.c. of $\mathrm{NaOH}$ (HCHO sol. had no correction) $5.75 \mathrm{HCl}$, or 5.95 c.c. $N /$ ro, equivalent to $5.95 \times 1.4$ or $8.33 \mathrm{mg}$. $\mathrm{N}$. Now, the 2.5 c.c. of solution contain practically $8.6 \mathrm{mg}$. of $\mathrm{N}$; the formaldehyde nitrogen, therefore, represents $98 . \mathrm{I}$ per cent of the total nitrogen.

The following results obtained with the liquid from a tryptic digestion of casein: 100 c.c. of filtered liquid, the untransformed casein being precipitated by a little acetic acid, contain $240 \mathrm{mg}$. total $\mathrm{N}$; 50 c.c. of this liquid, treated with $\mathrm{BaCl}_{2}$ and alcoholic $\mathrm{Ba}(\mathrm{OH})_{2}$, produced a quantity of $\mathrm{NH}_{3}$ that required 2.5 c.c. $\mathrm{N} /$ Io $\mathrm{H}_{2} \mathrm{SO}_{4}$ for neutralization, equivalent to $2.5 \times \mathrm{r} .4$ or $3.5 \mathrm{mg}$. $\mathrm{N}$, that is to say, $7 \mathrm{mg}$. of ammonia nitrogen per 100 c.c. of original liquid. The liquid, free from $\mathrm{NH}_{3}$, is brought to Ioo c.c., 50 c.c. are taken for analysis and made exactly neutral to litmus. Io c.c. of formaldehyde are then added. This solution required I3.0 c.c. $N /$ ro $\mathrm{NaOH}$; then 5.4 c.c. $N /$ ro $\mathrm{HCl}$; and, finally, 0.2 c.c. $N /$ Io $\mathrm{NaOH}$. The correction for the $\mathrm{HCHO}$ sol. was - o.I c.c.; $13.0-5.4+0.2-0.1=7.7$ c.c. $N /$ ro $\mathrm{NaOH}$ required, equivalent to $7.7 \times 1.4$ or $10.78 \mathrm{mg}$. $\mathrm{N}$; whence 10.78 $\times 4=43.12 \mathrm{mg}$. of formaldehyde $\mathrm{N}$ per. 100 c.c. original solution.

This digestion liquid therefore contains:

$\left.\begin{array}{l}2.9 \text { ammoniacal } \mathrm{N} \\ \text { 17.9 } \mathrm{N} \text { titratable by formaldehyde }\end{array}\right\}$ per roo total nitrogen.

The method just described requires some comments.

(r) As already observed, nitrogen titratable with formaldehyde represents only a minimum of the nitrogen contained in liberated peptides, due to the existence of di-and tri-nitrogenous bodies.

(2) Determinations made by following exactly the directions indicated give good results - varying between 89 and 99 , with an average of 97.5 per cent - for the estimate of the nitrogen contained in the different amino-acids; glycin, alanin, leucin, aspar- 
tic acid, ornithin, lysin, serin, histidin, etc. Yet, Sörensen found that with prolin, the results are less satisfactory. In a decinormal solution of this substance, 85 per cent of the nitrogen content can be found by this titration. On the other hand, with tyrosin more nitrogen is found than was calculated.

Another cause of error is the presence of the arginin grouping in the albuminoid molecule. We know that salts of guanidin, even after addition of formaldehyde, act like entirely neutral combinations, and that on the other hand arginin, although having two amino-groupings, nevertheless acts toward this reagent like a mono-acid base only. As this last body is not connected to the other parts of the albuminoid molecule except by its $\mathrm{CO}_{2} \mathrm{H}$ group or the neighboring $\mathrm{NH}_{2}$, the guanidin grouping $\mathrm{NH}_{2}-\mathrm{C}(\mathrm{NH})-\mathrm{NH}$ remaining free in the molecule itself, the passivity of this group with regard to formaldehyde does not at all interfere with the determination. But if the guanidin group is found connected to a $\mathrm{CO}_{2} \mathrm{H}$ group of the protein chain, the rupture of such a relationship will escape measurement by the formaldehyde test, and there is no means of estimating the magnitude of this error.

(3) We have seen that it is important first to neutralize to litmus the liquid to be examined, whereas the formaldehyde determination should be made in the presence of phenolphthalein. Sörensen has based his choice of these two indicators on theoretical reasons. Yet, the use of litmus, at the beginning, has not been adopted by all. In general, the figures found by starting with a neutrality to litmus are higher than those obtained with a neutrality to phenolphthalein. However, they may be less, the direction of this difference depending on the nature of the aminoacids present in the solution. In particular, the use of litmus at the time of neutralization causes a rather large error when the determination is made in the presence of large quantities of weak acids, such as carbonic or phosphoric acids. These acids are completely removed by treating the solution with barium chloride and hydroxide.

(4) Decinormal solutions either of $\mathrm{NaOH}$ or $\mathrm{Ba}(\mathrm{OH})_{2}$ may be 
used. However, their use is not entirely arbitrary. The solutions normally deprived of carbonate and phosphate are as easily tested by a solution of $\mathrm{NaOH}$ as by $\mathrm{Ba}(\mathrm{OH})_{2}$. $\mathrm{NaOH}$ is preferred to $\mathrm{Ba}(\mathrm{OH})_{2}$, with the omission of the previous treatment with $\mathrm{BaCl}_{2}+\mathrm{Ba}(\mathrm{OH})_{2}$, when it is a question of mixtures rich in phenylalanin, or when the liquid examined contains chiefly the products of the primary cleavage of proteins. The determination of tyrosin, however, is less satisfactory with $\mathrm{NaOH}$ than with $\mathrm{Ba}(\mathrm{OH})_{2}$.

(5) A difficulty which is often present in the application of the method of Sörensen comes from the coloration of the liquids to be analyzed. We have already indicated a method adopted to render comparable those determinations made in the control solution and the solution to be examined. The use of small quantities of coloring matter is suitable, however, only for slightly colored solutions. In other cases, a purification is effected in the following manner: To 25 c.c. of dark liquid add 4 c.c. of a $2 \mathrm{~N}$ solution of $\mathrm{BaCl}_{2}$; shake vigorously and then add 20 c.c. of a $\mathrm{N} / 3$ solution of $\mathrm{AgNO}_{3}$; shake again, dilute to 50 c.c., and filter. The precipitate of $\mathrm{AgCl}$ thus formed carries with it the larger part of the coloring matter.

\section{§ 9. Progress of Tryptic Peptonization.}

Formation of Amido Nitrogen. - To follow the chemical transformations produced in the course of a tryptic digestion, an

Degradation of Nitrogen in the Course of Tryptic Digestion.

\begin{tabular}{|c|c|c|c|c|c|}
\hline Duration of digestion. & $\begin{array}{l}\text { Albumose } \\
\text { percipit- } \\
\text { able by } \\
\mathrm{ZnSO}_{4}\end{array}$ & $\begin{array}{l}\text { Peptones } \\
\text { precipit- } \\
\text { able by } \\
\text { Phos. tung. }\end{array}$ & $\begin{array}{c}\text { Part not } \\
\text { precipit- } \\
\text { able by } \\
\text { Phos. tung. }\end{array}$ & $\begin{array}{l}\text { Amino } \\
\text { nitrogen } \\
\text { Formalde- } \\
\text { hyde. }\end{array}$ & $\begin{array}{l}\text { Nitrogen } \\
\text { precipit- } \\
\text { able by } \\
\text { tannin. }\end{array}$ \\
\hline 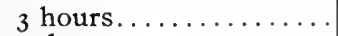 & 60.1 & I 7.6 & $22 \cdot 3$ & I I . I & 52.8 \\
\hline 2 days......... & I 6.4 & 26.9 & 56.7 & $2 I \cdot 3$ & IO.I \\
\hline 3 days........... & I6. I & $23 \cdot 5$ & 56.7 & 26.8 & 10.7 \\
\hline 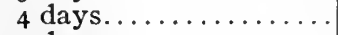 & 19.2 & $2 \mathrm{I}^{\circ} \cdot 7$ & 59.1 & $27 \cdot 7$ & 17.0 \\
\hline 5 days. . . . . . . . . . & 17.5 & $19 \cdot 3$ & 63.2 & 29.0 & I $5 \cdot 5$ \\
\hline I I days ............ & $14 \cdot 2$ & 20.4 & $65 \cdot 4$ & 34.6 & I 2.8 \\
\hline I6 days............ & 12.9 & $2 \mathrm{I} \cdot 5$ & $6_{5} .6$ & 34.8 & 10.8 \\
\hline 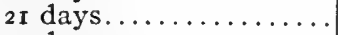 & 6.9 & 24.0 & $69 . x$ & 4 I.9 & 3.2 \\
\hline 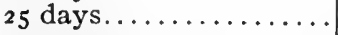 & 6.9 & $24 \cdot I$ & 69.0 & $4 I \cdot 5$ & 3.0 \\
\hline
\end{tabular}


alkaline 2 per cent solution of egg-albumin is placed in contact with trypsin at a temperature of $37^{\circ}$, in the presence of toluol or chloroform. In the accompanying experiment 0.5 g. of $\mathrm{Na}_{2} \mathrm{CO}_{3}$ were mixed with $0.6 \mathrm{~g}$. of Merck trypsin in a liter of water. At stated intervals samples were taken, filtered, and then submitted to analysis as described in the preceding chapter.

The columns, Albumose, Peptones, and Part not Precipitable by Phosphotungstic acid, combined give the total nitrogen contained in the filtrate. The portion not precipitable by phosphotungstic acid represents the amino-acids. We see that this quantity increases gradually after three hours of digestion - of Io parts total $\mathrm{N}$ in the filtrate, 22.3 is found in the form not precipitable by phosphotungstic acid. After twenty-five days, the corresponding portion now represents 69.0 per cent of the total nitrogen. This course differs radically from that caused by pepsin, and characterizes the action of trypsin. As control, the amino-acids are measured directly with $\mathrm{HCHO}$ solution. We see that their proportion likewise gradually increases from II.I per cent at the beginning, to 29 per cent after five days and, at the end, to $4 \mathrm{I} .5$ per cent. These figures indicate that the part not precipitable by phosphotungstic acid is not exclusively composed of mono-amino-acids, since its nitrogen content is always conspicuously higher than the nitrogen titratable with formaldehyde. Without doubt, these last figures are too small, but the error resulting from the percentage difference in nitrogen content between the mono-amino-acids and the di-amino-acids is not large enough to explain the discrepancy here found. Moreover, the formaldehyde nitrogen is not analyzed for in the part not precipitable by the tungstic reagent, but in the total digestion solution after filtration; it includes, therefore, the nitrogen of the hexonic bases, which are precipitated at the same time as the peptones. In spite of this increase, the figures of the fourth column are much lower than those of the third column.

From this table the irregular progress of the formation of peptones is very apparent. Abundant at the beginning of the action, the proportion of these products increases up to a certain 
maximum, then descends again, to reascend afterward and attain a second maximum. Here again we have an effect quite different from that of pepsin. With this last enzyme, the quantity of substances precipitable with the tungstic reagent increases regularly, to become fixed at the end of hydrolysis at a certain value, while with trypsin we obtain a more or less stable equilibrium in peptone, an equilibrium that is rot attained until after several fluctuations.

A similar digression is observed in the disappearance of albumoses. After descending from 60.I per cent to I6.I per cent, the nitrogen content of these substances ascends again to r9.2, to return to 14.2 , and to become fixed at 6.9 per cent. This transitory increase of albumose is observed likewise in the data contained under the heading, tannin. After three days, the nitrogen content of the tannin precipitate is ro.7. It rises the fourth day to I 7 per cent, to redescend afterward, and fall on the twenty-fifth day to 3.0 per cent. The reformation of albumose does not always appear in the course of digestions with a clearness as evident as that found in this table. However, if, at intervals of twelve hours, the variation of albumose nitrogen of a solution submitted to digestion is followed, discrepancies are frequently found amounting to 3 or 4 per cent.

Albumose is reformed at the expense of the peptones and of the products not precipitable by phosphotungstic acid. It is often very difficult to follow the increase in the proportion of albumose, for, in the course of tryptic digestions, the nitrogen of the filtered solution varies constantly, a part of that which was previously soluble taking again an insoluble form. In general, after three or six hours digestion in the presence of a sufficient quantity of trypsin, the coagulated albumin is completely dissolved and the liquid, before and after filtration, has the same nitrogen content. Then, the peptonization advancing, the liquid, formerly clear, becomes turbid in a more or less pronounced fashion. Moreover, the intensity of the turbidity varies with the time. If, in the course of a digestion, at different intervals of time the determination of nitrogen in the liquid is 
repeated before and after filtration, variable results are observed.

Precipitation of Nitrogen in the Course of a Tryptic Digestion.

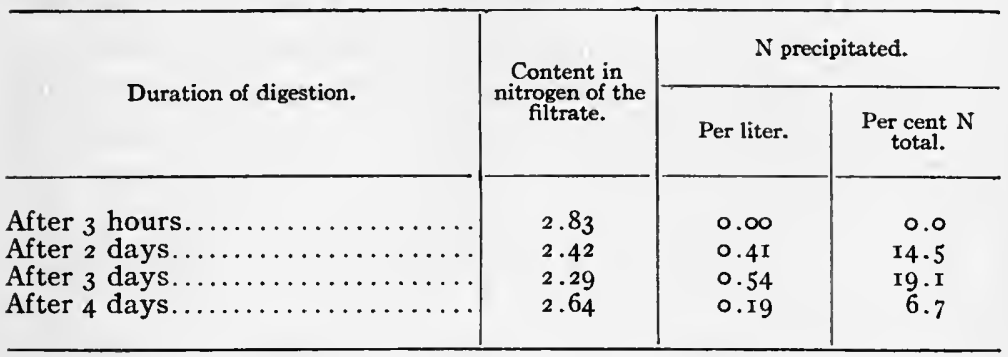

The substance precipitated in the course of tryptic digestion is plastein, a substance already mentioned in a preceding chapter. Its appearance here would indicate a synthetic action, a reaction apparently rendered possible by the changes which take place in the conditions of the medium during proteolysis. It is even very probable that the final arrest in hydrolysis caused by trypsin before the latter is complete, corresponds to a stable equilibrium which is established between the two opposite functions of this enzyme, its analy tic and its synthetic power. In every case, the albumoses and the peptones remaining after advanced hydrolysis by trypsin, when separated from the digestion liquid, can, in their turn, be further degraded by the enzyme; they are not, therefore, residual bodies, as Kühne thought, when he described them under the name of anti-peptones. The existence of such substances becomes very problematical, their confusion with the first products of decomposition - or of recombination - being explained moreover by the preceding experiments.

To summarize, from a purely chemical point of view, the action of trypsin is differentiated from that of pepsin- by the three following points:

(I) A great quantity of products titratable by formaldehyde are formed - simple amino-acids, tyrosin, tryptophane, etc.

(2) The limit of degradation of peptone is not reached through a regular progress, but through a series of oscillations. 
(3) A manifest synthetic tendency is observed, even in strong dilutions.

Formation of Amido Nitrogen in the Course of Tryptic Digestion. - Tryptic digestion does not differ from peptic digestion solely in the degree of the hydrolysis, but also in the character of the latter. While pepsin preferably liberates amide groupings, thus rendering a considerable quantity of $\mathrm{NH}_{3}$ easily liberable by magnesia, the action of trypsin relates much less to these links in the albuminoid molecule, the final quantity of ammonia capable of being carried off by boiling with alkali being much less.

Formation of Ammoniacal Nitrogen in Tryptic Digestion.

\begin{tabular}{|c|c|c|c|}
\hline Nature of the albuminoid matter. & $\begin{array}{l}\text { Duration of } \\
\text { digestion. }\end{array}$ & $\begin{array}{l}\text { Ammoniacal } \\
\text { nitrogen (per } \\
\text { cent total N). }\end{array}$ & $\begin{array}{l}\text { Ammoniacal } N \\
\text { and formalde. } \\
\text { hyde } N \text { (per } \\
\text { cent total } N \text { ). }\end{array}$ \\
\hline \multirow{3}{*}{ Egg-albumin. } & Days. & & \\
\hline & $\int \mathrm{I} 4$ & I. 4 & 30.1 \\
\hline & 73 & 4.6 & 60.2 \\
\hline \multirow{2}{*}{ Casein... } & $\left\{\begin{array}{l}\text { I4 } \\
\text {. }\end{array}\right.$ & 2.4 & 34.8 \\
\hline & 73 & $5 . \mathrm{I}$ & $4^{2} \cdot 5$ \\
\hline \multirow[t]{2}{*}{ Edestin. . } & $\{$ I 2 & 2.9 & 29.7 \\
\hline & 73 & 7.0 & 45.4 \\
\hline Gliadin. . & $\begin{cases}\text { II } \\
43\end{cases}$ & $\begin{array}{r}6.5 \\
-8\end{array}$ & 30.5 \\
\hline \multirow{2}{*}{ Gelatin. } & $\left\{\begin{array}{l}43 \\
21\end{array}\right.$ & $\begin{array}{r}12.8 \\
0.9\end{array}$ & $\begin{array}{l}35 \cdot 5 \\
20.9\end{array}$ \\
\hline & $\left\{\begin{array}{l}70 \\
0\end{array}\right.$ & 1.4 & 25.0 \\
\hline \multirow{2}{*}{ Witte peptone.. } & $\left\{\begin{array}{l}12 \\
n\end{array}\right.$ & 2.0 & 34.7 \\
\hline & 73 & $3 \cdot 3$ & 43.1 \\
\hline
\end{tabular}

Thus, while the proportion of ammonia nitrogen obtained with gliadin, for example, was 20.9 per cent after 43 days of peptic digestion (p. 243), with trypsin a liberation of only I 2.8 per cent was observed, and yet the total quantity of nitrogen (ammonia + $\mathrm{HCHO}$ ) is greater in the second case than in the first - 35.5 per cent, as against 33.3 per cent.

The difference between the peptic and tryptic reactions becomes still more evident when we compare the products of these two digestions brought to the same degree of hydrolysis, a condition estimated according to the value of the formaldehyde figure. 
Ammoniacal Nitrogen Formed in Peptic and Tryptic Digestions.

\begin{tabular}{|c|c|c|c|}
\hline Albuminoid. & $\begin{array}{l}\text { Degree of } \\
\text { hydrolysis } \\
\text { Formal. } N\end{array}$ & Pepsin. & Trypsin. \\
\hline 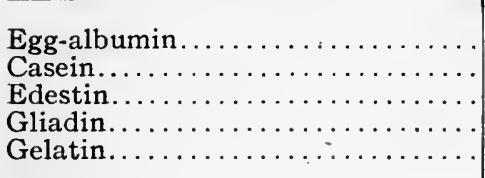 & $\begin{array}{c}\text { Per cent. } \\
\begin{array}{c}24 \\
24 \\
27 \\
30 \\
\text { I } 5\end{array}\end{array}$ & $\begin{array}{c}\text { Per cent. } \\
3.6 \\
6.1 \\
7.1 \\
\text { I } 8.5 \\
\text { I. } 3\end{array}$ & $\begin{array}{c}\text { Per cent. } \\
\begin{array}{l}\text { I. } 4 \\
\text { I .4 } \\
\text { I. } 8 \\
9.1 \\
0.9\end{array}\end{array}$ \\
\hline
\end{tabular}

Egg-albumin, with a content in formaldehyde nitrogen of 24 per cent, gives 3.6 per cent of ammoniacal nitrogen when the catalyst used is pepsin, and only I.4 per cent when trypsin is used. Similar results are obtained with the other albuminoids.

\section{$\S$ io. Anti-Trypsin.}

Blood serum prevents to a great degree the activity of trypsin. This anti-tryptic action of serum was established in 1894 by Fermi and Pernossi, and has since been made the object of numerous investigations. In particular, Glaessner found that the anti-proteolytic power of serum varied in the same individual during the course of the day. Zunz confirmed this result by showing, as we shall see further on, that the anti-fermenting action of the serum of a dog toward kinased pancreatic juice ordinarily increased several hours after the ingestion of raw horse meat.

Determination of the Anti-tryptic Power of a Serum. - The determination of the anti-tryptic power of serum is usually made according to the method of Marcus, which consists in making on plates of glass, covered with coagulated beef serum, a series of drops of varying mixtures of serum and trypsin. As long as the quantity of serum is insufficient, the albumin is attacked, and a little cupu e forms at the spot where the liquid is deposited; on the contrary, starting with the first drop where the beef serum remains intact, it may be concluded that the neutralizing action has been accomplished. The method of Fuld is also used, a method based on the effect of mixtures of serum and trypsin on 
casein, an effect observed after a precipitation with acetic acid. The neutralization of trypsin is indicated by the non-solution of the casein. Finally, the method of colored fibrin of Grützner and Gehrig is at times used. Achalme and Stévenin are able to measure, not the anti-tryptic power, but the anti-coagulating power, which we know to be in direct parallel with the former. They put 2 to 3 c.c. of milk in tubes and sterilize at $120^{\circ}$. Then they added various dilute mixtures of trypsin and serum at $\mathrm{I}-\mathrm{IO}$ in increasing quantities, and allowed to stand twenty hours at $50^{\circ}$, placing a drop of essence of mustard in the stoppers which close the tubes. The last tube coagulated indicates the limit of neutralization; the quantity of serum added to the first tube of the series remaining intact then determines its anti-tryptic value. This last method gives very good results.

In all these determinations, there is a very important point on which writers do not often insist enough, and which renders their results but little comparable, namely, the strength of the trypsin used. Achalme recommends a maceration of 5 to 7 per cent of pancreatin made in a physiological solution for 18 to 24 hours at $35^{\circ}$, in the presence of several drops of essence of mustard. The liquid is filtered and distributed in sterilized and sealed pipettes, which are kept in the dark. This trypsin is then tested with respect to its effect on the serum of an adult guinea pig. By using the method of Achalme, just described, a trypsin should be obtained, o.oI c.c. to 0.015 c.c. of which (or O.I c.c. to 0.I 5 c.c. of a I-IO dilution) is neutralized by about 0.01 c.c. (or O.I c.c. of I-IO dilution) of guinea-pig serum, or again by 0.015 c.c. of rabbit serum.

The anti-tryptic power of man is found to be between 4 and 5 . Different writers have found that the human serum of carriers of various neoplasms had an anti-protein power higher than the normal. Thus, A. Girault and Rubinstein have found with a certain number of patients ill with lesions of the stomach, such as gastric cancer, that the anti-tryptic index often reached 8, Io, and I2. However, this is not a specific character of cancer, for ulcers also give a strong anti-fermenting power. 
Guido Finzi submits the following figures on the anti-tryptic power of the serum of healthy domestic animals killed at abattoirs.

Anti-tryptic Power of the Serum of Domestic Animals.

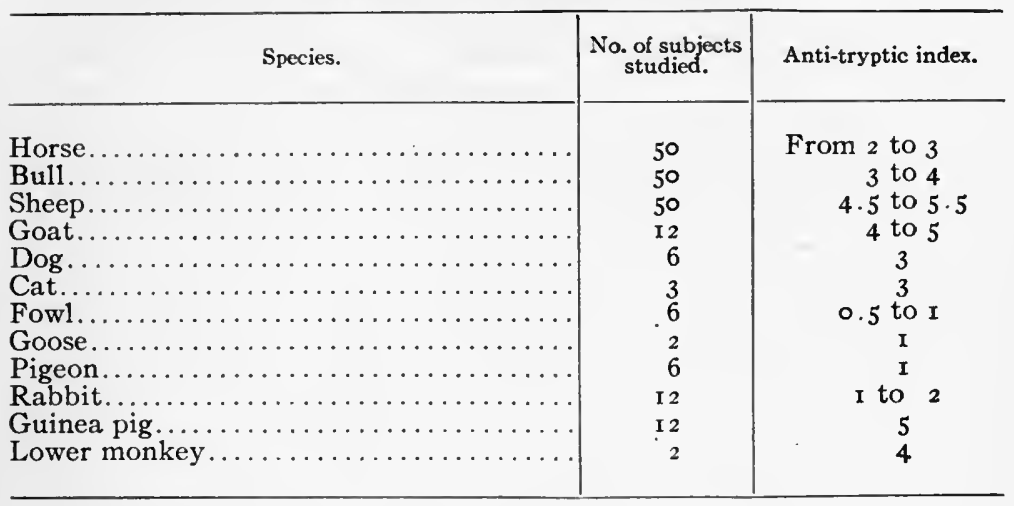

The anti-trypsin constituent of the serum is less resistant to heat than the anti-pepsin. Serum kept for a half-hour at $50^{\circ}$ loses about one-fifth of its anti-tryptic power. Kept for an hour between $60^{\circ}$ and $65^{\circ}$, it weakens considerably, and after 2 hours at $68^{\circ}$ the serum has lost all its neutralizing power toward trypsin. The sensitiveness of anti-trypsin toward temperature is moreover perceptibly reduced when fibrin or albumin is added to the serum.

To study the action of increasing quantities of serum on a pancreatic juice activated by enterokinase, Zunz proceeds as follows: To 2 c.c. of inactive juice, with the addition of 0.5 c.c. of a I per cent solution of enterokinase, he adds increasing quantities of blood serum of a dog, and measures the resulting action by the digestion undergone in 24 hours at $38^{\circ}$ by Mett tubes containing serum or coagulated white of egg.

In tube No. I without serum, $8 \mathrm{~mm}$. of albumin were digested; with o.I c.c. of serum, the anti-tryptic action is already manifest, and it gradually increases with the quantity of serum; in the presence of I c.c. of serum the tryptic action is completely arrested. The serum obtained during fasting is less active than after a meal. Further, the anti-proteolytic power of dog serum 
toward pancreatic juice activated by enterokinase generally increases $1 \frac{1}{2}$ hours after the meal. A maximum is attained after 3 hours, and begins again to decrease after 5 or 7 hours.

Anti-Proteolytic Power of Serum of a Dog Toward Kinased Pancreatic Juice.

\begin{tabular}{|c|c|c|}
\hline \multirow{2}{*}{ Liquids used. } & \multicolumn{2}{|c|}{$\begin{array}{l}\text { Lengths of albumin } \\
\text { digested in } \mathrm{mm} \text {. }\end{array}$} \\
\hline & Serum. & $\begin{array}{l}\text { White of } \\
\text { egg. }\end{array}$ \\
\hline $\begin{array}{r}2 \text { c.c. panc. juice to.5 c.c. enterok. }+0.5 \text { c.c. } \circ .8 \% \\
\mathrm{NaCl} \ldots \ldots \ldots \ldots \ldots \ldots \ldots\end{array}$ & 80 & $6=$ \\
\hline 2 c.c. panc. juice to.5 c.c. enterok. +o.1 c.c. $0.8 \%$ & 0.0 & 0.5 \\
\hline 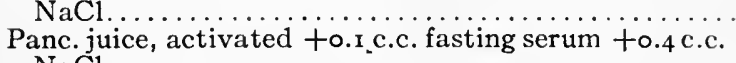 & 6.5 & $5 \cdot 5$ \\
\hline $\begin{array}{l}\mathrm{NaCl} \ldots \ldots \ldots \ldots \ldots \ldots \ldots \ldots \ldots \ldots \ldots \ldots \\
\text { Panc. juice, activated +o.2 c.c. fasting serum +o.3 c.c. }\end{array}$ & 2.5 & I. 5 \\
\hline $\begin{array}{l}\mathrm{NaCl} \ldots \ldots \\
\text { Panc. juice, activated }+0.3 \text { c.c. fasting serum }+0.2 \text { c.c. }\end{array}$ & 2.0 & 1.0 \\
\hline 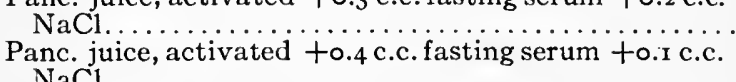 & I. 5 & 0.8 \\
\hline $\begin{array}{l}\mathrm{NaCl} . \ldots \ldots \ldots \ldots \ldots \ldots \ldots \ldots \ldots \ldots \ldots \ldots \ldots \\
\text { Panc. juice, activated }+0.5 \text { c.c. fasting serum. }\end{array}$ & $\begin{array}{l}1.2 \\
0.8\end{array}$ & $\begin{array}{l}0.5 \\
0.2\end{array}$ \\
\hline Panc. juice, activated to. 6 c.c. fasting serum........ & 0.5 & traces \\
\hline Panc. juice, activated + r.o c.c fasting serum......... & 0.0 & 0.0 \\
\hline $\begin{array}{l}\text { Panc, juice, activated to.1 c.c. fed serum +o.4 c.c. } \\
\mathrm{NaC1} \ldots \ldots \ldots \ldots \ldots\end{array}$ & 2.0 & 1.0 \\
\hline $\begin{array}{l}\text { Panc. juice, activated to.2 c.c. fed serum }+ \text { o. } 3 \text { c.c. } \\
\mathrm{NaCl} \ldots \ldots \ldots \ldots \ldots \ldots \ldots \ldots \ldots \ldots \ldots \ldots \ldots \ldots \ldots \ldots \ldots \ldots \ldots \ldots\end{array}$ & I.5 & 0.8 \\
\hline 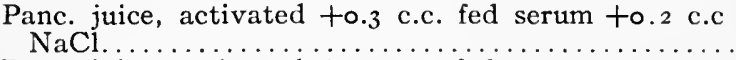 & 1.2 & 0.5 \\
\hline $\begin{array}{l}\text { Panc. juice, activated to.4 c.c. fed serum +o.1 c.c. } \\
\mathrm{NaCl} . \ldots \ldots \ldots \ldots \ldots \ldots \ldots \ldots \ldots\end{array}$ & 1.0 & 0.4 \\
\hline Panc. juice, activated +o.5 c.c. fed serum............ & 0.8 & 0.2 \\
\hline Panc. juice, activated +o. 6 c.c. fed serum........... & 0.5 & traces \\
\hline Panc. juice, activated + i.o c.c. fed serum.. & $\circ$ & 0.0 \\
\hline
\end{tabular}

Anti-trypsin acts like an enzyme, but it is necessary to ask, in its action on the pancreatic juice, which of the three factors is responsible, trypsinogen, enterokinase, or the resulting product, trypsin. According to the case, the action of blood serum is explained by the existence of an anti-trypsinogen, of an antikinase, or of an anti-trypsin. From the researches of Meyer, it appears that blood serum contains only one anti-trypsin. By preparation of an animal by means of repeated injections of 
trypsin, we succeed in increasing the anti-tryptic power of its blood, but we do not succeed at all by the same procedure, injecting inactive pancreatic juice or intestinal juice, in obtaining anti-trypsinogen or anti-kinase. However, Dastre and Delezenne are of a contrary opinion, and, according to them, the serum would act through an anti-kinase.

Effect of Anti-trypsin on Solutions of Trypsin Attenuated by Heating or Shaking. - Anti-trypsin may serve to estimate the proteolytic activity of a solution of trypsin. The serum retains its anti-fermenting power long enough for one to be able to make a type solution and, when need arises, thus bring by its means any solution of trypsin to the same standard. The use of antitrypsin in determinations offers still another great advantage. It shows modifications in the solution of enzyme which would have completely escaped notice in other methods. Thus the volume of trypsin solution corresponding to a definite quantity of serum will depend on the temperature to which the solution of trypsin has been previously brought. When we keep a trypsin solution for 25 minutes at $50^{\circ}$, it loses 25 per cent of its proteolytic power, that is to say, it would take a fourth more of this heated trypsin solution to produce, under the same conditions, the same effect as that produced by the original trypsin solution.

The enzyme thus attenuated should also be relatively less sensitive with regard to serum. Such is not observed. Before the trypsin was heated to $50^{\circ}$, I part of serum neutralized 5 parts of trypsin solution. Consequently, by using trypsin weakened by heating, which has lost one-fourth of its power, to neutralize I part of serum, would theoretically require 6.7 c.c. of this solution. In reality, I part of serum requires at least to parts of heated trypsin.

A similar divergence is observed with solutions of trypsin weakened by shaking. In the study of pepsin it has already been noted that a solution of enzyme loses practically all its proteolytic power by shaking. The cause of this diminution is explained, in part, by the fact that a portion of the enzyme remains stuck on the walls of the vessel, that another passed into the froth, and 
that a last is really destroyed. A similar phenomenon takes place with solutions of trypsin.

Deterioration of Trypsin with Shaking.

\begin{tabular}{|c|c|c|}
\hline & $\begin{array}{c}\text { Proteolytic } \\
\text { Power. }\end{array}$ & Loss. \\
\hline Control. & 100 & 0 \\
\hline $30 \mathrm{~m}$. shaking. & 75 & 25 \\
\hline $60 \mathrm{~m}$. shaking. . & 42 & $5^{8}$ \\
\hline
\end{tabular}

Thus, the solutions lose considerably their activity as the result of shaking. But the tests made with anti-trypsin show that the deterioration caused by shaking is still greater than direct analysis indicates:

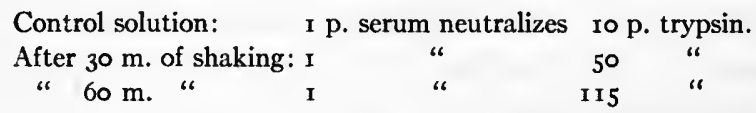

Analytical data, such as the preceding, based on the quantity of albumin dissolved in a given time, do not always show the actual content of active constituent in a trypsin. An enzyme very much weakened may still give a maximum result at a temperature very near that of its destruction. In a deteriorated solution, the quantity of active substance remaining cannot be measured by its direct action on the albuminoid material, for its activity is here apparently increased. Although the number of enzyme units are reduced, the digestive work done is such as to lead to the conclusion that there is a much greater quantity of enzyme present than really is the case. On the contrary, antitrypsin does not appear sensitive to all these secondary effects; a unit of anti-trypsin, irrespective of the conditions, always saturates a unit of trypsin present. As a result the data furnished by anti-trypsin always approach true values more closely than those obtained by ordinary analysis.

The attenuation of the activity of living cells cannot always be measured in the direct way, and on this point there is again a close analogy between the cells and the enzymes. In the course of the alcoholic fermentation of a mash of grain at a 
temperature of $28^{\circ}$, the temperature can be raised to $37^{\circ}$ for an hour without any apparent injury. Brought to $28^{\circ}$ again, the fermentation continues giving per hour the same gaseous liberation as before the liquid was brought to $37^{\circ}$. Yet, on closer study, we see that this short degree of overheating has nevertheless brought about deep changes, which remain for a certain time. Thus it is that yeast, which before it had been brought to $37^{\circ}$, withstood a definite quantity of antiseptic, now succumbs when the quantity is reduced by one-half. Furthermore, into such a mash, unheated and in full fermentation by a culture of lactic ferment, the introduction of one bacterium per roo cells of yeast does not cause acidification. On the contrary, the yeast which has undergone the action of $38^{\circ}$ for an hour no longer withstands the attack of the lactic ferment, and even if only one bacterium is added per 500 cells of yeast the liquid acidifies very quickly. Thus, in the action of enzymes, as in that of living cells, an attenuation of their activity cannot be directly measured, and only special reactions, like those of the anti-enzymes, the sensitiveness to antiseptics, or, again, the resistance toward other living cells, can show the whole extent of the change.

\section{$\S$ it. Determination of Trypsin.}

Methods Already Cited for the Determination of Pepsin. The general considerations which we have developed with regard to the determination of pepsin are found to apply equally well here. The same variety exists in the choice of albuminoid substrates as well as in the manner of estimating the progress of the reaction. It must, however, be noted that trypsin has an advantage over pepsin in that the pancreatic enzyme pushes digestion much further than the stomachal enzyme, and that it gives, among the products of hydrolysis, a certain quantity of amino-acids. Now, these acids are subject to exact analysis by the method of Sörensen. We have, then, in the determination of the amino-acid formed, a convenient and accurate means of measuring the activity of a trypsin. Aside from this fortunate exception, almost all the methods described for pepsin apply to 
the determination of trypsin. The methods of Grützner with colored fibrin, of Effront with white of egg, of Mett with small tubes full of coagulated albumin, are applicable, only the digestion, instead of being carried out in acid media, must take place in neutral, or better still, in very slightly alkaline media.

Method with Casein. - Casein has been proposed successively by Weis, Fuld, and others, as a suitable substrate for the determination of trypsin. The following is a procedure for the determination of the enzyme content of a pancreatin. I $\mathrm{g}$. of Hammarsten casein is dissolved in Io c.c. of $N / \mathrm{IO} \mathrm{NaOH}$ with the addition of a little water. (It is advantageous to heat slightly.) When solution is complete, neutralize exactly to litmus paper, which requires usually about 5 to 7 c.c. $N /$ ro $\mathrm{HCl}$. Then bring to a volume of roo c.c. with a 0.8 per cent solution of $\mathrm{NaCl}$. Also prepare a r.o per cent, a o.I per cent," and a 0.0 I per cent pancreatic solution in physiological salt solution. Finally, prepare a dilute solution of acetic acid by mixing 5 c.c. of glacial acid +45 c.c. of alcohol +50 c.c. of water. The determination is made in the following manner: In a series of tubes put $\mathrm{I}$ to 5 c.c. of pancreatic solution at various concentrations. Bring to a ro c.c. volume with the physiological salt solution, and add 2 c.c. of the I per cent solution of casein. Allow to stand an hour at $48^{\circ}$, and then add three drops of acetic acid solution and note turbidity or its absence. Take, for example, a series of tubes containing:

\begin{tabular}{|c|c|c|c|c|}
\hline I: I c.c pa & solution at & $1 \%$ & $0.0100 \mathrm{~g}$ & stance. \\
\hline II: $\frac{1}{10}$ c.c. & “ & ${ }_{1} \%$ & $0 . \infty 10$ & " \\
\hline III: $\frac{5}{10}$ c.c. & " & $0.1 \%$ & $0 . \infty 005$ & " \\
\hline IV: 3 c.c. & " & $0.01 \%$ & 0.0003 & " \\
\hline$V: 2.5$ c.c. & " & $0 . \infty 1 \%$ & 0.00025 & " \\
\hline VI: 2 c.c. & " & $0 . \infty 01 \%$ & $0 . \infty 002$ & " \\
\hline
\end{tabular}

If, after action of the enzyme and addition of the acid, it is found that I, II, III, IV are not turbid, while V and VI give a precipitate of casein, then $0.0003 \mathrm{~g}$. is the minimum quantity capable of digesting the casein present. Thus:

(I) $0.0003 \mathrm{~g}$. of trypsin digests $0.02 \mathrm{~g}$. of casein, or I g. of 
trypsin digests $66 \mathrm{~g}$. of casein. The strength of the trypsin is, therefore, 66.

(2) The unit of trypsin required to digest 2 c.c. of casein solution is 0.0003 .

Remarks. - I. It happens sometimes that after having mixed, according to the technique recommended, the enzyme and the casein solutions, and having placed the tube on the waterbath at $40^{\circ}$, at the end of a few minutes the appearance of a turbidity or even a precipitate is observed. This phenomenon is observed especially with microbic cultures made on milk, and is due to an enzymic action. In fact, if comparative experiments are made with boiled or unboiled proteolytic solutions, the precipitate appears only in tubes containing the unheated enzyme. The phenomenon is probably due to the precipitation of the casein under the influence of the rennet contained in the culture along with the trypsin. If a similar tube where the casein has been precipitated is maintained several hours at $40^{\circ}$, the precipitate finally redissolves. The method of measuring trypsin with casein would evidently not apply to such enzyme liquids. In fact, the liquid resulting from the mixture of the enzyme and the casein being already turbid, it is impossible to determine if the addition of acetic acid produces a new precipitate. Furthermore, if trypsin acts on precipitated, in place of dissolved, casein the experimental conditions cease to be comparable.

II. - Casein can also be used in another manner to measure trypsin. This procedure is especially suitable when a series of measurements are to be made. Prepare, as previously, a I per cent neutral solution of casein. Distribute it in roo c.c. portions in a series of flasks, and then add the enzyme solutions to be studied. Control tests are arranged by introducing the same quantities of boiled enzyme solutions. Add to each flask 2 c.c. of a mixture of chloroform and toluol, and allow to stand 24 or 48 hours at $40^{\circ}$. Then precipitate the remaining casein by a quantity of $\mathrm{N} /$ ro acetic acid, determined in advance and always the same. Dilute, filter, and determine the total nitrogen in the clear liquid. 
If a differentiation in the manner of attack upon albuminoid substrates by the various vegetable trypsins is required, it is necessary to determine in the filtered liquid, coming from the digestion of casein or albumin and separated from the excess of untransformed material, not only total nitrogen but also the distribution of this nitrogen among the different products of the degradation. Therefore, in an aliquot part of the liquid, a determination is made of the nitrogen precipitable by zinc sulphate; in another, a determination of the nitrogen precipitable by phosphotungstic acid; finally, in a third portion, a determination of the amino-nitrogen by Sörensen's method. In certain cases, it will even be well to determine the ammoniacal nitrogen, which is set free by boiling with $\mathrm{MgO}$, either at $10^{\circ}$, or better in a vacuum.* It is thus found not only that trypsin can have greater or less activities, but also that they often act in different ways.

Method of Jacoby. - This method is based on the same principle as that already described with regard to the analysis of pepsin. First of all an emulsin of ricin is prepared containing $\mathrm{I} \mathrm{g}$. of this substance and $1.5 \mathrm{~g}$. $\mathrm{NaCl}$ in 100 c.c. of water. Pour 2 c.c. of this liquid in a series of test tubes, in which is then added $0,0.1,0.2,0.3,0.5,0.7, \mathrm{I} .0$ c.c. of a I per cent solution of trypsin. Then bring all the volumes to 3 c.c., and add in each tube 0.5 c.c. of I per cent $\mathrm{NaOH}$. Allow to stand at $37^{\circ}$, and note the time required for the liquid to become clear. With o.I c.c. of I per cent trypsin of good quality, a complete clarification is obtained at the end of 6 hours.

\section{$\delta$ i 2. Pancreatic Juice.}

According to Glæssner, we can estimate at 500 or 800 c.c. the quantity of juice secreted in man in 24 hours. For a long time it has been believed that the activation of the pancreatic gland was provoked by the nervous system, and it was believed that

* For the necessary directions for making these different determinations, zinc sulphate nitrogen, and tungstic nitrogen, see page 234 ; for formaldehyde nitrogen, page 323 ; and for ammonia nitrogen, page 243 . 
the nerve centers commanding this secretion resided in the pylorus. Bayliss and Starling first showed that in the secretory mechanism a special agent was employed, secretin, which comes from the intestine and is brought to the pancreas by the blood. Definite proof of this latter fact has been obtained by Wertheimer, Launoy, Gopidsky, Zunz, Lalon, and others.

The pancreatic juice forms a viscous liquid, of alkaline reaction. To make 100 c.c. neutral to phenolphthalein, requires ro to 50 c.c. of $N /$ ro acid. The specific gravity varies from r.00448 to r. 00455 , and may reach 1.0098 . Its freezing point varies between $-0.46^{\circ}$ and $-0.49^{\circ}$. The analysis of the pancreatic juice of man gives the following results:

Analysis of Pancreatic Juice.

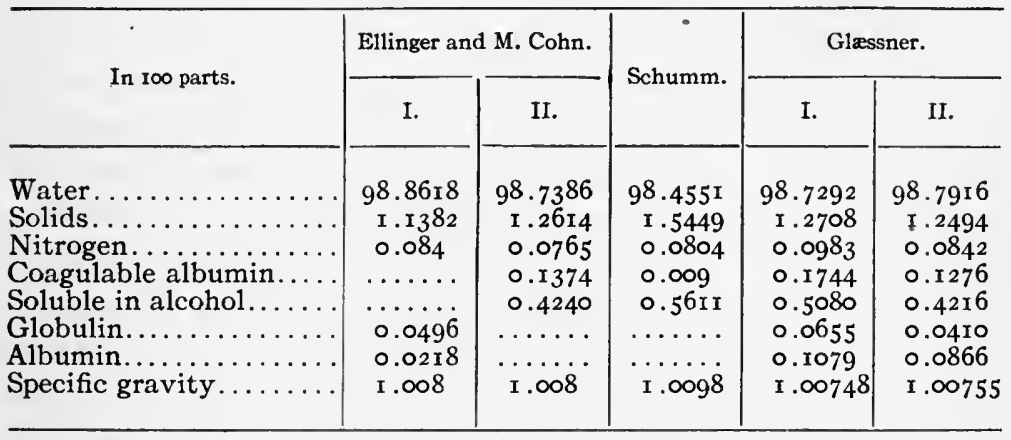

The ash analysis is of importance in that the juice contains from 0.9 to 0.95 per cent ash, equivalent to about half of the dry extract. In this ash are found carbonates, chlorides, sulphates, and a small amount of phosphates. Certain writers note the presence of calcium; others, its absence. This important point will be discussed later.

The mineral compositions of the pancreatic juices of the dog and the cow are given on the following page, the figures referring to one liter of pancreatic juice.

We shall see in another chapter that pure pancreatic juice, collected by catheterization of the canal of Wirsung, is always deprived of proteolytic power. It is rendered active by the 
addition of either enterokinase or of a calcium salt. It is, therefore, interesting to inquire whether the pancreatic juice itself contains calcium.

Mineral Composition of the Pancreatic Juice.

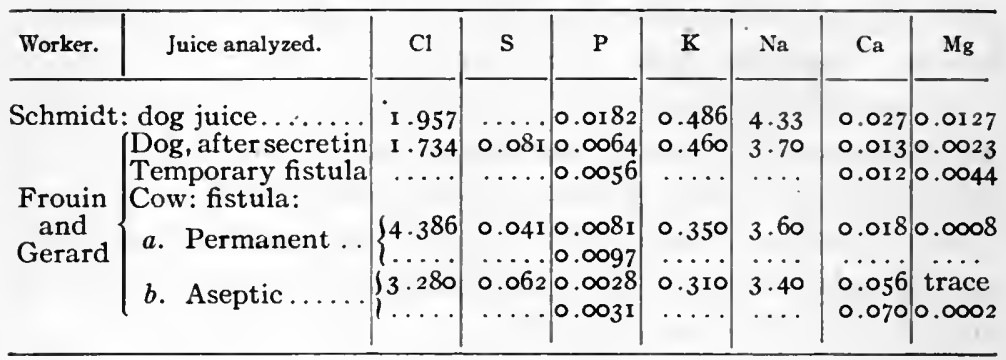

E. Pozerski, using the method of analysis devised by Grimmé (Thesis of Fribourg, I905), investigated the presence of this element in the pancreatic juice of a dog under the influence of various secretory agents. First he studied the juice of secretin. Analyses were made on twelve samples of different juices, all completely inactive toward coagulated egg-albumin. All gave negative tests for calcium. Then he examined the juice of pilocarpin. It is known that this alkaloid, like other substances, causes a secretion manifesting by itself a proteolytic action at times very intense. This juice, from various points of view frankly abnormal, always contains calcium in very appreciable quantity. Pozerski has even found that its proteolytic activity varies perceptibly in the same direction as its calcium content. The following are the results given by different juices of pilocarpin:

Relation Between the Calcium Content of the Juice of Pilocarpin and its Proteolytic action.

\begin{tabular}{|c|c|}
\hline Per cent calcium. & $\begin{array}{c}\text { Time for complete diges- } \\
\text { tion of given cube of } \\
\text { albumin by I c.c. }\end{array}$ \\
\hline $0.0119-0.0103$ & I2 to 24 hours \\
$0.0069-0.0053$ & 40 to 48 hours \\
$0.0021-0.0009$ & 4 to 6 days \\
\hline
\end{tabular}


These last juices, of very low calcium content, had moreover been obtained in cases where the injection of pilocarpin had been badly conducted and where, consequently, the animal had secreted juice of pilocarpin, and also juice of secretin.

Pure pancreatic juice, strictly inactive, is lacking in calcium and though in the analyses of Schmidt and Frouin, referred to above, calcium was found, although in very small amounts, it is because the juice examined was not strictly inactive. In fact, Delezenne, resorting to the spectrum analyses, was able to show in the majority of cases infinitesimal traces of calcium; but these cannot share in the activation except under some very special conditions, determined in a precise manner by Delezenne.

The nitrogenous constituent of the juice coagulates between $45^{\circ}$ and $90^{\circ}$. The neutralized juice becomes turbid at $45^{\circ}$ and gives a slight precipitate at $55^{\circ}$. The filtered liquid is again turbid at $62^{\circ}$ and precipitates at $70^{\circ}$. When this precipitate is removed, it is found that the filtrate again becomes turbid at $75^{\circ}$ and precipitates abundantly at $90^{\circ}$. The nitrogenous material is made up of globulin, albumin, albumoses and peptones. The juice, freshly collected, shows itself active to starch and fatty substances, but inactive, or active only to a very slight extent, toward albuminoid substances. On the contrary, it becomes proteolytic when intestinal juice is added. The juice, thus activated from the point of view of trypsin, likewise increases its amylolytic and lipolytic powers. The bile also exerts an accelerating action on the lipase and the amylase of the pancreatic juice.

When the secretion of the pancreatic juice is caused by means of repeated injections of secretin, it is found that during the draining, which may last for Io or I 2 hours, the enzyme content of the juice constantly diminishes. Yet, the decrease is much greater for the lipolytic and amylolytic powers than for the proteolytic power. At the same time, the alkalinity of the juice also decreases. With respect to this last variation, the following figures, estimated in fractions of normal liquor, are given by L. Morel and Terroine. 
Variation of Alkalinity of Pancreatic Juice of Secretin.

\begin{tabular}{|c|c|}
\hline & $\begin{array}{l}\text { Normality } \\
\text { in } \mathrm{Na}_{2} \mathrm{CO}_{2}\end{array}$ \\
\hline 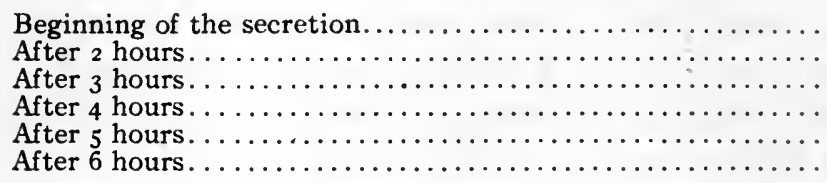 & $\begin{array}{l}\text { I } / 9 \\
1 / 10.4 \\
1 / 11.2 \\
1 / 11.3 \\
\text { I } / 12.2 \\
1 / 19.9\end{array}$ \\
\hline
\end{tabular}

In the course of the digestion, the composition of the juice varies as much from the point of view of its density and of its alkalinity as of its enzyme content. The maximum rate of secretion, as well as the maximum alkalinity, is reached 4 hours after a meal. The secretion, which gradually decreases in rate, is nevertheless prolonged for about 8 hours.

Babkin and Tichomirow found that an exact relation exists between the solid residue and the nitrogen content on the one hand, and between these two factors and the proteolytic power on the other. The following table combines the different pancreatic juices of dogs, classified according to their increasing proteolytic powers (determined by the method of Mett). It is to be noted that the solid residue and the nitrogen vary very perceptibly in the same direction.

Relation Between the Proteolytic Power of the Pancreatic Juice and its Content in Solid Residue and in Nitrogen.

\begin{tabular}{|c|c|c|c|c|c|}
\hline $\begin{array}{l}\text { Proteolytic } \\
\text { power. }\end{array}$ & $\begin{array}{l}\text { Per cent solid } \\
\text { residue. }\end{array}$ & $\begin{array}{c}\text { Per cent } \\
\text { nitrogen. }\end{array}$ & $\begin{array}{l}\text { Proteolytic } \\
\text { power. }\end{array}$ & $\begin{array}{l}\text { Per cent solid } \\
\text { residue. }\end{array}$ & $\begin{array}{c}\text { Per cent } \\
\text { nitrogen. }\end{array}$ \\
\hline I. 9 & I. 330 & $0.065^{8}$ & 3.6 & 2.126 & 0.1933 \\
\hline 2.3 & 1.570 & 0.1016 & 3.8 & 2.036 & $0.189 \mathrm{I}$ \\
\hline 2.4 & I. 470 & 0.0869. & 3.9 & 2.423 & 0.2410 \\
\hline 2.75 & I .683 & 0.1079 & 3.95 & 2.248 & 0.2088 \\
\hline 2.8 & I. 352 & 0.1016 & 4.75 & 2.612 & 0.2746 \\
\hline 2.8 & $1.45^{2}$ & $0.09 \mathrm{II}$ & 4.8 & 2.770 & 0.3047 \\
\hline $3 \cdot 1$ & I. $57^{2}$ & 0.1135 & 4.8 & 2.964 & 0.3236 \\
\hline 3.4 & I .869 & $0.147 \mathrm{I}$ & 5.0 & 3.104 & 0.3587 \\
\hline $3 \cdot 55$ & I .885 & 0.1926 & 5.2 & 3.288 & 0.4189 \\
\hline 3.6 & I.9I4 & 0.1695 & 7.1 & 5.180 & 0.6585 \\
\hline
\end{tabular}




\section{§ 13. Excitation of the Pancreatic Secretion.}

Pawlow first established the correlation which exists between the gastric and pancreatic secretions. According to him, this second secretion was due to a nervous reflex, resulting from innervation produced by various substances of the chyme, notably its acidity. This opinion is no longer accepted by physiologists. Bayliss and Starling have, in fact, shown experimentally that the nervous system does not intervene at all in the pancreatic secretion, but that this is caused solely by a humoral route, under the action of a special substance, secretin, which is contained in the intestine and which normally, in the course of digestion, is distributed in the blood and directly excites the cells of the pancreas.

First of all, this secretin originates in the intestine. If the duodenum-jejunal mucous membrane is macerated in dilute $\mathrm{HCl}$, $\mathrm{N} /$ Io for example, and after neutralization the solution so obtained is injected into the veins, a large pancreatic secretion results. This setting free of secretin also takes place in vitro. By injecting dilute hydrochloric acid or a solution of soap into the duodenum of an animal, it is found that its intestinal content, collected after a certain time and injected into another animal, causes pancreatic secretion. It is not even necessary to previously inject $\mathrm{HCl}$ or a solution of sodium oleate. The simple duodenum-jejunal content of an animal in the course of digestion, after filtration and elimination of the albumoses and the peptones, can, if it is introduced into the circulatory system of an animal, cause a pancreatic secretion to take place.

In the second place, it is easy to show that this secretin, liberated either normally or under the action of $\mathrm{HCl}$, passes into the blood before acting on the pancreas. We find, in fact, if we collect the blood which flows from the mesenteric veins of an animal in the act of digesting, and inject it into another fasting animal, that this blood brings about in this second animal a manifest pancreatic secretion. Also, the blood taken under identical conditions from an animal into whose intestine has been 
injected dilute $\mathrm{HCl}$ or a solution of soap, when injected into a second fasting animal, causes in the latter a pancreatic secretion. Therefore, the secretin comes from the intestine and acts on the pancreas through the blood as a medium. Finally, the demonstration may be completed by establishing the fact that artificial secretion caused by injections of secretin, from the qualitative and quantitative point of view, as from the point of view of general method, is quite comparable to that obtained in normal digestion.

\section{Normal and Artificial Secretions of Pancreatic Juice.}

I. - Secretions observed during digestion with animals with permanent fistulas.

\begin{tabular}{|c|c|c|}
\hline Foods. & $\begin{array}{c}\text { Length of } \\
\text { secretion. }\end{array}$ & $\begin{array}{c}\text { Quantity of } \\
\text { juice secreted. }\end{array}$ \\
\cline { 2 - 3 } & Hours. & c.c. \\
Bread and milk...... & 6 & I30 \\
Meat ............. & 6 & 206 \\
Bread and milk...... & 6 & 192 \\
\hline
\end{tabular}

II. - Secretions caused with animals with permanent fistulas by introduction of $\mathrm{HCl}$ into the stomach.

\begin{tabular}{|c|c|c|}
\hline $\begin{array}{l}\text { Quantity of } 0.5 \% \\
\mathrm{HCl} \text { introduced. }\end{array}$ & Length of secretion. & $\begin{array}{l}\text { Quantity of } \\
\text { juice secreted. }\end{array}$ \\
\hline $\begin{array}{l}\text { c.c. } \\
200\end{array}$ & $\begin{array}{l}\text { h. } \min . \\
\text { I } 50\end{array}$ & $\begin{array}{l}\text { c.c. } \\
\mathrm{I} 38\end{array}$ \\
\hline 200 & 220 & 154 \\
\hline 200 & 20 & 173 \\
\hline
\end{tabular}

III. - Secretions caused with animals with temporary fistulas by introduction of $\mathrm{HCl}$ in the duodenum.

\begin{tabular}{|c|c|c|}
\hline $\begin{array}{l}\text { Quantity of } 0.5 \% \\
\mathrm{HCl} \text { introduced. }\end{array}$ & Length of secretion. & $\begin{array}{l}\text { Quantity of juice } \\
\text { secreted. }\end{array}$ \\
\hline $\begin{array}{l}\text { c.c. } \\
210 \\
500 \\
280\end{array}$ & $\begin{array}{cc}\text { h. } & \text { min. } \\
4 & \text { ro } \\
4 & 7 \\
3 & \end{array}$ & $\begin{array}{l}\text { c.c. } \\
59.2 \\
14.7 \\
18.8\end{array}$ \\
\hline
\end{tabular}


IV. - Secretions caused with animals with temporary fistula by repeated injections of secretin.

\begin{tabular}{|c|c|}
\hline Length of the secretion. & Quantity of juice secreted. \\
\hline Hours. & c.c. \\
6 & 320 \\
8 & 1300 \\
8 & 400 \\
\hline
\end{tabular}

With regard to the chemical nature of the excito-secretorial substance, we know but very little. Secretin is not an enzyme product, for.it resists boiling; however, if kept for a long time at $120^{\circ}$, it is destroyed. Mineral acids and alkalis at $100^{\circ}$ also change it very rapidly. This material evidently belongs to the albuminoid group, for trypsin and erepsin digest it easily and cause it to lose its specific property. This destructive action of erepsin explains why secretin is not obtained when mucous membranes are macerated in pure water, and not in dilute acid. In the first case, the proteolytic enzyme digests the secretin which was already diffused in the liquid. This is certainly the case, for if a watery intestinal extract is added to a solution of secretin already prepared, the mixture loses in a few hours a great deal of its excito-secretory power.

Secretin pre-exists in the intestine; it is not, as was believed for some time, found in the state of pro-secretin, which a treatment with $\mathrm{HCl}$ would have the effect of transforming into active substance. The best proof of this fact is that secretin can be isolated without the use of acid, by treating the duodenal mucous membrane with boiling salt solutions, with Io per cent solutions of Witte peptone, with bile salts, with urea, and even by pressure.

Secretin acts on the pancreatic cells solely by the humoral path. The normal pancreatic secretion differs from all the other secretions in that it requires no nervous mechanism. Popielski has shown without doubt, that the maceration of duodenal mucous membrane, in addition to its excito-secretory power, also possesses the faculty of causing a vaso-dilation, whence 
results a lowering of arterial pressure. But it has since been established by numerous experimenters that these two physiological effects are not dependent on the same cause; we can, in fact, by means of boiling absolute alcohol, separate the crude secretin into two substances, one of which causes pancreatic secretion without lowering the blood pressure and the other of which brings about this lowering without having any influence on the pancreas. The distinction is therefore very clear.*

We have just established the fact that the excitation of the pancreatic secretion could be realized by injections of secretin into the blood, or again by introducing into the duodenum certain substances, like acids, soaps, neutral fats, ether, chloroform, chloral, alcohol, essence of mustard, or barium chloride. Most often, the pancreatic juice, obtained under the influence of excitants, is not directly proteolytic; this is notably the case with the juice which flows after intestinal injections of acid or of maceration of duodenal mucous membrane in $\mathrm{HCl}$. But it may also happen that the juice which is collected is active on egg albumin. This is the case in the secretion caused by injections of pilocarpin, of cholin, or even of Witte's peptone. It is very probable that, in these cases, the mechanism of action is entirely different from that studied with pure secretin, these bodies no longer acting like normal excitants but like true poisons of the cell. It is to be noted, furthermore, that the intestinal mucous membrane is not the only source of secretin. It has been found, in fact, that the macerations of liver, brain, kidney, thyroid, mesenteric ganglia, testicles, and other organs also cause, under definite conditions, a pancreatic secretion.

We have just seen that the direct injection of a peptone solution, or again the injection of a maceration of intestinal mucous membrane made in a ro per cent solution of peptone, causes pancreatic secretion. In fact, the action is not always so simple. Frouin found that if peptone is utilized, not as just described but with the addition of solutions of mineral or

* A complete exposition of this question, and all the related literature is found in the recent work of Terroine, The Pancreatic Secretion. 
organic acids, very different results are obtained. With $\mathrm{HCl}$, $\mathrm{H}_{2} \mathrm{SO}_{4}, \mathrm{HNO}_{3}$, the secretion is diminished; on the contrary, it is increased with citric, oxalic, and tartaric acid. If intestinal mucous membrane is macerated in solutions of mineral or organic acids, the addition of 5 per cent solution of peptone to each of them will in the first case diminish in vitro the formation of secretin (hence a slackening in the pancreatic secretion), while in the second, it perceptibly increases this formation. The results obtained should vary, then, with the method utilized. On the contrary, ro-2o per cent solutions of the carbohydrates, maltose, saccharose, and especially lactose, in 0.36 per cent $\mathrm{HCl}$ unmistakably increase the secretion produced by hydrochloric acid.

\section{§ 14. Zymogen and Trypsinogen. - Enterokinase.}

The extract of fresh pancreas possesses a very slight proteolytic power. With time and under the influence of conditions that are still but little understood, this power increases to reach a constant maximum. Kühne has given the name of zymogen to the inactive body which is afterward transformed into trypsin. The transformation of zymogen into trypsin has been attributed by different writers to the oxygen of the air. It was also thought for a long time that the acid reaction favored the appearance of this proteolytic enzyme. In reality, this can be observed just as well in alkaline media as in acid media. A solution of zymogen, with addition of alcohol, furnishes a precipitate which possesses great activity. The pancreas of animals killed in the course of digestion furnishes an extract capable of being activated very rapidly. On the contrary, the result appears to be less satisfactory with pancreas of animals which have fasted for a long time. The extracts of calf or dog pancreas in general activate quite easily. The pancreas of the cat gives, on the contrary, an extract difficult to activate. The extracts of pancreas made in glycerin solutions or in boiled water are inactive. Water not boiled, or slightly acidified by acetic or lactic acid, gives, on the 
contrary, extracts easily activated. The activation is accentuated by adding to these liquids extract of liver or amino-acids.

The pancreatic juice of man and of animals acts quite differently from corresponding extracts of the fresh pancreas. The pancreatic juice does not contain trypsin as such, but rather the zymogen, which does not activate under the same conditions as the zymogen of the pancreas. Kühne considers it then to be different from the zymogen of the pancreas, and gives it the name of trypsinogen. The pancreatic juice which is collected by the aid of a fistula is sometimes active, while in other cases it is not. Pawlow has drawn attention to this anomaly, and, in collaboration with Schepowalnikow in 1890 , established the very important fact that intestinal juice increases considerably the activity of pancreatic juice. In I902, Delezenne and Frouin recognized that the pancreatic juice in a pure state, collected with care from animals carrying a permanent fistula by catheterization from the canal of Wirsung, is always inactive toward coagulated albumin, and that the intestinal juice intervenes, not only as a favoring agent but as a real generator of trypsin. Delezenne has, therefore, established that trypsin is found in the pancreatic juice in the form of a zymogen, and that it is under the influence of a special activating substance contained in the intestinal juice that this zymogen is transformed into trypsin. To this activating substance, discovered by Pawlow, has been given the name of enterokinase. It appears to be related to the soluble enzymes, for an intestinal juice boiled and filtered loses its power of stimulating the pancreatic juice. On the contrary, the activation takes place very rapidly with a fresh juice at a temperature of $38^{\circ}$.

The data of Delezenne and of Pawlow have been since confirmed by Popielsky, Hekma, Zunz, and others. Different hypotheses as to the mechanism of the action of the intestinal juice have been offered. The most reasonable suggests a direct action of intestinal juice on the trypsinogen, without this touching at all the albuminoid matter which must then be transformed. This activation of pancreatic juice would result, therefore, from a 
special enzyme action, caused by an enzyme on a zymogen. The rôle of enterokinase would be confined solely to transforming trypsinogen into trypsin, without taking part directly in the resulting hydrolysis.

Enterokinase. - From the preceding, enterokinase is the active substance of the intestinal juice which transforms trypsinogen of the pancreatic juice into trypsin. It is found in abundance especially in the duodenum-jejunal portion of the intestine, but is also met with in the lymphatic ganglia and in the leucocytes. According to Dastre and Stassano, enterokinase is obtained by the following method: Dogs are killed five or six hours after an abundant meal of horse meat. The intestines are cut in pieces, washed, and the intestinal mucous membrane scraped with the back of a scalpel. The product obtained from this scraping is treated for some time with a 15 per cent solution of $\mathrm{Na}_{2} \mathrm{CO}_{3}$, is then filtered, and the clear liquid precipitated by acetic acid. The precipitate is dried at $40^{\circ}$, then powdered. This product contains the enterokinase mixed with the nucleoalbumins precipitated by the acid. I g. of crude enterokinase can stimulate from 300 to $400 \mathrm{~g}$. of pancreatic juice after a period of 24 hours in the incubator at $38^{\circ}$.

The normal intestinal juice differs perceptibıy with respect to its content in different enzymes from a maceration of mucous membrane. In the intestinal juice, besides kinase, we find amylase and maltase. In the maceration juice we find, besides these three active substances, invertin and lactase. These two last enzymes come from the epithelial lining cells or the débris of dead cells.

Zunz, in order to study the course of the activation of the pancreatic juice by enterokinase, makes use of a I per cent solution of powdered enterokinase in 0.5 per cent $\mathrm{Na}_{2} \mathrm{CO}_{3}$. In a series of reaction tubes he pours 2 c.c. of inactive pancreatic juice, adds increasing quantities of solution of enterokinase, and brings the volume to 3 c.c. by means of physiological salt solution. In the mixtures thus prepared, he plunges Mett tubes - containing blood serum or white of egg. He adds several drops 
of toluol and leaves the whole 24 hours at $38^{\circ}$. The following are the results he obtained:

Activation of Pancreatic Juice by Enterokinase.

\begin{tabular}{|c|c|c|c|c|c|}
\hline \multirow{2}{*}{$\begin{array}{l}\text { Quantity of } \\
\text { enterokinase } \\
\text { in drops. }\end{array}$} & \multicolumn{2}{|c|}{$\begin{array}{l}\text { Length of albumin digested, } \\
\text { in millimeters. }\end{array}$} & \multirow{2}{*}{$\begin{array}{l}\text { Quantity of } \\
\text { enterokinase } \\
\text { in drops. }\end{array}$} & \multicolumn{2}{|c|}{$\begin{array}{l}\text { Length of albumin digested, } \\
\text { in millimeters. }\end{array}$} \\
\hline & Blood serum. & White of egg. & & Blood serum. & White of egg. \\
\hline 0 & o & 0 & 6 & I 2. & 5.5 \\
\hline I & 2 & I & 7 & 13 & 6 \\
\hline 2 & 4 & 2 & 8 & 14 & 6.5 \\
\hline 3 & 6 & 3 & 9 & 14.5 & 7 \\
\hline 4 & 8 & 3.5 & 10 & 15 & 7 \\
\hline 5 & 10 & 4.2 & 20 & 15 & 7 \\
\hline
\end{tabular}

From this table it appears that the quantity of albumin digested depends directly on the quantity of enterokinase added to the pancreatic juice. Without enterokinase, no proteolytic action takes place. With one drop, $2 \mathrm{~mm}$. were digested, and with a double or triple quantity, a double or triple length of albumin was digested. Solutions of enterokinase heated for a half-hour at $60^{\circ}$ lost a noticeable part of their stimulating power. Brought to $75^{\circ}$, they are completely destroyed. The enzymic character of enterokinase is also shown by the fact that a very small quantity of this substance can activate, by a sufficient prolongation of the time of the test, considerable quantities of trypsinogen. It must also be added that the rate of stimulation is very nearly proportional to the quantity of kinase used.

Enterokinase is found not only in the intestinal juice but is a very widely distributed enzyme which Delezenne has noted in the poison of serpents, in mushrooms, especially the Amanitas, and in bacteria. This investigator has found, in fact, that if pancreatic juice is inoculated with the spores of Bacillus subtilis or Bacillus mesentericus, the liquid, after the bacteria have developed and when filtered, has acquired a pronounced proteolytic activity. Nevertheless, the bacterial culture, filtered before its mixture with the pancreatic juice, does not confer any activity on the latter. Finally, Hougardy has found that in milk there 
is likewise a substance capable of activating the pancreatic juice, a substance quite similar to the enterokinase of Pawlow. It is destroyed by heating for a half-hour at $75^{\circ}$, and bears the name of lactokinase.

\section{§ 15. Activation of Pancreatic Juice by Calcium.}

The activation of the pancreatic juice can thus be brought about without the intervention of enterokinase. In 1905, Larguier des Bancels showed that this activation can also be produced by the combined action of mineral salts and colloidal substances. To coagulated albumin impregnated with a colloid, quinolin-blue, he added inactive pancreatic juice with the addition of an alkaline-earth salt, and he found that under these conditions he obtained a solution of the albuminoid. In reality, the rôle of the colloid does not appear to be indispensable, at least according to the observations of Delezenne, who, at the same period, found that the activation of the pancreatic juice can be produced solely under the influence of calcium salts. In a series of tubes, containing inactive pancreatic juice, Delezenne adds increasing quantities of chloride, of nitrate and of acetate of calcium. He brings to equal volumes, and introduces coagulated albumin. While the tube which has not received calcium remains intact, digestion on the contrary proceeds very actively in the other tubes. However, it is noted that the maximum result is produced after a certain time of contact between the liquid and the calcium salt, and that the addition of a great excess of salt is plainly unfavorable to the reaction.

This rôle of calcium in activation has been studied very closely by Delezenne, and his results have since been confirmed by Zunz. From their experiments it appears that calcium salts furnish constant results. Without doubt, in certain cases, a slight activation is produced by salts of barium, strontium, magnesium and of cadmium, as well as of certain amino-acids, but the effect of these salts is irregular and in most cases uncertain. The effect of calcium salts, while specific, depends on the metallic 
ions contained in the liquid. Equimolal quantities of different salts of calcium have the same activating effect on the same pancreatic juice, but the activating quantities of calcium vary perceptibly with the origin of the juice tested.

For the most part, calcium salts at a concentration of 0.5 per cent give the most favorable action. It must be noted that the greater part of the calcium used, up to 80 per cent, serves to precipitate the carbonates and the other salts contained in the juice. The part really active on the zymogen is extremely small. As the precipitation of the salts from the juice can also be brought about by salts of barium, strontium, or magnesium, the optimum effect is reached with the aid of a very reduced quantity of calcium, the rest of the metal necessary being replaced by various members of the alkaline-earth group. The following is an observation that bears witness to the very great sensitiveness of pancreatic juice toward calcium ions. Pancreatic juice, completely inactive on gelatin and albumin, when brought in a dialyzer of collodion or vegetable parchment to a temperature of $39^{\circ}$, immediately acquires a very intense proteolytic activity, already perceptible at the end of 2 hours. Pozerski proved that this activation is due to the small quantity of calcium contained in the membrane. It is sufficient, in fact, to decalcify this by an immersion of 18 hours in $\mathrm{I}$ per cent $\mathrm{HCl}$ and then washing for 6 hours in distilled water, to obtain a dialyzer which remains entirely incapable of affecting an inactive pancreatic juice.

Activation of juice by calcium salts is not an instantaneous reaction. It manifests itself only in the long run and requires, in general, 6 to 8 hours to produce the maximum effect. Calcium salts, like enterokinase, transform trypsinogen by way of catalysis and take no part in the proteolytic action of the trypsin so formed. This point has been confirmed by the experiments of Zunz.

In No. I, the pancreatic juice has had no addition of calcium salt and the proteolytic action on the albumin, serum, or white of egg is zero even after 48 hours. In No. 2, the calcium salt has 
Activation of Pancreatic Juice by $\mathrm{CaCl}_{2}$.

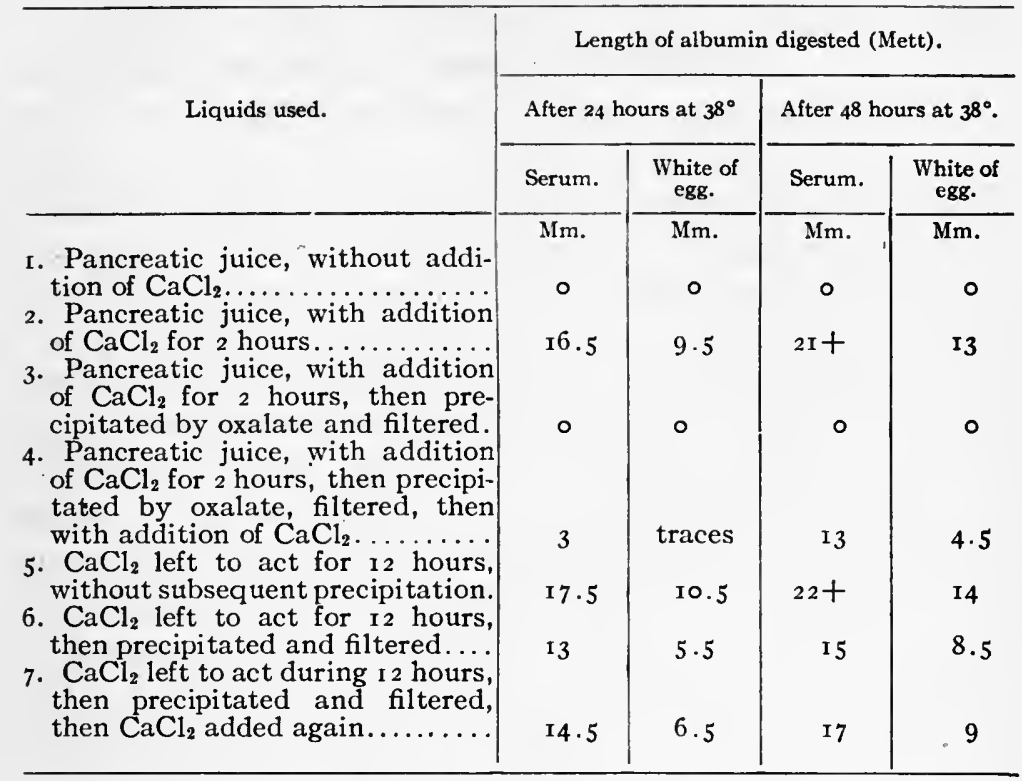

been added; there is a manifest action on the serum and the eggalbumin. In No. 3 , the salt has been allowed to act for only 2 hours, and then it has been eliminated; the juice is inactive. On the contrary, if the juice is left in contact with the calcium salt for I 2 hours, as in (6), we find that the filtrate, after precipitation, is endowed with the proteolytic power. The salt requires, therefore, a certain time to exert its action. Further, the non-intervention of the calcium salts in the final action of the trypsin formed is indicated in same test, No. 6. It is found, in fact, that the liquid, once activated, can be deprived of the calcium ions without losing its proteolytic power. In fact, the digestion is a little weaker in (6) than in (5), where the calcium ions have remained; but this comes from the fact that the precipitate of calcium oxalate formed has carried with it a little trypsin. The proof consists in adding again to the filtered liquid a little $\mathrm{CaCl}_{2}$, as in (7), whereby its activity is not perceptibly increased. The very slight increase observed comes from the fact that the juice, 
even after 12 hours contact with the calcium salts, still contains a little zymogen.

Not only is the activation of the pancreatic juice by calcium salts not instantaneous, but it also presents in its course a very characteristic property in that after a certain length of time, more or less considerable, this activation always takes place suddenly. Delezenne has shown this curious fact by taking a natural pancreatic juice which is strictly inactive, adding a sufficient quantity of $\mathrm{CaCl}_{2}$ to bring about the activation, and then maintaining it at $40^{\circ}$. When a portion of this mixture was taken from time to time and added to a solution of gelatin, and after ro minutes at $40^{\circ}$ the liquid was cooled to $10^{\circ}$, he found that solidification took place if the $\mathrm{CaCl}_{2}$ had not acted on the pancreatic juice for over a certain length of time, for example $4 \mathrm{~h} .35 \mathrm{~min}$. If the removal is done after $4 \mathrm{~h} .38 \mathrm{~min}$. the tube of gelatin no longer solidified upon cooling. The activation is then produced suddenly, in the space of a few minutes. If he precipitated the calcium before the pancreatic enzyme was activated, or after 4 h. $15 \mathrm{~min}$. for example, the activation would not take place; if he precipitated the calcium after the activation had been brought about, the active trypsin retained its digestive power.

This experiment shows a certain analogy between this phenomenon of activation and the formation of the thrombin in the coagulation of blood. There is another phenomenon which still better justifies this relation. Delezenne has found that the sudden activation of pancreatic juice by calcium salts is radically different when, instead of making the experiments in clean glass vessels, vessels of para ffined glass are used. The $\mathrm{CaCl}_{2}$ does not act until after several hours have elapsed. Moreover, once the juice is rendered active, the action on the gelatin, for example, can take place in a paraffined tube as well as in a tube not paraffined. It is the physical nature of the wall which is of influence, for the experiment succeeds just as well in a tube of glass as of platinum; the activation, which does not take place in a paraffined tube, does take place if the paraffin is scratched in such a way as to 
expose the glass. Moreover, a piece of paraffin placed in a glass tube does not hinder activation. Finally, the paraffin can be replaced by wax, resin, vaseline, oil, or any other substance rendering the wall incapable of being wet by water. It is seen that these effects are quite comparable with those already described with regard to the coagulation of blood. Although at the present time it would be difficult to pronounce on the cause of these anomalies, Delezenne thinks that electrical contact may perhaps intervene in this curious influence exerted by the wall.

Calcium ions being endowed with the same faculty of activating pancreatic juice as enterokinase, we are right in asking if the intestinal juice does not act simply by reason of the calcium ions that it contains. Pozerski has plainly shown that such is not the case and that the intestinal juice acts especially through its enterokinase, its calcium being in an organic complex and probably inactive. Having first collected juice from dogs having fistulas of Thiry, he found that the secretion, though mixed with much epithelial rubbish and leucocytes, contained 0.0059 per cent calcium. Then submitting this same intestinal juice for 2 hours to centrifugation, he separates the clear liquid from the insoluble parts. Pozerski finds that the liquid, carefully decanted, does not contain calcium or only in immeasurable quantities. However, added in small quantity to an inactive pancreatic juice, it is always very strongly enzymic. On the other hand, the precipitate formed by the elements from whence enterokinase is derived is very rich in calcium. On an average, this residue, including the adhering liquid, contains o.oro60.0265 per cent calcium. The writer does not say, however, whether a like emulsion would be capable of activating pancreatic juice.

\section{§ i6. Other Enzymic Properties of Pancreatic Juice.}

We have already noted a certain number of characteristics of pancreatic juice resulting from the three principal enzymes composing it. Other properties are as follows: 
I. - When pure pancreatic juice is submitted to the action of calcium ions, the juice acquires, in addition to a proteolytic power, the property of very energetically coagulating milk. This appearance of rennet, noted by Delezenne, takes place under exactly the same conditions as those previously determined with regard to the activation of trypsin. The same sort of behavior is found again here. After a more or less extended interval, the reaction takes place suddenly and is influenced by the nature of the walls. The coagulation of milk caused by the activated pancreatic juice strongly resembles, at least at the beginning of the action, the coagulation by gastric rennet. The clot is at first compact and coherent, but very rapidly, under the influence of the trypsin activated along with the rennet, becomes soft and is digested.

These facts are quite favorable to the theory that pepsin, trypsin, and rennet are perhaps one and the same enzyme, the differences observed in the digestive and coagulating properties of pancreatic juice activated by varied quantities of $\mathrm{CaCl}_{2}$ being capable of explanation as being due to the same influences of the medium as those previously described in the chapter relative to the supposed identity of pepsin and rennet (see page I99).

II. - In addition, even as rennet or pepsin have the property of coagulating concentrated solutions of peptone, pancreatic juice activated by calcium ions acquires a similar coagulating power. Delezenne and Mouton have found that inactive pancreatic juice remains without action on solutions of Witte's peptone. On the contrary, the conditions under which this plasteinic power appears and manifests itself under the influence of calcium ions, are also here exactly the same as those which govern the behavior of trypsin and rennet-lost time, sudden appearance of the property, progressive attenuation and parallel sensitiveness of the three actions, and destruction by heat at the same temperature. Finally, the parts of Witte's peptone soluble in strong alcohol at $75^{\circ}$ or $80^{\circ}$ G.L., which are the most apt to furnish plasteins under the influence of preparations of rennet or of pepsin, are likewise those which coagulate best with activated pancreatic juice. 
III. - The amylolytic power of pancreatic juice has also given rise to some interesting observations. Pozerski found that pancreatic juice, if left for some time at $40^{\circ}$, progressively loses its amylolytic power, and the quicker is the loss, the higher the trypsin content of the juice. In other words, if the juice is activated with a little $\mathrm{CaCl}_{2}$, this addition will have the effect of causing the amylase which was contained there to disappear very rapidly. A pancreatic juice alone, on the one hand, and this same juice with 0.3 c.c. of a solution $2 \mathrm{~N} \mathrm{CaCl}_{2}$ added per I c.c. of juice, on the other, was allowed to stand 8 hours at $40^{\circ}$. After this time, o.I c.c. of each of these liquids was allowed to act on 50 c.c. of a 2 per cent starch solution. The quantities of juice measured after 20 minutes were as follows:

Disappearance of Amylase in Pancreatic Juice Activated by Calcium Ions.

\begin{tabular}{|c|c|}
\hline & $\begin{array}{l}\text { Sugar } \\
\text { formed. }\end{array}$ \\
\hline 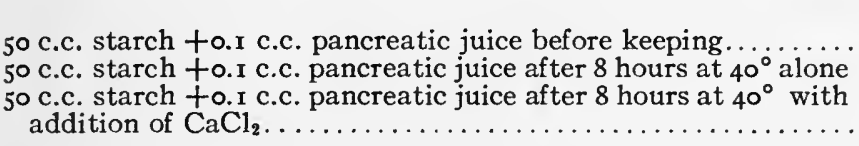 & $\begin{array}{l}\text { mg. } \\
208 \\
\mathrm{I} 83 \\
7\end{array}$ \\
\hline
\end{tabular}

This progressive disappearance of amylase in pancreatic juice activated by calcium ions is found likewise in juice treated with kinase. As, on the other hand, we know that kinased pancreatic juice acting on egg-albumin retains its amylolytic properties, we have been led to think that the products of the digestion of albuminoids, egg-albumin, edestin, muscle, and the like, under the influence of juice, considerably stimulate the saccharification of starch. Terroine and Weill have shown that this activation is due to the presence of amino-acids formed in the course of the proteolysis, especially to glycin, alanin, valin, leucin, phenylalanin, tyrosin, aspartic acid, arginin, histidin, and others. These acids act at a very weak concentration $-1 / 50,000$ to $1 / 100,000$ of glycin giving a very obvious acceleration, whereas $1 /$ I0, $\infty 0$ produces a rate of saccharification 30 to 40 times greater than 
in the case of juice alone. These facts, similar to those noted previously by Effront relative to the activation of amylase of malt by extractive materials of beer mash or similar previously boiled products, show how extensively very small quantities of substances formed by the action of an enzyme can influence the activity of another enzyme also present in the same medium, and how they are, consequently, of just the nature to show the complexity of the digestive phenomena. Roger and Simon have given a new example of this favorable action which an enzyme may exert on another enzyme secreted in a different portion of the alimentary tract, by showing that the amylase of the pancreatic juice may be activated by saliva previously destroyed by a contact of several hours with gastric juice, or may be activated simply by a neutralized gastric juice. In the two cases, the effect produced is probably due to the appearance of aminoacids formed by the action of trypsin on the stomachal pepsin.

The disappearance of the amylolytic power of the pancreatic juice under the influence of calcium salts without doubt affords an explanation of the fact, noted by Bierry and Victor Henri, that pancreatic juice of the dog, submitted to dialysis with distilled water, loses its saccharifying power, and that the addition of $\mathrm{NaCl}$ in part revives this power. The data furnished by these writers is given below.

Amylolytic Inactivity of Dialyzed Pancreatic Juice.

\begin{tabular}{|c|c|}
\hline & $\begin{array}{l}\text { Sugar formed after } \\
2 \text { hours at } 37^{\circ} \text {. }\end{array}$ \\
\hline 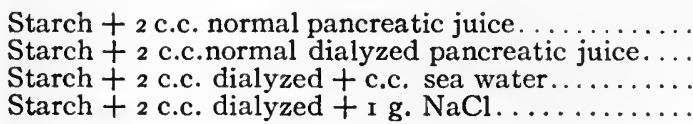 & $\begin{array}{l}0.525 \mathrm{~g} \text {. } \\
\text { No trace of sugar } \\
0.246 \mathrm{~g} \text {. } \\
0.216 \mathrm{~g} \text {. }\end{array}$ \\
\hline
\end{tabular}

Nothing similar has been found with the amylase of malt; this, although completely dialyzed, acts as well on starch paste as on undialyzed solution. It appears that this very great difference in the manner of action of these two enzymes toward dialysis is due to the fact that traces of calcium, which could 
remain in the collodion sac, have activated the trypsin of the pancreatic juice and have put it in condition to digest the amylase present, and that the reactivation of the dialyzed juice, under the influence of the $\mathrm{NaCl}$ added, is the result of the activating power which this salt possesses toward the small quantity of amylase which has escaped destruction.

Furthermore, Lisbonne has found that the pancreatic juice, as well as saliva, possesses the power of rapidly coagulating solutions of soluble starch at $130^{\circ}$, a property which Fernbach and Wolff noted in 1904 in the macerations of seeds of cereal, and which they attributed to a special enzyme, amylo-coagulase.

IV. - The study of the lipolytic power of the pancreatic juice has given Terroine the occasion to make some interesting observations. First of all, he found that pancreatic juice possessing a very slight proteolytic power, and which by preservation loses its lipasic activity only very slowly, on the other hand loses this property very rapidly if intestinal extract is added to the juice. To establish this fact, he measures the lipolytic activity by noting the number of c.c. of $N /$ ro $\mathrm{NaOH}$ necessary to neutralize the ro c.c. of oil used, after the juice has exerted its action for 4 hours. Here are the results found:

Disappearance of Lipasic Power in Kinased Pancreatic Juice.

\begin{tabular}{|c|c|}
\hline & $\begin{array}{l}\text { c.c. } N / 10 \\
\mathrm{NaOH} .\end{array}$ \\
\hline 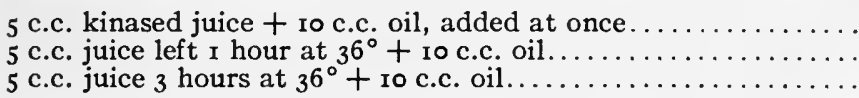 & $\begin{array}{r}29.4 \\
5.1 \\
2.0\end{array}$ \\
\hline
\end{tabular}

This diminution of activity is not produced with as great a velocity under physiological conditions, since the active juice is then in the presence of albuminoids requiring digestion.

Thus, in spite of the formation of basic products in the course of the digestion of albumin, the acidity of the liquid in the last test was still such as to require Ir.7 c.c. $N /$ IO $\mathrm{NaOH}$. The destruction of the lipase is then much less intense when the 
kinased juice can exert its action toward an albumin requiring digestion. We find here again the favorable influence that the products of proteolysis play in the manifestations of activity of the other enzymes likewise contained in the pancreatic juice.

V. - In addition to the three principal enzymes just described, two other enzymes have also been noted in the pancreatic juice. Bierry and Terroine have established the presence here of a maltase, an enzyme capable of transforming maltose into glucose in slightly acid media. In addition, Schaeffer and Terroine have shown that the pancreatic juice, which in the pure state is totally inactive toward coagulated albumin, is, however, directly active toward all the degraded protein products obtained either by chemical or enzymic action, both from the peptides and from a certain number of natural albumins like casein. These facts lead them to the conclusion that, far from being inactive proteolytically, pancreatic juice possesses an erepsin. Moreover, these investigators have been able to separate the trypsinogen from the erepsin by dialysis.

\section{BIBLIOGRAPHY.}

\section{PREPARATION AND PROPERTIES.}

C.. Bernard. Leçons de physiologie expérimentale, Paris, 1856.

Meissner. Zeits. f. rationnelle Medizin, 1856.

Heidenhain. Pflïg. Arch., I875, (I0), p. 558.

Danilewsky. Arch.f. Path. u. Anath., 1862, p. 279.

KüHNE. Virchows Arch., 1867, p. 130.

Escherich. Arch.f. klin. Medizin, 1885, (37), p. 196.

Hoppe-Seyler. Pflïg. Arch., (14), p. 395.

Mroczkowski. Biol. Centr., 1889-189o, (9), p. 154 .

LoEw. Pflüg. Arch., 1882, (27), p. 207.

\section{TRYPSIN.}

von Wrrrich. Ueber eine neue Methode zur Darstellung künstlicher Verdauungsproducte, Pflüg. Arch., I869, (2), p. 198; 1870, (3), p. 339.

FredericQ. B. Acad. Roy. de Belg., 1878, (46), p. 213 . Arch. de Zool. expérim., 1878 .

Podolinsky. Thèse, Breslau, 1876 .

Setschenow. Centralbl: f.d. med. Wissenschaft, 1887, p. 498.

Lewaschew. Bildung im Pankreas, Arch.f.d. ges. Physiol., I886, (37), p. 32.

Paschutin. Arch.f. Anat. u. Physiol., 1873, p. 383. 
Koudzewsky. Diss., St-Petersburg, 1890.

Grimmer. Zur Kenntniss der Wirkung der proteol. Enzyme der Nahrung, Bioch. Zeits., I907, (4), p. 80.

KüHNE. Verhandlungen der Heidelberger Natur. hist. mediz. Vereins, 1876, 1884.

Gulewitsch. Zeits. f. physiol. Chem., r899, (27), p. 544.

Dastre. Archiv. de Physiol., 1896, p. 122.

HaMmaRsten. Lehrbuch d. physiol. Chem., 1895.

K. MAYs. Zeits. f. physiol. Chem., 1903, (38), p. 230.

Hedin. Ueber die Aufnahme v. Trypsin durch verschiedene Substanzen, Zeits. $f$. physiol. Chem., 1907, (50), p. 497.

A. Benfey. Trypsin i. Säuglingsharn, Bioch. Zeits., rgo8, (1о), p. $45^{8}$.

Stutzer et Merres. Trypsinlösungen, Bioch. Zeits., 1908, (9), p. 127.

MOURRUT. Journ. de pharm. et chim., 1879, (30), p. 44I.

GENERAL ACTIONS.

Harlay. De l'application de la tyrosinase, Thèse, Paris, 1900.

Javillier. Les ferments protéolytiques, Thèse, Paris, I909.

Van de Velde u. Poppe. NaFl. Bioch. Zeits., I910, (28), p. I34.

F. Frank u. A. Schittenhelm. Im Magendarmkanal, Zeits. f. experim. Path.

u. Therap., 1910, (8), p. 237.

Hirata. Gehalt i. Harn u. Blut, Bioch. Zeits., r910, (27), p. 385.

M. Siegrried. Tryps. Verd. d. Caseins, Pflïg., Arch., 191 I, (1 36$)$, p. 185.

Hirayama. Nachweis u. Gehalt in Fäces, Zeits. f. experim. Path. u. Therap., I9II, (8), p. 624 .

Th. Brugsch u. Masuda. Nachweis u. Gehalt in Fäces, Zeits. f. experim. Path. u. Therap., I9I I, (8), p. 6 I 7 .

von Grützner. Wirkung als Ferment, Pflïg. Arch., I9I r, (I4I), p. 63.

Michaelis u. Davidsohn. Bioch. Zeits., I91 r, (36), p. 280.

WeINKoPFF. Trypsinverdauung der grampositiven und gramnegativen Bakterien, Zeits. f. Immunitätsforsch. u. experim. Therapie, I9I I, (I I), p. I.

KirchHerm. Giftwirkung und Verdauung lebenden Gewebes, Arch. f. experim. Path. u. Pharmak., I9II, (66), p. 352.

J. Lоeв. Wirkung, Bioch. Zeits., 1909, (19), p. 534.

J. Reich-herzberge. Wirkung auf Leim, Zeits. f. physiol. Chem., I901, (34), p. 120.

Salkowsky. Begriff Trypsin, Zeits. f. physiol. Chem., 1902, (35), p. 545.

A. Loeв. Versuche mit. Lab. u. Trypsin, Centralbl. f. Bakter. u. Parasitenk., 1903, (32), p. 472.

Oppenheimer u. Aron. Verhalten des genuinen Serums gegen die tryptische Verdauung, Beit. z. chem. Physiol. u. Path., I903, (4), p. 279.

Oppenhermer. Einwirk. d. Trypsinverdauung auf die Präzipitinreaction, Beit. z. chem. Physiol. u. Path., 1903, (4), p. 259.

E. Pólya. Wirkung des Trypsin auf das lebende Pankreas, Pfiug. Arch., 1908, (I2I), p. 483 .

StURzer u. MERres. Wirkung auf vegetabilische Eiweissstoffe. Bioch. Zeits, I908, (9), p. 127.

M. JАСову. Einwirkung auf Serum, Bioch. Zeits., I908, (10), p 232. 
Herzog u. Kasarnowsky. Diffusion, Bioch. Zeits., 1908, (Ir), p. 172.

Hedin. Enzymwirkung, Zeits. f. physiol. Chem., I9I0, (64), p. 82.

Glaessner u. Stauber. Trypsin u. Erepsin, Bioch. Zeits., 1910, (25), p. 204.

White u. Crozier. Journ. Amer. Chem. Soc., r91 I, (33), p. 2042.

Larres. Poison du suc pancréatique, Arch. de Farmacol. sperim., I912, (12), p. 37 .

\section{EFFECT OF PHYSICAL AND CHEMICAL AGENTS.}

Hans Richard Weiss. Zur Kenntnis der Trypsinverdauung, Zeits. f. physiol. Chem., I904, (40), p. 483.

Wolberg. Salze u. Alkaloīde, Pfiug. Arch., I88o, (22), p. 29 I.

Chittenden u. Gummins. Therapeut. u. toxische Substanzen; Pankreasferment, Maly Jahresberichte für Tierchemie, (15), p. 304.

Chittenden. Uransalze, Maly Jahresberichte, I 887 , (1 7 ), p. 475.

Coenen. Calomel im Darm, Maly Jahresberichte, 1887, (17), p. 273.

LINDBERGer. Einwirkung v. Säure, Maly Jahresberichte, I888, (13), p. 283.

LoEb. Electrolytische Dissociation, Bioch. Zeits., 1909, (19), p. 534.

Fernbach et Schoen. Mécanisme de fonctionnement, $C$. $R$., I9I I, (2), p. I33.

Kudo. Säuren, Alkalien, Neutralsalze, Bioch. Zeits., 1909, (I5), p. 473.

E. W. Scrmidr. Enzymat. Mitteilungen, Zeits. f. physiol. Chem., 1910, (67), p. 315 .

Delezenne et Lisbonne. Action des rayons ultra-violets, $C$. $R$., I91 2, (2), 788.

Froun et A. Compton. Inactivation de la trypsine par le dialyse, $C$. $R$., I9I I, (153), p. 1032.

H. R. WeIss. Zeits. f. physiol. Chem., 1904, (40), p. 480.

Vernon. Schutzwerte d. Proteide; Spaltungsproducte aus Trypsin, Journ. of Physiol., 1904, (31), p. 341. D'aprés: Chem. Centralbl., 1904, (1), p. I161.

W. M. Bayliss. Journ. of Physiol., 1907, (36), p. 221.

Robertson u. Schmidt. Alcali, Journ. of Biol. Chem., igo8, (5), p. 3.

Erdmann u. Lederer. HCl, Berl. klin. Woch., I909, (26), p. 1224.

W. E. Dixon u. P. Hamil. Substanzen, Journ. of Physiol., 1909, (38), p. 314.

T. Kudo. Electrizität, Bioch. Zeits., I909, (16), p. 233.

MichaELis. Electr. Ueberführung, Bioch. Zeits., I909, (I6), p. 486.

voN Fürth u. Schwarz. Sticksoffausscheidung; Eiweisszerfall, Bioch. Zeils., I909, (20), p. 384 .

Shaklee a. Meltzer. Agitation, Amer. Journ. of Physiol., r909, (25), p. 8r.

Minami. Schütteln u. Erwärmen, Bioch. Zeits., I91 2, (39), p. 75.

DE Souza. Temperatur, Journ. of Physiol., I91 I, (43), p. 374.

Kawashma. Lösungsmittel, Bioch. Zeits., I909, (23), p. 186.

Scrmidr. Ausschaltung d. Hitzeempfindlichkeit, Zeits. f. physiol. Chem., 1910, $(67)$, p. 314 .

Michaelis u. Davidsohn. H. Ionenconcentration, Bioch. Zeits., I9I1, (36), p. 280.

L. Pincussorn. Anorgan. Kolloïde, Bioch. Zeits., I91 2, (40), p. 307.

OHTA. Temperatur, Bioch. Zeits., I912, (44), p. 472.

Roeder. Wirkung Temperat., Bioch. Zeits., 1910, (24), p. 499.

Abderhalden U. Koelker. Zeits. $f$. physiol. Chom., 1907, (5I), p. 294. 
TRYPSIN.

H. L. Holzberg. Journ. of Biol. Chem., 1913, (14), p. 335 .

Bergelti et Bickel. Verhandl. des Kongress $f$. Med., Wiesbaden, 1906.

Løwenthal et Edelstein. Bioch. Zeits., 1908, (14), p. 484.

\section{ACTION OF ANTISEPTICS.}

IRGer. Quelques substances antiseptiques, Arch. de Physiol., I898, p. 672.

TAPPener. NaFl, Arch.f. Therm. u. Path., (27), p. 108.

Arthus et Huber. C. R., I892, (II5), p. 839.

R. Kaufmann. Protoplasmagifte, Zeits. f. physiol. Chem., I903, (39), p. 437.

FüRsT. Berl. klin. Woch., 1909, (2), p. 58.

FERMi. Arch.f. Hyg., (14), p. 1.

FoKker. Chloroforme, Forts. d. Med., I891, p. 93.

Lewin. Thymol, Antiseptic, Antiferment, Centralbl. f. de med. Wissens., 1875, (2I).

Efrront. Moniteur scient., 189o, (8), p. 449.

\section{ANTI-TRYPSIN.}

MARcus. Berl. klin. Woch., 1908, (14), p. 689.

Achalme. Propriétés pathogènes de la trypsine et le pouvoir antitrypsique du sérum, Ann. Inst. Pasteur, I9or, (1 5), p. 737.

Glaessner. Beit. z. chem. Physiol. u. Path., 1903, p. 79.

Brieger u. Trebing. Berl. klin. Woch., igo8, (22), p. I04I.

Ascoli u. Bezzola. Centralbl.f. Bacteriol., 1903, (33), p. 783.

Ehrenreich. Antifermente des Blutes, Diss., Wurzbourg, I904.

K̈̈MMERER. Antitrypsin, Zeits. f. Immunitätsforsch. u. exper. Therap., Igг I, (I I), p. 235 .

Kurt Meyer. Diabetes. Bioch. Zeits., 1909, (23), p. 69; r912, (40), p. 125.

G. EISNer. Zeits. f. Immunitätsforsch. u. exper. Therap., r Abt., I909, (1), p. 650 .

Dastre et Stassano. Les facteurs de la digestion pancréatique. Suc pancréatique, kinase et trypsine; anti-kinase, Arch. Intern. de Physiol., I904, p. 86.

Zunz. Acad. royale de Belgique, 1905, p. 606.

Bergman. Zeits.f. expcrim. Path., igo6, (3), p. 401.

Marcus. (I) Antitryps. Kraft d. Blutes, Berl. klin. Woch., I9o9, (4), p. 156.

Antitrypsin d. Blutes, Zeits. f. expcrim. Path. u. Therm., I909, (6), p. 879.

Fermi. Antitrypsin, Arch. de Farmacol. sperim., I909, (8), p. 407.

Meyer. Antitrypsin, Berl. klin. Woch., I909, (42), p. 189o.

JACH. Antitrypsin, Münch. med. Woch., 1909, (44), p. 2254.

Meyer. Trypsin u Antitrypsin, Bioch. Zeits., rgog, (23), p. 68.

Bauer. Antitrypsin, Zeits. f. Immunitätsforsch. u. exper. Therap., I. Teil, I9ro, (5), p. 186.

DöвlIN. (I) Natur d. Antitrypsin, Zeits. f. Immunitätsforsch. u. exper. Therap., I910, (4), p. 229. (2) Antitrypsin im Harn, Ibid., r910, (4), p. 224.

Becker. Antitrypsin i. Blut, Mïnch. med. Woch., 1909, (27), p. 1363.

ЈАсов. Antitrypsin i. Blut, Münch. med. Woch., r9oг, (27), p. г36r.

Rondoni. Antitrypsin i. Blut, Berl. klin. Woch., I910, (I2), 528.

Cobliner. Ueber Antitrypsin, Bioch. Zeits., r910, (25), p. 494. 
Braunstein u. Kepinow. Antitrypsin, Bioch. Zeits., I910, (27), p. 170.

Hugo KäMmerer u. I. Mogulesko. Antitrypsin gegen Pankreas- Hefe- Pyocyaneustrypsin, Zeits. f. Immunitätsforsch. u. exper. Therap., I911, (12), p. 16.

P. Achalme et Stevenin. Soc. Biol., igir, (1), pp. 333 et 480.

Guido Finzi. Soc. Biol., 1909, (r), p. 1007.

A. Girault et Rubinstein, Soc. Biol., 1912, (2), p. 205.

\section{LAW OF ACTION.}

Hedin. (1) Journ. of Physiol., 1905, (32), p. 47x. (2) Zur der Enzyme, Zeits.f. physiol. Chem., 1908, (57), 472. (3) Reaction zw. Enzymen u.' anderen Substanzen, Zeits. f. physiol. Chem., (82), p. 176.

ARRHENIUS. Immunochemie, Leipzig, 1907, p. 46.

Johonson-Blohm. Kolloïde, Zeits. $f$. physiol. Chem., (82), p. I79.

V. Henri et Larguier des Bancels. Loi de l'action de la trypsine sur la gélatine, C. R., 1903, (1), pp. 1088 et 1581; Soc. Biol., 1903, (55), p. 864.

S. G. Hedin. Journ. of Physiol., I906, (34), p. 370.

Hedrn. Zeits. f. physiol. Chem., 1908, (57), p. 468.

Michaelis u. Davidsohn. Isoelectr. Konstante, Bioch. Zeits., igr I, (36), p. 48 I.

Walters. (I) Journ. of Biol. Chem., I91 2, (11), p. 267. (2) Hydrolyse, Journ. of Biol. Chem., 191 2, (12), p. 43.

\section{CHEMICAL ACTIVITY AND ANALYSIS.}

M. Schwarzschild. Beit. z. chem. Physiol. u. Path., 1903, (4), p. 155.

W. Lohlein. Analyse, Beit. z. Chem. Physiol. u. Path., 1905, (7), p. I 20.

Levene u. Wallace. Zeits. f. physiol. Chem., 1906, (47), p. I43.

G. FERMr. Reagentien u. Versuchsmethoden z. Studium der proteol. Enzyme, Arch.f. Hyg., 1906, (55), p. 140.

Paul Hâri. Wasseraufnahme bei der tryptischen Verdauung, Pfiig. Arch., 1906, (II5), p. $5^{2}$.

Alonzo Englebert Taylor. Journ. of Biol. Chem., I907, (3), p. 87.

O. GRoss. Die Wirksamkeit, u. einfache Methode z. Bestimmung von Trypsin, Arch. f. exper. Pathol. u. Pharmak., 1907, (58), p. 157 .

Rogozinski. Beit. z. chem. Physiol. u. Path., I908, (I I), pp. 229-24I.

Fuld. Bestim. v. Trypsin, Arch. f. exper. Pathol. u. Pharmak., 1908, (58), p. 468.

M. Jасову. Nachweis d. Trypsins, Bioch. Zeits., 1908, (10), p. 229.

EMIL Fischer. Untersuchungen über Aminosäuren, Berlin, 1906.

Kossel. Ueber den gegenwärtigenstand d. Eiweisschemie, Ber. d. Deutsch. Chem. Ges., 1901, (3), p. 3214.

TAylor. Synthese v. Protamin d. Fermentwirkung, Journ. of Biol. Chem., 1909, (5), p. 381, d'après Chem. Centralblatt, 1909, (I), p. 1417.

Harlay. Thèse, Paris, rgoo.

Bourquelot. Journ. de Pharm. et Chimie, 1896, p. 97.

Nencki. Ber. d. Deutsch. Chem. Ges., 1895, (1), p. 560.

Goldschmidt. Nachweis u. Best. v. Trypsin, Deutsche Med. Woch., 1909, (35), p. 522.

Waldschumt. Bestimmung, Pflig. Arch., I912, (143), p. I89. 
Roberison. Journ. of Biol. Chem., I9I 2, (r2), p. 23.

Palladin. Best. d. Trypsins, Pflüg. Arch., r9 Io, (134), p. 337.

Ramsay. Anal. pancriatine, Journ. of Ind. a. Engin. Chem., I91 I, (3), p. 822.

SerRensen. Enzymstudien, Bioch.Zeits., 1907, (7), p. 45; 1909, (21), p. 13I; 1909, (23), p. $35^{2}$.

\section{PANCREATIC JUICE.}

DASTre. Ferment du pancréas, Soc. Biol., r893, p. 648; Arch. de Physiol., 1893, p. 774 .

Kart Glaessner. Menschl. Pankreassecret, Zeils. f. physiol. Chem., I904, (40), p. 477.

Schumm. Menschl. Pankreassecret, Zeits. f. physiol. Chem., I902, (36), p. 298.

Delezenne. Etude du suc pancréatique, Soc. Biol., voir tomes 53 à 57 ; I901-I905. Alex. Ellinger u. Max CoHn. Pankreassekrete d. Menschen, Zeits. f. physiol. Chem., 1905, (45), p. 28.

Wassilief. Arch.d. Sc. biolog., St-Pétersbourg, I893, p. 2 19.

Walter. Arch.d.Sc. biolog., St-Pétersbourg, r889, p. I.

Heidenhain. Pankreaskenntniss, Pflïg. Arch., 1875, (10), p. 557.

Babin et Tichomroff. Beziehung proteolyt. Kraft u. N-Gehalt im Saft d. Bauchspeichel-drüse, Zeits.f. physiol. Chem., I909, (62), p. 469.

WeIs. Pankreasverdauung, Virch. Arch., r876, p. 68.

Popielski. Pankreassaft, Centralbl. $f$. Physiol., igo3, (I 7), p. 65.

Mays. Trypsinwirkung, Zeits. f. physiol. Chem., 1906, (49), pp. I 29 et I80.

Frouin et Gérard. Composit. minérale du suc pancr., Soc. Biol., I9I 2, (I), p. 98.

Terronve. Disparit. du pouv. lipasique dans suc pancr. kinasé, Soc. Biol., I908, (2), p. 329 .

Bierry et Terroine. C. $R$., Ig06, (I), p. 476 .

Gley. Action des albumoses sur sécrétion pancréat., Soc. Biol., Igr I, (2), p. 82.

Gley et Pozerski. Soc. Biol., igi 2, (1), p. 560 .

Frourn. Soc. Biol., I904, (I), p. 806; 1905, (I), p. 1025; 1907, (2), p. 473; 1907, (2), p. 519; I910, (r), p. I76; IgI , (2), p. I5.

Pozerski. Soc. Biol., I906, (I), p. 1068; 1908, (I), p. 505; I9I I, (I), p. 2 I.

L. Morel et Terroine. Soc. Biol., igo9, (2), p. 36 .

Delezenne. Format. de présure dans suc pancréat. activé par $\mathrm{Ca}$, Soc. Biol., $1907,(2)$, p. 98.

Delezenne et Mouton. Soc. Biol., 1907, (2), p. 277.

Terroine et Weill. Soc. Biol., igo9, (1), p. 285.

Roger et Simon. Soc. Biol., r907, (r), p. I070; 1908, (1), p. 541 .

Bierry et V. Henri. Soc. Biol., I906, (I), p. 479.

Lisbonne. Coagul. de l'amidon par suc pancr., Soc, Biol., IgI I, (2), p. I40.

E. Terroine. Ia sécrétion pancréatique, I vol., Paris, Igr 3 .

Zunz AND Gyö̈gy. Bull. Soc. Sc. Belgique, March, I9I4.

\section{TRYPSINOGEN AND ENTEROKINASE,}

Schepowalnikow. La physiologie du suc intestinal, Thèse, St-Pétersbourg, 1899 . Popielski. Thèse, St-Pétersbourg, r 896 .

Hекмa. Maly's Journ. f. Tierchemie, (33), p. $5^{67}$. 
Delezenne et Frouin. Inactivité de la sécrétion physiol. pancréatique sur l'albumine, Soc. Biol., 1902, (54), p. 691.

Nirnon. Journ. of Physiol., 1901, (26), p. 405.

Bayliss a. Starling. Journ. of Physiol., I903, (30), p. 61; 1905, (32), p. 129.

KüHNE. Verhand. d. natur. med. Vereins zu Heidelberg, 1886, (3), p. 463.

Heidenhain. Kenntnis d. Pancreas, Pfiug. Arch., 1875, (10), p. 557.

Hekma. Umwandlung des Trypsinzymogens in Trypsin, Archiv. der Anat. und. Physiol., 1904, p. 343.

Delezenne. Activation par les sels de Ca, Soc. Biol., I905, (2), pp. 523, 614; 1907, (2), p. 274.

Loew. Pancreasfermente, Pfiug. Arch., 1882, (27), p. 207.

von Wrtrich. Darstellung künstl. Verdauungsprodukte, Pflïg. Arch., I869, (2), p. $198 ; 1870,(3)$, p. 339 .

Molyneux Hamill. Journ. of Physiol., I906, (33), pp. 476, 479.

WoHLgemuth. Bioch.Zeits., I906, (2), p. 264. Herzen Rückschlag des Trypsin zu Zymogen, Arch.f. d. ges. Physiol., I883, (30), p. 295.

Meyer. Ueber Trypsin u. Antitrypsin, Bioch. Zeits., I909, (23), p. 68.

Terroine. Z. Kenntniss der Fettspaltung d. Pankreassaft, Bioch. Zeits., 1909, (23), p. 404 .

Zunz. (I) Activation du suc pancréatique par les sels, Bull. Soc. Roy. d. Sc. médicales, 1906, pp. 28, 96 . (2) Id., 1907, pp. 2, 96. (3) Ann. Soc. Sci., 1907, t. I6, fasc. I, p. I.

Dastre et Stassano. Suc pancréatique, kinase et trypsinogène. Arch. intern. de Physiol., I904, p. 106.

Henri. Colloïdes kinase artificielle, Rev. gén. de Sc. pur. et appliq., I905, p. 640.

LARguier des Bancels. Activation du suc pancr. par les colloīdes, Soc. Biol., I905, (I), p. 987 ; (2), p. I3०.

Bayliss and Starting. Journ. of Physiol., 1902, pp. 230 et $325 ; 1905$, p. 129.

Delezenne. Soc. Biol., I901, p. I161. Bioch.Zeits., 1906, (2), p. 264; 1907, (4), p. 271.

Wohlgemuth. Activirung tryps. Fermente, Bioch. Zeits., 1906, (2), p. 264.

KARL MAYs. Wirkung, Zeits. f. physiol. Chem., 1906, (49), p. I89.

Delezenne. (I) Kinase microbienne, pouvoir digestif, C. R., I902, (2), p. 252. (2) Présence de la kinase dans champignons, $C$. $R$., 1903, (1), p. 167. (3) Activation du suc pancr. par les sels de Ca, $C$. $R$., 1907, (I), p. 388. (4) Nature des parois sur l'activation, $C . R$., 1907, (I), p. 506.

Hougardy. Lactokinase, Bull. Acad. Roy. Belgique, I906, p. 888.

IBRAhim. Beim Embryo u. Neugeborenen, Bioch. Zeits., I909, (22), p. 25.

Popielski. Eigenschaft d. Pancreassaftes, Centralbl. f. Physiol., 1903, p. 66.

Howell. Chem. Regulirung d. Vorgänge i. Körper. mittels aktivatiren Kinase, etc., Naturw. Rundschau, (25), p. 172.

Ayrton-Quart. The Activation of Pancreatic Juice. Jour. of Exper. Physiol, I909, vol. 2, p. 201.

DE Songe. The Activation of Pancreatic Juice. London, rgo6.

Mellamby and Woolley. The Ferment of the Pancreas. Jour. of Physiol., 1912, vol. 45, p. 270 ; 1913, vol. 46, p. I59.

(See also bibliography upon the functioning of the digestive glands.) 


\section{CHAPTER I (Continued). \\ FUNCTIONING OF THE DIGESTIVE GLANDS.}

\section{§ I. Variations in the Secretion of the Gastric and Pancreatic Glands.}

Action of Nutritive Substances on the Zymogenic Glands and on Their Enzymes. - The preceding chapters have dealt with the composition and the properties of the gastric and pancreatic juices, and we have found the activity of these juices to be due to the different enzymes which they contain. From the numerous experiments cited in the study of pepsin and trypsin, it appears that there exists a multitude of substances which produce considerable retarding effects on the proteolytic enzymes, while the agents which bring about a plainly favorable action on these enzymes are, as yet, not cleariy recognized. It has been seen, also, that the different albuminoid substrates do not act in the same way toward the proteolytic enzymes. Fibrin, egg-albumin, and globulin in the presence of the same quantities of pepsin, do not hydrolyze alike; certain of these products peptonize much more easily than others. We can, furthermore, find striking differences in the behavior of the same albuminoid substrate according to its physical state; thus it is that raw egg-albumin has a much less resistance than coagulated albumin.

These data furnish interesting indications with regard to the phenomenon of digestion; but it would be erroneous to conclude that these results, obtained in vitro reflect exactly all the intricacies of the complicated mechanism of normal digestion. In reality, the data thus far produced explain only a part of the problem. Experiments in vitro allow us to understand only in general outlines by what means and by what series of 
transformations a nutritive substance can be digested in the alimentary tract, but our chemical knowledge does not permit us to explain the differences in the digestibility of the various foods, nor the difficulties so frequently observed in the course of digestion.

To fathom rightly the mechanism of this important physiological function, it is necessary to study digestion from an entirely different point of view. We must not be content merely with establishing the influence of physical and chemical conditions on the enzymes contained in the digestive juices, but we must also especially study the reaction of these influences on the progress of secretion itself; in other words, we must determine the influence exerted by the foods on the formation of the juice, its secretion, and its composition. The importance of this influence results from a multitude of facts which indicate that the substances submitted to the action of pepsin, trypsin, and lipase can also materially effect the progress of the secretion, and perhaps the very formation of these enzymes. They can influence them favorably or unfavorably. According to the nourishment, it is possible to get less or more juice, and juice not always of the same quality. In addition, substances which show themselves inactive on the fermenting power of pepsin, trypsin, or other enzymes, may, on the contrary, act very strongly on the secretion of these enzymes. Furthermore, substances known to be paralyzing to an enzyme may counterbalance this action by increasing the quantity of secretion which they cause; inversely, a favoring substance, from the enzymic point of view, may be unfavorable as to the quantity of juice produced. The chemical agents may therefore act in a very complex manner, and the study of the influence of foods on the secretion is indeed of an even much greater importance than the study of the influence exerted on the enzymes themselves.

Work of the Pawlow School. - This new point of view has been introduced into physiology by Pawlow and his pupils. Thanks to his improvement of surgical technique, notably by the use of gastric and pancreatic fistulas, Pawlow has been 
able to follow the progress of digestion with great clearness. To obtain gastric juice in the pure state, not contaminated with food materials, he uses the method of œesophagotomy (see p. 256). While the animal receives and masticates the nourishment given him, food which is then cast out through an opening made in the osophagus, the gastric juice, which is simultaneously secreted, is collected through a stomachal fistula. The animal is actually nourished by the direct introduction of food into the stomach by means of a probang. The fictitious meal furnishes, under the conditions just described, a very active juice. The observations made by this arrangement have given important results, though this method cannot, as yet, give a true image of what normally occurs in the stomach.

It is evident that the enzyme secretion must take place differently in the pseudo-nutrition than in the real nutrition. It is to reply to this objection that Pawlow had recourse to a second method, which consists in dividing the stomach by means of resections and suitable sutures into two unequal parts, one being about ten times larger than the other. The larger alone receives the nourishment, while the smaller, excited by reflex action, has a fistula by which the juice which it secretes flows out. Pawlow, by making a fistula in each of the sections, has verified the fact that the secretion is produced in each in exactly the same manner, the quantities of juice being proportional to the volumes of the two stomachs but their relative activity being always the same. This delicate operation is borne well by dogs, and those treated in this way can serve for years for the same experiments. By varying these experiments, it has been possible to draw from this complex question a certain number of definite conclusions.

Influence of Feeding on the Quantity and the Composition of the Gastric Juice Secreted. - First of all, it has been established that there exists a very definite relation between the secretorial functions and the food absorbed. Thus Chigin, by measuring the quantity of gastric juice which flows after the ingestion of various foods, has found: 
Per 100 g. meat absorbed, there flows 26 c.c. gastric juice.

" 200 " " " " " 40 c.c. " "

" 400 " " " " " 106 c.c. " "

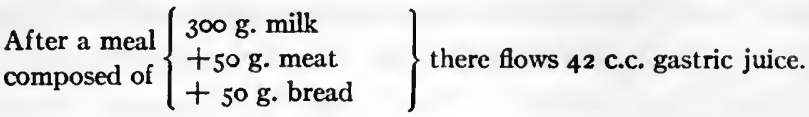

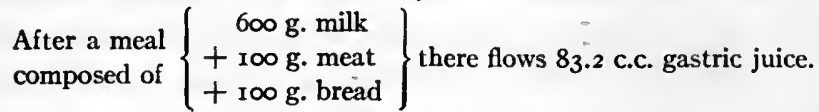

The quantity of juice secreted is therefore proportional to the actual need, the quantity increasing with the quantity of foods ingested. The production of gastric juice during the meal depends above all on the nature of the food. For materials having the same nitrogen content, there are secreted very different quantities of juice. The unit of nitrogen contained in bread corresponds to the maximum of juice secreted. Milk, for the same unit of nitrogen, brings about a smaller secretion, and this is still smaller for meat. The influence of the food on the secretion is shown not only in the quantity of juice elaborated, but also in the composition of this juice.

Influence of Feeding on the Gastric Secretion.

\begin{tabular}{|c|c|c|c|}
\hline Quantities of foods absorbed. & $\begin{array}{l}\text { Gastric juice } \\
\text { secreted. }\end{array}$ & $\begin{array}{c}\text { Digestive power } \\
\text { (Mett). }\end{array}$ & Digestive value. \\
\hline $\begin{array}{l}250 \mathrm{~g} . \text { bread.......... } \\
\text { roo g. meat.......... } \\
600 \mathrm{~g} . \text { milk......... }\end{array}$ & $\begin{array}{l}\text { c.c. } \\
42 \\
27 \\
34\end{array}$ & $\begin{array}{l}6 . \mathrm{I} 6 \\
4 \\
3 . \mathrm{I}\end{array}$ & $\begin{aligned}(6.16)^{2} \times 42 & =1600 \\
(4)^{2} \times 27 & =432 \\
(3.1)^{2} \times 34 & =327\end{aligned}$ \\
\hline
\end{tabular}

In the three cases mentioned, the quantities of nitrogen are the same, but the quantities of juice secreted and their content in pepsin are different. The bread, which is digested with more difficulty than milk, receives about five times more pepsin than the latter, and meat almost one-fourth more than milk. This influence of the chemical nature of the food on the quantity and the richness of the gastric juice is shown with more exactness when the juice secreted is analyzed every hour.

With bread, a very prolonged secretion is produced. Meat is next in order, while with milk the secretion is arrested after 
the 6th hour. As to the digestive power, marked differences can be noted. Definitely this table indicates that not only the quantity and the quality of the gastric juice varies with each food, but that the very manner of secretion is characteristic for each type of food.

Variations of Gastric Secretion with the Nature of the Food.

\begin{tabular}{|c|c|c|c|c|c|c|}
\hline \multirow{2}{*}{ Hours. } & \multicolumn{3}{|c|}{ Quantity of juice secreted per hour. } & \multicolumn{3}{|c|}{ Digestive power according to Mett. } \\
\hline & $\underset{(100 \text { g.) }}{\text { Meat }}$ & $\begin{array}{c}\text { Bread } \\
(250 \mathrm{~g} .)\end{array}$ & $\underset{(600 \mathrm{~g} .)}{\text { Milk }}$ & $\begin{array}{c}\text { Meat } \\
(100 \mathrm{~g} .)\end{array}$ & $\begin{array}{l}\text { Bread. } \\
(250 \mathrm{~g} .)\end{array}$ & $\begin{array}{l}\text { Milk. } \\
(600 \mathrm{g.})\end{array}$ \\
\hline $\begin{array}{l}\text { From o to I } \\
\text { From I to } 2 \\
\text { From } 2 \text { to } 3 \\
\text { From } 3 \text { to } 4 \\
\text { From } 4 \text { to } 5 \\
\text { From } 5 \text { to } 6 \\
\text { From } 6 \text { to } 7 \\
\text { From } 7 \text { to } 8 \\
\text { From } 8 \text { to } 9 \\
\text { From } 9 \text { to } 10\end{array}$ & $\begin{array}{l}\text { c.c. } \\
\text { I I . } 2 \\
\text { I I . } 3 \\
7.6 \\
5.1 \\
2.8 \\
2.2 \\
\text { I . } \\
0.6 \\
0.6 \\
0.6 \\
\end{array}$ & \begin{tabular}{r}
\multicolumn{1}{c}{ c.c. } \\
I0.6 \\
5.4 \\
4.0 \\
3.4 \\
3.3 \\
2.2 \\
2.6 \\
2.2 \\
0.9 \\
0.4 \\
\end{tabular} & $\begin{array}{l}\text { c.c. } \\
4.0 \\
8.6 \\
9.2 \\
7.7 \\
4.0 \\
0.5 \\
0.5 \\
0.5 \\
0.5 \\
0.5 \\
\end{array}$ & $\begin{array}{l}4.94 \\
3.03 \\
3.01 \\
2.87 \\
3.20 \\
3.5^{8} \\
2.25 \\
3.87 \\
3.87 \\
3.87\end{array}$ & $\begin{array}{l}6 \cdot 10 \\
7 \cdot 97 \\
7 \cdot 51 \\
6.19 \\
5 \cdot 29 \\
5 \cdot 72 \\
5 \cdot 48 \\
5 \cdot 50 \\
5 \cdot 75 \\
5 \cdot 75\end{array}$ & $\begin{array}{l}4.21 \\
2.35 \\
2.35 \\
2.65 \\
4.68 \\
6.12 \\
6.12 \\
6.12 \\
6.12 \\
6.12\end{array}$ \\
\hline Totals & 42.0 & 35.0 & 34.0 & & & \\
\hline
\end{tabular}

Influence on Pancreatic Secretion. - If the secretion of the pancreatic juice is studied by means of a fistula placed in the canal of Wirsung, we again find the same sensitiveness toward the food received. Here the question is more complex. The dependence relates, in fact: ist, to the general manner of the secretion, that is to say, to the rapidity with which the juice flows each hour into the intestine; 2nd, to the quantity of juice secreted, for example, per unit of nitrogen; 3 rd, to the content in trypsin, amylase, and lipase.

The following is a table showing this influence of the nature

Influence of Feeding on the Pancreatic Secretion.

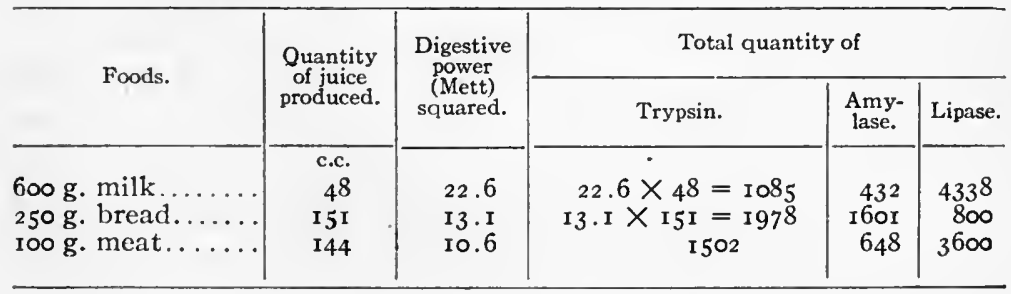


of the food on the quantity and the composition of the pancreatic juice secreted.

It is seen that for the proteolytic power of the pancreatic juice, the same order of classification is obtained as was the case with the gastric juice. Bread causes the maximum production of trypsin, meat is next in order, and then milk. As regards amylase, bread requires a large amount, meat less, and milk still less. For the lipolytic enzyme, it is milk, rich in fatty matter, which will require the largest quantity.

In a general way, Pawlow and his pupils have observed that the proteolytic activity of the pancreatic juice varies according to the diets to which the animals are submitted. But by what mechanism is this adaptation made? Various explanations could be given of this phenomenon. First of all, the differences of activity observed in the juices collected by the method of Pawlow might be due to the variations of the kinasic secretion furnished by the fragment of intestinal mucous membrane fixed to the skin with the opening of the canal of Wirsung. However, Frouin has shown that the intestinal juice collected by means of a permanent Thiry fistula in a dog (carnivorous), or in a bull (herbivorous), possesses practically the same kinasic activity per unit volume. On the other hand, the pancreatic juice of the same animal submitted to different diets, with addition of $\mathrm{I} / 20$ or $\mathrm{I} / \mathrm{IO}$ of its volume of intestinal juice, presents always practically the same digestive power for albumin; it is therefore not the trypsin content which varies. For example:

I. Pancreatic juice of a dog nourished for 2 months with raw meat exclusively $+\mathrm{I} / 20$ of its volume of filtered intestinal juice; the mixture digests $4 \mathrm{~mm}$. Mett tube in 15 hours.

2. Pancreatic juice of the same dog, submitted for a month to a bread diet, $+1 /$ ro of its volume of intestinal juice; the mixture digests 4 to $5 \mathrm{~mm}$. Mett tube in ${ }_{5}$ hours.

I and 2 show an equal activity. Since, on the other hand, animals nourished with bread secrete 4 to 5 times more juice than those nourished on meat, it follows that, under the bread diet, 
the total pancreatic secretion can digest 4 to 5 times more albumin; if there is an adaptation it would seem to be in the opposite direction to that which would at first be supposed. The justification of the results found by Pawlow, as Frouin has shown, probably consists in the fact that the minimum quantities of intestinal juice necessary to confer on the pancreatic juice the maximum of digestive power are very different. While it is sufficient to add to the juice, secreted under the influence of meat, I/500 or even I/ rooo of its volume of intestinal juice to give to it the maximum digestive activity, it.is necessary to add to the juice secreted by bread $\mathrm{I} / 20$ or $\mathrm{I} / \mathrm{I}$ o of its volume to reach the same result. This fact indicates clearly that, if it is true that in equal volume activated pancreatic juices have the same digestive powers, there is nevertheless a very great difference in activation under the influence of different diets.

\section{§ 2. Mechanism of Secretion.}

Our organs, in the course of digestion, furnish agents of transformation which are very different and which pour out on the foods as their intervention is required. The appearance of these agents takes place in a rational order. First of all, the food eaten is diluted by the aid of the salivary secretion, which, by its alkaline reaction, reduces the acidity of the foods and thus furnishes favorable conditions for the action of ptyalin. Moreover, to permit this enzyme to act, the flow of gastric secretion undergoes a slight retardation. Then, after the starchy foods have undergone a sufficiently advanced transformation, the gastric juice appears with its distinctly acid reaction, which greatly facilitates the action of pepsin. The quantity of juice which is thus produced is proportional to the quantity of food ingested. The rapidity of flow and the general manner of the secretion is regulated according to a definite plan, and according to actual needs. In this phase of the process, the albuminoid material is transformed into albumose and into peptone. The pancreatic juice then accomplishes the digestion 
of the nitrogenous foods and of the starches, at the same time that it decomposes the fatty materials. In the intervention of the pancreatic juice a still greater precision is attained, the adaptation to the needs of the digestive processes and of the reaction of the medium and of all the reagents taking place this time in an almost exact manner. It has been seen, in particular, that according to the food absorbed, there is a difference in the quantity and the duration of the secretion, as well as in the chemical properties of this juice.

Mechanical Action. Psychic Impulse. - Let us now see how this marvelous mechanism is regulated which automatically manufactures, in suitable quantities, all the various reagents, and pours them out judiciously at the opportune moment. It was for a long time believed that the secretions were due to the irritation produced by the foods in the course of their passage through the alimentary tract, and that a mechanical action then appeared, especially in the stomach itself. The inaccuracy of this opinion is now established. The gastric and intestinal glands do not react like the salivary glands to mechanical stimuli and, on the other hand, Pawlow has demonstrated that the introduction into the stomach of inert bodies, like sand or any other material not foods, does not change at all either the secretion or the reaction of the medium.

Moreover, the fact that the mechanical actions play no rôle in the elaboration of the digestive juices had already been proved by Bidder and Schmidt, who had found, with a dog provided with a gastric fistula, that the escape of juice could be produced by the simple sight of the nourishment, simply by reason of the desire to eat. This observation remained for a long time without confirmation, although it was in complete agreement with the data then possessed on salivary secretion. Taken up again by Pawlow and his pupils, it served as the basis for a whole series of extensive studies relative to psychic influences on secretions.

Unconscious Nutrition.-By adopting accurate experimentation methods and by taking favorable conditions, it was 
found that psychic influences play a rôle of the greatest importance. If meat is fed to a dog provided with a fistula and operated on with osophagotomy, there is observed, not only the fact of an abundant secretion which can be prolonged even for hours, but also the fact that, with sensitive subjects, the quantity of juice flowing depends both on the character and on the quantity of food placed before the animal. The sight of meat generally causes a more abundant secretion than the sight of bread, and, in addition, the sight of a large quantity of nourishment brings about a larger flow of juice than the sight of a small ration.

Sonotzky, by causing a dog to undergo for 5 minutes a pseudo-meal, found that the secretion which resulted was prolonged for hours. Without doubt, the maximum effect corresponds to the first five minutes, but it is to be noted that the secretion is still very perceptible after $3 \frac{1}{2}$ hours and that its digestive power at this point is always high.

Gastric Secretion Produced by a Pseudo-Meal.

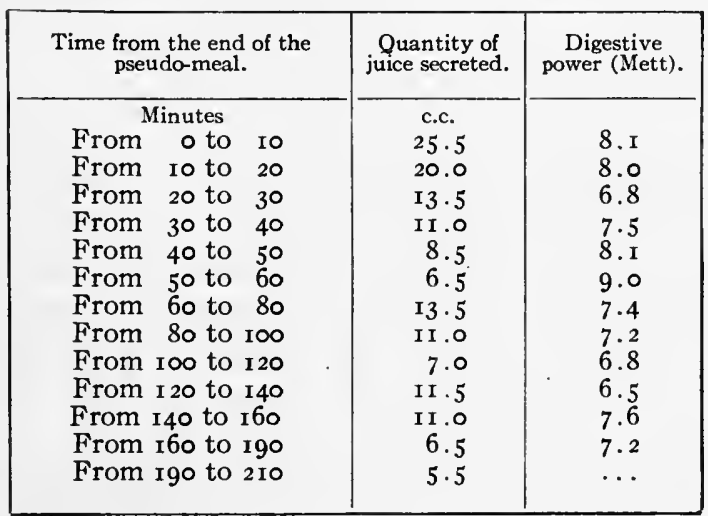

The rôle of psychic influences on secretion can likewise be shown in the course of actual digestion. For this purpose Labosoff utilizes the experimental arrangement already described, and which consists in dividing the stomach into two unequal parts, the food going into the larger portion while 
the secretion is collected by the aid of a fistula placed in the smaller one whose volume is about $\mathrm{r} /$ Io of the total stomach and is isolated from the whole of the digestive tube. Labosoff gives to a dog thus treated $400 \mathrm{~g}$. of meat, divided in 4 pieces. Four feedings take place at $\mathbf{I} \frac{1}{2}$ hour intervals. Each time that the animal sees and eats his ration, there is a re-acceleration of secretion; not only the volume secreted increases, but also the digestive power.

Psychic Influence Produced in the Course of a Normal Nutrition.

\begin{tabular}{|c|c|c|c|}
\hline Progress of the experiment. & Time in half-hours. & $\begin{array}{l}\text { Juice secreted } \\
\text { in the part } \\
\text { isolated. }\end{array}$ & $\begin{array}{c}\text { Digestive } \\
\text { power } \\
\text { (Mett). }\end{array}$ \\
\hline $\begin{array}{l}\text { Ioo grams of meat........ } \\
\text { Again too grams of meat. } \\
\text { Again roo grams of meat. }\end{array}$ & $\begin{array}{l}\text { From } 0 \text { to } I \\
\text { I to } 2 \\
2 \text { to } 3 \\
3 \text { to } 4 \\
4 \text { to } 5 \\
5 \text { to } 6 \\
6 \text { to } 7 \\
7 \text { to } 8 \\
8 \text { to } 9 \\
9 \text { to } 10 \\
\text { Io to } 11 \\
\text { II to } 12\end{array}$ & $\begin{array}{l}\text { c.c. } \\
3 \cdot 1 \\
5 \cdot 0 \\
4 \cdot 7 \\
5 \cdot 4 \\
5 \cdot 5 \\
4 \cdot 7 \\
6.0 \\
5 \cdot 4 \\
5 \cdot 9 \\
5.4 \\
5 \cdot 3 \\
4.2\end{array}$ & $\begin{array}{l}5.13 \\
4.63 \\
4.50 \\
4.88 \\
3.38 \\
2.75 \\
3.75 \\
2.50 \\
2.50 \\
3.88 \\
3.00 \\
2.50\end{array}$ \\
\hline
\end{tabular}

Chemical Action. - Psychic influence is not, however, the sole cause of the secretion. When nutritive material is introduced into the stomach of an animal by the aid of a fistula, it is found that this food is more or less digested, indicating that the secretion is made even in the absence of all psychical intervention. The secretion produced in this last case is due to the specific action of the nutritive substances, and it is observed that the quantity and quality of the juice secreted vary with the chemical nature of the substance absorbed. Indeed, two causes regulate the secretion of the gastric juice, one of purely psychic origin, the other of chemical origin. To study the unconscious secretion, that is to say the direct effect produced by the foods on the secretorial glands, Pawlow introduced $400 \mathrm{~g}$. of meat into the stomach of a dog operated upon as before, 
without having the animal see the food. The secretion, which began about 25 minutes after the introduction of the meat, took place as follows:

Gastric Secretion in the Course of Unconscious Nutrition.

\begin{tabular}{|c|c|c|}
\hline Duration. & $\begin{array}{l}\text { Juice secreted in } \\
\text { the part isolated. }\end{array}$ & $\begin{array}{l}\text { Digestive power } \\
\text { (Mett). }\end{array}$ \\
\hline 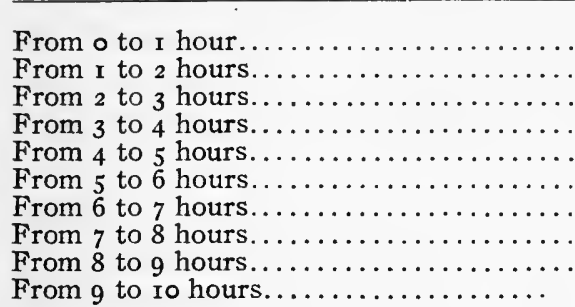 & $\begin{array}{r}\text { c.c. } \\
3 \cdot 7 \\
10.6 \\
9.2 \\
7.0 \\
5.6 \\
6.6 \\
7.5 \\
5 \cdot 3 \\
3.0 \\
2.0\end{array}$ & $\begin{array}{l}2.0 \\
1.63 \\
1.5 \\
1.88 \\
2.25 \\
2.63 \\
1.88 \\
2.0 \\
5.0 \\
\cdots\end{array}$ \\
\hline
\end{tabular}

This table, compared with the two preceding ones, shows that the chemical secretion differs considerably from the psychic secretion, not only from the point of view of the progress of the secretion itself, but also from the point of view of its pepsin content.

Rôle of the Psychic Impulse in Normal Nutrition. - Realizing these two influences, Pawlow proposed to determine the part which psychic secretion plays in normal nutrition. To this end, he introduced meat into the stomach of a dog, taking great care that the animal did not realize, either by sight or odor, that he was receiving nourishment. After the meat had remained a certain time, it was removed by means of a string to which it had been attached. The loss of weight furnished the measure of the digestion and, consequently, the value of the secretion in unconscious nutrition. By repeating this experiment with a dog excited at the same time by a pseudo-feeding, the effect of the two influences chemical and psychic combined was obtained, and it was found that the quantity of meat digested in the stomach in this second case is always greater than that digested in the course of unconscious nutrition, the difference of the two results furnishing an indirect measure of 
the purely psychic secretion. The dogs experimented with each received too $\mathrm{g}$. of meat through a gastric fistula. After I hour, the meat was taken away and weighed. It was found that in the case of the pseudo-meal $70 \mathrm{~g}$. of meat remained undigested, while without pseudo-feeding the weight of meat remaining was $94 \mathrm{~g}$. By unconscious feeding there was therefore digested only $6 \mathrm{~g}$. of meat, while digestion under the psycho-chemical influence brought the quantity up to $30 \mathrm{~g}$. In another experiment, lasting $1 \frac{1}{2}$ hours, unconscious nutrition gave $5.6 \mathrm{~g}$, , while with pseudo-feeding $\mathrm{I}_{5} \mathrm{~g}$. of meat were digested.

When the meat is left for 5 hours in the stomach for unconscious nutrition, $5^{8} \mathrm{~g}$. of meat is found digested and $85 \mathrm{~g}$. for nutrition accompanied by a pseudo-meal. Thus, even after a stay of 5 hours, there still exists a noticeable difference due to psychic influences. These various data show that among the causes producing the gastric secretion, psychic influences play a preponderating rôle. This influence is especially evident at the beginning, causing the appearance of an abundant juice, very strong in pepsin, while the unconscious nutrition brings about only a tardy secretion of a much weaker pepsin content. The importance of the two secretions, psychic and chemical, is still better summarized in the following table:

Experiment Made with Meat Given to Dogs.

\begin{tabular}{|c|c|c|c|c|c|c|c|}
\hline \multirow{2}{*}{$\begin{array}{l}\text { Time in } \\
\text { hours. }\end{array}$} & \multicolumn{2}{|c|}{ Normal nutrition. } & \multicolumn{2}{|c|}{ Unconscious nutrition. } & \multicolumn{2}{|c|}{ Pseudo-nutrition. } & \multirow{2}{*}{$\begin{array}{l}\text { Sum of } \\
\text { headings } \\
2 \text { and } 3 .\end{array}$} \\
\hline & $\begin{array}{c}\text { Juice se- } \\
\text { creted per } \\
\text { hour. }\end{array}$ & $\begin{array}{c}\text { Digestive } \\
\text { power. }\end{array}$ & $\begin{array}{l}\text { Juice se- } \\
\text { creted per } \\
\text { hour. }\end{array}$ & $\begin{array}{l}\text { Digestive } \\
\text { power. }\end{array}$ & $\begin{array}{l}\text { Juice se- } \\
\text { creted per } \\
\text { hour. }\end{array}$ & $\begin{array}{c}\text { Digestive } \\
\text { power. }\end{array}$ & \\
\hline $\begin{array}{l}\text { I } \\
2 \\
3 \\
4\end{array}$ & $\begin{array}{r}\text { c.c. } \\
\text { I } 2.4 \\
13.5 \\
7.5 \\
4.2\end{array}$ & \multirow[t]{2}{*}{$\begin{array}{l}5 \cdot 43 \\
3.63 \\
3.5 \\
3.12\end{array}$} & $\begin{array}{l}\text { c.c. } \\
5.0 \\
7.8 \\
6.4 \\
5.0\end{array}$ & \multirow[t]{2}{*}{$\begin{array}{l}2 \cdot 5 \\
2.75 \\
3 \cdot 75 \\
3 \cdot 75\end{array}$} & $\begin{array}{l}\text { c.c. } \\
7.7 \\
4.5 \\
0.6 \\
\ldots\end{array}$ & \multirow[t]{2}{*}{$\begin{array}{l}6.4 \\
5.8 \\
5.75 \\
\ldots .\end{array}$} & $\begin{array}{l}\text { c.c. } \\
12.7 \\
12.3 \\
7.0 \\
5.0\end{array}$ \\
\hline Totals & 37.6 & & 24.2 & & I 2.8 & & 37.0 \\
\hline
\end{tabular}

This table indicates various interesting facts. First of all, it is seen that the sum of the quantities of juice secreted in 
4 hours, on the one hand by unconscious nutrition and on the other by pseudo-nutrition, approach in a striking manner the total quantity of juice secreted in the course of normal nutrition, and that, further, this concordance is found again practically every hour. It is clear that the secretion during a normal meal results from two different influences. Secondly, the two effects are superimposed in a manner proportional to each of them. Thus, of the 12.4 c.c. of juice secreted in the first hour in the course of a normal meal, 7.7 c.c. are due to psychic action, that is to say to the appetite itself. In the second hour, of I3.5 c.c. secreted, there are no more than 4.5 c.c. due to the appetite; in the third hour, of 7.5 c.c., only 0.6 c.c. results from this influence, and at the end, in the course of the fourth hour, it is only the chemical influence which acts, the appetite having at this moment entirely disappeared.

These figures, it is understood, are not absolute. The quantity of juice secreted, produced by the same ration, will never have a fixed value. It will depend on the sensitiveness of the animals submitted to the tests and on their greater or less desire to eat. It will be influenced not only by sight but also by odor. It is by these impressions, sight and smell, that the appetite is increased, and the idea that eating is about to begin causes a secretorial excitation by a reflex mechanism analogous to that which causes us to close the eyes when confronted by a sudden movement. Sight and smell are, moreover, not the only factors which act on the secretorial glands. A special influence must also be attributed to the sensation which foodstuffs cause in the mouth, the taste of foods playing undoubtedly an important rôle in the psychic secretion. Finally, it must also be admitted that all the secretorial nerves, from the mouth to the stomach, are influenced by the passage of the food, and that by direct reason of the pleasure which we experience in ingesting this food. But an objection arises. It should, in fact, be observed that pseudo-nutrition, whi e giving an idea more or less exact of the effect of psychic influence on the secretion of the gastric juice in the course of normal digestion, cannot 
furnish a true measure of the quantity of juice secreted under this influence alone. In pseudo-nutrition, the excitation is much greater than in normal nutrition, where the sensation of actual satisfaction produced by the food absorbed is not slow to paralyze the imagination. As we eat appetite slackens, while pseudo-nutrition can be conducted for hours without assuaging the need of eating, so that we cause, in this case, the secretion of a much larger quantity of psychical juice.

These considerations would lead us to disbelieve the great importance ascribed to psychic secretion in normal nutrition. However such a conclusion would be erroneous. The last table given above proves that in normal nutrition the psychic effect plays a very important rôle. It is even possible that its influence is even more considerable than that which appears from the experiments previously cited. It is known, as a matter of fact, that during digestion the juice secreted varies constantly with respect to its digestive power. Whence comes this variation? Do both the two juices, psychic and chemical, change in composition, or only one? The following experiment of Labossof and Chigin furnishes the answer to this question:

Secretions Compared in Pseudo-Nutrition and Normal Nutrition.

\begin{tabular}{|c|c|c|c|c|}
\hline \multirow{2}{*}{ Time in hours. } & \multicolumn{2}{|c|}{ Pseudo-nutrition. } & \multicolumn{2}{|c|}{ Normal nutrition. } \\
\hline & Juice secreted. & Digestive power. & Juice secreted. & Digestive power. \\
\hline $\begin{array}{l}\text { I } \\
2 \\
3 \\
4\end{array}$ & $\begin{array}{l}\text { c.c. } \\
7.7 \\
4.5 \\
0.6 \\
\ldots .\end{array}$ & $\begin{array}{l}6.4 \\
5 \cdot 3 \\
5.75 \\
\ldots \ldots\end{array}$ & $\begin{array}{r}\text { c.c. } \\
11.2 \\
11.3 \\
7.6 \\
5.1\end{array}$ & $\begin{array}{l}4.94 \\
3.03 \\
3.01 \\
2.87\end{array}$ \\
\hline
\end{tabular}

It is seen that, in the course of normal digestion of meat, the quantity of juice secreted diminishes as the proteolytic action advances. Further, the digestive power, which at the beginning was 4.94 , has fallen to 2.87 . On the contrary in the pseudo-nutrition quite a different progress is observed. The digestive action of the psychic juice is almost arrested. It 
then appears from the preceding that the diminution which is found in the quantity and the quality of the juice elaborated in the course of normal nutrition comes almost exclusively from the arrest of psychic secretion. It is, therefore, the psychic impulse which produces the most active juice; beyond this juice, there is a secretion of a digestive power of only a secondary importance.

The secretion which is produced in addition to the psychic impulse is considered by Pawlow to be a specific secretion, due to the agencies of nourishment themselves. We will now see how this chemical influence is exerted on the secretorial glands. The method used in this research is the same as that which previously served in the study of unconscious nutrition. It is a question of placing the patient in conditions such that the rôle of appetite is completely excluded. This may be accomplished by introducing food into a dog by a fistula, while taking good care that the animal perceives nothing, either by sight or by smell. Nourishment may also be introduced while the animal sleeps, since it is known that the secretorial glands function normally even during sleep. A whole series of very varied arrangements are known for studying unconscious nutrition. Rjasanzeff, Chigin, Labosoff and others, while modifying the conditions, have reached results which are very similar, and have given us data of primary importance with regard to digestion. By introducing in the alimentary tract different food substances, it is found that each acts in a manner which is peculiar to itself and which is perfectly constant. The influence of these substances differs according to their chemicai nature, some acting on the secretorial glands causing a more or less rich secretion, others acting in an opposite direction, checking or completely arresting the secretion. Finally, there exists a whole class of foods which show themselves inactive and which in no way influence the secretorial glands. Moreover, it has been observed that the chemical action of nutritive substances takes place sometimes on the gastric glands, sometimes on the pancreatic gland. The substances active on 
the gastric glands may show themselves without action on the pancreas, and vice versa.

To summarize the numerous data which we possess on this important question, we will study separately the chemical effects, first on the glands of the stomach, and secondly on the pancreatic cells.

\section{§ 3. Chemical Influences on Gastric Secretion.}

Effect of water. - All foods do not influence gastric secretion. Among the substances which show themselves most active are water and raw meat. Water introduced into the stomach causes an acid secretion, though not a very abundant one; this is shown plainly only in the presence of a large quantity of water. To obtain an appreciable flow of gastric juice through a fistula with a dog of medium size, it is necessary to introduce 400 to 450 c.c. of water, while roo to $5_{50}$ c.c. give no result. This effect of water on the secretorial glands is extremely important from the point of view of digestion.

Effect of Meat. - Raw meat brings about a larger secretion than water. Introduced in the stomach of a dog, it causes a considerable secretion even after $\mathrm{I}_{5}$ or 30 minutes. Boiled meat is without effect. The fact is, then, that the secretorial substance is found in the extractive materials of the meat. The effect of Liebig's extract has been studied by Labossof. This investigator dissolved ro $\mathrm{g}$. of Liebig's extract in $\mathrm{r} 50 \mathrm{~g}$. of water and introduced the mixture in the stomach of a dog. The secretion started after $\mathrm{I}_{3}$ minutes. He found that:

The first hour there flows 5.3 c.c. juice of a digestive power $=4.25$

The second hour there flows 2.6 c.c. juice of a digestive power $=4.0$.

We have not yet been able to determine the chemical nature of the constituent of meat extract causing the secretion. We only know that this substance is among those parts insoluble in alcohol.

The different commercial peptones act very differently. According to Pawlow, only Chapoteau peptone possesses a secretorial power. It is quite probable that this peptone was obtained from raw meat, while the others have been manufactured 
from other albuminoid material, and that it is from this that the difference noted above results. It is also possible, as we shall see later, that this difference arises from the various degrees of hydrolysis to which the peptones examined have been submitted. The secretorial action of Liebig's extract or of peptone is considerably increased if these are mixed with other substances less active by themselves, such as starch. This increase of the secretorial power comes from the fact that the extract thus remains for a longer time in contact with the mucous membranes.

As other active substances, gelatin and milk may be mentioned. However the greater part of the foods appear to be without chemical effect on the secretorial glands. Thus albumin, liquid or coagulated, starch and sugar produce no secretion. Diluted in water, they furnish no more juice than the water itself.

Effect of Fats. - The effect of fatty materials and of starch merits a special attention. Oil, introduced into the stomach, is without effect, but the true rôle of fatty materials is shown only when their effect in the course of digestion is studied. When fatty materials are introduced into the stomach, either before or during the meal, there is always found a considerable slackening in the flow of juice. Thus with a dog, which ordinarily secretes in the first 2 or 3 hours after a meal of $400 \mathrm{~g}$. of meat, from ro.o to 15.0 c.c. of juice (collected in the isolated part of the stomach), the results will be quite different if, an hour before his meal, roo g. of oil are introduced into his stomach. There is no secretion during the first hours, and only after 2 or 3 hours do we find the appearance of a very slight flow of juice. The influence of the oil still persists even after it has been eliminated from the stomach.

This influence of fatty materials is felt, not only on the quantity of juice secreted, but also on its quality. The following is an experiment of Labossof which demonstrates this inhibition. On the left is indicated the progress of the secretion (juice collected in the isolated part of the stomach) with a dog after a 
meal of $400 \mathrm{~g}$. of meat, and on the right, the progress of the secretion with the same animal, which has received, $\mathrm{I} \frac{1}{2}$ hours before the meal, 75 c.c. of oil and then a meal of $400 \mathrm{~g}$. of meat:

Influence of Fatty Materials on Gastric Secretion.

\begin{tabular}{c|c|c|c|c}
\hline \multirow{2}{*}{ rime, hours. } & \multicolumn{2}{|c|}{ Ordinary meal. } & \multicolumn{2}{c}{ Meal preceded by an ingestion of oil. } \\
\cline { 2 - 3 } & Juice secreted. & Digestive power. & Juice secreted. & Digestive power. \\
\hline & c.c. & & & \\
I & 17.8 & 6.25 & 4.3 & 4.25 \\
2 & 13.8 & 4.50 & 5.3 & 3.00 \\
3 & 12.0 & 3.75 & 4.5 & 1.75 \\
4 & 8.5 & 3.38 & 3.8 & 1.75 \\
\hline
\end{tabular}

It is seen that the difference is not only quantitative, but also qualitative; the digestive power is considerably lowered following the ingestion of oil, and this diminution is felt from the first hour of the digestion. This last finding gives us an indication as to the nature of the process by which the oil exerts its effect. The change in the progress of the secretion is due in part to the diminution of the specific secretion, but especially to the great weakening of the psychic secretion. However, Pawlow offers a decisive proof of the influence exerted by oil on the psychic secretion. He submits a dog to a pseudo-meal for 6 minutes. The secretion normally begins and continues in the following way:

In the ist hour there is collected 4.0 c.c. juice of a digestive power $=4.75$.

In the 2 nd hour there is collected I. $O$ c.c. juice of a digestive power $=4.75$.

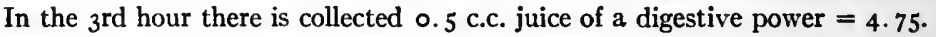

The digestive power is constant and equal to 4.75. The same dog then receives $100 \mathrm{~g}$. of oil in the stomach a half-hour before the pseudo-meal, and then the pseudo-nutrition is conducted as before for 6 minutes. No secretion is produced during the first two hours. The pseudo-nutrition is repeated. The secretion appears, but is very faint; it gives in the first hour r.8 c.c. of juice, of digestive power equal to 4.0 only. Thus, the presence of fatty materials in the stomach leads to a marked slackening in the secretion of psychic origin. This unfavor- 
able effect of fatty materials can again be shown by determining the secretion obtained by nourishing a dog on the one hand with milk, and on the other with cream.

Influence of Fatty Materials on the Gastric Secretion.

\begin{tabular}{|c|c|c|c|c|}
\hline \multirow{2}{*}{ Hours. } & \multicolumn{2}{|c|}{600 grams of milk. } & \multicolumn{2}{|c|}{600 grams of cream. } \\
\hline & Quantity secreted. & $\begin{array}{c}\text { Digestive } \\
\text { power. }\end{array}$ & Quantity secreted. & $\begin{array}{c}\text { Digestive } \\
\text { power. }\end{array}$ \\
\hline \multirow{9}{*}{$\begin{array}{c}\text { I } \\
2 \\
3 \\
4 \\
5 \\
6 \\
7 \\
8 \\
\text { Totals and } \\
\text { averages }\end{array}$} & $\begin{array}{l}\text { c.c. } \\
4.2\end{array}$ & 3.57 & $\begin{array}{l}\text { c.c. } \\
2.4\end{array}$ & 2.1 \\
\hline & 12.4 & 2.63 & 3.4 & 2.0 \\
\hline & 13.2 & 3.6 & 3.1 & 2.0 \\
\hline & 6.4 & 3.91 & 2.2 & 1. 75 \\
\hline & I. 5 & $7 \cdot 37$ & 2.2 & 2 \\
\hline & $\ldots$ & $\ldots$ & I. 8 & 1. 38 \\
\hline & $\cdots$ & $\cdots$ & 2.5 & $\begin{array}{l}1.88 \\
.62\end{array}$ \\
\hline & $\cdots$ & $\cdots$ & $\mathrm{I} \cdot 3$ & I.62 \\
\hline & 37.7 & 3.86 & 18.9 & I. 63 \\
\hline
\end{tabular}

The milk produces a slow secretion and one not very active because it contains fatty materials. The cream, which is much richer in fatty materials, will cause a still slower secretion, possessing a much less activity.

Effect of Starch. - The effect of starch is quite complicated. Introduced into the empty stomach, it produces no secretion, but mixed with other nutritive substances, it influences in a high degree the formation of juice. The effect produced by starch does not consist, as with the fats, in a slackening of the secretion, but rather in a change of the digestive power of the juice secreted. If we add starch to a ration of meat, we find in the secretion a modification quite different from that obtained with meat alone. The activity of the juice is considerably increased, even from the beginning of the digestion; furthermore, the secretion continues for a long time.

In a preceding paragraph it was noted that meat and bread produce gastric juices of very different activity. While bread causes the appearance of a very active juice, which increases still further in value in the first hour of digestion, meat, on the contrary, brings about only the secretion of a less active 
juice, whose digestive power moreover decreases very rapidly. The characteristic progress of the secretion produced by bread would be due, according to Pawlow, to the specific effect of the starch, so that it is possible by mixing meat and starch in suitable proportions, to realize a secretorial progress absolutely analogous to that furnished by bread:

Gastric Secretion Produced by Bread.

\begin{tabular}{|c|c|c|c|c|}
\hline \multirow{2}{*}{ Time in hours. } & \multicolumn{2}{|c|}{ Secretion obtained by $200 \mathrm{~g}$. bread. } & \multicolumn{2}{|c|}{$\begin{array}{l}\text { Secretion obtained by } 100 \mathrm{~g} . \text { meat } \\
+ \text { I0 g. starch }+150 \text { g. water. }\end{array}$} \\
\hline & Quant. juice. & Dig. power. & Quant. juice. & Dig. power. \\
\hline $\begin{array}{l}1 \\
2 \\
3 \\
4 \\
5 \\
6 \\
7 \\
8 \\
9\end{array}$ & $\begin{array}{r}\text { c.c. } \\
\text { II.9 } \\
4.1 \\
5.7 \\
4.5 \\
4.1 \\
1.6 \\
1.8 \\
0.8 \\
0.6\end{array}$ & $\begin{array}{l}5.22 \\
8.25 \\
6.69 \\
3.56 \\
3.62 \\
4.80 \\
5.50 \\
5.62 \\
\ldots .\end{array}$ & $\begin{array}{r}\text { c.c. } \\
\text { 13.5 } \\
\text { II.0 } \\
8.9 \\
4.9 \\
4.3 \\
1.9 \\
\text { 1.2 } \\
\ldots . \\
\ldots \ldots\end{array}$ & $\begin{array}{l}7.88 \\
7.0 \\
6.13 \\
5.63 \\
5.0 \\
6.5 \\
6.0 \\
\ldots \ldots \\
\ldots .\end{array}$ \\
\hline averages & 35.1 & 6.12 & 45.8 & 6.75 \\
\hline
\end{tabular}

The mixture of meat and starch produces then a gastric juice quite similar to that of bread, while meat alone produces a juice of a much smaller digestive power.

From the preceding, it appears that meat can be digested, if not completely, at least fairly thoroughly without the intervention of psychic influence, provided it possesses a suitable secretorial power. The specific effect of bread is of a very different order; its secretorial power is manifested only when it has stayed a sufficiently long time in the stomach. To begin digestion, it requires first of all a juice produced either by a psychic influence, or by water or by any other material endowed with a secretorial activity, as meat, for example. It is only subsequently that the specific power of bread appears when digestion is well declared. Bread, incapable of effect in the beginning, then becomes a secretorial agent of the first order due to certain products resulting from its own hydrolysis. In the 
natural digestion of bread the psychic effect plays a preponderating part in that it first excites the juice indispensable to the first operation, and thus allows the secretorial power of the bread to show itself afterward in all its intensity.

Effect of Salts. - Certain inorganic substances likewise influence secretion. The influence of salts has been studied by Cahn, Wohlgemuth, Kudo, and others. In particular, the last has found that meat which has been freshened slightly favors gastric secretion. The following table summarizes his observations:

Influence of Sodium Chloride on Gastric Secretion.

\begin{tabular}{|c|c|c|c|c|c|}
\hline Days. & Quantity of meat. & $\begin{array}{c}\text { Gastric } \\
\text { juice } \\
\text { secreted. }\end{array}$ & $\begin{array}{c}\text { Acidity } \\
\text { free HCl. }\end{array}$ & $\begin{array}{l}\text { Pepsin in } \\
\text { I c.c. }\end{array}$ & $\begin{array}{l}\text { Total content } \\
\text { in pepsin. }\end{array}$ \\
\hline I & $250 \mathrm{~g}$. not freshened. & $\begin{array}{l}\text { c.c. } \\
40\end{array}$ & 65 & 80 & 3200 \\
\hline 2 & $250 " 1 " \quad " \quad$ " $\quad \ldots$ & 43 & 55 & 80 & 3440 \\
\hline 3 & $250 "$ " & 38 & IIO & 125 & $475^{\circ}$ \\
\hline 4 & $25^{\circ}$ " freshened........ & 2 & $\circ$ & 800 & 1600 \\
\hline 5 & $250 “ \quad$ “ $\quad \ldots \ldots \ldots$ & 14 & I0 & 312 & 4312 \\
\hline 6 & $250 " 1$ & 13 & 0 & 312 & $4056^{\circ}$ \\
\hline 7 & $2.50 "$ " $"$ " $\ldots \ldots$ & 9 & 0 & 312 & 2808 \\
\hline 8 & 250 " not freshened.. & 21 & 72 & 200 & 4200 \\
\hline 9 & $250 " 1 \quad$ " & 48 & I 10 & 80 & 3840 \\
\hline 10 & $250 " 1$ & 73 & II 5 & 50 & $375^{\circ}$ \\
\hline
\end{tabular}

The digestive power was established by the Fuld method. It is seen that the absence of salt causes a considerable diminution of the secretion, ${ }^{*}$ and that the juice obtained under these conditions is of quite different composition, containing only traces of hydrochloric acid, though its content in pepsin has increased a great deal. This experiment thus offers an explanation as to the origin of hydrochloric acid in the stomach. On the other hand, as we shall see later with regard to the pancreatic secretion, this same salt also has an influence on the alkalinity of this juice in that the sodium chloride introduced into the alimentary tract is hydrolyzed to $\mathrm{HCl}$ and $\mathrm{NaOH}$, the one going to the gastric juice, the other to the pancreatic juice, so

* See also table of Frouin and Girard, p. 259. 
that the acidity and alkalinity of these two liquids are always equivalent.

According to Mayeda, Rosenblatt, Johan, Feigl, and Rollett, sodium carbonate and bicarbonate diminish gastric secretion. However, it would appear that this inhibitory action is due to the fact that the bicarbonate is introduced into a fasting stomach. If, on the contrary, this salt is ingested either immediately before or during a meal, it acts as an excitant of the gastric secretion. At least this is what Linossier and Lemoine found in 1894 with man, and in 1906 with dog. According to these experimenters, the excitation is stronger the more hydrochloric the subject. Therefore, before the experiment all salt food is avoided. The Ist day there is introduced into the small isolated stomach of a dog, by means of a tube, $500 \mathrm{~g}$. meat +250 c.c. distilled water; the 2 nd day the water is replaced by a I per cent solution of -sodium bicarbonate, or $2 \frac{1}{2} \mathrm{~g}$. of this salt. The following are the quantities of juice secreted in the small stomach during the two hours which follow each of these meals:

Influence of Sodium Bicarbonate on Gastric Secretion.

\begin{tabular}{|c|c|c|c|c|}
\hline Nourishment. & $\begin{array}{l}\text { Juice secreted } \\
\text { by the small } \\
\text { stomach. }\end{array}$ & $\begin{array}{l}\mathrm{HCl} \text { secreted } \\
\text { by the small } \\
\text { stomach. }\end{array}$ & Per cent, $\mathrm{HCl}$. & Pepsin (Mett). \\
\hline 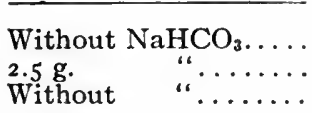 & $\begin{array}{c}\text { c.c. } \\
23.4 \\
59.6 \\
25.2\end{array}$ & $\begin{array}{c}\text { g. } \\
0.1095 \\
0.2878 \\
0.0972\end{array}$ & $\begin{array}{l}0.468 \\
0.483 \\
0.413\end{array}$ & $\begin{array}{l}\mathrm{mm} \\
4 \cdot 0 \\
2.3 \\
5.2\end{array}$ \\
\hline
\end{tabular}

The sodium bicarbonate therefore increases the secretion both in volume and in acidity but it lowers the digestive power.

Calcium and lithium carbonates in small quantities exert a favorable action. Carbonic acid, iron, and metallic aluminium act as excitants of the secretion, the effect of iron and aluminium being related to that of hydrogen. As other substances favoring the secretion, we may mention certain arsenical preparations, as well as medicinal preparations containing iodine.

Effront measures the secretorial powers of meat, of meat extracts, and of yeast according to the nitrogen content of the 
excrement. He first establishes the coefficient of assimilability of nitrogen in a vegetarian diet. Then he adds to the same meal the substances to be tested, the variation in nitrogen of the excrement indicating the influence which these substances have exerted on digestion, and consequently serving as a measure of their secretorial powers.

Secretorial Power Determined According to the Quantity of Nitrogen Absorbed.

\begin{tabular}{|c|c|c|}
\hline \multirow[b]{2}{*}{ Food. } & \multicolumn{2}{|c|}{ Excrement of 24 hours. } \\
\hline & $\begin{array}{c}\text { Content in } \\
\text { nitrogen. }\end{array}$ & $\begin{array}{l}\text { Per cent content } \\
\text { nitrogen contained } \\
\text { in vegetable food. }\end{array}$ \\
\hline 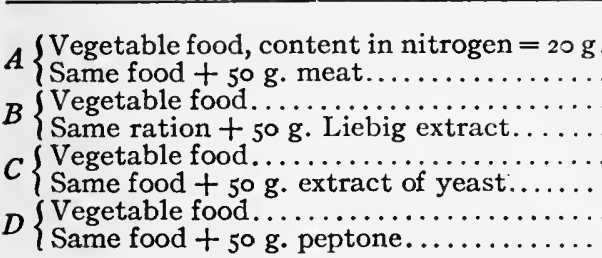 & $\begin{array}{l}g \cdot \\
4 \cdot 5 \\
4 \cdot I \\
5 \cdot 3 \\
3 \cdot 4 \\
5 \cdot 1 \\
5 \cdot 3 \\
5 \cdot 7 \\
2.8\end{array}$ & $\begin{array}{l}\text { Per cent. } \\
22.5 \\
19.0 \\
22.0 \\
\text { 18.0 } \\
24.8 \\
25.0 \\
24.9 \\
14.3\end{array}$ \\
\hline
\end{tabular}

The peptone used was obtained by the action of sulphuric acid on gluten. It contained 3.5 per cent of albumose nitrogen, 49 per cent of nitrogen precipitable by phosphotungstic acid, and 47.5 per cent of amino-nitrogen. Following an addition of peptone to the vegetable ration, it is found that the nitrogen of the excrement is considerably diminished. Of roo units of vegetable nitrogen there remains only I4 not absorbed, while the control tests indicate a value of between 22 and 24.9 units. The secretorial power of this peptone is greater than that of meat. On the contrary, the yeast extract is without effect. As for Liebig's extract, the figure which is found is a confirmation of the observations of Pawlow. These results with Liebig's extract are the more interesting in that this product, introduced into commerce as food, has become of more than a problematical efficacy from the day when it was perceived that animals fed with the extract alone succumbed more quickly than those left without nourishment. In reality, Liebig's extract, and for that 
matter simple broth, without being in any way a nutritive material can render great service as excitants of the gastric secretion.

\section{§ 4. Chemical Influences on Pancreatic Secretin.}

Pancreatic secretion is influenced to a very high degree by chemical agents. Their effects are still more complex and pronounced here than in the case of gastric secretion. This influence relates not only to the quantity of juice elaborated, but also to its concentration and its composition. The content of the juice in trypsin, lipase, and amylase varies considerably, according to whether it has been furnished under the impulse of such an agent. The variations observed in the content of enzymes; in the differences of general manner of secretion, and in the modifications of the concentration of the juice, are generally the consequence of the complex causes, and it is often difficult to relate the sum of the effects so obtained to these initial causes. The difficulty increases still more from the fact that the pancreatic secretion appears at the same time as the gastric secretion, and that the elaboration of this last directly influences the first. Yet, in a certain number of particular cases, which we shall examine, it has been possible to establish the direct influence of chemical substances on the pancreatic secretion.

Influence of Acids. - The most active agent, even in small doses, on pancreatic secretion is the reaction of the medium. Acidulated water introduced into the stomach immediately causes a pancreatic secretion whose intensity is in direct relation to the acid content. Thus it is that a fasting dog, provided with a pancreatic fistula, which has received into the stomach 200 c.c. of water acidulated to different degrees, secretes the following quantities of pancreatic juice: 
Influence of Acidity on the Pancreatic Secretion.

\begin{tabular}{|c|c|c|c|}
\hline \multirow{2}{*}{ Time in hours. } & \multicolumn{3}{|c|}{ Pancreatic juice secreted. } \\
\hline & $\begin{array}{c}0.05 \text { per cent } \\
\mathrm{HCl} .\end{array}$ & $\begin{array}{l}\text { o. I per cent } \\
\mathrm{HCl} \text {. }\end{array}$ & $\begin{array}{l}0.5 \text { per cent } \\
\mathrm{HCl} \text {. }\end{array}$ \\
\hline $\begin{array}{l}\text { I } \\
2 \\
3 \\
4\end{array}$ & $\begin{array}{c}\text { c.c. } \\
\ldots \\
\ldots \\
20.5 \\
\ldots\end{array}$ & $\begin{array}{c}\text { c.c. } \\
\ldots \ldots \\
25.7 \\
26.8 \\
32.5\end{array}$ & $\begin{array}{c}\text { c.c. } \\
70.8 \\
79.5 \\
82.5 \\
89.4\end{array}$ \\
\hline
\end{tabular}

The most favorable quantity of acid is 0.5 per cent. The gastric juice, containing 0.4 to 0.5 per cent $\mathrm{HCl}$, must consequently act on the pancreas in the same manner. Direct experiment has confirmed this hypothesis. When gastric juice is introduced into the stomach of a fasting dog, the pancreatic secretion at once appears and continues for 2 to 3 hours. The acidity of the stomach is, as we see, a factor of jrime importance in gastric and pancreatic digestions. In the first, the acid intervenes directly by favoring the action of the pepsin; in the second, its rôle, although indirect, is equally important in that it regulates the secretion.

Influence of Fatty Materials. - The action of oil on the pancreas presents a still more striking example of an agent acting differently on the pancreatic and gastric glands. The fatty material, as seen above, acts as paralyzer of the gastric secretion, while it shows very great energy in exciting the pancreas. It thus results that this substance, introduced into the stomach, will cause after 3 or 5 minutes a pancreatic secretion which will continue for hours, while the stomach will remain neutral without secreting anything. The secretion, brought about by the influence of the fats, differs perceptibly from that furnished by the acids. In the former, in fact, are found quantities of lipase much larger than in the latter. The two principal activators of the pancreas, the acids, and the fats, cause, moreover, secretions differing not only in their respective content in enzymes, but also in their general activities.

Influence of Bread and of Meat. - The effect of diet on the pancreatic secretion has been studied by Wassilieff. This 
investigator found that if the feeding with bread and milk is changed to a feeding with meat, the pancreatic secretion changes, both with respect to its general activity, and to its content in proteolytic enzyme.

Variation of Pancreatic Secretion with Diet.

\begin{tabular}{|c|c|c|c|c|}
\hline \multirow{2}{*}{ Time in hours. } & \multicolumn{2}{|c|}{ Milk + bread. } & \multicolumn{2}{|c|}{ Meat. } \\
\hline & Secretion per hour. & Digestive power. & Secretion per hour. & Digestive power. \\
\hline $\begin{array}{l}\text { I } \\
2 \\
3 \\
4 \\
5 \\
6\end{array}$ & $\begin{array}{r}\text { c.c. } \\
\text { I5 } \\
45 \\
30 \\
20 \\
9 \\
\text { I I } \\
\end{array}$ & $\begin{array}{l}3.50-0.50 \\
0.50-0.25 \\
1.50-2.75 \\
2.00-2.55 \\
3.00-4.50 \\
4.50-4.50\end{array}$ & $\begin{array}{l}\text { c.c. } \\
72 \\
46 \\
27 \\
25 \\
19 \\
17 \\
\end{array}$ & $\begin{array}{l}4.00-4 \cdot 75 \\
4.25-5 \cdot 50 \\
5.50-5.25 \\
5.25-5.50 \\
6 . \infty 0-6.50 \\
6.00-5.75\end{array}$ \\
\hline Total...... & 130 & $\ldots \ldots \ldots$ & 206 & $\ldots \ldots \ldots$ \\
\hline
\end{tabular}

Thus, while the dog is on the milk + bread diet, he secretes in all $\mathrm{I} 30$ c.c. of juice in 6 hours, and the digestive value in the last three cases was from 2.00 to 4.50 . The same dog, having passed to meat feeding, furnished in the same time a pancreatic secretion of 206 c.c., and furthermore the digestive value has considerably increased, since in the last three hours it has grown from 5.25 at the minimum to 6.50 at the maximum. There is found, moreover, with the change of diet, a corresponding variation in the amylolytic power.

Variation of the Amylolytic Power with the Nature of the Food.

\begin{tabular}{|c|c|c|c|c|c|}
\hline \multirow{2}{*}{ Time in hours. } & \multicolumn{2}{|c|}{ Amylolytic power. } & \multirow{2}{*}{ Time in hours. } & \multicolumn{2}{|c|}{ Amylolytic power. } \\
\hline & Milk + bread. & Meat. & & Milk + bread. & Meat. \\
\hline I & 15 & 6 & 4 & $2 I$ & 6 \\
\hline 2 & I I & 6 & 5 & 16 & 8 \\
\hline 3 & I9 & 7 & 6 & I 8 & 7 \\
\hline
\end{tabular}

A similar influence is found again in the variation of lipase. With a diet of fat the maximum content in this enzyme is reached. The correlation which exists between the composition of pancreatic juice and the diet is often difficult to verify. The indi- 
viduality of the subject experimented with in reality plays a large part. The dog, which has been kept for a long time on a certain diet, ends by secreting a pancreatic juice of a quite stable and independent composition up to a certain degree of change of diet. On the contrary, the dog which is used to a mixed and varied food is much more sensitive. It is, of course, on a dog of this class that the changes of juice according to the different diets must be studied.

Influence of Alkalis. - The preponderating influence of acids on the pancreatic secretion would lead to the conclusion that the alkalis play an unfavorable part in the secretion. In practice, the alkali salts exert this inhibitory action even in minimum doses. The following are data, due to Becker, on this influence. He introduces successively into the stomach of a dog, 250 c.c. of ordinary water and 250 c.c. of a 0.8 per cent solution of sodium bicarbonate and each hour measures the pancreatic secretion produced.

\begin{tabular}{|c|c|c|}
\hline Time. & Ordinary water. & $\mathrm{NaHCO}_{3}$ sol. \\
\cline { 2 - 3 } & c.c. & c.c. \\
\hline hours. & 5.6 & 4.2 \\
$\mathrm{I}$ & 9.9 & 0.6 \\
2 & 6.2 & 1.0 \\
\hline
\end{tabular}

Moreover, Becker has demonstrated that the effect of alkali is very persistent, and that the alkaline solution appears to influence the pancreas a long time after it has disappeared from the stomach. Thus, by giving to a dog a meal composed of milk and bread on the one hand, and on the other, the same meal, preceded 2 hours before by an ingestion of 400 c.c. of alkaline solution, the results obtained are shown in the table on page 398 .

We see thus, according to the progress of the secretions, that the ingestion, 2 hours before the meal, of 400 c.c. of alkaline solution, results in the diminishing the quantity of juice secreted by about 25 per cent with respect to the control experiments made before and after the experiment. 
Influence of Alkalinity on the Pancreatic Secretion.

\begin{tabular}{c|c|c|c}
\hline Time in hours. & $\begin{array}{c}\text { Meal composed of } \\
\text { milk + bread. }\end{array}$ & $\begin{array}{c}\text { Same meal preceded 2 } \\
\text { hours before by 400 c.c. } \\
\text { alkaline solution. }\end{array}$ & $\begin{array}{c}\text { Meal again without } \\
\text { alkaline water. }\end{array}$ \\
\cline { 2 - 3 } I & c.c. & c.c. & c.c. \\
2 & 46.6 & 32.2 & 42.1 \\
3 & 45.4 & 56.3 & 62.1 \\
4 & 53.5 & 21.5 & 46.4 \\
5 & 18.1 & 15.7 & 21.0 \\
6 & 22.4 & 12.0 & 14.5 \\
Totals & 18.7 & 14.4 & 13.9 \\
\hline
\end{tabular}

If we now compare the action of chemical agents on the gastric glands and on the pancreatic gland, we find that alkali acts unfavorably on the two secretions, and that acid, on the contrary, is indifferent toward the gastric juice, but very activating for the pancreatic secretion. Certain peptones [Ex. Liebig's extract] are very favorable, from the gastric point of view. They appear not to have direct action on the pancreatic secretion. The fats, very unfavorable to the gastric secretion, produce on the contrary a stimulating action on the pancreas. Water acts on the two secretions, but in an independent manner. In fact, the quantity which begins to activate the pancreatic secretion, is still inactive toward the gastric secretion. The carbohydrates, albumin, and cooked meat are without effect on the two secretions, but these substances become active due to the products of their hydrolysis.

Similarity Between the Salivary Glands and the Gastric and Pancreatic Glands. - We have just seen that the gastric and pancreatic glands, indifferent to purely mechanical influences, react to psychic and chemical influences. These two factors. appeared, à priori, to be very different in nature. One would be tempted to believe that innervation plays an important rôle on the psychic impulse and that it does not come into play in the chemical impulse. In reality, these two factors have the same origin. The chemical substances cause a specific secretion by their direct action on the mucous membranes, the sensation produced being transmitted by innervation to the glands; an active substance introduced into the organism by a 
route other than the stomach will remain without effect on the secretorial glands. The sight of food, the smell, even the desire to eat, in a word the appetite, excite the secretorial nerves by psychic influence. A similar excitation is caused by certain foods on the gastric mucous membranes, the latter being a chemical effect. These phenomena are the result of innervation, the mechanism being similar in the two cases, but the effect being different, both from the quantitative and the qualitative points of view. As to the chemical effect, the innervation produced by certain substances preferably reacts on the secretorial glands which furnish water and mineral substances. Other substances, on the contrary, cause a smaller secretion of liquid, but reach the enzyme reserve, and thus bring into the juice different enzymes as the situation demands. In the specific secretions, it is the peripheral extremities of the centripetal nerves of the gastric and intestinal mucous membranes which receive the first impulses, and these are then transmitted to the corresponding glands. The differences in the composition of the juices obtained is explained by the fact that the effect of different substances is selective, and that it reacts on one or the other of the nerve filaments to which specific secretions correspond.

Glinsky and Wulfson have shown that the observation made with regard to the gastric glands also relates to the salivary glands. The salivary secretion which is caused by a mechanical action differs from that which is produced by the psychic influence. Furthermore, a specific secretion is found to correspond to the different influences. The salivary glands give a secretion which acts, according to conditions, through its physical, chemical, and physiological properties. Without doubt, the saliva acts on starchy material by means of the ptyalin which it contains. This chemical action, which begins in the mouth and is continued for some time in the stomach, is far from being complete, or even partially so. The rôle of ptyalin, from the point of view of saccharification, is then rather-insignificant. The saliva, however, acts primarily by reason of its alkalinity, and especially through its physical effect. The solid substances introduced 
into the mouth are reduced to pap or dissolved, and the insoluble materials are engulfed in a viscous mass which will easily undergo deglutination. Under the influence of the saliva a sifting takes place among all the substances which enter the mouth, those which are resistant to nutrition being separated and soon instinctively cast out.

To produce these multiple functions, the salivary glands possess a system of innervation which is very complete and even more perfect than that of the gastric glands. According to the needs, the saliva becomes more liquid and more abundant, or more concentrated and more viscous. The saliva, which is poured on meat, will change in nature as soon as the buccal mucous membrane encounters another substance, alimentary or not. Decidedly, therefore, there exists a complete analogy between the innervation which reacts on the salivary glands and that which reacts on the alimentary canal.

It should also be noted that the saliva, according to Frouin, has a peculiar secretorial effect on the gastric glands. This investigator has found, in fact, that the ingestion by dogs of dog saliva or cow saliva greatly increases the quantity as well as the acidity and the digestive power of the gastric juice. The following are the results of two of his experiments.

The first relates to a dog with an isolated small stomach; there is introduced into his large stomach, regularly for 7 days, $500 \mathrm{~g}$. of raw meat, and it is found that the small stomach secretes on an average 58 c.c. of juice in 9 hours. By adding to the $500 \mathrm{~g}$. of meat, 100 c.c. of a mixture of parotid and submaxillary saliva from a dog, the small stomach gave 74 c.c. of juice in the same time. Further, the secretion is more active in the first hour and the flow is more prolonged. If the quantity of saliva is 200 c.c., the secretion in 9 hours is 82 c.c.

The second experiment relates to a dog with a sequestrated stomach. The animal is submitted to a diet composed of meat and of rice $+5 \mathrm{~g}$. $\mathrm{NaCl}$ per day. The secretion. averages 385 c.c. per day. If 100 c.c. of parotid and submaxillary dog saliva are then introduced, the secretion increases to 482 c.c. 
If, on the contrary, Ioo c.c. of cow parotid saliva are used, the secretion increases to 500 c.c. It is well, moreover, to add that this manifest increase of secretion is not due, as Frouin concludes, to the alkalinity of the saliva.

\section{$\S$ 5. Nutrition from the Secretorial Point of View.}

Chemists have a tendency to regard food solely from the point of view of its composition. They think they have already made a great concession by adding some indication as to the degree of digestibility of nitrogenous material, information based on the effect in vitro of proteolytic enzymes on these substances. These data, while of a certain interest, are nevertheless insufficient. They do not give us the exact expression for the actual value of the nutritive substances. In fact, the digestion in vitro, showing that the substances dissolve more or less easily, leads to the conclusion that a substance which requires a large quantity of gastric juice for digestion is not an economical food since the expenditure in active enzymes required must be compensated for by this food itself. In reality, however, it is erroneous to regard the question exclusively from this point of view.

The most digestible food is not always that which digests with the minimum quantity of juice, the important factor being the direct influence which this food exerts on the secretion, or, in other words, its secretorial power. In the discussion of secretion, reference was made to a dog which gave, in the course of an imaginary meal, a very large quantity of gastric juice without injury to its health. The secretorial glands, like every other organ, can be induced to perform a supplementary amount of work. Now the juice secreted during a meal must suffice for the materials submitted to digestion. The question is not, therefore, to determine if the food requires much or little juice, but simply to determine if this food is capable of causing, in a given time, the appearance of the quantity of juice necessary for its digestion. If this quantity, though very large, is secreted, 
the substance ingested is a good food. In other words, the degree of digestibility of a food cannot be measured by the quantity of juice necessary for its digestion in vitro, but rather by the quantity of juice which it is capable of causing to be secreted as the result of its psychic and chemical action.

The data just recorded in the preceding paragraph on the action of the secretorial glands throw light, we believe, upon the comparative values of foods, and at the same time furnish valuable indications as to nutrition in general. . The nutritive substances, according to their effects upon the secretorial glands, can be divided into three classes:

I. Those substances which excite appetite, that is to say those which cause psychic secretion.

2. Those substances which act directly on the glands through a chemical action.

3. Those substances inactive towards the glands, but which act through the products of their transformation.

Appetite is the most important factor in digestion. It furnishes an abundant juice, and one rich in active substances. At the sight and smell of well-prepared dishes, secretion begins. This influence should be prolonged as far as possible, and every subject of great distraction or of annoyance, serious conversations, absorbing reading, calls to the telephone, and the like are causes which diminish or inhibit psychic secretion. Spices and condiments should be mentioned as substances stimulating the appetite. Often an accelerating effect on the enzymes is attributed to these substances, but in reality they do not influence the active substances of the juices, nor even directly the mucous membranes or the secretorial glands. They act exclusively on the taste, and through contrast render more desirable the foods which, in the case of spices, will follow their ingestion, or, in the case of condiments, will be seasoned by them.

The effect obtained with these substances is, for the most part, purely individualistic. In warm countries, where people are nourished with sweet dishes, with starchy foods, and with vegetables cooked in oil, the use of pepper, onions, garlic and 
aromatic herbs is very widely practiced. The foodstuffs being uniform and tasteless, it is sought to provoke appetite by violent sensations. Pawlow explains the Russian custom of beginning the meal with a glass of brandy by the unconscious need which a man in these localities experiences for abandoning the daily cares, and for thus fortifying himself with the good humor so favorable to digestion. In the more refined world, the appetite is influenced by a thousand details, of an esthetic as well as culinary order. Not only are the menus varied and skillfully composed, but likewise the table, prepared carefully, will affect a certain style in its ornamentation. The changes of costume, which also results from the need of forgetting the preoccupations of the day, the flowers, the agreeable company, the hearing of subdued music, all should contribute to coax appetite and quiet satisfaction.

Among the substances acting directly by their contact and which thus bring about a specific secretion, are raw meat, broth, milk and water. In the list of substances which are directly inactive on the glands, but which, through their decomposition products, cause secretion, are cooked meat, egg-albumin, as well as all the nitrogenous vegetables. In the preparation of dishes, and especially in the composition of menus, we find again the constant effort to maintain the psychic secretion as long as possible. Furthermore, everything is done to create the conditions most favorable for chemical secretion.

In the most elementary meal, composed exclusively of bread, we cannot rely at all upon the psychic secretion. Bread, introduced into the stomach, can stay there for a long time without finding the necessary juice for digestion. On the contrary a thin soup, or simply water, brings a great relief. The liquid causes the first secretion, digestion begins, the nitrogenous substance of the bread undergoes hydrolysis, and the products formed then accelerate considerably the secretion through their specific influences. In a more abundant meal, we find in the successive order of the dishes the same effort to produce an abundant and rich juice. The meal begins with 
the hors-d'euvre, which excites the psychic secretion. Then chemical secretion is hastened by a soup, before taking the substantial dish composed of meat and vegetables. The appetite begins to diminish. The fatty materials ingested, while accelerating the pancreatic secretion, have retarded that of the stomach. The juice diminishes in quantity and in quality. At this point we have the dessert, consisting of ices and fruits, all succulent things which should again, by a contrast of taste, produce an excitation of the secretorial glands. Thus we seek by propitious conditions to prolong the appetite, and the most successful dinners, and at the same time the most digestible, are indisputably those where one is led to keep the appetite intact from one end of the meal to the other. As to the nourishment of the nursling, we have seen that the milk causes an abundant specific secretion, which causes it to digest almost automatically. Of all known foods, only milk can be digested without the aid of psychic juice. Here is a marvelous adaptation of the individual to the laws of nature, and this property of milk is moreover recognized by all physicians.

The various statements just made certainly contain nothing new. The desire for eating well and for digesting well has always occupied man since the most remote times. It is quite natural that we should thus have arrived at a partial perfection, even without the help of the physiologist. However, the theory of digestion just expounded can boast of the fact that it gives a scientific explanation to a whole series of well-established observations, and thus brings a real contribution to the problem of nutrition, a problem still far from being completely solved.

\section{BIBLIOGRAPHY ON THE DIGESTIVE GLANDS.}

PaWlow. Leçons sur les glandes sécrétorielles, St-Pétersbourg, 1897 .

Wassiliew. Arch. des. Sc. Biol., 1893, (2), p. 219.

Becker. Arch. des. Sc. Biol., 1893, (2), p. 433.

RozenblatT. Experim. Untersuchungen über die Wirkung von $\mathrm{NaCl}$ u. $\mathrm{Na}_{2} \mathrm{CO}_{3}$ auf Magensaftsecretion, Bioch. Zeits., 1907, (4), p. 500.

FeIgl. Einfluss der Arzneimittel auf die Magensecretion, Bioch. Zeits., 1907, (6), pp. 17, 47; 1908, (8), p. 467 . 
Mayeda. Ueber die Wirkung einiger Alcalien u. Lithiumsalze auf die Magensaftsecretion, Bioch. Zeits., I906, (2), p. 332.

Hoffmann u. Wintgen. Arch.f. Hygiene, (6r), p. 187.

Bayliss a. Starling. The mechanism of pancreatic secretion, Journ. of Physiol., I902, (28), p. 325 .

Rosemann. Beitrag z. Physiologie der Verdauung, Pfligg. Arch., I907, (118), p. 467 .

I. Feigl et A. Rolletr. Experim. Untersuchungen über d. Einfluss v. Arzneimittel auf die Magensaftsecretion, Bioch. Zeits., 1909, (19), p. 156.

Boldyrefr. Die Lipase des Darmsaftes u. ihre Charakteristik, Zeits. f. physiol. Chem., 1907, (50), p. 406.

Sommerfeld. Zur chem. Zusammensetzung des menschl. Magensaftes, Bioch. Zeits., 1908, (9), p. 352.

Kudo. Beziehungen zw. Magensaftmengen u. Pepsingehalt, Bioch. Zeits., 1909, (16), p. 219.

CaHn. Zeits. f. physiol. Chem., (10), p. 522.

WoHLgemuth. Arbeiten aus dem patholog. Institut, Berlin, igo6.

Jean Effront. Ueber Pepton, V. intern. Kongress $f$. angew. Chemie, Berlin, 1903, Band IV, p. 97.

W. BoldyreFF. Arch. d. Sciences biolog., I905, XI, (2).

Hirata. Zur Kenntnis der Fermentconcentration d. Pankreassaftes, Bioch. Zeits., I9I0, (24), p. 445.

Hoffmann u. Wintgen. Einwirk. von Fleisch u. Hefeextract auf die Secretion des Magensaftes beim Hunde, Arch.f. Hygiene, (6I), p. I87.

London. Zum Chemismus der Verdauung i. Thierkörper, Zeits.f. physiol. Chem., $1907,(53)$, p. 247.

Krocuan. Ueber die Wirkung einiger Arzneimittel, Zeits. f. physiol. Chem., (80), p. 23.

Damaskur. Einwirkung d. Fettes auf Secretion d. Pancreassaftes, Diss., i89o.

Connstein, Hoyer u. Wartenberg. Ueber ferment. Fettspaltung, Berichle d. Deuts. Chem. Ges., I902[3], (35), p. 3988.

G. Bruno. Die Galle als Agent d. Verdauung, Maly's Journ., 1897, p. 44 I.

Linossier et Lemoine. Soc. Biol., 1906, (I), p. 663.

Frouin. Soc. Biol., 1904, (I), p. 806; 1905, (I), p. 1025; 1907, (2), p. 473; 1907, (I), p. 80.

PopIElsky. Thèse, St-Pétersbourg, 1896.

Schepowalnikow. La physiologie du suc intestinal. Thèse, St-Pétersbourg, i899. 
CHAPTER II.

\section{TRYPSINS OF VARIOUS ORIGINS.}

\section{$\S$ I. Animal Trypsins and Proteases.}

Animal Trypsins. - In addition to the pancreatic juice of the vertebrates, where trypsin was first of all discovered, this proteolytic enzyme is encountered in the whole series of animals. Krukenberg recognized the presence of a proteolytic enzyme reacting in neutral media in the Sponges, the Echinoderms, the Insects, the Crustaceans, etc. Fredericq and Grofithus note the presence of trypsin in several invertebrates. Sellier finds in the digestive juice of the Cephalopods a protease giving tryptophane with casein. Yet this enzyme reacts especially well in slightly acid medium - O.I per cent to 0.2 per cent $\mathrm{HCl}$. Boussoure recognized in the intestines of various insects of the family of the Coleoptera the presence of a proteolytic enzyme capable of dissolving 5 per cent gelatin, but without appreciable action on coagulated egg-albumin. This enzyme is met in larger quantity in the Dytisci, insects purely carnivorous, than in the Melolonthians, which are clearly insectivorous.

Mesnil, studying the intracellular digestion of the Actinia, noted a special trypsin, actino-protease, in the mesenteric filaments of this organism. Likewise, Mouton, in his researches on the amœba, has described a digestive enzyme, amobo-enzyme, entirely comparable to trypsin. This latter writer isolated from the ground a species of amcba which he was able to obtain in mixed culture, associated with a single bacterial species, B. coli. By centrifugation of the amoba and digestion of the residue in glycerin, Mouton obtained an enzymic liquor, which, treated with alcohol, gives a precipitate which can be redissolved in water and which contains the amœbo-enzyme. This has a 
very evident proteolytic action comparable to that of trypsin, both in its optimum reaction and in the products of its activity. In fact, amobo-enzyme jellies gelatin, although the action stops at the albumoses. The reaction of the medium must lie between neutrality to phenolphthalein and neutrality to litmus. The enzyme is sensitive to the effect of temperature. Starting with $54^{\circ}$, its activity is very slight, and at $60^{\circ}$ it completely disappears. Amœbo-enzyme reacts well with fibrin, becoming adsorbed by it, as the enzymes which attack this substance generally are. The effect here is more advanced than in the case of gelatin, giving tryptic fermentation products - tyrosin and tryptophane. On coagulated albumin, the reaction, although noticeable, always remains weak. Nevertheless, all these reactions are indeed due to the enzyme under consideration, the colon bacillus being a bacterium which secretes no proteolytic enzyme, or at most only a very little.

Animal Proteases. - All the kinds of cells of the medullary series contain a very active protease. Since the work of Achalme in 1899, and that of Müller and Jochmann in 1906, we can affirm the existence of a proteolytic enzyme in the white corpuscles. If, for example, drops of blood coming from a leucomyelitis are placed on Loeffler plates prepared with coagulated beef serum and maintained at $55^{\circ}$, after 24 hours cup-like depressions are found where the blood had been deposited. This reaction is due to an enzyme derived from the polynuclear cells. Fiessinger and P. L. Marie have detected a similar enzyme in pus. This protease, which they have isolated, digests very well a ro per cent solution of albumin in distilled water, advancing the hydrolysis to the formation of amino-acids, leucin and tyrosin. It reacts best in alkaline media, and its action is checked only by large quantities of acetic acid. It is not destroyed by Io per cent formaldehyde solution, but heating to $75^{\circ}$ for 20 minutes is enough to render it inactive.

The same investigators, examining the cephalorachidian liquor of several cases of cerebro-spinal meningitis caused by meningococci, found that the organized bodies of these liquors possess 
a very strong proteolytic power. They have isolated the protease of these polynuclears and have found its properties to be very similar to those of the protease of pus. Further, these investigators have observed the very interesting fact that the anti-meningococcic serum contains, as moreover does the normal serum of man or of the horse, an anti-enzyme capable of strongly inhibiting the action of the leucocyte protease. This inhibition can be produced in vivo, intra-rachidian injections of antimeningococcic serum very quickly bringing about a considerable lowering of proteolytic power of the polynuclears, and at the same time a decrease in their number. On the other hand, it has been demonstrated that the injection of proteolytic enzyme provokes in the organism an acute and febrile reaction, similar to the general reactions of acute meningitis. Fiessinger and Marie ask if in this malady there is not, in addition to the microbic affection, an auto-intoxication, due to the proteases of the polynuclears as a result of cytolysis, and if the efficacy of the anti-meningococcic serum does not come both from its antibacterial and from its anti-enzymic action. This opinion would moreover be supported by the observation that the simple intrarachidian injection of serum which is not specific produces a plain and rapid amelioration of cerebro-spinal meningitis caused by meningococci.

\section{$\S$ 2. Vegetable Proteases.}

The first indications as to the presence of proteolytic enzymes in seeds were furnished by Gorup-Besanez, who found in malt, hemp, flax, and vetch the existence of substances whose activity is easily shown in the presence of 0.2 per cent $\mathrm{HCl}$. Green has studied various vegetable trypsins, especially those obtained from the cotyledons of Lupinus hirsutus and from the endosperm of Ricinus communis. With lupin, the study was on seeds that had germinated for four days. Green then separates the cotyledons, which he treats with glycerin. The extract so obtained is active in the presence of 0.2 per cent $\mathrm{HCl}$, digestion of 
the fibrin giving peptones, - tyrosin, and leucin. The optimum temperature is $40^{\circ}$. With 0.5 per cent $\mathrm{NaOH}$, the proteolytic enzyme is destroyed. The glycerin extract of seeds not germinated is inactive, but if $\mathrm{HCl}$ is added to the glycerin and the whole left some time at $40^{\circ}$, it is found that the extract becomes active. These seeds normally contain, therefore, a zymogen which is decomposed in the course of germination, or simply by the action of the acid.

The data of Besanez, confirmed and completed by Green, have been much disputed. Peptonization in the same seeds, that is to say the transformation of albuminoid material during germination, has been attributed to purely vital activity. The work of Fernbach and Huber, of Weiss, and especially that of Butkewitsch, has definitely established the existence of these vegetable proteases which give tyrosin, leucin, and other products of advanced hydrolysis. Butkewitsch has experimented on a certain number of seedlings of lupin, of vetch, and of Ricinus. Causing Lupinus angustifolius to germinate, he dries the young plants at $40^{\circ}$ and then leaves them for $\mathrm{I} 2$ days to auto-digest in the presence of water with addition of thymol. The control is made from the same seeds, previously submitted to boiling:

Autolysis of Germinated Lupin Seeds.

\begin{tabular}{|c|c|c|}
\hline Determinations. & Control. & $\begin{array}{l}\text { After an auto-digestion } \\
\text { of } 12 \text { days at } 40 \text { deg. }\end{array}$ \\
\hline 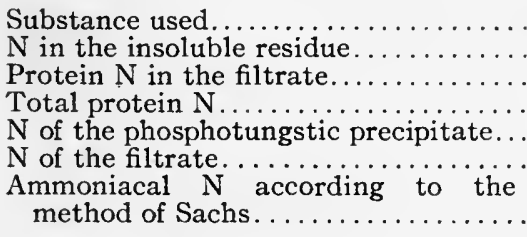 & 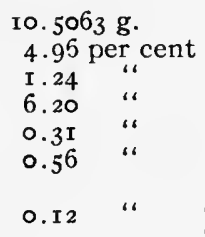 & $\begin{array}{l}\text { I0.9340 g. } \\
3.40 \text { per cent } \\
\text { I.50 “" } \\
4.90 \text { “" } \\
0.45 \text { “" } \\
1.72 \\
0.27 \text { “ }\end{array}$ \\
\hline
\end{tabular}

In the control test, there remains, after 12 days in the oven, 4.96 per cent of insoluble nitrogen. On the contrary, in seeds not boiled is found only 3.40 per cent. The digestive action has the special effect of increasing the proportion of nitrogen 
not precipitable by phosphotungstic acid, which nitrogen ranges from 0.56 per cent to $\mathrm{I} .72$ per cent.

The following table summarizes the influence of the reaction of the medium on the autolysis of germinated lupin:

\begin{tabular}{|c|c|c|c|}
\hline & $\begin{array}{l}\text { Neutral re- } \\
\text { action. }\end{array}$ & $\begin{array}{l}0.2 \text { per cent } \\
\text { HCl. }\end{array}$ & $\begin{array}{l}\text { o. I per cent } \\
\mathrm{Na}_{3} \mathrm{CO}_{3} \text {. }\end{array}$ \\
\hline 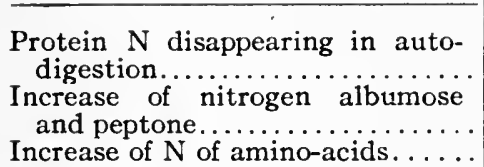 & $\begin{array}{l}\text { Per cent. } \\
\begin{array}{l}\text { I. } 53 \\
\text { O.I } 4 \\
\text { I. } 39\end{array}\end{array}$ & $\begin{array}{l}0.57 \\
0.35 \\
0.22\end{array}$ & $\begin{array}{l}0.81 \\
0.09 \\
0.72\end{array}$ \\
\hline
\end{tabular}

According to these tests, the optimum reaction is reached, not in an acid, but in a neutral medium. Alkali, as high as o.I per cent, is favorable, while the most unfavorable is a 0.2 per cent acid medium. Furthermore, according to the reaction of the medium, the ratio between nitrogen precipitable and nitrogen not precipitable by phosphotungstic acid also changes. While in alkaline media there is produced a large number of amino-acids, in acid media, on the contrary, only very few products of advanced hydrolysis are to be detected.

The ungerminated seeds contain protease in the form of its zymogen, and also the enzyme as such. Grimmer determined the content in proteolytic enzyme of the different seeds, his figures representing the per cent of its own albuminoid substance that the raw seed can digest during 6 hours at $40^{\circ}$ :

Richness in Proteolytic Enzymfs of Different Fodder Seeds.

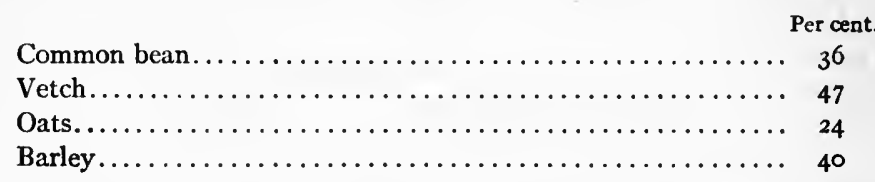

Consequently, when oats are allowed to digest in water at $40^{\circ}$, after 6 hours 24 per cent of the protein nitrogen contained in the seed has been transformed. On the contrary, in vetch the content in enzyme is greater, since 47 per cent of the 
nitrogen in place of 24 has been rendered soluble. The question as to whether the enzymes contained in the fodder seeds play a rôle in the digestion of animals which are nourished by them, has not yet been solved. However, à priori, it appears probable that the enzymes cannot help but facilitate nutrition. According to Vines, in ungerminated seeds, in peas, and in hemp there exists not only one active substance, but two, a peptase and an ereptase, which act successively on the albuminoid material of the seed. Yet, the co-existence of these two enzymes is not yet completely established.

Windisch and Schellhorn have studied in detail the proteolytic enzymes of barley. The ungerminated seeds, according to these investigators, contain enzymes already formed. However the enzymes of the ungerminated grain very easily render this substance soluble. The quantity of enzyme contained in barley depends, up to a certain degree, on its richness in nitrogen, the grains very rich in nitrogen containing the more enzyme. During soaking of barley, which is the preliminary work in germination, the quantity of proteolytic enzyme does not increase at all, the increase taking place only during germination. From this moment the digestive power gradually increases and it reaches the maximum when the seed is completely germinated, that is to say, at the period when the plumule is equal to the length of the stem.

The protease of malt also acts vigorously on vegetable albumin, gelatin, or casein, but it attacks egg-albumin much less easily. The water extract of germinated seed withstands the temperature of $60^{\circ}$, but its proteolytic power is destroyed at $70^{\circ}$. The malting weakens this power without totally destroying it. The peptonizing agent of the malt exerts its effect in a neutral, slightly alkaline, or slightly acid medium. The organic acids lactic or acetic, 0.2 per cent to 0.4 per cent, are very favorable to the activity of this enzyme. The extent of the proteolysis depends on the conditions under which it is produced. At low temperature, the digestion is slow but thorough, while at $45^{\circ}$ or $50^{\circ}$, it is more rapid and more superficial. 
As final products, we always have peptones, amino-acids, and amino-bases.

Another proteolytic enzyme, which is likewise very active, is found in figs, in particular in Ficus carica. Mussi has studied this enzyme, which he calls by the name of gradina. He has observed that it is found especially in the fruit, but also in the leaves and the other parts of the plant. According to this writer, this enzyme is inactive in neutral media, its maximum effect being shown in slightly acid solutions. It reacts on fibrin, as well as on other protein material. In Portugal, figs are used as culture media for yeast. That which has been prepared by the aid of musts coming from figs is very vigorous and keeps perfectly. These musts are even superior to those obtained with malt, from the standpoint of yield and quality of the yeast. These advantageous properties are explained by the existence of proteolytic enzymes which bring the nitrogen of the must to a condition very favorable to the yeast cells.

Green has isolated from Cucumis utilissimus a proteolytic enzyme which reacts especially in the presence of weak alkali, also well in a neutral medium and hardly at all in an acid reaction. It forms albumoses, peptones, leucin, and other amino products. The fruits of cucumis, which have a pineapple odor, are juicy. By squeezing the pulp one gets a slightly active liquid, but the residue is still very rich in protease, which can easily be extracted by means of a 3 per cent solution of $\mathrm{NaCl}$.

Gerber has detected a proteolytic enzyme, capable of coagulating milk and peptonizing albuminoid materials, in the sap of Broussonetia papyrifera L. (Paper Mulberry). The sap contains three kinds of enzymes: one, amylolytic; one, lipolytic and one, proteolytic. The enzyme composition of this juice resembles that of the pancreatic juice. These enzymes come into play in the chemistry of the plant from the moment when the reserves are utilized to form the young leaves. Gerber has found that this vegetable pancreatic juice diminished in activity in autumn and winter, but without having its proteolytic power disappear completely. The relative content in these three active 
substances is not constant, one or the other enzyme predominating, according to conditions. The protease of the sap exerts its optimum action at $85^{\circ}$. This high temperature distinguishes this enzyme from the others of the same class. We have just seen that the sap of Broussonetia coagulates milk at the same time that it digests albuminoid materials. This co-existence of two properties, however much they may differ, is a general characteristic of all the active juices. To be complete and to cite all vegetables where proteolytic enzymes have been detected we must recall all those cases where rennet has been found. These two enzymes are in fact always found associated, so that certain writers consider them as being one and the same enzyme. Thus, Javillier has found in rye-grass, besides rennet, a proteolytic enzyme capable of digesting gelatin, casein and the proteoses, but not fibrin nor coagulated egg-albumin. Harlay, utilizing the reaction of tyrosinase to distinguish peptic from tryptic digestions, a reaction realized in the case of vegetable juices, observes that proteolytic enzymes similar to animal pepsin are found especially in adult phanerogams, while enzymes similar to animal trypsin are found more frequently in certain vegetables of rapid growth, like mushrooms. He further recognizes that the proteolytic enzymes of germinating seeds appear to be analogous to those of mushrooms, possessing, consequently, a tryptic nature.

Enzymes of Insectivorous Plants. - Before taking up the subject of the proteases of mushrooms and of microbes, a few words concerning proteolytic enzymes of insectivorous plants are necessary. Although there has been noted a very large number of plants capable of killing and digesting the insects which allow themselves to be trapped in thẹir special organs, nothing, or at least very little, is known concerning the nature of the active substances brought into play in this special method of vital defense.

Among the best known insectivorous plants are Drosera rotundifolia of our climate and Nepenthes of the tropics. The data, often contradictory, which we have on this subject per- 
mits the following conclusions: Nepenthin, or juice of the urns of Nepenthes, does not act in neutral media. It does not dissolve albuminoid materials, fibrin or albumin, except in a medium giving an acid reaction. The proteolysis is then advanced as far as the formation of leucin, tyrosin, and tryptophane. A similar phenomenon is observed with the juice of Drosera, which can likewise be obtained in vitro, for example by maceration of the leaves in glycerin. In the plant, the juice secreted is normally neutral. In consequence of the irritation caused by the insect taken in the trap, there is a secretion of acid which renders the juice active. This acidification of the juice has, however, according to Labbé, another origin, being the result of a fermentation of the glucose contained in the secretion under the influence of external micro-organisms.

Moreover, this intervention of microbes in the phenomenon of digestion observed in the case of the so-called carnivorous plants must be much more common then we suppose, and this presumption cannot but throw a serious doubt on the conclusions presented in 1765 by Ellis, according to whom there would be about $35^{\circ}$ species of plants endowed with the property of digesting insects in order to nourish themselves.

Proteases of the Molds. - Proteolytic enzymes have been discovered by numerous investigators in the lower mushrooms. There are especially those described by Bourquelot, and again by Malfitano in Aspergillus niger. This mold secretes a protease which is found in the cell at every period in the plant growth, but which is not diffused in the liquid until the moment when the ripe plant begins to die. It can be obtained by macerating in water the dry and finely ground mycelium just before sporulation, and by precipitating the filtered liquid with alcohol. This enzyme is destroyed by heating for several hours at $70^{\circ}$. Its maximum activity is observed at about $40^{\circ}$. The most favorable reaction of the medium is that obtained in solutions of the monobasic phosphates, that is to say, a very slight acidity to litmus and neutrality to methyl orange. However, the protease acts in neutral media, though an alkaline reaction 
inhibits its action. This proteolytic enzyme liquefies and hydrolyzes gelatin, but does not attack coagulated albumin. Fibrin and, better still, albumin of blood serum are dissolved, digestion advancing as far as the peptones. Casein is similarly attacked. Malfitano has compared the action of protease to that of other proteolytic enzymes, pepsin, pancreatin and papain. He finds that their sensitiveness to the acid reaction shows the following differences: Pepsin acts in media slightly acid to methyl orange; protease exerts its maximum activity in media neutral to methyl orange, but still acid to litmus; papain acts in media intermediate between neutrality to methyl orange and neutrality to litmus; pancreatin acts in media intermediate between neutrality to litmus and neutrality to phenolphthalein. To summarize, the protease of aspergillus possesses the characteristics of a vegetable proteolytic enzyme, resembling closely papain and the trypsin of malt.

The appearance of proteases in molds is often dependent upon the conditions of the medium. According to Went, Monilia sitophila forms protease only in the presence of peptone, and not in a medium deprived of albuminoid material. According to Duclaux, Aspergillus glaucus furnishes protease in a culture containing calcium lactate and inorganic salts, while in a medium containing sugar without lactate no protease appears. On the contrary, in Aspergillus niger, the method of nutrition does not appear to have any influence on the secretion of proteolytic enzymes. .

\section{§ 3. Proteolytic Enzyme of Yeasts.}

Autophagy of Yeast. - Béchamp, Schutzenberger, Salkowski, and many others have found that by auto-digestion of yeast the nitrogenous material of the protoplasm undergoes an advanced hydrolysis with the formation of tyrosin, leucin, xanthin: and other amino-acids.

Effront has demonstrated that the chemical reactions produced during autophagy of yeast are due to the enzymes which 
are formed in the cells before their de-nutrition, and that the progress of the phenomenon depends on the chemical conditions of the medium. The digestion of the nitrogenous material takes place more rapidly in a 10 per cent or 15 per cent alcoholic solution than in pure water. Yeast, in alcoholic solution, loses in ro days about 90 per cent of its nitrogen, and after a very prolonged digestion, the cells retain only 7 per cent of the original nitrogen. The proteolytic enzyme of yeast is not diffused, or only with much difficulty, outside the living and normal cell. It is, therefore, an intra-cellular enzyme. However, the appearance of the enzyme in the external liquid is easily observed in the case of strong starvation, and especially when the cells are dead. Certain species of yeast, according to Lindner, cultivated on the surface in a nutritive gelatin medium, succeed in the long run in liquefying gelatin. Boullanger, while confirming these results, finds that such a liquefaction requires from 2 to 6 months according to the species, and that the yeast capable of dissolving gelatin also digests casein, the digestion, however, being extremely slow. Milk, inoculated with yeast and analyzed several months afterwards, contains still 28.4 to $3 \mathrm{I}$ g. per liter of casein, as compared with the $34 \mathrm{~g}$. initially present.

The action of yeașt is especially manifested by a dissolving of the casein as well as by a production of ammonia. The

Progress of the Transformation of Casein by Yeast.

\begin{tabular}{|c|c|c|c|c|c|c|c|c|c|}
\hline \multirow[b]{2}{*}{ Products measured. } & \multirow{2}{*}{ 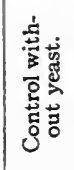 } & \multicolumn{8}{|c|}{ Various species of yeast. } \\
\hline & & 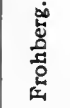 & 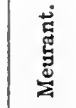 & 范 & 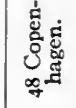 & 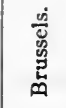 & 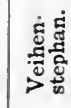 & 造 & 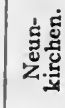 \\
\hline Lactose in $g$. per 1 . & 50.2 & 47.2 & 48.1 & 48.8 & 49.1 & 49.2 & 50.2 & & 49.3 \\
\hline Casein in sol. per 1. . & 5.2 & 16.2 & 15.8 & I2.I & II . 9 & II. 5 & II.I & 8.8 & 8.2 \\
\hline Casein in susp. per 1. & 29.4 & I 4.8 & I 2.6 & 18.3 & 16.9 & I9.I & 17.8 & 20.4 & 22.4 \\
\hline Total casein per $1 \ldots \ldots$ & 34.6 & 31.0 & 28.4 & 30.4 & 28.8 & 30.6 & 28.9 & 29.2 & 30.6 \\
\hline Min. mat. in sol. per 1. & 5.0 & 4.1 & 4.2 & 4.7 & 4.6 & $4 \cdot 3$ & 4.6 & 4.8 & 4.9 \\
\hline Min. mat. in sus. per $1 .$. & 2.3 & $3 \cdot 3$ & 3.4 & 2.8 & 3.1 & 3.2 & 2.8 & 2.4 & 2.6 \\
\hline Ammonia in cg. per & 7.6 & 24.8 & 52.0 & 33.8 & 46.4 & $29 \cdot 3$ & 47.5 & 38.8 & 29.7 \\
\hline
\end{tabular}


casein in solution in the control is 5.2 ; the quantity is increased to 15.8 with Meurant yeast, while the ammoniacal nitrogen is raised from 7.6 to $52 \mathrm{cg}$. per liter. In this test, it is very difficult to distinguish the action of dead yeast cells from that of the cells which have resisted the long stay in the milk. Dead yeast, or yeast in an advanced state of de-nutrition, abundantly secretes proteolytic enzymes, which in the normal state are retained in the protoplasm.

Zymogen. - The firm of Bal \& Co., of Antwerp, has manufactured for fifteen years a food for yeast, sold under the name of zymogen, which consists of peptonized casein. This commercial product, prepared according to the directions of Effront, results from the action of brewery yeast on freshly precipitated casein. 2 to $3 \mathrm{~kg}$. of moist casein are used per kilogram of liquid brewery yeast. The digestion takes place at $40^{\circ}$ in the presence of xylol. After 8 days, the casein is entirely transformed and the yeast completely consumed. Filtering to separate the cellulose residue, and concentrating up to $40^{\circ}$ Bé., a product is obtained containing 7 per cent to 9 per cent nitrogen.

Analysis of Zymogen rn 4.5 Per Cent Solution.

\begin{tabular}{|c|c|c|}
\hline & Per & $\infty$ of total nitro \\
\hline Ammoniacal nitrogen. ...... & 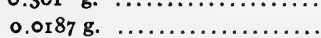 & $\cdots \cdots$ \\
\hline Nitrogen precipitable by $\mathrm{ZnSO}_{4} \ldots \ldots \ldots$. & $0.0140 \mathrm{~g}$. whence albumose: $\mathrm{N}$ & $0 . \mathrm{or}_{4} \mathrm{o}$ or 4.6 \\
\hline Nitrogen precipitable by pbosphotungstic & & \\
\hline acid (albumoses + peptones) . ........ & 0.1201 g. whence peptones: $\mathrm{N}$ & $0.106 \mathrm{r}$ or 35.4 \\
\hline Nitrogen precipitable by tannin........ & $0.0152 \mathrm{~g} \ldots \ldots \ldots \ldots \ldots \ldots \ldots$ & $\ldots \ldots$ or 5.0 \\
\hline Nitrogen not precipitable............. & & 0. 1809 or 60.3 \\
\hline Sörensen nitrogen (after removal of $\mathrm{NH}_{3}$ ). & o.r324 g., a & o. 1324 or 44.0 \\
\hline
\end{tabular}

From the distribution of the nitrogen, we must conclude that the protease of yeast gives a very thorough hydrolysis, resembling that of tryptic activity, although the reaction of the medium should be distinctly acid.

Hefanol. - N. Iwanoff has studied the proteolytic enzymes of hefanol, which is a commercial product, consisting of dead yeast with its enzymes preserved. It contains about 9.10 per cent total nitrogen and 6.3 per cent albumin nitrogen. Macerated for a half-hour with ro volumes of water, it gives a liquid 
which, after filtration, titrates $8 \mathrm{I} .4 \mathrm{mg}$. of albuminoid nitrogen per $100 \mathrm{~g}$. of liquid. This solution is very active with respect to proteolytic enzymes. If toluol is added and the solution left at $33^{\circ}$, the albuminoid nitrogen gradually disappears, and after 69 hours, 55 per cent of the albumin has been transformed. In the products of hydrolysis are found albumoses, peptones and amino-acids. By leaving hefanol in contact with water only a half-hour or an hour and then filtering, only a part of the proteolytic enzymes of this yeast are removed. A much more rapid and thorough autolysis is obtained if the product is left in water, instead of using the filtered maceration. After a digestion of 60 hours, ' 70 per cent of the albumin contained in the hefanol is found to be peptonized.

Optimum Temperature for Hefanol.

\begin{tabular}{|c|c|}
\hline Temperature. & $\begin{array}{l}\text { Per cent nitrogen } \\
\text { transformed. }\end{array}$ \\
\hline $\begin{array}{l}18^{\circ} \text { to } 19^{\circ} \\
32^{\circ} \text { to } 35^{\circ} \\
55^{\circ} \text { to } 56^{\circ} .\end{array}$ & $\begin{array}{l}24 \\
67 \cdot 3 \\
65\end{array}$ \\
\hline
\end{tabular}

This table shows that the strongest autolysis takes place in the neighborhood of $35^{\circ}$, which is thus the temperature most favorable to the reaction.

As to the influence of the phosphates on the protease of yeast, the same effect is observed here as was observed by Fernbach in the case of pancreatin. Dibasic phosphate is injurious, an equimolal mixture of monobasic phosphate $\mathrm{KH}_{2} \mathrm{PO}_{4}\left(0.76\right.$ per cent) and of dibasic phosphate $\mathrm{K}_{2} \mathrm{HPO}_{4}(0.979$ per cent) is without effect, while monopotassium phosphate is distinctly favorable. The following table, expressing these results, indicates that the favorable action of the acid phosphate is exerted just as well at $18^{\circ}$ as at $50^{\circ}$. Furthermore, its efficacy increases with the quantity used: 
Action of Phosphates on the Progress of the Proteolysis of Hefanol.

\begin{tabular}{|c|c|c|}
\hline \multirow{2}{*}{ Liquids used. } & \multicolumn{2}{|c|}{ Per cent of albumin transformed. } \\
\hline & At $10^{\circ}-18^{\circ} \mathrm{C}$. & At $47^{\circ}-50^{\circ} \mathrm{C}$. \\
\hline $\begin{array}{r}\text { Hefanol digested with water alone...... } \\
\text { Hefanol + water cont. }\left(\mathrm{K}_{2} \mathrm{HPO}_{4}\right): 0.98 \% \\
\left(\mathrm{KH}_{2} \mathrm{PO}_{4}\right): 0.76 \% \\
\text { Hefanol + water + }\left(\mathrm{K}_{2} \mathrm{HPO}_{4}\right): 0.98 \% \ldots \\
\left(\mathrm{KH}_{2} \mathrm{PO}_{4}\right): 0.38 \% \ldots \\
\text { Hefanol + water + }\left(\mathrm{KH}_{2} \mathrm{PO}_{4}\right): \mathbf{r} .52 \% \ldots\end{array}$ & $\begin{array}{l}12.7 \\
12.94 \\
7.9 \\
20.74 \\
27\end{array}$ & $\begin{array}{l}6 \mathbf{r} \cdot 96 \\
64.8 \\
26.8 \mathrm{r} \\
68 \\
76\end{array}$ \\
\hline
\end{tabular}

The favorable action of acid phosphate can be explained by the neutralization effect which it exerts on the bases or the amino-acids formed in the course of digestion. Although tyrosin or leucin have no injurious influence on the proteolysis of yeast, there exist however paralyzing bodies among the products of the autolysis which are sensitive to the action of monopotassium phosphate.

Influence of Extract of Yeast and of $\mathrm{KH}_{2} \mathrm{PO}_{4}$ ON the Protease of HEFANOL.

\begin{tabular}{|c|c|}
\hline Liquids used. & Per cent albumin digested. \\
\hline $\begin{array}{l}\text { Autodigestion of hefanol in water } \ldots \ldots \ldots \ldots \\
\text { Same in solution }\left(\mathrm{KH}_{2} \mathrm{PO}_{4}\right) \text { at } \mathrm{r} .52 \% \\
\text { Same with the product of autolysis of the } \\
\left.\text { hefanol. } \mathrm{KH}_{2} \mathrm{PO}_{4}\right) \ldots \ldots \ldots \ldots \ldots \ldots \ldots \ldots \ldots \ldots \ldots \ldots\end{array}$ & $\begin{array}{l}\left.\begin{array}{l}61.6 \\
71.7\end{array}\right\} \text { Difference }=10.1 \\
\left.\begin{array}{l}48.8 \\
76.6\end{array}\right\} \text { Difference }=27.8\end{array}$ \\
\hline
\end{tabular}

Thus, as could have been foreseen, in the products of autodigestion of hefanol are found substances which retard the action of the proteolytic enzyme, but it is of interest that the acid phosphate is extremely active with regard to them. The injurious influence of the extract of autolysis is likewise observed with the product brought to $100^{\circ}$, and in this case the acid phosphate still exerts a very favorable effect.

Acid phosphate possesses, furthermore, the very curious property of regenerating the protease which has been changed by 
the action of heat. This is evident from the following experiments: To $1.5 \mathrm{~g}$. of hefanol are added I50 c.c. of water and the solution is brought to different temperatures. The various samples are then left at $50^{\circ}$ for 27 hours. It is observed that a heating of $\mathrm{I}$ min. at $100^{\circ}$, or even at $75^{\circ}$, is enough to destroy the proteolytic enzyme. However, if the heating is done in the presence of phosphate, or even if this salt is added after the heating, a distinct digestion is still observed.

Regeneration of Protease by $\mathrm{KH}_{2} \mathrm{PO}_{4}$.

\begin{tabular}{|c|c|c|}
\hline & Liquids used. & $\begin{array}{l}\text { Albumin dig. at } \\
50 \text { deg. after } 27 \mathrm{~h} \text {. }\end{array}$ \\
\hline I. & 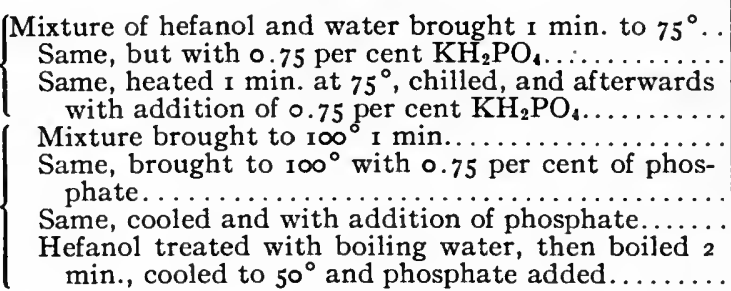 & $\begin{array}{r}0 \\
21 \cdot 3 \\
21 \cdot 3 \\
0.0 \\
3 \cdot 1 \\
5 \cdot 1 \\
0.0\end{array}$ \\
\hline
\end{tabular}

The regeneration of the protease does not therefore come from the fact that the presence of phosphate has raised the destructive temperature, since the subsequent addition of this salt exerts the same action as the addition during the heating. We may then conclude from these tests that an enzyme which, while having lost its proteolytic power. still retains its own structure characteristic of the active substance, can be regenerated by phosphate, while if this intimate structure has been destroyed by an energetic treatment, as in the last test (No. 7), the phosphate no longer acts. In this last test (No. 7), it is seen, furthermore, that it is not at all a question of the action of the phosphate on the albuminoids but rather a direct action of this salt on the proteolytic enzyme. 


\section{§ 4. Endotryptase of Yeast.}

In the juice of yeast, obtained by strong pressure according to the process of Büchner, M. Hahn has found a proteolytic enzyme which he designates by the name of endotryptase, reserving the general term endo-enzymes for the active substances which do not traverse the cellular membranes, and which consequently exert an intracellular action. The yeast juice obtained by pressure possesses a density of 1.027 to 1.057 . It contains 8.5 per cent to I4 per cent of solid matter and from 0.82 per cent to 1.45 per cent of total nitrogen. Of 100 parts of total nitrogen contained in the juice, about 60 are in the state of coagulable albumin. The rest is found in the form of derivatives corresponding to a very advanced proteolysis. Of the roo parts of non-albumin nitrogen, 30 parts were determined to be precipitable by phosphotungstic acid and 70 non-precipitable.

The proteolytic enzyme of this juice can be isolated by precipitation with alcohol. To I part of juice 8 parts of absolute alcohol are added. The coagulum is washed with absolute alcohol and is then treated again with water, which dissolves only a part of the precipitate. The filtrate is again treated with 8 volumes of alcohol. The precipitate, after washing with alcohol, is dried in a desiccator. The product so obtained is far from being pure and contains, besides proteolytic enzyme, quite large quantities of invertin which are difficult to remove. The product prepared by repeated precipitations with a mixture of alcohol and ether is generally more active. Thus is obtained a substance coagulable on boiling, with water, precipitable by neutral lead acetate and by mercuric nitrate, but not giving either the Millon or biuret reaction.

Influence of the Reaction of the Medium, of Alcohol and of Glycerin. - Endotryptase produces the maximum effect in an acid medium. In the neutral juice, submitted to autolysis, the albumin is digested much less easily than in the natural juice, which is always acid. The data relative to the influence 
of the reaction of the medium on the activity of endotryptase is summarized in the following table:

INFLUeNCE OF THE Reaction of the Medium on Endotryptase.

\begin{tabular}{|c|c|c|}
\hline & Liquids used. & $\begin{array}{l}\text { Per cent albumin } \\
\text { digested after I2 } \\
\text { hours. }\end{array}$ \\
\hline \multirow{3}{*}{$A$} & Natural juice with acid (control). & 71.9 \\
\hline & Juice neutralized with $\mathrm{NaOH} \ldots \ldots \ldots ̈$ & 57.0 \\
\hline & “ $\quad+0.2$ per cent $\mathrm{NaUH} . .$. & $\begin{array}{l}40.8 \\
27.7\end{array}$ \\
\hline & Natural juice with acid (control)......... & 61.9 \\
\hline$B$ & $"$ " $" 0.05$ per cent HĆl. & 80.8 \\
\hline & " +0.1 per cent $\mathrm{HCl}$. & 86.2 \\
\hline & " " $\quad+0.2$ per cent $\mathrm{HCl}$. & 88.8 \\
\hline & Neutralized juice (control)........... & 68.4 \\
\hline C & $\because \quad " \quad+0.2$ per cent $\mathrm{HCl} \ldots . .$. & $\begin{array}{l}92.2 \\
78.8\end{array}$ \\
\hline
\end{tabular}

Thus, the most favorable quantity of acid is 0.2 per cent $\mathrm{HCl}$. Sulphuric acid, in equimolal quantities, gives the same effect. Acetic acid, in equimolal quantities, is still more favorable than $\mathrm{HCl}$, but phosphoric acid is less favorable. than $\mathrm{HCl}$. The neutral reaction, and especially the alkaline reaction, exerts a retarding effect. The influence which salts produce on the activity of endotryptase is as follows:

INFLUENCE OF SALTS ON ENDOTRYPTASE.

\begin{tabular}{|c|c|}
\hline Liquids used. & $\begin{array}{l}\text { Albumin coagu- } \\
\text { lable, after } 35 \text { hours, } \\
\text { at } 37 \text { deg. }\end{array}$ \\
\hline 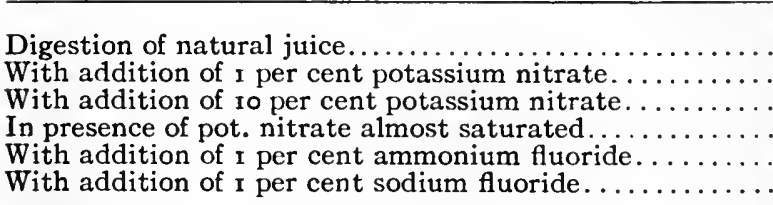 & $\begin{array}{l}\text { Per cent } \\
4.6 \\
2.2 \\
0.2 \\
2.5 \\
2.5 \\
21.8\end{array}$ \\
\hline
\end{tabular}

These salts, with the exception of sodium fluoride, therefore exert a favorable influence. Common salt in concentration of from 0.7 to $3 \%$ acts favorably, as do many other salts. Endotryptase appears to be less sensitive to antiseptics than are pepsin and trypsin. A 0.2 per cent solution of salicylic 
acid, 0.I per cent solutions of formaldehyde or chloroform, or 0.2 per cent -0.4 per cent of thymol or toluol, exert no unfavorable influence on the juice of pressed yeast. However, formaldehyde, in amounts as high as 0.5 per cent, becomes injurious. In the class of paralyzers we must place alcohol in the front rank.

Action of Alcohol on Endotryptase.

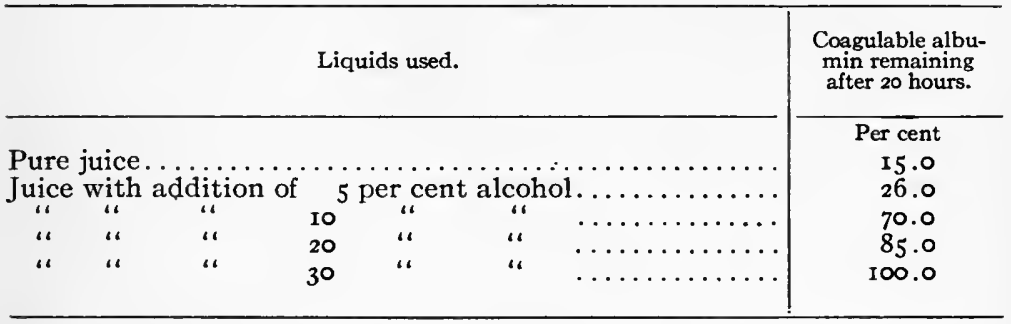

Thus, in the presence of 30 per cent alcohol, digestion is completely arrested, but even in the presence of 5 per cent the paralyzing influence is distinctly manifest. There is a difference from observations previously made with the protease of yeast in that glycerin and cane sugar, in large quantities, exert a very disastrous influence on the proteolysis.

\begin{tabular}{|c|c|}
\hline Liquids used. & $\begin{array}{l}\text { Per cent coagulable } \\
\text { albumin remain- } \\
\text { ing. }\end{array}$ \\
\hline 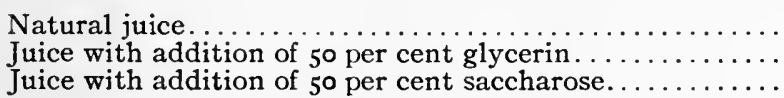 & $\begin{array}{r}9 \cdot 1 \\
63 \cdot 6 \\
30 \cdot 7\end{array}$ \\
\hline
\end{tabular}

The presence of air or of oxygen is without marked effect. Hydrocyanic acid, in small concentrations, has no action though at a concentration of I per cent it paralyzes but does not completely arrest the enzyme. During the autolysis of yeast juice, part of the organic phosphorus of the juice, at times as high as $5 / 6$, is found transformed into phosphoric acid. Even from the beginning of the digestion the formation of this acid is detected. Organic sulphur of the juice also undergoes a transformation, but the increase in $\mathrm{H}_{2} \mathrm{SO}_{4}$ is not very marked. Finally, 
under the action of endotryptase, very small quantities of ammonia are produced.

Characteristic Reactions of Endotryptase. - Endotryptase is related to pepsin, if considered from the point of view of the reaction of the medium, since the two enzymes give the maximum effect in a distinctly acid medium, and since for both, neutral and alkaline media are injurious. But, from the point of view of the extent of the hydrolysis, endotryptase must indisputably take its place in the group of trypsins; it must even be considered as an extreme member of the group, in that the digestion which it produces is more advanced than that produced by ordinary trypsin or pancreatin. The chemical reactions of endotryptase are characterized by the fact that, in the course of proteolysis, only a very little albumose is produced, and no peptones. The products formed are composed in great part of amino-acids not precipitable by phosphotungstic acid and of bases precipitable by this reagent.

Distribution of the Bases and the Acids in the Course of Digestion By Yeast Juice.

\begin{tabular}{|c|c|c|c|}
\hline Length of digestion. & $\begin{array}{l}\text { Per cent nitrogen } \\
\text { not coagulable. }\end{array}$ & $\begin{array}{l}\text { Per cent bases, } \\
\text { formed nitrogen } \\
\text { precipitable by } \\
\text { phos. tung. }\end{array}$ & $\begin{array}{l}\text { Per cent acids, } \\
\text { formed nitrogen } \\
\text { not precipitable by } \\
\text { phos. tung. }\end{array}$ \\
\hline 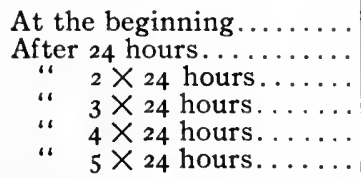 & $\begin{array}{r}33 \cdot 3 \\
85.8 \\
99 \cdot 2 \\
100 \\
100 \\
100\end{array}$ & $\begin{array}{l}32.4 \\
40.0 \\
34 \cdot 3 \\
34.6 \\
33.2 \\
26.2\end{array}$ & $\begin{array}{l}67.6 \\
60.0 \\
63 \cdot 7 \\
65 \cdot 4 \\
66.8 \\
73.8\end{array}$ \\
\hline
\end{tabular}

At the beginning, only $1 / 3$ of the total nitrogen of the liquid is found in the form of non-coagulable nitrogen; in the liquid free from the coagulable portion, it is found that of the nitrogen in solution, 32.4 per cent is precipitable by phosphotungstic acid and is composed almost exclusively of bases like histidin, arginin and lysin, no peptones at all being found and the quantity of albumose being very small. After four days of autodigestion when the coagulable nitrogen has completely disappeared, 
there is found again the same relation between the basic and the acidic nitrogen. On the contrary, in the peptic reaction as in the tryptic, quite a different progress is observed, notably a gradual disappearance of the portion precipitable by phosphotungstic acid. The constant absence of the biuret reaction with the products of digestion and the fact that peptone added to the juice rapidly disappears leads to the conclusion that in the yeast juice we are not dealing with a single proteolytic enzyme, but rather with different enzymes which together cause the hydrolysis.

The proteases of the yeast, whether those studied in connection with zymogen or those contained in hefanol, are evidently of the same nature as endotryptase. In all these cases we obtain the same reactions, characterized by a definite proportion of bases and of amino-acids. On the contrary, in the experiment of Boullanger cited above, other active substances are apparently concerned than those which constitute endotryptase, the products of digestion not being at all the same. The hydrolysis is not very thorough, and the extensive formation of ammonia does not correspond to an endotryptase reaction.

In conclusion, this study of the proteolytic enzymes of yeast is far from being complete. The subject has not been exhausted, and in the chapter on amidase we shall have occasion to return to it.

Combined Actions of Yeast Enzymes. - It has been previously stated that Iwanoff had observed that the liquor from the autolysis of hefanol, that is to say of dead yeast, possesses an anti-proteolytic action. Büchner and Hahn have found these inhibitory substances even in the juice of yeast. According to these investigators, these anti-enzymic products are not specific. They act on pepsin, trypsin, and on other proteolytic enzymes, which accordingly digest albumin or gelatin but poorly. To prove the anti-proteolytic action of the yeast juice on the digestion of gelatin by the juice itself, the procedure is as follows: Prepare a 9 per cent solution of gelatin, on the one hand in distilled water, on the other hand in boiled yeast juice. Put 
3 c.c. of these solutions in reaction tubes, and, after cooling and curdling, add to each of the tubes a mixture of 0.75 c.c. of juice and I c.c. of water. Put in several drops of toluol and leave the whole at $22^{\circ}$ :

Anti-Proteolytic Action of Yeast Juice.

\begin{tabular}{|c|c|c|c|}
\hline \multirow{2}{*}{ Liquids used. } & \multicolumn{3}{|c|}{ Liquefaction of gelatin. } \\
\hline & After $24 \mathrm{hrs}$. & After $48 \mathrm{hrs}$. & After $72 \mathrm{hrs}$. \\
\hline $\begin{array}{l}\text { Solution of gelatin in water...... } \\
\text { Solution of gelatin in infusion of } \\
\text { yeast............................. }\end{array}$ & $\left\{\begin{array}{l}\text { c.c. } \\
0.3 \\
0.3 \\
0 \\
0\end{array}\right.$ & $\begin{array}{l}\text { c.c. } \\
0.9 \\
0.9 \\
0.1 \\
0.1\end{array}$ & $\begin{array}{l}\text { c.c. } \\
1 \\
1 \\
0.1 \\
0.1\end{array}$ \\
\hline
\end{tabular}

As to the nature of the anti-tryptase of the juice, we possess interesting data. This substance, in solution, is very resistant to the action of heat; boiling, even though prolonged, does not destroy it. A previous alkalization or acidification is also without effect. In the ash of the yeast juice, none of the antiproteolytic substance is found. Furthermore, this is destroyed by the action of lipase. According to Büchner, the anti-body of yeast, which is called anti-tryptase or anti-protease, is an organic material belonging to the class of phospho-proteins.

This special substance intervenes in the yeast cell as a regulator of the activity of the enzymes in the same way as the reaction of the medium, which favors one or the other enzyme of the mixture. We owe to Büchner and to his collaborators our ideas as to the combined action and the reciprocal rôle of the active substances of the yeast cell. In the yeast juice are found active substances of very varied nature. In the first place, we find the substance capable of transforming sugar into alcohol and $\mathrm{CO}_{2}$; it is the zymase of Büchner, which is not, nevertheless, a well-determined individual, but is probably a complex mixture of enzymes affecting different fermentable sugars. Then we recognize the proteolytic enzymes; then invertin, maltase, a lipase, reducing enzymes, oxidases, catalases, and finally amidases. In addition to all these enzymes, more or less well 
characterized, we also find substances of very peculiar nature, the anti-protease and the co-enzyme.

Harden and Young have demonstrated that yeast juice active on sugar is found, after passage through a Martin filter, to be inactive toward gelatin. The filtered part and the part remaining in the filter separately show an inability to ferment sugar, but, when mixed the activity is restored. The same phenomenon has been observed by allowing yeast juice to dialyze through parchment paper. In the dialyzed liquid there is a substance which renders active the non-dialyzable zymase. This substance, indispensable to the activity of zymase, has received the name of co-enzyme. Alcoholic fermentation therefore results from the coöperation of two indispensable factors, the enzyme properly speaking, which is zymase, and the activating substance, which is the co-enzyme. This last withstands a temperature of $100^{\circ}$ and affords no characteristic reactions of enzymes. The co-enzyme is found again in boiled yeast juice, and can be prepared by exposing this juice to a temperature of $100^{\circ}$. It may also be prepared by allowing yeast to macerate in water. It is easy to demonstrate the existence of a co-enzyme, for a dialyzed juice, that is to say, the zymase remaining in the dialyzer may be activated by adding boiled yeast juice, i.e., juice deprived of enzyme.

The study of the co-enzyme has established the fact that this substance is in reality very unstable. Its activity diminishes perceptibly in the presence of 2.5 per cent $\mathrm{K}_{2} \mathrm{CO}_{3}$ at $35^{\circ}$, and is completely destroyed if maintained at $130^{\circ}$ for 4 hours. The co-enzyme is indifferent to the action of the proteolytic enzymes, but is destroyed, on the contrary, under the influence of lipase, due to the setting free of phosphoric acid. As noted, this substance resembles in certain ways, particularly in its chemical nature, the anti-protease referred to above, but differs from the latter in its sensitiveness to alkalis and its lower resistance to heat. Anti-protease, in fact, not only is not capable of activating zymase, but it is not even destroyed by $\mathrm{K}_{2} \mathrm{CO}_{3}$, nor by heating 4 hours at $130^{\circ}$. 
The combination co-enzyme + zymase, which causes alcoholic fermentation. is influenced by other active substances which also come from the yeast. Notably it is sensitive to the action of endotryptase, lipase, and anti-protease. Endotryptase digests and destroys zymase. The juice, allowed to stand, gradually deteriorates as a result of a digestive action of the tryptic enzyme on the zymase. All the conditions which favor the activity of endotryptase (acidity of the medium, temperature of $35^{\circ}-37^{\circ}$, etc.) are consequently detrimental to the preservation of zymase. On the contrary, conditions unfavorable to endotryptase become excellent for zymase. Thus it is that at a temperature from $5^{\circ}$ to $7^{\circ}$, yeast juice may be preserved for fifteen days, while at a temperature of $35^{\circ}$, after 5 or 6 days there is observed a noticeable diminution in its activity. Lipase, without having a direct effect on zymase, also influences the combination co-enzyme + zymase, since it reacts on the coenzyme as it did on anti-protease.

In the inactivation of the juice, from the point of view of alcoholic fermentation resulting from a more or less prolonged autolysis, two kinds of phenomena are observed. In the first case, the inactivation is produced because the co-enzyme has deteriorated. Under these conditions, the addition of boiled juice renews activity by restoring the required complement, the co-enzyme. Moreover, this addition of boiled juice will contribute greatly to the preservation of the fresh juice, for the co-enzyme and the anti-protease both protect the zymase from the destructive action of the endotryptase. This protection, however, will be of short duration. Lipase, present in the juice, will soon destroy the co-enzyme as well as the antibody, and a fresh addition of boiled juice will be necessary to cause a fresh activity in the deteriorated juice, a portion of zymase having been able to escape the action of the endotryptase.

The case is quite otherwise when yeast juice is left for a long time to itself. Here, not only does the co-enzyme deteriorate, but even the zymase itself is digested or destroyed by the endotryptase. Under these conditions, the addition of co- 
enzyme or of boiled juice will lead to no new activity of the juice. Thus, by considering exclusively the zymase, we see how the enzymes, endotryptase, lipase, and anti-protease, intervene directly in the preservation of the fermentive power of yeast juice. The reciprocal influence of the enzymes, the modifications of the composition of the medium produced by each of these active substances, throw a special light on the rôle of enzymes, and on their combined action, as well as on the resulting chemical complications.

\section{§ 5. Bacterial Proteases.}

There exists a whole class of bacteria which transform protein material. In bacterial culture-media are found not only the products of tryptic digestion, but also a large number of other products derived from albuminoids which are not met in peptic or tryptic hydrolyses, such as phenol, volatile fatty acids, indol, scatol, and others. The origin of these products was first attributed to the vital activity of the cells, but as our knowledge of enzymes broadened, we found that the work of disintegration of nitrogenous materials was accomplished by the aid, either of ecto-enzymes, capable of traversing the cellular membrane, or of endo-enzymes, exerting their action in the interior of the cells.

General Properties. - The bacteria produce various proteolytic enzymes. In the present chapter only the proteases will be considered, the consideration of the other proteolytic enzymes which also take part, and even still more actively in reducing complex albuminoid materials to relatively simple products, being postponed to a later chapter. The putrefactive ferment, B. mesentericus vulgaris, the vibrio of cholera, as well as a large number of other bacteria, secrete proteases. Geret and Hahn have shown the presence of these proteolytic enzymes in the bacillus of tuberculosis, in the bacillus of typhoid fever, and in Sarcina rosa. To obtain these enzymes the above experimenters applied the method of Büchner as utilized for the 
extraction of yeast juice. The juice, obtained by pressure from cultures of these bacteria, contains albumoses. When left to autodigestion, the quantity of the latter diminishes as a consequence of the action of the proteases contained in the liquid.

The progress of the secretion of proteases by bacteria depends especially on the reaction of the medium, as well as on its composition. According to the manner of nutrition, the quantity of protease furnished by the same bacterial species may undergo pronounced variations. Thus for $B$. viridis, $B$. anthracis, and $B$. lupuliperda, the secretion depends on the method of culture and on its age. B. mesentericus, cultivated on large surfaces, produces 20 times more protease than if cultivated below the surface, though the same medium be used. According to Fermi, bacterial proteases act in neutral or slightly alkaline media. Acidity of the medium is very injurious especially if a mineral acid is used. Proteases act on gelatin and on fibrin, but only with great difficulty on uncoagulated albumin.

The optimum temperature lies between $30^{\circ}$ and $40^{\circ}$. At the temperature of $4^{\circ}$ to $5^{\circ}$, there is no longer action, although even very low temperatures, $-200^{\circ}$ for example, do not destroy proteases. The active substance of protease, exposed for a prolonged time to the action of light, deteriorates greatly. The destruction temperature of proteases varies according to their origin. Proteases of $M s p$. cholerae, of B. anthracis, and of Vibrio Finkler-Prior withstand high temperatures. To destroy them the enzymes must be heated at $65^{\circ}$ to $70^{\circ}$ for an hour. The proteolytic enzymes of $B$. ramosus, of $B$. prodigiosus, of staphylococcus pyocyaneus aureus are, on the contrary, destroyed at $55^{\circ}$. The enzymes of $B$. pyocyaneus, of $B$. subtilis, of $B$. fluorescens, of $B$. megatherium lose their activity at $55^{\circ}$ to $60^{\circ}$. The bacterial ecto-proteases possess a very energetic dissolving power, but the proteolysis is not very advanced. On the contrary, the endo-proteases generally produce much more advanced hydrolyses. This peculiarity is perhaps explained by the fact 
that, in this last case, proteolytic enzymes other than the socalled proteases come into play.

Resistance to Heat of the Proteases of B. Pyocyaneus and B. Prodigiosus. - Karl Meyer has furnished interesting data on the proteases of $B$. prodigiosus, on the one hand, and of $B$. pyocyaneus, on the other. To obtain these enzymes, he cultivated these bacteria in very slightly alkaline nutritive broths. After a certain time, the liquors were filtered $-B$. pyocyaneus easily filters through paper, but porcelain filters must be used for $B$. prodigiosus. The maximum proteolytic activity was obtained with $B$. prodigiosus after 2 or 3 weeks, though $B$. pyocyaneus reached its maximum more rapidly. The two filtered liquids possessed very pronounced proteolytic powers and rapidly liquefied gelatin with the formation of amino-acids. For cultures of $B$. pyocyaneus, the addition of 4 per cent glycerin to the nutritive broth considerably accelerated the production of proteolytic enzymes. On the contrary, for $B$. prodigiosus, that was not necessary. With these two proteases the optimum medium corresponded to a slightly alkaline reaction. The enzymes were less active in neutral, and still less in acid media. By comparison with trypsin, these two protease-bacteria appeared to be more unfavorably influenced by alkali than is trypsin.

Interesting facts should be kept in mind as to the action of heat on these two proteolytic enzymes. First of all it is found that the prodigiosus protease resists a temperature of $100^{\circ}$, while the temperature of $56^{\circ}$ to $60^{\circ}$ considerably reduces its digestive power.

action of Heat on the Prodigiosus Protease.

\begin{tabular}{|c|c|c|c|c|c|c|c|}
\hline \multirow{2}{*}{$\begin{array}{l}\text { Quantity of pro- } \\
\text { digiosus pro- } \\
\text { tease. }\end{array}$} & \multirow{2}{*}{$\begin{array}{l}\text { Enzyme } \\
\text { not } \\
\text { heated. }\end{array}$} & \multicolumn{4}{|c|}{ Enzyme heated $30 \mathrm{~min}$. at } & \multicolumn{2}{|c|}{ Enzyme heated to $100^{\circ}$. } \\
\hline & & $56^{\circ}$ & $65^{\circ}$ & $75^{\circ}$ & $85^{\circ}$ & 5 minutes. & I5 minutes. \\
\hline $\begin{array}{l}\text { c.c. } \\
\text { I }\end{array}$ & $1 / I$ & $I / 2$ & $I / I$ & $\mathbf{I} / \mathbf{I}$ & I/I & $\mathrm{I} / \mathrm{I}$ & $I / I$ \\
\hline 0.5 & $I / I$ & $\mathrm{I} / 4$ & $I / I$ & I/I & $I / I$ & $I / I$ & $I / I$ \\
\hline 0.2 & $I / I$ & $1 / 4$ & $I / 2$ & $\mathrm{I} / \mathrm{I}$ & $I / I$ & $I / I$ & \\
\hline 0.1 & $I / I$ & $\circ$ & $\mathrm{I} / 4$ & $1 / 4$ & $I / 2$ & $1 / 2$ & $1 / 2$ \\
\hline 0.05 & $1 / 2$ & $\circ$ & $\ldots$ & $\ldots$ & $\ldots$ & $\ldots$ & $\ldots$ \\
\hline
\end{tabular}


Protease, both heated or not heated in quantities of I c.c. to 0.05 c.c., is added to a constant volume of a solution of casein. These are allowed to digest for a given time and then the state of the proteolysis is determined. I/I indicates a complete digestion, that is to say, the non-formation of a precipitate by a subsequent addition of acid; $1 / 2$ indicates an incomplete digestion, and $\circ$ the absence of digestion. Under the heading, "Enzyme not heated," we see that with o.1 c.c. of unheated protease a complete digestion is obtained. On the contrary, protease which has been kept $30 \mathrm{~min}$. at $56^{\circ}$ before being put in contact with casein is considerably weakened, and even with I c.c. only a partial digestion is observed. The relation between the control product and that which has been heated is as I to 0.05 . The destruction of the enzyme at $56^{\circ}$ is very rapid and, in certain cases, even after a 5 minute exposure, the enzyme activity is considerably weakened. The liquid once inactive does not regain its activity if heated to $100^{\circ}$. However the protease, heated first to $100^{\circ}$, can be kept for $15 \mathrm{~min}$. without a perceptible diminution of its proteolytic power being observed.

It might be concluded that the resistance to boiling results from the presence in the culture liquor of substances capable of protecting the enzyme against the effect of heat. However, cultures of $B$. prodigiosus, made in media free from albuminoids and thus containing very little colloidal substance, give the same result. To explain this resistance we can make two hypotheses. First of all it can be supposed that the enzyme, by its very nature, resists a temperature of $56^{\circ}$. It is however destroyed at this temperature, in consequence of the intervention either of another enzyme existing in the liquid, or of an anti-enzyme, these two agents of inactivation disappearing at a temperature near $100^{\circ}$. The second hypothesis is that anti-protease is formed in the liquid at a temperature of $56^{\circ}$, so that by bringing the solution suddenly to $100^{\circ}$, the formation of this substance is avoided. According to Béarn and Cramer, we could suppose here the intervention of zymoids, or kinds of antibodies which result from the action of a moderate heating 
on the enzymes. It is known, in fact, that in certain cases solutions of enzymes, kept at $56^{\circ}$, can be attacked by derived substances which paralyze the enzymes from which they were derived. This second hypothesis, however, has not been verified. By bringing a solution of prodigiosus protease to a temperature of $56^{\circ}$, no substance is produced capable of retarding the proteolytic action, for such a liquid, heated to $56^{\circ}$ and added to a new solution of protease, does not change at all the digestive power of the latter.

The following table shows the action of temperature on the pyocyaneus protease:

\begin{tabular}{|c|c|c|c|c|c|c|c|}
\hline \multirow{2}{*}{$\begin{array}{l}\text { Quantity of } \\
\text { pyocyaneus } \\
\text { protease. }\end{array}$} & \multirow{2}{*}{$\begin{array}{c}\text { Enzyme } \\
\text { not } \\
\text { heated. }\end{array}$} & \multicolumn{4}{|c|}{ Enzyme heated $30 \mathrm{~m}$. at } & \multicolumn{2}{|c|}{ Enzyme brought to $100^{\circ}$. } \\
\hline & & $56^{\circ}$ & $65^{\circ}$ & $75^{\circ}$ & $85^{\circ}$ & 5 minutes. & I5 minutes. \\
\hline $\begin{array}{l}\text { c.c. } \\
0.5\end{array}$ & $I / I$ & $\mathbf{I} / \mathbf{I}$ & $I / I$ & $I / I$ & ? & $I / I$ & $I / I$ \\
\hline 0.2 & $I / I$ & $\mathrm{I} / \mathrm{I}$ & $\mathrm{I} / \mathrm{I}$ & $\mathrm{I} / 2$ & $\mathrm{I} / 4$ & $1 / 2$ & $1 / 2$ \\
\hline 0.1 & $I / I$ & $I / I$ & $\mathrm{I} / 2$ & $\mathrm{I} / 4$ & 0 & $\mathrm{I} / 4$ & $1 / 4$ \\
\hline 0.05 & $I / 2$ & $\mathrm{I} / 2$ & $\ldots$ & $\ldots$ & 0 & $\ldots$ & $\cdots$ \\
\hline
\end{tabular}

Thus the pyocyaneus protease also resists a temperature of $100^{\circ}$. The length of heating is likewise without significance, though this enzyme is much less sensitive to the temperature of $56^{\circ}$ than the prodigiosus protease. While with the prodigiosus, the destruction temperature is $56^{\circ}$, here a temperature of $85^{\circ}$ is required.

It is, moreover, interesting to note that these two proteases, while resisting a boiling temperature, are only slightly affected under these conditions. At $100^{\circ}$ they are not destroyed, but they no longer react perceptibly. The optimum temperature for the two proteases is $37^{\circ}$.

Individuality of Bacterial Proteases: Their Antibodies. As to the individuality of the bacterial proteases, we possess also curious data. From rabbits, by means of repeated injections of increasing quantities of prodigiosus protease or of pyocyaneus protease, serums are obtained containing anti-proteases. These 
two antibodies are specific, and react exclusively on the corresponding proteases. Moreover, they are without action on trypsin. These anti-proteases withstand a temperature of $75^{\circ}$ for a half-hour without perceptibly weakening. At $85^{\circ}$ deterioration begins, and at $100^{\circ}$ the destruction is complete. A mixture of protease and of the corresponding anti-protease, in the proportion required in activation, again becomes active when heated to $100^{\circ}$, in consequence of the destruction of the antibody.

The anti-protease and the anti-trypsin contained in the serum differ not only from the physiological point of view, but also from the chemical point of view. The anti-protease is precipitated almost quantitatively when the serum is half saturated with ammonium sulphate. It is therefore found among the globulins. On the contrary, the anti-trypsin of the serum is precipitated along with the albumin. The two antibodies can thus be separated either by fractional precipitation or by heating. By heating the serum for a half-hour at $75^{\circ}$, the anti-trypsin is destroyed, while the anti-protease remains. It should be added that the anti-protease, as well as the antitrypsin, is weakened by a treatment with petroleum. The action, however, is too slight to permit the conclusion that the nature of these substances is lipoidal. The action of antiproteases on proteases is very rapid. The same results are obtained whether the anti-proteasic serum is allowed to act for some time on protease before adding the casein, or whether the three bodies are directly mixed together. In the action of antitrypsin on trypsin there is observed the Danysz phenomenon which consists in the fact that the quantity of antitoxin neutralized by a given quantity of toxin is larger when the latter is added in stages than when added all at once. With anti-protease, on the contrary, no such phenomenon is observed.

Proteases in Bacterial Cultures - Research Methods. In the examination of bacterial proteolytic enzymes, as well as in their quantitative estimation, filtered cultures are used. To the liquids, free from bacteria, 0.5 per cent to I per cent of 
toluol is added and the mixtures are vigorously shaken. In these solutions the proteolytic power is tested by means of albumin, fibrin, gelatin, peptone, or casein. To determine the enzyme power by means of gelatin, 7 per cent solutions of this substance are used with the addition of phenol or of thymol. Pour 3 or 4 c.c. of this solution into tubes of about 8-millimeter diameter, cool to obtain a complete solidification, and pour on it $\mathrm{I}$ to 2 c.c. of filtered culture. Mark the level of the separating surface and leave the tubes at ordinary temperature. The estimation of the quantity of enzyme is made according to the volume of gelatin liquefied. Gelatin colored with carmine may also be used in place of the gelatin solution. Plates of small dimensions are cut and are left in water for 24 hours to remove the acid and the impurities which they may contain. They are then suspended by a thread in the liquid to be analyzed. The intensity of the proteolysis is here measured by the coloration which the solution takes.

To make tests with fibrin or albumin, a definite quantity of these substances is taken and immersed in the filtered bacterial culture, with or without previous dilution, thymol being also added. The tubes are left at $37^{\circ}$, the liquids neutralized, and Io per cent of a saturated solution of $\mathrm{NaCl}$ is added. The solution is boiled, brought to constant volume, filtered, and the nitrogen in the filtrate determined. To characterize more closely the protease in the bacterial cultures the use of Witte's peptone is recommended. A 2 per cent solution of this product sterilized at 2 atmospheres for 30 minutes is used. After cooling a certain volume of the filtered culture, from I to Io per cent, is added. The total nitrogen in the mixture is determined as well as the tannin nitrogen and the Sörensen formaldehyde nitrogen. In a second test, prepared in the same way, digestion is allowed to proceed for 3 or 4 days at $38^{\circ}$. The liquid is then analyzed and the results compared. The difference in tannin nitrogen and formaldehyde nitrogen will indicate the extent of proteolytic action, and will especially reveal the presence in the liquid of enzymes other than the proteases, 
probably members of the erepsin family which give a larger quantity of degraded products.

In these tests, however, recourse must be had to the microscope, or better, the digestion liquids should be tested by inoculations in plate cultures to make sure that there is no infection in the course of analysis. The filtration often presents great. difficulties, especially when very little liquid is to be filtered. In this case the culture to be studied is kept for a half-hour at a temperature of $55^{\circ}$ to $60^{\circ}$, and then the tests are made in a casein solution as described in the chapter relative to the analysis of trypsin. Since the digestion is of short duration, an infection is very little to be feared here, but it may happen, as was observed in the case of the prodigiosus protease, that the enzyme is destroyed at this temperature. In the case of negative results it will therefore be well to repeat the tests at $50^{\circ}$ to make sure that no action is obtained.

\section{§ 6. Papain.}

Preparation. - It has been known for a long time that the sap of Carica papaya L., or melon tree, contains a constituent which renders meats tender and digests albuminoid substances. Vauquelin, in 1802 , studied this juice, but it was Wurtz, in 1879, who made known the curious properties, and showed that they were due to a soluble enzyme. The proteolytic enzyme of the pawpaw tree, designated by the various names of papain, papayotin, papaytin, or papayacin, is found distributed in all parts of the tree, in the roots, the trunk, the leaves, the fruits and the seeds. To obtain the active substance, an incision is made on the trunk of the tree, though a product of superior quality is obtained by pricking the fruits. The juice which flows hardens in the air and forms crude papain. A good primary material may likewise be prepared by pressing the fruits with a little water and letting the juice dry in the air. In both cases; the product is reduced to a fine powder of whitish appearance and serves for the preparation of commercial papain. For this 
purpose, the powder is dissolved in water, the liquid obtained is filtered, and treated with so volumes of alcohol. The coagulum, redissolved in water, is again precipitated by alcohol. The product, thus purified, is finally dried at low temperature, and is then pulverized. A papain of careful preparation can dissolve in I 2 hours almost 2000 times its weight of fibrin. However, we rarely find products capable of such activity.

Products of Hydrolysis. - Papain dissolves natural albuminoid material, such as muscles, diphtheretic false membranes, cancerous tissues, and their like. Papain decomposes peptone much more rapidly than does pepsin. The hydrolysis is also more thorough, promptly producing tryptophane and, in operations of long duration, tyrosin. However, tyrosin is not produced in papainic digestions with the same facility as in tryptic digestions. When a maceration of Russula delica is added to the digestion liquor of fibrin, casein or albumin obtained with papain, a red coloration passing slowly to green is obtained in place of dark-red. Furthermore, when albumin is exposed to the prolonged action of papain, crystals of tyrosin are not observed at the bottom of the vessels. As a matter of fact, the question as to the extent of the hydrolysis by the pawpaw enzyme is still quite obscure. Kutscher and Lohmann have indeed found leucin, aspartic acid, glycin, arginin, histidin, lysin, prolin, tryptophane, and tyrosin in the products formed, but their results, as moreover those of other experimenters who have reached the same conclusions, have been obtained in cases of very prolonged digestions, Io months and more, in the presence of chloroform and we may ask if there has not actually been contamination and bacterial intervention.

It nevertheless appears from the work of Emmerling and of Kutscher that there is really a production of amino-acids in the prolonged digestions and that, both from the standpoint of time as well as of the nature of the products formed, this progress of hydrolysis differs unmistakably from that of pepsin. The fact that papain belongs rather to the class of trypsins is indicated by the discovery, made by Abderhalden, that papain 
easily decomposes glycyl-l-tyrosin giving tyrosin, as does trypsin, while pepsin is inactive toward this same polypeptide. Mendel and Blood have proved the production of amino-acids under the influence of papain by adding hydrocyanic acid to the digestion liquid. The presence of very small quantities of $\mathrm{HCN}$, according to them, accelerates the action of papain on fibrin, albumin, and edestin, and by this artifice they succeeded in obtaining tyrosin and amino-acids even in digestions which were not prolonged.

The following, according to the experiments of Effront, are figures on the comparative activity of papain in a solution of peptone and in an emulsion of coagulated egg-albumin. A 2 per cent solution of Witte's peptone had added to it o.I per cent of papain and was left 95 hours in the oven, in the presence of chloroform and of xylol, and then was analyzed. For the various nitrogen percentages, before and after digestion, the following figures were found:

Analysis of Peptone Before and After the Action of Papain.

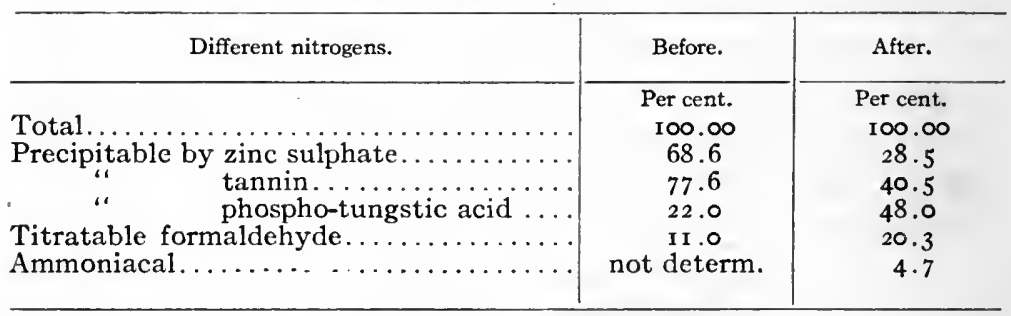

On the other hand, an emulsion of albumin was obtained by suspending 2 per cent of coagulated and finely divided eggalbumin in water with addition of 5 c.c. $\mathrm{HCl}(\mathrm{N})$ per 300 c.c. liquid. The following is the result of the analysis after 78 hours:

Analysis of an Emulsion of Albumin After Papainic Digestion.

$\mathrm{N}$ total ................................ 100

$\mathrm{N}$ precipitable by zinc sulphate................. 40

$\mathrm{N}$ precipitable by tannin...................... 50

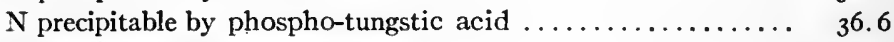

$\mathrm{N}$ titratable with formaldehyde................... 44.6 
These two tables show that papain acts differently on peptone and on albumin. In the first case there was true digestion, that is to say, a destruction of albumose nitrogen (precipitable by zinc sulphate) and an increase of peptone nitrogen (precipitable by the tungstic reagent), but the nitrogen of the aminoacids (titratable with formaldehyde) remained low. On the contrary, in the second case, the degradation was more advanced and the quantity of Sörensen nitrogen was found to be more than doubled.

Another characteristic which distinguishes papain from pepsin and relates it to trypsin is its easy adaptation to the reactions of the medium. Papain reacts in neutral media, and the presence of 0.005 per cent $\mathrm{HCl}$ or of 0.1 to 0.25 per cent $\mathrm{NaOH}$ is favorable to it. In the presence of 0.2 per cent $\mathrm{HCl}$ papain no longer reacts.

Effect of Temperature. - Papain in a dry state can, according to Wurtz, withstand a temperature of $105^{\circ}$ without losing its ability to dissolve fibrin. In solution it is naturally more sensitive but its resistance is still very high. Harlay, in order to study the influence of heat on papain, brought solutions of this enzyme to different temperatures for 30 minutes. Then after cooling, he put them with fibrin and left the whole 24 hours in the heater. The following are the results obtained, using $20 \mathrm{~g}$. of fibrin for each test:

Effect of Temperature on Papain.

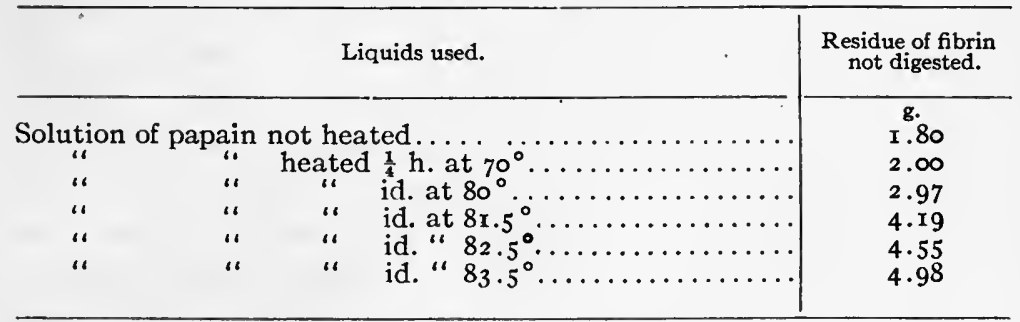

Papain can be heated for 30 minutes at $70^{\circ}$ without a perceptible weakening of its enzymic power. Here there is indeed a considerable difference from trypsin, since the latter is com- 
pletely destroyed by an exposure of a half-hour at only $65^{\circ}$. The destruction of papain would be almost complete at $82^{\circ}-83^{\circ}$. According to Harlay, the comparative effect of heat on the three proteolytic enzymes is as follows:

Pepsin is destroyed after a half-hour of heating at $68^{\circ}$.

Trypsin is destroyed after a half-hour of heating at $60-65^{\circ}$.

Papain is not weakened after a half-hour of heating at $70^{\circ}$.

Although between $70^{\circ}$ and $80^{\circ}$ the activity of papain is perceptibly diminished, this weakening of the digestive power, according to Harlay, involves no modification in the process of bydrolysis, that is to say the same products of degradation are made with this reduced enzyme activity as with the normal. However, this point needs to be verified by other experiments.

Pozerski in recent years has studied with much care the action of papain. From his researches it appears that the optimum temperature for this enzyme is high, generally very near that of its destruction. If at ordinary temperature papain and egg-albumin are placed together a change in the viscosity of the mixture very rapidly takes place. The albuminoid liquid no longer produces a persistent foam after shaking, and the determinations made with the viscosimeter show a pronounced change in the physical state of the liquid. However, no product of digestion is formed. At temperatures up to $50^{\circ}$ papain acts very feèbly on blood serum and egg-albumin in the natural state, and very few products of hydrolysis are to be detected. On coagulated albumin the action is easier and occurs even at a temperature of $40^{\circ}$ where an advanced decomposition is obtained.

The difference of action of papain on fresh and coagulated albumin has two explanations. First of all, this difference is due to the fact that the transformation of albumin is favorable to digestion, for in the course of coagulation physical and chemical changes are produced in the constitution of the albuminoid which make the attack by papain easier. Secondly, this difference is due to the fact that natural albumin offers a spccial resistance due to the specific action which it exerts on 
papain. Liquid egg-albumin neutralizes the action of this enzyme. This effect is shown at ordinary temperature, is found again with more intensity at $50^{\circ}$ or $60^{\circ}$, and then decreases in such a way that albumin heated for a half-hour to $80^{\circ}$ loses the property of reducing the activity of papain. This neutralizing action occurs as though an anti-enzyme were present, however, the existence of the latter has not been demonstrated. The weakening of the papain by natural egg-albumin or serum is observed in neutral or very slightly acid media, but the presence of 0.05 per cent of hydrochloric acid is enough to check the same entirely.

Phenomenon of Sudden Digestion. - In the action of papain on fresh albumin, as well as on animal tissues taken in . the natural state, a very curious phenomenon is observed which, at least in appearance, is in complete contradiction with what is known concerning the action of enzymes. We find particularly that the intensity of digestion, in the presence of a definite quantity of papain, decreases with the time of contact of the enzyme with the material to be digested. The following is one of the experiments cited by Pozerski: Make a series of mixtures containing 15 c.c. of egg-albumin diluted one-third with physiological salt solution and 2 c.c. of 2 per cent Merck papain solution. In one of the tubes make a control test, the 2 c.c. of papain having been boiled. Then add 2 drops of acetic acid and bring to $100^{\circ}$ to coagulate the albumin. The other tubes are left at the temperature of the laboratory $\left(\mathrm{I} 8^{\circ}\right)$ for various times, then treated as before with acetic acid and boiled. The precipitates collected on the.tared filters are washed, dried and weighed. See table on p. 442 .

In the control test, the enzyme having been immediately destroyed, the precipitate, weighing $338 \mathrm{mg}$. represents the quantity of total albumin contained in each tube. In test No. 2, the papain has been left I minute in direct contact with the albumin, then rapidly brought to $100^{\circ}$. The duration of heating, which has been from to to 20 seconds, has been quite sufficient to bring about a maximum effect. I $2 \mathrm{mg}$. of dissolved albumin 
have been obtained. If the mixture of albumin and papain is left not I minute but 5 , Io, or 30 minutes at ordinary temperature before bringing to boiling to destroy the enzyme, it is found that the quantity of albumin dissolved becomes not bigher, but, on the contrary, smaller, and that after 4 hours of contact, the albumin dissolved is not more than $4 \mathrm{I} \mathrm{mg}$., or hardly $\frac{1}{3}$ of the quantity dissolved in the test (2).

Digestion of Egg-Albumin by Papain.

\begin{tabular}{|c|c|c|}
\hline Time of contact before coagulation by heat. & $\begin{array}{l}\text { Weight of precipi- } \\
\text { tate collected. }\end{array}$ & $\begin{array}{l}\text { Quantity of albu- } \\
\text { min digested. }\end{array}$ \\
\hline 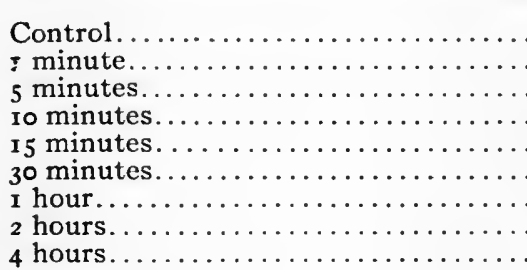 & $\begin{array}{l}\mathrm{mm} . \\
338 \\
226 \\
232 \\
243 \\
250 \\
259 \\
283 \\
289 \\
297\end{array}$ & $\begin{array}{r}\text { mm. } \\
\ldots . \\
\text { i } 12 \\
\text { I06 } \\
95 \\
88 \\
79 \\
55 \\
49 \\
41\end{array}$ \\
\hline
\end{tabular}

How can this anomaly be explained? In fact, under the conditions of the experiment, the digestion did not take place as long as the temperature was below $40^{\circ}$. It took place, on the contrary, suddenly between $80^{\circ}$ and $89^{\circ}$ during the 10 or 20 seconds used to bring the liquid to the boiling point, with a view of killing the enzyme and then making the determination. It is, moreover, to be noted that this important dissolving action during so short a time is accompanied by a thorough hydrolysis, with an extensive formation of peptones. Further, the preliminary contact at ordinary temperature of albumin and papain influences the reaction unfavorably; the very meager results obtained in the last tests are due to the fact that the anti-papainic effect of the unheated serum or of the unheated egg-albumin, an effect referred to above, is exerted for a very long time, 2 or 4 hours. Finally, in this experiment, there are two kinds of phenomena. The first, which takes place at each temperature between $0^{\circ}$ and $40^{\circ}$, consists in a reduction of the enzyme, this weakening increasing with the time. The second, 
which takes place between $80^{\circ}$ and $89^{\circ}$, is a process of very rapid digestion, but is, aside from that, similar to all the other enzymic effects.

In this phenomenon of sudden digestion, a certain relation is found between the quantities of enzyme used and the action produced during a given time. To be sure, there is not the simple proportionality which was observed in the case of the other enzymes, but the conditions here are infinitely more complicated. The temperature is not constant, and even the quantity of enzyme, although well defined at the beginning, is also essentially variable, since it constantly diminishes. However this may be, Pozerski finds that his figures practically obey the following rule: The quantity of material transformed is proportional to the square root of the quantity of enzyme added. It is only by pure coincidence that this law is identical with that of Schutz and Borissow, which relates to the action at a fixed temperature of trypsin on albumin placed in Mett tubes.

Pozerski proceeds as follows: To 15 c.c. of dog serum diluted $\mathrm{x} / 3$ in salt water, he adds varying quantities of the 2 per cent papain solution. All the mixtures are brought to the same volume with physiological salt solution, and then the various solutions, with addition of 2 drops of acetic acid, are rapidly brought to $100^{\circ}$. The precipitates are then collected and weighed:

Digestion of the Serum by Papain.

\begin{tabular}{|c|c|c|}
\hline $\begin{array}{c}\text { Quantity of } \\
\text { papain used. }\end{array}$ & $\begin{array}{c}\text { Weight of the pre- } \\
\text { cipitates. }\end{array}$ & $\begin{array}{c}\text { Quantity of albu- } \\
\text { min dissolved. }\end{array}$ \\
\hline Control & mg. & mg. \\
0.25 c.c. & 350 & 0 \\
0.5 & 280 & 70 \\
I & $25 \mathrm{I}$ & 99 \\
2 & 191 & 159 \\
4 & 146 & 204 \\
\hline
\end{tabular}

In all the tests just cited, giving results that have since been verified by Jonescu and by Sachs, the digestive action is obtained by the rapid passage of the solution examined from . 
ordinary temperature to that of boiling. It is evident that the reaction must be the function of a definite temperature, and not of the whole thermometric scale. This temperature has been found in the following way: A series of mixtures of I c.c. of egg-albumin, diluted I: 1 , and 2 per cent in juice of papayotin, were brought to increasing temperatures, $55^{\circ}, 60^{\circ}$, $65^{\circ} \cdots 95^{\circ}$. When the desired temperature was reached, to each sample was rapidly added an equal volume of ro per cent trichloracetic acid, which prevented any further enzymic action. Then the solutions were heated to $100^{\circ}$ and the precipitates collected.

Influence of Temperature on Papainic Digestion.

\begin{tabular}{|c|c|c|}
\hline $\begin{array}{c}\text { Temperatures } \\
\text { reached. }\end{array}$ & $\begin{array}{c}\text { Weight of precipi- } \\
\text { tates. }\end{array}$ & $\begin{array}{c}\text { Quantity of albu- } \\
\text { min digested. }\end{array}$ \\
\hline Control & mg. & mg. \\
55 & 558 & 0 \\
60 & 552 & 0 \\
65 & 550 & 0 \\
70 & 546 & 12 \\
75 & 535 & 28 \\
80 & 488 & 72 \\
85 & 351 & 207 \\
90 & 312 & 246 \\
95 & 280 & 338 \\
\hline
\end{tabular}

Thus digestion begins in the neighborhood of $60^{\circ}$, it increases notably at $80^{\circ}$, passes by a maximum at $90^{\circ}$, but is still very intense at $95^{\circ}$. On the whole, papain reacts especially well at a temperature very near that of its destruction. It is to be noted furthermore that the optimum temperature practically coincides with the coagulation temperature of albuminoids. It appears from this that in the sudden reaction just studied, it is not the unheated albumin which is digested by papain in the neighborhood of $80^{\circ}-85^{\circ}$, but rather the albumin already transformed by heat. This albumin, en route to coagulation, is found in a special condition which permits a more easy attack. In conclusion, it is well to note that this very sudden digestion likewise appears when a solution of papain is boiled for sterili- 
zation. Then a true autolysis takes place, so that albumoses and peptones are always encountered in these boiled products.

Anti-papain. - From the purely physiological point of view the serum of animals se sitized against papain is as susceptible to the enzyme as the serum of fresh animals, apparently no anti-enzyme being formed in the course of the sensitization. Yet the serum of sensitized rabbits acquires new properties. This serum possesses a specific precipitin and an antibody or sensitizer, capable of deviating the complement. But it is probable that these antibodies act rather on the very complex albuminoid material of the pawpaw juice rather than on the digestive enzyme itself. Finally, Pozerski has found that guinea-pigs, under the influence of repeated injections of very small quantities of papain, present very evident symptoms of anaphylaxis.

\section{$\S 7$. BROMELIN.}

This enzyme, noted by Marcano in r89r, has been studied by Chittenden, then by Pozerski. It is found in very abundant quantities in pineapples. To prepare it, the fruit is cut in slices, which are pressed. The juice obtained contains sugar, albuminoids, and organic acids, its acidity being from 0.45 per cent to 0.5 per cent. When treated with 2 volumes of alcohol, the precipitate which forms contains almost no enzyme. It is separated from the liquid and to the filtrate are again added 5 volumes of alcohol. The coagulum is then collected, dried and pulverized. $0.1 \mathrm{~g}$. of this powder dissolves I $\mathbf{g}$. of fibrin in 6 hours at $60^{\circ}$ in a slightly acid medium. The pineapples of the Azores are especially rich in bromelin. This enzyme acts on fibrin or coagulated albumin in neutral, slightly alkaline, or very slightly acid media.

Using neutral media 18 hours are required for the addition of $\mathrm{HNO}_{3}$ to bring about further precipitation. With $30 \mathrm{mg}$. of $\mathrm{HCl}$ per liter only $\mathrm{I} 2$ hours are required, the digestion being then much more rapid. If the quantity is increased to $60 \mathrm{mg}$. 
the favorable effect is diminished, and with $80 \mathrm{mg}$. per liter very perceptible retardation is observed.

Influence of Acidity on the Action of Bromelin on Fibrin at $40^{\circ}$.

\begin{tabular}{|c|c|}
\hline $\begin{array}{c}\mathrm{HCl} \text { in mg. per } \\
\text { liter. }\end{array}$ & $\begin{array}{c}\text { Length of digestion } \\
\text { estimated by ab- } \\
\text { sence of a nitric acid } \\
\text { precipitate. }\end{array}$ \\
\hline 0 & Hours. \\
\hline 0 & 18 \\
20 & 16 \\
30 & 14 \\
60 & 12 \\
80 & 16 \\
\hline
\end{tabular}

The optimum temperature for bromelin is at about $60^{\circ}$. According to Pozerski, bromelin, like papain, possesses a very great resistance to the action of heat. The activity of this enzyme is only slightly reduced by heating for a half-hour at $80^{\circ}$. It is necessary to bring it to a temperature of $100^{\circ}$ to observe the very rapid disappearance of its proteolytic power. This same investigator has found in bromelin some of the properties which he has observed in papain. In particular, the enzyme of pineapples acts differently toward fresh and coagulated albuminoids.

According to Effront, the action of bromelin on an emulsion of coagulated albumin in neutral reaction and at different temperatures is as follows:

Effect of Temperature on Bromelic Digestion.

\begin{tabular}{c|c||c|c}
\hline Temperature. & $\begin{array}{c}\text { Per cent albumin } \\
\text { digested. }\end{array}$ & Temperature. & $\begin{array}{c}\text { Per cent albumin } \\
\text { digested. }\end{array}$ \\
\cline { 2 - 3 } deg. & 7.9 & deg. & \\
40 & 9.6 & 60 & 12.5 \\
45 & 11.4 & 65 & 9.3 \\
50 & 11.9 & 70 & 8.3 \\
55 & $\ldots$ & $\cdots$ \\
\hline
\end{tabular}

The optimum temperature is, therefore, in the neigbborhood of $60^{\circ}$. If now pineapple juice is allowed to act on unheated egg-albumin and, at the end of varying intervals, the solutions 
are boiled, as has been described in the experiments with papain, there is observed again a sudden digestion, very intense and corresponding to an advanced hydrolysis. Here, again, the greater part of the transformation takes place at the moment of the passage from $80^{\circ}-90^{\circ}$. But by reason of the acidity of the natural pineapple juice, there is not observed the progressive reduction which was noted with papain. It is sufficient, however, to neutralize the acids of the juice or better to utilize the precipitated enzyme, to find that unheated albumin also exerts on bromelin a neutralizing action of anti-enzymic nature, quite comparable to that studied by Pozerski with the pawpaw enzyme.

Digestion of Horse Serum by Bromelin.

\begin{tabular}{|c|c|c|c|c|}
\hline \multirow{2}{*}{ Time of stay at $20^{\circ}$. } & \multicolumn{2}{|c|}{ Natural pineapple juice. } & \multicolumn{2}{|c|}{$\begin{array}{l}\text { Pineapple juice neutralized by } \\
\qquad \mathrm{Na}_{2} \mathrm{CO}_{3} .\end{array}$} \\
\hline & $\begin{array}{l}\text { Weight of pre- } \\
\text { cipitates. }\end{array}$ & $\begin{array}{l}\text { Quantity of } \\
\text { alb. digested. }\end{array}$ & $\begin{array}{l}\text { Weight of pre- } \\
\text { cipitates. }\end{array}$ & $\begin{array}{l}\text { Quantity of } \\
\text { alb. digested. }\end{array}$ \\
\hline Control & mg. & mg. & mg. & $\mathrm{mg}$ \\
\hline 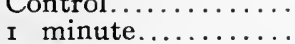 & $\begin{array}{l}324 \\
204\end{array}$ & $\ddot{1} 20$ & $\begin{array}{l}324 \\
\ldots\end{array}$ & $\cdots$ \\
\hline 5 minutes.......... & 206 & 118 & $2 \mathrm{I} 4$ & Iro \\
\hline I5 $"$ " $\quad \ldots \ldots \ldots$ & 203 & I 2 I & $\ldots$ & $\ldots$ \\
\hline 30 " $\quad \ldots \ldots \ldots$ & 205 & I I9 & 227 & 97 \\
\hline 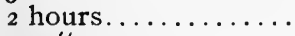 & $\ldots$ & $\ldots$ & 239 & 85 \\
\hline $4 \quad " \quad \ldots \ldots \ldots \ldots \ldots$ & 182 & 142 & 242 & 82 \\
\hline
\end{tabular}

The data as to the products of hydrolysis caused by bromelin are not very numerous. It appears, however, that, by its action bromelin resembles pepsin more closely than trypsin. Yet, there is found here a greater proportion of degraded products than in peptic reactions.

Reaction of Bromelin and Pepsin Compared.

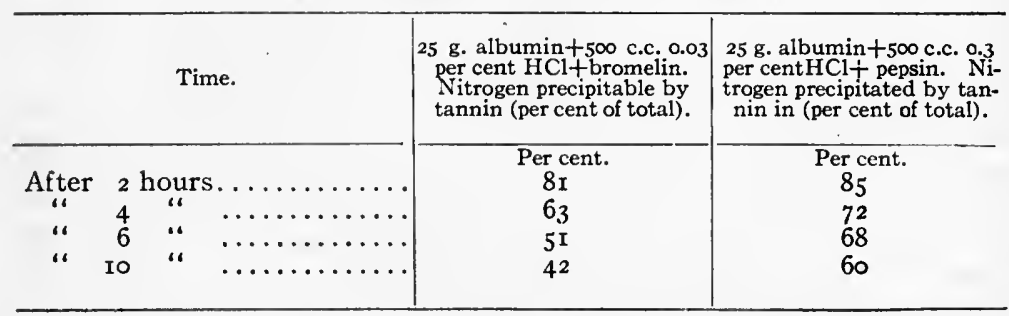


In bromelic digestion, albumoses and peptones are formed, but there is some doubt as to the formation of amino-acids and tyrosin. According to some writers, if the reaction is sufficiently prolonged, these substances appear in appreciable quantities; according to others, their existence is doubtful. However, Pozerski, in liquids resulting from sudden digestion, has never been able to show the presence of amino-acids.

\section{§ 8. Casease.}

This name has been given by Duclaux to the enzyme which destroys and liquefies the coagulum formed by rennet. This enzyme is difficult to classify for its properties are not characteristic. As Duclaux himself recognizes, if there is no decisive argument for retaining this enzyme and making of it a well-defined entity, neither is there any serious reason for eliminating it from the literature. We shall see, however, from a certain number of phenomena, that its existence appears very probable, and, until better informed, we will reserve a place for it among the proteolytic enzymes.

Casease is essentially of bacterial origin. To obtain it, Duclaux recommends the use of bacteria capable of living in milk, as Tyrothrix tenuis, but grown in broth instead of milk, since their secretions always contain a too great proportion of rennet mixed with casease. The precipitate which is obtained by treating the culture with alcohol is redissolved in water and contains in sufficient quantity the enzyme under consideration. If then a little of this solution is added to milk at $20^{\circ}-25^{\circ}$, there soon appears a coagulum, which moreover has not as firm an aspect as that obtained with calf rennet, and which slowly disintegrates to dissolve completely at the end of a few hours. The milk becomes almost clear, being no longer more than slightly opalescent. Casein, under these circumstances, has undergone a real digestion, and not only does the liquid no longer coagulate with rennet or with acids, but it now gives the reactions of peptones. 
The phenomenon observed, moreover, varies according to the respective proportions of rennet and of casease contained in the enzyme solution used, or again according to the temperature at which the experiment is performed. If there is an excess of casease and if the milk is sufficiently cold, coagulation no longer takes place and the casein undergoes transformation without being precipitated. If the enzyme, in place of acting on casein mixed with an emulsion, as in milk, or on a clot formed in the very heart of the digestive liquid and consequently impregnated in the entire mass by the enzyme, exerts its action on a coagulum already made by means of rennet, for example, the proteolysis will be less rapid, but will nevertheless follow the same path. This is especially observed in the ripening of cheeses, where the casein is progressively digested and peptonized under the influence of casease secreted by the lactic ferments scattered through the whole mass of the cheese, and by the molds and proteolytic bacteria which populate its surface.

According to Duclaux, when the temperature rises the activity of casease first increases and then decreases. The maximum action takes place in the neighborhood of $30^{\circ}$. In compensation, casease is still active at low temperatures where rennet no longer reacts, being active at about $4^{\circ}$ or $5^{\circ}$. Casease reacts especially well in neutral or slightly alkaline media. Like pepsin with fibrin, casease is adsorbed on the casein which it attacks and gradually gives to it a translucent aspect.

Casease is widely distributed. All bacteria producing rennet, and consequently capable of coagulating milk, are also producers of casease; for example, the Tyrothrix of Duclaux, the Bacillus subtilis, and the lactic ferments, the latter acting in previously neutralized media. Yeasts, as already noted in studying the work of Boullanger, are likewise capable of living in milk; but the general course of the digestion, in particular the formation of quite considerable quantities of ammonia, does not correspond at all to a casease reaction. Furthermore, this hydrolysis is very slow in taking place and varies also with the species of yeast considered. On the contrary, casease is 
frequently met in molds. Bourquelot and Herissey have noted casease in Aspergillus niger and Penicillium glaucum, and we have seen that the protease which Malfitano has taken from Aspergillus very easily digests casein. Again it has been found by Sartory in a great number of oömycete and ascomycete mushrooms. The higher mushrooms also contain this enzyme, and, according to Bourquelot, Psalliota campestris contains a considerable proportion while Amanita mappa is relatively poor in it. This mushroom casease, in slightly alkaline media, would transform the casein of milk into peptones with the formation of leucin and tyrosin.

Finally, the higher plants likewise secrete caseases. Javillier has shown the existence of a similar enzyme in the cell-juices of a large number of plants, notably Lolium perpeene, Urtica dioica, Euphorbia lathyris, Papaver album, etc. He has found in particular that the casease of rye-grass decomposes casein with the formation of tyrosin and tryptophane. The sap of Carica papaya and that of Ficus carica likewise digests casein. However, it must not be concluded that all the plants possessing rennet necessarily possess casease. Clover juice, which contains an active rennet, does not contain casease. Finally, casease must likewise be very widely distributed in the animal organism, since many tryptic fluids of animal origin are capable of digesting casein. Let us recall in particular that Duclaux has found casease in the pancreas by inoculating milk with a fragment of the gland which he had taken aseptically. Under these conditions the milk eventually became entirely digested.

In conclusion, casease has a mode of action which closely resembles that of trypsin, and in certain cases it appears to be confounded with trypsin. The existence of this enzyme is rendered problematical in that there is nothing characteristic in its chemical behavior, the conditions of medium, or of temperature which favors its activity. Furthermore, not a single case is known where casease acts exclusively on casein, while remaining without action on other albuminoid materials, such as 
albumin or fibrin. On the contrary, it is indisputable that there exists a whole class of proteases, notably those of bacterial origin, which evince a special predilection for casein, and that the very progress of the digestion of this substance under the influence of these enzymes, rapid solution, aspect of the liquid, and transparency, seems to be quite characteristic of their individuality. Since, furthermore, the name of casease already occupies an important place in literature, it is preferable to keep this name to designate an enzyme which acts easily on casein.

\section{$\S$ 9. Galactase.}

Fresh milk with the addition of an antiseptic undergoes very extensive transformations when left to itself for a long time. There is found a strong dissolving action on the nitrogenous constituents, with the formation of albumoses, peptones, and amino-acids. Van Slyke, H. A. Harding, and E. B. Hart have studied closely the auto-peptonization of milk and have obtained interesting data on the distribution of nitrogen in the milk thus kept protected from bacteria.

Distribution of Nitrogen in Milk (Per Cent Total Nitrogen).

\begin{tabular}{|c|c|c|c|}
\hline Length of preservation. & Soluble nitrogen. & $\begin{array}{c}\text { Albumose nitrogen } \\
\text { and peptone. }\end{array}$ & Amin nitrogen. \\
\hline 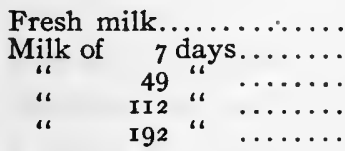 & $\begin{array}{r}9.33 \\
11.77 \\
21.59 \\
32.82 \\
37.83\end{array}$ & $\begin{array}{r}4.58 \\
7.00 \\
14.97 \\
17.86 \\
16.57\end{array}$ & $\begin{array}{l}4.75 \\
4.7 \\
6.62 \\
14.96 \\
21.06\end{array}$ \\
\hline
\end{tabular}

The peptonization which manifests itself in milk is attributed by many writers to the presence of a proteolytic enzyme. Babcock and M. Russell have studied the enzyme which intervenes under these conditions and have called it galactase.

To prepare this enzyme, whey is saturated with ammonium sulphate, the precipitate so obtained is dialyzed, and then concentrated at the temperature of $40^{\circ}$. The enzyme belongs to the group of trypsins, though it produces more ammonia 
than pancreatic trypsin. Galactase acts on casein in a neutral or slightly alkaline medium; in an acid medium the action is preceptibly retarded.

The optimum temperature is from $37^{\circ}$ to $42^{\circ}$, the destruction temperature is between $75^{\circ}$ and $80^{\circ}$. To destroy the enzyme in a neutral medium it is necessary to keep it 60 minutes at $72^{\circ}$. In a slightly acid medium, Io minutes is sufficient at the same temperature. Natural milk, kept for several seconds at $80^{\circ}$, loses its ability to decompose hydrogen peroxide and contains no more galactase.

Galactase is but slightly sensitive to the action of chloroform, ether, or sodium fluoride. On the contrary, formaldehyde destroys this enzyme very rapidly. This sensitiveness toward formaldehyde is greater than that shown by pepsin and trypsin. This is why this easy destruction of galactase may be used to separate it from its mixture with trypsin or pepsin.

The data on the origin of galactase are very contradictory. Some investigators have found this enzyme in all normal milks. Others maintain that it has only a bacterial origin, and that only the spores of the bacteria contained in the milk could produce this enzyme.

\section{$\S$ io. Glutenase.}

J. Baker and Hulton, in 1908, found in wheat flour a proteolytic enzyme capable of transforming the nitrogenous materials of the grain into various hydrolytic products, among which is tryptophane. This enzyme, which acts similarly on Witte's peptone, was identified with erepsin by these writers.

However, this conclusion does not appear acceptable, if it is true that the enzyme discovered by Baker and Hulton is identical with that found by G. Bertrand and Muterlich. These two latter investigators, in studying the phenomenon of coloration of brown bread, have shown that this characteristic browning is the result of the oxidation, caused by a special tyrosinase contained in the husks of wheat, of a tyrosinic chromogen, 
formed, in the course of the digestion of the nitrogenous substances of the grain, under the influence of a special proteolytic enzyme, likewise derived from the husks. This protease, which they have called glutenase, hydrolyzes not only the protein materials of the husks and of the gluten but also the casein of cow's milk, with the production of tyrosin.

This enzyme is distinguished from erepsin, and also from the caseases, in that it acts especially well in acid media. Inactive in alkaline media, it acts even in neutral media, though much better in the presence of a slight acidity. The organic acids (acetic or oxalic), as well as the mineral acids, serve to stimulate its digestive power. A 2 per cent solution of $\mathrm{HCl}$ appears very favorable. These are characteristic properties which really make this enzyme a well-defined type.

\section{§ i i. Gelatinase.}

It has been seen in the preceding chapter that a large number of bacteria liquefy and transform gelatin more or less thoroughly. It is generally considered that this action on gelatin, a substance resulting from the hydrolysis of ossein and which by its properties is quite plainly differentiated from the ordinary albuminoids, is due to the intervention of a special proteolytic enzyme, which has been described by different writers under the various names of gelatinase, glutinase, or gelatase. The active substance acting on gelatin is encountered not only in bacteria, but also in molds, mushrooms, in pancreatic juice, as well as in commercial trypsin.

According to Pollak, gelatinase may be separated from the pancreatic juice by the successive treatment with acid and alkali. To roo c.c. of pancreatic juice or of a solution of trypsin is added 50 c.c. $\mathrm{HCl}(N)$. After 36 hours the liquid is neutralized with $\mathrm{NaOH}(N)$ using methyl orange as the indicator (to reach this point generally requires 46 or 47 c.c. of $\mathrm{NaOH}$ ). A precipitate is then formed, which is separated by filtration. The filtrate is very active toward gelatin, while it is inactive toward the other albuminoids. 
Action of a Solution of Trypsin after Treatment with Acid and AlKali.

\begin{tabular}{|c|c|c|c|c|}
\hline Liquids used. & $\begin{array}{l}\text { Gelatin di- } \\
\text { gested (Mett). }\end{array}$ & $\begin{array}{l}\text { Album. serum } \\
\text { digested } \\
\text { (Mett). }\end{array}$ & $\begin{array}{l}\text { Egg-albumin } \\
\text { digested } \\
\text { (Mett). }\end{array}$ & Fibrin. \\
\hline $\begin{array}{l}\text { I }\left\{\begin{array}{l}\text { Control...... } \\
\text { Gelatinase. }\end{array}\right. \\
\text { II }\left\{\begin{array}{l}\text { Control..... } \\
\text { Gelatinase. }\end{array}\right.\end{array}$ & $\begin{array}{l}\mathrm{mm} . \\
\text { I5 } \\
\text { 10 } \\
\text { I I } \\
9\end{array}$ & $\begin{array}{l}\mathrm{mm} . \\
7 \\
0 \\
\cdots \\
\cdots\end{array}$ & $\begin{array}{c}\text { mm. } \\
\ldots \\
\text { io } \\
0\end{array}$ & $\begin{array}{l}\text { Complete dig. } \\
\text { No digestion } \\
\text { Complete dig. } \\
\text { No digestion }\end{array}$ \\
\hline
\end{tabular}

The controls are prepared with trypsin not treated with acid and alkali. On the contrary, the gelatinase is the same solution of trypsin which has undergone treatment. The successive addition of $\mathrm{HCl}$ and of $\mathrm{NaOH}$ results in causing the solution of trypsin to lose its property of dissolving albumin and fibrin, without, however, destroying its power of liquefying gelatin. To study the action of the proteolytic enzymes on gelatin, Minami caused different active substances to act on ro per cent solutions of gelatin. He then determined the nitrogen not precipitable by tannin, as well as the nitrogen of the amino-acids formed, using the formaldehyde method.

Action of Proteolytic Enzymes on Gelatin.

\begin{tabular}{|c|c|c|c|c|}
\hline \multirow{3}{*}{$\begin{array}{l}\text { Io per cent gelatin, quantity } \\
\text { digested in the presence of: }\end{array}$} & \multicolumn{4}{|c|}{ Distribution of the nitrogen. } \\
\hline & \multicolumn{2}{|c|}{ After 24 hours. } & \multicolumn{2}{|c|}{ After 48 hours. } \\
\hline & $\begin{array}{l}\text { N not precip. } \\
\text { by tannin. }\end{array}$ & $\begin{array}{l}\text { Formaldehyde } \\
\text { N. }\end{array}$ & $\begin{array}{l}\text { N not precip. } \\
\text { by tannin. }\end{array}$ & $\begin{array}{l}\text { Formaldehyde } \\
\text { N. }\end{array}$ \\
\hline $\begin{array}{l}0.25 \text { per cent } \mathrm{HCl} . . . \\
\text { Pepsin hydrochloride } \\
\text { Pancreatic extract... } \\
\text { Rhenania pancreatin. } \\
\text { PancreatoneRhenania }\end{array}$ & $\begin{array}{c}\text { Per cent. } \\
2.1 \\
3.2 \\
30.5 \\
22.5 \\
40.0\end{array}$ & $\begin{array}{c}\text { Per cent. } \\
3.6 \\
4.6 \\
\text { I } 5.8 \\
\text { I } 2.6 \\
18.3\end{array}$ & $\begin{array}{c}\text { Per cent. } \\
2.6 \\
4.7 \\
33.7 \\
31.4 \\
47.2\end{array}$ & $\begin{array}{c}\text { Per cent. } \\
4.3 \\
5.7 \\
\text { I } 6.5 \\
\text { I4 } 4.0 \\
20.8\end{array}$ \\
\hline
\end{tabular}

Pepsin hydrochloride acts freely at the beginning and its activity does not appreciably increase later. The different preparations of pancreatin show, on the contrary, a very energetic activity and with pancreatin, a hydrolysis is obtained indicating 40 per cent of nitrogen not precipitable with tannin and nearly 
20 per cent of amino-acids. From data furnished by Messernitzky on the gelatinase of Micrococcus prodigiosus, it appears that this enzyme acts on gelatin still more vigorously than pancreatone, since, after 24 hours, the nitrogen not precipitable by tannin is 60 per cent; with a prolonged action, the latter rises to 85 per cent. The formaldehyde nitrogen very quickly reaches I5 per cent, and in long digestions it increases to 40 per cent. The products of the action of the gelatinase of $M$. prodigiosus, includes glycin, as well as the other amino-acids.

The gelatinase of $M$. prodigiosus and pancreatone, while belonging to the same group of enzymes, differ, however, perceptibly in the extent of the reactions produced. This difference is still more clearly marked when the effect of other bacterial proteases on gelatin is considered. A large number of bacteria furnish proteases which liquefy gelatin, though giving very little formaldehyde nitrogen, and also very little nitrogen not precipitable by tannin. This last, when the action is prolonged, increases slowly to reach a maximum of ro per cent or 20 per cent of the total nitrogen.

It may be seen, therefore that the gelatinolytic enzymes do not all produce the same effect, and it is necessary to ask what in reality is the characteristic property of gelatinase. To answer this question, resulting from so large a variation in the chemical processes catalyzed, and considering as probable the existence of a gelatinase, we could, strictly speaking, state that this enzyme only liquefies gelatin and exerts only a very slight effect upon hydrolysis. Advanced digestions, observed with pancreatone or with prodigiosus gelatinase, would then be explained by the combined action of gelatinase and other proteolytic enzymes. The characteristic property of gelatinase, then, consists in the simple dissolving of gelatin, the products formed being subsequently attacked and digested by the ordinary proteolytic enzymes. The keystone of this argument would be to furnish proof that there really exist enzymes which act exclusively on gelatin, or else to show, as above, but by an irrefutable process that it is possible to separate gelatinase from other proteolytic 
enzymes. In reality, it is certain that some bacterial proteases act only on gelatin and are without effect on other albuminoid substances. But we always have very small results, which do not appear except in prolonged actions, and, under these circumstances, we may ask if the result found is not due to the special conditions of the medium which are favorable to the digestion of gelatin and unfavorable to the digestion of the other proteins, instead of being due to a true specificity of the enzyme.

This last theory finds quite a firm support in the experiments of Ascoli and Neppi. These investigators prepared gelatinase according to the method of Pollak. After having brought the solution to neutrality to methyl orange (MO), they allowed it to act on gelatin and various other albuminoids, and obtained again the results indicated by the writer, namely, that this enzyme, active on gelatin, is without action on horse serum, white of egg, and fibrin. But if in the first experiment the same liquid is made neutral to litmus $(T)$, in a second made slightly alkaline to this indicator, and finally, in a third experiment, is brought again to neutrality to methyl orange, in all these cases very different effects are observed. The liquids are always active toward gelatin, but appear endowed with very unequal activities toward other albuminoid substances.

Influence of the Reaction of the Medium on Gelatinase.

\begin{tabular}{|c|c|c|c|c|}
\hline Action of & $\begin{array}{l}\text { On gela- } \\
\text { tin. }\end{array}$ & $\begin{array}{c}\text { On } \\
\text { horse } \\
\text { serum. }\end{array}$ & $\begin{array}{c}\text { On } \\
\text { white } \\
\text { of egg. }\end{array}$ & On fibrin. \\
\hline $\begin{array}{l}\text { Gelatinase neutral to } \mathrm{MO} \ldots \ldots \ldots \\
\text { Gelatinase neutral to litmus....... }\end{array}$ & $\begin{array}{c}\mathrm{mm} . \\
9 \\
\text { II }\end{array}$ & $\begin{array}{c}\mathrm{mm} . \\
\circ \\
3\end{array}$ & $\begin{array}{c}\mathrm{mm} . \\
0 \\
6\end{array}$ & $\begin{array}{l}\text { Nothing } \\
\text { Digest. compl. }\end{array}$ \\
\hline " slightly alkal. to litmus. & 10 & $\begin{array}{l}3 \\
1.5\end{array}$ & $\ldots$ & ............. \\
\hline again made neut. to MO. & & 0 & $\circ$ & No digestion \\
\hline
\end{tabular}

It is seen from this table that the separation of gelatinase has not been realized by the method of Pollak and that everything in this assumed specificity must be referred to different conditions of the medium. Variations of a similar nature are observed 
when, instead of changing the reaction of the gelatinase, the latter is submitted to dialysis.

To summarize, the question of the individuality of gelatinase is far from being clearly answered, and, until the contrary is proved, we may class this enzyme with the other proteases studied hitherto, the property of dissolving and of digesting gelatin being common to a whole series of enzymes. Although the term gelatinase has come into the literature, it does not appear to answer to a well-defined enzyme, and it is preferable, in future, not to use it.

\section{BIBLIOGRAPHY ON THE TRYPSINS AND DIFFERENT PROTEASES.}

\section{ANIMAL AND PLANT TRYPSINS.}

V. Gorup-Besanez. In den Wickensamen, Ber. der. Deuts. Chem. Ges., 1874, p. 1478.

I. R. Green. Philos. Transactions of the Royal Soc. of London, 1887 , (178) B, p. 39.

FréDÉricQ. Bull. Acad. Bruxelles, 1878, (46), p. 213; Arch. de Zool. exp., 1878, (7), p. 399 .

Vines. Ann. of Botany, 1903, pp. 237 et 597; 1904, p. 290; 1905, p. 149; 1906, p. I7I; I908, p. I03.

Neumeister. In jugendlichen Pflanzen, Zeits. f. Biologie, r894, p. 447.

LASzczynski. Proteol. Enz. i. Malz, Centralbl. f. d. ges. Brauwezen, i899, p. 6.

LoE. Im Malz, Der Bierbrauer, I899, p. 6.

Fernbach et Hubert. Diast. protéol. du malt, $C$. $R$., I900, (I), p. I 783 .

Butkewrtsch. Im gekeimten Samen, Zeits. f. physiol. Chem., r9o1, (32), p. 9.

Windisch u. Schellhorn. Enzym in Gerste, Woch. f. Brauerei, r9oo, (r 7), p. 29.

Grimmer. Bioch. Zeits., I907, (4), p. 80.

MEYer. Antibakterienprotease, Bioch. Zeits., (32), pp. 278, 286.

Malfitano. Aspergillus niger, Ann. Inst. Past., (14), p. 60.

Harlay. Ferment protéol. du grain, $C . R ., 1900,(2)$, p. 623.

Mouton. Amibo-diastase, Ann. Inst. Past., I902, (16), p. 457.

Mesnil. Actino-protéase, Ann. Inst. Past., I9or, (1 5), p. 352.

Gerber. C. R., I9II, (I), p. I6II.

JAVILlier. C. $R$., I903, (I), p. IOI3.

LABBÉ. Du rôle des micro-organismes dans les phénomènes de digestions observées chez le Drosera rotundifilia, Thèse, Ec. Ph. Paris, 1904.

Weiss. Over Proteolytiske Enzymer, Copenhague, 1902.

KäMMERER. Zeits. f. Immunitatsforsch. u. experim. Therapie, IgI I, (1 I), p. 235.

Fiessinger et P. L. Marie. Soc. Biol., I9o9, (r), pp. 864 et 915.

Boussoure. Protéases chez les insectes, C. R., I9I I, p. 228. 
Sellier. Protéases des Céphalopodes, Soc. Biol., r907, (2), p. 705.

KRUKenBerg. Unters. d. phys. Inst. Heidelberg, 1878, (2), pp. I et 26r; 1882, (2), pp. 339 et 366 .

\section{TRYPSINS OF YEAST.}

BüCHNER. 'Die Zymasgärung, Berlin, 1907.

Salkowsky. Zeits.f. physiol. Chem., (13), p. 506; (34), p. I60.

Kutscher. Zeits. f. physiol. Chem., 1901, (32), p. 59.

Gromow. Zeits. f. physiol. Chem., 1906, (48), p. 87.

Martin Hahn. Ber. d. Dents. Chem. Ges., I898, (31), p. 200.

Wróblewsky. Journ.f. Prak. chem., (64), p. 49.

Boullanger. Ann. Inst. Past., I897, (I I), p. 720.

Beyerinck. Centralbl.f. Bact. u. Parasitenk., i 897, p. 524.

Buchner u. Klatte. Bioch. Zeits., I908, (8), p. 520.

L. Rosenthaler. Bioch. Zeits., (26), p. 4.

Lintner. Centralbl.f. Bact. u. Parasitenk., I899, (5), p. 798.

LINTNER. Microbiolog. Betribskontrol, Berlin.

Iwanow. Zeits.f. Garungsphysiol., (1), p. 23 I.

Efrront. Bull. Soc. Chim., (33), p. 847. Monit. Scient., 1905, p. 485.

\section{BACTERIAL TRYPSINS.}

K. Meyer. Ueber Antibakterienproteasen, Bioch. Zeits., (32), p. 283.

Emmerding u. Reiser. Zur Kenntniss eiweissspaltende Bakterien, Ber. d. Deuts. Chem. Ges., I902, (I), p. 700.

Tissier et Martelly. Recherches sur la putréfaction de la viande de boucherie, Ann. Inst. Past., 1902, (16), p. 865.

MesernitzKy. Bioch. Zeits., I911, (3I), p. Iog.

Hata. Centralbl.f. Bakteriologie Ref., I904, (34), p. 208.

\section{TRYPSINS OF VARIOUS ORIGINS.}

von DUngern. Münch. med. Woch., I898, (38), p. II57.

Wassermann. Zeits.f. Hygiene, I896, (22), p. 263.

Geret u. Hahn. Zum Nachweis des im Hefepresssaft enthaltenen proteol. Enzym, Ber. d. Deuts. Chem. Ges., I898, (31), pp. 202, 2335.

Macfadyen. Journ. of Anatom. and Physiol., i 892, (26), p. 409.

Fermi. Arch.f. Hygiene, (14), p. I; Centralbl.f. Bakter u. Parasit., I Abt., (52), p. 252.

Bürgers, Schermann u. Schreiber. Zeits. f. Hyg. u. Infekt. Krankh., Igir, (70), p. IIg.

\section{PAPAIN.}

Vauquelin. Examen chim. suc papayer, Ann. Chim. et Phys., 1802, (18), p. 267. WÜRTZ. C. R., I879, (89), p. 425; I880, (90), p. 1379; г880, (91), p. 787; 1881, (93), p. I I07.

Emmerling. Ber. der Deuts. Chem. Ges., igo2, (35), pp. 695 et Ior 2.

SACHs. Verdauung v. rohem Hühnereiweiss d. Papaïn, Zeits. f. physiol. Chem., $1907,(51)$, p. 488. 
Kutscher u. Lohmann. Zeits. f. physiol. Chem., i905, (46), p. $3^{8} 3$.

Abderhalden u. Terinuchi. Einwirk. auf Peptide, Zeits. f. physiol. Chem., rgo6, (49), p. 2 I.

JONESCU. Bioch. Zeits., 1906, (2), p. 177.

Claudio Fermi. Arch. d. Farmacol., igr I, (2), p. 360.

Seligmann. Zeits. f. Immunitätsforsch. $u$. Therap., I91 2, (14), p. 4 I9.

RIPPETOE. Journ. of Ind. and Engin. Chem., I912, (4), p. 517.

Stenitzer. Bioch. Zeits., I908, (9), p. 383.

Pozerski. Contr. à l'etude de la papainne, Ann. Inst. Past., r9o9, (23), p. 205.

Fermi. Ueber die wutgift-zerstörende Wirkung d. Papains u. d. Milchsaftes von Ficus carica, Centralbl.f. Bact. u. Parasitenk., I Abt., 1909, (52), p. 265, Arch. Farmacol. sperim., I9I I, (I I), p. 360 .

Mendel u. Blood. Einige Besonderheiten der proteolyt. Wirkung d. Papaīn, Journ. of Biol. Chem., ıوro, (8), p. I77, d'après Chem. Centralbl., ı9ro, (2), p. 1318 .

Harlay. Thèse, Paris, rgoo.

\section{BROMELIN.}

Pozersky. Digestion brusque produite par la broméline, Ann. Inst. Past., (23), p. $34 \mathrm{I}$.

Marcono. Bull. of Pharmacy, 1891, p. 74.

Chittenden. Journ. of Physiol., (15), p. 249.

\section{GELATINASE.}

Mesernitzky. Zersetzung der Gelatinose durch Microc. prodigiosus, Bioch. Zeits., I910, (29), p. II I.

MrNami. Ueber die Einwirkung d. Enzyme d. Magens des Pankreas. u. d. Darmschleimhaut auf Gelatine, Bioch. Zeits., I9II, (34), p. 254.

Reich-Herzberge. Zeits.f. physiol. Chem., I90I, (34), p. I2I.

Victor Henri et LARguier des Bancels. Action de la trypsine sur la gélatine et la caséine, Soc. Biol., 1903, (55), p. 866.

Mavrojannis. Soc. Biol., 1903, (55), p. 1605.

Charasow. Action de la formaline sur la gélatine fluidifiée par les fibromes, Bioch. Centralbl., Rep., 1905.

Malfitano. Soc. Biol., (55), p. 843.

Ascoli et Neppr. Ueber die Specifität d. Glutinase, Zeits. f. physiol. Chem., I908, (56), p. I35.

Pollak. Hofm. Beitr., 1905, (6), p. 95.

\section{CASEASE.}

Duclaux. Mémoire sur le lait, Ann. Inst. Agron., I882.

Bourquelot et Herissey. Bull. Soc. Myc., I899, (15), p. 60.

SARTORY. Soc. Biol., I908, (I), p. 789. 


\section{GALACTASE.}

Van Slyke, H. A. Harding et E. Hart. Journ. Amer. Chem. Soc., 1901; 1903, (25), p. 1243 .

Babcock et Russell. Centralbl.f. Bact. u. Parasitenk., 1900, (6), pp. 17, 45, 79. BÉchaMp. C. R. Acad., r894.

GLUTENASE.

Baker and Hulton. Journ. of the Soc. Chem. Ind., 1908, (27), p. 368.

G. Bertrand et Muterlich, $C$. $R$., I907, (144), pp. I 285 , et 1444 . 


\section{CHAPTER III.}

\section{ANTIGENS, ANTIBODIES, COMPLEMENT.}

\section{Products of Vital Defense and of Immunization.}

The preceding chapters on anti-pepsin, anti-trypsin and antirennet have already given us occasion to indicate how the organism reacts when different enzymes are introduced into it. In reality, the phenomenon is quite general. Each time that a foreign substance, enzyme, toxin, microbes, blood corpuscles, or various cells, penetrates into a living being, the latter opposes itself to the invasion by the elaboration of special products, which in some manner neutralize the effect of the disturbing agent. It may be said that this phenomenon is a consequence of the universal law that to every action there corresponds a reaction. This question, which is still far from being completely settled is one of those which occupies the physiologists of the present time. It raises a problem of the greatest interest, as much from the practical as from the theoretical point of view; namely, that of immunity, a problem whose solution involves among other things, the correct diagnosis and treatment of various infectious maladies.

Phenomenon of Hæmolysis. - Although a certain number of phenomena, relating to the study of antibodies come within the scope of this treatise, only brief descriptions are necessary to make clear the portions of more direct interest. The phenomenon of hæmolysis was discovered in 1899 by Bordet. If repeated injections are made into the peritoneum of an animal $A$, a rabbit for example, of the red corpuscles of an animal $B$, for example, the sheep, suspended in physiological salt solution, the blood corpuscles of $B$ being toxic for the organism $A$, the latter reacts, producing substances capable, under certain conditions, of neutralizing the injurious action of the red corpuscles. In 
fact, if at the end of a certain time, to the serum of animal $A$ thus sensitized, red corpuscles of the animal $B$ are added, it is now found that the former has acquired the power of agglutinating and then of destroying these cells. This destruction, which thus takes place in vitro, is evidenced by the fact that the hemoglobin of the blood corpuscles diffuses outside the cellular protoplasm and colors the whole of the liquid a beautiful red. The phenomenon is called hamolysis, and the serum of $A$ is said to be hæmolytic toward the species $B$.

This phenomenon must not be confounded with that observed when any blood corpuscles are placed in water. As a result of plasmolysis, the protoplasmic sac breaks under the influence of the excessive osmotic pressure and allows the contents to flow into the external liquid, which thus colors bright red. There is indeed hæmolysis but this is entirely physical and is produced only in hypotonic media. On the contrary, the Bordet phenomenon appears in a solution of serum, perfectly isotonic. In addition the hæmolytic serum of $A$ is specific; it acts only on the corpuscles of the animal species $B$, which have served by peritoneal injections in making the preparation. In the example given above, the serum of the rabbit thus sensitized will hæmolyze only the corpuscles of sheep, and not those of cattle, of guinea-pigs, of dogs, etc.

The Pfeiffer Experiment: Phenomenon of Bacteriolysis. The phenomenon just indicated is almost identical with that which had been formerly described under the name Pfeiffer Experiment. In 1894 , it was shown that if a culture of cholera vibrios is injected into the peritoneum of a guinea-pig vaccinated against cholera - a vaccination obtained by previous injection either of several successive cultures of vibrios attenuated by heat or of an anti-cholera serum - examination of a little of peritoneal fluid showed that the vibrios, instead of being developed, as would have been the case in a fresh animal, have, on the contrary, not multiplied at all - they are immobile, agglutinated, and they have the tendency to disappear. This phenomenon, which is specific for the infection experimented 
with, takes place within the organism but it is not due solely to the play of vital forces as Pfeiffer believed. In fact, it is also observed in vitro. Thus Metchnikoff, and then Bordet in 1895 , observed it with the cholera vibrio. Some years later, Dopter observed it with the dysentery bacillus, and Max Gruber with the Eberth bacillus. We know how Widal has utilized this reaction for the serum diagnosis of typhoid fever.

What is then the mechanism of this reaction, which is, as will be seen later, much more general than has so far been indicated? It is to Bordet that 'we owe the major part of our knowledge on this subject. His investigations, moreover, complete the earlier ones of Ehrlich and of Buchner, or were confirmed afterward by the researches of Ehrlich and Morgenroth, Sachs, Wassermann, and others. Bordet proved that if the hæmolytic serums of sensitized animals (for example, rabbit serum active on sheep blood), or immunized bacteriolytic serums of animals (for example, the serum of a guinea-pig immunized against the cholera vibrio), are heated higher than $70^{\circ}$, these serums lose their property either of dissolving blood corpuscles, or of agglutinating and digesting the corresponding bacteria. These facts, along with other ', establish, therefore, the enzymic nature of these bio-chemical substances elaborated by the organism for its resistance. If now these serums are not heated beyond $70^{\circ}$, but only for a half hour at $55^{\circ}-65^{\circ}$, it is found that they are still agglutinants but that they have lost their properties, either hæmolytic or bacteriolytic. However, these properties have not been destroyed; they can reappear if to these heated serums a little fresh, unheated animal serum is added. Thus the serums which have been heated to $56^{\circ}$ may be reactivated.

Sensitizer and Alexin. - How are the above facts to be explained? They are due to the existence in the serum of prepared or immunized animals, of two substances endowed with unequal resistances toward the influence of temperature, and whose combined action confers on these serums either hæmolytic or bacteriolytic properties.

The following are the names given to these two substances by 
the different writers who have investigated this problem. The explanation of the different terms will follow from the subsequent description of their properties. The substance resisting a temperature of $55^{\circ}-56^{\circ}$ bears the names of sensibilatrice (Bordet); fixater, antibody, amboceptor (Ehrlich); lysin, hamolysin or bacteriolysin (Duclaux) ; immunisin (Duclaux) or immunizing body (Ehrlich). That substance which is destroyed at the temperature of $55^{\circ}-56^{\circ}$ is called alexin (Buchner and Bordet); complement (Ehrlich); or again cytase (Metchnikoff and Buchner). Thus lysin is thermostable, while alexin is thermolabile. However, there are other differences. The sensibilatrice is a product of immunization; it is specific for red corpuscles or for injected bacteria, that is to say the agent which has caused its formation in the organism. Further, it is capable of fixing itself without injury on the red corpuscles or the corresponding bacteria, and upon them alone. On the contrary, alexin is not specific; it is a substance common to all the serums, fresh or immunized. It cannot be fixed directly on the corpuscles or the bacteria, but for this purpose requires the mediation of the sensibilatrice. It thus completes the action of this latter, since it is by means of the coöperation of these two substances that hæmolysis or bacteriolysis is made possible.

These facts may be explained by an illustration. A rabbit is prepared by 5 successive injections at 7 -hour intervals of 5 to 8 c.c. of sheep red corpuscles, washed in physiological salt solution. The serum of this rabbit will possess the ability to hæmolyze in vitro the sheep corpuscles. This serum is heated to $56^{\circ}$. The alexin is killed and the serum loses all hæmolytic power; it can still agglutinate the blood-corpuscles, but it no longer dissolves them. However, these corpuscles are capable of fixing hæmolysin which has resisted the heating at $56^{\circ}$. They are impregnated and thus become sensitized. In fact, if these corpuscles, collected by centrifuging, are washed repeatedly with an 0.8 per cent solution of $\mathrm{NaCl}$ and are then put with

* What Duclaux designates here under the name of lysin, corresponds rather to what is today called alexin. 
any unheated serum of rabbit, of sheep or of guinea-pig, these corpuscles will, in their turn, absorb the alexin of this serum and will soon undergo hæmolysis. The heated rabbit serum which has previously served for sensitizing the sheep corpuscles has been entirely deprived of its fixater if the relative quantities of each of the portions have been proportional, and can no longer serve for sensitizing fresh sheep corpuscles, nor the corpuscles of any other animal.

A different procedure is as follows: Fresh sheep corpuscles are placed in a fresh serum, that of guinea-pig for example. They will fix no trace of alexin and consequently will not hæmolyze. On the contrary, if the sheep corpuscles have been previously sensitized and are added in suitable quantities to a fresh guinea-pig serum, they will absorb the whole of the alexin contained in this serum, and will consequently make it incapable of hæmolizing a second lot of fresh sensitized sheep corpuscles. It is said that these first corpuscles have fixed the complement contained in the guinea-pig serum. It will be seen later how this property is utilized for the diagnosis of various infectious maladies. The above is an example of the phenomenon of hæmolysis. The results would have been exactly the same if cholera vibrios had been used in place of sheep corpuscles, and immunized guinea-pig serum in place of rabbit serum. The vibrios would have undergone bacteriolysis under the same conditions. As the complement is not specific but can be absorbed just as well by any sensitized.bacterial species as by sensitized red corpuscles, the two phenomena may be combined and an immunized serum may be deprived of its alexin by means of corresponding bacteria, and made incapable of causing the hæmolysis of sensitized corpuscles that are subsequently added. This is the principle of the method of fixation of the complement, to be discussed later.

Yet a reservation is necessary here. The phenomena of hæmolysis and of bacteriolysis, such as have just been described, do not occur with all this clearness except when the conditions are most favorable. In fact, the choice of animals such as the 
rabbit or the guinea-pig was not without reason. We find in fact that the serum of many animals contains natural hæmolysins or bacteriolysins toward other animal species or toward certain bacteria, and formed before any immunization. Thus the serum of eel is hæmolytic for blood corpuscles of all mammals, and human serum contains hæmolysins for sheep corpuscles. Likewise certain animals are not susceptible to certain microbic infections in consequence of the natural presence in their blood of the corresponding bacteriolysins. On the contrary, it has been observed that rabbit serum is not hæmolytic with respect to sheep corpuscles except after sensitization, and that the serum of guinea-pig contains only a minimum of natural hæmolysins with respect to the majority of blood corpuscles. Rabbit serum has, furthermore, the advantage of containing after the sensitization of the animal a small proportion of agglutinin in proportion to the hæmolysin formed, which facilitates the manipulations, especially the centrifugation of the sensitized corpuscles. Still another precaution to be taken is to isolate the corpuscles and inject these only after washing and suspending in salt solution. As a matter of fact, the serum contains albumins, whose introduction in the organism causes by the same process of vital reaction just noted the formation of special antibodies, called precipitins because of their property of giving a precipitate in the corresponding serum. Now, this would disturb the clearness of the reactions observed. In conclusion, the most favorable temperature for these manifestations is from $37^{\circ}-38^{\circ}$. At $0^{\circ}$ the phenomenon of hæmolysis does not occur. However, the sensitizer is still capable of being fixed at this temperature on the corresponding corpuscles, so that it is possible, by putting corpuscles at $0^{\circ}$ in a sensitized and unheated serum and by centrifuging after 24 hours, to isolate the antibody of this serum and preserve the alexin in solution. This process of separation is sometimes used in laboratories.

Bordet's Conception - Side Chain Theory of Ehrlich. As to the actual mechanism of these hæmolyzing or bacteriolyzing actions, two hypotheses have been formulated. The first, 
due to Bordet, likens the preceding phenomena to those of dyeing. According to Bordet, alexin is similar to a dye which cannot fix itself on the material to be dyed, the red corpuscle or the bacterium, until the latter has been supplied with a mordant by the sensitizer. This special mordant is specific for the material to be dyed. On the contrary, the dye would be common and capable of coloring different materials supplied with a mordant. The analogy of the phenomena of hæmolysis or of bacteriolysis to that of dyeing is continued also in that the sensitized corpuscles are capable of depriving the serum of the whole of its alexin, exactly as a stuff supplied with a mordant drains the bath of its dye.

The second hypothesis, due to Ehrlich and Morgenroth; is an extension and adaptation to the phenomena of immunity of the general theory of Ehrlich, known under the name of side chain theory. According to Ehrlich, living matter is composed of cellular units formed around a sort of fundamental nucleus, and possessing a multifude of specific functional groups that form the extremities of the lateral chains or receptors. These receptors play an active part in the various life processes, especially in that of nutrition, for they are charged with att acting and fixing on the protoplasmic molecule the assimilable particles which serve for the maintenance and the reconstruction of the living cell. To them Ehrlich attributes a very important part in the defense of the organism against various poisons, and consequently in immunization. $\mathrm{He}$ believes that the molecule of toxin contains a toxic group, the toxophore, and another group capable of combining with the receptors or fixing agents of the living cell, to which latter group he gives the name of haptophore. For a toxic molecule to exert its injurious action on a cell, it is necessary that it be first fixed by its haptophore group upon the corresponding receptor of this same cell. The free toxophore group can then act.

Ehrlich further believes that the number of these functional groupings is very large and is capable of increasing if the vital conditions are favorable. It appears that, if in consequence of a penetration into the organism of any toxin, all or almost all 
of these receptors have been saturated by the haptophore functions of the toxin, the cell will be killed. But if the number of receptors saturated is small and insufficient to compromise the vitality of the cell, the latter will defend itself by forming new receptors and will even exceed the requirement, so that the lateral chains, very much multiplied, will become detached from the generating cell and scattered in the fluids, for example the blood. These free receptors then become capable of fixing on themselves toxins introduced into the organism, and form true antitoxins. Thus we understand how, by arranged injections of a poison into an animal, we succeed in developing in it a very great resistance against the action of this poison, and in conferring an immunity which is even transmissible to a certain degree to other animals.

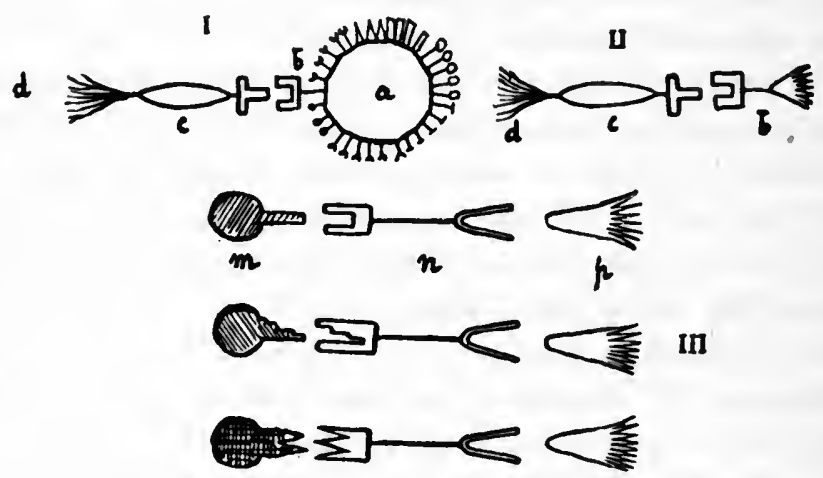

Figure I: Toxin $c$ saturates one of the functional groups $b$ of the cell $a ; d$ is the toxophore group.

Figure II: The functional group $b$ has left the cell $a$ and has become the antitoxin of the toxin $c$.

Figure III: The various microbes or blood corpuscles are represented by $m$, of variable form. The sensitizers $n$ have antigenaphile groupings, capable of exactly adapting themselves to the corresponding receptors of $m$. As to the complementophile groupings, they are all the same. The action on $m$ of alexin $p$ is possible only after reunion of these three parts, $m, n, p$. 
Ehrlich and Morgenroth have thus adapted the preceding theory to the phenomena of hæmolysis and bacteriolysis. The amboceptor or antibody, formed in the prepared or immunized animal by the liberation of the side chain corresponding to the inoculated blood corpuscles or microbes, has two functional groups. One, the haptophore or antigenophile group, is specific, and of such form that it can adapt itself to the receptor grouping of the corresponding microbe or blood corpuscle; the other, the complementophile group, is destined to receive the alexin. It is of the same form in all the amboceptors. The alexin or complement is of a unique form, capable of fixing itself by its amboceptophile group on all the amboceptors. The other group, in the form of a plume or of a torch, is the zymophore group to which the complement owes its digestive action toward microbes. According to Ehrlich, these fixations are the result of a true chemical attraction and will act like chemical combinations. The amboceptor will have a great affinity for the corresponding bacteria or blood corpuscles, which will permit it to combine with it even at a temperature of $0^{\circ}$, while its complementophile group will have a much less affinity for alexin. The preceding figures, and their discussion, show how the various facts which have just been described may be represented diagrammatically.

Precipitins and Anti-precipitins. - Up to the present, we have spoken almost exclusively of phenomena of hæmolysis and bacteriolysis. In reality, the reaction which an organism opposes to the introduction of any foreign substance is of a still more general order. We have already had occasion in other chapters to speak of anti-enzymes, anti-rennet, anti-pepsin, and other antibodies which appear in the serum of an animal in which an injection of the corresponding enzyme has been made, a subject that will be discussed later. There is, however, another kind of antibody which has been only mentioned in the preceding pages and which merits further discussion here, namely, the precipitins.

Kraus, in 1897 , first discovered the existence of such substances. Since their discovery they have been studied by 
Bordet, Tchistowitch, Ehrlich, Wassermann and others, and the number now known is very large. If there is injected into an animal a solution of any albumin, serum, milk, egg-albumin, or of a broth of filtered culture, it is found that the serum of the animal inoculated has acquired the property of precipitating the solutions of albumin used to make the injection. These precipitins are specific; for example, we have sero-precipitins, lacto-precipitins; anti-globulin precipitins, anti-albumose precipitins, etc.

These substances have properties very similar to those of the coagulating enzymes. They are, however, distinguished by their mode of action. While the enzymes act catalytically, without appearing finally in the products of the reaction, the precipitins on the contrary really combine with the substances which they precipitate, as Myers, Michaelis, Eisenberg and many others have found. In this, moreover, they act as do the various products of vital reaction, hæmolysins, bacteriolysins, etc., which have just been studied. They also resemble the latter substances in their behavior toward a rise in temperature. Although this effect is less clear with precipitins than in the case of the preceding lysins, it has nevertheless been established that the precipitating power of a serum is due to the combined action of two substances possessing an unequal resistance to the action of heat. In general, the precipitins withstand easily an elevation of temperature and are destroyed only at temperatures about $70^{\circ}$. Thus, it has been found that a precipitin, for example lacto-precipitin found in milk, heated to $70^{\circ}$ no longer has the power of precipitating the solution of corresponding albumin, but that moreover, when added to this liquid it hinders precipitation with a fresh lacto-serum precipitant, whose effect is thus annulled. Thus there is produced an inactivated anti-serum, in the same way as heating a toxin to a suitable temperature produces a toxoid or attenuated toxin, a non-toxic substance but one capable of neutralizing in vitro the corresponding antitoxin, or capable of causing this antitoxin to appear in the serum of an animal into which it is inoculated. 
In addition, a precipitin, for example lacto-serum of rabbit, the precipitant of goat milk, is a poison for an organism sensitive to its action, consequently for the goat itself. It appears that this organism reacts by elaborating an anti-precipitin capable of preventing the precipitin from reacting. This anti-precipitin will then oppose the precipitation of goat milk by the lactoserum inoculated, and furthermore, added in vitro to the corresponding lacto-serum, it will neutralize its effect. In the same manner, moreover, a serum hæmolytic for one species (rabbit serum hæmolytic for sheep corpuscles), injected in sheep, will cause in this animal the formation of an antibody capable of opposing the hæmolysis of its corpuscles under the influence of the original serum. This kind of second order antibody is formed, as Bordet has recognized, by an anti-hamolysin, antialexin or anti-cytase, specific for the corresponding active bodies.

Anti-alexin. - Ehrlich has found that if there is injected into an animal a normal serum of another species, previously heated to $55^{\circ}$ and consequently deprived of its alexin, this serum nevertheless brings about in the organism so treated the appearance of an anti-alexin. This, added to normal serum, will saturate the alexin which is contained and will consequently hinder the latter from inducing the hæmolysis of sensitized corpuscles which are put with it. This result shows that the effect of heat has not been to destroy completely the alexin as might be supposed, but only, according to Ehrlich, to deprive it of its digestive power, in other words, of its zymophore grouping. This is exactly what takes place when a toxin is heated moderately. Its toxophore grouping is destroyed and there is formed, as seen above, a non-toxic toxoid, capable nevertheless of bringing about in an organism the formation of the corresponding antitoxin. This property is, moreover, the basis of one of the most popular methods for conferring a definite immunity on an individual.

Toxins and Poisons: Their Antibodies. - In addition to anti-enzymes, precipitins, anti-precipitins, anti-hæmolysins and antialexins, there also exist many other antibodies. Thus it 
is that the microbic toxins, such as the diphtheric toxin, the tetanic toxin, etc., injected into an animal cause the appearance of specific antitoxins or immunizing serums in the serum of the latter, as indicated in the work of Roux, Behring, Kitasato and of many others. Likewise, the inoculation of vegetable toxins, like abrin or ricin, also develops by vital reaction in the subjects treated the power of producing specific antibodies (Warden, Ehrlich, etc.).

Finally, the poisons secreted by some serpents, batrachia, fishes, etc., are also capable, like the preceding toxins, of being transformed into vaccines, for example by reducing their virulence under the action of heat and thus conferring immunity (Calmette, Roux, Phisalix, and Bertrand, etc.).

Cyto-toxins. - In the last examples the inoculations were made with organic substances, of cellular origin without doubt but entirely dissolved and deprived of every particle in suspension. The reaction will take place also if cellular elements are injected directly. We have already seen how an organism reacts to the introduction of blood-corpuscles of species other than its own or of various microbes. It at once secretes agglutinins, kinds of coagulating enzymes, more or less specific, and then lysins which digest and dissolve progressively the foreign body introduced. A like phenomenon is observed if an animal is submitted to repeated injections of white corpuscles, spermatozoids, nerve cells, hepatic cells, etc. The serum of the animal thus prepared acquires the power first of agglutinizing, and then of rapidly dissolving these various cells, and is thus leuco-toxic, spermo-toxic, neuro-toxic or hepta-toxic, etc.

Metchnikoff has given the name of cyto-toxins to the active substances contained in these serums. They have been studied by this investigator as well as by Bordet, Lansteiner, Moxter and others. These different investigators have recognized that these cyto-toxic serums are composed of specific cyto-agglutinins and cyto-lysins, these last requiring in order to act the concurrence of alexin, common to all fresh or prepared serums, exactly as was previously necessary for the action of hæmolysin or of bacteriol- 
ysin. Metchnikoff has found, moreover, that we can prepare antibodies of these substances (Ex., anti-leucotoxin) by injecting a leuco-toxin in repeated small quantities into an animal. The serum of the latter acquires the property of neutralizing the action of the leuco-toxin inoculated.

Finally, the manner of formation of these substances in the organism is general and very simple. Each time that we introduce into a living being, in the form either of a solution or of solid particles, a foreign substance toxic for it, the former immediately reacts by secreting an antagonistic substance having for effect the incorporation and solution of the insoluble parts and the precipitation or neutralization of the soluble poisons. In order to act, all these antibodies require a complement which exists in all the unheated sera. In contrast to the term of antibody, the name of antigen has been given to the immunogen substances capable of causing the formation of antibodies in an organism so treated.

\begin{tabular}{|c|c|}
\hline Injected substances. & $\begin{array}{l}\text { Antagonistic substances elab- } \\
\text { orated. }\end{array}$ \\
\hline 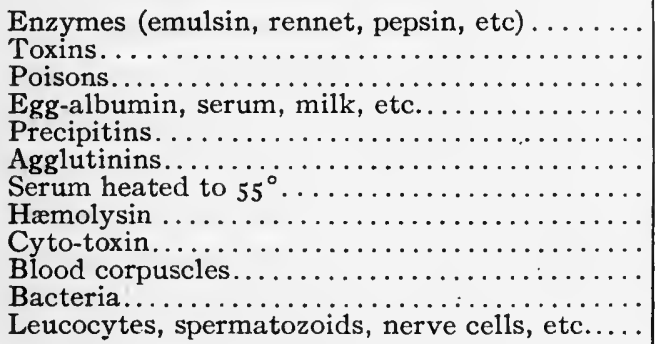 & $\begin{array}{l}\text { Anti-enzymes } \\
\text { Antitoxins } \\
\text { Anti-poisons } \\
\text { Precipitins } \\
\text { Anti-precipitins } \\
\text { Anti-agglutinins } \\
\text { Anti-alexin } \\
\text { Anti-hæmolysin } \\
\text { Anti-cytotoxin } \\
\text { Hæmolysins } \\
\text { Bacteriolysins } \\
\text { Cyto-lysins }\end{array}$ \\
\hline
\end{tabular}

What is now the nature of these three factors for immunity? We do not know. All that we can say is that on the one hand alexin or cytase appears to come from the cells, and especially from leucocytes whose important rôle in phagocytosis is known, and that on the other hand all the antigens, in spite of their great diversity, bear a certain relation to the albuminoids. Thus the vegetable toxins and the poisons, protein in nature, are still capable of producing antibodies, while the alkaloids, likewise toxic for the cells but of quite different chemical nature, do not 
produce antibodies. As to the formation itself of these antibodies or sensitizers, we can say that it apparently obeys a very general law. Not only in the inorganic world do we see a broken crystal repair the facets removed and even grow additional ones if we plunge it in its mother liquor, but also in the animal world we find that a destruction or a suppression of a certain number of cells of an organism at once brings about by vital reaction an excess development of those which remain; there is an overproduction so that the wound is soon enclosed with a scar pad, and in the case of certain batrachia, for example, the amputation of a member causes the appearance of two or more others. It is by virtue of this increase of vital activity, an observation developed by Weigert under the name of Theory of Overproduction, that the lateral chains of Ehrlich, partially saturated by a foreign toxic substance, can reform and multiply the more - up to a certain limit - the more repeated the attacks against the cell.

Relation Between the Phenomena of Vital Reaction and Those of Digestion. - It would seem possible to unite into a single concept all these facts, which, though they appear to be very complex, in reality relate to a single mode of vital defense. The organism, submitted to an attack, fights the foreign substance introduced, bacterium or toxin, by dissolving and by digesting it. The whole is only a digestion phenomenon. Only albuminoid material is susceptible of being digested, and every known antigen is albuminoidal. Now, the proteolytic cnzyme that possesses this function of dissolving the antigen is alexin, an enzyme that exists in every natural or prepared serum. In order to react, this enzyme requires a fixing agent, which is moreover specific to the antigen considered. The preparation or immunization will consist only in producing in the organism treated this sensitizing substance capable of rendering active the alexin already present. Properly speaking, the substance really acting would be alexin; the sensitizer of Bordet would be rather the complement.

In fact, this necessity of a second product to allow proteolytic action to appear is not surprising, nor is it new. It is found in the 
influence which calcium salts exert on the different enzyme coagulations, especially on that of casein by rennet, or again in the indispensable part which is played by acids in the activity of pepsin on other enzymes. It is true that these complementary substances are not specific and that, further, they resist the action of heat. There is, however, another enzyme, trypsin, which in a pure state, as Delezenne has shown, is without action on albuminoid material. It digests them, on the contrary, if they are impregnated with a second enzyme complementary to the first, the enterokinase of Pawlow. This active substance sensitizes the protein materials and thus allows trypsin to become fixed and, consequently, the phenomenon of proteolysis to take place.

Related Substances, Isolysins, Isoprecipitins, Anaphylaxis, etc. - Although the results obtained in this domain of vital reactions are already very numerous and can be co-ordinated into a sufficiently well established theory, there are still many facts to be first confirmed and then explained and many questions to be elucidated. Only a brief mention will be made here of some of these.

In the preceding discussions, it has been said that alexin was found in all sera. Generally these sera give typical alexin reactions, but there are cases where they appear to have a relative specificity, so that certain writers strongly urge a plurality of the cytases. Moreover, it is known that the anticytases are specific for the serum of the animal which served to make the preparation. The alexins would then have a sort of reduced specificity, analogous to that previously mentioned in connection with fibrinase.

There is still another series of vital reactions that remain as yet undiscussed. Each time that it was desired to prepare an antibody, a species different from that which furnishes the immunogen substance was taken. Now, it has been found in special cases that not only the same species can react but also the same individual. Thus the blood corpuscles of goat $A$, injected into a goat $B$, cause the formation of isolysins in the serum of $B$, capable of dissolving the corpuscles of the goat $A$ and of a certain number of other goats, but not of all goats. 
Moreover, to these isolysins there correspond anti-isolysins, specific for the isolysins under consideration. There exists then a difference between the cells identically situated in the same species, those of the individual $A$ being biologically different from those of the individual $B$. Likewise, isoprecipitins are known, for example, substances obtained, by injecting serum from rabbit $B$ into another rabbit $A$. The serum of $B$ becomes a precipitant for that of $A$ and for those of a certain number of other rabbits. In addition, autoantibodies are known, for example autolysins, substances which confer on the serum the ability to dissolve the blood corpuscles of the same organism which produced them. Likewise the existence of autoprecipitins has been advanced by certain authors.

In conclusion, there remains still the closely related subject of anaphylaxis. This phenomenon, discovered in 1902 by $\mathrm{Ch}$. Richet and studied successively by Arthus, Pirquet and Schick, Th. Smith, Besredka and others, is characterized by the curious property which certain poisons possess, of increasing instead of diminishing the sensitiveness of the organism to their action. We find, in fact, when we undertake in the usual way the sensitization of animals by repeated injections of corpuscles, bacteria, or of toxins, that the greater part of the organisms so treated soon accustom themselves to them, and then become immune, but that on the contrary a small proportion acquire a hypersensitiveness so that they are rapidly killed by the poison in quantities much smaller than that which is fatal for fresh animals. In fact, it would seem that the toxic substance injected had caused anaphylaxis in all the individuals, but that this had disappeared more rapidly in some cases than in others. Where it still persists, later injections have caused death in place of immunity. It appears from various publications on the question that anaphylaxis is due to special antibodies (toxogenic substance. of Richet or sensibilisin of Besredka), to which anti-anaphylactic substances correspond. It should be added that, according to Richet, anaphylaxis is the first stage of prophylaxis; it hastens the reaction of the organism against microbic poisons and thus 
causes the more rapid formation of antitoxins, from whence will result immunity.

\section{APPLICATIONS.}

I. Application of Precipitins. - Although most of the methods based on the use of precipitins that have been proposed for the characterization of the different albuminoids have proved uncertain or erroneous, there are some which are fairly accurate and which render great service. Among these may be mentioned the Wassermann test for the identification of blood. If, for example, it is desired to determine if suspected spots are due to human blood, a solution of the incriminated spot in salt water is tested with a serum precipitant for human blood. If the reaction is positive, it must be remembered, however, that other human albuminoid materials, like milk, pus, saliva, albuminous urines, etc., may also give a precipitate under these conditions. This reaction is characteristic of man, with the exception that the blood of anthropoid apes also reacts like that of man.

Analogous procedures have been proposed for the identification of butcher's meats (Jess and Uhlenhuth), for the identification of bones, for the determination of commercial albumins in fraud investigations, etc.

2. Application of Agglutinins. - It has already been seen that the injection of blood corpuscles or microbes into an animal develops in addition to hæmo- or bacteriolysins, an agglutinin, a substance on which has developed the rôle of agglutinating the foreign cellular elements, which the lysins then dissolve. The absolute specificity of these agglutinins is disputed. However, there are cases (Ex., the Eberth bacillus), where this specificity is real. The phenomenon of agglutination has been utilized for the diagnosis of a certain number of infectious diseases. Widal, in particular, has thus developed his method of the sero-diagnosis of typhoid fever, which renders valuable service. This investigator has recognized, in fact, that this phenomenon of agglutination is relatively precocious in the course of this disease, that it appears from the beginning of the infection and that it thus permits a 
diagnosis of the nature of the evil at the very beginning. These results have been since extended to other pathogenic bacteria, especially by Arloing and Courmont to the tuberculosis bacillus.

3. Fixation of the Complement-Method of Bordet and Gengou. - Let us recall in a few words the principle of this method. Take on the one hand a culture of a certain virulent microbe $M$, and on the other hand a serum $S$ of an animal rendered immune against this pathogenic agent. The culture is introduced into the serum which has been previously heated to $56^{\circ}$. The microbes are sensitized in such a way that by next placing them in a fresh guinea-pig serum they absorb all the alexin there contained. On the other hand, sensitized blood corpuscles, for example corpuscles of sheep previously immersed in a prepared rabbit serum, when heated to $56^{\circ}$ and introduced into the guinea-pig serum, which has been previously deprived of its complement by the microbes, will not be hæmolyzed, the corpuscles not being able to fix the absent alexin. This reaction is said to be positive, for the microbes $M$ have effectively fixed the complement of the guinea-pig serum. If, on the contrary, the microbes $M$ and the immunizing serum $S$ did not correspond to the same pathogenic agent, the microbes not being sensitized in the heated serum $S^{\prime}$, will not be able to fix the complement of the guinea-pig serum, and the hæmolysis of the red corpuscles will become possible. The reaction is then said to be negative. This method (Bordet and Gengou) can be used, either to determine the nature of certain antibodies contained in a serum, in other words to diagnose the infectious disease which produced them, or to identify the species of certain antigens.

The advantage of this method lies in its simplicity, a simple examination of the tubes without the aid of the microscope alone being required to determine hæmolysis. However, a certain technique is evidently necessary to prevent errors of interpretation and permit this method to give all the results which may be expected. In particular, it is indispensable to measure previously the activity of the various reagents in order to know in what proportions they must be used. It is clear, in fact, that if the 
quantity of antigen sensitized is too small relative to that of guinea-pig serum utilized, all the alexin will not be absorbed, the deflection will not be complete and a partial hæmolysis of the red corpuscles may result, leading to a wrong conclusion. On the other hand, too much antigen must not be used, for this, even without the aid of its sensitizer, is capable of absorbing a little alexin, and consequently of slightly fixing the complement.

In practice, the procedure is as follows:

First determine:

(I) The value of the elements of the hæmolytic system, constituted ordinarily by blood corpuscles of sheep and prepared rabbit serum, heated to $56^{\circ}$ (corpuscles + sensitizer). The strength of these two reagents must be such that, by addition of the necessary complement, hæmolysis will take place in a halfhour at $37^{\circ}$.

(2) The value of the alexin power of a guinea-pig serum (sensitized corpuscles + guinea-pig alexin);

(3) The absorption power or power of spontaneous fixation - of the non-sensitized antigen (antigen + guinea-pig alexin).

All these reactions take place in small tubes at $37^{\circ}$, if necessary diluting the liquids with a physiological salt solution to bring the volume up to 2 c.c.

Prepare the following mixture in the proportions determined by the previous tests:

(a) Specific antigen + serum to be studied (previously heated a half-hour at $55^{\circ}-5^{\circ}$ ) + fresh guinea-pig serum rich in alexin. The whole is left for I hour at $37^{\circ}$. Also prepare a series of controls.

(b) Add to the different tubes the following hæmolytic system, made up of sheep corpuscles in suspension in a rabbit serum hæmolytic for these corpuscles but previously heated to $5^{\circ}$. These as well as the controls are put back in the incubator for a half-hour, and the appearance of hæmolysis determined. If at the end of a half-hour the mixtures have remained intact in the first tubes while the controls are completely hæmolyzed, the reaction is called positive. 
The following is, according to P. F. Armand-Delille (Techniques du diagnostic par la méthode de déviation du complément, Paris, I9I I, p. 99), the typical arrangement of an experiment for the fixation of the complement with the necessary controls. In this experiment for antigen, use is made of a culture of cholera vibrios belonging to a species deprived of hæmolytic properties; for antibody, an anti-cholera serum, such as that prepared by Salimbeni at the Pasteur Institute; for alexin, guinea-pig serum, collected the day before, diluted one-quarter in physiological salt solution or pure water if it dates back more than eight days (being preserved in a refrigerator); finally, for hæmolytic system, sheep corpuscles washed three times with salt water, brought to the volume of the original blood, then diluted to 5 per cent in physiological salt solution, and rabbit serum prepared by intraperitoneal injections of sheep corpuscles.

I. Study of the Fixation of the Complement by the Cholera Vibrios.

\begin{tabular}{|c|c|c|c|c|c|c|c|}
\hline \multirow[b]{2}{*}{ Tests. } & \multicolumn{4}{|c|}{ I. } & \multicolumn{3}{|c|}{ II. } \\
\hline & $\begin{array}{l}\text { Emul- } \\
\text { sion of } \\
\text { vib. }\end{array}$ & $\begin{array}{l}\text { Anti- } \\
\text { chol. } \\
\text { serum } \\
\text { heated } \\
\text { to } 55^{\circ} \text {. }\end{array}$ & $\begin{array}{l}\text { Alex. } \\
\text { serum } \\
\text { of } \\
\text { g. pig, } \\
\text { new, } \\
\text { dil. } 1 / 4 .\end{array}$ & $\begin{array}{l}\text { Phy- } \\
\text { siol. } \\
\text { solu- } \\
\text { tion. }\end{array}$ & $\begin{array}{l}\text { Sheep } \\
\text { corp. } \\
\text { dil. to } \\
5 \text { per } \\
\text { cent. }\end{array}$ & $\begin{array}{l}\text { Hæmol. } \\
\text { rabbit } \\
\text { serum } \\
\text { heated } \\
\text { to } 55^{\circ} .\end{array}$ & $\begin{array}{l}\text { Results after a } \\
\text { half-hour at } \\
37^{\circ}-38^{\circ} \text {. }\end{array}$ \\
\hline Tube $a$ & $\begin{array}{l}\text { c.c. } \\
\text { o.I }\end{array}$ & $\begin{array}{l}\text { c.c. } \\
0.2\end{array}$ & $\begin{array}{l}\text { c.c. } \\
\text { o.I }\end{array}$ & $\begin{array}{l}\text { c.c. } \\
0.5\end{array}$ & $\begin{array}{l}\text { c.c. } \\
\text { I }\end{array}$ & $\begin{array}{l}\text { c.c. } \\
\text { o.I }\end{array}$ & Slight hæm. \\
\hline "b.... & 0.2 & 0.2 & 0.1 & 0.4 & I & 0.1 & No \\
\hline$" \quad c \ldots \ldots$ & 0.3 & 0.2 & O.I & 0.3 & I & O.I & No " " \\
\hline 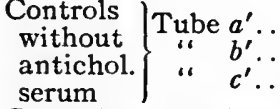 & $\begin{array}{l}0.1 \\
0.2 \\
0.3\end{array}$ & $\begin{array}{l}0.0 \\
0.0 \\
0.0\end{array}$ & $\begin{array}{l}0.1 \\
0.1 \\
0 . I\end{array}$ & $\begin{array}{l}0.7 \\
0.6 \\
0.5\end{array}$ & $\begin{array}{l}\text { I } \\
\text { I } \\
\text { I }\end{array}$ & $\begin{array}{l}0.1 \\
0.1 \\
0.1\end{array}$ & $\begin{array}{l}\text { Total hæm. } \\
\text { Almost “" }\end{array}$ \\
\hline $\begin{array}{l}\text { Control, serum alone } \\
\text { Control, hæmolytic } \\
\text { system.......... }\end{array}$ & $\begin{array}{l}0.0 \\
0.0\end{array}$ & $\begin{array}{l}0.2 \\
0.0\end{array}$ & 0.1 & 0.7 & I & $\begin{array}{l}0.1 \\
0.1\end{array}$ & $\begin{array}{c}\text { Total hæm. } \\
\text { ॥ } ~\end{array}$ \\
\hline
\end{tabular}

Table I having shown that anti-cholera serum fixes the complement in the presence of the cholera vibrios used as antigen, it is proposed in Table II to test the richness in antibody, that is to say, the power of fixing or deflecting the anti-cholera serum. For that purpose, the necessary and sufficient quantity of antigen (in the actual experiment 0.2 c.c. of the emulsion of vibrios) is placed in contact with varying quantities of anti-cholera serum, 
making besides a control series with normal heated serum (of the same kind). From Table II it is seen that the deflection is already very large with 0.1 c.c. of serum, but that it is not absolute until 0.2 c.c. were used. On the contrary, the normal serum is not deflected until larger quantities, for example 0.4 to 0.5 c.c., were used and then only slightly.

II. Titration for the Quantity of Antibody in ANTi-cholera

SERUM.

\begin{tabular}{|c|c|c|c|c|c|c|c|}
\hline Tests. & $\begin{array}{l}\text { Emul- } \\
\text { sion of } \\
\text { vib. }\end{array}$ & $\begin{array}{l}\text { Anti- } \\
\text { chol. } \\
\text { serum, } \\
\text { heated. }\end{array}$ & Alexin. & $\begin{array}{c}\text { Phy- } \\
\text { sio!. sol }\end{array}$ & $\begin{array}{c}\text { Cor- } \\
\text { puscles }\end{array}$ & $\begin{array}{l}\text { Ham. } \\
\text { serum, } \\
\text { heated. }\end{array}$ & Results after hour at $370^{\circ}$ \\
\hline $\begin{array}{c}\text { Sample } \\
\text { "، } \\
\text { “ } \\
\text { " }\end{array}$ & $\begin{array}{l}\text { c.c. } \\
0.2 \\
0.2 \\
0.2 \\
0.2 \\
0.2\end{array}$ & $\begin{array}{l}\text { c.c. } \\
0.1 \\
0.2 \\
0.3 \\
0.4 \\
0.5\end{array}$ & $\begin{array}{l}\text { c.c } \\
0.1 \\
0.1 \\
0.1 \\
0.1 \\
0 . I \\
0.1\end{array}$ & $\begin{array}{l}\text { c.c. } \\
0.5 \\
0.4 \\
0.3 \\
0.2 \\
0.1\end{array}$ & $\begin{array}{l}\text { c.c. } \\
\text { I.0 } \\
\text { I.O } \\
\text { I.O } \\
\text { I.O } \\
\text { I.0 }\end{array}$ & $\begin{array}{l}\text { c.c. } \\
0.1 \\
0.1 \\
0.1 \\
0.1 \\
0.1\end{array}$ & $\begin{array}{l}\text { No hæmolysis (slight) } \\
\text { " } \text { " fixation } \\
\text { of complement } \\
\text { No hæmolysis } \\
\text { “ }\end{array}$ \\
\hline & & $\begin{array}{l}\text { Nor- } \\
\text { mal } \\
\text { serum } \\
\text { heated. }\end{array}$ & & & & & \\
\hline $\begin{array}{c}\text { Control } \\
، \\
، \\
،\end{array}$ & $\begin{array}{l}0.2 \\
0.2 \\
0.2 \\
0.2 \\
0.2\end{array}$ & $\begin{array}{l}\text { c.c. } \\
0.1 \\
0.2 \\
0.3 \\
0.4 \\
0.5\end{array}$ & $\begin{array}{l}0.1 \\
0.1 \\
0.1 \\
0.1 \\
0.1\end{array}$ & $\begin{array}{l}0.6 \\
0.5 \\
0.4 \\
0.3 \\
0.2\end{array}$ & $\begin{array}{l}1.0 \\
1.0 \\
1.0 \\
1.0 \\
1.0\end{array}$ & $\begin{array}{l}0.1 \\
0 . I \\
0 . I \\
0 . I \\
0 . I\end{array}$ & $\begin{array}{l}\text { Total hæmolysis } \\
\text { "، } \\
\text { Incomplete "، } \\
\text { Partial " }\end{array}$ \\
\hline
\end{tabular}

III. Control (Sample Observation Based on Preceding and CONTROL).

\begin{tabular}{|c|c|c|c|c|c|c|c|}
\hline & $\begin{array}{l}\text { Vi- } \\
\text { brios. }\end{array}$ & $\begin{array}{l}\text { Anti- } \\
\text { chol. } \\
\text { serum, } \\
\text { heated. }\end{array}$ & Alexin. & $\begin{array}{l}\text { Phy- } \\
\text { siol. sol. }\end{array}$ & $\begin{array}{c}\text { Cor- } \\
\text { puscles. }\end{array}$ & $\begin{array}{l}\text { Hæm. } \\
\text { serum, } \\
\text { heated. }\end{array}$ & $\begin{array}{c}\text { Results after thour at } \\
\text { at } 37^{\circ} \text {. hour }\end{array}$ \\
\hline \multirow[t]{2}{*}{$\left.\begin{array}{c}\text { Sample ob- } \\
\text { servation }\end{array}\right\}$} & $\begin{array}{l}\text { c.c. } \\
0.2\end{array}$ & $\begin{array}{l}\text { c.c. } \\
0.2\end{array}$ & $\begin{array}{l}\text { c.c. } \\
\text { O. I }\end{array}$ & $\begin{array}{l}\text { c.c. } \\
0.4\end{array}$ & $\begin{array}{l}\text { c.c. } \\
\text { I .O }\end{array}$ & $\begin{array}{l}\text { c.c. } \\
\text { o.I }\end{array}$ & No hæmolysis \\
\hline & $\begin{array}{l}\text { Bacilli } \\
\text { (Eberth) }\end{array}$ & & & & & & \\
\hline \multirow[t]{2}{*}{ Control..... } & $\begin{array}{l}\text { c.c. } \\
0.2\end{array}$ & 0.2 & 0.1 & 0.4 & I.O & 0.1 & Total hæmolysis \\
\hline & Vibrios. & & & & & & \\
\hline Control..... & $\begin{array}{l}\text { c.c. } \\
0.2\end{array}$ & 0.2 & 0.1 & 0.5 & I.O & 0.1 & Total hæmolysis \\
\hline
\end{tabular}


Finally, it remained to demonstrate the specificity of the reaction to prove that anti-cholera serum alone fixes the complement in the presence of the corresponding antigen, that this antigen does not absorb alexin if it is not given an amboceptor, and finally that the anti-cholera serum is not fixed on alexin in the presence of another antigen. The control table indicates that while the anti-cholera serum fixes the complement in the presence of the necessary quantity of cholera vibrios, it remains without effect if another antigen is used, such as Eberth's bacillus (taken in a quantity which in the presence of 0.2 c.c. of anti-typhoid serum fixes the complement). It shows further that another specific serum, the anti-typhoid serum, does not fix the complement in the presence of the same quantity of vibrios, since the fixation of the complement easily occurs in the presence of the corresponding antigen (emulsion of Eberth's bacilli).

The method of Bordet and Gengou has been applied to the diagnosis of a certain number of diseases and of the specificity of some microbic species. Thus Widal and Lesourd have applied it to the study of the Eberthian infection, especially for diagnosing typhoid fever and establishing a distinction between true typhoid and a paratyphoid; Cohen, of Brussels, has utilized it for the diagnosis of cerebro-spinal meningitis; Dopter made use of it in the study of dysentery; Bordet has used it to verify the specificity of the microbe of whooping cough, etc. Finally, Wassermann has utilized this method for the diagnosis of syphilis. This application being the most extensive and the most practical, we shall say a few words about it in concluding this chapter.

It should be noted, first of all, that in all these fixation procedures, we can substitute, for bacterial bodies the extracts of these substances or toxins, as antigen. Thus tuberculin permits the examination of the antibodies contained in the serum of tuberculous subjects. Likewise for syphilis, the extracts of syphilitic organs can be used as antigens, and in the presence of the antibodies contained in the serum of infected individuals, will produce the fixation. Since the publication of the work of Wassermann, there have been numerous modifications introduced 
in the original method. The following is the usual procedure for the Wassermann Test. As syphilitic antigen (derived from Treponema pallidum), use preferably an alcoholic solution of fœtus liver or of a hereditary syphilitic new-born child. First make sure that it does not hinder hæmolysis, even in larger quantities (double doses) than those required for the reaction, and that further it fixes the complement in the presence of a serum syphilitic to the desired degree. Then test the absorbing power of the antigen alone toward alexin. As to the serum, it will be obtained by coagulation of the blood of the patient to be examined. This blood will have to be taken aseptically, either by direct puncture of a vein, for example at the bend of the elbow, or by means of cupping with scarification. The quantity

Example of Wassermann Reaction.

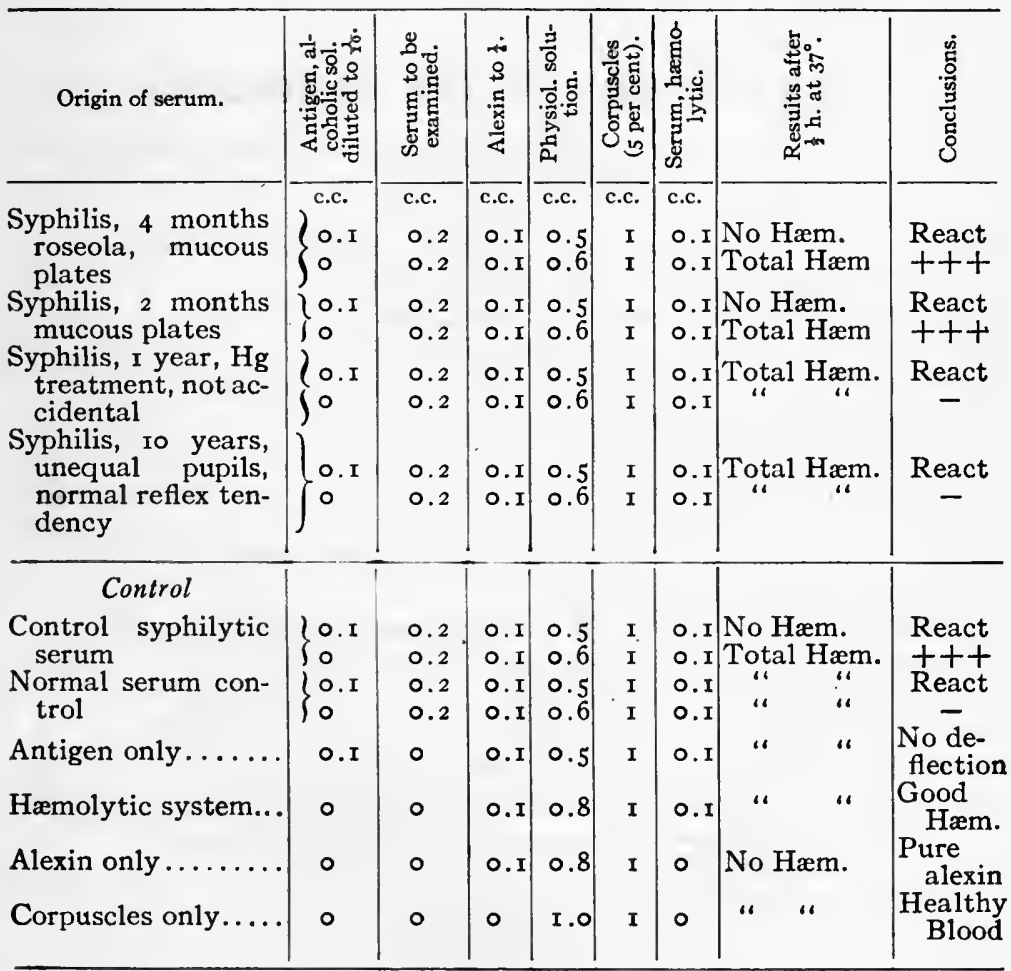


of blood collected must be from 8 to ro c.c. Coagulation will take place in the dark, but not in the refrigerator. When the clot is well retracted, at the end of about 24 hours decant the serum with a sterile pipette, distribute it in tubes, and heat these 30 minutes at $55^{\circ}-56^{\circ}$. After that, this serum may be immediately studied or preserved several days in the refrigerator. If the tubes were perfectly sterile, they may be kept thus for months or years without losing their antibodies. Finally, the alexin and the hæmolytic system are the same as those used in the general method.

The experiment is then arranged with the necessary controls, as has been previously described in the experiments with the cholera vibrios (tables I and II). The preceding table gives the result of the examination of serum of several patients in the Broca Hospital in Paris.

\section{BIBLIOGRAPHY ON THE ANTIBODIES.}

Buchner. Centralbl. f. Bact., 1889 et 1890. Arch. f. Hygiene, 1890 et 1893. Mïnch. med. Woch., 1891 et 1894 .

Metchnikoff. Ann. Inst. Past., 1894, 1895, 1900.

EHRLICH. Bedurfniss des Organismus, Berlin, 1885. Klinisch. Jahrbuch, I897. EhrLich et Morgenroth. Berl. Klin. Woch., 1899.

PFeIfFer. Zeits.f. Hyg., r894. Deuts. med. Woch., I894.

Bordet. . Ann. Inst. Past., 1895, I898, 1899, I900, 1901. Journ. de Méd.

Bruxelles, 1906. Berl. klin.Woch., 1907. Zeits.f. Imm., 1909.

Bordet et Gengou. Ann. Inst. Past., 1901, r9o6.

SACHs. Deuts. med. Woch., 1905 .

Wassermann. Congrès méd. int. de 19oo, Paris. Berl. klin. Med., 1907. Wien klin. Med., r9o8.

Kraus. Gesellsch. der Aerzte in Wien, 1897. Wien klin. Woch., I897.

Roux. Ann. Inst. Past., r888-1889.

WARden et WAdELI. Non bacillar nature of Abrus poison, Calcutta, I884.

Calmettes. Ann. Inst. Past., 1894-1895.

Phisalix et Bertrand. Soc. Biol., I894.

Widal. Arch. intern. de Ph. et Thêr., (6).

Widal et Lesourd. Soc. méd. des hôpitaux, rgor.

Armand Delille. Antigènes, Anticorps, Deviation du complément, Paris, IgIr.

Pozzi-Escot. Les Actualités chimiques, ${ }^{\text {os }} 3,4,7$, Paris, I906-r907.

Cohen. Presse médicale, rgog.

Dopter. Ann. Inst. Past., I905. 
PART IV.

EREPSINS. 



\section{CHAPTER I.}

\section{INTESTINAL EREPSIN.}

\section{$\S$ i. Preparation and Properties of Erepsin.}

Salvioli, in 1880 , found that the intestinal mucous membrane exerts a very pronounced effect on peptone and that the latter rapidly disappears from the solution. This fact was confirmed later by Hofmeister and by other observers, and was for a long time attributed to a synthetic reaction. It was thought that peptone, in contact with the wall of the intestine, was reformed into albumin. In 1899 , Cappareli attributed this transformation to an enzymic action, and Cohnheim, in rgor, proved that this disappearance of the peptone results, not from a condensation, but from a decomposition which is produced by a special enzyme to which he gave the name of erepsin. The final products of digestion of peptone are non-biuretic substances corresponding, consequently, to an advanced hydrolysis, and are composed chiefly of amino-acids.

Distribution. - Erepsin is widely distributed in the animal as well as in the vegetable kingdom. It is found in the intestinal juice, but still more abundantly in extracts obtained by the maceration of the intestinal mucous membrane. It has also been found in the pancreas, the kidney, the spleen, the blood, the muscle, the brain, etc. Cohnheim has demonstrated that different organs deprived of blood appear to be very rich in erepsin, and he was thus led to make a sort of topography of the distribution of this enzyme. He found in particular that:

$3.7 \mathrm{~g}$. of kidney will transform in 20 hours $0.5 \mathrm{~g}$. peptone

$4.8 \mathrm{~g}$. of lung will transform in 58 hours $0.5 \mathrm{~g}$. peptone

ro. $8 \mathrm{~g}$. of muscle will transform in 68 hours $0.5 \mathrm{~g}$. peptone

There is then in the kidney about 3.7 times as much erepsin as in the lung and about ro times as much in the muscle. In the large 
intestine itself erepsin is not uniformly distributed, but is especially abundant in the jejunum, very ittle being found in the duodenum. Erepsin has also been found in different seeds, as well as in wheat flour. It has been found in the leaves of Brassica oleracea and in several mushrooms, especially Glamerella ruformaculans and $S$ phoeropsis molorum.

To detect the presence of this enzyme in organs, advantage is taken of its property of being very easily adsorbed by fibrin, and especially by elastin.

Preparation and Properties. - Erepsin is commonly obtained from the large intestine of a dog scarified in full digestion. After having washed the intestine, the mucous membrane is scraped, the product obtained crushed with sand, and the residuum allowed to macerate for some time in physiological salt solution with addition of toluol. Two parts of maceration liquor are then added to 3 parts of a saturated solution of ammonium sulphate. The precipitate formed is allowed to settle and is then filtered off. The coagulum is suspended in water and the ammonium sulphate removed by dialysis. After filtration, a solution of enzyme containing only a few albuminoids is obtained.

Blood succeeded in preparing erepsin from Brassica oleracca. The crushed leaves are reduced to a paste and pressed. The juice, with addition of toluol, is dialyzed against water, then precipitated by ammonium sulphate. The coagulum is collected after 24 hours, dissolved in water, and is then dialyzed again until all the sulphate has disappeared. There remains behind a liquid neutral to litmus and very rich in erepsin.

The dried mucous membrane of the large intestine may be used as source of erepsin. The tissue is dehydrated with alcohol, and finally dried over sulphuric acid. The product obtained withstands a temperature of $100^{\circ}$ for more than an hour without deterioration and shows great activity.

Erepsin does not act on natural albuminoid materials, such as albumin, fibrin, and edestin, but it appears to be active toward albumoses and peptones. It easily decomposes deutero- and proto-albumoses, but hetero-albumoses are much more resistant. 
This enzyme likewise transforms casein and the histone of thymus gland. Erepsin gives its maximum effect in neutral or very slightly alkaline reaction; it is in fact much more sensitive to alkalinity than trypsin. Furthermore, an acid reaction is completely inhibitory, erepsin remaining inactive even in a liquid neutral to methyl orange. Its optimum temperature is from $28^{\circ}$ to $30^{\circ}$. Heated to $63^{\circ}$, it is rapidly destroyed.

Individuality of Erepsin. - The question has been much disputed as to whether erepsin is really a well-characterized enzyme and it was long confounded with trypsin. In reality trypsin, as well as pancreatin, always contains slight quantities of erepsin. It has also been believed that erepsin is found in the zymogenic state and that it is the pancreas which renders it active. This opinion was refuted by Cohnheim who showed that a dog which survived for 7 days the extirpation of the pancreas, nevertheless gave an extract of intestinal mucous membrane which still acted very vigorously on peptone, although it was inactive on albumin and fibrin.

The individuality of erepsin is at the present time especially demonstrated by the chemical reactions which it induces, reactions which differ essentially from those induced by trypsin. We shall return later to this very important point in the study of proteolytic degradation under the influence of various enzymes. Glässner and Stauber have shown the difference which exists between trypsin and erepsin by making use of blood serum as a reagent. In a previous chapter, it was seen that serum contains an anti-trypsin capable of neutralizing the effect of trypsin. If serum is added to a mixture of trypsin and erepsin, a mixture which digests simultaneously coagulated albumin and peptone, it is found that the dissolving action on white of egg is lost, while the ability to produce non-biuretic bodies persists. The two enzymic actions are then capable of being differentiated by this means. A difference is also observed between trypsin and erepsin when these enzymes act on toxins. According to Sieber and Simonowsky, one part of pancreatic juice will destroy 10,000 to 100,000 lethal doses of diphtheretic 
toxin, while an equivalent quantity of erepsin will destroy only 50 doses. Further intestinal juice containing erepsin is without action on tetanus toxin; on the contrary, $0.06 \mathrm{~g}$. of trypsin can destroy up to 10,000 lethal doses of this substance.

Chemical Activity. - The chemical action induced by pepsin consists of a hydration followed by a splitting. The protein molecule, whose weight is about 6000 , formed by the union of a large number of various amino-acids connected through their $\mathrm{CO}_{2} \mathrm{H}$ and $\mathrm{NH}_{2}$ groups, gradually disintegrates. Under the influence of pepsin, the chain breaks at different weak points and acid and amine functions appear, but the fragments formed still represent very complex polypeptids, such as albumoses, peptones, and bases. Trypsin brings a more efficacious assistance to the reaction by liberating the amino-acids and by attacking albuminoid material. This biochemical agent rapidly frees tyrosin and indol nuclei, but there will still remain large fragments of the broken chain which will resist its action. Thus tyrosin and tryptophane, appear in the digestion liquid together with the complex polypeptids, albumoses, and peptones as well as a certain number of amino-acids of a relatively simple structure, such as leucin, aspartic acid, alanin, etc. Finally, the action of trypsin is distinguished from that of pepsin by the molecular weight of the products formed. The compounds obtained after the action of trypsin have a molecular weight averaging much less than the corresponding weight for a peptic action. However, this fact does not prevent there still being found in the liquid at the end of tryptic digestion nitrogenous compounds of large molecular weight. The same albumoses and the same peptones form in the two reactions, but a larger proportion of these is digested in tryptic reaction, which brings them furthermore to a much more advanced stage of simplification.

The enzymes belonging to the erepsin group still further accentuate the results of hydrolysis by freeing. the various amino-acids which were previously retained in the polypeptid complexes. It is true that these enzymes carry their action no further on natural albuminoids, but rather on products which 
have already undergone an initial hydrolysis. In a solution of albumin which has been submitted to peptic digestion three different portions can be separated: (I) Albumoses, which are precipitated by $\mathrm{ZnSO}_{4}$; (2) peptones, which are precipitated by phosphotungstic acid; (3) amino-acids and analogous products, which remain in the tungstic solution. This third fraction is largely made up of amino-acids, but their molecular size is variable. In addition to substances already largely degraded, there are some which are quite complex, and which are susceptible of being further transformed under the influence of erepsin. As to the other two portions, erepsin is capable also of digesting them, but with a different intensity. It is the albumoses that resist most highly the influence of this enzyme, and it is curious to observe that among these substances it is precisely those that most easily disappear in peptic and tryptic digestions that are with most difficulty transformed in ereptic hydrolysis.

Products of Advanced Hydrolysis of Different Albuminoid Materials, According to Abderhalden.

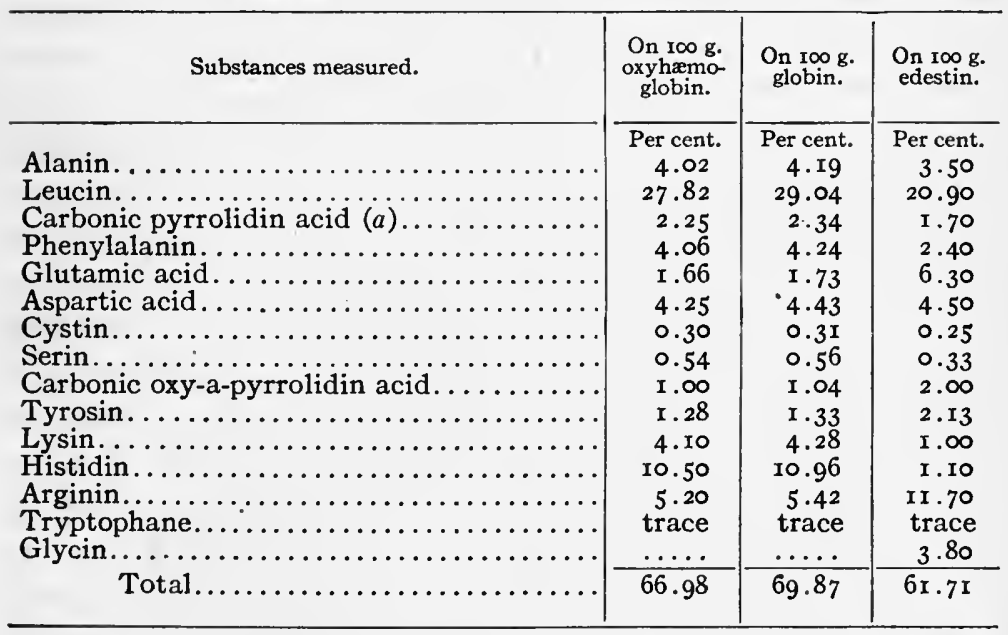

From these facts it appears that, to a certain extent, pepsin and trypsin may be substituted for each other. Both lead to the formation of albumoses and peptones from natural albuminoids. 
On the contrary, erepsin deals with secondary products. It does not have the same characteristics. It is not an enzyme for albuminoids properly speaking, and it cannot replace the two preceding enzymes. With this new class of enzyme, we descend in the scale of degradation of albuminoids, and thus reduce all the nitrogenous compounds, natural or already decomposed, to bodies of a very simple structure, crystallizable and no longer having the characteristics of the intermediate peptids. In fact, the final product obtained in ereptic digestions is entirely comparable to that which results from the energetic action of concentrated $\mathrm{HCl}$ or of 30 per cent $\mathrm{H}_{2} \mathrm{SO}_{4}$ on the different protein materials.

Identification of Ereptic Activity. - It was previously seen that tryptic activity is easily distinguished from peptic activity by the presence in the case of trypsin of products easy to detect, such as tyrosin and tryptophane. We should expect to have also among the final products which result from the action of erepsin, one or more easily recognizable substances, which would be characteristic for the reaction. Unfortunately this is not the case. The products obtained through the activity of erepsin cannot be differentiated from those coming from trypsin.

It is possible, however, to distinguish ereptic activity from peptic and tryptic activities by means of phosphotungstic acid. This reagent, by giving precipitates of a composition varying with the liquids and with the time, permits a following of the intensity of the hydrolysis and of thus recognizing one or the other of these three digestive enzymes. A 3 per cent solution of albumin is divided into two portions; to one, after acidification with 0.2 per cent $\mathrm{HCl}$, pepsin is added; to the other, after being made alkaline with 0.2 per cent $\mathrm{Na}_{2} \mathrm{CO}_{3}$, trypsin is added. After 48 hours of action, a certain quantity of the second liquid is removed and to it erepsin is added. Thus three digestions are in progress. In each of them a little toluol is added and they are left 20 hours at $27^{\circ}$. The course of the hydrolysis is determined through the following phosphotungstic reagent: Io g. phosphotungstic acid +100 c.c. water +50 c.c. $\mathrm{H}_{2} \mathrm{SO}_{4}$ diluted to $\frac{1}{4}$. To make a 
Comparative Progress of Hydrolysis According to the Tungstic Reagent.

\begin{tabular}{c|c|c|c}
\hline \multirow{2}{*}{ Length of the action. } & \multicolumn{2}{|c}{ Per cent nitrogen precipitable by phosphotungstic acid. } \\
\cline { 2 - 4 } & Pepsin. & Trypsin. & Erepsin. \\
\hline Days. & & & \\
2 & 49 & 43 & 49 \\
4 & 52 & 43 & 43 \\
8 & 46 & 45 & 40 \\
10 & 48 & 38 & 35 \\
20 & 55 & 36 & 21 \\
\hline
\end{tabular}

determination, to 25 c.c. of the liquid to be tested, roo c.c. of the tungstic reagent are added, and the mixture allowed to stand $\mathrm{I} 2$ hours. After filtering, the precipitate is washed three times with the reagent, and finally the Kjeldahl nitrogen is determined in the product collected. This nitrogen represents the albumoses and the peptones.

Thus, under the influence of erepsin, the nitrogen precipitable by the tungstic reagent gradually diminishes. Of the total nitrogen contained in the liquid, there remains after 20 days only 2 I per cent still precipitable; the difference has been transformed into amino-acids. At the end of the same time trypsin leaves 36 per cent precipitable nitrogen, and pepsin 55 per cent. Furthermore, a very irregular progress is observed for pepsin. In the course of proteolysis, substances are formed which, at first not precipitable, afterward become precipitable. With trypsin this fluctuation is less apparent, and with erepsin the digestion is quite regular. The very small content in precipitable nitrogen in the liquid obtained with erepsin is interesting. Furthermore, the difference does not concern merely the quantity of nitrogen contained in the precipitates. The composition of these precipitates is also variable, according to whether we have a peptic, a tryptic, or an ereptic digestion. In the first case the precipitates are composed of peptids of the most complex nature. They contain, besides very large quantities of albumoses, relatively few peptones. In the second case, the precipitates contain almost no albumoses, still less peptones, and already many simple 
polypeptids. Finally, the tungstic precipitates obtained after an advanced ereptic action are composed almost entirely of bases. They contain only very few polypeptids and may even be completely lacking in them.

Henriques, to differentiate the peptic, tryptic, and ereptic activities, determines in the products of hydrolysis the content in polypeptids still untransformed. For this purpose he begins by determining in each of the liquids after digestion the quantity of formaldehyde nitrogen which has appeared. Then he submits these liquids to the action of concentrated $\mathrm{HCl}$, this treatment having as a result the hydrolysis of the remaining peptids to the amino-acid state. A new determination of the formaldehyde nitrogen is now made. The difference found between the two determinations before and after the action of $\mathrm{HCl}$ represents the polypeptid nitrogen. Hydrolysis by $\mathrm{HCl}$ is made in the following manner: To 50 c.c. of liquid are added 50 c.c. concentrated $\mathrm{HCl}$. It is evaporated almost to dryness on the water-bath, then 50 c.c. of concentrated $\mathrm{HCl}$ are added and the solution is again brought to dryness. Add I50 c.c. water and evaporate a third time. The residue is dissolved in water and brought again to the original volume.

The Progress of Hydrolysis According to the Content in Peptid Nitrogen Remaining.

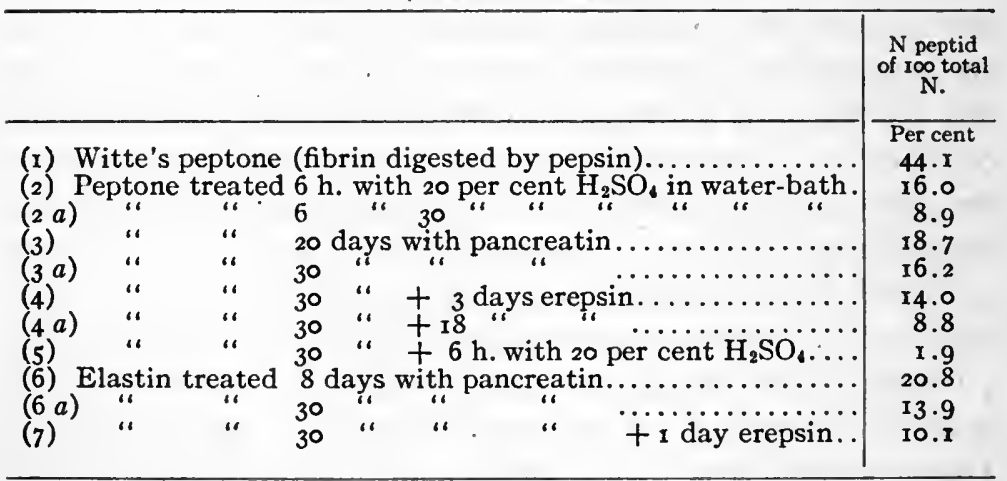

It appears from this table that Witte's peptone, the product of peptic hydrolysis of fibrin, contains still a very large quantity 
of polypeptids. These, under the prolonged action of 30 per cent $\mathrm{H}_{2} \mathrm{SO}_{4}$, are lowered to 8.97 , a proportion practically the same as that found after having caused pancreatin and erepsin to act successively on this same peptone. Thus erepsin appears to be the most energetic agent of proteolytic transformations, and its action can be compared only with that of acids. But to reach a more or less complete hydrolysis with this enzyme, it is necessary first of all to treat thoroughly the albuminoid material with trypsin and then to prolong the action of erepsin for a very long time, about 2 or 3 months. Naturally, by reason of the destruction of erepsin, it will be necessary to replace it in the liquids from time to time. This need of a quite thorough digestion before beginning the action of erepsin finds an analogy in the action of $\mathrm{H}_{2} \mathrm{SO}_{4}$. We can, in fact, conclude from tests (2) and (5) that the result of hydrolysis by acid is not independent of the method of its use, the action on a product already digested by pancreatin and erepsin being much more advanced than that exerted on a product not previously treated.

The following are the results of the analysis of several digestion products, according to Sörensen. The contents in formalde-

Accumulations of Amino-acids in the Course of Peptic, Tryptic, AND EREPTIC Digestions.

\begin{tabular}{|c|c|c|c|c|c|c|c|}
\hline \multirow{2}{*}{ Nos. } & \multirow{2}{*}{ Protein subst. used. } & \multirow{2}{*}{ Enzyme used. } & \multicolumn{4}{|c|}{$\begin{array}{l}\text { Per cent formaldehyde nitro- } \\
\text { gen of total nitrogen. }\end{array}$} & \multirow{2}{*}{$\begin{array}{l}\text { Percent } \\
\text { tannin } \\
\text { nitro- } \\
\text { gen } \\
\text { precip. } \\
\text { after } \\
\text { I50 hr. }\end{array}$} \\
\hline & & & At first. & $\begin{array}{l}\text { After } \\
6 \mathrm{hr} \text {. }\end{array}$ & $\begin{array}{l}\text { After } \\
30 \mathrm{hr} \text {. }\end{array}$ & $\begin{array}{l}\text { After } \\
\text { I50 hr. }\end{array}$ & \\
\hline I & Witte's peptone. & Pepsin & I6.6 & & 19.8 & 23.04 & 67.2 \\
\hline 2 & Casein......... & & 14.9 & 19.0 & 20.9 & $23.6^{\circ}$ & 67.3 \\
\hline 2 & Album. of egg coag. & “ & 5.9 & 8.5 & 12.8 & 17.9 & 46.7 \\
\hline 2 & Witte's peptone... & Pancreatin & I5.9 & 22.1 & 31.5 & $39 \cdot 3$ & $35 \cdot 3$ \\
\hline 2 & & & 16.4 & 28.8 & $39 \cdot 3$ & 53.8 & 20.7 \\
\hline 2 & Casein. & " & $\mathrm{I} 5.8$ & 33.8 & 42.8 & 50.4 & 19.9 \\
\hline 2 & & “ & 16.5 & $34 \cdot 3$ & 43.6 & 50.7 & 15.2 \\
\hline 2 & Egg-alb. part. digested & “ & 13.5 & 17.7 & $24 \cdot 3$ & 30.4 & 32.1 \\
\hline 2 & Egg-alb. part. digested & “ & 14.1 & 24.8 & $35 \cdot 5$ & 48.8 & $5 \cdot 1$ \\
\hline Io & Witte's peptone ...... & Erepsin & 20.0 & 22.8 & 31.8 & $5^{8} .9$ & 34.8 \\
\hline II & & & 19.5 & 29.3 & 50.6 & 84.7 & 13.6 \\
\hline I2 & Casein.... & “ & $\mathbf{1 8 . 9}$ & 22.1 & 53.2 & $89 \cdot 3$ & I 2.2 \\
\hline
\end{tabular}


hyde nitrogen of the liquids, numbers which are considered to represent the nitrogen of the amino-acids formed, have been determined in the different phases of hydrolysis of various materials under the influence of the three digestive enzymes. Furthermore, the nitrogen content of the various precipitates obtained with tannin in the final liquicis of the reaction are recorded so as to be able to compare the two analytical procedures.

The preceding table indicates that digestion, even with erep$\sin$, is not complete, and that there still remains after the action about ro per cent, and of ten more, of nitrogen not measurable by formaldehyde. This quantity does not correspond entirely to the polypeptids. It must, in fact, be observed that in the hydrolysis of protein materials by concentrated acids, we observe a similar phenomenon, and that we never succeed in finding in such liquids roo per cent formaldehyde nitrogen. This discrepancy is explained in two ways: First of all, the method of Sörensen, although very convenient and capable of rendering valuable service, is not exact and does not lend itself to perfectly exact quantitative analyses. In addition, there exists a constant error in the facts that what is determined is not nitrogen but carboxyl groupings, and that we assume that a nitrogen corresponds to each $\mathrm{CO}_{2} \mathrm{H}$. This is true for the larger part of aminoacids formed, but there are also others that contain several atoms of nitrogen, and yet these count only as mono-nitrogenous. Formaldehyde nitrogen will then represent a minimum of the aminoacid nitrogen. We must also take into consideration the fact that in hydrolysis with erepsin or with acids, there are produced, besides the amino-acids, nitrogenous compounds which are no longer decomposable by acids, and which likewise are not titratable with formaldehyde. We may even find that the discrepancy noted above is very. slight compared with that usually noticed. Effront several times had occasion to hydrolyze completely albuminoids with $\mathrm{HCl}$, and in such a way that there remained in the tungstic precipitates, which were made from time to time to follow the reaction, no substances capable of being 
further degraded by the acid, and yet he was never able to measure in these liquids with formaldehyde more than 75 per cent to 77 per cent of the total nitrogen. Yet, it is possible to obtain better figures and to titrate almost the whole of the nitrogen if the acid is allowed to act, not on the natural albumin but on the product from advanced enzymic action, provided that under these conditions the hydrolysis takes place in a different manner.

The results indicated in the last column prove that tannin cannot serve to differentiate the three digestions. We find, for example, by comparing Numbers 9 and 12 that the proportion of nitrogen precipitable by tannin is less for pancreatin than for erepsin, 5.I and $\mathrm{I} 2.2$ respectively, which tends to show that the hydrolysis is advanced further in the first case than in the second, though the results furnished by formaldehyde, 48.8 and 89.3 , show the reverse. Tannin can in fact very well serve for detecting the beginning of the degradation. In the case of the albumoses and peptones, its indications are conformable with actual conditions, but later this reagent becomes insufficient for it no longer precipitates certain polypeptids which, while having a less complexity than those which appeared at the beginning, are nevertheless nowhere near being transformed into simple amino-acids.

Analysis of Erepsin. - To measure the activity of an erepsin the colorimetric method is often used. A very dilute solution of strongly alkaline $\mathrm{CuSO}_{4}$ colors violet blue under the influence of albumoses and peptones; this is the biuret reaction. Now, as erepsin reacts, the coloration produced by the digestion liquid is less and less intense, and finally, when the peptones have entirely disappeared, the solution furnishes no further coloration.

Procedure. - Add to Io c.c. of a Io per cent $\mathrm{NaOH}$ solution 3 or 4 drops of a 2 per cent solution of $\mathrm{CuSO}_{4}$. Then add with care, drop by drop, the solution of peptone to be digested, until a deep coloration is produced. Note the volume by a mark on the tube; this is the blank test. On the other hand, take ro c.c. of the same solution of peptone, add to it I c.c. of the enzyme liquor to be analyzed, leave a certain time at $37^{\circ}$, then make with this second liquid the biuret reaction exactly in the same manner 
as before, using the same quantities of liquid. The comparison of the intensities of the shades, made either with the colorimeter or with the spectrophotometer, gives an estimate of the richness in enzyme of the liquid examined. It is possible to reach the same result by bringing the liquids to the same tint by adding water to that which is the darker.

\section{§ 2. Actions Exerted by Proteolytic Enzymes upon Each OTHER.}

According to the opinion of Fischer, there exists an intimate relation between the structure of enzymes and the substances on which they react. On the basis of this opinion, proteolytic enzymes must have a structure similar to that of the albuminoids, and one can foresee that enzymes themselves will be digested by other active substances. There already exists in the literature a certain number of observations, dating years back, relative to the action of trypsin on pepsin. Harlay, to show the reciprocal actions of these two enzymes, makes use of a solution containing 0.I g. of pepsin + o.I g. of pancreatin and 25 c.c. of distilled water. In a control test, the pancreatin is destroyed by a prolonged boiling. After cooling, the pepsin and chloroform are added, and the solution brought to a volume of 25 c.c. We then have two liquids; in one, the two enzymes are in the active state; in the other, the trypsin has been destroyed. The two flasks are kept 2 hours at $40^{\circ}$, then 15 hours at the ordinary temperature. Then to each solution are added 2.5 c.c. $N /$ Io $\mathrm{HCl}+5$ g. of cooked and dried fibrin, containing about 40 per cent of dry material. These are allowed to digest for a certain time at $40^{\circ}$, and then the residue of undigested fibrin is weighed. As a control, the dry extracts in 5 c.c. of the filtered liquid are also determined.

The digestive power of pepsin has then been considerably diminished by contact with trypsin. Analogous results are found when pepsin and papain are left together either in neutral or in slightly acid media; papain destroys or weakens the pepsin. 
Action of Trypsin on Pepsin.

\begin{tabular}{|c|c|c|}
\hline & $\begin{array}{l}\text { Weight of } \\
\text { fibrin not } \\
\text { digested. }\end{array}$ & $\begin{array}{l}\text { Weight of dry } \\
\text { extract in } 5 \text { c.c. } \\
\text { filtrate. }\end{array}$ \\
\hline $\begin{array}{l}\text { Action of pepsin alone } \ldots \ldots \ldots \ldots \ldots \ldots \ldots \\
\text { Action of the solution containing pepsin }+ \text { pan- } \\
\text { creatin } . \ldots \ldots \ldots \ldots \ldots \ldots \ldots \ldots \ldots \ldots\end{array}$ & $\begin{array}{l}\text { g. } \\
0.7 \\
0.99 \mathrm{I}\end{array}$ & $\begin{array}{c}\text { g. } \\
0.085 \\
0.037\end{array}$ \\
\hline
\end{tabular}

On the contrary, pepsin exerts no action either on trypsin or on papain, and these last two enzymes do not react on each other.

Effront has sought to determine the influence of erepsin on trypsin. He dissolves $0.2 \mathrm{~g}$. of trypsin and $0.4 \mathrm{~g}$. of erepsin in 500 c.c. of water. The filtered liquid has toluol added to it, and is left at $30^{\circ}$; at different times a sample of a constant volume is taken, a certain quantity of coagulated and finely divided albumin is added, and this test is allowed to digest for 2 hours at $50^{\circ}$. Then the nitrogen is determined in the filtered liquid. On the other hand, a control experiment is established by destroying by boiling the erepsin which is to be added to the trypsin.

INFLUeNCE OF EREPSiN ON TRYPSin.

\begin{tabular}{|c|c|c|}
\hline \multirow{2}{*}{$\begin{array}{l}\text { Length of } \\
\text { action. }\end{array}$} & \multicolumn{2}{|c|}{$\begin{array}{l}\text { Content in nitrogen of the } \\
\text { filtered liquid. }\end{array}$} \\
\hline & $\begin{array}{l}\text { With erepsin } \\
\text { destroyed. }\end{array}$ & $\begin{array}{l}\text { With erepsin } \\
\text { not destroyed. }\end{array}$ \\
\hline $\begin{array}{c}\text { Hours. } \\
2 \\
4 \\
8 \\
12 \\
23 \\
35 \\
50\end{array}$ & $\begin{array}{r}\text { mg. } \\
\text { I } 30 \\
\text { I } 28 \\
\text { I } 20 \\
\text { I } 15 \\
\text { I } 12 \\
109 \\
96\end{array}$ & $\begin{array}{r}\text { mg. } \\
136 \\
\text { I } 19 \\
91 \\
85 \\
61 \\
31 \\
21\end{array}$ \\
\hline
\end{tabular}

It appears from these figures that after 2 hours the erepsin exerts no action at all on the trypsin. But as the contact is prolonged, the unfavorable influence declares itself and is accentuated. In the control test, where the erepsin has been destroyed by boiling, the trypsin also is weakened in the long run; 
from $130 \mathrm{mg}$., the content of nitrogen in solution falls to $96 \mathrm{mg}$. But the diminution is markedly greater with the other test.

It is curious to find that in this reciprocal action of proteolytic enzymes, it is the most active enzyme, from the point of view of degradation in hydrolysis, which destroys that which is less active. Pepsin is digested by trypsin, which itself disappears under the influence of erepsin.

\section{BIBLIOGRAPHY ON INTESTINAL EREPSIN.}

Hofmeister. Zeits.f. physiol. Chem., i88I, (6), p. 5 I.

Cappareli. Sulla transform. peptoni, Alli. della Acad. Gioenia die Scienze en Catania, 1899, (1 2).

Castle and Loewenhart. Amer. Chem. Journ., 1900, (24), p. 491.

EuIse Roubitscher. Zeits.f. experim. Pathol. u. Therap., Igor, (4), p. 675.

Cohnherm. Die Umwandlung des Eiweisses d. d. Darmwand, Zeits. f. physiol. Chem., rgor, (33), p. 45 r.

Salaskin. Zeits.f. physiol. Chem., igo2, (35), p. 4 I9.

Nadine Sieber u. Schumoff Snonowski. Wirkung d. Erepsins im Darmsaft, Zeits. f. physiol. Chem., I902, (36), p. 255.

Kutscher u. Seemann. Zeits.f. physiol. Chem., igo2, (35), p. 45 I.

Cohnherm. Trypsin u. Erepsin, Zeits. f. physiol. Chem., 1902, (35), pp. 135, 417; (36), p. I3.

LaMbert. Soc. Biol., 1903, (55), p. 4 I9.

Weinland. Zeits. f. Biol., I903, (44), p. 292.

Bayliss and Starling. Journ. of Physiol., 1903, (30), p. 6 r.

M. Nakayama. Ueber das Erepsin, Zeits.f. physiol. Chem., 1904, (4I), p. 349.

Vervon. Journ. of Physiol., 1905, (32), p. 33; (33), p. 81. Zeils.f. physiol. Chem., $1907,(50)$, p. $44 \mathrm{I}$.

Abderhalden u. Teruuchi. Zeits. $f$. physiol. Chem., 1906, (49), p. I3.

Cohnhenr. Spaltung d. Nahrungeiweisses i. Darm, Zeits. f. physiol. Chem., I906, (49), p. 67.

Karl Mays. Beitr. z. Kenntniss d. Trypsinwirkung, Zeits. f. physiol. Chem., I906, (49), p. 18I.

FoA. Arch. de Physiol., rgo8, (5).

Henriques. Die Eiweissynthese im Thierorganismus, Zeits. f. physiol. Chem., 1908, (54), p. $4 \mathrm{II}$.

NÜRENBERG. Bioch. Zeits., I909, (16), p. 106.

O. Cohnherm et D. Pletnew. Ueber den Gehalt blutfreier Organe an Erepsin, Zeits. f. physiol. Chem., г910, (69), p. Iog.

Blood. Journ. of Biol. Chem., I9I0, (8), p. 215.

Giuseppe Amanteau. Alli Reale Accad. Lincei, Roma, I9II, (5), p. 20. Arch.d. Farmacol. sperim., Roma, I9II, (12), p. 562. 
Glässner u. Stauber. Beziehungen zw. Trypsin u. Erepsin, Bioch. Zeils., igio, (25), p. 204.

Sörensen. Ezymstudien, Bioch. Zeits., I907, (7), p. 45.

WEINLAND. Journ.f. Biol., (27), p. 392.

ACTION OF THE ENZYMES UPON EACH OTHER.

V. A. Harlay. Thèse, Paris, I900, p. 7 I.

William Roberts. The digestive ferments, 1880 .

Wroblewski, Bednarski u. Wojczynski. Beit. z. Chem. Phys. u. Pathol., Igor, (I), p. 290. 


\section{CHAPTER II.}

\section{PEPTOLYTIC ENZYMES.}

Distribution. - The polypeptids of Fischer, products of synthesis, cannot be compared with the natural derivatives obtained by hydrolysis of albuminoid materials. However, they approach them closely, and a whole class of these substances are hydrolyzed by certain peptolytic enzymes (this generic name designating the active substances capable of exerting an action on the polypeptids). These enzymes, at the present time, are not yet well characterized. According to the classification which we have adopted, we must place them in the group of enzymes which decompose peptone with abundant formation of amino-acids. In reality, certain of these enzymes present all the characteristics of intestinal erepsin, but the class of peptolytic enzymes also includes substances which do not act at all on the peptones, so that it is better to make of them a separate group.

The interest in the study of these enzymes centers especially in the fact that as primary material products are used whose composition and structure is known, and that thus the progress of the hydrolysis can be followed with infinitely more accuracy than with the albuminoid materials whose complexity is much greater. It is to Abderhalden and to his pupils that we owe a whole series of very interesting data which bring to light the decomposition of polypeptids by enzymes, and which in a general way give valuable indications on the different active substances.

We do not know, at the present time, a single polypeptid that can be hydrolyzed by pepsin, but we know a large number of them that are decomposed under the influence of the active pancreatic juice. We find peptolytic enzymes in the juice of vegetable and animal tissues. This sort of enzyme is much more widely distributed than the secretorial enzymes. In the action of peptolytic enzymes on polypeptids there comes into play a number of factors. All the polypeptids are not equally sensitive to the 
enzymes. Their structure and their composition play an important part. Thus we observe results that vary according to the amino-acids which enter into their constitution, and also according to the disposition of the latter. In particular, while alanyl-glycin

$$
\mathrm{CH}_{3}-\underset{\mathrm{NH}_{2}}{\mathrm{CH}}-\mathrm{CO}-\mathrm{NH}-\mathrm{CH}_{2}-\mathrm{CO}_{2} \mathrm{H}
$$

is split, its isomer, glycyl-alanin

$$
\mathrm{NH}_{2}-\mathrm{CH}_{2}-\mathrm{CO}-\mathrm{NH}-\underset{\mathrm{CH}_{3}}{\mathrm{CH}}-\mathrm{CO}_{2} \mathrm{H}
$$

is not.

Investigation of Peptolytic Enzymes. - Abderhalden introduced the optical method in the investigation of peptolytic enzymes. With the exception of glycin, the products resulting from the hydrolysis of the polypeptids rotate polarized light. The decomposition of the racemic polypeptids is then shown by the appearance of rotatory power, while the decomposition of polypeptids, already active, is accompanied by a change in the rotation. To identify a peptolytic reaction, it consequently suffices to follow in a polarimeter the variation of the rotatory power. A definite quantity of polypeptid is dissolved in a given volume of an enzymic solution. Toluol is added, the solution brought to $37^{\circ}$, the reading taken, and then the temperature is kept constant. The change in rotation after a certain time indicates hydrolysis. When the rotatory power of the polypeptid used is known as well as those of the hydrolytic products, a very exact quantitative estimate of the progress of the hydrolysis can be made.

Diminution of Rotatory Power of a Solution

of Glycyl-L-Tyrosin.

6 c.c. sol. (\$3 mol.) glycyl-l-tyrosin $+x$ c.c. liver juice.

\begin{tabular}{c|c||c|c}
\hline Time in minutes. & Angle, deg. & Time in minutes. & Angle, deg. \\
I & +0.76 & 25 & +0.50 \\
5 & 0.72 & 30 & 0.47 \\
15 & 0.60 & 35 & 0.43 \\
20 & 0.55 & 40 & 0.38 \\
\hline
\end{tabular}


In optical tests which are required to remain under observation for a long time, the use of polypeptids with a tyrosin nucleus is avoided, since the solution often is colored in consequence of the intervention of oxidases, and this coloration, as well as the turbidity due to the tyrosin deposited, hinders the polarimetric reading. The polypeptid wnich is most commonly used for optical observation is d-alanyl-glycyl-glycin. On the contrary, by the aid of polypeptids with tyrosin base, we can easily show the presence of peptolytic enzymes by the rapid determination of tyrosin which is easily isolated. The weight of tyrosin obtained can even indicate the degree of hydrolysis. For these tests, in place of synthetic polypeptids, the La Roche peptone from the factory of Hoffman and La Roche at Basle is often used. This peptone is obtained by treatment of silk waste with cold 70 per cent sulphuric acid, and is very rich in tyrosinic.polypeptids. For the analysis, a to to 50 per cent solution of the same peptone is brought to a slightly alkaline reaction with $\mathrm{Na}_{2} \mathrm{CO}_{3}$, a certain volume of liquid containing the peptolytic enzyme is added, and the solution is kept with a little toluol at a temperature of $37^{\circ}$. From time to time a sample is taken, allowed to cool, and the crystallization observed.

To investigate these enzymes when they are present in large quantities in the organs, Abderhalden also recommends the use of a 25 per cent solution of silk peptone. The anatomical specimens to be studied are washed in physiological salt solution, are then plunged into the peptone solution with addition of toluol, and are left at a temperature of $37^{\circ}$. After 6 to 12 hours small crystals of tyrosin are found adhering to the tissues, representing, however, only a small part of the tyrosin which is in the liquid. The tissues, afterward hardened by alcohol, form preparations which may be preserved. By this method it has been possible to detect the presence of peptolytic enzymes in the kidney, the heart, the stomach, the intestine, and other organs. When the investigation of peptolytic enzymes in vegetable tissues is required, polypeptids containing tryptophane are utilized. These polypeptids do not give any coloration with bromine, a 
coloration which appears, on the contrary, as soon as hydrolysis begins. In this test the use of Io per cent solutions of glycyl-1tryptophane, with addition of toluol is recommended. The thin slice to be analyzed is introduced and left for 20 to 48 hours.

Influence of Physical and Chemical Conditions. - The progress of digestion is much influenced by physical and chemical conditions. Let us first examine the effect of temperature.

Influence of Temperature on Hydrolysis of Glycyl-L-Tyrosin.

\begin{tabular}{|c|c|c|c|c|}
\hline \multicolumn{3}{|c|}{$\begin{array}{l}\text { I c.c. sol. glycyl-1-tyrosin ( }\left(\sigma^{2} \sigma 0 \mathrm{~mol} \text { ) }\right)+\text { I c.c. yeast } \\
\text { juice }+4.5 \text { c.c. water. }\end{array}$} & \multirow{2}{*}{\multicolumn{2}{|c|}{$\begin{array}{c}\text { I c.c. sol. glycyl-1-tyrosin ( } 60^{2} \mathrm{~mol} \text { mol.) } \\
+2 \text { c.c. pancreatic juice }+3.5 \\
\text { c.c. water. }\end{array}$}} \\
\hline \multirow{2}{*}{ Temperature. } & \multirow{2}{*}{ Length of action. } & \multirow{2}{*}{ Rotation. } & & \\
\hline & & & Direct reading. & Corrected. \\
\hline 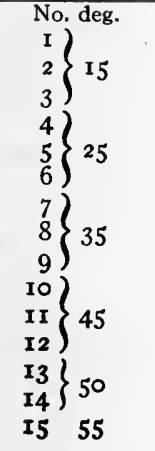 & $\begin{array}{c}\text { minutes. } \\
\text { o } \\
\text { I } 5 \\
60 \\
\text { I5 } \\
30 \\
60 \\
\text { I } 5 \\
45 \\
55 \\
\text { I5 } \\
45 \\
55 \\
\text { I0 } \\
35 \\
\text { I0 }\end{array}$ & $\begin{array}{r}\text { deg. } \\
+0.29 \\
0.28 \\
0.27 \\
0.25 \\
0.22 \\
0.16 \\
0.20 \\
0.08 \\
\ldots . . \\
0.15 \\
0.07 \\
\ldots . . \\
0.10 \\
\ldots .08 \\
0.08\end{array}$ & $\begin{array}{r}\text { deg. } \\
+0.18 \\
0.18 \\
0.18 \\
0.15 \\
\ldots . .8 \\
0.08 \\
0.13 \\
\ldots .06 \\
0.06 \\
0.10 \\
\ldots \ldots \\
0.05 \\
\ldots . .5 \\
0.05 \\
\ldots . .\end{array}$ & $\begin{array}{c}\text { deg. } \\
+0.30 \\
0.30 \\
0.30 \\
0.27 \\
\ldots . .2 \\
0.20 \\
0.25 \\
\ldots \ldots \\
0.18 \\
0.22 \\
\ldots . . \\
0.07 \\
\ldots \ldots \\
0.17 \\
\ldots . .\end{array}$ \\
\hline
\end{tabular}

It is seen that the optimum temperature for yeast juice is $55^{\circ}$, while pancreatic juice gives the maximum effect at $45^{\circ}$. Hydrolysis of polypeptids takes place in neutral reaction. The presence of free acid is very injurious. A very slight alkalinity appears to be favorable, but we note here a much greater sensitiveness to alkali than in the action of trypsin. The presence of small quantities of sodium cyanide, 0.03 per cent for example, favors hydrolysis of glycyl-1-tyrosin. A larger quantity, or o.I5 per cent, almost completely arrests action. $0.20 \% \mathrm{NaCl}$ has no effect. $\mathrm{CaCl}_{2}$ is, on the contrary, often very favorable. The presence of glycin as well as of other products of hydrolysis is 
very injurious. Nevertheless, as might be expected since it is a question not of one but of a whole group of enzymes, there is no absolute rule and the effect of a substance may differ from one polypeptid to another. Thus, sodium fluoride in 3 per cent doses is favorable to hydrolysis of glycyl-l-tyrosin by yeast juice, while the same quantity is unfavorable when d-leucyl-glycin is used.

Individuality of Peptolytic Enzymes. - The known polypeptids can be divided into two large groups, one hydrolyzable by enzymes, the other indifferent. The following is a list of the principal polypeptids, divided into two classes according to the action of kinased pancreatic juice:

\begin{tabular}{|c|c|}
\hline Hydrolyzable Polypeptids. & Non-hydrolyzable Polypeptids. \\
\hline 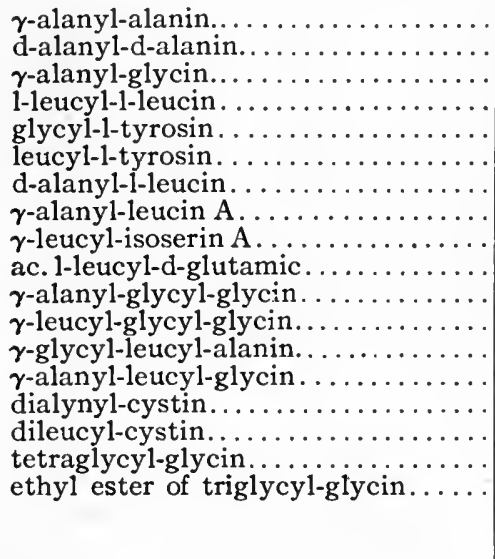 & $\begin{array}{l}\text { d-alanyl-l-alanin } \\
\text { l-alanyl-d-alanin } \\
\text { glycyl-alanin } \\
\text { l-leucyl-d-leucin } \\
\text { d-leucyl-l-leucin } \\
\text { leucyl-leucin } \\
\text { leucyl-alanin } \\
\text { alanyl-leucin B } \\
\text { glycyl-glycin } \\
\text { l-leucyl-glycin } \\
\text { leucyl-glycin } \\
\text { aminobutyryl-glycin } \\
\text { ac. aminobutyryl-aminobutyric A } \\
\text { ac. aminobutyryl-aminobutyric B } \\
\text { amino-isovaleryl-glycin } \\
\text { glycyl-phenylalanin } \\
\text { leucyl-prolin } \\
\text { diglycyl-glycin } \\
\text { triglycyl-glycin } \\
\text { dileucyl-glycin }\end{array}$ \\
\hline
\end{tabular}

All the peptolytic enzymes do not react with polypeptids hydrolyzable by pancreatic juice, and here, up to a certain point, we can indicate an action peculiar to each individual. In these investigations, it is useful first of all to bring to the same standard the liquids to be analyzed. Take, for example, two active liquids which act on glycyl-tyrosin. Their enzymic content will be different, provided the quantity of tyrosin liberated in a given time from the same quantity of polypeptid and of active liquid 
is different. To equalize the enzymic powers, dilute the liquid, which is rich in enzyme, to the standard of the poorer liquid. The two solutions thus equalized are then tried on a second hydrolyzable polypeptid. In the case of complete identity, the two liquids act absolutely in the same way; on the contrary, if their enzymic composition is not similar, a difference in the action will be observed, either one of the liquids reacting while the other remains without action, or the hydrolysis caused by the two enzymes differing from the point of view of the quality or the quantity of the products formed.

The pancreatic extract obtained by strong pressure, and also the pancreatic juice are both active on d-alanyl-l-glycin. Now the test of these two active liquids on d-glycyl-l-alanin reveals a radical difference, since the extract is active and the juice is inactive. I-leucyl-glycyl-d-alanin hydrolyzes with yeast juice as well as with pancreatic juice, but the course of the reaction is not the same. In the presence of the pancreatic juice there are first formed d-alanin and l-leucyl-glycin, which then hydrolyze, while yeast juice first produces i-leucin and glycyl-dalanin. The polypeptids with alanin base are hydrolyzed by kinased pancreatic juice, as well as by the juice of mushroom. The polypeptids of i-alanin are disposed of by yeast juice, but not by pancreatic juice. The juice of Mucor mucedo is inactive on leucyl-d-leucin, while Rhizopus tonkinensis, Allescheria gayonii and Aspergillus wentii are more or less active on this compound.

The use of polypeptids permits a substantial differentiation between the closely related active substances. The comparative effect on d-alanyl-d-alanin by pancreatic juice, intestinal juice, and yeast juice is given in the table [Page 508].

Therefore, of these three juices the pancreatic juice is without action, the intestinal juice is slightly active, while the yeast juice acts very energetically. Pancreatin reacts vigorously on leucylalanin while kinased pancreatic juice is without effect. The behavior with polypeptids indicates, then, a difference between these two products, and the existence in one of an enzyme which is not found in the other. 
HydRolysis OF D-ALANYL-D-ALANin.

\begin{tabular}{|c|c|c|c|c|c|}
\hline \multicolumn{2}{|c|}{$\begin{array}{c}\text { Pancreatic juice } 0.45 \mathrm{~g} . \text { di- } \\
\text { peptid }+6 \text { c.c. pancreatic } \\
\text { juice. }\end{array}$} & \multicolumn{2}{|c|}{$\begin{array}{l}\text { Intestinal juice o.45 g. di- } \\
\text { peptid }+6 \text { c.c. intestinal } \\
\text { juice. }\end{array}$} & \multicolumn{2}{|c|}{$\begin{array}{l}\text { Yeast juice } 0.45 \mathrm{~g} \text {. dipeptid } \\
+0.72 \text { c.c. juice }+5.28 \text { c.c. } \\
\text { water. }\end{array}$} \\
\hline Time. & Angle. & Time. & Angle. & Time. & Angle. \\
\hline $\begin{array}{l}\text { At first. } \\
6 \text { hours }\end{array}$ & \begin{aligned} \multicolumn{1}{c}{ deg. } \\
-1.24 \\
1.23\end{aligned} & $\begin{array}{l}\text { At first. } \\
8 \text { hours }\end{array}$ & \begin{tabular}{r}
\multicolumn{1}{c}{ deg. } \\
-1.38 \\
$\mathbf{1} .01$
\end{tabular} & $\begin{array}{l}\text { At first. } \\
20 \mathrm{~min} .\end{array}$ & \begin{aligned} \multicolumn{1}{c}{ deg. } \\
$-\mathbf{I} .35 \\
\mathbf{I} .26\end{aligned}$ \\
\hline $12 "$ & I. 22 & $9 \lll$ & 0.93 & $50 " 1$ & 1.07 \\
\hline 24 & I . 22 & 24 & 0.13 & $280 "$ " & 0.02 \\
\hline 36 & 1.20 & & $\ldots \ldots$ & 400 " & $\circ$ \\
\hline
\end{tabular}

Thus, the use of polypeptids gives slight distinctions which would be very difficult to obtain by other means. The advantage of this method resides in the fact that it is very quick and very simple, and that, in certain cases, it indicates with a great facility a difference in the progress of hydrolysis, a difference which would have been very difficult to detect by any other procedure. For example, take d-alanyl-glycyl-glycin. It can split in two ways, namely: (I) d-alanin + glycyl-glycin; (2) d-alanyl-glycin + glycin. In the first case, the rotatory power of the liquid is diminished in consequence of the production of optically inactive glycyl-glycin. In the second case, on the contrary, d-alanyl-glycin is formed, which is endowed with a rotatory power $(\alpha)_{20}^{\mathrm{D}}=+50^{\circ}$. Then a simple reading of the polarimeter determines the progress of the reaction and thus fixes the identity of the enzyme examined, since this polypeptid will be decomposed in one of two directions according to the origin of the enzyme.

It has already been noted above that polypeptid enzymes are found in all the juices of vegetable and animal tissues, and that the gastric juice, even when neutralized, is without action on the polypeptids. However, it is observed that in certain cases the gastric juice is active. It has been established that this activity of the juice depends on the manner of nutrition. According to Abderhalden, this result is due to a retrogression of the active substances of the intestine toward the stomach. We know, in 
fact, that each time that we give fat to an animal, the lipolytic enzyme of pancreatic origin appears in the stomach. It is by an analogous process that we would explain the abnormal presence of peptolytic enzymes in the gastric juice. Peptolytic enzymes have also been found in the blood, located in the corpuscles, in the plates, and sometimes in the plasma. The serum and the plasma of the dog, the horse, and the calf are without action on glycyl-1-tyrosin. Rabbit plasma contains, on the contrary, enzymes active on this dipeptid. The content in peptolytic enzymes of rabbit serum increases perceptibly with subcutaneous injections of serum from an animal of another species. We can thus obtain a dog plasma and serum rich in peptolytic enzymes by injections, either of serum from another animal or of solutions of egg-albumin:

Comparative Action on Glycyl-L-Tyrosin of Normal Plasma and Plasma of Treated Animals.

\begin{tabular}{c|c|c|c|c|c}
\hline \multicolumn{3}{c|}{ Normal dog plasma. } & \multicolumn{3}{c}{ Plasma of dog which has been treated by } \\
egg-albumin.
\end{tabular}

There is, then, a very great difference between normal serum and serum which has been obtained after repeated injections at certain intervals of egg-albumin, or of serum of another animal. For these analyses I g. of glycyl-l-tyrosin and Io g. plasma are left in contact at $37^{\circ}$ in the presence of toluol. After a certain time of action the albumin is eliminated by mastic and kaolin, and determinations are made in the filtered liquid of the products formed, as well as of the polypeptid not transformed. The introduction of foreign albuminoid materials into blood will cause the formation of peptolytic enzymes in the plasma, or at least pro- 
voke the excretion of these enzymes which the blood corpuscles already contain. Results of the same order have been observed when the animals are prepared by injections with silk peptone. Horse serum and that of rabbit are normally inactive toward this peptone, but after preparation their serum decomposes this peptone with the production of tyrosin.

The method of Abderhalden is especially applicable to the study of the endocellular enzymes which regulate the internal digestion of organs, and which plainly appear when the organs are left to autolysis. The proteolytic endocellular enzymes are of different types. The individuality of these substances is especially indicated by their decomposing action toward the polypeptids.

To summarize, the investigations undertaken in this direction have already furnished, as we have seen, a few decisive results. This method has not as yet given complete satisfaction, since the procuring of polypeptids in a pure state is still at present a difficult task, and since furthermore we do not possess sufficiently complex bodies of this nature. When the chemistry of the polypeptids is sufficiently developed this method will show, without any doubt, all that is right to expect of it. In conclusion, the following is, according to Abderhalden and his pupils, the action of the different juices of organs on various polypeptids:

Polypeptids split

I. Juice of the liver:

Polypeptids not split

(a) of cattle

glycyl-glycin

leucyl-leucin

anh. of glycin

leucyl-phénylalanin (spl. asym.)

r. leucyl-leucin

leucyl-glycyl-glycin

alanyl-glycyl-glycin

d. I. leucyl-glycin

glycyl-d-l-alanin

(b) of dog

glycyl-glycin

glycyl-1-tyrosin 
Polypeptids split

Polypeptids not split

I. Juice of the liver:

(c) of rabbit

d-l-leucyl-glycin (spl. asym.)

glycyl-d-l-alanin (id.)

glycyl-glycin

2. Juice of muscle:

(a) of cattle

glycyl-glycin

d-l-leucyl-glycin

glycyl-d-l-alanin (small)

(b) of dog

glycyl-glycin

glycyl-1-tyrosin

(c) of rabbit

like the juice of the liver

3. Juice of kidney:

(a) of dog

glycyl-glycin

(b) of rabbit

like the juice of the liver

4. Plasma:

(a) of cattle

d-l-alanyl-glycin

glycyl-1-tyrosin

diglycyl-glycin

glycyl-d-l-alanin (small)

(b) of horse (plasma and serum)

d-l-alanyl-glycin

glycyl-1-tyrosin

triglycyl-glycin

d-1-leucyl-glycyl-glycin

glycyl-d-l-alanin

glycyl-d-l-leucin

diglycyl-glycin

d-1-alanyl-glycyl-glycin

(c) of man (serum)

glycyl-1-tyrosin

\section{Blood corpuscles of horse}

d-l-alanyl-glycin

glycyl-1-tyrosin

d-l-alanyl-glycyl-glycin

glycyl-d-1-leucin 
Polypeptids split

Polypeptids not split

diglycyl-glycin

d-l-alanyl-glycin

glycyl-d-l-alanin

6. Crystalline humor of pig

d-l-alanyl-glycin glycyl-l-tyrosin glycyl-d-l-alanin diglycyl-glycin

7. Brain of calf

d-I-alanyl-glycin diglycyl-glycin glycyl-d-l-alanin glycyl-I-tyrosin

\section{BIBLIOGRAPHY ON THE PEPTOLYTIC ENZYMES.}

Emil Fischer u. Em. Abderhatden. Ueber das Verhalten verschiedener Polypeptide gegen Pankreassaft u. Magensaft, Zeits. für physiol. Chem., I905, (46), p. 52 ; $1907,(51)$, p. 264.

Emil Fischer. Unters. über Aminosäuren u. Polypeptide, Berlin, 1906.

Emil Abderhalden u. Koelker. Zeils. f. physiol. Chem., I907, (5r), p. 294.

E. Abderhalden u. W. Manwaring. Zeils. f. physiol. Chem., 1908, (55), p. 379. Abderhalden u. F. Lussana. Zeits. f. physiol. Chem., I908, (55), p. 39 r. Abderhalden u. Koelker. Zeits. $f$. physiol. Chem., igo8, (55), p. 424. Abderhalden. Zeits. f. physiol. Chem., 1908, (57), pp. 320, 329, 332, 342; I909, (59), pp. 230, 249, 293; (60), p. 41 5; (6r), pp. 200, 421; (62), pp. I 20, I 16, I45, 243; I910, (64), pp. 100, 426, 429; (66), pp. I 20, 137, 265, 277; (68) pp. 312, 416. 


\section{CHAPTER III.}

\section{ENZYMES OF THE NUCLEO-PROTEINS.}

Nucleo-proteins, Nucleins, Nucleic Acids. - Among the various complex compounds encountered in the organism, there is a class of substances which contain, besides the four usual constituents of albuminoid materials, - carbon, hydrogen, oxygen, and nitrogen,- - two other elements, sulphur and phosphorus. These substances, important constituents of living cells particularly of the nuclei, are designated under the name of nucleoproteins. Insoluble in water, they dissolve in dilute alkali (about I to 5 parts per thousand), and are then reprecipitated upon the addition of acetic acid or a very small quantity of $\mathrm{HCl}$. They give the color reactions of albuminoids, but are dextro-rotary and not laevo-rotary like the albumins.

Nucleo-proteins are easily obtained from the pancreas, from the leucocytes of the thymus gland, from the brain, from the liver, as well as from many of the other organs. Their content in phosphorus varies between 0.5 per cent and 3.0 per cent. The nucleo-proteins are regarded as the result of the combination of a nuclein, a compound richer in phosphorus than the original nucleo-protein, and of a different albuminoid material, which may be either a protamine, a histone, or a true albumin as is the case of the nucleo-proteins taken from the sperm or the cellular nuclei of the higher animals. Thus the nucleo-proteins can be decomposed into their two constituents under the influence of the proteolytic enzymes or of weak acids. The protein is dissolved in the form either of an acid albumin or of albumose, while the nuclein remains, being insoluble in acidulated water. Nucleins are obtained from the tissues or substances rich in nucleo-proteins, which have been previously submitted to the action of the gastric juice or of dilute $\mathrm{HCl}$. Nucleins have been thus prepared with the soft roe of fishes, beer yeast, the 
cerebral substance, etc. Their chemical composition varies according to the origin:

Composition of SOME NuCleins.

\begin{tabular}{|c|c|c|c|c|c|}
\hline & Yeast. & Roe. & Brain. & Yolk of egg. & Milk. \\
\hline 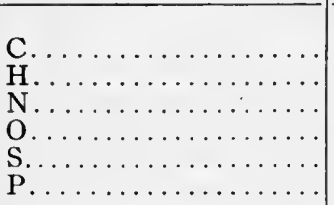 & $\begin{array}{r}\% \\
40.81 \\
5 \cdot 38 \\
15 \cdot 98 \\
31.26 \\
0.38 \\
6.19\end{array}$ & $\begin{array}{r}\% \\
36.1 \\
5.1 \\
13.1 \\
\cdots \\
\cdots .6 \\
9.6\end{array}$ & $\begin{array}{r}\% \\
50.5 \\
7.8 \\
13.2 \\
\cdots \\
\cdots \\
2.1\end{array}$ & $\begin{array}{r}\% \\
42.11 \\
6.08 \\
14.33 \\
31.05 \\
0.55 \\
5.19\end{array}$ & $\begin{array}{r}\% \\
48.5 \\
7.1 \\
13 \cdot 3 \\
\cdots . \\
\cdots .6 \\
4.6\end{array}$ \\
\hline
\end{tabular}

The composition of the last two substances is quite similar to that of the first three; however, these do not belong at all to the same family as the former. The substances extracted from the yolk of egg and from milk form what is called the para-nucleins, and will be described later.

The nucleins, in their turn, under the influence of alkalis split into an albuminoid material and into acids still richer in phosphorus than the original nucleins, the nucleic or nucleinic acids. These acids are amorphous compounds. Generally insoluble in water and alcohol, they are soluble in dilute alkalis but are not precipitable from these solutions by acetic acid. Yet they are reprecipitated by the addition of a diluted mineral acid, like 5 per cent $\mathrm{HCl}$. While the nucleins contain sulphur, the nucleic acids do not. These two compounds also differ in their phosphorus content, the percentage of this element in the nucleic acids reaching 9 to Io per cent. For nucleic acid of yeast the formula $\mathrm{C}_{40} \mathrm{H}_{69} \mathrm{~N}_{16} \mathrm{O}_{22} \cdot 2\left(\mathrm{P}_{2} \mathrm{O}_{5}\right)$ has been given. The nucleic acids have the property, in acid solution, of precipitating the albuminoids to give compounds similar to the nucleins. They do not give either the biuret or Millon reaction. Like the nucleoproteins, they are dextro-rotary.

The constitution of these acids is also very complex. Kossel has shown that under the action of mineral acids or diluted and hot alkalis these bodies decompose: (I) into phosphoric acid; (2) into xanthin or purin bases, such as xanthin, hypoxanthin, 
guanin, and adenin; (3) into pyrimidin bảses, as thymin, cytosin, and uracil; (3) finally into a sugar, pentose, or hexose. The presence in the decomposition products of xanthin bases is quite characteristic; it permits the establishment of a very clear distinction between the compounds just described and others which by their properties much resemble them. Although the latter do not interest us directly at this point, we will, nevertheless, say a word to make clear the difference just indicated.

In addition to the true nucleo-proteins we do, in fact, encounter similar compounds, designated by the name of paranucleo-proteins or pseudo-nucleo-proteins, or sometimes again nucleo-albumins, compounds whose principal representatives are the casein of milk and the vitellin of egg yolk, and which have the property, like true nucleo-proteins, of being split under the influence of the gastric juice into albuminoid material and into insoluble phosphoretted compounds, the para- or pseudo-nucleins. These hydrolyzed para-nucleins furnish only albuminoid derivatives and phosphoric acid, and never xanthin bases. The successive splitting of the nucleo-proteins may be represented by the following diagram:

$$
\underbrace{\text { Nucleo-protein }}_{\text {Protein nucleic acid ......... }}\left\{\begin{array}{l}
\begin{array}{l}
\text { Phosphoric acid. } \\
\text { Xanthin bases. } \\
\text { Pyramidin bases. } \\
\text { Sugars. }
\end{array}
\end{array}\right.
$$

The nucleo-proteins under the influence of enzymes are successively transformed into nucleins, into nucleic acids, then into xanthin bases, into phosphoric acid, etc. But only the first phase of the transformation, that which corresponds to the formation of protein and nuclein, can be realized by pepsin and trypsin. The nucleins resist the action of the digestive juices, both gastric and pancreatic. The transformation of the nucleins first into nucleic acids, then into phosphoric acid and into purin bases, is induced in living cells, on the contrary, by the endocellular enzymes, which bear the name of nucleases.

Nucleases, Preparation. - We owe to Schützenberger the first data on the decomposition of the nucleins. This investiga- 
tor found, in fact, that beer yeast, left to autolyse at about $37^{\circ}$ very rapidly furnishes guanin, hypoxanthin, xanthin, and phosphoric acid. Kossel has shown that these products come from the decomposition of the nucleins. The facts discovered by Schützenberger have been confirmed by Salomon, Salkowski, Kutscher, Schwiening, and many others. These writers have found, furthermore, that in auto-digestion of animal organs the decomposition products of nucleins are likewise found, as well as free phosphoric acid and characteristic bases. It has also been noted that during the germination of seeds the vegetable nucleoproteins undergo the same hydrolysis. Araki has shown that in the transformation of nucleic substances two distinct phenomena must be recognized. In a first phase the insoluble nuclein is dissolved, and in a second phase real digestion takes place. The dissolving of nucleic material can be brought about by pepsin and trypsin. Under the influence of these enzymes, there is observed also a very slight appearance of phosphoric acid, but the greater part of the phosphorus remains in the organic compound which is separated.

The most thorough hydrolysis is realized by means of the nucleases. These enzymes are found in a great number of organic juices, in serum, in yeast juice prepared by the Buchner method, in cultures of Aspergillus, of Penicillium and of, Mucor. Iwanoff has isolated the active enzyme of different mold-fungi. These are cultivated in broths containing nucleic acids as nitrogenous substrate. After the development of molds, the culture liquids are active toward nucleic acid, but are inactive toward albumin. Emmerlich and Loew have noted similar phenomena with certain pathogenic bacteria. Salomon has found in the auto-digestion of the liver, the muscles, and other organs the appearance of the same enzymes. Abderhalden and Schittenhelm have tried pancreas juice on thymo-nuclein on the one hand, and pancreatic juice on the other. They found that while the second liquid decomposes nuclein with the production of xanthin bases, the first causes the nucleic substance to undergo a much less radical transformation. The thymo-nucleic acid, 
which was slightly soluble in water and of a gelatinous appearance, becomes more soluble and no longer jellies. Furthermore, this substance, originally non-dialyzable, now passes through a parchment wall.

Kikkoji found nuclease in an edible Japanese mushroom, Cortinellus edodes. The mushroom, submitted to a strong pressure, furnishes a slightly acid liquid which rapidly decomposes sodium nucleate with the formation of guanin, adenin, and phosphoric acid. The course of the reaction has been followed by the liberation of phosphorus. To $2.5 \mathrm{~g}$. of sodium nucleate, previously dissolved in 150 c.c. of warm water, are added after cooling 25 c.c. of mushroom juice and a little toluol, and the mixture is then left at $37^{\circ}$.

Decomposition of Sodium Nucleate.

\begin{tabular}{|c|c|}
\hline Length of the digestion. & $\begin{array}{c}\mathrm{P}_{2} \mathrm{O}_{6} \text { appearing in 20 c.c. } \\
\text { liquid. }\end{array}$ \\
\hline hrs. & g. \\
24 & $0.014 \mathrm{I}$ \\
48 & 0.0206 \\
72 & $0.025 \mathrm{I}$ \\
96 & 0.0276 \\
$\mathrm{I} 20$ & 0.0287 \\
\hline
\end{tabular}

Sachs has investigated the conditions favorable to the isolation of the nuclease of the pancreas. Ioo g. of pancreas are minced and left with 200 c.c. of water with addition of toluol. From time to time a filtered sample is taken, and the clear liquid tested on fibrin, as well as on sodium nucleate.

Effect of an Extract of Pancreas on Fibrin and on Sodium NuCleate.

\begin{tabular}{|c|c|c|}
\hline Length of extraction. & $\begin{array}{l}\text { Minimum time for dissolving a } \\
\text { flake of fibrin. }\end{array}$ & $\begin{array}{l}\text { Minimum time for causing the } \\
\text { jelly of sodium nucleate to } \\
\text { disappear. }\end{array}$ \\
\hline $\begin{array}{l}\text { hrs. } \\
\text { I6 } \\
\text { I8 } \\
22 \\
25 \\
41\end{array}$ & $\begin{array}{l}2 \text { to } 3 \text { hours } \\
\text { I hour } \\
45 \text { to } 30 \text { minutes } \\
20 \text { minutes } \\
20\end{array}$ & $\begin{array}{l}4 \text { to } 6 \text { hours } \\
4 \text { to } 6 \text { " } \\
9 \text { to Io " } \\
\text { I6 to I8 “ } \\
\text { After } 24 \text { hours, no } \\
\text { liquefaction }\end{array}$ \\
\hline
\end{tabular}


The pancreas, after 16 hours of digestion, gives a liquid very active toward nucleic acid. The action on fibrin is feeble. With the length of the extraction the nucleases diminish, while trypsin increases. After $4 \mathrm{I}$ hours of maceration, the liquid obtained no longer digests nucleic acid. It appears from these tests that even in the passage of nucleic acid from the gelatinous to the soluble state a special enzyme intervenes. It is seen, further, that in the pancreas there exists, besides trypsin, a nuclease. But this latter acts in vitro, especially during the first period of the maceration. It disappears, in fact, quite rapidly from the extracted liquid in consequence, apparently, of a secondary action of the trypsin upon it.

The nuclease of pancreas in dry form is prepared in the following manner: I kilogram of pancreas is reduced to paste with sand, then submitted to the action of a Buchner press. The juice which immediately flows out is saturated with ammonium sulphate. The precipitate obtained is washed with alcohol and with ether, and then dried. In this form it preserves very well. This product rapidly liquefies the jelly of nucleic acid to give xanthin bases and phosphoric acid.

Properties. - Tests by dialysis have shown that the active substance does not pass through the filtering wall. A fresh pancreatic extract, rich in nuclease, is always slightly acid. By neutralizing or acidifying it further with acetic acid, we get an injurious effect from the point of view of the enzyme. The slightly acid reaction then appears favorable, but it must not be excessive. In every case, alkalinity is very detrimental. G. Pighini has applied the optical method for the investigation of nucleases. We know that nucleic acids rotate the plane of polarized light very strongly to the right. On the contrary, the products of decomposition do not give any rotation, or at least only a very slight one. Pighini used nucleic acid made by Merck. r.6 g. of this substance is dissolved in roo c.c. of physiological salt solution ( 0.85 per cent $\mathrm{NaCl}$ ). Special conditions are created by making the liquor slightly alkaline by the addition either of small quantities of $\mathrm{NH}_{3}$ or of $\mathrm{Na}_{2} \mathrm{CO}_{3}$. Then 2 c.c. of the liquid 
to be analyzed, for instance serum, are added and the variation of the rotatory power is determined in a Soleil saccharimeter.

Action of Serum of Various Origins on Nucleic Acid.

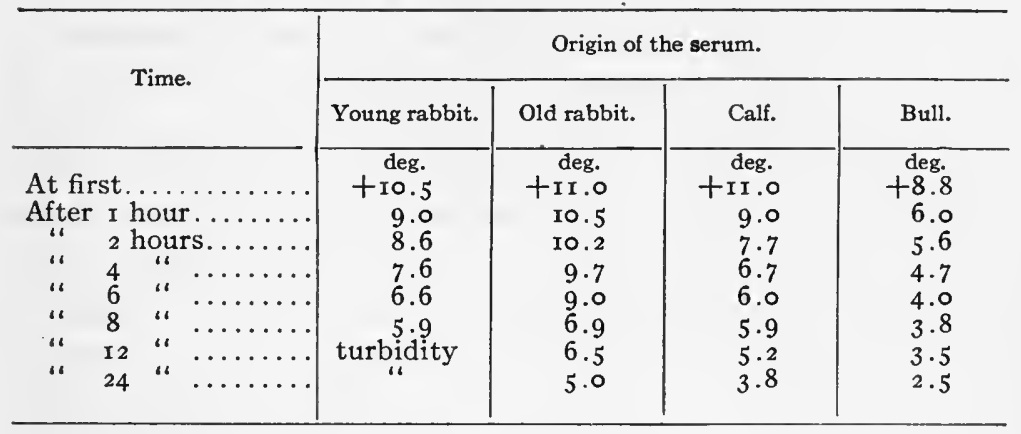

The action at the beginning is seen to be very intense, then to slacken progressively. Moreover, the same liquids, kept for some time at $65^{\circ}$, no longer react on nucleic acid. According to the author, who has made more exact determinations, the law of decrease of rotatory powers is a logarithmic function analogous to that of all enzymic activities.

Individuality of Nucleases. - Among the xanthin bases derived from nucleic acids, we find adenin, guanin, sarcin or hypoxanthin, and xanthin. Nucleic acid which comes from thymus gland gives adenin exclusively. Those taken from other tissues give by hydrolysis two, three, or four of the bases mentioned above. There are, then, several different nucleic acids. Kossel claims the existence of four nucleic acids corresponding to each of the bases; sarcylic acid gives sarcin, xanthic acid gives xanthin, adenylic acid gives adenin, and guanylic acid, guanin. The four nucleic acids will each have their own enzyme. There will then exist a guanylase, an adenylase, a xanthiase and a sarcylase. In support of this opinion, we may cite the fact that certain juices of organs are active on nucleic acids of one special origin, and not on others. From the point of view of the individuality of these nucleolytic enzymes, we must also take into consideration the fact that the transformation of nucleic acids 
into phosphoric acid and xanthin bases takes place by successive stages.

The polynucleotids are first hydrolyzed to mononucleotids. This reaction probably corresponds to the transformation of gelatinous nucleic acid into dialyzable acid, a modification which takes place without liberation of phosphoric acid. Then, as Levene and his students have shown, the mononucleotid is split into phosphoric acid and a nitrogenous compound, nucleonosin, which itself is lacking in phosphorus. The nucleonosin in its turn is decomposed into carbohydrate and xanthin bases. Thus guanylic acid, hydrolyzed in neutral solution and at a temperature of $130^{\circ}$, gives phosphoric acid and a sort of pentoside, guanosin, $\mathrm{C}_{10} \mathrm{H}_{13} \mathrm{O}_{5} \mathrm{~N}_{5}$, a well-defined crystalline substance which, under the influence of acids of moderate concentration, gives guanin and a pentose. Compounds analogous to guanosin have been obtained from nucleic acids of other origins, and, further, the presence of nucleosids among the cellular digestion products of nucleo-proteins has been detected.

Levene, who made remarkable investigations on the chemistry of the nucleins, believes that to the three phases of the decomposition of nucleins correspond three classes of different enzymes: nucleinases, nucleotidases, nucleosidases. The first transform nucleic molecules into nucleotids without liberation of phosphoric acid; the second split nucleotids into nucleosids and phosphoric acid; finally, the third decompose nucleosids into carbohydrate and xanthin bases.

In view of this classification, and considering as demonstrated the existence of the four nucleic acids of Kossel, we see that the number of nucleases must be very large. However, though experimental data on the subject are lacking, it is nevertheless beyond doubt that we have here a whole class of very different enzymes. Even as the albuminoid molecule disintegrates by degrees under the influence of several enzymes, which finally bring it to the state of very simple compounds, it is more than probable that the nuclein molecule also possesses a series of enzymes capable of causing it to undergo the successive transforma- 
tions which we have just described. Our knowledge on the chemistry of the nucleins is still too little advanced for us to be able at present to give a decisive proof.

\section{BIBLIOGRAPHY ON THE ENZYMES OF THE NUCLEO-PROTEINS.}

SCHÜTZENBERGER. C. R., 1874, (78), p. 493.

Salomon. Arch.f. Anatomie u. Physiologie, 188r, p. 166.

Kossec. Die Nucleine u. ihre Spaltungsproducte, Strassburg, 1881. Zeits.f. phys. Chem., 1882, (7), p. 7 .

Salkowsky. Zeits. f. physiol. Chem., 1889, (13), p. 527. Z. Klin. Med., Suppl. I890, (I 7$)$, p. 77 .

IwaNoFr. Vork. im Schimmelpilzen, Zeits.f. physiol. Chem., 1903, (39), p. 43.

Araki. Ueber enzymat. Zersetzung d. Nucleïnstoffe, Zeits. f. physiol. Chem., $1903,(38)$, p. 87 .

A. Schittenhely. Der Nucleinstoffwechsel u. seine Fermenten beim Menschen u. Thiere, Zeits. f. phsiol. Chem., I905, (46), p. 364; 1909; (63), p. 275.

SAChs. Gewinnung u. Wirkzamkeit, Zeits. f. physiol. Chem., 1905, (46), p. 337.

P. A. Levene et JacoBs. Ueber Guanylsäure, Ber. d. Deuts. chem. Ges., r 909 , (42), p. 2469 .

W. Jones. Ueber die Beziehung der auswässerigen Organextracten gewonnenen Nucleïnfermente zu den physiol. Vorgängen im lebenden Organismus, Zeits. f. physiol. Chem., s9ro, (65), p. 385 .

G. Pighini. Zeits.f. physiol. Chem., I910, (70), p. 85. Bioch. Zeits., (33), p. 190.

NeUBERg. Ueber Erkennung von enzymat. Nucleïns-Spaltung durch Polarisation, Bioch. Zeits., I910, (30), p. 505.

Walter Jones. Journ. of Biol. Chem., I9I1, (9), pp. 135, 169.

Levene et Medigzeceanu. Journ. of Biol. Chem., I9i I, (9), p. $3^{89}$.

A. Schttenhelm u. Wiener. Verdauung u. Resorption von Nucleïnsäure im Magen d. Kindes, Zeits. f. physiol. Chem., rgr r, (72), p. 459.

Hahn et Geret. Zeits. f. Biol., I900, (40), p. II 7 .

Kutscher. Zeits. f. physiol. Chem., I90I, (32), p. 66; (34), p. II6.

Schwiening. Virch. Arch., 1894, (136), p. 444.

Abderhalden u. Schttenhelm. Nucleinsäure im tierischen Organismus, Zeits. f. physiol. Chem., (47), p. $45^{2}$.

KıкKoJI. Vorkommen v. nucleīnspalt. Fermenten, Zeits. f. physiol. Chem., 1907, (51), p. 201.

EMmerlich u. Loew. Zeits. f. Hygiene, r899, (3r), p. r.

Schittenhelm. Ueber die Fermente d. Nucleïnstoffwechsels i. menschl. Organe, Zeits. f. physiol. Chem., (63), p. 256. 


\section{CHAPTER IV.}

\section{ARGINASE.}

In the course of the hydrolysis of albuminoids by $\mathrm{HCl}$ or proteolytic enzymes, as many as eighteen different atomic groupings are detached from the original molecule that was formed by linear chains, benzene, pyridin, indolin, and other nuclei. Among the nitrogenous fragments encountered is the interesting compound arginin, which is derivative of guanidin:

$\mathrm{NH}_{2}-\mathrm{C}(\mathrm{NH})-\mathrm{NH}-\mathrm{CH}_{2}-\mathrm{CH}_{2}-\mathrm{CH}_{2}-\mathrm{CH}\left(\mathrm{NH}_{2}\right)-\mathrm{CO}_{2} \mathrm{H}$.

This body is formed in great quantity in the decomposition of salmin, which is a protamine extract of sturgeon roe. In fact, two-thirds of the total nitrogen of this simple albuminoid is found in the form of arginin. According to Kossel, the arginin grouping must be considered as essentially albuminoid. Arginin resists the action of acids, as well as that of pepsin, trypsin, and erepsin, but is decomposed under the influence of alkalis with the formation of urea. The fact that arginin is not always found in the products of autolysis of animal organs, as well as the fact that erepsin can in certain cases act on protamines, led Kossel to the conclusion that the animal organism must contain a special enzyme capable of decomposing arginin into urea and ornithin, according to the reaction:

$$
\begin{array}{r}
\mathrm{NH}_{2}-\mathrm{C}(\mathrm{NH})-\underset{\text { arginin }}{\mathrm{NH}}-\mathrm{C}_{3} \mathrm{H}_{6}-\mathrm{CH}\left(\mathrm{NH}_{2}\right)-\mathrm{CO}_{2} \mathrm{H}+\mathrm{H}_{2} \mathrm{O} \\
=\underset{\text { urea }}{\mathrm{CO}\left(\mathrm{NH}_{2}\right)_{2}}+\mathrm{NH}_{2}-\mathrm{C}_{3} \mathrm{H}_{6}-\underset{\text { ornithin }}{\mathrm{CH}\left(\mathrm{NH}_{2}\right)-\mathrm{CO}_{2} \mathrm{H} .}
\end{array}
$$

This hypothesis was fully confirmed by experiment. Kossel submitted a solution of arginin carbonate to the action of juice of liver on the one hand, and to that of liver reduced to fragments on the other. He added toluol to the two liquids and left the whole at $37^{\circ}$. To analyze the products formed, the solutions are brought to the boiling point, which eliminates the albumin and 
in the filtrate, whose nitrogen content is known, the fraction precipitable by phosphotungstic acid is determined. This tungstic precipitate contains arginin, as well as the ornithin which has already appeared. These two substances can moreover be measured by treating the precipitate with $\mathrm{Ba}(\mathrm{OH})_{2}$ which liberates the bases, and then by making a separation of the latter by means of silver sulphate. The silver salts are then decomposed by $\mathrm{BaCl}_{2}$. On the other hand, the urea which corresponds to the arginin decomposed in the reaction can be easily measured in the fraction not precipitated by the tungstic reagent. The following are the results so obtained:

Decomposition of Arginin by the Juice of Liver.

\begin{tabular}{|c|c|c|c|c|c|c|}
\hline Quantity of liver. & Volume & $\begin{array}{l}\text { Length of } \\
\text { digestion. }\end{array}$ & $\begin{array}{l}\text { Argin- } \\
\text { in used }\end{array}$ & $\begin{array}{l}\text { Arginin de- } \\
\text { composed. }\end{array}$ & $\begin{array}{l}\text { Ornithin } \\
\text { formed. }\end{array}$ & $\begin{array}{l}\text { Urea } \\
\text { formed. }\end{array}$ \\
\hline $\begin{array}{l}25 \text { c.c. juice pre- } \\
\text { viously boiled... }\end{array}$ & $\begin{array}{l}\text { c.c. } \\
\text { I000 }\end{array}$ & h. & g. & g. & Traces & 0.03 \\
\hline $25 \mathrm{~g}$. pulp of liver. . & 1000 & 5 & 3.6 & 3.4 & I.9 & 0.9 \\
\hline & 1000 & 5 & 0.0 & 0.0 & Traces & Traces \\
\hline I0 " 4 & 300 & 5 & 5.0 & 5.0 & 2.6 & I. 9 \\
\hline $\begin{array}{l}20 \text { c.c. juice of liver.. } \\
\text { I0 }\end{array}$ & 400 & 5 & I.3 & I. 2 & 0.8 & 0.5 \\
\hline brought to $100^{\circ} \ldots$ & 300 & 36 & 0.6 & 0.08 & Traces & 0.03 \\
\hline
\end{tabular}

As seen, the action is really due to an active substance which is destroyed at $100^{\circ}$. The hydrolysis is extremely rapid in comparison with that obtained with the other enzymes, since in 6 hours $5 \mathrm{~g}$. of material can be destroyed. This special enzyme has received the name of arginase.

Preparation. - To prepare arginase, reduce a dog liver to pap, mix thoroughly with infusorial earth, and submit the whole to pressure. For 100 parts of liver, about 45 parts of juice are obtained. In this liquid, precipitate the arginase by a mixture of 2 parts alcohol and 1 part ether. The coagulum is washed in ether, and then dried over sulphuric acid. Ioo parts of liver give about $2.5 \mathrm{~g}$. of active powder, 0.I g. of which completely decomposes $2 \mathrm{~g}$. of arginin in 24 hours. This product is only partially soluble in water, the insoluble parts being especially 
active. The solutions, as well as the powder, may be kept very well for several months. Arginase may also be obtained from a maceration of liver made in water acidulated with acetic acid. $250 \mathrm{~g}$. of liver are put in a liter of water containing 0.2 per cent of acetic acid. After standing 16 hours at $37^{\circ}$ it is then filtered. In the clear liquid the enzyme is precipitated by a mixture of alcohol and ether, or by a solution of ammonium sulphate. The coagulum is collected and dried in the first case; in the second, it is suspended in water and dialyzed.

Arginase is encountered especially in the kidney, the thymus gland, and the lymphatic ganglia. It is also found, but in less amounts, in the intestinal mucous membrane. On the contrary, it is absent from the pancreatic juice, the muscle and the blood. Finally, it is very abundant in yeast juice.

To follow the action of arginase, use is made of a 0.5 per cent or I per cent solution of an arginin salt (Ex.: carbonate). The active substance to be analyzed is added, and immediate determinations are made in the liquid of the relation between the total nitrogen and the nitrogen not precipitable by phosphotungstic acid. Then, after 3 or 4 hours' stay in the thermostat, the nonprecipitable nitrogen is again determined. The increase of this ratio indicates the decomposition of the arginin present.

\section{BIBLIOGRAPHY ON ARGINASE.}

Kossel and Dakin. 'Ueber die Arginase, Zeits. f. physiol. Chem., I904, (4r), p. $322 ;(42)$, p. $18 \mathrm{I}$.

Alex. KIesel. Zeils. f. physiol. Chem., r909, (60), p. 46I.

Charles Richet. C. R., I894, (I I8), p. I I 25. Diastase uréo-poítique, Soc. Biol., I894, (I), p. 525 .

Shiga. Ueber einige Hefefermente, Zeits. f. physiol. Chem., 1904, (42), p. 505.

DAKIN. The action of arginase upon Creatin and other Guanidin derivatives, Journ. of Biol. Chem., 1907, (3), p. 435. 


\section{CHAPTER V.}

\section{CREATINO-CREATASE, CREATASE, CREATINASE.}

By studying the auto-digestion of muscular juice, we find immediately an increase of the creatin, an increase which results from the splitting of certain nitrogenous compounds whose evolution we cannot, moreover, very well follow. However, the creatin formed does not accumulate in the liquid, but progressively disappears. This is due to the fact that the creatin present is transformed into creatinin, and that these two compounds, creatin and creatinin, are then decomposed in a more complete manner in consequence of the action of certain biochemical agents.

Transformation of Creatin in the Autolysis of Muscular Juice.

\begin{tabular}{|c|c|c|c|c|}
\hline \multirow{2}{*}{$\begin{array}{l}\text { Quantity of } \\
\text { muscular juice } \\
\text { used. }\end{array}$} & \multirow{2}{*}{ Water. } & \multirow{2}{*}{ Length of autolysis. } & \multicolumn{2}{|c|}{ Quantity of } \\
\hline & & & Creatinin. & $\begin{array}{l}\text { Creatin } \\
\text { creatinin. }\end{array}$ \\
\hline $\begin{array}{l}\text { c.c. } \\
5 \\
5 \\
5 \\
5 \\
5\end{array}$ & $\begin{array}{l}\text { c.c. } \\
0 \\
5 \\
5 \\
5 \\
5\end{array}$ & $\begin{array}{l}\text { At beginning } \\
\text { After } 114 \text { hours } \\
\text { “. } 160 \text { “، } \\
\text { “ } 227 \text { “ } \\
\text { “ } 91 \text { days }\end{array}$ & $\begin{array}{l}\text { mg. } \\
2.8 \\
4.1 \\
5.2 \\
2.9 \\
2.8\end{array}$ & $\begin{array}{c}\text { mg. } \\
19.2 \\
23.0 \\
21.0 \\
16.6 \\
10.2\end{array}$ \\
\hline
\end{tabular}

Thus, we find first an increase in the quantity of creatin present, since after I 14 hours we measure $23.0-4.1=18.9 \mathrm{mg}$. of this body, in place of $19.2-2.8=16.4 \mathrm{mg}$. measured at the beginning. But this quantity then diminishes to produce creatinin, whose proportion will increase. Furthermore, the creatin, and even the creatinin, will be destroyed in another way, since the sum of these two substances grows smaller. The transitory increase in the content in creatin, and the ultimate destruction of the creatin and the creatinin can be better shown if creatin is added to muscular juice. 
Transformation of Creatin in the Autolysis of Muscular Juice.

\begin{tabular}{c|c|c|c|c}
\hline $\begin{array}{c}\text { Quantity of } \\
\text { juice. }\end{array}$ & Creatin added. & Duration of autolysis. & $\begin{array}{c}\text { Creatinin } \\
\text { found. }\end{array}$ & $\begin{array}{c}\text { Creatin+creat. } \\
\text { inin found. }\end{array}$ \\
\cline { 1 - 2 } c.c. & mg. & At beginning & mg. & mg. \\
5 & 0 & After 40 hours & 3.8 & 19.2 \\
5 & 8.6 & " 155 “" & 9.7 & $3 \mathrm{I} .4$ \\
5 & 8.6 & " 91 days & 3.9 & 16.8 \\
5 & 8.6 & & & 16 \\
\hline
\end{tabular}

Although Voit first stated that the transformation in the organism of creatin into creatinin took place in the kidneys, $\mathrm{E}$. Gérard, in r9or, showed that this reaction could be made in vitro by the aid of a water extract of horse kidney, and that it was apparently due to a soluble ferment. Gottlieb and Stangassinger have confirmed these data. They have found a transformation of creatin into creatinin, under the influence of the extracts of different organs, and at the same time have established that these liquids heated to $100^{\circ}$ lose all their activity. The active substance causing the decomposition of creatin, and which we will call creatino-creatase, can be precipitated from the juices of organs, either by a mixture of alcohol and ether or by ammonium sulphate. This enzyme acts in neutral or very slightly acid media. The addition to the juice of 1.5 c.c. of $\mathrm{N} / 10 \mathrm{NaOH}$ per Ioo of juice greatly retards the action. Creatino-creatase is encountered especially in the kidney and the liver. It is precipitated from the juices of these organs together with arginase, but it should be noted that there are certain organs from which arginase is absent and which, however, contain creatino-creatase.

The chemical reaction accelerated by creatino-creatase is expressed by the following equation:

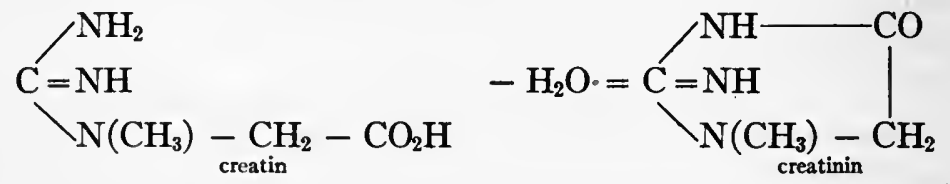

According to Gottlieb and Stangassinger, in the disappearance of creatin three different enzymes intervene; the one just described, which transforms creatin into creatinin, and then two 
others, creatase and creatinase, which decompose creatin and creatinin respectively.

The existence of the last two enzymes is very problematical; in fact, the chemical effect which they do produce is not exactly known. The disappearance of these compounds may even be the result of the action of oxidases or other enzymes which are already known. However, it appears certain that the transformation of creatin into creatinin is due to an enzyme, and the fact is all the more remarkable that the transformation in question is of a quite different character from that which is generally observed. Without doubt, pepsin and trypsin, while having as a general function the hydrolysis of albuminoid materials, can also in certain cases cause the reverse reaction and the production of condensation compounds, the plasteins, of which we have already spoken. But in the case of creatino-creatase the enzyme normally dehydrates the molecule, and the corresponding process of hydration is not known. From this point of view creatino-creatase deserves a special place in the discussion of proteolytic enzymes. It should, moreover, be noted that this enzyme, extracted from the kidney, continues to show the dehydrating action characteristic of the juice of this organ. Abelous and Ribaut have realized some years ago the synthesis of hippuric acid from glycin and benzoic acid by means of water macerations of horse kidney.

\section{BIBLIOGRAPHY ON CREATASE.}

Gotrieb et Stangassinger. Ueber das Verhalten des Kreatins bei der Autolysen Zeits. f. phys. Chem., 1907, (52), p. I; (55), p. 322.

GERARD. Transform. de la créatine en créatinine par un ferment soluble déshydratant l'organisme, C. $R$., I901, (1), p. I53.

Abelous et Ribaut. Sor. Biol., I900, (52), p. 543.

Vort. Zeits. f. Biol., (4), p. I77. 

PART V.

AMIDASES. 



\section{AMIDASES.}

\section{$\S$ I. Historical. - Distribution. - Properties.}

\section{Physiological Rôle.}

We have seen in the preceding chapters that trypsin and erepsin induce the conversion of proteins to amino-acids, and a complete hydrolysis of the albuminoid material is obtained through the combined action of these two enzymes. The aminoacids formed are not, however, directly assimilable; their utilization in plants and by micro-organisms requires in most cases another decomposition, which takes place through the mediation of special catalysts, bearing the name of amidases or again that of de-amidases. This class of enzymes acts on the products of tryptic and ereptic digestion and reduces the quaternary substances to ternary substances by transforming the amide and amine nitrogen into ammonia, and by producing volatile fatty acids, oxyacids, phenols, and alcohols.

For a very long time it has been known that in the presence of certain bacteria the digestion of nitrogenous substances is accompanied by a formation of ammonia, of organic acids, and of alcohols. The chemistry of this reaction has been previously studied by Nencki, but the catalysts which intervene in this decomposition escaped discovery. The first result obtained in the investigation of active substances, and one not highly conclusive, is due to Musculus, who in 1874 succeeded in isolating the soluble enzyme that transforms urea into ammonia. This enzyme, which bears the name urease, is the subject of a very extensive study on the part of Miquel. Urease plays a part which is very important in the dunghill and in the soil, but it is without effect on substances derived from tryptic or ereptic hydrolyses. The existence of an enzyme capable of acting on these products was first discovered in 1898 by Loewi. This investigator, in verifying the 
tests of Ch. Richet on the formation of urea in the liver after death, recognized that the maceration of crushed liver acted on glycin and on leucin, giving rise to compounds susceptible of being easily transformed into ammonia. The data furnished by Loewi have been confirmed by Jacobi in 1900 . According to Jacobi, the autolysis of the liver in the presence of amide bodies and of toluol also produces ammonia. In 1902, Gonnerman found that macerations of liver or of kidney are active on succinimide, benzamide and phthalimide, yet the quantities of ammonia formed in all these tests are small. Gonnerman likewise attributes to trypsin the ability to decompose succinimide, benzamide and phthalimide; emulsin acts only on phthalimide. However, these latter facts seem doubtful.

In I904, S. Lang studied the deamidization of glycin, leucin, tyrosin, phenylamine, acetamide, glucosamine, and urea. The amidases, according to this investigator, are distributed in all the animal organs. The comparative study of macerations of different animal organs from the point of view of their action on amines and amides, has indicated perceptible qualitative and quantitative differences. With glycin, the macerations of intestine or of pancreas are three times as active as those of liver. Kidney and testicles have the same action as the liver. On the contrary, the liquids obtained from the spleen or the lymphatic glands are without effect. With tyrosin, the kidney gives the maximum action; the liver, on the contrary, does not act. With asparagin and glutamine, results are obtained with macerations of lymphatic glands, of testicles, of intestine, of spleen, and of pancreas, but the action is confined to the amide nitrogen. With acetamide, the pancreas does not exert any action; on the contrary, the kidneys and the liver are plainly active. It is to be noted that the decomposition of acetamide is very slight, only ro per cent of the nitrogen being transformed. With glucosamine, the pancreas does not act, the kidneys give the maximum effect, and the intestines give a limited action. In the tests of Lang the activity of the macerations of organs has been studied, both in the presence of toluol and without the addi- 
tion of an antiseptic. This investigator has found that toluol considerably retards the transformation. He finds, in fact, that the maceration of liver with addition of amino-acids produces without antiseptic as much ammonia. in an hour as it would give in 12 days in the presence of toluol.

The data furnished by Loewi, Gonnerman, and S. Lang have thus shown in different animal organs the presence of catalysts acting on the amide compounds. On the other hand, in 1904, Schibata gave the proof of the existence of amidases in Aspergillus. Two years later Effront studied amidases of yeasts and of butyric ferments. Finally the work of Kiesel and Butkewitsch disclosed the presence and the rôle of the amidases in plants.

According to Schibata, the dried and crushed mycelium of Aspergillus niger, as well as the mycelium treated with acetone, contains an active substance which gives ammonia with acetamide, oxamide, benzamide, and asparagin. Dried Aspergillus niger is also active toward urea, *

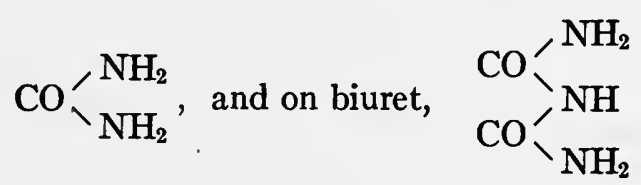

but it is inactive on ethylurethane,

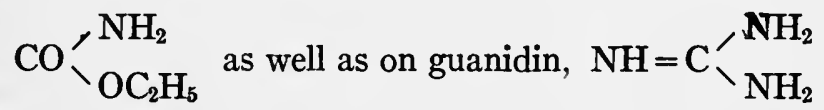

It has just been stated that Butkewitsch and Kiesel established the presence of these enzymes in plants, and it appears from their studies that the amidases intervene in the assimilation of nitrogenous materials by vegetables. In fact, if we consider a germinated plant, Vicia Faba for example, we find that it furnishes by autolysis, in the presence of chloroform and toluol, very appreciable quantities of ammonia. The analysis of the juice before and after autolysis gives, according to Kiesel, the following figures:

* The dried mycelium of Aspergillus also decomposes hippuric acid into its two constituents, but it is without action on the glycin so produced. 
Composition of the Juice of Vicia faba.

\begin{tabular}{|c|c|c|c|c|}
\hline \multirow[b]{2}{*}{ Nitrogen measured. } & \multicolumn{2}{|c|}{ Before autolysis. } & \multicolumn{2}{|c|}{ After autolysis. } \\
\hline & $\underset{\text { juice. }}{O \text { On Ioo g. of }}$ & $\begin{array}{l}\text { Per cent of } \\
\text { total nitro- } \\
\text { gen. }\end{array}$ & On roo g. of & $\begin{array}{l}\text { Per cent of } \\
\text { total nitro- } \\
\text { gen. }\end{array}$ \\
\hline 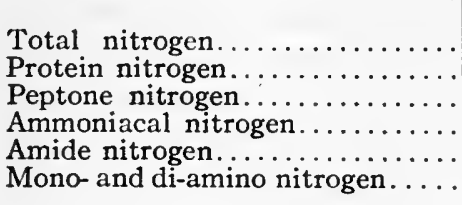 & $\begin{array}{l}\mathrm{g} . \\
0.543 \\
0.112 \\
0.030 \\
0.013 \\
0.121 \\
0.263\end{array}$ & $\begin{array}{r}100 . \infty \\
20.63 \\
5.52 \\
2.40 \\
22.29 \\
49.17\end{array}$ & $\begin{array}{l}\text { g. } \\
0.55 \mathrm{r} \\
0.101 \\
0.055 \\
0.075 \\
0.104 \\
0.216\end{array}$ & $\begin{array}{r}100.00 \\
18.33 \\
9.98 \\
13.61 \\
18.87 \\
39.20\end{array}$ \\
\hline
\end{tabular}

The ammoniacal nitrogen increases during autolysis from 2.40 per cent to $13.6 \mathrm{I}$ per cent, but this quantity of ammonia produced considerably exceeds that which could be formed by the peptonization of protein materials. Neither can it be attributed to the transformation of amide nitrogen. On the contrary, the appearance of this substance coincides with the disappearance of mono- and di-amino nitrogen. It is, then, perfectly natural to suppose that its formation results from the decomposition of the amino-acids. On the other hand, Butkewitsch has succeeded in following very closely the genesis and the evolution of ammonia in the course of vegetation. We know that during germination, amide bodies such as asparagin and glutamin are produced in abundance. Now the accumulation of these amides in plants cannot be the result of the action of proteolytic enzymes, the quantities found there being much higher than could arise from peptic and tryptic activity. On the contrary, the increase of amide bodies takes place simultaneously with the disappearance of the mono-amino-acids. These observations should allow Schulz and Loewi to explain the formation of amides during germination.

Schulz and Loewi are of the opinion that amino-acids coming from the digestion of albuminoid reserves are decomposed by amidases with the liberation of ammonia, and that the amides then form, due to a synthetic activity. of the cell. The presence of a small quantity of ammonia in the germinated 
seeds is moreover favorable to this opinion. Butkewitsch has added an experimental proof to the theory of Loewi. By referring to the work of Claude Bernard on anesthesia, he caused seeds to germinate in the presence of toluol. The anesthesia preventing all vital reconstruction from taking place, the germinated seeds can no longer elaborate amides, and the product formed (in this case, ammonia) under the influence of the enzyme must remain intact in the plant. Such was actually found to be the case: By causing Lupinus luteus to germinate in the presence of toluol, he found in the seeds 12 times the ammonia found under normal conditions. Butkewitsch arrives at a similar result by suppressing in the germinating seeds the carbohydrate reserve. Ammonia nitrogen accumulates and the formation of amides does not take place, this time in consequence of a want of the elements necessary for the germs to effect their synthesis.

As to the presence of amidases in blood as well as their rôle in assimilation, we find very interesting data in the work of Medwedew. Oxalated blood, left between $36^{\circ}$ and $38^{\circ}$, shows perceptible changes in its ammonia content. These variations are observed even under conditions of perfect asepsis. The animals which have undergone thyro-parathyroidectomy give a blood whose content in ammonia gradually increases during the first 24 hours. This is not the case with the blood of animals that have fasted for a long time. In consequence of denutrition, this blood has an ammonia content three times as high as that found under normal conditions, which points to a diminution in this substance on preserving. This decline is especially noticeable at first. After six hours of rest a 40 per cent to 45 per cent deficit is observed, after which the blood remains fairly stable.

The accumulation of ammonia in the blood of animals that have been operated upon follows a very regular course, and corresponds practically to a logarithmic curve whose constant, calculated by assuming the quantity of active substance fixed, is well verified by experiment. The sudden arrest in the 
increase of ammonia after 24 hours is explained by the exhaustion of the material on which the catalyst acts. In other words, this arrest corresponds to the moment when no more amide bodies capable of producing ammonia remain. The reverse action, that is to say, the disappearance of ammonia which is regularly found in the blood of iasting animals, would be due, on the contrary, to the intervention of a synthetic enzyme which would fix the ammonia into a true combination. The origin of this catalyst, or rather the first cause of this transformation, must be found in the thyroid apparatus, an organ which furnishes an enzyme endowed with a condensing power, or rather an organ which would act directly by bringing to the amidases certain new elements that permit them to perform a reversible action.

In normal blood, there is also observed an increase of ammonia in the course of its preservation, but this phenomenon is of a different character. At first the reaction is very slow, and is accentuated only in the long run, in consequence apparently of an addition of active substance flowing into the medium to keep pace with the reaction. It appears from this special course that deamidization takes place likewise in the plasma, and that the catalysts, at first imprisoned in the blood corpuscles, are slowly released. This last conclusion is experimentally verified by the fact that blood, hæmolyzed by saponin acts entirely differently from normal blood:

Increase of Ammonia in Normal Blood and in Hæamolyzed Blood.

\begin{tabular}{|c|c|c|c|c|}
\hline \multirow{2}{*}{$\begin{array}{l}\text { Length in } \\
\text { hours. }\end{array}$} & \multicolumn{2}{|c|}{ Normal blood oxalated. } & \multicolumn{2}{|c|}{ Blood oxalated + saponin. } \\
\hline & $\begin{array}{l}\mathrm{NH}_{3} \text { in } 100 \mathrm{~g} . \\
\text { blood. }\end{array}$ & Increase in $\mathrm{NH}_{3}$. & $\begin{array}{l}\mathrm{NH}_{3} \text { in roo g. } \\
\text { blood. }\end{array}$ & Increase in $\mathrm{NH}_{3}$. \\
\hline $\begin{array}{c}0 \\
3 \frac{1}{2} \\
55^{\frac{1}{2}} \\
7 \frac{1}{2} \\
10 \frac{1}{2} \\
22 \frac{1}{2} \\
24\end{array}$ & $\begin{array}{c}\mathrm{mg} . \\
\circ .5 \mathrm{I} \\
\ldots \ldots \\
\circ .59 \\
\ldots \ldots \\
\ldots \ldots \\
\mathrm{I} .43 \\
\ldots \ldots\end{array}$ & $\begin{array}{c}\mathrm{mg} . \\
\ldots \ldots \\
\ldots \ldots \\
\circ .08 \\
\ldots \ldots \\
\ldots \ldots \\
\ldots .9^{2} \\
\ldots\end{array}$ & $\begin{array}{c}\text { mg. } \\
0.5 \text { I } \\
\text { I.05 } \\
\ldots . . \\
\text { I.33 } \\
\text { I.45 } \\
\ldots \ldots \\
\text { I. } 55\end{array}$ & $\begin{array}{c}\text { mg. } \\
\ldots . .5 \\
\circ .54 \\
\cdots .3 . \\
0.82 \\
0.94 \\
\ldots \ldots \\
I .04\end{array}$ \\
\hline
\end{tabular}


$\mathrm{n}$ hæmolyzed blood, the increase in ammonia follows the same law as that found in the blood of animals deprived of the thyroid apparatus, that is to say it takes place according to a logarithmic law. From data of Medwedew, we can then conclude that blood corpuscles contain amidases, which are more or less rapidly poured out into the plasma according to the condition of the animal. Yet the action of the amidases of the blood is counterbalanced by a synthetic action, which immobilizes the ammonia formed, and which is produced by the thyroidal secretions. These two enzymes, capable of reversed effects, are constantly found in normal blood.

The presence of amidases in animal tissues, and especially in blood, and in higher plants as well as in micro-organisms, has attracted the attention of physiologists to the rôle which these enzymes play in nature. It is generally supposed that these enzymes are the regulators of the reaction of the medium. To them is due, therefore, the constant alkalinity in the blood and in the tissues. But it is also beyond doubt that these active substances intervene in animal nutrition, as they intervene in vegetable nutrition. The theories as to the assimilation of albuminoids have for some time undergone manifold modifications, as will be seen more in detail later. It was first believed that proteins were directly assimilable and that they could be utilized for reconstructive work as well as for the maintenance of the individual if they were found in a soluble state. This theory was abandoned, and we came to believe that it is the albumoses which are the true assimilable products. Then the direct utilization of these substances has been doubted, and the property of being directly absorbed was attributed to the peptones.

At present, much more value is attached to the final products of hydrolysis, to the mono- and di-amino-acids, than to all the substances which precede them in the degradation of nitrogenous foods. Many writers believe that these products through a retrogressive metamorphosis in the digestive mucous membranes form the various albuminoids appropriate to the 
needs of the organism. In reality, there is nothing to prove that the amino-acids enter directly into the circulation; on the contrary, there is every reason to think that these products are previously decomposed, provided that the enzymes which transform them are also present.

During plant germination, the albuminoids are submitted to the action of proteolytic enzymes. These last are the ones which produce amino compounds along with very small quantities of ammonia. Under the action of amidases, the aminoacids are transformed into ternary substances and into ammonia, which serve for the synthesis of albuminoid material, as well as for the formation of amides such as asparagin and glutamin; these last, moreover, form albuminoids as required. In the animal kingdom, the final products of the metamorphosis of albuminoid substances are chiefly uric acid and urea. With mammals, the formation of this last substance appears, at least in a certain measure, as a consequence of a dehydration in the liver of ammonium carbamate and carbonate, these substances themselves resulting from the decomposition by the amidases of the amino-acids of digestive origin. The uric acid results from a direct disintegration of the nucleoproteins, and probably also, as has been plainly established in the case of birds, from a true synthesis realized by aid of ammonical salts and ternary substances resulting from the action of the amidases. Urea would thus be, at least in the case of mammals where its formation is important, a residuary ammonia, rendered inoffensive by its immediate transformation into carbamide. In plants the same ammonia is found in the form of amides such as asparagin.

Finally, the amidases, which constantly intervene in the animal and vegetable kingdoms, must be considered as digestive enzymes; it is they which produce the final hydrolysis and decomposition of the amino-acids, and thus render them directly assimilable. 


\section{§ 2. Chemicai Activity of Ammases.}

In all the previous experiments the formation of ammonia was of very little importance. Furthermore, the enzyme had a very limited activity, and it has not been possible to analyze the products formed with the exception of the ammonia. It follows that the chemical reaction induced by these enzymes could not be established experimentally. The principal difficulty in this kind of investigation lies in the fact that the alkaline medium necessary for the activity of amidase very easily becomes infected. It is also to be noted that the amidases are intracellular enzymes, and that they are obtained with great difficulty in the liquids. Furthermore, these enzymes are considerably weakened by antiseptics.

In order to determine the intermediate products and those formed at the end of the reaction, and to establish the chemical process by which the amidases react, it is necessary to make a thorough study of this decomposition, and to determine the experimental conditions permitting a definite reaction to take place. This was accomplished by Effront in 1905. He demonstrated that amidase is found abundantly in beer yeast, as well as in a butyric ferment obtained from the soil. The maximum effect is obtained with cultures with a vitality reduced either by alkalis or by other chemical or physical conditions. The active liquid, filtered from the ferment or from the yeast, still produces an action on the amino-acids. This action ceases when the liquid is brought to a temperature of $100^{\circ}$. The activity of amidase coming from living cultures of butyric ferment is considerably accelerated by aeration and by a strong alkalinity of the medium. Under these conditions a very thorough enzymic action is observed, and in a majority of cases a complete deamidization of the amino-acids experimented with. Effront succeeded in isolating and identifying in various decompositions all the products formed, and in establishing the reaction equations involved. From this study the following facts appear: 
I. The monobasic acids deamidize under the influence of amidase, and are transformed into ammonia salts. Ex.:

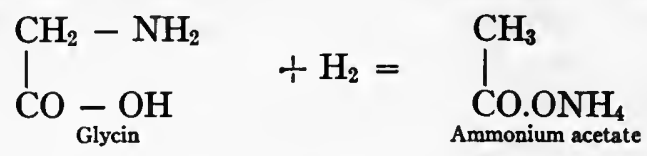

2. Betain is transformed under the influence of amidase into the acetate of trimethylamine:

$$
\mathrm{OH}-\underset{\text { Hydrated betain (or oxynevrin) }}{\mathrm{N}}\left(\mathrm{CH}_{3}\right)_{3}-\mathrm{CH}_{2}-\mathrm{CO}_{2} \mathrm{H}+\mathrm{H}_{2}-\mathrm{H}_{2} \mathrm{O}=\underset{\text { Acetate of trimethylamine }}{\mathrm{CH}_{3}} \cdot \mathrm{CO}_{2} \mathrm{H}, \mathrm{N}\left(\mathrm{CH}_{3}\right)_{3} \text {. }
$$

3. The polybasic acids undergo a molecular degradation upon deamidization. Ex.:

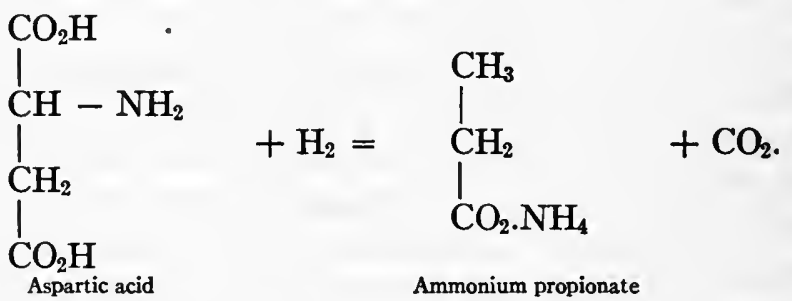

4. In asparagin, the deamidization begins with the amide group and ends with the same result as with aspartic acid. The molecular simplification has also been observed with glutamic acid:<smiles>NC(CCC(=O)O)C(=O)O</smiles><smiles>CCCC(N)=O</smiles>

All these transformations are the result of a reduction. The hydrogen necessary comes from a decomposition of water. This latter reaction evidently cannot take place except in the presence of easily oxidizable substances which appropriate step by step the oxygen liberated. 
The presence of amidase in yeast and its secretion in the course of the autolysis of the latter have since been confirmed by Fürth and Friedeman. On the other hand, the fact indicated by Effront that the enzymes appear more abundantly in bacteria of a reduced vitality, has been again noted by Berghaus; this investigator has found, further, the presence of amidase in the following bacteria: B. coli com., Proteus, Prodigiosus, B. faecalis alc. Finally, the transformation of glutamic acid into butyric acid has been also observed in putrefaction, by Brasch and $\mathrm{C}$. Neuberg. It should be observed, in passing, that in this last fermentation, the same enzyme intervenes as that which acts on beer yeast. Previous to his last publication and especially in 1907, Neuberg had submitted an hypothesis as to the transformation of glutamic acid into butyric acid. This hypothesis, formulated during his study on the origin of petroleum, is fully verified by what we now know concerning amidases. (See the chapter on the Origin of the Petroleum.) The transformation of asparagin into aspartic acid in putrefaction has likewise been studied by this investigator. He finds, besides the reaction noted above, the formation of succinic acid as a secondary product. Finally, the decomposition of amino-isovalerianic acid into valerianic acid has also been noted by Neuberg in putrefaction.

In all the reactions just cited there is a formation of ammonia as a result of a reduction, and also the production of volatile fatty acids. It must, however, be observed that amidases can also act in a different manner on amino-acids; the liberation of ammonia can be accompanied by a production of an oxyacid or by the formation of an alcohol. In general, the deamidization is caused either by a hydrolysis or by a reduction, these two processes being, moreover, capable of taking place simultaneously. The following is a scheme of the three types of reactions:

(a) Hydrolysis:

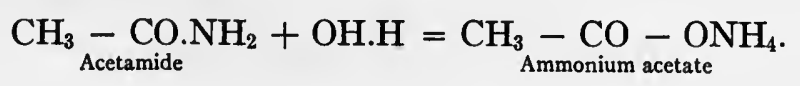


(b) Reduction:

$\mathrm{CH}_{2} \cdot \mathrm{NH}_{2}$

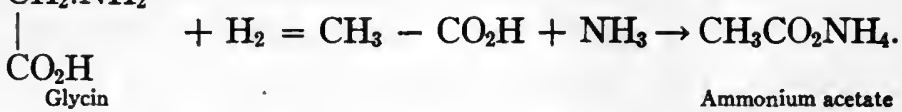

(c) Hydrolysis and reduction:

$$
\begin{aligned}
\mathrm{CO}_{2} \mathrm{H}-\mathrm{CH}_{2} & -\underset{\text { Asparagin }}{\mathrm{CH}}\left(\mathrm{NH}_{2}\right)-\mathrm{CONH}_{2}+\mathrm{H}_{2} \mathrm{O}+\mathrm{H}_{2} \\
& =\mathrm{CH}_{3}-\mathrm{CH}_{2}-\mathrm{CO}-\mathrm{ONH}_{4}+\mathrm{NH}_{3}+\mathrm{CO}_{2} . \\
& \mathrm{NH}_{3}+\mathrm{H}_{2} \mathrm{O}+\mathrm{CO}_{2}=\left(\mathrm{NH}_{4}\right)_{2} \mathrm{CO}_{3} .
\end{aligned}
$$

In the reactions where oxyacid or alcohol is formed, the amidase acts by hydrolysis. Ex.:

$\mathrm{CO}_{2} \mathrm{H} \cdot \mathrm{CH}_{2} \cdot \mathrm{CH}\left(\mathrm{NH}_{2}\right) \cdot \mathrm{CONH}_{2}+{ }_{2} \mathrm{H}_{2} \mathrm{O}$

Asparagin

$$
=\mathrm{NH}_{4} \mathrm{O}-\mathrm{OC}-\underset{\text { Ammonium malate }}{\mathrm{CH}_{2}}-\mathrm{CHOH}-\mathrm{CO}-\mathrm{ONH}_{4} \text {. }
$$

It is probable that the oxyacids formed in the organism come from the decomposition of amino-acids, in consequence of the intervention of a special amidase.

The transformation of amino-acids into alcohols has been studied principally by Ehrlich. In 1878 , Nencki had described the transformation of amino-acids into alcohols and oxyacids; but his investigations concerned especially certain processes of putrefaction. Ehrlich has observed reactions analogous to those found by Nencki by using beer yeast. When a solution of sugar containing amino-acids is allowed to ferment with yeast, there is soon found in the liquid noticeable quantities of higher alcohols. The alcohols formed correspond to the acids used. The reaction thus takes place according to the following general scheme:

R.CH. $\left(\mathrm{NH}_{2}\right) \cdot \mathrm{CO}_{2} \mathrm{H}+{ }_{2} \mathrm{H}_{2} \mathrm{O}=\mathrm{R} \cdot \mathrm{CH}_{2} \mathrm{OH}+\left(\mathrm{NH}_{4}\right) \mathrm{HCO}_{3}$. Thus leucin, tyrosin, and phenylalanin react as follows:

$$
\begin{aligned}
& \mathrm{CH}_{3} \backslash \mathrm{CH}-\mathrm{CH}_{2}-\mathrm{CH}\left(\mathrm{NH}_{2}\right) \mathrm{CO}_{2} \mathrm{H}+{ }_{2} \mathrm{H}_{2} \mathrm{O} \\
& =\underset{\mathrm{CH}_{3}}{\mathrm{CH}_{3} \backslash} \backslash \mathrm{CH} \cdot \mathrm{CH}_{2}-\mathrm{CH}_{2} \mathrm{OH}+\left(\mathrm{NH}_{4}\right) \mathrm{HCO}_{3} .
\end{aligned}
$$




$$
\begin{gathered}
\mathrm{C}_{6} \mathrm{H}_{4}(\mathrm{OH})-\mathrm{CH}_{2}-\underset{\text { Tyrosin }}{\mathrm{CH}}\left(\mathrm{NH}_{2}\right)-\mathrm{CO}_{2} \mathrm{H}+{ }_{2} \mathrm{H}_{2} \mathrm{O} \\
=\mathrm{C}_{6} \mathrm{H}_{4}(\mathrm{OH})-\underset{\text { Oxyphenyl ethyl alcohol }}{\mathrm{CH}_{2}-\mathrm{CH}_{2}-\mathrm{OH}}+\left(\mathrm{NH}_{4}\right) \mathrm{HCO}_{3} . \\
\mathrm{C}_{6} \mathrm{H}_{5} \mathrm{CH}_{2}-\underset{\text { Phenylalanin }}{\mathrm{CH}\left(\mathrm{NH}_{2}\right)-\mathrm{CO}_{2} \mathrm{H}+{ }_{2} \mathrm{H}_{2} \mathrm{O}} \\
\quad=\mathrm{C}_{6} \mathrm{H}_{5}-\mathrm{CH}_{2}-\mathrm{CH}_{2} \mathrm{OH}+\left(\mathrm{NH}_{4}\right) \mathrm{HCO}_{3} .
\end{gathered}
$$

The resulting alcohols undergo a transformation at the moment of their appearance. Thus, glutamic acid does not yield oxybutyric acid but succinic acid. Ehrlich has shown, in fact, that the succinic acid of an alcoholic fermentation comes from the glutamic acid and not from the sugar, as was formerly believed.

$$
\begin{aligned}
\mathrm{CO}_{2} \mathrm{H}- & \mathrm{CH}\left(\mathrm{NH}_{2}\right)-\mathrm{CH}_{2}-\mathrm{CH}_{2}-\mathrm{CO}_{2} \mathrm{H}+{ }_{2} \mathrm{H}_{2} \mathrm{O} \\
= & \mathrm{CH}_{2} \mathrm{OH}-\mathrm{CH}_{2}-\underset{\text { Oxybutyric acid }}{\mathrm{CH}_{2} \mathrm{H}}+\left(\mathrm{NH}_{4}\right) \mathrm{HCO}_{3} .
\end{aligned}
$$

- This last by oxidation gives succinic acid:

$\mathrm{CH}_{2} \mathrm{OH}$. $\mathrm{CH}_{2} \cdot \mathrm{CH}_{2} \cdot \mathrm{CO}_{2} \mathrm{H}+\mathrm{O}_{2}=\mathrm{CO}_{2} \mathrm{H}_{\text {Succinic acid }} \mathrm{CH}_{2} \cdot \mathrm{CH}_{2} \cdot \mathrm{CO}_{2} \mathrm{H}+\mathrm{H}_{2} \mathrm{O}$.

According to Ehrlich oxyglutaric acid, $\mathrm{CO}_{2} \mathrm{H}$.CHOH. $\left(\mathrm{CH}_{2}\right)_{2}$.$\mathrm{CO}_{2} \mathrm{H}$, would be formed as an intermediate product. But, knowing the tendency for the carboxyl group of glutamic acid to be eliminated, this explanation is not at all plausible, and furthermore this body has not been found in the products of alcoholic fermentation.

The ammonia formed in the presence of yeast in the reaction studied by Ehrlich is not found at all in the free state like that coming from the other amidases previously studied. The absence of free ammonia in the present case recalls the conditions of germination. In the germinated grains very little ammonia is found while germination produces intense deamidization. In both these two cases there is, however, a real and intense formation of ammonia, but in this latter case it has been synthesized into the living cell.

At the beginning of his investigation on yeast, Ehrlich explained the transformations observed by vital activity, and 
refused to admit any enzymic intervention. In consequence of publications which appeared on this subject, he changed his opinion, and affirmed then that the enzyme of yeasts producing the higher alcohols is an intracellular enzyme, incapable of entering into solution and consequently of acting only in living cells. This conception is, however, entirely erroneous.

The beer yeast diluted in weak alcohol or in dilute solutions of $\mathrm{NaF}$ preserves its vitality for a very long time. Without doubt, in these liquids freed from cells and containing sometimes more than 90 per cent of the total nitrogen of the yeast, there is indeed found ammonia and higher alcohols (amyl and butyl): in the filtrate coming from a yeast autophaged for six months 42 per cent of the total nitrogen has been found in the ammoniacal state; it has been demonstrated, furthermore, that this ammonia was combined with volatile acids (especially butyric acid) to form salts. But, on the other hand, the solution coming from autophagy of yeast in the presence of sodium fluoride acts also on leucin and asparagin, forming ammonia, volatile acids, and higher alcohols. Now, this action is obtained with the carefully filtered liquid, which is consequently free from all yeast cells. The enzyme giving the higher alcohols can, then, under certain conditions, enter into solution just like the other amidases. Beer yeast is not the only micro-organism producing amyl alcohol. Nawaisky has found that $B$. proteus vulg. causes leucin to ferment, giving caproic acid, valerianic acid, and amyl alcohol. He has also established the fact that dead bacteria act like living cells, and that the effect is proportional to the weight of enzyme used.

From the different experiments made on yeast we may conclude, then, that the cells contain two different classes of amidases, the one giving alcohols, the other volatile acids, these two enzymes being capable, according to the conditions, of reacting on the same amine bodies. We must also remember the curious property noted by Chodat. According to this writer, oxidizing enzymes, such as tyrosinase, can act like amidases. The liberation of ammonia then progresses parallel 
to the formation of compounds belonging to the aldehyde group. Thus, in the presence of tyrosinase, glycin will be transformed according to the following equation:

$$
\mathrm{NH}_{2}-\underset{\text { Glycin }}{\mathrm{CH}_{2}}-\mathrm{CO}_{2} \mathrm{H}+\frac{1}{2} \mathrm{O}_{2}+\mathrm{H}_{2} \mathrm{O}=\underset{\text { Formaldehyde }}{\mathrm{HCHO}}+\mathrm{NH}_{4} \mathrm{HCO}_{3} \text {. }
$$

Reactions of the same nature are observed with alanin, phenylglycin, and other similar substances. Although the formation of aldehyde and the resulting liberation of ammonia is very slight and requires further confirmation, this decomposition is nevertheless interesting, for differing entirely from those thus far studied, it suggests a possible interpretation of the nature of the action of amidases, an interpretation which deserves further experimentation.

\section{§ 3. UREASE.}

The transformations which urine undergoes spontaneously have for a long time attracted the attention of investigators. Vauquelin and Dumas first recognized that the change of reaction found in urine exposed to the air corresponds to a transformation of urea into ammonium carbonate:

$$
\mathrm{CO}\left(\mathrm{NH}_{2}\right)_{2}+{ }_{2} \mathrm{H}_{2} \mathrm{O}=\left(\mathrm{NH}_{4}\right)_{2} \mathrm{CO}_{3} \text {. }
$$

This phenomenon is similar to a fermentation, and its origin was attributed to the organic substances forming the deposit of decomposed urine. This theory has been supported especially by Jacquemart. In I860 Müller originally sought to explain the formation of ammonia in urine by the intervention of microorganisms, but he did not offer decisive proof in support of this new theory. However, at about the same period, Pasteur isolated Torula urea, which Van Tieghem later described under the name of Micrococcus urea. The data furnished by Pasteur and Van Tieghem were later confirmed by the discovery of numerous micro-organisms capable of transforming urea. Among others are: Urococcus, Urosarcina, Planosarcina, Micrococcus urece liquefaciens, various urobacilli, etc. We owe espe- 
cially to Miquel the identification of many of these special bacterial species.

The urea ferments are widely distributed in nature, being found in the air, in the dunghill, in sewage and in city mud. To cultivate these ferments, a solution of peptone with addition of $0.2-0.3$ per cent of urea is used. According to Machida, the addition of magnesium sulphate to the culture medium greatly favors the development of bacteria. The fermentation of urea takes place especially well at a temperature of $30^{\circ}$; at $5^{\circ}$ almost no ammonia is formed; at $60-70^{\circ}$, the cells of Urococcus succumb, though bacterial spores can be kept alive up to $80^{\circ}$.

The catalyst intervening in the decomposition of urea was discovered in 1874 by Musculus, who found that ammoniacal urine, filtered and evaporated in a vacuum, is capable of causing the fermentation of fresh urea, giving a thick and viscous product similar to that obtained by precipitating decomposed urine with alcohol. Musculus thus established that the production of ammonia is not due exclusively to the ferment, but claims that it results from the action of a special substance of enzymic nature secreted in the bladder. Thus, while finding the presence of the enzyme, Musculus did not comprehend the relation which exists between the bacterium and the active substance. It was Miquel who definitely demonstrated that the enzyme acting on urea is indeed secreted by a micro-organism. This special catalyst was first described under the name of urase, then under that of urease.

To isolate urease, a solution of peptone is fermented by a culture of pure uro-bacteria with addition of from 2 to $3 \mathrm{~g}$. of urea per liter; the culture, kept at $30^{\circ}$ becomes very turbid. After 3 or 4 days this is filtered through a porous cup, and the clear liquid, free from bacteria, can serve as a solution of urease. This liquor is very active. A liter can transform per hour as much as $120 \mathrm{~g}$. of urea. Protected from the air, these solutions of urease can be preserved for months. The addition of two volumes of alcohol gives a precipitate which, washed in 
alcohol at $50^{\circ}$, becomes less and less soluble while still maintaining a certain activity. Yet by this treatment with alcohol about half the active substance is lost. The enzyme may also be procured by inducing in the liquid a precipitate with calcium salts. Thus almost all the enzyme is carried down, though it is very difficult afterward to free the urease collected from the adhering calcium salts.

Kossowicz found urease in molds. Thus Aspergilius niger, Aspergillus glaucus, Penicillium crustaceum, Mucor boidin, and many others destroy urea with the formation of ammonia. Shibata has detected this enzyme in mushrooms. Finally, Emmerling and Reiser recognized that $B$. fluorescens liquefaciens is also capable of transforming urea into ammonium carbonate.

According to Takeuchi, urease is widespread in the vegetable kingdom. Germinated soja beans (Glycin-hispida) contain an enzyme which acts on urea in the same manner as the bacterial urease. The active substance can be extracted by a maceration in water. The extract from I $\mathrm{g}$. of soja beans transforms in 20 hours the urea contained in a liter of urine. Soja urease acts exclusively on urea, being inactive on other amine and amide combinations. On the contrary, according to some writers, bacterial urease contained in certain culture broths acts also on asparagin. Yet this is not true of the urease extracted by precipitation of these liquids, and the action observed on aminoacids must apparently be attributed to the intervention of other amidases accompanying the urease.

Influence of Physical and Chemical Conditions. - The optimum temperature for the activity of urease lies between $48^{\circ}$ and $50^{\circ}$. This enzyme, however, is very sensitive to thermal

Variation of the Activity of Urease with the Temperature.

\begin{tabular}{|c|c|c|}
\hline Duration of action. & Temperature. & Content in urease. \\
\hline $\begin{array}{ll} & \text { hrs. } \\
\because & 2 \frac{1}{2} \\
& 2 \frac{1}{2} \\
& 2 \frac{1}{2} \\
& 2 \frac{1}{2}\end{array}$ & $\begin{array}{l}{ }^{\circ} \mathrm{C} . \\
14 \\
40 \\
4 \mathrm{I} \cdot 5 \\
56\end{array}$ & $\begin{array}{r}100.00 \\
95.68 \\
91.36 \\
46.05\end{array}$ \\
\hline
\end{tabular}


influences. Thus the optimum temperature is also a destructive temperature.

A solution of urease kept for $2 \frac{1}{2}$ hours at $14^{\circ}$ still preserves intact its original enzymic content, which is expressed here by the value 100. At a temperature of $4 \mathrm{I} .5^{\circ}$ the content in urease is 91.36 ; at $56^{\circ}$ it is only 46 ; finally, at $65^{\circ}$ the destruction becomes very rapid. On the other hand, the following are the losses of activity of a solution of urease kept for to minutes at different temperatures:

$\begin{array}{ccccc}\text { After to m. at } 64^{\circ} \text {, the standard in urease falls from roo to } 97.8 \\ \text { " } & 66^{\circ} & \text { " } & \text { " } & 36.7 \\ \text { " } & 70^{\circ} & \text { " } & \text { " } & 25.89 \\ \text { " } & 75^{\circ} & \text { " } & \text { " } & 0\end{array}$

Urease withstands relatively strong alkalinity. 0.5 per cent of $\left(\mathrm{NH}_{4}\right)_{2} \mathrm{CO}_{3}$ does not paralyze its activity, though $4 \mathrm{~g}$. of $\mathrm{NaOH}$ per liter of solution is injurious. This enzyme is much more sensitive to an acid reaction; thus, in the presence of I g. of mineral acid per 5 liters of the liquid, the urease is paralyzed. The degree of sensitiveness of urease to the reaction of the medium is shown by the following experiment: A solution of this enzyme has added to it 4 per cent hydrochloric acid. At the end of ro minutes, this is neutralized. It is then found that this treatment is sufficient to destroy completely the active substance.

Glycerin and sugar activate urease. To show the effect of sugar, Miquel proceeds in the following manner: In a control experiment he adds to an active solution 8 per cent of urea; then in the real test the active solution is diluted $\frac{1}{4}$ with a syrup containing 20 per cent of sugar, and this mixture has added to it the same quantity of urea as the control test. It is left to act at a temperature of $48^{\circ}-50^{\circ}$, and the quantity of urea remaining in the two samples after various intervals is determined.

Thus, after 24 hours, $5 \mathrm{~g}$. of urea are destroyed in the presence of sugar, while the control test shows a disappearance of only $1.96 \mathrm{~g}$. The sugar thus acts as protector of the enzyme 
EFfect of Sugar on Urease.

\begin{tabular}{|c|c|c|}
\hline $\begin{array}{c}\text { Length of action } \\
\text { in hours. }\end{array}$ & $\begin{array}{c}\text { Experiment without } \\
\text { sugar. Per cent urea } \\
\text { disappearing in } \\
\text { liquid. }\end{array}$ & $\begin{array}{c}\text { Experiment with } \\
\text { sugar. Percent urea } \\
\text { disappearing in } \\
\text { liquid. }\end{array}$ \\
\hline & I.5I & r.91 \\
I & $\mathbf{1 . 9 6}$ & 3.75 \\
2 & $\mathbf{1 . 9 6}$ & 5.02 \\
3 & 1.96 & 5.00 \\
\hline
\end{tabular}

against the action of temperature, a phenomenon which Effront has already observed with many other substances in connection with different enzymes.

In the following table are recorded the effects of sodium chloride and other compounds on urease:

\begin{tabular}{|c|c|c|}
\hline Substances used. & Quantity per 1. of liquid. & Enzymic value. \\
\hline Sodium chloride........ & $\left\{\begin{array}{l}\mathrm{g} . \\
\mathrm{1}^{2} .5 \\
60\end{array}\right.$ & $\begin{array}{r}100 \\
90\end{array}$ \\
\hline Alcohol. & $\left\{\begin{array}{r}10 \\
200\end{array}\right.$ & $\begin{array}{l}90 \\
30\end{array}$ \\
\hline Boric acid. & $\left\{\begin{array}{l}0.2 \\
0.5\end{array}\right.$ & $2 \mathrm{I}$ \\
\hline Phenol. & $\left\{\begin{array}{r}0.5 \\
10.0\end{array}\right.$ & $\begin{array}{l}12 \\
82 \\
64\end{array}$ \\
\hline Corrosive sublimate. . & $\left\{\begin{array}{l}0.001 \\
0.002\end{array}\right.$ & 50 \\
\hline Copper sulphate....... & 0.05 & $\circ$ \\
\hline
\end{tabular}

Thus, urease is an enzyme that is very sensitive to chemical conditions of the medium; the presence of very small quantities of copper sulphate, of sublimate, even of boric acid inhibits its activity. Salicylic acid in small quantities also produces a paralyzing action. Benzoic acid is less active. A solution of urease, saturated with chloroform, loses at the end of 8 days more than 50 per cent of its activity. This sensitiveness to antiseptics is the reason why we cannot make tests of long duration with this enzyme, the results being always more or less in error due to the antiseptic used. We can, however, avoid this inconvenience by working in a sterile medium with a solution filtered through a porcelain filter. 


\section{§ 4. Guanase and Adenase.}

Of the two phenomena which occur in the transformations undergone by nitrogenous material in the animal organism, on the one hand, the phenomenon of anabolism, and on the other, that of katabolism, the former is the more completely understood. Without doubt, the ultimate phase of nutrition, namely, the reconstruction of tissues, escapes us in its details; but we can at least conceive how nature can arrive at this result by the reversed activity of enzymes already studied. On the contrary, the data relative to the formation of the nitrogenous products of excretion are much more vague. By what chemical process are the numerous substances which are found in varying proportions in the urine elaborated? Whence comes the uric acid and the various purin bases, xanthin, guanin, adenin, the creatin and creatinin, the allantoin, the hippuric acid, and finally the urea? We can give to these manifold questions only provisional answers, which up to the present rest more on presumptions than on sure proof. Apparently, the production of compounds of so varied a nature results from the activity of appropriate enzymes; but of those possible we know only a very small number, and we understand these very imperfectly. Until better informed, we should attribute the greater part of these syntheses to the action of purely vital forces.

The physiological rôle of the amidases, and how these enzymes intervene in the elaboration of materials by whose aid urea and uric acid, the two principal nitrogenous constituents of the urine, can be formed, has already been discussed. Ammonium salts represent, in fact, one of the principal sources of urea for, converted to ammonium carbonate and carbamate, they are rapidly dehydrated by the cells of the kidneys to form urea. It would even appear that uric acid, at least with animals such as birds and reptiles that produce large quantities of this substance, also has its origin in the liver, the hepatic organ of birds being capable of transforming ammonia salts, accompanied or not accompanied by ternary substances, into uric acid and not 
into urea. Yet the principal cause of the formation of uric acid in mammals results from the disintegration of the nucleoproteins forming their various tissues. We have previously described in the chapter on the nucleo-proteases the various products which successively arise under the influence of the numerous enzymes which attack this group of substances so scattered throughout the organism. It was found that pyrimidin and xanthin bases appear at the end of nuclein hydrolysis. Now, the chemical relationship which exists between the latter products and uric acid is evident from the following formulæ:

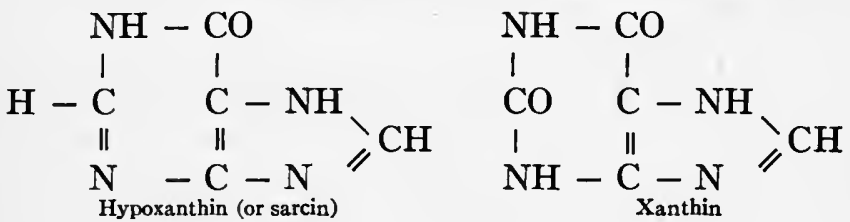

$$
\begin{aligned}
& \mathrm{N}=\mathrm{C}\left(\mathrm{NH}_{2}\right) \\
& \stackrel{\text { I }}{\mathrm{CH}} \stackrel{\mathrm{C}}{\mathrm{C}}-\mathrm{NH}
\end{aligned}
$$

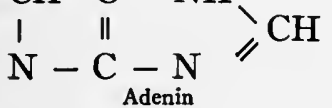

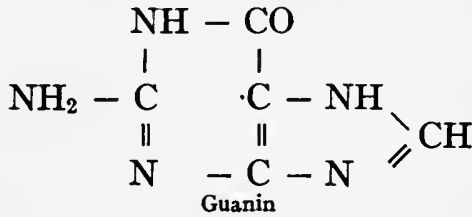

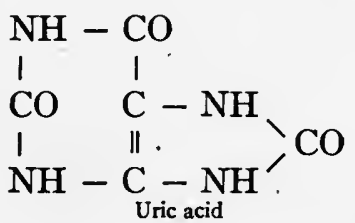

The various members of the purin group are capable of being transformed one into the other, or of giving new compounds, under the influence of various enzymes. Among these, five are well known. They are all found in the liver, an organ which has already been shown to play a very important rôle in the synthesis of urea in the blood. However, they are sometimes encountered in other animal organs. These enzymes are: (I) xanthynoxydase; (2) uricase; (3) arginase; (4) guanase; (5) adenase.

(I) Xanthynoxydase is an oxidizing enzyme, discovered by 
Schittenhelm. It helps to transform hypoxanthin first into xanthin, and then into uric acid; we thus have:

$$
\underset{\text { Hypoxanthin }}{\mathrm{C}_{5} \mathrm{H}_{4} \mathrm{ON}_{4}} \rightarrow \underset{\text { Xanthin }}{\mathrm{C}_{6} \mathrm{H}_{4} \mathrm{O}_{2} \mathrm{~N}_{4}} \rightarrow \underset{\text { Uric acid }}{\mathrm{C}_{5} \mathrm{H}_{4} \mathrm{O}_{3} \mathrm{~N}_{4}} \text {. }
$$

Its activity explains the presence of uric acid in urine, and also why the uric acid content in the urine of an animal increases when the latter is fed with material rich in purins.

(2) Uricase in reality belongs to a group of enzymes called uricolytics, which, however, all act, in different ways, on uric acid. Uricase decomposes uric acid, giving allantoin:

$$
\mathrm{CO}^{\prime} \stackrel{\mathrm{NH}-\mathrm{CO}}{\stackrel{\mathrm{I}}{\mathrm{CH}}-\mathrm{CH}-\mathrm{NH}-\mathrm{CO}-\mathrm{NH}_{2},}
$$

according to the reaction:

$$
\underset{\text { Uric acid }}{\mathrm{C}_{5} \mathrm{H}_{4} \mathrm{~N}_{4} \mathrm{O}_{3}}+\mathrm{O}+\mathrm{H}_{2} \mathrm{O}=\underset{\text { Allantoin }}{\mathrm{C}_{4} \mathrm{H}_{6} \mathrm{~N}_{4} \mathrm{O}_{3}}+\mathrm{CO}_{2} \text {. }
$$

As, on the other hand, allantoin can be transformed in vitro under the influence of oxidizing agents, into urea and oxalic acid, it has been thought that in the organism urea could come from the oxidation of uric acid. But this hypothesis does not rest on decisive proofs.

L. Brunton has also detected in the liver the presence of an enzyme which transforms uric acid into glycin, ammonia, and carbonic acid, according to the equation:

$$
\begin{gathered}
\mathrm{C}_{5} \mathrm{H}_{4} \mathrm{~N}_{4} \mathrm{O}_{3}+{ }_{5} \mathrm{H}_{2} \mathrm{O}=\mathrm{NH}_{2} \mathrm{CH}_{2} \mathrm{COOH}+{ }_{3} \mathrm{CO}_{2}+{ }_{3} \mathrm{NH}_{3} . \\
{ }_{2} \mathrm{NH}_{3}+\mathrm{CO}_{2}+\mathrm{H}_{2} \mathrm{O}=\left(\mathrm{NH}_{4}\right)_{2} \mathrm{CO}_{3} .
\end{gathered}
$$

Finally, an enzyme is known that is capable of transforming uric acid into urea and tartronic acid:

$$
\underset{\text { Uric acid }}{\mathrm{C}_{5} \mathrm{H}_{4} \mathrm{~N}_{4} \mathrm{O}_{3}}+{ }_{4} \mathrm{H}_{2} \mathrm{O}=\underset{2}{\mathrm{CH}_{4} \mathrm{ON}_{2}}+\underset{\text { Trea }}{\mathrm{C}_{3} \mathrm{H}_{4} \mathrm{O}_{5}} \text {. }
$$

All these uricolytic enzymes contribute to the elimination of the uric acid from the organism, and thus afford a certain interest, both from the point of view of the establishment in an individual of the losses in urinary nitrogen in all its forms, and of the etiology 
of various pathological conditions. Some investigators believe, in fact, that the origin of gout, a disease which is known to be due to an accumulation of uric acid, comes from an absence or a diminution of these uricolytic enzymes, which normally exist in sufficient quantities in the organism. Yet this hypothesis is not admitted by all.

It should be added that in addition to these transformations due to enzymes taken from organs, certain aerobic bacteria are also capable of destroying uric acid, the reaction being:

$$
\underset{\text { Uric acid }}{\mathrm{C}_{5} \mathrm{H}_{4} \mathrm{~N}_{4} \mathrm{O}_{3}}+8 \mathrm{H}_{2} \mathrm{O}+\mathrm{O}_{3}=\underset{\text { Ammonium acid carbonate }}{4 \mathrm{NH}_{4} \mathrm{HCO}_{3}}+\mathrm{CO}_{2}
$$

In this process several enzymes must intervene. Often it is observed that urea forms as an intermediate product, to rapidly disappear under the influence of the urease present in the culture broth.

(3) Arginase, already described, decomposes arginin, giving urea and ornithin. Now, arginin, a hexonic base, is a substance which is found in the products of the hydrolysis of the major part of albuminoid material. It is thus conceivable that by this method of division a certain quantity of protein nitrogen can escape the transformation to urea.

(4) Guanase and Adenase, finally, are true amidases. In their activity the first affects guanin, giving:

$$
\underset{\text { Guanin }}{\mathrm{C}_{5} \mathrm{H}_{5} \mathrm{ON}_{5}}+\mathrm{H}_{2} \mathrm{O}=\underset{\text { Xanthin }}{\mathrm{C}_{5} \mathrm{H}_{4} \mathrm{O}_{2} \mathrm{~N}_{4}}+\underset{\text { Ammonia }}{\mathrm{NH}_{3}}
$$

and the second acts on adenin, giving:

$$
\underset{\text { Adenin }}{\mathrm{C}_{5} \mathrm{H}_{5} \mathrm{~N}_{5}}+\mathrm{H}_{2} \mathrm{O}=\underset{\text { Hypoxanthin }}{\mathrm{C}_{5} \mathrm{H}_{4} \mathrm{O}_{2} \mathrm{~N}_{4}}+\underset{\text { Ammonia }}{\mathrm{NH}_{3}}
$$

These two enzymes have been described by W. Jones, who recognized their presence in different animal organs. Yet their individuality has been much disputed. Schittenhelm thinks that they are but one substance. It has been possible, however, to show that this was not the case by making a comparative analysis of different organs. It is found that certain extracts are active toward guanin, and are inactive toward adenin: 
Presence of Guanase and of Adenase in Different Organs.

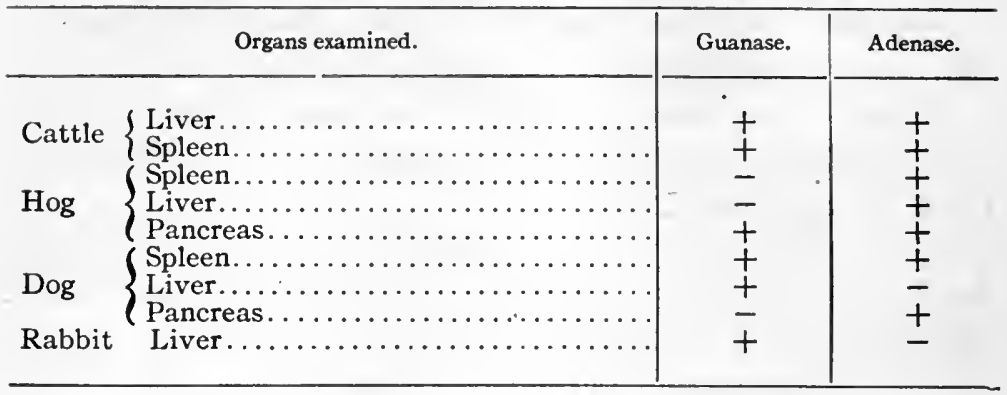

Similar results were obtained from other sources. Thus, W. Jones and Straghn have demonstrated that the water extract of yeast transforms guanin, whereas it does not act on adenin. On the other hand, Müller has observed that adenase is not met in any extract of human organs, while guanase is always present in the liver. Finally, Wells has sought these two enzymes in the human fœtus. He has found guanase in the fœtus of I or 2 months, while adenase appears only in the fœtus of 5 months. All these points are very uncertain; it could not be otherwise, since the distribution of these two enzymes not only varies in the different organs but also changes, as just seen, with the age of the subjects examined. This would be, we think, an added proof of the individuality of these two enzymes.

\section{§ 5. ButYRo-AMIDASE.}

Effront isolated from a butyric ferment culture an amidase acting on acetamide and asparagin. The culture medium is composed of a solution of albumin hydrolyzed by sulphuric acid up to the complete disappearance of the albumoses. After the sulphuric acid is removed with $\mathrm{Ba}(\mathrm{OH})_{2}$, the liquid is brought to a nitrogenous content of I.5 per cent, and is then made alkaline with sodium carbonate, Io g. per liter. Thus prepared, the liquid receives a culture of butyric ferment, obtained from a factory for recovering nitrogenous wastes. The fermentation is conducted in the presence of air at a temperature of $48^{\circ}$. As soon 
as the liquor reaches a content in ammonia liberable by magnesia corresponding to 50 per cent of total nitrogen, the culture is increased by adding to the preceding broth 4 volumes of fresh liquid of the same composition as above. By successive additions a volume of 25 liters is reached. Then the liquid is left to itself for 40 days. Cooling to $15^{\circ}, 25 \mathrm{~g}$. of colophane, previously dissolved in sodium carbonate, are added. After standing for some time, the clear liquid is decanted, and all the bacteria are found agglomerated in the deposit. This deposit is centrifuged, washed in alcohol and in ether, and then dried over. sulphuric acid. The powder obtained is pulverized, and then with the addition of a $\frac{1}{20} \mathrm{~N}$ solution of $\mathrm{Na}_{2} \mathrm{CO}_{3}$ is maintained at $45^{\circ}$ for 3 hours. 4 to 5 volumes of alcohol are then added and the mixture thrown on a filter. It must be filtered several times in order to get a perfectly transparent liquid. The precipitate is again washed with alcohol and with ether, and then dried. The dry product is finally triturated with water, and filtered through a porcelain filter.

The filtrate thus obtained is active on asparagin and acetamide. With asparagin, the formation of ammonia is limited to the amide grouping, but with the products of proteolysis much more perfect results are obtained. For these tests use hydrolyzed products, giving 74 to 75 per cent of formaldehyde nitrogen according to Sörensen's method, and utilize a solution containing $100 \mathrm{mg}$. of nitrogen. To the liquid, after sterilization, are added $0.5 \mathrm{~g}$. of sodium carbonate and a definite quantity of butyro-amidase. The mixture is kept at $50^{\circ}$ for 4 hours and then the ammoniacal nitrogen in the solution is determined.

With $3 \mathrm{cg}$. of active substance, $34 \mathrm{mg}$. of ammonia are obtained per $100 \mathrm{mg}$. of total nitrogen. Further increase of the quantity of enzyme then produces no perceptible effect. About a third of the total nitrogen is thus easily deamidized. The same phenomenon is observed in a more concentrated liquid. In a solution containing $150 \mathrm{mg}$. of nitrogen, a certain proportionality is found between the quantity of enzyme used 
and the ammonia formed, up to the moment when the liquid shows a content in ammonia of $48 \mathrm{mg}$. Beyond this limit, the amidase produces almost no effect.

Progress of Deamidization of a Sol
OF Hydrolyzed Albumin.
\begin{tabular}{|c|c|}
\hline $\begin{array}{c}\text { Quantity of enzyme } \\
\text { used. }\end{array}$ & $\begin{array}{c}\text { Ammoniacal nitro } \\
\text { gen formed per Io } \\
\text { g. liquid. }\end{array}$ \\
\hline cg. & mg. \\
I & I2 \\
2 & 28 \\
3 & 34 \\
6 & 36 \\
Io & 39 \\
\hline
\end{tabular}

When definite quantities of asparagin are added to the products of hydrolysis of albumin, very interesting results are obtained. The deamidization of the mixture is then very pronounced:

Action of Amidase on a Mixture of Products of Hydrolysis and of Asparagin.

\begin{tabular}{|c|c|}
\hline Composition of the medium. & $\begin{array}{l}\text { Ammonia formed } \\
\text { after } 8 \text { hours. }\end{array}$ \\
\hline Nitrogen of products of hydrolysis of albumin, $150 \mathrm{mg} .$. & $\begin{array}{l}\text { mg. } \\
\ldots\end{array}$ \\
\hline Nitrogen in the form of asparagin.......... $200 \mathrm{mg}$. & $\ldots$ \\
\hline Total nitrogen, $\ldots \ldots \ldots \ldots \ldots \ldots \ldots \ldots \ldots \ldots \ldots \ldots \ldots \ldots$ & 252 \\
\hline
\end{tabular}

It is still to be noted that the butyro-amidase does not furnish the reaction ordinarily found when the experiment takes place in the presence of bacteria which secrete the enzyme. We succeed in the latter case, with the living ferment, in deamidizing up to 96 per cent of the total nitrogen contained in the products of the proteolysis. Now the enzymes isolated from their bacteria react solely on certain substances which form only about $\frac{1}{3}$ of the total weight. Butyro-amidase reacts in two different ways on asparagin, according to the conditions of the medium. With water alone, as seen above, the action is limited to the amide grouping. On the contrary, in the presence of 
oxidizable products the deamidization is complete, as the preceding table shows. It appears, in fact, from the latter, that the ammonia formed arises partly from the total nitrogen of the asparagin, and partly from only a third of the original protein nitrogen.

The limited effect which we have just found with butyroamidase acting on the products of hydrolysis of the nitrogenous materials, can be explained by the hypothesis that the bacterium secretes numerous specific enzymes, each acting on different substances. Butyro-amidase, thus isolated, would contain only certain enzymes, and not all those which are necessary for the evidently more exacting fermentation. Although the amidases attack a series of substances of similar structure, they set ammonia free by different methods. We are, therefore, quite naturally led to believe that in this process several catalysts intervene, for it would be inadmissible that the same enzyme should be called upon to fill such varied functions. We will then conclude by saying that to each of the different chemical processes there corresponds a well-determined type of catalyst, whether the reaction be one of hydrolysis, or one of reduction. Unfortunately, in the present state of the question, this opinion cannot be confirmed by any experimental proof.

Although the study of these enzymes offers great interest from the physiological point of view as well as from the practical, it also offers great difficulties by reason of the impossibility of being able to isolate these active substances in a satisfactory manner. Further, the limited data which we possess with regard to them, explicable by the short time which has elapsed since their discovery, is not such as to facilitate investigations into a domain, which, however, later promises a good harvest. We have sketched in the preceding paragraphs only a few of the properties of these curious enzymes. In the chapter on putrefaction, we shall have occasion to return to the chemical work performed by the amidases. The enzymes secreted by the putrefactive bacteria are, in fact, endowed with an activity much more varied even than those studied hitherto, and they are 
capable of performing transformations which are characteristic of this kind of fermentation. Accordingly, their study has been referred to this special chapter.

\section{BIBLIOGRAPHY ON THE AMIDASES.}

\section{General Properties. Butyro-Amidase.}

O. Lawr. Die Harnstoffbildende Fermente der Leber, Zeits. f. physiol. Chem., (25), p. 5 II.

Ch. Richet. Sur la formation de l'urée dans le foie après la mort, $C . R$., r894, (II8), p. II 25 .

JACOBY. Ueber die fermentative Eiweissspaltung und Ammoniakbildung in der Leber, Zeits. f. physiol. Chem., I900, (30), p. 148.

M. Gonnerman. (I) Verseifbarkeit von Säureamiden und Säureaniliden durch Fermente, Pflüg. Arch., (89), p. 493. - (2) Verseifbarkeit von Säureamiden und Aminosäuren durch Fermente, A poth. Zeitung, 1903, (18), p. 192.

S. LANG. Ueber Desamidierung im Tierkörper, Beitr. z. chem. Physiol., 1904, (5), p. $32 \mathrm{I}$.

K. Shibata. Ueber das Vorkommen von amidespaltenden Enzymen bei Pilzen, Beitr. z. chem. Physiol., I904, (5), p. 384.

EFrront. (r) Brevet belge $\mathrm{n}^{\circ}$ I8921 2, déposé en 1906, addition 20102. - (2) Sur l'utilisation de l'azote dans les résidus de distillerie, Monit. Scient., 1908, p. 429. - (3) Sur la fermentation d'acides amidés, Monit. Scient., r9o9, p. 145. - (4) Sur les acides amidés, $C$. R., 1908, (I), p. 779. - (5) Sur la fermentation ammoniacale, C. R., I909, (r), p. $238 .-(6)$ Action du ferment Culgare sur les substances protéiques, $C . R .$, I910, (2), p. $1007 .-(7)$ Sur la fermentation putride, Monit. Scient., I9II, p. 489 .

FUrth u. FriedemanN. Ueber die Verbreitung asparaginspaltender Organfermente, Bioch. Zeits., I910, (26), p. 435.

Berghaus. Ueber die Ammoniakbildung bei einiger Bacterienarten, Arch. $f$. Hyg., 1908, (64), pp. 1-32.

Hans Pringsherm. Ueber Pilzdesamidase, Bioch. Zeits., rgo8, (12), p. 15.

W. Brasch u. C. Neuberg. Biochemische Umwandlung der Glutaminsäure in n. Buttersäure, Bioch. Zeits., I908, (13), p. 299.

C. Neuberg. (I) Die Entstehung des Erdöls, Königl. Preuss. Akad d. Wissensch., mai 1907.- (2) Verhalten von racemischer Glutaminsäure bei der Fäulnis, Bioch. Zeits., I909, p. 433.

C. Neuberg u. Cesare Coppezzuoli. Biochemische Umwandlung von Asparagin und Asparaginsäure in Propionsäure und Bernsteinsäure, Bioch. Zeits., 1909, (I8), p. 425 .

C. Neuberg u. Karezag. Verhalten von Aminoisovaleriansäuren bei der Fäulnis, Bioch. Zeits., I909, (18), p. 435.

Butkewirsch. (I) Ammoniak als Umwandlungsprodukt Stickstoffhaltiger Stoffe in höheren Pflanzen, Bioch. Zeits., 1909, (16), p. 41 1. - (2) Ammoniak-abspaltung im Pflanzen, Zeits.f. physiol. Chem., rgo9, (63), p. ró3. 
Kresel. (I) Ueber fermentative Ammoniakbildung im höheren Pflanzen, Zeits. f. physiol. Chem., 1909, (60), p. 452. - (2) Autolytische Argininzerzetzung im Pflanzen, Ibid., 1909, (60), p. 460.

E. Schulze. Ueber die Umzetzung der Eiweissstoffe im lebenden Pffanzen, Zeits. f. physiol. Chem., 1898, 24, p. 18.

O. Law. Die chemische Energie der lebenden Zellen, München, I899.

EHRLICH. (I) Ueber die Entstehung des Fuselöls, Zeits.d. Ver. der deutsch. Zuckerindustrie, (55), p. 592. - (2) Ueber die Bedingungen der Fuselölbildung und über ihren Zusammenhang mit dem Eiweissaufbau der Hefe, Ber.d.deuts. Chem. Ges., 1907, (40), p. I027. - (3) Entstehung der Bernsteinsäure bei der alkoholischer Gährung, Zeits. d. Ver. der deutsch. Zuckerindustrie, I909, p. 646.

Effront. (I) Sur les conditions de la formation d'alcool amylique dans les fermentations à l'aide de levure de bière, $C . R . d u I^{\text {er }}$ Congrès internat. de Sucrerie et Distillerie, Liége, 1905. - (2) Sur l'autophagie de la levure de bière, Bull. Soc. Chim. Paris, r 905 , p. 847 .

Nawiasky. Umsetzung von Aminosäuren durch Bac. Proteus vulgaris, Arch. $f$. Hyg., 1908, (66), pp. 209, 243.

Akikazu Suwa. Ueber das Schiksal der N-freien Abkommlingen der aromatischen Aminosäuren in normalen Organismus, Zeits. f. physiol. Chem., Igr I, (72), p. II3.

CAstoro. Ueber Vorkommen von Ammoniak in Keimpflanzen und über seine Bildung bei der Autolyse solcher Pflanzen, Zeits. f. physiol. Chem., (50), p. 525 .

W. Gulewitsch. Ueber das Verhalten von Trypsin gegen einfache organische Verbindungen, Zeits. f. physiol. Chem., (27), p. 54I.

R. Сhорat. Neue Untersuchungen über die oxydirenden Fermente, Arch. de la Soc. phys., Genève, (35), p. 140 .

Medwedew. Ueber Desamidirungvorgang in Blute von Thieren, Zeits.f. physiol. Chem., I9II, (72), p. 446.

O. Neubauer. Deuts. Arch.f. klin. Medizin, I909, p. $21 \mathrm{I}$.

KNOOP. Zeits. f. physiol. Chem., ז910, p. 489 .

\section{UREASE.}

JacQuemart. Note sur la fermentation urinaire, Ann. Chim. et Phys., i843, p. I49.

LAUBE. Virchow's Arch., 1895, (100), p. 540.

Musculus. Sur le ferment de l'urée, $C . R$., 1874, (78), p. 132; 1876, (82), p. 334.

Pasteur. C. R., I860, (50), p. 849; Ann. Chim. et Phys., I862, (64), p. $5^{2}$.

SCHUTZENBERger et Bourgeois. C. R., 1876, (82), p. 262.

Guignard. Sur la transformation ammoniacale, Thèse, Paris, 1883.

MACHIDA. Bul. of the Central agricol Exper. Station, Japon, 1907, p. I.

Miquel. (I) Bull. Soc. chim., I878, (29), p. 387; I879, (3I), p. 391; (32), p. 126.

(2) Sur le ferment soluble de l'urée, C. $R$., I89o, (I II), p. 397.

Müller. Zeits. f. praktische Chem., I860, (8I), p. 467 .

LEA, Sheridan. Journ. of Physiol., I885, (6), p. 136.

Schmedeburge. Ueber Spaltung und Synthesen in Tierkeper, Arch. exper. Path. u. Pharm., I881, (14), p. 382.

Van Tieghem. Sur la fermentation ammoniacale, Thèse, Paris, 1864; C. R., 1864, (58), p. 212. 
BeIJERINCK. Vergleichende Untersuchungen über die Anhaufung von Urebacterien, d'après Centralblatt, I902, (2), p. I44.

TAKeUChI. Einige techn. Anwendung der Urease, Chem. Zeit., I9I I, (35), p. 408.

Kossowicz. Zeils. f. Gärungphysiologie, 1912, (1), p. 60.

Shibata. Beilr. z. chem. Physiol. u. Path., 1904, (5), p. 32 I.

Emmering u. Reiser. Ber. d. deuts. Chem. Ges., 1902, (35), p. 700.

\section{GUANASE AND ADENASE.}

Walter Jones. Ueber das Enzym der Thymusdrüse, Zeits. $f$. physiol. Chem., 1904, (4I), p. I01; 1904, (42), p. 35; Ueber Guanase, Ibid., I905, (45), p. 84.

W. Jones u. Partridge. Zeils. f. physiol. Chem., 1904, (42), p. 343.

W. Jones u. Winternitz. Zeits. $f$. physiol. Chem., I905, (44), p. I.

W. Jones u. Ausrrian. Zeits. f. physiol. Chem., Igo6, (48), p. I Io; Journ. of Biol. Chem., r907, (3), p. 228.

A. Schtrtenhelm. Zeits.f. physiol. Chem., I904, (42), p. 25I; (43), p. 228; 1905, (45), pp. I21 et I52; (46), p. 354; 1906, (48), p. 57 I; 1908, (57), p. 21.

A. Schittenhelm u. Schmid. Zeils. $f$. physiol. Chem., i906, (50), p. 30.

Batteli u. Stern. Untersuch. über Uricas in den Thiergeweben, Bioch. Zeils., I909, (19), p. 219.

A. Schittenhelm. Die uricolytische Fermente, Zeits. f. physiol. Chem., 1905, (45), p. 160.

L. Brunton. Zeits. f. Physiologie, I905, (19), p. I.

Straghn et W. Jones. Journ. of Biol. Chem., I909, (6), p. 245.

W. Jones. Journ. of Biol. Chem., (9), p. 169.

C. Vögtrin u. W. Jones. Ueber Adenase und ihre Beziehung zu der Entstehung von Hypoxanthin in Organismus, Zeits. f. physiol. Chem., I910, (66), p. 250.

Miller et W. Jones. Ueber der Fermente der Nucleins bei der Gicht, Zeils. $f$. physiol. Chem., r9o9, (6I), p. 395.

Gideon Wells and Herry Corper. Journ. of Biol. Chem., Igio, (6), p. 486.

Gideon Wells. Journ. of Biol., I910, (7), p. I7I. 
PART VI.

APPLICATIONS. 



\section{CHAPTER I.}

\section{$\S$ i. Officinal Pepsin: Its Therapeutic Use.}

\section{Commercial Peptones.}

In 1855, Corvisart first recommended the use of pepsin in therapeutics. Boudault, in 1857 , prepared a powder with pepsin base which was much in fashion at that period. The success caused a great many imitations to appear, which soon led to a complete discrediting of this remedy. From suits entered against manufacturers of pepsin in France at this time, it appears that there had been then delivered to the public products completely inactive, unclean, and dangerous. A commission formed in 1859 in France by the Society of Pharmacy regulated the manufacture of medicinal pepsin. Although its requirements were very moderate $(0.4$ to $0.75 \mathrm{~g}$. of pepsin must dissolve $7 \mathrm{~g}$. of fibrin in 12 hours), the situation was not perceptibly improved. The writings of Panum, of Mourrut, of Hofmeister, and of many others indicate, in fact, that pharmacies in France and other countries continued for a very long time to furnish products of a doubtful value.

In a thesis by Konovaloff, published in 1893 at St. Petersburg, we find the analysis of several pepsins of different brands. The data published by the author are all the more interesting since they allow us to compare these pharmaceutical products with the gastric juice of a dog.

We find that Io c.c. of gastric juice, prepared by the method of Pawlow, dissolves in 4 hours at $40^{\circ} 9.8 \mathrm{~g}$. of coagulated egg-albumin. Now, a similar result is obtained only with a small number of commercial pepsins, if we take into account the dilutions of active substances. Nevertheless it should be noted that two circumstances render difficult a comparison of the values of these products. First of all, the most favorable 
Analysis of Different Commercial Pepsins.

\begin{tabular}{|c|c|c|c|c|c|}
\hline Name of pepsin. & $\begin{array}{l}\text { Most favor- } \\
\text { able } \\
\text { acidity. }\end{array}$ & $\begin{array}{l}\text { Dis. Pow. } \\
\text { Quant. alb. } \\
\text { dis. in } 4 \mathrm{~h} \text {. } \\
\text { by I g. pep. }\end{array}$ & $\begin{array}{l}\text { Liq. pow. No. } \\
\text { mm. alb. liq. } \\
\text { (Mett method). }\end{array}$ & $\begin{array}{l}\text { Equiv. in } \\
\text { c.c. of gas. } \\
\text { juice of } \\
\text { Ioo g. of } \\
\text { dif. pep. }\end{array}$ & $\begin{array}{l}\text { Actual } \\
\text { content } \\
\text { in } \\
\text { pepsin. }\end{array}$ \\
\hline Gastric juice of a dog. & $0.2 \%$ & $\begin{array}{c}9.8 \mathrm{~g} . \\
\text { with ro } \\
\text { c.c. gas- } \\
\text { tric juice }\end{array}$ & $\begin{array}{l}9 \text { mm. with } \\
\text { undiluted } \\
\text { juice }\end{array}$ & 100 & $0.15 \%$ \\
\hline $\begin{array}{l}\text { Pepsin-glycerin } \\
\text { MerCK (Darmstadt) }\end{array}$ & 0.3 & 7.0 & $2.5(\mathrm{sol} .25 \%)$ & 25 & 0.037 \\
\hline $\begin{array}{l}\text { Pepsin-essence LIEB- } \\
\text { REICH (Berlin) ...... }\end{array}$ & 0.3 & 6.6 & $2.5 \quad$ (id.) & 20 & 0.03 \\
\hline & $0.2-0.3$ & 8.4 & $5($ sol. $6 \%)$ & 600 & 0.9 \\
\hline $\begin{array}{l}\text { WITTE (Rostock).... } \\
\text { Peps. granulatum }\end{array}$ & $0.2-0.3$ & 7.64 & 5 (id.) & 300 & 0.45 \\
\hline $\begin{array}{l}\text { WITre (Rostock).... } \\
\text { P. hydrochloratum } \\
\text { Ioo\% soluble MERCK }\end{array}$ & $0.3-0.4$ & 8.12 & 4.5 (id.) & 400 & 0.6 \\
\hline $\begin{array}{l}\text { (Darmst.). } \\
\text { Peps. cum amylo }\end{array}$ & 0.2 & 8.34 & $6 \quad$ (id.) & 550 & 0.82 \\
\hline $\begin{array}{l}\text { Merck (Darmst.)... } \\
\text { P. germanicum purum }\end{array}$ & 0.3 & $5 \cdot 72$ & $4 \quad$ (id.) & 150 & 0.25 \\
\hline $\begin{array}{l}\text { LAMATSCH MERCK } \\
\text { (Darmst.) . . } \\
\text { P. acid }\end{array}$ & $0.2-0.5$ & $7 \cdot 9$ & 2.5 (id.) & 300 & 0.45 \\
\hline $\begin{array}{l}\text { Boudault ....... } \\
\text { P. neutre gallicum }\end{array}$ & 0.4 & $5 \cdot 98$ & (id.) & 160 & 0.24 \\
\hline $\begin{array}{l}\text { Bouda ULT . . . . . . } \\
\text { Pepsinum purum bri- }\end{array}$ & 0.4 & 6.14 & (id.) & 200 & 0.3 \\
\hline $\begin{array}{l}\text { tann. FERRIS_(Bris- } \\
\text { tol)................ } \\
\text { P. concentratum } \\
\text { LANGENBEK-JENSEN }\end{array}$ & 0.4 & 7.91 & $7 \quad$ (id.) & 300 & 0.45 \\
\hline $\begin{array}{c}\text { (Copenhag.) } \\
\text { Pepsin-extract }\end{array}$ & I & 8.2 & (id.) & 500 & 0.75 \\
\hline 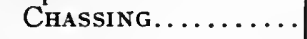 & 0.5 & 7.07 & $4 \cdot 5$ (id.) & 250 & 0.37 \\
\hline
\end{tabular}

acidity for the action of pepsin varies with each of the preparations. For gastric juice the optimum acidity is 0.2 per cent, but the reaction still takes place very well when the quantity of $\mathrm{HCl}$ is diminished or increased. On the contrary, the activity of the commercial products is found to be much more dependent on the reaction of the medium. The limits for a tolerated acidity are very restricted, and in the case of Langenbek's pepsin we even reach degrees of acidity which are not found at all in the normal stomach.

In addition in estimating the value of pepsins, we must 
take into consideration not only the dissolving power of the product, but also its actual peptonizing power. We very often encounter preparations capable of easily dissolving albuminoid materials, but of digesting them poorly. Natural gastric juice, on the contrary, dissolves and peptonizes at the same time. In test No. I, after 4 hours the filtered liquid does not become turbid upon nęutralization and gives no precipitate at all after the addition of nitric acid, while all the pepsins of commerce act otherwise under these conditions. The heading, "Equivalent in c.c. of gastric juice per roo g. of different pepsins," furnishes the exact relation which exists between the pharmaceutical pepsins and the gastric juice, a relation established not according to the dissolving power, but according to the true digestive power. We thus find that Ioo g. of the most active pepsin (pepsinum russicum) represent 600 c.c. of gastric juice. The heading, "Content in real pepsin" is based on the assumption that gastric juice contains 0.45 per cent of solid material, of which $\frac{2}{3}$ are inert substances. The quantity of real pepsin in gastric juice will then be o.I 5 per cent. According to the comparative results furnished in the preceding column, we see that pepsin No. 4, the most active, contains in reality 0.9 per cent of active matter and 99.I per cent of inert substances.

The Russian Codex of r89r prescribes the following conditions for the analyses of pepsin: "O.I g. of pepsin must digest in 4 hours, at $40^{\circ}$, ro g. of cooked and sifted egg-albumin, diluted in 100 c.c. of 0.25 per cent $\mathrm{HCl}$." In this test, we are limited only in regard to the solution, without taking into account the digestion of the liquid. In the Codices of other countries (Germany, England, Belgium, Italy, etc.) it is also the dissolving, under given conditions, of the white of egg used that is made the basis for the determination. Here, for example, is the official test of pepsin, described in the Belgian Codex (I906 edition):

"Keep for ro minutes in boiling water a fresh hen's egg. After cooling, take away the white, which is separated, by caus- 
ing it to pass through sieve No. 10.* Dissolve by trituration O.I g. of pepsin in $100 \mathrm{~g}$. of water containing 0.25 per cent of hydrochloric acid. Pour the solution in a flask of about 200 c.c. capacity. Add to it Io g. of white of egg, shake and heat in the vapor-bath at the temperature of $40^{\circ}$, shaking from time to time. After an hour of digestion the white of egg is dissolved, leaving only some small flakes."

Pepsin conforming to the Russian test and, still more, to the Belgian test, unquestionably represents a very active pepsin, on condition that the solution be really complete and that no appreciable residue remains, with the exception of some fragments of membrane. It then becomes a question of estimating complete solution, a difficult problem in that between complete solution and apparent solution there is an enormous difference, which may require a duration of several hours. We must then substitute for this imperfect criterion a more precise method for distinguishing complete digestion. Two reactions can accomplish this aim. The first method is based on the precipitation of acid albumins when the liquid submitted to the action of pepsin is neutralized. When digestion is sufficiently advanced, exact neutralization does not bring about any precipitation. The second method depends upon the appearance of a precipitate when a little nitric acid is added to the digestion liquid. If no turbidity occurs under these conditions, the hydrolysis has already gone as far as the total transformation of the heteroalbumoses.

These two methods, which were introduced by Petit, are practically on an equal footing, although the first corresponds to a slightly more advanced action. Yet, as it is easier to execute the second correctly, it is this latter which has been adopted in the French Codex.

The great difference which exists between the dissolving and peptonizing powers was established by Effront on six pharmaceutical products of different origins.

\footnotetext{
* Io meshes per centimeter of length.
} 


\begin{tabular}{|c|c|c|c|c|}
\hline \multicolumn{2}{|r|}{ Origin of the pepsin. } & $\begin{array}{l}\text { Length } \\
\text { of solu- } \\
\text { tion. }\end{array}$ & $\begin{array}{l}\text { Disappearance of } \\
\text { acid albumin. }\end{array}$ & $\begin{array}{c}\text { Disappearance of } \\
\text { the precip. with } \\
\mathrm{HNO}_{3} \text {. }\end{array}$ \\
\hline $\begin{array}{l}\text { (I) } \\
\text { (2) } \\
\text { (3) } \\
\text { (4) } \\
\text { (5) } \\
\text { (6) }\end{array}$ & 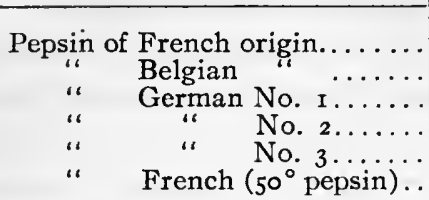 & $\begin{array}{l}\text { hrs. } \\
4 \\
\text { I } \frac{1}{2} \\
3 \\
6 \\
8 \\
\text { I }\end{array}$ & $\begin{array}{ll}\text { I } 2 & \text { hrs. } \\
\text { I4 } \\
20 \\
35 \\
50 \\
7\end{array}$ & $\begin{array}{l}\text { i } \\
\text { I3 } 3 \\
\text { I8 } \\
40 \\
50 \text { (incomplete) } \\
6\end{array}$ \\
\hline
\end{tabular}

For the tests from (I) to (5), were used ro g. of egg-albumin, cooked and sifted, roo c.c. of 0.25 per cent $\mathrm{HCl}$ and $0 . \mathrm{I}$ g. of pepsin, the temperature being at $40^{\circ}$. Test No. 6 was made at the temperature of $50^{\circ}$. The solution of the white of egg is very rapid in tests (I), (2), (3), and (6); yet the disappearance of acid albumin progresses quite slowly. It takes 3 to 7 times longer for the entire transformation of this substance than for the solution of albumin. With Io c.c. of the gastric juice of dog we get, under the same conditions, the disappearance of acid albumin in 4 hours. To reach the same point in 12 hours, 2 c.c. of gastric juice is quite sufficient. Of the products analyzed, Nos. (I), (2), (3), while answering the requirements of the Codex, are in comparison with the gastric juice absolutely inferior products. Ioo g. of the most active pepsin (No. I) correspond to 2 liters of gastric juice, which represents about $2.8 \mathrm{~g}$. of true pepsin and $98.2 \mathrm{~g}$. of inert matter.

According to the latest French Codex, that of 1908, the text relative to the analysis of pepsin is as follows:

"Pepsin must come up to the following test:

Pepsin: ten centigrams............................ o.

Distilled water: fifty-eight grams fifty centigrams........... 58.50

Diluted hydrochloric acid: one gram fifty centigrams.......... I.50

Dried fibrin $(a)$ : two grams fifty centigrams............... 2.50

In a flask with large opening introduce the fibrin, the distilled water, and the dilute hydrochloric acid.* Then place this flask in a heater, or in a vapor-bath heated to $50^{\circ}$, until the mixture shall have reached the same temperature. Add then the

* The quantity prescribed by the Codex represents 0.25 per cent $\mathrm{HCl}$. 
pepsin and let it digest for six hours, taking care, at the beginning, to shake frequently until complete solution of the fibrin, and thereafter at about every hour. Let it cool and filter. Io c.c. of the liquor thus obtained, at ordinary temperature, becomes turbid upon the addition of twenty drops of standard nitric acid.*

(a) In this test the dried fibrin may be replaced with ro g. of fibrin dried in the open air. The proportion of distilled water must then be reduced to $5^{\mathrm{I}}$ g."

From the preceding, we then conclude that French officinal pepsin must have a standard equal to roo. We know, in fact, that the standard of a pepsin represents the number of grams of fresh fibrin which a gram of this pepsin is capable of transforming in 6 hours at $50^{\circ}$ into products incoagulable by nitric acid. The protein material submitted to the action of pepsin is hog fibrin, which has been washed, dried in the air, desiccated at $40^{\circ}$, either in a heater, or in a current of dry air, and then pulverized. This product keeps well, remains constant in value, and is attacked by pepsin as well as fresh fibrin. We can also use fresh fibrin, but the degree of humidity of the product obtained may then vary considerably according to the degree of the drying. On an average, to g. of fibrin washed and dried gives $2.5 \mathrm{~g}$. of dry fibrin.

Fibrin prepared under these conditions gives entire satisfaction. However, in France this result was not reached at the first attempt. This is why, in view of the difficulty of procuring a fibrin easily preserved and always comparable to itself, pharmacologists of other countries have adopted another method of attack. We have already seen that Russia makes use of coagulated white of egg. It is the same with England, Austria, Belgium, the United States, Italy, and Switzerland. To give results always identical, the cooking of the egg-albumin should be done in the minimum time, and the coagulum obtained should be brought to a perfect state of division. Further, we must use this substance immediately after its preparation.

* This acid is a mixture of about $\mathrm{I}$ mol. $\mathrm{HNO}_{3}$ and 2 mols. $\mathrm{H}_{2} \mathrm{O}$. 
The use of casein and gelatin, also requiring great care, has not been adopted by any country.

There is another point worthy of attention. The quantity of water used in the preceding test is specified. The fact is that the peptonizing power of a pepsin diminishes when the concentration of the digestive medium increases. We see that account is taken of this factor by using desiccated fibrin or dried fibrin, and that in the latter case the proportion of water directly added is reduced. There is another detail of some importance. It is recommended not to add the pepsin in the mixture of fibrin, water, and acid until the latter shall have taken the temperature of the heater. As a matter of fact, pepsin in the presence of $\mathrm{HCl}$ undergoes a diminution of activity, and if this weakening is not taken into account it is important that it should always be produced under the same conditions.

These few remarks show the care with which the different experimental conditions have been specified. The temperature of the reaction, its length, the determination of the end of the digestion, have all been chosen as being the most favorable for a good operation. Like care is not found in the Codices of all the other countries and, because of this lack of precision and also of the diversity of experimental conditions adopted, we experience great embarrassment in comparing the activities of several pepsins of different origins.

Having given the requirement of the French Codex, it is indisputable that the pharmacies of France will always have products superior to those furnished in the other countries. However, it must be noted that these products are still far from being as active as sample ( $\mathrm{I}$ ), examined above. In fact, the enzymic power of this latter having been determined by means of cooked albumin, which we know to be much more difficult to digest than the fibrin recommended by the French Codex, pepsin (I) can be considered as a product of maximum activity.

Therapeutic Use. - Pepsin is used either in the form of a powder, or of an extract. Amylose pepsin and lactose pepsin, which figure in the Codex of 1908, are mixtures of pepsin with 
starch or sugar of milk. They must have a standard equal to 40. The extracts of pepsin are solutions of pepsin made in wine. In view of the injurious influence which alcohol exerts on the enzyme, it is preferable to reduce as far as possible the percentage of alcohol, and in order to avoid the deterioration of the liquid, a certain quantity of glycerin is added.

The following is the formula of the elixir described in the Codex of I908:

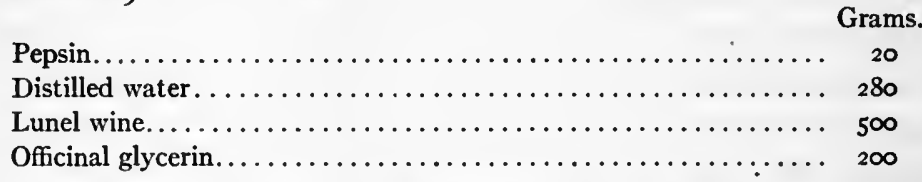

This extract in Io-g. amounts should dissolve Io $\mathrm{g}$. of dried fibrin under conditions of temperature and of time analogous to those prescribed for the pepsin test. It preserves in a satisfactory manner.

In addition to these preparations of pepsin, we can also use gastric juice itself. We find on the market commercial dog juices, or again those of hog, collected by fistulas in an aseptic manner and endowed with great proteolytic powers. The gastric juice of a dog can be preserved for a very long time without deterioration. It does not putrefy on long keeping and consequently affords infinitely more of a guarantee than most of the pharmaceutical products of pepsin base.

The quantity of pepsin used in therapeutics is from I to $4 \mathrm{~g}$. a day. That of gastric juice, recommended by Pawlow, is 30 c.c. per meal. Now 30 c.c. of gastric juice is equivalent to $5 \mathrm{~g}$. of pepsin of the best quality; with such proportions, the advantage will always be with the gastric juice. But its true efficacy consists especially in the fact that it is more easily adapted to the variation in acidity of the stomach, and that furthermore there are not introduced with it into the stomach foreign bodies capable themselves of causing trouble.

Commercial Peptones. - We find in commerce under the name of peptones a certain number of products which result from the action of proteolytic enzymes on albuminoid materials 
of various kinds. Among these are Witte peptone, Chapoteaut peptone, etc. Most often pepsin is the active substance used, although pancreatic peptones are also prepared, and even papainic peptones (Antweiler's peptone).* The raw materials ordinarily utilized are beef or horse meat, fibrin, white of egg, casein, etc. Peptic digestion takes place in the presence either of $\mathrm{HCl}$, of phosphoric, or of tartaric acids. The following is, according to Catillon, a peptone preparation adopted by the Codex (supplement r895):

I kg. of beef, free from fat and finely minced, is allowed to digest at a temperature of $50^{\circ}$ for 6 to 8 hours with 5 liters of water, with addition of $50 \mathrm{~g}$. of officinal hydrochloric acid and $20 \mathrm{~g}$. of a pepsin extract capable of dissolving 50 times its weight of fibrin. It is frequently stirred and kept at a constant temperature. The mixture gradually becomes fluid-like and, after some hours, transparent. The operation is complete when ro c.c. of the filtered and cooled liquid no longer becomes turbid upon the addition of 30 drops of officinal nitric acid. Filter and saturate the clear solution with sodium bicarbonate. Finally, evaporate in the vapor-bath until a formation of a surface film is observed. The saturated solution of peptone has a density of I.I5; it contains half of its weight of liquid peptone and corresponds to 4 to 5 times its weight of meat.

This medicinal peptone is very salty from the large quantity of $\mathrm{NaCl}$ resulting from the neutralization of the $\mathrm{HCl}$ used. Petit, to remedy this inconvenience, replaces this acid by tartaric acid, the elimination of the latter taking place almost completely in the form of the only slightly soluble cream of tartar.

In a general way the commercial peptones, especially those of peptic origin, in spite of their names contain only very little true peptone, and are made up largely of albumoses. The

* We also find commercial peptones of a brand of which Kemmerich is the type, obtained by the action of superheated steam on meat; they are composed of albuminoids that are very little transformed, being simply dissolved. On the other hand, the La Roche peptone, as previously described (p. 525), is a product of an entirely different nature, since it results from the advanced hydrolysis of silk waste under the influence of concentrated sulphuric acid. 
following is an analysis of Witte peptone, which was obtained by the action of pepsin on fibrin. Although this is not a constant product and its composition varies from one sample to another, we observe that the proportion of nitrogen precipitable by zinc sulphate is always large:

Analysis of Witte's Peptone.

Of total nitrogen:

Per cent

Nitrogen precipitable by $\mathrm{ZnSO}_{4}$ : albumoses............. 57.5 to 68.6

Nitrogen precipitable by phosphotungstic acid: albumoses + peptones............................... 90.5 to $9 \mathrm{I}$ Ammoniacal nitrogen . . . . $\ldots \ldots \ldots \ldots \ldots \ldots \ldots \ldots \ldots \ldots, 0.4$ to 1.4 Formaldehyde nitrogen (after elimination of $\left.\mathrm{NH}_{3}\right) \ldots \ldots \ldots \ldots$ II to 12

Without doubt, the pancreatic peptones, which are prepared in the same way as the peptic peptones except that the digestion liquid in place of being acidified is made slightly alkaline with $\mathrm{Na}_{2} \mathrm{CO}_{3}$, contain a large quantity of degraded products (Ex: tyrosin and tryptophane), though here again the hydrolysis is never very far advanced. Moreover, the two kinds of peptones are distinguished by the same procedures as those which have been previously described, especially by the use of bromine water, which gives a yellow precipitate in the case of peptic peptones, and in the case of pancreatic peptones a deep violetred coloration, passing to brown with an excess of reagent. It should be noted, furthermore, that these products are often adulterated by the addition of starch, dextrin, glucose, lactose, gelatin, etc.*

These peptones are usually sold in the form of very light amorphous powders, very hygroscopic, of a slight acrid and bitter flavor, and with an odor resembling that of spoiled broth. Soluble in water, they are incompletely precipitable by alcohol. These products are said to be easily assimilable, and accordingly are used in the diet of tuberculous, cancerous, and debilitated patients. According to the case, a dose of 5 to $50 \mathrm{~g}$. of dry peptone is prescribed per day. We also use them in an associated

* To analyze peptones, the reader is referred to the thesis of Javillier already cited: Sur Les Ferments Proteolytiques. 
form, especially in connection with various metallic salts. These are the peptonates, of which the best known are those of iron or mercury, and in which the metals have more or less concealed specific reactions. Finally, peptones are found in some medicinal iodin preparations, the iodin being partially in the organic complex.

In addition to these purely therapeutic applications, the peptones are also frequently used in bacteriological laboratories for preparing culture broths. We will recall that the Zymogen, referred to on page 4I 7 , and which forms an excellent food for yeasts, is an industrial peptone obtained by the action of proteolytic enzymes of yeast on freshly precipitated casein. Likewise, a solution of peptone that is rich in very degraded nitrogenous products can be prepared by leaving yeast for some time to autolysis, but the liquid so obtained will also contain excretion products which are often toxic for micro-organisms.

\section{BIBLIOGRAPHY.}

Boudault. Schmidt's Jahresbuch, 1857, p. 290. - Mémoire sur la pepsine, Journ.

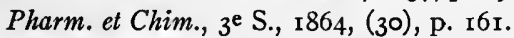

L. Panum. Wiener Sitzungsberichte d. Acad., I871, p. 64 .

HeIтz. Arch. pharmac., I871, (196), p. г30.

HofMeIster. Deuts. Mediz. Woch., 1875, p. 3.

Mourrut. Journ. Pharm. et Chim., I879, (30), p. 44I.

Hammarsten. Schmidt's Jahresbuch, 1877, p. I73.

Ewald. Zeits.f. klin. Med., I879, p. 23 I.

Coоmbs. Zeits. d. Apoth. Vereines, I886, p. 38.

Konowalofr. Thèse, St-Pétersbourg, I893.

Bourquelot et Herissey. Journ. Pharm. et Chim., r903, (17), p. 164.

P. Thibault. Etude sur les prép. officin. de pepsine. Thèse pharm., Paris, Igoz.

A. Petit. Etude sur la pepsine, Paris, i88I.

A. Perrt. Sur les peptones, Journ. Pharm. et Chim., 5e S., r881, (3), p. 529. 


\section{§ 2. Chemical and Enzymic Exploration of the Stomach.}

I. In consequence of advances realized in our knowledge of the mechanism of digestion, it had been thought that perhaps the analysis of the contents of the stomach would give valuable notes on the pathology of this organ, as well as on the origin of various diseases accompanying a defective nutrition. But the results obtained in this way were not encouraging, and the partisans of the stomach exploration turned to other methods of a more physical nature, such as the determination of the molecular concentration of the gastric liquid, gastroscopy, and radiography. Without doubt, the study of gastric chemistry cannot always offer an infallible control for the functioning of this organ, and, by itself, would not afford a sure diagnosis of the more or less normal state of the stomachal mucous membrane. However, the discredit into which this sort of investigation has fallen is undeserved, resulting, as it does, from errors in application rather than in the principle itself. In fact, a score of years ago, the methods of analysis of gastric contents, both as to its chemical composition as well as its enzymic content, were very imperfect. However, later methods, much surer and more accurate, have succeeded in establishing results that are worthy of consideration.

In a preceding chapter we have studied the gastric juice and have given its composition, this juice coming from animals provided with a fistula. The stomachal contents of man is collected by means of sounds introduced into the alimentary canal, either after fasting, or after a test meal. The test meal of Ewald is composed of 60 to $80 \mathrm{~g}$. of stale white bread and of $\frac{1}{4}$ of a liter of weak tea. The sounding is done $\mathrm{I}$ hour after the meal. Under normal conditions, about 50 c.c. of liquid are obtained. To determine exactly the volume of liquid contained in the stomach, a small part is first collected, whose acidity is determined. The stomach is then washed, and the acidity of this dilute liquid, of known volume, is determined. From these two acidities, by a very simple calculation, the total 
volume of the gastric liquid may be estimated. We can also introduce sodium phosphate or iron salts into the test meal, and the determination of these products in the gastric juice affords a means for calculating the total volume of stomachal liquid. Lœper uses as test meal the following mixture: $60 \mathrm{~g}$. of fresh white of egg, representing $8 \mathrm{~g}$. of pure albumin, are diluted with water and coagulated; 15 to 20 gr. of glucose are added, and the whole is brought up to about 200 c.c. The sounding is made 25 to 30 minutes after ingestion and about 80 c.c. of gastric liquid are taken for analysis.

II. The examination of the stomachal contents is concerned especially with the quality and the quantity of the enzymes, as well as with the acidity, and often this study of the gastric secretion is completed with the determination of the nitrogenous materials which it contains. With regard to the latter, the least important of the three, the chemical analysis of the gastric liquid includes the determination of the following substances: (I) albumin remaining; (2) peptones; (3) amino-acids and other nitrogenous substances.

The procedure, according to Lœper, is as follows: In a first portion of gastric juice, the total albumin is measured by the Esbach reaction. In another portion of equal volume, the true albumins are precipitated by hot ferric chloride and sodium acetate, and the peptones are measured colorimetrically in the filtrate with Fehling's solution. It is evident that for the information drawn from these analyses to have any value, it is necessary that the test meal shall be kept constant, both as to its chemical composition as well as to the action which it can exert on the stomachal mucous membrane. It is for this reason that Lœper always uses the meal of coagulated white of egg with addition of glucose, as seen above. The amino-acids and the other nitrogenous products are determined by first precipitating the albumins and the peptones by the addition of three times their volume of alcohol at $90^{\circ}$. After standing, the liquids are centrifuged. The nitrogen found in the clear liquid indicates the approximate quantity of non-biuretic bodies. 
This method of analysis is obviously rudimentary. Very satisfactory methods, however, exist for determination of the nitrogenous compounds in their different forms. They have been described above and will only be mentioned again; precipitations by zinc sulphate, by tannin, by phosphotungstic acid, and the formaldehyde method of Sörensen. In the clinics, however, expeditious procedures are preferred, though the latter are uncertain, not at all comparable, and often badly applied. It is not astonishing that the results obtained under these conditions are not in agreement with those which might be expected. The following figures were obtained after ingestion of the test meal of Lœper, corresponding to $8 \mathrm{~g}$. of albumin:

Analysis of Stomachal Contents.

\begin{tabular}{|c|c|c|c|}
\hline Diseases of subjects examined. & $\begin{array}{l}\text { Albumin } \\
\text { remaining. }\end{array}$ & Peptones. & $\begin{array}{l}\text { Amino } \\
\text { acids. }\end{array}$ \\
\hline 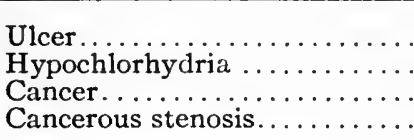 & $\begin{array}{l}\text { g. } \\
\text { I } \\
7 \\
6 \\
6\end{array}$ & $\begin{array}{l}\text { Very abund. } \\
\text { Small amt. } \\
\text { Small amt. } \\
\text { Small amt. }\end{array}$ & $\begin{array}{l}\cdots \cdots \\
\text { Traces } \\
\text { Traces }\end{array}$ \\
\hline
\end{tabular}

The presence of amino-acids in the gastric juice does not appear to result from the peculiar activity of the mucous secretion, but rather from the action of the pancreatic juice flowing back into the stomach, from the action of bacteria, and also from certain products secreted by morbid cells. Thus the abundance of amino-acids often shows, as we shall see, the existence of a stenosis or of a cancer.

In a fasting stomach very little gastric juice is normally found. By the tube-washing method, using slightly alkaline water, it is found that in a healthy organ this operation brings only a little mucus, only slight traces of $\mathrm{HCl}$, and very little chlorides. In a pathological condition, on the other hand, we may find blood, as well as albumin, peptones, and non-biuretic peptic derivatives, certain nucleo-proteins, more or less specific, and finally, various substances like urea, sugar, acetone, oxalic acid, etc. Gastric digestion is advanced much further in cer- 
tain diseases than was formerly thought to be the case. According to Emerson, 50 per cent of total nitrogen of the albumin dissolved in the gastric juice of sick people with slight gastric disturbance is found in the form of peptones or other more advanced products. Fischer finds amino compounds, tyrosin, leucin, arginin, etc., in the gastric contents of sick people affected with cancers of the stomach, compounds not found in normal subjects. We should note, in passing, the coincidence which exists, in the case of gastric cancers, between the presence of amino-acids on the one hand, and the absence of $\mathrm{HCl}$ on the other. This is due to the hydrolysis of the albumin molecule; the further its degradation is pushed, the more acid is required to neutralize the amine groupings which appear. We believe that the peptones come directly from certain tumors or inflammatory lesions of the stomach, or that they come from the transformation of a diffused albumin by the gastric juice. The amino-acids may result from the action of the enzymes of the tumor on its own albumin. This special proteolytic enzyme of tumors is, moreover, quite similar to erepsin.

We owe to Zunz some interesting facts upon the quantity of amine $\mathrm{N}$ in the stomach contents. This author used for several years the method of Sörenson and more recently that of Van Slyke. ${ }^{*}$ The figures obtained by the latter are here given:

Aliphatic Amine Nitrogen in the Gastric Contents of Man.

\begin{tabular}{|c|c|c|}
\hline Group of persons examined. & $\begin{array}{c}\text { Per cent amine } N \text { of } \\
\text { total } N \text {. }\end{array}$ & Degree of hydrolysis. \\
\hline 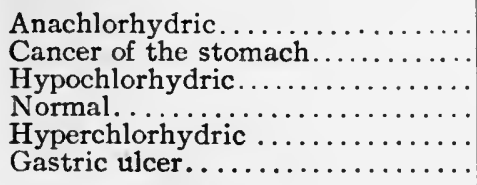 & $\begin{array}{l}4.14 \\
6.8 \mathrm{I} \\
4.23 \\
4.33 \\
3.85 \\
5.07\end{array}$ & $\begin{array}{r}7.25 \\
16.06 \\
7.61 \\
8.27 \\
7.06 \\
15.42\end{array}$ \\
\hline
\end{tabular}

* The method of Van Slyke is based on the action of $\mathrm{HNO}_{2}$ upon the aminogrouping of amino-acids. There is produced under these conditions gaseous nitrogen: the gas is collected and measured and its volume represents the aliphatic amine nitrogen titratable by the above method. While straight chained aminoacids, such as leucin, give one molecule of nitrogen, and proline and oxy-proline do not act at all, lysin, on the other hand, furnishes two molecules of nitrogen. 
The degree of hydrolysis is furnished by the ratio between the amine nitrogen found in the stomach contents before and after complete hydrolysis with ${ }_{5} \mathrm{~N} \mathrm{HCl}$ under pressure. Thus, with anachlorhydrics, there was found by direct analysis 4.I4 per cent of $\mathrm{N}$, and after treatment with the acid 56.26 per cent, thus giving for the degree of hydrolysis, 7.25. This table confirms what we have previously stated, that is, that the disintegration of nitrogenous material is carried relatively far in the case of ulcers and especially of gastric cancer since in the affection one observes that the degree of hydrolysis is about two times greater than if digestion is normal.

III. We will now consider the study of the enzymic content of the stomach. First of all, we must establish the stomach content in acids - total acid, free acid, and combined acid. In clinical laboratories, the total acidity is determined by titrating with a $\mathrm{N}$ / Io solution of $\mathrm{NaOH}$, in the presence of phenolphthalein. This total acidity corresponds to the free hydrochloric acid, which turns red Congo paper blue, to fixed organic acids, like lactic acid, which has the property of turning yellow the violet solution obtained with phenol and ferric chloride, and finally, to volatile acids, like acetic acid, butyric acid, etc. The determination of hydrochloric acid in the three states, free, combined inorganic, and combined organic, is generally made according to the method of the three capsules, developed by Hayem and,Winter. Pour into each of three capsules 5 c.c. of gastric juice. Add to the first $\mathrm{Na}_{2} \mathrm{CO}_{3}$ and ignite. The determination of the chlorides formed gives the total chloride. The second is simply ignited and gives the fixed inorganic chlorin. Finally, the third is evaporated at $100^{\circ}$. Free $\mathrm{HCl}$ escapes and a determination of the remaining chlorin furnishes the combined chlorin, inorganic and organic. From these three results the free $\mathrm{HCl}$ may be deduced. This procedure is quite rapid, but gives a doubtful precision. On the other hand, we observe that no allusion has been made to the characterization and to the quantitative determination of the volatile acids. However, the method of Duclaux is adequate for this purpose. 
In spite of this criticism, the results which are thus obtained are interesting. In a general way, we find that the total acidity in the course of a normal digestion increases from about 0.05 per cent to about 0.2 per cent at the end of the first hour, and then diminishes. The free hydrochloric acid reaches a maximum of $0.05^{-0.06}$ per cent, and the combined hydrochloric acid 0.15-0.18 per cent. As to organic acids, they are only found in traces. In the case of illness, these figures undergo considerable modifications. In the case of cancer, for example, $\mathrm{HCl}$ is deficient and the chlorides are weak; on the contrary, lactic acid increases. On the other hand, in cases of ulcer and hyperhydrochloria, the total acidity is always very high.

In the investigation of the enzymes, we ordinarily use the Mett method, previously described (p. 252), for the estimation of pepsin, and for the measurement of the rennet, the usual procedure (p. I43), which consists in adding in tubes containing equal volumes of milk increasing quantities of gastric liquid. The proportion of enzyme is judged according to the rapidity of coagulation. There also exist in the gastric juice other enzymes, like amylase and lipase, but it is not established that their presence is really due to a stomach secretion. On the contrary, the determination of the pepsin and of the rennet is of very great importance. Formerly, in applying the two preceding methods, it had been possible to establish in the pathological cases that the richness of the gastric juice in pepsin is not always proportional to that in $\mathrm{HCl}$. Thus it is that this cancer enzyme remains at a concentration often high and out of proportion with that of $\mathrm{HCl}$; in sclerous gastritis, on the contrary, the absence of acid and of pepsin is the rule. On the other hand, rennet has been found especially in breast-fed children, and is often absent in cancers.

For some years we have had at our disposal, for the analysis of peptonizing and coagulating enzymes, the much more delicate methods that have already been described in preceding chapters. These are: for pepsin, the Fuld method, with edestin (p. 253), and that of Jacoby, with ricin (p. 255); for rennet, the method 
of Morgenroth, based on a previous activity of the enzyme at $\circ^{\circ}$ (p. 143), and that of Blum and Fuld, based on the use of Ekenberg's * milk powder (p. 144). On the other hand, there is another procedure, developed by Meunier, which is likewise very satisfactory. It consists in adding to 5 c.c. of sterilized milk, 5 c.c. of a I per cent solution of $\mathrm{CaCl}_{2}$, and then 5 c.c. of gastric juice diluted $\frac{1}{10}, \frac{1}{100}$, or $\frac{1}{1000}$, so that the coagulation takes place in the first ten minutes at a temperature of $40^{\circ}$. Meunier adopts, as the coagulating unit of a gastric juice, that quantity of milk which is coagulated in exactly ro minutes by I c.c. of pure juice. It is calculated according to the formula: $F=\frac{D \times \text { Io }}{m}$, where $D$ is the dilution and $m$ the time. If the juice is diluted $\frac{1}{100}$ and the length of coagulation is $8 \mathrm{~min}$., the coagulating value will then be 125 . By the aid of his method, Meunier obtained the following results:

Coagulating Value of Some Gastric Jujces.

1000-2000: normal juice.

500-1000: slight disturbances with good prognosis.

100- 500: alcoholic gastritis; old stomachal ulcer; old case of hypersecretion.

o- Io: chronic gastritis. Cancer.

In the chapter on the possible identity of pepsin and rennet, it was stated that there ordinarily existed a narrow relation between the quantities of these two enzymes present in the stomach. Blum and Fuld even believe that the variations of secretion of these are parallel, and that it is sufficient to determine one of them to know the proportion of the other. On the following page are some of the figures which these investigators offer in support of this assertion, and which, with some exceptions, are especially conclusive.

In view of such conclusive results, we can say that if gastric exploration has not so far given in practice all that was expected of it, it is because the problem was badly attacked, as much from the chemical as the enzyme point of view, and that on the

* This product is sold by The Ekenberg Milk Product Co., Ltd., London, Victoria buildings 37, Queen Victoria street. 
Comparative Contents in Pepsin and in Rennet of Various Gastric Juices.

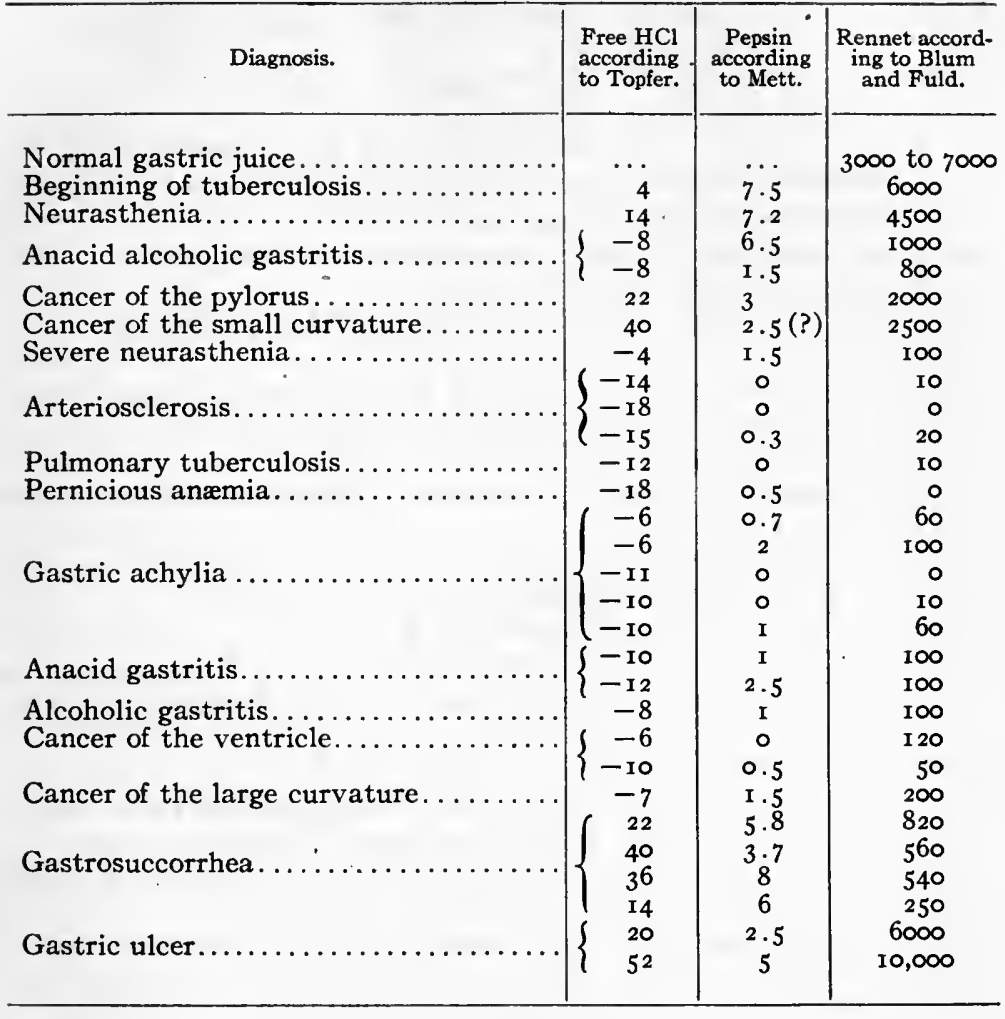

contrary, it is very probable that if we obtained in clinical laboratories really accurate analyses of gastric contents we should get indications very useful for the diagnosis of a large number of diseases. 


\section{BIBLIOGRAPHY.}

LCPER. Leçons de pathalogie digestive, Paris, I9I2.

G. Leven. La dysepsie, Paris, I9r3.

Emerson. Deuts. Arch. f. klin. Medizin, (72).

Fischer. Deuts. Arch. f. klin. Medizin, (93).

L. Blum u. E. Fuld. Ueber eine neue Methode der Labbestimmung u. über das Verhalten des menschlischen Magenlabs unter normalen u. pathologischen Zuständen, Berl. klin. Woch., 1905, $\left(44^{\mathrm{a}}\right)$, p. 107.

Linossier. Recherche et dosage de la pepsine dans le contenu gastrique. Journ. de physiol. et de pathol. gén., (I), p. $28 \mathrm{I}$.

Meunier. Digestion lactée: rôle du lab-ferment, Bull. gén. de thérap., rgo4, p. 683 .

Robin et Gouraud. Du ferment lab, Bull. gén. de thérap., 1902, (143), p. 197.

Vortruba et Mixa. Le chimisme gastrique dans divers cas morbides, Bull. gén. de thérap., 1903, (146), p. 495.

Gluzinski. Zur Frühdiagnose des Carcinoms, Grenzgebiete der Medizin u. Chirurgie, (10), p. I.

UMBER. Berl. klin. Woch., 1905, p. 89.

EINHORN. Arch. f. Verdauungskrankheiten, (I), p. I58; (7), p. II.

Martins u. Labarsch. Achylia Gastrica, Wien, I897.

Kuttner. Zur Frage der Achylia Gastrica, Zeits. f. klin. Medizin, (45), p. x.

Prollew. Zur Pepsinfrage bei Achylia Gastrica, Arch. $f$. Berdauungskrankheiten, (5), p. $\mathrm{I} 5 \mathrm{I}$.

GläsSNer. Berl. med. Woch., I902, p. 675.

E. Zunz. Recherche sur l'azote titrable dans le contenu stomacal par la methode de Soerensen. Int. Beitr. z. Pathol. u. Therap. d. Ernähungsstörugen, t. 2, p. 372, 1910. Même recherche par la methode de Van Slyke, Id. Bd. 5, Heft I, I913.

D. Van Slyke. The quantitative determination of aliphatic amino-groups. Journ. Biol.-Chem., IX, p. 185, I9I I XII, p. 275, 191 2.

For the determination of acid in the stomach contents, see:

BiCKEL. Bioch. Zeits., I906, (I), p. i 56 .

Steensma. Bioch. Zeits., Ig08, (8), p. 210.

Lamblisg. Dictionn. de chimie de Würtz, $2^{\mathrm{e}}$ suppl,, $2^{\circ}$ t., p. 473. 
§ 3. Rôle of Proteolytic Enzymes in the Preservation of Grains and Flours, as well as in Breadmaking.

Flour, used in breadmaking, contains saccharifying enzymes and proteolytic enzymes. These enzymes come from the grain itself, as well as the bacterial flora which accompany it. It is known, in fact, that in the course of the ripening of grains active substances develop, which keep for a very long time after desiccation. As to the bacterial species which are found on their surface, they are very varied, particularly in the case of wheat and rye. Now many of these germs, such as $B$. mesentericus and $B$. proteus, have highly accentuated proteolytic properties. The number of bacteria which can be counted in I g. of grain varies from 200,000 to $10,000,000$. The greater part of these remains in the bran, but the flour also contains quite considerable quantities, from 20,000 to 30,000 per gram of substance.

From the point of view of the preservation of grains or of flours, the enzymes and the bacteria play an extremely important part. Dried grains, brought to a water content of 8 to ro per cent, generally preserve quite well in dry air or air which is not very humid, but in the presence of moist air, and especially if the temperature rises, the seeds absorb water, principally on the surface, and the conditions are just as favorable for the development of bacteria as for the awakening of all the enzymic activities. In fact, we find that the grain in a silo becomes heated, deteriorates, and that its respiration is considerably increased:

INFLUENCE OF THE HUMIDITY ON GRAI
\begin{tabular}{|c|c|}
\hline $\begin{array}{c}\text { Content in } \\
\text { water. }\end{array}$ & $\begin{array}{c}\mathrm{CO}_{2} \text { per kg. of } \\
\text { grain in } 24 \\
\text { hours. }\end{array}$ \\
\hline $\begin{array}{c}\text { Per cent. } \\
10\end{array}$ & $\begin{array}{c}\text { Mg. } \\
20\end{array}$ \\
33 & 0.3 to I .5 \\
\hline 350 \\
\hline
\end{tabular}


A similar phenomenon is also observed with flours which have been preserved. When these are heated, we observe an increase of the acidity due in part to the decomposition of fatty materials, and in part to the formation of volatile acids at the expense of the sugar and the starchy substances. According to Marion and Monget, the acidity of deteriorated flours comes especially from the decomposition of the gluten by enzymes, a reaction which entails the appearance of an acid substance. While fresh wheat contains from 0.004 to 0.023 per cent acid, calculated in lactic acid, the acidity in spoiled flours varies from 0.14 to 0.5 per cent. The acid content of deteriorated rye flour may reach 0.5 per cent, whereas the healthy product contains only 0.045 per cent.

The spoiling of grains and of flours is especially indicated by the change which takes place in the nature of the gluten which these materials contain, a change due largely to proteolytic enzymes. According to Fleurent, gluten is composed of three substances: gliadin, glutenin, and conglutin. This last plays a lesser rôle than the other two in the transformation, but nevertheless its proportion also changes. While in wheat of good quality we find $\mathrm{I}$ to 2 per cent conglutin in roo parts of total albumins, in wheat of vitreous grains of mediocre value we find as much as 15 and 16 per cent.

The quality of a flour, from the point of view of breadmaking, depends on the relation which exists between the gliadin and the glutenin. These two substances may be distinguished from the fact that the first is soluble in hot alcohol $\left(70^{\circ} \mathrm{G}\right.$. L.), whereas the second is insoluble.

Content in Gliadin and in Glutenin of Different Flours.

\begin{tabular}{|c|c|c|c|}
\hline & Gluten in flour. & $\begin{array}{l}\text { Gliadin in } 100 \\
\text { parts gluten. }\end{array}$ & $\begin{array}{l}\text { Glutenin in ro } \\
\text { parts gluten. }\end{array}$ \\
\hline Rye. & $\begin{array}{l}\text { Per cent. } \\
8.26\end{array}$ & $\begin{array}{l}\text { Per cent. } \\
8.14\end{array}$ & $\begin{array}{l}\text { Per cent. } \\
92.83\end{array}$ \\
\hline Maize. & 10.63 & 47.50 & 52.50 \\
\hline Barley.. & 13.82 & 15.60 & 84.90 \\
\hline Rice.............. & 7.86 & $14 \cdot 31$ & 85.70 \\
\hline Buckwheat. .................. & 7.26 & 13.08 & 86.92 \\
\hline Wheat.................... & 7.47 & 75.25 & 24.75 \\
\hline
\end{tabular}


The relation between gliadin and glutenin is thus quite different in the various cereals. Thus in wheat of good quality the relation is 3 to $I$, while in buckwheat it is almost $I$ to 7 .

According to Kosutany, these two substances, gliadin and glutenin, are capable of being transformed into each other by an enzymic intervention. Gliadin would be a reduced hydrate of glutenin, and inversely the latter would be an oxidized anhydride of gliadin. These transformations, according to Kosutany, take place in the course of the ripening of the grain, as well as in the various circumstances attending its treatment and preservation. According to the value of the ratio $\frac{\text { gliadin }}{\text { glutenin }}$ the product will improve or deteriorate, a flour of good quality corresponding, as Fleurent has found, to a proportion of 3 of the first constituent to $\mathrm{I}$ of the second.

First of all, it is easy to show that in breadmaking the gluten is hydrated: If in a paste of freshly prepared flour the gluten is at once isolated, it is found that the latter contains 43.65 per cent of dry substance. If the paste is diluted a halfhour before isolation of the gluten, it is found that the gluten obtained contains only 42.36 per cent of dry material. If 2 hours elapse before treating the paste, a gluten is isolated having not more than 41.02 per cent of dry material. The gluten is then hydrated, since the products obtained contain less and less dry substance.

This hydration takes place at the expense of the glutenin, which is thus transformed into gliadin. Inversely, if we dehydrate the nitrogenous substance of the wheat by means of 40 per cent alcohol we find that the proportion of gliadin remaining in the alcoholic solution diminishes with the length of the digestion:

\begin{tabular}{c|c|c|c}
\hline \multirow{2}{*}{$\begin{array}{c}\text { Length of the diges- } \\
\text { tion. }\end{array}$} & \multicolumn{3}{|c}{ Quantity of nitrogen dissolved. } \\
\cline { 2 - 4 } & I. & II. & III. \\
\hline Hours. & Per cent. & Per cent. & Per cent. \\
I & 0.979 & 0.773 & 0.757 \\
3 & 0.924 & 0.724 & 0.730 \\
6 & 0.903 & $0.66 \mathrm{r}$ & 0.692 \\
\hline
\end{tabular}


These transformations take place in various circumstances. Thus, in the course of the germination of grain under the influence of the enzymes which have appeared, the glutenin is hydrated to give gliadin. The proportion of the latter is then too great, and the flour prepared with germinated seeds is always a product of inferior quality, for it gives a dough which does not hold together and which falls in the baking. On the contrary, the inverse transformation can be observed during the grinding of grain. If the product begins to get heated, the gliadin is dehydrated, at the same time that it oxidizes. Glutenin is formed, the ratio $\frac{\text { gliadin }}{\text { glutenin }}$ is again changed, and the flour obtained is also of an inferior quality, for the bread which it makes does not rise well and has a compact crumb.

According to circumstances, it is found that during their conservation, flours gain or lose in actual value. If a dry flour is placed in a warm room in the presence of dry air, we shall have oxidation and dehydration of the gliadin, which will consequently yield glutenin, and the product will improve. On the contrary, if a flour of good quality is kept in a moist and warm atmosphere, the glutenin is hydrated and transformed into gliadin, and the whole deteriorates.

Finally, in the actual breadmaking, the transformation of glutenin into gliadin takes place. If the dough is left to itself, we find that it becomes softer and softer in consequence of a hydration, as shown above. During kneading, unquestionably a favorable change takes place in the nature of the gluten. It is believed that this change is due to the action of proteolytic enzymes brought by the grain. But this intervention of enzymes takes place also in the course of manufacture. We know, in fact, that to make the dough rise, we use a leaven prepared from beer yeast. The latter, by causing the sugar normally contained in the flour or formed in the course of kneading to ferment, gives carbonic acid and alcohol, which by being liberated or volatilized during baking give to the bread a certain porosity. Now, experience has shown that we get good results only with 
compressed yeast specially prepared for bakery purposes. The use of yeast from breweries, or again that of artificial leavens, like ammonium carbonate or a mixture of bicarbonate of soda and tartaric acid, a very common recipe a few years ago in America, has almost gone out of practice.

This recognized advantage in favor of compressed yeast compared with ordinary brewery yeast apparently comes from a different and more favorable action of the proteolytic enzymes of this yeast on gluten. Without doubt, this question is not yet completely settled, but it deserves the greatest attention, since the use of yeast in baking is a very costly procedure. In fact, without even taking into account the price of this yeast, we must note that its action is correlated with a deficit in fermentable substance, estimated at 2 per cent in the case of brewery yeast or spontaneous leaven, and again at 0.75 per cent or I per cent in the case of compressed yeast. This relatively slight percentage, however, represents a considerable loss in nutritive substances if we consider the enormous quantity of bread made in the world every day. If, then, it could be really established that a great part of the utility of yeast came from the nature of the proteolytic enzymes which they secrete, it would be a great advance in the baking industry to substitute for these yeasts artificial leavens, composed, on the one hand, of products generating carbonic gas and, on the other, suitable proteolytic enzymes.

\section{BIBLIOGRAPHY.}

T. Kosutany. Der ungarische Weizen und das ungarische Mehl, Budapest, I907.

Fleurent. C. R., 1896, (123), p. 327 .

E. Boutroux. Le pain et la panification, Encyclopedie de Chim. indust., Paris, I897.

BaLland. C. R., 1896, (122), p. 1496. 


\section{§ 4. Rôle of Proteolytic Enzymes in Brewing.}

I.

Nitrogenous materials play an important part in the making of beer as well as after its manufacture. In the first phase, they are necessary to the nourishment and to the development of the yeast. In the second, conjointly with the remaining carbohydrates, they assure the nutritive value of the finished beer, and furthermore contribute in a large measure to the viscosity and to the special taste of the product obtained. But it is important that this residual nitrogen shall fulfil certain conditions from qualitative and quantitative points of view, so that the beer shall not become turbid or deteriorate during its preservation under various physical or biological influences. All the work of brewing, as we shall see, is exactly regulated so as to fulfil these numerous desiderata.

Germination. - Barley grain contains nitrogen in approximately from I to 2 per cent of its weight according to the origin, the quality, etc. This nitrogen corresponds to various substances. Among these there is a certain number of proteins soluble in water, whose weight amounts to about 15 to 16 per cent of the total weight of the albuminoids contained in barley. These soluble reserves, however, are only slightly useful for the embryo. The proteolytic enzymes in the course of germination elaborate new ones, which can then serve for the development of the young plant. Naturally, these enzymes are also found in the malt, which the brewer uses for quite other purposes than those intended by nature. The following are data relative to the distribution of nitrogen in the different parts of the grain, considering the content of total nitrogen of barley as 100:

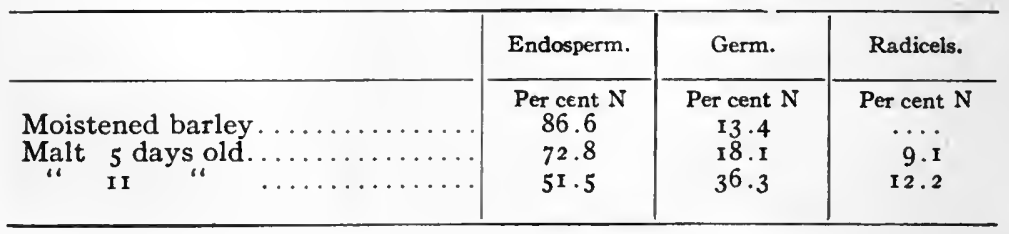


Thus, during malting there is a very important displacement of the nitrogen of the endosperm toward the plantlet in development. This displacement reaches $\frac{86.6-72.8}{86.6} \times 1 \infty$ or nearly 16 per cent of total nitrogen of the endosperm in the first five days of germination, and under the conditions of the experiment it even surpasses 40 per cent at the moment when the malt is ready to be treated in the kiln. In practice, under normal conditions, there is about 35 to 38 per cent of reserve nitrogen of the endosperm which passes by the scutellum into the young plant in the course of the ordinary period of malting. These figures apply only to the nitrogenous reserves which have been reduced by the enzymes to their simplest form, without including the more complex bodies. It is probable that in a well-made malt, which has germinated from Io to I2 days, at least 60 to 70 per cent of the protein reserves have been converted, while in the same period only I6 to 17 per cent of the starch reserve has been attacked. The proteolytic activity is intense.

To consider now the finished malt, free from its radicels, in place of the whole germinated grain, we find that in the manner of the preceding experiment, the distribution of the nitrogen is represented as follows:

Nitrogen of the endosperm $=58.7$ per cent of total nitrogen of the malt. " embryo $=41.3$

The nitrogen always soluble and not coagulable by heat is distributed in the following manner:

Nitrogen of the endosperm $=52.2$ per cent of soluble nitrogen.

" embryo $=47.5$

Thus one-half the soluble nitrogen of the malt is furnished by the germ, in spite of the small fraction, about ro per cent, of the total weight of the grain represented by the germ. On the contrary, the greater part of the coagulable nitrogen comes from the endosperm, this form of nitrogen having undergone a less advanced degradation to soluble substances which are 
displaced by dialysis than is the case with those of the germ. From the preceding, it appears that the dissolving of the nitrogenous material in the course of germination increases rapidly with the development of the plumules. If the weight of the latter increases slightly, the quantity of soluble nitrogen will increase a great deal, whence the necessity of stopping germination at the exact moment when it is judged sufficient.

In a general way, the quantity of proteolytic enzyme contained in the barley grain would appear to be in direct proportion to the quantity of nitrogenous materials to be dissolved; in other words, the activity would be proportional to the result to be produced. This, at least, is what appears from the fact that the more nitrogenous the barley the more soluble nitrogen the malt contains, so that for identical experimental conditions a constant portion of the nitrogen of the barley is found again in the malt in a soluble form. It is hard to give an exact proof of this fact, for it is very much influenced by the point to which the malting has been carried. However, the following results have been obtained by Brown with barleys coming from the experimental Station of Rothamsted:

Relation of Soluble Nitrogen Extracted from Malt to Total Nitrogen of Barley.

\begin{tabular}{|c|c|c|c|c|}
\hline \multirow{2}{*}{$\begin{array}{l}\text { Per cent } \\
\text { nitrogen in } \\
\text { barley. }\end{array}$} & \multirow{2}{*}{$\begin{array}{l}\text { Per cent soluble } \\
\text { nitrogen in malt. }\end{array}$} & \multirow{2}{*}{$\begin{array}{l}\text { Per cent nitrogen } \\
\text { in malt. }\end{array}$} & \multicolumn{2}{|c|}{$\begin{array}{l}\text { Soluble nitrogen extract from malt } \\
\text { at } 65^{\circ} \text { expressed in per cent. }\end{array}$} \\
\hline & & & Nitrogen in malt. & Nitrogen in barley. \\
\hline $\begin{array}{l}1.31 \\
1.46 \\
1.37 \\
1.38 \\
1.69\end{array}$ & $\left.\begin{array}{l}0.53 \\
0.57 \\
0.54 \\
0.55 \\
0.69\end{array}\right\} 0.55$ & $\begin{array}{l}1.22 \\
1.22 \\
1.27 \\
1.28 \\
1.64\end{array}$ & $\begin{array}{l}43.8 \\
45 \cdot 5 \\
45 \cdot 2 \\
42.2 \\
42 \cdot 3\end{array}$ & $\left.\begin{array}{l}34.3 \\
35.8 \\
36.4 \\
35.6 \\
35.6\end{array}\right\}$ \\
\hline
\end{tabular}

The first four samples of barley have an average nitrogen percentage of 1.367 . The malt obtained contains 0.55 per cent of soluble nitrogen. The fifth sample has a percentage of 1.69 and its malt contains 0.69 per cent soluble nitrogen. But the ratio of soluble nitrogen of the malt to the total nitrogen of the 
barley is constant -35.5 per cent in the first case, 35.6 per cent in the second.

In practice, it has been found that barleys rich in albuminoids are just as good for the preparation of the wort as poor barleys. However, in the American manufacture, the preference is given to a barley relatively rich in albumin for the production of bottled beer, stable from the points of view of preservation, foam, and full flavor.

Treatment in the Kiln. - It is known that a slow drying of the malt, so that the heat can act before all the moisture has disappeared, results in an increase in the soluble carbohydrates contained in the finished malt of from 25 to 30 per cent, as compared with that found in case of a careful, more rapid expulsion of the water. There is nothing similar in the behavior of the nitrogenous substances. Neither a slow desiccation, nor a final converting of the malt into caramel, changes the quantity of soluble nitrogen which can later be extracted from the malt by brewing.

Brewing. - Beer worts contain a certain quantity of nitrogenous substances which are derived from the malt and remain constantly soluble even after boiling. These substances, in a wort entirely of malt, represent from 5 to 6 per cent of the solid extract whose weight is from Io to $15 \mathrm{~g}$. per roo c.c. of wort. A small fraction of these come direct from the barley where they pre-existed. The greater part has been formed: (I) in the course of the germination, that is to say, in the malting; (2) in the course of the preparation of the wort, that is to say, in the brewing.

As to brewing, the effect of the temperature on the extraction of the soluble nitrogen of the malt is very marked. If the temperature of the mixture rises from $15^{\circ}$ to $100^{\circ}$, the quantity of soluble nitrogen increases slightly during the temperature interval $15^{\circ}$ to $37.8^{\circ}$, then rapidly during the interval $37.8^{\circ}$ to $43.3^{\circ}$, and finally very slowly, reaching a maximum at $49^{\circ}$. At this optimum temperature for the activity of peptonizing enzymes about 40 per cent of the total nitrogen of the malt is dissolved and 
becomes non-coagulable, 23 per cent coming from the soluble nitrogen pre-existing in the malt and $\mathrm{I} 7$ per cent resulting from proteolysis. Between $49^{\circ}$ and $60^{\circ}$ the quantity of soluble nitrogen diminishes somewhat, for the enzymes begin to deteriorate. The diminution continues up to $66^{\circ}$, the temperature adopted in industrial brewing. It is a critical temperature, for if it is slightly exceeded the enzymes act with difficulty, and at $82^{\circ}$ they do not act at all.

Nitrogenous Extract of an English Malt at Different

Temperatures.

(Length of brewing $=3$ hours.) .

\begin{tabular}{|c|c|c|c|}
\hline \multirow{2}{*}{$\begin{array}{c}\text { Temperature of } \\
\text { brewing. }\end{array}$} & \multirow{2}{*}{$\begin{array}{l}\text { Nitrogen coagulable } \\
\text { in ro c.c. of wort. }\end{array}$} & \multicolumn{2}{|c|}{ Nitrogen not coagulable (always soluble). } \\
\hline & & Per 100 c.c. of wort. & $\begin{array}{l}\text { Per Io of total nitro } \\
\text { gen of malt. }\end{array}$ \\
\hline $\begin{array}{l}{ }^{\circ} \mathrm{C} . \\
15.5 \\
37.8 \\
43.3 \\
48.9 \\
60.0 \\
65.5 \\
68.3 \\
82.2 \\
100.0\end{array}$ & $\begin{array}{c}\text { g. } \\
0.0191 \\
0.0200 \\
0.0179 \\
0.0175 \\
0.0084 \\
0.0070 \\
0.0046 \\
0.0074 \\
0.0021\end{array}$ & $\begin{array}{c}\text { g. } \\
0.06487 \\
0.08540 \\
0.10313 \\
0.10710 \\
0.09940 \\
0.09240 \\
0.08312 \\
0.06044 \\
0.06160\end{array}$ & $\begin{array}{c}\text { Per cent. } \\
23.0 \\
37.1 \\
30.5 \\
39.0 \\
37.4 \\
34.8 \\
31.3 \\
22.7 \\
23.0\end{array}$ \\
\hline
\end{tabular}

The duration of the brewing is influential, depending also on the temperature. Thus at $49^{\circ}$ proteolysis is not ended at the end of 3 hours, while after 2 hours at $66^{\circ}$ the quantity of soluble nitrogen does not increase.

A question of the greatest importance in the brewery is that of water. If we examine the influence exerted by the composition of the water used in brewing on the quantity of soluble nitrogen extracted at $65^{\circ}$, we find that distilled water dissolves a much larger quantity of soluble nitrogen than a water containing certain mineral salts, like the calcium and the alkali carbonates. The differences, as Fernbach and Hubert have shown, must be attributed to the transformation of the phosphates of the malt from primary (mono-metallic) into secondary (bi-metallic), under the influence of carbonates, a reaction which causes a partial inhibj- 
tion of the proteolytic enzymes. On the contrary, the addition to distilled water of a small quantity of $\mathrm{CaCl}_{2}$ increases the quantity of soluble nitrogen. This salt, by transforming the secondary phosphates of the malt into primary phosphates, accelerates the activity of the enzymes. The following are figures relative to these observations; soluble nitrogen formed in a brewing where the water used had been previously distilled has been taken as a basis for comparison.

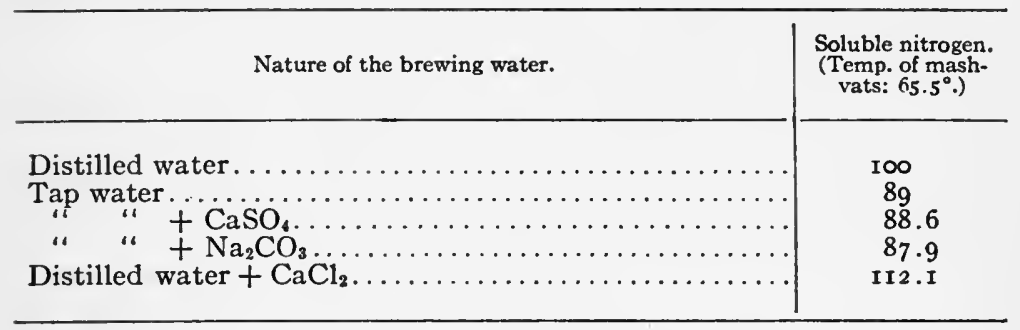

Fermentation. - So far in connection with worts we have considered only the always soluble nitrogen in its entirety, without establishing any difference between the various substances which it represents. All these soluble nitrogenous materials do not behave in the same manner toward yeast, and are not all assimilable, as we shall see. First, however, a few words are necessary concerning the nature of the non-coagulable nitrogenous constituents of malt. Take a malt extract, made cold, then boiled, and treat it with phosphotungstic acid. About one-half of the nitrogen is precipitated, the other half remaining in the filtrate. It is generally believed that the precipitated portion contains albumoses and peptones, plus a certain quantity of organic bases like betain and cholin. To the unprecipitated portion is attributed the amides, amino-acids, and finally a small quantity of $\mathrm{NH}_{3}$. Brown, in studying this method of separation, finds the method to be erroneous. Not all the unprecipitated nitrogen corresponds to amino-acids, but barely $\frac{1}{4}$ of it, or even $\frac{1}{8}$ or 12.5 per cent of the total nitrogen of the original malt extract. Brown even reduces this limit, and 
finally estimates that the actual quantity of nitrogen in the amides and the amino-acids coming from the malt extract is not more than 8.5 per cent, in place of 50 per cent as was formerly believed. This amide nitrogen corresponds to asparagin, leucin, tyrosin, and allantoin, derived from urea, all bodies which have been isolated from wort.

On the other hand, Brown has determined the ammoniacal nitrogen, as well a's the other different forms of nitrogen, and the following is, according to him, the composition of a malt extract made cold, then boiled *:

Per cent of total nitrogen of the wort.

Ammoniacal nitrogen.......................... 3.5

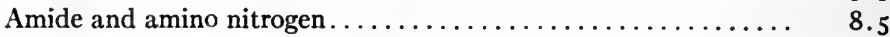

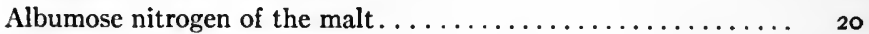

Peptone nitrogen..............................

Nitrogen of organic bases...................... 4

Remaining unexplained nitrogen. ................. 33

Total .................................... $\frac{33}{100}$

The albumoses and the peptones, which represent about half of the soluble nitrogen of the malt extract, will, according to Brown, give the largest part of the nitrogen assimilable by

* In a publication previous to that of Brown, Milar had already determined the composition of a boiled malt extract and had found very similar figures:

Per cent.

Nitrogen precipitated by phosphotungstic acid $\ldots \ldots \ldots \ldots \ldots$ 5I

Not precipitated....................... 49

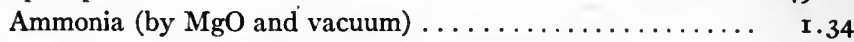

Amides (hydrolysis by $\mathrm{HCl}$ then measured as $\mathrm{NH}_{3}$ ) ..... 3.44

Mono-amine acids (by nitrous acid) ............. 9.15

Unclassified........................... 35.07

The unclassified compounds belong to different groups. They are asparagin, betain, cholin, peptones, and albumoses. These albumoses are precipitable by $\mathrm{ZnSO}_{4}$, but do not give the biuret reaction; the peptones likewise do not give this reaction.

According to Osborne, the hordein of barley is the protein which is transformed chiefly into non-coagulable soluble substances. These nitrogenous substances are evidently in greater quantity in the malt than in the barley, though the relative proportion of each in nitrogen is practically the same. Yet, as for the nitrogen not precipitable by phosphotungstic acid, it contains three times as much ammonia nitrogen as that of the barlev. 
the yeast. In general, these two products resemble, with slight differences, similar ones obtained with enzymes of animal origin. It is impossible to separate these substances which represent the different stages of the proteolytic degradation. But we are given an idea as to their disintegration by the aminoindex, that is to say by the quantity of nitrogen which these substances liberate under the action of nitrous acid, the formation of nitrogen being the greater the more $\mathrm{NH}_{2}$ groupings there are in the molecule. Brown thus distinguishes:

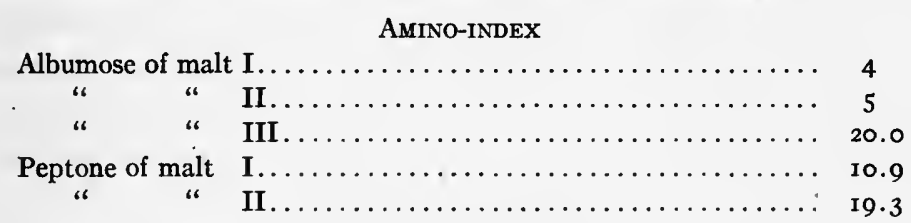

The albumoses I and II, which have here the same aminoindex, have very different properties in other respects, which justify the distinction introduced. Brown, having thus established the approximate composition of a wort, from the standpoint of its nitrogenous constituents, determines the quantity of assimilable nitrogen which it contains by submitting it several times to the action of yeast, under conditions such that the arrest of fermentation shall be due solely to the disappearance of assimilable nitrogen; in other words, he makes sure that the wort contains sufficient sugar, that it is aerated, and that the quantity of alcohol formed cannot be injurious. A determination of the nitrogen before and after then gives the nitrogen taken by the yeast. The quantities of assimilable nitrogen in a malt extract made with cold water, and then boiled are:

Total nitrogen of the original extract. ................ I0

Nitrogen taken by the first 3 fermentations. . 5 I. $0+7.5+$ I. $7=60.2$

By repeating this operation several times, this quantity can be raised to $62-63$ per cent. Of this 62 per cent of assimilable nitrogen, 5I per cent were abstracted at the first fermentation, and are consequently easily assimilable, ro per cent are assimilable with difficulty, and about 40 per cent are not assimilable at 
all. We shall see later the opinion of Brown as to the chemical nature of these three kinds of nitrogens. But first it should be stated that this quantity of assimilable nitrogen varies with the conditions of the treatment of the malt.

If, in place of a malt extract made with cold water, we examine a boiled brewery wort prepared at the usual temperature, (about $65^{\circ}$ ), the quantity of assimilable nitrogen is found to be smaller (54.6 per cent approximately, in place of 60 per cent). The reason for this decrease is that the raising of the temperature at which the brewing is done has not only caused the quantity of non-coagulable soluble nitrogen in the wort to vary, but has also changed this nitrogen by making it more or less assimilable. In other words, the quantity of assimilable nitrogen will pass through a maximum with the temperature.

Influence of Temperature on the Nature of the Nitrogen.

\begin{tabular}{|c|c|c|c|c|}
\hline \multirow{2}{*}{ Nature of the nitrogen. } & \multicolumn{4}{|c|}{ Brewing done at } \\
\hline & $16.4^{\circ}$ & $45^{\circ}$ & $65.5^{\circ}$ & $71^{\circ}$ \\
\hline 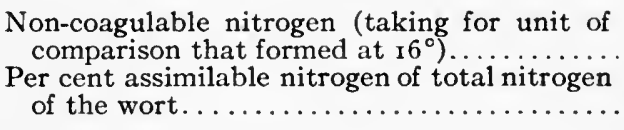 & $\begin{array}{l}100 \\
60.5\end{array}$ & $\mid \begin{array}{l}\text { I5I.8 } \\
6 \mathrm{I} .3\end{array}$ & $\begin{array}{r}159.9 \\
55.4\end{array}$ & $\begin{array}{r}147.6 \\
47.2\end{array}$ \\
\hline
\end{tabular}

These differences arise from the fact that during brewing there is produced a digestion which can increase the proportion of soluble substances, but which does not increase to the same degree the quantity of assimilable substances. In other words, the elevation of the temperature by weakening the enzymes has preserved their dissolving power, but has perceptibly diminished their peptonizing action. It results from this that we can increase the assimilability of hot-brewed worts by adding to them a proteolytic enzyme which will bring about peptonization, for example the active substance contained in the young malt stalks. The increase of assimilable nitrogen realized thus is from Io to I2 per cent. Naturally, a similar 
addition made in worts obtained at low temperature will cause no change. These results explain the rôle of the so-called yeast nutrients, such as malt flour, which are sometimes added in the vats at the moment of fermentation. These products would act only indirectly by peptonizing a larger quantity of the nitrogenous substances necessary to the development of the yeast.

To summarize, the assimilable nitrogen contained in the wort is the sum of that which existed previously in the malt and that which is formed in the course of brewing. That which was already in the malt comes especially from the germs, but it cannot be determined exactly, since by an extraction with even cold water a hydrolysis is produced which changes the character of the nitrogen. However, we find that by an exhaustion of the germs with water at $65^{\circ}$, these parts of the grain give as much as 30 per cent of their total nitrogen in the assimilable form, or about 65 per cent of the soluble nitrogen. In other words, of the assimilable nitrogen extracted from the malt, 51.6 per cent, or more than half, comes from the germs.

Distribution of the Different Nitrogens in Malt.

Determinations made by infusion at $65 \cdot 5^{\circ}$.

\begin{tabular}{|c|c|c|}
\hline Different nitrogens of the malt. & $\begin{array}{l}\text { Nitrogen } \\
\text { coming from } \\
\text { the germs. }\end{array}$ & $\begin{array}{l}\text { Nitrogen } \\
\text { coming from } \\
\text { the endosperm. }\end{array}$ \\
\hline $\begin{array}{l}\text { Total nitrogen. } \\
\text { Nitrogen always soluble }(40 \text { per cent total } \mathrm{N}) \\
\text { Or in percentage } \ldots \ldots \ldots \ldots \ldots \ldots \\
\text { Assimilable nitrogen }(24 \text { per cent of total } \mathrm{N}) \ldots \\
\text { Or in percentage } \ldots \ldots \ldots \ldots \ldots \ldots \ldots \ldots\end{array}$ & $\begin{array}{c}\text { Per cent. } \\
4 \text { I . } 3 \\
\text { I } 9.0 \\
47.5 \\
\text { I } 2.4 \\
5 \text { I .6 }\end{array}$ & $\begin{array}{c}\text { Per cent. } \\
58.7 \\
21.0 \\
52.5 \\
\text { II } .6 \\
48.4\end{array}$ \\
\hline
\end{tabular}

The quantity of assimilable nitrogen contained in the malt is evidently influenced by the duration of the germination. It appears to be modified also by the temperature of the treatment in the kiln, an elevation of temperature having for result the weakening of the activity of the enzymes, and consequently the diminishing of the proteolysis which will take place during the brewing. In spite of all these causes of variations, we 
can say, however, that, in a general way, the relation of assimilable nitrogen to the soluble nitrogen in a wort is practically constant when the extraction is always made under the same conditions. What is the nature of this assimilable nitrogen? Taking as a basis the similarity of the figures representing, on the one hand, the composition of a malt extract (table, p. 594), and, on the other hand, the various portions of nitrogen taken or left by the yeast, Brown thinks that the $5^{I}$ per cent of easily assimilable nitrogen corresponds to the $5^{\mathrm{I}}$ per cent of albumoses and of peptones; that the ro per cent of nitrogen assimilable with difficulty represents the amides, the amino-acids, the organic bases, and the ammonia; and finally, that the non-assimilable nitrogen is the nitrogen not explained in the preceding table. This is only one opinion, which is moreover in contradiction with the fact noted by Brown himself that the quantity of assimilable nitrogen increases the further proteolysis is carried in a wort. Neither is it confirmed by the results obtained by precipitating a wort by tannic acid, a reagent which is known to give insoluble combinations with albumoses and certain complex polypeptids. A boiled wort, not impregnated with hops, is treated with tannic acid in such a way as to obtain the maximum precipitation. Then the following determinations were made: (I) in the original wort, the nitrogen easily assimilable (taken by the first fermentation), the nitrogen less assimilable (taken by the second fermentation), finally the non-assimilable nitrogen; (2) in the filtered liquid after the precipitation, the residual nitrogen and the assimilable nitrogen.

Relation Between the Assimilable Nitrogen in a Wort and the Nitró́en Precipitable by Tannin.

Nitrogen precipitated by tannic acid $=25.6$ per cent of total nitrogen.

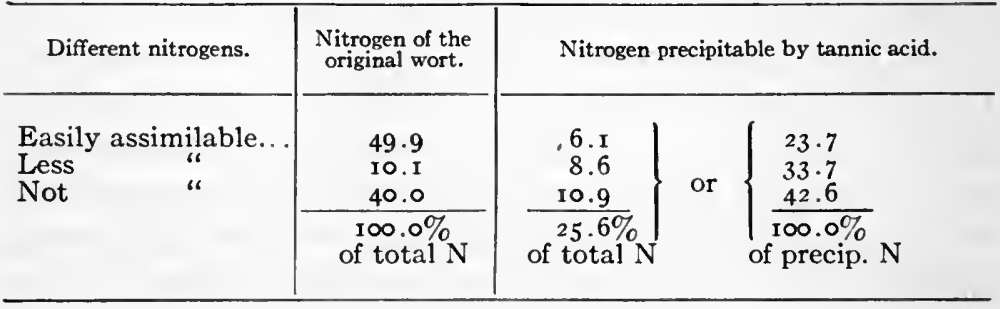


This table shows that about one-fourth of the total soluble nitrogen of a wort is precipitated by tannic acid when this reagent is added in slight excess. Now, 23.7 per cent belongs to the easily assimilable nitrogenous bodies, 33.7 per cent to the less assimilable bodies, and 42.6 per cent to the non-assimilable bodies. We see that the nitrogen of the albumoses, nitrogen which is precipitable by tannin, is far from corresponding to the total assimilable nitrogen. What is then the cause of this disagreement? In all this study, there is a grave criticism to make, namely that Brown takes no account of the enzymes of the yeast itself. However, these certainly act, and so that we should regard the assimilable nitrogen of a wort as resulting from a double proteolysis, one due to the malt enzymes and the other to the yeast enzymes. It would be interesting to separate these two actions. We should very probably throw some light on the chemical nature of the nitrogenous bodies which are really absorbed by the yeast.

In fact, if we find in a wort at the beginning a certain proportion of amino-acids $(A)$, and after fermentation another proportion $\left(A^{\prime}\right)$, it does not follow that the difference $A-A^{\prime}$ represents exactly the quantity assimilated by the yeast. This may well have been produced at the expense of more complex nitrogenous substances, so that the quantity really absorbed will be in fact much larger. On the contrary, as the albumoses diminish, it does not necessarily result that these have been assimilated, as they may have been only hydrolyzed. One of the natural functions of yeast is to secrete proteolytic enzymes. Feeding on a mixture of albumose and amino-acids, it transforms the first and absorbs the second. From the disappearance of the albumoses, we cannot deduce their assimilation.

Finally, the opinion of Brown that the most assimilable nitrogen corresponds to the albumoses and to the peptones, does not appear well founded. It is, moreover, in contradiction with what we know in a general way of the alimentation of yeast. This cell, as likewise do many others, prefers among all the nitrogenous substances non-biuretic products to biuretic 
products, and peptones to albumoses. The more the degradation is advanced - up to a certain limit - the more easily is the product assimilable. The experiments of Effront on compressed yeast, which we shall report more fully in a subsequent. chapter, are very conclusive on this point. As to the actual determination of assimilable nitrogen, we can also make some criticisms of the procedure. In particular, it appears incorrect to conclude from the fact that the yeast does not absorb nitrogenous material in a culture medium which has undergone four or five successive fermentations, that the nitrogenous substances remaining are not really assimilable. The yeast may very well have secreted poisons which hinder its later development. To be sure, Brown answers this objection by saying that there has been no auto-intoxication, but only a want of assimilable substances, since by introducing asparagin into the liquid, the fermentation again becomes possible. But this argument has only a relative value. We know, in fact, that in a medium with the addition of antiseptic (Ex: sodium fluoride), yeast has difficulties from the beginning, and that an addition of nitrogenous material corrects the paralyzing action of this salt.

Let us now see how yeast acts in an industrial way in a brewery wort. Here the conditions differ from those of the laboratory, in particular, aeration is insufficient. It results from this that the yeast does not absorb all the assimilable nitrogen contained in the wort, but only from 20 to 36 per cent:

Loss of Nitrogen Easily Assimilable in Industrially Fermented WORTS.

\begin{tabular}{c|c|c|c|c}
\hline $\begin{array}{c}\text { Nitrogen in the } \\
\text { wort, } \\
\text { g. per 100 c.c }\end{array}$ & $\begin{array}{c}\text { Nitrogen in the } \\
\text { beer drawn off. } \\
\text { g. per 100 c.c. }\end{array}$ & $\begin{array}{c}\text { Nitrogen as- } \\
\text { similated by } \\
\text { yeast, } \\
\text { g. per 100 c.c. }\end{array}$ & $\begin{array}{c}\text { Assimilated nitrogen } \\
\text { expressed in per cent } \\
\text { of total nitrogen of the } \\
\text { wort. }\end{array}$ & $\begin{array}{c}\text { Nitrogen as- } \\
\text { similable left } \\
\text { in the beer. } \\
\text { gr. per 100 c.c. }\end{array}$ \\
\hline (I) 0.10472 & 0.08216 & 0.02256 & & \\
(2) 0.09086 & 0.06620 & 0.02466 & 21.6 & 0.0213 \\
(3) 0.08750 & 0.05600 & 0.03150 & 27.6 & 0.0140 \\
(4) 0.07000 & 0.05600 & 0.01400 & 36.0 & 0.0052 \\
(5) 0.10500 & 0.08400 & 0.02100 & 20.0 & 0.0154 \\
& & 20.0 & 0.0231 \\
\hline
\end{tabular}


If we assume, as average value, that the easily assimilable nitrogen is 42 per cent of the nitrogen of the original wort, we see that there remains 22 to 6 per cent of nitrogen, or about $0.0052 \mathrm{~g}$. to $0.023 \mathrm{~g}$. of easily assimilable nitrogen per roo c.c. of beer manufactured. It is to be noted that if the wort originally contains much assimilable nitrogenous material, the yeast always takes only the same portion, under industrial conditions only, for in the laboratory we easily succeed in making it absorb the whole.

The different results which we have just set forth, and which are due to $\mathrm{H}$. Brown, relate only to yeast of top fermentation. Petit, by an analogous method, has determined in a wort the quantities of assimilable protein and assimilated protein (which disappeared in the main fermentation) per roo parts of extract in the course of a bottom fermentation. Some of the figures found are:

\begin{tabular}{|c|c|c|}
\hline $\begin{array}{c}\text { Different } \\
\text { worts. }\end{array}$ & $\begin{array}{c}\text { Assimilable pro- } \\
\text { tein per cent of } \\
\text { extract. }\end{array}$ & $\begin{array}{c}\text { Assimilated pro- } \\
\text { tein per cent of } \\
\text { extract. }\end{array}$ \\
\hline & I.50 & I.04 \\
I & $\mathbf{I} .59$ & I.IO \\
3 & I.77 & I.22 \\
\hline
\end{tabular}

Although expressed in a different manner, we see that these results are entirely comparable with those given by Brown. Petit has also investigated the influence which different industrial methods of brewing can have in practice on the quantities of assimilable and assimilated nitrogen. He has submitted malt to the following operations at $60^{\circ}-65^{\circ}:$ (I) to brewing with two steepings, (2) to brewing with one steeping, (3) to a working up cold, then to an infusion in the mash-tub. He has found that the quantities of assimilable nitrogen are not influenced by the method of brewing when the malt used is entirely dissolved and plainly forced. On the contrary, with a barley which is very little germinated, and showing a coarse and imperfect dissolution, it is the brewing by working up cold and then infusion with progressive reheating which gives the most soluble 
nitrogen and consequently a better penetration of the water, though the quantity of assimilable nitrogen remains the same. The method of malting can give very different quantities of assimilable nitrogen in the worts and the beers according to the manner in which it is conducted. In particular, a barley steeped with water containing potassium nitrate produces a larger quantity of assimilable nitrogen, but the quantity of nitrogen really assimilated by the yeast is not increased.

Hopping. - (Impregnation with Hops.) - Since hops contain a certain quantity of tannin, it was interesting to investigate the effect which they can exert on the nitrogen of the worts. When the wort is boiled with hops added in the usual proportions, the precipitation of soluble nitrogen which results is small, but is yet very appreciable, amounting to about 4 to 5 per cent of the noncoagulable nitrogen of the wort. On the other hand, the hops themselves give nitrogen to the wort and in an amount almost equal to that which has been eliminated. The final' result is, then, that the wort, impregnated with hops or not, contains the same quantity of soluble nitrogen. However, the nitrogen of the wort precipitable by the hops belongs to the group of non-assimilable nitrogen; on the contrary, 60 per cent of the nitrogen furnished directly by the hops is assimilable. It appears that in the last analysis, the boiling with hops serves to increase slightly the assimilable nitrogen contained in the wort, an increase which is, under normal conditions, about 2.5 per cent of the total nitrogen of the wort.

Stability. - Let us first of all indicate the nitrogen content of a finished beer. The proportion of total nitrogenous materials varies between $\mathrm{I}$ g. per liter for pale beers which are very light, to $7.5 \mathrm{~g}$. for some Belgian or English beers. The nitrogen content is thus about 0.16 to $1.2 \mathrm{~g}$. per liter, or on the average, 0.7 per cent. We also find nitrogen in the liquid in all its forms, from albumin up to amino-acids and organic bases. Thus, according to Miskowsky, a Pilsener beer containing $36.96 \mathrm{~g}$. of extract per liter and $0.308 \mathrm{~g}$. of nitrogen, would give on analysis: 


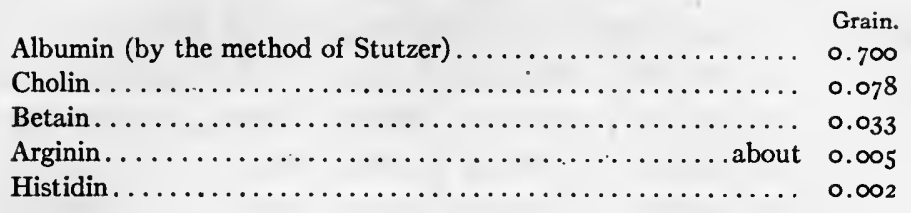

But to come to the conditions for stability, we have just seen that in practice the yeast never absorbs all the assimilable nitrogen. A greater or less quantity remains, and this residual assimilable nitrogen plays a very important rôle in the future history of the beer. It permits the multiplication of some yeast cells which have been carried down at the moment of drawing off, and which afterward will be deposited in the casks, or again it. permits the development of abnormal yeasts or of various bacteria which are the frequent causes of the deterioration of the product. These dangers, moreover, represent only one of the factors of this complicated problem of the stability of beer. The absorption of oxygen in the course of the transfusions, the greater or less quantity of carbonic acid, the content in carbohydrates, the alcoholic richness of the beer, the possible contaminations, are also factors which intervene to a greater or less marked degree.

The question as to the quantity and quality of soluble nitrogen which mur ${ }^{\wedge}$ remain in the beer has been very much discussed of late. Certainly, from the point of view of the nutritive value of this beverage, the residual nitrogen has its advantages, but it may happen in the actual manufacture that in consequence of ill-defined transformations a part of this is precipitated and on keeping, gives to the stored beer a disagreeable taste. And first of all, there is a point to which Petit (of Nancy) has called attention. Without doubt, a part of the nitrogen peptonized by the enzymes of the malt and of the yeast is not absorbed in the course of the fermentation and forms residual nitrogen which we find again in the finished beers. In addition, there is another portion which comes from the content of the cells and which, after having undergone transformation within them, is diffused through the liquid in the form of amino-acids, bases, etc. Finally 
there is also assimilable nitrogen, created in the beer itself after delivery, by the cells which are still to be found there. These two causes of production depend greatly on conditions of temperature to which the beer is submitted.

As to the nitrogen excreted by the yeast, this action will be very feeble, or nil, in very cold low fermentation cellars. On the contrary, if the beer is exposed, even in the brewery, to a high temperature, the quantity of nitrogen diffused in the liquid will increase, first because the yeast present has multiplied, and secondly because the velocity of diffusion has become greater. Furthermore, for the same number of cells, we find that the diffusion takes place more rapidly when these remain in suspension in the liquid, a condition which is frequently fulfilled. As for the production of assimilable nitrogen at the expense of the albuminoids remaining in the delivered beer, this action presupposes the presence of numerous and energetic yeast cells. We may therefore say that this production will be greater in top fermentation than in bottom fermentation. In the latter method of manufacture, a beer having been kept for a long time in a very cold cellar and well clarified in the cask, will give only very few cells, which in addition will be weakened by a long inanition. On the contrary, a young beer, which has a vigorous secondary fermentation or which presents a still imperfect clarification, will contain numerous and active yeasts, capable of attacking the albuminoids and of thus producing a certain quantity of assimilable nitrogen. These considerations show that there is a very evident difference, from the point of view of the content of beers in degraded nitrogen, between the top and the bottom fermentation, a difference which can explain the more or less easy alterability in products manufactured by either of these two processes.

What is then the rôle of residual nitrogen in the preservation of beer? It has been previously stated that the assimilable nitrogen, that is to say, that nitrogen which corresponds to a very degraded form, could favor the subsequent development of yeast or of bacteria and thus contribute to the deterioration 
of the product. .Furthermore, the nitrogen in the combined state can also be the cause of a turbidity of the liquid, in consequence of a modification which it assumes under the influence of special physical or chemical conditions, particularly that of cold. We know, in fact, that at the end of the main fermentation, and during storage, some of the remaining albuminoids are mechanically eliminated by clarification, or later are deposited as a result of the low temperature to which the beer is submitted. Different operations which the beer is made to undergo, such as saturation with carbonic acid or "krausening" can also change the quantity of precipitated nitrogen, and influence in a beneficial manner the qualities of the product. Now, according to Rohde, the turbidity which is sometimes produced after long keeping is.due to a simple precipitation of the nitrogenous material. This point will be discussed later.

However, important progress from the point of view of the stability of beer has been recorded in recent years, due to the introduction into the brewery of the Wallerstein (of New York) process. This process consists in adding to beers in the cellars a small quantity of proteolytic enzymès, such as pepsin, papain, or bromelin, with the object of preventing this liquid from later becoming turbid. This means of stabilization is at the present time much used in the United States, and during the past years has been applied to the treatment of the greater part of the beer made in that country. The keeping quality of bottled beer is, in fact, of the greatest importance in America, where it is the custom to drink this liquid very cold and very clear. These beers must be capable of being kept for some time on ice without showing turbidity or the least deposit. Now pasteurization does not give all the results desired. Further, this treatment gives a special taste which is objectionable. The results obtained by "wallerization" are, on the contrary, very satisfactory. The quantity of enzyme used in this process is from I to $2 \mathrm{~g}$. per hectoliter. These quantities cannot be exceeded in practice for this would lead to diametrically opposite results. On the contrary, it is 
curious to note that the enzyme, although introduced in very small quantity, nevertheless keeps for several months, and that it can also be revealed by analysis at the end of this time.

In the course of the year I9I3, the writer received from a brewery of New York two lots of beer of the same brew, one having undergone "wallerization," the other not having been treated. The analysis of these two lots, which were allowed to stand at the temperature of the laboratory, was made four months after the date of shipping. All the bottles treated had remained clear. On the other hand, the controls all contained a considerable deposit, and the liquid became turbid upon shaking and did not become clear again until after a prolonged standing. Further, the beers which had been treated were of a fresh, perfect taste; the others, on the contrary, had a flavor denoting a marked deterioration. The nitrogen contents and the contents in extract and in maltose of the two beers were the same. However, a difference could be found in the distribution of the nitrogen in the different samples:

Analysis of a New York BeER.

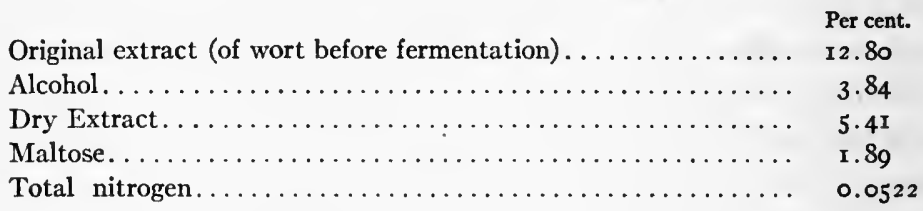

Distribution of Nitrogen in the Treated and the Non-Treated BEER.

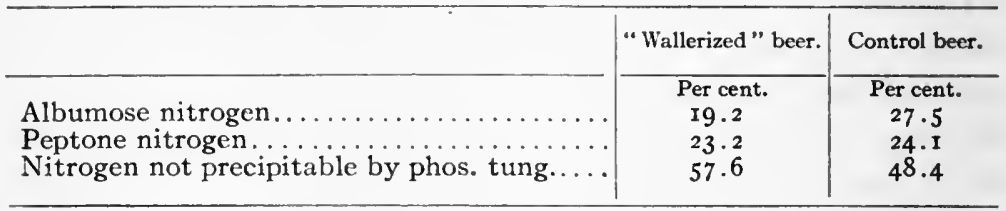

The content in formaldehyde nitrogen was the same in the two beers; namely, I 5.06 per cent. Furthermore, the presence of proteolytic enzymes was determined with edestin by the Fuld method, modified by Wallerstein (see p. 255). The treated 
beer gave a plainly positive reaction, while the other was perfectly inactive.

The addition of proteolytic enzymes to the beer gives an unquestioned result from the point of view of stability, but the mechanism of the action is yet to be elucidated. Beers, treated or not treated, have after filtration practically the same nitrogen content; moreover, the deposits contain only an insignificant trace of nitrogen. We may then say that the albuminoid substance does not intervene directly in the formation of turbidity, as is often believed. It is probable that the peptonization produced by the enzyme added serves to keep in equilibrium certain organic and inorganic substances, which under ordinary conditions are precipitated.

\section{II.}

As we have just seen, biochemical catalysts play a very important rôle in brewing. To the enzymes of the malt, which comprise various enzymes for the carbohydrates as well as for the albuminoids, are added the active substances of the yeast, which contain, in addition to zymase, very energetic proteolytic enzymes. The art of brewing consists merely in utilizing in a judicious manner all the catalysts which come into play in the manufacture of beer, accelerating or inhibiting at the right moment the activity of this or that enzyme. Brewing has been raised to the rank of an applied science, and a veritable army of investigators have devoted their entire activity to it. Recent data on albuminoid materials, as well as on the carbohydrates, researches in bacteriology and the study of enzymes, have contributed much to the development of this industry, bringing practical improvements both in economical manufacture and in preservation.

Although the advances realized are considerable, they are far from having exhausted the field of investigation. This field is, on the contrary, unlimited and new horizons readily open to the gaze of the investigator. At present, we utilize as 
rationally as possible the catalysts present in the primary material, and there remains little to be done in this direction. But the time has come to ask if the factors brought into play in this procedure are really the only ones which can satisfy all the desiderata and if there is not here an opportunity to utilize other agents.

Beer must be considered primarily as a liquid food. In addition to taste and appearance, unquestionably very important factors, it must not only answer an immediate need of drinking, but must also bring to the organism the maximum nutrition. We can even say that the true excuse for its existence lies in the nourishment offered. On the other hand, beer should not be an expensive food, but a cheap one.

The manufacture of beer as now practiced is, in fact, much too costly. Malting is an operation which involves considerable losses in nutritive materials. The catalysts which are developed during this part of the manufacture, although very expensive, are far from performing all the necessary work. The amylase of the malt has two functions: the one, liquefaction; the other, saccharification. Now, these two effects are exerted at different temperatures, and the saccharification cannot be conducted in the desired way without neglecting the liquefaction. Consequently, undissolved starch always remains in the husks. On the other hand, the proteolytic enzymes are abundant but of a quality not suited to the work desired. They can induce an advanced hydrolysis, but exert only a poor dissolving action.

As a result, during the malting a considerable proportion of the nutritive substance is lost. Then, we leave in the husks as much as 3 per cent of carbohydrates, and more than 50 per cent of the nitrogen contained in the grains. Furthermore, that portion of nitrogen which it has been possible to dissolve is not kept wholly in the wort. We are relentless, with all the arms forged by science, in reducing it to the strict minimum, so as to assure the preservation of the final product. Of the Io to $I_{3} \mathrm{~kg}$. of proteins contained in $100 \mathrm{~kg}$. of grain, there passes into the finished beer from 1.5 to $3.5 \mathrm{~kg}$., or 2 to $5 \mathrm{~g}$. 
of various nitrogenous substances per liter of this liquid. This nitrogen content is insufficient to be included in a consideration of nutrition.

When we study brewing a little more closely, we find an exaggerated respect on the part of the specialists in this branch for all the so-called natural products and practices which must be used in the course of this industry. The malt, the hops, and the yeast are the three fundamental constituents that characterize beer. Without these, no beer is possible. The defenders of this opinion forget that beer is not a natural beverage, like wine, but that it forms, on the contrary, an artificial product created by man, and that nothing requires us to keep to the same formula.

The procedure long ago instituted for the manufacture of beer unquestionably represents an admirable discovery, full of ingenuity. The early brewers, artisans rather than scientists, by purely empirical means arrived at a procedure sufficiently satisfactory. Since then science has entered the field. The greatest benefit derived from science has been the explanation of the results acquired through experimentation. Without doubt, it has also rendered the manufacture much easier and more stable by establishing methods for control and for analysis. Finally, science has contributed in large measure toward assuring the preservation of the products. But it must be granted that the advances realized are unquestionably out of proportion to the labor involved.

The brewing academies of Germany, Austria, America, etc., bear the stamp of institutions created for the maintenance of the brewing traditions - traditions whose exclusive basis is the holy trinity, Hops, Malt, Yeast. The conservative spirit of the present science of brewing condemns it to sterility. If, in fact, we assume that only the three elements cited above can come into play in the manufacture of beer, the door of all investigation is forever closed. The various combinations which it is possible to obtain by varying the conditions of medium, temperature, concentration, etc., have been or will be tried, but 
in any event we can say that experiments of this character will never lead to anything very essential. The losses in the course of germination, the bad liquefactions, the destruction of the important albuminoid material, cannot be avoided in this process.

To arrive at new results, we must enlarge the scope of investigation and have recourse to new conceptions. Is the malt, which is the basis of all the precedures, really indispensable in the preparation of the wort? From the theoretical point of view nothing prevents the liquefying, saccharifying, or the peptonizing of the starch materials or the albuminoids of raw grains with special enzymes obtained from materials less costly than barley. For example, by first submitting raw barley to an enzyme liquefying starch we should no longer afterward require a saccharifying enzyme to bring the wort to the desired sugar content. The saccharified wort can also be peptonized by suitably chosen enzymes. We should in this way, by the use of catalysts obtained outside of the malt, arrive at a complete solution of the starch and the nitrogen contained in the grain, and should advance the hydrolysis of these products to points that would be better suited to the requirements of the succeeding operations. Thus peptonization could be conducted in such a manner that the nitrogenous material in solution would not later coagulate, either upon boiling or upon fermentation. Wort, prepared under these conditions with the addition of hops, might contain from 15 to $20 \mathrm{~g}$. of albuminoids per liter, in place of the 2 to $3 \mathrm{~g}$. which it contains today.

It is evident that the system recommended above has not already been adopted. We do not even pretend that, at the first attempt, we are going to make a beer of a given type by suppressing the malt, but we do think that the procedure just indicated is that which must be adopted if we wish to prepare beers which, while being more economical than those now made, will nevertheless possess a much higher nutritive value. It is certain that by seeking in this direction, we shall easily succeed, without offending either the taste or the customs of the public, 
in furnishing beers which will possess these new qualities and, nevertheless, will remain very similar to the existing brands.

To summarize, we must hereafter demand as a desideratum the production of a nutritive and economical beer; and to realize this end, we shall recommend the use of enzymes, taken not from the malt, but cultivated on much cheaper materials than barley.

\section{BIBLIOGRAPHY.}

H. Brown. La question de l'azote dans la brasserie, Journ. of the Institute of Brewing, 1909, (13), p. 394. Daprès Monit. Scientif., I9 10, pp. 5, 88, 2 I 7, 645 . Perrt. Bull. de l'Assoc. des Chimistes de sucrerie, r9o9-1910, p. 485.

RoHDE. La Bière, juillet I9ro, p. 83 .

Miskowsky. Composés azotés de la bière, Zeits. f. d. ges. Brauw., 1906, p. 309.

MIIAR. Recherches sur les substances azotées solubles du malt, Trans. of the guinness Research, r9o6, p. 166.

Fernbach et Hubert. 'Diast. protéol. du malt, C.R., rgoo, (r), p. I783; (2), p. 293.

Windisch u. Schellhorn. Enzym in Gerste, Woch. f. Brauerei, r900, (17), p. 29. Wallerstein. Brevet: U. S. Pat.: 995,820 


\section{§ 5. Role of Proteolytic Enzymes in Grain Distillation.}

Proteolytic enzymes intervene in an efficient manner in the manufacture of alcohol from grain, since it is they that render assimilable by the yeast a part of the proteins contained in the raw product. The principal difficulty with this industry being to arrive at a complete liquefaction of the starch, it is customary to add to the grains treated at the moment of preparation of the mash, quite a large quantity of malt, about 8 to to per cent, in order to assure a complete exhaustion of the starchy material. In addition, this malt gives assimilable nitrogen necessary to the yeast, for it brings with it not only products already degraded, formed in the course of the germination, but also tryptases which exert their dissolving action on the albuminoids contained in the ungerminated grain. Now, if we consider exclusively the nutrition of the yeast, it is observed that the proportion of the malt used in distilleries could be considerably reduced: I to 2 kilos of this substance contain, in fact, enough nutritive nitrogenous material to ensure the complete fermentation of too kilos of ungerminated grain. The use of large quantities of malt is not justified, as we have seen above, except through the necessity of introducing into the brewing vat a sufficient quantity of liquefying and saccharifying catalysts indispensable for the complete transformation of the starch. But this procedure presents great inconveniences. First of all, malting causes a considerable destruction of the fermentable substances, and this disappearance correspondingly diminishes the yield in alcohol. Furthermore, it is quite expensive. Finally, the addition of large quantities of proteolytic enzymes to the nitrogenous material of the grain brings about a too extensive dissolving of the latter, thus causing a considerable loss in nitrogen.

In broad outlines, distilling, as ordinarily carried out, involves the following operations: The grains are first of all cooked in water under a pressure of four atmospheres for two or three hours; then the temperature is lowered to $60^{\circ}$ and a malt infu- 
sion is added to the mass. For an hour the mixture is kept at this temperature. Saccharification ended, the mash is cooled to $20^{\circ}-25^{\circ}$, yeast is added, and the whole is allowed to ferment. This is followed by a distillation. In rural plants, the waste ("slop") coming from the stills serves as cattle food. In industrial distilleries, they are passed to the filter press, the cakes obtained are dried, and are then sold in the form of dry husks. The waste liquors which flow from the filter are sent to the river. As industrial distilleries develop, the quantity of waste liquors thus thrown away is very large. These residual liquids, as a result of the improvements introduced constantly in the work of recovery, contain but little fermentable material, 8 to ro kilos per cubic meter. On the contrary, their nitrogen content is from 800 to $1200 \mathrm{~g}$. per cubic meter. A grain distillery which daily produces 300 to 400 hecto liters of alcohol therefore loses in this way about $35^{\circ}$ to $45^{\circ} \mathrm{kg}$. of protein nitrogen, valued at between 450 and 700 francs (\$90-\$140). The loss in nitrogen, which represents up to 33 per cent of the total nitrogen of the grains, comes from the solution of the albuminoids, caused in part by the cooking under pressure, but more especially by the action of the proteolytic enzymes of the malt.

By the "amylo" process it was hoped to avoid this loss in nitrogen though the results have not proved very satisfactory. This procedure, much developed of late, was conceived in 1898 by Calmette, but has been developed especially by Boidin. It consists essentially in saccharifying the grains by special molds. In the beginning, Amylomyces Rouxii was used; later the Mucors $\alpha$ and $\beta$ were used; now only Rhizopus delemar is employed. Thanks to the efforts of Boidin, who for several years studied in detail all the phases of the process, the great difficulties which the writer mentioned in I899 in his book "The Enzymes and Their Applications" have finally been overcome. Now malt is no longer used in the amylo process. Liquefaction and saccharification are produced wholly by Mucedineae, a process giving perfect results and an excellent yield. However, as in the case with malt, there is here again the same fault 
from the point of view of the utilization of the nitrogen of the raw products.

Rhizopus delemar acts on the grain as do the proteolytic enzymes of the malt. Mash, fermented by the amylo process, gives from 0.8 to I.I g. of soluble nitrogen, per liter, for an alcohol content of 7-8 per cent. This nitrogen is completely lost when the husks are filtered. The catalysts which intervene in the work of Rhizopus are of the same nature as those which are formed in the germination of grain. In the work with amylo, as in that with malt, we have amylo-liquefying, saccharifying, and proteolytic enzymes. From the quantitative point of view, the proteolytic activity is the same in the two processes. In the malt the amylo-liquefying enzymes, in the mold the saccharifying enzymes, predominate. The weak side of the two processes is the loss which the nitrogen of the grain undergoes, a loss which amounts to one-third of the total. In addition, both involve the necessity of cooking the grain under high pressure since the enzymes of the malt, like those of the amylo, exert their action incompletely on unconverted starch. Now this cooking implies serious inconveniences. In addition to the cost of fuel, cooking turns to caramel the sugar pre-existing in the grains and makes it unfermentable. It also partially saponifies the fatty substances, setting free a rich nauseous oil which much depreciates the products.

All these reasons have led Boidin and Effront to seek another method of working with grain. Their new process, which applies as much to amylo as to malt, is based on the use of bacterial enzymes which are amylo-liquefying. The secretion of the active substances by the micro-organisms is a function of the medium in which they live. By gradually accustoming the bacteria to the chemical and physical conditions determined, we get them to produce in large quantities the enzymes, which they elaborate only very feebly under ordinary conditions. Thus it is that certain bacteria become capable, by being placed in a suitable medium, of secreting in 24 hours 60 times more enzyme capable of liquefying starch, than that which would be furnished 
in the same time by cells of the same species cultivated in the usual nutritive broth.

The amylo-liquefying enzyme, which appears to be the most advantageous, is obtained by the culture of certain species of $B$. mesentericus in an alkaline medium, and in the presence of a strong aeration. The resulting liquid keeps for a long time and is hard to infect. This solution of enzyme has a value of $I$ to I000, that is to say, one part of liquid will liquefy rooo parts of grain. By concentrating the culture broth in a vacuum or by precipitating the active substance, we can prepare products of a strength even 6 times greater.

In the new process, the procedure is as follows: The grain, previously soaked in alkaline baths, is coarsely ground. The ground mass has added to it 2 volumes of water, and is brought to $75^{\circ}-80^{\circ}$. At this temperature, the amylo-liquefying enzyme, prepared in the factory by means of acclimated ferments, is added. The enzyme is left to act for a half-hour to an hour, and then the mixture, which has become entirely liquid, is sent to the cooker where it remains some time at a temperature of I $10^{\circ}-120^{\circ}$. In the work with amylo, the mash coming from the cooker is sent directly to the fermentation vat, where it is cooled under conditions of perfect sterility, and then is sown first with Rhizopus delemar, which saccharifies the liquid, and then with yeast, which causes it to ferment. The mold, on the other hand, dissolves and peptonizes a certain quantity of nitrogenous materials, which, from the fact that the mash has not been brought to a very high temperature, remains very small although in sufficient quantities for the nourishment of the yeast. In the work with malt, the liquid obtained above is cooled in open vats to $60^{\circ}, \mathrm{r}$ to 2 per cent of malt is added, and the whole is allowed to saccharify for an hour. Upon cooling, yeast, acclimated to hydrofluoric acid, is added to the mash thus prepared. Due to the previous liquefaction of the starch, we can from the beginning produce a complete fermentation with only $\mathrm{r} .5$ to 2 per cent of malt. The advantages of the suppression of cooking are shown in the first place by an increase of the yield in alcohol, 
an increase estimated at from $\mathrm{I} .5$ to 2 liters of alcohol per $100 \mathrm{~kg}$. of grain. In the second place, there is a poverty of the mash in extractive substances, since the density of the fermented liquid is from $-\mathbf{I} .0$ to $-\mathbf{I} .5^{\circ}$, Balling. Finally, the most important result is that the mash, which ordinarily gives at the same concentration I.2 g. of nitrogen per liter, now gives only 0.1 to $0.3^{*} \mathrm{~g}$.

To summarize, the manufacture of alcohol from grain, both in the old way with malt, as well as in the newer one with amylo, involves large losses in nitrogen due to a too extensive dissolving of the proteins contained in the saccharified grain. The originality of the last process consists in bringing about the liquefaction of the starch by an enzyme, which brings its action to bear only on the starch, leaving the whole of the albuminoids in the husks, free to provide the nitrogenous requirement of the yeast either by a small and strictly necessary addition of malt, or by a very careful action of Rhizopus on the grain already deprived of its starch, one or the other of these two operations being furthermore justified by the necessity of producing a total saccharification of the mash.

\section{Manufacture of Compressed Yeast.}

In the manufacture of compressed yeast, the proteolytic enzymes play a much more important rôle than in the distillation of alcohol from grain. The quantity of yeast harvested, and its quality from the point of view of breadmaking, depends on the proportion and the nature of the nitrogen contained in the wort. In the process of manufacture of compressed yeast with clear wort, allowing a strong aeration, we get returns exceeding sometimes 30 per cent of the weight of the grain used. Fresh yeast contains about 2 per cent of nitrogen and so is often richer in proteins than the raw materials used. The albuminoid substances of the raw grain form a mediocre nourishment for the yeast. Their preliminary transformation is therefore

\footnotetext{
* For more ample details consult the provisional French patent No. 49,520.
} 
indispensable. It is not sufficient merely to dissolve the nitrogenous material and make it non-coagulable, but it must also undergo quite a deep-seated change, with the formation of aminoacids and yet without the decomposition being carried too far, since ammoniacal salts and certain amino-acids are not favorable to good yields. The influence of the degree of simplification of the nitrogenous constituents on the yield in yeast is obvious from experiments of Effront with gluten, brought to different conditions of hydrolysis by the aid of trypsin, pepsin, and of sulphuric acid. For these tests $100 \mathrm{~g}$. of glucose are dissolved in $\mathbf{I} .5$ liters of water. As nutritive materials, I g. of yeast ash is added, and a quantity of gluten more or less peptonized, and corresponding to I.5 g. of nitrogen. After sterilization, inoculation with a pure yeast culture is affected, and the solution is allowed to ferment at $20^{\circ}$ while a current of sterile air passes over it.

Influence of the Degree of Hydrolysis of The Gluten on the YIELD IN YeAST.

\begin{tabular}{|c|c|c|c|}
\hline $\begin{array}{l}\text { Catalysts which intervened } \\
\text { in the hydrolysis. }\end{array}$ & $\begin{array}{l}\text { Estimate of the de- } \\
\text { gree of hydrolysis } \\
\text { according to the } \\
\text { formaldehyde } \\
\text { nitrogen. }\end{array}$ & $\begin{array}{l}\text { Yeast yielded per } \\
\text { roo grams of fer- } \\
\text { mented sugar. }\end{array}$ & $\begin{array}{c}\text { Nitrogen in the yeast } \\
\text { harvested. }\end{array}$ \\
\hline 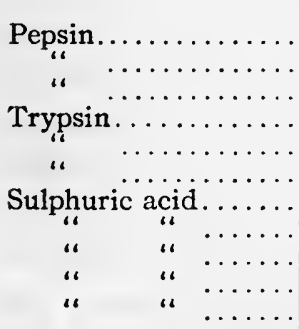 & $\begin{array}{c}\text { Per cent. } \\
9 \\
14 \\
21 \\
20 \\
32 \\
44 \\
25 \\
45 \\
60 \\
65 \\
72\end{array}$ & $\begin{array}{l}\text { g. } \\
10.2 \\
13.6 \\
16.2 \\
18.0 \\
19.6 \\
20.0 \\
17.2 \\
22.5 \\
17.0 \\
15.0 \\
12.0\end{array}$ & $\begin{array}{cc} & \text { mg. } \\
& 195 \\
251 \\
296 \\
328 \\
\text { Not measured } \\
\text { “ } \\
\text { Not measured } \\
\text { 380 } \\
\\
230\end{array}$ \\
\hline
\end{tabular}

The return in yeast increases as the hydrolysis is accentuated; the gluten which was peptonized up to only 9 per cent of formal- . dehyde nitrogen furnishes $10.2 \mathrm{~g}$. of yeast, containing in all 195 mg. of nitrogen. A thorough peptonization with pepsin leads to a yield of $16.2 \mathrm{~g}$. of yeast, which has taken from the nutritive 
medium $296 \mathrm{mg}$. of nitrogen. Hydrolysis with trypsin gives better results, and that with acids leads to the maximum. However, we must avoid pushing hydrolysis to its extreme limit, having found that a standard of 60 in formaldehyde nitrogen will unfavorably influence the yield.

The necessity of giving to the yeasts albuminoid material which is sufficiently degraded explains the use of large proportions of germinated grain. In the manufacture of yeast 50 per cent, and sometimes more, of malt is used. Furthermore, most manufacturers add to the mash large quantities of rootlets rich in amines and amides, which thus supplement the insufficient supply of nutritive material. The different processes now existing for the manufacture of yeast are all based on the utilization of the proteolytic enzymes contained in the germinated grain. The raw materials, malt and rootlets, give to the wort relatively large quantities of degraded nitrogenous substances, and these again increase, due to the activity of the tryptases of the malt on the proteins of the raw grain. However, the results obtained industrially are far from being satisfactory from the point of view of the raw materials, since the use of malt and of rootlets is very costly and does not give a constant yield.

Effront and Boidin have sought to replace malt by active substances of microbic origin, better suited to the work which it is desired to accomplish. The principle of the new process for compressed yeast is in reality the same as that developed in the first part of this chapter with regard to the manufacture of alcohol from grain. By a gradual habituation to the determined conditions of the medium, certain species of Tyrothrix are caused to secrete proteases abundantly. Thus an active liquid is obtained, possessing a proteolytic power 4 to 5 times as great as that of the malt. In the presence of bacterial proteases and in a favorable medium, the nitrogenous constituents of raw grain are almost totally dissolved, and hydrolysis to any desired degree is rendered easy.

The preparation of the mash for compressed yeast is accom- 
plished in the following manner: Crushed grains are liquefied with amylo-liquefying bacterial enzymes at a temperature of $75^{\circ}-80^{\circ}$, as previously explained (p. 614). The mash, rendered semi-fluid, is brought by sodium hydroxide or ammonia to a certain alkalinity, and then bacterial proteases are added. Peptonization, which is performed at a temperature of $45^{\circ}-50^{\circ}$, lasts from 2 to 4 hours and as much as 95 per cent of the total nitrogen of the grain is hydrolyzed and dissolved. The degree of hydrolysis is regulated at will, either by the quantity of enzyme used, by the choice of temperature, or finally by the length of the action. Peptonization ended, the liquid is brought to $110^{\circ}-$ I $20^{\circ}$; then, after cooling to $60^{\circ}$, it is saccharified with 5 or 6 per cent of malt. The process, while considerably favoring the yield, gives a very strong yeast and a perfect conservation.*

By the rational use of bacterial enzymes, we succeed in freeing distilleries and yeast factories from the necessity of working with malt, and thus avoid the inconveniences previously enumerated. We find, in fact, that the amylo-liquefying enzymes, kept in the presence of salts or with the addition of protein material, change in character in the long run. Their liquefying ability becomes slightly lessened while a pronounced saccharifying power appears. This deviation in the activity of catalysts is sufficiently pronounced to make it possible, in the two industries in question, to replace the malt completely, even as a saccharifying agent by active cultures more or less modified. From the theoretical point of view, the changes produced by conservation in the properties of the amylo-bacterial enzyme is likewise of interest. They recall certain facts observed with pepsin and rennet and, unless we accept the transformation of one enzyme into another, they are more favorable to the hypothesis that the liquefying and saccharifying powers belong to a single individual substance, which acts according to cirsumstances, either with its two functions simultaneously, or with one or the other, the double function being the more usual.

* For further details, consult the French patent No. 433,I I9. 


\section{§ 6. Enzymes in the Cheese Industry.}

The manufacture of cheeses involves a certain number of practices imposed by ancient custom, which we formerly did not dare to dispense with for fear of coming to grief. The systematic study of the processes used has formed for a score of years the object of special investigations. If it is true that the good results obtained are often due to judiciously applied mechanical treatments, it is none the less certain that the preparation . and the ripening of cheeses depend entirely on the action of proteolytic enzymes, and that an irregular intervention of these enzymes gives very inferior products. The attention of the microbiologists has therefore been directed toward defining the rôle of the enzymes, toward investigating and defining the optimum conditions for their activity, and toward explaining the expedients employed in this or that factory. This is the task undertaken by Duclaux and Freudenreich, by Lezé, Mazé, Gorini, and many others. For some years these investigators, armed with data previously acquired by a strict analysis of the various phenomena observed, have resolutely advanced in the manner of applications; numerous improvements have already been added to the processes which empiricism had established and which appeared intangible. The practice has begun in the cheese industry of utilizing selected ferments to regulate the ripening of the products and to bring these products to a constantly satisfactory quality.

It is not within the scope of this work to give even a cursory description of the various methods of cheese manufacture. It will be sufficient to indicate the principle in its most general aspects. Milk, skimmed or not skimmed, is first of all coagulated under conditions determined by the nature of the products desired. The curd is then more or less completely separated from the whey, either by a simple mechanical division (soft cheeses), or by a stirring effected during a moderate heating (cooked cheeses), and is then collected and put in the mould. Draining ensues and, if it is necessary, the excess whey is expelled by means of a 
slight or strong pressure (Dutch cheese). Then the cheese, if it is not eaten fresh, is salted and left to ripen. This is done under the influence of the enzymes carried in the milk, by action of rennet, or by enzymes secreted by micro-organisms. In certain cases, it is accompanied by an abundant development of molds on the surface of the cheese (Brie cheese); in others, this superficial vegetation is prevented by frequent washings of the crust which then becomes hard and encloses then only a bacterial flora. In other cases (Roquefort cheese), there is incorporated in the curds spores of Penicillium whose development also contributes to the transformation of the mass. The ripening of cheeses takes place in places of low or moderate temperature and in a dry or moist atmosphere, according to the case in hand.

The different cheeses are classified as follows:

Fresh cheeses: Little Swiss, Half-salt, Bondon, etc.

Soft
cheeses $\left\{\begin{array}{c}\text { Fermented } \\ \text { or ripened } \\ \text { cheeses }\end{array}\left\{\begin{array}{c}\text { By means of } \\ \text { superficial } \\ \text { molds } \\ \text { With washed } \\ \text { crust }\end{array}\right\}\right.$ Brie, Coulommiers, Camembert, etc.

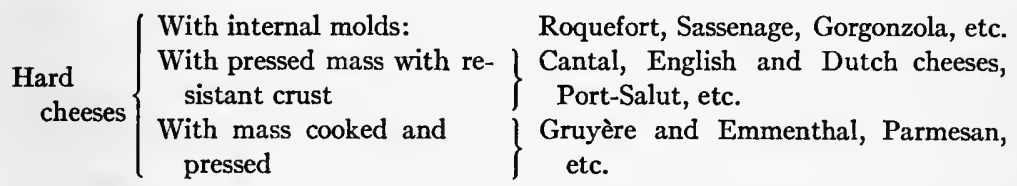

Let us now study more closely the action of the proteolytic enzymes, and let us see how the changes made in the conditions under which they act can influence the nature of the products which are derived.

Coagulation. - We know that the rapidity of coagulation of milk is a function of the quantity of rennet used, of the temperature, and of the acidity of the liquid. On the other hand, the consistency of the curd formed also is a function of the experimental conditions, an insufficient quantity of rennet acting at low temperature in an acid milk giving a soft curd, while an excess of rennet put in contact with a normal milk at 
a temperature of $30^{\circ}$ quickly gives a very firm and elastic curd, which even becomes friable if the temperature of the milk is above $35^{\circ}$. Thus, for the preparation of double-cream Swiss cheese very little rennet is used, and at a temperature of $15^{\circ}$ to $18^{\circ}$, in such a way that the coagulation is produced in about 20 hours. The curds obtained are very oily. On the contrary, in cheeses of firm consistency, coagulation is much more rapid. For Dutch, the coagulation takes place in about 30 minutes, at $3 \mathrm{I}^{\circ}$, with an excess of rennet. For Brie, whose curds must have a medium consistency, coagulation takes place at $30^{\circ}$, but in about 2 hours. For Camembert, repeated coagulations are made in such a way as to have a more oily cheese; curdling takes place in 3 hours at about $30^{\circ}$. Now, to work more regularly, the milk is often brought to an acidity always constant for the same cheese but varying with the species, by the addition before coagulation of a definite quantity of lactic culture. According to Mazé, the acidity which best suits the manufacture of Brie requires 7 decigrams of fermentation lactic acid per liter of milk; Camembert requires from Io to 12 decigrams; cheeses with pressed curd require from 3 to 6 , according to the temperature and the part which mechanical actions play in the draining.

The curd obtained must be separated from the accompanying whey. This is done by dividing the mass, and the removal of the liquid is much hastened by heating. The coagulum is finally reduced to small elastic grains that crack between the teeth. This is what is attained in the preparation of Gruyère, where the milk, after coagulation, is gently heated and carried gradually to a temperature of between $55^{\circ}$ and $60^{\circ}$, which is maintained for about 30 minutes. Yet not all the whey must be driven out, for this is useful in the subsequent operations, and if not enough remains the fermentations which are to bring about the ripening of the cheese will not succeed.

Liquid extracts of rennet are generally used for coagulating purposes. These commercial products should be regularly standardized and used fresh, so as to be free from bacterial trypsins 
whose dissolving action on the nitrogenous material would diminish the yield of curd. However, in the manufacture of Gruyère, use is made of macerations of dried calf stomach linings made in a special liquid called recuite. This is whey carried to the boiling point in the presence of "aisy," in such a way as to coagulate the soluble casein which it contains, the precipitate formed being then separated. Aisy is an acid culture broth, which can be obtained spontaneously by leaving at $35^{\circ}$ a mixture of whey previously separated from its soluble casein, a little vinegar, and good milk. Aisy is ordinarily kept in stock by replacing the quantity of this liquid emoved for use by the same volume of boiling whey, in such a way that the temperature of the whole rises to about $70^{\circ}$. This moderate heating produces a partial sterilization of the mass which destroys a number of bacteria, but which spares others, the latter being the ones to which aisy owes its efficacy. It is certain that in the preparation of whey with aisy, these bacteria very resistant to the action of heat are not destroyed and that they are found again in the rennet, whence they pass into the curds, and that there they will later contribute to the ripening of the Gruyère. The proof of this is that if we seek to replace the macerations of rennets in these culture liquids by commercial extracts of rennet, the results obtained are not as satisfactory, and in particular the flavor of the products is always inferior. Therefore, the bacteria of the aisy, and also those which are found in the rennets, play a useful part. With regard to the ripening of Gruyère more will be said later.

Ripening. - In prepared cheese, draining and sometimes pressure have reduced to the right proportion the quantity of whey remaining. Salting, always performed by scattering salt on the outside of the curd, above, below, and on the sides, is done to modify the flavor advantageously, at the same time that it will regulate the fermentations by playing the part of a weak antiseptic, and also by varying the humidity of the mass. In fact, it favors the desiccation of the product by drawing the water of the interior toward the surface layers. 
The cheese is then placed in a room of suitable temperature and humidity where it is watched, turned, put on dry straw, etc. The object of this continual care is to direct in a favorable manner the fermentations which take place during its ripening.

The transformation of curd into cheese is accomplished by the influence of various enzymes: ( $i$ ) galactase, a special trypsin, secreted by the mammary gland simultaneously with the milk, an active substance of which we have previously had occasion to speak; (2) pepsin, which is always found associated with rennet and which some writers even confound with it; (3) various tryptases, or caseases, secreted by molds, bacteria, yeasts, etc., which develop on the surface or in the interior of the mass. To these enzymes, which dissolve casein and bring it to the simpler state of peptones, amino-acids, and ammonia, it is proper to add: (4) amidases, that produce the formation of volatile acids; (5) lactase and lactacidase, enzymes secreted by lactic bacilli, and which transform lactose into glucose and then into lactic acid; (6) lipases, which apparently decompose a part of the fatty material; (7) finally oxidases, which burn the organic wastes, especially the acids.

The question concerning the rôle played by proteolytic enzymes in the ripening of cheeses is much debated. At first; their action was considered predominant. Now there is a tendency to attribute to them a much less importance; certain writers even without denying their intervention consider them useless, not to say really harmful. As a matter of fact, in Brie and Camembert cheeses for example, a very advanced peptonization results in rendering the mass semi-fluid and in giving to it a disagreeable bitter taste. The entire art of cheesemaking consists in this case in reducing to a minimum the action of the proteolytic enzymes. But it should not be concluded that cheese is a simple insoluble extract of milk formed by fat and by casein degraded as little as possible. The products of the solution of the nitrogenous material, however small they may be, nevertheless play a very important rôle in the production of the flavor, which we know is very different in different types, 
precisely because of a slight variation in the relative proportion of the different derivatives of hydrolysis. Without being able to describe definitely at the present time the mechanism by which the aroma of a cheese is elaborated, it is very probable that the amino acids contribute in large part to this synthesis. It is established, in fact, that in malting, the aromatic odor which is developed results from the action of the carbohydrates on the amino-acids, both being contained in the germinated barley. In cheeses a similar phenomenon occurs, the degraded products of the albuminoid hydrolysis giving with the bodies re ulting from the decomposition of fats special ethers, endowed with the characteristic flavor.

Moreover, microbes excel in engendering these traces of savory and volatile substances, to which the culture broths owe their organo leptic properties, at times so typical. Certain bacteria produce a strong burnt taste; others, a very pronounced bitterness; many molds smell musty, etc. The lactic ferments have, in this respect, a very remarkable power. It is to them, in particular, that fresh butter owes its delicate taste, for the ripening of the cream before churning depends on the development of these ferments in the whey which still accompanies the fat globules. This special aroma can moreover arise in the presence of fatty material other than milk. The manufacturers of margarine utilize this property in making an emulsion of their fats with whey, or even with pure milk, and by submitting the whole to lactic fermentation. The products thus acquire, up to a certain point, the taste of real butter. The lactic ferments, therefore, undoubtedly intervene in the production of the aroma of cheeses. Yet they are not the only ones to act, and it appears very probable that each variety of cheese would have, furthermore, its own bacterial species, some favorable, others injurious, which contribute toward its distinctive qualities.

As to the ripening of cheeses, the curd being made up of casein and a greater or less quantity of adhering cream and whey, let us first of all examine the influence of this latter component, whose rôle is of first order. Under the action of the 
lactic ferments, which have already begun their work before coagulation, the lactose is transformed into lactic acid. This, in a certain measure, plays the part of an antiseptic. However, its presence in too great excess injures the ripening. In cream cheeses, which retain 50 to 60 per cent of whey, the quantity of lactic acid produced is very great. It will gradually disappear by the way of biochemical combustion effected by the molds developed on the surface of the mass. Yet, if the quantity of 'whey left in the curd, and consequently that of the lactose contained there, is too great, there will be produced a too abundant cryptogamic vegetation, whose peptonizing action may become harmful. On the contrary, if there is not enough remaining, the ferments fare badly. In cheeses with compressed curd, the proportion of lactic acid formed is much reduced, and it becomes useless to oxidize the little which remains. The mass then continues slightly acid. On the contrary, in the soft cheeses, the acid reaction finally disappears, the mass becoming alkaline. The proteolytic enzymes then find a medium favorable to their activity. Sometimes, however, the change of reaction is due not to a destruction of the lactic acid, but to a neutralization by the ammonia produced.

The transformation of the casein takes place first of all by the action of the galactase. Then, when the acidity becomes too great, this enzyme ceases to act. However, the proteolytic properties of the rennet then come into play and exert themselves, although feebly, during a great part of the ripening. During this interval, the acidity of the mass is progressively neutralized by the molds or by the ammonia present. The latter is formed by the peptonizing action of superficial fungi, or even is absorbed from the exterior, some practitioners of experience being accustomed to scatter a little ammonia in the cellars where the cheeses are ripening. Lindet and Ammann have, in fact, recognized that the introduction into the curd rnass of a very small quantity of ammonia considerably stimulates the ripening. Then, when the reaction of the mass has become almost neutral, the true proteolytic enzymes intervene. 
These are tryptases secreted by the molds or by the bacteria contained originally in the milk, such as tyrothrix and yeast, and the casease secreted by the lactic ferments themselves. We know, in fact, that these are capable under certain conditions of peptonizing the albuminoid materials, and especially casein. A direct proof is offered by Mazé. The experiment is made, on one hand with Str ptococcus lebenis, which is encountered in the product known by the names of Bulgarian buttermilk, leben, yoghourt, etc., and on the other hand with an industrial lactic ferment. Milk, sterilized at $120^{\circ}$, is inoculated with the two bacteria, and after a certain time the filterable nitrogen is determined. Thus, the ratio $R=\frac{\text { Total nitrogen }}{\text { Filterable nitrogen }}$ is obtained. Controls are made by precipitating the casein with I per cent lactic acid.

Dissolving of Casein by Lactic Ferments.

\begin{tabular}{|c|c|c|c|}
\hline Age of the cultures. & Control milks. & Ferment ( $\mathrm{I}$ ). & Ferment (2). \\
\hline $\begin{array}{c}\text { Days. } \\
\text { I } \\
2 \\
6 \\
\text { I0 }\end{array}$ & $\begin{array}{r}R=\text { 10. } 66 \\
\text { 10.49 } \\
\text { 10.59 } \\
\quad \ldots \ldots\end{array}$ & $\begin{array}{r}R=10.56 \\
9 . \infty 0 \\
8.35 \\
\ldots \ldots\end{array}$ & $\begin{array}{r}R=10.20 \\
8.30 \\
7.97 \\
7.20\end{array}$ \\
\hline
\end{tabular}

This table indicates that solution, although slight, takes place in acid media, and that furthermore it takes place better, the weaker the acidity of the culture. Moreover, not only has the casein been dissolved, but the insoluble residue also shows properties which are not possessed by the coagulum obtained with pure lactic acid. Naturally, if the reaction is neutral or alkaline, the casease, as well as all the trypsins, acts much more vigorously, and then we might fear a too advanced peptonization, and consequently commercial products of inferior quality. If the curd has been pressed, the acidity is slight and becomes incapable of preventing the proteolytic activity of the lactic ferments. To illustrate these general 
data the refining of some well-known cheeses may be taken as examples.

Examples: Soft cheeses. - These cheeses give rise to the greatest variety of microbial interventions. The curds, prepared as previously described and left to themselves, begin a series of transformations. The lactic ferments, which are disseminated in the entire mass, decompose the lactose and give lactic acid which the surface molds will cause to disappear, either by oxidizing it or by neutralizing it. Among the oxidizing agents, it is proper to mention the penicillia, with which are associated certain yeasts, mycoderma, and sometimes oidia. The composition o this flora depends on the working conditions, and it is possible to modify its components to a certain extent. Thus, according to Mesnil, the salting of Brie cheese is of great importance, a quantity of 6 to $9 \mathrm{~g}$. of ordinary salt (with I I per cent water) per liter of the milk which enters into the preparation of the curd being the best proportion to use, since below 6 g. the cheese is often found contaminated by Oidium lactis, which liquefies it when it gets its full growth, and since above 9 g. the mass is dry, brittle and possesses an unpleasant taste.

Normally, the cheese, put in the drying room, is invaded by a white mold, which is that of penicillium. According to Roger, the true mold of Brie cheese in Penicillium candidum, whose spores remain white. According to Mazé, this mold is rather the mold of Bondon and of Coulommiers, while it is Pen. album which is developed here, as well as in Camembert. .Often it is $P$. glaucum which intervenes, though this last gives a poorer cheese. Afte 15 or 20 days, the cheeses are put down in the cellar where the temperature is at about $12^{\circ}$. The curd is ripened and softened, at the same time that yellowish. spots appear, then reddish spots formed by the so-called red ferments. According to Mazé, these arrest the action of the fungi which, by a too great development, would secrete sufficient casease to dissolve the casein near the crust and thus render the mass non-homogeneous. They also result in rendering the curds slightly alkaline by liberating ammonia, and in protecting the 
mass of the cheese against the penetration of oxygen which would rapidly cause rancidity of the fatty material. As these ferments prefer broken down nitrogenous material to insoluble casein, it follows that their activity causes all the substances capable of giving a bad taste to the cheese to disappear. On the other hand, they give a special flavor, recalling that of consommé. The ferments forming the red spots belong to the family of Tyrothrix. According to Roger, the essential bacterium of the red spots of Brie is Bacillus firmaticus, a very active producer of casease. So, for good results, its action should be modified by another bacterium, likewise isolated by the same investigator, and called Micrococcus meldensis. Finally, in addition to these multiple actions of biochemical nature due to the molds, it is proper to add another, entirely physical in nature. The filamentous mold fungi, from the fact that they increase the surface of the cheese, contribute in a large measure to assuring the drying of the mass by eliminating by evaporation the excess humidity.

When the acidity has almost disappeared, the lactic ferments scattered through the whole mass intervene through the casease which they secrete. According to Mazé, the ripening of the curd is due to lactic ferments, with which are associated some bacteria producing tryptases, and is not due to the action of the surface fungi, penicillia, and red ferments. If it is true that the partial dissolving of the casein begins at the surface layers to slowly extend toward the deeper portions, it is not, as certain writers believe, because the peptonization is due to the enzymes of the molds, but rather because these micro-organisms have destroyed or neutralized the lactic acid contained in these regions, and thus have made the medium more favorable to the action of the casease of the lactic ferments everywhere present. The ripening of the cream cheeses takes place in neutral or alkaline media. The ammonia produced by the red ferments slowly penetrates into the mass, and peptonization follows a parallel course from the exterior toward the interior. Without doubt, the molds also secrete many proteolytic enzymes, and even in 
very large quantities, but these tryptases cannot easily be diffused throughout the thickness of the curd. They are fixed by the insoluble casein, on which they act directly. If, consequently, too much enzyme is formed in these surface layers, it accumulates on the spot and cannot attack ihe underlying parts without being previously liberated by the solution of the retaining portions. One portion of the curd, situated immediately under the crust, then becomes fluid without the interior of the cheese having reached maturity, and the product is of inferior quality. To summarize, according to Mazé, the ripening of Brie cheese takes place through the activity of lactic ferments; the aroma is that of butter and of cream, to which is added that of consommé due to the red fermentation. As to the consistency, it should be that of butter, for the soluble casein is distributed in the whole mass and plays the part of the interstitial substance.

For the preparation of Camembert, there is little to change in the preceding, unless it is that the secondary fermentations must be slightly different from those of Brie, since the aroma of these cheeses is not quite the same. The almost exclusive part which we have just seen attributed to the lactic ferments is not, however, accepted by all. Ebstein, in particular, s of the opposite opinion. According to him, the curd of Camembert contains lactic ferments, which are found especially in the inside, while the soft outside contains Tyrothrix. The complete ripening would consist in the penetration of the tyrothrix to the regions populated by the lactic ferments. It is then the tyrothrices which are the true agents of ripening. Always, according to the same writer, we encounter in Brie cheese not only lactic ferments but also yeasts, Penicillium glaucum and $P$. album. Now it is to this last that must be attributed the preponderating action. The ripening of Brie, according to Ebstein, takes place not through the influence of the lactic ferment, but rather through that of the mold.

Cheeses with Washed Cake. - In this kind of cheese, the oxidation of the lactic acid is not complete, since the development of the surface molds is prevented by continual washings 
of the surface with salt water. However, the ripening takes place just the same, due to the neutralization of the lactic acid by the ammonia produced by a special bacterial flora which is developed, in spite of the washings, on the surface which is kept constantly moist. Under these conditions, the ripening goes on in a medium at first slightly acid, then alkaline. The presence of ammonia, however, gives to this kind of product a bitter taste which is not liked by all consumers.

Cheeses with Mass Spotted with Green. - The ripening of Roquefort, Gorgonzola cheeses, etc. takes place in a special manner. In the course of manufacture, there are introduced into the mass the spores of a particular mold, Penicillium Roquefortii. The spores then quickly develop and push their mycelium in all directions, this penetration being favored by the presence of air bubbles, and of numerous punctures made in the mass. The lactic acid is then in part oxidized, and in part neutralized by the ammonia which is formed under these circumstances. The proteolytic enzymes secreted by the penicillium, as well as the casease of the lactic ferment aid in the ripening which takes place at a temperature of $8^{\circ}$ and lasts for about two months.

Cheeses with Pressed Curd. - With all these cheeses, molds are not required to destroy the lactic acid, since this is no longer found in excess. The ripening therefore takes place in slightly acid media, but at a temperature a little higher than that required for Brie or Camembert. The ripening also lasts longer.

Cheeses with Cooked Curd. - In these cheeses the ripening no longer takes place under the influence of the casease secreted by the ordinary lactic bacteria, since during the cooking of the curds, which are heated for at least 20 minutes at $60^{\circ}$, these microbes are destroyed. However, we have seen that cheesemakers are accustomed to add with the rennet a certain quantity of aisy, a culture broth containing the acidifying bacteria required to assure the ripening of the curd. Although we as yet are not well acquainted with these bacteria, we know that they cause in the mass a liberation of hydrogen and carbonic acid gases, bringing about the formation of the "eyes of Gruyère," and that further they push the peptonization of the casein to a 
more advanced stage. Due to their activity, the ripening process continues for a very long time, about ro days in a cold cellar $\left(10^{\circ}\right)$, then several months in a warm cellar $\left(16^{\circ}-18^{\circ}\right)$ under rather moist conditions.

According to Lafar, Emmenthal cheese is made in three stages. In the first there is produced a partial solution of the casein by the action of Micrococcus casei liquefaciens, or by analogous bacteria of the family of tyrothrix. These act also at the beginning of the second stage of the process, which is the acidification that is brought about by Streptococcus lacticus. Finally, in the third stage the lactic ferment intervenes to cause the odor and taste of the product. In this last phase, certain yeasts and butyric ferments may also play a part. On the other hand, it should be noted that, following the investigations of Freudenreich in Switzerland, for some years pure ferments have been used for the manufacture of Emmenthal. These are prepared by allowing rennet to macerate in a mixture of culture broths coming, either from a special lactic ferment obtained by Freudenreich, or from a mycoderm isolated by Thöni the natural rennet used in the preparation of this highly esteemed type of Gruyère. The results thus reached appear to be very satisfactory.

Composition. - In conclusion, the Table p. 633 shows the composition of the different kinds of cheeses from figures borrowed from Lindet, Ammann, and Brugier. (Page 633.)

We see that the percentage of soluble nitrogen varies, and that there is not always even a relation between soluble nitrogen and ammonia nitrogen. Thus Camembert, which shows 86.I per cent of the total nitrogen as soluble nitrogen, has I4.2 per cent of this soluble nitrogen converted into ammonia, while Gorgonzola, wnich has only a much smaller percentage of soluble nitrogen, 27.2 per cent, nevertheless gives 17.1 per cent of ammonia nitrogen. In this second case, the peptonization is less extended, but more advanced. 
Analysis of Different Cheeses.

\begin{tabular}{|c|c|c|c|c|c|c|}
\hline \multirow[b]{2}{*}{ Kinds. } & \multicolumn{4}{|c|}{ Per $100 \mathrm{~g}$. of cheese. } & \multirow{2}{*}{$\begin{array}{l}\text { Sol. N. } \\
\text { per cent } \\
\text { of total. }\end{array}$} & \multirow{2}{*}{$\begin{array}{l}\text { Ammoni- } \\
\text { acal N. per } \\
\text { cent sol. N. }\end{array}$} \\
\hline & Water. & Fats. & $\begin{array}{l}\text { Tot. } \\
\text { nit. } \\
\text { mat. }\end{array}$ & Ammonia. & & \\
\hline 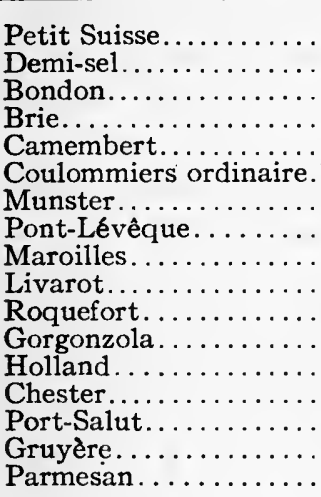 & $\begin{array}{l}\text { gr. } \\
54.6 \\
49.6 \\
54 \cdot 3 \\
53 \cdot 5 \\
53.8 \\
53.0 \\
52.4 \\
51.0 \\
40.3 \\
52.2 \\
36.9 \\
41.5 \\
42.6 \\
31.1 \\
38.1 \\
35.7 \\
34.0\end{array}$ & $\begin{array}{l}\text { gr. } \\
35 \cdot 0 \\
34 \cdot 0 \\
23 \cdot 0 \\
22 \cdot 5 \\
22.0 \\
21 \cdot 5 \\
24 \cdot 4 \\
23 \cdot 1 \\
33 \cdot 5 \\
15.0 \\
29 \cdot 5 \\
29.0 \\
20.0 \\
32 \cdot 3 \\
24 \cdot 5 \\
28.0 \\
23.0\end{array}$ & $\begin{array}{l}\text { gr. } \\
7.3 \\
\text { I I .8 } \\
\text { I } 6.1 \\
\text { I } 8.0 \\
\text { I } 7.1 \\
\text { I } 6.9 \\
\text { I } 5 \cdot 5 \\
\text { I } 7.8 \\
20.2 \\
25.9 \\
20.5 \\
\text { I9.7 } \\
23.9 \\
30.9 \\
24.8 \\
28.9 \\
35.0\end{array}$ & $\begin{array}{l}\text { gr. } \\
0.00 \\
\text { traces } \\
0.11 \\
0.18 \\
0.23 \\
0.26 \\
0.19 \\
0.13 \\
0.32 \\
0.36 \\
0.14 \\
0.17 \\
0.02 \\
0.20 \\
0.02 \\
0.05 \\
0.14\end{array}$ & $\begin{array}{r}3.2 \\
12.2 \\
32.9 \\
58.1 \\
86.1 \\
60.7 \\
53.2 \\
43.9 \\
59.4 \\
55.9 \\
47.5 \\
27.2 \\
22.3 \\
30.1 \\
20.2 \\
22.9 \\
21.7\end{array}$ & $\begin{array}{r}\ldots \ldots \\
\ldots \ldots .6 \\
10.6 \\
13.1 \\
14.2 \\
16.0 \\
12.3 \\
8.1 \\
14.2 \\
15.5 \\
8.9 \\
17.1 \\
2.0 \\
11.4 \\
2.3 \\
4.7 \\
9.9\end{array}$ \\
\hline
\end{tabular}

This table, aside from the quantity of ammonia formed, does not indicate the distribution of the soluble nitrogen among the different forms. The following is a summary of ten analyses made on Gruyères by Jensen and Plattner which show that the quantities of soluble nitrogen are, as stated above, relatively very large. These Gruyères, nine months old, contained from 3 I.5 to 35 per cent of water and from 25.8 to 29.7 per cent of nitrogenous substances, the latter being distributed as follows:

\begin{tabular}{|c|c|c|}
\hline Different stages of nitrogen. & Per 100 total nitrogen. & Per too soluble nitrogen. \\
\hline 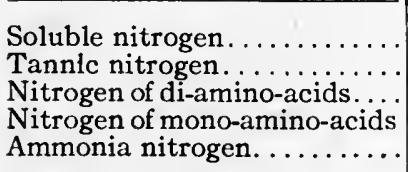 & \begin{tabular}{|l} 
From 28.2 to 36 \\
From 6.2 to 12.2 \\
From 2.9 to 7.8 \\
From 11.2 to 18.1 \\
From 1.7 to 5.3
\end{tabular} & 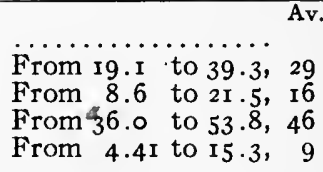 \\
\hline
\end{tabular}


The striking thing is the large percentage of soluble nitrogen, of which the nitrogen of the mono-amino-acids represents about one-half. Now, we know that Gruyère owes its ripening to special lactic bacteria, which give, especially with casein, monoamino-acids and only very little ammonia or other compounds. O. Jensen and Plattner explain the large quantity of soluble albuminoid substances also by the probable presence of acid albuminates, formed directly with the nitrogenous material of the cheese.

Jensen and Plattner have likewise studied the cheese called "Brique d'Allgau." They have found that the proportion of soluble nitrogen can reach 90 per cent of the total nitrogen. The content in ammonia nitrogen is likewise high, representing some II per cent of the total nitrogen. It is by reason of this strong alkalinity that the solution of the albuminoids has been so thorough. In fact, we observe that 75.5 per cent of soluble nitrogen is precipitable by tannic acid, and corresponds to alkaline albuminates and primary albumoses. The quantities of mono-amino nitrogen ( 7 per cent) and of di-amino nitrogen (5.4 per cent of the soluble nitrogen) are on the contrary very small.

In conclusion, the study of the manufacture of cheeses has furnished very important results, and considerable advances have been realized in this branch of the dairy industry. In France especially, at the instigation of Mazé, dairymen have instituted reforms, and at the present time are deriving real advantages, from the point of view of the value of the products, from the use of selected enzymes. Some prepare even Brie cheeses of a quality yielding large profits in an ordinary way, with the aid of pasteurized milk inoculated with pure lactic ferments. It is certain that the example will spread, and we may regard the day as near when the technique of cheesemaking will be established on an entirely scientific basis, comparable with the methods which already characterize numerous other fermentation industries. 


\section{BIBLIOGRAPHY.}

P. Mazé. (1) Les microbes dans l'industrie fromagère, Ann. Inst. Past., 1905, (I9), pp. $37^{8}$ et $48 \mathrm{I}$. (2) Technique fromagère, Ibid., I9 Io, (24), pp. 395, 435 et 543. (3) Les ferments lactiques et le lait, Rev. Scient., 19r3, (2), p. 228.

O. Jensen et E. Plattner. Contribution à l'analyse des fromages, Mon Scient., 1908, (22), p. 252; d'après Ziets. f. Untersuchung d. Nahrungs- u. Genunmittel, (12), p. г93.

DE FREUDENREICH. Etude sur l'Emmenthal, Landwirs. Jahrbuch der Schweiz, I906 (?).

Duclaux. Le lait, I vol., Paris, I894.

LezÉ. (I) Les industries du lait, I vol., Paris, 1904. (2) Préparation et maturation des caillés de fromagerie, I vol., Paris, 1905.

Henseval. Les microbes du lait et de ses dérivés, I broch., Lierre, I903.

LindeT. Le lait, la crème, le beurre et les fromages, I vol., Paris, I907.

C. Martin. Laiterie, I vol., (Encyclop. agricole), Paris, r9r3.

F. LAFAR. Handbuch der technische Mykologie, 4 vol., Jena, I904-I907. 


\section{§ 7. Rôle of Proteolytic Enzymes in Tanning.}

Proteolytic enzymes intervene many times in the course of the numerous operations to which hides are submitted while being transformed into leather. To understand the rôle of enzymes in tanning, it is important first of all to recall the fundamentals of this industry. The hide, before being submitted to the action of tanning materials, must receive a suitable preparation. We know that the hide of an animal is formed of different layers, namely: on he external side, the epidermis, with the hair; below, the dermis or chorion, which is found to be separated from the epidermis by a thin layer of cells representing the vitreous or hyaline membrane; finally, on the internal side, some fragments of flesh and of adipose tissue, as well as the muscles adhering to the skin. Of all these layers, only that which is central, that is to say the dermis, is suitable for tanning, so this layer must be freed of the useless layers by a suitable treatment. The dermis is, in fact, formed by a padded layer of different fibers, mingled with connective cells, the whole united by an interstitial substance called the coriin. When the hide is on the animal, the coriin kept in a semi-fluid state, permits the fibers to slide easily upon each other, and confers on the whole mass the suppleness and elasticity which characterize this part of the body. But when the hide is removed, either it is kept moist and quickly putrefies, or else it is dried, and then the coriin hardens, the fibers become horny, the whole stiffens and loses its great flexibility.

To ensure to the hide, on the one hand its preservation, and on the other hand a certain elasticity, that is to say, to transform the hide into leather, it is necessary then: ( 1 ) To separate the dermis from the useless parts, epidermis and subcutaneous tissue which accompany it, but sparing the hyaline membrane, which will form the outside of the leather; (2) to eliminate from the dermis the coriin and its connective cells, in order to separate the different fibers from each other and even to reduce these to smaller and more numerous fibrils; (3) to re-cover each of these 
fibers with a special material, called tannin, capable of ensuring its non-putrefaction, of preventing its sticking to its neighbors, while in fact it even favors a certain sliding upon them.

It is not necessary to consider here the third series of operations, which corresponds to the tanning properly speaking since the proteolytic enzymes do not intervene in this part of the work. In the first two series the hairy skin is brought to a special state called tripe hide, which is made up solely of the swollen dermis, covered with the hyaline membrane and reduced to a loose network of dissociated fibrils. To obtain this result, the skin is submitted to the following operations:

(I) Soaking (Plumping soak), which consists in cleansing the fresh skins received from the slaughter house or in letting the dry skins soak for some time, so as to swell them and give them back their original suppleness.

(2) Depilation and hair removal, by which the dermis is separated from the epidermis and the hairs which accompany it.

(3) Fleshing, which eliminates the flesh and the fatty parts remaining attached to the dermis. This operation, which is effected either by hand with suitable knives, or by a machine, does not concern us here.

(4) Cleansing in the tubs, a treatment which results not only in the freeing of the hide from traces of lime which this contains if it has been depilated by means of this substance, but also in giving to it, if necessary, a still greater suppleness.

Soaking. - Normally, this operation must be carried on in running water, or water which is frequently changed. However, to stimulate the action on hides which are very dry and particularly hard, recourse is sometimes had to a soaking in vats where the water is not changed. These baths, rich in organic matter, are the source of a true putrefaction. A rich microbic flora develops there, secreting proteolytic enzymes, which exert on the hide a very energetic dissolving action. This naturally softens much more quickly under these conditions, than with soaking in pure water, but this increase in speed is attained to the detriment of the quality of the product. 
Depilation. - Depilation is accomplished in several ways: (I) by sweat ng; (2) by the use of lime; (3) by the use of alkaline sulphides; (4) by the use of arsenic sulphide. The first process results in a special fermentation, to which we shall return in a moment. Depilation with lime, or barking, appears to be based on the combined action of enzymes and the caustic used. As to the third and fourth processes; they are purely chemical, and, for this reason we shall speak no further of them.

- To understand the mechanism of depilation, it is first necessary to describe the construction of the epidermis and the manner in which the hairs are fastened to the surface of the hide. The epidermis is composed of several layers of different cells, some of which are living, and the others, the most superficial, are dead. The first, under normal conditions, form what is called the $\mathrm{Mal}$ pighian layer. Among them, the deepest layer of cells, that which is in direct contact with the dermis, forms the vitreous membrane, of which we have already spoken. The second, which results from the transformation and the death of the cells of the Malpighian layer, form the horny layer. They are made up especially of keratin, a very complex albuminoid, insoluble in water and dilute acids, not attacked by gastric and pancreatic juices, but soluble in hot alkaline liquors, as well as in alkaline and alkaline-earth sulphide solutions. As to the hair, the horn, the scales, etc., their fundamental substance is also keratin. The hairs are fastened to the hide by a peculiar kind of insertion. At their roots which penetrate quite far into the integument, the epidermis sinks into the dermis, in such a way as to form for the hair a real sheath, at the bottom of which the hair is held in place by means of an enlargement of the bulb. Therefore, in order to pull out the hairs, it is necessary to dissolve these bulbs at the same time that the epidermis is more or less disorganized. In the process of heating, as well as in barking, it is the enzymes secreted by the bacteria, aided by the lime added, that perform this function. In depilation with sulphides, it is the chemical substances which dissolve the keratin and ensure an easy depilation. 
In practice, one or the other of these two processes is utilized. Depilation with sweating is especially utilized for large cattle hides to be used for sole leather, or else for sheepskins whose wool it is desired to keep. Cattle hides, fresh or soaked, are extended on one another in such a way as to make a pile of about I meter in depth and are left to themselves until the hair comes out easily. To avoid having the fermentation progress too quickly, especially in summer, and so change the dermis substance, between each of the hides is scattered a certain quantity of salt. In winter, on the contrary, the hides are placed in a slightly heated room. With sheep-hides, the only difference is that the hides are suspended in a closed room, in place of being placed in a heap. In consequence of a beginning of putrefaction, the proteolytic enzymes secreted soon disorganize the Malpighian layer, which is the least resistant of all the parts of the hide, and soon the hairs offer no further resistance to being pulled out. We find, in fact, that hides suitably sterilized and kept moist in an aseptic medium, do not depilate, and that to arrive at this result bacterial intervention is necessary. Schmitz-Dumont, on the other hand, claims to be able to isolate from hides submitted to sweating a streptococcus, which is developed at the expense of the Malpighian layer, which liberates ammonia, and which would, according to him, be the active agent of depilation. On the other hand, Villon thinks that it is the bulb of the hair itself which undergoes the first decomposition. This writer, in fact, claims to have proved that in the course of sweating, the special albuminoid material, pillin, which forms the bulb, is the seat of a particular fermentation due to a type of bacterium which he calls the pillin bacterium, and that by the action of this micro-organism different nitrogenous products are formed, especially ammonia, whose dissolving action on the epidermis layer is well known. Moreover, whatever may be the bacterial species which intervenes here, whatever may be that part of the hide. which first undergoes its action, or whatever the mechanism by which it operates, it is certain that a proteolysis is established in the course of depilation, and that at the same time the destruction of the epidermis results from the direct action 
exerted on it by the bacteria and the enzymes which they secrete, as well as from the dissolving action of the ammoniacal products formed in these reactions.

The data relative to barking are less precise. We may state, first of all, that the process of depilation with lime consists in immersing the hides for some time in a series of baths or pits, usually three in number, containing milk of lime of increasing strengths. The first pit, called the dead pit, is filled with a lime milk which has already served for several operations. In this bath the hides, washed and soaked, are immediately plunged. After some hours or some days, according to the character of work, the hides are passed into the second pit, called the gray pit, which contains a lime milk which has likewise been used, but not as much as the preceding. After another lapse of time, the hides are withdrawn and are immersed in the third bath, or new pit, which contains a lime milk freshly prepared. Here they are left until the hair has ceased to adhere to the dermis. Naturally, in the normal course of events, a rotation is established among the three baths; the dead pit, after having served for the treatment of a definite number of skins, is thrown away and is replaced by a fresh lime milk and so becomes the new pit; at this point, the pit which has up to that time been considered as new becomes the gray pit, and the latter is changed into the dead pit.

What is then the action of these pits on the hide, and particularly on the epidermis? For a long time it was thought that the lime acted only by reason of its alkalinity, and that the caustic sufficed to disorganize the Malpighian layer and bring about a quick falling of the hair. The use of three pits then arose from the necessity of acting not too suddenly but rather in a progressive manner on the hide. However, this opinion does not seem to be in harmony with experience. We always, in fact, encounter in the pits a great number of bacteria, and it seems probable that these intervene effectively in the destruction of the epidermis. Villon even claims to have succeeded in isolating the pillin bacteria from lime-treated hides. According to him, barking results from the decomposition of the nitrogenous substance of 
the bulb, pillin, under the influence of this special micro-organism, the lime serving only to keep the putrefying bacteria away from the bath. Villon, in the support of this theory, cites the fact that sterilized hides in contact with lime do not depilate, while the action takes place as soon as a culture of pillin bacterium, which withstands very well the alkalinity produced by the lime water, is added to these hides. It should be added that this experiment is corroborated by a practice common with many tanners, which consists in adding a certain quantity of dead pit to a new pit, in such a way, it appears, as to hasten in the latter the development of the pillin bacteria. If this is not always done, it is because, as a matter of fact, this inoculation takes place spontaneously by the passage of the hides from one pit to the next.

Another indication that very energetic hydrolytic agents actually intervene in the barking is that drawn from the analysis of lime baths which have served for many depilations. Schroeder and Schmitz-Dumont, by analyzing a pit which had been used for several months in a tannery, found not only a large quantity of nitrogenous organic matter, albumin and immediate derivatives, but also considerable amounts of volatile acids, ammonia, and trimethylamine. Now we know that these are the ultimate products of protein hydrolysis, and that they result especially from the enzymic activity of amidases. It thus appears much more logical to believe that their formation here is the consequence of proteolytic fermentations, rather than the effect of the simple chemical action of the lime on the albuminoid material of the hide. In addition, this enrichment of the pits in organic matter evidently takes place at the expense of the components of the hide. Now, Eitner found that the loss of dry weight by a barked hide is greater when an older bath is used, one consequently richer in bacteria and in dissolving enzymes. Furthermore, the disintegration of the hide is more rapid if the operation is performed at a higher temperature. All these data harmonize well with the idea that the proteolytic enzymes also play an active rôle in depilation with lime, and it is 
very probable that if we really seek enzymes in these liquids we shall find them present.

In the depilated hides that have just undergone heating or barking, the hair still holds to the dermis, but a very slight pull is enough to detach them from it. This detaching, called dehairing, is done by means of hand utensils or special machines. Then the hides are rinsed, fleshed and again washed. At this point, the hide is made up of the recovered dermis and the hyaline membrane, a membrane which, more resistant than the rest of the epidermis to the dissolving action of the depilatory agents, has been preserved. The treatments which the hides will then undergo vary according to the nature of the hide and the kind of leather for which it is intended. In general, these treatments are mechanical or purely chemical, and are of no interest to us here. On the other hand, there are cases where the treated hides must acquire a certain suppleness. They are then made to undergo the action of microbic tubs (fermentation). For example, when goat-hides or sheep-hides are treated with a view of tanning them with sumach to transform them into morocco or its imitation, they are submitted, after barking and fleshing, to the action of a bran tub ("drench"). The mixture of bran and water, which soon becomes the site of an intense lactic fermentation, causes a special action on the hide. First of all, due to the fermentation gases, there is produced in the very heart of the dermis a swelling and an internal loosening, which isolates the fibers, distends the tissue, and gives to it a suitable suppleness. This action is, in the beginning, entirely mechanical. Then, when the tub has become acid, it causes a real removal of lime, that which remained in the skin dissolving at this point in the organic ac ds formed. Further than this, this tub affords only a moderate interest, since it is established that the proteolytic enzymes here play no rôle, the substance of the dermis being in no way influenced by this treatment.

Excrement Tubs. - The excrement tubs ("bate"), on the contrary, exert a very energetic action, since they effect a true digestion of the hide, which first of all softens, but which then may deteriorate profoundly if the attack is prolonged too far. Two 
kinds of tubs are used, those of dog dung and those of pigeon dung. These products do not act in just the same manner, but the cause of these variations is not yet well determined. However, the difference is not very great, and to simplify we will speak only of dog dung, which is moreover the one most used. This tub is used only in leather dressing for hides which require a great.suppleness, as for example goat-hides, which are tanned in chrome and then made into glacé kid for shoes, or again those of kid or of sheep, which, after dressing in a mixture of alum, salt, yolk of egg, and flour, are used for glove-making. This tub is prepared as follows: Dog dung, generally imported from Asia Minor, is put in barrels and is sprinkled with a certain quantity of water in such a way as to moisten it and bring about a fermentation. After about a month, a certain quantity of this paste is taken, diluted in water, and soon afterward the clear liquid is decanted. It is in this maceration that the hides coming from barking and fleshing are plunged. It is found that the hide at the same time that it is purged of lime which it still contained, is very quickly cleansed, becomes flabby, and falls. Under the microscope, it is observed that the starred or connective cells have disappeared, and that the superficial fibers even begin to dissolve. It is necessary, however, not to prolong the action, for the hide would then become disorganized and end by losing all firmness.

To what is this double action due? The elimination of the lime results very probably from the presence in the tub of phosphates and butyrates as well as other organic salts, which tend to increase the solubility of the alkaline-earth constituents of the bath. As to the loosening of the tissue, it is due solely to the action of enzymes, as well as to the nitrogenous products which are derived from their action. These conclusions are the result of the investigations of Eitner, Popp, Becker, and finally of Wood. In the action of the tub, three factors are to be considered: (I) the bacteria; (2) the organic and inorganic material forming the culture medium; (3) the digestive enzymes coming from the animal, which have been cast out by him in his excrement. 
As to the rôle of the bacteria, we possess interesting data. First of all, it is certain that the bacteria which intervene here are not those characteristic of putrefaction; in fact, these latter, instead of producing on the hide a favorable action, attack and change it rapidly. At present, it cannot be said that they are really useful species. Popp and Becker have succeeded, it is true, in isolating from tubs of dog dung three kinds of bacteria, which, in contact with " tripe," give it the desired suppleness. However, we cannot prove that these bacteria are truly specific for the action under consideration. It even appears that the number of species capable of playing a favorable part is much larger than was formerly thought. Wood succeeded, in fact, in obtaining from the tubs 90 species of bacteria which, each taken separately, had no action on the hide, but which, judiciously associated, gave the characteristic effect of a good tub. Certain writers even think that these useful bacteria are found not only in the tubs of dog dung, but that they are also met elsewhere. Thus Wood was able to isolate from wool grease two bacteria, which by a simultaneous action showed a great efficacy on the hide, an efficacy even superior to that of the natural tubs. Similarly, Nördlinger succeeded in obtaining good results simply by using micro-organisms which were spontaneously developed on cooked slices of potatoes left for several days at $37^{\circ}$.

However, whatever is the nature of the bacterial species which here come into play, undoubtedly these act through the enzymes which they secrete, as well as through the ammoniacal products which are formed by their action. Wood found, in fact, that if such a tub in full power is taken and the volatile products are isolated by distillation in a vacuum, and the enzymes by precipitation with alcohol, the liquid which remains has lost all its properties, while the mixture of the two parts previously separated, the amines and the enzymes, exerts a very favorable action on the hide. Another indication that the bacteria do not act so much by their vegetative life as by the products to which they give rise comes from the fact that fresh tubs are without action on "tripe," and that it is necessary to cause the excrements 
to undergo a preliminary fermentation in order for them to acquire their special solvent action. The fact is that during the preparatory work the bacteria which pre-existed in the dung have elaborated enzymes and transformed the materials on which they lived, thus bringing the whole to a state of maximum power.

On the other hand, certain accidents in the use of tubs result from the inopportune development of injurious bacteria which are always found associated with the useful bacteria in the excrements. Thus a bad preliminary fermentation can favor the multiplication of a particular bacterium, which causes the production of black tub, whose action on the hide is very disastrous. In the same manner, the undecanted tubs, which, however, are more active than clear tubs, are not to be recommended, because of the harmful bacteria which accumulate in the foul deposit and which, by fixing themselves on the hide, may spot and even pierce it.

Now as to the effect of the inert materials which are present in the dung, Eitner, since $\mathrm{I} 88 \mathrm{r}$, has regarded these merely as nutritive substances for the microbes which accompany them. This would, according to him, be the reason for the different effects of bates of various origins, as those of $d o g$ and of pigeon dung. The nutritive media being different, the bacterial species which are developed there will also be different. On this basis, Eitner has even prepared artificial tubs by substituting for the excrementitial materials inorganic compounds such as phosphates of potassium and of calcium, magnesium sulphate and ammonium tartrate, which he added to old tubs of dog dung. These culture broths, after a certain period of development, acted on the hide exactly as do natural tubs. In spite of this positive result, obtained with purely inorganic substances (ammonium tartrate can be omitted from the preceding mixture), it appears rather that the tubs also exert, by reason of the organic materials which they contain, a direct action on the dermis. In particular, it has been established that the amino-acids and the ammonia here formed cause the hide to fall in a very evident manner, and 
that on the other hand the ammonium tartrate contributes in a large measure to the complete elimination of the lime.

Finally, the enzymes remaining in the excrements at the moment of their expulsion intervene, in their turn, when these tubs are used. Among the proteolytic enzymes which are found there are trypsin and erepsin. The alkalinity of the medium in which they are placed is very favorable to their activity, and there is no doubt but that they also contribute to the hydrolysis of the complex nitrogenous materials still contained in the excrements, and to the preparation of a favorable medium for the development of the useful bacteria of the tubs.

To summarize, we see that the tubs of dog dung act especially through the products of secretion and through the enzymic transformations which are elaborated within them. It is then quite natural to seek to reproduce their action by means of artificial tubs, of a less repulsive character and a more certain effect. This has been done in a fairly satisfactory manner, the several difficulties which have yet to be overcome being of a practical rather than theoretical order. The first tubs to be prepared were of cultures ofmicro-organisms taken from natural tubs, these cultures made wholesale on suitable organic media, especially in meat broths. Use was also made of gelatin peptonized by lactic acid, a product which was then inoculated with bacteria coming from an infusion of wool grease. Then, when it was perceived that the natural tubs acted especially through the enzymes secreted, the use of these broths was rendered easier by reducing their volume by evaporating in a vacuum. Thus, at present, extensive use is made of a product obtained by concentrating at $50^{\circ}$ under reduced pressure, the result of the culture on a suitable medium of different bacteria extracted from dog dung. This thick liquid, after having been diluted in water, exerts a very favorable effect on the hide, since, from its very preparation, it contains no bacteria. Unfortunately, this preparation is still relatively expensive. 


\section{BIBLIOGRAPHY.}

A. M. Villon. Traité de la fabrication des cuirs, Paris, I889.

L. Meunier et C. VANey. La tannerie, Paris, I903.

Schmtz-Dumont. Débourrage à l'échauffe, Mon. Scient., i897, p. 312.

Schroeder et Schmitz-Dumont. Dingler's, r896.

EITNER. Gerb., 1895, pp. 157 et 159 .

Eitner. Confits d'excréments, Gerb., I890, p. 87.

Wood. Journ. of the Soc. chem. Indust., 1898, p. 1011; 1899, p. 990. 


\section{§ 8. Rôle of Amidases in the Formation of Petroleum.}

The knowledge of the rôle played by amidases in the ultimate transformation of the products of the degradation of albuminoid material, permits a better understanding of one of the points still remaining obscure in one of the two ruling theories as to the formation of petroleum. At the present time it is generally believed that these natural hydrocarbons are the result of reactions at high temperature between substances either purely inorganic or organic; or, since these two reactions are not mutually exclusive, it are very probable that both intervene in the formation of petroleum.

According to the first theory, there are produced by the action of heat in the bosom of the earth, metallic carbides, which, when subsequently decomposed by steam, produce hydrocarbons. These, by reason of the high temperature and the strong pressure to which they are subjected, combine with free hydrogen and are transformed into saturated hydrocarbons. They are also condensed to give rise to products of higher molecular weight. The net result would be the formation of substances resembling the natural petroleums. It is to be noted that in this synthesis, it is not even necessary to have very high temperatures, the experiments of Sabatier and of Senderens having shown the possibility of transforming, in the presence of powdered nickel heated only to $200^{\circ}$ or $300^{\circ}$, a mixture of acetylene and hydrogen into hydrocarbons identical with the different known petroleums. Moreover, volcanic phenomena might favor these reactions by bringing together the different reagents required.

The second theory is of more direct interest. According to this theory, the petroleums arise from a decomposition, accompanied by the production of heat, of animal and vegetable débris, especially hat of marine origin. This theory is based on various observations, of which the principal is the frequent presence in petroleum wells, along with rock salt, of organic remains, such as those of fishes and mollusks, or those of marine plants. The neigh- 
borhood of these petroliferous veins with those of coal or of bituminous schists allows the supposition that both have the same origin. The value of the arguments given in favor of this theory has been disputed by geologists, but it is not necessary to judge as to that here. It is popularly conceded that this manner of formation is, if not general, at least almost certain for some veins, such as the Canadian veins, for example.

This theory moreover rests on various experiments. Engler, in 1888 , by distilling under pressure fatty material coming from marine animals, such as cod liver oil, succeeded in obtaining a liquid resembling petroleum. The distillation began at $320^{\circ}$ under ro atmospheres of pressure and ended at $400^{\circ}$ under 4 atmospheres. Of the products distilled, comprising about 70 per cent of the material used, and weighing $492 \mathrm{~kg}$., about $\frac{1}{6}$ were combustible gases, and $\frac{5}{6}$ an oily liquid, composed of saturated and of a few unsaturated hydrocarbons. By redistillation and fractionation, of this crude liquid a purer product could be separated closely resembling ordinary refined petroleum. This experiment is moreover comparable to that made by Cahours in I875, who found that the distillation of fatty acids with superheated steam gives rise to a whole series of saturated hydrocarbons, from pentane to duodecane.

Thus, according to these data, the petroleums would result from the pyrogenation of fatty materials, and these could in turn arise from the disintegration and the putrefaction of various marine animals, which, due to submarine currents or other modes of transportation, accumulated in certain parts of the globe. The different constituents forming the organic tissues were decomposed each in its turn. The carbohydrates were oxidized or dehydrated, while the nitrogenous material was hydrolyzed and simplified. Only the fats would have resisted this disorganization. To realize all these transformations, the assistance of the micro-organism would have been necessary. This is not a mere supposition, since B. Renault found the presence of bacteria in the fossil débris of all the periods, Jurassic, Permian, Carboniferous, etc. 
All this reasoning appeared very plausible until, in I900, Walden recorded a grave objection. Following up an old observation of Biot ( 1835 ) he found that natural petroleums possess a very obvious rotatory power, while the products of synthesis are inactive. The hypothesis of Engler thus appeared fallacious. It, at least, had need of revision, and that is what Neuberg did some years later. Neuberg had the idea that perhaps the rotatory power of natural petroleums was due to the presence, in the fatty materials from whence they are derived, of optically active fatty acids, such as isovaleric acid and isocaproic acid,

$$
\begin{aligned}
& \mathrm{CH}_{3} \\
& \mathrm{C}_{2} \mathrm{H}_{5}
\end{aligned}>\mathrm{CH}-\mathrm{CO}_{2} \mathrm{H} \text { and } \begin{aligned}
& \mathrm{CH}_{3} \\
& \mathrm{C}_{2} \mathrm{H}_{5}
\end{aligned}>\mathrm{CH}-\mathrm{CH}_{2}-\mathrm{CO}_{2} \mathrm{H},
$$

substances which are known to be formed in the course of the putrefaction of nitrogenous materials.

Practically, by distilling under pressure a mixture of oleic acid and a little isovaleric acid, Neuberg obtained a product, which, after purification, presented all the characteristics of a true petroleum, including the dextro-rotatory power. Even with this artificial product the increase of rotatory power is found to be a function of the boiling point, an increase which had already been observed with natural petroleums. Thus it is that by fractionating a previously purified distillate, an average sample of which in a tube of 2 decim. gave a rotation of $+\mathrm{r}^{\circ} \mathrm{Io}^{\prime}$, we get the following fractions:

(I) That collected up to $125^{\circ}$ causes rotation of $+0.2^{\circ}$.

(2) That collected from $125^{\circ}$ to $230^{\circ}$ causes rotation of $+0.5^{\circ}$.

(3) That collected from $230^{\circ}$ to $320^{\circ}$ causes rotation of $+0.7^{\circ}$.

Also natural naphthas show a rotation extending from $+0.2^{\circ}$ to $2.3^{\circ}$.

This experiment then answers the only objection which can be made to the theory of Engler, and furthermore renders very probable the formation of petroleums starting with fatty materials. We could, however, inquire by what process these substances are formed at the period when the work of Neuberg 
appeared, although he took care to verify experimentally the fact that albuminoid material on being purified gives many volatile fatty acids. We now know that they are due to the action of amidases acting on simple amino-acids; thus it is that glutamic acid on splitting gives butyric acid and ammonia according to the equation:

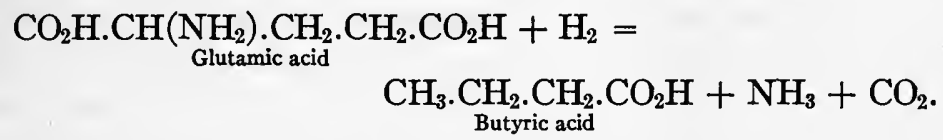

The putrefactive bacteria, which obviously must have acted in former times as they do at present, secrete proteolytic enzymes as well as amidases. In succession, these various enzymes exerted their action on the nitrogenous materials of marine animals to give finally, among numerous derivatives, volatile fatty acids, of which some were endowed with a rotatory power. These optically active substances, mixed with fats which had resisted bacterial decomposition, and subjected to the combined action of a high temperature and a strong pressure, formed the natural petroleums.

\section{BIBLIOGRAPHY.}

L. C. Tassart. Exploitation du pétrole, Paris, Igo8.

C. Neuberg. Die Enststehung des Erdöls, Sitzungsber. der könig. preuss. Akad. der Wissensch, I6 May, r907.

C. Engler. Ber. d. deuts. Chem. Ges., I888, (21), p. I816; I889, (22), p. 595.

P. Walden. Nalurw. Rundschau, I900, (15), No. 12-16. 


\section{$\S$ 9. Putrefaction.}

When any albuminoid material is allowed to stand under suitable conditions of moisture and of temperature, it decomposes very rapidly. The nitrogenous substance disintegrates, becomes soluble, simplifies chemically, and at the same time the mass acquires a repulsive odor due, among other products, to the sulphuretted hydrogen and to the trimethylamine and indol derivatives here formed. This phenomenon, known for a long time, formed the basis for the work of Pasteur on spontaneous generation, a subject since studied by numerous investigators. It has been recognized that the transformations which take place in the course of putrefaction are the work of various bacteria which bring about a true digestion of the material through the proteolytic enzymes which they secrete. Yet this special fermentation differs from natural digestion due to the activity of trypsin and erepsin in that the former yields gaseous products $\left(\mathrm{CO}_{2}, \mathrm{CH}_{4}, \mathrm{~N}_{2}, \mathrm{H}_{2} \mathrm{~S}, \mathrm{PH}_{3}\right)$; mercaptans, volatile acids, and aromatic acids, amines, phenol, :ndol, and scatol, and finally ptomaines, products not found in natural digestions.

To explain the presence of these substances as being characteristic of putrefaction, they were formerly regarded as the result of the vegetative activity of the bacteria in question, that is to say, as excreted products elaborated in the cell by an unknown mechanism. A great step was made in this direction upon the recognition of the existence of amidases and their rôle in the decomposition of amino-acids, an activity already discussed in a previous chapter. Thereafter it was concluded that putrefaction is purely an enzymic phenomenon in which various trypsins and amidases of bacterial origin intervene, and that the different products formed in the course of this thorough decomposition of albuminoid material are all the result of well-known chemical reactions. However, these general considerations must now be further defined.

The different bacteria which are encountered in a spontaneous putrefaction belong to various species. Among the most active have been noted: The family of Proteus, B. putrificus coli (Bienstock), B. perfringens (Veillon and Zuber), Micro- 
coccus flavus liquefaciens (Fluegge), B. gracilis putidus (Tissier and Martelly), B. diplococcus griseus non liquefaciens (Tissier and Martelly), B. bifermentans sporogenes (Tissier and Martelly), B. coli communis (Escherich), Streptococcus pyogenes (Doléris and Pasteur) and Staphylococcus pyogenes albus (Rosenbach), etc. These bacteria are very widely distributed. The following is, according to Cantu, the distribution of Bacillus proteus in nature:

Distribution of B. Proteus.

\begin{tabular}{|c|c|c|c|}
\hline Substances analyzed. & Examinations. & Positive tests. & Percentages. \\
\hline Air of the laboratory. & 100 & o & o \\
\hline Potable water....... & 80 & I & I. 25 \\
\hline Raw sausages... & 30 & ro & $33 \cdot 3$ \\
\hline Cheeses........... & 20 & 3 & 15 \\
\hline Melon............. & 30 & 7 & $23 \cdot 3$ \\
\hline Rotten meat....... & 22 & 22 & 100 \\
\hline Dung............. & 25 & 20 & 80 \\
\hline Normal human dejecta....... & 50 & 15 & 30 \\
\hline Diarrhœal human dejecta.... & 40 & I 6 & 40 \\
\hline Garden soil. . . . . . . $\ldots \ldots \ldots \ldots$ & 52 & 23 & 44.2 \\
\hline
\end{tabular}

According to this table, B. proteus is found especially in substances undergoing decomposition. Its presence is constant in rotten meat, is very frequent in dung, and is met in large amounts in dejecta, even normal ones. However, it may likewise be detected in foods that are wholesome in appearance.

The putrefactive bacteria are ordinarily anaerobic, like $B$. putrificus coli; nevertheless there are also very active ones which are aerobic, like $B$. coli communis. Moreover these different species do not all develop together but successively. Martelly studied the changes which a $\mathrm{e}$ produced in the putrefactive flora, collected at several consecutive periods, on fresh meat exposed to the air. He found first of all Microc. flarus, Staphylococcus albus, B. coli, Diploc. griseus, etc. Then, at the end of 3 or 4 days, when the acid reaction is less strong and the attack on the albumins is sufficiently pronounced, $B$. perfringens and $B$. sporogenes appeared. At the end of 8 to ro days he detected the presence of $B$. putidus, B. putrificus, Proteus zenkeri, etc. Finally, the first species to appear disappeared later, or formed spores, and after 3 months there remained only $B$. putrificus, B. putidus and Diploc. griseus. 
This evolution in the flora evidently corresponds to differences in the secretions of the bacterial species considered, those appearing last not being able to develop on natural albuminoid material, but rather on substances already transformed. We shall, moreover, see a new proof of this diversity of enzyme secretions when we study the chemical reactions influenced by the different putrefactive bacteria, each acting in pure cultures on media of the same composition. First, it is necessary to describe all of the products elaborated in the course of a putrefaction. We should, in fact, consider this as an associated process, in which numerous bacterial species succeeding each other collaborate, as we have previously seen to be the case with meat left to itself.

Chemical Activity in the Course of Putrefaction. - If the enzymic secretions from the different putrefactive bacteria are examined, it is found that none of them contains pepsin, that is to say, none contains an enzyme capable of acting in distinctly acid media. On the other hand, several contain trypsins more or less active, those of Staphylococcus pyog. albus, B. putrificus, and $B$. perfringens being particularly easy to characterize. It is to be noted in passing that Proteus vulgaris secretes trypsin abundantly, while Proteus zenkeri does not secrete it at all. Furthermore, we sometimes find certain differences in the activity of these enzymes, some attacking fibrin and not gelatin, others doing the opposite. However, under the influence of these various trypsins, the albuminoid material is dissolved in the form of albumoses, peptones, and amino-acids. Other bacterial species of a peptolytic nature then intervene, and by means of their erepsin advance the hydrolysis of the products formed. It should, however, be noted that the hydrolysis produced by putrefactive enzymes, while more thorough than that effected by the ordinary digestive enzymes, pepsin or trypsin, is not yet complete. Even in very advanced putrefactions, nitrogenous compounds are always found that still give the reactions for albuminoids. Finally the amidases, which are likewise secreted by all these bacteria, enter in and bring about the formation of volatile acids and amines, as well as phenol and indol derivatives. 
The following are the numerous substances which appear in the course of a putrefaction in addition to the gaseous products already noted and the residual peptones: (I) ammonia and amines: ethylamine, propylamine, and trimethylamine; (2) volatile acids, comprising all the members of the fatty series up to caproic acid. They are sometimes normal acids, sometimes their isomers; propionic acid is less frequent than the others; formic acid is quite rare; acetic and butyric acid are especially common. (3) Aromatic acids and oxyacids, like phenylpropionic, oxyphenylacetic, and oxyphenylpropionic acids; (4) phenol, indol, scatol, pyrrol, and its derivatives, these bodies sometimes being in very small quantities, or even completely absent; (5) sulphur derivatives, like methyl-mercaptan; (6) various amino-acids, leucin, tyrosin, tryptophane, and sometimes glycin, creatinin, etc.; (7) various ptomaines, like putrescin and cadaverin, the guanidins, cholin and neurin, pyridin, hydrocollidin, etc.

It has already been shown, in the chapter on the amidases, by what chemical reactions ammonia and the volatile fatty acids are produced. The liberation of the fatty amines, like monoethylamine, probably results from the activity of special amidases, acting this time on amino-acids substituted in the $\mathrm{NH}_{2}$ group. At least that is what is observed with trimethylamine, which is derived from betain. The aromatic acids and oxyacids come from the decomposition of amino-acids with a benzene or indol nucleus, in the same manner as occurs with the corresponding members in the fatty series. We have, for example:

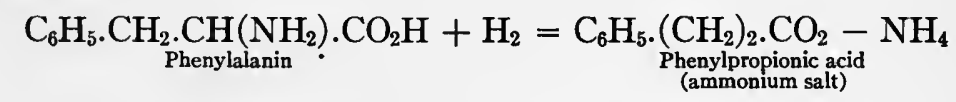

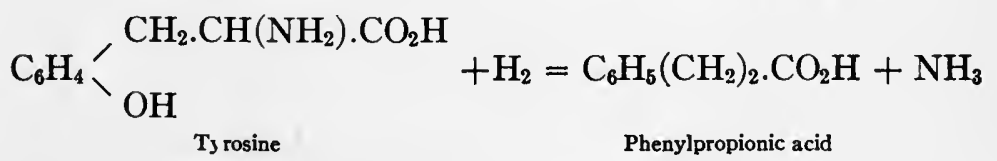

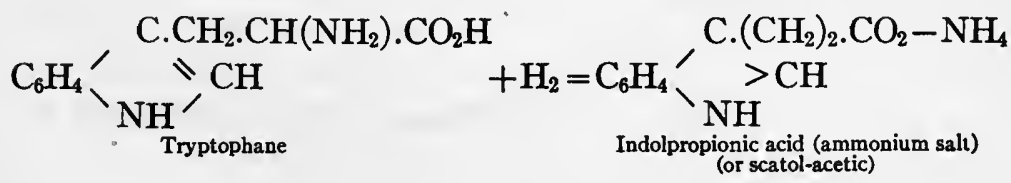


However, it is to be noted that the deamidization of aromatic amino-acids is often accompanied by a diminution in the number of carbon atoms in the chain united to the nucleus. Now we have previously recognized that the amidases act by reduction or by hydrolysis. In the first case, the production of hydrogen resulting from the decomposition of the water entails a corresponding liberation of oxygen, which at once unites with the easily oxidizable substances present. Generally, it is the carbohydrates present in the medium that are thus oxidized and are converted into $\mathrm{CO}_{2}$ and $\mathrm{H}_{2} \mathrm{O}$. But this oxygen can also be taken up by the aromatic amino-acids. Thus:

$$
\begin{aligned}
& \mathrm{C}_{6} \mathrm{H}_{5} \cdot\left(\mathrm{CH}_{2}\right)_{2} \cdot \mathrm{CO}_{2} \mathrm{H}+\frac{3}{2} \mathrm{O}_{2}=\mathrm{C}_{6} \mathrm{H}_{5} \cdot \mathrm{CH}_{2} \cdot \mathrm{CO}_{2} \mathrm{H}+\mathrm{CO}_{2}+\mathrm{H}_{2} \mathrm{O} \\
& \text { Phenylpropionic acid } \\
& \text {.Phenylacetic acid } \\
& \mathrm{C}_{6} \mathrm{H}_{5} \cdot \mathrm{CH}_{2} \cdot \mathrm{CO}_{2} \mathrm{H}+\frac{3}{2} \mathrm{O}_{2}=\mathrm{C}_{6} \mathrm{H}_{5} \mathrm{CO}_{2} \mathrm{H}+\mathrm{CO}_{2}+\mathrm{H}_{2} \mathrm{O} \text {. } \\
& \text { Phenylacetic acid } \\
& \text { Benzoic acid }
\end{aligned}
$$

In the same way $p$-oxyphenolpropionic acid gives $p$-oxyphenylacetic acid, and indolpropionic acid furnishes indolacetic or scatolcarbonic acid.

It is to a process of this nature that is due the formation of cresol and phenol, these bodies resulting from the decomposition of aromatic oxyacids, which thus play the part of intermediate products. We have:

$$
\begin{aligned}
& \underset{\text { p-oxyphenylacetic acid }}{\mathrm{C}_{6} \mathrm{H}_{4}} \underset{\mathrm{OH}}{>\mathrm{CH}_{2} \mathrm{CO}_{2} \mathrm{H}}=\mathrm{C}_{6} \mathrm{H}_{4} \underset{\text {-cresol }}{>} \underset{\mathrm{OH}}{\mathrm{CH}_{3}}+\mathrm{CO}_{2} . \\
& \underset{\text { p-cresol }}{\mathrm{C}_{6} \mathrm{H}_{4}} \underset{\mathrm{OH}}{>} \underset{\mathrm{OH}}{\mathrm{CH}_{3}}+\frac{3}{2} \mathrm{O}_{2}=\underset{\text { p-oxybenzoic acid }}{\mathrm{C}_{6} \mathrm{H}_{4}} \underset{\mathrm{OH}}{>\mathrm{CO}_{2} \mathrm{H}}+\mathrm{H}_{2} \mathrm{O} \text {. } \\
& \underset{\text { p-oxybenzoic acid }}{\mathrm{C}_{6} \mathrm{H}_{4}} \underset{\mathrm{OH}}{\mathrm{CO}_{2} \mathrm{H}}=\underset{\text { Phenol }}{\mathrm{C}_{6} \mathrm{H}_{5} \mathrm{OH}}+\mathrm{CO}_{2} .
\end{aligned}
$$

Likewise, the formation of scatol and indol is explained by analogous reactions: 


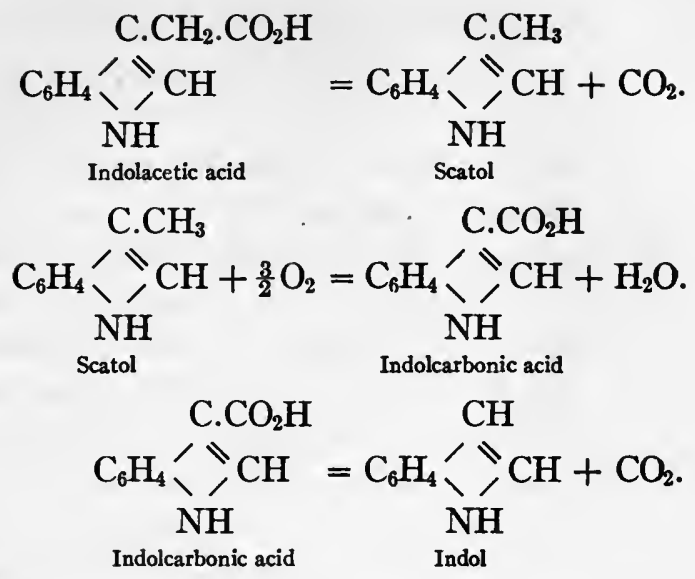

Thus, indol and scatol result from the progressive transformation of tryptophane. F. Hopkins and W. Cole have verified this experimentally by showing that the action of the bacteria on tryptophane that is added to a gelatin undergoing putrefaction gives indol, scatol, scatol-carbonic acid, and scatol-acetic acid. If the fermentation is well carried out, the scatol may reach 65 per cent of the theoretical yield. Moreover, E. and H. Salkowski had already previously recognized that the basic material for the production of scatol is scatol-carbonic acid, which they had isolated from products of putrefaction of albuminoids.

The preceding reactions also explain the formation of certain aromatic amines. Thus:

$$
\begin{aligned}
& \mathrm{C}_{6} \mathrm{H}_{5} \cdot \mathrm{CH}_{2} \cdot \mathrm{CH}\left(\mathrm{NH}_{2}\right) \mathrm{CO}_{2} \mathrm{H}=\mathrm{C}_{6} \mathrm{H}_{5} \cdot\left(\mathrm{CH}_{2}\right)_{2} \cdot \mathrm{NH}_{2}+\mathrm{CO}_{2} \text {. } \\
& \text { Phenylalanin Phenylethylamine }
\end{aligned}
$$

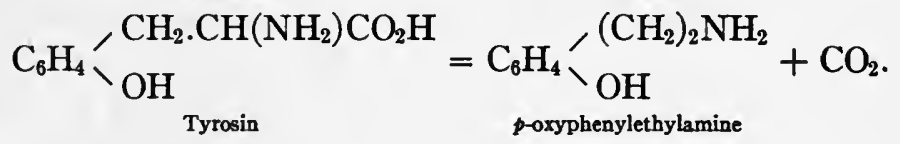

Sometimes a simplification of the carbon chain is accomplished by other means than by the loss of $\mathrm{CO}_{2}$. Thus, when tyrosin is allowed to putrefy in place of the $p$-oxyphenylpropionic acid, there is often found a certain quantity of $p$-oxyphenylacetic acid: 


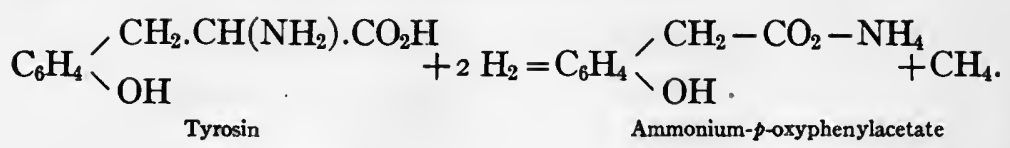

The above reaction explains the formation of the methane gas that is found in albuminoid putrefactions.

It has just been shown hat the action of amidases on aromatic derivatives is often accompanied by an oxidation. The following is a new example, though here the deamidization is followed by a fixation on the benzene nucleus of one or more hydroxyl groups. Thus the formation of phenol at the expense of the phenylalanin has been observed on different occasions. When phenylalanin is allowed to ferment in the presence of small quantities of peptone, there is found in the liquid oxyphenylpropionic acid, oxyphenylacetic acid, as well as phenol. Now, the quantity of oxyacids formed considerably exceeds in weight that which corresponds to the tyrosin of the peptone; the phenylalanin is thus transformed into oxyacids according to the reactions:

$$
\begin{gathered}
\mathrm{C}_{6} \mathrm{H}_{5} \cdot \mathrm{CH}_{2} \cdot \mathrm{CH}\left(\mathrm{NH}_{2}\right) \cdot \mathrm{CO}_{2} \mathrm{H}+\mathrm{H}_{2}=\mathrm{C}_{6} \mathrm{H}_{5} \cdot\left(\mathrm{CH}_{2}\right)_{2} \cdot \mathrm{CO}_{2}-\mathrm{NH}_{4} \cdot \\
\mathrm{C}_{6} \mathrm{H}_{5} \cdot\left(\mathrm{CH}_{2}\right)_{2} \cdot \mathrm{CO}_{2} \mathrm{H}+\mathrm{O}=\mathrm{C}_{6} \mathrm{H}_{4} \backslash \underset{\mathrm{OH}}{\left(\mathrm{CH}_{2}\right)_{2} \cdot \mathrm{CO}_{2} \mathrm{H}} \\
\text { Phenylpropionic acid } \\
\text { Oxyphenylpropionic acid }
\end{gathered}
$$

Here a reduction and an oxidation has taken place successively on the same nucleus with a net result equivalent to a simple hydrolysis. Thus, the oxyphenylpropionic acid, by progressive oxidation, loses I, 2, and 3 atoms of carbon, and is finally converted into phenol.

The formation of homogentisinic acid, or dioxyphenylacetic acid,

$$
\mathrm{C}_{6} \mathrm{H}_{3} \backslash \underset{(\mathrm{OH})_{2(1.4)}}{\mathrm{CH}_{2} . \mathrm{COOH}}
$$

gives us the proof of a deamidization followed by the attaching of two hydroxyl groups. Falta and Langstein have demonstrated that the quantity of this acid appearing in alcaptonuria considererably exceeds the quantity of tyrosin disappearing, and they 
have found by experiment that the phenylalanin produces dioxyacids in the same manner as does tyrosin.

From the preceding, it should be remembered that tyrosin, phenylalanin, tryptophane, and the amino-acids substances that remain unchanged in he hydrolyses realized by the digestive enzymes, are, on the contrary, the substances which are the most easily transformed in putrefactions, since they give rise to numerous derivatives, oxyacids, indol, and phenols. One of the characteristics of the action o the amidases secreted by the putrefactive bacteria appears to be the fact that they address themselves preferably to the aromatic substances which have come from proteolysis.

In addition to these various products, more or less characteristic of putrefaction, there are still others which come from the action of amidases on the diamines resulting from the thorough decomposition of the albuminoid molecule. Thus it is that arginin, the hexonic base which we encounter in most of the products of proteolysis, is transformed first by the action of arginase into urea and into ornithin:

$$
\begin{aligned}
\mathrm{NH}=\mathrm{C}\left(\mathrm{NH}_{2}\right) & -\mathrm{NH}-\left(\mathrm{CH}_{2}\right)_{3}-\mathrm{CH}\left(\mathrm{NH}_{2}\right)-\mathrm{CO}_{2} \mathrm{H}+\mathrm{H}_{2} \mathrm{O} \\
& =\mathrm{CO}\left(\mathrm{NH}_{2}\right)_{2}+\mathrm{NH}_{2} \cdot\left(\mathrm{CH}_{2}\right)_{3} \cdot \mathrm{CH}\left(\mathrm{NH}_{2}\right) \cdot \mathrm{CO}_{2} \mathrm{H}
\end{aligned}
$$

and that the ornithin thus formed is reduced under the influence of a special amidase to amino-valeric acid, a substance constantly recovered in the products of putrefaction:

$$
\mathrm{NH}_{2} \cdot\left(\mathrm{CH}_{2}\right)_{3} \cdot \mathrm{CH}\left(\mathrm{NH}_{2}\right) \cdot \mathrm{CO}_{2} \mathrm{H}+\mathrm{H}_{2}=\underset{\text { Aminthin }}{\mathrm{NH}_{2} \cdot\left(\mathrm{CH}_{2}\right)_{4} \cdot \mathrm{CO}_{2} \cdot \mathrm{NH}_{4}}
$$

Yet, ornithin can also be decomposed in another manner and thus give putrescin or tetramethylene-diamine:

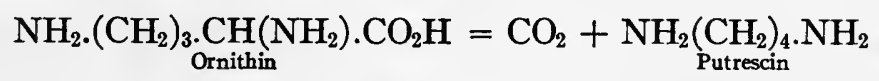

In the same way lysin, which is another hexonic base, is transformed into cadaverin and $\mathrm{CO}_{2}$ :

$$
\mathrm{NH}_{2} \cdot\left(\mathrm{CH}_{2}\right)_{4} \cdot \underset{\text { Lysin }}{\mathrm{CH}}\left(\mathrm{NH}_{2}\right) \cdot \mathrm{CO}_{2} \mathrm{H}=\mathrm{CO}_{2}+\underset{\text { Cadaverin }}{\mathrm{NH}_{2}} \cdot\left(\mathrm{CH}_{2}\right)_{5} \cdot \mathrm{NH}_{2}
$$


The method of formation of these two ptomaines represents a reaction typical of putrefaction, and results from the exceptional rather than the normal behavior of amidase. We have already noted a like transformation with regard to the production of phenylethylamine from phenylalanin. As another example, by allowing amino isovaleric acid to putrefy Neuberg and Karczag have observed that in addition to valeric acid there is also produced isobutylamine.

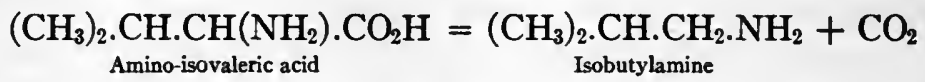

Putrescin and cadaverin are formed by introducing in a putrefying medium either arginin, the ornithin which is derived from it, or lysin. However, these ptomaines, once formed, offer a very pronounced resistance to the amidase: added to a nutritive medium composed of peptone and asparagin, they remain unchanged, even after the nutritive materials are totally exhausted. These experiments have been made with $B$. proteus and $B$. bienstockii. However, it should be noted that in spontaneous putrefaction of albuminoid material, we find no gradual or regular accumulation of ptomaines; we should conclude from this that in natural fermentations there exist agents which destroy these ptomaines. But, at all events these are not ordinary amidases.

Neurin, another ptomaine which is also found in the products of putrefaction, is derived, by loss of water, from cholin, which is the constituent base of the lecithins and of the phosphoretted fats distributed in numerous tissues and very abundantly in in the brain:

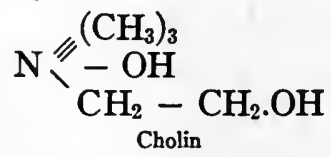$$
\mathrm{N} \underset{\text { Neurin }}{\stackrel{\left(\mathrm{CH}_{3}\right)_{3}}{-\mathrm{OH}}}
$$

Putridin, $\mathrm{C}_{11} \mathrm{H}_{26} \mathrm{~N}_{2} \mathrm{O}_{3}$, which is another toxic material of illdefined structure, was discovered by Ackermann in the products of advanced decomposition. 
Finally pyrrol and its derivatives result from the decomposition of hemoglobin. As to the pyridin compounds, whose presence is still more exceptional, they come also from the transformation of special nitrogenous compounds.

As to the origin of the sulphur compounds in putrefaction, we know but very little. However, this fact is to be noted, that whereas by the action of the digestive enzymes - pepsin, trypsin, and erepsin - the sulphur contained in albuminoid material does not undergo noticeable transformation, on the other hand, under the influence of putrefactive enzymes this sulphur undergoes a change, and we get noticeable quantities of sulphur derivatives such as $\mathrm{H}_{2} \mathrm{~S}$ and the mercaptans. These last substances result apparently from the activity of reducing enzymes, exhibiting a special affinity for sulphur, on the cystein and taurin groups liberated by proteolysis, an activity of the same nature, doubtless, as that characteristic of the bacteria that decompose sulphates, or again of some of the philothionic bacteria.

As to the formation of gaseous products, we also are totally lacking in accurate data with the exception of that concerning carbon dioxide and methane. We have seen that methane can arise from the reduction of tyrosin, and that, on the other hand, carbonic acid appears in most of the reactions previously described. This gas likewise results from the oxidation of various substances, the combustion being effected by means of the oxygen coming from the decomposition of water. However, we know nothing as to the mechanism by which nitrogen, hydrogen, hydrogen sulphide, and hydrogen phosphide are formed. At the most, in such a reducing medium, we can comprehend that hydrogen is separated by the action of special reductases either in the free state or combined with sulphur or phosphorus. But as to the nitrogen, if it really comes from albuminoid material, a fact which is not established with certainty, its formation remains as yet totally unexplained. It is true that we should rather attribute the formation of this gas to the reduction of the nitrates present in the putrefaction liquids. 
Variation in the Products Which Appear. - The proportion of the numerous products noted above varies perceptibly from one fermentation to the other. Sometimes this result is the consequence of a desired change in the experimental conditions. In other cases, when the cultures used are not pure, it might be that the variation is due rather to a modification in the flora which had produced the effect under consideration. For example, Simnitzki made the following observations by inoculating meat, suspended in slightly alkaline solution, with cultures of putrefactive bacteria. These cultures were not pure, but were obtained from a very active natural putrefaction. Comparative tests were made, both without addition of sugar and in the presence of 25 and $50 \mathrm{~g}$. of sugar per $100 \mathrm{~g}$. of albuminoid matter. The analyses, made after 4 days, gave:

Putrefaction of Meat With and Without Sugar.

\begin{tabular}{|c|c|c|c|}
\hline Products measured. & $\begin{array}{l}\text { Without addi- } \\
\text { tion of sugar. }\end{array}$ & $\begin{array}{l}\text { With } 25 \text { per. } \\
\text { cent of sugar. }\end{array}$ & $\begin{array}{l}\text { With } 50 \text { per } \\
\text { cent of sugar }\end{array}$ \\
\hline \multirow{3}{*}{ Material decomposed $\ldots \ldots \ldots \ldots$} & \multicolumn{3}{|c|}{ Of albumin introduced. } \\
\hline & $\begin{array}{l}\text { Per cent. } \\
89\end{array}$ & $\begin{array}{c}\text { Per cent. } \\
74\end{array}$ & $\begin{array}{l}\text { Per cent. } \\
26.92\end{array}$ \\
\hline & \multicolumn{3}{|c|}{ Of albumin decomposed. } \\
\hline 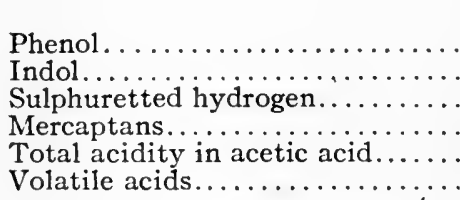 & $\begin{array}{l}\text { Per cent. } \\
0.43 \\
0.38 \\
0.17 \\
0.09 \\
25.20 \\
13.48\end{array}$ & $\begin{array}{l}\text { Per cent. } \\
0.23 \\
0.23 \\
0.19 \\
0.03 \\
24.40 \\
14.00\end{array}$ & $\begin{array}{l}\text { Per cent. } \\
0.00 \\
0.00 \\
0.004 \\
0 . \infty \\
9.20 \\
1.70\end{array}$ \\
\hline Ammonia............... $\left\{\begin{array}{l}a \\
b\end{array}\right.$ & $\begin{array}{r}5.40 \\
34.00\end{array}$ & $\begin{array}{r}2.90 \\
18.49\end{array}$ & $\begin{array}{l}0.80 \\
5.20\end{array}$ \\
\hline
\end{tabular}

$a$ : Of $100 \mathrm{~g}$. of albuminoid matter decomposed.

$b$ : Of $100 \mathrm{~g}$. total nitrogen.

We observe that the formation of the different substances characteristic of putrefaction, among others that of phenol and indol, is greatly influenced by the addition of sugar. Thus, with $5 \circ$ per cent sugar none of these substances is produced (Column III). 
As these experiments were made with mixtures of various micro-organisms, an objection immediately arises. The variation in the proportion of the products elaborated might result from a modification in the flora, the strictly proteolytic bacteria having given place to those more greedy for carbohydrates. This objection could be justified up to a certain point from the fact that the quantity of decomposed albuminoid material is much smaller in the case where the fermentation is carried out in the presence of an excess of sugar than when the fermentation takes place in the absence of this substance. This objection, however, does not appear to be valid. This difference of effect is the consequence of the double faculty which certain putrefactive bacteria possess of attacking at the same time both sugars and albuminoids. Péré found, in fact, that $B$. coli communis, which is, as we know, an active ferment for sugar, evinces curious peculiarities when cultivated on a mixed medium of sugar and nitrogen. In the presence of a very small quantity of nitrogenous material, ammoniacal salts or peptone, the sugar is transformed into lactic acid. If the quantity of peptone exceeds 0.4 per cent, the formation of lactic acid does not take place. As to the peptone, the bacteria do not touch this substance to an appreciable extent except when the sugar has disappeared. As long as there is sugar there is no indol, the bacterium apparently preferring to nourish itself with carbon in the form of sugar material rather than with the peptone. Martelly has continued this study by cultivating $B$. coli in media containing increasing quantities of peptone and of glucose without the addition of calcium carbonate. After 8 days incubation, during which the reaction was noted each day, the products were analyzed. The figures given correspond to I000 parts of liquid: 
action of B. Coli on Peptone With and Without Sugar.

\begin{tabular}{|c|c|c|c|c|c|}
\hline Glucose. & Peptone. & Reaction of the medium. & $\begin{array}{c}\text { Acidity in } \\
\mathrm{H}_{2} \mathrm{SO}_{1}\end{array}$ & $\begin{array}{l}\text { Alkalinity } \\
\text { in } \mathrm{NH}_{\mathrm{s}}\end{array}$ & Indol. \\
\hline $\mathbf{I}$ & 10 & Always alkaline & $\ldots$ & I.39 & Plain react. \\
\hline 2 & ro & id. & $\ldots$ & $\mathrm{I} .2 \mathrm{I}$ & id. \\
\hline 5 & ro & id. & $\ldots$ & 1.04 & traces \\
\hline I0 & 10 & Acid, the 3 rd day alk. & $\ldots$ & 0.40 & o \\
\hline 15 & I0 & Always acid & 0.46 & $\ldots$ & o \\
\hline 20 & I0 & id. & 0.46 & $\ldots$ & ० \\
\hline 30 & IO & id. & 0.46 & $\ldots$ & $4=$ \\
\hline 35 & I0 & id. & 0.46 & $\cdots$ & $\circ$ \\
\hline
\end{tabular}

This table shows that indol is formed only in cases where the quantity of glucose is small, an excess preventing the decomposition of the albuminoid material from taking place up to this limit. However, the peptone is always attacked, for in all the tests, whether the reaction is alkaline or acid, ammonia free or combined is present in the liquid. From this experiment it appears, further, that the fermentation of sugar stops when the acidity has become equivalent to $0.46 \mathrm{~g} . \mathrm{H}_{2} \mathrm{SO}_{4}$ per liter, that is to say when $15 \mathrm{~g}$. of glucose have been added, since the excess remains in the liquid. But if only to g. of sugar per liter are added all disappears. It is found under these circumstances that the quantity of peptone may be increased, for example to $40 \mathrm{~g}$. per liter per to g. of sugar, without getting a formation of indol. There is here a deviation, under the influence of the sugar, from the usual method of fermentation of peptone by $B$. coli, which is to give indol. We have previously observed a similar deviation under the influence of small quantities of peptone from the normal method of fermentation of sugar by this bacterium, namely for the formation of lactic acid.

We may conclude from the preceding that the presence of indol is not an infallible characteristic of putrefaction. Numerous aerobes and even certain anaerobes, like $B$. putrificus, which cause the disintegration of albumin to the most simple substances, do not, however, yield phenol or indol. The production of indol indicates merely a mode of attack, a particular direction given to the dismemberment. In other words, it corresponds 
only to the secretion of an amidase specific for this transformation. Finally, from the point of view of intestinal digestion, these data are likewise interesting, for they show us that a variation in diet, an excess of carbohydrate substances for example, may modify in one direction or another the fermentations which originate in the intestine, and which contribute to the increase or diminution of the production of these ultimate compounds in general so toxic for the organism.

As to the other substances found in the course of putrefaction, we likewise possess some data as to their formation. Ammonia, especially, although always present in the products of putrefaction, can undergo very considerable variations as to the quantity present. It can attain, in exceptional conditions, as high as 40 per cent of the total nitrogen. We shall have occasion moreover, to return to this question when discussing the industrial utilization of nitrogenous residues. Besides ammonia, as indicated, we also encounter amines. Thus Emmerling claims to have shown the presence of trimethylamine in a large number of putrefactions. In particular, when Proteus vulgaris was made to act on gluten, trimethylamine and phenol were found in the liquid; by using Staphylococcus pyogenes, there was formed, in addition to trimethylamine and phenol, indol and scatol. Sometimes we find trimethylamine, without scatol, indol, or sulphuretted hydrogen. Such is the case with $B$. fluorescens liquefaciens, which gives, tri- and also monomethylamine from gluten. It should, however, be noted that Effront has analyzed for trimethylamine, by means of the very exact method of François (C. R., r907, (r), p. 567), in a large number of putrefactions obtained with albumin, fibrin, gluten, etc., and that he has never detected it. Only those materials containing betain, like beet waste liquors, are capable of giving rise to this tri-substituted amine.

The production of volatile fatty acids in putrefaction is beyond doubt, since a whole industry has been created in order to recover these products through the fermentation of the byproducts of the distillery. Emmerling, by subjecting albumin 
to putrefaction, obtains 0.3 per cent of acetic acid, 0.06 per cent of propionic acid, $\mathbf{x} .25$ per cent of butyric acid, and 0.06 per cent of the higher acids, in addition to 0.4 per cent of trimethylamine. Yet Effront has found that the quantity of these volatile acids formed, as well as their composition, varies with the nature of the bacteria used. The species plainly characterized as putrefactive, which we know to be incapable of contributing in large measure to the liberation of ammonia, are very poor producers of acid as compared with other bacteria better suited to this sort of work due to their higher content in the corresponding amidases. This subject will be discussed further in the chapter on Recovery.

Among the products of putrefaction, there are some which are ordinarily toxic, and which are sometimes regarded as being characteristic of putrefaction. These are the ptomaines. With the exception of some, like putrescin and cadaverin, which are actually produced by the action of special amidases, it appears that these complex substances are not derived directly from albuminoids, but are rather cellular products, which not only are secreted by the putrefactive bacteria but are also encountered, although in a less dangerous state, as leucomaines in healthy tissues, such as fresh meat for example. It thus appears, in passing, that in the disputed question as to a satisfactory criterion for putrefaction, toxicity should not take precedence over the chemical changes produced. It must even be noted that the injurious substances are always intermediate products. It is, in fact, much more dangerous to eat meat just beginning to putrefy than that which is completely decomposed. This fact is of great importance from the point of view of medico-legal investigations, since, if haste is not made in analyzing the suspected foods, the transformations go on and the incriminating substance may escape investigation. This is probably the basis for the opinion given out by Duclaux, that if the phenol and indol, already appearing at the beginning of putrefaction, are not found at the end, it is because they have disappeared in the course of fermentation. However, 
the facts do not justify this opinion; when we do not find indol present, it is because it was never formed.

We have just noted some of the variations which occur in the composition of the products of putrefaction according to experimental conditions, especially according to modifications of the bacterial species and the medium in which they react. The following experiments by Martelly show that the principal natural albuminoids are more or less attacked by the various putrefactive bacteria which are most widely distributed. The substances studied were inoculated with pure cultures, then the flasks were emptied of air and sealed, with the exception of the corresponding tests made with $B$. proteus which are aerrobic. After a month at $37^{\circ}$, the broths were analyzed, with the following results:

Putrefaction of Various Albumins.

\begin{tabular}{|c|c|c|c|c|c|}
\hline Albumins. & $\begin{array}{l}\text { Bac. putri- } \\
\text { ficus. }\end{array}$ & $\begin{array}{l}\text { Bac. colico- } \\
\text { genes. }\end{array}$ & $\begin{array}{l}\text { Bac. sporo- } \\
\text { genes. }\end{array}$ & $\begin{array}{l}\text { Bac. perfrin- } \\
\text { gens. }\end{array}$ & $\begin{array}{l}\text { Bac. proteus } \\
\text { vulg. }\end{array}$ \\
\hline . & \multicolumn{5}{|c|}{ Material dissolved (per cent of material introduced). } \\
\hline $\begin{array}{l}\text { Blood albumin...... } \\
\text { Fibrin. ............ } \\
\text { Alb. of yolk of egg... } \\
\text { Vegetable albumin. }\end{array}$ & $\begin{array}{l}91 \\
88 \\
97 \\
6 \mathbf{I}\end{array}$ & $\begin{array}{l}88 \\
88 \\
97 \\
64\end{array}$ & $\begin{array}{l}86 \\
88 \\
98 \\
72\end{array}$ & $\begin{array}{r}13 \\
0 \\
22 \\
28\end{array}$ & $\begin{array}{l}66 \\
40 \\
85 \\
44 \cdot 5\end{array}$ \\
\hline & \multicolumn{5}{|c|}{ Dissolved nitrogen (per cent of nitrogen introduced). } \\
\hline 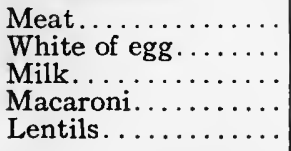 & $\begin{array}{r}82 \\
100 \\
93 \\
45 \\
33\end{array}$ & $\begin{array}{r}89 \\
100 \\
97 \\
62 \\
57\end{array}$ & $\begin{array}{r}100 \\
100 \\
98 \\
64 \\
53\end{array}$ & $\begin{array}{l}\text { No growth } \\
\text { Id. } \\
72 \\
\text { No growth } \\
\text { Id. }\end{array}$ & $\begin{aligned} 18 \\
2 \\
26 \\
45 \\
2.8\end{aligned}$ \\
\hline
\end{tabular}

We thus see that the solution of various substances, due to the activity of the same bacterium, varies, and that in a general way the most powerful proteolytics are obligatory anaërobic basteria. However, if the amino-acids formed are determined according to the method of Sörensen, we find that the percentages are very variable and do not correspond at all with that expected, judging from the amounts dissolved. 
Amino-acids Formed in Putrefaction.

\begin{tabular}{|c|c|c|c|c|c|}
\hline Albumins. & $\begin{array}{l}\text { B. putrifi- } \\
\text { cus. }\end{array}$ & $\begin{array}{l}\text { B. colica- } \\
\text { genes. }\end{array}$ & $\begin{array}{l}\text { B. sporo- } \\
\text { genes. }\end{array}$ & $\begin{array}{l}\text { B. perfrin- } \\
\text { gens. }\end{array}$ & B. proteus. \\
\hline & \multicolumn{5}{|c|}{ Amino-acids (calc. in glycin.) per cent of dissolved album. } \\
\hline $\begin{array}{l}\text { Blood albumin......... } \\
\text { Alb. of yolk of egg..... } \\
\text { Vegetable alb......... }\end{array}$ & $\begin{array}{l}\text { I. } .60 \\
\text { I. } 40 \\
0.28\end{array}$ & $\begin{array}{l}1.40 \\
1.30 \\
0.18\end{array}$ & $\begin{array}{l}\text { I. } 80 \\
\text { I.60 } \\
\cdots\end{array}$ & $\begin{array}{l}1.60 \\
2.50 \\
0.50\end{array}$ & $\begin{array}{l}0.43 \\
0.34 \\
0.55\end{array}$ \\
\hline & \multicolumn{5}{|c|}{ Amino-acids (calc. in glycin.) per cent of dissolved nitrogen. } \\
\hline 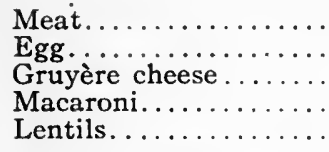 & $\begin{array}{l}2.00 \\
1.08 \\
1.70 \\
1.00 \\
3.50\end{array}$ & $\begin{array}{l}\text { I. } .70 \\
1.08 \\
1.70 \\
0.77 \\
4.00\end{array}$ & $\begin{array}{l}0.69 \\
\cdots \\
\cdots \\
0.62 \\
0.90\end{array}$ & $\begin{array}{l}\text { No growth } \\
\text { Id. } \\
\text { Id. } \\
\text { Id. } \\
\text { Id. }\end{array}$ & $\begin{array}{l}0.50 \\
\ldots \\
\ldots \\
\cdots \\
\cdots\end{array}$ \\
\hline
\end{tabular}

Thus the tests conducted with lentils, in which only a slight solution of nitrogenous material occurred, nevertheless indicated a very strong proteolytic action.

These data further confirm the great diversity of enzymes coming into play in these complex bacterial reactions, and they explain satisfactorily why it is impossible to give a simple and unchangeable scheme for putrefaction. Both the quality and the quantity of the products formed are dependent upon the nature of the acting enzymes, which, themselves, are functions of the species of bacteria present, of the albuminoid substance to be transformed, and also of the physical and chemical conditions of the medium.

\section{BIBLIOGRAPHY.}

Tissier et Martelly. Ann. Inst. Past., I902, (I6), p. 855.

Martelly. La digestion animale et la putrefaction, Thèse, Paris, 1902.

Cantu. Ann. Inst. Past., 1912, (25), p. 526.

Simnitzki. Zeits. f. physiol. Chem., 1903, (39), p. 99.

NeNcki. Journ. f. prakt. Chem., (1 7), p. I05.

PÉré. Ann. Inst. Past., I892, (6).

Emmerting. Ber. d. deuts. Chem. Ges., 1896, (29), p. 2721 ; I902, (35), p. 700 . ACKermann. Zeits. f. physiol. Chem., 1908, (54), p. I.

Martelly. Ann. Inst. Past., I9I2, (26), p. 525.

EFrront. Sur la fermentation putride, Mon. Scient., I9II, (2), p. 489. 
Bienstock. Arch.f. Hyg., 1901, (39), p. 390.

Bachmann. Dissert., Marbourg, I899. Maly Jahresberichte, 1900, (30), p. 186. E. u. H. Salkowski. Ber. d. deuts. Chem. Ges., 1880, (13), pp. 189 et 2217.

T. Hopkins a. S. Cole. Journ. of Physiol., I901, (27), p. 418; 1902, (29), p. 45 I. Falta u. Langstern. Zeits. $f$. physiol. Chem., r903, (37). p. 515.

C. Neuberg u. Karczag. Bioch. Zeits., I909, (18), p. 435. 


\section{$\S$ io. Lactobacillin and Simlar Products.}

The putrefactive bacteria, which play such an important rôle in the disorganization of organic material, are always found in the alimentary canal of man. Ingested with the food, they enter the intestine, a medium most favorable for their development. Their predominance and their virulence here are the indirect cause of a large number of diseases attending a faulty nutrition. Located in the intestine, they soon modify the glandular secretions, and passing into the pancreas and the liver, they sooner or later cause much trouble. Metchnikoff has made a masterly study of the intestinal flora of man. Of the large number of putrefactive ferments he found that only three were constantly present. These three were the bacillus first described by Welch and Nuttal, B. welchii, B. sporogenes, and the $B$. putrificus of Bienstock. To these he later added $B$. proteus, which is likewise always present and at times to excess. These four species predominate in the intestine.

In a general way, it is the putrefactive bacteria which elaborate the different products whose effect is manifested sometimes, although rarely, by an acute poisoning, but more often by a slow and chronic poisoning affecting the kidneys, the liver, the brain, and the arteries. Their influence is recognized in the origin of enteritis, nephritis, arterio-sclerosis, and some of the other diseases due to an internal intoxication. The principal toxic agents are phenol and indol. They are produced only in small quantities; but as this production is uninterrupted, their presence finally becomes disastrous to the organism.

The intestinal flora contains, furthermore, a series of nonpathogenic micro-organisms which, by the production of enzymes acting on the carbohydrates, the fats, or the albuminoids, contribute to the digestion of the foods which have escaped the action of the gastric and pancreatic secretions. Before the part played by these latter microbes became known it was at one time even believed that their aid was indispensable to animal life. 
The question as to this indispensability has been made the basis of numerous investigations, which have recently led to a definite solution. Thus, it has been proved that animals nourished with sterile foods can develop normally, without intestinal fermentation of a fetid or a gaseous nature. If then it is true that the intestinal flora has its use, it is not absolutely necessary; and as, moreover, its favorable action is counterbalanced by the accidents which it causes, it is easily understood why we seek to overcome it. Unfortunately, the means for attaining a direct suppression are lacking, for we cannot think, on the one hand, of using sterile foods, or on the other hand, of making antiseptic in a complete fashion the whole of the intestine. There then remain only indirect means, which consist in influencing the bacterial flora by changes of a chemical or biological nature brought about in the intestinal environment.

The intensity of putrefaction does not depend exclusively upon the greater or less quantity of putrefactive bacteria ingested with the food, but depends rather on the nature of the medium to be encountered in the intestine. The albuminoid materials, according to their origin, act differently with putrefactive bacteria; some easily decompose, others offer more or less resistance. The resulting chemical reactions, in particular the formation of phenol and indol, are likewise subject to wide variations, depending not only upon the albuminoid material used, but also upon the physical and chemical conditions of the medium, as seen in a preceding chapter. Furthermore, it must be noted that the presence of antagonistic enzymes in the intestine is likewise capable of influencing the course of putrefaction in one direction or another.

The diet imposed upon the sick, in cases of infectious diseases, is in reality only a means of creating an intestinal medium unfavorable, either to the development of putrefactive bacteria, or to the formation of toxins or other injurious substances. In the chapter on the functioning of the digestive glands, it was shown that, of all complete foods, milk is the only one 
which can be digested without the intervention of the psychic reflex. To this valuable property is also added that of not being putrescible. Exposed to the air, it is rapidly invaded by organisms producing lactic acid which protect it from putrefaction. Furthermore, the casein of milk, free from sugar, keeps much better than any other albuminoid food material. Milk is then quite naturally chosen as nutriment in all cases of intestinal infection.

Clotted milk, in the. forms of Yoghourt, Leben, Koumis, or of Képhir, is very much valued as food in certain parts of Bulgaria, Russia and Egypt, as well as in many other countries. Fermented milk, in fact, offers great advantages in various ways. First of all, it is unquestionable that the casein of the milk in this condition is more digestible than that of natural milk. Furthermore, due to its special aromatic flavor, fermented milk can be taken in larger quantities than ordinary milk. Finally, it keeps much better than the latter, an interesting fact from the economic point of view. To these essential qualities must be added the very important one indicated by Metchnikoff and Combe. These investigators see in fermented milk an antiputrefactive agent of great value. In reality, clinical experiments confirm the fact that sour milk considerably influences the progress of digestion in the large intestine. The putrefactions, so frequent in this region, are diminished or completely arrested. According to Combe, the antiputrefactive property is due to the lactic acid contained in the fermented milk; the casein, more or less coagulated during fermentation, arrives in the intestine while impregnated with lactic acid, and there causes a change of medium very unfavorable to the putrefactive bacteria. Metchnikoff, while not neglecting the rôle of the lactic acid carried by the milk, attributes more importance to the acid formed on the spot in the intestine itself. According to this scientist, the mechanism of the action consists in the change produced in the bacterial flora of the intestine. The lactic ferment introduced by the milk becomes implanted, develops, and thus opposes the development of 
the putrefactive micro-organisms, the latter finally disappearing.

The conception of Metchnikoff leads us to consider fermented milk, not exclusively as a food, but especially as a carrier of a biological agent indirectly favoring digestion. This opinion is interesting by reason of the great consequences involved. Yoghourt, Koumis and Képhir are prepared in the different countries under very unfavorable conditions. Besides the essential ferment which gives to the milk its special properties, there also develops a large number of foreign bacteria which hinder the principal fermentation and influence the product from the point of view of taste and preservation. As to the antiputrefying action, the wild flora of the fermented milk no longer come into play, but only certain species capable of developing in the intestine, and of striving victoriously with the putrefactive microbes.

The problem was then to isolate in pure cultures the bacteria of clotted milk, and to inoculate sterilized milk with these pure micro-organisms. For Yoghourt, at least, this problem is believed to be entirely and practically solved. Massol first isolated a lactic ferment, and Bertrand studied its action on casein and sugars. Considering this bacterium as the active agent of the product, it was called Bulgarian ferment. Yoghourt can now be prepared scientifically and according to a certain standard.

The theory of Metchnikoff has likewise resulted in introducing into medicinal practice the use of antiputrefactive ferments. As the specific action of fermented milk resides in the bacteria present, and not in the milk itself, the latter has been replaced by selected bacteria, capable of propagating themselves in the intestine. In this connection many new preparations have been proposed. Among these, Lactobacillin occupies the foremost place. It is presented to the public in the form of compressed products or powder, and there also exist cultures in milk and in sterile malt. Lactobacillin, from the first, was considered as a medicine, and its good effects have been estab- 
lished in a large number of cases. It is especially under the first two forms that the public has welcomed it. A diet, consisting of a lozenge or a powder taken after each meal is, in fact, much easier to follow than that based on the ingestion of fermented milk, whose flavor is not agreeable to all and which is certainly less convenient to use. According to the specifications given with the product, Lactobacillin contains Bulgarian ferment mixed with ordinary lactic ferments. An unquestionable proof of the success of this medicine consists in the fact that a whole series of similar products have been put on the market. At Brussels, especially, three factories make compressed products of medicinal lactic ferments. The writer has had occasion to experiment with the effect of Lactobacillin. In many instances he was able to note its efficacy in enteritis. On the other hand, the good results produced by Lactobacillin are controlled by the proportion of phenol, indol and of oxyacids; the observations made in this direction by various investigators fully confirm the data furnished by Metchnikoff and Combe.

Thus the hypothesis of Metchnikoff on the change in intestinal flora caused by fermented milk is verified by bacteriological and chemical analyses. This point appears to be beyond all discussion. But it still remains necessary to verify the mechanism of the action and to see if really, as is supposed, the therapeutic effect of fermented milk comes from the lactic acid. Bulgarian ferment which has been isolated from Yoghourt is a very active producer of acid. Inoculated into normal milk, it rapidly and almost completely transforms the lactose into lactic acid. From this it is deduced that in the organism a similar phenomenon takes place. This conclusion, however, requires verification. Above all else, it would be necessary to show that the ferment produces the same effect in the large intestine as it does in vitro. It would also be necessary to establish the presence in this region of appreciable quantities of free lactic acid after a treatment with fermented milk. These proofs have not been found. Moreover, we know that the content of the large intestine has, on the contrary, an alkaline 
reaction and that, further, the sugar necessary for the formation of lactic acid does not exist at this part of the alimentary canal.

The writer has arrived at quite a different conception of the process after tests which he undertook with Bulgarian ferment, as well as with the different medicinal lactic ferments proposed in recent years. Bertrand had studied the action of the Bulgarian ferment, either in its natural medium, milk, or in artificial media with additions of large quantities of sugar. The results obtained under these conditions led him to conclude that the principal function of this micro-organism is to produce acid, while its proteolytic properties are but slight. The very accurate data of Bertrand have been erroneously applied to intestinal fermentation. The conditions which this microbe encounters in the intestine are entirely different from those realized in Bertrand's experiments. The ferment here finds no more sugar, and so it produces no lactic acid; but its proteolytic activity, which formerly was entirely latent, is now, on the contrary, accelerated. This bacterium, which was at first a very excellent ferment for carbohydrates, has become a ferment for nitrogenous materials. In view of its new rôle, it secretes tryptases and amidases abundantly, and thoroughly transforms the residues from albuminoid foods. The antiputrefactive action of Bulgarian ferment would then not consist in the formation of lactic acid, but rather in the struggle for the nitrogenous medium, a strife for which it is better equipped than the putrefactive bacteria.

The development of the proteolytic properties of Bulgarian ferment can be observed by cultivating this bacterium in sterilized milk, with the addition of sterile calcium carbonate. A flask of milk is inoculated, and then left in the incubator at $40^{\circ}$. After Io days, a second flask is inoculated with fermented milk coming from the first and this is repeated every ro days. The following are the results of analyses of the various cultures: 


\begin{tabular}{|c|c|c|c|c|c|c|}
\hline \multicolumn{4}{|c|}{ No. of the culture and experim. conditions. } & \multirow{2}{*}{$\begin{array}{l}\text { Total nitrogen. } \\
\text { Gram, per cent. } \\
0.500\end{array}$} & \multirow{2}{*}{$\begin{array}{c}\text { Soluble nitro- } \\
\text { gen. } \\
\text { Gram, per cent. } \\
\text { O. I09 }\end{array}$} & \multirow{3}{*}{$\begin{array}{c}\begin{array}{c}\text { Soluble nitro- } \\
\text { gen, per ccnt } \\
\text { total nitrogen. }\end{array} \\
\text { Per cent. } \\
21.8\end{array}$} \\
\hline \multirow[t]{2}{*}{ Ist } & Without & bon & e.......... & & & \\
\hline & With & “ & & 0.500 & 0.130 & \\
\hline \multirow[t]{2}{*}{$3 \mathrm{rd}$} & Without & $“$ & & 0.475 & O.IOI & $21 \cdot 3$ \\
\hline & With & “ & & 0.475 & 0.147 & 30.9 \\
\hline \multirow[t]{2}{*}{$5^{\text {th }}$} & Without & $"$ & & $0.4 \delta_{5}^{5}$ & 0.099 & 20.4 \\
\hline & With & “ & & 0.485 & 0.168 & 34.6 \\
\hline \multirow[t]{2}{*}{$8 \mathrm{th}$} & Without & “ & & 0.498 & 0.110 & 22.0 \\
\hline & With & at & & 0.498 & ‥ 189 & $37 \cdot 9$ \\
\hline
\end{tabular}

All the cultures without addition of carbonate have remained thick and white; the cultures with carbonate assume a yellow color and become less viscous. Although No. I filters with great difficulty, No. 8 easily passes through a filter paper. After eight cultures in normal milk, the ferment retains its properties, the quantity of dissolved nitrogen being practically the same as in the first culture. On the contrary, with the milk which had received carbonate, we find a considerable change in the dissolved nitrogen; the filtrate of the 8th culture contains already 37.9 per cent of the nitrogen in the soluble state.

The increase in the proteolytic power is still better shown if we determine the distribution of the nitrogen in the milks filtered after fermentation:

\begin{tabular}{|c|c|c|}
\hline $\begin{array}{c}\text { No. of the culture and experim. } \\
\text { conditions. }\end{array}$ & $\begin{array}{c}\text { Protein nitro- } \\
\text { gen (per cent } \\
\text { of soluble } \\
\text { nitrogen). }\end{array}$ & $\begin{array}{c}\text { Nitrogen non- } \\
\text { precipitable by } \\
\text { phosphotungstic } \\
\text { acid. }\end{array}$ \\
\hline 8th Without carbonate \\
$\begin{array}{c}\text { With } \\
\text { "“ }\end{array}$ & 45.1 & 55 \\
\hline
\end{tabular}

Thus, in the milk containing carbonate the soluble nitrogen is composed almost exclusively of more or less complex aminoacids. Moreover, it is observed that in tests with carbonate there is a great deal more of volatile acids and of ammonia than in the tests without carbonate.

These data on the proteolytic properties of the Bulgarian ferment find confirmation in the recent work of Barthel who 
inoculates sterilized milk containing $\mathrm{CaCO}_{3}$ with different ferments taken from Yoghourt and, after 60 days, analyzes the liquids.

Proteolytic Action of Ferments of Yoghourt.

\begin{tabular}{|c|c|c|c|c|}
\hline - & Cultures. & $\begin{array}{l}\text { Soluble nitro- } \\
\text { gen, per cent of } \\
\text { total nitrogen. }\end{array}$ & $\begin{array}{l}\text { N not ppt. by } \\
\text { P.P.T., per cent } \\
\text { of tot. nit. }\end{array}$ & $\begin{array}{l}\text { Nitrogen as } \\
\mathrm{NH}_{3} \\
\text { per cent of } \\
\text { total nitrogen. }\end{array}$ \\
\hline $\begin{array}{c}\text { Ferment } \\
",\end{array}$ & $\begin{array}{l}\text { I of Yoghourt. } \\
\text { II } \\
\text { III }\end{array}$ & $\begin{array}{l}48.21 \\
47.67 \\
58.38\end{array}$ & $\begin{array}{l}40.17 \\
37 \cdot 76 \\
45 \cdot 53\end{array}$ & $\begin{array}{l}3 \cdot 32 \\
4 \cdot 33 \\
4 \cdot 71\end{array}$ \\
\hline
\end{tabular}

Culture III was obtained from paste lactobacillin, furnished by the society "Le Ferment" of Paris. Barthel has identified the bacterium which is contained in it with the Bulgarian ferment of Bertrand. Species I was isolated from a preparation of Yoghourt sold by the firm Groll of Vienna. Finally, ferment II was taken from a similar commercial product, made by "Hygiene-Laboratorium" of Berlin-Wilmersdorf. The three cultures, as we see, have a very pronounced proteolytic power. While Bertrand supposes that Bulgarian ferment dissolves very little casein, culture III reveals, on the contrary, $5^{8.38}$ per cent of soluble nitrogen and 45.53 per cent of total nitrogen in a state not precipitable by phosphotungstic acid. The almost complete absence of albumoses and of peptones indicates a very thorough hydrolysis, due to the erepsin, and probably also to the amidases secreted by the bacterium. In addition, the final arrest of the transformation would be explained by the accumulation of amino-acids in the culture liquid.

The experiments just mentioned relate solely to Bulgarian ferment. The tests made with other medicinal lactic ferments have led to results of the same character, but show an effect still more accentuated. The proteolytic properties of these ferments are very pronounced. They are manifested not only in milk containing carbonate, but also in normal milk, in media free from sugars, and finally on coagulated albumin. Several samples of the same milk have been fermented with 
different medicinal lactic ferments. In these tests, the milk had added to it precipitated and sterilized calcium carbonate. Its analysis was made after 5 days of fermentation:

\begin{tabular}{|c|c|c|c|c|c|}
\hline Kind of ferment. & $\begin{array}{c}\text { Total } \mathrm{N} \text { of } \\
\text { ferm. milk, } \\
\text { mg., } \\
\text { per cent. }\end{array}$ & $\begin{array}{c}\mathrm{N} \text { in } \\
\text { filtrate, } \\
\text { mg.. } \\
\text { per cent. }\end{array}$ & $\begin{array}{l}\text { Amm. N } \\
\text { in filtrate, } \\
\text { mg.. } \\
\text { per cent. }\end{array}$ & $\begin{array}{c}\text { Protein } N \\
\text { in filtrate, } \\
\text { mg.. } \\
\text { per cent. }\end{array}$ & $\begin{array}{l}\mathrm{CaO} \text { in the } \\
\text { filtrate, } \\
\text { mg.., } \\
\text { per cent. }\end{array}$ \\
\hline I Lactobacillin (lozenge) & 567 & 420 & 22 & 91 & 249 \\
\hline $\begin{array}{l}2 \text { Lactobacillin (powder) } \\
3 \text { Bulgarian ferm., Gripe- }\end{array}$ & 490 & 420 & 22 & 85 & 243 \\
\hline 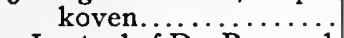 & 520 & $45^{\circ}$ & 29 & 85 & 173 \\
\hline 4 Lacteol of Dr. Boucard & 504 & 217 & 14 & $5^{6}$ & 1190 \\
\hline
\end{tabular}

The products I, 2 and 3 have a very obvious action on protein material. The filtered milk is completely free from casein and the greater part of the nitrogen is found in solution. Lacteol alone, by its action, is related to the Bulgarian ferment studied by Bertrand. Like the latter, it is an active producer of acid, although it possesses more definite proteolytic properties, since it succeeds in dissolving 40 per cent of the nitrogen without previous precipitation. Similar tests have been made with milk not containing calcium carbonate; the action on casein is less rapid, but the final result is the same.

Having noted the large proportions of volatile acids present in milk fermented by the aid of commercial cultures, it has been observed that the total weight of acids formed always exceeds that of the sugar destroyed, a fact which plainly indicates that the acids do not come solely from the sugar, but also from the protein material. A quantitative analysis has revealed the presence of succinic, malic and acetic acids. It is only. with Bertrand's ferment and Boucard's Lacteol that lactic acid has been obtained.

Thus, only Bulgarian ferment and Boucard's Lacteol are true lactic ferments. None of the other products, compressed or powdered, contains bacteria in the active state belonging to this great group, but, on the other hand, all contain a bacillus 


\begin{tabular}{|c|c|c|c|}
\hline Cultures used. & $\begin{array}{c}\text { Amine } \\
\text { nitrogen, } \\
\text { per cent } \\
\text { total } \\
\text { nitrogen. }\end{array}$ & $\begin{array}{c}\text { Total acid- } \\
\text { ity N/Io } \\
\text { NaOH per } \\
\text { 10o c.c. of } \\
\text { liquid. }\end{array}$ & $\begin{array}{c}\text { Volatile } \\
\text { acidity } \\
\mathrm{N} / \mathrm{Io} \mathrm{NaOH} \\
\text { per Io c.c. } \\
\text { of liquid. }\end{array}$ \\
\hline 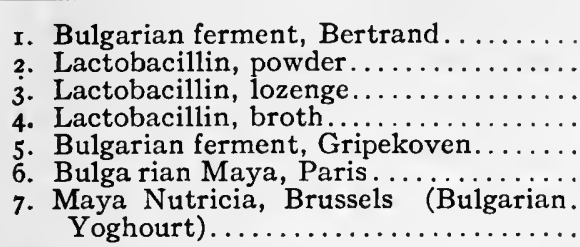 & $\begin{array}{l}16 \\
87 \\
86 \\
73 \\
87 \\
71 \\
86\end{array}$ & $\begin{array}{r}\text { c.c. } \\
280 \\
\text { Iro } \\
85 \\
95 \\
108 \\
78 \\
97\end{array}$ & $\begin{array}{l}\text { c.c. } \\
\text { 18 } \\
88 \\
80 \\
60 \\
82 \\
55 \\
78\end{array}$ \\
\hline
\end{tabular}

which the writer has called B. pseudolactic.* However, in the course of 1912, he analyzed fifty samples of Lactobacillin, compressed and powdered, of different origins, and he has never found lactic ferments present in them. B. pseudolactic, which is found in various preparations, belongs to the family of Mesentericus. It forms spores which are very resistant, and under the microscope appears to be identical with $\mathrm{Bul}$ garian ferment. Inoculated in a solution of peptone, it forms a scum after 24 hours at $40^{\circ}$, which falls at the end of several days to the bottom of the tube without causing the liquid to become turbid. This bacterium is without action on starch, but it secretes proteases which dissolve and peptonize coagulated albumin. It also produces an amidase which acts on asparagin. In the peptone culture, about 40 per cent of the total nitrogen is found in the form of ammonia. This micro-organism is then an energetic destroyer of nitrogenous material.

To summarize, the rapid solution of casein observed in milk inoculated with Lactobacillin or similar products, the formation in these cultures of considerable quantities of amine nitrogen, the presence of ammonia nitrogen and of large quantities of volatile acids, denote an intense, rapid, and advanced proteolysis. This activity best explains the mechanism of the action

* The constant presence of this same ferment in commercial products of such varied origin leads to the conclusion that Lactobacillin, which was the first in date, has served, more or less wittingly, as the basis for the preparation of the others. 
of these ferments in the intestine. It should be added that the conception, according to which the efficacy of these medicinal products resides in their proteolytic power, finds confirmation in the results obtained by Dr. Biernocki, who found that the addition of Lactobacillin to food causes a perceptible diminution of the feces and at the same time causes them to become poorer in nitrogen. The increase in the coefficient of assimilation of foods in the intestine is easily explained by the proteolytic function of the ferments introduced.

In the study of Bulgarian ferment certain errors have crept in from which a great misunderstanding has resulted. The ferment with proteolytic functions, which was isolated from Lactobacillin, was originally described by Bertrand under the name of Bulgarian ferment. To avoid all confusion, he afterwards designated it by the name $B$. pseudolactic. This microbe was studied in pure culture, and was identified with the ferment found in the following pharmaceutical preparations: Bulgarian ferment, Gripekoven; Bulgarian Maya, Paris; Maya nutricia, Brussels (Bulgarian Yoghourt); Lactobacillin in lozenges and in powder. This bacterium was found in pure culture in most cases; in others, it was found associated with a lactic ferment. B. pseudolactic was found neither in paste Lactobacillin nor in liquid Lactobacillin (culture in milk). These are the only commercial products which contain the ferment studied by Bertrand.

The constant presence of a proteolytic enzyme in industrial Bulgarian ferments, and the fact that the laboratory Bulgarian ferment studied by Bertrand, when cultivated in the presence of calcium carbonate, also develops its proteolytic properties to a pronounced degree, at one time led the writer to suppose here also the presence of the same bacterium which was undergoing biochemical variations under the influence of the conditions of the environment. Since then, a more thorough study has demonstrated that there is, in reality, a difference between Bulgarian ferment of Bertrand and B. pseudolactic contained in commercial preparations, and that they must be regarded as two entirely distinct species. 
However, these discoveries do not at all affect the question as to the chemistry of the pharmaceutical lactic ferment. The true Bulgarian ferment, as we have seen, possesses proteolytic properties whose manifestation requires certain conditions. Planted in the intestine, it exerts there its influence in the same manner as the lactic ferment influences the ripening of cheese, and this activity is also accentuated with time, the ferment acquiring a sort of enthusiasm for this new work. It must be noted, however, that in most. of the tests made in hospitals, Lactobacillin has been used in lozenges, in powder, or even in milk which has been made to ferment by the aid of these substances, so that the universal reputation of Lactobacillin is due solely to the pseudolactic ferment which it contains.

With people who use Yoghourt or similar preparations, intestinal diseases are not frequent. According to Metchnikoff, sour milk is even an excellent preventive against premature old age. In the beneficent action of this special lacteal diet several factors take part. First of all, consumed in large proportions, as in the case of Yoghourt, the fermented milk acts especially as a nitrogenous food that is hard to putrefy. It replaces meat, the digestion of which produces nitrogenous wastes that form a very good food for putrefactive agents. Furthermore, it also manifests its usefulness by its acid reaction. A clot of casein impregnated with acid is very hard to neutralize, even by its introduction into an alkaline medium, whence the special resistance which it offers to the putrefactive enzymes. Finally, milk bacteria, finding themselves in a medium free from sugar, develop their proteolytic properties, so that the amino-acids resulcing from their activity are all the more quickly absorbed. The net result of all these factors is that the putrefactive bacteria encounter in the intestine a soil unsuited to their multiplication, and so are eliminated more or less rapidly.

The use of pharmaceutical bacterial cultures may lead to similar results, but the conditions which they offer are less favorable. Of the three factors intervening in the lacteal diet, only the last comes into play and, here again, the result 
depends exclusively on the proteolytic power of the ferment used. Bulgarian ferment, in milk culture and ingested at the rate of Io to $20 \mathrm{~g}$. a day, necessarily produces a very slow effect. It becomes acclimated with difficulty in the intestinal medium, but once its proteolytic properties are developed, it will show a very noticeable dissolving and peptonizing activity toward albuminoid material. On the contrary, the organisms of Lactobacillin, in lozenges or in powder, as well as similar products, grow much more easily in the intestine, for. this medium is very favorable to them, both in its reaction and in the presence of nitrogenous material and the absence of carbohydrates. This explains their great efficacy as antiputrefactive agents.

\section{BIBLIOGRAPHY.}

G. Bertrand. C. $R$., I9io, p. I I6I.

Bertrand et Weisweiller. Action du ferment bulgare sur le lait, Ann. Inst. Pasi., 1906, (20), p. 977.

Cомве. L'auto-intoxication intestinale, Paris, 1907.

EFrRont. (I) Action du ferment bulgare sur les substances protéiques et amidées, C. R., 1910, (I5I), p. 1007. (2) Sur le ferment bulgare, C. R., I9Ir, (I), p. 125 .

Metchnikoff. Études sur la flore intestinale, Ann. Inst. Past., Igo8, (22), p. 929; 1910, (24), p. 755 ; 19I, (26), p. 825 .

C. BARTHEL. Zeits. für Gärungsphysiologie, I913, (2), p. 216.

Brernocki. See Article by Dr. Laroche in the Journal de Diététique, rgri, $\mathbf{n}^{\circ} .7$. M. Bertrand. Influence du régime sur la formation d'indol dans l'organisme, Ann. Inst. Past., I913, (27), p. 77. 
$\S$ II. Catalysts of the Soll.

Bacterial Flora of the Soil. - The bacterial flora of the soil represents the principal agent of the assimilation of nitrogenous substances by plants. Sterilized ground gives poor returns, even in the presence of manures. On the contrary, the harvest produced by a soil depends, in great part, on the quantity and the quality of the micro-organisms which are found there. The richness of the soil in bacteria is in direct relation with its degree of humidity and its chemical composition. For instance, the number of bacteria found in a soil varies with its origin:

Micro-organisms per Gram of Soll.

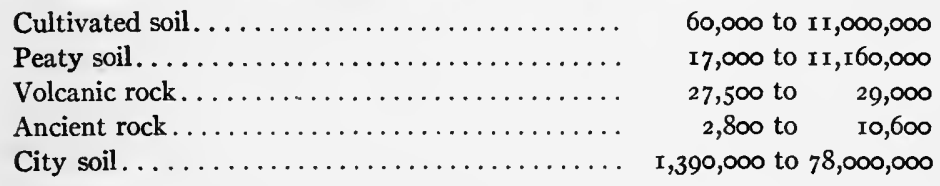

On the other hand, it has been found that these bacteria become less numerous as we go deeper into the soil, disappearing almost entirely at a depth of 3 to 4 meters:

$\begin{array}{lc}\text { Depth. } & \text { Number of bacteria. } \\ 0.30 \text { meter } & \text { I,800,000 } \\ 0.60 \text { " } & 300,000 \\ \text { I } ~ & 20,000\end{array}$

These figures, however, are by no means absolute, since they are modified by the large number of organisms that live in the ground, such as insects, larvæ, earth-worms, etc., as well as by the roots of plants, which, from the fact of their very deep penetration into the soil, favor the dissemination of bacteria, and at the same time influence the development of the latter by slightly changing the substrate through their secretions.

The importance of micro-organisms in the transformations taking place in the soil is evident from the following experiment of Müntz and Coudon, who have shown that if dried blood is added to a slightly calcareous earth, which is then sterilized at $120^{\circ}$, its inoculation with soil bacteria causes the appearance of considerable quantities of ammonia: 
INFLUence of Bacteria on Transformation in the Soll.

\begin{tabular}{|c|c|c|c|}
\hline Different lots of earth. & $\begin{array}{l}\text { Initial content } \\
\text { in } \mathrm{NH}_{3} \text { per Ioo } \\
\mathrm{g} \text {. earth. }\end{array}$ & $\begin{array}{c}\text { After } 70 \text { days } \\
\text { content in NH } \\
\text { per } 100 \text { g. earth. }\end{array}$ & $\begin{array}{l}\text { Ammonia } \\
\text { formed per 100 } \\
\text { g. earth. }\end{array}$ \\
\hline 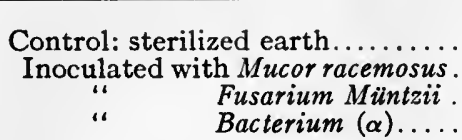 & $\begin{array}{l}\mathrm{mg} . \\
\mathrm{I} 6.3 \\
\mathrm{I} 6.3 \\
\mathrm{I} 6.3 \\
\mathrm{I} 6.3\end{array}$ & $\begin{array}{l}\mathrm{mg} . \\
16.3 \\
43.5 \\
36.1 \\
33.4\end{array}$ & $\begin{array}{l}\mathrm{mg} . \\
0 \\
27.2 \\
19.8 \\
17.1\end{array}$ \\
\hline
\end{tabular}

There is no doubt that the fertility of a soil depends much more on the bacterial flora which it contains than on the inorganic fertilizers or manures which may be applied. The very utility of the latter does not depend solely upon the materials which it brings, but depends also to a large extent upon its microbic composition. A gram of manure contains as many as 70 million micro-organisms, and these exert a marked influence on the transformations taking place in the soil, as Stoklasa has shown by comparing harvests obtained both with natural manures and with sterilized manures.

The different species of micro-organisms contained in the earth vary chiefly with its composition. In acid soils and soils rich in humus, molds and yeasts predominate, as Marchal has demonstrated. Aspergillus terricola and Cephalothecium roseum are found in abundance. In other soils, such bacteria as: B. putrificus, B. fluorescens, Proteus punctatum, Bact. erythrogenes, B. mycoïdes, B. subtilis, etc., ${ }^{*}$ are found.

Chemical Reactions in the Soil. - Bacteriology is constantly being enriched by the discovery of new species of bacteria that have been isolated from the soil, and most of these are already well studied. However, it was quickly found that our artificial cultures do not lend themselves to the isolation of all species. The transformations which are accomplished in the soil result almost entirely from actions of symbiosis, which offers to the different micro-organisms a medium appropriate to each, and it is often very difficult to reproduce these media.

* One will find in the excellent work of Kossovicz: Agriculturmykologie, Berlin, 1912, much information on this subject, as well as all the related literature. 
in the laboratory. Nevertheless, in spite of the still incomplete data which we possess, it is possible to follow in general outline the chemical reactions which take place in the soil. As the result of the investigations of Winogradsky, Beijerinck, Gayon, Omeliansky, Boullanger and Massol, Kossowicz, and others, who have devoted themselves especially to the study of special media capable of favoring the development of one species to the detriment of the others, it has been possible to isolate from the soil a certain number of bacteria showing very characteristic properties. The composition of the culture media originated by bacteriologists is very instructive. It throws a bright light, not only on the nature of the activity of the organism which is there developed, but also on the origin of the energy which it utilizes. The nutritive media suitable for the soil bacteria may be divided into four classes:

I. Media exclusively inorganic, containing ammonia or nitrites;

2. Media containing nitrates and organic carbon;

3. Media rich in carbohydrates, but not containing combined nitrogen;

4. Media composed of nitrogenous organic substances.

The following are two examples of the first type of culture medium:

With Ammonia Base.

With Nitrite BASE.

\begin{tabular}{|c|c|c|c|}
\hline$\left(\mathrm{NH}_{4}\right)_{2} \mathrm{SO}_{4}$. & 0.2 & $\mathrm{KNO}_{2}$. & 0.10 \\
\hline $\mathrm{K}_{2} \mathrm{HPO}_{4} \ldots \ldots \ldots \ldots \ldots \ldots$ & 0.1 & $\mathrm{~K}_{2} \mathrm{HPO}_{4} \ldots \ldots \ldots \ldots \ldots$ & 0.05 \\
\hline $\mathrm{NaCl} \ldots \ldots \ldots \ldots \ldots \ldots$ & 0.2 & $\mathrm{MgSO}_{4} \ldots \ldots \ldots \ldots \ldots \ldots$ & 0.05 \\
\hline $\mathrm{MgSO}_{4} \ldots \ldots \ldots \ldots \ldots \ldots$ & 0.05 & $\mathrm{Na}_{2} \mathrm{CO}_{3} \ldots \ldots \ldots \ldots \ldots$ & 0.1 \\
\hline $\mathrm{MgCO}_{3} \ldots \ldots \ldots \ldots \ldots \ldots$ & 1.0 & $\mathrm{NaCl} . \ldots \ldots \ldots \ldots \ldots \ldots$ & 0.05 \\
\hline $\mathrm{FeSO}_{4} \ldots$ & 0.04 & $\ldots \ldots \ldots \ldots \ldots$ & 0.04 \\
\hline$\ldots$ & $\infty .0$ & $\mathrm{H}_{2} \mathrm{O}$. . & $\infty 0.0$ \\
\hline
\end{tabular}

We note that these solutions are slightly alkaline. Furthermore, if we inoçulate with soil the medium which contains ammonia, we find that this is transformed into nitrite, then into nitrate. If we start with the medium containing nitrous nitrogen, we immediately arrive at nitrate. The bacteria which are developed in the two cases then do not require organic 
carbon, as that results from the very composition of the medium. They have the power of assimilating the inorganic carbon of the $\mathrm{CO}_{2}$, and it is the $\mathrm{MgCO}_{3}$ and $\mathrm{Na}_{2} \mathrm{CO}_{3}$ that serve as the source of carbon, an element indispensable to the construction of their protoplasmic substance. The energy required to decompose the $\mathrm{CO}_{2}$ is drawn from the heat which is liberated in the oxidation of the ammoniacal or nitrous nitrogen:

$$
\begin{aligned}
2 \mathrm{NH}_{3}+3 \mathrm{O}_{2} & =3 \mathrm{H}_{2} \mathrm{O}+\mathrm{N}_{2} \mathrm{O}_{3}+\mathrm{I}_{57} \text { calories. } \\
\mathrm{N}_{2} \mathrm{O}_{3}+\mathrm{O}_{2} & =\mathrm{N}_{2} \mathrm{O}_{5}+37 \text { calories. }
\end{aligned}
$$

The two principal reactions, assimilation of carbon and oxidation of nitrogen, are intimately connected. Winogradski, in fact, has established that, in this kind of fermentation, for I g. of carbon assimilated there are about $3^{6} \mathrm{~g}$. of nitrogen oxidized:

\begin{tabular}{|c|c|c|c|}
\hline & I & 2 & 3 \\
\hline Carbon assimilated. & $\begin{array}{l}\text { mg. } \\
19.7\end{array}$ & $\begin{array}{l}\mathrm{mg} . \\
24 \cdot 4\end{array}$ & $\begin{array}{l}\text { mg. } \\
26.4\end{array}$ \\
\hline 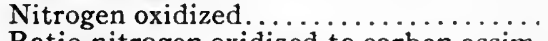 & 722.0 & 815.0 & 928.0 \\
\hline $\begin{array}{l}\text { Ratio nitrogen oxıdized to carbon assim- } \\
\text { ilated } \ldots \ldots \ldots \ldots \ldots \ldots \ldots \ldots \ldots \ldots \ldots \ldots \ldots\end{array}$ & & $36: 1$ & $35: x$ \\
\hline
\end{tabular}

Relation between Nitrogen Oxidized and Carbon Assimilated.

For the second type of medium, containing nitrates and organic carbon, we can take as example that proposed by Giltay:

Mediug with Nitrate Base.

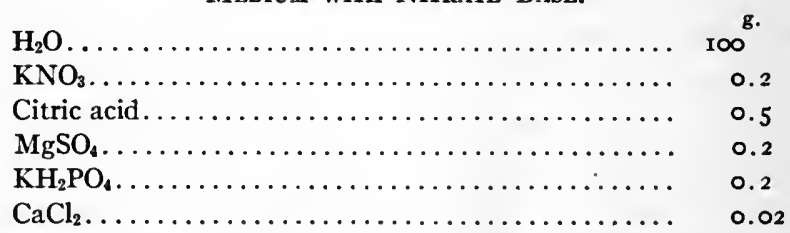

Traces of $\mathrm{FeCl}_{3}$ with enough $\mathrm{NaOH}$ to obtain a slightly alkaline reaction.

Moreover, we also use organic compounds other than citric acid, glucose or starch serving equally as well. In this sort of medium denitrifying bacteria develop, that is to say, a species that reduces nitrates to the state of lower oxides or even to the form of gaseous nitrogen. These bacteria perform an endo- 
thermic reaction, which is compensated for by the combustion of the organic material on which they live..

The third class, free from combined nitrogen, is also characterized by the predominance of carbohydrate.

Medium Free from Combined Nitrogen.

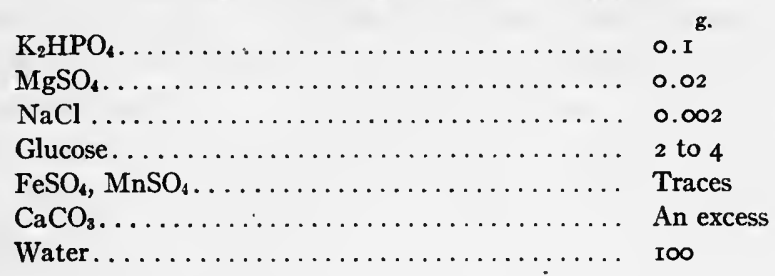

This medium is utilized for the culture of extremely important micro-organisms from the point of view of agriculture, namely, the bacteria that fix nitrogen. These bacteria withdraw this element from the atmosphere and convert it into assimilable organic substance, thus aiding to render vegetation to a certain extent independent of the nitrogen introduced into the soil in the form of fertilizers. The energy required is obtained, as in the preceding case, from the decomposition of the organic material present.

Finally, the fourth class can be obtained by adding $0.1 \mathrm{~g}$. of potassium phosphate and an equal quantity of magnesium chloride to a 2 per cent solution of peptone. This medium, rich in ammonia and fatty acids, is especially suited to the development of proteolytic soil bacteria.

To summarize, we find in the soil: (I) bacteria which decompose nitrogenous organic material to give ammonia; (2) oxidizing bacteria, some of which oxidize ammonia to the nitrite state, others oxidize this intermediate nitrite to nitrate; (3) bacteria that fix atmospheric nitrogen and which help to store this nitrogen in the ground or the plant; (4) reducing bacteria, which destroy nitrates. The organisms belonging to the first three groups render valuable aid toward fertilizing the soil. As to the denitrifying bacteria, their rôle appears less favorable. We shall see later their possible utility. Let us 
now say that the losses in free nitrogen which they may occasion in cultivated lands are relatively very slight.

In the pages which follow we shall give a short survey of the properties of these various bacteria - ammoniacal, nitrifying, nitrogen fixing and finally denitrifying.

Ammoniacal Fermentation of the Soil. - The decomposition of the nitrogenous substance of the soil takes place by means of both aërobic and anaërobic micro-organisms. Putrefactive and urea-splitting bacteria especially intervene, but the flora remains very varied. Among the more common species are: $B$. subtilis, B. mesentericus, Bact. punctatum, B. putrificus, B. fluorescens, Proteus erythrogenes, $B$. mycoïdes, as-well as different butyric ferments. The humiferous soils contain chiefly molds, as we have already stated. The digestion of nitrogenous material is very intense in summer; in winter it slackens, but it does not completely stop, even during freezing, for at this time special bacteria intervene that are adapted to low temperatures. The catalysts involved in this transformation are the bacterial trypsins and amidases. As to urease, it exerts its action especially on the organic nitrogen introduced by the manuring.

Ammoniacal fermentation is regarded by certain scientific agriculturists as the result of the complete oxidation of the protein substance, a true combustion caused by the microorganisms, which oxidizes the complex material directly to $\mathrm{CO}_{2}$, $\mathrm{H}_{2} \mathrm{O}$ and $\mathrm{NH}_{3}$. However, the decomposition of albuminoids by anaërobes always produces intermediate substances. There is no reason to suppose that the catalysts which the aërobes utilize are of another nature than those employed by the anaërobes. Ammoniacal fermentation, whether caused by the one or the other, is always the result of a thorough hydrolysis. The protein nitrogen is brought by successive hydrolyses to the amino nitrogen and then to ammonia nitrogen state. To each molecule of ammonia formed there corresponds a molecule of either a volatile acid, an oxyacid, or else an alcohol. In anaërobic fermentation, the products of the fatty series appear to be stable: one finds them almost intact in the medium. On 
the contrary, in aërobic fermentation the destruction of this material goes on gradually, resulting in more or less rapid mineralization.

This disintegration of the albuminoid substance, which converts almost the whole of the organic nitrogen into ammonia, results from the combined action of numerous micro-organisms, of different species and nature. The products elaborated by one class of bacteria often serve as th Thus the volatile acids and oxyacids furnished in the course of ammoniacal fermentation constitute a nutritive material for the ferments of cellulose, bacteria abundantly represented in the soil. Calcium acetate, as well as its homologues, react under these conditions in the following manner:

$$
\underset{\text { Calcium acetate }}{\left(\mathrm{C}_{2} \mathrm{H}_{3} \mathrm{O}_{2}\right)_{2} \mathrm{Ca}}+\mathrm{H}_{2} \mathrm{O}=\mathrm{CaCO}_{3}+\mathrm{CO}_{2}+\underset{\text { Methane }}{\mathrm{CH}_{4}} \text {. }
$$

Moreover, the methane appearing in this decomposition will be, in its turn, taken up by other micro-organisms, such as Bac. methanicus, Bact. pyocyaneum, and various bacteria swarming in pools and decaying matter. The volatile substances, such as hydrogen, phenol, amines, etc., which arise in the course of putrefaction, can no longer be considered as wastes resulting from the evolution of albuminoid matter. We know, for example, that hydrogen is oxidized by Hydrogenomonas flava and Hydrogenomonas vitrea (both studied by Niklewski), and that there are a certain number of bacteria, like the $B$. methanicus of Kaserer, already mentioned, and the $B$. oligocarbophilus of Beijerinck, which borrow their carbon from gaseous organic products. On the other hand, the formation and the disappearance of ptomaines during putrefaction, variations observed by several investigators, indicate that even these products can be utilized. We may, finally, by analogy say as much for the various gases liberated from the soil, Trillat having demonstrated that these favor in a very marked way the development of the bacterial flora of the air.

The progress of ammoniacal fermentation of the soil depends 
on the condition in which the organic nitrogen is found. The partially hydrolyzed albuminoid products are rapidly decomposed. The natural albuminoids, on the contrary, disintegrate slowly; it is moreover to this resistance that must be attributed the superiority of organic nitrogen as a fertilizer. The nitrogenous substances which are not capable of being converted into ammonia nitrogen by enzymes never become fertilizing substances. Thus, calcium cyanamide, which can be converted into ammonia by certain ureases, according to the equation

$$
\mathrm{CaCN}_{2}+{ }_{3} \mathrm{H}_{2} \mathrm{O}={ }_{2} \mathrm{NH}_{3}+\mathrm{CaCO}_{3}
$$

may serve for fertilization, while dicyandiamide, unattacked by amidase, cannot be used for this purpose.

Nitrification. - The ammonia which has accumulated in the soil forms a source of nitrogen for a great many microorganisms, as well as for certain higher plants. However, the latter show a preference for nitrogen which is in the form of a nitrate. The utilization of the ammonia contained in the soil is intimately related to the phenomenon of nitrification, a phenomenon in which two groups of oxidizing bacteria intervene, one bringing its action to bear on the original ammonia, the other on the nitrite resulting from it. This transformation has been known for a very long time, and has even been taken advantage of in the establishment of saltpetre works in different countries. However, the mechanism of the reaction remained without satisfactory explanation up to the time of Pasteur.

In 1879 , Schloesing and Müntz established in an indisputable manner that nitrification is due to bacterial intervention. The bacteria involved have since been isolated by Winogradsky and Frankland, as well as by Warington. Many varieties exist that are capable of causing the oxidation of ammonia, the most familiar of which are Nitrosococcus and Nitrosomonas, the second being distinguished from the first by being motile and by an ability to form zoöglea. These bacteria have an optimum temperature of $37^{\circ}$, and are destroyed in a few minutes at $42^{\circ}$. The presence of organic materials in the culture is very injurious. 
Quantities of Substances which Retard Nitrous Fermentation.

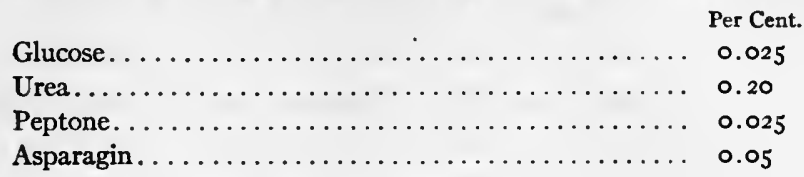

According to Boullanger and Massol, an excess of ammonia is very unfavorable, 3 to $5 \%$ of ammonium sulphate completely inhibiting the action. The accumulation of the nitrite formed offers much greater danger, since 0.8 to r.o per cent of this substance causes a perceptible retardation. As to nitrate, it influences much less strongly the progress of fermentation. Since the bacteria cannot utilize nitrogen except in the ammoniacal form, protein nitrogen and amine nitrogen must undergo a preliminary transformation. On the other hand, the ammonia can be in the form of the chloride or sulphate, or even of the organic salts, though the carbonates disappear with more difficulty than the sulphates.

Oxidation of nitrites into nitrates is accomplished by a bacterium, isolated by Winogradsky, which bears the name of Nitrobacter. This micro-organism has an optimum temperature identical with that of nitrous bacteria though it shows a little higher resistance to the action of heat. To destroy it, it is necessary to keep it for 5 minutes at $55^{\circ}$. Further, the cultures of Nitrobacter require a slightly greater alkalinity. Usually we add o.I per cent of sodium carbonate to their medium.

The presence of nitrites in amounts exceeding I per cent is manifestly injurious to the nitro-bacteria. The presence of nitrates is less injurious, and the culture may contain up to 2 per cent of sodium nitrate or 2.5 per cent of magnesium nitrate without being influenced. As to organic materials, they are less disturbing in the cultures of Nitrobacter than in those of the nitrous bacteria. $15 \mathrm{cg}$. of ammonia per liter begin to inhibit activity. However, the adult bacterium can withstand relatively large quantities of ammonia. Nitrification, in laboratory cultures, takes place at first very slowly. Then it is stimu- 
lated, and after several days its activity is three or four times as intense as the original.

No nitrous acid, even in traces, is found in the soil. On the other hand, independently of nitrogen in the form of humus (about $2 \mathrm{~g}$. $\mathrm{N}$ per $\mathrm{kg}$. of soil) or in the form of ammonia (about Io $\mathrm{mg}$. $\mathrm{N}$ per $\mathrm{kg}$. of soil), we find this element in the form of nitrates, and sometimes even in quite large quantities $(0.2 \mathrm{~g}$. and more of $\mathrm{N}$ per $\mathrm{kg}$. of soil). The reason for this is that the nitrous and nitric bacteria in the soil work simultaneously, the nitrites being oxidized as fast as they appear. At first sight, the conditions which are realized in nature do not appear to be favorable to the parallel occurrence of nitrous and nitric fermentations, the presence of carbohydrates being detrimental to the development of nitrous enzymes at the same time that the existing ammonia retards the transformation of the nitrites into nitrates. In reality, the conditions in the soil are quite different from those in artificial cultures; symbiosis favors the two phases of oxidation, which then proceed together and with a great activity, the work being further increased, as Omélianski has shown, by the presence in the soil of putrefactive bacteria and other producers of ammonia. Furthermore, the organic material, injurious in pure cultures, not only is no longer injurious in the ground, but even increases the intensity of nitrification. Müntz, in fact, proved that the addition of humus to a soil accelerated the appearance of nitrates. The same effect is observed, moreover, when ammonium sulphate is added to a soil.

In conclusion, it is proper to add that these nitrifying bacteria, which play so large a part in agriculture, are also, together with the bacteria of ammoniacal fermentation, the active agents of the purification of sewage by the spreading on fields or by the use of bacterial filters, methods which have been studied in detail by $\mathrm{H}$. Mills, by Schioesing and Frankland, by Müntz and Lainé, and especially by Calmette, to whom we owe a masterly investigation of the subject.

Assimilation of Atmospheric Nitrogen. - It is especially to the French scientists that agricultural bacteriology owes its 
fundamental ideas. It is they who first studied the phenomena of nitrification and denitrification, as well as that of the assimilation of nitrogen. One of the most renowned, Berthelot, in 1885 , explained the mechanism of the enrichment of the soil in nitrogen. By comparative observations made on sterilized and on normal soils he established the fact that the fixation of nitrogen takes place through the mediation of micro-organisms. Then Schloesing, Jr., showed that the nitrogen absorbed is really the elementary nitrogen obtained from the atmosphere, and not the combined nitrogen which is also found there. Finally Beijerinck, some years later, isolated Bacillus radicicola, which is the principal fixing agent for nitrogen. From the work of Winogradsky, Pringsheim, and of Beijerinck and his pupils, we have come to know a whole series of micro-organisms which intervene in the enrichment of the soil in combined nitrogen through the assimilation of the elementary nitrogen of the air.

Aërobes and anaërobes aid in this work. In the first place we must mention Clostridium, such as: C. pastorianum, $C$. giganteum, C. americanum; furthermore, Granulobacter spharicum, G. reptans, G. polymyxa; Azotobacter of Beijerinck and Van Delden, etc. Assimilation takes place also through the different species belonging to the Mesentericus group. According to Löhnis and Pillai, Bact. acidi lactici, B. lactis innocuus, Bact. lactis viscosum also possess this property. The molds and yeasts are also capable, under certain conditions, of absorbing atmospheric nitrogen. According to Kossowicz, Monilia candida, Oidium lactis and Dematium pullulans can be cultivated in an inorganic medium free from combined nitrogen. Lipman, as well as Zikes, class in the same group certain yeasts, such as Sacch. Pastorianus III, Mycoderma vini and others, which can live and reproduce themselves in. a medium almost entirely free from combined nitrogen.

The moist soils on which molds and algæ grow are rich in nitrogen. This assimilation, as Kossowicz has plainly shown by means of a pure culture of Chorella vulgaris, results from a work of symbiosis which takes place between these lower plants 
and the micro-organisms. The plants furnish to the bacteria carbohydrate material which they themselves have synthesized; the bacteria utilize the energy put at their disposal by the decomposition of this carbohydrate, to fix the nitrogen by which the plant profits.

The bacteria which assimilate nitrogen, such as Azotobacter, contain as much as 19 per cent of protein and 2.5 to 2.97 per cent of phosphorus. They develop in a medium poor in nitrogen, but rich in assimilable carbon, and require the presence of phosphoric acid, of calcium, of magnesium, and of potassium. The fixation of nitrogen takes place especially in the upper layers of the soil, but the process goes on to a depth of 50 and even $80 \mathrm{~cm}$. The aërobes, such as Azotobacter, are found in the upper layers; the anaërobes, like certain Clostridia, on the contrary, react in the deeper layers. Moreover these two species of bacteria often live in symbiosis, one serving as protector of the other. The degree of fixation of nitrogen by bacteria depends on the quantity of carbonaceous material consumed. Azotobacter, in a pure culture, does not succeed in decomposing enough carbohydrate to permit a large fixation. On the contrary, 'in mixed cultures, containing bacteria that decompose carbonaceous material, assimilation takes place more rapidly and with more intensity.

The quantity of nitrogen assimilated by the algæ and the molds is ordinarily considered as being too small to be taken into account. Only bacteria are of real interest from the point of view of the enrichment of the soil in combined nitrogen. And yet, of all these, the most important, as we shall see, are those which are capable of living in symbiosis with the plant.

From the earliest times, it has been noted that certain crops, such as those of leguminous plants, improve the soil, and that others, like those of cereals, exhaust it. This observation for a long time served as the basis of agriculture, before the exact cause was known. More recent studies have revealed that the mechanism of this enrichment is due to an assimilation of nitrogen, an assimilation originating in the radicular nodules 
of certain plants, swellings which were formerly considered as being due to an objectionable parasitic disease. In 1879 , Franck observed that these excrescences do not form in sterile lands, and in 1886, Hellriegel and Wilfarth established the relation which exists between these nodules and the nitrogenous enrichment of the soil.

The bacterial flora of the nodules was studied by Beijerinck, Stoklasa, Schloessing, Jr., Laurent, Prazmowski, and others. These investigators recognized that the active bacterium is B. radicicola. This micro-organism has an optimum temperature lying, according to the variety, between $10^{\circ}$ and $40^{\circ}$. It resists desiccation, so that cultures on maltose-agar keep for years without losing their activity, as Edwards has demonstrated. In the ground, the bacterium can live and assimilate nitrogen without being in direct contact with the plant cells, though it prefers to live in the plant.

The formation of the nodules is due to the penetration into the root of leguminous plants of the motile bacterium, which becomes attached to the plant cells by means of a specific secretion that varies with the race of the bacteria. Moreover, the products elaborated by the root of the plant likewise play a part in this phenomenon of mutual influence. The bacteria, attached preferably on the root hairs, penetrate to the subcortical layers, where they form colonies, which soon are surrounded by a sheath. These colonies, thus mingled with absorbing root hairs, attach themselves to the plant cells and produce a swelling. At this point, the bacteria are converted into ramified or bacteroid forms, mucous filaments appear, the cortical cells divide, and the whole forms the nodule. These nodules represent that part of the plant that is most rich in nitrogen. They contain as much as 7.5 per cent nitrogen, and are filled with granular substances, as well as bacteria that stain red with iodine. The fixed nitrogen is transformed into protoplasmic substance, then into a reserve material utilizable by the plant. At the period of the formation of the mucous filaments, that is to say, when the bacteroids first become visible, the assimila- 
tion of nitrogen is at its maximum. This continues during the entire growth of the plant, and then in autumn the tubercles empty, and the bacteria are detached in the form of small rods and return into the ground, to begin again, with the new season, their evolution as fixing agents for nitrogen.

Different races of Bacillus radicicola exist. Lupines and sainfoin contain some which are different from those encountered in beans, peas, clover, etc. Furthermore, the manner of grouping of the swellings varies from one plant to another; sometimes they are small and numerous, sometimes they are less numerous but are larger in size. This second variety is the more advantageous, in that it corresponds to a larger assimilation of nitrogen. The nodule bacteria develop in soils rich in carbon and poor in combined nitrogen. The presence of nitrates is injurious; the presence of humus, on the contrary, increases the activity. Furthermore, they require phosphate and potassium in the soil. In general the tubercles appear on that part of the roct most exposed to the air, though always the ease with which the bacterium penetrates into the radicular system and is fixed there depends on specific secretions which it and the plant furnish. The nodules can be reproduced by inoculation.

The catalysts that intervene in the assimilation of nitrogen by micro-organisms are unknown. They form a field of investigation still unexplored. However, it has been established that enzymes play a considerable part in the symbiosis of bacteria with plants. The bacterium is attached to the plant by means of active substances, and it is by means of the secretions of the radicular system that the utilization of the nitrogenous reserve of bacterial origin takes place.

To summarize, in the enrichment of the soil nitrogen; three types of micro-organisms intervene:

(I) The bacteria of the nodules of leguminous plants which act by way of symbiosis with the plant, in this process the two elements of the association being in almost absolute dependence on each other. Previous to the formation of the swellings, the bacteria absorb the nitrogen of the roots; then, 
when the tubercles are formed, the bacteroids fix the atmospheric nitrogen and the plant profits by the same, while the latter, in exchange, gives to the micro-organism the carbohydrate indispensable for the assimilation of the nitrogen.

(2) The bacteria which live in symbiosis with certain green algæ, like Nostoc punctiforme, the mechanism of whose action is similar to that of the bacteria of the leguminous plants. As to the various molds, such as Aspergillus niger and Penicillium glaucum, which also absorb atmospheric nitrogen, they appear to accomplish this in the manner of micro-organisms of the following third type.

(3) Finally, bacteria, such as the varieties of Amylobacter, Azotobacter and Clostridium, which fix nitrogen in the presence of substances furnishing energy by their own decomposition. These can assimilate the nitrogen of the air without the aid of other living cells. In this latter group must also be classed: B. asterosporus, B. saccharobutyricus, Granulobacter pectinovorum, etc. In liquid media, the fixation of nitrogen is accompanied by a considerable decomposition of sugar:

Clostridium decomposes I g. sugar, to fix $2 \mathrm{mg}$. nitrogen.

Azotobacter

I $\mathrm{g}$.

9 to ro mg. nitrogen.

Stoklasa finds that, with Azotobacter chroococcum, I6 $5 \mathrm{~g}$. of sugar were required to fix I $\mathrm{g}$. of nitrogen. We see that the relation between the nitrogen fixed and the sugar decomposed is far from being constant. It depends, in fact, on many conditions, among others being the fact that the young cultures are much more favorable to the fixation of nitrogen than the old.

Denitrification in the Soil. - To complete the study of the cycle accomplished by nitrogen in the ground, it is necessary to give some data as to denitrification. In I868, Schoenbein discovered the existence of micro-organisms capable of reducing nitrates to nitrites, and then to ammonia. In 1882, Gayon and Dupetit isolated bacteria, designated by them by $\alpha$ and $\beta$, that reduce nitrates to the form of free nitrogen. At the same time, Dehérain and Maquenne, who studied very closely the 
chemical mechanism of denitrification, furnished the first analyses of the gases formed. By inoculating with earth, they caused a solution of sugar containing nitrate to ferment, and so obtained the following figures for the gases formed.

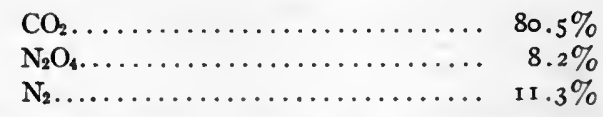

In addition to these gases, we sometimes find hydrogen evolved. According to the preceding writers, Gayon and Dehérain, the bacteria active in this reduction are anaërobic. This question was taken up later by Kossowicz, Stoklasa, Lebedew, Grimbert, Fred, etc., who studied denitrification from both the bacteriological and chemical points of view. The denitrifying bacteria are very widely distributed in the soil and are found in manure as well as on straw. Various species capable of decomposing nitrates are known. The true denitrifiers reduce nitrates to free nitrogen; the indirect denitrifiers produce only nitrous acid, which, with the amide substances of the soil, give free nitrogen. There are, furthermore, species which are inactive toward nitrate, and which reduce only the oxides of nitrogen. Finally, other denitrifiers reduce nitrate to ammonia; among the latter are: B. subtilis, B. mesentericus, B. proteus vulgaris, etc. The property of reducing nitrates is then very common, and we even find that certain Saccharomyies and some molds also possess it.

Gayon regards the phenomenon of denitrification as the result of an internal oxidation. The micro-organisms, according to him, transfer oxygen from the nitrate to a carbohydrate, the latter being consumed. We should have:

$$
\begin{aligned}
4 \mathrm{KNO}_{3}+{ }_{5} \mathrm{C}+2 \mathrm{H}_{2} \mathrm{O} & ={ }_{4} \mathrm{KHCO}_{3}+2 \mathrm{~N}_{2}+\mathrm{CO}_{2} . \\
4 \mathrm{KNO}_{2}+3 \mathrm{C}+\mathrm{H}_{2} \mathrm{O} & =2 \mathrm{KHCO}_{3}+2 \mathrm{~N}_{2}+\mathrm{K}_{2} \mathrm{CO}_{3} .
\end{aligned}
$$

On the contrary, the indirect denitrifiers which have recourse to the amide nitrogen give a reaction such as the following:

$$
\underset{\text { Urea }}{\left(\mathrm{NH}_{2}\right)_{2} \mathrm{CO}}+\underset{\text { Nitrous acid }}{2 \mathrm{HNO}_{2}}=2 \mathrm{~N}_{2}+\mathrm{CO}_{2}+3 \mathrm{H}_{2} \mathrm{O} \text {. }
$$


The conditions of the medium play a very large part in the progress of denitrification; they influence especially the formation of carbonic acid, and that of the oxides and free nitrogen. In the first place, the presence of an easily oxidizable carbohydrate is indispensable. Sugar favors denitrification. According to Stoklasa, xylose is equally favorable, though arabinose is a little less so. Certain denitrifying bacteria can even utilize sulphur as a source of energy. Thus Thiobacillus denitrificans transforms this element into sulphate according to the equation:

$$
\begin{aligned}
6 \mathrm{KNO}_{3}+5 \mathrm{~S}+{ }_{2} \mathrm{CaCO}_{3} \\
\quad=3 \mathrm{~K}_{2} \mathrm{SO}_{4}+{ }_{2} \mathrm{CaSO}_{4}+3 \mathrm{~N}_{2}+{ }_{2} \mathrm{CO}_{2} .
\end{aligned}
$$

The catalysts intervening in denitrification are but little known. Irving and Hankinson having detected the presence of an enzyme capable of reducing nitrates in the leaves and the roots of green plants, one might hope to find the same enzymes in the denitrifying bacteria. Such is not the case, all experiments in this direction having given negative results. The denitrifying bacteria, on the contrary, contain an oxidase. On the other hand, Stoklasa and Vitek have found an alcoholase in a denitrifying ferment. According to these investigators, the alcohol produced by this zymase may have a part in the denitrification:

$$
\mathrm{C}_{2} \mathrm{H}_{5} \mathrm{OH}+2 \mathrm{~N}_{2} \mathrm{O}_{3}=2 \mathrm{~N}_{2}+{ }_{2} \mathrm{CO}_{2}+3 \mathrm{H}_{2} \mathrm{O} \text {. }
$$

The function of denitrifying bacteria, widely distributed in the ground, is not entirely defined. We only know that they are found there ordinarily under conditions very unfavorable for producing intense action.

Moreover, it is very probable that in symbiosis in the soil they do not exert their destructive action to the end, thereby bringing the nitrate nitrogen to a free state, but that they play rather the part of regulators in the phenomenon of nitrification by moderating oxidation and by thus preventing a too great formation of nitrates, which would be lost with the drain- 
age waters. Finally, these bacteria could even act as fixation agents for nitrogen, as has been observed in certain cases.

Chemical Nitrogenous Fertilizers and Green Fertilizers.

Plants borrow all their nitrogen from the ground. The continual removal of this element by cultivation would logically exhaust the soil and render it sterile if there did not exist important compensations. Boussingault was the first to establish the balance-sheet of nitrogen in agricultural operations. Maquenne ("Course of Vegetable Physics at the Museum") thus distributes nitrogen among the different requirements of a hectare in cultivation during an entire year:

Balance of Nitrogen per Hectare per Year.

\begin{tabular}{|c|c|}
\hline \begin{aligned} & \multicolumn{1}{c}{ Nitrogen taken. } \\
& Harvest. . . . W . . \end{aligned} & $\begin{array}{l}\mathrm{Kg} . \\
80\end{array}$ \\
\hline Drainage................. & 50 \\
\hline Ammonia set free.......... & 20 \\
\hline Nitrogen liberated.$\ldots \ldots \ldots \ldots$ & \\
\hline
\end{tabular}

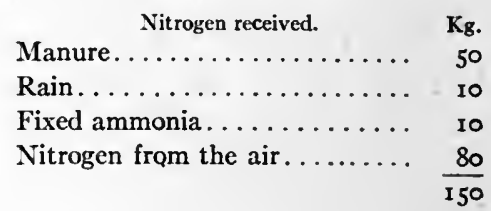

However, this writer gives these figures only as an average of different observations, each being susceptible of large variations. Let us consider the losses. A harvest of cereals removes per hectare from 40 to 80 kilos of nitrogen; on the other hand, the nitrates, when not absorbed by the roots of the plants, are not retained by the soil. The drainage waters then carry off a certain quantity, which can be estimated, according to Dehérain, at from 5 to 100 kilos in nitrogen per hectare per year, according to the barrenness of the soil and to climatic conditions. Finally, we must also mention the diffusion into the air of a part of the ammonia of the soil and the liberation of gaseous nitrogen coming from the action of the denitrifiers.

To compensate for these various losses, three factors intervene: Ist, the rain and the dew, which bring to the soil the combined atmospheric nitrogen; 2nd, the bacteria which assimilate free nitrogen; 3 rd, manure and artificial nitrogenous fertilizers. The effect of the dew and the rains, without being of an absolute efficacy, is however important, a more or less constancy in the phenomenon being assumed: 
Nitrogen Content of the Atmospheric Water Per Liter.

$\mathrm{Mg}$.

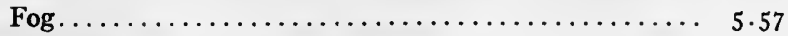

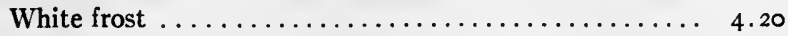

Dew................................ 5.00

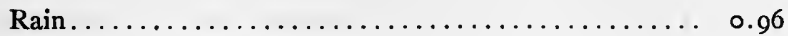

Hail........................... 2.77

The nitrogen brought by the rain and the dew is found in the form of nitrate, and sometimes in the form of ammonia. The nitric acid results from the action. of electric discharges on the constituents of the atmosphere. The ammonia comes from the ocean and especially from the decomposition of organic dusts. The quantities thus formed in the ground vary with the year, the climate, and the region, being estimated at from 2 to 22 kilos of nitrogen per year per hectare.

The income in nitrogen through bacterial agency is generally much larger than that coming from the rain or the dew. According to F. Löhnis, this gain can be estimated at from ro to 40 kilos per year per hectare, though it is certain that in many cases this estimate is much too large.

This fixation is due, not only to the bacteria which are found in the bare ground, but also to those which develop in the organic residues from former cultivation, and to those which live in symbiosis with the algæ and the higher plants. Thus Henry found that dried oak leaves, left in the air for a year, became the object of an intense bacterial action. Whereas they originally contained only 0.947 per cent of nitrogen, they contained at the end 1.727 per cent. In beech leaves, the proportion is from I.I to I.5 per cent. On the other hand, the use of green manures, that is to say, the turning under of a harvest sufficiently developed with suitable leguminous plants, a practice recommended by G. Ville under the name of sideration, brings to the soil a quantity of nitrogen estimated at between roo and 300 kilos per hectare. We see that, by this latter process, we can entirely meet and even exceed the demands of the soil for nitrogen. However, in intense cultivation, the use of leguminous plants and green manures is not always possible, and then the only resources 
at one's disposal are manure, some waste products, or finally artificial nitrogenous fertilizers.

Manure restores to the land a part of the nitrogen and the inorganic material, especially phosphorus and potassium, which the harvests have removed; at the same time it furnishes useful bacteria. Although the nitrogen of the manure, about $5 \mathrm{~g}$. per kg., is not always utilized and there often is a 50 per cent loss, the use of this fertilizer is nevertheless very beneficial. It not only introduces a fresh and active culture of bacteria which fixate nitrogen, but also organic material which will serve the bacteria for nourishment. Finally, by its physical structure, it changes the compactness of the soil, facilitates its aëration, and helps it to retain the moisture.

As artificial nitrogenous fertilizers, either ammonia or nitrates are used. In reality, these products are natural, and not at all artificial, for they come from the decomposition of animal or vegetable material. Ammonia is recovered in the manufacture of coke or in the distillation of organic residues, that which has a purely synthetic origin not being recovered, in spite of the great efforts made in this direction in the course of the last few years.

As to nitrates, they come especially from immense saltpetre beds situated in South America. It is supposed that these veins result from the putrefaction of deposits of algæ, or else from the decomposition of banks of guano. In either case, the organic vegetable or animal nitrogen has been transformed into ammonia, and then into nitrates under the influence of various bacteria. Here, again, the industrial nitrates, in spite of their growing importance, are in a relatively small proportion as compared with those of natural origin. The following is a table which gives the quantities of nitrogenous fertilizers used in agriculture; we see that they are considerable:

World Consumption of Nitrogenous Fertilizers in igio.

Sodium Nitrate ......................................... Tons.

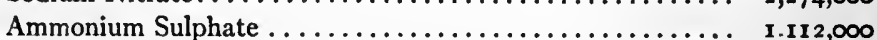

Calcium Nitrate....................... 10,800 
The agricultural expenditures for the purchase of nitrogenous fertilizers amount to more than twenty million dollars a year, and are gradually increasing, so that the early exhaustion of the nitrate veins is predicted. To provide against this eventuality, enormous efforts have been made to realize the synthesis of ammonia or nitric acid from the nitrogen of the air, and now it is certain that the industry will be able one day to furnish, in one form or the other, all the nitrogen necessary for agriculture. However the technical solution will not solve the economic problem. Synthetic ammonia and nitrate, in view of the cost of the energy required, will always be as expensive as it is today, and agriculture will continue to pay too high a price for artificial fertilizers. Under these circumstances, it appears more logical to seek to solve the question, not through the chemical, but rather through the biological route.

Biological Fertilization. - We have seen that a sterilized soil nourishes only a poor vegetation, and that, on the other hand, the harvest depends largely on the bacterial flora contained in the soil. In reality, the most important factor is not so much the presence of an abundance of bacteria, or even the nature of these, but rather the equilibrium which regulates their multiple activities. Thus it is that the nitrifying bacteria, so beneficial to the fertility of the soil, become injurious if they are predominant, for then they accomplish a too intense work, bringing about a production of nitrate which runs the risk of not being assimilated by the plant. Also, when present in too large an excess, they may retard the development of the species which controls the decomposition of the carbohydrate material, and accordingly may inhibit the functioning of the micro-organisms which fix atmospheric nitrogen. We do not yet possess an infallible means for preserving a favorable equilibrium between the bacterial flora of the soil. However, various investigations have been made in this direction, and the progress already realized is very encouraging.

The problem can be regarded from two different standpoints. To aid in the selection of useful bacteria, we can proceed 
either by the direct elimination of the injurious species, or by the introduction of useful species characterized by great resistance. A rational drainage, the breaking up and constant stirring of the soil, the dressing with lime, the addition of certain inorganic fertilizers or of manure, the changes produced by the rotation of crops, are all indirect means used to maintain equilibrium among the various micro-organisms. We may also mention, in this connection, the work of Hiltner relative to the action of carbon-disulphide on the soil, in which he pointed out the efficacy of certain antiseptics in changing the bacterial flora contained in a soil. Furthermore, Stoklasa has shown quite recently that radioactivity exerts an advantageous influence on the general circulation of nitrogen, favoring the bio-synthetic work of the bacteria which fixate, and retarding, on the contrary, the action of the reducers of nitrates. Finally, the application of manure or even soil rich in bacteria is also a means which leads to the same result as the preceding.

Although the nitrifying bacteria most often find favorable conditions in the soil, the accumulators of nitrogen often encounter numerous antagonists. Also, the introduction into the soil of species endowed with a great activity, from the point of view of assimilation of free nitrogen and accustoming themselves to the conditions of the medium, is an economic problem of enormous import. In this question, as in so many others, empiricism has preceded science. For a long time, in many countries, the soil of leguminous plants was carried over to the soils of poor quality to improve them. Having given conclusive results, this practice has gradually spread. However, to be efficacious, it is necessary that the quantity of soil introduced shall be large, from 30 to 40 hectolitres per hectare. As the transportation of these soils is not always convenient, it has been attempted to remedy this inconvenience by the use of pure cultures of bacteria. Large German manufacturers of chemical products first provided bacterial preparations designed for this purpose.

The Alinit of Caron, made at Elberfeld, was the first attempt made in this direction. The fact that it has not given the 
expected results is because the bacterium chosen was not a good assimilator of nitrogen. On the contrary, Nitragin, manufactured at the Höchst factory for dyes, has, after many trials, found application in agriculture. It is prepared by means of bacteria coming from leguminous plants, and is of an unquestioned utility in virgin soils, in soils which have not yet grown leguminous plants, and also in those where this kind of plant grows only with difficulty. As a general rule, this bacterial culture, and other similar ones, gives good results in lands poor in nitrogen and rich in carbohydrate material, the presence of potassium and of phosphates being always necessary. The best returns are obtained in the cultivation of certain species of leguminous plants. The difficulty in the use of this product consists in the fact that, during the germination of the seeds, there are secreted substances harmful to the bacteria, so that these die before having penetrated into the roots. To obviate this inconvenience, it has been advised to let germination proceed quite far before inoculating the bacteria, for at a certain point of growth this danger disappears. Another means, although not practical, of inhibiting the action of these injurious secretions consists in adding peptone or whey to the soil.

The almost insurmountable difficulties which Nitragin encountered at the beginning have been, in great part, overcome by the choice of suitable species, and by a rational use. Agar, on which the first cultures were made, has been replaced by cotton. Wadding is plunged in a bacterial culture, is then dried, and the preparation is delivered to commerce in the form of dry cotton. It is accompanied by two packages of nutritive substances, the first containing sugar, potassium acid phosphate and magnesium sulphate, the second containing ammonium phosphate. To render the culture active, it is only necessary, at the moment of use, to plunge the wadding into water and to add to it the contents of the first package. At the end of 24 hours at $20^{\circ}$, the second package is added and the whole is left to ferment for some time. The liquid, thus prepared, is used to moisten the seeds to be used for sowing. The liquid can 
also be incorporated in sand or earth, which is then spread on the field. The bacteria of Nitragin are a mixture of various species taken from different leguminous plants; they can therefore serve for various crops.

In addition to Nitragin, we also find, as commercial products, Azotogen, Formogen, etc. We possess some data on the yields obtained with all these bacterial cultures. The opinions are very diverse, and in certain cases, the inoculated ground would give a plus-value mounting to $200 \mathrm{~kg}$. of nitrogen per hectare. These good effects have been obtained especially with leguminous plants and by means of nitrogen-fixing agents living in symbiosis with the plant. But with cereals the results have been less favorable, for the assimilators of nitrogen, which directly utilize the carbohydrates of the soil, do not lend themselves well to inoculation.

The great problem then remains of inducing nitrogen accumulators to intervene in the cultivation of cereals, without having to pass through the intermediate phase of the leguminous plants.

To advance in this direction, it is not sufficient to find in nature bacteria of great activity, to isolate and to propagate them. Such researches could lead us to the discovery of species suitable to certain soils under certain physical and chemical conditions of the environment. We cannot expect to find a species capable of fitting all possible requirements, for if such a micro-organism existed, it would already have supplanted all the others. The properties acquired by a microbic species are always the result of its environment. The predominating quality of fixing atmospheric nitrogen is counterbalanced by the tendencies which the bacterium has of living on its own nitrogenous reserve, or else of utilizing the combined nitrogen contained in the surrounding medium. To obtain cultures whose power of assimilating nitrogen shall be developed to an extreme, we must first of all bring about the conditions most favorable for this function, and then it is necessary, by a progressive accustoming of the bacterium to these conditions, to increase and stabilize this specific property. This accustoming 
should also be applied to the rational and economical utilization of carbon, a factor varying with the species, with the age of the bacterium, and also with the composition of the medium.

Future of Biological Fertilization. - After having chosen a suitable species, investigations should strive for: Ist, increasing the resistance of this bacterium in its struggle with other unacclimated species; 2nd, increasing its power of assimilating nitrogen; 3 rd, reducing to a minimum the decomposition of carbonaceous material. The goal, at first sight, seems unattainable. However, if we examine it closely, we see that it is only a question of accentuating properties already inherent in the species, that is to say, of changing, at least quantitatively, the catalysts which intervene in this work, all being variations that have already been realized in a satisfactory manner in other branches of fermentations. Are not industrial ferments for the most part domesticated micro-organisms, bent by a sort of education to the work which is required of them? The yeasts of top and bottom fermentation used in brewing are not cells identical with those which are encountered in the wild state; they have acquired their special properties in consequence of the artificial conditions to which they have been subjected for a very long time. The distillery yeasts, and those of the brewery and the bakery, are three types of ferments having the same origin, and yet possessing absolutely distinct specific properties. Their differentiation comes just as much from the nature of the catalysts which they bring into play as from the actual proportion of these catalysts.

Acclimation to special media may also cause the development and the secretion of enzymes which under ordinary conditions are not found in appreciable quantities. Thus, Effront found that yeasts which are ordinarily inactive on dextrins acquire the property of secreting amylase, and consequently of causing this carbohydrate to ferment, when they are acclimated to nitrates. By the choice of a suitable medium, we increase at will the fermenting power of yeast or its power of growth. It is true that the properties thus acquired by a long accustoming 
cannot be considered as definite, for they disappear in the long run if we change the conditions of the environment; but the modification resists for months, sufficiently long to be utilized with profit industrially.

The influence of acclimation on chemical activity is also observed in ammoniacal fermentation. We know, scientifically speaking, of no ammonia bacterium capable of transforming in 24 hours $\mathrm{I} .5$ to $2 \mathrm{~g}$. of amino-acid per liter into ammoniacal nitrogen. Yet this work is accomplished industrially today by butyric ferments acclimated according to the Effront process. ${ }^{*}$ In the same manner efforts have been directed toward the acclimation of yeasts to antiseptics and other conditions of environment. A progressive training succeeded in radically changing the sensitiveness of Saccharomyces to the reactions of the environment, and in causing yeast to withstand quantities of antiseptic at least a hundred times greater than in the beginning. At the same time, a profound change was effected in the progress of the cellular work, which had the interesting result of perceptibly reducing the production of glycerin, of succinic acid, and of higher alcohols.

Working in another field, Muntz and Lainé, by studying in detail the conditions for the formation of natural saltpeters, have succeeded in instituting a rational treatment which improves the yield in the ratio of 60 to $\mathrm{I}$. This new process is based on the utilization of peat as raw material, and makes use of special arrangements which the inventors have called inclined niterbeds. These first receive a solution of ammonium sulphate, 7.5 g. per liter. Then, by means of a continual enrichment of the already nitrated liquid with fresh sulphate, they finally arrive at solutions containing as much as $200 \mathrm{~g}$. of potassium nitrate per liter. We see that these results are obtained here again by successive acclimations of the bacterium to increasing quantities of sulphate and of nitrate, the nitric bacterium of the saltpeters of Muntz and Lainé very probably differing from the bacterium of the soil from which they were obtained.

* See the following paragraph on the Recovery of Nitrogenous Wastes. 
All these facts indicate plainly that certain properties of micro-organisms can be increased or diminished. A more thorough study of the bacteria which assimilate nitrogen will disclose likewise the means for increasing this peculiar property which they already possess, and which is all the more valuable in that upon it depends the agricultural fortune of a large number of countries. Without doubt, while the experiments made in this direction have not as yet led to definite results, there is room to hope that success will come in the near future.

\section{BIBLIOGRAPHY.}

ANDré. Chimie agricole, $\mathrm{I}$ vol., Paris, IgI 2.

BEIJERINCK. (I) Centralbl. f. Bakt., I892, (II), p. 69.-(2) Ueber oligonitrophile Mikroben, Ibid., r9or, (7), p. $5^{67}$.

Beijerinck u. Van Delden. (I) Assimilation des freien Stickstoffs durch Bacterien, Centralbl. f. Bakt., I902, (9), p. 33. - (2) Ueber eine farblose Bacterie deren $\mathrm{CO}^{2}$ Nahrung aus der atmosphärischen Luft berührt, Ibid., I903, (Io), p. 34 .

Beijerinck u. Minckman. Bildung und Verbrauch von Stickoxydul durch Bacterien, Centralbl.f. Bakt., rgog, (25), p. 30.

Berthelot. Chimie végétale et agricole, 4 vol., Paris, 1899.

Berthelot et ANDRÉ. (I) Observations sur la proportion et le dosage de $\mathrm{NH}^{3}$ dans le sol, C. R., r886. - (2) Recherches nouvelles sur les microorganismes fixateurs d'azote, Ibid., 1893.

Boullanger et Massol. Microbes nitrificateurs, Ann. Inst. Past., 1903, p. 492; 1904, p. I8I.

CALMETTE. Recherches sur l'épuration biologique et chimique des eaux d'égout, 5 vols., Paris.

Dehérain. Chimie agricole, I vol., Paris, I902.

Dehérain et Maquenne. Sur la réduction des nitrates dans la terre arable, $C$. $R$., (95), p. 854. Bull. Soc. Chim., 1882, (39), p. 49.

G. FERMI. Ueber die Gegenwart von Enzymen in Boden, im Wasser u. in Staub, Centralbl. f. Bakt., 1910, (26), p. 330.

P. et G. Frankland. Philos. Transact. Royal Soc. London, 1890, (18r), 107. GaYon. Sur la fermentation du fumier, $C$. R., r884, (98), p. 528 .

Gayon et Dupetit. (I) Sur la fermentation des nitrates, C. R., 1882, (95), p. 644 . - (2) Recherches sur la réduction des nitrates, Nancy, 1886.

Grimbert. Étude du Bacillus orthobutylicus, Ann. Inst. Past., 1893, p. 354.

Hellriegel u. Wilfarth. Beilageheft z. Zeits. d. Ruibenzuckerindustrie, 1888. ED. Kayser. Microbiologie agricole, I vol., Paris, IgrI.

Kossowicz. (1) Einfiihrung in die Mykol. d. Genussmittel u. in die Gärungsphysiologie, Berlin, I9Ir. - (2) Agriculturmykologie, Berlin, I9I 2. 
LAURENT. (I) Recherche sur la valeur comparative des nitrates et des sels ammoniacaux, Ann. Inst. Past., 1889, p. 362. - Réduction des nitrates, Ibid., I89o, p. 741 .

LEBEDEFr. (I) Ueber die Assimilation von $\mathrm{C}$ bei wasserstoffoxydirenden Bakterien, Bioch. Zeits., 1907, p. 1.-(2) Ber. d. deuts. Bot. Ges., 1909, (27), p. 569 .

Lemoigne. Bactéries dénitrificantes des lits percolateurs, C. R., Igri, (152), p. 1873 .

MazÉ. Les phénomènes de fermentation sont des actes de digestion, Ann. Inst. Past., 191 r, pp. 288, 36 .

LöHns. Handbuch d. landw. Bakteriologie, Berlin, I9ro.

Marchal. Bull. Acad. de Belgique, 1893, pp. 738, 765 .

MUNTZ et LAINÉ. Recherches sur la nitrification intensive, Ann. de l'Inst. nation. agronomique, 1907, (6).

NAWIASKy. Arch.f. Hyg., I907, p. 6I; 1908, p. 209.

Niklewski. Centralbl.f. Bakt., igo8, p. 469 .

Omelianski. Sur la fermentation de la cellulose, 1895, (2), p. 653.

Prazmowski. Landwirthsch. Versuch. Stat., I890, p. I6r.

E. Roux. Les engrais et les amendements, I vol., 1896, Paris.

Schlcesing. Réduction des nitrates, $C . R$., 1873 , (77), p. 353.

Schlcesing et Muntz. Sur la nitrification par les ferments organisés, $C$. $R$., I878, p. 892 .

Schlcesing, fils. Contribution à l'étude de la nitrification dans les soıs, $C . R$, I889, pp. 618 et $673 ; 1897,(2)$, p. 824 .

Stoklasa. (I) Centralbl. f. Bakt., 1898, p. 817; 1900, p. 22. - (2) Assimilation von $\mathrm{N}$ durch Azotobacter und Radiobakter, Zeits. f. Riibenzuckerindust., I906, p. 815. - (3) Influence de la radioactivité sur les microorganismes fixateurs d'azote ou transformateurs de matières azoteés, C. R., 1913, (I57), p. 879 .

Suzuki. Entstehung der Stickoxyde bei Denitrification, Centralbl. f. Bakt., I9II, (3I), p. 27.

Trillat et Sauton. Action des gaz putrides sur les microbes, C. R., Igog, (2), p. 875 .

Winogradsky. Recherches sur les organismes de la nitrification, Ann. Inst. Past., 1891, p. 577; 1893, (116), p. 1385 .

\section{§ I2. Recovery of Nitrogenous Wastes.}

The study of bacterial amidases has led the writer to seek a method for recovering and utilizing the residual nitrogen of various industries. This problem is concerned with two distinct propositions: (I) the transformation of organic nitrogen, such as that contained in the waste liquors from distilleries or in the scum from sugar-refineries, into ammonia and volatile fatty 
acids; (2) the utilization of non-nitrogenous organic substances of these residues for fixing atmospheric nitrogen through bacterial agencies. The transformation of residues into ammonia and fatty acids can be realized through the amidases of bacteria, as well as by those of yeast. Evidence of the activity of the latter is obtained when compressed yeast acts on solutions of amino-acids in the presence of strong amounts of alkali. In 800 g. of water, containing 60 c.c. of normal $\mathrm{NaOH}, 20$ g. of asparagin or of another amino-acid and $80 \mathrm{~g}$. of compressed yeast are introduced. The solution is kept at $40^{\circ}$ for eight or ten days, and then the liquid is brought to a volume of one liter:

Action of Yeast on Amino-ACIDS.

\begin{tabular}{|c|c|c|c|c|}
\hline Amino-acids. & $\begin{array}{c}\text { Nitrogen of } \\
\text { filtrate } \\
\text { (per liter). }\end{array}$ & 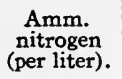 & $\begin{array}{c}\text { Amine } \\
\text { nitrogen } \\
\text { (per liter). }\end{array}$ & $\begin{array}{c}\text { Volatile } \\
\text { fatty acids } \\
\text { (per liter). }\end{array}$ \\
\hline 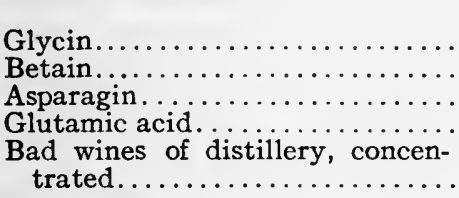 & $\begin{array}{l}\text { g. } \\
5.20 \\
3.64 \\
4.77 \\
3.04 \\
6.15\end{array}$ & $\begin{array}{l}\text { g. } \\
4.63 \\
\text { I.30 } \\
4.00 \\
2.81 \\
2.60\end{array}$ & $\begin{array}{l}\text { g. } \\
0.00 \\
2.25 \\
0.00 \\
0.00 \\
3.05\end{array}$ & $\begin{array}{l}\text { g. } \\
24.80 \\
16.96 \\
17.19 \\
20.40 \\
34.00\end{array}$ \\
\hline
\end{tabular}

In these experiments, in addition to the nitrogen of the amino-acids, there was that resulting from the autophagy of the yeast. Now, of the I300 mg. of nitrogen introduced with the $80 \mathrm{~g}$. of yeast used, there remained on the filter, according to the experiment, only 30 to $300 \mathrm{mg}$. of insoluble nitrogen. The remaining nitrogen, as well as that coming from the aminoacids, has been transformed wholly into ammonia in the course of fermentation. On the other hand, the quantity of volatile acids formed is proportional to the ammonia nitrogen produced. Finally, we may find that the nature of the acids appearing depends on the amino-acid used: 
Variation of the Composition of Acids According to the AminoACID USED.

\begin{tabular}{|c|c|c|c|c|}
\hline Amino-acid used. & $\begin{array}{l}\text { Average } \\
\text { molecular } \\
\text { weight of } \\
\text { acids. }\end{array}$ & $\begin{array}{c}\text { Acetic acid, } \\
\text { per cent. }\end{array}$ & $\begin{array}{l}\text { Propionic } \\
\text { acid, } \\
\text { per cent. }\end{array}$ & $\begin{array}{l}\text { Butyric } \\
\text { acid, } \\
\text { per cent. }\end{array}$ \\
\hline 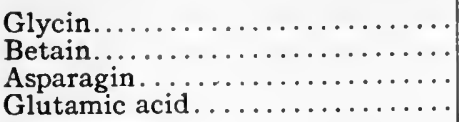 & $\begin{array}{l}65 \cdot 4 \\
65 \cdot 3 \\
72.03 \\
80.0\end{array}$ & $\begin{array}{l}61 \cdot 7 \\
64 \cdot 3 \\
16.6 \\
14 \cdot 1\end{array}$ & $\begin{array}{l}30.6 \\
25.6 \\
77.2 \\
19.2\end{array}$ & $\begin{array}{r}7.6 \\
10.1 \\
16.0 \\
66.7\end{array}$ \\
\hline
\end{tabular}

The percentage of acids furnished by the fermentation of amino-acids approaches the theoretical, the discrepancies observed resulting from the nitrogenous substances introduced with the yeast:

\begin{tabular}{|c|c|c|c|c|c|c|}
\hline \multirow{2}{*}{ Amino-acid used. } & \multicolumn{2}{|c|}{ Acetic acid } & \multicolumn{2}{|c|}{ Propionic acid } & \multicolumn{2}{|c|}{ Butyric acid } \\
\hline & calc. & found. & calc. & found. & calc. & found. \\
\hline 100 parts of glycin..... & 80.0 & 76.5 & & & .. & \\
\hline 100 parts of betain. ....... & 51.2 & $54 \cdot 3$ & $\ldots$ & & $\ldots$ & .... \\
\hline roo parts of asparagin. .... & & $\ldots$ & $49 \cdot 32$ & 66.0 & & \\
\hline ro parts of glutamic acid. & $\ldots$ & $\ldots$ & $\ldots$. & $\ldots$ & 59.8 & 68.0 \\
\hline
\end{tabular}

In the experiments with yeast, it is the amidases in reserve inside the cells which act. Necessarily, we are led to use large quantities in order to obtain results. To obviate this inconvenience, which would render the process not very practical, bacteria are utilized that are capable of secreting amidases in abundance. The bacterial species chosen must be well determined, for not all the ammonia ferments lend themselves to the exacting work required by industry. Moreover, it is for many reasons indispensable that the bacterium adopted shall be acclimated to the conditions of the environment so that the formation and the secretion of its catalysts shall be favored as much as possible. In fact, bacterial activity producing ammonia is always very slow. The bacteria are very sensitive to their own products, and the deamidization which they cause is never 
complete. It is only after having been trained to withstand alkalinity and increasing temperatures that they lose, to some extent, the preceding defects. Moreover, the introduction into the culture medium of earth and certain salts, like those of aluminium, wonderfully reinforces the virulence and rapidity of action of the ammonia bacteria. Aëration also exerts a good influence on this kind of fermentation.

It has just been stated that the ammonia-producing bacteria do not all lend themselves to an intense activity. Thus, if we abandon to putrefaction a solution of an albuminoid, we see that the quantity of ammonia gradually increases. Then, having arrived at about a third of the total nitrogen, the production stops, although the fermentation, properly speaking, still continues. We observe similar results with pure cultures of ammonia ferments. A nutrient medium, composed of asparagin, peptone, and calcium carbonate, and containing 0.49 per cent of nitrogen, is inoculated with pure cultures. After a 30 day fermentation at $40^{\circ}$, the ammonia in the liquid is determined, as well as the volatile acids:

\section{Production of Ammonia and Volatile Acids by Putrefactive and OTHER BACTERIA.}

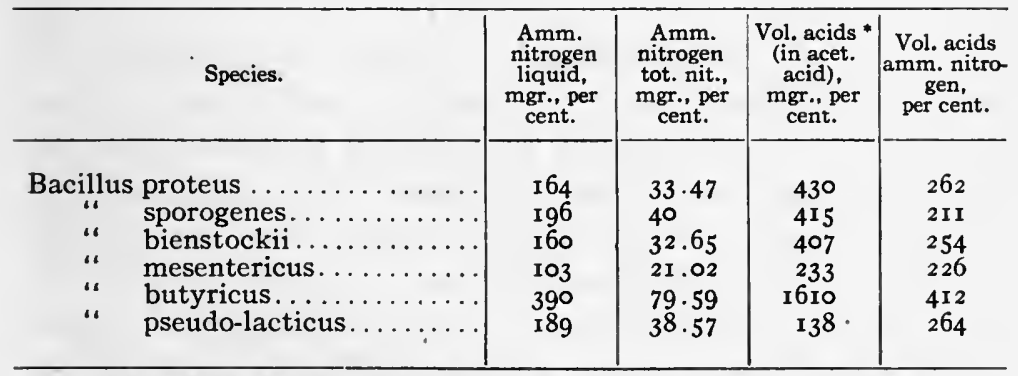

- This table shows that the different bacterial species produce variable quantities of volatile acids; elsewhere we have found that they are also capable of changing greatly the quantitative composition of the latter.

Of the putrefactive bacteria it is $B$. sporogenes that produces the maximum of ammonia; the quantity, however, does not exceed 40 per cent. A much more thorough action is realized with $B$. butyricus, which gives 79.59 per cent under the same 
conditions of time and medium. However, this high percentage of ammonia cannot be found again when the nitrogen content of the liquid is increased, the products formed being themselves very injurious to the bacteria:

\section{Intensity of Ammoniacal Fermentation According} to the Nitrogen Content.

\begin{tabular}{|c|c|c|}
\hline $\begin{array}{c}\text { Nitrogen con- } \\
\text { tent of the } \\
\text { medium per } \\
\text { liter. }\end{array}$ & \multicolumn{2}{|c|}{$\begin{array}{c}\text { Ammoniacal nitrogen per roo } \\
\text { total nitrogen. }\end{array}$} \\
\cline { 2 - 3 } & After ro days. & After 6o days. \\
\hline g. & Per cent. & Per cent. \\
5 & Io & 81 \\
IO & 9 & 49 \\
I5 & I I & 38 \\
20 & 9 & 33 \\
\hline
\end{tabular}

We see then that the production of ammonia depends not only on the bacterial species, but also on the concentration of the medium in nitrogenous substances. Furthermore, it is also related to the temperature. For actions of short duration, the optimum is $55^{\circ}$. Thus, in a fermentation of 24 hours duration, we obtain from 40 to 50 per cent more ammonia at this temperature than at $40^{\circ}$. But the results observed in longer fermentations are more favorable at the temperature of $40^{\circ}$ than at that of $55^{\circ}$. We can then conclude that the production and the secretion of amidases are favored by quite high temperatures, but that in the long run this weakens the bacteria. However, this sensitiveness to the concentration of the medium and to the elevation of the temperature disappears with acclimation.

This is accomplished by the method used before by the writer for the acclimation of yeast to different antiseptics. The fundamental principle consists in always working with young cultures. The cultures are renewed as often as possibleevery 24 hours or at least every 48 hours. The rejuvenation is accomplished by means of a very copious inoculation, using from 20 to 25 parts of fermented liquor per roo parts of fresh liquor. The rise in temperature and the increase in density or in alkalinity takes place very slowly, and only when an already 
marked progress has been acquired. The results obtained by a successive acclimation to density and to temperature, and corresponding to a fermentation of 6 days in length, are summarized in the following table:

Accustoming of Butyric Ferments to Rising Temperatures and INCREASING CONCENTRATIONS.

\begin{tabular}{|c|c|c|c|}
\hline Dates. & $\begin{array}{l}\text { Content in } \\
\text { nitrogen of the } \\
\text { medium per } \\
\text { liter. }\end{array}$ & $\underset{\text { temperature. }}{\text { Optimum }}$ & $\begin{array}{r}\text { Ammoniacal } \\
\text { nitrogen in } 24 \\
\text { hours per liter. }\end{array}$ \\
\hline 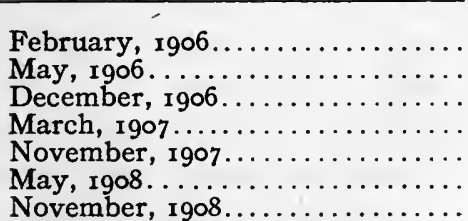 & $\begin{array}{r}\text { g. } \\
5.0 \\
7.0 \\
8.3 \\
9.6 \\
10.8 \\
14.0 \\
\text { I6.0 }\end{array}$ & $\begin{array}{l}{ }^{\circ} \mathrm{C} . \\
42 \\
47 \\
5 \mathrm{I} \\
52 \\
53 \\
54 \\
55\end{array}$ & $\begin{array}{l}\text { g. } \\
0.15 \\
0.65 \\
0.95 \\
\text { I. } 10 \\
\text { I. } 20 \\
\text { I. } .30 \\
\text { I. } .45\end{array}$ \\
\hline
\end{tabular}

From I906 to 1908 , acclimation resulted in an increase of from 5 to $16 \mathrm{~g}$. per liter in the nitrogen content of the mash treated. At the same time an increase was observed in the virulence of the bacteria. This gave originally, in 24 hours, $0 . x 5 \mathrm{~g}$. of ammoniacal nitrogen, and it ended by giving $\mathrm{x} .45 \mathrm{~g}$. It also became much more resistant to heat: its optimum temperature, which was $42^{\circ}$, was finally raised to $55^{\circ}$. However, in this condition, the ferment does not yet show the maximum activity. The yield is not theoretical, and to obtain a better result, recourse must be had to a strong aëration and to additions of culture earth, as well as salts of aluminium.

Industrially, the process is applied in the following manner: Distillery liquors, with a density of $10^{\circ}$ to $13^{\circ}$ Beaumé, made alkaline with Io to $12 \mathrm{~g}$. caustic per liter, are brought to the temperature of $55^{\circ}$, and are then put aside to ferment, either with a prepared yeast, or by mixing, as is the practice in beet distilleries. The duration of the fermentation is from 4 to 6 days. The acclimated ferment, once introduced into the factory, has a preponderance over all the foreign ferments; this is why the fermentation takes place with a great regularity, although unsterilized mashes and open vats are used, and for years no 
disturbance has occurred in the progress of the work. The ammonia obtained, containing also a certain quantity of trimethylamine, is at most 85 per cent of the total nitrogen put to ferment. In this calculation the nitrogen contained in the yeast of the slop, which under the existing conditions is not transformed into ammonia, is not included.

The fermentation ended, the ammonia is separated from the amines by distillation in the presence of an excess of alkali, and is absorbed in sulphuric acid. The liquid is then concentrated to $40^{\circ}$ Beaumé, sulphuric acid added, and is treated for the recovery of the volatile acids. Freed from these by heating, the residue contains, in addition to potassium sulphate, acids such as succinic and malic. These substances are found in the mother liquor from the crystallization of the potassium sulphate. The slop resulting from the manufacture of $\mathrm{x}$ hectolitre of alcohol gives from 3 to 4 kilos of crystallizable organic acids.

The recovery plant of Nesle (Somme), where this process is applied, ${ }^{*}$ and which utilizes the waste liquors from a single distillery, produces daily from 1000 to 1400 kilos of nitrogen in the form of ammonia and trimethylamine, and from 10,000 to $12, \infty 00$ kilos of volatile acids. The separation of the ammonia and the trimethylamine is very easy, and takes place generally almost quantitatively, the first of these substances being transformed into sulphate, the second being converted into potassium cyanide. As to the volatile acids, they are composed of acetic acid and butyric acid in almost equal proportions. It is likewise possible to separate in a satisfactory way these two products, and the factories at Nesle produce almost chemically pure glacial acetic acid, and butyric acid with a boiling point very near the normal figure.

It should, however, be noted that the relative proportions of the two nitrogenous derivatives are variable. When we work with liquors from grains, the ammonia formed is free from $*$ The
$189,212]$. 
amines; on the contrary, with the wastes of molasses we get considerable quantities of trimethylamine.

Another procedure is followed when it is desired to produce ammonia and trimethylamine exclusively. The liquors, after the ammoniacal fermentation, are distilled to recover the volatile nitrogenous products. Then to the liquid is added culture earth rich in azotobacter, and the whole is allowed to ferment at $40^{\circ}$ in a continuous current of air. At this stage there is produced simultaneously an active combustion of carbohydrate material and a fixation of atmospheric nitrogen, the latter being converted to the organic state. It is thus found that in a fermentation lasting 5 or 6 days the mixture is enriched by 3 to $4 \mathrm{~g}$. of nitrogen per liter. At this point the fixed nitrogen is treated by one of the two following procedures: (r) The fermentation ended, the liquid is allowed to settle, is decanted and then the sediment remaining is sent to the filter-press. The cakes so obtained, when dried, form a complex nitrogenous fertilizer of great value. (2) After decanting the clear liquid, the sediment is diluted with a little fresh slop, is heated at $100^{\circ}$ for an hour, and then is put in mash vats undergoing active ammoniacal fermentation. Under these circumstances, the organic nitrogen fixed by the bacteria is transformed into ammonia nitrogen, which thus enters again into the process.

From the economic point of view the process has considerable importance. We should, in fact, note that the waste liquors filtered from grains and those of beets are at the present time poured into the river, and that those of molasses are treated in the furnaces for the recovery of the potassium. Now, the organic nitrogen annually lost in this manner in distilleries is very valuable. By dumping the liquor from beets and grain in the sewers, we lose more than two million kilos of organic nitrogen; on the other hand, the calcining of the molasses liquor entails a loss of 16 million kilos. Finally, the non-utilization of these residues entails the loss of $\mathrm{I} 20$ to $\mathrm{I} 30$ million kilos of fatty acids that could have been recovered by fermentation. The value in round numbers of the products thus lost annually may be 
estimated at from io to 14 million dollars for Europe alone. The volatile acids formed in this decomposition will always have a market, even if they reach a very large production, for they constitute an excellent material for the manufacture of acetone. The fermentation process is very simple indeed and gives constant yields. The principal improvement needed at the present time is in the mechanical installation of the refinery. An industry of this importance, when it has just been created, always requires modifications in the mechanical appliances, and its more or less rapid development will depend on the ingenuity with which these changes are introduced.

\section{BIBLIOGRAPHY.}

Erfront. (1) Sur la fermentation des acide amidés, Mon. Scient., rgog, p. 145. - (2) Sur l'utilisation de l'azote des résidus de distillerie, Ibid., I908, p. 429.

$\S$ I3. Artificlal Nitrogenous Foods. Nutritive Value of Products of Advanced Hydrolysis of Protein SubSTANCES.

Synthesis of Albuminoid Substances in the Organism. - We have known for a long time that protein foods, before entering into the circulation, undergo the action of many enzymes: The products formed through the influence of peptic and tryptic digestions thus serve for the reconstruction of the various albuminoids of the organism. These general data however raise a series of secondary questions which become more and more pressing as our knowledge in the domain of the constitution and the hydrolysis of albuminoids is enlarged. Not long ago it was believed that the products giving the biuret reaction were the only ones that intervened in the synthesis of albuminoids in the animal, the crystalline products, of a relatively simple structure, which appeared in tryptic digestion being regarded as waste possessing an entirely secondary importance.

This opinion was in accord with the preconceived idea that was entertained concerning the hydrolysis of proteins. 
The study in vitro of proteolytic enzymes gave an entirely erroneous picture of digestion as it really occurs in the animal organism. First of all, digestion in vitro, with pepsin or with trypsin, takes place very slowly. Furthermore, the hydrolysis is not very thorough, even after a time sufficient for natural digestion to be complete. We could then very easily conclude that the work of the proteolytic enzymes was limited to splitting the complex protein molecules into substances still very high in molecular weight, which retain all the properties of the initial albuminoid material, and which can easily be reconstructed into the same. The analyses of the alimentary bolus in the different phases of digestion also gave results favorable to the hypothesis that peptones were capable of regenerating albuminoid material, since it is known that the content of the stomach and of the first portions of the intestine is chiefly made up of albumoses and peptones with very little of the more thoroughly hydrolyzed material.

It should be recognized that the idea of the direct assimilation of peptones and their later transformation into albuminoids found serious support in a celebrated experiment of Hofmeister and Neumeister, who proved that fresh intestinal tissue possesses the property of rendering non-biuretic a solution of peptone. The disappearance of the peptones under these circumstances was interpreted as involving a reconstruction of the original albumin. However, this argument of Hofmeister was confuted in I90I by an experiment of Cohnheim, who proved that the absence of the non-biuretic reaction does not entail the retrogression of the peptone into albumin, but, on the contrary, is due to a more complete hydrolysis of this peptone under the influence of the erepsin contained in the tissue.

However that may be, the work of Pawlow has contributed much to the modification of our ideas as to the limit of the degradation of the albuminoid molecules under the influence of the proteolytic enzymes. Hitherto, in the study of the protein digestion, we used only preparations coming from extracts of organs. These products were naturally of slight activity, and 
their very methods of preparation involved a contamination with a quantity of impurities which influenced, not only the velocity of the reaction, but also the final result. Pawlow, by perfecting the technique in the application of fistulas to animals, rendered their use practical, and so allowed us to procure pure gastric and pancreatic juices and in abundant quantities. The active substances obtained in this way digest the albuminoid very rapidly, and thus give crystalline substances much more quickly. The experiments made in vitro with these natural juices leave the impression that the transformation of proteins in the alimentary canal leads to an advanced molecular degradation, and that the result of the analysis of the alimentary bolus, of which we have previously spoken, should receive a different interpretation. The albumoses and the peptones, which were found in the intestinal content, should be regarded as intermediate products on the way to transformation, while the ultimate products of digestion, the amino-acids, are absorbed very rapidly and regenerated into animal albuminoids. Moreover, we also find amino-acids in the advanced portions of the intestine.

This new conception explains the interest which has been attached for some time to the study of the amino-acids and the other products of the advanced hydrolysis of protein substances, substances whose nutritive value raises interesting problems both from the theoretical and from the practical standpoint. The synthesis of albuminoids, although now recorded as a definitely acquired fact in certain recent texts on physiology, is still far from being realized. The artificially prepared polypeptides, of high molecular weight, while allowing themselves to be hydrolyzed by certain biochemical catalysts, can not be considered as albuminoid, but are only more or less distantly related to certain of the products derived from proteolysis. Furthermore, with these few fragments of so complex a molecule, it does not appear possible to reconstruct the original one, any more than it is possible to remake a broken vase from its incomplete fragments. In reality, the question stands quite differently. If the organism can really utilize a mixture of all 
the amino-acids resulting from the activity of the tryptic and ereptic fermentations, or from the hydrolysis with inorganic acids as the basis for the synthesis of the protein material, a large avenue is opened which would then lead to artificial nitrogenous feeding, a problem which has always been regarded as unsolvable.

Work of Effront. - Since I888, the writer had occasion to determine the food value of various nitrogenous products. At this period, the problem of the use of molasses for the nourishment of cattle occupied to a high degree the sugar manufacturer and the agriculturist. The practice was then widespread, although the molasses was valued solely from the point of view of its sugar content and that of its potassium salt. In the course of his researches on this subject, the writer was able to establish the nitrogenous balance in animals having received large quantities of molasses with their usual food. What influence does this product exert on the nitrogenous feeding? was the question. It was generally thought that salts were unfavorable to the utilization of the proteins of the fodder.

Now, the conclusion drawn from a very large number of analyses of urine and feces was that this hypothesis is in no way confirmed. In fact, the results quite unexpectedly indicated that the nitrogen of the molasses is likewise assimilated. These facts were in complete contradiction with the prevalent idea that the protein substance alone was the nutritive material, the nitrogenous materials of the molasses, composed almost entirely of amino-acids and non-biuretic compounds, not being classed among the nutritive substances at this period.

In order to clear up this point, the writer made nutrition tests on dogs, using products of the advanced acid hydrolysis of various proteins, products whose composition is similar to that of the nitrogenous substances contained in molasses, and which were moreover mixed with starch in such a way as to form a food comparable to molasses itself. In the year 1899, it had been found possible to keep a dog for 18 days in nitrogenous equilibrium by feeding with a mixture of sugar, starch, 
fat, and the products of acid hydrolysis containing no albumose. To these preliminary tests a very great value has been attached from the economic standpoint. The price of meat being on an average 70 cents a kilo, and its content in nitrogen being but 3.5 per cent, we see that a kilogram of meat nitrogen costs five dollars. Now, the nitrogen of dried blood is sold at the rate of 40 cents a kilo; the same is true for fodder; the residual nitrogen of distilleries and breweries comes to 30 cents a kilo. If then we succeed in utilizing these substances in feeding, we realize a considerable economy, even by replacing only a third or. a half of the meat by products coming from the hydrolysis of the nitrogenous residues of animal or vegetable origin. Furthermore, from the point of view of concentrated nourishment, so useful for sick people and convalescents, the strengthening substances, such as peptones and albumoses, which are recommended by physicians, in addition to being very questionable in their effects, are very expensive. Their nitrogen brings a much higher price than that of meat, and consequently they are not procurable by the poorer classes. On the contrary, the extracts of hydrolysis, possessing a great nutritive value and obtainable at a moderate price, could render real service.

The first tests of acid hydrolyses were made with albumins of beef blood. To the albumin, dissolved in water, is added 50 per cent sulphuric acid in such a way that the mixture contains 350 to 400 g. of acid per liter. The product, now very pasty, is left in the steam-bath at $90^{\circ}-95^{\circ}$ from 8 to ro hours, until it contains no more albumose and no longer gives the biuret reaction. At this point, the liquid is diluted with water, filtered, treated with lime, and filtered again. The remaining sulphates are precipitated with barium chloride, and the filtrate is evaporated in a vacuum. The product obtained has an astringent taste which improves considerably with keeping. To get products more perfect from the point of view of taste, a current of air is allowed to pass into the liquid during boiling, before evaporating in a vacuum. This aëration of the filtered mass has proved beneficial, especially for the extracts of 
malt, of hay, etc. The product obtained with albumin contained from 9 to r r per cent of nitrogen. The analysis is as follows:

\begin{tabular}{|c|c|c|}
\hline Per roo of nitrogen. & Product A. & Product B. \\
\hline Nitrogen precip. by zinc sulphate. & 0.26 & $0 . \infty$ \\
\hline " $"$ by $\tan n i n \ldots \ldots \ldots \ldots \ldots$ & I.22 & I.60 \\
\hline “ by phosphotungstic reagent & 38.01 & $23 . \infty$ \\
\hline
\end{tabular}

The product A, analyzed by the Sörensen method, contains 60.04 per cent of the total nitrogen as amine nitrogen. When completely hydrolyzed with hydrochloric acid, it gives 75.5 per cent amine nitrogen. The product $\mathrm{A}$, then, still contains 20.6 per cent of polypeptides. It is to be noted also that $A$, which still precipitates with zinc sulphate, no longer gives the biuret reaction. It, however, belongs to the type of least hydrolyzed products, though its composition differs radically from the commercial products sold under the name of peptones. All these last contain at least from 30 to 40 per cent albumoses, and the tungstic precipitate gives a decided biuret reaction. In the product $B$, we find only 23.0 per cent of the total nitrogen as tungstic nitrogen. Here the hydrolysis is pushed to the extreme. It is this type which has served in the tests to which we shall return later.

As primary material for the preparation of food extracts, we can also use malt, hay or clover. Malts must be completely cleaned, and should contain at the most not more than 6 per cent of water in order to preserve well. The product obtained with malts or hay is a thick, brown syrup, of a pronounced odor recalling meat extract. Before the malts are allowed to react with the acid, they must be separated as far as possible from the sugar and the starch which they contain. This result is attained by lactic fermentation. For this purpose, I part of malt and 2 parts of water are heated under a pressure of 2 atmospheres. The mass is cooled to $50^{\circ}$, pieces of marble are added to neutralize the acid, and the mixture is inoculated with a lactic 
ferment. The fermentation lasts 2 or 3 days. Then the liquid is separated from the insoluble residue, and this last is pressed and mixed with dilute sulphuric acid (I volume of pressed residue +2 volumes of acid +2 volumes of water). The rest of the operation is conducted as above. From the point of view of the taste of the product, it is recommended not to complete the evaporation in a single operation, but to stop when the syrup reaches $30^{\circ}$ Bé. Let it stand, separate from it the inorganic salts which have been deposited, subject to a strong aëration, and afterwards regulate the concentration.

Tests made with hay have a special interest. The nitrogen of this material hydrolyzes with a surprising facility, and the albumose disappears rapidly under the action of cold acid. The procedure is as follows: In a glass vat, stirred with paddles, put roo kilograms of sulphuric acid concentrated to $57^{\circ}-59^{\circ}$ Bé.; then add the finely cut hay in portions of $10 \mathrm{~kg}$. each. $5^{\circ}$ $\mathrm{kg}$. of hay can easily be added to roo kg. of acid, without the mass becoming too thick. At this point, it is well to reheat the mixture to keep it at $40^{\circ}$. As soon as the liquid is brought to this temperature, continue to introduce the hay until the movement of the paddles is made impossible. With a good apparatus, 250 to $300 \mathrm{~kg}$. of hay can be introduced into $100 \mathrm{~kg}$. of acid. The kneading ended, allow the mixture to stand for I 2 hours. Then, dilute with water, send to the filter-press, neutralize the acid with lime, and finish as before. By this method, the acid dissolves up to 85 or 90 per cent of the dry material contained in the fodder. There remains about io per cent of residue not very rich in nitrogen.*

The products of the advanced hydrolysis of the albumin obtained by the preceding method have been experimented with in 1899 at the Hospital Saint-Jean, at Brussels, by Professor Stiénon, and by Dr. Pechère. The following are the conclusions of Professor Stiénon:

* These products resulting from advanced hydrolysis of proteins by acid have retained the name of peptones, although they have nothing in common with the commercial substances thus designated. 
The experiments were carried out on four subjects. In the first two subjects, ages 31 and 53 , the urea emitted in 24 hours was regularly determined. With the other two, regular determinations of urea were not made, but each day investigations were made as to whether any trouble had been caused by the peptone administered in the daily dose of 30 and of 60 grams. No trouble was found, digestive or otherwise, in any of the subjects experimented with; the peptone was easily taken in broth and caused no anomaly in appetite or in digestion; the stools were not influenced. With the two subjects for whom the regular changes of the elimination of nitrogenous residues of the urine were followed, it was found:

rst. That a dose of $50 \mathrm{~g}$. of peptone, added to the ordinary diet for 5 days, increased the amount of eliminated nitrogenous material $(7.52 \mathrm{~g}$. in one case and $4.8 \mathrm{I} \mathrm{g}$. in the other).

2nd. That a dose of $60 \mathrm{~g}$. of peptone, added to the daily diet for 7 days while the quantity of meat was being diminished by about a half, caused a larger elimination of nitrogenous residues than that of an ordinary diet without peptone. Differences: with the Ist subject, Ir.34 g.; with the $2 n d$ subject, $7.48 \mathrm{~g}$.

$3 r d$. That the weight of the subjects experimented with remained stationary in one case and increased in the other. From experiments made, it is then permissible to conclude that the peptone studied has no injurious influence on the economy; that furthermore it is properly absorbed and assimilated and can, in a certain measure, replace the meat of the normal diet.

On the other hand, the investigations made by Dr. Pechère are reported as follows:

In the first place, three patients convalescing from typhoid fever were observed. Having first established the average daily elimination of nitrogenous material (urea) in the urine, there was added to the diet a certain quantity of the product to be experimented with, diluted in broth. Then the elimination of urea was measured, and the influence of the diet thus modified on the weight of the body was studied:

I. - A. S., age 22, convalescent of typhoid fever for fifteen days.

A. -3 days of ordinary diet:

Daily average of urea............. 23.60 grams

Weight of body................. 48 kilograms

B. - During the 7 following days, daily addition of 60 grams of the peptone tested to the ordinary diet:

Average urea................ 28.88 grams

Weight at the end of 7 days $\ldots \ldots \ldots \ldots \ldots 49.350$ kilograms

Or an average increase of $5.28 \mathrm{~g}$. of urea, and an increase in weight of $1350 \mathrm{~g}$.

C. - During the 7 following days, suppression of the peptone replaced by 100 grams of meat:

Average urea.................. 23.25 grams

Weight at the end of the 7 days........ 50. 165 kilograms

It was then found that there was a diminution in urea of $5.63 \mathrm{~g}$. from the corresponding period, and an increase in weight of $815 \mathrm{~g}$. only. 
II. - J. V., age I6, convalescent from typhoid fever for fifteen days.

A. -3 days of ordinary diet:

Average urea................. 22.15 grams

Weight of subject. . . . . . . .

B. - During 7 days, daily addition of 60 grams of peptone to the ordinary diet:

Average urea................. 26.96 grams

Weight at the end of the 7 days........ 45.200 kilograms

Or an average increase of $4.8 \mathrm{I} \mathrm{g}$. of urea and an increase of weight of $1.740 \mathrm{~kg}$.

C. - The peptone is suppressed and replaced by 100 grams of meat daily:

The 7 th day of this diet the patient weighs $49.500 \mathrm{~kg}$. He has therefore gained 700 grams only.

The measurement of urea made during the last 5 days gives an average of $2 \mathrm{I} .98 \mathrm{~g}$. per day, or an average diminution of urea from the preceding period of $4.98 \mathrm{~g}$.

III. - J. B., age $\mathrm{I} 7$, convalescent from typhoid fever for fifteen days. Weight of body: $53.320 \mathrm{~kg}$.

A. - During 7 days, daily addition of 60 grams of peptone to the ordinary diet:

Weight of body after 7 days: $54.010 \mathrm{~kg}$. Which makes an increase of 690 or $98.50 \mathrm{~g}$. per day.

B. - Suppression of the peptone for 2 days. Weight of body: $54.100 \mathrm{~kg}$. Increase: 90 grams, or 45 grams per day.

C. - Daily addition to his diet for 7 days of 20 grams of peptone and of 100 grams of meat:

Weight of body: $54.730 \mathrm{~kg}$., or an increase of $630 \mathrm{~g}$, , or $90 \mathrm{~g}$. per day.

D. - He is again put on the ordinary diet for 2 days:

Weight: $54.815 \mathrm{~kg}$., or an increase of $85 \mathrm{~g}$., or $42.50 \mathrm{~g}$. per day.

E. - We add to his diet 60 grams of peptone per day, during 7 days:

Weight: $55.620 \mathrm{~kg}$., or an increase of $805 \mathrm{~g}$., or $115 \mathrm{~g}$. per day.

To summarize, the administration of peptone has in the first two cases brought an increase of the proportion of urea, and caused in the three cases an increase in weight. This last is constant during convalescence from typhoid fever; but we shall note, especially by examination of the 3 rd observation, the favorable influence of the administration of the peptone.

IV. - Daily administering for a month of Io to 30 grams of peptone to a neurasthenic whose appetite was poor: at the end of the month, increase of 320 grams in weight.

V. - Daily administering for a month of about $\mathrm{I}_{5}$ grams of peptone to three well children, ages $7,8 \frac{1}{2}$, and $9 \frac{1}{2}$, respectively.

It was not possible to make sure of the weights, but it was possible to discover that the children were not at all inconvenienced by the almost daily ingestion of the product. 
VI. - Daily administering for 20 days of to grams of peptone to three scrofulous children, age $2,2 \frac{1}{2}$, and 3 , respectively.

The weight increased respectively, 120 grams, 185 grams, and 160 grams during 20 days, while during a period of a month without the administration of peptone the increase in weight was 80 grams, 115 grams, and 95 grams.

Dr. Pechère arrives at the following conclusions:

"In none of the cases under observation did the giving of the peptone prepared by M. Effront give rise to any disturbance of appetite or digestion. The stools always remained normal. The weight increased further when we added the peptone to the ordinary diet; the same is true of urea in the urine. This product, according to these findings, has then no ill effect on the economy and represents a food of great value."

The peptones obtained by means of hay or clover are likewise without injurious effect on the organism. The writer took for several months daily doses of 50 to $80 \mathrm{~g}$., and maintained good health. On the other hand, these products may serve as food extract. It is necessary to add only a small quantity of them to soups, sauces, etc., to give a pronounced and very agreeable meat flavor.

The preceding results being very encouraging, the writer believed it possible to make use of his products on a large scale. Since then, patents have been taken out in different countries, especially in Belgium, May 23, 1903 (No. 170,510), with an addition (No. I73,652) dated November I3, I903. Unfortunately, the current opinion in the scientific and medical world was then unfavorable to its use, and in the face of strong opposition the writer completely abandoned the problem. However, the fundamental idea was good, the best proof for which being the fact that the problem was taken up again with much skill and energy by Abderhalden and his pupils, who, assuredly, had no knowledge of either the experiments made in Brussels, or the patents taken out since 1903 , since they never refer to them in their publications. Moreover, the work of the latter has cleared up a certain number of questions which the researches of the writer had not been able to solve.

However, the knowledge was thus gained that the products of advanced hydrolysis can be utilized for the nitrogenous 
feeding of man, and this discovery gave rise to a whole series of questions of great importance. Thus we may ask to what extent albuminoid substances can be hydrolyzed without losing their nutritive power. Should decomposition be advanced to its extreme limit, or is it better to stop with certain polypeptides of relatively simple structure? Furthermore, do the nitrogenous wastes each, separately, retain all the constituents to be reconstructed into albuminoid substances, or is the presence of the entire mixture necessary for the organism to be able to reform a protein molecule? If all the products of hydrolysis are not indispensable, which are the ones that play the chief part from the point of view of assimilation? Finally, may we use indiscriminately for this work all protein material, or must preference be given to certain ones?

The different albuminoid materials, while having a similar structure and composition, nevertheless possess each its own individuality, which results from a slight modification in the relation of the amine groupings, either in the fatty or of the aromatic series. These specific differences appear especially in the course of hydrolysis. If the analyses of serum-albumin and globulin are compared, it is seen that the quantities of the products obtained are evidently not identical, but that they are of the same order of magnitude. This is not true of gelatin and gliadin. Among the decomposition products of gliadin, a large excess of glutamic acid is observed, while lysin is absent. As to the products furnished by gelatin, they contain prolin, oxyprolin, serin, and finally arginin and lysin, but we do not find histidin. Finally, gelatin is distinguished by the absence of tyrosin and histidin; aside from glycin, which is formed in great quantity, the other amino-acids are rarely found.

From the differences observed in the products of hydrolysis of the various albuminoids, can we draw any practical conclusions as to the nutritive value of these same proteins? In particular, as regards gelatin, which cannot be regarded as an albuminoid food properly speaking, should we seek the cause of its nondigestibility in the excess of glycin formed, or in the relatively 
small quantity of the other amino-acids present, or again in the absence of tyrosin or any other combination indispensable to the later reconstruction of albuminoid material in the organism? These manifold ideas, given out in 1904 by Abderhalden, show the extent of the field open to experimentation, and indicate the direction of the numerous researches which have been carried out by him and his pupils in recent years.

Work of Abderhalden on the Assimilation of Nitrogenous Material. - The first observations to be published concerning the nutritive value of the products of advanced protein hydrolysis were made in 1902 by Otto Loewi. The experiments were made on a young dog fed with starch, sugar, and non-biuretic products coming from pancreatic digestion. The nitrogenous equilibrium of the animal could be maintained for 25 days, and at the end of this diet there was even found a slight increase in weight. The results obtained led this investigator to suppose that the nutritive albumins are completely disintegrated in the intestine, and that the synthesis of the albuminoid substances in the organism is made with the final products of hydrolysis.

The experiments of Loewi were continued in I904 by Abderhalden, in collaboration with Rona. The tests were made with mice. As nitrogenous material, they used the following preparations: (a) Casein peptonized with pancreatin for 2 months. The product still contained about $\mathrm{I}_{5}$ per cent of polypeptides and gave a slight biuret reaction; (b) casein treated I month with pepsin and 2 months with pancreatin. It contained about 8 per cent of polypeptides and no longer gave the biuret reaction; (c) Casein boiled to hours with 25 per cent sulphuric acid. These nitrogenous products, free from ammonia, were neutralized, evaporated in a vacuum, and then had added to them sugar and sodium carbonate. One lot of mice was fed with undigested casein. This control lot lived 16 to 30 days. The mice fed with the product $(a)$ behaved like those of the control lot. As to the last two lots, corresponding to the products $(b)$ and $(c)$, they died, one after 5 to 6 days, the other after 5 to 9 days. These tests appeared therefore to show that the products of 
advanced hydrolysis, though giving only a slight biuret reaction, were capable of maintaining life in mice, while the non-biuretic products had lost all nutritive power. A similar conclusion is drawn from the negative results obtained with the products of acid hydrolysis, namely that this method of hydrolyzing brings about a disintegration too advanced to give assimilable products.

On the whole these first experiments were not very conclusive, but led to another series of tests which were carried on from r904 to rgr2. First of all, in r905 the experiments on dogs were repeated, using as nitrogenous foods caseins hydrolyzed by pancreatin and by acid. The hydrolysis with pancreatin was accomplished in the following manner: To I kilo of casein, suspended in a liter of water, was added $50 \mathrm{~g}$. of pancreatin and a little toluol. The liquid, made slightly alkaline, was left 2 months and a half at $36^{\circ}$. At this point it had become slightly acid and no longer gave the biuret reaction. It, however, still contained Io per cent of polypeptides. After neutralization, it was evaporated in a vacuum. The final product titrated 8:5 per cent of nitrogen. For the experiments, this product is mixed with fat free from nitrogen and with starch, and a paste is made which the dog takes willingly. He moreover behaves normally during the whole dieting, even a slight increase in weight being observed at the end. This result then confirmed those obtained by Loewi, according to which the amino-acids and the nonbiuretic nitrogenous compounds coming from pancreatic hydrolysis are capable of ensuring the nitrogenous balance of an animal.

The hydrolysis by acid is accomplished as follows: I kilogram of casein is boiled for I 2 hours with 5 liters of 25 per cent $\mathrm{H}_{2} \mathrm{SO}_{4}$. The acid is then precipitated quantitatively with $\mathrm{BaCl}_{2}$. The liquid is filtered, the filtrate neutralized with $\mathrm{Na}_{2} \mathrm{CO}_{3}$, and the liquor concentrated in a vacuum. The residue from the evaporation gives Io per cent of nitrogen. This product, with addition of fat and carbohydrates, is not acceptable to the dog, and his weight figures indicated that this food has not succeeded in maintaining his nitrogenous equilibrium. 
Abderhalden thinks that the negative results can be attributed to three causes. In the first place hydrolysis by acid is too advanced, and the products obtained are no longer assimilable. In the second place the action of the acid determines the formation of injurious foreign substances. In the third place the partial racemization of the products obtained is unfavorable. These three hypotheses have been subjected to a very thorough examination. As it was, above all else, a question of knowing whether the products of advanced hydrolysis can maintain nitrogenous equilibrium, Abderhalden took special pains with his study of the properties and with his analyses of the products obtained, in order to be sure that he was working with pure amino-acids, free from complex polypeptides.

The tests made since 1907 on meat, casein, milk, etc., hydrolyzed by enzymes or acids until only amino-acids are obtained, led Abderhalden to very conclusive results. The following is a typical experiment carried out with meat. The enzyme - preparation used was obtained thus: The minced meat is subjected for 6 weeks to the action of the gastric juice of a dog. Then it is rendered alkaline and treated with pancreatic juice and pancreatin. The tryptic action lasts 4 weeks. After the mass ceases to give the biuret reaction, there is added to it an intestinal extract which is allowed to act 4 weeks longer. To determine the degree of hydrolysis, the meat to be experimented on is treated with 3 volumes of concentrated hydrochloric acid for 8 hours at the boiling temperature. By the esterification method, it has been found that 40.8 per cent of the protein substances contained in meat were converted into mono-aminoacids. The meat digested by the enzymes gave almost the same figure, or 41.05 per cent. Moreover, mono-amino-acids are found in the portion not precipitated by phosphotungstic acid. Now, the precipitate furnished by this reagent, decomposed by $\mathrm{BaCl}_{2}$ and hydrolyzed by the concentrated acids, does not give appreciable quantities of mono-amino-acids, a fact which confirms the absence of polypeptides in the product of the digestion. 
The acid hydrolysis of the meat was made in the following manner: The meat was allowed to boil I week with ro per cent sulphuric acid, and then was kept for 2 hours and a half on a steam-bath with 25 per cent sulphuric acid. The acid was then eliminated with $\mathrm{BaCl}_{2}$, the solution was filtered, the filtrate was evaporated to dryness, and to the residue was added a little tryptophane.

The experiments with the digested meat were made on a dog 6 years of age, and weighing $8.620 \mathrm{~kg}$.

His weight, after he had been kept fasting for 17 days, had fallen to 7.120 kg. At first, during a period of 10 days, the animal was given a ration composed of cane sugar, glucose, fat and digested casein. The daily quantity of digested casein was 25 grams, and this dose was then increased to 33 grams during the 5 succeeding days. The dog, which was at the end of its fast totally exhausted, regained his strength and looked healthy, but his weight did not increase, remaining at 7000 grams.

In a second period, which lasted $2 \mathrm{I}$ days, the casein was replaced by meat digested by enzymes, in quantities increasing from 30 to $4 \mathrm{I}$ grams. The weight of the dog increased gradually, to reach in the end 8400 grams.

Finally, in a third period of 5 days, the meat was replaced by milk that had, been completely hydrolyzed by enzymes, and the average weight remained at 8500 grams.

The fact that the digested meat and the hydrolyzed milk were more favorable than the casein is explained by the presence of other substances that favor assimilation, especially salts. In another series of tests the same result was obtained when fats were substituted for carbohydrates. In all these experiments, it is always the digested meat that gives the best results. Results obtained with digested casein are less satisfactory, and results with casein or meat hydrolyzed by sulphuric acid are thoroughly unsatisfactory. However, by changing the technique and by taking more care in the preparation of the products and in the choice of method of feeding the animals, almost similar results were obtained, irrespective of the hydrolyzed material utilized or even of the conditions of treatment.

The obvious conclusion to be drawn from these tests is that the products of advanced hydrolysis, mono-amino-acids derived from the degradation of protein material, can preserve the 
animal in nitrogenous equilibrium. Nevertheless, we can always make the same criticism of all these results, namely, that, in addition to the amino-acids, the hydrolysis produces a large number of unknown nitrogenous substances which are neither polypeptides nor amino-acids, and which, however, probably intervene in assimilation, and perhaps play an important part in the synthesis of the nitrogenous material by the organism. To remove this objection, it was necessary to nourish an animal by using as nitrogenous food only pure amino-acids, associated with perfectly definite ternary substances. Many attempts were made to attain this object. The first experiments were not successful, but after many trials a mixture was obtained that gave quite satisfactory results. Its composition is as follows:

$\begin{aligned} & \text { Grams } \text { glycin } \\ & 5 \text { (d) alanin } \\ & \text { Io } \\ & 3 \text { (l) serin } \\ & 2 \text { (l) cystin } \\ & 5 \text { (d) valin } \\ & \text { Io (l) leucin } \\ & 5 \text { (d) isoleucin } \\ & 5 \text { (l) aspartic acid } \\ & \text { I (d) glutamic acid } \\ & 5 \text { (l) phenylalanin } \\ & 5 \text { (l) tyrosin } \\ & 5 \text { (l) lysin (carbonate) } \\ & 5 \text { (d) arginin (carbonate) } \\ & \text { Io (l) prolin } \\ & 5 \text { (l) histidin } \\ & 5 \text { (l) tryptophane } \\ & \text { I00 grams amino-acids }\end{aligned}$

\begin{tabular}{|c|c|c|}
\hline & & ms Nitrogen. \\
\hline containing & $\ldots \ldots \ldots \ldots \ldots \ldots$ & 0.9335 \\
\hline & $\ldots \ldots \ldots \ldots \ldots \ldots$ & I. $573^{\circ}$ \\
\hline “ & $\ldots \ldots \ldots \ldots \ldots$ & 0.4002 \\
\hline “ & $\ldots \ldots \ldots \ldots \ldots \ldots$ & 0.2330 \\
\hline “ & $\ldots \ldots \ldots$ & 0.5980 \\
\hline “ & ... & $x .069$ \\
\hline “ & $\ldots \ldots \ldots \ldots \ldots$ & 0.5345 \\
\hline “ & $\ldots \ldots \ldots \ldots$ & 0.5265 \\
\hline " & $\ldots \ldots \ldots \ldots \ldots \ldots$ & $\mathrm{I} .425$ \\
\hline “ & $\ldots \ldots \ldots \ldots \ldots \ldots$ & 0.4245 \\
\hline “ & $\ldots \ldots \ldots \ldots \ldots \ldots$ & 0.387 \\
\hline “ & $\ldots \ldots \ldots \ldots$ & 0.9585 \\
\hline " & $\ldots \ldots \ldots$ & 1.6090 \\
\hline " & $\ldots \ldots \ldots \ldots \ldots \ldots$ & 1.2170 \\
\hline “ & $\ldots \ldots \ldots \ldots \ldots \ldots$ & I. 298 \\
\hline “ & $\ldots \ldots \ldots \ldots$ & 0.686 \\
\hline taining & . $\ldots \ldots \ldots \ldots \ldots \ldots$ & I 3.87 \\
\hline $1 \mathrm{~m}$ & ssured directly: & 14.25 g.) \\
\hline
\end{tabular}

By using this mixture of absolutely pure products, with the addition of sugar, fat, etc., it was possible to keep the animal for quite a long time in nitrogenous equilibrium, although this nourishment was tolerated with difficulty and produced vomitings, diarrhœa, etc.

The best results were obtained when the diets were alternated. By giving to a dog first digested meat, then digested casein, then 
amino-acids, etc., that is, by varying the manner of feeding while always using products of hydrolysis, it was possible to keep this animal 74 days in a good state of health, and even to observe a considerable increase in weight. The following table summarizes these last experiments:

\begin{tabular}{|c|c|c|c|c|c|c|c|}
\hline \multirow{2}{*}{ Days. } & \multirow{2}{*}{$\begin{array}{l}\text { Weight of } \\
\text { animal. }\end{array}$} & \multirow{2}{*}{ Food. } & \multicolumn{3}{|c|}{ Nitrogen content } & \multirow{2}{*}{$\begin{array}{c}\text { Total } \\
\text { nitrogen } \\
\text { in the } \\
\text { excreta. }\end{array}$} & \multirow{2}{*}{$\begin{array}{l}\text { Nitrogen } \\
\text { balance. }\end{array}$} \\
\hline & & & of the food. & of the urine. & of the feces. & & \\
\hline $\begin{array}{l}\text { I } \\
4 \\
7\end{array}$ & $\left.\begin{array}{c}\text { g. } \\
7500 \\
7600 \\
7700\end{array}\right\}$ & I & $\left\{\begin{array}{c}\mathrm{g} . \\
\text { I3. I5 } \\
\text { I3. I5 } \\
\text { I3. } 55\end{array}\right.$ & $\begin{array}{c}\text { g. } \\
\text { II . } 25 \\
\text { I } 2.66 \\
\text { I } 2.32\end{array}$ & $\begin{array}{l}\text { g. } \\
0.21 \\
0.12 \\
0.08\end{array}$ & $\begin{array}{c}\text { g. } \\
\text { II. } 46 \\
\text { I2.78 } \\
\text { I } 2.40\end{array}$ & $\begin{array}{l}\text { g. } \\
+ \text { r. } .69 \\
+0.37 \\
+0.75\end{array}$ \\
\hline $\begin{array}{r}8 \\
12\end{array}$ & $\left.\begin{array}{l}7800 \\
7850\end{array}\right\}$ & II & $\left\{\begin{array}{l}2.10 \\
2.10\end{array}\right.$ & $\begin{array}{l}2.20 \\
1.90\end{array}$ & $\begin{array}{l}0.06 \\
0.08\end{array}$ & $\begin{array}{l}2.26 \\
1.98\end{array}$ & $\begin{array}{l}-0.16 \\
+0.12\end{array}$ \\
\hline 25 & 7440 & V & 2.08 & I. 89 & 0.13 & 2.02 & +0.06 \\
\hline 40 & 7615 & VII & 2.25 & $3 \cdot 5^{\circ}$ & 0.12 & 3.62 & -1.37 \\
\hline 50 & 7425 & IX & 2.06 & 3.68 & 0.14 & 3.82 & $-1 \cdot 76$ \\
\hline $\begin{array}{l}62 \\
74\end{array}$ & $\left.\begin{array}{l}7825 \\
8700\end{array}\right\}$ & $\mathrm{XI}$ & $\left\{\begin{array}{l}6.60 \\
6.60\end{array}\right.$ & $\begin{array}{l}3.15 \\
4.50\end{array}$ & $\begin{array}{l}0.42 \\
0.2 \mathrm{I}\end{array}$ & $\begin{array}{l}3.57 \\
4.71\end{array}$ & $\begin{array}{l}+3.03 \\
+1.89\end{array}$ \\
\hline
\end{tabular}

Mixture I. $100 \mathrm{~g}$. oi hydrolyzed horse meat $+10 \mathrm{~g}$. of bone ashes.

" II. $15 \mathrm{~g}$. of hydrolyzed horse meat $+30 \mathrm{~g}$. of fat $+50 \mathrm{~g}$. of glucose $+5 \mathrm{~g}$. of bone ashes.

" V. $2 \mathrm{I}$ g. of caseine+tryptophane+fat, glucose, etc., as above.

“ VII. $20 \mathrm{~g}$. of hydrolyzed gelatin $+2 \mathrm{~g}$. of hydrolyzed nucleic acids +fat, glucose, etc.

"IX. I8 g. of mixture of amino-acids +fat, glucose, etc.

" XI. $45 \mathrm{~g}$. of hydrolyzed beef+fat, etc.

The thorough knowledge which we possess at present as to the nature of albuminoid material permits us to attack the question of nitrogenous assimilation from an entirely new point of view. The various protein foods do not all behave alike upon hydrolysis. Qualitative and quantitative differences exist in the mono-amino-acids formed, as can be easily understood when we determine the proportions of glutamic acid contained in the products of hydrolysis:

\section{Content in Glutamic Acid.}

White of $\operatorname{egg} . \ldots \ldots \ldots \ldots \ldots \ldots \ldots \ldots$. 8.0 to 9.0

Meat protein.................. ro. 5

Serum albumin of horse............ 7.7

Serum globulin ................ 8.5

Gliadin........................ 36.0 to 37.0 
From these differences in the composition of the products of digestion, we can draw conclusions as to the mechanism for the assimilation of nitrogenous foods. Especially can we see if there is a relation between the structure of the albumin entering into the circulation and that of the nutritive albumin. If this relation exists, we should find in blood all the peculiarities of the absorbed albumin, and in the case of gliadin, a very high content in glutamic acid. If, on the contrary, we find no relation, it is because the synthesis takes place by way of selection and thus not all the products of hydrolysis are necessary. After the elimination of certain of them, the composition of the plasma would then become practically constant. A large number of experiments made by feeding horses with gliadin have brought scientists to the conclusion that the proteins of the serum maintain a constant composition absolutely independent of the particular nature of the ingested proteins.

For testing nutrition by means of simple amino-acids described above, we have utilized a very complex mixture which greatly resembles the natural products of the hydrolysis of peptones. Most of the substances entering into this mixture have been studied separately, especially from the point of view of the influence which each may exert on the assimilation of the whole. It has been attempted experimentally to see whether all the products of hydrolysis are indispensable, or whether they can be replaced by each other. Although these tests are very delicate and are difficult to perform, they have nevertheless given definite results. Glycin appears to play an entirely secondary. part and another acid can be substituted for it. On the contrary, tryptophane appears to be a substance that is absolutely necessary. The following is an experiment on this subject:

A certain quantity of hydrolyzed casein is divided into 3 parts, A, B, C. The part A contains all the products of hydrolysis, B is lacking in tryptophane, and in $\mathrm{C}$ tryptophane has first been removed, and then subsequently replaced. The three products are tested as to their respective nutritive value for animals. The results obtained establish that $A$ and $B$ have a very different influence. Whereas A keeps the anima' in nitrogenous equilibrium, the product B shows 
itself unassimilable, and causes the animal to suffer a loss of weight and a rapid starvation. With the product $\mathrm{C}$, the animal regains its health and assimilation becomes normal.

The withdrawal of tryptophane therefore has the result of preventing albuminoid synthesis from a mixture of amino-acids, and renders this mixture null as to nutritive value.

In the tests with the products $\mathrm{A}$ and $\mathrm{C}$, we do not find perceptible quantities of amino-acids in the urine. On the contrary, in test B without tryptophane, we find appreciable quantities of tyrosin, alanin, glycin, and other amino-acids. Yet, the quantity of the acids thus recovered represents only a small proportion of the amine nitrogen absorbed, the greater part of the latter being transformed into urea.

This formation of urea to the detriment of the amino-acids not assimilated, throws a particular light on the mechanism of nitrogenous assimilation. The albumin of the plasma is formed by means of amino-acids coming from the protein foods, and this assimilation takes place by way of selection. Only the particles entering into the blood albumin are absorbed, as we have seen in the experiment above with gliadin. These are the real nutritive nitrogenous substances. The others, which cannot contribute toward maintaining the constant composition of the albumin of the plasma, are eliminated. These wastes are consumed and transformed into urea, behaving here like carbohydrates. The formation of residual nitrogen at the expense of nutritive nitrogen will take place with a much greater intensity in the assimilation of the cells, since these construct their varied proteins with the albuminoid substance of the plasma.

Of all the nutritive albuminoid materials, gelatin has been recognized for a long time as being the least assimilable. Two causes explain the indigestibility of this substance. First, it is more difficult of attack by the digestive enzymes than the other albuminoids, and secondly, it is too deficient in alanin, leucin, hystidin, and in phenylalanin to be able to furnish plasma albumin. It was to be foreseen that if we added the required amino-acids to hydrolyzed gelatin, this body would behave like a normal food substance. The investigations attempted in this direction have given positive though still incomplete results. Rona and Müller proved that gelatin, with the addition of tyrosin 
and tryptophane, can replace 40 per cent of the albuminoid food. Abderhalden, by adding the required amino-acids to hydrolyzed gelatin, effected an assimilability that permitted the substitution of this mixture for 65 per cent of the necessary proteins.

In conclusion, it should be noted that experiments relative to the nutritive value of the products of hydrolysis have been made on man. A boy of twelve years of age, who had burned his throat by swallowing caustic soda and who was fed by means of a fistula and clyster, was used for the tests. These tests, using the products of hydrolysis alone, lasted I $_{5}$ days. The patient received daily: (I) by the fistula: $90 \mathrm{~g}$. oats $+150 \mathrm{~g}$. fat $+50 \mathrm{~g}$. glucose $+25 \mathrm{~g}$. starch; (2) by clyster: Io g. oats $+72.4 \mathrm{~g}$. digested meat. This corresponded in all to a quantity of ingested nitrogen equivalent to $9.34 \mathrm{~g}$. per day. On the other hand, the total nitrogen eliminated averaged $6.7 \mathrm{~g}$. a day. It is observed that the nitrogenous balance is normal, since the weight of the body increased from $26.9 \mathrm{~g}$. to $27.4 \mathrm{~g}$. The hydrolyzed meat used in these experiments was obtained by a digestion of 6 weeks with pancreatic juice, and of 5 weeks with intestinal juice. The product did not give the biuret reaction.

These various results leave the conviction that the assimilation of nitrogenous materials in the organism is preceded by a very advanced hydrolysis. Still it is not impossible that certain polypeptides, coming from the union of amino-acids of a relatively simple structure, are also capable of intervening in the synthesis of albuminoids to the same degree as the mono-acids. Abderhalden and his pupils have sought by direct analysis of the digestive tracts, and on animals provided with fistulas, to follow the progress of hydrolysis during digestion. That the results have not been quite conclusive is due probably to the differences in the rapidity of absorption of the various substances. However, from the numerous analyses made by this experimenter, it appears that if no appreciable quantities of amino-acids are found in the stomach, they are at least encountered in quite considerable quantities in the duodenum.

To summarize, from all the data which we have just men- 
tioned it appears that there is ample proof that the organism draws all its nitrogenous constituents from the products of the advanced hydrolysis of proteins. These may result either from the actual process of digestion, or from artificial means, like the action in vitro of proteolytic enzymes or the action of concentrated acids. In all events, these are directly assimilable substances and should be considered as food material of great nutritive value.

In fact, it has been established that a mixture of aminoacids, containing qualitatively and quantitatively all the principal products of the complete hydrolysis of proteins, can replace the albuminoid foods, and, as such, maintain the animal organism in nitrogenous equilibrium. Furthermore, certain of these amino-acids can be substituted for each other; but there are others, such as tryptophane, tyrosin, prolin, etc., whose presence is indispensable. These facts are of a great practical importance, for they allow us to look forward to the recovery and the utilization of residual nitrogenous products, with a view to a rational and economical nutrition. In the cost of food for man, the part relative to nitrogenous foods remains the most important. The economy which can be realized in this direction is certainly much greater than that arising from the introduction of margarine, for example. Artificial nitrogenous food will evidently bring about a great reduction in the consumption of meat, though it will never entirely do away with the latter. It can be used in different forms in the preparation of dishes without revolutionizing either the method of nutrition, the taste, or the appearance of the foods.

\section{BIBLIOGRAPHY.}

Otro Loewi. Ueber Eiweisssynthese in Tierkörper, Centralbl. f. Physiol., I902, (15), p. 592 .

Kutscher u. LeEman. Beitrage zur Kenntniss der Verdauung in Dunndarm, Centralbl. f. Physiol., I901, (5), p. 275.

Conherm. Die Umwendlung des Eiweisses durch die Darmwande, Zeits. $f$. physiol. Chem., r9or, (33), p. 45 I.

Kutscher. Die Endprodukte der Trypsinverdauung, 1899, Habilitationschr., Strasbourg. 
Henderson Dean. Americ. Journ. of Pharmacy, (9), p. 386.

Henriques u. Hansen. Zeits. f. physiol. Chem., I905, (44), p. I 7.

WoHLgemuth. Ueber das Verhalten Stereoisomerischer Substanzen im tierischen Organismus, Ber. d. deuts. Chem. Ges., r905, (38), p. 2064.

Alfred Schittenhelm. Verfutterungsversuche, Zeits. f. exper. Path. u. Therap., I906, (2), p. 560 .

Kaufman. Ueber den Ersatz von Eiweiss durch Leim im Stoffwechsel, Pflüg. Arch., I905, ch. IX, sect. I.

RoNa u. Müller. Ueber den Ersatz von Eiweiss durch Leim, Zeits. f. physiol. Chem., 1907, (50), p. 265.

Vort u. Zisterer. Die Wertigkeit des Caseins und seiner Spaltungsprodukte, Zeits. f. Biol., 1900, (2), p. 457.

Kopp. Ueber den physiologischen Abbau der Säuren und Synthese einer Aminosäure im Körper, Zeits. $f$. physiol. Chem., I9ı I, p. 489.

EFrront. (I) Sur la valeur nutrutive des produits d'hydrolyse des substances protéiques, Mon Scient., I912, p. 425. - (2) Brevet belge, $\mathrm{n}^{\circ}$ I $70{ }_{5}$ Io, déposé le 23 mai 1903 pour: "Procédé de fabrication d'un extrait alimentaire au moyen de substances protéiques." - (3) Brevet de perfectionnement belge, $n^{\circ} 173652$ déposé le 13 novembre igo3.

Abderhalden. Les travaux sur ce sujet, faits par Abderhalden et son Ecole, sont exposés dans les nombreux mémoires insérés au Zeits. $f$. physiol. Chem., de 1905 à I912. 



\section{INDEX}

PAGE

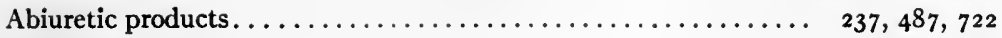

Acclimatization......................... 708, 7 I 4

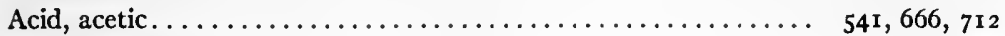

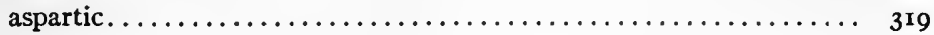

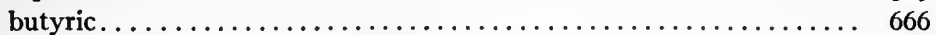

glutamic.......................... 319, 543, 71 2, 734

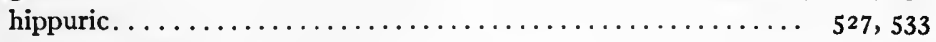

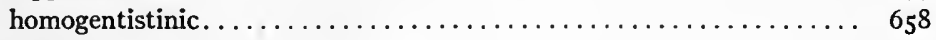

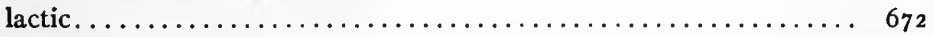

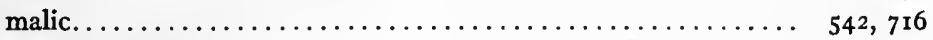

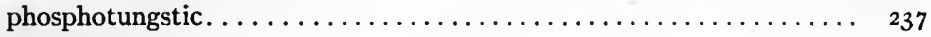

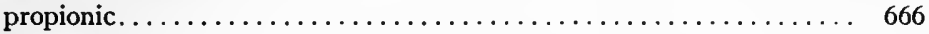

succinic............................ 543, 716

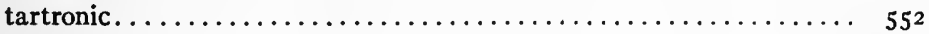

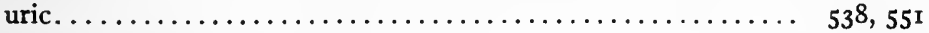

Acids, amino-.......................... 242, 281, 31 7, 495

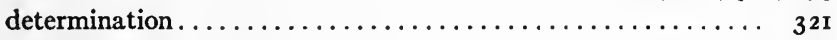

products of hydrolysis.............. 237, 28I, 328, 490

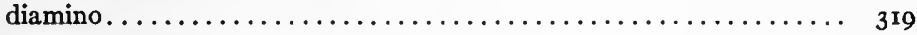

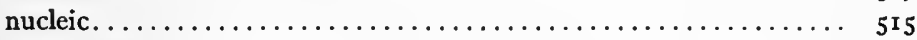

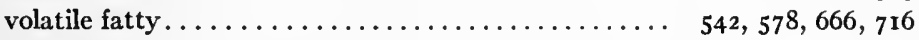

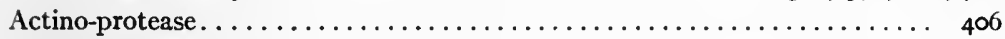

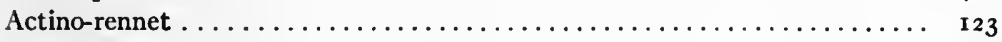

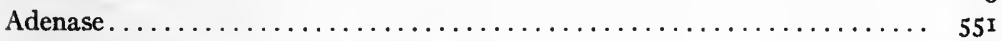

Adenylase . . . . . . . . . .

Adsorption of enzymes....................... I7 I, 173, 303, 488

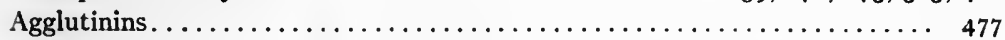

Aisy, 1 . . . . . . . . . .

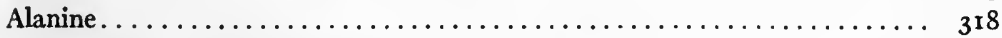

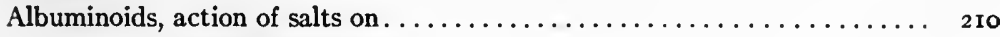

general properties.................

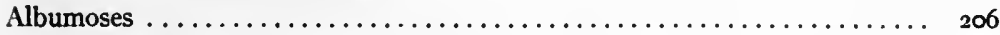

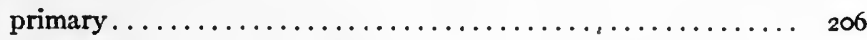

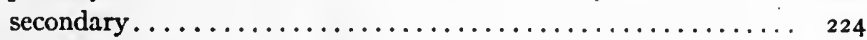

Alcohol, isoamylic. . . . . . . . .

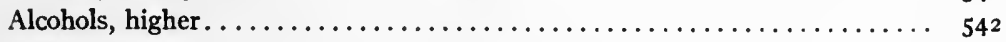

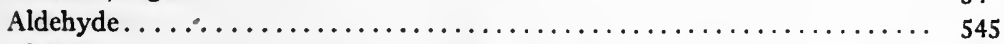

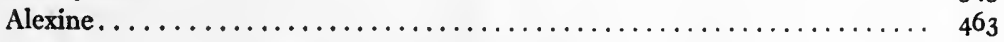

Alinit........................................... 704 
Amboceptor .

Amidases

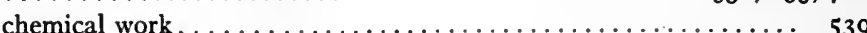

Amido-nitrogen

Ammonia

25,328

Amobo-diastase.

Amphopeptone

Amygdalin . 206

Amylo-liquefying enzyme.

Amylo-process

614,615

Anaphylaxis

Antialexine

Antibodies

Anticoagulants

433,471

Anticytase.

Antiferment

Antigens

461,473

Antileucotoxin. . . . . . . . .

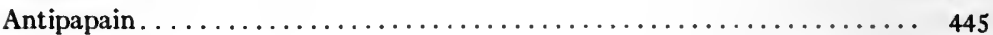

Antipeptones. . . . . . 207. 208, 331

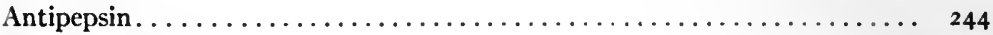

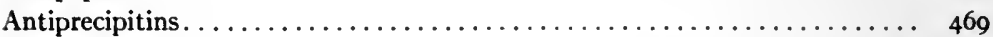

Antiprotease. . . . . . .

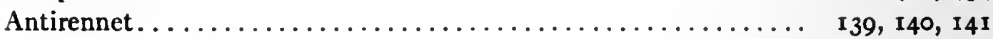

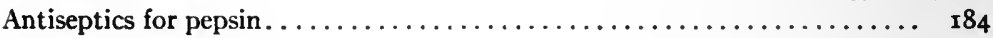

for rennet............................ 108

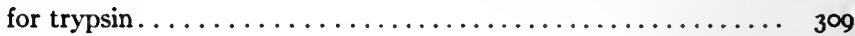

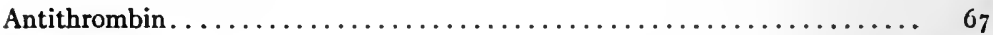

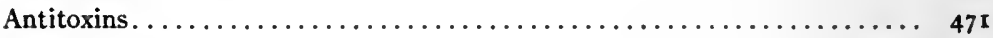

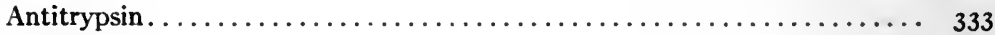

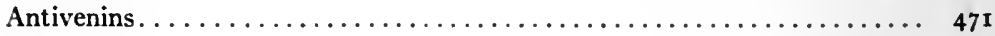

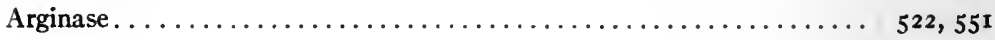

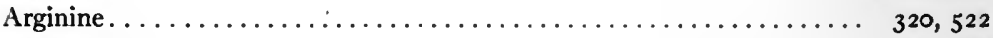

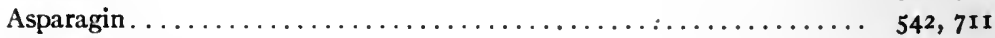

Aspergillus niger. ....................... 4I4

Assimilation of atmospheric nitrogen. . . . . . .

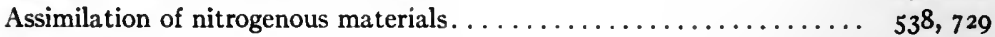

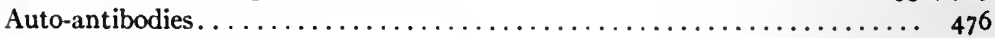

Autolysins. . . . . . . . . . . . .

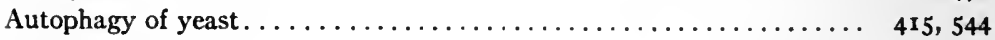

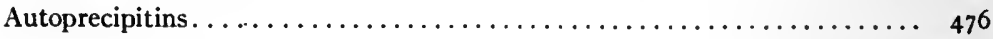

Bacteriolysis.................................. 465

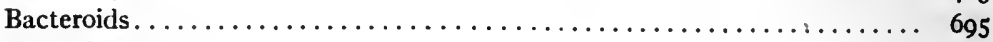

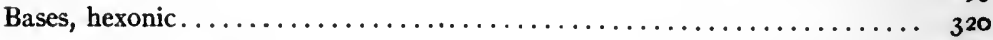

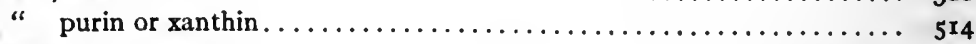

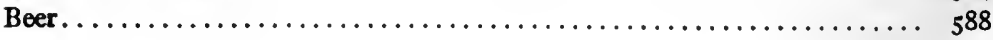


Betaine.

Biuret (reaction)

Blood, analysis.

amidases in

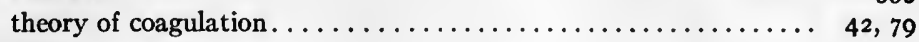

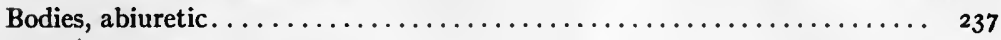

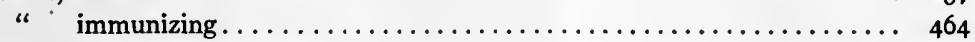

Bread, influence on secretion. . . . . . . . . . . . .

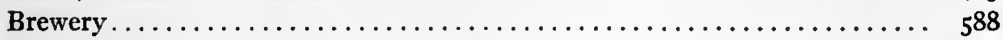

Bromelin . . . . . . . . . . .

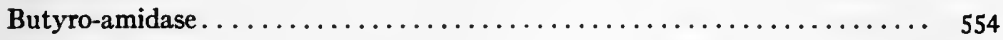

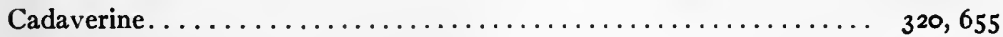

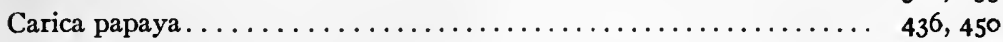

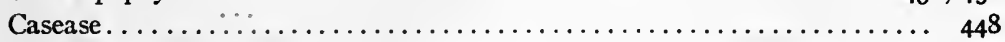

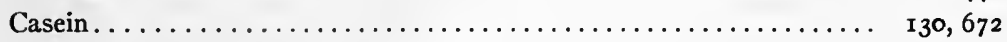

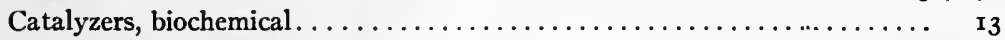

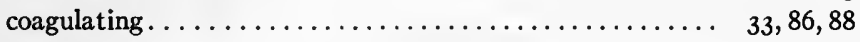

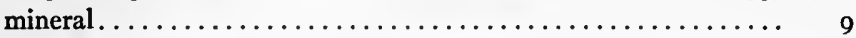

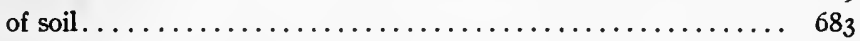

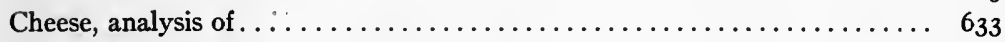

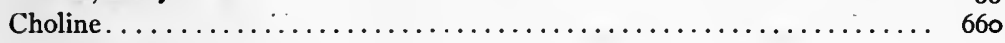

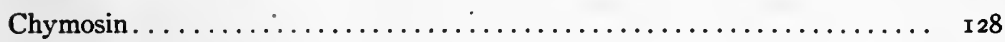

Classification of enzymes...........................

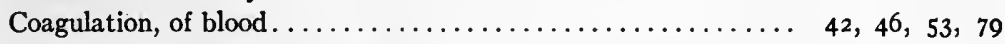

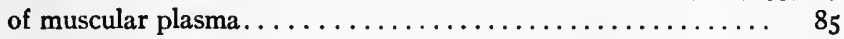

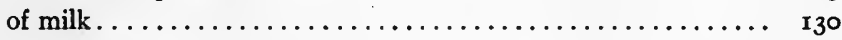

Coagulose. . . . . . . . . . . . . . .

Coenzyme. . . . . . . . . . . . . . . . .

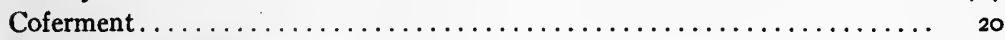

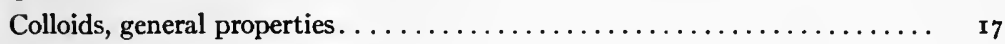

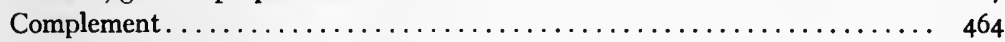

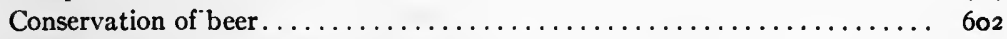

Contact, action on coagulation of blood ....................... $6_{2}$

" " " activation of pancreatic juice $\ldots \ldots \ldots \ldots \ldots \ldots \ldots \ldots . \ldots \ldots 55$

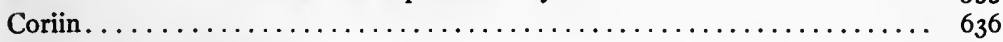

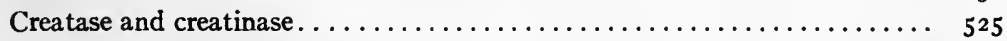

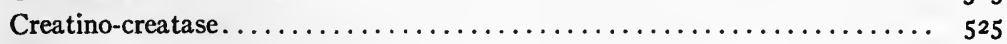

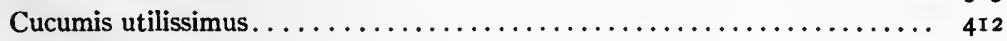

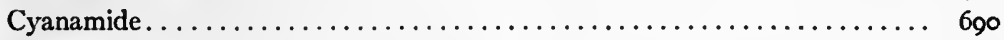

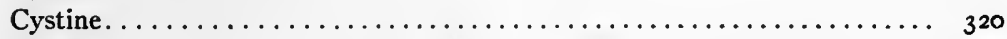

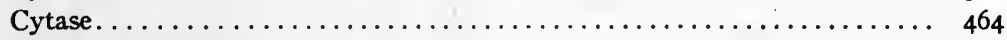

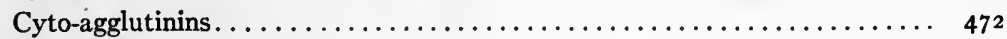

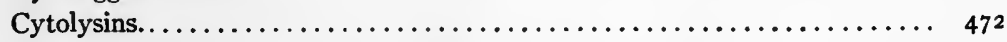

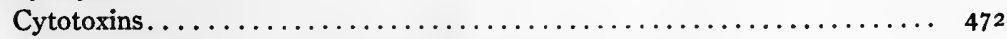

Deflection of the complement, method of Bordet \& Gengou . . . . . . . 477

Denitrification................................ 697 
Deutero-albumoses

Diastases (see Enzymes)

Distillery, grain

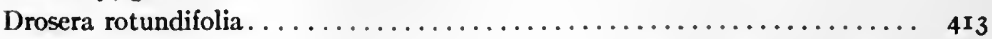

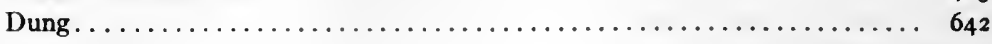

Ectoenzymes................................. 429

Edestin . . . . . . . . . . . . . .

Endo-enzymes.................................. 429

Endo-tryptase. . . . . . . . . . . . .

Enterokinase. . . . . . . . . . . . .

Enzymes, action upon each other........................... 498

adsorption..................... I7 173

amylo-liquefying................... $6 \mathbf{1 4}_{4}, 6 \mathbf{1 5}$

attenuation by heat.............. I6I, 335, 470, 478, 479

classification..................... 21,27

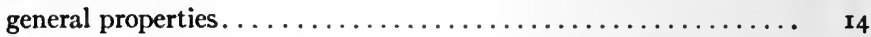

in ammoniacal fermentation. .............. 531, 555, 708

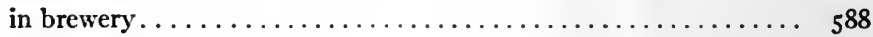

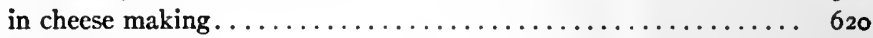

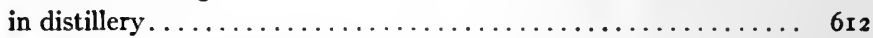

in putrefactive fermentation. . . . . . . $6{ }^{2}$

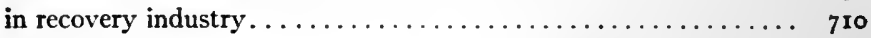

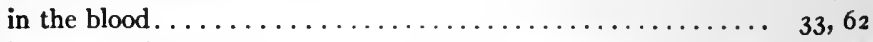

in the gastric juice................... $151,256,563,574$

in the pancreatic juice.................... 293, 352

in the tannery . . . . . . . . . . . . . . . . . $66_{36}$

in yeast manufacture $\ldots \ldots \ldots \ldots \ldots \ldots \ldots \ldots \ldots \ldots \ldots \ldots \ldots \ldots \ldots$

mechanism of action . . . . . . . . .

of nucleoproteids........................... $55^{13}$

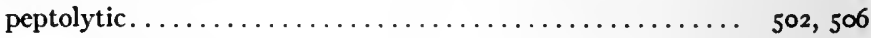

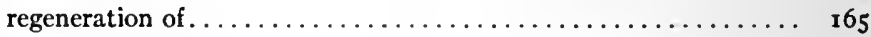

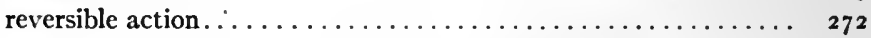

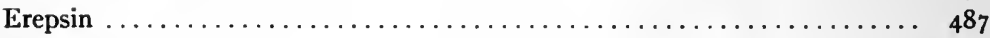

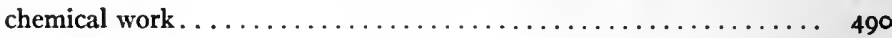

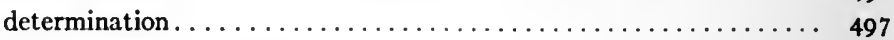

Ereptase............................. 4II

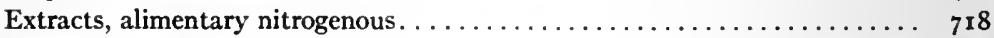

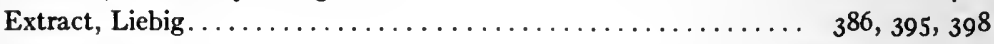

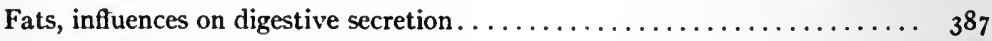

Fatty materials in distillery............................. 6r 4

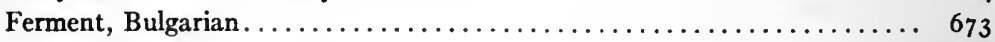

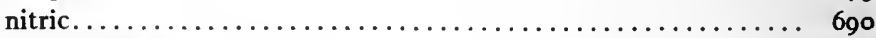

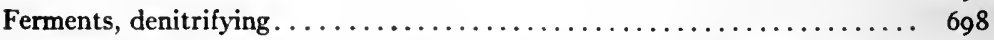

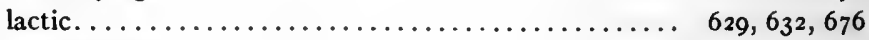

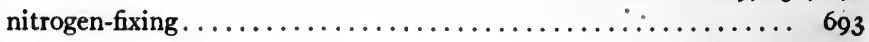




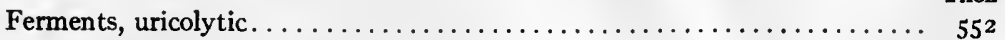

Fermentation, ammoniacal ........................ 665, 688, 710

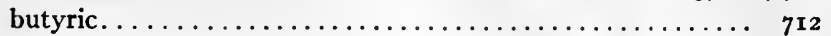

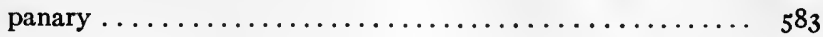

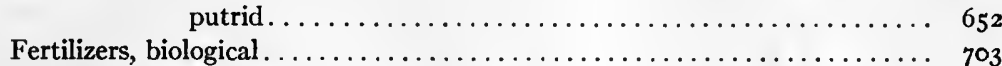

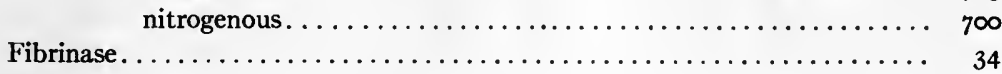

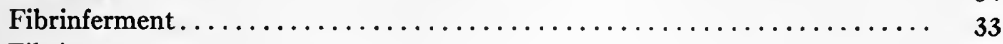

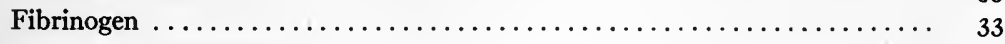

measurement.......................... 8ז

Ficus carica......................... I $27,4 \mathrm{I} 2,45^{\circ}$

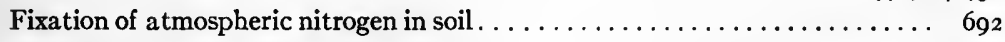

nitrogen in recovery processes................... $7 \mathrm{I} 7$

Food value of products of advanced hydrolysis . . . . . . . . . . . . 724

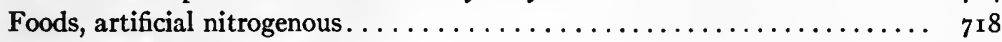

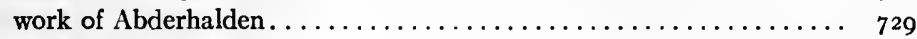

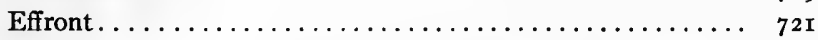

Fractional precipitation. . . . . . . . .

Galactase. .................................. $45^{\mathrm{I}}$

Galium verum. . . . . . . . . . . .

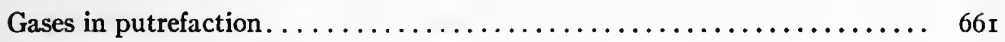

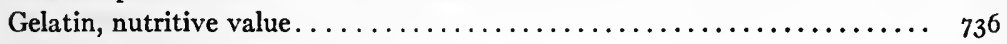

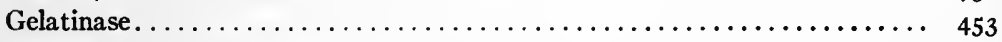

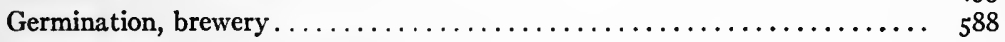

Glands, digestive (functioning) $\ldots \ldots \ldots \ldots \ldots \ldots \ldots \ldots \ldots \ldots \ldots \ldots \ldots \ldots \ldots \ldots \ldots \ldots$

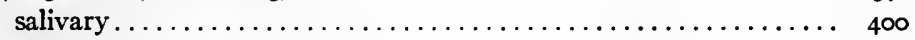

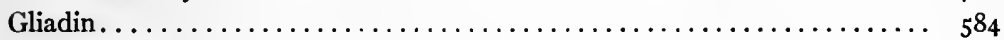

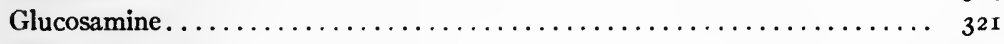

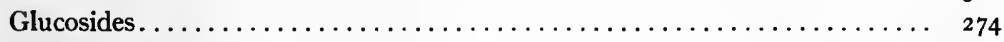

Glutamic acid ........................... 319, 543, 712, 734

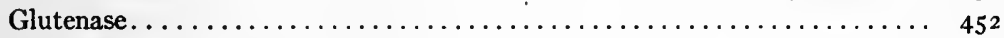

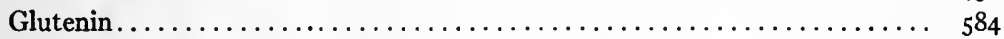

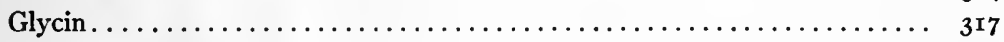

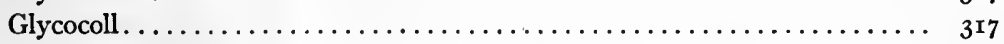

Glycyl-1-tyrosine.................................... 307

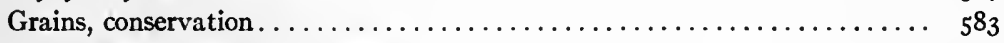

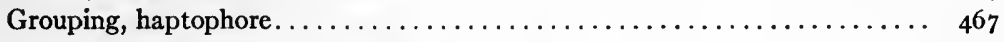

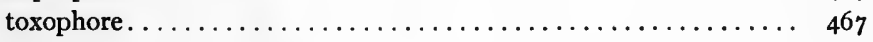

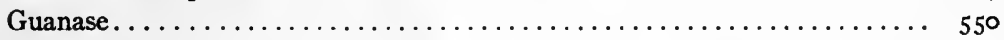

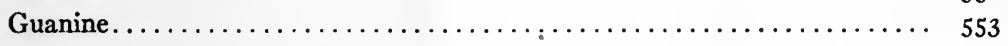

Hefanol...................................... $\mathbf{4}^{\mathrm{I}} 7$

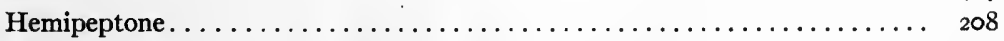

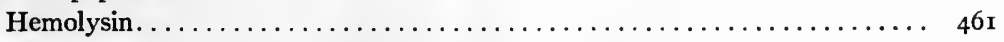

Hemolysis. . . . . . . . . . . . . . . . . . . . . . 
Hepatothrombin.

Hetero-albumose.

207,208

Histidin . . . . . . . . . . .

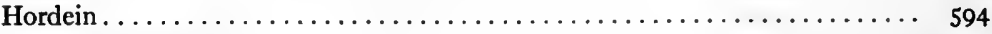

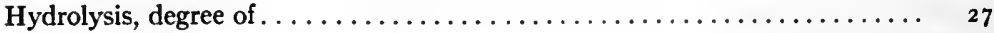

Impulse, psychic, in nutrition.................... 38 I

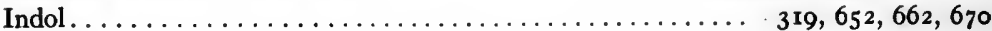

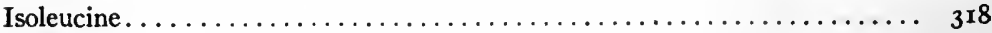

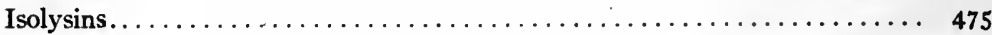

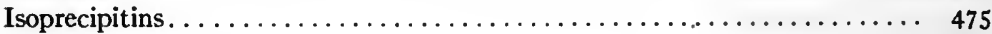

Juice, enterokinase. . . . . . . . . . . . . . . . . . . . 354

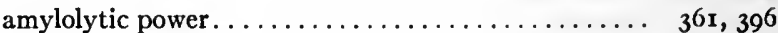

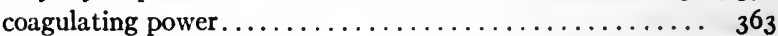

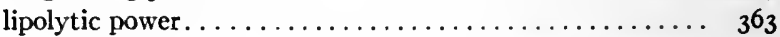

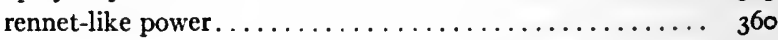

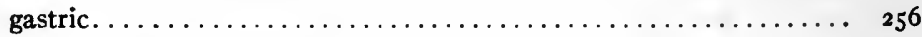

(action of temperature) $\ldots \ldots \ldots \ldots \ldots \ldots \ldots \ldots \ldots \ldots, \quad 260$

chemical influences on secretion. . . . . . . . . . . . . $33^{86}$

properties........................... $\quad{ }_{256}$

variations of the secretions according to the nature of the foods. . 373

influence of foods and chemical substances.......... 375, 391, 393

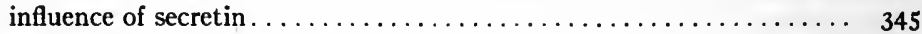

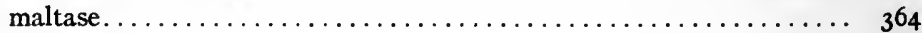

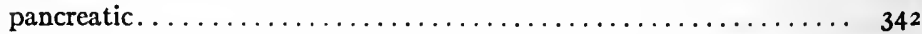

activation by calcium. .................. 355

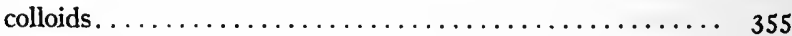

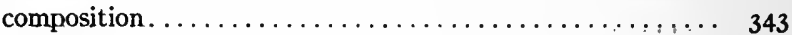

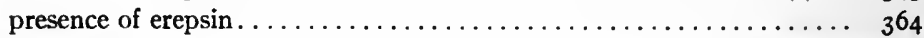

trypsinogen . . . . . . . . .

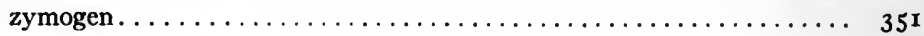

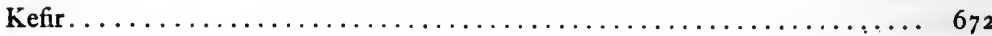

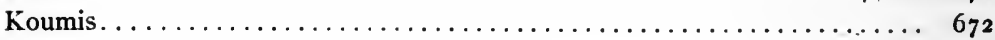

Lab ferment (see Rennet)

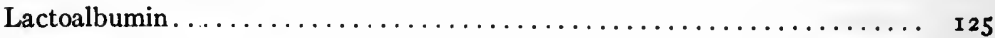

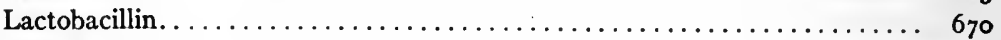

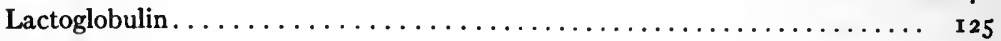

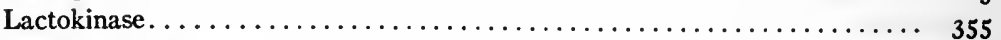

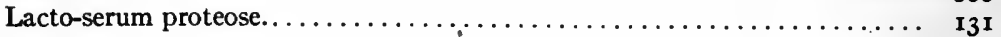

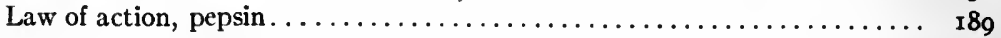

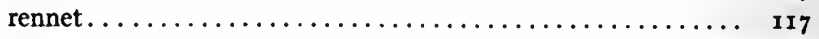

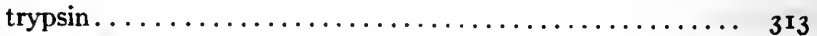

Schütz-Borissow ............................ 195

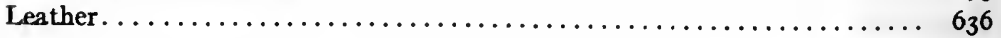


Leben.

Lecithin (coagulation of blood) $\ldots \ldots \ldots \ldots \ldots \ldots \ldots \ldots \ldots \ldots \ldots \ldots, \mathbf{5 2}^{2}$

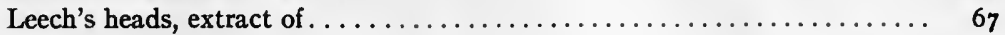

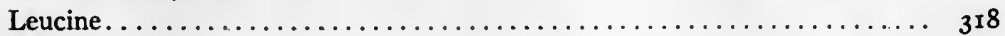

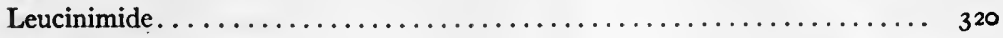

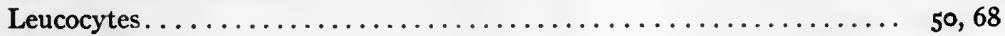

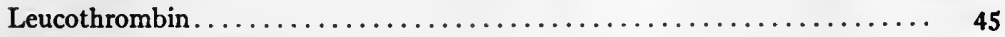

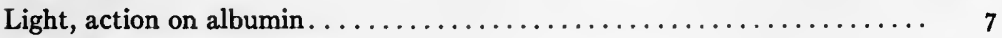

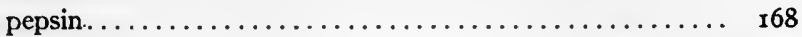

rennet. . . . . . . . . . .

Lipoids. . . . . . . . . . . . . .

Lysin .................................... 659

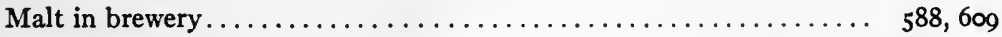

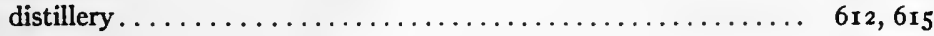

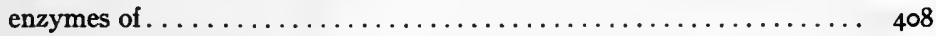

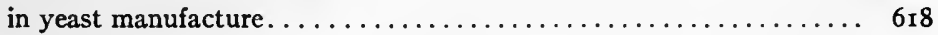

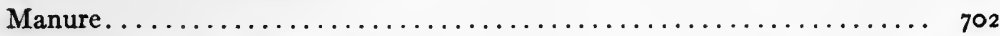

Meat, influence on secretions...................... 386, 393

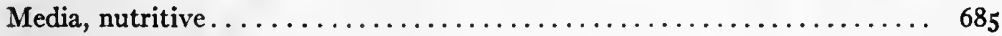

Method of Abderhalden............................. 5 10

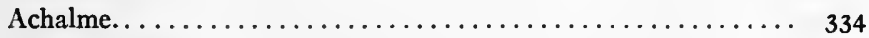

Blum and Fuld $\ldots \ldots \ldots \ldots \ldots \ldots \ldots \ldots \ldots \ldots \ldots \ldots \ldots \ldots \ldots \ldots \ldots$

Bordet and Gengou........................ 477

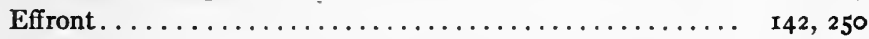

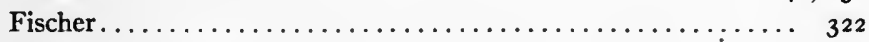

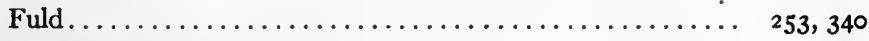

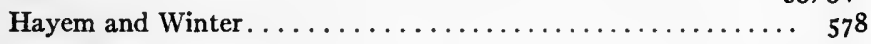

Hofmeister.......................... 214

Grützner........................... 250

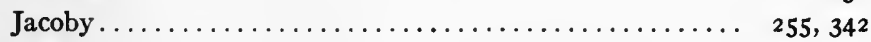

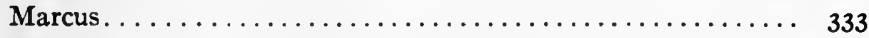

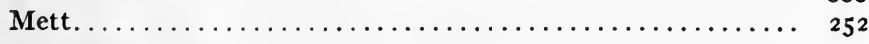

Morgenroth ..........................

Nencki and Zaleski. . . . . . . . . . . . . .

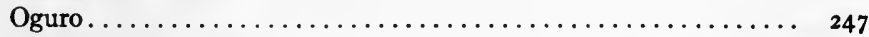

Sörensen........................... 322

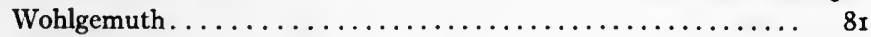

Zunz........................... 221, 245

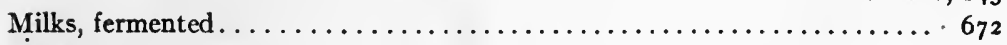

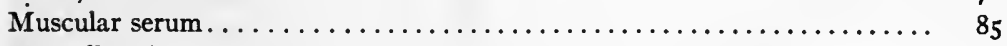

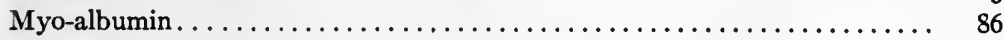

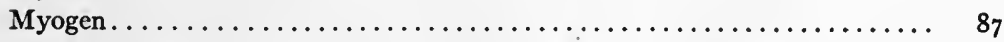

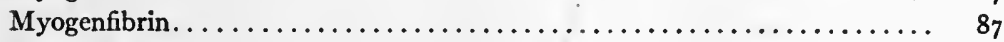

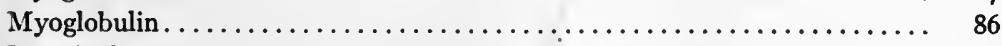

Myosin-ferment. . . . . . . . . . . . . . 
Myosin-fibrin

Myosinogen.

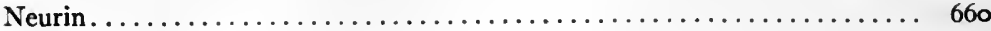

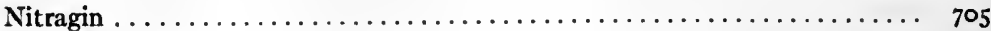

Nitrification. .............................. 690

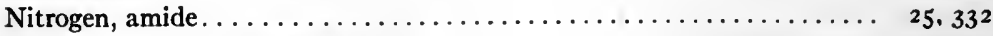

" (pepsin digestion) ................... 242

ammoniacal . . . . . . . . . $\ldots \ldots \ldots \quad 332,540,555,665,713$

formaldehyde................ $24,240,285,287,322$

peptides.............................. 494

rôle in breweries. .......................... $5_{588}$

" $"$ distilleries and yeast factories................ $6 \mathrm{r}_{2}$

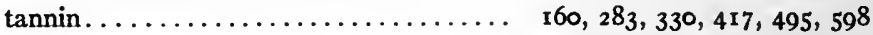

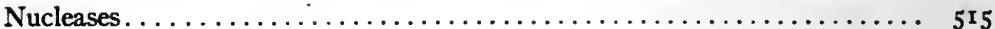

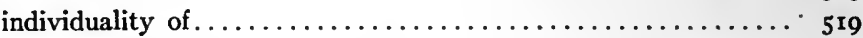

Nuclcins. . . . . . . . . . . . . . . . . . . . . . . . . . $5^{13}$

Nucleinase. . . . . . . . . . . . . . . . . . . . . . . . . 520

Nucleonosin. ................................ 520

Nucleoproteins. .............................. $5^{13}$

Nucleosidase. . . . . . . . . . . . . . . . . . . . . . . . . . 520

Nucleotidase. ................................ 520

Nutrients for yeast. . . . . . . . . . . . . . . . . . . . . 597

Nutrition (from the point of view of secretion) $\ldots \ldots \ldots \ldots \ldots \ldots \ldots$.

unconscious......................... 378

Ornithine. ................................. 320

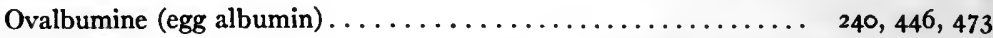

Oxproline ............................. 318

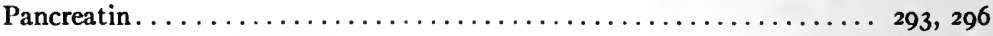

Papain, action of temperature . . . . . . . . . . . . . . 439

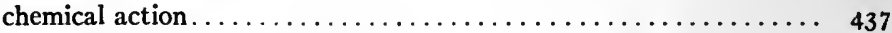

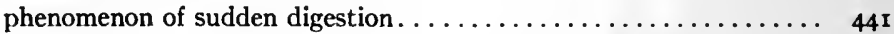

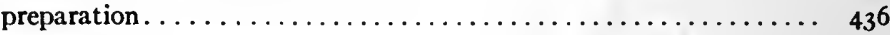

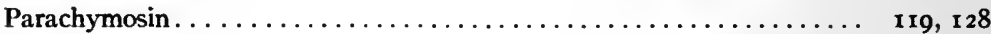

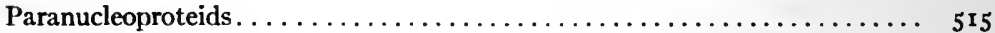

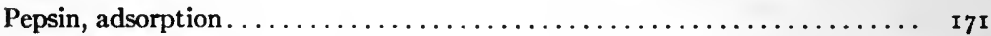

analysis. . . . . . . . . . . . . . . $249,5 \ldots \ldots \ldots \ldots$

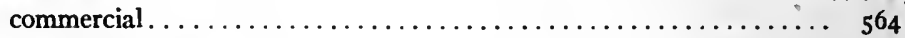

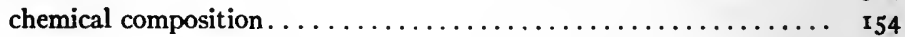

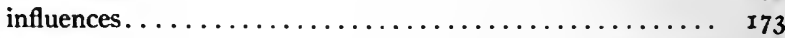

work. .................... 206,242

identity with rennet . . . . . . . . . . . . . . . . . I 29, 199

law of action $\ldots \ldots \ldots \ldots \ldots \ldots \ldots \ldots \ldots \ldots \ldots \ldots \ldots \ldots$

non-destruction during work . . . . . . . . . . . . . $\ldots \ldots 8$ 


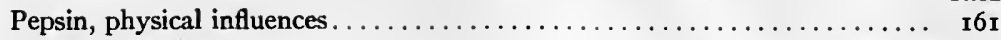

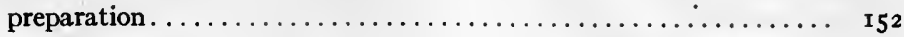

regeneration of $\ldots \ldots \ldots \ldots \ldots \ldots \ldots \ldots \ldots \ldots \ldots \ldots \ldots \ldots \ldots \ldots \ldots \ldots$

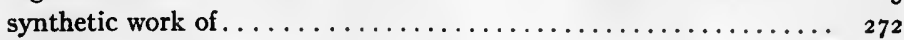

use in therapeutics. . . . . . . . . . . . . . . . . ${ }_{569}$

Peptase. . . . . . . . . . . . . . . .

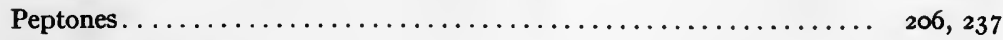

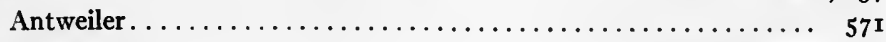

Chapoteaut. ..................... 386, 571

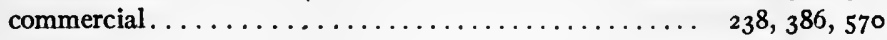

Effront..................... 417, 724

Kemmerich....................... 57 I

Laroche (silk) ..................... 504, 57 I

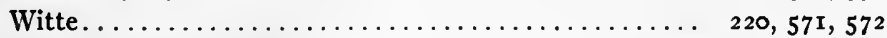

Peptonization (method with edestine) $\ldots \ldots \ldots \ldots \ldots \ldots \ldots \ldots \ldots \ldots \ldots$

Petroleum, origin . . . . . . . . . . . . . . . . . . . . . . . 648

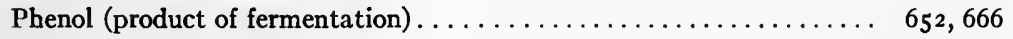

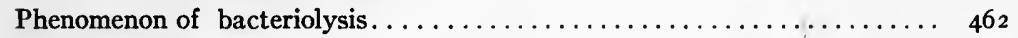

hemolysis. ...................... 462

Phenylalanin $\ldots \ldots \ldots \ldots \ldots \ldots \ldots \ldots \ldots \ldots \ldots \ldots \ldots \ldots \ldots \ldots \ldots$

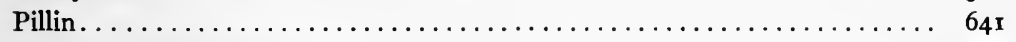

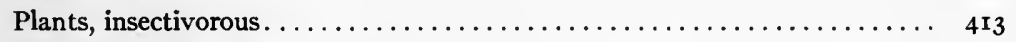

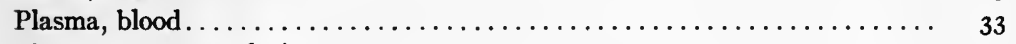

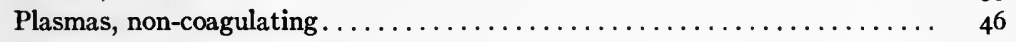

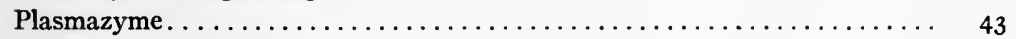

Plates, blood ............................. 4I, 50

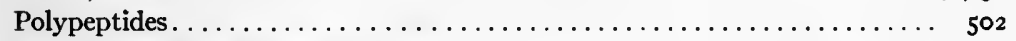

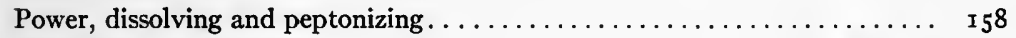

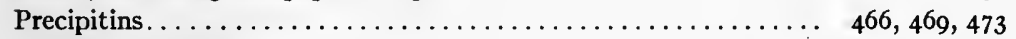

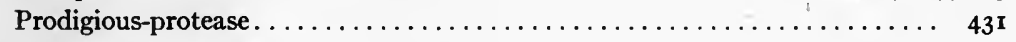

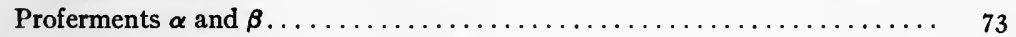

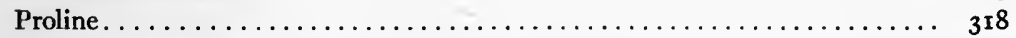

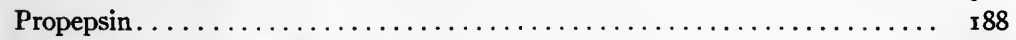

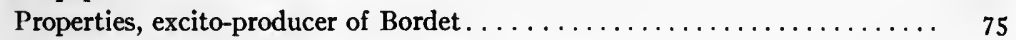

Prorennet. ............................... II3

Proteases, actino-protease. . . . . . . . . . . . . . . . . . . . . 406

amcbo-diastase........................ 406

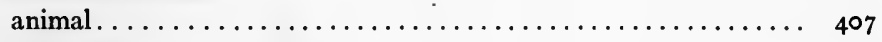

antiproteases. ......................... 433

microbic......................... 429, 62

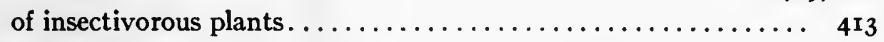

of molds. ............................ $4{ }^{14}$

of yeasts......................... 4I 5,420

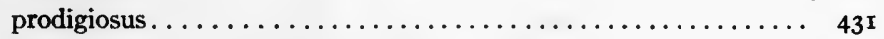

pyocyaneus.......................... 43 I

vegetable............................... 408 


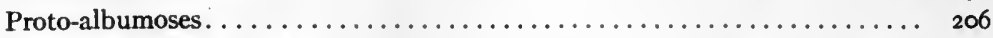

Psychic effect in nutrition.......................... 378

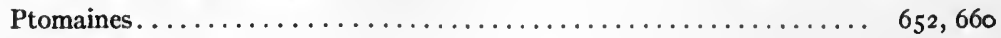

Ptyalin.................................... 186, 377

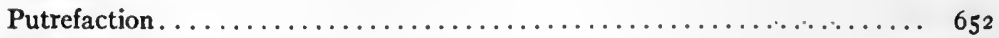

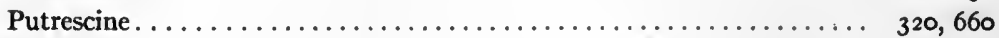

Putridine............................................. 660

Pyocyaneus-protease................................ 431

Pyrrolic derivatives.......................... 318,660

Reaction, Danilewsky ............................ ${ }^{274}$

Danysz.................................. 434

of tryptophane............................... 299

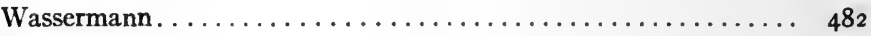

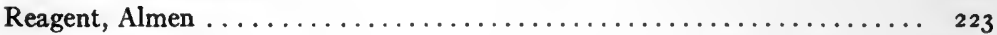

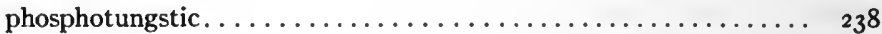

tannin............................... 160

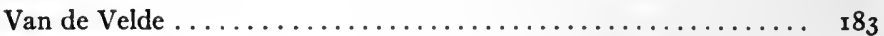

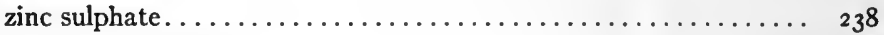

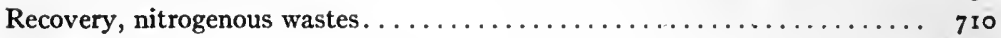

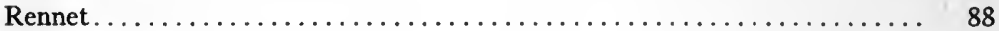

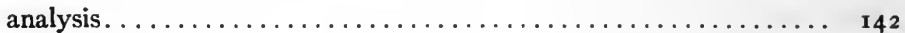

chemical influences........................... 100

chemical work ........................... 130

identity of different rennets.................... 123

identity with pepsin (see Pepsin)

law of action........................ II

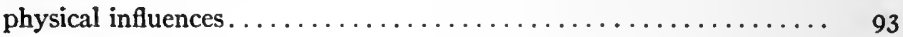

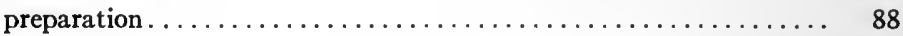

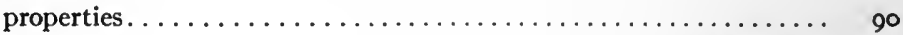

Ricin........................... 164, 248, 256, 342

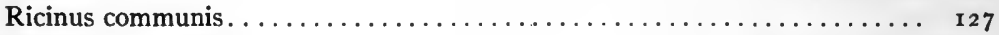

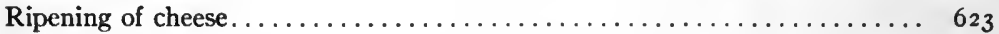

Russula delica............................... 299, 437

negricans............................. 299

Saliva................................. 362, 363, 398

Sarcylase. .................................... 519

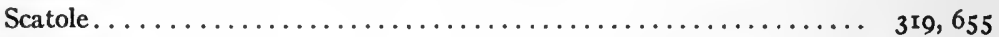

Secretin. . . . . . . . . . .

Secretion of enzymes..................... 185, 186, 313,541

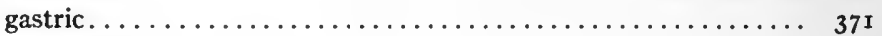

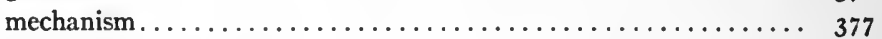

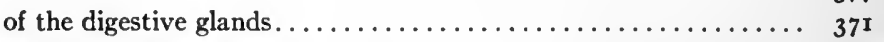

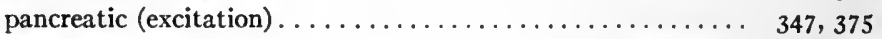

work of Pawlow......................... 372,719 
Serin

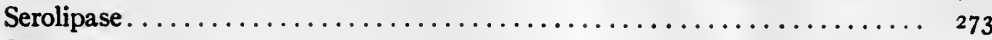

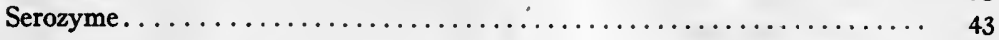

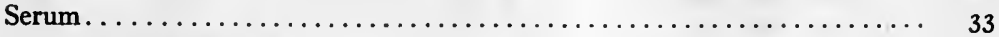

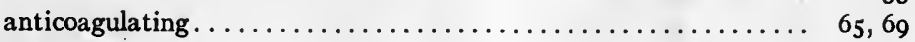

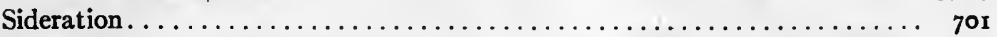

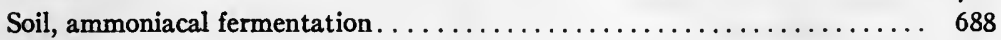

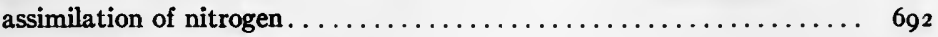

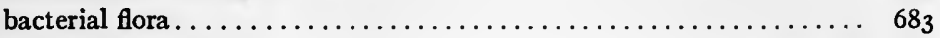

chemical work ................................ $68_{4}$

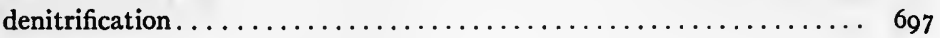

nitrification. . . . . . . . . .

Starch, influence on secretion. . . . . . . . . . . . . . . . . . . 389

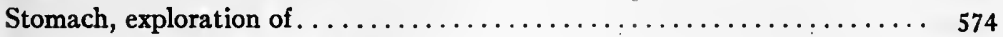

Substances, anticoagulating. $\ldots \ldots \ldots \ldots \ldots \ldots \ldots \ldots \ldots \ldots \ldots \ldots \ldots, 6_{7}$

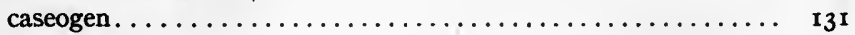

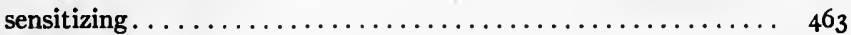

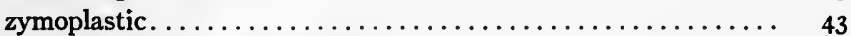

Sulphur-containing products of putrefaction $\ldots \ldots \ldots \ldots \ldots \ldots \ldots \ldots, 66 \mathrm{r}$

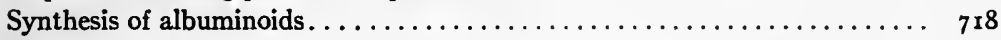

Syntonin.............................. 207, 208

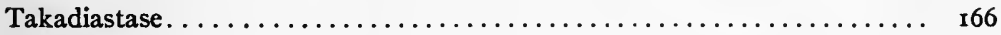

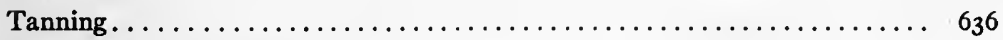

Theory of overproduction $\ldots \ldots \ldots \ldots \ldots \ldots \ldots \ldots \ldots \ldots \ldots \ldots \ldots \ldots, 474$

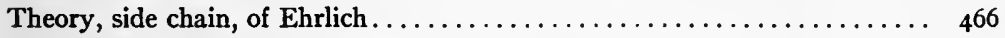

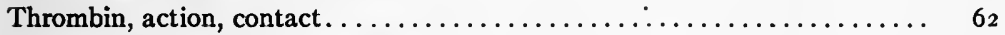

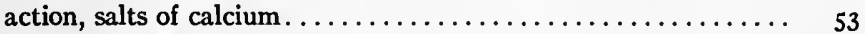

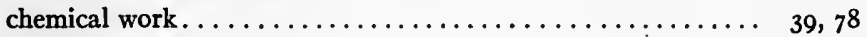

measurement................... 8 r

origin $. \ldots \ldots \ldots \ldots \ldots \ldots \ldots \ldots \ldots \ldots \ldots \ldots \ldots \ldots \ldots \ldots \ldots \ldots \ldots \ldots, 48$

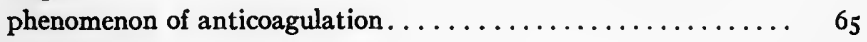

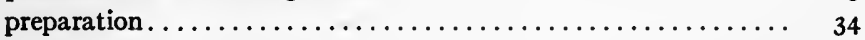

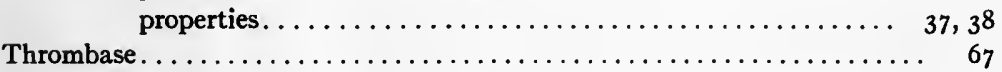

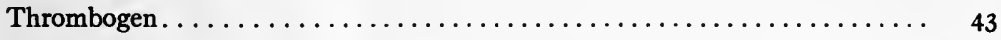

Thrombokinase. .................. 43, 51, 79

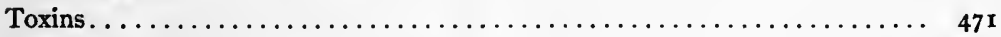

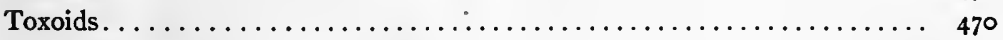

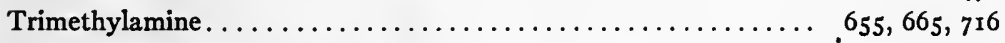

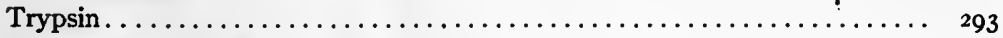

Trypsin, action of antiseptics.......................... 309

" " temperature ......................... 300

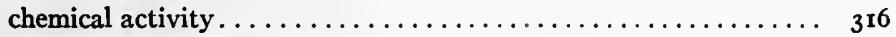

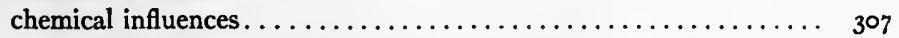

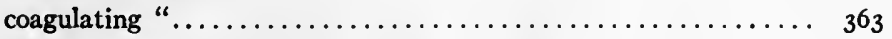

law of action. . . . . . . . . . . 
Trypsin, lipolytic action . PAGE

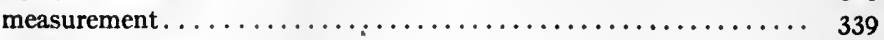

physical influences.......................... 300

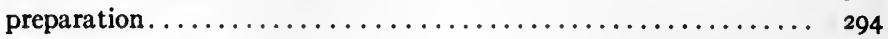

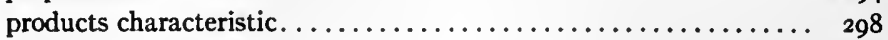

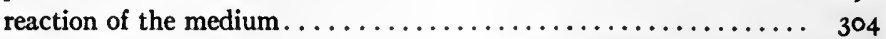

reversible action. ..................... 275, 330

Trypsins, pancreatic. .............................. ${ }^{293}$

Tryptophane. . . . . . . . . . . . .

Tyrosinase ..................................... ${ }^{298}$

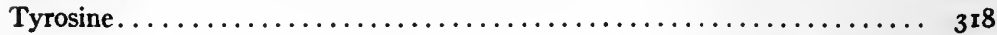

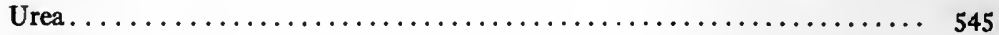

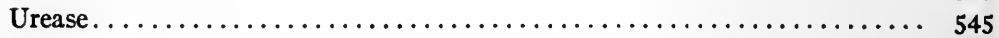

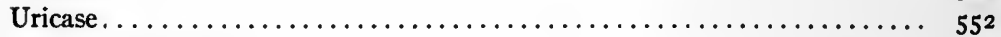

Uricolytic ferments. . . . . . . . . . . . . .

Venins............................... 47 r

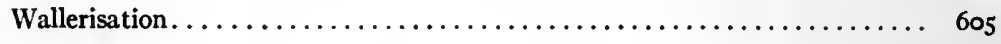

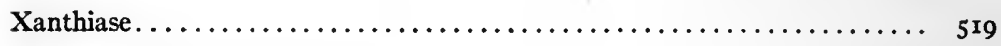

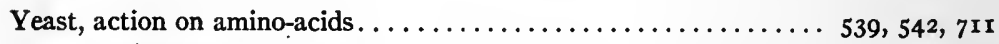

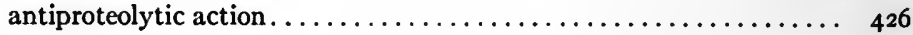

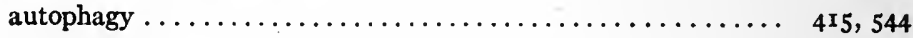

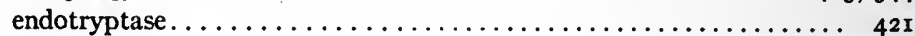

extracts of . . . .

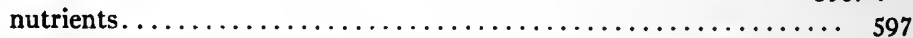

pressed................................... 616

proteolytic enzymes.......................... 415

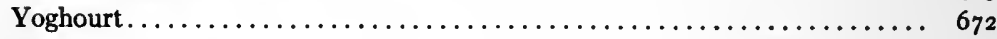

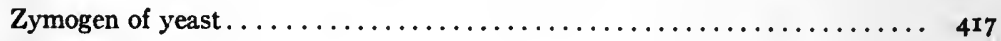

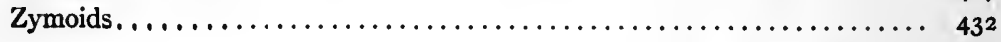







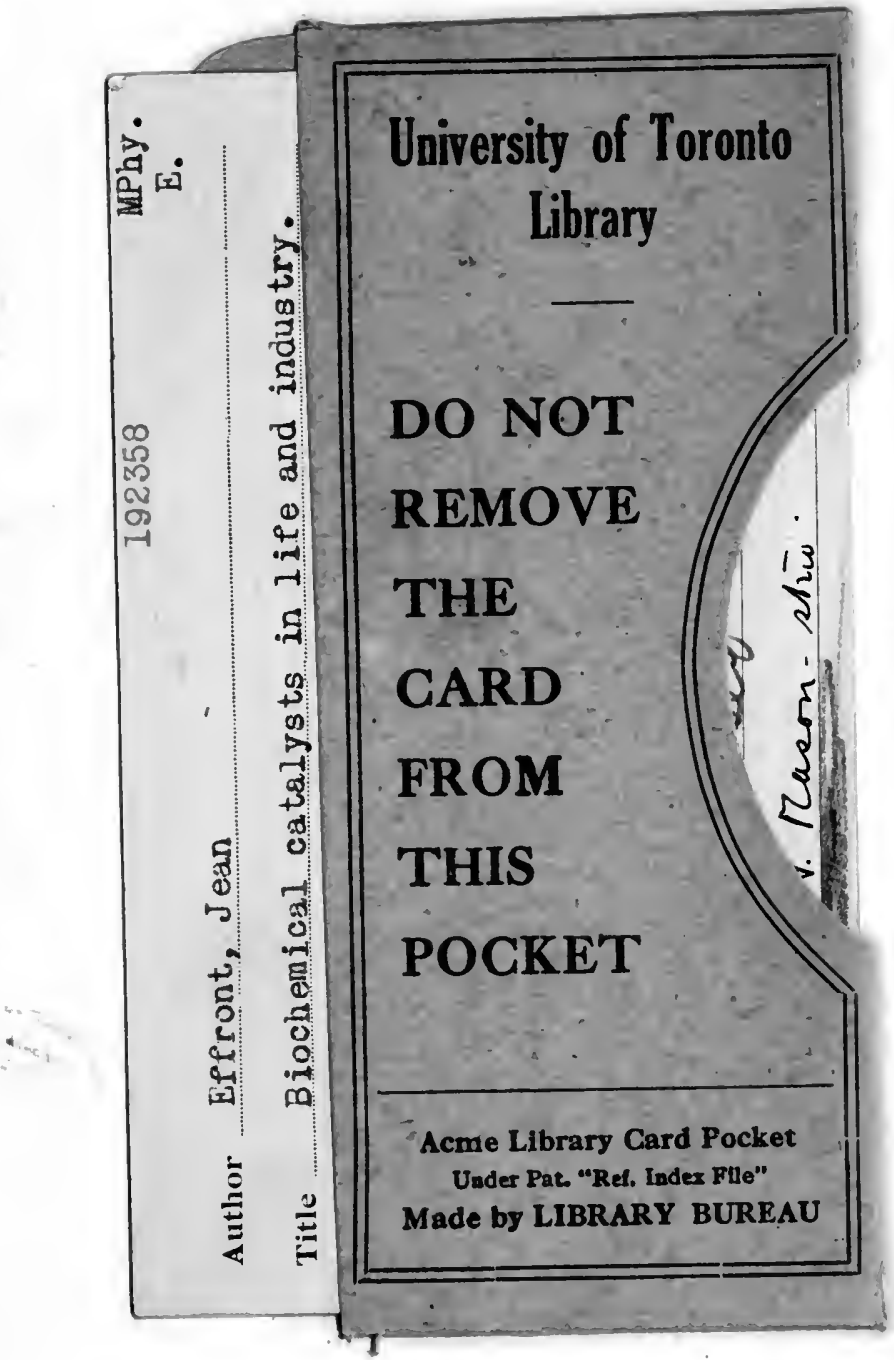


